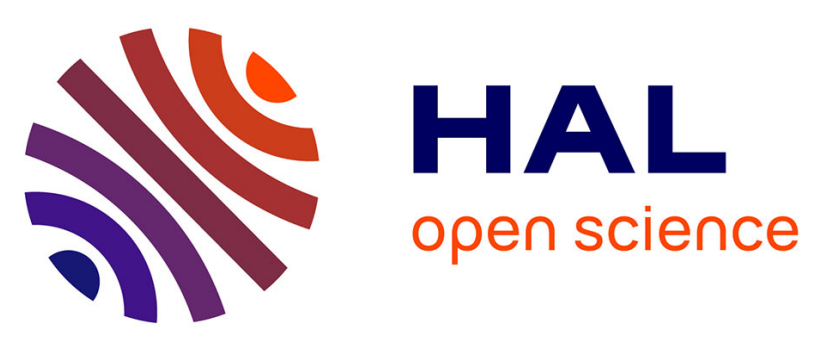

\title{
Towards first principles prediction of pKa: COSMO-RS and the cluster-continuum approach
}

\author{
Frank Eckert, Michael Diedenhofen, Andreas Klamt
}

\section{To cite this version:}

Frank Eckert, Michael Diedenhofen, Andreas Klamt. Towards first principles prediction of pKa: COSMO-RS and the cluster-continuum approach. Molecular Physics, 2010, 108 (03-04), pp.229-241. 10.1080/00268970903313667 . hal-00580701

\section{HAL Id: hal-00580701 https://hal.science/hal-00580701}

Submitted on 29 Mar 2011

HAL is a multi-disciplinary open access archive for the deposit and dissemination of scientific research documents, whether they are published or not. The documents may come from teaching and research institutions in France or abroad, or from public or private research centers.
L'archive ouverte pluridisciplinaire HAL, est destinée au dépôt et à la diffusion de documents scientifiques de niveau recherche, publiés ou non, émanant des établissements d'enseignement et de recherche français ou étrangers, des laboratoires publics ou privés. 


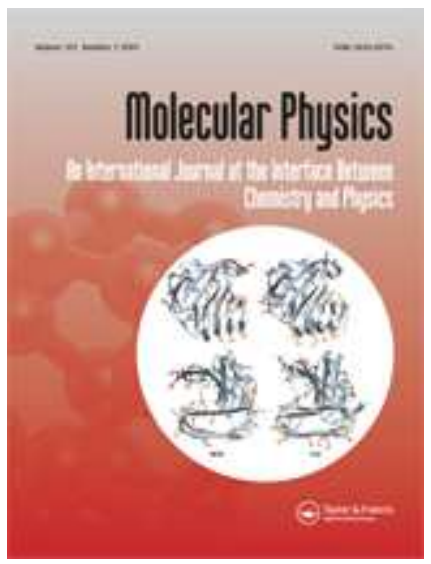

\section{Towards first principles prediction of pKa: COSMO-RS and the cluster-continuum approach}

\begin{tabular}{|c|c|}
\hline Journal: & Molecular Physics \\
\hline Manuscript ID: & TMPH-2009-0227.R1 \\
\hline Manuscript Type: & Special Issue Paper - In honour of Prof Werner 60th birthday \\
\hline $\begin{array}{r}\text { Date Submitted by the } \\
\text { Author: }\end{array}$ & 04-Sep-2009 \\
\hline Complete List of Authors: & $\begin{array}{l}\text { Eckert, Frank; COSMOlogic GmbH \& Co KG } \\
\text { Diedenhofen, Michael; COSMOlogic GmbH \& Co KG } \\
\text { Klamt, Andreas; COSMOlogic GmbH \& Co KG; University of } \\
\text { Regensburg, Institute of Physical and Theoretical Chemistry }\end{array}$ \\
\hline Keywords: & $\begin{array}{l}\text { pKa, COSMO, COSMO-RS, explicit solvation, cluster-continuum } \\
\text { model }\end{array}$ \\
\hline
\end{tabular}

\section{(5) ScholaroNE \\ Manuscript Central}


1

2

3

4

5

6

7

8

9

10

\title{
Towards first principles prediction of $\mathrm{p} K_{\mathrm{a}}$ :
}

\section{COSMO-RS and the cluster-continuum approach}

\author{
Frank Eckert ${ }^{a^{*}}$, Michael Diedenhofen ${ }^{a}$, Andreas Klamt ${ }^{a, b}$ \\ ${ }^{a}$ COSMOlogic GmbH \& Co KG, Burscheider Str. 515, D-51381 Leverkusen, Germany \\ ${ }^{b}$ University of Regensburg, Institute of Physical and Theoretical Chemistry, 93040 Regensburg, \\ Germany \\ *Corresponding Author. Email: eckert@ cosmologic.de. Phone: +492171731680. Fax: +492171731689.
}

\begin{abstract}
:
The COSMO-RS method, a post quantum chemistry extension of the quantum chemical dielectric continuum solvation model COSMO with a statistical thermodynamics treatment for realistic solvation simulation, has been applied to the prediction of aqueous $\mathrm{p} K_{\mathrm{a}}$ of acids and bases. The combination of the COSMO-RS approach to $\mathrm{p} K_{\mathrm{a}}$ prediction with the cluster-continuum approach (explicit solvation of the solute compound with one ore more solvent molecules) was used on three data sets consisting of 94 acids and 75 bases. Correlation of the calculated free energies of dissociation in water with the experimental aqueous $\mathrm{p} K_{\mathrm{a}}$ of the solute acids and bases in their bare state and explicitly solvated by one or two solvent molecules showed an increase of the regression slope with the number of explicit solvent molecules thus showing a regression slope that is closer to the theoretical value than the slope found for bare solutes. It was found that the cluster-continuum approach is limited to a $\mathrm{p} K_{\mathrm{a}}$ range of strong to moderately weak acids and bases, because the optimizations of the solvent-solute complexes of the ionic species of very weak acids (such as the anion of tert-butanol) did not lead to the desired complexes but yielded dissociation products.
\end{abstract}


Keywords: $\mathrm{p} K_{\mathrm{a}}$; acidity; basicity; COSMO; COSMO-RS; explicit solvation; cluster-continuum model;

\section{Introduction}

The transfer of protons is one of the central processes in biology, organic, and inorganic chemistry. Consequently, the detailed analysis of the thermodynamics of the proton transfer reaction and the prediction of dissociation constants of acids and bases in different solvents have found substantial interest in many areas of chemistry and biochemistry. Experimental measurement of aqueous phase $\mathrm{p} K_{\mathrm{a}}$ values nowadays has become an inexpensive standard application ${ }^{1}$. However, there are broad classes of chemicals that are not readily amenable to experimental characterization (e.g. reaction intermediates, very strong and very weak acids or bases with a $\mathrm{p} K_{\mathrm{a}}$ outside the $\mathrm{p} K_{\mathrm{a}}$ range that can be conveniently measured). Therefore, considerable and ongoing effort has been devoted to the development of first principle prediction methods for $\mathrm{p} K_{\mathrm{a}}$ values. The computational prediction of high quality gas phase dissociations constants has become feasible with high level (post-Hartree-Fock) ab initio quantum chemistry methods, which nowadays can be as good or even more accurate than the experimentally challenging measurements of this property ${ }^{2-6}$. In the liquid phase, which is of much larger practical importance, the situation seems reversed: at least for solvent water there is a large number of reliable experimental dissociation constant data available, but the theoretical prediction from first principles is extremely challenging because of the strong solvation effects of the neutral and ionic species involved in the proton transfer reaction. Thus the prediction of dissociation constants can be seen as the ultimate challenge for quantum chemistry (QC) based solvation models, where the general goal is to improve the reliability of the computational approach so as to have a realistic comparison with the available experimental data. A large number of attempts in this direction have been reported, but a consistent and generally applicable method is still lacking. Nevertheless, the rapid development of efficient QC methods in recent years has opened new perspectives for the modeling of acid-base equilibria in solution 
and the rigorous prediction of the associated $\mathrm{p} K_{\mathrm{a}}$ values in the liquid. The effect of the solvent on the proton transfer reaction can be included in quantum chemical computations in two ways: either the solvent molecules are accounted for explicitly, as in the molecular dynamics (MD) simulation framework ${ }^{7}$, or implicitly, using a continuum solvation model. Due to its considerable computational cost, the MD based explicit solvation approach yet remained restricted to a limited number of small systems. Of the different implicit QC methodologies available for the computation of $\mathrm{p} K_{\mathrm{a}}$ values the Dielectric Continuum Solvation Methods (DCSMs) have become most popular ${ }^{9}$, because they are able to describe long range electrostatic interactions of solutes with a solvent at moderate computational cost in the context of quantum chemical programs ${ }^{10-17}$. Thus QC based DCSM methods may help to shed some light on the particulars of the relationship between molecular structure and $\mathrm{p} K_{\mathrm{a}}$ value. In DCSMs the interactions of a solute molecule with the solvent are treated by means of putting the solute in a cavity that is surrounded by a dielectric continuum, which represents the solvent. Solvent-related properties like the size and shape of the cavity are treated as adjustable parameters and obtained from physically motivated modeling procedures. DCSMs are distinguished by the construction of the cavity and how the solute-solvent interactions are treated. Despite the well known deficiencies of DCSM methods, (i.e. the neglect of hydrogen bonding and the inadequate treatment of the short range electrostatics ${ }^{10,18-21}$, which can be much stronger in ions than in neutrals and thus can introduce a large asymmetry to the solvation energy of an acid compared to its conjugate base) it is possible to correlate the quantum chemical dissociation free energy of a solvated molecule $\Delta G_{\text {diss }}$ with its $\mathrm{p} K_{\mathrm{a}}$ via a linear free energy relationship (LFER) ${ }^{10}$ :

$$
\mathrm{p} K_{\mathrm{a}}=c_{1} \Delta G_{\mathrm{diss}}+c_{2}
$$

From the basic thermodynamics $c_{1}$ is expected to be unity if $\Delta G_{\text {diss }}$ would be calculated without a systematic error and the LFER axis intercept $c_{2}$ is expected to be equal to $-\log [\text { Solvent }]^{22}$. Looking in detail into the DCSM studies, ${ }^{10-17}$ in the regression of $\mathrm{p} K_{\mathrm{a}}$ values versus the calculated dissociation free energy $\Delta G_{\text {diss }}$ the studies report slopes that are significantly lower than the theoretically expected value 


\section{Molecular Physics}

of $1 / R T \ln (10)$, which was first noted and discussed by Klamt et al. ${ }^{10}$. Such a behavior has been reported for aqueous ${ }^{10-12}$ and non-aqueous acids ${ }^{10,13-15,23}$ as well as for bases. ${ }^{16,17,24}$ This drawback is common to all simple DCSMs unless considerable effort is taken in the (often physically hardly justifiable) adjustment of numerous additional and often physically doubtful parameters of the DCSM. Atom type or hybridization specific cavity radii and cavity definitions that depend on the charge of the molecule are examples of such parameters ${ }^{25}$. Although such models became quite popular and successful applications for water and nonaqueous solvents have been reported ${ }^{10-17,22-27}$, it remains doubtful if the predictive power of such empirical adjustments persists for more complex chemically multifunctional solutes or for solutes such as free radicals, zwitterions or excited states ${ }^{23}$. In particular empirical models are very likely to fail if electron delocalization is important in the anions or cations involved in the proton exchange reaction ${ }^{8,28}$. Pliego and Riveros ${ }^{29}$ showed that the error of such DCSM methods in $\mathrm{p} K_{\mathrm{a}}$ predictions can become as large as $7 \mathrm{p} K_{\mathrm{a}}$ units when solutes with different functional groups are considered. They proposed that the neglect of specific solute-solvent interactions may cause this significant error ${ }^{29}$.

A promising approach to the $\mathrm{p} K_{\mathrm{a}}$ problem, which does not artificially modify the cavity to try to reproduce hydrogen bonding and short-range solute-solvent interaction behavior that is not accounted for by the DCSM, is the addition of explicit solvent molecules to the solute ions ${ }^{29-34}$, which in the context of $\mathrm{p} K_{\mathrm{a}}$ prediction by DCSM's has been reported in the literature as "Cluster-Continuum"29,30,33, "Supermolecule",32,34, or "Implicit-Explicit Solvation",31 approach. The solute and its according ionic form are represented by a cluster of the neutral and ionic solute molecule with one or more surrounding solvent molecules that form a partial or full solvation shell around the compound, accounting for local solute-solvent interactions such as Hydrogen Bonding (HB) and electrostatic charge-dipole interactions in a physical way. Compared to simple DCSMs, this approach appears to increase the slope of the aqueous $\mathrm{p} K_{\mathrm{a}}$ LFER with each added explicit water ${ }^{29-34}$, but its practical application leads to some ambiguities and problems: there is no natural choice of the number of solvent molecules that represent the solvent shell, retaining some level of arbitrariness involved, where a choice has to be taken. 
However, what in practice might turn out to be the much harder problem, is the optimization of the solute-solvent cluster. For complex, multifunctional solutes, as most chemically or biologically interesting drug-like compounds are, it is very difficult and computationally demanding to find the global minimum of the weakly bonded solute-solvent complex ${ }^{34}$. If the solvent itself is a complex multifunctional compound, or if a mixture of several solvent compounds is used, it easily may become impossible to find and optimize the global minimum of the cluster at all. To avoid the problematic optimization of weakly bound complexes Adam ${ }^{34}$ suggested to use only one or two explicit solvent moelcules only if there are strong hydrogen bonds between the solute and solvent, so that the resulting complex is stable in the gas phase and thus can be observed experimentally ${ }^{34}$. Unfortunately this criterion does not resolve the problem of getting the right number of explicit solvent molecules, nor does it seem to be applicable to complex multifunctional compounds or compounds with electron delocalization, where it might not be easy to decide if there is strong or weak bonding in the complex.

To be able to overcome the arbitrariness involved in the choice of the number of explicit solvent molecules added to the solute, Ding et al. ${ }^{32}$ proposed for each solute to pick from the set of bare, monoor di-solvated solutes that species, which minimizes the calculated total free energy of dissociation, thus introducing an additional local descriptor of the chemical nature of the solute-solvent interactions. They argue that the minimum dissociation energy species should be the compound with the highest thermodynamic stability in the framework of the quantum chemical dielectric continuum solvation model used. This approach reflects some known defects of DCSM's for protic and polar solvents, in particular the neglect of Hydrogen Bonding interactions between solute and solute, which are introduced when explicit solvent molecules are added. Thus for protic and polar solvents such as water or dimethylsulfoxide the addition of a solvent sphere may lead to improved correlation of $\Delta \mathrm{G}_{\text {diss }}$ with experimental $\mathrm{p} K_{\mathrm{a}}$, while solvents of low polarity are well represented by the DCSM on the bare solute compound. It must be noted, that while being physically plausible, this criterion is hardly applicable in 
practical predictions, because for any solute it involves the conformational search and optimization of bare solutes, mono-, di- and probably even higher solvated solute-solvent clusters.

A different criterion for the choice of the number of explicit solvent molecules was introduced by Pliego and Riveros ${ }^{29}$. In their reasoning the cluster with the number of explicit solvent molecules that minimizes the free energy of solvation of the anionic or cationic form of the solute acid or base, respectively, should lead to the best predictions of the property based on the cluster-continuum model, based on the assumption that for a rigid cluster, the complex with minimum free energy of solvation will be closest the real free energy of solvation of the ion in solution. They found that for aqueous $\mathrm{p} K_{\mathrm{a}}$ typically three explicit water molecules minimize the free energy of solvation of the anions or cations. Apart from the significant theoretical and practical problems that are raised by finding the global minimum of a cluster consisting of four weakly bonded compounds, the free energy of solvation of the ionic form only partly reflects the physics of the dissociation reaction, totally neglecting the solvation of the neutral solute and the influence of the quantum chemical method, both of which are accounted for by the criterion of Ding et al. $^{32}$.

Another set of rather heuristic criteria for explicit solvation of acids was proposed by Kelly et al. ${ }^{31}$. In combination with their post-DSCM method $\mathrm{SM}^{35}$, they proposed to add a single explicit water molecule to the anionic form of a solute acid only (semi-solvation), either if the anion consists of less than three atoms, or if the charge of the anionic form of the acid is localized on one or more oxygen atoms in a way that the oxygen(s) bear a more negative partial charge than the oxygen of the solvent water. Obviously, for both criteria a certain amount of arbitrariness remains. In particular, the quite arbitrary partition of the solute anions into species that form a strong hydrogen bond with water (the ones whose oxygens have a more negative partial charge than the water oxygen) and those who do not, is doubtful. If explicit solvation is trying to compensate the shortcomings of the DCSM, which does not account for Hydrogen Bonding interactions at all, the division into strong and weak hydrogen bonds does not seem useful. Neither does this separation make sense if one tries to account for shortcomings of 
the underlying quantum chemistry or DFT methods, which have or don't have the same problems for strong and weak Hydrogen Bonds.

The DCSM-based implicit/explicit $\mathrm{p} K_{\mathrm{a}}$ models already mentioned use the combination of a gas phase dissociation energy of the cluster compounds (the explicit solvation part) computed by a high-level QC method, with the cluster free energy of solvation ${ }^{36-38}$ computed by a DCSM model (the implicit solvation part), which connects the gas phase to the liquid state. These approaches all rely on explicit solvation to correct the shortcomings of the DCSM method. In this work we chose a different approach: the Conductor-like Screening Model for Real Solvents (COSMO-RS) ${ }^{18-21}$. COSMO-RS goes beyond the DCSM concept in that it combines the electrostatic advantages and the computational efficiency of the DCSM COSMO $^{39}$ with a statistical thermodynamics method for local interaction of surfaces, which takes into account local deviations from dielectric behavior as well as hydrogen bonding. In this approach all information about solutes and solvents is extracted from initial QC-COSMO calculations, and only very few parameters have been adjusted to experimental values of partition coefficients and vapor pressures of a wide range of neutral organic compounds. COSMO-RS is capable of predicting partition coefficients, vapor pressures, and solvation free energies of neutral compounds with a root mean square error (rmse) of $0.3 \log$-units and better and a lot of experience has been gathered during the past years about its surprising ability to predict mixture thermodynamics ${ }^{18-20}$. Although COSMO-RS could successfully be applied to the first principles prediction of aqueous acidity ${ }^{10}$ and basicity ${ }^{24}$ as well as acid and base $\mathrm{p} K_{\mathrm{a}}$ in non-aqueous solvents ${ }^{10,28}$, there remained some problematic aspects: most prominently, the correlation of COSMO-RS predicted free energy of dissociation with experimental $\mathrm{p} K_{\mathrm{a}}$ showed a slope that is lower than the one expected from thermodynamic theory ${ }^{10,24}$. I.e. COSMO-RS was not able to solve the slope problem of the underlying DCSM COSMO. In addition it was found that the systematic deviations known from DCSMs for aliphatic amine compounds were also present in COSMO-RS predictions of aqueous base $\mathrm{p} K_{\mathrm{a}}{ }^{24}$. This study tries to assess if the combination of the COSMO-RS method for predicting $\mathrm{p} K_{\mathrm{a}}$ with the explicit solvation approach is able to resolve these issues. In addition, the performance and stability of different criteria for the choice of the number of 
explicit solvent molecules is tested. We decided to perform this study on the data sets for aqueous acids and base $\mathrm{p} K_{\mathrm{a}}$ already published ${ }^{10,24}$. The standard COSMO-RS method implemented in the COSMOtherm program ${ }^{40}$ based on Turbomole DFT/COSMO calculations ${ }^{41-46}$ was used for this purpose.

\section{Theoretical calculations}

Our theoretical calculations of $\Delta G_{\text {diss }}$ of aqueous acids are based on the reaction model

$$
\mathrm{AH}+\mathrm{H}_{2} \mathrm{O} \rightarrow \mathrm{A}^{-}+\mathrm{H}_{3} \mathrm{O}^{+}
$$

The theoretical calculations of $\Delta G_{\text {diss }}$ of aqueous bases are based on the reaction model

$$
\mathrm{B}+\mathrm{H}_{3} \mathrm{O}^{+} \rightarrow \mathrm{BH}^{+}+\mathrm{H}_{2} \mathrm{O}
$$

Unlike the DCSM methods mentioned, COSMO-RS is able to directly calculate the free energy of each species in aqueous solution. Thus no gas phase energies were computed. We applied our standard procedure for COSMO-RS calculations to all four species appearing in eqs. 2 and 3, which consists of two steps:

1) Full DFT geometry optimization with the Turbomole program package ${ }^{43}$ using B-P density functional ${ }^{44,45}$ with TZVP quality basis set using the RI approximation. ${ }^{46}$ During these calculations the COSMO continuum solvation model was applied in the conductor limit $(\varepsilon=\infty)$. Optimized elementspecific radii from the COSMO-RS parameterizations ${ }^{19,20}$ have been used for the COSMO cavity construction. Such calculations end up with the self-consistent state of the solute in the presence of a virtual conductor that surrounds the solute outside the cavity.

2) COSMO-RS calculations have been done using the COSMOtherm program ${ }^{36}$. In these calculations the deviations of the real solvent, in this case water, compared to an ideal conductor are taken into account in a model of pair-wise interacting molecular surfaces. For this purpose, electrostatic energy differences and hydrogen bonding energies are quantified as functions of the local COSMO polarization 
charge densities $\sigma$ and $\sigma^{\prime}$ of the two interacting surface pieces. The chemical potential differences arising from these interactions are evaluated using an exact statistical thermodynamics algorithm for independently pair-wise interacting surfaces, which is implemented in COSMOtherm. More detailed descriptions of the COSMO-RS method are given elsewhere ${ }^{18-21}$.

If more than one cluster conformation or different deprotonation or protonation sites were considered to be potentially relevant for the neutral, anionic, or cationic form of the acids AH and bases B, several conformations were calculated in step 1 and a thermodynamic Boltzmann average over the total Gibbs free energies of the conformers was consistently calculated by the COSMOtherm program in step 2. For this study the geometries of all molecules taken from refs. 10 and 24 have been re-optimized on BP/TZVP/COSMO level. In addition all compounds have been carefully rechecked for possible conformations, and if necessary, additional conformations have been added to the COSMOtherm calculations in step 2 .

To avoid the human bias and ambiguity involved in the construction of the clusters of the solute with one or two explicit water molecules, their geometries were created with a novel COSMO-RS based procedure: starting from the optimized BP/TZVP/COSMO geometries of the bare solute molecule and the water molecule, the pair-interaction energies of all possible contacts of the cavities of the two molecules are scanned and the minimum energy contact, which for both molecules is the site where the contact probability between the molecule is largest and thus the site where a cluster is most likely to be formed, will be identified. Along the bond defined by this contact site, the solvent molecule is arranged at different torsional angles between $0^{\circ}$ and $360^{\circ}$, in steps of $45^{\circ}$ and the resulting cluster geometries were optimized independently at BP/TZVP/COSMO level of theory. Of the cluster conformer thus found, the minimum energy cluster is used in the COSMO-RS computation of step 2. The clusters with two explicit water molecules are created the same way, using the mono-solvated solute cluster as starting structure instead of the bare solute. This procedure is applicable in the same manner to neutral and ionic species, and to any solvent or solute (which itself can be a cluster). Thus it can be automated quite 


\section{Molecular Physics}

easily. Although the cluster generation procedure via COSMO-RS created cluster geometries is not a global optimization procedure and thus can not guarantee that the optimized clusters are the absolute minimum energy structures of the phase space spanned by the atomic coordinates of the complexes, it provides a reliable, consistent - and what is even more important for practical application: simple, automatic, and unbiased - procedure for finding cluster geometries that are very likely to be the best (and minimum energy) structures in the framework of the QC/COSMO-RS approach used. For a small subset of the clusters, a global approach to finding the minimum energy conformations of the clusters was tested: $a b$ initio MD simulated annealing of the geometries on BP/TZVP/COSMO level of theory, as provided by Turbomole ${ }^{43}$. For the compounds tested (tert-butanol, oxalic acid, indole, melamine) the MD procedure was not able to find lower energy cluster conformations than the COSMO-RS contact probability based method, which is strong indicator for the soundness of the COSMO-RS provided geometries. The optimized geometries of all the acids, bases, ions and explicitly solvated clustercompounds are given in the Supplementary Material section of this journal.

For all acids AH, the Gibbs free energy of dissociation $\left(\Delta G_{\text {diss }}\right)$ has been calculated as the difference of the total free energy of the anion $\mathrm{A}^{-}$and the neutral acid $\mathrm{AH}$. The free energy difference of $\mathrm{H}_{3} \mathrm{O}^{+}$and $\mathrm{H}_{2} \mathrm{O}$ is a constant additional contribution:

$$
\Delta G_{\text {diss }}=G_{\text {tot }}\left(A^{-}\right)-G_{t o t}(A H)+\left[G_{t o t}\left(H_{3} O^{+}\right)-G_{t o t}\left(H_{2} O\right)\right]
$$

For all bases $B$, the Gibbs free energy of dissociation $\left(\Delta \mathrm{G}_{\mathrm{diss}}\right)$ has been calculated as the difference of the total free energy of the protonated cation $\mathrm{BH}^{+}$and the base $\mathrm{B}$. To this free energy difference the free energy difference of of $\mathrm{H}_{3} \mathrm{O}^{+}$and $\mathrm{H}_{2} \mathrm{O}$ is a constant additional contribution:

$$
\Delta G_{d i s s}=G_{t o t}(B)-G_{t o t}\left(B H^{+}\right)+\left[G_{t o t}\left(H_{3} O^{+}\right)-G_{t o t}\left(H_{2} O\right)\right]
$$

From the calculation procedure described above, we get $G_{\text {tot }}\left(\mathrm{H}_{3} \mathrm{O}^{+}\right)-G_{\mathrm{tot}}\left(\mathrm{H}_{2} \mathrm{O}\right)=1124.47 \mathrm{~kJ} / \mathrm{mol}$. This value is in good agreement with literature estimates ${ }^{23}$. Zero point vibrational energies are not taken into account. Consequently, the geometries optimized in step 1 were not analyzed for the nature of the stationary point of the optimized geometry. We make the common assumption that the difference in zero 


\section{Page 11 of 461}

point energy between the neutral and the deprotonated acid or protonated base is small and systematic ${ }^{10}$.

1

2

3

4

5

6

7

8

9

10
All regressions of the dissociation free energy vs. experimental $\mathrm{pKa}$ have been cross-validated by a "leave-one-out" cross-validation procedure. The cross-validated regression coefficients $g^{2}$ are given together with the regression coefficients $r^{2}$

\section{Aqueous Acids}

For the purpose of finding the LFER coefficients of eq. 1, a data set of 94 acids in solvent water was used. The data set consists of the reference data set of 5 alcohols, 23 carboxylic acids, 8 inorganic acids, 16 phenols, and 12 heterocyclic compounds as reported in ref. 10. A set of 30 multifunctional substituted benzoic acids ${ }^{51}$ has been added to the initial data set, to be able to asses the performance of the explicit solvation on more challenging solutes. The acids experimental $\mathrm{p} K_{\mathrm{a}}$ values range from 0 to 19. No compound has been removed from the data set for any reason during the study.

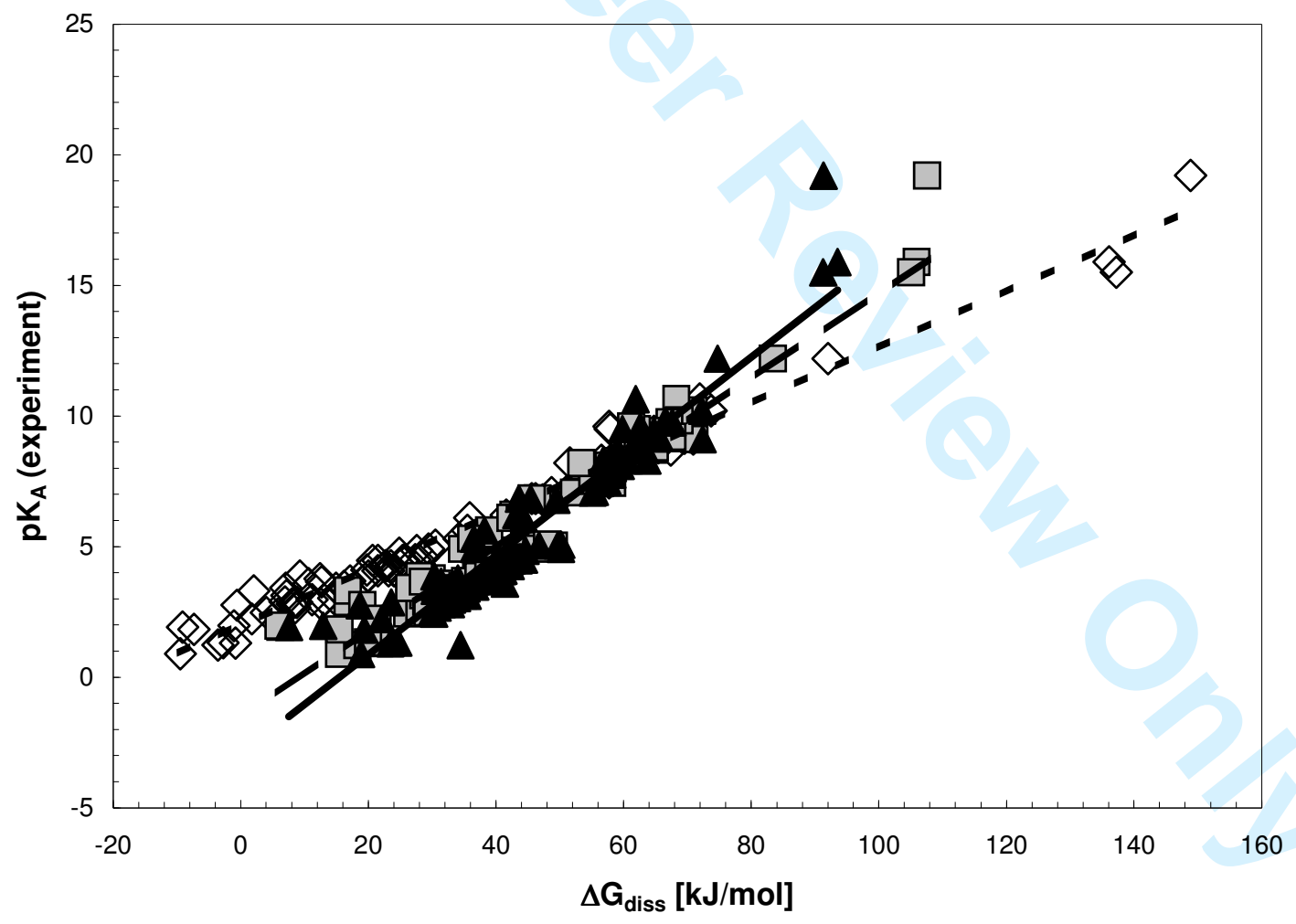

Formatted

Formatted

Deleted: $q$ 
FIGURE 1: Calculated Gibbs free energy of dissociation vs. experimental acids $\mathrm{p} K_{\mathrm{a}}$ in solvent water. Open rhombus: bare acids and anions. Dotted line: regression line (eq. 4) for bare acids $\left(r^{2}=0.978, q^{2}=0.976, c_{1}=0.107, c_{2}=1.94\right)$. Filled squares: acids and anions with one explicit water molecule. Striped line: regression line (eq. 5) for monosolvated compounds $\left(r^{2}=0.935, \underline{q^{2}=0.932}, c_{1}=0.164, c_{2}=-1.56\right)$. Filled triangles: acids and anions with two explicit water molecules, respectively. Solid line: regression line (eq. 6) for $d i$-solvated compounds $\left(r^{2}=0.899, \underline{q^{2}=0.893}\right.$, $\left.c_{1}=\mathbf{0 . 1 9 0}, c_{2}=\mathbf{- 2 . 9 4}\right)$.

The results for all 94 acids are shown in Table 1 and Figure 1. The free energies of all individual species are given in the Supplementary Material section of this journal. The regression of the calculated aqueous Gibbs free energy of dissociation $\left(\Delta G_{\text {diss }}\right)$ vs. experimental $\mathrm{p} K_{\mathrm{a}}$ for the bare neutral and ionic compounds results in a correlation coefficient of $r^{2}=0.978\left(q^{2}=0.976\right)$. The regression equation for the bare acids $\mathrm{p} K_{\mathrm{a}}$ in aqueous solution reads

$\mathrm{p} K_{\mathrm{a}}=0.107( \pm 0.01) \Delta G_{\text {diss }}+1.94( \pm 0.1)$

If we would have omitted the free energy difference of $\mathrm{H}_{3} \mathrm{O}^{+}$and $\mathrm{H}_{2} \mathrm{O}$, which we calculate as

$-1124.47 \mathrm{~kJ} / \mathrm{mol}$, in the definition of $\Delta \mathrm{G}_{\mathrm{diss}}$ we would have got a regression constant of $\hat{c}_{2}=-118.75$. Including this energy difference, the calculated axis intercept of 1.9 is reasonably close to the theoretical value of $c_{2}=-\log \left[\mathrm{H}_{2} \mathrm{O}\right]=-1.74$. In accordance with previous work ${ }^{10}$ on aqueous acidity of bare compounds we found that the slope of the regression is lower than the theoretical value of $1 / \mathrm{R} \operatorname{Tn}(10)=$ 0.1759

The regression equation for the mono-solvated neutral and ionic compounds $\left(r^{2}=0.935, q^{2}=0.932\right)$ reads

$\mathrm{p} K_{\mathrm{a}}=0.164( \pm 0.01) \Delta G_{\text {diss }}-1.56( \pm 0.1)$

which omitting the free energy difference of $\mathrm{H}_{3} \mathrm{O}^{+}$and $\mathrm{H}_{2} \mathrm{O}$, yields a regression constant of $\hat{c}_{2}=-185.42$. Thus, in comparison with the bare compound regression, the slope of the mono-solvated solutes is closer to the theoretical value. Yet the slope remains to be somewhat smaller than the theoretical value. 
Moreover, the correlation of $\Delta \mathrm{G}_{\text {diss }}$ with the experimental $\mathrm{p} K_{\mathrm{a}}$ data is worse than for the bare compounds. If the explicit solvation is applied to the ionic form of the compound set only (semisolvation, as proposed by Kelly et $a l^{31}$ ) the slope of the regression (with $\left.r^{2}=0.929, q^{2}=0.926\right)$ ) decreases to $c_{1}=0.139$ and the regression constant is $c_{2}=27875.09$, which omitting the free energy difference of $\mathrm{H}_{3} \mathrm{O}^{+}$and $\mathrm{H}_{2} \mathrm{O}$, yields $\hat{c}_{2}=-317.98$. Thus the semi-solvation expectably yields to a regression that is somewhat in between the regression of the bare and the mono-solvated solutes.

The regression equation for the $d i$-solvated neutral and ionic compounds $\left(r^{2}=0.899, q^{2}=0.893\right)$ reads

$\mathrm{p} K_{\mathrm{a}}=0.190( \pm 0.01) \frac{\Delta G_{\text {diss }}}{R T \ln (10)}-2.94( \pm 0.1)$

which omitting the free energy difference of $\mathrm{H}_{3} \mathrm{O}^{+}$and $\mathrm{H}_{2} \mathrm{O}$, yields a regression constant of $\hat{c}_{2}=-216.54$. Both slope and regression constant are closer to the theoretical values for the $d i$-solvated compounds compared to the bare compounds or the mono-solvated compounds, the slope is even higher than the theoretical value. Again, the correlation of $\Delta \mathrm{G}_{\text {diss }}$ with the experimental $\mathrm{p} K_{\mathrm{a}}$ data for $d i$-solvated compounds is worse than for the mono-solvated and bare compounds.

These results corroborate the claims given in the literature ${ }^{29-34}$ that the explicit solvation approach is able to solve the slope problem ${ }^{10}$ of the quantum chemical DCSM's. However, they lead to new problems: First, even when quite careful choice of the minimum energy structure of the solvent-solute cluster is applied, the overall correlation deteriorates with increasing number of explicit solvent molecules. Second, even though the slope of the $\Delta \mathrm{G}_{\mathrm{diss}}$ vs. $\mathrm{p} K_{\mathrm{a}}$ correlations is closer to the theoretical slope for the mono- or di-solvated solutes, it is still somewhat different, and for the di-solvated solutes even overshoots the theoretical value. A convergence of the slope appears to be reached at $d i$-solvation, because the change in slope from mono- to di-solvation is much smaller than from bare solutes to monosolvation. 
If, following Ding et $a l^{32}$, we introduce the additional solute specific category of minimal $\Delta \mathrm{G}_{\text {diss }}$ in the choice of the cluster or bare solute for the correlation with $\mathrm{p} K_{\mathrm{a}}$, a look at the $\Delta \mathrm{G}_{\mathrm{diss}}$ values in Table 1 reveals an important trend: while for strong and medium acids with $\mathrm{p} K_{\mathrm{a}}<10$ the bare solute is preferred, weak acids show minimum dissociation free energy if they are solvated with one or, for very weak acids with $\mathrm{p} K_{\mathrm{a}}>12$, two explicit water molecules. When looking at dissociation energy as computed on the DCSM level only (i.e. BP/TZVP/COSMO without COSMO-RS), the doubly solvated solute is preferred throughout the entire $\mathrm{p} K_{\mathrm{a}}$ range. This implies that a major portion of the interaction effects that are missing in the DCSM already is taken into account by COSMO-RS, which however has problems with very weak acids. This flaw can be explained quite well in the framework of COSMO-RS theory: as yet, non-local long range electrostatic effects and strong hydrogen bonding are not explicitly taken into account by COSMO-RS ${ }^{47}$. Now, the dissociation of very weak acids leads to anions where the charge is very concentrated and often localized on only one atom. It is exactly this type of ions where the COSMO-RS neglect of non-local electrostatic effects and strong hydrogen bonding may lead to increased errors - and, in turn, explicit solvation captures some of these neglected interactions. Thus the combination of COSMO-RS computed free energies of dissociation with the approach of Ding et $a l^{32}$, does not seem to be very promising: the overall correlation of the minimum $\Delta \mathrm{G}_{\text {diss }}$ with $\mathrm{p} K_{\mathrm{a}}$ is worse than the one for the bare solutes $\left(r^{2}=0.915, q^{2}=0.912\right)$ and it leads to a slope that is significantly lower than the theoretical: $c_{1}=0.127$ and the regression constant is $c_{2}=1.54$, which omitting the free energy difference of $\mathrm{H}_{3} \mathrm{O}^{+}$and $\mathrm{H}_{2} \mathrm{O}$, yields $\hat{c}_{2}=-141.58$.
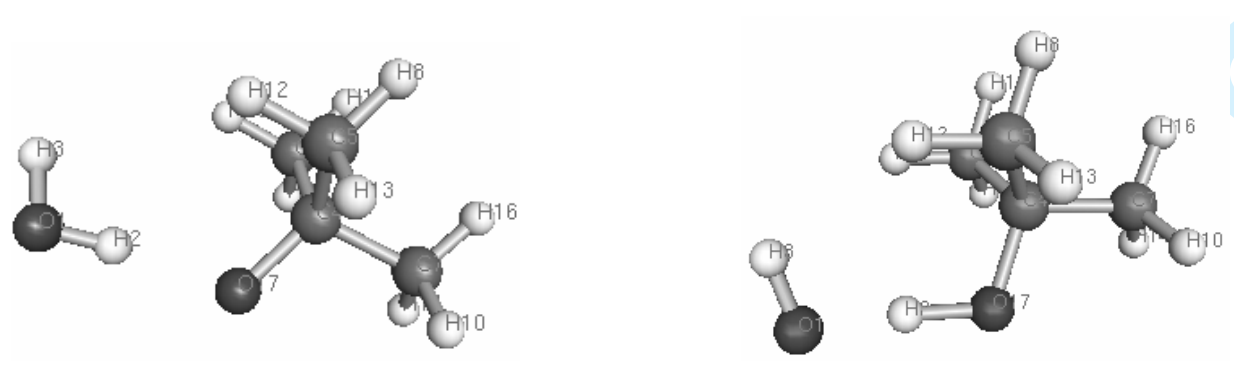
FIGURE 2: Optimized geometries for $\left(\mathrm{CH}_{3}\right)_{3} \mathrm{C}-\mathrm{O}^{(-) \ldots} \mathrm{H}-\mathrm{O}-\mathrm{H}$ and $\left(\mathrm{CH}_{3}\right)_{3} \mathrm{C}-\mathrm{OH}{ }^{\cdots(-)} \mathrm{O}-\mathrm{H}$.

Critical inspection of Table 1 and Figure 1 shows that there is one significant outlier in the regression of the mono- and di-solvated compounds, but not in the regression of the bare ions. It is tert-butanol, the weakest acid in the data set. A closer look at the optimized geometries of the explicitly solvated tertbutanol and tert-butoxy-anion revealed that the quantum chemical optimization of the tert-butoxy-anion - water and di-water clusters yielded an unexpected structure: Instead of the anion - water cluster $\left(\mathrm{CH}_{3}\right)_{3} \mathrm{C}-\mathrm{O}(-) \cdots \mathrm{H}-\mathrm{O}-\mathrm{H}$, a complex of tert-butanol with an hydroxy-anion $\left(\mathrm{CH}_{3}\right)_{3} \mathrm{C}-\mathrm{OH}{ }^{\cdots(-)} \mathrm{O}-\mathrm{H}$, or in the case of two explicit water molecules the anion $\left(\mathrm{CH}_{3}\right)_{3} \mathrm{C}-\mathrm{OH}^{\cdots \cdots}{ }^{\cdots-} \mathrm{O}-\mathrm{H}^{\cdots} \mathrm{H}-\mathrm{O}-\mathrm{H}$, was optimized (see Figure 2). The exact same artifact can also be observed in the data published by Kelly et al. ${ }^{31}$. A deeper investigation of the configurational space of the tert-butoxy-anion cluster showed that any free optimization of the tert-butoxy-anion - water cluster always yielded in a tert-butanol - hydroxy-anion cluster. A constrained optimization of the tert-butoxy-anion - water complex with the contacting hydrogen atom of the water molecule fixed at the equilibrium bond distance of the isolated water molecule with a subsequent potential energy surface scan along the $\mathrm{O}^{(-) \cdots} \mathrm{H}-\mathrm{O}-\mathrm{H}$ bond resulted in a minimum energy structure with the expected $\left(\mathrm{CH}_{3}\right)_{3} \mathrm{C}-\mathrm{O}^{(-) \ldots} \mathrm{H}-\mathrm{O}-\mathrm{H}$ geometry, which however was 73 $\mathrm{kJ} / \mathrm{mol}$ higher in energy than the $\left(\mathrm{CH}_{3}\right)_{3} \mathrm{C}-\mathrm{OH}^{\cdots}{ }^{\cdots-(-)} \mathrm{O}-\mathrm{H}$ structure and thus lead to an even worse regression with the experimental $\mathrm{p} K_{\mathrm{a}}$. Obviously, for the anions of very weak acids like tert-butanol, which have their negative charge sharply localized on one atom, the hydrogen-transfer of a hydrogen atom from the water to the anionic site leads to an energetically much favoured structure, compared to the hydrogen bonded anion - water cluster expected from the explicit solvation model - at least on the quantum chemical level of theory employed in this study. This implies that the cluster-continuum model can not be applied to very weak acids like tert-butanol. Thus the applicability range of the clustercontinuum model seems to be restricted to acids of medium strength, (again that is, for the quantum chemical BP//TZVP//COSMO level of theory used in this study). As a consequence, tert-butanol was 
removed from the regression. The new correlation for 93 acids leads to the following regression equations: The regression equation for the bare solutes $\left(r^{2}=0.975, q^{2}=0.973\right)$ reads

$\mathrm{p} K_{\mathrm{a}}=0.105( \pm 0.01) \Delta G_{\text {diss }}+1.99( \pm 0.1)$

which omitting the free energy difference of $\mathrm{H}_{3} \mathrm{O}^{+}$and $\mathrm{H}_{2} \mathrm{O}$, yields a regression constant of $\hat{c}_{2}=-116.40$.

The regression equation for the mono-solvated neutral and ionic compounds $\left(r^{2}=0.934, q^{2}=0.929\right)$ reads

$\mathrm{p} K_{\mathrm{a}}=0.157( \pm 0.01) \Delta G_{\text {diss }}-1.32( \pm 0.1)$

which omitting the free energy difference of $\mathrm{H}_{3} \mathrm{O}^{+}$and $\mathrm{H}_{2} \mathrm{O}$, yields a regression constant of $\hat{c}_{2}=-177.83$.

The regression equation for the $d i$-solvated neutral and ionic compounds $\left(r^{2}=0.907, q^{2}=0.901\right)$ reads

$\mathrm{p} K_{\mathrm{a}}=0.180( \pm 0.01) \Delta G_{\text {diss }}-2.56( \pm 0.1)$

which omitting the free energy difference of $\mathrm{H}_{3} \mathrm{O}^{+}$and $\mathrm{H}_{2} \mathrm{O}$, yields a regression constant of $\hat{c}_{2}=-205.20$.

Thus even if the problematic compound is removed from the data set, the general trends of decreasing correlation quality and non-theoretical slope with increasing number of explicit solvent molecules remained.

\section{Aqueous Bases}

For the purpose of finding the eq. 1 LFER correlations of the base protonation reaction, a data set of 43 bases was taken from ref. 24. Experimental base $\mathrm{p} K_{\mathrm{a}}$ values range between -5 and 14 . This set consists of 35 bases that are protonated at a nitrogen atom, namely five anilines, four amidines, six benzodiazepines, four guanidines, and 16 five- or six-ring heterocyclic compounds, and eight bases that 
are protonated at a carbon atom, namely four pyrroles and four indoles. For the pyrroles and indoles we

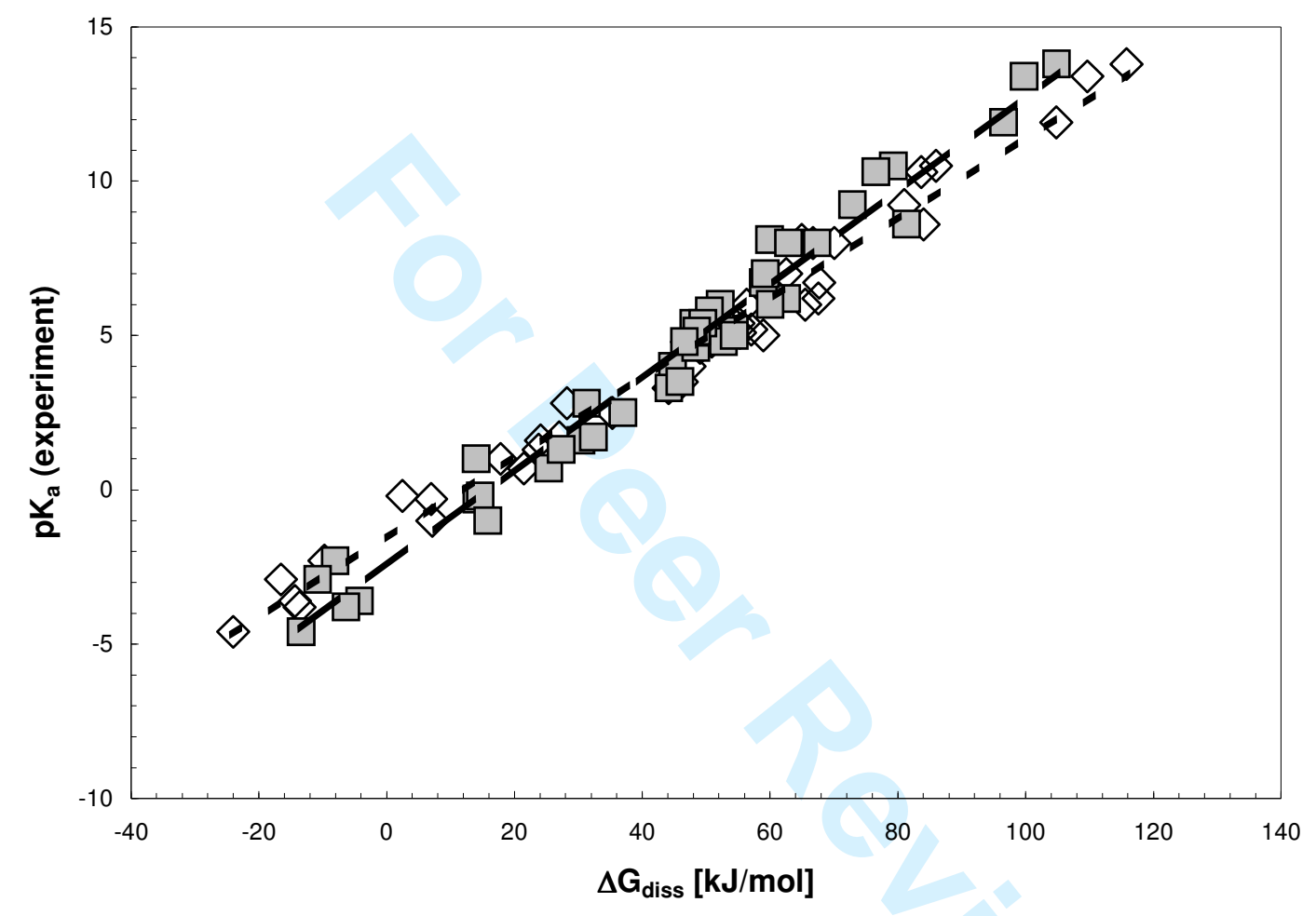

FIGURE 3: Calculated Gibbs free energy of dissociation vs. experimental bases $\mathrm{p} K_{\mathrm{a}}$ in solvent water. Open rhombus: bare bases and cations. Dotted line: regression line (eq. 10) for bare bases $\left(r^{2}=0.981, q^{2}=0.979, c_{1}=0.130, c_{2}=-1.57\right)$.

Filled squares: bases and cations with one explicit water molecule. Striped line: regression line (eq. 11) for monosolvated compounds $\left(r^{2}=0.973, q^{2}=0.970, c_{1}=0.153, c_{2}=-2.48\right)$.

The results for all 43 bases are shown in Table 2 and Figure 3. The free energies of all the individual species are given in the Supplementary Material section of this journal. The regression of the calculated 
aqueous Gibbs free energy of dissociation $\left(\Delta G_{\text {diss }}\right)$ vs. experimental base $\mathrm{p} K_{\mathrm{a}}$ for the bare neutral and cationic compounds results in a correlation coefficient of $r^{2}=0.981\left(q^{2}=0.979\right)$. The regression equation for the bare bases $\mathrm{p} K_{\mathrm{a}}$ in aqueous solution reads

$\mathrm{p} K_{\mathrm{a}}=0.130( \pm 0.01) \frac{\Delta G_{\mathrm{diss}}}{R T \ln (10)}-1.57( \pm 0.1)$

If we would have omitted the free energy difference of $\mathrm{H}_{3} \mathrm{O}^{+}$and $\mathrm{H}_{2} \mathrm{O}$ in the definition of $\Delta \mathrm{G}_{\text {diss }}$ we would have got a regression constant of $\hat{c}_{2}=-147.96$ Including this energy difference, the calculated axis intercept of 1.9 is very close to the theoretical value of $c_{2}=-\log \left[\mathrm{H}_{2} \mathrm{O}\right]=-1.74$. In accordance with previous work ${ }^{24}$ on aqueous basicity of bare compounds we found that the slope of the regression is lower than the theoretical value of $1 / \mathrm{RT} \ln (10)=0.1759$.

The regression equation for the mono-solvated base compounds $\left(r^{2}=0.973, q^{2}=0.970\right)$ reads

$\mathrm{p} K_{\mathrm{a}}=0.153( \pm 0.01) \frac{\Delta G_{\text {diss }}}{R T \ln (10)}-2.47( \pm 0.1)$

which omitting the free energy difference of $\mathrm{H}_{3} \mathrm{O}^{+}$and $\mathrm{H}_{2} \mathrm{O}$, yields a regression constant of $\hat{c}_{2}=-174.24$. Thus, in comparison with the bare compound regression, the slope of the mono-solvated solutes is closer to the theoretical value. Yet the slope remains to be significantly smaller than the theoretical value. Moreover, the correlation of $\Delta \mathrm{G}_{\text {diss }}$ with the experimental $\mathrm{p} K_{\mathrm{a}}$ data is worse than for the bare compounds.

If, again following Ding et al. ${ }^{32}$, we introduce the additional solute specific category of minimal $\Delta \mathrm{G}_{\mathrm{diss}}$ in the choice of the cluster or bare solute for the correlation with $\mathrm{p} K_{\mathrm{a}}$, the $\Delta \mathrm{G}_{\mathrm{diss}}$ values in Table 2 follow the same trend that was spotted for the acidic solutes: for strong bases with base $\mathrm{p} K_{\mathrm{a}}<5$ the bare solute is preferred, while weak bases show minimum dissociation free energy for the solvated molecules, which is essentially the same behavior as recognized for acids. The overall correlation of the minimum $\Delta \mathrm{G}_{\text {diss }}$ with base $\mathrm{p} K_{\mathrm{a}}$ is worse than the one for the bare solutes $\left(r^{2}=0.974 \underline{q^{2}=0.972}\right)$ and it leads to a 
slope that is significantly lower than the theoretical: $c_{1}=0.140$ and the regression constant is $c_{2}=-1.61$, which omitting the free energy difference of $\mathrm{H}_{3} \mathrm{O}^{+}$and $\mathrm{H}_{2} \mathrm{O}$, yields $\hat{c}_{2}=-159.17$.

Another important aspect of the base $\mathrm{p} K_{\mathrm{a}}$ prediction using DCSM's is the systematic deviation that is found for aliphatic amines ${ }^{24}$. This group of compounds is known to cause considerable problems in most quantum chemical continuum solvation models ${ }^{21,48-50}$, which also has consequences in the COSMO-RS prediction of $\mathrm{p} K_{\mathrm{a}}$, namely a systematic deviation for secondary and tertiary aliphatic amines. COSMORS computations of $\Delta \mathrm{G}_{\mathrm{diss}}$ of bare and mono-solvated bases is given in Table 3 for 32 primary, secondary and tertiary aliphatic amines. The $\mathrm{p} K_{\mathrm{a}}$ values for these compounds have been calculated using eq. 10 for bare solutes and eq. 11 for mono-solvated solutes, respectively. The root mean square error (RMSE) is 1.38 for bare amine solutes and 1.76 for mono-solvated amines. The systematic deviation of the secondary and tertiary aliphatic amines is more pronounced for the mono-solvated amines than for the bare amines. Thus it must be concluded that the explicit solvation approach is not capable of curing the flaws that DCSM's and COSMO-RS show for aliphatic amines.

\section{Conclusions}

Linear relations between $\mathrm{p} K_{\mathrm{a}}$ and the free energy of dissociation in aqueous solution $\Delta \mathrm{G}_{\mathrm{diss}}$, as computed by the post-DCSM method COSMO-RS on the basis of quantum chemical DFT/COSMO calculations, have been obtained for the deprotonation and protonation reactions of acids and bases, respectively. Without any special adjustments of radii or other parameters this led to a prediction model for acid and base $\mathrm{p} K_{\mathrm{a}}$ values in water. This allows for prediction of absolute aqueous acid and base $\mathrm{p} K_{\mathrm{a}}$ values with an average error of typically less than $0.6 \mathrm{p} K_{\mathrm{a}}$ units. From the observation that the slope of the experimental $\mathrm{p} K_{\mathrm{a}}$ versus theoretical $\Delta G_{\mathrm{diss}}$ was found to be significantly lower than the theoretical value $1 / R T \ln (10)$ if bare solutes and their respective ionic species were used in the COSMO-RS computation, the explicit solvation approach, as first proposed by Pliego and Riveros ${ }^{29,38}$, was used to comprise local 


\section{Molecular Physics}

interaction effects that play an important role in the protonation reaction, but are not, or not sufficiently accounted for by the COSMO-RS method. The application of the COSMO-RS method to the calculation of acids $\mathrm{p} K_{\mathrm{a}}$ in acetonitrile ${ }^{28}$ showed a better performance of the COSMO-RS model on acids that produced anions with delocalized charges, as they are less affected by solvation, while acids that formed localized anions showed a systematic deviation. This observation is corroborated by a recent study, which used multipole derived charge analysis to assess the degree of charge delocalization in anion species formed from aqueous dissociation reactions ${ }^{8}$. Both findings are, at least in part, related to the capability of the solvent to solvate and thus stabilize the anions or cations. Apparently this effect is not captured sufficiently by COSMO-RS and its underlying QC/DCSM method DFT/COSMO if there are short-range interactions in the solvation of ions with localized charges. In this study it was found that the explicit solvation approach in combination with COSMO-RS was able to remove these deficits in parts: while the slope of the $\mathrm{p} K_{\mathrm{a}}$ versus $\Delta G_{\mathrm{diss}}$ correlation was closer to the theoretical slope when the number of explicit solvent atoms in the calculations was increased, the correlation decreased, which may have been expected due to the increasing number of ambiguities with increasing number of explicit solvent molecules. Moreover, it was found that for weak acids, such as tert-butanol, the quantum chemical geometry optimization of the solvent-solute complex of the according tert-butoxy anion was not possible, which makes the explicit solvation approach inapplicable for such compounds. In addition, the systematic deviation found for aliphatic amines was not resolved by the cluster method. Application of only mono- or only di-solvated clusters of neutral and ionic solute species lead to an improved, yet not fully theoretical slope of the $\mathrm{p} K_{\mathrm{a}}$ versus $\Delta G_{\mathrm{diss}}$ correlation. Other criteria proposed for the choice of the number of explicit solvent molecules used, such as semi-solvation of only ions ${ }^{31}$, or from a list of bare, mono- or $d i$-solvated solutes, choice of the species that minimizes the free energy of dissociation ${ }^{32}$ performed worse than the simple decision to use one or two explicit solvent molecule for all solutes involved. Thus the choice of the explicit solvent molecules remains ambiguous and must be considered an unsolved issue. From a practical standpoint it remains difficult or even impossible to find the global minimum energy cluster geometries for weakly bonded clusters with more than one explicit solvent 
molecules. Even for the medium number of compounds used in this study it was impossible to apply global conformational search procedures for finding the cluster geometries of each solute and ion. To avoid this general problem, an automatic procedure, based on COSMO-RS contact interaction energies was proposed, creating "good" or at least sufficiently low energy cluster geometries, without aguarantee of obtaining the actual global minimum, however. Thus it can be concluded that the combination of COSMO-RS with the explicit solvation methodology leads to a theoretical improvement in the slope of the correlation of the free energy of dissociation with $\mathrm{p} K_{\mathrm{a}}$ and thus a better understanding of the physics of the protonation reaction in solution, but suffers from serious disadvantages that - at least for now prevent it from being a practical prediction method for everyday production work: the simple linear relation between $\mathrm{p} K_{\mathrm{a}}$ and the dissociation free energies of the bare solutes and ions proved to be the more robust approach for $\mathrm{p} K_{\mathrm{a}}$ prediction.

\section{Supplementary Material}

Free energies of all species involved in the computation of the dissociation energies (Tables S1, S2, and S3). List of optimized geometries used in the free energy calculations (Table S4). 


\section{References}

1. A. Albert, E. P. Serjeant, The Determination of Ionization Constants Chapman and Hall, New York, (1984).

2. P. Burk, I. A. Koppel, I. Koppel, I. Leito, O. Travnikova, Chem. Phys. Lett., 323, 482 (2000).

3. Y. Seo, Y. Kim, Y. Kim, Chem. Phys. Lett., 340, 186 (2001).

4. $\quad$ E. K. Pokon, M. D. Liptak, S. Feldgus, G. C. Shields, J. Phys. Chem. A., 105, 10483 (2001).

5. $\quad$ K. B.Wiberg, J. Org. Chem., 67, 4787 (2002).

6. $\quad$ K. M. Ervin, V. F. DeTuri, J. Phys. Chem. A., 106, 9947 (2002).

7. M. Sprik, Chem. Phys., 258, 139 (2000).

8. J. Ho, M. L. Coote, J. Chem. Theory Comput., 5, 295 (2009).

9. C. J. Cramer, D. G. Truhlar, Chem. Rev., 99, 2161 (1999).

10. A. Klamt, F. Eckert, M. Diedenhofen, M. E. Beck, J. Phys. Chem. A, 107, 9380 (2003).

11. G. da Silva, E. M. Kennedy, B.Z. Dlugogorski, J. Phys. Chem. A, 110, 11371 (2006).

12. C. Gossens, A. Dorcier, P. J. Dyson, U. Rothlisberger, Organometallics, 26, 3969 (2007).

13. Y. Fu, L.Liu, R.-Q.Li, R. Liu, Q.-X. Guo, J. Am. Chem. Soc., 126, 814 (2004).

14. G. I. Almerindo, D. W. Tondo, J. R. Pliego Jr., J. Phys. Chem. A, 108, 166 (2004).

15. A. Yu, Y. Liu, Z. Li, J.-P. Cheng, J. Phys. Chem. A, 111, 9978 (2007).

16. J. I. Mujika, J. M. Mercero, X. J. Lopez, J. Phys. Chem. A, 107, 6099 (2003).

17. H. Lu, X. Chen, C.-G. Zhan, J. Phys. Chem. B, 111, 10599 (2007).

18. F. Eckert, A. Klamt, AIChE J., 48, 369 (2002).

19. A. Klamt, F. Eckert, Fluid Phase Equilibria, 172, 43 (2000).

20. A. Klamt, J. Phys. Chem., 99, 2224 (1995).

21. A. Klamt, V. Jonas, T. Bürger, J. C. W. Lohrenz, J. Phys. Chem., 102, 5074 (1998).

22. J. R. Pliego Jr., Chem. Phys. Letters, 367, 145 (2003).

23. D. M. Chipman, J. Phys. Chem. A., 106, 7413 (2002).

24. F. Eckert, A. Klamt, J. Comput. Chem., 27, 11 (2006).

25. V. Barone, M. Cossi, J. J. Tomasi, Chem. Phys., 107, 3210 (1997).

26. E. Westphal, J. R.Pliego Jr., J. Phys. Chem. A, 111, 10068 (2007).

27. J.-N. Li, Y. Fu, L. Liu, Q.-X. Guo, Tetrahedron, 62, 11801 (2006). 
28. F. Eckert, I. Leito, I. Kaljurand, A. Kütt, A. Klamt, M. Diedenhofen, J. Comp. Chem., 30, 799 (2009).

29. J. R. Pliego Jr., J. M. Riveros, J. Phys. Chem. A, 106, 7434 (2002).

30. E. Westphal, J. R. Pliego Jr., J. Chem. Phys., 123, 74508 (2005).

31. C. P. Kelly, C. J. Cramer, D. G. Truhlar, J. Phys. Chem. A, 110, 2493 (2006).

32. F. Ding, J. M. Smith, H. Wang, J. Org. Chem., 74, 2679 (2009).

33. Y. Fu, L. Liu, R.-Q. Li, R. Liu, Q.-X. Guo, J. Am. Chem. Soc., 126, 814 (2004).

34. K. R. Adam, J. Phys. Chem. A, 106, 11963 (2002).

35. C. P. Kelly, C. J. Cramer, D. G. Truhlar, J. Chem. Theor. Comput., 1, 1133 (2004).

36. P. Claverie, J. P. Daudey, J. Langlet, B. Pullman, D. Piazzola, M. J. Huron, J. Phys. Chem., 82, 405 (1978).

37. L. C. G. Freitas, R. L. Longo, A. M. Simas, J. Chem. Soc., Faraday Trans., 88, 189 (1992).

38. J. R. Pliego Jr., J. M. Riveros, J. Phys. Chem. A, 105, 7241 (2001).

39. A. Klamt, G. Schüürmann, J. Chem. Soc. Perkins Trans. 2, 799 (1993).

40. F. Eckert, A. Klamt, COSMOtherm, Version C2.1, Revision 01.08 COSMOlogic GmbH\&CoKG, Leverkusen, Germany, (2008) see also URL: http://www.cosmologic.de.

41. R. Ahlrichs, M. Bär, M. Häser, H. Horn, C. Kölmel, Chem. Phys. Letters, 162, 165 (1989).

42. A. Schäfer, A. Klamt, D. Sattel, J. C. W. Lohrenz, F. Eckert, Phys. Chem. Chem. Phys., 2, 2187 (2000).

43. TURBOMOLE V6.0 (2009), a development of University of Karlsruhe and Forschungszentrum Karlsruhe GmbH, 1989-2007, TURBOMOLE GmbH, since 2007; available from http://www.turbomole.com.

44. A. D. Becke, Phys. Rev. A, 38, 3098 (1988).

45. J. P. Perdew, Phys. Rev. B, 33, 8822 (1986).

46. K. Eichkorn, O. Treutler, H. Öhm, M. Häser, R. Ahlrichs, Chem. Phys. Letters, 242, 652 (1995).

47. A. Klamt, COSMO-RS: From Quantum Chemistry to Fluid Thermodynamics and Drug Design, Elsevier, Amsterdam, (2005).

48. B. Marten, K. Kim, C. Cortis, R. A. Friesner, R. B. Murphy, N. Ringnalda, D. Sittkoff, B. Honig, J. Phys. Chem., 100, 11775 (1996).

49. P.-Y. Morgantini, P. J. Kollman, J. Am. Chem. Soc., 117, 6057 (1995).

50. A. Klamt, F. Eckert, M. Hornig, M. Beck, T. Bürger, J. Comp. Chem., 23, 275 (2002).

51. H.C. Brown, et al., in E. A. Braude, F. C. Nachod, Determination of Organic Structures by Physical Methods, Academic Press, New York, (1955). 
TABLE 1: Experimental $\mathrm{p} K_{\mathrm{a}}$ and Gibbs free energies of dissociation of bare, mono-solvated and disolvated aqueous acids.

1

\begin{tabular}{|c|c|c|c|c|}
\hline Compound & $\mathbf{p K A}(\exp )^{a}$ & $\mathbf{G}_{\text {diss }}(\text { bare })^{b}$ & $\Delta \mathbf{G}_{\text {diss }}\left(\mathbf{H}_{\mathbf{2}} \mathbf{O}\right)^{b}$ & $\Delta \mathbf{G}_{\text {diss }}\left(2 \mathbf{H}_{2} \mathbf{O}\right)^{b}$ \\
\hline Ethanol & 15.9 & 136.08 & 105.94 & 93.53 \\
\hline 2,2,2-trichloroethanol & 12.2 & 92.04 & 83.43 & 74.74 \\
\hline formic acid & 3.77 & 12.43 & 29.97 & 34.06 \\
\hline acetic acid & 4.75 & 24.83 & 39.69 & 42.86 \\
\hline Nitromethane & 10.2 & 73.69 & 71.03 & 72.31 \\
\hline dichloroacetic acid & 1.3 & -0.81 & 21.69 & 23.47 \\
\hline trichloroacetic acid & 0.89 & -9.46 & 15.42 & 18.86 \\
\hline n-pentanoic acid & 4.84 & 27.56 & 42.44 & 44.66 \\
\hline 2,2-dimethylpropanoic acid & 5.05 & 30.50 & 44.45 & 46.77 \\
\hline benzoic acid & 4.27 & 23.53 & 39.46 & 40.93 \\
\hline oxalic acid & 1.23 & -3.61 & 18.30 & 34.47 \\
\hline maleic acid & 1.83 & -7.35 & 15.52 & 19.36 \\
\hline fumaric acid & 3.03 & 14.59 & 33.21 & 33.91 \\
\hline acrylic acid & 4.26 & 22.85 & 38.68 & 42.33 \\
\hline bromoacetic acid & 2.87 & 8.51 & 28.40 & 30.93 \\
\hline chloroacetic acid & 2.81 & 8.07 & 28.65 & 31.53 \\
\hline cyanoacetic acid & 2.44 & 5.88 & 26.08 & 30.29 \\
\hline fluoroacetic acid & 2.66 & 8.52 & 28.07 & 30.84 \\
\hline iodoacetic acid & 3.13 & 7.22 & 28.64 & 32.52 \\
\hline 2-hydroxypropanoic acid & 3.87 & 9.23 & 28.01 & 30.36 \\
\hline 2-chloropropanoic acid & 2.8 & 12.58 & 29.71 & 32.95 \\
\hline 3-chloropropanoic acid & 4.1 & 19.76 & 36.88 & 38.34 \\
\hline nitroacetic acid & 1.32 & -2.75 & 19.99 & 24.66 \\
\hline 2-acetylbutanedioic acid & 2.86 & 8.81 & 16.73 & 23.54 \\
\hline carbonic acid & 3.58 & 10.60 & 31.62 & 41.37 \\
\hline hypochlorous acid & 7.43 & 57.75 & 58.25 & 56.35 \\
\hline hypobromous acid & 8.7 & 67.41 & 64.64 & 59.08 \\
\hline hypoiodous acid & 10.64 & 71.94 & 68.28 & 61.87 \\
\hline nitrous acid & 3.3 & 2.04 & 16.80 & 30.42 \\
\hline sulfurous acid & 1.92 & -9.09 & 6.22 & 7.57 \\
\hline phosphoric acid & 1.97 & -1.03 & 5.92 & 12.89 \\
\hline boric acid & 9.12 & 70.92 & 71.10 & 72.38 \\
\hline pentachlorophenol & 4.9 & 29.41 & 34.67 & 36.55 \\
\hline 2,3,4,6-tetrachlorophenol & 5.62 & 35.52 & 38.96 & 38.19 \\
\hline 2,3,4-trichlorophenol & 7.1 & 51.03 & 53.73 & 55.52 \\
\hline 2,3-dichlorophenol & 7.76 & 55.05 & 56.73 & 58.24 \\
\hline 2,4,5-trichlorophenol & 7.07 & 48.73 & 52.22 & 55.17 \\
\hline 2,4,6-trichlorophenol & 6.21 & 41.62 & 42.76 & 43.19 \\
\hline 2,4-dichlorophenol & 8.09 & 56.88 & 57.71 & 59.56 \\
\hline 2,5-dichlorophenol & 7.51 & 53.01 & 55.10 & 57.45 \\
\hline 2,6-dichlorophenol & 6.79 & 47.36 & 47.81 & 49.47 \\
\hline 2-chlorophenol & 8.29 & 60.88 & 60.80 & 63.71 \\
\hline 3,4-dichlorophenol & 8.68 & 62.51 & 62.24 & 61.19 \\
\hline 3,5-dichlorophenol & 8.27 & 57.87 & 58.91 & 58.01 \\
\hline 3-chlorophenol & 8.78 & 65.41 & 64.91 & 63.21 \\
\hline
\end{tabular}




\section{Page 25 of 461}

Molecular Physics

\begin{tabular}{|c|c|c|c|c|}
\hline 4-chlorophenol & 9.14 & 69.57 & 67.63 & 65.17 \\
\hline phenol & 9.82 & 70.87 & 68.82 & 66.57 \\
\hline 5-nitrouracil & 5.3 & 34.18 & 36.17 & 36.53 \\
\hline cis-5-formyluracil & 6.84 & 46.20 & 46.58 & 43.60 \\
\hline thymine & 9.75 & 68.64 & 67.21 & 67.64 \\
\hline trans-5-formyluracil & 6.84 & 45.61 & 45.64 & 45.44 \\
\hline uracil & 9.42 & 64.78 & 64.13 & 64.79 \\
\hline fluorouracil & 8 & 54.99 & 58.34 & 57.85 \\
\hline methylthiouracil & 8.2 & 51.60 & 53.38 & 57.35 \\
\hline phenytoin & 8.3 & 56.53 & 59.71 & 56.83 \\
\hline 3,3-methylphenylglutarimide & 9.2 & 64.17 & 67.65 & 65.55 \\
\hline 3,3-dimethylsuccinimide & 9.5 & 57.89 & 62.08 & 59.80 \\
\hline dimethadione & 6.1 & 35.86 & 42.26 & 44.33 \\
\hline phthalimide & 8.3 & 58.09 & 61.04 & 60.47 \\
\hline succinimide & 9.6 & 57.76 & 61.20 & 62.62 \\
\hline phthalic acid & 2.76 & -0.61 & 19.06 & 18.70 \\
\hline methanol & 15.5 & 137.22 & 105.05 & 91.27 \\
\hline hypophosphorous acid & 2.23 & 1.72 & 21.44 & 22.11 \\
\hline 4-methylbenzoic acid & 4.47 & 25.56 & 40.87 & 42.31 \\
\hline 4-ethylbenzoic acid & 4.47 & 25.40 & 41.26 & 42.49 \\
\hline 4-propylbenzoic acid & 4.47 & 25.51 & 41.08 & 42.11 \\
\hline 4-(1-methylethyl)-benzoic acid & 4.47 & 25.44 & 40.40 & 43.60 \\
\hline 4-butylbenzoic acid & 4.47 & 25.52 & 41.27 & 41.64 \\
\hline 4-(1,1-dimethylethyl)-benzoic acid & 4.4 & 25.51 & 41.39 & 42.06 \\
\hline 4-phenylbenzoic acid & 4.07 & 23.99 & 40.19 & 41.02 \\
\hline 4-fluorobenzoic acid & 4.07 & 23.17 & 39.27 & 40.84 \\
\hline 4-chlorobenzoic acid & 4.07 & 21.50 & 38.01 & 39.74 \\
\hline 4-bromobenzoic acid & 4.47 & 21.52 & 38.14 & 40.56 \\
\hline 4-iodobenzoic acid & 4.47 & 20.68 & 37.66 & 39.99 \\
\hline 4-methoxybenzoic acid & 4.5 & 27.33 & 42.67 & 44.36 \\
\hline 4-hydroxybenzoic acid & 4.58 & 28.58 & 42.70 & 43.75 \\
\hline 4-nitrobenzoic acid & 3.4 & 14.92 & 34.05 & 35.39 \\
\hline 4-hydroxymethylbenzoic acid & 4.27 & 22.68 & 38.95 & 40.09 \\
\hline 4-cyanobenzoic acid & 3.47 & 16.19 & 34.72 & 36.76 \\
\hline 4-aminobenzoic acid & 4.92 & 36.24 & 46.32 & 50.17 \\
\hline 4-methylaminobenzoic acid & 5.04 & 36.15 & 49.09 & 49.67 \\
\hline 4-dimethylaminobenzoic acid & 5.07 & 35.04 & 48.01 & 50.23 \\
\hline 4-trifluoromethylbenzoic acid & 3.67 & 19.09 & 36.47 & 38.60 \\
\hline 4-sulfamoylbenzoic acid & 3.67 & 17.14 & 35.17 & 36.75 \\
\hline 4-carbamoylbenzoic acid & 3.87 & 20.01 & 37.27 & 39.77 \\
\hline salicylic acid & 2.97 & 13.13 & 31.28 & 32.75 \\
\hline 2,4-dihydroxybenzoic acid & 3.11 & 18.08 & 34.85 & 35.57 \\
\hline 5-iodo-2-hydroxybenzoic acid & 2.89 & 6.91 & 29.06 & 31.52 \\
\hline 3-hydroxybenzoic acid & 4.08 & 24.19 & 38.86 & 41.19 \\
\hline mandelic acid & 3.41 & 7.04 & 26.43 & 30.56 \\
\hline 3-hydroxy-2-naphtoic acid & 2.97 & 11.16 & 30.58 & 33.13 \\
\hline anthracene-9-carboxylic acid & 3.65 & 12.65 & 28.61 & 31.41 \\
\hline pamoic acid & 2.47 & 4.01 & 29.28 & 30.03 \\
\hline tert-butanol & 19.2 & 148.88 & 107.60 & 91.32 \\
\hline
\end{tabular}

${ }^{a} p \mathrm{~K}_{a}^{E x p}$ :Experimental aqueous $\mathrm{pKa}$ value of acids, taken from refs. 10, 51. 
${ }^{b} \Delta G_{\text {diss }}$ : Gibbs free energies of dissociation calculated from eq. 4 in $\left[\mathrm{kJ} \cdot \mathrm{mol}^{-1}\right]$.

1

2

3

4

5

6

7

8

9

10

11

12

13

14

15

16

17

18

19

20

21

22

23

24

25

26

27

28

29

30

31

32

33

34

35

36

37

38

39

40

41

42

43

44

45

46

47

48

49

50

51

52

53

54

55

56

57

58

59

60

URL: http://mc.manuscriptcentral.com/tandf/tmph 
TABLE 2: Experimental base $\mathrm{p} K_{\mathrm{a}}$ and Gibbs free energies of dissociation of bare and monosolvated aqueous bases.

\begin{tabular}{|c|c|c|c|}
\hline Compound & $\mathbf{p K}_{\mathbf{A}}(\operatorname{Exp})^{c}$ & ${ }^{a} \Delta \mathbf{G}_{\text {diss }}(\text { bare })^{b}$ & $\Delta \mathbf{G}_{\text {diss }}\left(\mathbf{H}_{2} \mathbf{O}\right)^{b}$ \\
\hline hydroxyimidazo(2,3-a)isoindole & 8.6 & 84.03 & 81.42 \\
\hline imidazo[2,3-b]thioxazole & 8 & 70.10 & 67.38 \\
\hline tetrahydrozoline & 10.5 & 85.97 & 79.31 \\
\hline tolazoline & 10.3 & 83.64 & 76.58 \\
\hline 4-chloroaniline & 4 & 47.44 & 44.78 \\
\hline 4-methoxyaniline & 5.2 & 57.04 & 54.43 \\
\hline 4-nitroaniline & 1 & 17.79 & 14.03 \\
\hline aniline & 4.6 & 49.04 & 48.42 \\
\hline p-toluidine & 5.1 & 55.31 & 49.08 \\
\hline 1,3-dihydro-1-methyl-5-phenyl-1,4-benzodiazepin-2-one & 3.3 & 44.14 & 44.16 \\
\hline 1,3-dihydro-3-hydroxy-1-methyl-5-phenyl-1,4-benzodiazepin-2-one & 1.6 & 24.12 & 30.42 \\
\hline 1,3-dihydro-3-hydroxy-5-phenyl-1,4-benzodiazepin-2-one & 1.7 & 27.01 & 32.37 \\
\hline 1,3-dihydro-5-phenyl-1,4-benzodiazepin-2-one & 3.5 & 46.25 & 45.91 \\
\hline 2,3-dihydro-1-methyl-5-phenyl-1,4-benzodiazepine & 6.2 & 67.59 & 62.62 \\
\hline 3-hydro-2-methylamine-4-oxy-5-phenyl-1,4-benzodiazepine & 4.8 & 50.84 & 52.73 \\
\hline clonidine & 8.1 & 64.95 & 59.94 \\
\hline debrisoquin & 11.9 & 104.81 & 96.56 \\
\hline guanidine & 13.8 & 115.78 & 104.87 \\
\hline methylguanidine & 13.4 & 109.68 & 99.79 \\
\hline 2-aminopyridine & 6.71 & 67.69 & 58.98 \\
\hline 2-aminothiazole & 5.4 & 55.09 & 48.11 \\
\hline 2-methylimidazole & 8 & 66.68 & 62.94 \\
\hline 3-aminopyridine & 6 & 65.51 & 60.04 \\
\hline 4-aminopyridine & 9.23 & 81.02 & 72.89 \\
\hline 4-methylpyridine & 6 & 56.34 & 52.25 \\
\hline benzimidazole & 5.8 & 52.15 & 50.55 \\
\hline imidazole & 7 & 62.51 & 59.27 \\
\hline isoquinoline & 5.4 & 52.84 & 49.51 \\
\hline melamine & 5 & 58.94 & 54.44 \\
\hline pyrazine & 0.7 & 21.47 & 25.39 \\
\hline pyrazole & 2.5 & 35.36 & 36.96 \\
\hline pyridine & 5.14 & 51.55 & 48.55 \\
\hline pyrimidine & 1.3 & 23.74 & 27.33 \\
\hline quinoline & 4.8 & 46.99 & 46.62 \\
\hline thiazole & 2.8 & 28.25 & 31.32 \\
\hline 1-methylindole & -2.3 & -9.71 & -8.02 \\
\hline 1-methylpyrrole & -2.9 & -16.56 & -10.76 \\
\hline 2-methylindole & -0.3 & 6.96 & 14.10 \\
\hline 2-methylpyrrole & -0.2 & 2.42 & 14.66 \\
\hline 3-methylindole & -4.6 & -24.06 & -13.34 \\
\hline 3-methylpyrrole & -1 & 7.11 & 15.84 \\
\hline indole & -3.6 & -14.37 & -4.23 \\
\hline pyrrole & -3.8 & -13.64 & -6.38 \\
\hline
\end{tabular}


${ }^{a} p \mathrm{~K}_{a}^{E x p}:$ Experimental aqueous base $\mathrm{pKa}$ value taken from ref. 24.

${ }^{b} \Delta G_{\text {diss: }}$ : Gibbs free energies of dissociation calculated from eq. 5 in [kJ.mol ${ }^{-1}$.

2

3

4

5

6

7

8

9

10

11

12

13

14

15

16

17

18

19

20

21

22

23

24

25

26

27

28

29

30

31

32

33

34

35

36

37

38

39

40

41

42

43

44

45

46

47

48

49

50

51

52

53

54

55

56

57

58

59

60

URL: http://mc.manuscriptcentral.com/tandf/tmph 
TABLE 3: Experimental aqueous base $\mathrm{p} K_{\mathrm{a}}$ and Gibbs free energies of dissociation of bare and mono-solvated aliphatic amine bases.

\begin{tabular}{|c|c|c|c|c|c|c|c|c|}
\hline Compound & Base-Type & $\mathbf{p} \mathbf{K}_{\mathrm{A}}(\operatorname{Exp})^{a}$ & $\Delta \mathbf{G}_{\text {diss }}(\text { bare })^{b}$ & $\Delta \mathbf{G}_{\text {diss }}\left(\mathbf{H}_{2} \mathbf{O}\right)^{b}$ & ${ }^{b} k_{\mathrm{a}}{ }^{\text {calc }}(\text { bare })^{c}$ & ${ }^{c} \mathrm{pk}_{\mathrm{a}}{ }^{\text {calc }}\left(\mathrm{H}_{2} \mathrm{O}\right)^{d}$ & ${ }^{d} \Delta \mathrm{pk}_{\mathrm{a}}$ (bare) & $\Delta p k_{a}\left(H_{2} \mathbf{O}\right)$ \\
\hline methylamine & primary & 10.6 & 104.07 & 88.78 & 11.97 & 11.09 & -1.37 & -0.49 \\
\hline ethylamine & primary & 10.9 & 100.39 & 86.62 & 11.50 & 10.76 & -0.60 & 0.14 \\
\hline n-propylamine & primary & 10.7 & 99.45 & 85.81 & 11.37 & 10.64 & -0.67 & 0.06 \\
\hline n-butylamine & primary & 10.8 & 99.17 & 85.81 & 11.34 & 10.64 & -0.54 & 0.16 \\
\hline n-pentylamine & primary & 10.6 & 98.93 & 85.84 & 11.31 & 10.64 & -0.71 & -0.04 \\
\hline n-hexylamine & primary & 10.6 & 98.92 & 85.18 & 11.30 & 10.54 & -0.70 & 0.06 \\
\hline n-heptylamine & primary & 10.7 & 98.84 & 85.08 & 11.29 & 10.53 & -0.59 & 0.17 \\
\hline n-octylamine & primary & 10.7 & 98.76 & 85.15 & 11.28 & 10.54 & -0.58 & 0.16 \\
\hline iso-propylamine & primary & 10.6 & 95.54 & 83.76 & 10.86 & 10.32 & -0.26 & 0.28 \\
\hline sec-butylamine & primary & 10.56 & 91.95 & 81.99 & 10.40 & 10.05 & 0.16 & 0.51 \\
\hline tert-butylamine & primary & 10.7 & 93.11 & 81.53 & 10.55 & 9.98 & 0.15 & 0.72 \\
\hline neo-pentylamine & primary & 10.21 & 94.59 & 80.82 & 10.74 & 9.87 & -0.53 & 0.34 \\
\hline cyclohexylamine & primary & 10.6 & 95.11 & 82.76 & 10.81 & 10.17 & -0.21 & 0.43 \\
\hline dimethylamine & secondary & 10.7 & 96.12 & 79.30 & 10.94 & 9.64 & -0.24 & 1.06 \\
\hline diethylamine & secondary & 11.1 & 88.89 & 78.44 & 10.00 & 9.51 & 1.10 & 1.59 \\
\hline di-n-propylamine & secondary & 11 & 87.51 & 76.91 & 9.82 & 9.28 & 1.18 & 1.72 \\
\hline di-iso-propylamine & secondary & 11.1 & 85.10 & 76.66 & 9.50 & 9.24 & 1.60 & 1.86 \\
\hline di-n-butylamine & secondary & 11.4 & 87.21 & 76.31 & 9.78 & 9.19 & 1.62 & 2.21 \\
\hline di-iso-butylamine & secondary & 10.5 & 77.93 & 64.94 & 8.57 & 7.45 & 1.93 & 3.05 \\
\hline di-sec-butylamine & secondary & 11.01 & 80.87 & 75.10 & 8.95 & 9.00 & 2.06 & 2.01 \\
\hline tert-butylcyclohexylamine & secondary & 11.23 & 81.90 & 73.15 & 9.09 & 8.70 & 2.14 & 2.53 \\
\hline trimethylamine & tertiary & 9.8 & 80.69 & 66.81 & 8.93 & 7.73 & 0.87 & 2.07 \\
\hline dimethylethylamine & tertiary & 10.2 & 78.60 & 67.65 & 8.66 & 7.86 & 1.54 & 2.34 \\
\hline dimethyl-n-propylamine & tertiary & 9.99 & 77.48 & 66.97 & 8.51 & 7.76 & 1.48 & 2.23 \\
\hline dimethyl-iso-propylamine & tertiary & 10.3 & 77.00 & 68.84 & 8.45 & 8.04 & 1.85 & 2.26 \\
\hline dimethyl-n-butylamine & tertiary & 10.02 & 77.22 & 66.78 & 8.48 & 7.73 & 1.54 & 2.29 \\
\hline dimethyl-iso-butylamine & tertiary & 9.91 & 74.43 & 65.50 & 8.11 & 7.53 & 1.80 & 2.38 \\
\hline dimethyl-sec-butylamine & tertiary & 10.4 & 77.84 & 68.69 & 8.56 & 8.02 & 1.84 & 2.38 \\
\hline dimethyl-tert-butylamine & tertiary & 10.52 & 80.82 & 71.46 & 8.95 & 8.45 & 1.57 & 2.07 \\
\hline triethylamine & tertiary & 10.8 & 78.65 & 71.56 & 8.66 & 8.46 & 2.14 & 2.34 \\
\hline tri-n-propylamine & tertiary & 10.7 & 78.96 & 69.69 & 8.71 & 8.17 & 1.99 & 2.53 \\
\hline tri-n-butylamine & tertiary & 10.89 & 78.47 & 69.36 & 8.64 & 8.12 & 2.25 & 2.77 \\
\hline
\end{tabular}

${ }^{a} p K_{a}{ }^{E x p}$ :Experimental aqueous base pKa value taken from ref. 24 .

${ }^{b} \Delta G_{\text {diss }}$ : Gibbs free energies of dissociation calculated from eq. 5 in [kJ.mol ${ }^{-1}$ ].

${ }^{c} \mathrm{pk}_{\mathrm{a}}{ }^{\text {calc }}$ (bare): pKa of bare amine bases calculated from eq. 10 .

${ }^{d} \mathrm{pk}_{\mathrm{a}}{ }^{\text {calc }}\left(\mathrm{H}_{2} \mathrm{O}\right)$ : pKa of mono-solvated amine bases calculated from eq. 11. 


\title{
Supplementary Material to:
}

\section{First principles prediction of pKa:}

\section{COSMO-RS and the cluster-continuum approach}

\author{
Frank Eckert $^{a}{ }^{*}$, Michael Diedenhofen ${ }^{a}$, Andreas Klamt ${ }^{a, b}$
}

\begin{abstract}
${ }^{a}$ COSMOlogic GmbH \& Co KG, Burscheider Str. 515, D-51381 Leverkusen, Germany
${ }^{b}$ University of Regensburg, Institute of Physical and Theoretical Chemistry, 93040

Regensburg, Germany

* Corresponding Author. Email: frank.eckert@cosmologic.de. Phone: +492171731680. Fax: +492171731689.
\end{abstract}

KEYWORDS: $\mathrm{p} K_{\mathrm{a}}$; acidity; basicity; COSMO; COSMO-RS; density functional theory; 


\begin{tabular}{|c|c|c|c|c|c|c|}
\hline $\mathbf{p K}_{\mathbf{A}}(\operatorname{Exp})^{a}$ & $\mathbf{G}(\mathbf{A H})^{b}$ & $\mathbf{G}\left(\mathbf{A}^{-}\right)^{b}$ & $\mathbf{G}\left(\mathbf{A H}-\mathbf{H}_{2} \mathbf{O}\right)^{b}$ & $\mathbf{G}\left(\mathrm{A}^{-}-\mathrm{H}_{2} \mathbf{O}\right)^{b}$ & $\mathbf{G}\left(\mathrm{AH}-2 \mathrm{H}_{2} \mathrm{O}\right)^{b}$ & $\mathbf{G}\left(\mathrm{A}^{-}-2 \mathrm{H}_{2} \mathrm{O}\right)^{b}$ \\
\hline 15.9 & -407192.75 & -405932.1933 & -607976.05 & -606745.64 & -808761.49 & -807543.49 \\
\hline 12.2 & -4027063.85 & -4025847.339 & -4227856.50 & -4226648.60 & -4428641.71 & -4427442.50 \\
\hline 3.77 & -498419.13 & -497282.2218 & -699210.35 & -698055.91 & -899997.73 & -898839.19 \\
\hline 4.75 & -601684.32 & -600535.0143 & -802473.84 & -801309.68 & -1003259.56 & -1002092.23 \\
\hline 10.2 & -643528.89 & -642330.7273 & -844302.27 & -843106.77 & -1045086.79 & -1043890.01 \\
\hline 1.3 & -3014912.86 & -3013789.195 & -3215708.21 & -3214562.05 & -3416493.77 & -3415345.82 \\
\hline 0.89 & -4221505.27 & -4220390.258 & -4422303.25 & -4421163.36 & -4623090.73 & -4621947.40 \\
\hline 4.84 & -911383.95 & -910231.9169 & -1112173.20 & -1111006.29 & -1312958.43 & -1311789.29 \\
\hline 5.05 & -911379.72 & -910224.755 & -1112168.43 & -1110999.50 & -1312954.03 & -1311782.78 \\
\hline 4.27 & -1105183.13 & -1104035.132 & -1305972.59 & -1304808.66 & -1506757.71 & -1505592.30 \\
\hline 1.23 & -993682.13 & -992561.2674 & -1194476.68 & -1193333.90 & -1395275.89 & -1394116.95 \\
\hline 1.83 & -1196956.99 & -1195839.864 & -1397753.04 & -1396613.05 & -1598539.50 & -1597395.67 \\
\hline 3.03 & -1196967.63 & -1195828.574 & -1397758.39 & -1396600.71 & -1598553.49 & -1597395.11 \\
\hline 4.26 & -701679.58 & -700532.255 & -902469.38 & -901306.23 & -1103254.40 & -1102087.59 \\
\hline 2.87 & -7358183.68 & -7357050.701 & -7558976.50 & -7557823.62 & -7759760.56 & -7758605.15 \\
\hline 2.81 & -1808304.94 & -1807172.396 & -2009097.83 & -2007944.70 & -2209882.06 & -2208726.05 \\
\hline 2.44 & -843914.94 & -842784.5871 & -1044708.49 & -1043557.94 & -1245493.73 & -1244338.97 \\
\hline 2.66 & -862284.69 & -861151.695 & -1063077.34 & -1061924.80 & -1263861.99 & -1262706.68 \\
\hline 3.13 & -630170.59 & -629038.8935 & -830964.67 & -829811.56 & -1031750.65 & -1030593.65 \\
\hline 3.87 & -902477.35 & -901343.6465 & -1103269.20 & -1102116.71 & -1304053.44 & -1302898.61 \\
\hline 2.8 & -1911547.21 & -1910410.158 & -2112338.93 & -2111184.75 & -2313123.36 & -2311965.94 \\
\hline 4.1 & -1911557.21 & -1910412.972 & -2112347.78 & -2111186.43 & -2313131.32 & -2311968.50 \\
\hline 1.32 & -1138790.10 & -1137668.378 & -1339585.59 & -1338441.12 & -1540370.81 & -1539221.67 \\
\hline 2.86 & -1601062.73 & -1599929.447 & -1801854.80 & -1800713.60 & -2002637.00 & -2001488.99 \\
\hline 3.58 & -696063.66 & -694928.5828 & -896855.56 & -895699.46 & -1097648.86 & -1096483.02 \\
\hline 7.43 & -1407217.85 & -1406035.623 & -1608010.03 & -1606827.30 & -1808796.91 & -1807616.09 \\
\hline 8.7 & -6957132.58 & -6955940.694 & -7157923.29 & -7156734.17 & -7358708.79 & -7357525.24 \\
\hline 10.64 & -229155.38 & -227958.9666 & -429946.84 & -428754.09 & -630733.26 & -629546.92 \\
\hline 3.3 & -540300.54 & -539174.0233 & -741089.51 & -739948.23 & -941886.43 & -940731.54 \\
\hline 1.92 & -1641240.08 & -1640124.693 & -1842029.71 & -1840899.02 & -2042817.45 & -2041685.41 \\
\hline 1.97 & -1691557.18 & -1690433.734 & -1892350.57 & -1891220.18 & -2093144.26 & -2092006.90 \\
\hline 9.12 & -663171.83 & -661976.44 & -863958.05 & -862762.47 & -1064744.19 & -1063547.34 \\
\hline 4.9 & -6840598.39 & -6839444.506 & -7041383.83 & -7040224.69 & -7242169.77 & -7241008.74 \\
\hline 5.62 & -5633988.50 & -5632828.508 & -5834773.01 & -5833609.58 & -6035558.82 & -6034396.15 \\
\hline 7.1 & -4427367.41 & -4426191.903 & -4628156.05 & -4626977.85 & -4828942.19 & -4827762.20 \\
\hline 7.76 & -3220743.41 & -3219563.891 & -3421531.41 & -3420350.20 & -3622317.22 & -3621134.50 \\
\hline 7.07 & -4427376.94 & -4426203.737 & -4628166.28 & -4626989.59 & -4828952.12 & -4827772.48 \\
\hline 6.21 & -4427374.18 & -4426208.085 & -4628158.50 & -4626991.26 & -4828944.52 & -4827776.86 \\
\hline 8.09 & -3220750.81 & -3219569.449 & -3421538.79 & -3420356.61 & -3622324.86 & -3621140.83 \\
\hline 7.51 & -3220753.12 & -3219575.638 & -3421541.64 & -3420362.07 & -3622327.12 & -3621145.20 \\
\hline 6.79 & -3220742.78 & -3219570.947 & -3421526.15 & -3420353.87 & -3622311.80 & -3621137.85 \\
\hline 8.29 & -2014114.41 & -2012929.057 & -2214901.51 & -2213716.24 & -2415687.32 & -2414499.14 \\
\hline 8.68 & -3220747.30 & -3219560.318 & -3421535.27 & -3420348.56 & -3622321.71 & -3621136.04 \\
\hline 8.27 & -3220756.86 & -3219574.512 & -3421545.42 & -3420362.03 & -3622331.64 & -3621149.16 \\
\hline 8.78 & -2014119.59 & -2012929.705 & -2214907.33 & -2213717.95 & -2415693.09 & -2414505.40 \\
\hline 9.14 & -2014118.05 & -2012924.008 & -2214905.55 & -2213713.45 & -2415690.81 & -2414501.17 \\
\hline 9.82 & -807476.99 & -806281.6423 & -1008263.81 & -1007070.52 & -1209049.16 & -1207858.12 \\
\hline 5.3 & -1626604.26 & -1625445.605 & -1827385.29 & -1826224.65 & -2028170.63 & -2027009.62 \\
\hline 6.84 & -1387126.03 & -1385955.365 & -1587905.80 & -1586734.75 & -1788690.61 & -1787522.54 \\
\hline 9.75 & -1192743.49 & -1191550.382 & -1393523.03 & -1392331.35 & -1594308.61 & -1593116.50 \\
\hline 6.84 & -1387126.80 & -1385956.72 & -1587906.25 & -1586736.14 & -1788690.49 & -1787520.58 \\
\hline
\end{tabular}

\section{TABLE S1: Experimental $\mathrm{p} K_{\mathrm{a}}$ and Gibbs free energies of bare, mono-solvated and di- solvated aqueous acids.}

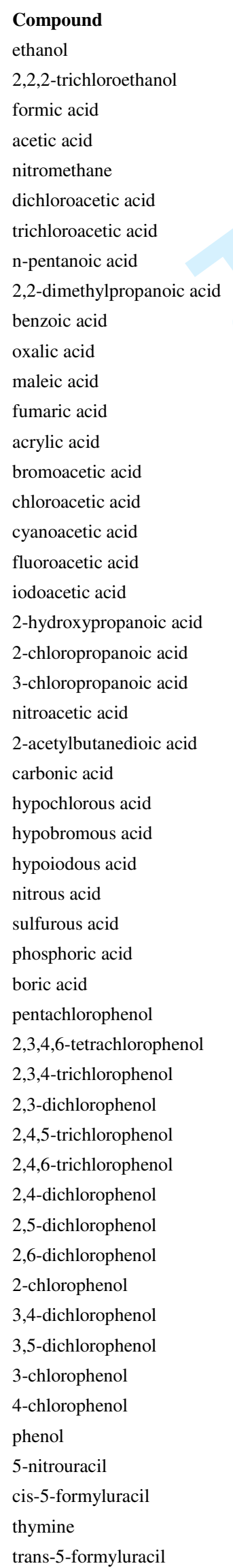


uracil

fluorouracil

methylthiouracil

phenytoin

3,3-methylphenylglutarimide

3,3-dimethylsuccinimide

dimethadione

phthalimide

succinimide

phthalic acid

methanol

hypophosphorous acid

4-methylbenzoic acid

4-ethylbenzoic acid

4-propylbenzoic acid

4-(1-methylethyl)-benzoic acid

4-butylbenzoic acid

4-(1,1-dimethylethyl)-benzoic acid

4-phenylbenzoic acid

4-fluorobenzoic acid

4-chlorobenzoic acid

4-bromobenzoic acid

4-iodobenzoic acid

4-methoxybenzoic acid

4-hydroxybenzoic acid

4-nitrobenzoic acid

4-hydroxymethylbenzoic acid

4-cyanobenzoic acid

4-aminobenzoic acid

4-methylaminobenzoic acid

4-dimethylaminobenzoic acid

4-trifluoromethylbenzoic acid

4-sulfamoylbenzoic acid

4-carbamoylbenzoic acid

salicylic acid

2,4-dihydroxybenzoic acid

5-iodo-2-hydroxybenzoic acid

3-hydroxybenzoic acid

mandelic acid

3-hydroxy-2-naphtoic acid

anthracene-9-carboxylic acid

pamoic acid

tert-butanol $\begin{array}{llllllll}9.42 & -1089497.97 & -1088308.718 & -1290277.91 & -1289089.31 & -1491063.39 & -14\end{array}$

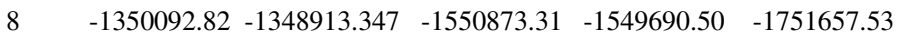

$\begin{array}{lllllll}8.2 & -2040569.26 & -2039393.19 & -2241345.72 & -2240167.86 & -2442134.11\end{array}$

$\begin{array}{lllllll}8.3 & -2202875.30 & -2201694.294 & -2403655.06 & -2402470.88 & -2604440.43\end{array}$

$\begin{array}{lllllll}9.2 & -1760440.91 & -1759252.26 & -1961218.09 & -1960025.97 & -2162001.52\end{array}$

$\begin{array}{lllllll}9.5 & -1153722.82 & -1152540.452 & -1354502.51 & -1353315.96 & -1555287.70\end{array}$

$\begin{array}{lllllll}6.1 & -1248067.53 & -1246907.195 & -1448849.90 & -1447683.17 & -1649635.83\end{array}$

$\begin{array}{lllllll}8.3 & -1347506.31 & -1346323.738 & -1548286.07 & -1547100.56 & -1749071.94\end{array}$

$\begin{array}{lllll}-947253.37 & -946071.131 & -1148032.98 & -1146847.30 & -1348818.45\end{array}$

$\begin{array}{llllll}-1600451.16 & -1599327.289 & -1801244.53 & -1800101.00 & -2002026.77\end{array}$ $\begin{array}{llllll}-303944.85 & -302683.1629 & -504728.69 & -503499.17 & -705514.01\end{array}$

$\begin{array}{llllll}-1296181.52 & -1295055.33 & -1496980.74 & -1495834.83 & -1697768.92\end{array}$ $\begin{array}{llllll}-1208429.73 & -1207279.699 & -1409219.11 & -1408053.76 & -1610003.58\end{array}$ $\begin{array}{llllll}-1311661.86 & -1310511.979 & -1512451.32 & -1511285.58 & -1713236.34\end{array}$ $\begin{array}{llllll}-1414896.30 & -1413746.319 & -1615685.65 & -1614520.09 & -1816470.40\end{array}$ $\begin{array}{llllll}-1414894.16 & -1413744.25 & -1615682.93 & -1614518.05 & -1816469.17\end{array}$ $\begin{array}{llllll}-1518129.79 & -1516979.803 & -1718919.20 & -1717753.46 & -1919703.85\end{array}$ $\begin{array}{llllll}-1518120.53 & -1516970.554 & -1718910.22 & -1717744.36 & -1919694.43\end{array}$ $\begin{array}{llllll}-1711933.61 & -1710785.145 & -1912723.38 & -1911558.71 & -2113508.03\end{array}$ $\begin{array}{llllll}-1365814.05 & -1364666.409 & -1566603.79 & -1565440.04 & -1767388.73\end{array}$ $\begin{array}{llllll}-2311823.82 & -2310677.843 & -2512613.70 & -2511451.22 & -2713399.04\end{array}$ $\begin{array}{llllll}-7861698.99 & -7860552.997 & -8062488.85 & -8061326.24 & -8263274.78\end{array}$ $\begin{array}{llllll}-1133680.18 & -1132535.028 & -1334470.31 & -1333308.18 & -1535256.61\end{array}$ $\begin{array}{llllll}-1405956.62 & -1404804.816 & -1606745.65 & -1605578.51 & -1807530.40\end{array}$ $\begin{array}{llllll}-1302780.78 & -1301627.725 & -1503568.82 & -1502401.65 & -1704361.16\end{array}$ $\begin{array}{llllll}-1642310.23 & -1641170.834 & -1843101.90 & -1841943.38 & -2043886.92\end{array}$ $\begin{array}{llllll}-1405982.04 & -1404834.881 & -1606771.60 & -1605608.18 & -1807556.39\end{array}$ $\begin{array}{llllll}-1347430.97 & -1346290.307 & -1548222.28 & -1547063.09 & -1749007.41\end{array}$ $\begin{array}{llllll}-1250609.21 & -1249448.504 & -1451396.14 & -1450225.35 & -1652183.29\end{array}$ $\begin{array}{llllll}-1353813.87 & -1352653.242 & -1554600.86 & -1553427.29 & -1755385.15\end{array}$ $\begin{array}{llllll}-1457011.07 & -1455851.555 & -1657798.34 & -1656625.86 & -1858582.64\end{array}$ $\begin{array}{llllll}-1990349.42 & -1989205.856 & -2191139.87 & -2189978.93 & -2391925.76\end{array}$ $\begin{array}{llllll}-2691002.97 & -2689861.36 & -2891793.37 & -2890633.73 & -3092578.83\end{array}$ $\begin{array}{lllllll}-1548296.73 & -1547152.255 & -1749086.91 & -1747925.16 & -1949872.98\end{array}$ $\begin{array}{llllll}-1302781.05 & -1301643.449 & -1503572.26 & -1502416.50 & -1704357.38\end{array}$ $\begin{array}{llllll}-1500379.49 & -1499236.936 & -1701169.59 & -1700010.27 & -1901962.21\end{array}$ $\begin{array}{llllll}-1331275.18 & -1330143.798 & -1532067.89 & -1530914.36 & -1732854.39\end{array}$ $\begin{array}{llllll}-1302772.24 & -1301623.573 & -1503560.22 & -1502396.89 & -1704353.11\end{array}$ $\begin{array}{llllll}-1405965.98 & -1404834.467 & -1606758.61 & -1605607.71 & -1807544.47\end{array}$ $\begin{array}{lllllll}-1706239.82 & -1705104.186 & -1907031.97 & -1905876.92 & -2107818.67\end{array}$ $\begin{array}{llllll}-1912066.61 & -1910929.486 & -2112858.36 & -2111705.28 & -2313643.82\end{array}$ $\begin{array}{lllll}-3512537.22 & -3511408.744 & -3713327.51 & -3712173.75 & -3914119.46\end{array}$ $\begin{array}{llllll}-613681.06 & -612407.7033 & -814465.54 & -813233.47 & -1015246.84\end{array}$
$-1489874.12$ $-1750475.21$ $-2440952.29$ $-2603259.12$ $-2160811.49$ $-1554103.43$ $-1648467.03$ $-1747887.00$ $-1347631.36$ $-2000883.60$ $-704298.27$ $-1696622.33$ $-1608836.79$ $-1712069.38$ $-1815303.82$ $-1815301.09$ $-1918537.73$ $-1918527.90$ $-2112342.53$ $-1766223.42$ $-2712234.83$ $-8262109.75$ $-1534092.14$ $-1806361.57$ $-1703192.94$ $-2042727.05$ $-1806391.84$ $-1747846.18$ $-1651008.64$ $-1754211.00$ $-1857407.94$ $-2390762.68$ $-3091417.61$ $-1948708.74$ $-1703200.15$ $-1900802.17$ $-1731698.40$ $-1703187.44$ $-1806389.44$ $-2106661.06$ $-2312487.94$ $-3912964.95$ $-1014031.04$

${ }^{a} p K_{a}^{E x p}$ :Experimental pKa value of acids in water..

${ }^{b} G$ : Gibbs free energies in $\left[\mathrm{kJ} \cdot \mathrm{mol}^{-1}\right]$. 


\section{TABLE S2: Experimental base $\mathrm{p} K_{\mathrm{a}}$ and Gibbs free energies of bare and mono-solvated aqueous bases.}

\begin{tabular}{|c|c|c|c|c|c|}
\hline Compound & $\mathbf{p K} \mathbf{K}_{\mathbf{A}}(\operatorname{Exp})^{a}$ & $\mathbf{G}(\mathbf{B})^{b}$ & $\mathbf{G}\left(\mathbf{B H}^{+}\right)^{b}$ & $\mathbf{G}\left(\mathbf{B}-\mathbf{H}_{2} \mathbf{O}\right)^{b}$ & $\mathbf{G}\left(\mathbf{B H}^{+}-\mathbf{H}_{2} \mathbf{O}\right)^{b}$ \\
\hline hydroxyimidazo(2,3-a)isoindole & 8.6 & -1747137.48 & -1748345.98 & -1947921.18 & -1949127.07 \\
\hline imidazo[2,3-b]thioxazole & 8 & -2088269.45 & -2089464.03 & -2289054.14 & -2290245.99 \\
\hline tetrahydrozoline & 10.5 & -1613792.59 & -1615003.04 & -1814578.71 & -1815782.50 \\
\hline tolazoline & 10.3 & -1307246.76 & -1308454.87 & -1508033.01 & -1509234.06 \\
\hline 4-chloroaniline & 4 & -1961939.83 & -1963111.75 & -2162726.60 & -2163895.85 \\
\hline 4-methoxyaniline & 5.2 & -1056058.83 & -1057240.34 & -1256843.90 & -1258022.80 \\
\hline 4-nitroaniline & 1 & -1292458.99 & -1293601.26 & -1493249.66 & -1494388.17 \\
\hline aniline & 4.6 & -755300.38 & -756473.89 & -956084.38 & -957257.27 \\
\hline p-toluidine & 5.1 & -858541.01 & -859720.79 & -1059327.74 & -1060501.29 \\
\hline 1,3-dihydro-1-methyl-5-phenyl-1,4-benzodiazepin-2-one & 3.3 & -2108407.13 & -2109575.74 & -2309189.75 & -2310358.39 \\
\hline 1,3-dihydro-3-hydroxy-1-methyl-5-phenyl-1,4-benzodiazepin-2-one & 1.6 & -2305987.13 & -2307135.73 & -2506764.53 & -2507919.42 \\
\hline 1,3-dihydro-3-hydroxy-5-phenyl-1,4-benzodiazepin-2-one & 1.7 & -2202786.39 & -2203937.88 & -2403564.50 & -2404721.34 \\
\hline 1,3-dihydro-5-phenyl-1,4-benzodiazepin-2-one & 3.5 & -2005206.89 & -2006377.61 & -2205989.94 & -2207160.32 \\
\hline 2,3-dihydro-1-methyl-5-phenyl-1,4-benzodiazepine & 6.2 & -1913920.45 & -1915112.51 & -2114706.02 & -2115893.12 \\
\hline 3-hydro-2-methylamine-4-oxy-5-phenyl-1,4-benzodiazepine & 4.8 & -2253639.16 & -2254814.48 & -2454417.22 & -2455594.42 \\
\hline clonidine & 8.1 & -3762692.20 & -3763881.63 & -3963474.07 & -3964658.48 \\
\hline debrisoquin & 11.9 & -1452665.25 & -1453894.53 & -1653451.72 & -1654672.75 \\
\hline guanidine & 13.8 & -539448.31 & -540688.56 & -740232.78 & -741462.12 \\
\hline methylguanidine & 13.4 & -642651.50 & -643885.65 & -843437.72 & -844661.98 \\
\hline 2-aminopyridine & 6.71 & -797461.96 & -798654.13 & -998245.68 & -999429.14 \\
\hline 2-aminothiazole & 5.4 & -1639571.03 & -1640750.59 & -1840353.64 & -1841526.22 \\
\hline 2-methylimidazole & 8 & -697390.05 & -698581.20 & -898175.66 & -899363.08 \\
\hline 3-aminopyridine & 6 & -797438.34 & -798628.33 & -998225.57 & -999410.08 \\
\hline 4-aminopyridine & 9.23 & -797457.41 & -798662.91 & -998245.21 & -999442.57 \\
\hline 4-methylpyridine & 6 & -755276.85 & -756457.67 & -956063.31 & -957240.03 \\
\hline benzimidazole & 5.8 & -997614.32 & -998790.94 & -1198398.63 & -1199573.66 \\
\hline imidazole & 7 & -594137.28 & -595324.27 & -794922.61 & -796106.36 \\
\hline isoquinoline & 5.4 & -1055491.82 & -1056669.13 & -1256277.98 & -1257451.97 \\
\hline melamine & 5 & -1172700.67 & -1173884.09 & -1373479.11 & -1374658.03 \\
\hline pyrazine & 0.7 & -694144.42 & -695290.37 & -894927.94 & -896077.80 \\
\hline pyrazole & 2.5 & -594086.49 & -595246.32 & -794869.01 & -796030.45 \\
\hline pyridine & 5.14 & -652027.79 & -653203.81 & -852813.94 & -853986.95 \\
\hline pyrimidine & 1.3 & -694163.24 & -695311.45 & -894946.47 & -896098.28 \\
\hline quinoline & 4.8 & -1055495.90 & -1056667.36 & -1256280.84 & -1257451.93 \\
\hline thiazole & 2.8 & -1494148.96 & -1495301.69 & -1694932.21 & -1696088.00 \\
\hline 1-methylindole & -2.3 & -1058643.42 & -1059758.17 & -1259414.79 & -1260531.24 \\
\hline 1-methylpyrrole & -2.9 & -655168.18 & -656276.09 & -855937.98 & -857051.70 \\
\hline 2-methylindole & -0.3 & -1058683.99 & -1059815.42 & -1259462.09 & -1260600.66 \\
\hline 2-methylpyrrole & -0.2 & -655207.87 & -656334.76 & -855980.94 & -857120.07 \\
\hline 3-methylindole & -4.6 & -1058679.73 & -1059780.14 & -1259454.52 & -1260565.65 \\
\hline 3-methylpyrrole & -1 & -655199.67 & -656331.26 & -855973.02 & -857113.34 \\
\hline indole & -3.6 & -955437.39 & -956547.49 & -1156212.31 & -1157332.55 \\
\hline pyrrole & -3.8 & -551960.55 & -553071.38 & -752735.34 & -753853.44 \\
\hline
\end{tabular}

${ }^{a} p \mathrm{~K}_{a}^{E x p}$ :Experimental pKa value of bases in water.

${ }^{b} G$ : Gibbs free energies in $\left[\mathrm{kJ} \cdot \mathrm{mol}^{-1}\right]$. 
TABLE S3: Experimental aqueous base $\mathrm{p} K_{\mathrm{a}}$ and Gibbs free energies of bare and monosolvated aliphatic amines.

\begin{tabular}{|c|c|c|c|c|c|c|}
\hline Compound & Base-Type & $\mathbf{p K}_{\mathrm{A}}(\operatorname{Exp})$ & G(B) & $\mathbf{G}\left(\mathbf{B H}^{+}\right)$ & $\mathbf{G}\left(\mathbf{B}-\mathrm{H}_{2} \mathrm{O}\right)$ & $\mathbf{G}\left(\mathbf{B H}^{+}-\mathbf{H}_{2} \mathbf{O}\right)$ \\
\hline methylamine & primary & 10.6 & -251766.85 & & -452559.44 & \\
\hline ethylamine & imary & 10.9 & -355007.93 & -356230.91 & -555799.60 & -557010.6987 \\
\hline propylamine & & 10.7 & -458241.12 & -459463.14 & -659032.76 & -660243.044 \\
\hline butylamine & imary & 10.8 & -561474.48 & -562696.40 & -762266.01 & -763476.2888 \\
\hline pentylamine & imary & 10.6 & -664708.10 & -665929.85 & -865499.55 & -866709.865 \\
\hline hexylamine & imary & 10.6 & -767941.75 & -769163.33 & -968733.09 & -969942.734 \\
\hline heptylamine & rimary & 10.7 & -871175.29 & -872396.82 & -1071966.63 & -1073176.187 \\
\hline & primary & 10.7 & $-9744 c$ & -97563 & -1175200.31 & 409.932 \\
\hline ylamine & & 10.6 & -4582 & & & 1431 \\
\hline & & 10.56 & & & & \\
\hline & & & & & & \\
\hline & & & & & & \\
\hline & & & -76 & & & \\
\hline & & & & & & \\
\hline & & & & & & \\
\hline amine & & 11 & $-76^{7}$ & & & \\
\hline amine & & & -767 & & & 1912 \\
\hline & & & -974 & & & 8.07 \\
\hline & & & -9743 & -97 & & .403 \\
\hline mine & & 11.01 & -9743 & -975 & & .912 \\
\hline lohexylamine & condary & 11.23 & -1177 & -117 & & \\
\hline & & & -4581 & & & \\
\hline & & & & & & \\
\hline & & & -66 & -66 & & 592 \\
\hline & & & & & & \\
\hline & & & & & & \\
\hline & & & & & & \\
\hline & & & & & & \\
\hline & & & & & & \\
\hline & & & & & & \\
\hline i-n & & & & -1078783.91 & -1278373.11 & -1279567.279 \\
\hline i-n-butylamine & tertiary & 10.89 & -1387289.99 & -1388483.44 & -1588073.28 & -1589267.115 \\
\hline
\end{tabular}
${ }^{a} p \mathrm{~K}_{a}{ }^{E x p}$ :Experimental $\mathrm{pKa}$ value of bases in water.

${ }^{b} G$ : Gibbs free energies in $\left[\mathrm{kJ} \cdot \mathrm{mol}^{-1}\right]$. 
TABLE S4: Optimized Geometries (sdf-format). Different conformers of one compound are denoted by subsequent index numbers $0,1,2, \ldots$

\section{S4 a) Bare Acids \& Anions}

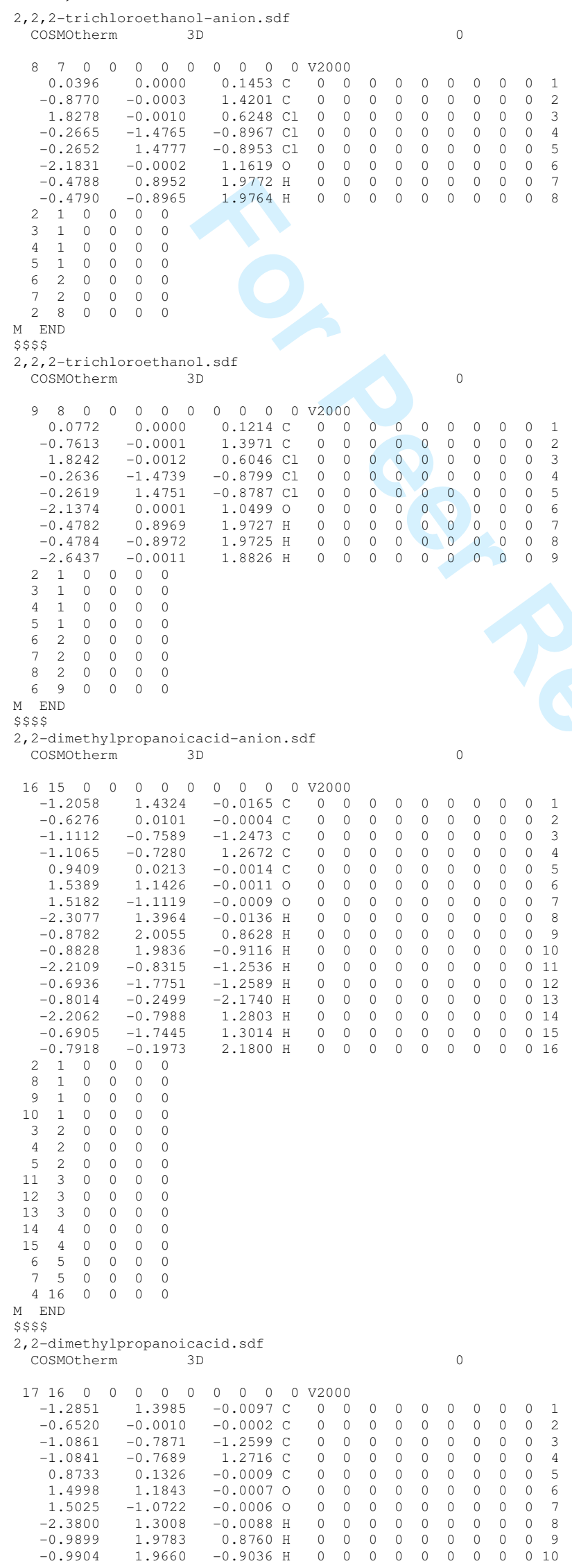



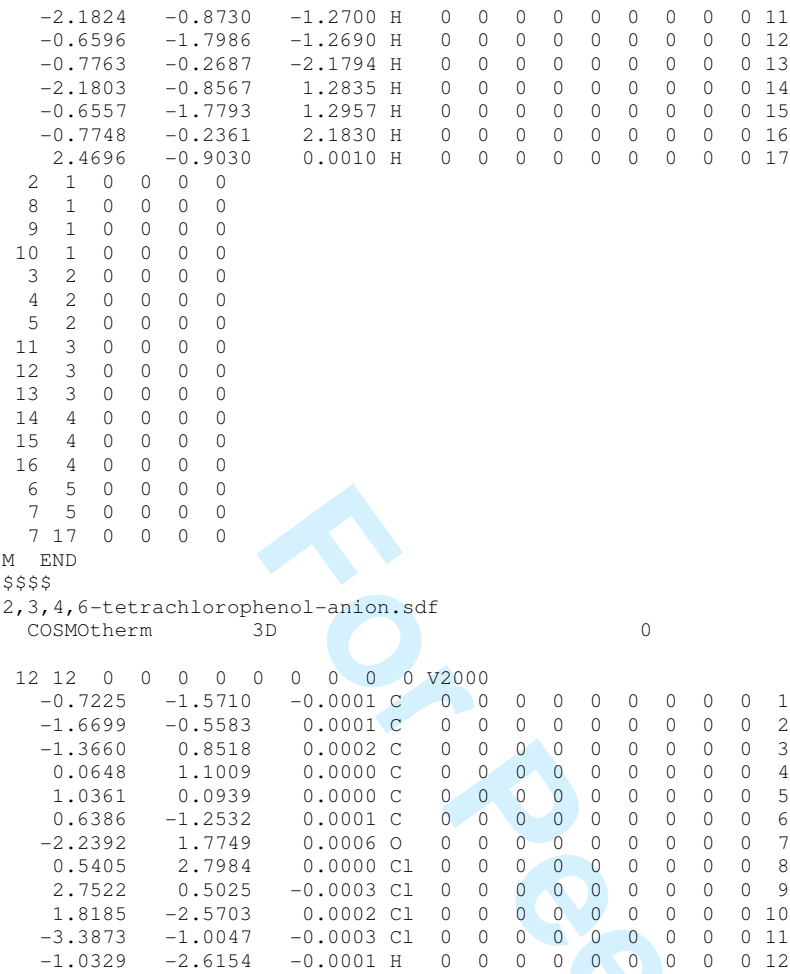

$-1.0329-2.6154$

$\begin{array}{lllll}2 & 1 & 0 & 0 & 0 \\ 6 & 1 & 0 & 0 & 0\end{array}$

$\begin{array}{llllll}6 & 1 & 0 & 0 & 0 & 0 \\ 3 & 2 & 0 & 0 & 0 & 0\end{array}$

$\begin{array}{llllll}11 & 2 & 0 & 0 & 0 & 0\end{array}$

$\begin{array}{lllllll}7 & 3 & 0 & 0 & 0 & 0 \\ 7 & 3 & 0 & 0 & 0 & 0\end{array}$

$\begin{array}{lllll}5 & 0 & 0 & 0 & 0\end{array}$

$\begin{array}{llll}4 & 0 & 0 & 0\end{array}$

$\begin{array}{lllll}5 & 0 & 0 & 0 & 0\end{array}$

$\begin{array}{rrrrr}6 & 0 & 0 & 0 & 0 \\ 12 & 0 & 0 & 0 & 0\end{array}$

1
END

$\$ \$ \$ \$$

2, 3, 4, 6-tetrachlorophenol.sdf

cosmotherm

$\begin{array}{llllllllllll}13 & 13 & 0 & 0 & 0 & 0 & 0 & 0 & 0 & 0 & 0 & \mathrm{~V} 2000\end{array}$

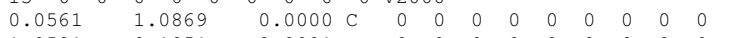

$\begin{array}{llllllllllllll}1.0591 & 0.1051 & -0.0001 & \mathrm{C} & 0 & 0 & 0 & 0 & 0 & 0 & 0 & 0 & 0 & 2\end{array}$

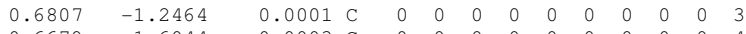

$\begin{array}{llllllllllllll}-0.6679 & -1.6044 & 0.0003 & C & 0 & 0 & 0 & 0 & 0 & 0 & 0 & 0 & 0 & 4\end{array}$

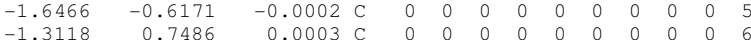

$\begin{array}{lllllllllllll}1.8867 & -2.5231 & 0.0002 & \mathrm{Cl} & 0 & 0 & 0 & 0 & 0 & 0 & 0 & 0 & 0\end{array}$

$\begin{array}{llllllllllllll}-2.3000 & 1.6709 & 0.0007 & 0 & 0 & 0 & 0 & 0 & 0 & 0 & 0 & 0 & 0 & 8\end{array}$

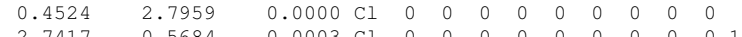

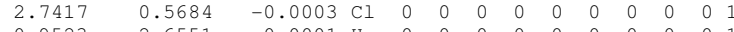

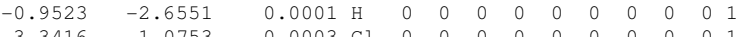

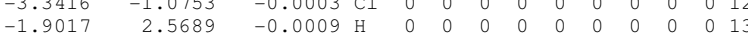

$-1.901700$

$\begin{array}{lllllll}6 & 1 & 0 & 0 & 0 & 0\end{array}$

$\begin{array}{lllll}1 & 0 & 0 & 0 & 0\end{array}$

$\begin{array}{lllll}3 & 0 & 0 & 0\end{array}$

$\begin{array}{lllll} & 2 & 0 & 0 & 0\end{array}$

$\begin{array}{lllll}3 & 0 & 0 & 0\end{array}$

4000

$\begin{array}{lllll}4 & 0 & 0 & 0 & 0\end{array}$

$\begin{array}{llllll}5 & 5 & 0 & 0 & 0 & 0\end{array}$

$\begin{array}{lllll}6 & 0 & 0 & 0 & 0\end{array}$

END

2,3,4-trichlorophenol0-anion.sdf

Cosmotherm 3D

$\begin{array}{llllllllllll}12 & 12 & 0 & 0 & 0 & 0 & 0 & 0 & 0 & 0 & 0 & \mathrm{v} 2000\end{array}$

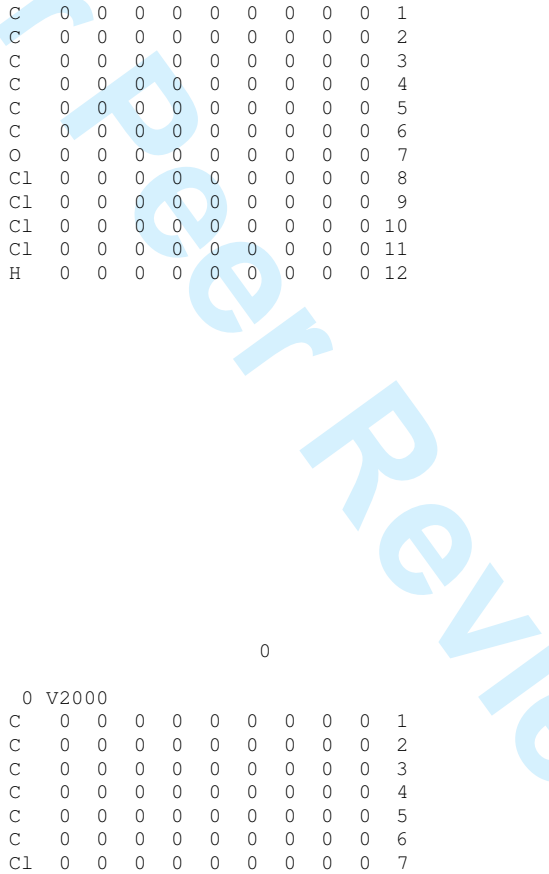

$\begin{array}{llllllllllll}0.0002 & \mathrm{C} & 0 & 0 & 0 & 0 & 0 & 0 & 0 & 0 & 0 & 1\end{array}$

$\begin{array}{llllllllllllll}2.0277 & -0.8240 & -0.0005 & \mathrm{C} & 0 & 0 & 0 & 0 & 0 & 0 & 0 & 0 & 0 & 2\end{array}$

$\begin{array}{llllllllllllll}-0.3328 & -0.2935 & -0.0001 & \mathrm{C} & 0 & 0 & 0 & 0 & 0 & 0 & 0 & 0 & 0 & 4\end{array}$

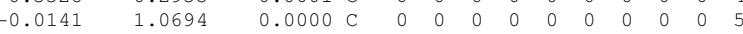

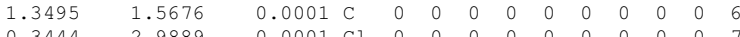

$\begin{array}{lrlllllllllll}1.3444 & -2.9889 & 0.0001 & \mathrm{Cl} & 0 & 0 & 0 & 0 & 0 & 0 & 0 & 0 & 0 \\ 1.6345 & 2.8151 & 0.0005 & 0 & 0 & 0 & 0 & 0 & 0 & 0 & 0 & 0 & 0\end{array}$

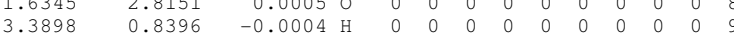

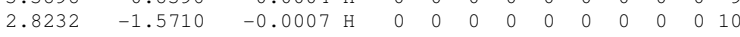

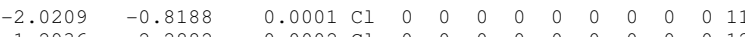

2.2882

$\begin{array}{llllll}-1 & 1 & 0 & 0 & 0 & \\ 6 & 1 & 0 & 0 & 0 & 0\end{array}$ 

$\begin{array}{rrrrrr}9 & 1 & 0 & 0 & 0 & 0 \\ 3 & 2 & 0 & 0 & 0 & 0 \\ 10 & 2 & 0 & 0 & 0 & 0 \\ 4 & 3 & 0 & 0 & 0 & 0 \\ 7 & 3 & 0 & 0 & 0 & 0 \\ 5 & 4 & 0 & 0 & 0 & 0 \\ 11 & 4 & 0 & 0 & 0 & 0 \\ 6 & 5 & 0 & 0 & 0 & 0 \\ 8 & 6 & 0 & 0 & 0 & 0 \\ 5 & 12 & 0 & 0 & 0 & 0 \\ \text { M END } & & & & \end{array}$ 


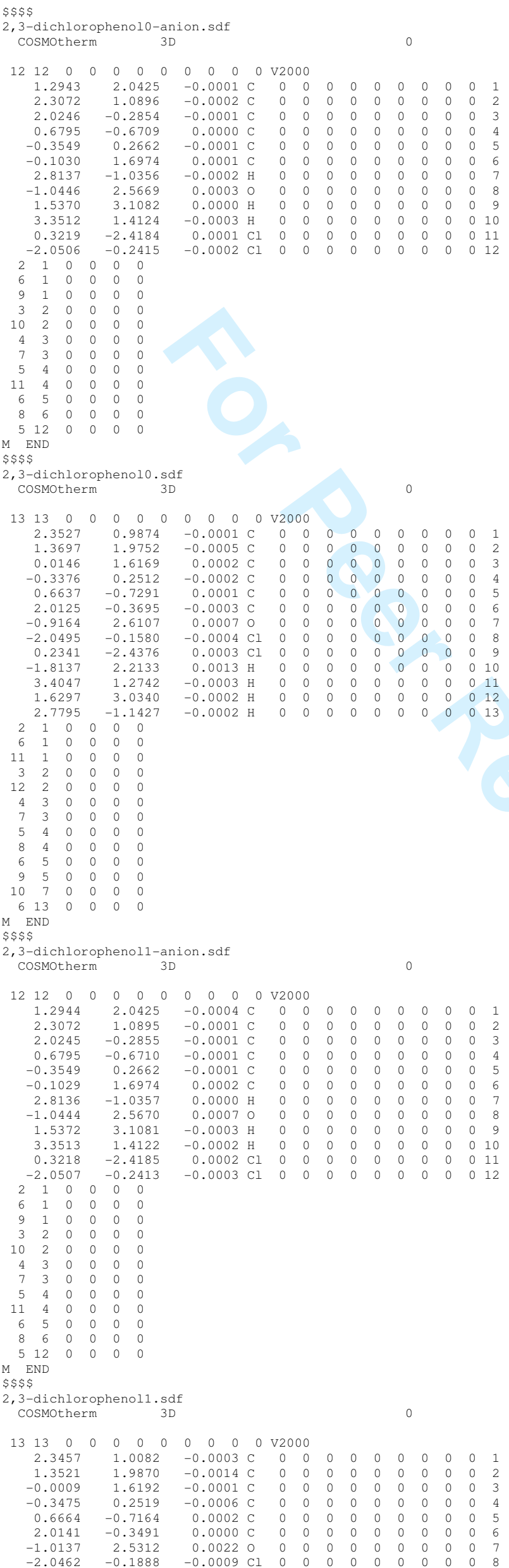




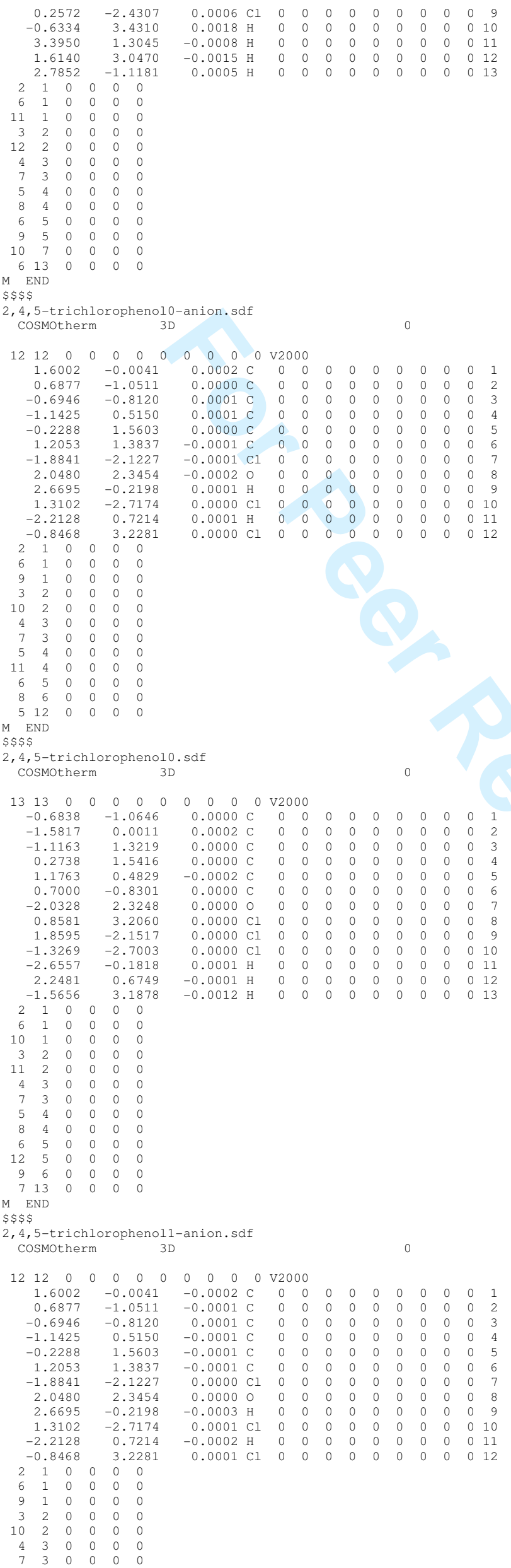




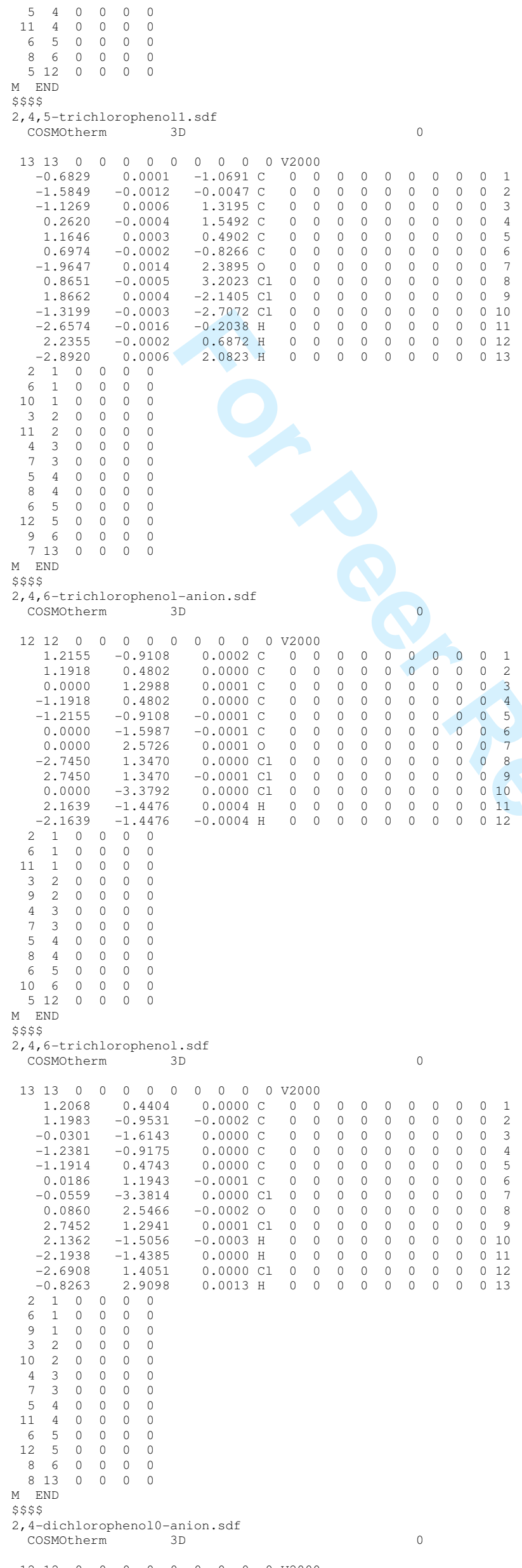




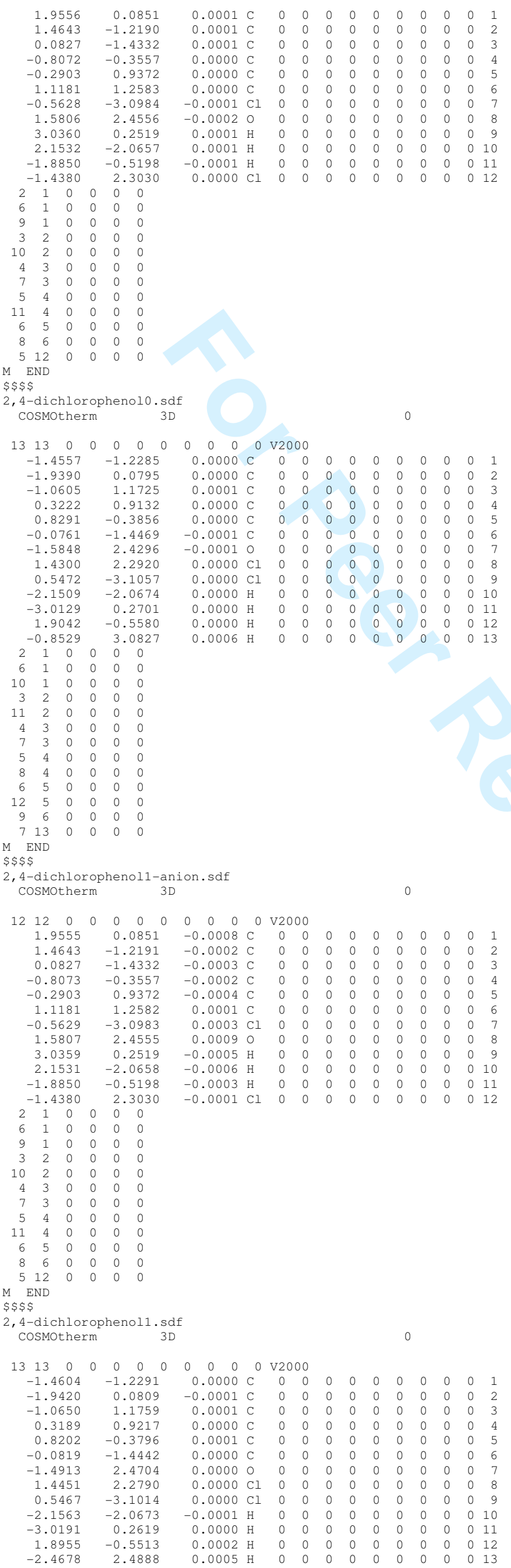




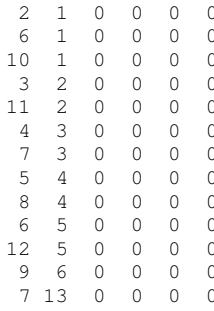




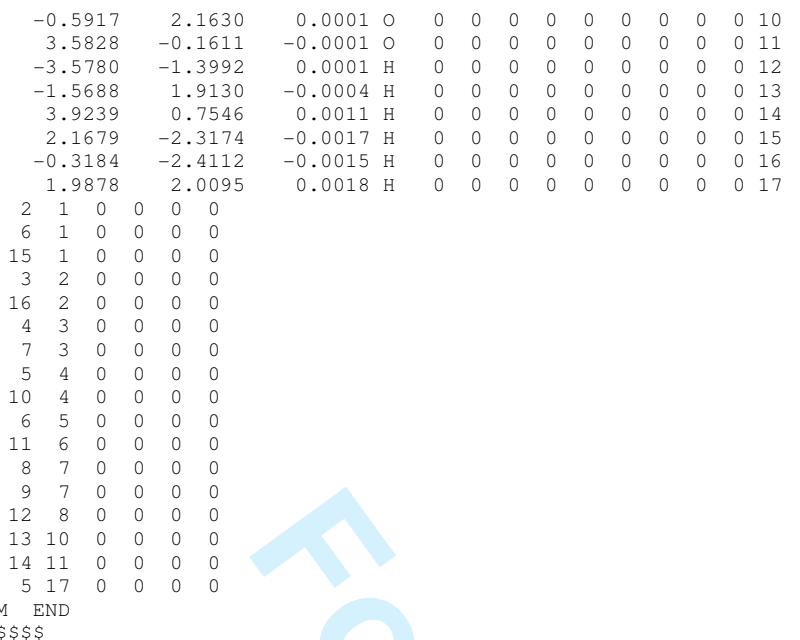




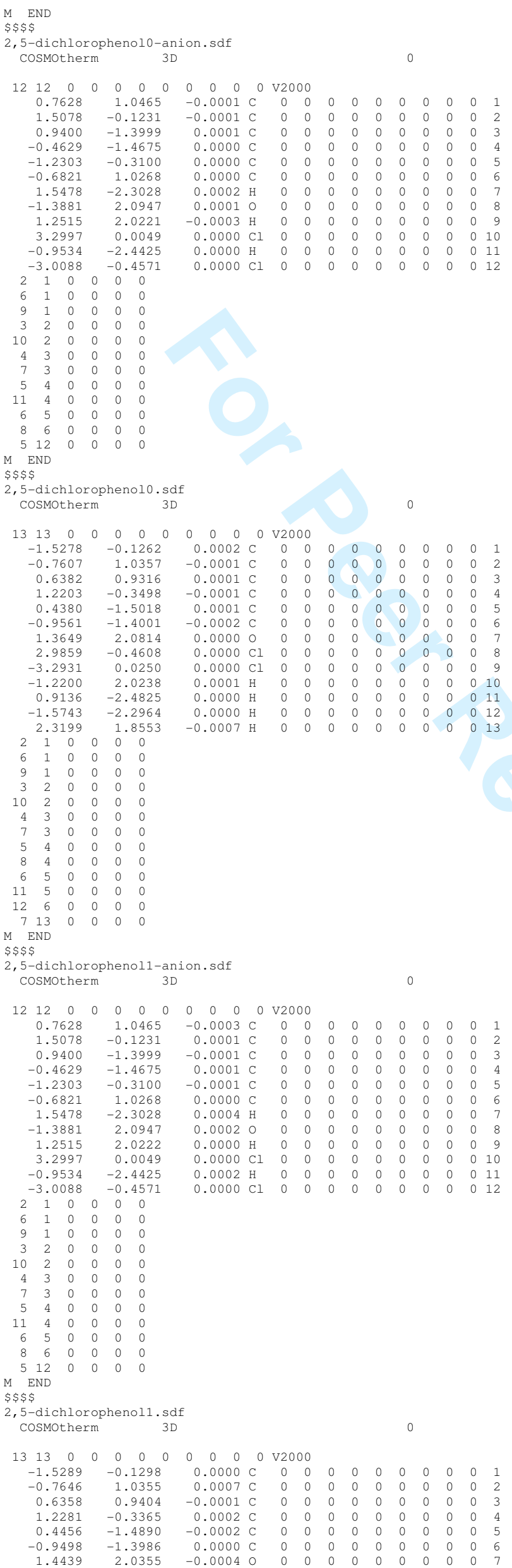




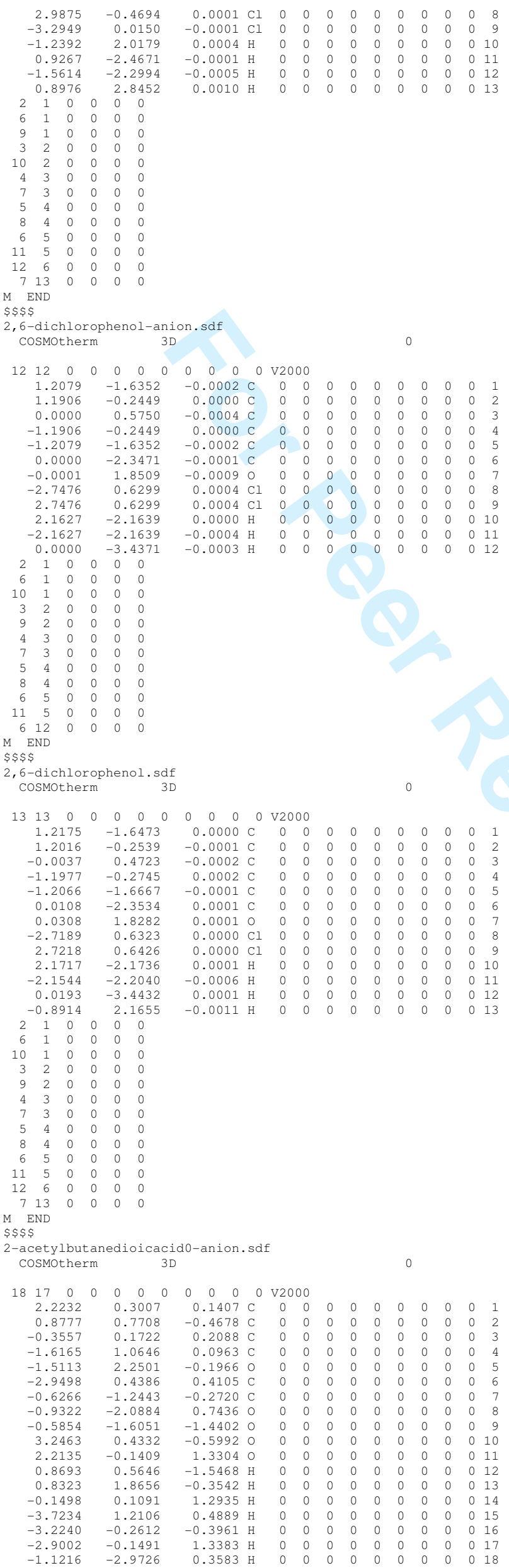




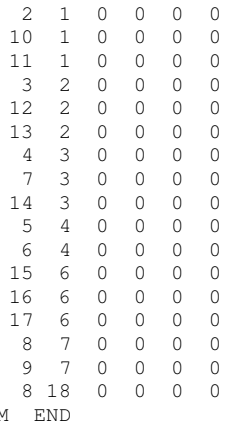




\section{Page 47 of 461}

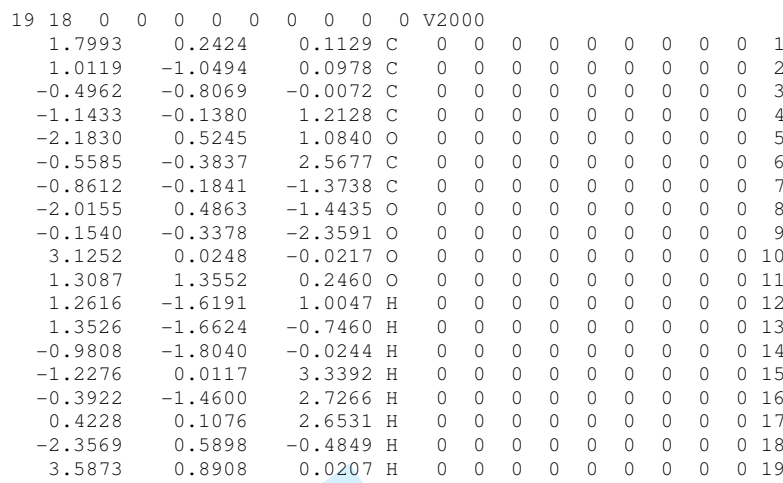




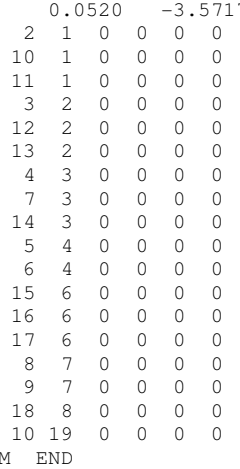




\section{Page 49 of 461}

1
2
3
4
5
6
7
8
9
10
11
12
13
14
15
16
17
18
19
20
21
22
23
24
25
26
27
28
29
30
31
32
33
34
35
36
37
38
39
40
41
42
43
44
45
46
47
48
49
50
51
52
53
54
55
56
57
58
59
60

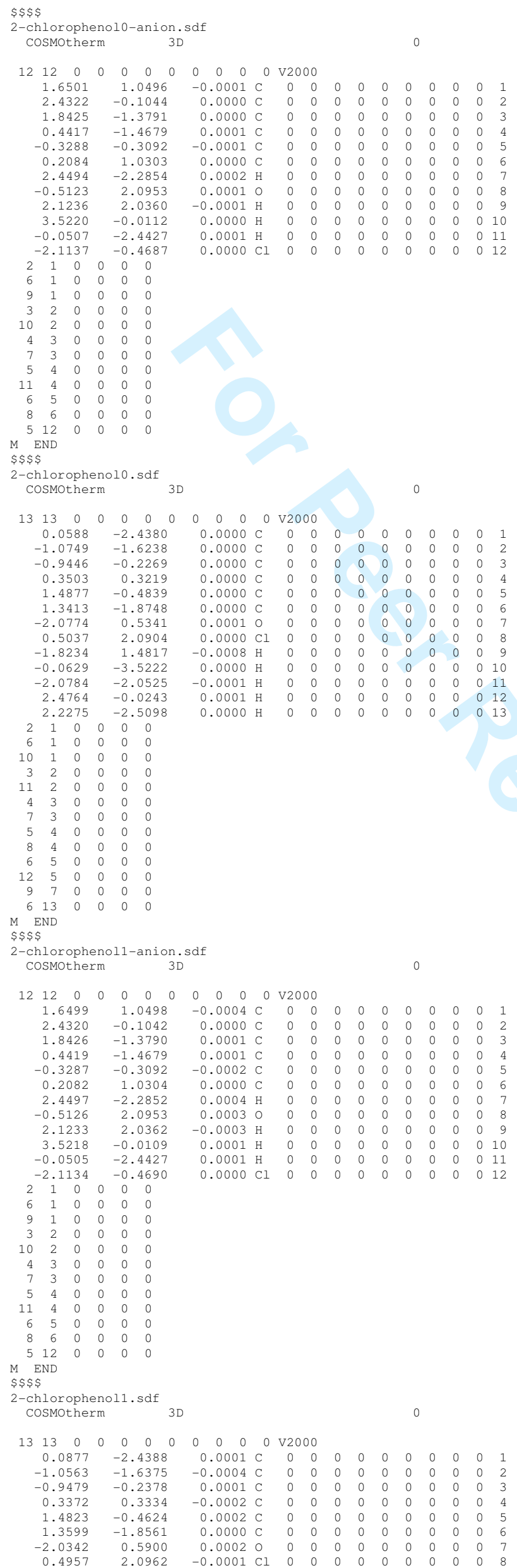




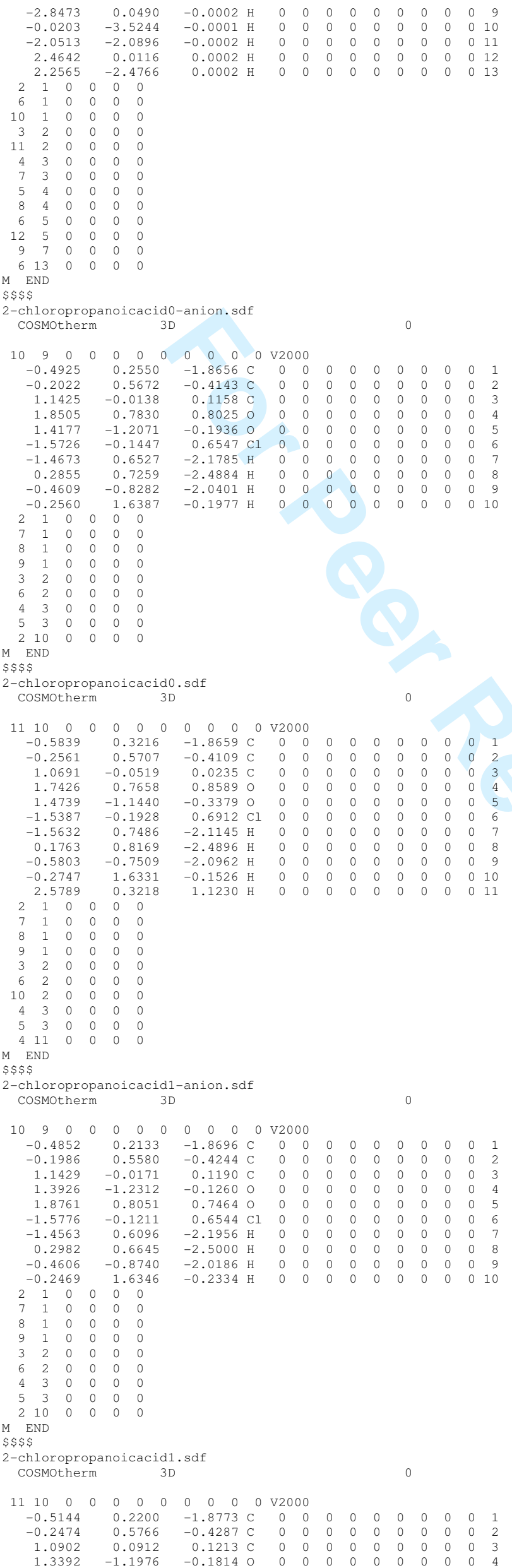




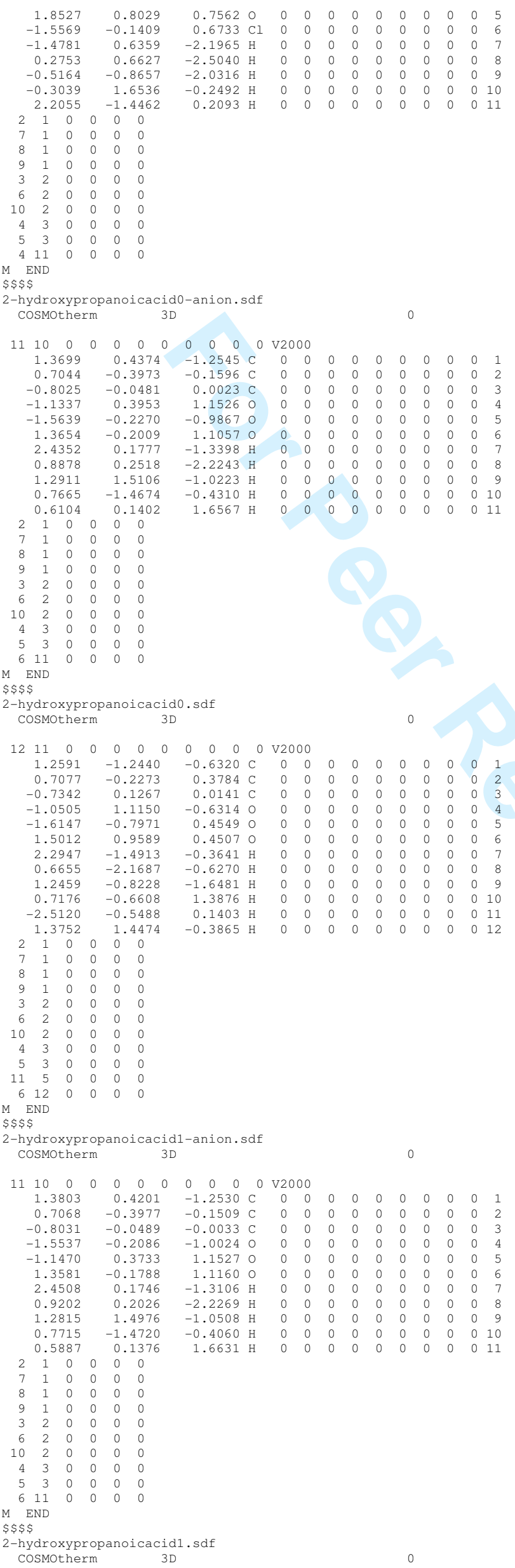



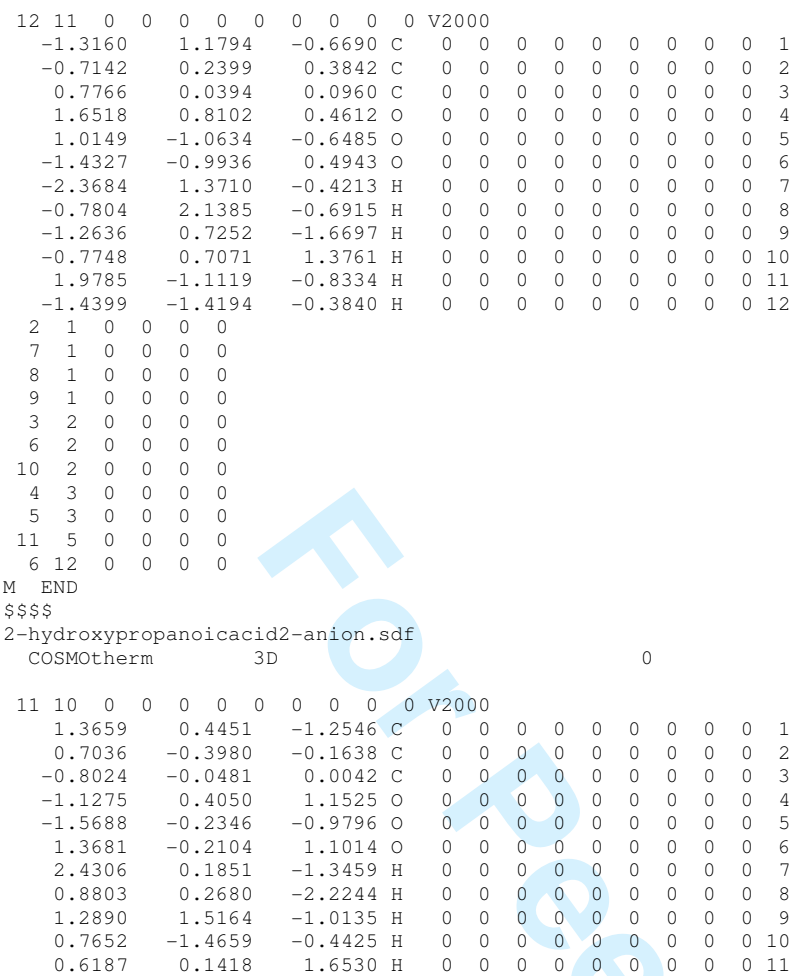

0.1418

$\begin{array}{llllll}2 & 1 & 0 & 0 & 0 & 0 \\ 7 & 1 & 0 & 0 & 0 & 0\end{array}$

8 1 1100000

$\begin{array}{lllll}1 & 0 & 0 & 0 & 0\end{array}$

$\begin{array}{lllll}2 & 0 & 0 & 0 & 0 \\ 2 & 0 & 0 & 0 & 0\end{array}$

$\begin{array}{llllll}2 & 0 & 0 & 0 & 0\end{array}$

$\begin{array}{lllll}3 & 0 & 0 & 0 & 0 \\ 3 & 0 & 0 & 0 & 0\end{array}$

$\begin{array}{lllll}11 & 0 & 0 & 0 & 0\end{array}$

$M$ END

$\$ \$$

-hydroxypropanoicacid2.sdf

$\begin{array}{llllllllllll}12 & 11 & 0 & 0 & 0 & 0 & 0 & 0 & 0 & 0 & 0 & \mathrm{~V} 2000\end{array}$

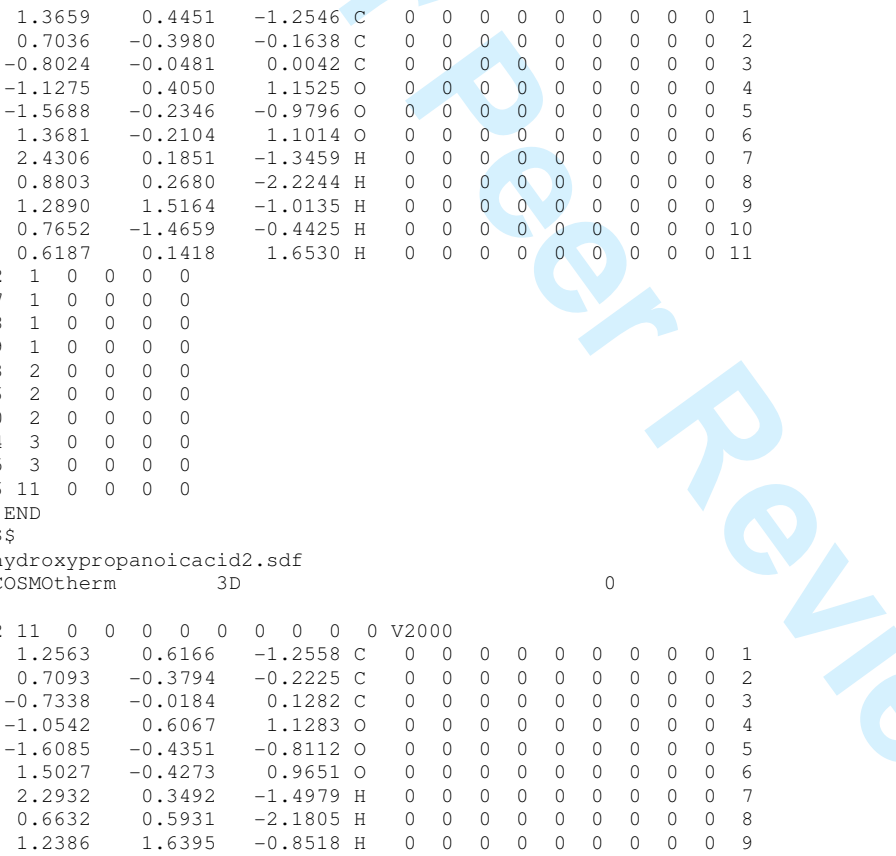

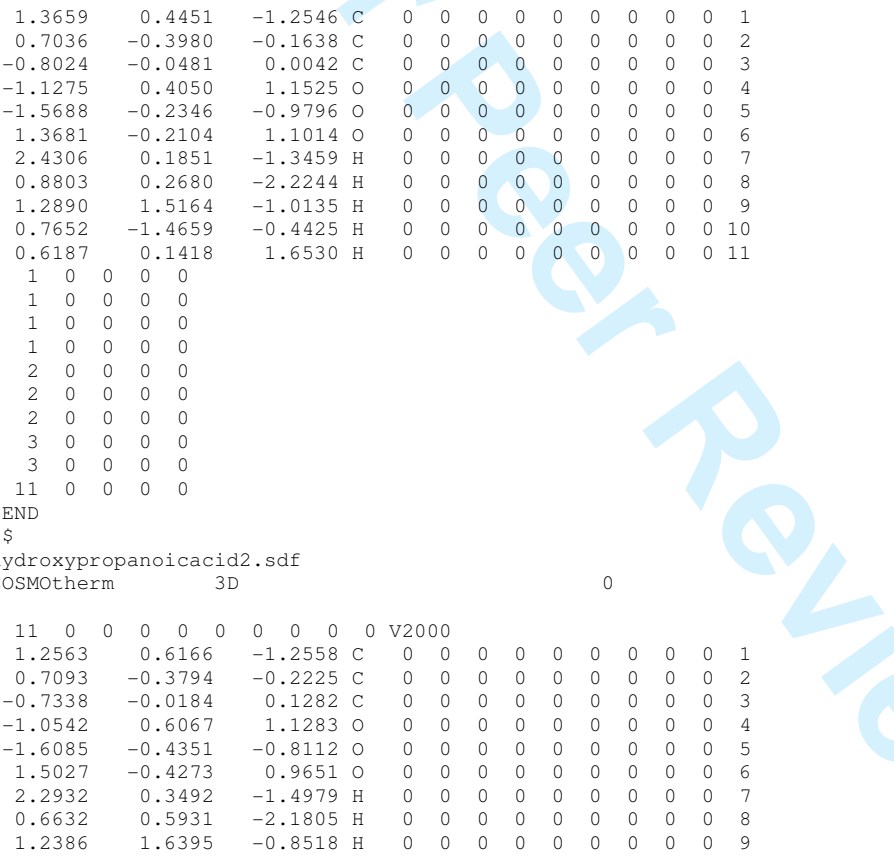

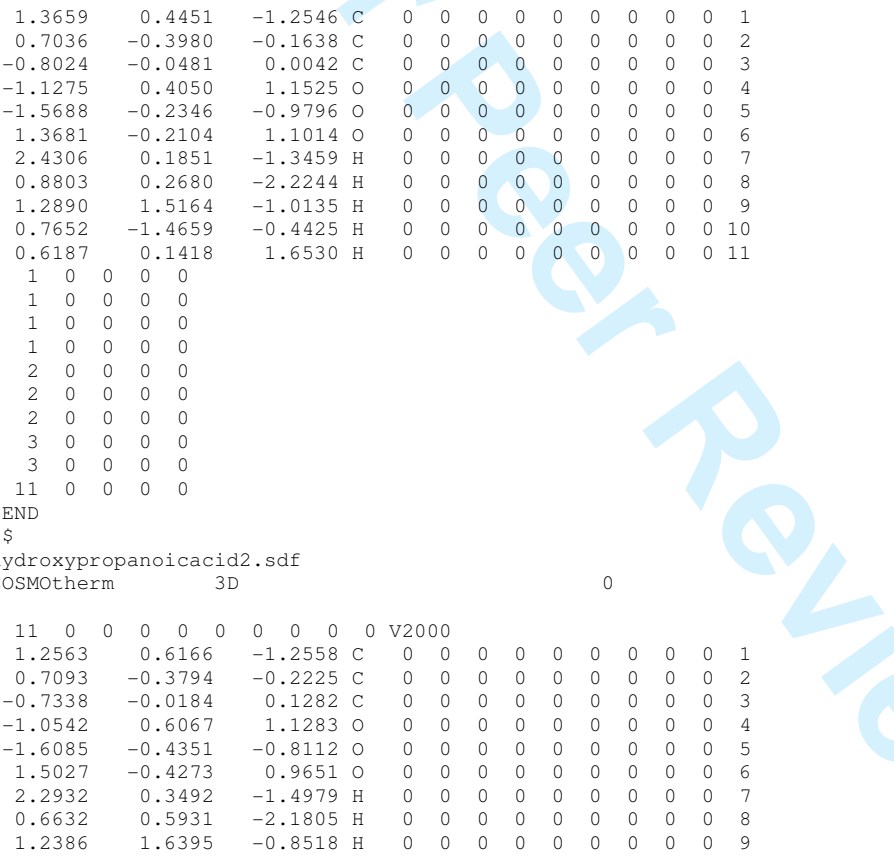

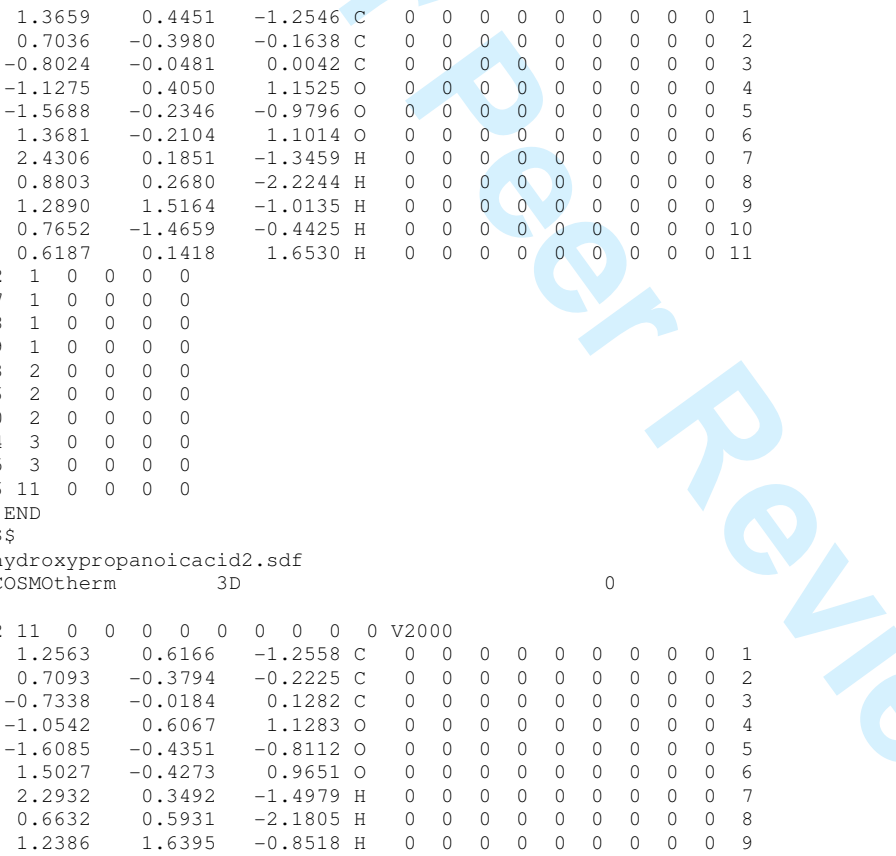

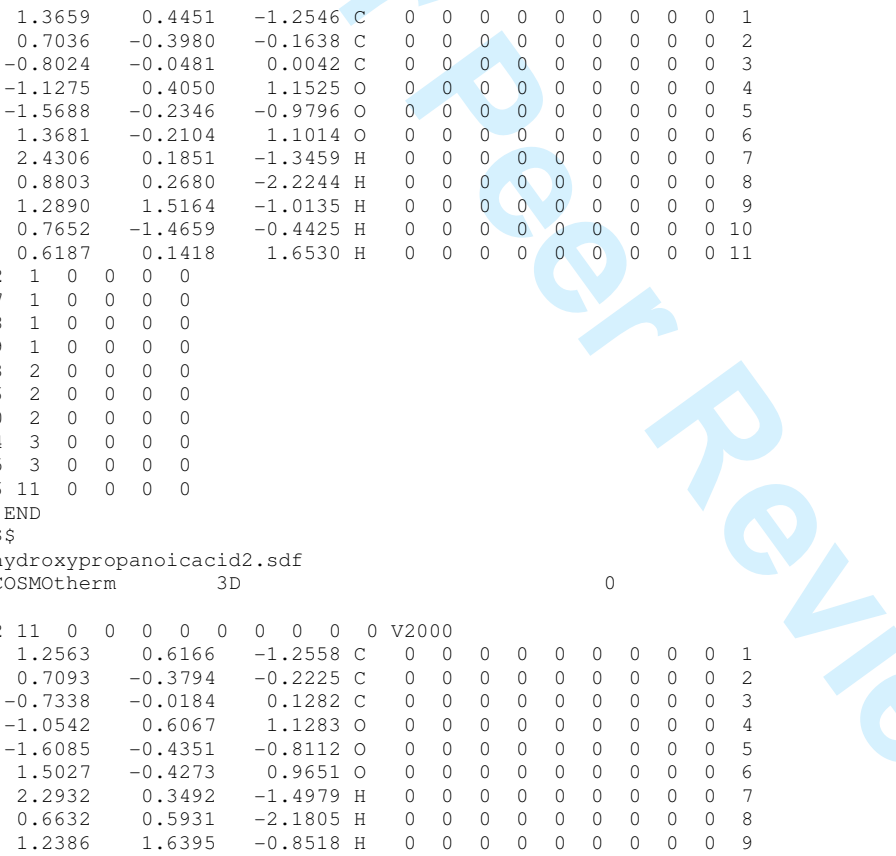

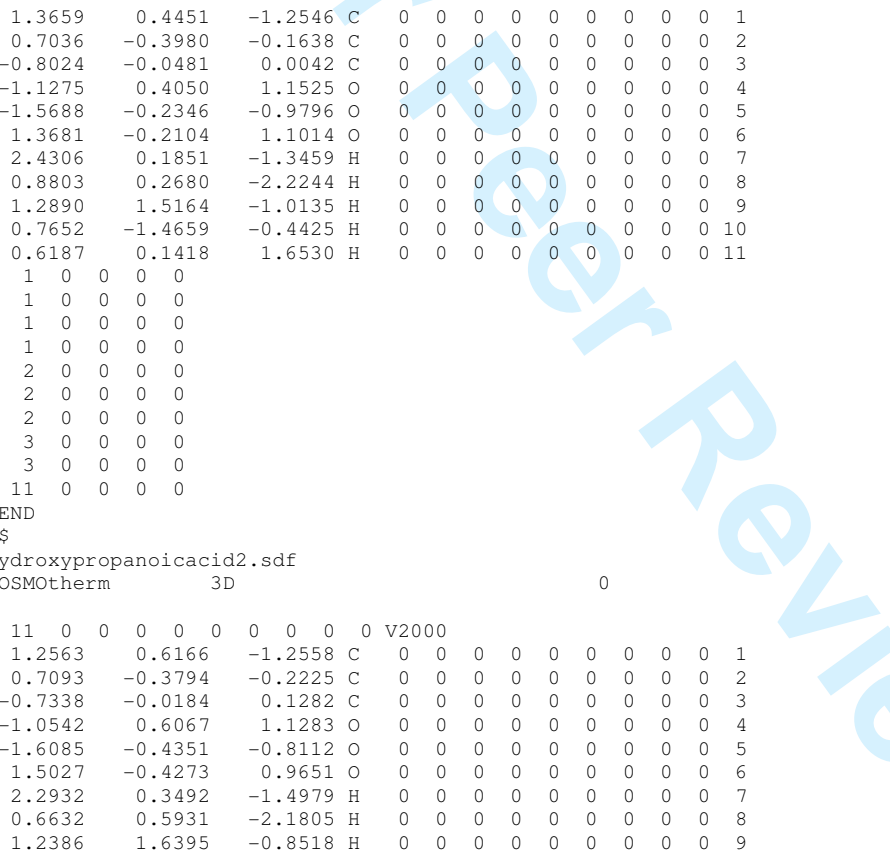

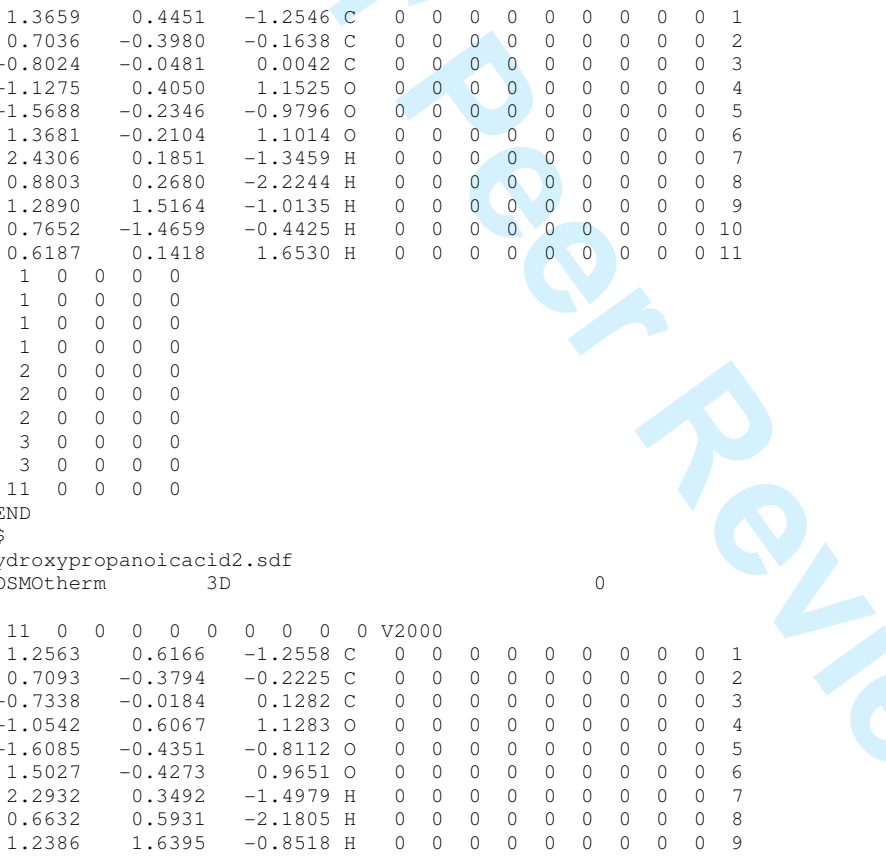

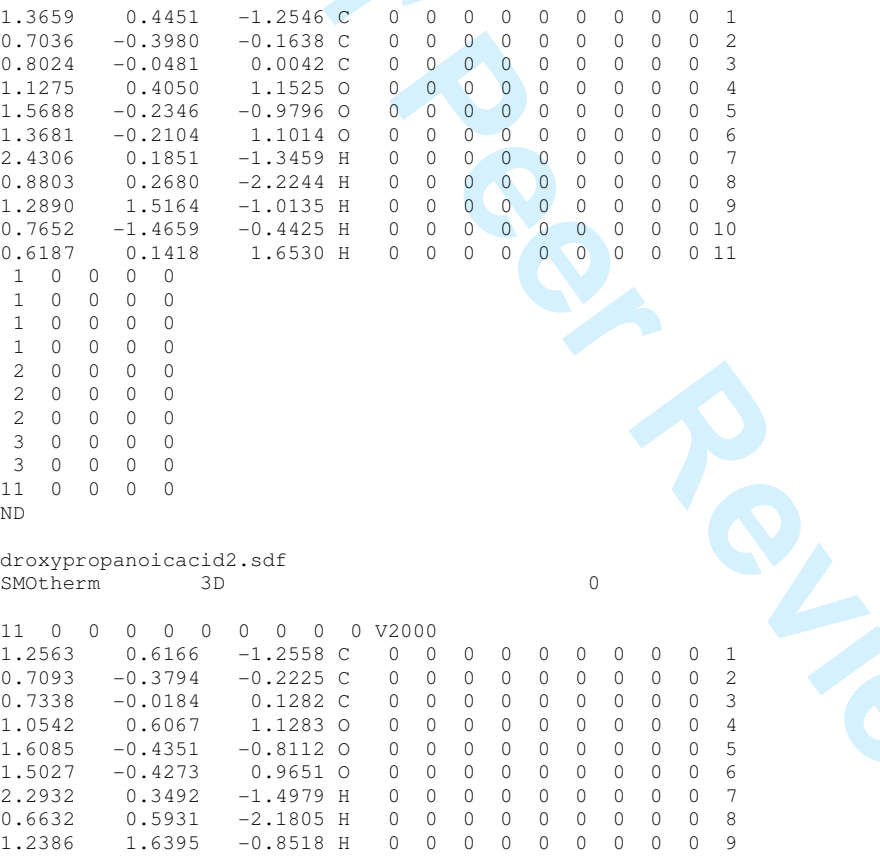

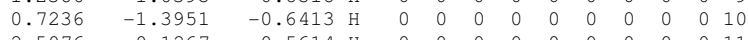

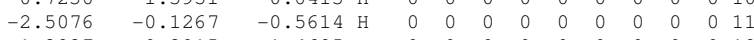

$1.3237-0.3915$

$\begin{array}{llllll}2 & 1 & 0 & 0 & 0 & 0 \\ 7 & 1 & 0 & 0 & 0 & 0\end{array}$

$\begin{array}{lllllll}1 & 0 & 0 & 0 & 0\end{array}$

$\begin{array}{lllll}1 & 0 & 0 & 0 & 0\end{array}$

$\begin{array}{lllll}2 & 0 & 0 & 0 & 0\end{array}$

$\begin{array}{lllllllll}6 & 2 & 0 & 0 & 0 & 0 & 0\end{array}$

$\begin{array}{llllll}4 & 3 & 0 & 0 & 0 & 0\end{array}$

$\begin{array}{lllll}3 & 0 & 0 & 0 & 0\end{array}$

$\begin{array}{lllll}12 & 0 & 0 & 0 & 0\end{array}$

END

,3-dimethylsuccinimide0-anion.sdf

cosmotherm

$\begin{array}{llllllllllll}17 & 17 & 0 & 0 & 0 & 0 & 0 & 0 & 0 & 0 & 0 & \mathrm{~V} 2000\end{array}$

$\begin{array}{llllllllll}0 & 0 & 0 & 0 & 0 & 0 & 0 & 0 & 0 & 1\end{array}$

$\begin{array}{lllllllllllll}0.0 .0001 & c & 0 & 0 & 0 & 0 & 0 & 0 & 0 & 0 & 0 & 0 & 2\end{array}$

$\begin{array}{llllllllllllll}-0.4141 & 0.9411 & -0.0001 & \mathrm{C} & 0 & 0 & 0 & 0 & 0 & 0 & 0 & 0 & 0 & 3\end{array}$

$\begin{array}{lllllllllllll}0.9388 & 1.1404 & 0.0004 & \mathrm{~N} & 0 & 0 & 0 & 0 & 0 & 0 & 0 & 0 & 0\end{array}$

$\begin{array}{llllllllllllll}1.5653 & -0.0734 & 0.0000 & \mathrm{C} & 0 & 0 & 0 & 0 & 0 & 0 & 0 & 0 & 0 & 5\end{array}$

$\begin{array}{llllllllllllll}-1.6317 & -0.8790 & 1.2624 & \mathrm{C} & 0 & 0 & 0 & 0 & 0 & 0 & 0 & 0 & 0 & 6\end{array}$

$\begin{array}{llllllllllllll}1.6321 & -0.8795 & -1.2621 & \mathrm{C} & 0 & 0 & 0 & 0 & 0 & 0 & 0 & 0 & 0 & \end{array}$

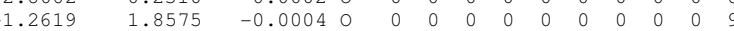

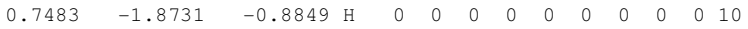

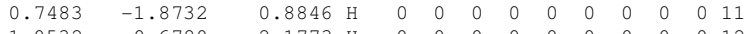

$\begin{array}{llllllllllllll}-1.0532 & -0.6780 & 2.1773 & \mathrm{H} & 0 & 0 & 0 & 0 & 0 & 0 & 0 & 0 & 0 & 12\end{array}$

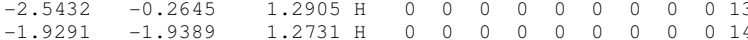

$\begin{array}{lllllllllllll}-1.0540 & -0.6781 & -2.1771 & \mathrm{H} & 0 & 0 & 0 & 0 & 0 & 0 & 0 & 0 & 0 \\ -15\end{array}$

$\begin{array}{lllllllllllllll}-2.5439 & -0.2656 & -1.2898 \mathrm{H} & 0 & 0 & 0 & 0 & 0 & 0 & 0 & 0 & 0 & 15\end{array}$

$\begin{array}{lllllllllllllll}-1.9288 & -1.9396 & -1.2726 & \mathrm{H} & 0 & 0 & 0 & 0 & 0 & 0 & 0 & 0 & 0 & 17\end{array}$

$\begin{array}{llllll}1 & 0 & 0 & 0 & 0\end{array}$ 


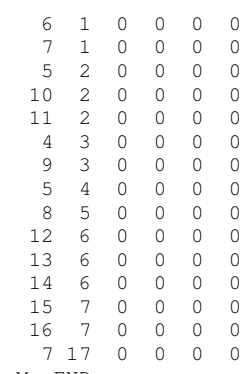




\section{Page 55 of 461}

$\begin{array}{rrrrrr}4 & 3 & 0 & 0 & 0 & 0 \\ 9 & 3 & 0 & 0 & 0 & 0 \\ 5 & 4 & 0 & 0 & 0 & 0 \\ 8 & 5 & 0 & 0 & 0 & 0 \\ 12 & 6 & 0 & 0 & 0 & 0 \\ 13 & 6 & 0 & 0 & 0 & 0 \\ 14 & 6 & 0 & 0 & 0 & 0 \\ 15 & 7 & 0 & 0 & 0 & 0 \\ 16 & 7 & 0 & 0 & 0 & 0 \\ 17 & 7 & 0 & 0 & 0 & 0 \\ 9 & 18 & 0 & 0 & 0 & 0\end{array}$ $M \quad$ END

\$\$\$ $\$$-methylphenylglutarimide0-anion.sdf

cosmotherm $3 \mathrm{D}$

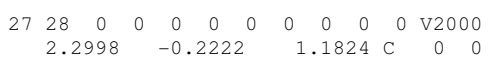

$\begin{array}{llllllllllllll}2.2998 & -0.2222 & 1.1824 & \mathrm{C} & 0 & 0 & 0 & 0 & 0 & 0 & 0 & 0 & 0 & 1\end{array}$

$\begin{array}{rrrrrrrrrrrrrr}2.9743 & -0.2331 & 0.0011 & \mathrm{~N} & 0 & 0 & 0 & 0 & 0 & 0 & 0 & 0 & 0 & 2 \\ 2.3027 & -0.2092 & -1.1818 & \mathrm{C} & 0 & 0 & 0 & 0 & 0 & 0 & 0 & 0 & 0 & 3\end{array}$

$\begin{array}{llllllllllllll}2.3027 & -0.2092 & -1.1818 & \mathrm{C} & 0 & 0 & 0 & 0 & 0 & 0 & 0 & 0 & 0 & 3 \\ 0.7730 & -0.1887 & -1.2395 & \mathrm{C} & 0 & 0 & 0 & 0 & 0 & 0 & 0 & 0 & 0 & 4\end{array}$

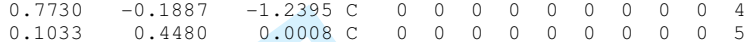

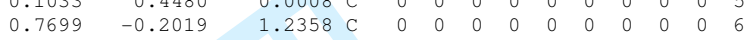

$\begin{array}{llllllllllllll}2.9287 & -0.2351 & -2.2718 & 0 & 0 & 0 & 0 & 0 & 0 & 0 & 0 & 0 & 0 & 7\end{array}$

$\begin{array}{llllllllllllll}2.9225 & -0.2599 & 2.2736 & 0 & 0 & 0 & 0 & 0 & 0 & 0 & 0 & 0 & 0 & 8\end{array}$

$\begin{array}{rrrrrrrrrrrrrr}-1.4053 & 0.1477 & -0.0019 & \mathrm{C} & 0 & 0 & 0 & 0 & 0 & 0 & 0 & 0 & 0 & 9 \\ 0.3945 & 1.9607 & 0.0089 & \mathrm{C} & 0 & 0 & 0 & 0 & 0 & 0 & 0 & 0 & 0 & 10\end{array}$

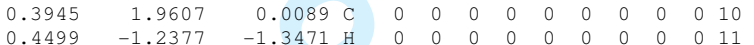

$\begin{array}{llllllllllllll}0.4719 & 0.3297 & -2.1614 & \mathrm{H} & 0 & 0 & 0 & 0 & 0 & 0 & 0 & 0 & 0 & 12\end{array}$

$\begin{array}{lllllllllllll}0.4464 & -1.2521 & 1.3308 \mathrm{H} & 0 & 0 & 0 & 0 & 0 & 0 & 0 & 0 & 0 & 13\end{array}$

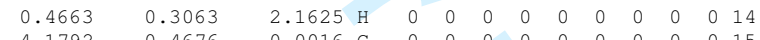

$\begin{array}{llllllllllllll}-4.1792 & -0.4676 & -0.0016 & \mathrm{C} & 0 & 0 & 0 & 0 & 0 & 0 & 0 & 0 & 0 & 15\end{array}$

$\begin{array}{llllllllllllll}-3.2250 & -1.4932 & -0.0162 & \mathrm{C} & 0 & 0 & 0 & 0 & 0 & 0 & 0 & 0 & 0 & 16\end{array}$

$\begin{array}{rrrrrrrrrrrrrr}-1.8620 & -1.1863 & -0.0163 & \mathrm{C} & 0 & 0 & 0 & 0 & 0 & 0 & 0 & 0 & 0 & 17 \\ -2.3778 & 1.1625 & 0.0125 & \mathrm{C} & 0 & 0 & 0 & 0 & 0 & 0 & 0 & 0 & 0 & 18\end{array}$

$\begin{array}{lllllllllllllll}-3.7472 & 0.8609 & 0.0125 & \mathrm{C} & 0 & 0 & 0 & 0 & 0 & 0 & 0 & 0 & 0 & 19\end{array}$

$\begin{array}{llllllllllllll}1.4788 & 2.1389 & 0.0099 & \mathrm{H} & 0 & 0 & 0 & 0 & 0 & 0 & 0 & 0 & 0 & 20\end{array}$

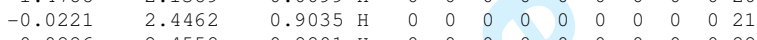

$\begin{array}{llllllllllllll}-0.0226 & 2.4558 & -0.8801 & \mathrm{H} & 0 & 0 & 0 & 0 & 0 & 0 & 0 & 0 & 0 & 22\end{array}$

$\begin{array}{llllllllllllll}-5.2449 & -0.7040 & -0.0015 & \mathrm{H} & 0 & 0 & 0 & 0 & 0 & 0 & 0 & 0 & 0 & 23 \\ -3.5430 & -2.5377 & -0.0274 & \mathrm{H} & 0 & 0 & 0 & 0 & 0 & 0 & 0 & 0 & 0 & 24\end{array}$

$\begin{array}{lllllllllllllll}-1.1437 & -2.0094 & -0.0273 & \mathrm{H} & 0 & 0 & 0 & 0 & 0 & 0 & 0 & 0 & 0 & 25\end{array}$

$\begin{array}{llllllllllllll}-2.0768 & 2.2096 & 0.0238 & \mathrm{H} & 0 & 0 & 0 & 0 & 0 & 0 & 0 & 0 & 0 & 26\end{array}$

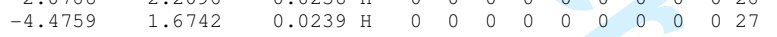

$\begin{array}{llllll}2 & 1 & 0 & 0 & 0 & 0\end{array}$

$\begin{array}{llllll}1 & 0 & 0 & 0 & 0\end{array}$

$\begin{array}{lllllll}3 & 2 & 0 & 0 & 0 & 0\end{array}$

$\begin{array}{llllll}4 & 3 & 0 & 0 & 0 & 0\end{array}$

$\begin{array}{llllll}5 & 4 & 0 & 0 & 0 & 0\end{array}$

$\begin{array}{llllll}11 & 4 & 0 & 0 & 0 & 0\end{array}$

$\begin{array}{llllll}12 & 4 & 0 & 0 & 0 & 0 \\ 6 & 5 & 0 & 0 & 0 & 0\end{array}$

$\begin{array}{lllllll}9 & 5 & 0 & 0 & 0 & 0\end{array}$

$\begin{array}{llllll}10 & 5 & 0 & 0 & 0\end{array}$

$\begin{array}{lllllllll}1 & 6 & 0 & 0 & 0 & 0 & 0 \\ 1 & 6 & 0 & 0 & 0 & 0\end{array}$

$\begin{array}{llllll}7 & 9 & 0 & 0 & 0\end{array}$

$\begin{array}{rrrrrr}18 & 9 & 0 & 0 & 0 & 0\end{array}$

$2010 \quad 0 \quad 0000$

$2110 \quad 000000$

$\begin{array}{llllll}22 & 10 & 0 & 0 & 0 & 0 \\ 16 & 15 & 0 & 0 & 0 & 0\end{array}$

$\begin{array}{llllll}19 & 15 & 0 & 0 & 0 & 0\end{array}$

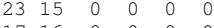

$\begin{array}{llllll}17 & 16 & 0 & 0 & 0 & 0 \\ 24 & 16 & 0 & 0 & 0 & 0\end{array}$

$2517 \quad 0 \quad 001000$

$\begin{array}{llllll}19 & 18 & 0 & 0 & 0 & 0 \\ 26 & 18 & 0 & 0 & 0 & 0\end{array}$

$\begin{array}{lllllllllll}19 & 27 & 0 & 0 & 0 & 0\end{array}$

M END

$\$ \$ \$ \$$

,3-methylphenylglutarimide0.sdf

cosmotherm

$\begin{array}{llllllllllll}28 & 29 & 0 & 0 & 0 & 0 & 0 & 0 & 0 & 0 & 0 & \mathrm{~V} 2000\end{array}$

\begin{tabular}{|c|c|c|c|c|c|c|c|c|c|c|c|c|}
\hline 2.2522 & 0.2135 & -1.2480 & $\mathrm{C}$ & 0 & 0 & 0 & 0 & 0 & 0 & 0 & 0 & 0 \\
\hline 2.8693 & 0.2514 & -0.0002 & $\mathrm{~N}$ & 0 & 0 & 0 & 0 & 0 & 0 & 0 & 0 & 0 \\
\hline 2.2516 & 0.2192 & 1.2475 & C & 0 & 0 & 0 & 0 & 0 & 0 & 0 & 0 & 0 \\
\hline 0.7401 & 0.2137 & 1.2521 & C & 0 & 0 & 0 & 0 & 0 & 0 & 0 & 0 & 0 \\
\hline 0.0954 & -0.4377 & 0.0007 & C & 0 & 0 & 0 & 0 & 0 & 0 & 0 & 0 & 0 \\
\hline 0.7407 & 0.2080 & -1.2533 & C & 0 & 0 & 0 & 0 & 0 & 0 & 0 & 0 & 0 \\
\hline 2.9356 & 0.2250 & 2.2705 & 0 & 0 & 0 & 0 & 0 & 0 & 0 & 0 & 0 & 0 \\
\hline 2.9367 & 0.2144 & -2.2707 & 0 & 0 & 0 & 0 & 0 & 0 & 0 & 0 & 0 & 0 \\
\hline-1.4162 & -0.1437 & 0.0001 & C & 0 & 0 & 0 & 0 & 0 & 0 & 0 & 0 & 0 \\
\hline 0.3978 & -1.9483 & 0.0042 & C & 0 & 0 & 0 & 0 & 0 & 0 & 0 & 0 & 0 \\
\hline 3.8930 & 0.2645 & 0.0001 & $\mathrm{H}$ & 0 & 0 & 0 & 0 & 0 & 0 & 0 & 0 & 0 \\
\hline 0.4335 & 1.2696 & 1.3357 & $\mathrm{H}$ & 0 & 0 & 0 & 0 & 0 & 0 & 0 & 0 & 0 \\
\hline 0.4124 & -0.2854 & 2.1738 & $\mathrm{H}$ & 0 & 0 & 0 & 0 & 0 & 0 & 0 & 0 & 0 \\
\hline 0.4341 & 1.2634 & -1.3422 & $\mathrm{H}$ & 0 & 0 & 0 & 0 & 0 & 0 & 0 & 0 & 0 \\
\hline 0.4135 & -0.2954 & -2.1728 & $\mathrm{H}$ & 0 & 0 & 0 & 0 & 0 & 0 & 0 & 0 & 0 \\
\hline-4.1878 & 0.4534 & -0.0017 & C & 0 & 0 & 0 & 0 & 0 & 0 & 0 & 0 & 0 \\
\hline-3.2414 & 1.4858 & -0.0067 & C & 0 & 0 & 0 & 0 & 0 & 0 & 0 & 0 & 0 \\
\hline-1.8766 & 1.1878 & -0.0059 & C & 0 & 0 & 0 & 0 & 0 & 0 & 0 & 0 & 0 \\
\hline-2.3783 & -1.1667 & 0.0056 & C & 0 & 0 & 0 & 0 & 0 & 0 & 0 & 0 & 0 \\
\hline-3.7489 & -0.8727 & 0.0052 & C & 0 & 0 & 0 & 0 & 0 & 0 & 0 & 0 & 0 \\
\hline 1.4818 & -2.1310 & 0.0048 & $\mathrm{H}$ & 0 & 0 & 0 & 0 & 0 & 0 & 0 & 0 & 0 \\
\hline-0.0173 & -2.4399 & -0.8867 & $\mathrm{H}$ & 0 & 0 & 0 & 0 & 0 & 0 & 0 & 0 & 0 \\
\hline-0.0174 & -2.4358 & 0.8972 & $\mathrm{H}$ & 0 & 0 & 0 & 0 & 0 & 0 & 0 & 0 & 0 \\
\hline-5.2549 & 0.6832 & -0.0023 & & 0 & 0 & 0 & 0 & 0 & 0 & 0 & 0 & 0 \\
\hline-3.5660 & 2.5280 & -0.0116 & $\mathrm{H}$ & 0 & 0 & 0 & 0 & 0 & 0 & 0 & 0 & 0 \\
\hline-1.1644 & 2.0164 & -0.0102 & & 0 & 0 & 0 & 0 & 0 & 0 & 0 & 0 & 0 \\
\hline & & & & & & & & & & & & \\
\hline
\end{tabular}




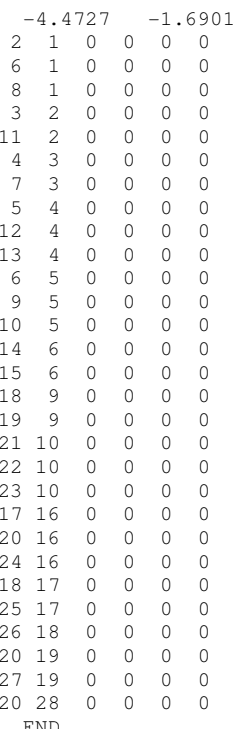

$\begin{array}{llllllllllll}0.0097 & \mathrm{H} & 0 & 0 & 0 & 0 & 0 & 0 & 0 & 0 & 0 & 28\end{array}$ 


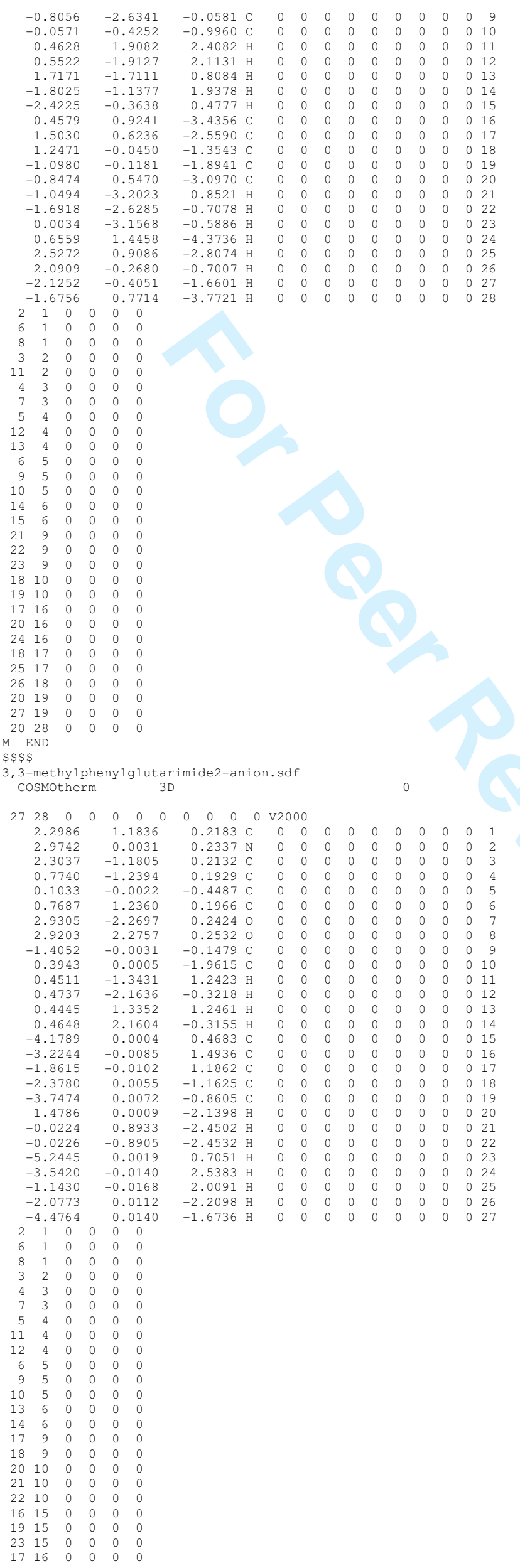




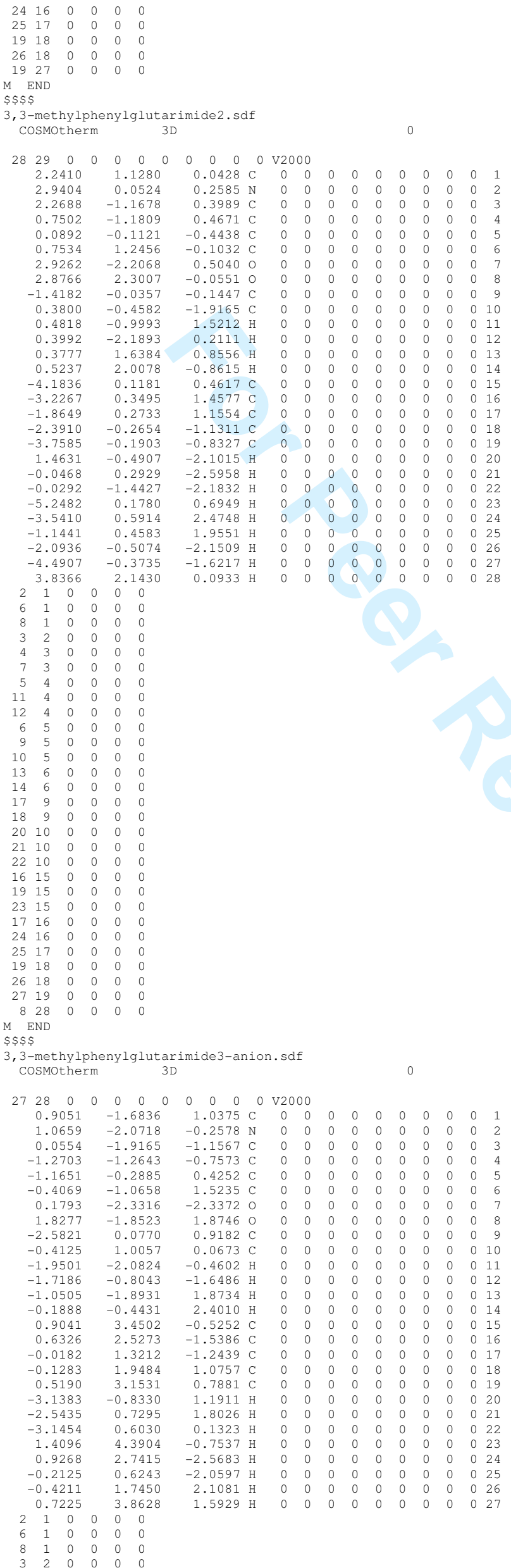




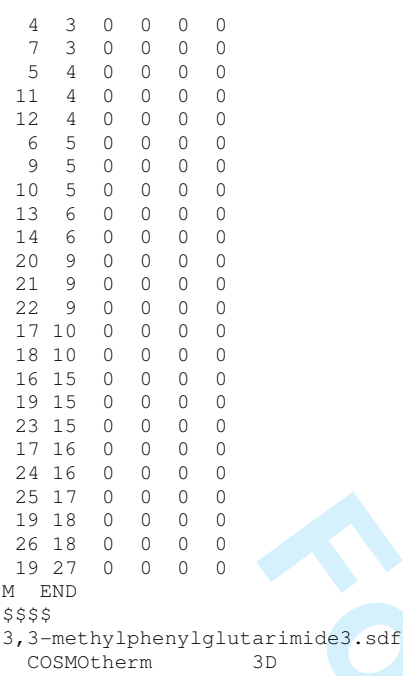

$\begin{array}{lllllllllllll}28 & 29 & 0 & 0 & 0 & 0 & 0 & 0 & 0 & 0 & 0 & \mathrm{~V} 2000\end{array}$

$\begin{array}{llllllllllllll}0.7502 & 0.9846 & 1.7241 & \mathrm{C} & 0 & 0 & 0 & 0 & 0 & 0 & 0 & 0 & 0 & 1\end{array}$ $\begin{array}{llllllllllllll}1.0544 & -0.2387 & 2.0498 & \mathrm{~N} & 0 & 0 & 0 & 0 & 0 & 0 & 0 & 0 & 0 & 2\end{array}$ $\begin{array}{llllllllllllll}0.1049 & -1.2436 & 1.8253 & \mathrm{C} & 0 & 0 & 0 & 0 & 0 & 0 & 0 & 0 & 0 & 3 \\ -1.2454 & -0.8771 & 1.2279 & \mathrm{C} & 0 & 0 & 0 & 0 & 0 & 0 & 0 & 0 & 0 & 4\end{array}$

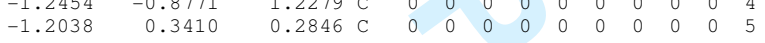
$\begin{array}{lllllllllllllll}-0.5254 & 1.4661 & 1.1031 & \mathrm{C} & 0 & 0 & 0 & 0 & 0 & 0 & 0 & 0 & 0 & 6\end{array}$ $\begin{array}{llllllllllllll}0.3705 & -2.4045 & 2.1489 & 0 & 0 & 0 & 0 & 0 & 0 & 0 & 0 & 0 & 0 & 7\end{array}$ $\begin{array}{llllllllllllll}1.6382 & 1.9548 & 1.9611 & 0 & 0 & 0 & 0 & 0 & 0 & 0 & 0 & 0 & 0 & 8\end{array}$

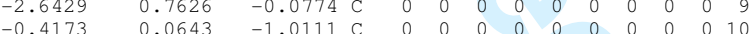
$\begin{array}{rrrrrrrrrrrrrr}-0.4173 & 0.0643 & -1.0111 & \mathrm{C} & 0 & 0 & 0 & 0 & 0 & 0 & 0 & 0 & 0 & 10 \\ -1.9050 & -0.6263 & 2.0777 & \mathrm{H} & 0 & 0 & 0 & 0 & 0 & 0 & 0 & 0 & 0 & 11\end{array}$ $\begin{array}{llllllllllllll}-1.6774 & -1.7689 & 0.7567 & \mathrm{H} & 0 & 0 & 0 & 0 & 0 & 0 & 0 & 0 & 0 & 12\end{array}$ $\begin{array}{rrrrrrrrrrrrrr}-1.1956 & 1.7717 & 1.9260 & \mathrm{H} & 0 & 0 & 0 & 0 & 0 & 0 & 0 & 0 & 0 & 13\end{array}$

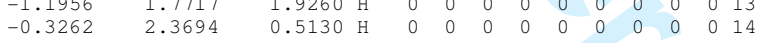
$\begin{array}{rrrrrrrrrrrrrrrr}-0.3262 & 2.3694 & 0.5130 & \mathrm{H} & 0 & 0 & 0 & 0 & 0 & 0 & 0 & 0 & 0 & 13 \\ 0.9560 & -0.3803 & -3.4514 & \mathrm{C} & 0 & 0 & 0 & 0 & 0 & 0 & 0 & 0 & 0 & 15\end{array}$ $\begin{array}{llllllllllllll}0.6538 & -1.4494 & -2.6044 & \mathrm{C} & 0 & 0 & 0 & 0 & 0 & 0 & 0 & 0 & 0 & 16\end{array}$ $\begin{array}{rrrrrrrrrrrrrrrrr}0.0264 & -1.2281 & -1.3994 & \mathrm{C} & 0 & 0 & 0 & 0 & 0 & 0 & 0 & 0 & 0 & 17\end{array}$ $\begin{array}{rrrrrrrrrrrrrr}-0.0264 & -1.2281 & -1.3994 & \mathrm{C} & 0 & 0 & 0 & 0 & 0 & 0 & 0 & 0 & 0 & 17 \\ -0.1103 & 1.1290 & -1.8805 & \mathrm{C} & 0 & 0 & 0 & 0 & 0 & 0 & 0 & 0 & 0 & 18\end{array}$ $\begin{array}{rrrrrrrrrrrrrrrr}-0.1103 & 1.1290 & -1.8805 & \mathrm{C} & 0 & 0 & 0 & 0 & 0 & 0 & 0 & 0 & 0 & 17 \\ 0.5673 & 0.9135 & -3.0834 & \mathrm{C} & 0 & 0 & 0 & 0 & 0 & 0 & 0 & 0 & 0 & 19\end{array}$ $\begin{array}{llllllllllllll}-3.2244 & 0.9734 & 0.8327 & \mathrm{H} & 0 & 0 & 0 & 0 & 0 & 0 & 0 & 0 & 0 & 20 \\ -2.6437 & 1.6660 & -0.7034 & \mathrm{H} & 0 & 0 & 0 & 0 & 0 & 0 & 0 & 0 & 0 & 21\end{array}$ $\begin{array}{rrrrrrrrrrrrrr}-2.6437 & 1.6660 & -0.7034 & \mathrm{H} & 0 & 0 & 0 & 0 & 0 & 0 & 0 & 0 & 0 & 21 \\ -3.1486 & -0.0389 & -0.6349 & \mathrm{H} & 0 & 0 & 0 & 0 & 0 & 0 & 0 & 0 & 0 & 22\end{array}$ $\begin{array}{rrrrrrrrrrrrrr}-3.1486 & -0.0389 & -0.6349 & \mathrm{H} & 0 & 0 & 0 & 0 & 0 & 0 & 0 & 0 & 0 & 22 \\ 1.4873 & -0.5512 & -4.3894 & \mathrm{H} & 0 & 0 & 0 & 0 & 0 & 0 & 0 & 0 & 0 & 23\end{array}$ $\begin{array}{llllllllllllllll}0.9471 & -2.4652 & -2.8767 & \mathrm{H} & 0 & 0 & 0 & 0 & 0 & 0 & 0 & 0 & 0 & 24\end{array}$ $\begin{array}{llllllllllllll}-0.2485 & -2.0883 & -0.7674 & \mathrm{H} & 0 & 0 & 0 & 0 & 0 & 0 & 0 & 0 & 0 & 25\end{array}$

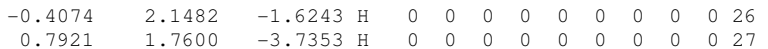

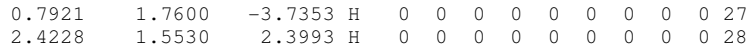
$\begin{array}{llllll}2 & 1 & 0 & 0 & 0 & 0\end{array}$

$\begin{array}{llllll}6 & 1 & 0 & 0 & 0 & 0 \\ 8 & 1 & 0 & 0 & 0 & 0 \\ 3 & 2 & 0 & 0 & 0 & 0\end{array}$

$\begin{array}{lllllll}8 & 1 & 0 & 0 & 0 & 0 \\ 3 & 2 & 0 & 0 & 0 & 0 & 0\end{array}$

$\begin{array}{llllll}2 & 0 & 0 & 0 & 0 & 0 \\ 3 & 0 & 0 & 0 & 0 & -1\end{array}$

$\begin{array}{lllllll}7 & 3 & 0 & 0 & 0 & 0\end{array}$

$\begin{array}{llllll}5 & 4 & 0 & 0 & 0 & 0 \\ 1 & 4 & 0 & 0 & 0 & 0\end{array}$

$\begin{array}{llllll}12 & 4 & 0 & 0 & 0 & 0\end{array}$

$6 \begin{array}{llllll}6 & 5 & 0 & 0 & 0 & 0 \\ 9 & 5 & 0 & 0 & 0 & 0\end{array}$

$\begin{array}{lllllll}0 & 5 & 0 & 0 & 0 & 0\end{array}$

$\begin{array}{llllll}3 & 6 & 0 & 0 & 0\end{array}$

$\begin{array}{llllll}4 & 6 & 0 & 0 & 0 & 0\end{array}$

$\begin{array}{lllllll}21 & 9 & 0 & 0 & 0\end{array}$

$\begin{array}{lllllll}2 & 9 & 0 & 0 & 0 & 0\end{array}$

$\begin{array}{lllll}10 & 0 & 0 & 0\end{array}$

$\begin{array}{llllll}8 & 10 & 0 & 0 & 0 & 0\end{array}$

$\begin{array}{llllll}15 & 15 & 0 & 0 & 0 & 0 \\ 19 & 15 & 0 & 0 & 0 & 0\end{array}$

$\begin{array}{lllllll}23 & 15 & 0 & 0 & 0 & 0\end{array}$

$\begin{array}{llllll}7 & 16 & 0 & 0 & 0 & 0\end{array}$

$\begin{array}{llllll}24 & 16 & 0 & 0 & 0 & 0 \\ 25 & 17 & 0 & 0 & 0 & 0\end{array}$

$\begin{array}{llllll}19 & 18 & 0 & 0 & 0\end{array}$

$\begin{array}{lllllll}26 & 18 & 0 & 0 & 0 & 0 \\ 27 & 19 & 0 & 0 & 0 & 0\end{array}$

$\begin{array}{llllll}8 & 28 & 0 & 0 & 0 & 0\end{array}$

$M$ END

$\$ \$ \$$

3,4-dichlorophenol-anion.sdf

Cosmotherm 3D

$\begin{array}{rrrrrrrrrrrrrrrr}12 & 12 & 0 & 0 & 0 & 0 & 0 & 0 & 0 & 0 & 0 & V 2000 & & & & \\ -0.5191 & -1.7123 & 0.0002 & \mathrm{C} & 0 & 0 & 0 & 0 & 0 & 0 & 0 & 0 & 0 & 1 \\ -1.8648 & -1.3605 & 0.0003 & \mathrm{C} & 0 & 0 & 0 & 0 & 0 & 0 & 0 & 0 & 0 & 2 \\ -2.3003 & 0.0124 & 0.0000 & \mathrm{C} & 0 & 0 & 0 & 0 & 0 & 0 & 0 & 0 & 0 & 3 \\ -1.2363 & 0.9879 & 0.0002 & \mathrm{C} & 0 & 0 & 0 & 0 & 0 & 0 & 0 & 0 & 0 & 4 \\ 0.1019 & 0.6136 & 0.0000 & \mathrm{C} & 0 & 0 & 0 & 0 & 0 & 0 & 0 & 0 & 0 & 5 \\ 0.4872 & -0.7354 & 0.0002 & \mathrm{C} & 0 & 0 & 0 & 0 & 0 & 0 & 0 & 0 & 0 & 6 \\ -3.5428 & 0.3554 & -0.0004 & 0 & 0 & 0 & 0 & 0 & 0 & 0 & 0 & 0 & 0 & 7 \\ 1.3394 & 1.8991 & 0.0000 & \mathrm{Cl} & 0 & 0 & 0 & 0 & 0 & 0 & 0 & 0 & 0 & 8 \\ 2.1895 & -1.2346 & -0.0002 & \mathrm{Cl} & 0 & 0 & 0 & 0 & 0 & 0 & 0 & 0 & 0 & 9 \\ -0.2299 & -2.7651 & 0.0003 & \mathrm{H} & 0 & 0 & 0 & 0 & 0 & 0 & 0 & 0 & 0 & 10 \\ -2.6264 & -2.1442 & 0.0002 & \mathrm{H} & 0 & 0 & 0 & 0 & 0 & 0 & 0 & 0 & 0 & 11 \\ -1.5013 & 2.0464 & 0.0000 & \mathrm{H} & 0 & 0 & 0 & 0 & 0 & 0 & 0 & 0 & 0 & 12\end{array}$




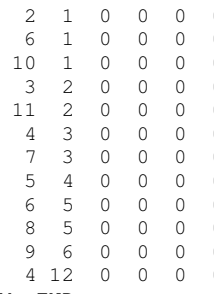




\section{Page 61 of 461}

$\begin{array}{rlllll}9 & 13 & 0 & 0 & 0 & 0\end{array}$
$\begin{aligned} & \text { M END } \\ & \text { \$\$\$ }\end{aligned}$
$\begin{aligned} & \text { 3-Chlorophenol-anion.sdf } \\ & \text { COSMOtherm }\end{aligned}$
3D

$\begin{array}{llllllllllll}12 & 12 & 0 & 0 & 0 & 0 & 0 & 0 & 0 & 0 & 0 & \mathrm{~V} 2000\end{array}$

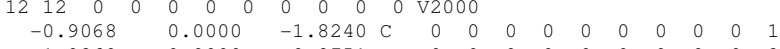

$\begin{array}{llllllllllllll}-1.9260 & 0.0000 & -0.8751 & \mathrm{C} & 0 & 0 & 0 & 0 & 0 & 0 & 0 & 0 & 0 & 2\end{array}$

$\begin{array}{llllllllllllll}-1.6532 & -0.0001 & 0.5399 & \mathrm{C} & 0 & 0 & 0 & 0 & 0 & 0 & 0 & 0 & 0 & 3 \\ -0.2522 & -0.0001 & 0.8965 & \mathrm{C} & 0 & 0 & 0 & 0 & 0 & 0 & 0 & 0 & 0 & 4\end{array}$

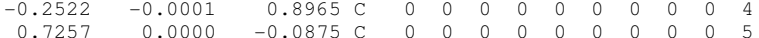

$\begin{array}{rrrrrrrrrrrrrr}0.4525 & 0.0000 & -1.4586 & \mathrm{C} & 0 & 0 & 0 & 0 & 0 & 0 & 0 & 0 & 0 & 6 \\ -2.5837 & 0.0001 & 1.4353 & 0 & 0 & 0 & 0 & 0 & 0 & 0 & 0 & 0 & 0 & 7\end{array}$

$\begin{array}{rrrrrrrrrrrrrr}-2.5837 & 0.0001 & 1.4353 & 0 & 0 & 0 & 0 & 0 & 0 & 0 & 0 & 0 & 0 & 7 \\ 2.4537 & 0.0000 & 0.4269 & \mathrm{Cl} & 0 & 0 & 0 & 0 & 0 & 0 & 0 & 0 & 0 & 8\end{array}$

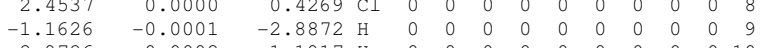

$\begin{array}{rrrrrrrrrrrrrr}-2.9726 & 0.0002 & -1.1917 & \mathrm{H} & 0 & 0 & 0 & 0 & 0 & 0 & 0 & 0 & 0 & 10 \\ 0.0165 & -0.0001 & 1.9543 & \mathrm{H} & 0 & 0 & 0 & 0 & 0 & 0 & 0 & 0 & 0 & 11\end{array}$

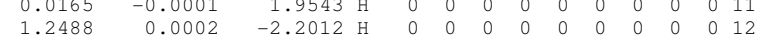

$\begin{array}{cccccc}1 & 1 & 0 & 0 & 0 & 0\end{array}$

$\begin{array}{llllll}6 & 1 & 0 & 0 & 0 & 0 \\ 9 & 1 & 0 & 0 & 0 & 0\end{array}$

$3 \begin{array}{lllll}3 & 0 & 0 & 0\end{array}$

$\begin{array}{llllll}0 & 2 & 0 & 0 & 0 \\ 4 & 3 & 0 & 0 & 0\end{array}$

$\begin{array}{lllll}3 & 0 & 0 & 0\end{array}$

$\begin{array}{llllll}5 & 4 & 0 & 0 & 0 & 0 \\ 1 & 4 & 0 & 0 & 0\end{array}$

$\begin{array}{llllll}6 & 5 & 0 & 0 & 0 & 0 \\ 8 & 5 & 0 & 0 & 0 & 0\end{array}$

$\begin{array}{llllll}6 & 12 & 0 & 0 & 0 & 0\end{array}$

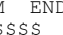

3-chlorophenol.sdf

$\begin{array}{llllllllllll}13 & 13 & 0 & 0 & 0 & 0 & 0 & 0 & 0 & 0 & 0 & \mathrm{~V} 2000\end{array}$

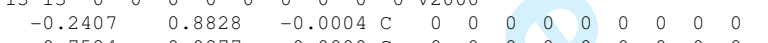

$\begin{array}{llllllllllllll}0.7584 & -0.0877 & 0.0000 & \mathrm{C} & 0 & 0 & 0 & 0 & 0 & 0 & 0 & 0 & 0 & 2\end{array}$

$\begin{array}{llllllllllllll}0.4796 & -1.4560 & -0.0002 & \mathrm{C} & 0 & 0 & 0 & 0 & 0 & 0 & 0 & 0 & 0 & 3\end{array}$

$\begin{array}{llllllllllllll}-0.8642 & -1.8488 & 0.0002 & \mathrm{C} & 0 & 0 & 0 & 0 & 0 & 0 & 0 & 0 & 0 & 4\end{array}$

$\begin{array}{rrrrrrrrrrrrrr}-1.8954 & -0.9061 & -0.0002 & \mathrm{C} & 0 & 0 & 0 & 0 & 0 & 0 & 0 & 0 & 0 & 5 \\ -1.5800 & 0.4614 & 0.0000 & \mathrm{C} & 0 & 0 & 0 & 0 & 0 & 0 & 0 & 0 & 0 & 6\end{array}$

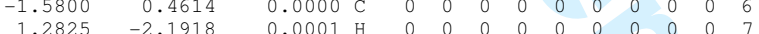

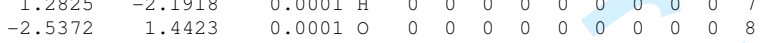

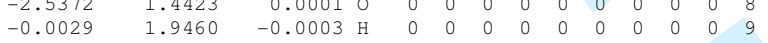

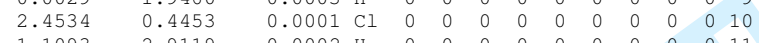

$\begin{array}{llllllllllllll}-1.1093 & -2.9119 & 0.0002 & \mathrm{H} & 0 & 0 & 0 & 0 & 0 & 0 & 0 & 0 & 0 & 11 \\ -2.9395 & -1.2257 & 0.0002 & \mathrm{H} & 0 & 0 & 0 & 0 & 0 & 0 & 0 & 0 & 0 & 12\end{array}$

$\begin{array}{rrrrrrrrrrrrrr}-2.9395 & -1.2257 & 0.0002 & \mathrm{H} & 0 & 0 & 0 & 0 & 0 & 0 & 0 & 0 & 0 & 12 \\ -3.4228 & 1.0313 & 0.0011 & \mathrm{H} & 0 & 0 & 0 & 0 & 0 & 0 & 0 & 0 & 0 & 13\end{array}$

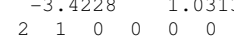

$\begin{array}{llllll}1 & 0 & 0 & 0 & 0\end{array}$

$\begin{array}{lllllll}1 & 0 & 0 & 0 & 0 \\ 3 & 2 & 0 & 0 & 0 & 0\end{array}$

$\begin{array}{llll}2 & 0 & 0 & 0\end{array}$

$\begin{array}{lllllll}4 & 3 & 0 & 0 & 0 & 0\end{array}$

$\begin{array}{llllll}3 & 0 & 0 & 0 & 0\end{array}$

$\begin{array}{lllll}4 & 0 & 0 & 0\end{array}$

\begin{tabular}{llllll}
6 & 5 & 0 & 0 & 0 & 0 \\
\hline & 5 & 0 & 0 & 0 & 0
\end{tabular}

$\begin{array}{lllll}6 & 0 & 0 & 0 & 0\end{array}$

END

\$\$\$

-chloropropanoicacid0-anion.sdf

$\begin{array}{cccccccccccc}10 & 9 & 0 & 0 & 0 & 0 & 0 & 0 & 0 & 0 & 0 & \mathrm{v} 2000\end{array}$

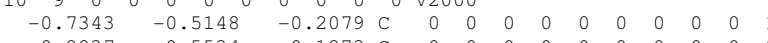

$\begin{array}{llll}0.5534 & 0.1273 \mathrm{C} & 0 \\ 0.0307 & 0.0239 & c & 0\end{array}$

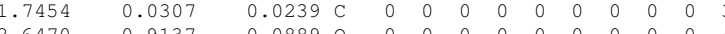

$\begin{array}{llllllllllllll}1.6470 & 0.9137 & -0.0889 & 0 & 0 & 0 & 0 & 0 & 0 & 0 & 0 & 0 & 0 & 4 \\ 1.9260 & -1.2239 & 0.0852 & 0 & 0 & 0 & 0 & 0 & 0 & 0 & 0 & 0 & 0 & 5\end{array}$

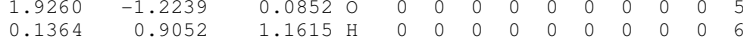

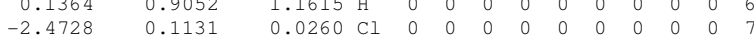

$\begin{array}{lllllllllllll}-0.6935 & -0.8257 & -1.2564 \mathrm{H} & 0 & 0 & 0 & 0 & 0 & 0 & 0 & 0 & 0 & 8\end{array}$

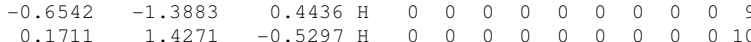

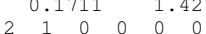

$\begin{array}{lllll}1 & 0 & 0 & 0 & 0\end{array}$

$\begin{array}{lllll}1 & 0 & 0 & 0 & 0\end{array}$

$\begin{array}{llllll}1 & 0 & 0 & 0 & 0 \\ 3 & 0 & 0 & 0 & 0\end{array}$

$\begin{array}{lllll}2 & 0 & 0 & 0\end{array}$

$\begin{array}{lllllll}5 & 3 & 0 & 0 & 0 & 0\end{array}$

$\begin{array}{lllll}10 & 0 & 0 & 0 & 0\end{array}$

M END

3-chloropropanoicacido.sdf

cosmotherm 3D

$\begin{array}{llllllllllll}11 & 10 & 0 & 0 & 0 & 0 & 0 & 0 & 0 & 0 & 0 & \mathrm{~V} 2000\end{array}$

$\begin{array}{rrrrrrrrrrrrrr}-0.7923 & -0.5857 & -0.0217 & \mathrm{C} & 0 & 0 & 0 & 0 & 0 & 0 & 0 & 0 & 0 & 1 \\ 0.2456 & 0.5226 & 0.0172 & \mathrm{C} & 0 & 0 & 0 & 0 & 0 & 0 & 0 & 0 & 0 & 2 \\ 1.6531 & -0.0326 & 0.0040 & \mathrm{C} & 0 & 0 & 0 & 0 & 0 & 0 & 0 & 0 & 0 & 3 \\ 2.5754 & 0.9591 & -0.0115 & 0 & 0 & 0 & 0 & 0 & 0 & 0 & 0 & 0 & 0 & 4 \\ 1.9487 & -1.2190 & 0.0089 & 0 & 0 & 0 & 0 & 0 & 0 & 0 & 0 & 0 & 0 & 5 \\ 0.1318 & 1.1389 & 0.9219 & \mathrm{H} & 0 & 0 & 0 & 0 & 0 & 0 & 0 & 0 & 0 & 6 \\ -2.4809 & 0.1364 & 0.0019 & \mathrm{Cl} & 0 & 0 & 0 & 0 & 0 & 0 & 0 & 0 & 0 & 1 \\ -0.7342 & -1.1790 & -0.9385 & \mathrm{H} & 0 & 0 & 0 & 0 & 0 & 0 & 0 & 0 & 0 & 8 \\ -0.7353 & -1.2411 & 0.8518 & \mathrm{H} & 0 & 0 & 0 & 0 & 0 & 0 & 0 & 0 & 0 & 9 \\ 0.1343 & 1.2009 & -0.8417 & \mathrm{H} & 0 & 0 & 0 & 0 & 0 & 0 & 0 & 0 & 0 & 10 \\ 3.4673 & 0.5474 & -0.0132 & \mathrm{H} & 0 & 0 & 0 & 0 & 0 & 0 & 0 & 0 & 0 & 11\end{array}$

URL: http://mc.manuscriptcentral.com/tandf/tmph 


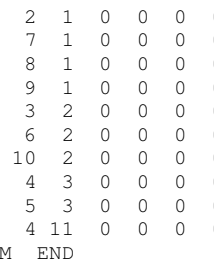




\section{Page 63 of 461}

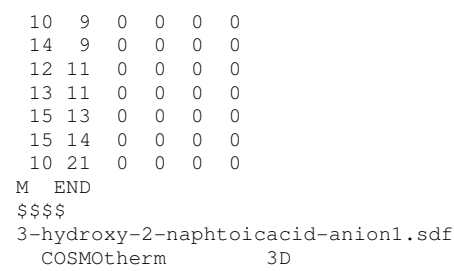

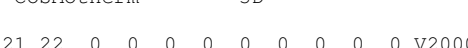

\begin{tabular}{|c|c|c|c|c|c|c|c|c|c|c|c|c|}
\hline 3.8241 & 0.2396 & 0.0002 & C & 0 & 0 & 0 & 0 & 0 & 0 & 0 & 0 & 0 \\
\hline 3.5381 & -1.1515 & -0.0037 & C & 0 & 0 & 0 & 0 & 0 & 0 & 0 & 0 & 0 \\
\hline 2.2266 & -1.5890 & -0.0048 & C & 0 & 0 & 0 & 0 & 0 & 0 & 0 & 0 & \\
\hline 1.1491 & -0.6584 & -0.0023 & C & 0 & 0 & 0 & 0 & 0 & 0 & 0 & 0 & \\
\hline 1.4396 & 0.7504 & 0.0011 & C & 0 & 0 & 0 & 0 & 0 & 0 & 0 & 0 & \\
\hline 2.8014 & 1.1699 & 0.0026 & C & 0 & 0 & 0 & 0 & 0 & 0 & 0 & 0 & \\
\hline-0.2090 & -1.0632 & -0.0021 & C & 0 & 0 & 0 & 0 & 0 & 0 & 0 & 0 & \\
\hline-1.2771 & -0.1760 & -0.0004 & C & 0 & 0 & 0 & 0 & 0 & 0 & 0 & 0 & \\
\hline-0.9661 & 1.2277 & 0.0021 & C & 0 & 0 & 0 & 0 & 0 & 0 & 0 & 0 & \\
\hline 0.3551 & 1.6607 & 0.0034 & & 0 & 0 & 0 & 0 & 0 & 0 & 0 & 0 & 0 \\
\hline-2.7009 & -0.7650 & -0.0006 & C & 0 & 0 & 0 & 0 & 0 & 0 & 0 & 0 & \\
\hline-2.7797 & -2.0344 & 0.0179 & 0 & 0 & 0 & 0 & 0 & 0 & 0 & 0 & 0 & 0 \\
\hline-3.6838 & 0.0323 & -0.0194 & 0 & 0 & 0 & 0 & 0 & 0 & 0 & 0 & 0 & 0 \\
\hline-1.9847 & 2.1470 & 0.0045 & 0 & 0 & & 0 & 0 & 0 & 0 & 0 & 0 & \\
\hline-1.5800 & 3.0371 & 0.0086 & $\mathrm{H}$ & 0 & 0 & 0 & 0 & 0 & 0 & 0 & 0 & 0 \\
\hline 4.8633 & 0.5744 & 0.0014 & & 0 & 0 & 0 & 0 & 0 & 0 & 0 & 0 & 0 \\
\hline 4.3581 & -1.8718 & -0.0057 & $\mathrm{H}$ & 0 & 0 & 0 & 0 & 0 & 0 & 0 & 0 & 0 \\
\hline 1.9988 & -2.6578 & -0.0077 & & 0 & 0 & 0 & 0 & 0 & 0 & 0 & 0 & 0 \\
\hline 3.0268 & 2.2390 & 0.0057 & & 0 & 0 & 0 & 0 & 0 & 0 & 0 & 0 & 0 \\
\hline-0.4519 & -2.1277 & -0.0029 & & 0 & 0 & 0 & 0 & 0 & 0 & 0 & 0 & 0 \\
\hline 0.5635 & 2.7353 & 0.0064 & H & 0 & 0 & 0 & 0 & 0 & 0 & 0 & 0 & 0 \\
\hline
\end{tabular}

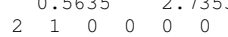

$\begin{array}{llllll}2 & 1 & 0 & 0 & 0 & 0 \\ 6 & 1 & 0 & 0 & 0 & 0\end{array}$

$\begin{array}{cccccc}16 & 1 & 0 & 0 & 0 & 0\end{array}$

$\begin{array}{rrrrrrr}3 & 2 & 0 & 0 & 0 & 0 \\ 17 & 2 & 0 & 0 & 0 & 0\end{array}$

$\begin{array}{llllll}4 & 3 & 0 & 0 & 0\end{array}$

$\begin{array}{rrrrrr}18 & 3 & 0 & 0 & 0 \\ 5 & 4 & 0 & 0 & 0\end{array}$

$\begin{array}{llllll}5 & 4 & 0 & 0 & 0 & 0 \\ 7 & 4 & 0 & 0 & 0 & 0\end{array}$

$\begin{array}{llllll}6 & 5 & 0 & 0 & 0 & 0\end{array}$

$\begin{array}{lllll}10 & 5 & 0 & 0 & 0\end{array}$

$\begin{array}{rrrrrrrr}19 & 6 & 0 & 0 & 0 & 0 \\ 8 & 7 & 0 & 0 & 0\end{array}$

$\begin{array}{llllll}20 & 7 & 0 & 0 & 0\end{array}$

$\begin{array}{rrrrrr}9 & 8 & 0 & 0 & 0 & 0 \\ 11 & 8 & 0 & 0 & 0 & 0\end{array}$

$\begin{array}{cccccc}11 & 8 & 0 & 0 & 0 & 0 \\ 10 & 9 & 0 & 0 & 0 & 0\end{array}$

$\begin{array}{llllll}14 & 9 & 0 & 0 & 0\end{array}$

$\begin{array}{llllll}12 & 11 & 0 & 0 & 0 \\ 13 & 11 & 0 & 0 & 0 & 0\end{array}$

$\begin{array}{llllll}15 & 14 & 0 & 0 & 0 \\ 10 & 21 & 0 & 0 & 0\end{array}$

END

\$\$\$

3-hydroxy-2-naphtoicacido.sdf

cosmotherm

$\begin{array}{llllllllllll}22 & 23 & 0 & 0 & 0 & 0 & 0 & 0 & 0 & 0 & 0 & \mathrm{~V} 2000\end{array}$

$\begin{array}{lllllllllllll}3.8316 & 0.2671 & -0.0020 & \mathrm{C} & 0 & 0 & 0 & 0 & 0 & 0 & 0 & 0 & 0\end{array}$

$\begin{array}{llllllllllllll}3.5657 & -1.1293 & -0.0012 & \mathrm{C} & 0 & 0 & 0 & 0 & 0 & 0 & 0 & 0 & 0 & 2\end{array}$

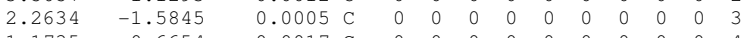

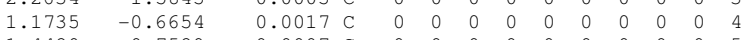

$\begin{array}{rrrrrrrrrrrrrr}1.4420 & 0.7529 & 0.0007 & \mathrm{C} & 0 & 0 & 0 & 0 & 0 & 0 & 0 & 0 & 0 & 5 \\ 2.8000 & 1.1855 & -0.0005 & \mathrm{C} & 0 & 0 & 0 & 0 & 0 & 0 & 0 & 0 & 0 & 6\end{array}$

$\begin{array}{rrrrrrrrrrrrrrr}-0.1693 & -1.0991 & 0.0018 & \mathrm{C} & 0 & 0 & 0 & 0 & 0 & 0 & 0 & 0 & 0 & 7\end{array}$

$\begin{array}{llllllllllllll}-1.2292 & -0.1949 & 0.0012 & \mathrm{C} & 0 & 0 & 0 & 0 & 0 & 0 & 0 & 0 & 0 & 8\end{array}$

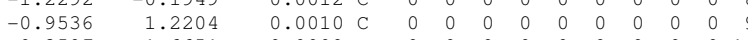

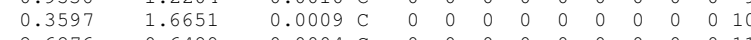

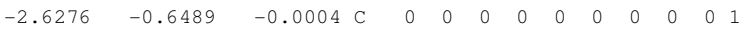

$\begin{array}{llllllllllllll}-2.7910 & -1.9852 & -0.0001 & 0 & 0 & 0 & 0 & 0 & 0 & 0 & 0 & 0 & 0 & 12\end{array}$

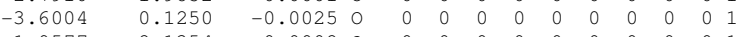

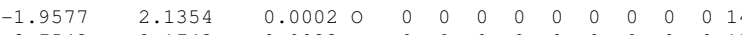

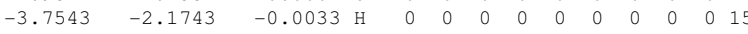

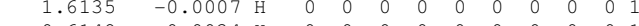

$\begin{array}{lllllllllllllll}4.8663 & 0.6148 & -0.0034 \mathrm{H} & 0 & 0 & 0 & 0 & 0 & 0 & 0 & 0 & 0 & 0 & 0 & 16\end{array}$

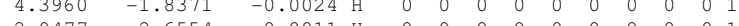

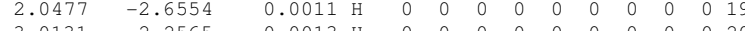

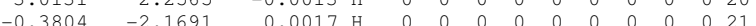

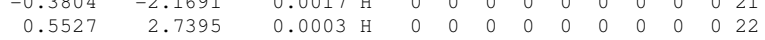

$2 \begin{array}{llllll}2 & 1 & 0 & 0 & 0 & 0\end{array}$

$\begin{array}{rrrrr}6 & 1 & 0 & 0 & 0 \\ 17 & 1 & 0 & 0 & 0\end{array}$

$\begin{array}{llllll}18 & 2 & 0 & 0 & 0 & 0\end{array}$

$\begin{array}{rrrrrr}4 & 3 & 0 & 0 & 0 & 0 \\ 19 & 3 & 0 & 0 & 0 & 0\end{array}$

$\begin{array}{ccccccccc}12 & 3 & 0 & 0 & 0 & 0\end{array}$

$\begin{array}{lllllll}7 & 4 & 0 & 0 & 0 & 0 & 0\end{array}$

$\begin{array}{llllll}6 & 5 & 0 & 0 & 0 & 0\end{array}$

$\begin{array}{lllllll}20 & 6 & 0 & 0 & 0 & 0\end{array}$

$\begin{array}{rrrrrr}8 & 7 & 0 & 0 & 0 & 0 \\ 21 & 7 & 0 & 0 & 0 & 0 \\ 9 & 8 & 0 & 0 & 0 & 0\end{array}$

$\begin{array}{rrrrrr}9 & 8 & 0 & 0 & 0 & 0\end{array}$

$\begin{array}{llllll}10 & 9 & 0 & 0 & 0\end{array}$

$\begin{array}{llllll}14 & 9 & 0 & 0 & 0 & 0\end{array}$

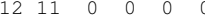

$\begin{array}{llllll}13 & 11 & 0 & 0 & 0 & 0 \\ 15 & 12 & 0 & 0 & 0 & 0\end{array}$ 


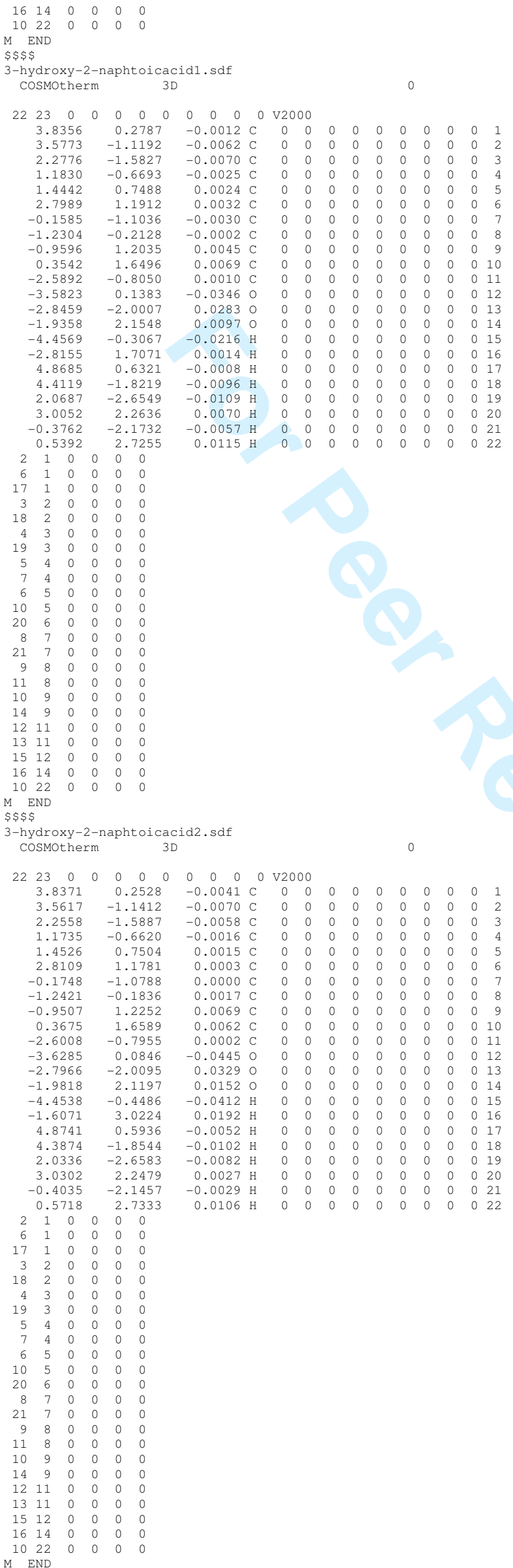




\section{Page 65 of 461}

$\$ \$ \$$

3-hydroxybenzoicacid-anion.sdf

$\begin{array}{llllllllllll}15 & 15 & 0 & 0 & 0 & 0 & 0 & 0 & 0 & 0 & 0 & \mathrm{~V} 2000\end{array}$

$\begin{array}{llllllllllllll}1.1373 & -1.8685 & 0.0015 & \mathrm{C} & 0 & 0 & 0 & 0 & 0 & 0 & 0 & 0 & 0 & 1\end{array}$

$\begin{array}{llllllllllllll}-0.2128 & -1.4995 & 0.0010 & C & 0 & 0 & 0 & 0 & 0 & 0 & 0 & 0 & 0 & 2 \\ -0.5718 & -0.1417 & 0.0003 & C & 0 & 0 & 0 & 0 & 0 & 0 & 0 & 0 & 0 & 3\end{array}$

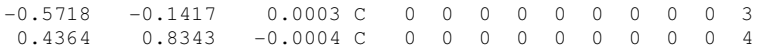

$\begin{array}{llllllllllllll}0.4364 & 0.8343 & -0.0004 & \mathrm{C} & 0 & 0 & 0 & 0 & 0 & 0 & 0 & 0 & 0 & 4 \\ 1.7858 & 0.4590 & -0.0003 & \mathrm{C} & 0 & 0 & 0 & 0 & 0 & 0 & 0 & 0 & 0 & 5\end{array}$

$\begin{array}{rrrrrrrrrrrrrr}1.7858 & 0.4590 & -0.0003 & \mathrm{C} & 0 & 0 & 0 & 0 & 0 & 0 & 0 & 0 & 0 & 5 \\ 2.1433 & -0.8978 & 0.0003 & \mathrm{C} & 0 & 0 & 0 & 0 & 0 & 0 & 0 & 0 & 0 & 6\end{array}$

$\begin{array}{rrrrrrrrrrrrrrr}2.1433 & -0.8978 & 0.0003 & \mathrm{C} & 0 & 0 & 0 & 0 & 0 & 0 & 0 & 0 & 0 & 5 \\ -2.0464 & 0.2811 & -0.0001 & \mathrm{C} & 0 & 0 & 0 & 0 & 0 & 0 & 0 & 0 & 0 & 7\end{array}$

$\begin{array}{rrrrrrrrrrrrrr}2.8078 & 1.3840 & -0.0015 & 0 & 0 & 0 & 0 & 0 & 0 & 0 & 0 & 0 & 0 & 8 \\ -2.2906 & 1.5276 & 0.0029 & 0 & 0 & 0 & 0 & 0 & 0 & 0 & 0 & 0 & 0 & 9\end{array}$

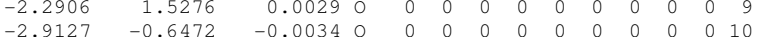

$\begin{array}{llllllllllllllll}-2.9127 & -0.6472 & -0.0034 & 0 & 0 & 0 & 0 & 0 & 0 & 0 & 0 & 0 & 0 & 10 \\ 1.4154 & -2.9246 & 0.0021 & \mathrm{H} & 0 & 0 & 0 & 0 & 0 & 0 & 0 & 0 & 0 & 11\end{array}$

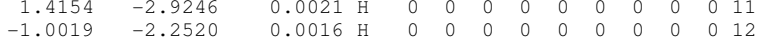

$\begin{array}{rrrrrrrrrrrrrr}-1.0019 & -2.2520 & 0.0016 & \mathrm{H} & 0 & 0 & 0 & 0 & 0 & 0 & 0 & 0 & 0 & 12 \\ 0.1502 & 1.8890 & -0.0006 & \mathrm{H} & 0 & 0 & 0 & 0 & 0 & 0 & 0 & 0 & 0 & 13\end{array}$

$\begin{array}{rrrrrrrrrrrrrrr}3.1984 & -1.1768 & 0.0004 \mathrm{H} & 0 & 0 & 0 & 0 & 0 & 0 & 0 & 0 & 0 & 14\end{array}$

$\begin{array}{ccccccccccccccc}2.4256 & & 2.2821 & -0.0002 & \mathrm{H} & 0 & 0 & 0 & 0 & 0 & 0 & 0 & 0 & 0 & 15 \\ 2 & 0 & 0 & 0 & 0\end{array}$

$\begin{array}{llllll}6 & 1 & 0 & 0 & 0 & 0\end{array}$

$\begin{array}{llllll}3 & 2 & 0 & 0 & 0 & 0\end{array}$

$\begin{array}{lllllll}2 & 2 & 0 & 0 & 0 & 0 \\ 4 & 3 & 0 & 0 & 0 & 0\end{array}$

$\begin{array}{llllllllll}7 & 3 & 0 & 0 & 0 & 0\end{array}$

$5 \quad 4 \quad 000000$

$\begin{array}{llllll}13 & 4 & 0 & 0 & 0 & 0 \\ 6 & 5 & 0 & 0 & 0 & 0\end{array}$

8 5 5100000

$\begin{array}{rrrrrr}14 & 6 & 0 & 0 & 0 & 0 \\ 9 & 7 & 0 & 0 & 0 & 0\end{array}$

$\begin{array}{llllll}10 & 7 & 0 & 0 & 0 & 0\end{array}$

$8 \quad 15$

$\$ \$ \$ \$$

3-hydroxybenzoicacid.sdf

Cosmotherm $3 \mathrm{D}$

$\begin{array}{llllllllllll}16 & 16 & 0 & 0 & 0 & 0 & 0 & 0 & 0 & 0 & 0 & \mathrm{~V} 2000\end{array}$

$\begin{array}{llllllllllllll}1.1774 & -1.8678 & 0.0016 & \mathrm{C} & 0 & 0 & 0 & 0 & 0 & 0 & 0 & 0 & 0 & 1\end{array}$

$\begin{array}{llllllllllllll}-0.1768 & -1.5251 & 0.0010 & \mathrm{C} & 0 & 0 & 0 & 0 & 0 & 0 & 0 & 0 & 0 & 2\end{array}$

$\begin{array}{llllllllllllll}-0.5459 & -0.1696 & 0.0001 & \mathrm{C} & 0 & 0 & 0 & 0 & 0 & 0 & 0 & 0 & 0 & 3\end{array}$

$\begin{array}{lllllllllllll}0.4386 & 0.8340 & -0.0004 & \mathrm{C} & 0 & 0 & 0 & 0 & 0 & 0 & 0 & 0 & 0\end{array}$

$\begin{array}{rrrrrrrrrrrrrr}1.7917 & 0.4753 & -0.0004 & \mathrm{C} & 0 & 0 & 0 & 0 & 0 & 0 & 0 & 0 & 0 & 5 \\ 2.1635 & -0.8788 & 0.0005 & \mathrm{C} & 0 & 0 & 0 & 0 & 0 & 0 & 0 & 0 & 0 & 6\end{array}$

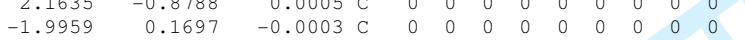

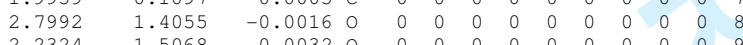

$\begin{array}{rrrrrrrrrrrrrr}-2.2324 & 1.5068 & 0.0032 & 0 & 0 & 0 & 0 & 0 & 0 & 0 & 0 & 0 & 0 & 9 \\ -2.9043 & -0.6553 & -0.0035 & 0 & 0 & 0 & 0 & 0 & 0 & 0 & 0 & 0 & 0 & 10\end{array}$

$\begin{array}{rrrrrrrrrrrrrr}-2.9043 & -0.6553 & -0.0035 & 0 & 0 & 0 & 0 & 0 & 0 & 0 & 0 & 0 & 0 & 10 \\ 1.4729 & -2.9179 & 0.0025 & \mathrm{H} & 0 & 0 & 0 & 0 & 0 & 0 & 0 & 0 & 0 & 11\end{array}$

$\begin{array}{rrrrrrrrrrrrrr}1.4729 & -2.9179 & 0.0025 & \mathrm{H} & 0 & 0 & 0 & 0 & 0 & 0 & 0 & 0 & 0 & 11 \\ -0.9497 & -2.2932 & 0.0016 & \mathrm{H} & 0 & 0 & 0 & 0 & 0 & 0 & 0 & 0 & 0 & 12\end{array}$

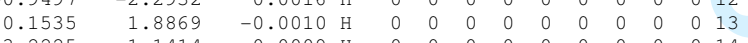

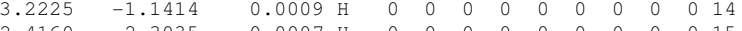

$\begin{array}{rrrrrrrrrrrrrr}2.4160 & 2.3035 & -0.0007 & \mathrm{H} & 0 & 0 & 0 & 0 & 0 & 0 & 0 & 0 & 0 & 15 \\ -3.2051 & 1.6365 & 0.0030 & \mathrm{H} & 0 & 0 & 0 & 0 & 0 & 0 & 0 & 0 & 0 & 16\end{array}$

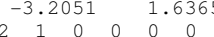

$6 \begin{array}{llllll}6 & 1 & 0 & 0 & 0 & 0\end{array}$

$\begin{array}{llllll}1 & 1 & 0 & 0 & 0 & 0\end{array}$

$\begin{array}{llllllll}2 & 0 & 0 & 0 & 0\end{array}$

$\begin{array}{llllll}4 & 3 & 0 & 0 & 0 & 0\end{array}$

$\begin{array}{llllll}3 & 0 & 0 & 0 & 0\end{array}$

$\begin{array}{llllll}5 & 4 & 0 & 0 & 0 & 0 \\ 3 & 4 & 0 & 0 & 0 & 0\end{array}$

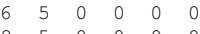

$\begin{array}{llllll}4 & 6 & 0 & 0 & 0\end{array}$

$\begin{array}{llllll}9 & 7 & 0 & 0 & 0 & 0\end{array}$

$\begin{array}{lllllllll}9 & 7 & 0 & 0 & 0 & 0\end{array}$

$\begin{array}{llllll}8 & 16 & 0 & 0 & 0 & 0\end{array}$

END

$\$ \$ \$ \$ 1$-dimethylethyl)-benzoicacid-anion, sdf

(1,1-dimethylethyl)-benzoicacid-anion.sdf

$\begin{array}{llllllllllll}26 & 26 & 0 & 0 & 0 & 0 & 0 & 0 & 0 & 0 & 0 & \mathrm{~V} 2000\end{array}$

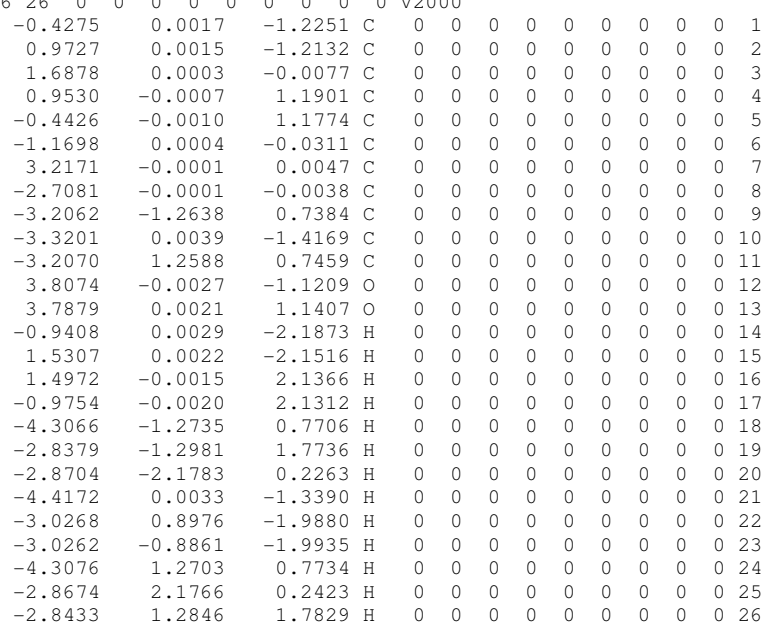




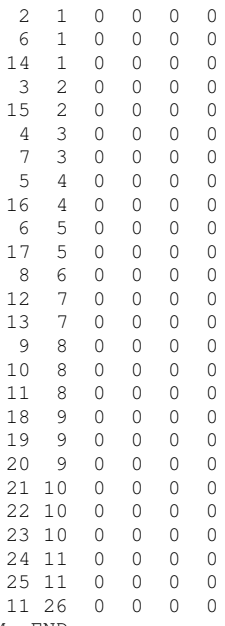




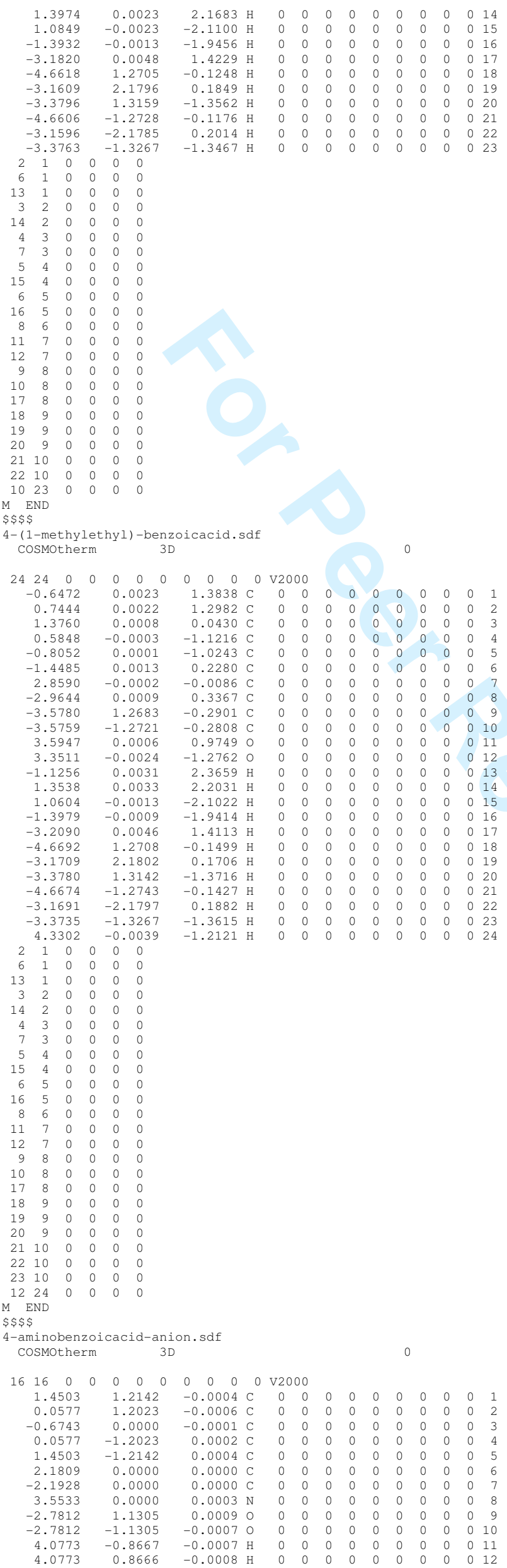



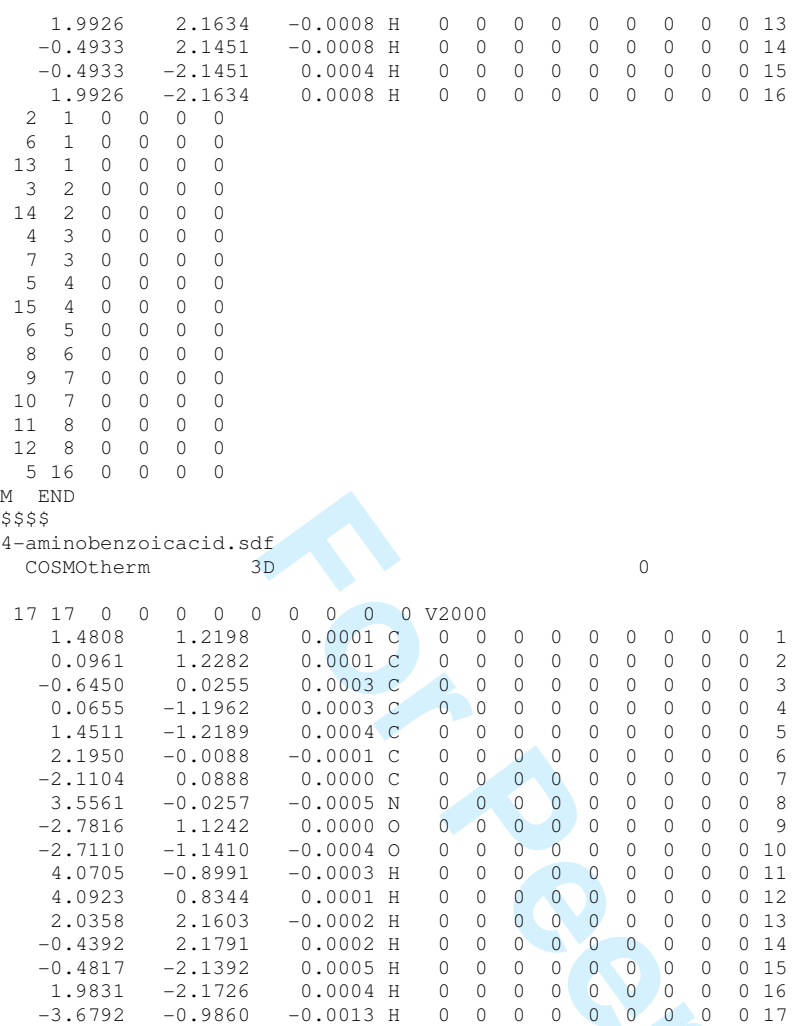

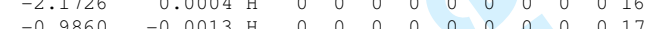
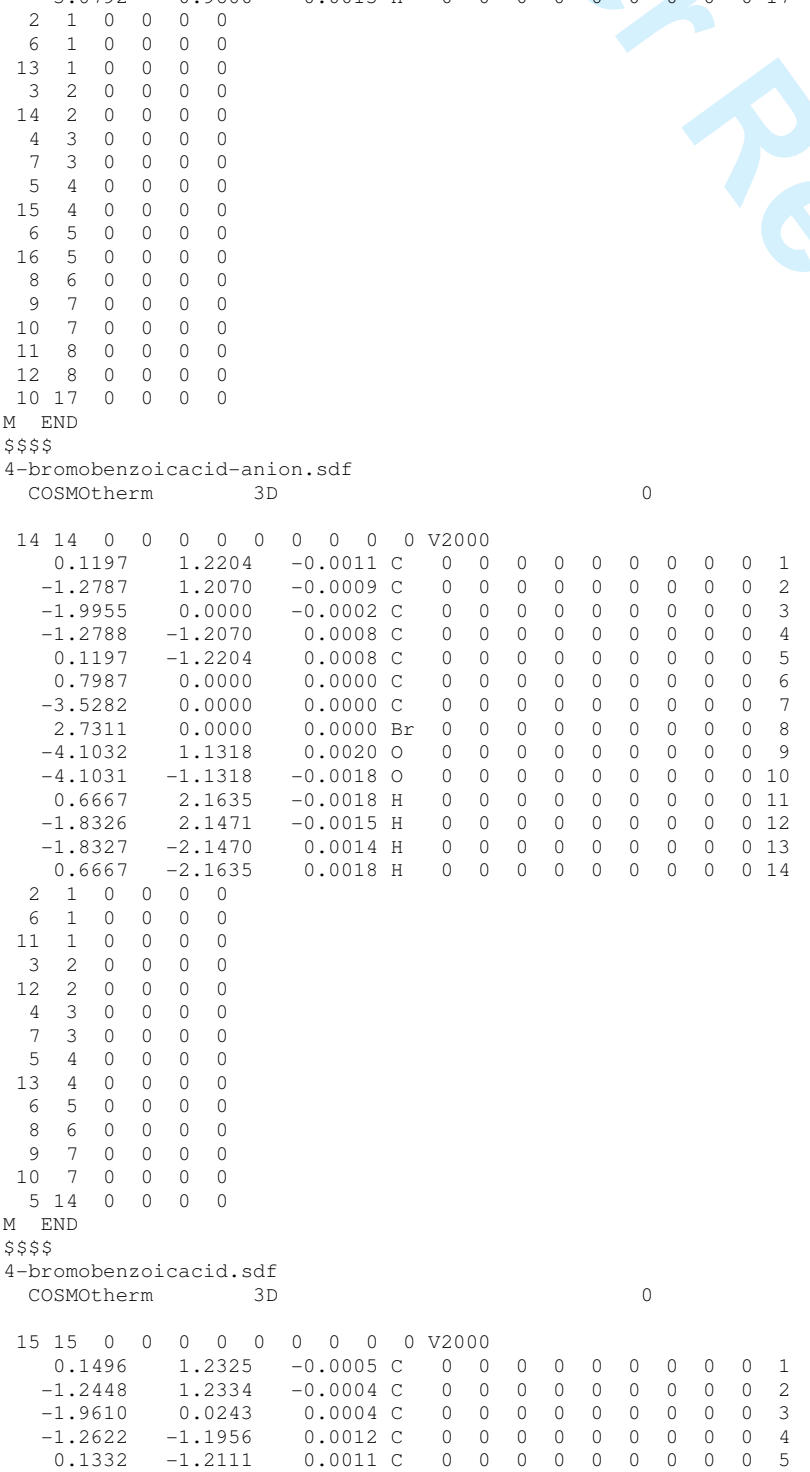


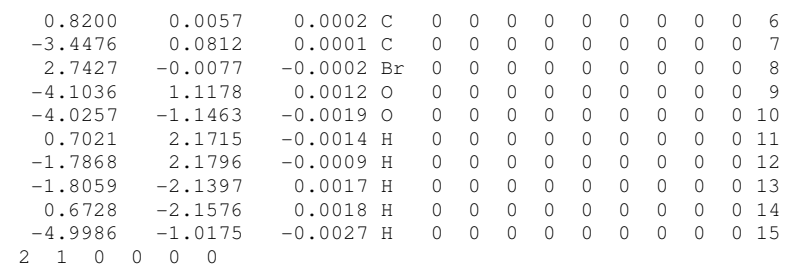


$\begin{array}{llllllllllllll}-0.5814 & -0.7484 & -2.0951 & \mathrm{H} & 0 & 0 & 0 & 0 & 0 & 0 & 0 & 0 & 0 & 17\end{array}$

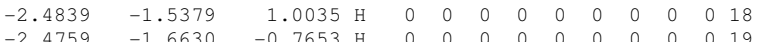

$\begin{array}{rrrrrrrrrrrrrr}-2.8484 & -1.6630 & -0.7653 & \mathrm{H} & 0 & 0 & 0 & 0 & 0 & 0 & 0 & 0 & 0 & 19 \\ -2.8484 & 0.8217 & -0.9393 & \mathrm{H} & 0 & 0 & 0 & 0 & 0 & 0 & 0 & 0 & 0 & 20\end{array}$

$\begin{array}{llllllllllllll}-2.8640 & 0.9428 & 0.8238 & \mathrm{H} & 0 & 0 & 0 & 0 & 0 & 0 & 0 & 0 & 0 & 21\end{array}$

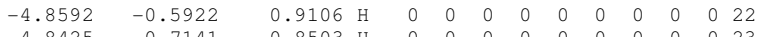

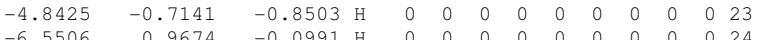

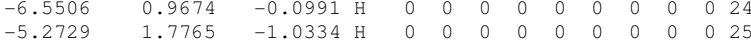

$\begin{array}{lllllllllllllll}-5.2898 & 1.8988 & 0.7396 & \mathrm{H} & 0 & 0 & 0 & 0 & 0 & 0 & 0 & 0 & 0 & 25\end{array}$

$\begin{array}{llllllllllllll}1.9381 & 0.69832 & -1.0725 & \mathrm{H} & 0 & 0 & 0 & 0 & 0 & 0 & 0 & 0 & 0 & 26\end{array}$

$\begin{array}{lllllll}2 & 1 & 0 & 0 & 0 & 0\end{array}$

$\begin{array}{lllllll}6 & 1 & 0 & 0 & 0 & 0\end{array}$

$14 \quad 1 \quad 000100$

$\begin{array}{llllll}3 & 2 & 0 & 0 & 0 & 0\end{array}$

$\begin{array}{llllll}5 & 2 & 0 & 0 & 0 & 0 \\ 4 & 3 & 0 & 0 & 0 & 0\end{array}$

$\begin{array}{lllllll}7 & 3 & 0 & 0 & 0 & 0\end{array}$

$\begin{array}{lllllll}5 & 4 & 0 & 0 & 0 & 0\end{array}$

$\begin{array}{llllll}6 & 5 & 0 & 0 & 0 & 0\end{array}$

$\begin{array}{llllll}7 & 5 & 0 & 0 & 0 & 0\end{array}$

$\begin{array}{lllllll}8 & 6 & 0 & 0 & 0 & 0 \\ 2 & 7 & 0 & 0 & 0 & 0\end{array}$

$\begin{array}{ccccc}13 & 7 & 0 & 0 & 0 \\ 9 & 8 & 0 & 0 & 0\end{array}$

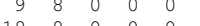

$\begin{array}{llllll}18 & 8 & 0 & 0 & 0 \\ 19 & 8 & 0 & 0 & 0 & 0\end{array}$

$\begin{array}{llllll}10 & 9 & 0 & 0 & 0 & 0\end{array}$

$\begin{array}{llllll}21 & 9 & 0 & 0 & 0 & 0\end{array}$

$\begin{array}{llllll}11 & 10 & 0 & 0 & 0 & 0 \\ 22 & 10 & 0 & 0 & 0 & 0\end{array}$

$\begin{array}{llllll}22 & 10 & 0 & 0 & 0 & 0 \\ 23 & 10 & 0 & 0 & 0 & 0\end{array}$

$\begin{array}{llllll}24 & 11 & 0 & 0 & 0 & 0\end{array}$

$\begin{array}{llllll}25 & 11 & 0 & 0 & 0 & 0 \\ 26 & 11 & 0 & 0 & 0 & 0\end{array}$

$\begin{array}{lllllll}13 & 27 & 0 & 0 & 0\end{array}$

END

4-carbamoylbenzoicacid-anion.sdf

Cosmotherm

$\begin{array}{llllllllllll}18 & 18 & 0 & 0 & 0 & 0 & 0 & 0 & 0 & 0 & 0 & \mathrm{v} 2000\end{array}$

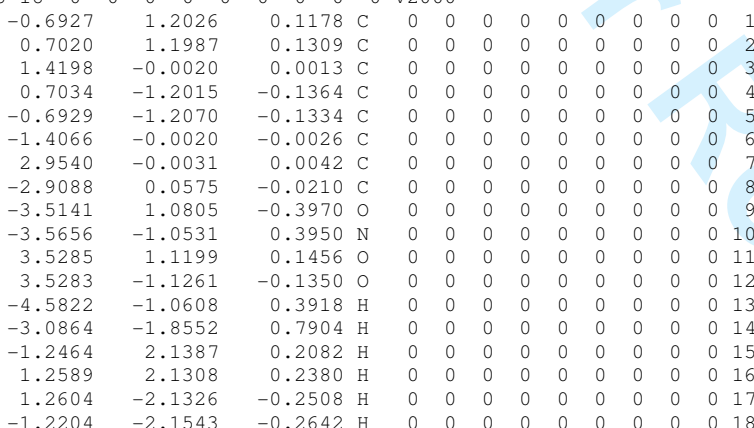

$\begin{array}{llllll}2 & 1 & 0 & 0 & 0 & 0\end{array}$

$\begin{array}{llllll}6 & 1 & 0 & 0 & 0 & 0\end{array}$

$\begin{array}{llllll}5 & 1 & 0 & 0 & 0 & 0 \\ 3 & 2 & 0 & 0 & 0 & 0\end{array}$

$\begin{array}{llllllllll}6 & 2 & 0 & 0 & 0 & 0\end{array}$

$\begin{array}{llllll}4 & 3 & 0 & 0 & 0 & 0\end{array}$

$\begin{array}{lllll}3 & 0 & 0 & 0 & 0\end{array}$

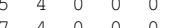

$\begin{array}{llllll}6 & 5 & 0 & 0 & 0\end{array}$

$8 \begin{array}{lllll}5 & 0 & 0 & 0\end{array}$

$\begin{array}{llllll}1 & 7 & 0 & 0 & 0 & 0\end{array}$

$\begin{array}{lllllll}2 & 8 & 0 & 0 & 0\end{array}$

$\begin{array}{lllllll}10 & 8 & 0 & 0 & 0 & 0\end{array}$

$\begin{array}{llllll}3 & 10 & 0 & 0 & 0 & 0\end{array}$

$\begin{array}{llllll}4 & 10 & 0 & 0 & 0 & 0\end{array}$

END

$\$ \$ \$$

-carbamoylbenzoicacid.sdf

cosmotherm

$\begin{array}{llllllllllll}19 & 19 & 0 & 0 & 0 & 0 & 0 & 0 & 0 & 0 & 0 & \mathrm{v} 2000\end{array}$

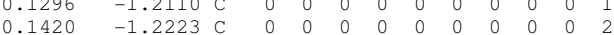

$\begin{array}{cccccccccccc}c & 0 & 0 & 0 & 0 & 0 & 0 & 0 & 0 & 2 \\ 0 & 0 & 0 & 0 & 0 & 0 & 0 & 0 & 0 & 3\end{array}$

$\begin{array}{llllllllllllll}1.3870 & 0.0040 & -0.0214 & \mathrm{C} & 0 & 0 & 0 & 0 & 0 & 0 & 0 & 0 & 0 & 3\end{array}$

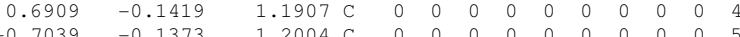

$\begin{array}{llllllllllllll}-0.7039 & -0.1373 & 1.2004 & \mathrm{C} & 0 & 0 & 0 & 0 & 0 & 0 & 0 & 0 & 0 & 5\end{array}$

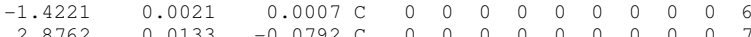

$\begin{array}{rrrrrrrrrrrrrr}2.8762 & 0.0133 & -0.0792 & \text { C } & 0 & 0 & 0 & 0 & 0 & 0 & 0 & 0 & 0 & 6 \\ -2.9289 & -0.0228 & -0.0476 & C & 0 & 0 & 0 & 0 & 0 & 0 & 0 & 0 & 0 & 8\end{array}$

$\begin{array}{llllllllllllll}-3.5313 & -0.4307 & -1.0575 & 0 & 0 & 0 & 0 & 0 & 0 & 0 & 0 & 0 & 0 & 9\end{array}$

$\begin{array}{llllllllllllll}-3.5744 & 0.4188 & 1.0571 & \mathrm{~N} & 0 & 0 & 0 & 0 & 0 & 0 & 0 & 0 & 0 & 10\end{array}$

$\begin{array}{llllllllllllll}3.5295 & 0.1470 & -1.1084 & 0 & 0 & 0 & 0 & 0 & 0 & 0 & 0 & 0 & 0 & 11\end{array}$

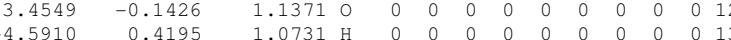

$\begin{array}{lllllllllllllll}-4.5910 & .41950 & 1.0731 & \mathrm{H} & 0 & 0 & 0 & 0 & 0 & 0 & 0 & 0 & 0 & 12 \\ -1.2887 & 0.8380 & 1.8434 & \mathrm{H} & 0 & 0 & 0 & 0 & 0 & 0 & 0 & 0 & 0 & 14\end{array}$

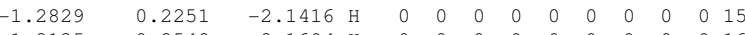

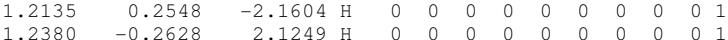

$\begin{array}{lllllllllllll}-1.2250 & -0.2720 & 2.1496 \mathrm{H} & 0 & 0 & 0 & 0 & 0 & 0 & 0 & 0 & 0 & 18\end{array}$

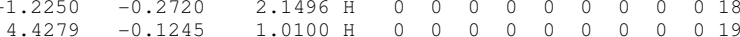




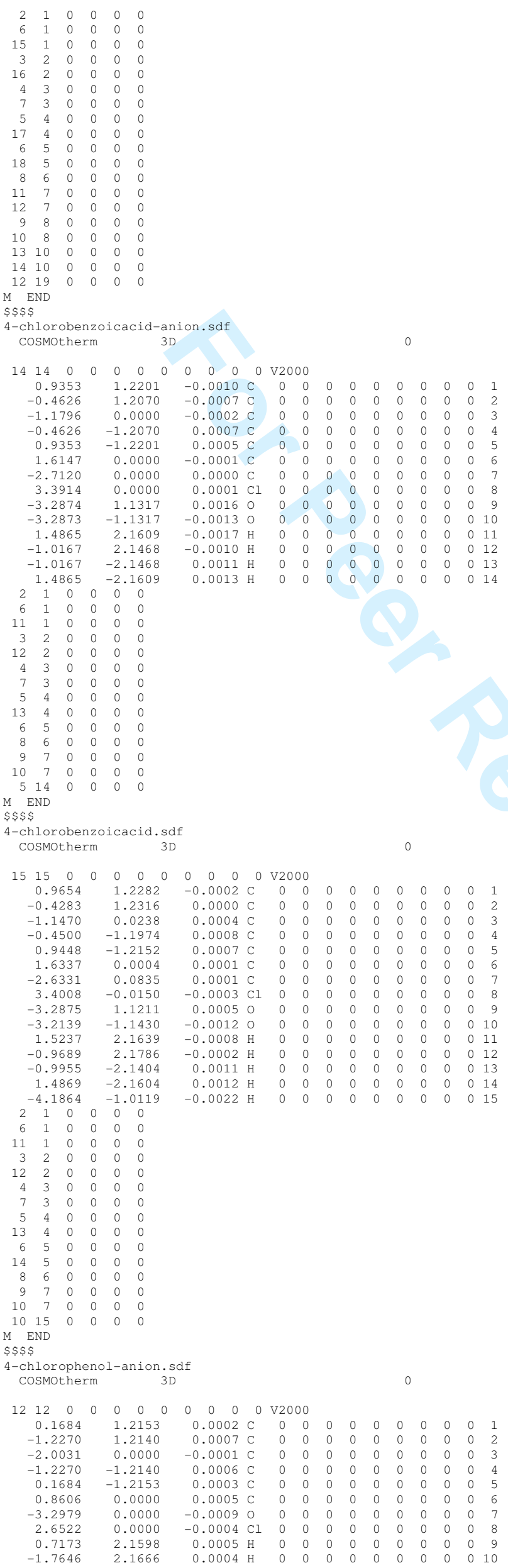




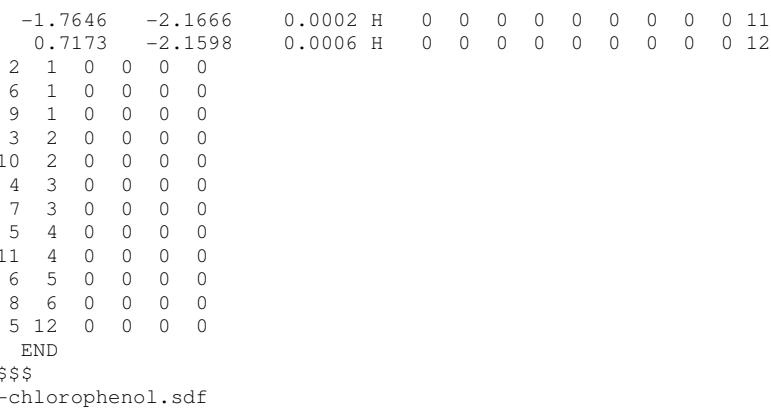

4-chlorophenol.sdf
CosMotherm

$\begin{array}{llllllllllll}13 & 13 & 0 & 0 & 0 & 0 & 0 & 0 & 0 & 0 & 0 & \mathrm{v} 2000\end{array}$

$\begin{array}{llllllllllllll}1.2136 & 1.1990 & -0.0003 & \mathrm{C} & 0 & 0 & 0 & 0 & 0 & 0 & 0 & 0 & 0 & 1\end{array}$ $\begin{array}{llllllllllllll}1.2156 & -0.1970 & 0.0001 & \mathrm{C} & 0 & 0 & 0 & 0 & 0 & 0 & 0 & 0 & 0 & 2\end{array}$ $\begin{array}{llllllllllllllll}-0.0015 & -0.8823 & -0.0001 & \mathrm{C} & 0 & 0 & 0 & 0 & 0 & 0 & 0 & 0 & 0 & 3\end{array}$ $\begin{array}{llllllllllllll}-1.2180 & -0.1991 & -0.0001 & \mathrm{C} & 0 & 0 & 0 & 0 & 0 & 0 & 0 & 0 & 0 & 4\end{array}$ $\begin{array}{rrrrrrrrrrrrrr}-1.2163 & 1.1989 & -0.0001 & C & 0 & 0 & 0 & 0 & 0 & 0 & 0 & 0 & 0 & 5 \\ -0.0014 & 1.9006 & 0.0001 & C & 0 & 0 & 0 & 0 & 0 & 0 & 0 & 0 & 0 & 6\end{array}$ $\begin{array}{llllllllllllllll}0.0001 & -2.6600 & 0.0001 & \mathrm{Cl} & 0 & 0 & 0 & 0 & 0 & 0 & 0 & 0 & 0\end{array}$ $\begin{array}{llllllllllllll}0.0597 & 3.2715 & 0.0001 & 0 & 0 & 0 & 0 & 0 & 0 & 0 & 0 & 0 & 0 & 8\end{array}$

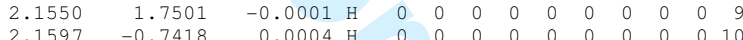
$\begin{array}{rrrrrrrrrrrrr}2.1597 & -0.7418 & 0.0004 \mathrm{H} & 0 & 0 & 0 & 0 & 0 & 0 & 0 & 0 & 0 & 10 \\ -2.1622 & -0.7437 & -0.0003 \mathrm{H} & 0 & 0 & 0 & 0 & 0 & 0 & 0 & 0 & 0 & 11\end{array}$ $\begin{array}{llllllllllllll}-2.1644 & 1.7416 & 0.0000 \text { H } & 0 & 0 & 0 & 0 & 0 & 0 & 0 & 0 & 0 & 12 \\ -0.8451 & 3.6379 & 0.0012 & \text { H } & 0 & 0 & 0 & 0 & 0 & 0 & 0 & 0 & 0 & 12\end{array}$ $2 \begin{array}{llllll}2 & 1 & 0 & 0 & 0 & 0\end{array}$

$\begin{array}{llllll}6 & 1 & 0 & 0 & 0 & 0\end{array}$ $\begin{array}{llllll}9 & 1 & 0 & 0 & 0 & 0\end{array}$ $\begin{array}{lllllll}3 & 0 & 0 & 0 & 0 \\ 3 & 2 & 0 & 0 & 0 & 0\end{array}$ $\begin{array}{llllllll}4 & 3 & 0 & 0 & 0 & 0\end{array}$ $\begin{array}{lllll}3 & 0 & 0 & 0 & 0 \\ 4 & 0 & 0 & 0 & 0\end{array}$

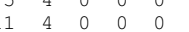

$\begin{array}{llllll}5 & 0 & 0 & 0 & 0\end{array}$

$\begin{array}{lllllll}2 & 5 & 0 & 0 & 0 & 0\end{array}$

$\begin{array}{llllll}13 & 0 & 0 & 0 & 0\end{array}$ END

$\$ \$ \$ \$$

-cyanobenzoicacid-anion.sdf cosmotherm 3D

$\begin{array}{llllllllllll}15 & 15 & 0 & 0 & 0 & 0 & 0 & 0 & 0 & 0 & 0 & \mathrm{~V} 2000\end{array}$ $\begin{array}{llllllllllllll}1.0942 & 1.2228 & -0.0008 & \mathrm{C} & 0 & 0 & 0 & 0 & 0 & 0 & 0 & 0 & 0 & 1\end{array}$

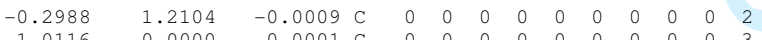
$\begin{array}{rrrrrrrrrrrrrr}-1.0116 & 0.0000 & -0.0001 & \mathrm{C} & 0 & 0 & 0 & 0 & 0 & 0 & 0 & 0 & 0 & 3 \\ -0.2989 & -1.2105 & 0.0007 & \mathrm{C} & 0 & 0 & 0 & 0 & 0 & 0 & 0 & 0 & 0 & 4\end{array}$ $\begin{array}{lllllllllllllll}1.0941 & -1.2230 & 0.0007 & \mathrm{C} & 0 & 0 & 0 & 0 & 0 & 0 & 0 & 0 & 0 & 5\end{array}$ $\begin{array}{llllllllllllll}1.7976 & -0.0001 & 0.0000 & \mathrm{C} & 0 & 0 & 0 & 0 & 0 & 0 & 0 & 0 & 0 & 6\end{array}$ $\begin{array}{llllllllllllll}-2.5482 & 0.0000 & 0.0000 & \mathrm{C} & 0 & 0 & 0 & 0 & 0 & 0 & 0 & 0 & 0 & 7\end{array}$

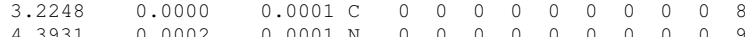
$\begin{array}{lllllllllllll}4.3931 & 0.0002 & 0.0001 & \mathrm{~N} & 0 & 0 & 0 & 0 & 0 & 0 & 0 & 0 & 0\end{array}$ $\begin{array}{llllllllllllll}-3.1184 & 1.1326 & 0.0017 & 0 & 0 & 0 & 0 & 0 & 0 & 0 & 0 & 0 & 0 & 10\end{array}$ $\begin{array}{llllllllllllll}-3.1185 & -1.1324 & -0.0015 & 0 & 0 & 0 & 0 & 0 & 0 & 0 & 0 & 0 & 0 & 11 \\ 1.6426 & 2.1655 & -0.0015 & & 0 & 0 & 0 & 0 & 0 & 0 & 0 & 0 & 0 & 12\end{array}$ $\begin{array}{llllllllllllll}1.6426 & 2.1655 & -0.0015 & \mathrm{H} & 0 & 0 & 0 & 0 & 0 & 0 & 0 & 0 & 0 & 12\end{array}$

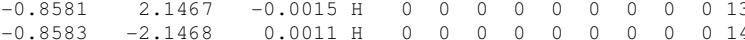
$\begin{array}{lllllllllllll}1.6425 & -2.1657 & 0.0014 \mathrm{H} & 0 & 0 & 0 & 0 & 0 & 0 & 0 & 0 & 0 & 15\end{array}$ $\begin{array}{llllllll}2 & 1 & 0 & 0 & 0 & 0\end{array}$

$\begin{array}{llllllllll}6 & 1 & 0 & 0 & 0 & 0\end{array}$

$\begin{array}{lllllll}12 & 1 & 0 & 0 & 0 & 0\end{array}$

$\begin{array}{llllll}3 & 2 & 0 & 0 & 0\end{array}$

$\begin{array}{llllll}3 & 2 & 0 & 0 & 0\end{array}$

$\begin{array}{lllll}3 & 0 & 0 & 0 & 0\end{array}$

$\begin{array}{lllll}3 & 0 & 0 & 0\end{array}$

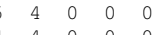

$\begin{array}{lllllll}5 & 5 & 0 & 0 & 0 & 0\end{array}$

$8 \begin{array}{lllll}6 & 0 & 0 & 0\end{array}$

$\begin{array}{llllll}7 & 0 & 0 & 0 & 0\end{array}$

$\begin{array}{lllll}8 & 0 & 0 & 0 & 0\end{array}$

215

\$S\$

4-cyanobenzoicacid.sd

Cosmotherm

$\begin{array}{lllllllllllll}16 & 16 & 0 & 0 & 0 & 0 & 0 & 0 & 0 & 0 & 0 & \mathrm{~V} 2000\end{array}$

$\begin{array}{lllllllllllll}0 & 0 & 0 & 0 & 0 & 0 & 0 & 1\end{array}$

$\begin{array}{lllllllllllll}-0.2673 & 1.2329 & -0.0008 & \mathrm{C} & 0 & 0 & 0 & 0 & 0 & 0 & 0 & 0 & 0\end{array}$

$\begin{array}{llllllllllllll}-0.9802 & 0.0222 & 0.0003 & \mathrm{C} & 0 & 0 & 0 & 0 & 0 & 0 & 0 & 0 & 0 & 3\end{array}$

$\begin{array}{llllllllllllll}-0.2881 & -1.2013 & 0.0016 & \mathrm{C} & 0 & 0 & 0 & 0 & 0 & 0 & 0 & 0 & 0 & 4\end{array}$

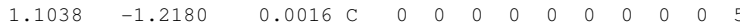

$\begin{array}{llllllllllllll}1.8126 & -0.0002 & 0.0000 & \mathrm{C} & 0 & 0 & 0 & 0 & 0 & 0 & 0 & 0 & 0 & 6\end{array}$

$\begin{array}{llllllllllllll}-2.4728 & 0.0823 & 0.0002 & \mathrm{C} & 0 & 0 & 0 & 0 & 0 & 0 & 0 & 0 & 0 & 7 \\ 3.2415 & -0.0126 & -0.0003 & \mathrm{C} & 0 & 0 & 0 & 0 & 0 & 0 & 0 & 0 & 0 & 8\end{array}$

$\begin{array}{llllllllllllll}4.4086 & -0.0224 & -0.0004 & \mathrm{~N} & 0 & 0 & 0 & 0 & 0 & 0 & 0 & 0 & 0 & 9\end{array}$

$\begin{array}{llllllllllllll}-3.1199 & 1.1225 & 0.0023 & 0 & 0 & 0 & 0 & 0 & 0 & 0 & 0 & 0 & 0 & 10\end{array}$

$\begin{array}{llllllllllllll}-3.0487 & -1.1426 & -0.0029 & 0 & 0 & 0 & 0 & 0 & 0 & 0 & 0 & 0 & 0 & 11 \\ 1.6784 & 2.1677 & -0.0024 & \mathrm{H} & 0 & 0 & 0 & 0 & 0 & 0 & 0 & 0 & 0 & 12\end{array}$

$\begin{array}{lllllllllllllllll}-0.8127 & 2.1767 & -0.0024 & \mathrm{H} & 0 & 0 & 0 & 0 & 0 & 0 & 0 & 0 & 0 & 0 & 11 \\ -0.0016 & \mathrm{H} & 0 & 0 & 0 & 0 & 0 & 0 & 0 & 0 & 0 & 13\end{array}$

$\begin{array}{lllllllllllllllllll}-0.8374 & -2.1416 & 0.0026 & \mathrm{H} & 0 & 0 & 0 & 0 & 0 & 0 & 0 & 0 & 0 & 14\end{array}$

$\begin{array}{lllllllllllllllll}1.6434 & -2.1648 & 0.0028 & \mathrm{H} & 0 & 0 & 0 & 0 & 0 & 0 & 0 & 0 & 0 & 15\end{array}$

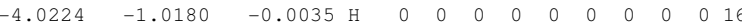

$2.10224-1.0180$ 
$\begin{array}{rrrrrr}6 & 1 & 0 & 0 & 0 & 0 \\ 12 & 1 & 0 & 0 & 0 & 0 \\ 3 & 2 & 0 & 0 & 0 & 0 \\ 13 & 2 & 0 & 0 & 0 & 0 \\ 4 & 3 & 0 & 0 & 0 & 0 \\ 7 & 3 & 0 & 0 & 0 & 0 \\ 5 & 4 & 0 & 0 & 0 & 0 \\ 14 & 4 & 0 & 0 & 0 & 0 \\ 6 & 5 & 0 & 0 & 0 & 0 \\ 15 & 5 & 0 & 0 & 0 & 0 \\ 8 & 6 & 0 & 0 & 0 & 0 \\ 10 & 7 & 0 & 0 & 0 & 0 \\ 11 & 7 & 0 & 0 & 0 & 0 \\ 9 & 8 & 0 & 0 & 0 & 0 \\ 11 & 16 & 0 & 0 & 0 & 0 \\ 16 & & & & & \end{array}$ $M$ END

-dimethylaminobenzoicacid-anion.sdf Cosmotherm

$\begin{array}{llllllllllll}22 & 22 & 0 & 0 & 0 & 0 & 0 & 0 & 0 & 0 & 0 & \mathrm{~V} 2000\end{array}$

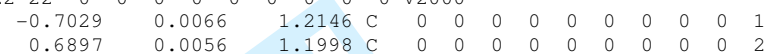
$\begin{array}{rrrrrrrrrrrrrr}0.6897 & 0.0056 & 1.1998 & \mathrm{C} & 0 & 0 & 0 & 0 & 0 & 0 & 0 & 0 & 0 & 2 \\ 1.4242 & 0.0002 & -0.0001 & \mathrm{C} & 0 & 0 & 0 & 0 & 0 & 0 & 0 & 0 & 0 & 3\end{array}$ $\begin{array}{llllllllllllll}0.6898 & -0.0050 & -1.1999 & \mathrm{C} & 0 & 0 & 0 & 0 & 0 & 0 & 0 & 0 & 0 & 4\end{array}$ $\begin{array}{llllllllllllll}-0.7028 & -0.0049 & -1.2148 & \mathrm{C} & 0 & 0 & 0 & 0 & 0 & 0 & 0 & 0 & 0 & 5\end{array}$

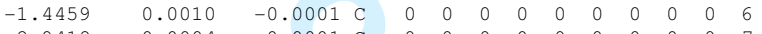
$\begin{array}{llllllllllllll}2.9418 & -0.0004 & 0.0001 & \mathrm{C} & 0 & 0 & 0 & 0 & 0 & 0 & 0 & 0 & 0 & 7\end{array}$ $\begin{array}{llllllllllllll}-2.8221 & 0.0019 & -0.0001 & \mathrm{~N} & 0 & 0 & 0 & 0 & 0 & 0 & 0 & 0 & 0 & 8 \\ -3.5580 & 0.0040 & -1.2595 & \mathrm{C} & 0 & 0 & 0 & 0 & 0 & 0 & 0 & 0 & 0 & 9\end{array}$

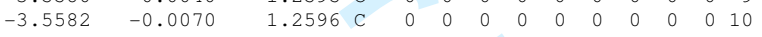

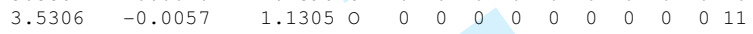
$\begin{array}{llllllllllllll}3.5308 & 0.0046 & -1.1304 & 0 & 0 & 0 & 0 & 0 & 0 & 0 & 0 & 0 & 0 & 12\end{array}$

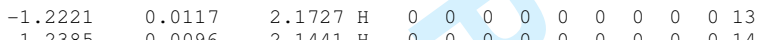

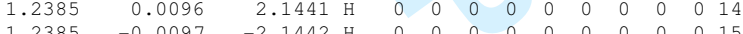

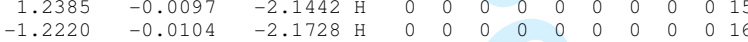

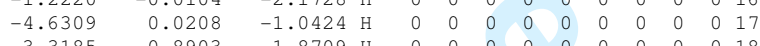

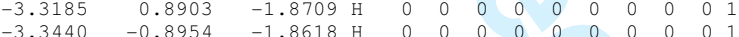
$\begin{array}{llrlllllllllll}-3.3440 & -0.8954 & -1.8618 & \mathrm{H} & 0 & 0 & 0 & 0 & 0 & 0 & 0 & 0 & 0 & 19 \\ -4.6312 & -0.0178 & 1.0424 & \mathrm{H} & 0 & 0 & 0 & 0 & 0 & 0 & 0 & 0 & 0 & 20\end{array}$ $\begin{array}{lllllllllllllll}-3.3221 & -0.8985 & 1.8650 & \mathrm{H} & 0 & 0 & 0 & 0 & 0 & 0 & 0 & 0 & 0 & 21\end{array}$ $\begin{array}{rrrrrrrrrrrrrr}-3.3221 & -0.8985 & 1.8650 & \mathrm{H} & 0 & 0 & 0 & 0 & 0 & 0 & 0 & 0 & 0 & 21 \\ -3.3407 & 0.8873 & 1.8680 & \mathrm{H} & 0 & 0 & 0 & 0 & 0 & 0 & 0 & 0 & 0 & 22\end{array}$

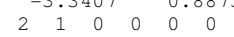
$\begin{array}{llllll}2 & 1 & 0 & 0 & 0 & 0 \\ 6 & 1 & 0 & 0 & 0 & 0\end{array}$ $\begin{array}{rrrrrr}6 & 1 & 0 & 0 & 0 & 0 \\ 13 & 1 & 0 & 0 & 0 & 0\end{array}$ 


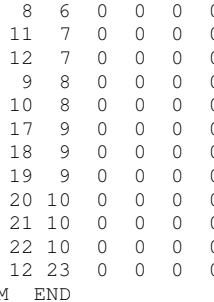




\section{Page 75 of 461}

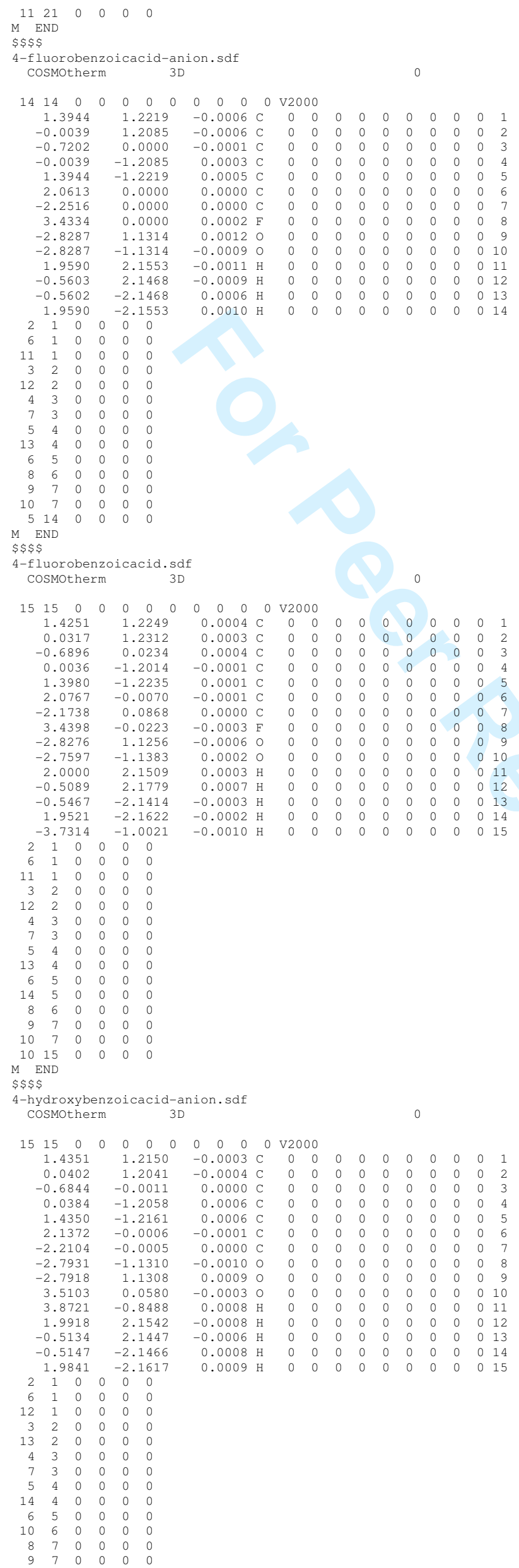




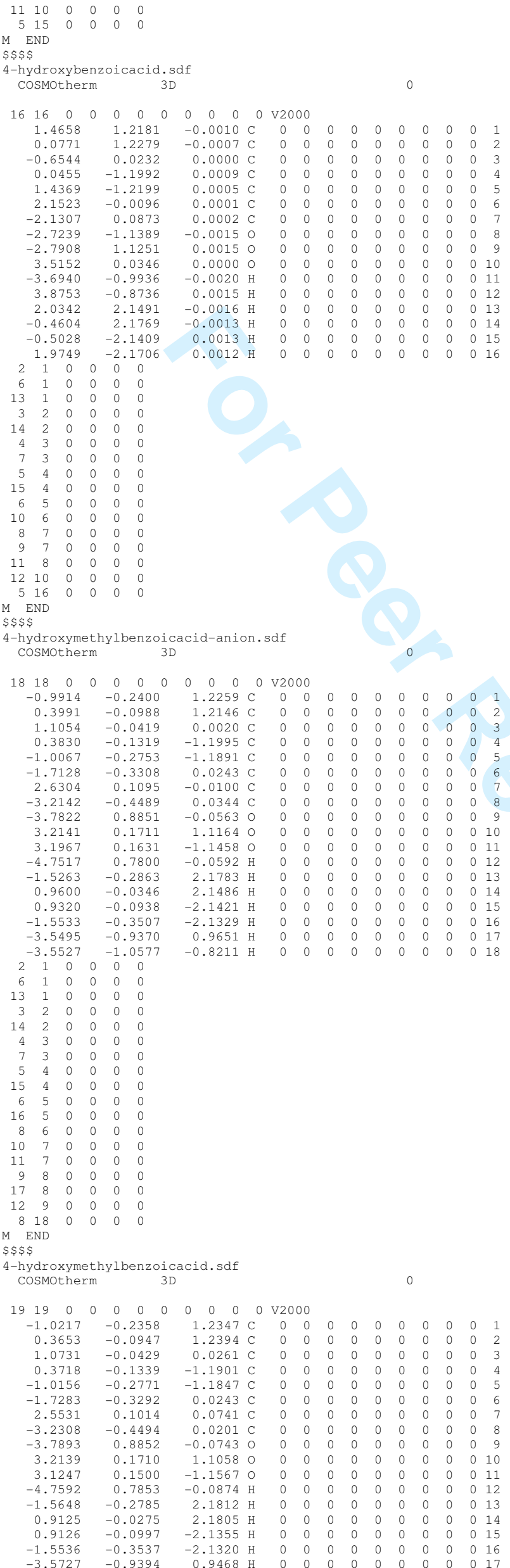




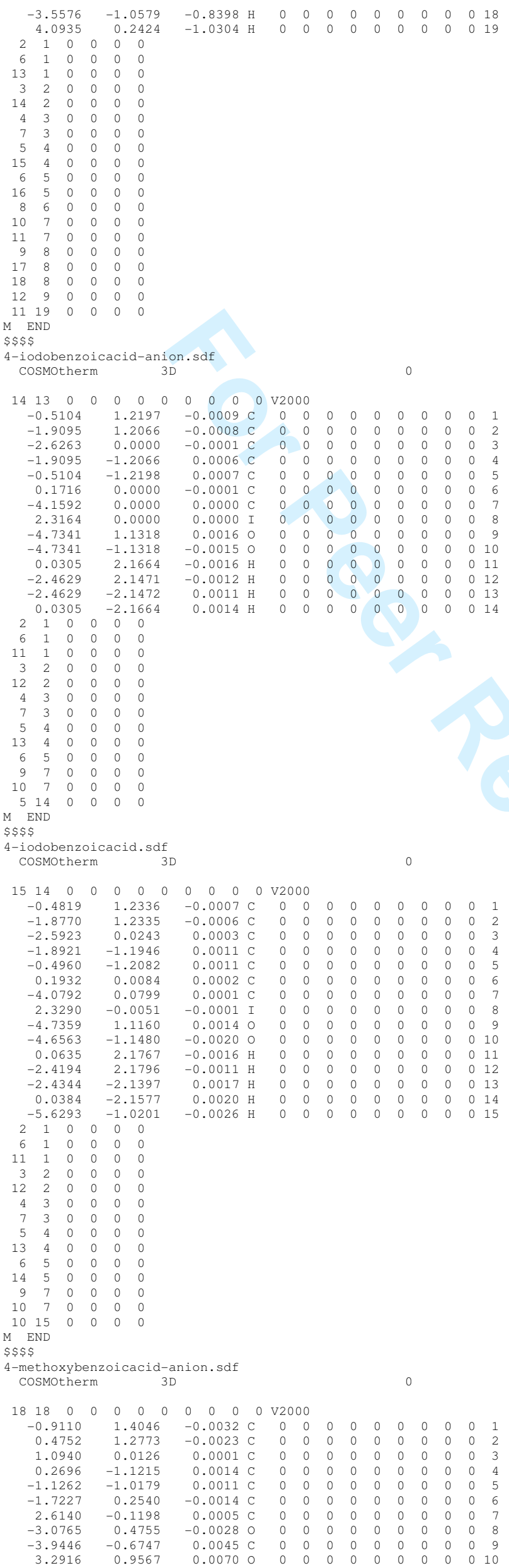




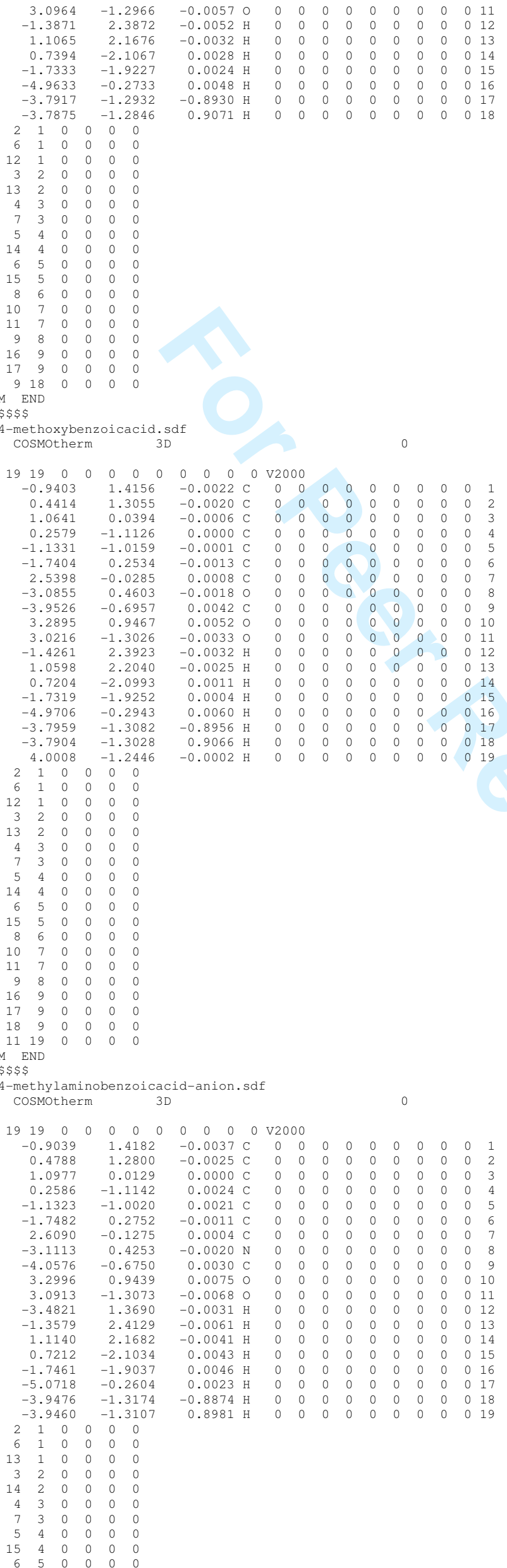


$\begin{array}{rrrrrr}16 & 5 & 0 & 0 & 0 & 0 \\ 8 & 6 & 0 & 0 & 0 & 0 \\ 10 & 7 & 0 & 0 & 0 & 0 \\ 11 & 7 & 0 & 0 & 0 & 0 \\ 9 & 8 & 0 & 0 & 0 & 0 \\ 12 & 8 & 0 & 0 & 0 & 0 \\ 17 & 9 & 0 & 0 & 0 & 0 \\ 18 & 9 & 0 & 0 & 0 & 0 \\ 9 & 19 & 0 & 0 & 0 & 0 \\ M & \text { END } & & & & \end{array}$

$\$ \$ \$$

4-methylaminobenzoicacid.sdf
Cosmotherm

$\begin{array}{llllllllllll}20 & 20 & 0 & 0 & 0 & 0 & 0 & 0 & 0 & 0 & 0 & \mathrm{~V} 2000\end{array}$

$\begin{array}{llllllllllllll}-0.9334 & 1.4307 & -0.0018 & \mathrm{C} & 0 & 0 & 0 & 0 & 0 & 0 & 0 & 0 & 0 & 1\end{array}$

$\begin{array}{llllllllllllll}0.4431 & 1.3104 & -0.0015 & \mathrm{C} & 0 & 0 & 0 & 0 & 0 & 0 & 0 & 0 & 0 & 2 \\ 1.0685 & 0.0409 & -0.0005 & \mathrm{C} & 0 & 0 & 0 & 0 & 0 & 0 & 0 & 0 & 0 & 3\end{array}$

$\begin{array}{rrrrrrrrrrrrrr}1.0685 & 0.0409 & -0.0005 & \mathrm{C} & 0 & 0 & 0 & 0 & 0 & 0 & 0 & 0 & 0 & 3 \\ 0.2459 & -1.1058 & 0.0001 & \mathrm{C} & 0 & 0 & 0 & 0 & 0 & 0 & 0 & 0 & 0 & 4\end{array}$

$\begin{array}{rrrrrrrrrrrrrr}-1.1383 & -1.0018 & 0.0002 & C & 0 & 0 & 0 & 0 & 0 & 0 & 0 & 0 & 0 & 5 \\ -1.7654 & 0.2739 & -0.0006 & C & 0 & 0 & 0 & 0 & 0 & 0 & 0 & 0 & 0 & 6\end{array}$

$\begin{array}{rrrrrrrrrrrrrr}-1.7654 & 0.2739 & -0.0006 & \mathrm{C} & 0 & 0 & 0 & 0 & 0 & 0 & 0 & 0 & 0 & 6 \\ 2.5323 & -0.0343 & 0.0005 & \mathrm{C} & 0 & 0 & 0 & 0 & 0 & 0 & 0 & 0 & 0 & 7\end{array}$

$\begin{array}{rrrrrrrrrrrrrr}2.5323 & -0.0343 & 0.0005 & \mathrm{C} & 0 & 0 & 0 & 0 & 0 & 0 & 0 & 0 & 0 & 7 \\ -3.1193 & 0.4097 & -0.0002 & \mathrm{~N} & 0 & 0 & 0 & 0 & 0 & 0 & 0 & 0 & 0 & 8\end{array}$

$\begin{array}{llllllllllllll}-4.0629 & -0.6965 & 0.0019 & \mathrm{C} & 0 & 0 & 0 & 0 & 0 & 0 & 0 & 0 & 0 & 9\end{array}$

$\begin{array}{llllllllllllll}3.2983 & 0.9335 & 0.0035 & 0 & 0 & 0 & 0 & 0 & 0 & 0 & 0 & 0 & 0 & 10\end{array}$

$\begin{array}{llllllllllllll}3.0151 & -1.3154 & -0.0023 & 0 & 0 & 0 & 0 & 0 & 0 & 0 & 0 & 0 & 0 & 11\end{array}$

$\begin{array}{llllllllllllll}-3.4979 & 1.3514 & -0.0007 & \mathrm{H} & 0 & 0 & 0 & 0 & 0 & 0 & 0 & 0 & 0 & 12\end{array}$

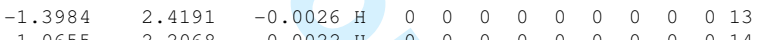

$\begin{array}{rrrrrrrrrrrrrr}1.0655 & 2.2068 & -0.0022 & \mathrm{H} & 0 & 0 & 0 & 0 & 0 & 0 & 0 & 0 & 0 & 14 \\ 0.7021 & -2.0961 & 0.0010 & \mathrm{H} & 0 & 0 & 0 & 0 & 0 & 0 & 0 & 0 & 0 & 15\end{array}$

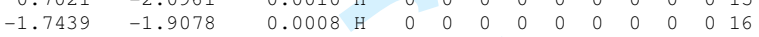

$\begin{array}{llllllllllllll}-5.0772 & -0.2837 & 0.0043 & \mathrm{H} & 0 & 0 & 0 & 0 & 0 & 0 & 0 & 0 & 0 & 17\end{array}$

$\begin{array}{llllllllllllll}-3.9482 & -1.3307 & -0.8921 & \mathrm{H} & 0 & 0 & 0 & 0 & 0 & 0 & 0 & 0 & 0 & 18\end{array}$

$\begin{array}{llllllllllllll}-3.9439 & -1.3308 & 0.8951 & \mathrm{H} & 0 & 0 & 0 & 0 & 0 & 0 & 0 & 0 & 0 & 19\end{array}$

$3.9935-1.2516$

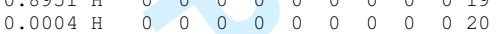

$\begin{array}{llllll}2 & 1 & 0 & 0 & 0 & 0 \\ 6 & 1 & 0 & 0 & 0 & 0\end{array}$

$\begin{array}{cccccc}13 & 1 & 0 & 0 & 0 & 0\end{array}$

$\begin{array}{lllllll}3 & 2 & 0 & 0 & 0 & 0 & 0 \\ 14 & 2 & 0 & 0 & 0 & 0 & 0\end{array}$

$\begin{array}{llllll}4 & 3 & 0 & 0 & 0 & 0\end{array}$

$\begin{array}{llllll}3 & 0 & 0 & 0 & 0 & 0\end{array}$

$\begin{array}{llllll}4 & 0 & 0 & 0 & 0\end{array}$

$\begin{array}{llllll}5 & 4 & 0 & 0 & 0 & 0 \\ 6 & 5 & 0 & 0 & 0 & 0\end{array}$

$\begin{array}{lllllll}6 & 5 & 0 & 0 & 0 & 0\end{array}$

$8 \begin{array}{llllll}8 & 6 & 0 & 0 & 0 & 0\end{array}$

$\begin{array}{llllllll}1 & 7 & 0 & 0 & 0 & 0\end{array}$

$\begin{array}{llllll}9 & 8 & 0 & 0 & 0 & 0\end{array}$

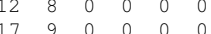

$\begin{array}{lllllll}8 & 9 & 0 & 0 & 0 & 0\end{array}$

$\begin{array}{rrrrrr}19 & 9 & 0 & 0 & 0 & 0 \\ 1 & 20 & 0 & 0 & 0 & 0\end{array}$

END

\$\$\$

4-methylbenzoicacid-anion.sdf

COSMOtherm

$\begin{array}{llllllllllll}17 & 17 & 0 & 0 & 0 & 0 & 0 & 0 & 0 & 0 & 0 & \mathrm{~V} 2000\end{array}$

$\begin{array}{llllllllllllll}1.4618 & 1.2047 & -0.0101 & \mathrm{C} & 0 & 0 & 0 & 0 & 0 & 0 & 0 & 0 & 0 & 1\end{array}$

$\begin{array}{llllllllllllll}0.0640 & 1.2046 & -0.0045 & \mathrm{C} & 0 & 0 & 0 & 0 & 0 & 0 & 0 & 0 & 0 & 2\end{array}$

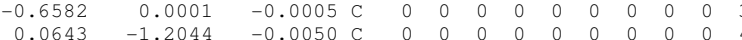

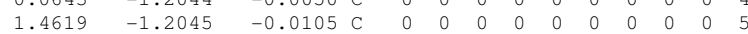

$\begin{array}{llllllllllllll}2.1880 & 0.0003 & -0.0095 & \mathrm{C} & 0 & 0 & 0 & 0 & 0 & 0 & 0 & 0 & 0 & 6\end{array}$

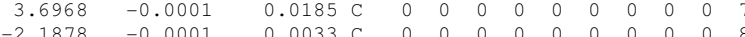

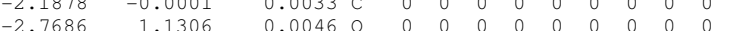

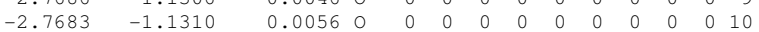

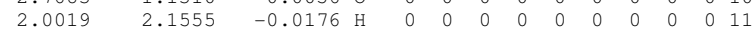

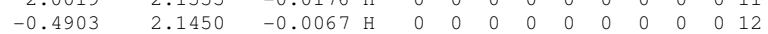

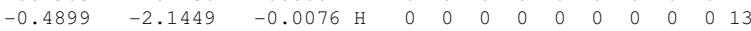

$\begin{array}{llllllllllllll}2.0019 & -2.1553 & -0.0185 & \mathrm{H} & 0 & 0 & 0 & 0 & 0 & 0 & 0 & 0 & 0 & 14\end{array}$

$\begin{array}{lllllllllllllll}4.0700 & -0.0358 & 1.0553 & \mathrm{H} & 0 & 0 & 0 & 0 & 0 & 0 & 0 & 0 & 0 & 0 & 15\end{array}$

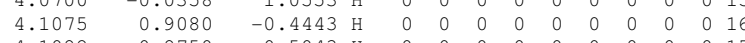

$\begin{array}{llllll}2 & 1 & 0 & 0 & 0 & 0\end{array}$

$\begin{array}{llllllllll}6 & 1 & 0 & 0 & 0 & 0\end{array}$

$\begin{array}{llllll}1 & 1 & 0 & 0 & 0 & 0 \\ 3 & 2 & 0 & 0 & 0 & 0\end{array}$

$\begin{array}{llllll}2 & 2 & 0 & 0 & 0 & 0\end{array}$

$\begin{array}{lllll}3 & 0 & 0 & 0 & 0\end{array}$

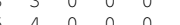

$\begin{array}{lllllllll}3 & 4 & 0 & 0 & 0\end{array}$

$\begin{array}{lllllll}6 & 5 & 0 & 0 & 0 & 0 \\ 4 & 5 & 0 & 0 & 0 & 0\end{array}$

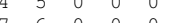

$\begin{array}{lllllll}7 & 6 & 0 & 0 & 0 & 0 & 0 \\ 15 & 7 & 0 & 0 & 0\end{array}$

$\begin{array}{llllll}16 & 7 & 0 & 0 & 0 & 0 \\ 9 & 8 & 0 & 0 & 0 & 0\end{array}$

$\begin{array}{lllllll}0 & 8 & 0 & 0 & 0 & 0\end{array}$

$\begin{array}{lllll}17 & 0 & 0 & 0 & 0\end{array}$

END

4 -methylbenzoicacid.sdf

$\begin{array}{llllllllllll}8 & 18 & 0 & 0 & 0 & 0 & 0 & 0 & 0 & 0 & 0 & v 2000\end{array}$

$\begin{array}{rrrrrrrrrrrrrr}1.4921 & 1.2082 & -0.0093 & \mathrm{C} & 0 & 0 & 0 & 0 & 0 & 0 & 0 & 0 & 0 & 1 \\ 0.0990 & 1.2280 & -0.0040 & \mathrm{C} & 0 & 0 & 0 & 0 & 0 & 0 & 0 & 0 & 0 & 2 \\ -0.6281 & 0.0242 & -0.0002 & \mathrm{C} & 0 & 0 & 0 & 0 & 0 & 0 & 0 & 0 & 0 & 3 \\ 0.0707 & -1.1972 & -0.0047 & \mathrm{C} & 0 & 0 & 0 & 0 & 0 & 0 & 0 & 0 & 0 & 4\end{array}$ 


$\begin{array}{rrrrrrrrrrrrrr}1.4648 & -1.2064 & -0.0103 & \mathrm{C} & 0 & 0 & 0 & 0 & 0 & 0 & 0 & 0 & 0 & 5 \\ 2.2014 & -0.0076 & -0.0092 & \mathrm{C} & 0 & 0 & 0 & 0 & 0 & 0 & 0 & 0 & 0 & 6 \\ 3.7072 & -0.0262 & 0.0169 & \mathrm{C} & 0 & 0 & 0 & 0 & 0 & 0 & 0 & 0 & 0 & 7 \\ -2.1104 & 0.0868 & 0.0029 & \mathrm{C} & 0 & 0 & 0 & 0 & 0 & 0 & 0 & 0 & 0 & 8 \\ -2.7686 & 1.1239 & 0.0040 & 0 & 0 & 0 & 0 & 0 & 0 & 0 & 0 & 0 & 0 & 9 \\ -2.6992 & -1.1391 & 0.0051 & 0 & 0 & 0 & 0 & 0 & 0 & 0 & 0 & 0 & 0 & 10 \\ 2.0430 & 2.1513 & -0.0164 & \mathrm{H} & 0 & 0 & 0 & 0 & 0 & 0 & 0 & 0 & 0 & 11 \\ -0.4397 & 2.1767 & -0.0056 & \mathrm{H} & 0 & 0 & 0 & 0 & 0 & 0 & 0 & 0 & 0 & 12 \\ -0.4783 & -2.1388 & -0.0072 & \mathrm{H} & 0 & 0 & 0 & 0 & 0 & 0 & 0 & 0 & 0 & 13 \\ 1.9940 & -2.1619 & -0.0182 & \mathrm{H} & 0 & 0 & 0 & 0 & 0 & 0 & 0 & 0 & 0 & 14 \\ 4.0735 & -0.0657 & 1.0559 & \mathrm{H} & 0 & 0 & 0 & 0 & 0 & 0 & 0 & 0 & 0 & 15 \\ 4.1283 & 0.8773 & -0.4442 & \mathrm{H} & 0 & 0 & 0 & 0 & 0 & 0 & 0 & 0 & 0 & 16 \\ 4.1065 & -0.9081 & -0.5023 & \mathrm{H} & 0 & 0 & 0 & 0 & 0 & 0 & 0 & 0 & 0 & 17 \\ -3.6703 & -0.9992 & 0.0065 & \mathrm{H} & 0 & 0 & 0 & 0 & 0 & 0 & 0 & 0 & 0 & 18 \\ 1 & 0 & & & & & & & & & & & & \end{array}$




\section{Page 81 of 461}

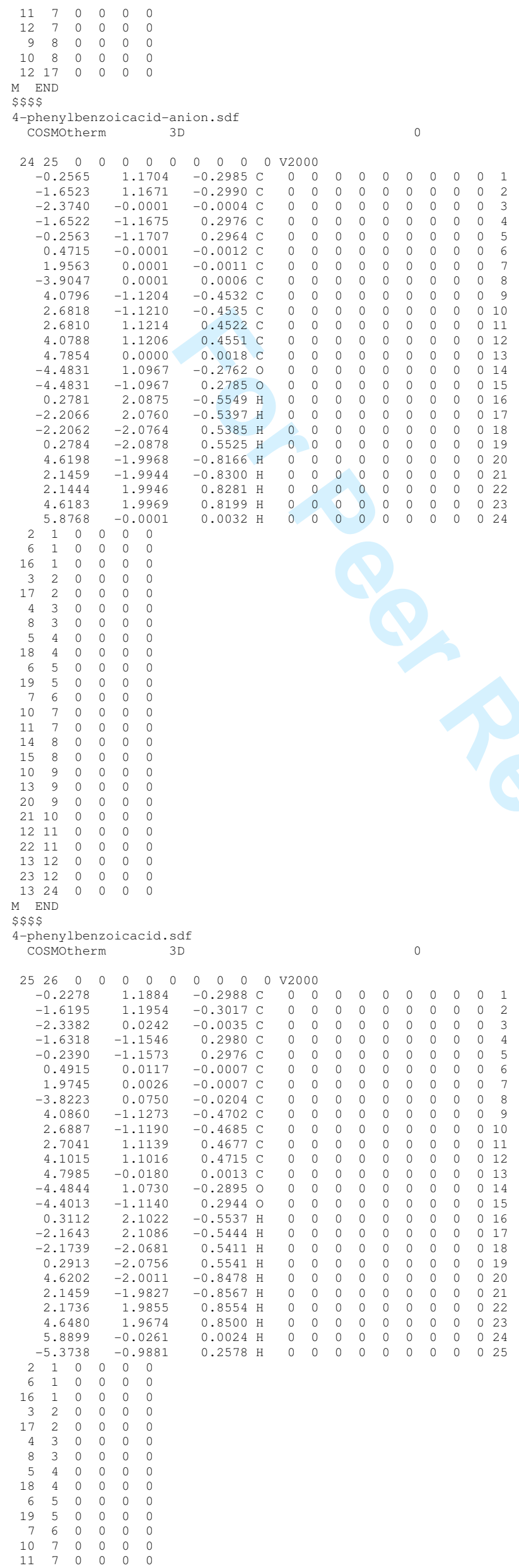




\section{Page 83 of 461}

$\begin{array}{rrrrrr}8 & 6 & 0 & 0 & 0 & 0 \\ 11 & 7 & 0 & 0 & 0 & 0 \\ 12 & 7 & 0 & 0 & 0 & 0 \\ 9 & 8 & 0 & 0 & 0 & 0 \\ 17 & 8 & 0 & 0 & 0 & 0 \\ 18 & 8 & 0 & 0 & 0 & 0 \\ 10 & 9 & 0 & 0 & 0 & 0 \\ 19 & 9 & 0 & 0 & 0 & 0 \\ 20 & 9 & 0 & 0 & 0 & 0 \\ 21 & 10 & 0 & 0 & 0 & 0 \\ 22 & 10 & 0 & 0 & 0 & 0 \\ 23 & 10 & 0 & 0 & 0 & 0 \\ 12 & 24 & 0 & 0 & 0 & 0 \\ M \\ M\end{array}$

$\$ \$ \$$

4-sulfamoylbenzoicacid-anion.sdf

Cosmotherm 3D

$\begin{array}{llllllllllll}19 & 19 & 0 & 0 & 0 & 0 & 0 & 0 & 0 & 0 & 0 & \mathrm{~V} 2000\end{array}$

$\begin{array}{llllllllllllll}-0.0481 & -0.0551 & 1.2165 & \mathrm{C} & 0 & 0 & 0 & 0 & 0 & 0 & 0 & 0 & 0 & 1\end{array}$

$\begin{array}{llllllllllllll}1.3476 & -0.0233 & 1.2076 & \mathrm{C} & 0 & 0 & 0 & 0 & 0 & 0 & 0 & 0 & 0 & 2 \\ 2.0623 & 0.0002 & -0.0011 & \mathrm{C} & 0 & 0 & 0 & 0 & 0 & 0 & 0 & 0 & 0 & 3\end{array}$

$\begin{array}{rrrrrrrrrrrrrr}2.0623 & 0.0002 & -0.0011 & \mathrm{C} & 0 & 0 & 0 & 0 & 0 & 0 & 0 & 0 & 0 & 3 \\ 1.3532 & -0.0029 & -1.2129 & \mathrm{C} & 0 & 0 & 0 & 0 & 0 & 0 & 0 & 0 & 0 & 4\end{array}$

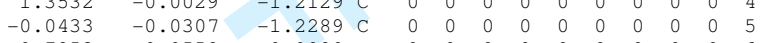

$\begin{array}{llllllllllllll}-0.7253 & -0.0552 & -0.0080 & \text { C } & 0 & 0 & 0 & 0 & 0 & 0 & 0 & 0 & 0 & 6\end{array}$

$\begin{array}{rrrrrrrrrrrrrr}3.5989 & 0.0249 & 0.0035 & \mathrm{C} & 0 & 0 & 0 & 0 & 0 & 0 & 0 & 0 & 0 & 7 \\ -2.5391 & -0.0849 & -0.0073 & \mathrm{~S} & 0 & 0 & 0 & 0 & 0 & 0 & 0 & 0 & 0 & 8\end{array}$

$\begin{array}{lrrrrrrrrrrrrr}-2.5391 & -0.0849 & -0.0073 & \mathrm{~S} & 0 & 0 & 0 & 0 & 0 & 0 & 0 & 0 & 0 & 8 \\ -2.9886 & -0.7789 & 1.2362 & 0 & 0 & 0 & 0 & 0 & 0 & 0 & 0 & 0 & 0 & 9\end{array}$

$\begin{array}{llllllllllllll}-2.9977 & -0.5703 & -1.3423 & 0 & 0 & 0 & 0 & 0 & 0 & 0 & 0 & 0 & 0 & 10\end{array}$

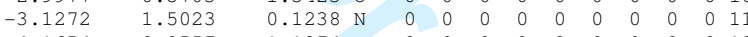

$\begin{array}{llllllllllllll}4.1654 & 0.0557 & 1.1374 & 0 & 0 & 0 & 0 & 0 & 0 & 0 & 0 & 0 & 0 & 12\end{array}$

$\begin{array}{llllllllllllll}4.1733 & 0.0117 & -1.1270 & 0 & 0 & 0 & 0 & 0 & 0 & 0 & 0 & 0 & 0 & 13\end{array}$

$\begin{array}{llllllllllllll}-2.8241 & 1.9378 & 1.0011 & \mathrm{H} & 0 & 0 & 0 & 0 & 0 & 0 & 0 & 0 & 0 & 14 \\ -2.8282 & 2.0721 & -0.6747 & \mathrm{H} & 0 & 0 & 0 & 0 & 0 & 0 & 0 & 0 & 0 & 15\end{array}$

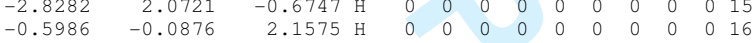

$\begin{array}{llllllllllllll}1.9036 & -0.0251 & 2.1459 & \mathrm{H} & 0 & 0 & 0 & 0 & 0 & 0 & 0 & 0 & 0 & 17\end{array}$

$\begin{array}{llllllllllllll}1.9135 & 0.0087 & -2.1486 & \mathrm{H} & 0 & 0 & 0 & 0 & 0 & 0 & 0 & 0 & 0 & 18\end{array}$

210.0411

$\begin{array}{llllll}2 & 1 & 0 & 0 & 0 & 0 \\ 6 & 1 & 0 & 0 & 0 & 0\end{array}$

$\begin{array}{rrrrrrr}16 & 1 & 0 & 0 & 0 & 0\end{array}$

$\begin{array}{lllllll}3 & 2 & 0 & 0 & 0 & 0\end{array}$

$\begin{array}{llllllll}7 & 3 & 0 & 0 & 0 & 0\end{array}$

$\begin{array}{llllll}4 & 0 & 0 & 0 & 0 & 0\end{array}$

$\begin{array}{lllll}8 & 4 & 0 & 0 & 0\end{array}$

$8 \begin{array}{lllllll}8 & 6 & 0 & 0 & 0 & 0\end{array}$

$\begin{array}{lllllllll}2 & 7 & 0 & 0 & 0 & 0\end{array}$

$\begin{array}{lllll}3 & 7 & 0 & 0 & 0\end{array}$

$\begin{array}{llllll}9 & 8 & 0 & 0 & 0 & 0 \\ 0 & 8 & 0 & 0 & 0 & 0\end{array}$

$\begin{array}{lllll}1 & 8 & 0 & 0 & 0\end{array}$

$\begin{array}{llllll}14 & 11 & 0 & 0 & 0 & 0 \\ 15 & 11 & 0 & 0 & 0 & 0\end{array}$

$\begin{array}{lllllll}5 & 19 & 0 & 0 & 0 & 0\end{array}$

$M$ END

$\$ \$ \$$

4-sulfamoylbenzoicacid.sdf
Cosmotherm

$\begin{array}{llllllllllll}20 & 20 & 0 & 0 & 0 & 0 & 0 & 0 & 0 & 0 & 0 & \mathrm{~V} 2000\end{array}$

$\begin{array}{llllllllllllll}-0.0765 & -0.0461 & 1.2276 & \mathrm{C} & 0 & 0 & 0 & 0 & 0 & 0 & 0 & 0 & 0 & 1\end{array}$

$\begin{array}{llllllllllllll}1.3166 & -0.0166 & 1.2336 & \mathrm{C} & 0 & 0 & 0 & 0 & 0 & 0 & 0 & 0 & 0 & 2\end{array}$

$\begin{array}{llllllllllllll}2.0303 & 0.0073 & 0.0234 & \mathrm{C} & 0 & 0 & 0 & 0 & 0 & 0 & 0 & 0 & 0 & 3 \\ 1.3407 & 0.0074 & -1.2007 & \mathrm{C} & 0 & 0 & 0 & 0 & 0 & 0 & 0 & 0 & 0 & \end{array}$

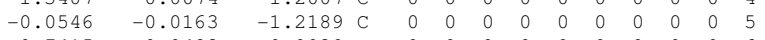

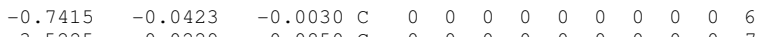

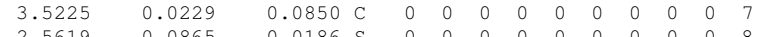

$\begin{array}{llllllllllllll}-2.5619 & -0.0865 & -0.0186 & \mathrm{~S} & 0 & 0 & 0 & 0 & 0 & 0 & 0 & 0 & 0 & 8 \\ -3.0022 & -0.8491 & 1.1851 & 0 & 0 & 0 & 0 & 0 & 0 & 0 & 0 & 0 & 0 & 9\end{array}$

$\begin{array}{lllllllllllllll}-3.0022 & -0.8491 & 1.1851 & 0 & 0 & 0 & 0 & 0 & 0 & 0 & 0 & 0 & 0 & 0\end{array}$

$\begin{array}{lllllllllllll}-3.1552 & 1.4840 & -1.3819 & 0 & 0 & 0 & 0 & 0 & 0 & 0 & 0 & 0 & 0\end{array}$

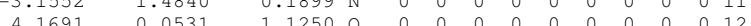

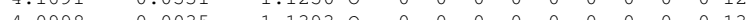

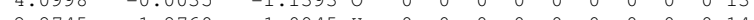

$\begin{array}{lllllllllllllll}-2.8745 & 1.8760 & 1.0945 & \mathrm{H} & 0 & 0 & 0 & 0 & 0 & 0 & 0 & 0 & 0 & 14\end{array}$

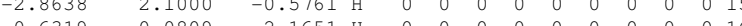

$\begin{array}{llllllllllllllll}-0.6319 & -0.0809 & 2.1651 \mathrm{H} & 0 & 0 & 0 & 0 & 0 & 0 & 0 & 0 & 0 & 0 & 15 \\ 1.8601 & -0.0215 & 2.1785 & \mathrm{H} & 0 & 0 & 0 & 0 & 0 & 0 & 0 & 0 & 0 & 16\end{array}$

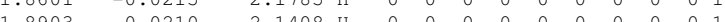

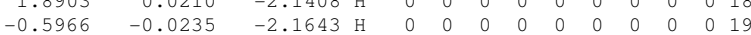

$\begin{array}{llllllllllllll}5.0733 & 0.0090 & -1.0139 \mathrm{H} & 0 & 0 & 0 & 0 & 0 & 0 & 0 & 0 & 0 & 0 & 20\end{array}$

$2 \quad 1 \quad 0 \quad 0000$

$\begin{array}{rrrrrrr}6 & 1 & 0 & 0 & 0 & 0 \\ 16 & 1 & 0 & 0 & 0 & 0\end{array}$

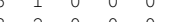

$\begin{array}{llllllll}2 & 2 & 0 & 0 & 0 & 0 & 0 & 0 \\ 7 & 2 & 0 & 0 & 0\end{array}$

$\begin{array}{lllll}3 & 0 & 0 & 0\end{array}$

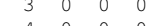

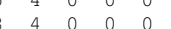

$\begin{array}{llllll}5 & 0 & 0 & 0 & 0 \\ 5 & 0 & 0 & 0 & 0\end{array}$

861000000

$\begin{array}{lllllllll}3 & 7 & 0 & 0 & 0 & 0\end{array}$

$\begin{array}{llllll}9 & 8 & 0 & 0 & 0 & 0\end{array}$

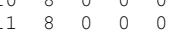

$1411 \quad 0 \quad 0000$

$\begin{array}{llllll}15 & 11 & 0 & 0 & 0 & 0\end{array}$

1 END

$\$ \$ \$$ 
-trifluoromethylbenzoicacid-anion.sdf

$\begin{array}{llllllllllll}17 & 17 & 0 & 0 & 0 & 0 & 0 & 0 & 0 & 0 & 0 & \mathrm{~V} 2000\end{array}$

\begin{tabular}{|c|c|c|c|c|c|c|c|c|c|c|c|c|}
\hline $\begin{array}{lll}17 & 0\end{array}$ & $\begin{array}{lll}0 & 0 & 0\end{array}$ & $\begin{array}{lll}0 & 0 & 0\end{array}$ & 0 & $\sqrt{2} 20$ & & & & & & & & \\
\hline 0.2651 & 1.2178 & -0.0294 & $\mathrm{C}$ & 0 & 0 & 0 & 0 & 0 & 0 & 0 & 0 & 0 \\
\hline-1.1306 & 1.2087 & -0.0163 & C & 0 & 0 & 0 & 0 & 0 & 0 & 0 & 0 & 0 \\
\hline-1.8443 & 0.0002 & -0.0123 & C & 0 & 0 & 0 & 0 & 0 & 0 & 0 & 0 & 0 \\
\hline-1.1288 & -1.2076 & -0.0292 & C & 0 & 0 & 0 & 0 & 0 & 0 & 0 & 0 & 0 \\
\hline 0.2667 & -1.2144 & -0.0443 & C & 0 & 0 & 0 & 0 & 0 & 0 & 0 & 0 & 0 \\
\hline 0.9647 & 0.0024 & -0.0470 & C & 0 & 0 & 0 & 0 & 0 & 0 & 0 & 0 & 0 \\
\hline-3.3801 & -0.0007 & 0.0105 & C & 0 & 0 & 0 & 0 & 0 & 0 & 0 & 0 & 0 \\
\hline 2.4672 & 0.0010 & -0.0013 & C & 0 & 0 & 0 & 0 & 0 & 0 & 0 & 0 & 0 \\
\hline 2.9450 & -0.0595 & 1.2888 & F & 0 & 0 & 0 & 0 & 0 & 0 & 0 & 0 & 0 \\
\hline 3.0147 & 1.1232 & -0.5539 & $\mathrm{~F}$ & 0 & 0 & 0 & 0 & 0 & 0 & 0 & 0 & 0 \\
\hline 3.0157 & -1.0668 & -0.6547 & F & 0 & 0 & 0 & 0 & 0 & 0 & 0 & 0 & 0 \\
\hline-3.9530 & 1.1310 & 0.0062 & 0 & 0 & 0 & 0 & 0 & 0 & 0 & 0 & 0 & 0 \\
\hline-3.9513 & -1.1331 & 0.0336 & 0 & 0 & 0 & 0 & 0 & 0 & 0 & 0 & 0 & \\
\hline 0.8061 & 2.1649 & -0.0321 & $\mathrm{H}$ & 0 & 0 & 0 & 0 & 0 & 0 & 0 & 0 & 0 \\
\hline-1.6881 & 2.1462 & -0.0074 & $\mathrm{H}$ & 0 & 0 & 0 & 0 & 0 & 0 & 0 & 0 & 0 \\
\hline-1.6851 & -2.1458 & -0.0292 & $\mathrm{H}$ & 0 & & 0 & 0 & 0 & 0 & 0 & 0 & 0 \\
\hline 0.8089 & -2.1609 & -0.0588 & $\mathrm{H}$ & 0 & 0 & 0 & 0 & 0 & 0 & 0 & 0 & 0 \\
\hline
\end{tabular}

$\begin{array}{lccccc}2 & 1 & 0 & 0 & 0 & 0\end{array}$

$\begin{array}{rrrrrr}6 & 1 & 0 & 0 & 0 & 0 \\ 14 & 1 & 0 & 0 & 0 & 0 \\ 3 & 2 & 0 & 0 & 0 & 0\end{array}$

$\begin{array}{llllll}3 & 2 & 0 & 0 & 0 & 0\end{array}$

$\begin{array}{llllll}15 & 2 & 0 & 0 & 0 & 0\end{array}$

$\begin{array}{llllllll}4 & 3 & 0 & 0 & 0 & 0 \\ 7 & 3 & 0 & 0 & 0 & 0\end{array}$

$\begin{array}{llll}0 & 0 & 0\end{array}$

$\begin{array}{llllll}16 & 4 & 0 & 0 & 0 & 0\end{array}$

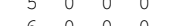

$\begin{array}{lllll}6 & 0 & 0 & 0 & 0 \\ 7 & 0 & 0 & 0\end{array}$

$\begin{array}{llllll}7 & 0 & 0 & 0 & 0\end{array}$

$\begin{array}{llllll}9 & 8 & 0 & 0 & 0 & 0 \\ 0 & 8 & 0 & 0 & 0 & 0\end{array}$

$\begin{array}{llllll}1 & 8 & 0 & 0 & 0 & 0\end{array}$

517

$4-$ trifluoromethylbenzoicacid.sdf

Cosmotherm thylbenzoica

$\begin{array}{lllllllllllll}18 & 18 & 0 & 0 & 0 & 0 & 0 & 0 & 0 & 0 & 0 & \mathrm{~V} 2000\end{array}$

$\begin{array}{ccccccccccccccccc}18 & 0 & 0 & 0 & 0 & 0 & 0 & 0 & 0 & 0 & \mathrm{~V} 2000 \\ 0.2943 & 1.2296 & -0.0284 & \mathrm{C} & 0 & 0 & 0 & 0 & 0 & 0 & 0 & 0 & 0\end{array}$

$\begin{array}{llllllllllllll}-1.0989 & 1.2345 & -0.0147 & \mathrm{C} & 0 & 0 & 0 & 0 & 0 & 0 & 0 & 0 & 0 & 2\end{array}$

$\begin{array}{llllllllllllllll}-1.8109 & 0.0244 & -0.0116 & \mathrm{C} & 0 & 0 & 0 & 0 & 0 & 0 & 0 & 0 & 0 & 3\end{array}$

$\begin{array}{llllllllllllllll}-1.1147 & -1.1960 & -0.0271 & \mathrm{C} & 0 & 0 & 0 & 0 & 0 & 0 & 0 & 0 & 0 & 3 \\ 0.2793 & -1.2053 & -0.0420 & \mathrm{C} & 0 & 0 & 0 & 0 & 0 & 0 & 0 & 0 & 0 & 4\end{array}$

$\begin{array}{rrrrrrrrrrrrrr}0.2793 & -1.2053 & -0.0420 & \mathrm{C} & 0 & 0 & 0 & 0 & 0 & 0 & 0 & 0 & 0 & 5 \\ 0.9810 & 0.0077 & -0.0434 & \mathrm{C} & 0 & 0 & 0 & 0 & 0 & 0 & 0 & 0 & 0 & 6\end{array}$

$\begin{array}{rrrrrrrrrrrrrr}-3.3026 & 0.0808 & 0.0076 & C & 0 & 0 & 0 & 0 & 0 & 0 & 0 & 0 & 0\end{array}$

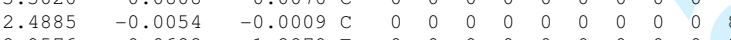

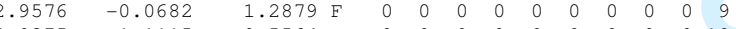

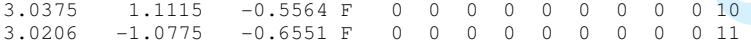

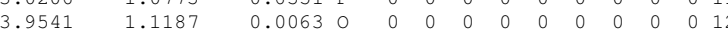

$\begin{array}{llllllllllllll}-3.8768 & -1.1453 & 0.0288 & 0 & 0 & 0 & 0 & 0 & 0 & 0 & 0 & 0 & 0 & 13\end{array}$

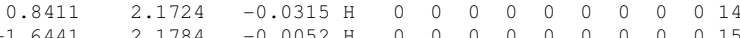

$\begin{array}{llllllllllllll}-1.6441 & 2.1784 & -0.0052 & \mathrm{H} & 0 & 0 & 0 & 0 & 0 & 0 & 0 & 0 & 0 & 15\end{array}$

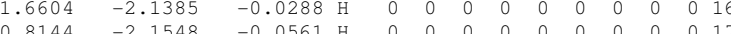

$\begin{array}{rrrrrrrrrrrrrr}0.8144 & -2.1548 & -0.0561 & \mathrm{H} & 0 & 0 & 0 & 0 & 0 & 0 & 0 & 0 & 0 & 17 \\ -4.8504 & -1.0215 & 0.0396 & \mathrm{H} & 0 & 0 & 0 & 0 & 0 & 0 & 0 & 0 & 0 & 18\end{array}$

$\begin{array}{llllll}2 & 1 & 0 & 0 & 0 & 0\end{array}$

$\begin{array}{llllll}6 & 1 & 0 & 0 & 0 & 0\end{array}$

$\begin{array}{llllll}4 & 1 & 0 & 0 & 0 & 0 \\ 3 & 2 & 0 & 0 & 0 & 0\end{array}$

$\begin{array}{lllllll}5 & 2 & 0 & 0 & 0 & 0\end{array}$

$\begin{array}{lllllll}4 & 3 & 0 & 0 & 0 & 0\end{array}$

$\begin{array}{lllll}3 & 0 & 0 & 0\end{array}$

$\begin{array}{llllll}5 & 4 & 0 & 0 & 0 & 0\end{array}$

$\begin{array}{llllll}6 & 5 & 0 & 0 & 0 & 0\end{array}$

$\begin{array}{llllll}7 & 5 & 0 & 0 & 0\end{array}$

$\begin{array}{lllllll}8 & 6 & 0 & 0 & 0 & 0\end{array}$

$\begin{array}{lllllll}2 & 7 & 0 & 0 & 0 & 0\end{array}$

$\begin{array}{llll}0 & 0 & 0 & 0\end{array}$

$\begin{array}{lllllll}10 & 8 & 0 & 0 & 0 & 0\end{array}$

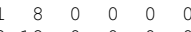

$\begin{array}{lll}13 & 18 \\ \text { END }\end{array}$

$\$ \$ \$ \$$

-fluorouracilo-anion.sdf

cosmotherm 3D

$\begin{array}{llllllllllll}11 & 11 & 0 & 0 & 0 & 0 & 0 & 0 & 0 & 0 & 0 & \mathrm{v} 2000\end{array}$

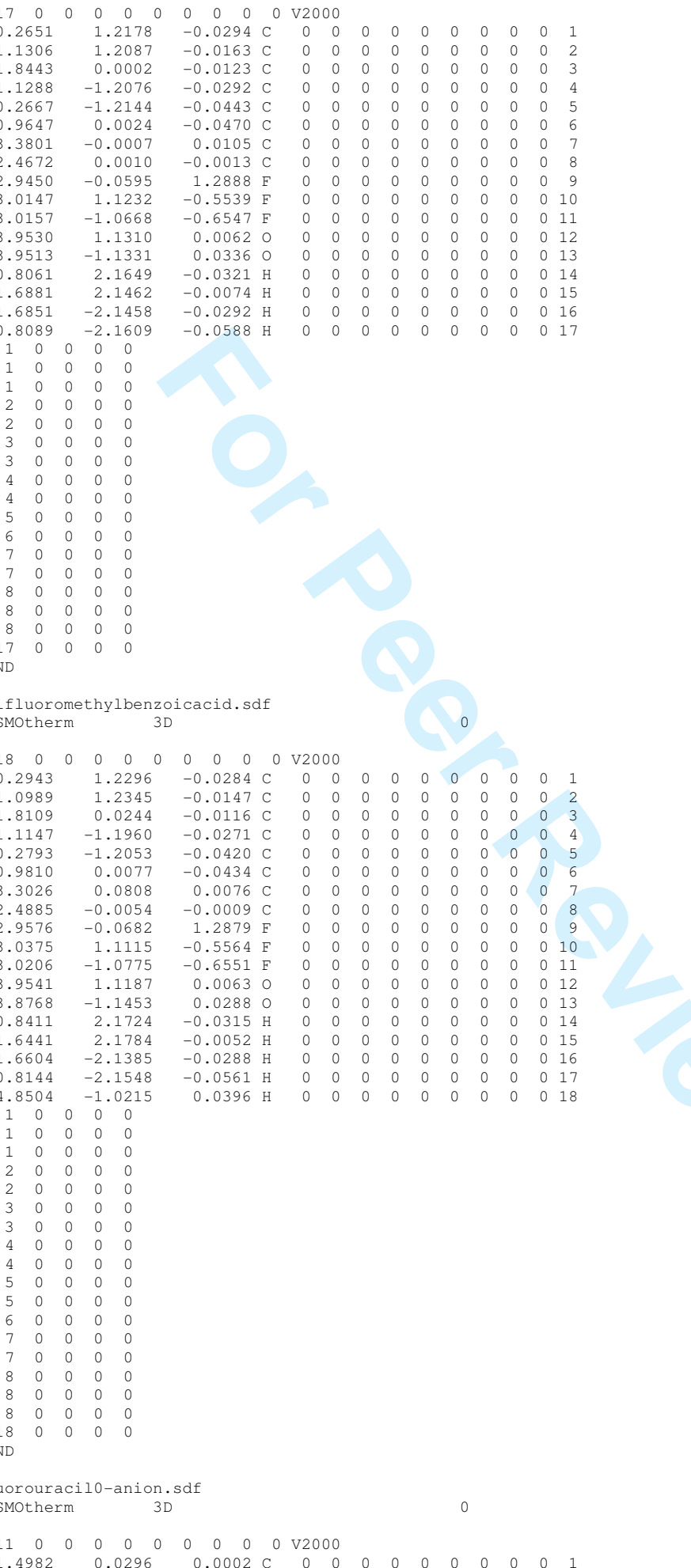

0.0002 C $0 \begin{array}{lllllllll}0 & 0 & 0 & 0 & 0 & 0 & 0 & 0 & 0\end{array}$

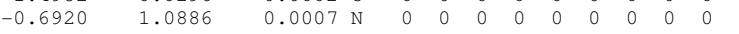

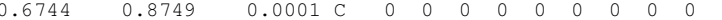

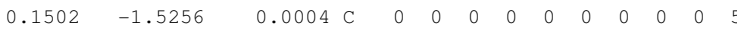

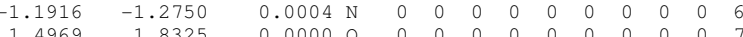

$\begin{array}{rrrrrrrrrrrrrr}1.4969 & 1.8325 & 0.0000 & 0 & 0 & 0 & 0 & 0 & 0 & 0 & 0 & 0 & 0 & \\ -2.8349 & 0.3365 & -0.0009 & 0 & 0 & 0 & 0 & 0 & 0 & 0 & 0 & 0 & 0 & 8\end{array}$

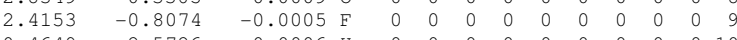

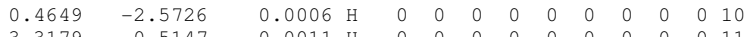

$-3.3179-0.5147$

$\begin{array}{llllllll}1 & 0 & 0 & 0 & 0\end{array}$

$\begin{array}{lllllll}8 & 1 & 0 & 0 & 0 & 0\end{array}$

$\begin{array}{llllll}3 & 2 & 0 & 0 & 0\end{array}$

$3 \begin{array}{llllll}3 & 0 & 0 & 0 & 0 \\ 3 & 0 & 0 & 0 & 0\end{array}$

$\begin{array}{lllll}4 & 0 & 0 & 0 & 0\end{array}$ 


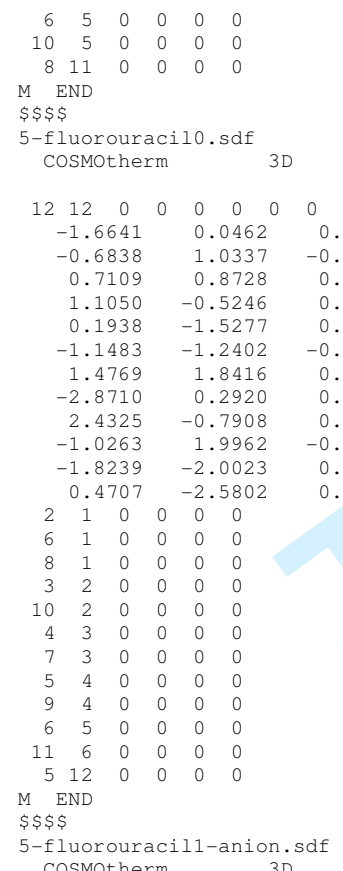

$\begin{array}{llllllllllll}11 & 11 & 0 & 0 & 0 & 0 & 0 & 0 & 0 & 0 & 0 & v 2000\end{array}$

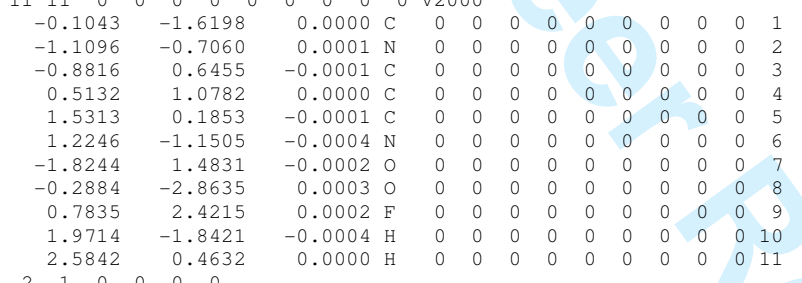

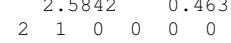

$\begin{array}{llllll}6 & 1 & 0 & 0 & 0 & 0 \\ 8 & 1 & 0 & 0 & 0 & 0 \\ 3 & 2 & 0 & 0 & 0 & 0\end{array}$

$\begin{array}{llllll}8 & 1 & 0 & 0 & 0 & 0 \\ 3 & 2 & 0 & 0 & 0 & 0\end{array}$

$\begin{array}{llllll}4 & 3 & 0 & 0 & 0 & 0 \\ 7 & 3 & 0 & 0 & 0 & 0\end{array}$

$\begin{array}{llllllll}7 & 4 & 0 & 0 & 0 & 0 & 0\end{array}$

$\begin{array}{llllll}9 & 4 & 0 & 0 & 0 & 0 \\ 6 & 5 & 0 & 0 & 0 & 0\end{array}$

$\begin{array}{rrrrrr}0 & 6 & 0 & 0 & 0 & 0 \\ 5 & 11 & 0 & 0 & 0 & 0\end{array}$

END

$\$ \$ \$ \$$

Cosmotherm

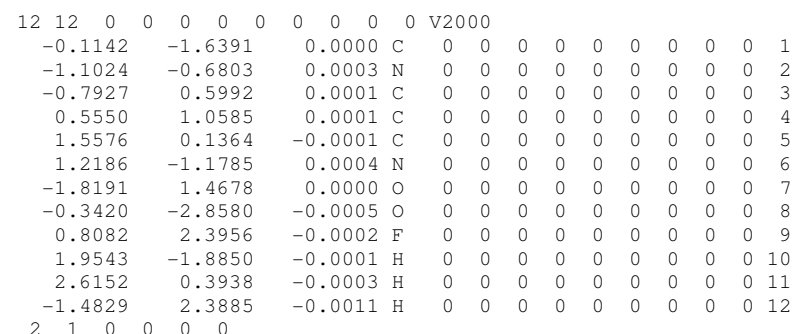

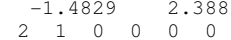

$\begin{array}{llllll}6 & 1 & 0 & 0 & 0 & 0\end{array}$

$\begin{array}{llllll}8 & 1 & 0 & 0 & 0 & 0 \\ 3 & 2 & 0 & 0 & 0 & 0\end{array}$

$\begin{array}{llllll}4 & 3 & 0 & 0 & 0\end{array}$

$\begin{array}{lllllll}7 & 3 & 0 & 0 & 0 & 0 \\ 5 & 4 & 0 & 0 & 0 & 0\end{array}$

$\begin{array}{llllll}4 & 0 & 0 & 0 & 0\end{array}$

$\begin{array}{llllll}6 & 5 & 0 & 0 & 0 & 0 \\ 1 & 5 & 0 & 0 & 0 & 0\end{array}$

$\begin{array}{lllllll}10 & 0 & 0 & 0 & 0\end{array}$

$\begin{array}{llllll}7 & 12 & 0 & 0 & 0 & 0\end{array}$

$M$
$\$ \$ \$ \$$
$5-5 N D$

5-fluorouracil2-anion.sdf

$\begin{array}{llllllllllll}11 & 11 & 0 & 0 & 0 & 0 & 0 & 0 & 0 & 0 & 0 & \mathrm{~V} 2000\end{array}$

$\begin{array}{rrrrrrrrrrrrrrr}1.6487 & -0.0175 & 0.0000 & \mathrm{C} & 0 & 0 & 0 & 0 & 0 & 0 & 0 & 0 & 0 & 1 \\ -1.6826 & 1.0118 & -0.0004 & \mathrm{~N} & 0 & 0 & 0 & 0 & 0 & 0 & 0 & 0 & 0 & 2 \\ 0.7089 & 0.8650 & 0.0000 & \mathrm{C} & 0 & 0 & 0 & 0 & 0 & 0 & 0 & 0 & 0 & 3 \\ 1.0793 & -0.5178 & 0.0000 & \mathrm{C} & 0 & 0 & 0 & 0 & 0 & 0 & 0 & 0 & 0 & 4 \\ 0.1329 & -1.5144 & 0.0001 & \mathrm{C} & 0 & 0 & 0 & 0 & 0 & 0 & 0 & 0 & 0 & 5 \\ -1.2065 & -1.3076 & 0.0001 & \mathrm{~N} & 0 & 0 & 0 & 0 & 0 & 0 & 0 & 0 & 0 & 6 \\ 1.4743 & 1.8547 & 0.0002 & 0 & 0 & 0 & 0 & 0 & 0 & 0 & 0 & 0 & 0 & 7 \\ -2.8626 & 0.3027 & 0.0001 & 0 & 0 & 0 & 0 & 0 & 0 & 0 & 0 & 0 & 0 & 8\end{array}$ 


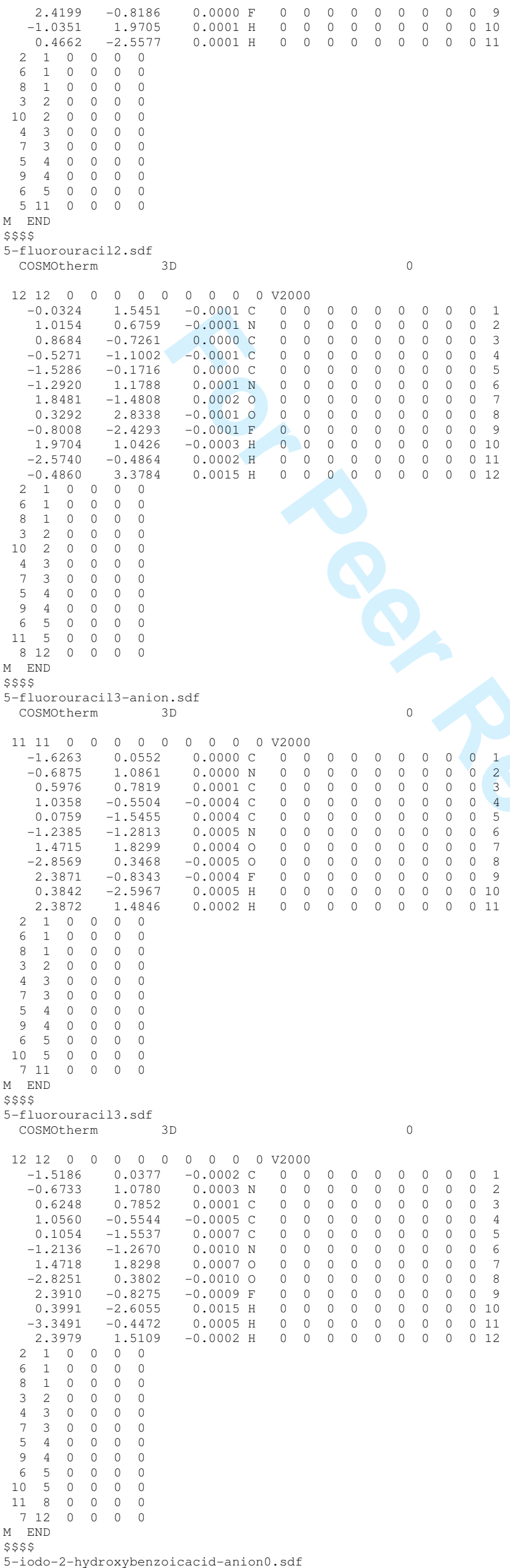




\section{Page 87 of 461}

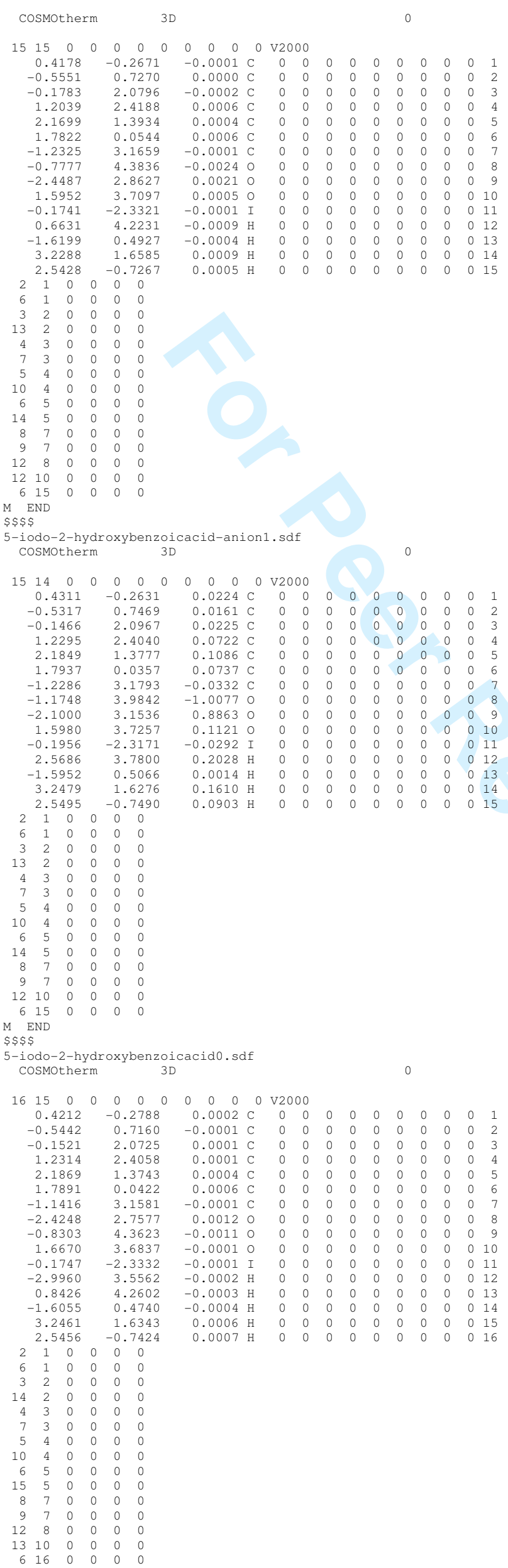




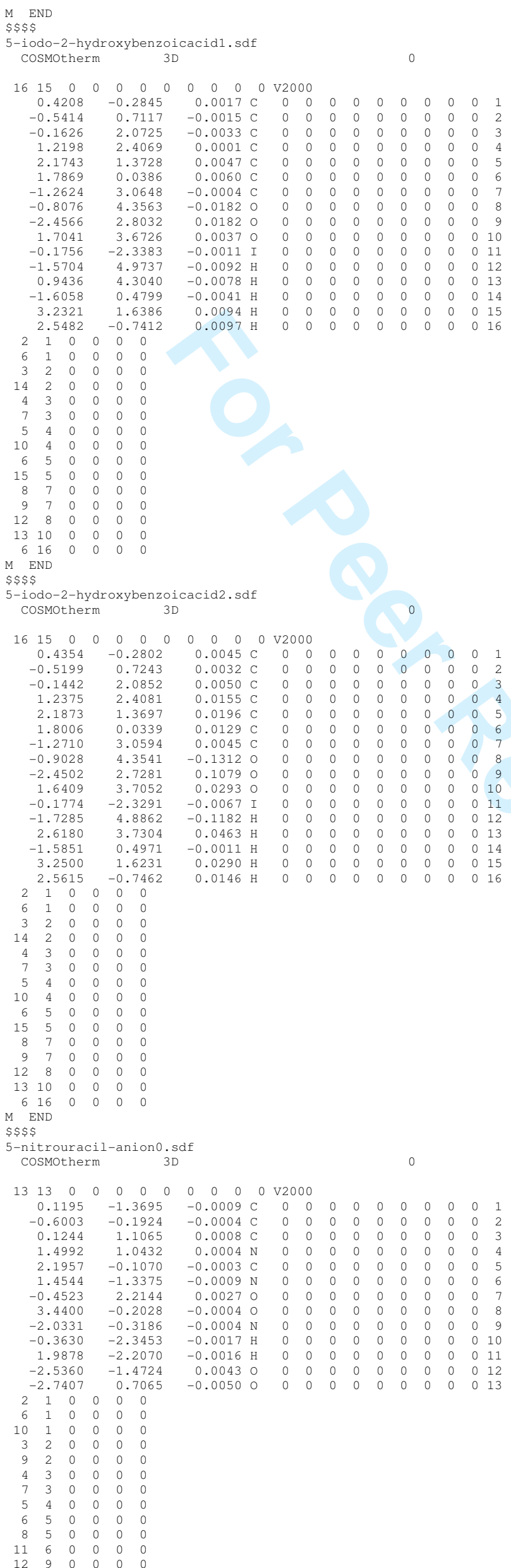




\section{Page 89 of 461}

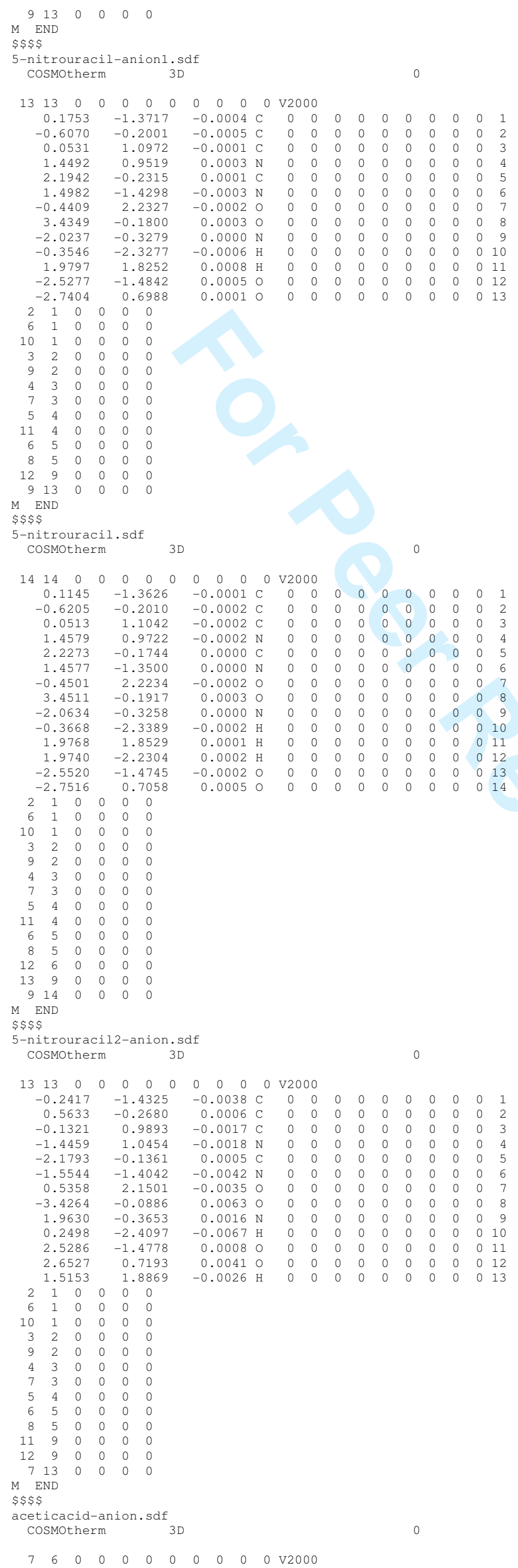




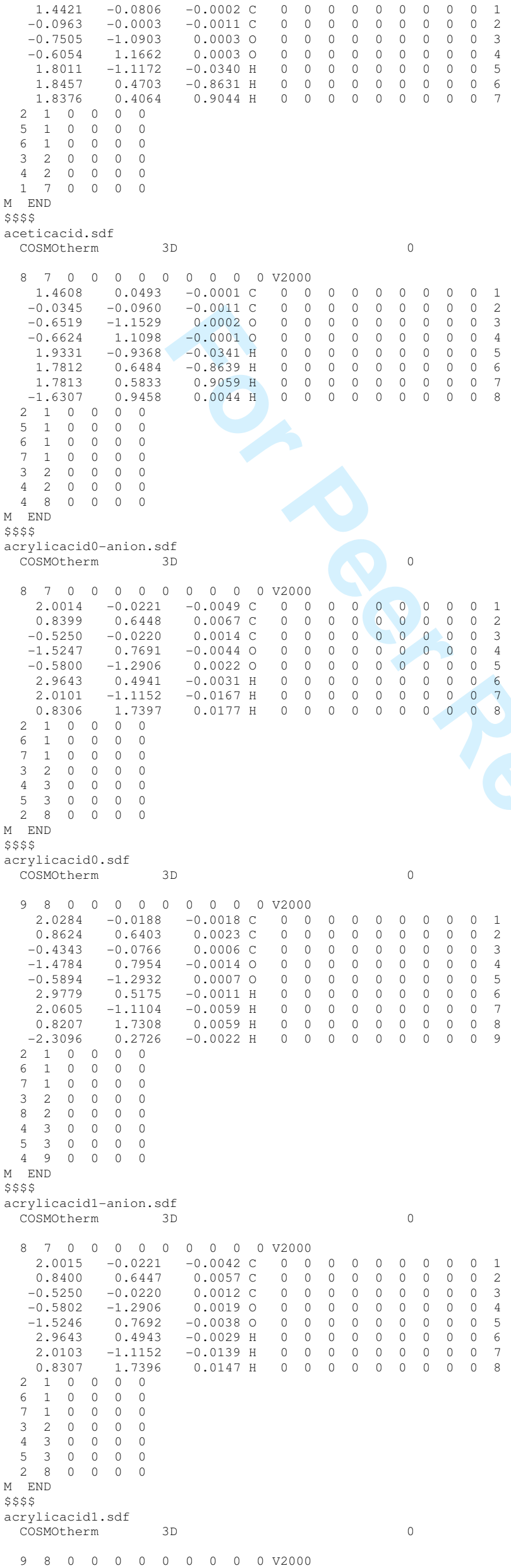




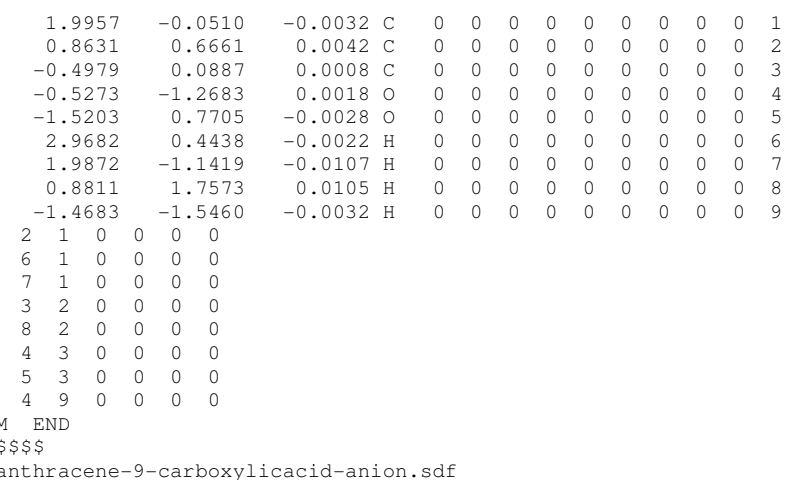

anthracene-9-carboxylicacid-anion.sdf

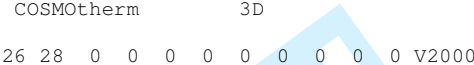

$\begin{array}{cccccccccccccccccc}26 & 28 & 0 & 0 & 0 & 0 & 0 & 0 & 0 & 0 & 0 & \mathrm{~V} 2000 \\ 3.6682 & -1.3855 & -0.0205 & \mathrm{C} & 0 & 0 & 0 & 0 & 0 & 0 & 0 & 0 & 0 & 1\end{array}$

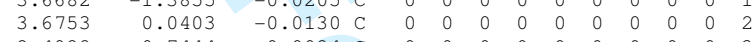
$\begin{array}{llllllllllllll}2.4922 & 0.7444 & -0.0024 & \mathrm{C} & 0 & 0 & 0 & 0 & 0 & 0 & 0 & 0 & 0 & 3\end{array}$ $\begin{array}{llllllllllllll}1.2272 & 0.0730 & 0.0011 & \mathrm{C} & 0 & 0 & 0 & 0 & 0 & 0 & 0 & 0 & 0 & 4\end{array}$ $\begin{array}{llllllllllllll}1.2232 & -1.3777 & -0.0064 & \mathrm{C} & 0 & 0 & 0 & 0 & 0 & 0 & 0 & 0 & 0 & 5 \\ 2.4758 & -2.0717 & -0.0165 & \mathrm{C} & 0 & 0 & 0 & 0 & 0 & 0 & 0 & 0 & 0 & 6\end{array}$ $\begin{array}{rrrrrrrrrrrrrr}2.4758 & -2.0717 & -0.0165 & \text { C } & 0 & 0 & 0 & 0 & 0 & 0 & 0 & 0 & 0 & 6 \\ 0.0000 & 0.7784 & 0.0000 & \text { C } & 0 & 0 & 0 & 0 & 0 & 0 & 0 & 0 & 0 & 7\end{array}$ $\begin{array}{llllllllllllll}-1.2272 & 0.0730 & -0.0011 & \mathrm{C} & 0 & 0 & 0 & 0 & 0 & 0 & 0 & 0 & 0 & 8\end{array}$ $\begin{array}{llllllllllllll}-1.2232 & -1.3777 & 0.0064 & \mathrm{C} & 0 & 0 & 0 & 0 & 0 & 0 & 0 & 0 & 0 & 9\end{array}$ $\begin{array}{rrrrrrrrrrrrrr}0.0000 & -2.0662 & -0.0001 & \mathrm{C} & 0 & 0 & 0 & 0 & 0 & 0 & 0 & 0 & 0 & 10\end{array}$ $\begin{array}{llllllllllllll}-2.4922 & 0.7444 & 0.0035 & \mathrm{C} & 0 & 0 & 0 & 0 & 0 & 0 & 0 & 0 & 0 & 11\end{array}$

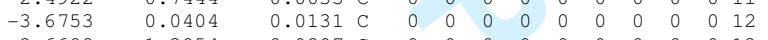

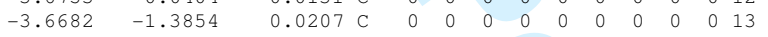
$\begin{array}{llllllllllllll}-2.4758 & -2.0716 & 0.0158 & \mathrm{C} & 0 & 0 & 0 & 0 & 0 & 0 & 0 & 0 & 0 & 14\end{array}$

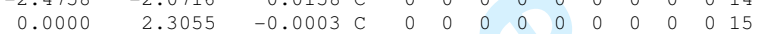
$\begin{array}{llrllllllllllll}0.1316 & 2.8724 & 1.1246 & 0 & 0 & 0 & 0 & 0 & 0 & 0 & 0 & 0 & 0 & 16\end{array}$

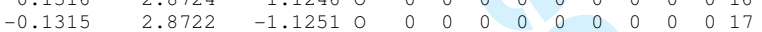

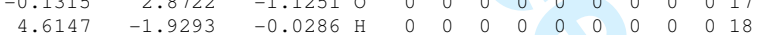

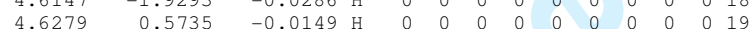
$\begin{array}{lllllllllllllllll}4.6279 & 0.5735 & -0.0149 & \mathrm{H} & 0 & 0 & 0 & 0 & 0 & 0 & 0 & 0 & 0 & 19 \\ 2.5085 & 1.8358 & 0.0100 & H & 0 & 0 & 0 & 0 & 0 & 0 & 0 & 0 & 0 & 20\end{array}$ $\begin{array}{lllllllllllllll}2.50845 & -3.1643 & -0.0216 & \mathrm{H} & 0 & 0 & 0 & 0 & 0 & 0 & 0 & 0 & 0 & 21\end{array}$ $\begin{array}{lllllllllllllllll}2.4645 & -3.1643 & -0.0216 & H & 0 & 0 & 0 & 0 & 0 & 0 & 0 & 0 & 0 & 21 \\ -0.0001 & -3.1594 & -0.0004 & H & 0 & 0 & 0 & 0 & 0 & 0 & 0 & 0 & 0 & 22\end{array}$ $\begin{array}{lllllllllllllllll}-0.0001 & -3.1594 & -0.0004 & \mathrm{H} & 0 & 0 & 0 & 0 & 0 & 0 & 0 & 0 & 0 & 22\end{array}$ $\begin{array}{llllllllllllllll}-2.5085 & 1.8359 & -0.0082 & \mathrm{H} & 0 & 0 & 0 & 0 & 0 & 0 & 0 & 0 & 0 & 23 \\ -4.6279 & 0.5736 & 0.0166 & \mathrm{H} & 0 & 0 & 0 & 0 & 0 & 0 & 0 & 0 & 0 & 24\end{array}$

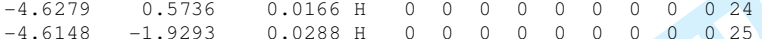
$\begin{array}{llllllllllllll}-4.6148 & -1.9293 & 0.0288 & \mathrm{H} & 0 & 0 & 0 & 0 & 0 & 0 & 0 & 0 & 0 & 25 \\ -2.4646 & -3.1643 & 0.0214 & \mathrm{H} & 0 & 0 & 0 & 0 & 0 & 0 & 0 & 0 & 0 & 26\end{array}$ $\begin{array}{llllll}2 & 1 & 0 & 0 & 0 & 0\end{array}$ $\begin{array}{llllll}6 & 1 & 0 & 0 & 0 & 0\end{array}$ $\begin{array}{rlllll}8 & 1 & 0 & 0 & 0 & 0 \\ 3 & 2 & 0 & 0 & 0 & 0\end{array}$ $\begin{array}{llllll}9 & 2 & 0 & 0 & 0 & 0\end{array}$ $\begin{array}{llllll}4 & 3 & 0 & 0 & 0 & 0 \\ 20 & 3 & 0 & 0 & 0 & 0\end{array}$

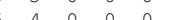
$\begin{array}{lllll}4 & 0 & 0 & 0\end{array}$ $\begin{array}{lllllll}6 & 5 & 0 & 0 & 0 & 0\end{array}$ $\begin{array}{lllllll}1 & 6 & 0 & 0 & 0 & 0\end{array}$ $8 \begin{array}{llllll}8 & 7 & 0 & 0 & 0 & 0\end{array}$ $\begin{array}{lllllll}9 & 8 & 0 & 0 & 0 & 0\end{array}$ $\begin{array}{llllll}1 & 8 & 0 & 0 & 0 & 0\end{array}$ $\begin{array}{lllllll}10 & 9 & 0 & 0 & 0 & 0\end{array}$ $\begin{array}{lllllll}14 & 9 & 0 & 0 & 0 & 0 \\ 22 & 10 & 0 & 0 & 0 & 0\end{array}$ 121100000 $\begin{array}{llllll}23 & 11 & 0 & 0 & 0 & 0\end{array}$ $\begin{array}{llllll}24 & 12 & 0 & 0 & 0 & 0\end{array}$ $\begin{array}{llllll}16 & 15 & 0 & 0 & 0 & 0\end{array}$ $\begin{array}{llllll}16 & 15 & 0 & 0 & 0 & 0 \\ 17 & 15 & 0 & 0 & 0 & 0\end{array}$ $\begin{array}{llllll}4 & 26 & 0 & 0 & 0 & 0\end{array}$ END

$\$ \$ \$$

anthracene-9-carboxylicacid.sdf COSMOtherm

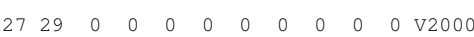

$\begin{array}{rrrrrrrrrrrrrr}29 & 0 & 0 & 0 & 0 & 0 & 0 & 0 & 0 & V 2000 & & & 0 & \\ 3.6791 & -1.3995 & -0.0593 & \mathrm{C} & 0 & 0 & 0 & 0 & 0 & 0 & 0 & 0 & 0 & 1 \\ 3.6874 & 0.0247 & -0.0481 & \mathrm{C} & 0 & 0 & 0 & 0 & 0 & 0 & 0 & 0 & 0 & 2 \\ 2.5112 & 0.7397 & -0.0188 & \mathrm{C} & 0 & 0 & 0 & 0 & 0 & 0 & 0 & 0 & 0 & 3 \\ 1.2424 & 0.0757 & 0.0083 & \mathrm{C} & 0 & 0 & 0 & 0 & 0 & 0 & 0 & 0 & 0 & 4 \\ 1.2384 & -1.3738 & -0.0187 & \mathrm{C} & 0 & 0 & 0 & 0 & 0 & 0 & 0 & 0 & 0 & 5 \\ 2.4839 & -2.0777 & -0.0466 & \mathrm{C} & 0 & 0 & 0 & 0 & 0 & 0 & 0 & 0 & 0 & 6 \\ 0.0009 & 0.7631 & 0.0151 & \mathrm{C} & 0 & 0 & 0 & 0 & 0 & 0 & 0 & 0 & 0 & 7 \\ -1.2326 & 0.0622 & 0.0164 & \mathrm{C} & 0 & 0 & 0 & 0 & 0 & 0 & 0 & 0 & 0 & 8 \\ -1.2115 & -1.3881 & 0.0038 & \mathrm{C} & 0 & 0 & 0 & 0 & 0 & 0 & 0 & 0 & 0 & 9 \\ 0.0172 & -2.0616 & -0.0185 & \mathrm{C} & 0 & 0 & 0 & 0 & 0 & 0 & 0 & 0 & 0 & 10 \\ -2.5098 & 0.7083 & 0.0767 & \mathrm{C} & 0 & 0 & 0 & 0 & 0 & 0 & 0 & 0 & 0 & 11 \\ -3.6768 & -0.0216 & 0.0936 & \mathrm{C} & 0 & 0 & 0 & 0 & 0 & 0 & 0 & 0 & 0 & 12 \\ -3.6516 & -1.4452 & 0.0581 & \mathrm{C} & 0 & 0 & 0 & 0 & 0 & 0 & 0 & 0 & 0 & 13 \\ -2.4483 & -2.1077 & 0.0163 & \mathrm{C} & 0 & 0 & 0 & 0 & 0 & 0 & 0 & 0 & 0 & 14 \\ 0.0136 & 2.2602 & 0.0615 & \mathrm{C} & 0 & 0 & 0 & 0 & 0 & 0 & 0 & 0 & 0 & 15 \\ 0.5507 & 2.9260 & 0.9380 & 0 & 0 & 0 & 0 & 0 & 0 & 0 & 0 & 0 & 0 & 16 \\ -0.6269 & 2.8249 & -0.9915 & 0 & 0 & 0 & 0 & 0 & 0 & 0 & 0 & 0 & 0 & 17 \\ 4.6234 & -1.9458 & -0.0812 & \mathrm{H} & 0 & 0 & 0 & 0 & 0 & 0 & 0 & 0 & 0 & 18 \\ 4.6407 & 0.5558 & -0.0673 & \mathrm{H} & 0 & 0 & 0 & 0 & 0 & 0 & 0 & 0 & 0 & 19 \\ 2.5472 & 1.8286 & -0.0069 & \mathrm{H} & 0 & 0 & 0 & 0 & 0 & 0 & 0 & 0 & 0 & 20 \\ 2.4613 & -3.1695 & -0.0629 & \mathrm{H} & 0 & 0 & 0 & 0 & 0 & 0 & 0 & 0 & 0 & 21 \\ 0.0232 & -3.1545 & -0.0345 & \mathrm{H} & 0 & 0 & 0 & 0 & 0 & 0 & 0 & 0 & 0 & 22\end{array}$




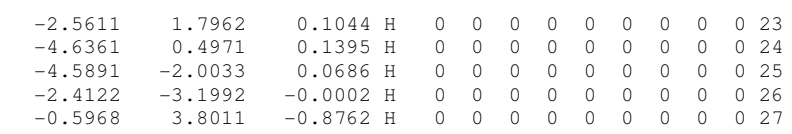

$\begin{array}{llllll}2 & 1 & 0 & 0 & 0 & 0 \\ 6 & 1 & 0 & 0 & 0 & 0\end{array}$

$\begin{array}{cccccc}18 & 1 & 0 & 0 & 0 & 0\end{array}$

$\begin{array}{lllllll}3 & 2 & 0 & 0 & 0 & 0\end{array}$

$\begin{array}{lllllll}19 & 2 & 0 & 0 & 0 & 0\end{array}$

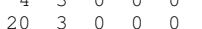

$\begin{array}{lllllll}5 & 4 & 0 & 0 & 0 & 0\end{array}$

$\begin{array}{llllll}7 & 4 & 0 & 0 & 0 & 0 \\ 6 & 5 & 0 & 0 & 0 & 0\end{array}$

$\begin{array}{llllll}10 & 5 & 0 & 0 & 0 & 0\end{array}$

$\begin{array}{llllll}21 & 6 & 0 & 0 & 0 & 0\end{array}$

$\begin{array}{cccccc}15 & 7 & 0 & 0 & 0 & 0\end{array}$

$\begin{array}{llllll}9 & 8 & 0 & 0 & 0 & 0\end{array}$

$\begin{array}{llllll}11 & 8 & 0 & 0 & 0 & 0 \\ 10 & 9 & 0 & 0 & 0 & 0\end{array}$

$\begin{array}{lllllll}14 & 9 & 0 & 0 & 0 & 0\end{array}$

$\begin{array}{llllll}22 & 10 & 0 & 0 & 0 & 0 \\ 12 & 11 & 0 & 0 & 0 & 0\end{array}$

$\begin{array}{lllllll}12 & 11 & 0 & 0 & 0 & 0 & 0 \\ 23 & 11 & 0 & 0 & 0 & 0 & 2\end{array}$

1312

$24 \quad 12$ 0 120000

$\begin{array}{llllll}14 & 13 & 0 & 0 & 0 & 0\end{array}$

$\begin{array}{llllll}25 & 13 & 0 & 0 & 0 & 0 \\ 26 & 14 & 0 & 0 & 0 & 0\end{array}$

$\begin{array}{llllll}16 & 15 & 0 & 0 & 0 & 0 \\ 17 & 15 & 0 & 0 & 0 & 0\end{array}$

$\begin{array}{llllll}17 & 27 & 0 & 0 & 0 & 0\end{array}$

END

benzoicacid-anion.sdf

cosmotherm $3 \mathrm{D}$

$\begin{array}{llllllllllll}14 & 14 & 0 & 0 & 0 & 0 & 0 & 0 & 0 & 0 & 0 & \mathrm{~V} 2000\end{array}$

$\begin{array}{llllllllllllll}1.9046 & 1.2115 & -0.0040 & \mathrm{C} & 0 & 0 & 0 & 0 & 0 & 0 & 0 & 0 & 0 & 1\end{array}$

$\begin{array}{llllllllllllll}0.5055 & 1.2086 & -0.0037 & \mathrm{C} & 0 & 0 & 0 & 0 & 0 & 0 & 0 & 0 & 0 & 2\end{array}$

$\begin{array}{llllllllllllll}-0.2109 & 0.0000 & -0.0003 & \mathrm{C} & 0 & 0 & 0 & 0 & 0 & 0 & 0 & 0 & 0 & 3\end{array}$

$\begin{array}{lllllllllllll}0.5056 & -1.2087 & 0.0036 & \mathrm{C} & 0 & 0 & 0 & 0 & 0 & 0 & 0 & 0 & 0\end{array}$

$\begin{array}{llllllllllllll}1.9046 & -1.2114 & 0.0041 & \mathrm{C} & 0 & 0 & 0 & 0 & 0 & 0 & 0 & 0 & 0 & 5\end{array}$

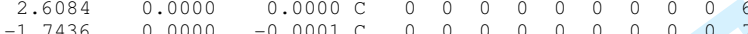

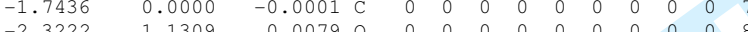

$\begin{array}{rrrrrrrrrrrrrrr}-2.3222 & 1.1309 & 0.0079 & 0 & 0 & 0 & 0 & 0 & 0 & 0 & 0 & 0 & 0 & 0 & 7 \\ -2.3222 & -1.1309 & -0.0077 & 0 & 0 & 0 & 0 & 0 & 0 & 0 & 0 & 0 & 0 & 9\end{array}$

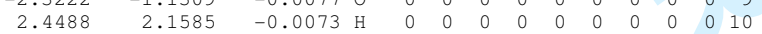

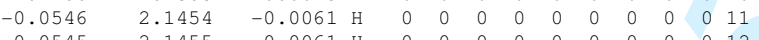

$\begin{array}{llllllllllllll}-0.0545 & -2.1455 & 0.0061 & \mathrm{H} & 0 & 0 & 0 & 0 & 0 & 0 & 0 & 0 & 0 & 12 \\ 2.4489 & -2.1585 & 0.0076 & \mathrm{H} & 0 & 0 & 0 & 0 & 0 & 0 & 0 & 0 & 0 & 13\end{array}$

$\begin{array}{rrrrrrrrrrrrrrrrr}2.4489 & -2.1585 & 0.0076 & \mathrm{H} & 0 & 0 & 0 & 0 & 0 & 0 & 0 & 0 & 0 & 12 \\ 3.7003 & 0.0000 & 0.0002 & \mathrm{H} & 0 & 0 & 0 & 0 & 0 & 0 & 0 & 0 & 0 & 14\end{array}$

2.700000

$\begin{array}{llllll}5 & 1 & 0 & 0 & 0 & 0\end{array}$

$\begin{array}{llllll}1 & 0 & 0 & 0 & 0 \\ 0 & 1 & 0 & 0 & 0\end{array}$

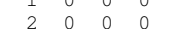

$\begin{array}{lllllll}1 & 2 & 0 & 0 & 0 & 0\end{array}$

$\begin{array}{llllll}4 & 3 & 0 & 0 & 0 \\ 7 & 3 & 0 & 0 & 0 & 0\end{array}$

$\begin{array}{lllll}3 & 0 & 0 & 0 & 0\end{array}$

$\begin{array}{llllll}4 & 0 & 0 & 0 & 0\end{array}$

$\begin{array}{llllll}5 & 0 & 0 & 0 & 0\end{array}$

$\begin{array}{llllll}5 & 0 & 0 & 0 & 0 \\ 7 & 0 & 0 & 0 & 0\end{array}$

$\begin{array}{rllll}7 & 0 & 0 & 0 & 0\end{array}$

END

$\$ \$ \$$

benzoicacid.sdf

Cosmotherm

$\begin{array}{lllllllllllll}15 & 15 & 0 & 0 & 0 & 0 & 0 & 0 & 0 & 0 & 0 & \mathrm{v} 2000\end{array}$

$\begin{array}{rrrrrrrrrrrrrr}-2.6184 & 0.0254 & -0.0002 & \mathrm{C} & 0 & 0 & 0 & 0 & 0 & 0 & 0 & 0 & 0 & 1 \\ -1.8989 & 1.2269 & 0.0001 & \mathrm{C} & 0 & 0 & 0 & 0 & 0 & 0 & 0 & 0 & 0 & 2 \\ -0.5026 & 1.2078 & 0.0003 & \mathrm{C} & 0 & 0 & 0 & 0 & 0 & 0 & 0 & 0 & 0 & 3\end{array}$

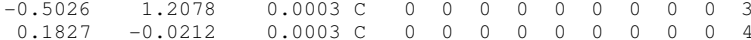

$\begin{array}{rrrrrrrrrrrrrr}-.1827 & -0.0212 & 0.0003 & C & 0 & 0 & 0 & 0 & 0 & 0 & 0 & 0 & 0 & 4 \\ -0.5444 & -1.2252 & 0.0000 & C & 0 & 0 & 0 & 0 & 0 & 0 & 0 & 0 & 0 & 5\end{array}$

$\begin{array}{rrrrrrrrrrrrrr}-0.5444 & -1.2252 & 0.0000 & C & 0 & 0 & 0 & 0 & 0 & 0 & 0 & 0 & 0 & 5 \\ -1.9395 & -1.2002 & -0.0002 & C & 0 & 0 & 0 & 0 & 0 & 0 & 0 & 0 & 0 & 6\end{array}$

$\begin{array}{lllllllllllll}-3.7097 & 0.0442 & -0.0004 & \mathrm{H} & 0 & 0 & 0 & 0 & 0 & 0 & 0 & 0 & 0\end{array}$

$\begin{array}{llllllllllllll}-2.4277 & 2.1814 & 0.0001 & \mathrm{H} & 0 & 0 & 0 & 0 & 0 & 0 & 0 & 0 & 0 & 8\end{array}$

$\begin{array}{rrrrrrrrrrrrrr}0.0568 & 2.1430 & 0.0004 & \mathrm{H} & 0 & 0 & 0 & 0 & 0 & 0 & 0 & 0 & 0 & 9 \\ 1.6688 & -0.0928 & 0.0001 & \mathrm{C} & 0 & 0 & 0 & 0 & 0 & 0 & 0 & 0 & 0 & 10\end{array}$

$\begin{array}{lllllllllllllll}-0.0051 & -2.1733 & 0.0001 & \mathrm{H} & 0 & 0 & 0 & 0 & 0 & 0 & 0 & 0 & 0 & 11\end{array}$

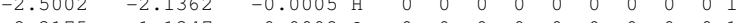

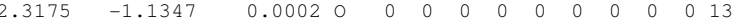

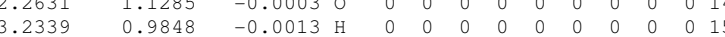

$\begin{array}{llllll}2 & 1 & 0 & 0 & 0 & 0\end{array}$

$\begin{array}{llllll}6 & 1 & 0 & 0 & 0 & 0\end{array}$

$\begin{array}{llllll}7 & 1 & 0 & 0 & 0 & 0 \\ 3 & 2 & 0 & 0 & 0 & 0\end{array}$

$\begin{array}{lllll}8 & 2 & 0 & 0 & 0\end{array}$

$\begin{array}{lllllll}4 & 3 & 0 & 0 & 0 & 0\end{array}$

$5 \begin{array}{llllll}9 & 4 & 0 & 0 & 0\end{array}$

$\begin{array}{cccccc}10 & 4 & 0 & 0 & 0 & 0\end{array}$

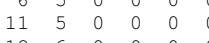

$\begin{array}{llllll}12 & 6 & 0 & 0 & 0\end{array}$

$\begin{array}{lllllll}13 & 10 & 0 & 0 & 0 & 0 \\ 14 & 10 & 0 & 0 & 0 & 0\end{array}$

1415 


\section{Page 93 of 461}

\$\$\$

boricacid-anion1.sdf
Cosmotherm

$\begin{array}{lccccccccccc}6 & 5 & 0 & 0 & 0 & 0 & 0 & 0 & 0 & 0 & 0 & \mathrm{~V} 2000\end{array}$

$\begin{array}{llllllllllllll}0.0002 & -0.1057 & 0.0001 & \text { B } & 0 & 0 & 0 & 0 & 0 & 0 & 0 & 0 & 0 & 1\end{array}$

$\begin{array}{rrrrrrrrrrrrrr}-1.1647 & 0.7346 & -0.0001 & 0 & 0 & 0 & 0 & 0 & 0 & 0 & 0 & 0 & 0 & 2 \\ 0.0032 & -1.4237 & 0.0000 & 0 & 0 & 0 & 0 & 0 & 0 & 0 & 0 & 0 & 0 & 3\end{array}$

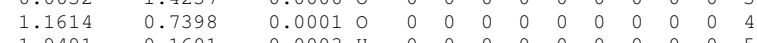

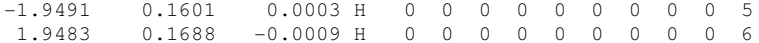

$\begin{array}{llllll}2 & 1 & 0 & 0 & 0 & 0\end{array}$

$\begin{array}{llllll}2 & 1 & 0 & 0 & 0 & 0 \\ 4 & 1 & 0 & 0 & 0 & 0\end{array}$

$\begin{array}{lllll}1 & 0 & 0 & 0 & 0\end{array}$

$\begin{array}{lllll}6 & 0 & 0 & 0 & 0\end{array}$

M END

$\$ \$ \$$

boricacid.sdf

CosMotherm 3D

$\begin{array}{lccccccccccc}7 & 6 & 0 & 0 & 0 & 0 & 0 & 0 & 0 & 0 & 0 & \mathrm{v} 2000\end{array}$

$\begin{array}{llllllllllllll}0.0053 & -0.0066 & 0.0000 & \text { B } & 0 & 0 & 0 & 0 & 0 & 0 & 0 & 0 & 0 & 1\end{array}$

$\begin{array}{rrrrrrrrrrrrrr}-1.2053 & 0.6601 & 0.0001 & 0 & 0 & 0 & 0 & 0 & 0 & 0 & 0 & 0 & 0 & 2\end{array}$

$\begin{array}{rrrrrrrrrrrrrr}-0.0808 & -1.3864 & -0.0001 & 0 & 0 & 0 & 0 & 0 & 0 & 0 & 0 & 0 & 0 & 3 \\ 1.1765 & 0.7316 & 0.0000 & 0 & 0 & 0 & 0 & 0 & 0 & 0 & 0 & 0 & 0 & 4\end{array}$

$\begin{array}{llllllllllllll}-1.0879 & 1.6261 & -0.0014 & \mathrm{H} & 0 & 0 & 0 & 0 & 0 & 0 & 0 & 0 & 0 & 5\end{array}$

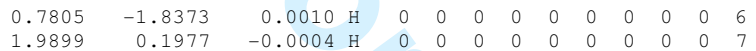

$\begin{array}{llllll}2 & 1 & 0 & 0 & 0 & 0\end{array}$

$\begin{array}{lllll}1 & 0 & 0 & 0 & 0\end{array}$

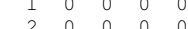

$\begin{array}{lllll}3 & 0 & 0 & 0 & 0 \\ 7 & 0 & 0 & 0 & 0\end{array}$

END

$\$ \$ \$$

bromoaceticacido-anion.sdf

cosmotherm $3 \mathrm{D}$

$\begin{array}{lllllllllllll}7 & 5 & 0 & 0 & 0 & 0 & 0 & 0 & 0 & 0 & 0 & \mathrm{~V} 2000\end{array}$

$\begin{array}{lllllllllllll}-0.0136 & -1.2577 & -0.0025 & \mathrm{Br} & 0 & 0 & 0 & 0 & 0 & 0 & 0 & 0 & 0\end{array}$

$\begin{array}{lllllllllllll}0.8722 & 0.5466 & 0.0191 & \mathrm{C} & 0 & 0 & 0 & 0 & 0 & 0 & 0 & 0 & 0\end{array}$

$\begin{array}{llllllllllllll}-0.0719 & 1.7667 & 0.0012 & \mathrm{C} & 0 & 0 & 0 & 0 & 0 & 0 & 0 & 0 & 0 & 3\end{array}$

$\begin{array}{cccccccccccccc}-1.3200 & 1.6304 & 0.0066 & 0 & 0 & 0 & 0 & 0 & 0 & 0 & 0 & 0 & 0 & 4\end{array}$

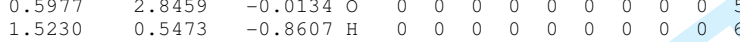

$\begin{array}{lllllllllllll}1.4815 & 0.5444 & -0.9284 \mathrm{H} & 0 & 0 & 0 & 0 & 0 & 0 & 0 & 0 & 0\end{array}$

$\begin{array}{lllllll}3 & 2 & 0 & 0 & 0 & 0\end{array}$

$\begin{array}{lllll}2 & 0 & 0 & 0 & 0\end{array}$

$\begin{array}{llllll}3 & 0 & 0 & 0 & 0\end{array}$

END

$\$ \$ \$$

bromoaceticacido.sdf

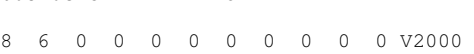

$\begin{array}{llllllllllllll}-0.0176 & -1.2512 & -0.0041 & \mathrm{Br} & 0 & 0 & 0 & 0 & 0 & 0 & 0 & 0 & 0 & 1\end{array}$

$\begin{array}{lllllllllllll}0.9088 & 0.4930 & 0.0289 & \mathrm{C} & 0 & 0 & 0 & 0 & 0 & 0 & 0 & 0 & 0\end{array}$

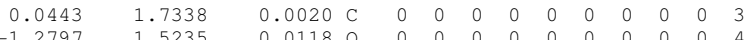

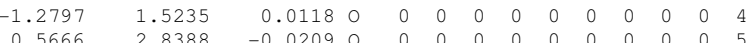

$\begin{array}{llllllllllllll}0.5666 & 2.8388 & -0.0209 & 0 & 0 & 0 & 0 & 0 & 0 & 0 & 0 & 0 & 0 & 5 \\ 1.5735 & 0.5077 & -0.8405 & \mathrm{H} & 0 & 0 & 0 & 0 & 0 & 0 & 0 & 0 & 0 & 6\end{array}$

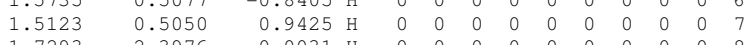

$-1.7293 \quad 2.3976$

$\begin{array}{lllllll}3 & 2 & 0 & 0 & 0 & 0 & 0 \\ 6 & 2 & 0 & 0 & 0 & 0\end{array}$

$\begin{array}{lllll}7 & 0 & 0 & 0 & 0\end{array}$

$\begin{array}{lllll}3 & 0 & 0 & 0 & 0\end{array}$

$\begin{array}{ccccccccc}3 & 0 & 0 & 0 & 0 & 0\end{array}$

END

\$\$\$

bromoaceticacid1-anion.sdf

Cosmotherm $3 \mathrm{D}$

$\begin{array}{llllllllllll}7 & 5 & 0 & 0 & 0 & 0 & 0 & 0 & 0 & 0 & 0 & \mathrm{v} 2000\end{array}$

$\begin{array}{llllllllllllll}1.2577 & 0.0136 & 0.0025 & \mathrm{Br} & 0 & 0 & 0 & 0 & 0 & 0 & 0 & 0 & 0 & 1\end{array}$

$\begin{array}{llllllllllllll}-0.5466 & -0.8722 & -0.0187 & \mathrm{C} & 0 & 0 & 0 & 0 & 0 & 0 & 0 & 0 & 0 & 2\end{array}$

$\begin{array}{rrrrrrrrrrrrrr}-1.7667 & 0.0721 & -0.0012 & C & 0 & 0 & 0 & 0 & 0 & 0 & 0 & 0 & 0 & 3 \\ -2.8459 & -0.5978 & 0.0132 & 0 & 0 & 0 & 0 & 0 & 0 & 0 & 0 & 0 & 0 & 4\end{array}$

$\begin{array}{llllllllllllll}-1.6303 & 1.3200 & -0.0064 & 0 & 0 & 0 & 0 & 0 & 0 & 0 & 0 & 0 & 0 & 5\end{array}$

$\begin{array}{lllllllllllll}-0.5470 & -1.5225 & 0.8615 & \mathrm{H} & 0 & 0 & 0 & 0 & 0 & 0 & 0 & 0 & 0\end{array}$

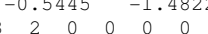

$\begin{array}{lllll}2 & 0 & 0 & 0 & 0\end{array}$

$\begin{array}{lllll}3 & 0 & 0 & 0 & 0\end{array}$

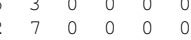

$\underset{\$ \$ \$ \$}{M}$

bromoaceticacidl.sdf

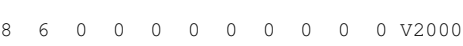

$\begin{array}{llllllllllllllll}1.2585 & 0.0141 & 0.0035 & \mathrm{Br} & 0 & 0 & 0 & 0 & 0 & 0 & 0 & 0 & 0 & 1\end{array}$

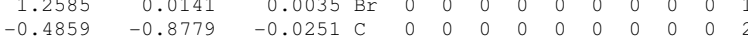

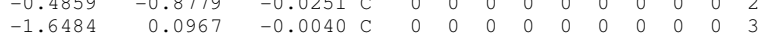

$\begin{array}{llllllllllllll}-2.8035 & -0.6071 & 0.0172 & 0 & 0 & 0 & 0 & 0 & 0 & 0 & 0 & 0 & 0 & 4\end{array}$

$\begin{array}{rrrrrrrrrrrrrr}-1.5899 & 1.3116 & -0.0088 & 0 & 0 & 0 & 0 & 0 & 0 & 0 & 0 & 0 & 0 & 5 \\ -0.5283 & -1.5368 & 0.8482 & \mathrm{H} & 0 & 0 & 0 & 0 & 0 & 0 & 0 & 0 & 0 & 6\end{array}$

$\begin{array}{rrrrrrrrrrrrrr}-0.5283 & -1.5368 & 0.8482 & \mathrm{H} & 0 & 0 & 0 & 0 & 0 & 0 & 0 & 0 & 0 & 6 \\ -0.5231 & -1.4852 & -0.9357 & \mathrm{H} & 0 & 0 & 0 & 0 & 0 & 0 & 0 & 0 & 0 & 7\end{array}$ 


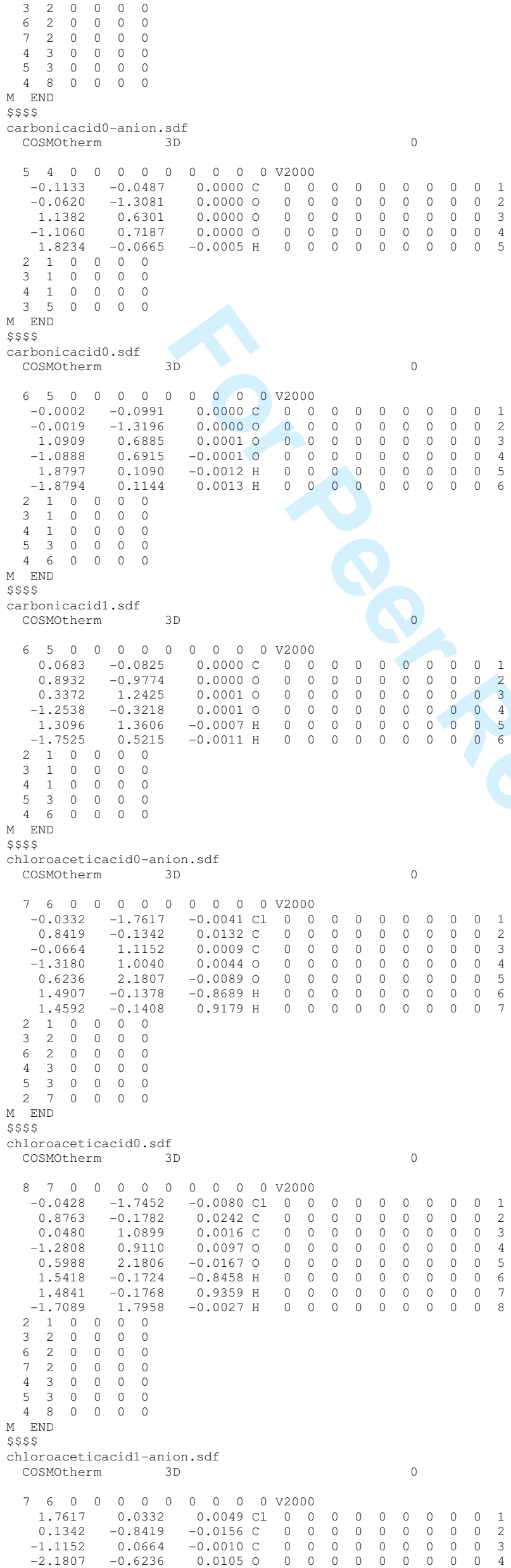




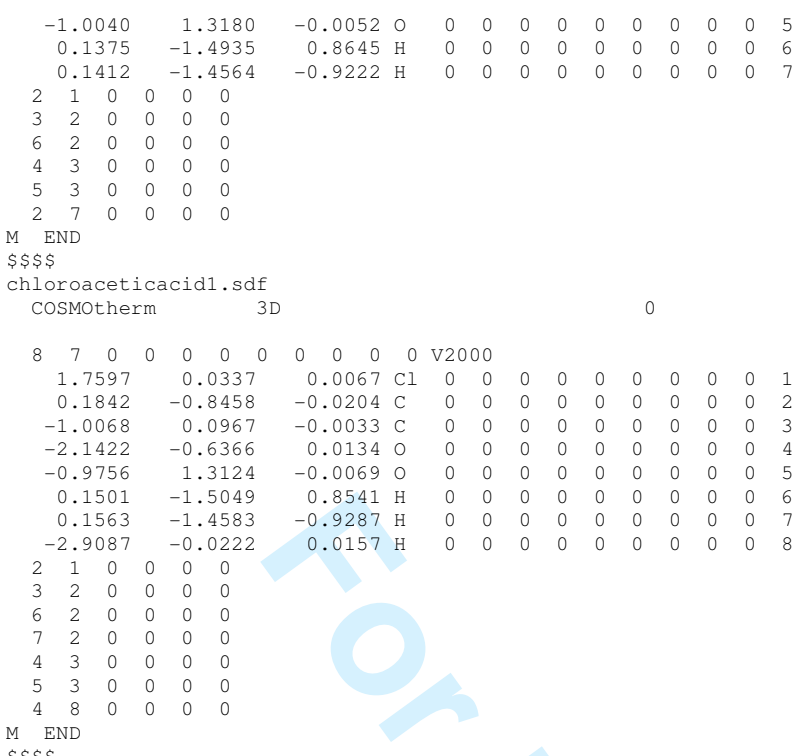

Cis-5-formyluracil-aniono.sdf

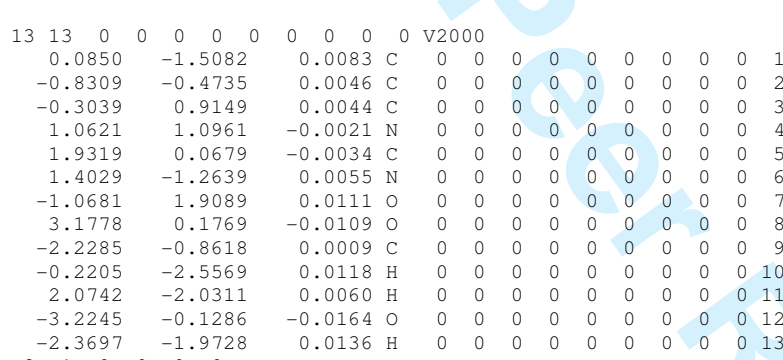

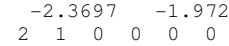

$\begin{array}{rrrrrr}6 & 1 & 0 & 0 & 0 & 0 \\ 10 & 1 & 0 & 0 & 0 & 0\end{array}$

$\begin{array}{rrrrrr}10 & 1 & 0 & 0 & 0 & 0 \\ 3 & 2 & 0 & 0 & 0 & 0\end{array}$

$\begin{array}{llllll}9 & 2 & 0 & 0 & 0\end{array}$

$\begin{array}{llllll}4 & 3 & 0 & 0 & 0 & 0 \\ 7 & 3 & 0 & 0 & 0 & 0\end{array}$

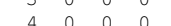

$\begin{array}{llllll}6 & 5 & 0 & 0 & 0 & 0\end{array}$

$\begin{array}{llllll}1 & 5 & 0 & 0 & 0 & 0\end{array}$

$\begin{array}{rrrrrr}12 & 9 & 0 & 0 & 0 & 0 \\ 9 & 13 & 0 & 0 & 0 & 0\end{array}$

M END

$\$ \$ \$ \$$

cis-5-formyluracil-anion1.sdf

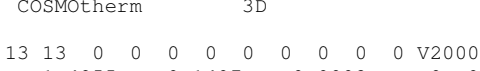

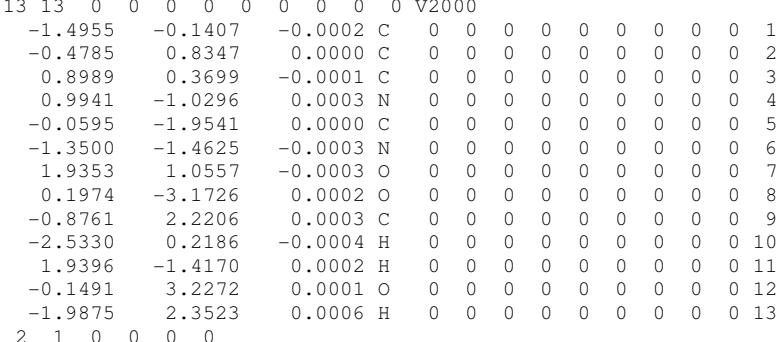

$\begin{array}{llllll}2 & 1 & 0 & 0 & 0 & 0 \\ 6 & 1 & 0 & 0 & 0 & 0\end{array}$

$\begin{array}{rrrrrr}6 & 1 & 0 & 0 & 0 \\ 10 & 1 & 0 & 0 & 0\end{array}$

$\begin{array}{llllll}3 & 2 & 0 & 0 & 0 \\ 9 & 2 & 0 & 0 & 0 & 0\end{array}$

$\begin{array}{llllll}9 & 2 & 0 & 0 & 0 & 0 \\ 4 & 3 & 0 & 0 & 0 & 0\end{array}$

$\begin{array}{llllll}3 & 3 & 0 & 0 & 0 & 0\end{array}$

$\begin{array}{llllll}5 & 4 & 0 & 0 & 0 \\ 1 & 4 & 0 & 0 & 0 & 0\end{array}$

$\begin{array}{cccccc}11 & 4 & 0 & 0 & 0 & 0 \\ 6 & 5 & 0 & 0 & 0 & 0\end{array}$

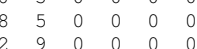

$\begin{array}{rrrrrr}12 & 13 & 0 & 0 & 0 & 0\end{array}$

$M$ END
$\$ \$ \$ \$$

Cis-5-formyluracil.sdf

$\begin{array}{llllllllllll}14 & 14 & 0 & 0 & 0 & 0 & 0 & 0 & 0 & 0 & 0 & \mathrm{~V} 2000\end{array}$

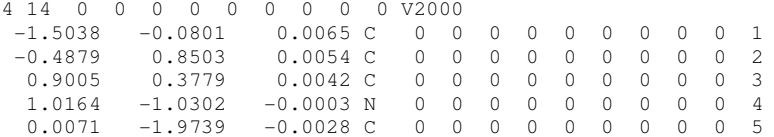




$\begin{array}{rrrrrrrrrrrrrr}-1.2761 & -1.4096 & 0.0020 & \mathrm{~N} & 0 & 0 & 0 & 0 & 0 & 0 & 0 & 0 & 0 & 6 \\ 1.9182 & 1.0687 & 0.0078 & 0 & 0 & 0 & 0 & 0 & 0 & 0 & 0 & 0 & 0 & 7 \\ 0.1927 & -3.1862 & -0.0082 & 0 & 0 & 0 & 0 & 0 & 0 & 0 & 0 & 0 & 0 & 8 \\ -0.8756 & 2.2589 & 0.0034 & \mathrm{C} & 0 & 0 & 0 & 0 & 0 & 0 & 0 & 0 & 0 & 9 \\ -2.5527 & 0.2200 & 0.0091 & \mathrm{H} & 0 & 0 & 0 & 0 & 0 & 0 & 0 & 0 & 0 & 10 \\ 1.9688 & -1.4009 & -0.0013 & \mathrm{H} & 0 & 0 & 0 & 0 & 0 & 0 & 0 & 0 & 0 & 11 \\ -2.0573 & -2.0655 & 0.0007 & \mathrm{H} & 0 & 0 & 0 & 0 & 0 & 0 & 0 & 0 & 0 & 12 \\ -0.1210 & 3.2302 & -0.0153 & 0 & 0 & 0 & 0 & 0 & 0 & 0 & 0 & 0 & 0 & 13 \\ -1.9821 & 2.4079 & 0.0186 & \mathrm{H} & 0 & 0 & 0 & 0 & 0 & 0 & 0 & 0 & 0 & 14\end{array}$

$-1.9821-2.4079$

$\begin{array}{lllll}2 & 1 & 0 & 0 & 0 \\ 6 & 1 & 0 & 0 & 0\end{array}$

$\begin{array}{rrrrrrr}10 & 1 & 0 & 0 & 0 & 0\end{array}$

$\begin{array}{llllll}3 & 2 & 0 & 0 & 0 & 0\end{array}$

$\begin{array}{lllllll}9 & 2 & 0 & 0 & 0 & 0 \\ 4 & 3 & 0 & 0 & 0 & 0\end{array}$

$\begin{array}{llllll}3 & 0 & 0 & 0 & 0 & 0\end{array}$

$\begin{array}{cccccc}5 & 4 & 0 & 0 & 0 & 0 \\ 1 & 4 & 0 & 0 & 0 & 0\end{array}$

$\begin{array}{llllll}5 & 0 & 0 & 0 & 0\end{array}$

$8 \begin{array}{llllll}8 & 5 & 0 & 0 & 0 & 0\end{array}$

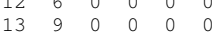

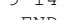

$M \quad$ END

cis-5-formyluracil2-anion.sdf

Cosmotherm 3D

$\begin{array}{llllllllllll}13 & 13 & 0 & 0 & 0 & 0 & 0 & 0 & 0 & 0 & 0 & \mathrm{v} 2000\end{array}$

$\begin{array}{llllllllllllll}-1.5697 & -0.2689 & 0.0010 & \text { C } & 0 & 0 & 0 & 0 & 0 & 0 & 0 & 0 & 0 & 1\end{array}$

$\begin{array}{llllllllllllll}-0.6003 & 0.7615 & 0.0008 & \mathrm{C} & 0 & 0 & 0 & 0 & 0 & 0 & 0 & 0 & 0 & 2\end{array}$

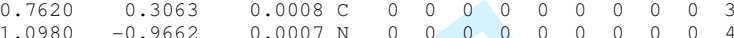

$\begin{array}{llllllllllllll}0.0784 & -1.9169 & -0.0003 & \mathrm{C} & 0 & 0 & 0 & 0 & 0 & 0 & 0 & 0 & 0 & 5\end{array}$

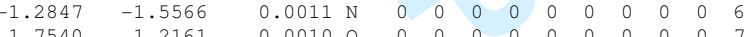

$\begin{array}{llllllllllllll}0.3755 & -3.1331 & -0.0021 & 0 & 0 & 0 & 0 & 0 & 0 & 0 & 0 & 0 & 0 & 8\end{array}$

$\begin{array}{llllllllllllll}-0.9264 & 2.1462 & -0.0008 & \mathrm{C} & 0 & 0 & 0 & 0 & 0 & 0 & 0 & 0 & 0 & 9\end{array}$

$0.0048-0.0014 \mathrm{H}-0$

$\begin{array}{llllllllllllll}-0.0615 & 3.0674 & -0.0016 & 0 & 0 & 0 & 0 & 0 & 0 & 0 & 0 & 0 & 0 & 10 \\ -2.0016 & 2.4161 & -0.0015 & \mathrm{H} & 0 & 0 & 0 & 0 & 0 & 0 & 0 & 0 & 0 & 1\end{array}$

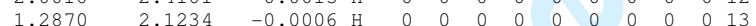

201000000

$\begin{array}{llllll}6 & 1 & 0 & 0 & 0 & 0\end{array}$

$\begin{array}{lllllll}10 & 1 & 0 & 0 & 0 & 0\end{array}$

$\begin{array}{llllll}2 & 0 & 0 & 0 & 0\end{array}$

$\begin{array}{lllll}2 & 0 & 0 & 0 \\ 3 & 0 & 0 & 0 & 0\end{array}$

$\begin{array}{llllllll}3 & 0 & 0 & 0 & 0\end{array}$

$\begin{array}{llll}4 & 0 & 0 & 0\end{array}$

$\begin{array}{ccccccc}5 & 0 & 0 & 0 & 0\end{array}$

$\begin{array}{llllll}1 & 9 & 0 & 0 & 0\end{array}$

$\begin{array}{rrrrrr}2 & 9 & 0 & 0 & 0 & 0 \\ 7 & 13 & 0 & 0 & 0 & 0\end{array}$

END

\$\$\$

cyanoaceticacido-anion.sdf

CosMotherm 3D

$\begin{array}{llllllllllll}8 & 7 & 0 & 0 & 0 & 0 & 0 & 0 & 0 & 0 & 0 & \mathrm{~V} 2000\end{array}$

$\begin{array}{llllllllllllll}1.5333 & -0.2852 & -0.0038 & \mathrm{C} & 0 & 0 & 0 & 0 & 0 & 0 & 0 & 0 & 0 & 1\end{array}$

$\begin{array}{llllllllllllll}0.2303 & -0.9179 & -0.0502 & \mathrm{C} & 0 & 0 & 0 & 0 & 0 & 0 & 0 & 0 & 0 & 2\end{array}$

$\begin{array}{llllllllllllll}-0.9912 & 0.0851 & -0.0028 & \mathrm{C} & 0 & 0 & 0 & 0 & 0 & 0 & 0 & 0 & 0 & 3 \\ -0.7398 & 1.3183 & -0.0254 & 0 & 0 & 0 & 0 & 0 & 0 & 0 & 0 & 0 & 0 & \end{array}$

$\begin{array}{lllllllllllll}-2.1144 & -0.4860 & 0.0450 & 0 & 0 & 0 & 0 & 0 & 0 & 0 & 0 & 0 & 0\end{array}$

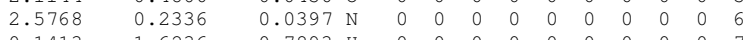

$\begin{array}{llllllllllllll}0.1413 & -1.6236 & 0.7893 & \mathrm{H} & 0 & 0 & 0 & 0 & 0 & 0 & 0 & 0 & 0 & 7\end{array}$

$\begin{array}{lll}0.1417 & -1.5105\end{array}$

$-0.9747 \mathrm{H}$

$\begin{array}{llllll}2 & 1 & 0 & 0 & 0 & 0 \\ 6 & 1 & 0 & 0 & 0 & 0 \\ 3 & 2 & 0 & 0 & 0 & 0\end{array}$

$\begin{array}{llllll}2 & 0 & 0 & 0 & 0\end{array}$

$2 \quad 000000$

$\begin{array}{llllll}3 & 0 & 0 & 0 & 0\end{array}$

$\begin{array}{ccccccccc}2 & 3 & 0 & 0 & 0 & 0\end{array}$

END

yanoaceticacido.sdf

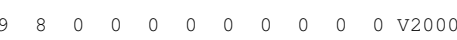

$\begin{array}{lllllllllllll}1.5649 & -0.2644 & -0.0017 & \mathrm{C} & 0 & 0 & 0 & 0 & 0 & 0 & 0 & 0 & 0\end{array}$

$\begin{array}{llllllllllllll}0.2567 & -0.8959 & -0.0431 & \mathrm{C} & 0 & 0 & 0 & 0 & 0 & 0 & 0 & 0 & 0 & 2\end{array}$

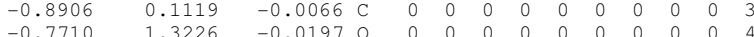

$\begin{array}{rrrrrrrrrrrrrr}-0.7710 & 1.3226 & -0.0197 & 0 & 0 & 0 & 0 & 0 & 0 & 0 & 0 & 0 & 0 & 4 \\ -2.0666 & -0.5426 & 0.0362 & 0 & 0 & 0 & 0 & 0 & 0 & 0 & 0 & 0 & 0 & 5\end{array}$

$\begin{array}{llllllllllllll}2.6204 & -0.5426 & 0.0362 & 0 & 0 & 0 & 0 & 0 & 0 & 0 & 0 & 0 & 0 & 5 \\ 0.1204 & 0.2221 & 0.0332 & \mathrm{~N} & 0 & 0 & 0 & 0 & 0 & 0 & 0 & 0 & 0\end{array}$

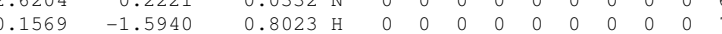

$\begin{array}{rrrrrrrrrrrrrr}0.1698 & -1.5003 & -0.9600 & \mathrm{H} & 0 & 0 & 0 & 0 & 0 & 0 & 0 & 0 & 0 & 8 \\ -2.7918 & 0.1203 & 0.0472 & \mathrm{H} & 0 & 0 & 0 & 0 & 0 & 0 & 0 & 0 & 0 & 9\end{array}$

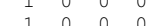

$\begin{array}{lllll}2 & 0 & 0 & 0 & 0\end{array}$

$\begin{array}{lllll}2 & 0 & 0 & 0 & 0\end{array}$

$\begin{array}{lllll}3 & 0 & 0 & 0 & 0\end{array}$

END

cyanoaceticacid1-anion.sdf

cosmotherm

$3 \mathrm{D}$

$\begin{array}{lllllllllllll}8 & 7 & 0 & 0 & 0 & 0 & 0 & 0 & 0 & 0 & 0 & \mathrm{~V} 2000\end{array}$ 


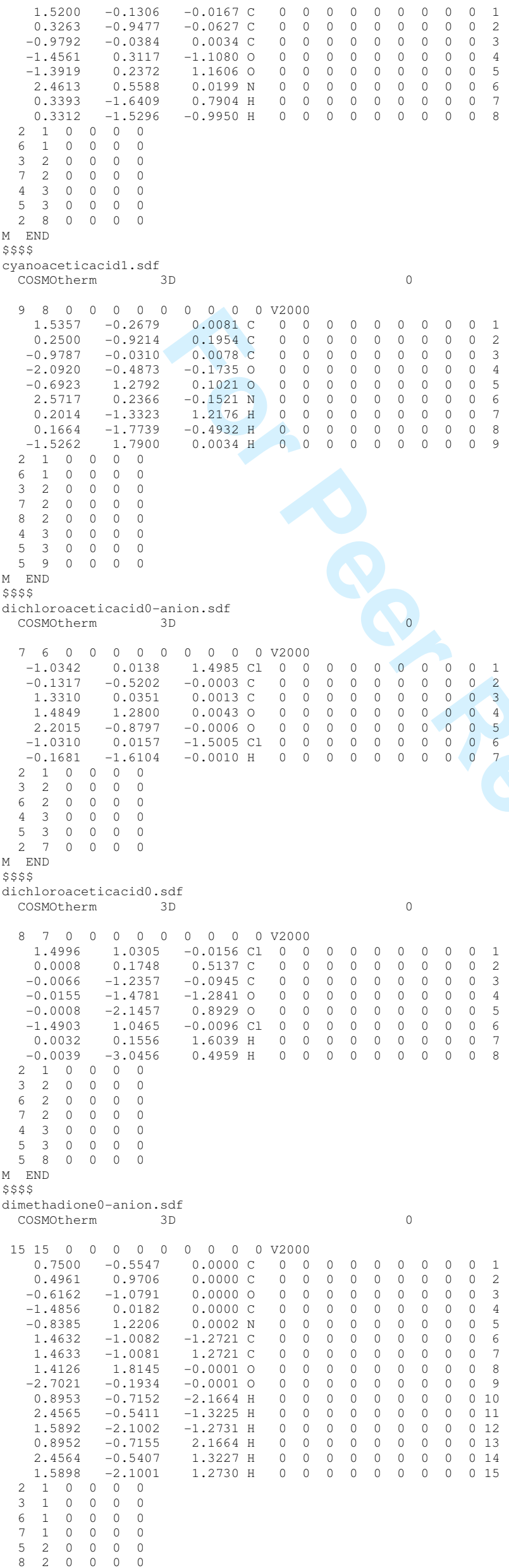




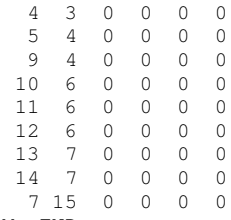




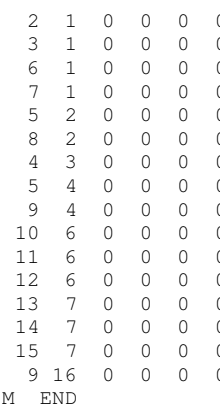


$\begin{array}{llllll}4 & 1 & 0 & 0 & 0 & 0\end{array}$

$\begin{array}{ccccc}1 & 0 & 0 & 0 & 0 \\ 1 & 0 & 0 & 0 & 0\end{array}$

$\begin{array}{lllll}2 & 0 & 0 & 0 & 0 \\ 2 & 0 & 0 & 0 & 0\end{array}$

$\begin{array}{lllll}8 & 0 & 0 & 0 & 0\end{array}$

$M$ END

ethanol.sdf

cosmotherm

$3 \mathrm{D}$

$\begin{array}{llllllllllll}9 & 8 & 0 & 0 & 0 & 0 & 0 & 0 & 0 & 0 & 0 & \mathrm{~V} 2000\end{array}$

$\begin{array}{llllllllllllll}1.2720 & 0.0000 & 0.2530 & \mathrm{C} & 0 & 0 & 0 & 0 & 0 & 0 & 0 & 0 & 0 & 1\end{array}$

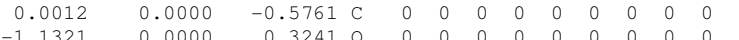

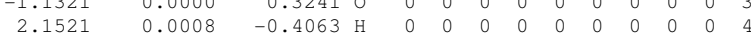

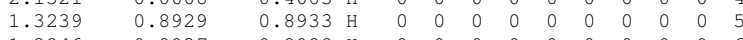

$\begin{array}{lllllllllllll}1.3246 & -0.8937 & 0.8922 \mathrm{H} & 0 & 0 & 0 & 0 & 0 & 0 & 0 & 0 & 0 & 6\end{array}$

$\begin{array}{rrrrrrrrrrrrrr}-0.0318 & 0.8925 & -1.2258 \mathrm{H} & 0 & 0 & 0 & 0 & 0 & 0 & 0 & 0 & 0 & 8\end{array}$

$\begin{array}{lll}-1.9393 & -0.0004 & -0.2211 \mathrm{H}\end{array}$

$\begin{array}{lllllll}2 & 1 & 0 & 0 & 0 & 0\end{array}$

$\begin{array}{lllll}5 & 1 & 0 & 0 & 0\end{array}$

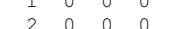

$2=0000$

$\begin{array}{lllll}2 & 0 & 0 & 0 & 0 \\ 9 & 0 & 0 & 0 & 0\end{array}$

$M \quad$ END

fluoroaceticacido-anion.sdf

cosmotherm 3D

$\begin{array}{llllllllllll}7 & 6 & 0 & 0 & 0 & 0 & 0 & 0 & 0 & 0 & 0 & \text { V2 } 2000\end{array}$

$\begin{array}{llllllllllllll}-0.0701 & -1.8291 & -0.0065 & \mathrm{~F} & 0 & 0 & 0 & 0 & 0 & 0 & 0 & 0 & 0 & 1\end{array}$

$\begin{array}{llllllllllllll}0.7532 & -0.6667 & 0.0096 & \mathrm{C} & 0 & 0 & 0 & 0 & 0 & 0 & 0 & 0 & 0 & 2\end{array}$

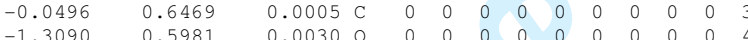

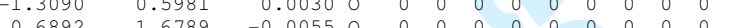

$\begin{array}{lllllllllllll}1.4027 & -0.7141 & -0.8765 & \mathrm{H} & 0 & 0 & 0 & 0 & 0 & 0 & 0 & 0 & 0\end{array}$

$\begin{array}{lll}1.3718 & -0.7184 & 0.9175 \mathrm{H}\end{array}$

$\begin{array}{llllll}1 & 0 & 0 & 0 & 0\end{array}$

$\begin{array}{llllll}1 & 2 & 0 & 0 & 0 & 0\end{array}$

$\begin{array}{llllll}2 & 0 & 0 & 0 & 0\end{array}$

$\begin{array}{llllllll}0 & 0 & 0 & 0\end{array}$

END

fluoroaceticacido.sdf

$8 \begin{array}{lllllllllllll}8 & 7 & 0 & 0 & 0 & 0 & 0 & 0 & 0 & 0 & 0 & \mathrm{~V} 2000\end{array}$

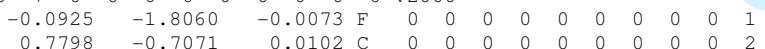

$\begin{array}{rrrrrrrrrrrrrr}0.7798 & -0.7071 & 0.0102 & \mathrm{C} & 0 & 0 & 0 & 0 & 0 & 0 & 0 & 0 & 0 & 2 \\ 0.0619 & 0.6286 & 0.0006 & \mathrm{C} & 0 & 0 & 0 & 0 & 0 & 0 & 0 & 0 & 0 & 3\end{array}$

$\begin{array}{rrrrrrrrrrrrrr}0.0619 & 0.6286 & 0.0006 & \mathrm{C} & 0 & 0 & 0 & 0 & 0 & 0 & 0 & 0 & 0 & 3 \\ -1.2773 & 0.5276 & 0.0039 & 0 & 0 & 0 & 0 & 0 & 0 & 0 & 0 & 0 & 0 & 4\end{array}$

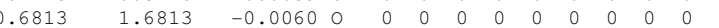

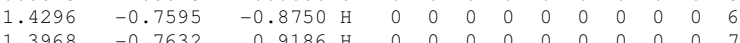

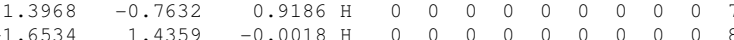

1.435

$\begin{array}{llllll}1 & 0 & 0 & 0 & 0\end{array}$

$\begin{array}{llllll}2 & 0 & 0 & 0 & 0\end{array}$

$\begin{array}{lllll}2 & 0 & 0 & 0 & 0\end{array}$

5 3 $30 \begin{array}{llll}0 & 0 & 0\end{array}$

FND

$\$ \$ \$$

fluoroaceticacidl-anion.sdf

CosMotherm 3D

$\begin{array}{llllllllllllll}6 & 0 & 0 & 0 & 0 & 0 & 0 & 0 & 0 & 0 & \mathrm{v} 2000\end{array}$

$\begin{array}{lllllllllll}0.0005 \text { F } & 0 & 0 & 0 & 0 & 0 & 0 & 0 & 0 & 0 & 1\end{array}$

$\begin{array}{lllllllllllll}0.6666 & -0.7533 & -0.0008 & \mathrm{C} & 0 & 0 & 0 & 0 & 0 & 0 & 0 & 0 & 0\end{array}$

$\begin{array}{llllllllllllll}-0.6469 & 0.0496 & -0.0001 & C & 0 & 0 & 0 & 0 & 0 & 0 & 0 & 0 & 0 & \\ -1.6789 & -0.6890 & 0.0004 & 0 & 0 & 0 & 0 & 0 & 0 & 0 & 0 & 0 & 0 & 3\end{array}$

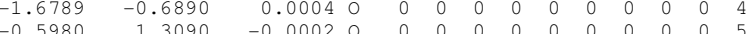

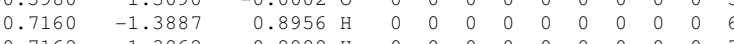

$0.7162-1.3862$

$\begin{array}{llllll}2 & 0 & 0 & 0 & 0\end{array}$

$\begin{array}{llllll}2 & 0 & 0 & 0 & 0\end{array}$

$\begin{array}{lllll}3 & 0 & 0 & 0 & 0\end{array}$

$\begin{array}{llllll}7 & 0 & 0 & 0 & 0\end{array}$

END

fluoroaceticacid1.sdf

cosmotherm

$\begin{array}{llllllllllll}8 & 7 & 0 & 0 & 0 & 0 & 0 & 0 & 0 & 0 & 0 & \mathrm{v} 2000\end{array}$

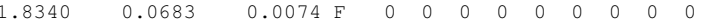

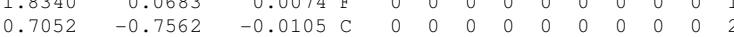

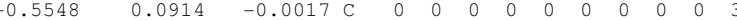

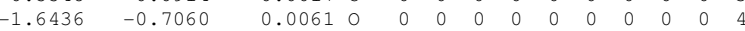

$\begin{array}{llllllllllllll}-0.5847 & 1.3082 & -0.0032 & 0 & 0 & 0 & 0 & 0 & 0 & 0 & 0 & 0 & 0 & 5\end{array}$

$\begin{array}{lllllllllllll}0.7247 & -1.4072 & 0.8754 & \mathrm{H} & 0 & 0 & 0 & 0 & 0 & 0 & 0 & 0 & 0 \\ 0.7308 & -1.3750 & -0.9193 & \mathrm{H} & 0 & 0 & 0 & 0 & 0 & 0 & 0 & 0 & 0\end{array}$

$\begin{array}{rrrrrrrrrrrrrr}0.7308 & -1.3750 & -0.9193 & \mathrm{H} & 0 & 0 & 0 & 0 & 0 & 0 & 0 & 0 & 0 & \\ -2.4473 & -0.1412 & 0.0045 & \mathrm{H} & 0 & 0 & 0 & 0 & 0 & 0 & 0 & 0 & 0 & 8\end{array}$

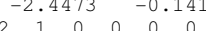

$\begin{array}{llllllll}3 & 0 & 0 & 0 & 0\end{array}$ 


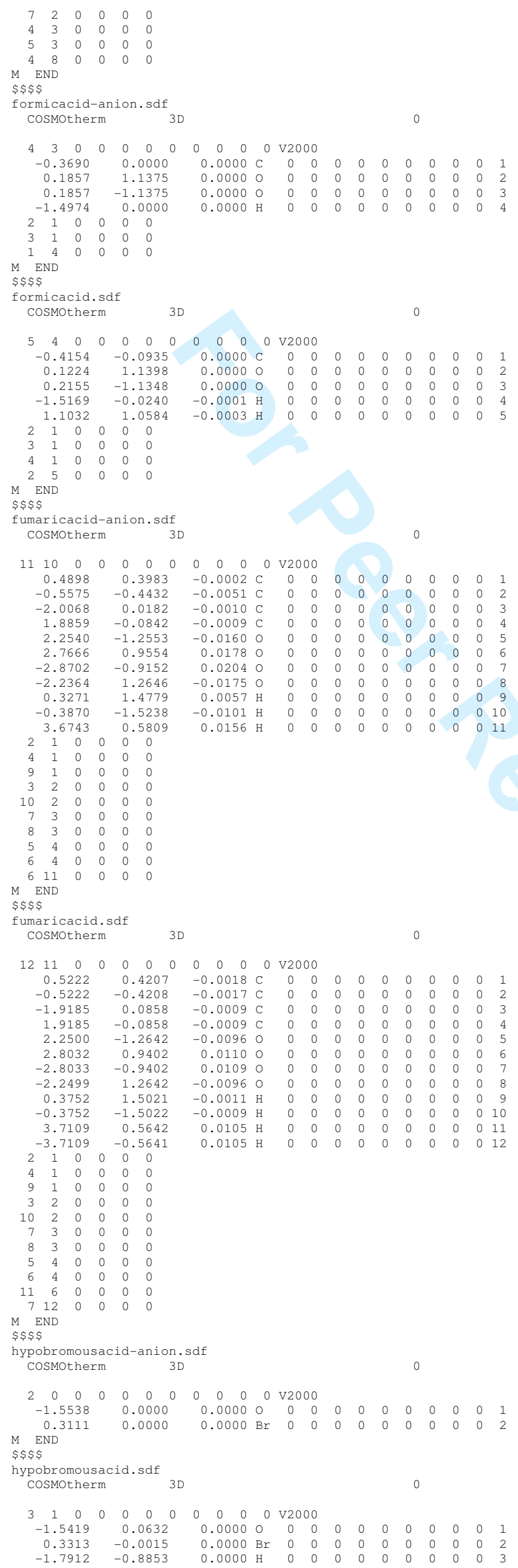




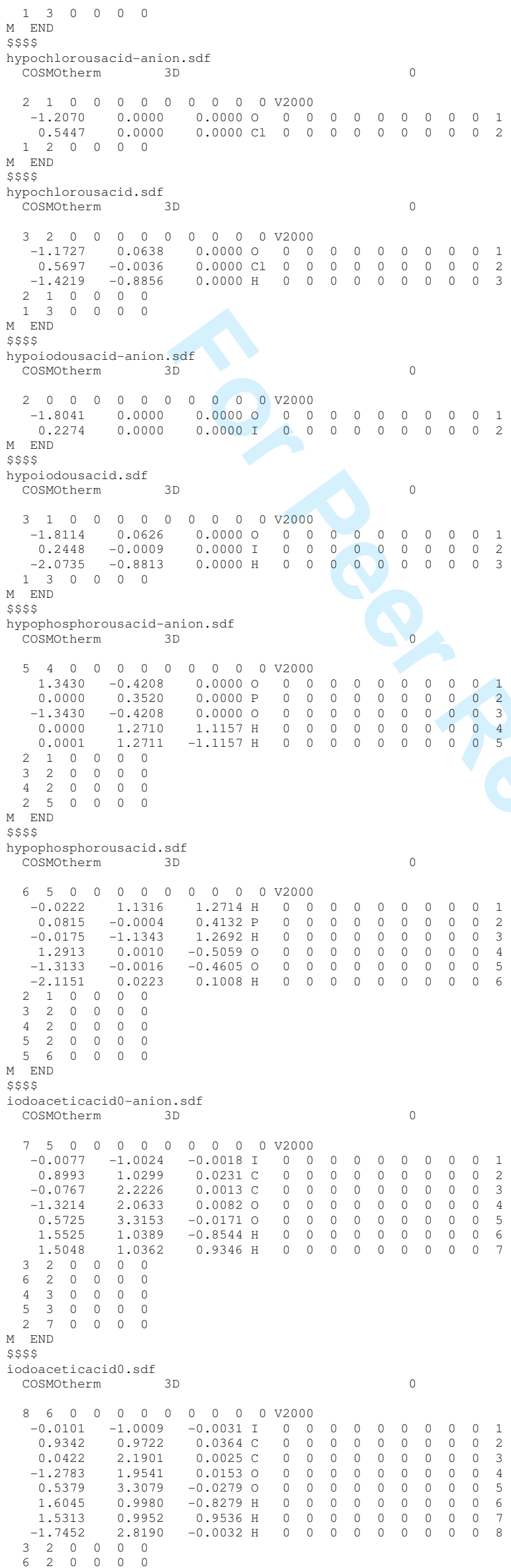




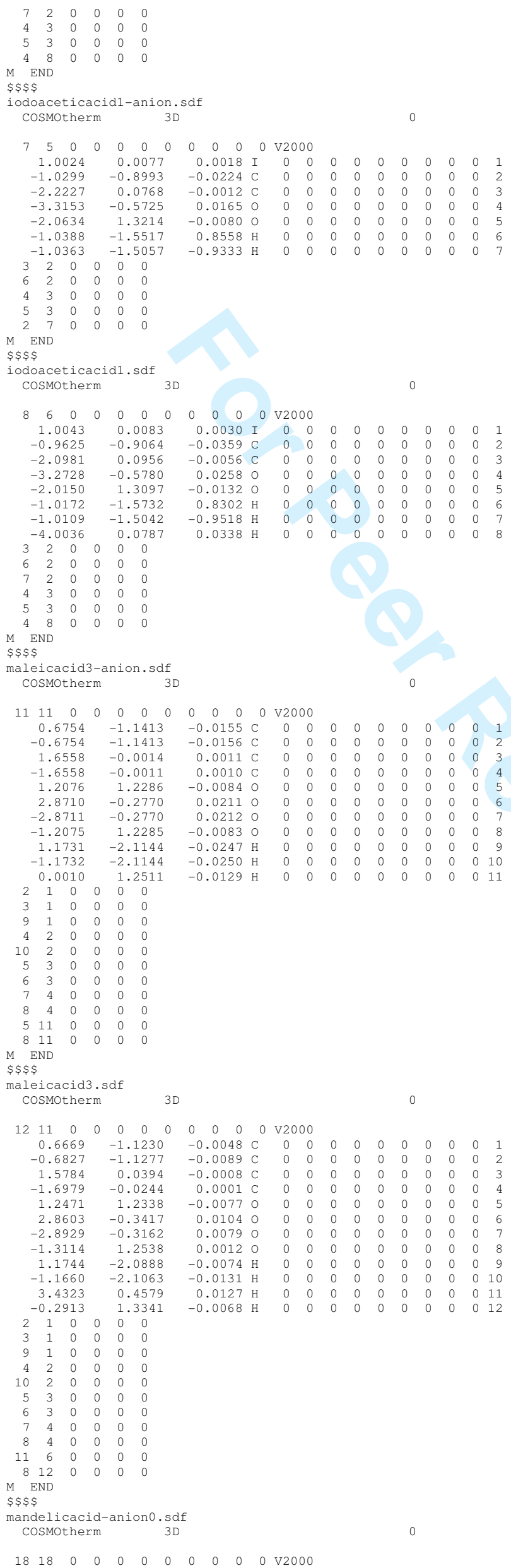


$\begin{array}{lrrrrrrrrrrrrr}-3.1021 & -0.0161 & -0.4438 & \mathrm{C} & 0 & 0 & 0 & 0 & 0 & 0 & 0 & 0 & 0 & 1 \\ -2.6068 & -0.9577 & 0.4658 & \mathrm{C} & 0 & 0 & 0 & 0 & 0 & 0 & 0 & 0 & 0 & 2\end{array}$

$\begin{array}{llllllllllllll}-1.2691 & -0.9026 & 0.8755 & C & 0 & 0 & 0 & 0 & 0 & 0 & 0 & 0 & 0 & 3\end{array}$

$\begin{array}{llllllllllllll}-0.4077 & 0.0894 & 0.3821 & \mathrm{C} & 0 & 0 & 0 & 0 & 0 & 0 & 0 & 0 & 0 & 4\end{array}$

$\begin{array}{llllllllllllll}-0.9151 & 1.0345 & -0.5251 & \mathrm{C} & 0 & 0 & 0 & 0 & 0 & 0 & 0 & 0 & 0 & 5 \\ -2.2509 & 0.9826 & -0.9357 & \mathrm{C} & 0 & 0 & 0 & 0 & 0 & 0 & 0 & 0 & 0 & 6\end{array}$

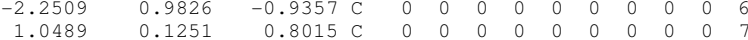

$\begin{array}{llllllllllllll}1.5025 & 1.4599 & 1.0622 & 0 & 0 & 0 & 0 & 0 & 0 & 0 & 0 & 0 & 0 & 8\end{array}$

$\begin{array}{lllllllllllllll}1.9744 & -0.5071 & -0.3011 & \mathrm{C} & 0 & 0 & 0 & 0 & 0 & 0 & 0 & 0 & 0 & 9\end{array}$

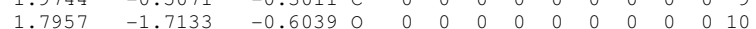

$\begin{array}{lllllllllllllllll}1.79437 & 0.2936 & -0.7770 & 0 & 0 & 0 & 0 & 0 & 0 & 0 & 0 & 0 & 0 & 11\end{array}$

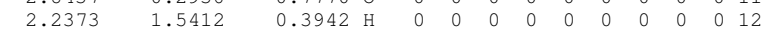

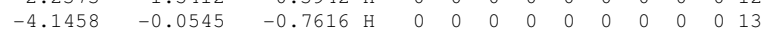

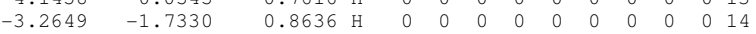

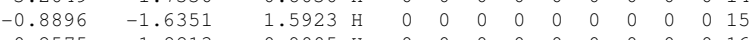

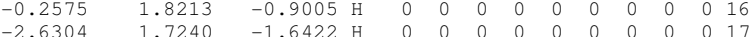

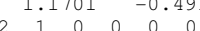

$6 \quad 1 \quad 000000$

$\begin{array}{llllll}3 & 1 & 0 & 0 & 0 & 0\end{array}$

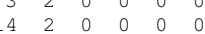

$\begin{array}{llllll}4 & 3 & 0 & 0 & 0\end{array}$

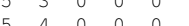

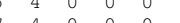

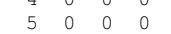

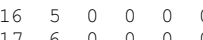

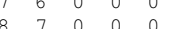

$\begin{array}{lllllll}9 & 7 & 0 & 0 & 0 & 0\end{array}$

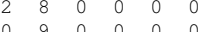

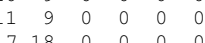

END

mandelicacid-anion1.sdf

COSMOtherm

$\begin{array}{lllllllllllll}18 & 18 & 0 & 0 & 0 & 0 & 0 & 0 & 0 & 0 & 0 & \mathrm{v} 2000\end{array}$

$\begin{array}{cccccccccccccccc}18 & 0 & 0 & 0 & 0 & 0 & 0 & 0 & 0 & 0 & & & 2000 \\ 0.0608 & -0.4269 & -3.1006 & \mathrm{C} & 0 & 0 & 0 & 0 & 0 & 0 & 0 & 0 & 0 & 1\end{array}$

$\begin{array}{lllllllllllllll}0.0608 & -0.4269 & -3.1006 & \mathrm{C} & 0 & 0 & 0 & 0 & 0 & 0 & 0 & 0 & 0 & \\ -1.0239 & -0.8298 & -2.3116 & \mathrm{C} & 0 & 0 & 0 & 0 & 0 & 0 & 0 & 0 & 0\end{array}$

$\begin{array}{llllllllllllll}-1.0239 & -0.8298 & -2.3116 & \mathrm{C} & 0 & 0 & 0 & 0 & 0 & 0 & 0 & 0 & 0 & 2 \\ -1.1116 & -0.4262 & -0.9744 & \mathrm{C} & 0 & 0 & 0 & 0 & 0 & 0 & 0 & 0 & 0 & 3\end{array}$

$\begin{array}{rrrrrrrrrrrrrrr}-1.1116 & -0.4262 & -0.9744 & \mathrm{C} & 0 & 0 & 0 & 0 & 0 & 0 & 0 & 0 & 0 & 3 \\ -0.1166 & 0.3845 & -0.4051 & \mathrm{C} & 0 & 0 & 0 & 0 & 0 & 0 & 0 & 0 & 0 & 4\end{array}$

$\begin{array}{lllllllllllllll}0.9624 & 0.7928 & -1.2062 & \mathrm{C} & 0 & 0 & 0 & 0 & 0 & 0 & 0 & 0 & 0 & 5\end{array}$

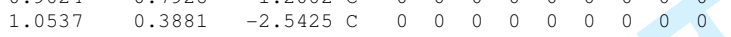

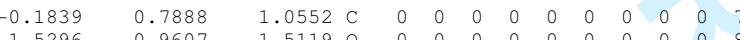

$\begin{array}{rrrrrrrrrrrrrr}-1.5296 & 0.9607 & 1.5119 & 0 & 0 & 0 & 0 & 0 & 0 & 0 & 0 & 0 & 0 & 8 \\ 0.5178 & -0.2840 & 1.9694 & \mathrm{C} & 0 & 0 & 0 & 0 & 0 & 0 & 0 & 0 & 0 & 9\end{array}$

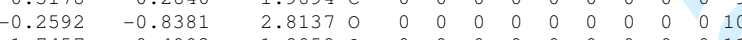

$\begin{array}{llllllllllllll}1.7457 & -0.4903 & 1.8058 & 0 & 0 & 0 & 0 & 0 & 0 & 0 & 0 & 0 & 0 & 11\end{array}$

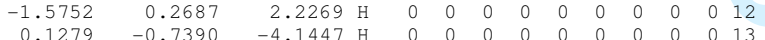

$\begin{array}{llllllllllllll}-1.8063 & -1.4604 & -2.7395 & \mathrm{H} & 0 & 0 & 0 & 0 & 0 & 0 & 0 & 0 & 0 & 14\end{array}$

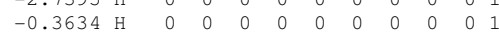

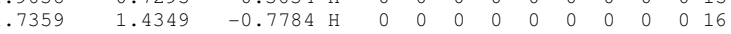

$\begin{array}{llllllllllllll}1.7359 & 1.4349 & 0.7168 & -3.1525 \mathrm{H} & 0 & 0 & 0 & 0 & 0 & 0 & 0 & 0 & 0 & 17\end{array}$

$1.89753-1.7318$

$\begin{array}{llllll}2 & 1 & 0 & 0 & 0 & 0\end{array}$

$\begin{array}{llllll}2 & 1 & 0 & 0 & 0 & 0\end{array}$

$\begin{array}{llllll}6 & 1 & 0 & 0 & 0 & 0\end{array}$

$\begin{array}{cccccc}3 & 2 & 0 & 0 & 0 & 0 \\ 2 & 0 & 0 & 0 & 0\end{array}$

$\begin{array}{llllll}4 & 3 & 0 & 0 & 0 & 0\end{array}$

$\begin{array}{llllll}5 & 3 & 0 & 0 & 0 & 0 \\ 5 & 4 & 0 & 0 & 0 & 0\end{array}$

$\begin{array}{lllll}4 & 0 & 0 & 0 & 0\end{array}$

$\begin{array}{lllll}5 & 0 & 0 & 0 & 0\end{array}$

$\begin{array}{lllll}7 & 0 & 0 & 0\end{array}$

$\begin{array}{lllll}7 & 0 & 0 & 0\end{array}$

$\begin{array}{llll}8 & 0 & 0 & 0\end{array}$

$\begin{array}{llll}0 & 0 & 0 & 0\end{array}$

$\begin{array}{llllll}1 & 9 & 0 & 0 & 0 & 0\end{array}$

END

\$\$\$

mandelicacido.sdf

3D

$\begin{array}{llllllllllll}19 & 19 & 0 & 0 & 0 & 0 & 0 & 0 & 0 & 0 & 0 & \mathrm{~V} 2000\end{array}$

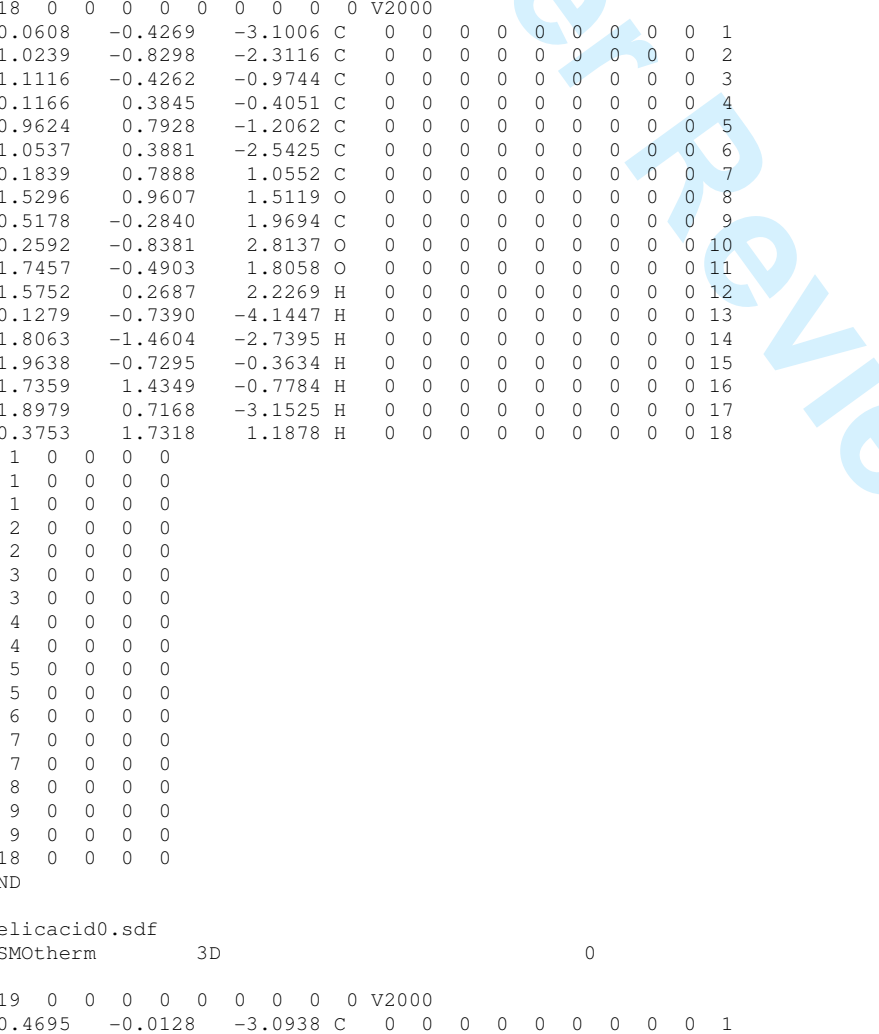

$\begin{array}{llllllllllll}0 & 0 & 0 & 0 & 0 & 0 & 0 & 0 & 1\end{array}$

$\begin{array}{llllllllllllll}-0.5099 & -0.9012 & -2.6371 & \mathrm{C} & 0 & 0 & 0 & 0 & 0 & 0 & 0 & 0 & 0 & 2 \\ -0.9291 & -0.8572 & -1.3021 & \mathrm{C} & 0 & 0 & 0 & 0 & 0 & 0 & 0 & 0 & 0 & 3\end{array}$

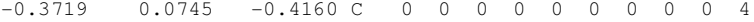

$\begin{array}{llllllllllllll}0.6076 & 0.9668 & -0.8790 & \mathrm{C} & 0 & 0 & 0 & 0 & 0 & 0 & 0 & 0 & 0 & 5\end{array}$

$\begin{array}{llllllllllllll}1.0251 & 0.9238 & -2.2122 & \mathrm{C} & 0 & 0 & 0 & 0 & 0 & 0 & 0 & 0 & 0 & 6 \\ -0.8301 & 0.1233 & 1.0384 & \mathrm{C} & 0 & 0 & 0 & 0 & 0 & 0 & 0 & 0 & 0 & 7\end{array}$

$\begin{array}{llllllllllllll}-0.8301 & 0.1233 & 1.0384 & \mathrm{C} & 0 & 0 & 0 & 0 & 0 & 0 & 0 & 0 & 0 & 7\end{array}$

$\begin{array}{llllllllllllll}-1.2043 & 1.4376 & 1.4489 & 0 & 0 & 0 & 0 & 0 & 0 & 0 & 0 & 0 & 0 & 8 \\ 0.2804 & -0.3854 & 1.9636 & 0 & 0 & 0 & 0 & 0 & 0 & 0 & 0 & 0 & 0 & 9\end{array}$

$\begin{array}{llllllllllllll}0.2804 & -0.3854 & 1.9636 & \mathrm{C} & 0 & 0 & 0 & 0 & 0 & 0 & 0 & 0 & 0 & 9\end{array}$

$\begin{array}{rrrrrrrrrrrrrr}0.5243 & -1.6952 & 1.8157 & 0 & 0 & 0 & 0 & 0 & 0 & 0 & 0 & 0 & 0 & 10 \\ 0.8728 & 0.3499 & 2.7423 & 0 & 0 & 0 & 0 & 0 & 0 & 0 & 0 & 0 & 0 & 11\end{array}$

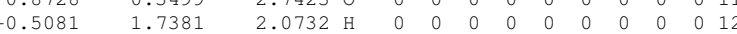

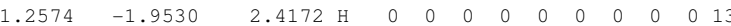

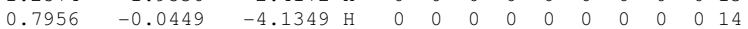

$\begin{array}{llllllllllllll}-0.9532 & -1.6274 & -3.3210 & \mathrm{H} & 0 & 0 & 0 & 0 & 0 & 0 & 0 & 0 & 0 & 15\end{array}$

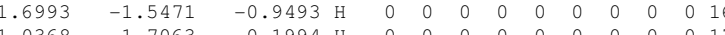

$\begin{array}{lllllllllllllllll}1.0368 & 1.7063 & -0.1994 & \mathrm{H} & 0 & 0 & 0 & 0 & 0 & 0 & 0 & 0 & 0 & 17\end{array}$

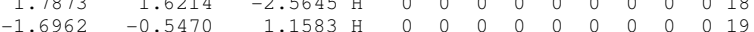

$\begin{array}{lllllllll}2 & 1 & 0 & 0 & 0 & 0\end{array}$

$\begin{array}{lllllllll}6 & 1 & 0 & 0 & 0 & 0\end{array}$ 
$\begin{array}{rrllll}14 & 1 & 0 & 0 & 0 & 0 \\ 3 & 2 & 0 & 0 & 0 & 0 \\ 15 & 2 & 0 & 0 & 0 & 0 \\ 4 & 3 & 0 & 0 & 0 & 0 \\ 16 & 3 & 0 & 0 & 0 & 0 \\ 5 & 4 & 0 & 0 & 0 & 0 \\ 7 & 4 & 0 & 0 & 0 & 0 \\ 6 & 5 & 0 & 0 & 0 & 0 \\ 17 & 5 & 0 & 0 & 0 & 0 \\ 18 & 6 & 0 & 0 & 0 & 0 \\ 8 & 7 & 0 & 0 & 0 & 0 \\ 9 & 7 & 0 & 0 & 0 & 0 \\ 12 & 8 & 0 & 0 & 0 & 0 \\ 10 & 9 & 0 & 0 & 0 & 0 \\ 11 & 9 & 0 & 0 & 0 & 0 \\ 13 & 10 & 0 & 0 & 0 & 0 \\ 7 & 19 & 0 & 0 & 0 & 0 \\ 19 & & & & & \end{array}$ $M \quad$ END

$\$ \$ \$$

mandelicacidl.sdf
Cosmotherm

$\begin{array}{llllllllllll}19 & 19 & 0 & 0 & 0 & 0 & 0 & 0 & 0 & 0 & 0 & \mathrm{~V} 2000\end{array}$

$\begin{array}{lrrrrrrrrrrrrr}0.1979 & 0.4805 & -3.0994 & \mathrm{C} & 0 & 0 & 0 & 0 & 0 & 0 & 0 & 0 & 0 & 1 \\ 0.4300 & -0.8489 & -2.7293 & \mathrm{C} & 0 & 0 & 0 & 0 & 0 & 0 & 0 & 0 & 0 & 2\end{array}$

$\begin{array}{llllllllllllll}0.4300 & -0.8489 & -2.7293 & \mathrm{C} & 0 & 0 & 0 & 0 & 0 & 0 & 0 & 0 & 0 & 2 \\ 0.2618 & -1.2454 & -1.3977 & \mathrm{C} & 0 & 0 & 0 & 0 & 0 & 0 & 0 & 0 & 0 & 3\end{array}$

$\begin{array}{llllllllllllll}-0.1413 & -0.3175 & -0.4264 & \mathrm{C} & 0 & 0 & 0 & 0 & 0 & 0 & 0 & 0 & 0 & 4\end{array}$

$\begin{array}{llllllllllllll}-0.3696 & 1.0173 & -0.8035 & \mathrm{C} & 0 & 0 & 0 & 0 & 0 & 0 & 0 & 0 & 0 & 5 \\ -0.2046 & 1.4125 & -2.1344 & \mathrm{C} & 0 & 0 & 0 & 0 & 0 & 0 & 0 & 0 & 0 & 6\end{array}$

$\begin{array}{lrrrrrrrrrrrrr}-0.2046 & 1.4125 & -2.1344 & \mathrm{C} & 0 & 0 & 0 & 0 & 0 & 0 & 0 & 0 & 0 & 6 \\ -0.3661 & -0.7714 & 1.0123 & \mathrm{C} & 0 & 0 & 0 & 0 & 0 & 0 & 0 & 0 & 0 & 7\end{array}$

$\begin{array}{lllllllllllllll}-1.7475 & -0.7717 & 1.3922 & 0 & 0 & 0 & 0 & 0 & 0 & 0 & 0 & 0 & 0 & 8\end{array}$

$\begin{array}{lllllllllllllr}0.5261 & 0.0462 & 1.9577 & \mathrm{C} & 0 & 0 & 0 & 0 & 0 & 0 & 0 & 0 & 0 & 9\end{array}$

$\begin{array}{rrrrrrrrrrrrrr}-0.1464 & 1.0468 & 2.5666 & 0 & 0 & 0 & 0 & 0 & 0 & 0 & 0 & 0 & 0 & 10 \\ 1.7173 & -0.1690 & 2.1168 & 0 & 0 & 0 & 0 & 0 & 0 & 0 & 0 & 0 & 0 & 11\end{array}$

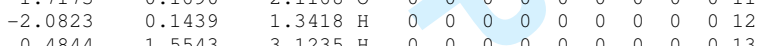

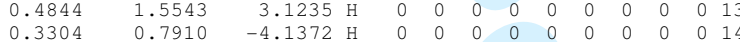

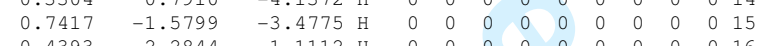

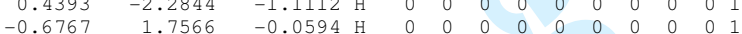

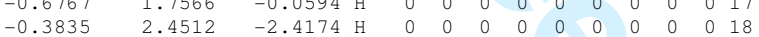

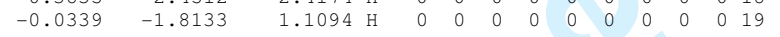

$\begin{array}{llllll}2 & 1 & 0 & 0 & 0 & 0\end{array}$

$\begin{array}{llllll}6 & 1 & 0 & 0 & 0 & 0\end{array}$

$\begin{array}{llllll}14 & 1 & 0 & 0 & 0 & 0\end{array}$

$\begin{array}{rrrrrrr}3 & 2 & 0 & 0 & 0 & 0 \\ 15 & 2 & 0 & 0 & 0 & 0\end{array}$

$\begin{array}{llllll}4 & 3 & 0 & 0 & 0 & 0\end{array}$

$\begin{array}{llllll}6 & 3 & 0 & 0 & 0 & 0 \\ 5 & 4 & 0 & 0 & 0 & 0\end{array}$

$\begin{array}{lllll}7 & 4 & 0 & 0 & 0\end{array}$

$\begin{array}{llllll}6 & 5 & 0 & 0 & 0 & 0 \\ 7 & 5 & 0 & 0 & 0 & 0\end{array}$

$\begin{array}{lllll}8 & 6 & 0 & 0 & 0\end{array}$

$\begin{array}{llllll}8 & 7 & 0 & 0 & 0 & 0 \\ 9 & 7 & 0 & 0 & 0 & 0\end{array}$

$\begin{array}{llllll}9 & 7 & 0 & 0 & 0 & 0\end{array}$

$\begin{array}{llllll}12 & 8 & 0 & 0 & 0 & 0 \\ 10 & 9 & 0 & 0 & 0 & 0\end{array}$

$\begin{array}{llllll}11 & 9 & 0 & 0 & 0 & 0\end{array}$

$\begin{array}{rrrrrr}13 & 10 & 0 & 0 & 0 & 0 \\ 7 & 19 & 0 & 0 & 0 & 0\end{array}$

M END

$\$ \$ \$ \$$

methanol-anion.sdf

\section{$\begin{array}{llllllllllll}5 & 4 & 0 & 0 & 0 & 0 & 0 & 0 & 0 & 0 & 0 & \mathrm{~V} 2000\end{array}$}

$\begin{array}{llllllllllllll}-0.6612 & 0.0000 & 0.0000 & \mathrm{C} & 0 & 0 & 0 & 0 & 0 & 0 & 0 & 0 & 0 & 1\end{array}$

$\begin{array}{llllllllllllll}0.7119 & 0.0000 & 0.0000 & 0 & 0 & 0 & 0 & 0 & 0 & 0 & 0 & 0 & 0 & 2\end{array}$

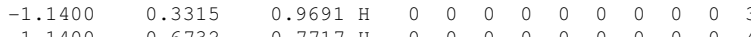

$\begin{array}{lrrrrrrrrrrrrr}-1.1400 & 0.6732 & -0.7717 & \mathrm{H} & 0 & 0 & 0 & 0 & 0 & 0 & 0 & 0 & 0 & 4 \\ -1.1402 & -1.0049 & -0.1973 & \mathrm{H} & 0 & 0 & 0 & 0 & 0 & 0 & 0 & 0 & 0 & 5\end{array}$

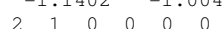

$\begin{array}{lllllll}3 & 1 & 0 & 0 & 0 & 0\end{array}$

$\begin{array}{llllll}4 & 1 & 0 & 0 & 0 & 0\end{array}$

END

\$\$

methanol.sdf

$\begin{array}{llllllllllll}6 & 5 & 0 & 0 & 0 & 0 & 0 & 0 & 0 & 0 & 0 & \mathrm{v} 2000\end{array}$

$\begin{array}{lllllllllllll}-0.7382 & 0.0127 & 0.0000 & \mathrm{C} & 0 & 0 & 0 & 0 & 0 & 0 & 0 & 0 & 0\end{array}$

$\begin{array}{rrrrrrrrrrrrrr}0.7000 & -0.0651 & 0.0000 & 0 & 0 & 0 & 0 & 0 & 0 & 0 & 0 & 0 & 0 & 2 \\ -1.1138 & -1.0183 & 0.0001 & \mathrm{H} & 0 & 0 & 0 & 0 & 0 & 0 & 0 & 0 & 0 & 3\end{array}$

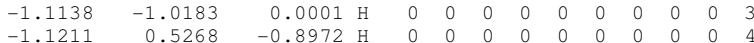

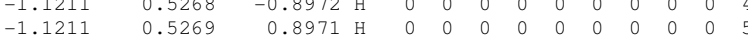

$\begin{array}{llllllllllllll}1.0413 & 0.8466 & 0.0000 & \mathrm{H} & 0 & 0 & 0 & 0 & 0 & 0 & 0 & 0 & 0 & 6\end{array}$

$\begin{array}{llllll}2 & 1 & 0 & 0 & 0 & 0\end{array}$

$\begin{array}{lllll}1 & 0 & 0 & 0 & 0\end{array}$

$\begin{array}{llllll}1 & 0 & 0 & 0 & 0\end{array}$

$\begin{array}{lllll}1 & 0 & 0 & 0 & 0 \\ 6 & 0 & 0 & 0 & 0\end{array}$

$\$ \$ \$$

methylthiouracilo-anion.sdf

cosmotherm

$\begin{array}{llllllllllll}14 & 14 & 0 & 0 & 0 & 0 & 0 & 0 & 0 & 0 & 0 & \mathrm{~V} 2000\end{array}$

$\begin{array}{lllllllllllll}-0.8379 & -0.0328 & 0.0001 & \mathrm{C} & 0 & 0 & 0 & 0 & 0 & 0 & 0 & 0 & 0\end{array}$

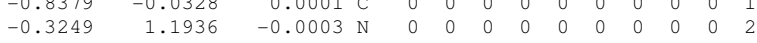

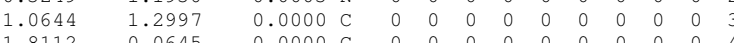

$\begin{array}{lllllllllllll}1.8112 & 0.0645 & 0.0000 & \mathrm{C} & 0 & 0 & 0 & 0 & 0 & 0 & 0 & 0 & 0\end{array}$

$\begin{array}{rrrrrrrrrrrrrr}1.1467 & -1.1460 & 0.0000 & \mathrm{C} & 0 & 0 & 0 & 0 & 0 & 0 & 0 & 0 & 0 & 5 \\ -0.2236 & -1.2205 & 0.0003 & \mathrm{~N} & 0 & 0 & 0 & 0 & 0 & 0 & 0 & 0 & 0 & 6\end{array}$ 
$\begin{array}{llllllllllllll}1.6084 & 2.4440 & 0.0001 & 0 & 0 & 0 & 0 & 0 & 0 & 0 & 0 & 0 & 0 & 7\end{array}$

$\begin{array}{llllllllllllll}2.9018 & 0.1062 & 0.0000 & \mathrm{~S} & 0 & 0 & 0 & 0 & 0 & 0 & 0 & 0 & 0 & 8 \\ 1.8724 & -2.46000 & \mathrm{H} & 0 & 0 & 0 & 0 & 0 & 0 & 0 & 0 & 0 & 9\end{array}$

$\begin{array}{llllllllllllll}1.8724 & -2.4645 & -0.0002 & \mathrm{C} & 0 & 0 & 0 & 0 & 0 & 0 & 0 & 0 & 0 & 10\end{array}$

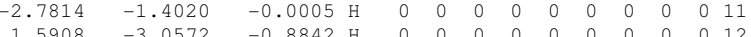

$\begin{array}{lllllllllllllll}1.5908 & -3.0572 & -0.8842 & \mathrm{H} & 0 & 0 & 0 & 0 & 0 & 0 & 0 & 0 & 0 & 12 \\ 2.9609 & -2.3248 & -0.0018 & \mathrm{H} & 0 & 0 & 0 & 0 & 0 & 0 & 0 & 0 & 0 & 13\end{array}$

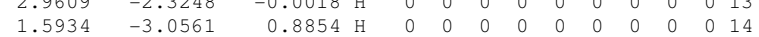

$\begin{array}{lllllll}2 & 1 & 0 & 0 & 0 & 0\end{array}$

$\begin{array}{lllll}1 & 0 & 0 & 0 & 0\end{array}$

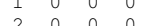

$\begin{array}{llllllll}3 & 0 & 0 & 0 & 0\end{array}$

$\begin{array}{lllll}3 & 0 & 0 & 0 & 0\end{array}$

$\begin{array}{lllllll}4 & 4 & 0 & 0 & 0 & 0\end{array}$

$\begin{array}{llllll}6 & 5 & 0 & 0 & 0\end{array}$

$\begin{array}{lllllll}1 & 8 & 0 & 0 & 0 & 0\end{array}$

$\begin{array}{llllll}2 & 10 & 0 & 0 & 0 & 0\end{array}$

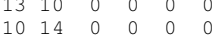

1 END

$\$ \$ \$$

methylthiouracilo.sdf

3D

$\begin{array}{lllllllllllll}15 & 15 & 0 & 0 & 0 & 0 & 0 & 0 & 0 & 0 & 0 & \mathrm{v} 2000\end{array}$

$\begin{array}{llllllllllllll}-0.9915 & -0.0143 & 0.0002 & \text { C } & 0 & 0 & 0 & 0 & 0 & 0 & 0 & 0 & 0 & 1\end{array}$

$\begin{array}{llllllllllllll}-0.2822 & 1.1540 & 0.0010 & \mathrm{~N} & 0 & 0 & 0 & 0 & 0 & 0 & 0 & 0 & 0 & 2\end{array}$

$\begin{array}{llllllllllllll}1.1275 & 1.2923 & 0.0001 & \mathrm{C} & 0 & 0 & 0 & 0 & 0 & 0 & 0 & 0 & 0 & 3\end{array}$

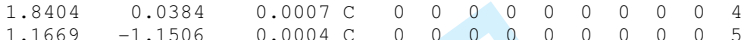

$\begin{array}{llllllllllllll}-0.2154 & -1.1440 & 0.0002 & \mathrm{~N} & 0 & 0 & 0 & 0 & 0 & 0 & 0 & 0 & 0 & 6\end{array}$

$\begin{array}{rrrrrrrrrrrrrr}1.6263 & 2.4258 & -0.0010 & 0 & 0 & 0 & 0 & 0 & 0 & 0 & 0 & 0 & 0 & 7 \\ 2.6818 & -0.0750 & -0.0003 & \mathrm{~S} & 0 & 0 & 0 & 0 & 0 & 0 & 0 & 0 & 0 & 8\end{array}$

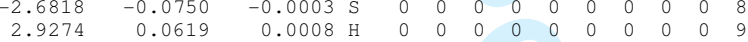

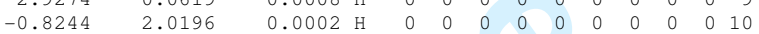

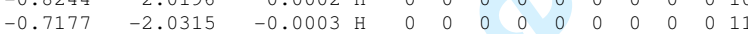

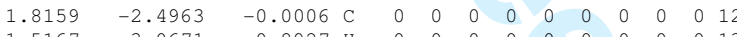

$\begin{array}{lllllllllll}\mathrm{H} & 0 & 0 & 0 & 0 & 0 & 0 & 0 & 0 & 0 & 13 \\ & 0 & 0 & 0 & 0 & 0 & 0 & 0 & 0 & 0 & 14\end{array}$

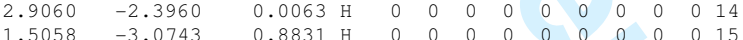

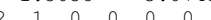

$\begin{array}{llllllll}2 & 1 & 0 & 0 & 0 & 0\end{array}$

$\begin{array}{llllll}1 & 1 & 0 & 0 & 0 & 0\end{array}$

$\begin{array}{lllll}2 & 0 & 0 & 0 & 0\end{array}$

$\begin{array}{lllll}3 & 0 & 0 & 0 & 0\end{array}$

$\begin{array}{lllll}4 & 0 & 0 & 0 & 0\end{array}$

$\begin{array}{lllll}4 & 0 & 0 & 0\end{array}$

$\begin{array}{lllll}5 & 0 & 0 & 0\end{array}$

$\begin{array}{rrrrrrr}11 & 6 & 0 & 0 & 0 & 0 \\ 3 & 12 & 0 & 0 & 0 & 0\end{array}$

$\begin{array}{lllllll}14 & 12 & 0 & 0 & 0 & 0 & 0\end{array}$

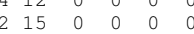

M END

methylthiouracill-anion.sdf

Cosmotherm 3D

$\begin{array}{llllllllllll}14 & 14 & 0 & 0 & 0 & 0 & 0 & 0 & 0 & 0 & 0 & \mathrm{~V} 2000\end{array}$

$\begin{array}{llllllllllllll}-0.9476 & 0.0740 & 0.0002 & \mathrm{C} & 0 & 0 & 0 & 0 & 0 & 0 & 0 & 0 & 0 & 1\end{array}$

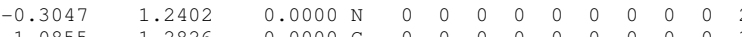

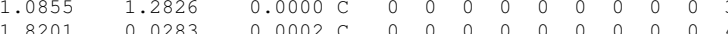

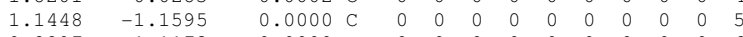

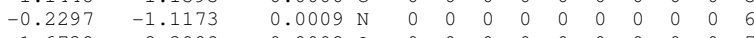

$\begin{array}{lllllllllllllll}1.6738 & 2.3982 & -0.0002 & 0 & 0 & 0 & 0 & 0 & 0 & 0 & 0 & 0 & 0 & 7\end{array}$

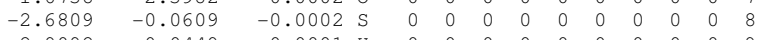

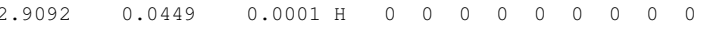

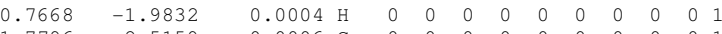

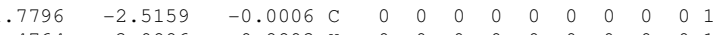

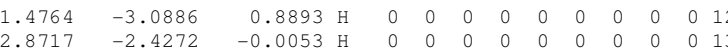

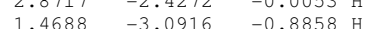

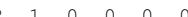

$\begin{array}{llllll}1 & 0 & 0 & 0 & 0\end{array}$

$\begin{array}{llllll}1 & 0 & 0 & 0 & 0\end{array}$

$\begin{array}{llllll}2 & 0 & 0 & 0 & 0\end{array}$

$\begin{array}{lllllll}3 & 0 & 0 & 0 & 0 \\ 3 & 0 & 0 & 0 & 0\end{array}$

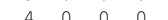

$1 \begin{array}{lllllll}1 & 5 & 0 & 0 & 0 & 0 & 1 \\ 10 & 6 & 0 & 0 & 0 & 0\end{array}$

$\begin{array}{llllllll}1 & 0 & 0 & 0 & 0\end{array}$

$\begin{array}{llllll}3 & 11 & 0 & 0 & 0 & 0\end{array}$

$\begin{array}{llllll}11 & 14 & 0 & 0 & 0 & 0\end{array}$

S\$S

methylthiouracill.sdf

$\begin{array}{llllllllllll}15 & 15 & 0 & 0 & 0 & 0 & 0 & 0 & 0 & 0 & 0 & \mathrm{~V} 2000\end{array}$

$\begin{array}{llllllllllllllll}-0.9747 & 0.0419 & -0.0001 & \mathrm{C} & 0 & 0 & 0 & 0 & 0 & 0 & 0 & 0 & 0 & 1\end{array}$

$\begin{array}{llllllllllllllllll}1.0337 & 1.1901 & -0.0003 & C & 0 & 0 & 0 & 0 & 0 & 0 & 0 & 0 & 0 & 3\end{array}$

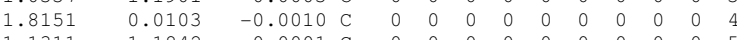

$\begin{array}{llllllllllll}1.1311-1.1842 & -0.0001 & 0 & 0 & 0 & 0 & 0 & 0 & 0 & 0 & 0\end{array}$

$\begin{array}{rrrrrrrrrrrrrr}0.2344 & -1.1349 & 0.0004 & \mathrm{~N} & 0 & 0 & 0 & 0 & 0 & 0 & 0 & 0 & 0 & 6 \\ 1.6839 & 2.3652 & 0.0005 & 0 & 0 & 0 & 0 & 0 & 0 & 0 & 0 & 0 & 0 & 7\end{array}$ 


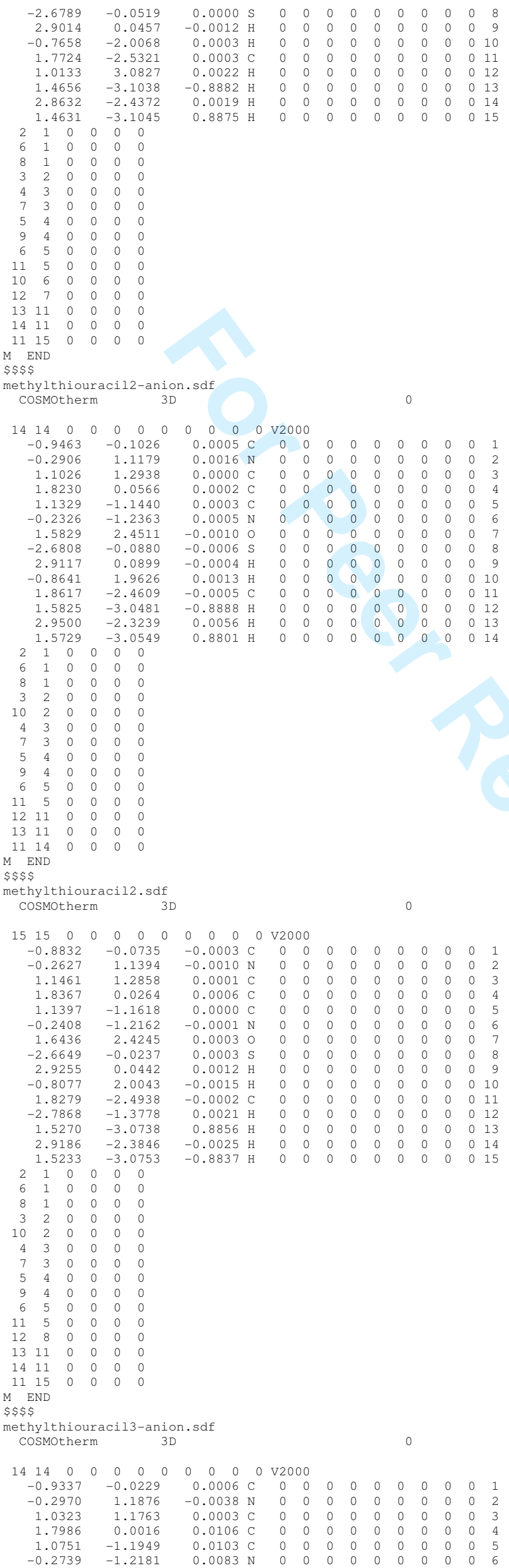


$\begin{array}{llllllllllllll}1.6019 & 2.4178 & -0.0049 & 0 & 0 & 0 & 0 & 0 & 0 & 0 & 0 & 0 & 0 & 7\end{array}$

$\begin{array}{rrrrrrrrrrrrrr}2.8888 & 0.0182 & -0.0014 & \mathrm{~S} & 0 & 0 & 0 & 0 & 0 & 0 & 0 & 0 & 0 & 8 \\ 1.7758 & -2.5269 & -0.0172 & \mathrm{H} & 0 & 0 & 0 & 0 & 0 & 0 & 0 & 0 & 0 & 9\end{array}$

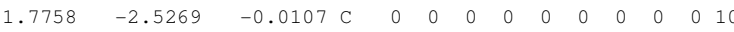

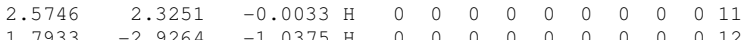

$\begin{array}{rrrrrrrrrrrrrr}1.7933 & -2.9264 & -1.0375 & \mathrm{H} & 0 & 0 & 0 & 0 & 0 & 0 & 0 & 0 & 0 & 12 \\ 2.8138 & -2.4442 & 0.3363 & \mathrm{H} & 0 & 0 & 0 & 0 & 0 & 0 & 0 & 0 & 0 & 13 \\ 1.2378 & -3.2528 & 0.6138 & \mathrm{H} & 0 & 0 & 0 & 0 & 0 & 0 & 0 & 0 & 0 & 14\end{array}$

$\begin{array}{llllll}2 & 1 & 0 & 0 & 0 & 0\end{array}$

$8 \begin{array}{llllll}1 & 1 & 0 & 0 & 0 \\ 8 & 1 & 0 & 0 & 0\end{array}$

$\begin{array}{llllll}3 & 2 & 0 & 0 & 0\end{array}$

$\begin{array}{lllllllll}4 & 3 & 0 & 0 & 0 & 0\end{array}$

$\begin{array}{llllll}7 & 0 & 0 & 0 & 0 \\ 5 & 4 & 0 & 0 & 0 & 0\end{array}$

$\begin{array}{llllll}5 & 4 & 0 & 0 & 0 & 0\end{array}$

$\begin{array}{llllll}6 & 5 & 0 & 0 & 0 & 0\end{array}$

$\begin{array}{llllll}1 & 7 & 0 & 0 & 0 & 0\end{array}$

$1210 \quad 00000$

$\begin{array}{llllll}13 & 10 & 0 & 0 & 0 & 0 \\ 10 & 14 & 0 & 0 & 0 & 0\end{array}$

$M$ END

\$\$

methylthiouracil3.sdf

cosmotherm

$\begin{array}{lllllllllllll}15 & 15 & 0 & 0 & 0 & 0 & 0 & 0 & 0 & 0 & 0 & \mathrm{v} 2000\end{array}$

$\begin{array}{llllllllllllll}-0.8636 & -0.0436 & 0.0005 & \text { C } & 0 & 0 & 0 & 0 & 0 & 0 & 0 & 0 & 0 & 1\end{array}$

$\begin{array}{llllllllllllll}-0.3057 & 1.1771 & -0.0026 & \mathrm{~N} & 0 & 0 & 0 & 0 & 0 & 0 & 0 & 0 & 0 & 2\end{array}$

$\begin{array}{llllllllllllll}1.0314 & 1.1961 & -0.0001 & \mathrm{C} & 0 & 0 & 0 & 0 & 0 & 0 & 0 & 0 & 0 & 3\end{array}$

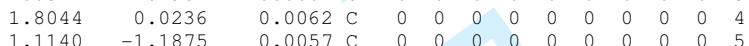

$\begin{array}{rrrrrrrrrrrrrr}1.1140 & -1.1875 & 0.0057 & \mathrm{C} & 0 & 0 & 0 & 0 & 0 & 0 & 0 & 0 & 0 & 5 \\ -0.2445 & -1.2274 & 0.0044 & \mathrm{~N} & 0 & 0 & 0 & 0 & 0 & 0 & 0 & 0 & 0 & 6\end{array}$

$\begin{array}{llllllllllllll}1.5698 & 2.4358 & -0.0025 & 0 & 0 & 0 & 0 & 0 & 0 & 0 & 0 & 0 & 0 & 7\end{array}$

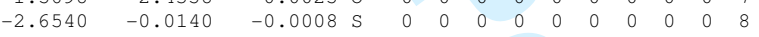

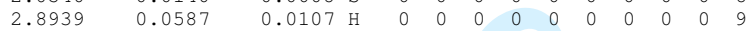

$\begin{array}{llllllllllllll}1.8253 & -2.5078 & -0.0059 & \mathrm{C} & 0 & 0 & 0 & 0 & 0 & 0 & 0 & 0 & 0 & 10\end{array}$

$\begin{array}{lllllllll}0 & 0 & 0 & 0 & 0 & 0 & 1\end{array}$

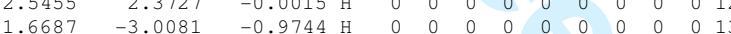

$\begin{array}{lllllllllllllllll}2.9032 & -2.3871 & 0.1542 & \mathrm{H} & 0 & 0 & 0 & 0 & 0 & 0 & 0 & 0 & 0 & 13\end{array}$

$\begin{array}{llllllllllllllll}1.4135 & -3.1685 & 0.7702 & \mathrm{H} & 0 & 0 & 0 & 0 & 0 & 0 & 0 & 0 & 0 & 15\end{array}$

2.1130000

$\begin{array}{llllllll}6 & 1 & 0 & 0 & 0 & 0\end{array}$

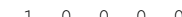

$\begin{array}{lllll}2 & 0 & 0 & 0 & 0 \\ 3 & 0 & 0 & 0 & 0\end{array}$

$\begin{array}{llllll}3 & 0 & 0 & 0 & 0\end{array}$

$\begin{array}{llllll}4 & 0 & 0 & 0 & 0\end{array}$

$\begin{array}{lllllll}5 & 5 & 0 & 0 & 0 & 0\end{array}$

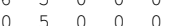

$\begin{array}{llllll}2 & 7 & 0 & 0 & 0\end{array}$

$\begin{array}{rrrrrrrr}1 & 8 & 0 & 0 & 0 & 0 \\ 1 & 10 & 0 & 0 & 0 & 0\end{array}$

$\begin{array}{lllllll}1 & 10 & 0 & 0 & 0 & -0 & 0\end{array}$

$10 \begin{array}{lllll}15 & 0 & 0 & 0 & 0\end{array}$

END

-pentanoicacid-anion.sdf

Cosmotherm 3D

$\begin{array}{llllllllllll}16 & 15 & 0 & 0 & 0 & 0 & 0 & 0 & 0 & 0 & 0 & \mathrm{~V} 2000\end{array}$

$\begin{array}{llllllllllllll}3.4621 & 0.3252 & 0.0088 & \mathrm{C} & 0 & 0 & 0 & 0 & 0 & 0 & 0 & 0 & 0 & 1\end{array}$

$\begin{array}{llllllllllllll}2.2171 & -0.5690 & -0.0087 & \mathrm{C} & 0 & 0 & 0 & 0 & 0 & 0 & 0 & 0 & 0 & 2\end{array}$

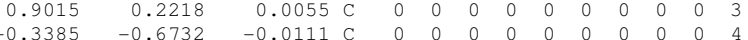

$\begin{array}{llllllllllllll}-1.7095 & 0.0430 & -0.0004 & \mathrm{C} & 0 & 0 & 0 & 0 & 0 & 0 & 0 & 0 & 0 & 5\end{array}$

$\begin{array}{llllllllllllll}-1.7358 & 1.3116 & -0.0065 & 0 & 0 & 0 & 0 & 0 & 0 & 0 & 0 & 0 & 0 & 6\end{array}$

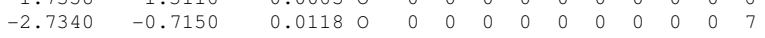

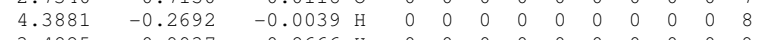

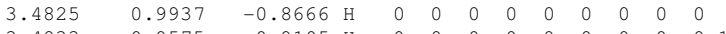

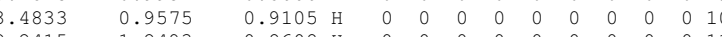

$\begin{array}{llllllllllllll}2.2415 & -1.2493 & 0.8608 & \mathrm{H} & 0 & 0 & 0 & 0 & 0 & 0 & 0 & 0 & 0 & 1\end{array}$

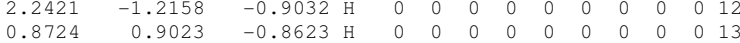

$\begin{array}{llllllllllllll}0.8724 & 0.9623 & -0.8623 \mathrm{H} & 0 & 0 & 0 & 0 & 0 & 0 & 0 & 0 & 0 & 13 \\ -0.8725 & 0.8696 & 0.8982 \mathrm{H} & 0 & 0 & 0 & 0 & 0 & 0 & 0 & 0 & 0 & 14\end{array}$

$\begin{array}{llllllllllllll}-0.3237 & -1.3597 & 0.8537 & \mathrm{H} & 0 & 0 & 0 & 0 & 0 & 0 & 0 & 0 & 0 & 15\end{array}$

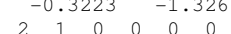

$\begin{array}{lllllll}2 & 1 & 0 & 0 & 0 & 0 \\ 8 & 1 & 0 & 0 & 0 & 0\end{array}$

$\begin{array}{llllllll}8 & 1 & 0 & 0 & 0 & 0\end{array}$

$\begin{array}{llllll}9 & 1 & 0 & 0 & 0 & 0 \\ 3 & 1 & 0 & 0 & 0 & 0\end{array}$

$\begin{array}{lllllll}3 & 2 & 0 & 0 & 0 & 0\end{array}$

$\begin{array}{llllllll}1 & 2 & 0 & 0 & 0 & 0 \\ 2 & 2 & 0 & 0 & 0 & 0\end{array}$

$\begin{array}{lllll}3 & 0 & 0 & 0 & 0\end{array}$

$\begin{array}{llllll}3 & 3 & 0 & 0 & 0\end{array}$

$\begin{array}{lllllll}4 & 3 & 0 & 0 & 0 & 0 \\ 5 & 4 & 0 & 0 & 0 & 0\end{array}$

$\begin{array}{llllll}5 & 4 & 0 & 0 & 0 & 0\end{array}$

$\begin{array}{llllll}4 & 0 & 0 & 0 & 0 \\ 5 & 0 & 0 & 0 & 0\end{array}$

$\begin{array}{lllll}5 & 0 & 0 & 0 & 0\end{array}$

4
END

$\$ \$ \$ \$$

-pentanoicacid.sdf

cosmotherm

$\begin{array}{llllllllllll}17 & 16 & 0 & 0 & 0 & 0 & 0 & 0 & 0 & 0 & 0 & \mathrm{~V} 2000\end{array}$

$\begin{array}{llllllllllllll}3.4908 & 0.3137 & 0.0067 & \mathrm{C} & 0 & 0 & 0 & 0 & 0 & 0 & 0 & 0 & 0 & 1\end{array}$

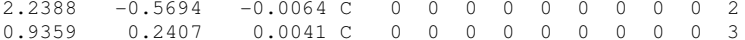

$\begin{array}{rrrrrrrrrrrrrr}0.9359 & 0.2407 & 0.0041 & C & 0 & 0 & 0 & 0 & 0 & 0 & 0 & 0 & 0 & 3 \\ -0.3026 & -0.6552 & -0.0086 & C & 0 & 0 & 0 & 0 & 0 & 0 & 0 & 0 & 0 & 4\end{array}$ 


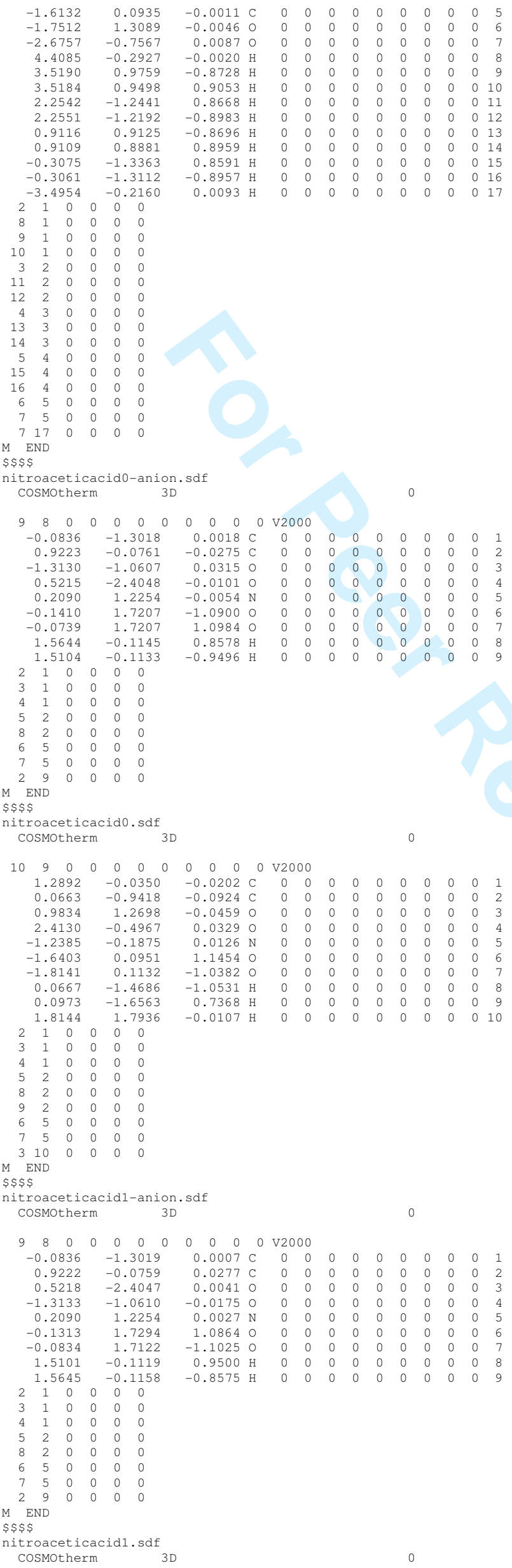




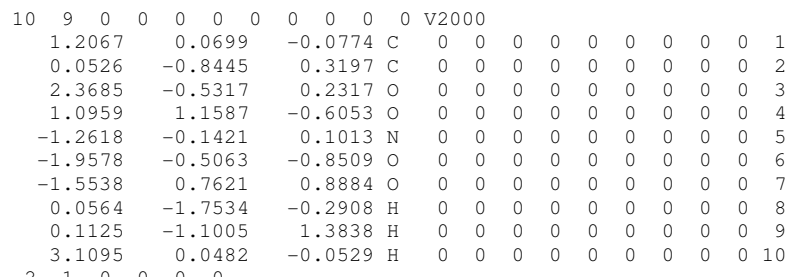




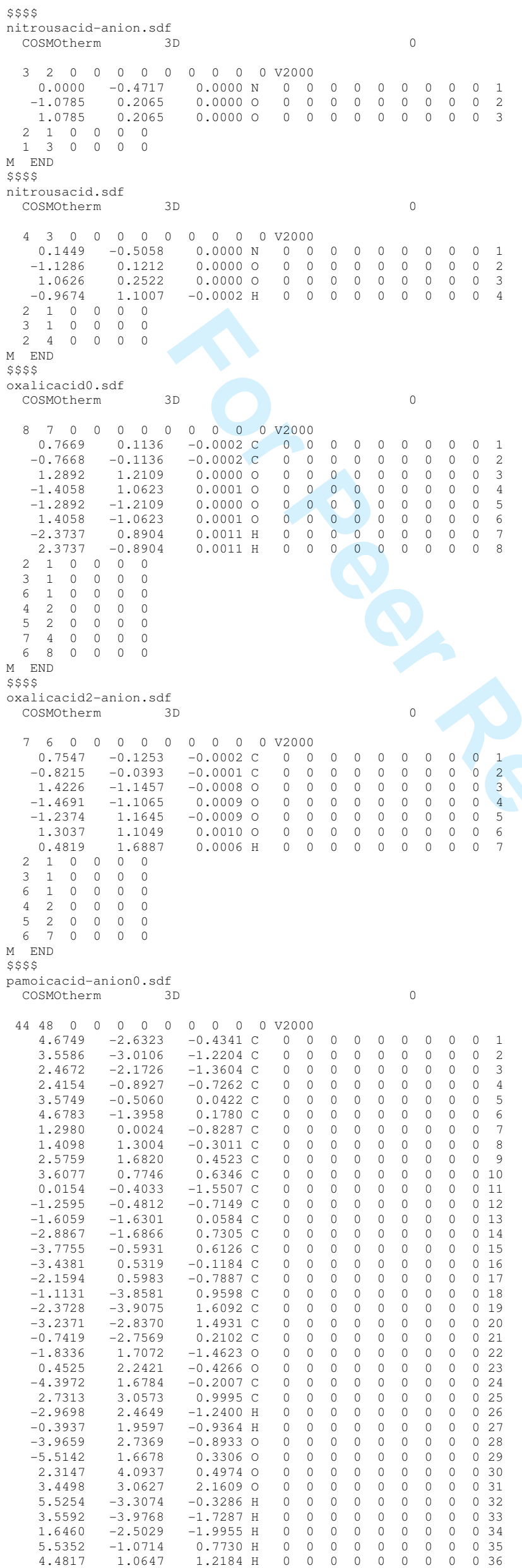


$\begin{array}{llllllllllllll}0.1649 & -1.3713 & -2.0423 & \mathrm{H} & 0 & 0 & 0 & 0 & 0 & 0 & 0 & 0 & 0 & 37\end{array}$

$\begin{array}{lllllllllllllll}-0.1737 & 0.3154 & -2.3641 & \mathrm{H} & 0 & 0 & 0 & 0 & 0 & 0 & 0 & 0 & 0 & 38\end{array}$

$\begin{array}{lllllllllllllll}-4.7459 & -0.6292 & 1.1128 & \mathrm{H} & 0 & 0 & 0 & 0 & 0 & 0 & 0 & 0 & 0 & 39\end{array}$

$\begin{array}{llllllllllllll}-0.4254 & -4.7009 & 1.0557 & \mathrm{H} & 0 & 0 & 0 & 0 & 0 & 0 & 0 & 0 & 0 & 40 \\ -2.6513 & -4.7839 & 2.1965 & \mathrm{H} & 0 & 0 & 0 & 0 & 0 & 0 & 0 & 0 & 0 & 41\end{array}$

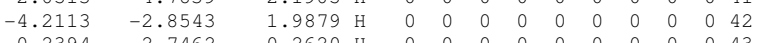

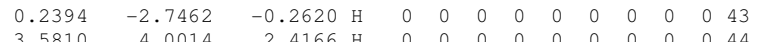

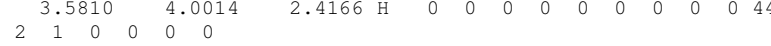




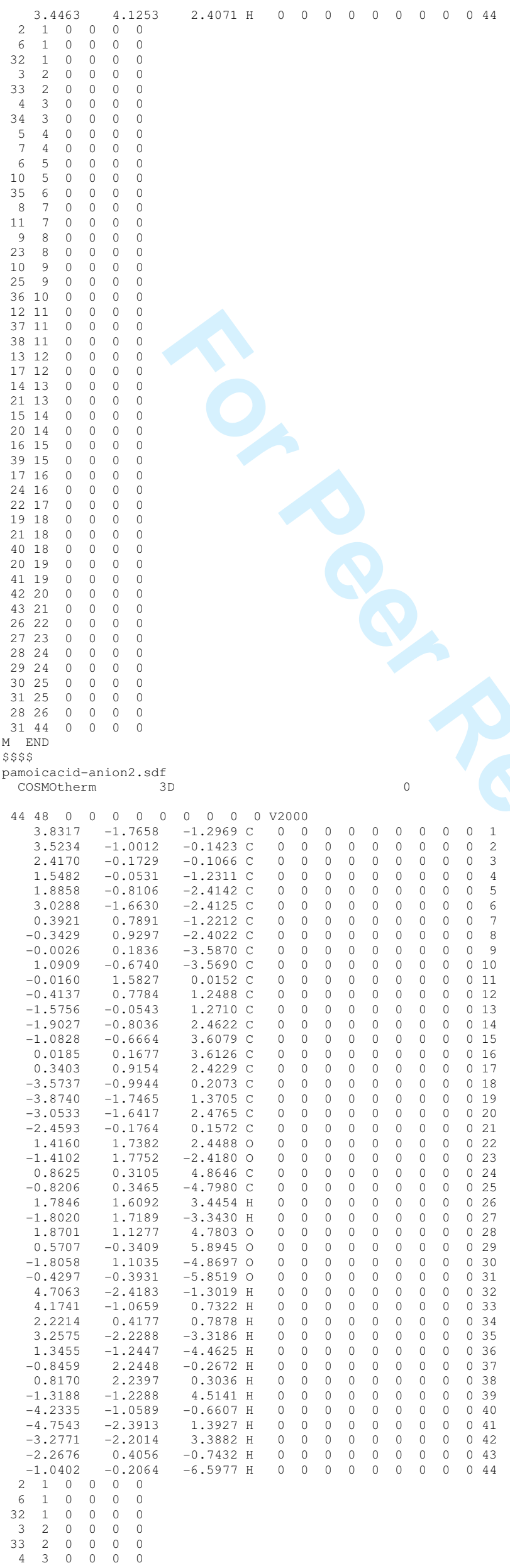

URL: http://mc.manuscriptcentral.com/tandf/tmph 


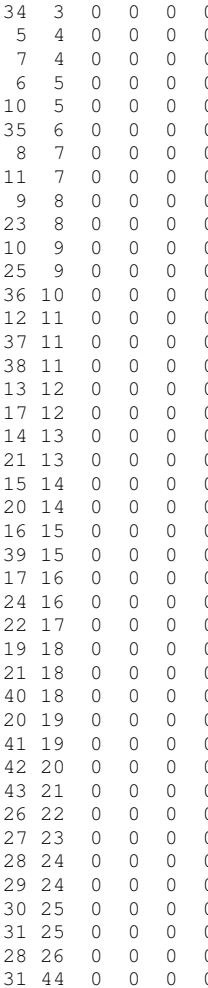

3144

$\$ \$ \$$

pamoicacid-anion3.sdf

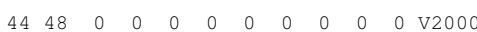

$\begin{array}{llllllllllllll}4.9618 & 1.1051 & -2.1600 & \mathrm{C} & 0 & 0 & 0 & 0 & 0 & 0 & 0 & 0 & 0\end{array}$

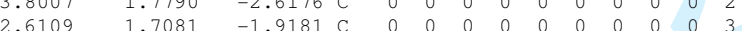

$\begin{array}{llllllllllllll}2.6109 & 1.7081 & -1.9181 & \text { C } & 0 & 0 & 0 & 0 & 0 & 0 & 0 & 0 & 0 & 3 \\ 2.4981 & 0.9599 & -0.7059 & C & 0 & 0 & 0 & 0 & 0 & 0 & 0 & 0 & 0 & 4\end{array}$

$\begin{array}{llllllllllllll}2.4981 & 0.9599 & -0.7059 & C & 0 & 0 & 0 & 0 & 0 & 0 & 0 & 0 & 0 & 3 \\ 3.6899 & 0.2773 & -0.2483 & C & 0 & 0 & 0 & 0 & 0 & 0 & 0 & 0 & 0 & 5\end{array}$

$\begin{array}{llllllllllllll}4.9005 & 0.3702 & -0.9959 & \mathrm{C} & 0 & 0 & 0 & 0 & 0 & 0 & 0 & 0 & 0 & 6\end{array}$

$\begin{array}{lllllllllllll}1.2832 & 0.8562 & 0.0445 & \mathrm{C} & 0 & 0 & 0 & 0 & 0 & 0 & 0 & 0 & 0\end{array}$

$\begin{array}{lllllllllllllll}1.2957 & 0.1437 & 1.2490 & \mathrm{C} & 0 & 0 & 0 & 0 & 0 & 0 & 0 & 0 & 0 & 8 \\ 2.4778 & -0.5458 & 1.6973 & c & 0 & 0 & 0 & 0 & 0 & 0 & 0 & 0 & 0 & 9\end{array}$

$\begin{array}{llllllllllllll}2.4778 & -0.5458 & 1.6973 & c & 0 & 0 & 0 & 0 & 0 & 0 & 0 & 0 & 0 & 9 \\ 3.6444 & -0.4676 & 0.9458 & c & 0 & 0 & 0 & 0 & 0 & 0 & 0 & 0 & 0 & 10\end{array}$

$\begin{array}{lllllllllllll}1.5595 & -0.3772 \mathrm{C} & 0 & 0 & 0 & 0 & 0 & 0 & 0 & 0 & 0 & 10 \\ 0.7051 & -0.5046 & \mathrm{c} & 0 & 0 & 0 & 0 & 0 & 0 & 0 & 0 & 0 & 11\end{array}$

$\begin{array}{llllllllllllll}-1.3446 & -0.3854 & -1.4209 & \text { C } & 0 & 0 & 0 & 0 & 0 & 0 & 0 & 0 & 0 & 13\end{array}$

$\begin{array}{llllllllllllllllll}-2.5650 & -1.1556 & -1.5059 & \mathrm{C} & 0 & 0 & 0 & 0 & 0 & 0 & 0 & 0 & 0 & 14\end{array}$

$\begin{array}{llllllllllllll}-3.6614 & -0.7954 & -0.6869 & \mathrm{C} & 0 & 0 & 0 & 0 & 0 & 0 & 0 & 0 & 0 & 15\end{array}$

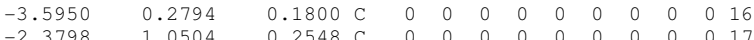

$\begin{array}{rrrrrrrrrrrrrr}-2.3798 & 1.0504 & 0.2548 & \mathrm{C} & 0 & 0 & 0 & 0 & 0 & 0 & 0 & 0 & 0 & 17 \\ -0.3948 & -1.8195 & -3.1709 & \mathrm{C} & 0 & 0 & 0 & 0 & 0 & 0 & 0 & 0 & 0 & 18\end{array}$

$\begin{array}{llllllllllllll}-1.5921 & -2.5764 & -3.2398 & C & 0 & 0 & 0 & 0 & 0 & 0 & 0 & 0 & 0 & 19\end{array}$

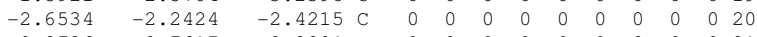

$\begin{array}{llllllllllllll}-0.2736 & -0.7617 & -2.2881 & \mathrm{C} & 0 & 0 & 0 & 0 & 0 & 0 & 0 & 0 & 0 & 21\end{array}$

$\begin{array}{llllllllllllll}-2.3396 & 2.1171 & 1.0856 & 0 & 0 & 0 & 0 & 0 & 0 & 0 & 0 & 0 & 0 & 22\end{array}$

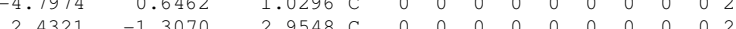

$-3.33071=-1.30707$

$-0.3706-2.1407$

$\begin{array}{llllllllllllll}-4.8440 & -0.0417 & 0.9621 & 0 & 0 & 0 & 0 & 0 & 0 & 0 & 0 & 0 & 0 & 29\end{array}$

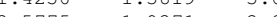

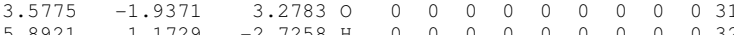

$\begin{array}{lllllllllllll}3.8462 & 2.3647 & -3.5377 \mathrm{H} & 0 & 0 & 0 & 0 & 0 & 0 & 0 & 0 & 0 & 33\end{array}$

$\begin{array}{lllllllllllll}1.7488 & 2.2484 & -2.3078 \mathrm{H} & 0 & 0 & 0 & 0 & 0 & 0 & 0 & 0 & 0 & 34\end{array}$

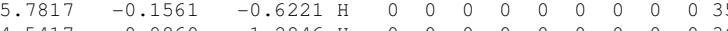

$\begin{array}{llllllllllllll}4.5417 & -0.9860 & 1.2846 & \mathrm{H} & 0 & 0 & 0 & 0 & 0 & 0 & 0 & 0 & 0 & 36\end{array}$

$\begin{array}{lllllllllllll}0.1664 & 2.0827 & -1.3287 \mathrm{H} & 0 & 0 & 0 & 0 & 0 & 0 & 0 & 0 & 0 & 37\end{array}$

$\begin{array}{rrrrrrrrrrrrrr}-0.2195 & 2.3430 & 0.3625 & \mathrm{H} & 0 & 0 & 0 & 0 & 0 & 0 & 0 & 0 & 0 & 38\end{array}$

$\begin{array}{llllllllllllll}-4.5886 & -1.3705 & -0.7371 \text { H } & 0 & 0 & 0 & 0 & 0 & 0 & 0 & 0 & 0 & 39\end{array}$

$\begin{array}{llllllllllllll}0.4428 & -2.0756 & -3.8233 & \text { H } & 0 & 0 & 0 & 0 & 0 & 0 & 0 & 0 & 0 & 40\end{array}$

$\begin{array}{llllllllllllll}-1.6728 & -3.4098 & -3.9399 & \mathrm{H} & 0 & 0 & 0 & 0 & 0 & 0 & 0 & 0 & 0 & 41\end{array}$

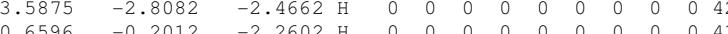

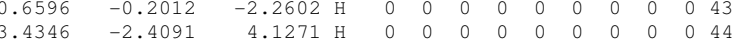

$\begin{array}{llllll}2 & 1 & 0 & 0 & 0 & 0\end{array}$

$\begin{array}{lllllll}6 & 1 & 0 & 0 & 0 & 0\end{array}$

$\begin{array}{llllll}2 & 1 & 0 & 0 & 0 & 0 \\ 3 & 2 & 0 & 0 & 0 & 0\end{array}$

$\begin{array}{llll}3 & 2 & 0 \\ 3 & 2 & 0 \\ 3 & 3 & -10 & -1 \\ 4 & 3 & 0\end{array}$

$\begin{array}{lllllll}4 & 3 & 0 & 0 & 0 & 0\end{array}$

$\begin{array}{llllll}4 & 3 & 0 & 0 & 0 & 0\end{array}$

$\begin{array}{lllllll}2 & 4 & 0 & 0 & 0 & 0\end{array}$

$\begin{array}{lllll}5 & 0 & 0 & 0 & 0\end{array}$

$35 \quad 6 \quad 600000$

$8 \begin{array}{llllll}8 & 7 & 0 & 0 & 0 & 0\end{array}$ 


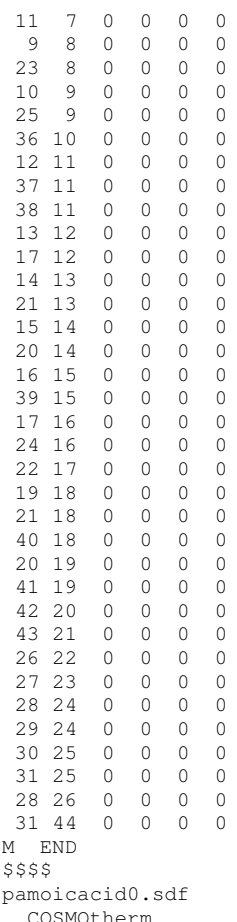

COSMOtherm

$\begin{array}{llllllllllll}45 & 48 & 0 & 0 & 0 & 0 & 0 & 0 & 0 & 0 & 0 & \mathrm{v} 2000\end{array}$

$\begin{array}{llllllllllllll}4.9793 & -2.1643 & 1.0661 & \mathrm{C} & 0 & 0 & 0 & 0 & 0 & 0 & 0 & 0 & 0 & 1\end{array}$

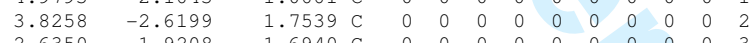

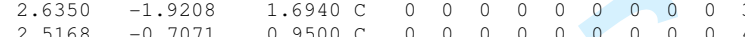

$\begin{array}{lllllllllllllll}2.5168 & -0.7071 & 0.9500 & \mathrm{C} & 0 & 0 & 0 & 0 & 0 & 0 & 0 & 0 & 0 & 4 \\ 3.7019 & -0.2486 & 0.2571 & \mathrm{C} & 0 & 0 & 0 & 0 & 0 & 0 & 0 & 0 & 0 & 5\end{array}$

$\begin{array}{lllllllllllllll}4.9132 & -0.9961 & 0.3378 & \mathrm{C} & 0 & 0 & 0 & 0 & 0 & 0 & 0 & 0 & 0 & 6\end{array}$

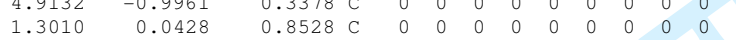

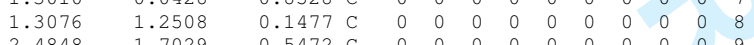

$\begin{array}{rrrrrrrrrrrrrr}2.4848 & 1.7029 & -0.5472 & \mathrm{C} & 0 & 0 & 0 & 0 & 0 & 0 & 0 & 0 & 0 & 9 \\ 3.6509 & 0.9501 & -0.4810 & \mathrm{C} & 0 & 0 & 0 & 0 & 0 & 0 & 0 & 0 & 0 & 10\end{array}$

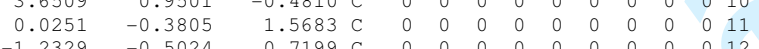

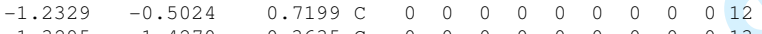

$\begin{array}{llllllllllllll}-1.3295 & -1.4270 & -0.3635 & \mathrm{C} & 0 & 0 & 0 & 0 & 0 & 0 & 0 & 0 & 0 & 13 \\ -2.5461 & -1.5111 & -1.1396 & \mathrm{C} & 0 & 0 & 0 & 0 & 0 & 0 & 0 & 0 & 0 & 14\end{array}$

$\begin{array}{lllllllllllllll}-3.6404 & -0.6959 & -0.7908 & \mathrm{C} & 0 & 0 & 0 & 0 & 0 & 0 & 0 & 0 & 0 & 15\end{array}$

$\begin{array}{llllllllllllll}-3.5678 & 0.1783 & 0.2879 & \mathrm{C} & 0 & 0 & 0 & 0 & 0 & 0 & 0 & 0 & 0 & 16 \\ -2.3516 & 0.2656 & 1.0558 & \mathrm{C} & 0 & 0 & 0 & 0 & 0 & 0 & 0 & 0 & 0 & 17\end{array}$

$\begin{array}{llllllllllllll}-0.3832 & -3.1831 & -1.7852 & \mathrm{C} & 0 & 0 & 0 & 0 & 0 & 0 & 0 & 0 & 0 & 18\end{array}$

$\begin{array}{llllllllllllll}-1.5769 & -3.2501 & -2.5499 & \mathrm{C} & 0 & 0 & 0 & 0 & 0 & 0 & 0 & 0 & 0 & 19\end{array}$

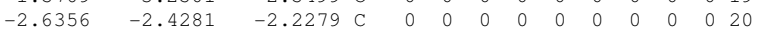

$\begin{array}{llllllllllllll}-0.2617 & -2.3006 & -0.7288 & \mathrm{C} & 0 & 0 & 0 & 0 & 0 & 0 & 0 & 0 & 0 & 21\end{array}$

$\begin{array}{llllllllllllll}-2.2806 & 1.1039 & 2.1213 & 0 & 0 & 0 & 0 & 0 & 0 & 0 & 0 & 0 & 0 & 22\end{array}$

$\begin{array}{llllllllllllllll}0.1738 & 1.9923 & 0.1290 & 0 & 0 & 0 & 0 & 0 & 0 & 0 & 0 & 0 & 0 & 23\end{array}$

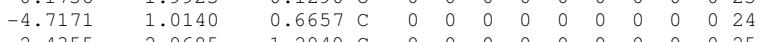

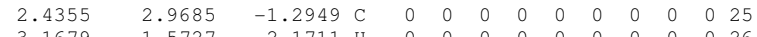

$\begin{array}{rrrrrrrrrrrrrr}-3.1679 & 1.5727 & 2.1711 & \mathrm{H} & 0 & 0 & 0 & 0 & 0 & 0 & 0 & 0 & 0 & 26 \\ 0.3728 & 2.7986 & -0.4358 & \mathrm{H} & 0 & 0 & 0 & 0 & 0 & 0 & 0 & 0 & 0 & 27\end{array}$

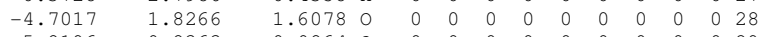

$\begin{array}{llllllllllllll}-5.8196 & 0.8363 & -0.0864 & 0 & 0 & 0 & 0 & 0 & 0 & 0 & 0 & 0 & 0 & 29\end{array}$

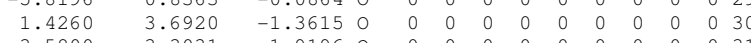

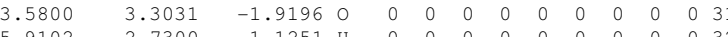

$\begin{array}{llllllllllllllll}3.9102 & -2.7300 & 1.1251 \mathrm{H} & 0 & 0 & 0 & 0 & 0 & 0 & 0 & 0 & 0 & 31 \\ 3.8759 & -3.5422 & 2.3357 & \mathrm{H} & 0 & 0 & 0 & 0 & 0 & 0 & 0 & 0 & 0 & 33\end{array}$

$\begin{array}{llllllllllllll}3.8759 & -3.5422 & 2.3357 \mathrm{H} & 0 & 0 & 0 & 0 & 0 & 0 & 0 & 0 & 0 & 33 \\ 1.7796 & -2.3090 & 2.2461 & 0 & 0 & 0 & 0 & 0 & 0 & 0 & 0 & 0 & 34\end{array}$

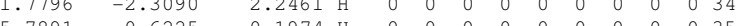

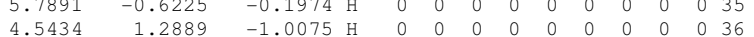

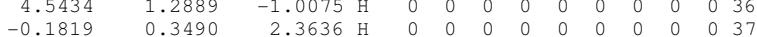

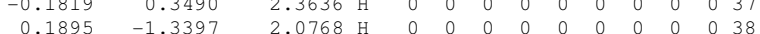

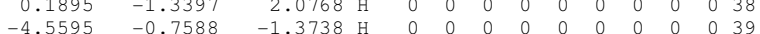

$\begin{array}{lllllllllllllll}0.4514 & -3.8414 & -2.0348 \mathrm{H} & 0 & 0 & 0 & 0 & 0 & 0 & 0 & 0 & 0 & 40\end{array}$

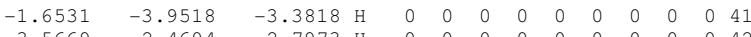

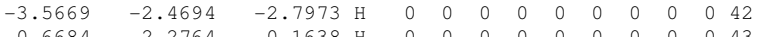

$\begin{array}{llllllllllllll}0.6684 & -2.2764 & -0.1638 & \mathrm{H} & 0 & 0 & 0 & 0 & 0 & 0 & 0 & 0 & 0 & 43\end{array}$

$\begin{array}{rrrrrrrrrrrrrr}-6.5154 & 1.4436 & 0.2464 & \mathrm{H} & 0 & 0 & 0 & 0 & 0 & 0 & 0 & 0 & 0 & 44 \\ 3.4360 & 4.1572 & -2.3817 & \mathrm{H} & 0 & 0 & 0 & 0 & 0 & 0 & 0 & 0 & 0 & 45\end{array}$

$\begin{array}{rrrrr}1 & 0 & 0 & 0 & 0\end{array}$

$\begin{array}{llllll}6 & 1 & 0 & 0 & 0\end{array}$

$\begin{array}{lllllll}32 & 1 & 0 & 0 & 0 & 0 \\ 3 & 2 & 0 & 0 & 0 & 0\end{array}$

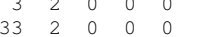

$\begin{array}{rrrrrr}4 & 3 & 0 & 0 & 0 \\ 34 & 3 & 0 & 0 & 0\end{array}$

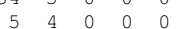

$\begin{array}{llllll}7 & 4 & 0 & 0 & 0 & 0 \\ 6 & 5 & 0 & 0 & 0 & 0\end{array}$

$\begin{array}{lllllll}10 & 5 & 0 & 0 & 0 & 0\end{array}$

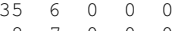

$\begin{array}{rrrrrr}8 & 7 & 0 & 0 & 0 & 0 \\ 11 & 7 & 0 & 0 & 0 & 0\end{array}$

$\begin{array}{llllll}9 & 8 & 0 & 0 & 0 & 0\end{array}$

$\begin{array}{rrrrrr}23 & 8 & 0 & 0 & 0 \\ 10 & 9 & 0 & 0 & 0 & 0\end{array}$

$\begin{array}{lllllll}25 & 9 & 0 & 0 & 0 & 0\end{array}$

$36 \begin{array}{llllll}36 & 10 & 0 & 0 & 0\end{array}$ 
$\begin{array}{llllll}12 & 11 & 0 & 0 & 0 & 0\end{array}$

$\begin{array}{llllll}37 & 11 & 0 & 0 & 0 & 0\end{array}$

$\begin{array}{llllll}38 & 11 & 0 & 0 & 0 & 0 \\ 13 & 12 & 0 & 0 & 0 & 0\end{array}$

$\begin{array}{lllllll}13 & 12 & 0 & 0 & 0 & 0 \\ 17 & 12 & 0 & 0 & 0 & 0\end{array}$

$\begin{array}{llllll}14 & 13 & 0 & 0 & 0 & 0\end{array}$

$\begin{array}{llllll}21 & 13 & 0 & 0 & 0 & 0 \\ 15 & 14 & 0 & 0 & 0 & 0\end{array}$

$20 \begin{array}{lllll}14 & 0 & 0 & 0\end{array}$

$\begin{array}{llllll}16 & 15 & 0 & 0 & 0 & 0 \\ 39 & 15 & 0 & 0 & 0 & 0\end{array}$

1716 16 000

$\begin{array}{llllll}24 & 16 & 0 & 0 & 0\end{array}$

$\begin{array}{lllllll}22 & 17 & 0 & 0 & 0 & 0\end{array}$

$\begin{array}{llllll}19 & 18 & 0 & 0 & 0\end{array}$

$\begin{array}{lllllll}40 & 18 & 0 & 0 & 0 & 0\end{array}$

$\begin{array}{lllllll}20 & 19 & 0 & 0 & 0 & 0\end{array}$

$\begin{array}{llllll}41 & 19 & 0 & 0 & 0 & 0\end{array}$

$\begin{array}{lllll}42 & 20 & 0 & 0 & 0\end{array}$

$\begin{array}{llllll}43 & 21 & 0 & 0 & 0 \\ 26 & 22 & 0 & 0 & 0\end{array}$

$\begin{array}{llllll}27 & 23 & 0 & 0 & 0 & 0\end{array}$

$\begin{array}{lllll}28 & 24 & 0 & 0 & 0 \\ 29 & 24 & 0 & 0 & 0\end{array}$

$\begin{array}{llllll}30 & 25 & 0 & 0 & 0\end{array}$

$\begin{array}{llllll}31 & 25 & 0 & 0 & 0 \\ 44 & 29 & 0 & 0 & 0\end{array}$

$\begin{array}{lllllll}31 & 45 & 0 & 0 & 0 & 0\end{array}$

$M \quad$ END

pamoicacidl.sdf

Cosmotherm

$\begin{array}{llllllllllll}45 & 48 & 0 & 0 & 0 & 0 & 0 & 0 & 0 & 0 & 0 & \text { V2 } 2000\end{array}$

$\begin{array}{lllllllllllll}5.0150 & -2.1073 & 1.1004 & \mathrm{C} & 0 & 0 & 0 & 0 & 0 & 0 & 0 & 0 & 0\end{array}$

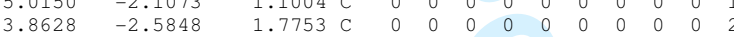

$\begin{array}{llllllllllllll}2.6605 & -1.9065 & 1.7748 \mathrm{C} & 0 & 0 & 0 & 0 & 0 & 0 & 0 & 0 & 0\end{array}$

$\begin{array}{lllllllllllllllllllll}2.5261 & -0.6981 & 0.9547 \mathrm{C} & 0 & 0 & 0 & 0 & 0 & 0 & 0 & 0 & 0 & 4\end{array}$

$4.9326-0.9452$

$1.2881 \quad 1.2348$

$3.6414 \quad 0.9731$

$0.0272-0.4131$

$-1.2344-0.5233$

$-2.5389-1.5117$

$-3.6317-0.7038$

$\begin{array}{ll}-3.5708 & 0.1609 \\ -2.3551 & 0.2378\end{array}$

$\begin{array}{lr}-2.3551 & 0.2378 \\ -0.3683 & -3.1622\end{array}$

$-1.5639-3.2261$

$-2.6274-2.4157$

$-0.2488-2.2919$

$-2.2451-1.0475$

$0.1443 \quad 1.9602$

$2.3933-2.9598$

$-3.0944 \quad 1.5367$

$\begin{array}{lllllllllllllll}-5.8461 & 0.8740 & -0.4615 & \mathrm{H} & 0 & 0 & 0 & 0 & 0 & 0 & 0 & 0 & 0 & 27\end{array}$

$\begin{array}{lllllllllllllll}-4.6703 & 1.7904 & 1.6490 & 0 & 0 & 0 & 0 & 0 & 0 & 0 & 0 & 0 & 0 & 29\end{array}$

$\begin{array}{llllllllllllll}1.3718 & 3.6652 & -1.3940 & 0 & 0 & 0 & 0 & 0 & 0 & 0 & 0 & 0 & 0 & 30\end{array}$

$\begin{array}{llllllllllllll}3.5336 & 3.3066 & -1.9418 & 0 & 0 & 0 & 0 & 0 & 0 & 0 & 0 & 0 & 0 & 31\end{array}$

$\begin{array}{llllllllllllll}5.9552 & -2.6564 & 1.1681 & \mathrm{H} & 0 & 0 & 0 & 0 & 0 & 0 & 0 & 0 & 0 & 32 \\ 3.9250 & -3.5029 & 2.3625 & \mathrm{H} & 0 & 0 & 0 & 0 & 0 & 0 & 0 & 0 & 0 & 33\end{array}$

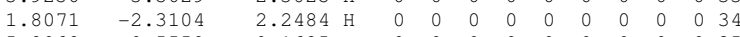

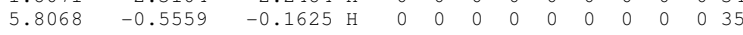

$\begin{array}{lllllllllllll}4.5327 & 1.3273 & -0.9934 \mathrm{H} & 0 & 0 & 0 & 0 & 0 & 0 & 0 & 0 & 0 & 36\end{array}$

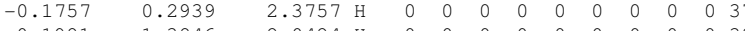

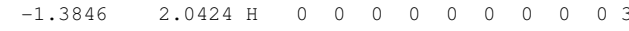

$\begin{array}{llllllllllll}-0.7533 & -1.3665 \mathrm{H} & 0 & 0 & 0 & 0 & 0 & 0 & 0 & 0 & 0 & 39\end{array}$

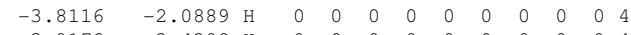

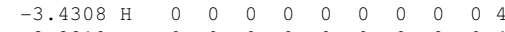

$\begin{array}{llllllllllllll}-0.5539 & -2.4554 & -2.8219 & \mathrm{H} & 0 & 0 & 0 & 0 & 0 & 0 & 0 & 0 & 0 & 42\end{array}$

$\begin{array}{rrrrrrrrrrrrrr}0.6831 & -2.2687 & -0.1991 & \mathrm{H} & 0 & 0 & 0 & 0 & 0 & 0 & 0 & 0 & 0 & 43 \\ -5.5163 & 2.2662 & 1.7943 & \mathrm{H} & 0 & 0 & 0 & 0 & 0 & 0 & 0 & 0 & 0 & 44 \\ 3.3760 & 4.1531 & -2.4133 & \mathrm{H} & 0 & 0 & 0 & 0 & 0 & 0 & 0 & 0 & 0 & 45\end{array}$

$\begin{array}{ll}-3.5760 & 4.2662 \\ 3 & 4.1531\end{array}$

$\begin{array}{llllll}2 & 1 & 0 & 0 & 0 & 0\end{array}$

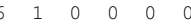

$\begin{array}{llllllll}3 & 2 & 0 & 0 & 0 & 0\end{array}$

$\begin{array}{llllll}3 & 2 & 0 & 0 & 0\end{array}$

$\begin{array}{lllllll}3 & 2 & 0 & 0 & 0 & 0\end{array}$

$\begin{array}{lllll}4 & 3 & 0 & 0 & 0\end{array}$

$\begin{array}{lllll}4 & 0 & 0 & 0 & 0\end{array}$

$\begin{array}{lllll}4 & 0 & 0 & 0 & 0\end{array}$

$\begin{array}{llllll}1 & 5 & 0 & 0 & 0 \\ 3 & 5 & 0 & 0 & 0\end{array}$

$\begin{array}{llllll}35 & 6 & 0 & 0 & 0 & 0\end{array}$

$\begin{array}{llllll}1 & 7 & 0 & 0 & 0 & 0\end{array}$

$\begin{array}{llllll}9 & 8 & 0 & 0 & 0 & 0\end{array}$

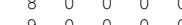

$\begin{array}{llllll}25 & 9 & 0 & 0 & 0 & 0\end{array}$

$\begin{array}{llllll}36 & 10 & 0 & 0 & 0\end{array}$

$\begin{array}{lllllll}12 & 11 & 0 & 0 & 0 & 0\end{array}$

$\begin{array}{llllll}38 & 11 & 0 & 0 & 0 & 0\end{array}$

$\begin{array}{llllll}13 & 12 & 0 & 0 & 0 & 0\end{array}$ 


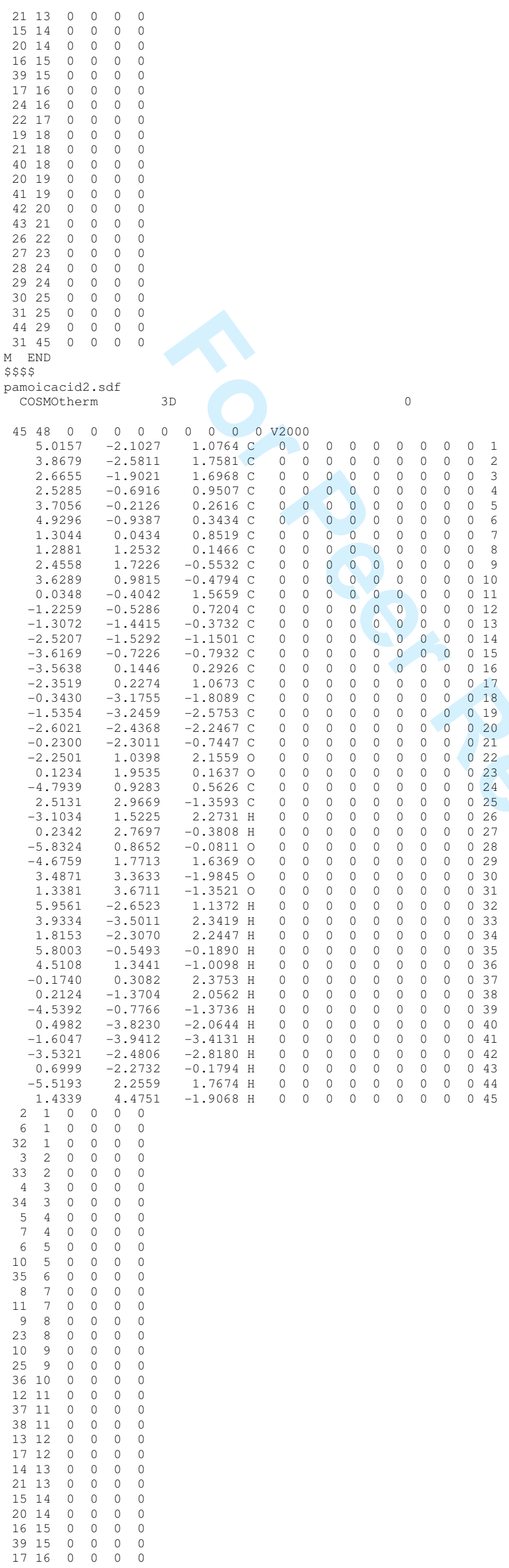




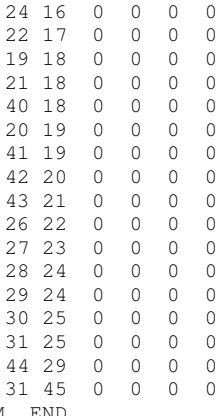

pamoicacid3.sdf

$$
\text { cosmotherm 3D }
$$

$\begin{array}{llllllllllll}45 & 48 & 0 & 0 & 0 & 0 & 0 & 0 & 0 & 0 & 0 & \mathrm{~V} 2000\end{array}$

\begin{tabular}{|c|c|c|c|c|c|c|c|c|c|c|c|c|}
\hline 4.7874 & 0.5722 & -2.5413 & & 0 & 0 & 0 & 0 & 0 & 0 & 0 & 0 & 0 \\
\hline 3.6428 & -1.3041 & -2.9453 & C & 0 & 0 & 0 & 0 & 0 & 0 & 0 & 0 & 0 \\
\hline 2.5259 & -1.3882 & -2.1349 & $\mathrm{C}$ & 0 & 0 & 0 & 0 & 0 & 0 & 0 & 0 & 0 \\
\hline 2.4801 & -0.7485 & -0.8597 & & 0 & 0 & 0 & 0 & 0 & 0 & 0 & 0 & 0 \\
\hline 3.6631 & 0.0292 & -0.4456 & & 0 & 0 & 0 & 0 & 0 & 0 & 0 & 0 & 0 \\
\hline 4.7938 & 0.0474 & -1.3101 & & 0 & 0 & 0 & 0 & 0 & 0 & 0 & 0 & 0 \\
\hline 1.3447 & -0.7984 & 0.0134 & & 0 & & 0 & 0 & 0 & 0 & 0 & 0 & 0 \\
\hline 1.4573 & -0.2551 & 1.2942 & & 0 & 0 & 0 & 0 & 0 & 0 & 0 & 0 & 0 \\
\hline 2.6286 & 0.46 & 1.7120 & & 0 & & 0 & 0 & 0 & 0 & 0 & 0 & 0 \\
\hline 3.6953 & 0.5685 & 0.8290 & & 0 & & 0 & 0 & 0 & 0 & 0 & 0 & 0 \\
\hline 0.0459 & -1.5009 & -0.3706 & & 0 & 0 & 0 & 0 & 0 & 0 & 0 & 0 & 0 \\
\hline-1.2319 & -0.6638 & -0.4358 & 0 & 0 & & 0 & 0 & 0 & 0 & 0 & 0 & 0 \\
\hline 1.510 & & -1.5715 & & 0 & & 0 & 0 & 0 & 0 & 0 & 0 & \\
\hline-2.8118 & 0.7674 & -1.7167 & c & 0 & & 0 & 0 & 0 & 0 & 0 & 0 & 0 \\
\hline-3.78 & & -0.7208 & & & & 0 & 0 & 0 & 0 & & & \\
\hline-3.5 & -0.20 & 0.4136 & $\mathrm{C}$ & & & 0 & 0 & 0 & 0 & 0 & 0 & 0 \\
\hline-2.2 & -0.8 & 0.5554 & C & & & 0 & 0 & 0 & 0 & 0 & 0 & 0 \\
\hline-0.8 & 1 . & -3.6994 & $\mathrm{C}$ & & & 0 & 0 & 0 & 0 & 0 & 0 & 0 \\
\hline-2.1 & 1 & -3.8335 & $\mathrm{C}$ & & & 0 & 0 & 0 & 0 & 0 & 0 & 0 \\
\hline-3.1 & 1.57 & -2.8553 & $\mathrm{C}$ & & & 0 & 0 & 0 & 0 & 0 & 0 & 0 \\
\hline-0.5 & 0.4110 & -2.6038 & $\mathrm{C}$ & & & 0 & 0 & 0 & 0 & 0 & 0 & 0 \\
\hline-2.0 & -1.58 & 1.6590 & 0 & & & 0 & 0 & 0 & 0 & 0 & 0 & 0 \\
\hline 0.3 & -0.4414 & 2.1371 & 0 & & & 0 & 0 & 0 & 0 & 0 & 0 & 0 \\
\hline-4.5966 & -0.30 & 1.4248 & c & & & 0 & 0 & 0 & 0 & 0 & 0 & 0 \\
\hline 2.796 & & 3.0399 & $\mathrm{C}$ & & & 0 & 0 & 0 & 0 & 0 & 0 & 0 \\
\hline-1.0557 & -1.45 & 1.9249 & $\mathrm{H}$ & & & 0 & 0 & 0 & 0 & 0 & 0 & 0 \\
\hline 0.5820 & -0.0 & 3.0078 & $\mathrm{H}$ & & & 0 & 0 & 0 & 0 & 0 & 0 & 0 \\
\hline-5.7886 & -0.31 & 1.1279 & 0 & & & 0 & 0 & 0 & 0 & 0 & 0 & 0 \\
\hline-4.1 & -0.3 & 2.71 & 0 & & 0 & 0 & 0 & 0 & 0 & 0 & 0 & 0 \\
\hline 3.7 & & 3.3988 & 0 & c & & 0 & 0 & 0 & 0 & 0 & 0 & 0 \\
\hline 1.722 & & 3.8685 & 0 & & 0 & 0 & 0 & 0 & 0 & 0 & 0 & 0 \\
\hline 5.657 & -0.5 & -3.1970 & $\mathrm{H}$ & $c$ & & 0 & 0 & 0 & 0 & 0 & 0 & 0 \\
\hline 3.6424 & -1.8 & -3.9104 & $\mathrm{H}$ & & & 0 & 0 & 0 & 0 & 0 & 0 & 0 \\
\hline & -1.9 & & $\mathrm{H}$ & c & & 0 & 0 & 0 & 0 & 0 & 0 & 0 \\
\hline 5.6704 & 0. & -0.9696 & $\mathrm{H}$ & & & 0 & 0 & 0 & 0 & 0 & 0 & 0 \\
\hline 4.5824 & & & $\mathrm{H}$ & 0 & & 0 & 0 & 0 & 0 & 0 & 0 & 0 \\
\hline-0.1 & -2.3 & & $\mathrm{H}$ & & & 0 & 0 & 0 & 0 & 0 & 0 & 0 \\
\hline & & -1.3 & $\mathrm{H}$ & 0 & 0 & 0 & 0 & 0 & 0 & 0 & 0 & 0 \\
\hline-4.7779 & 0.985 & -0.8298 & $\mathrm{H}$ & & & 0 & 0 & 0 & 0 & 0 & 0 & 0 \\
\hline & & & $\mathrm{H}$ & 0 & & 0 & 0 & 0 & 0 & 0 & 0 & 0 \\
\hline-2.3824 & 2.39 & -4.7037 & & & & 0 & 0 & 0 & 0 & 0 & 0 & 0 \\
\hline-4.0970 & 2.01 & -2.9392 & $\mathrm{H}$ & 0 & & 0 & 0 & 0 & 0 & 0 & 0 & 0 \\
\hline & -0.0134 & -2.5283 & & 0 & & 0 & 0 & 0 & 0 & 0 & 0 & 0 \\
\hline-4.9820 & -0.3580 & 3.2743 & & 0 & & 0 & 0 & 0 & 0 & 0 & 0 & 0 \\
\hline 1.8790 & 1.3566 & 4.7284 & & 0 & & 0 & & & & 0 & 0 & \\
\hline
\end{tabular}

$1.8790-1.3566$

$\begin{array}{llllll}2 & 1 & 0 & 0 & 0 & 0\end{array}$

$\begin{array}{llllll}6 & 1 & 0 & 0 & 0 & 0 \\ 3 & 1 & 0 & 0 & 0 & 0\end{array}$

$\begin{array}{llllll}3 & 2 & 0 & 0 & 0 & 0\end{array}$

$\begin{array}{lllll}33 & 2 & 0 & 0 & 0\end{array}$

$\begin{array}{llllll}4 & 3 & 0 & 0 & 0 & 0\end{array}$

$\begin{array}{lll}0 & 0 & 0 \\ 0 & 0 & 0\end{array}$

$\begin{array}{lllll}4 & 0 & 0 & 0 & 0\end{array}$

$\begin{array}{llll}0 & 0 & 0 & 0 \\ 0 & 0 & 0 & 0\end{array}$

$\begin{array}{lllllll}35 & 6 & 0 & 0 & 0\end{array}$

$\begin{array}{llllll}8 & 7 & 0 & 0 & 0\end{array}$

$\begin{array}{ccccccc}11 & 7 & 0 & 0 & 0 & 0\end{array}$

$\begin{array}{llllll}23 & 8 & 0 & 0 & 0\end{array}$

$\begin{array}{llllll}10 & 9 & 0 & 0 & 0 & 0 \\ 25 & 9 & 0 & 0 & 0 & 0\end{array}$

$\begin{array}{llllll}36 & 10 & 0 & 0 & 0\end{array}$

$\begin{array}{llllll}36 & 10 & 0 & 0 & 0\end{array}$

$\begin{array}{llllll}12 & 11 & 0 & 0 & 0 & 0\end{array}$

$\begin{array}{lllll}0 & 0 & 0 & 0\end{array}$

$\begin{array}{lllllll}38 & 11 & 0 & 0 & 0 & 0\end{array}$

$17 \begin{array}{llllll}12 & 0 & 0 & 0 & 0\end{array}$

$\begin{array}{llllll}14 & 13 & 0 & 0 & 0 & 0\end{array}$

$\begin{array}{llllll}21 & 13 & 0 & 0 & 0 & 0\end{array}$

$20 \begin{array}{llllll}14 & 0 & 0 & 0 & 0\end{array}$

$\begin{array}{llllll}16 & 15 & 0 & 0 & 0 & 0\end{array}$

$\begin{array}{llllll}39 & 15 & 0 & 0 & 0 & 0\end{array}$

$\begin{array}{lllll}17 & 16 & 0 & 0 & 0\end{array}$

$\begin{array}{llllll}22 & 17 & 0 & 0 & 0\end{array}$

$\begin{array}{llllll}19 & 18 & 0 & 0 & 0 & 0\end{array}$

$\begin{array}{llllll}21 & 18 & 0 & 0 & 0 & 0\end{array}$

$\begin{array}{llllll}20 & 19 & 0 & 0 & 0 & 0\end{array}$ 


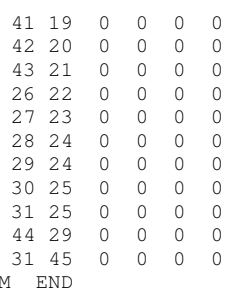


$\begin{array}{llllll}29 & 24 & 0 & 0 & 0 & 0\end{array}$

$\begin{array}{llllll}30 & 25 & 0 & 0 & 0 & 0 \\ 31 & 25 & 0 & 0 & 0 & 0\end{array}$

$\begin{array}{llllll}44 & 29 & 0 & 0 & 0 & 0 \\ 31 & 45 & 0 & 0 & 0 & 0\end{array}$

31
$M \quad$ END

$\$ \$ \$$

pentachlorophenol-anion.sdf

cosmotherm 3D

$\begin{array}{llllllllllll}12 & 12 & 0 & 0 & 0 & 0 & 0 & 0 & 0 & 0 & 0 & \mathrm{~V} 2000\end{array}$

$\begin{array}{llllllllllllll}0.4489 & 1.2096 & 0.0000 & \mathrm{C} & 0 & 0 & 0 & 0 & 0 & 0 & 0 & 0 & 0 & 1\end{array}$

$\begin{array}{lllllllllllllll}-0.9459 & 1.2075 & 0.0001 & \mathrm{C} & 0 & 0 & 0 & 0 & 0 & 0 & 0 & 0 & 0 & 2\end{array}$

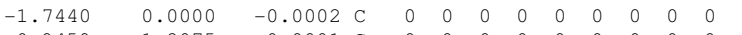

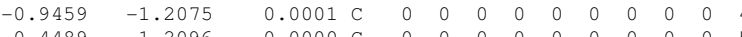

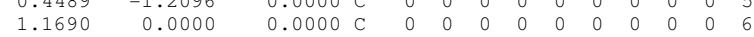

$\begin{array}{llllllllllllll}-3.0121 & 0.0000 & -0.0005 & 0 & 0 & 0 & 0 & 0 & 0 & 0 & 0 & 0 & 0 & 7\end{array}$

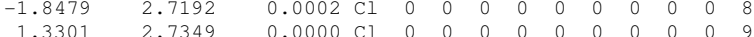

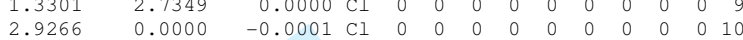

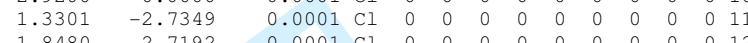

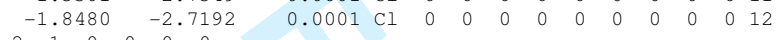

$\begin{array}{llllll}2 & 1 & 0 & 0 & 0 & 0\end{array}$

$\begin{array}{lllllllll}9 & 1 & 0 & 0 & 0 & 0\end{array}$

$\begin{array}{lllllllll}9 & 1 & 0 & 0 & 0 & 0 \\ 3 & 2 & 0 & 0 & 0 & 0\end{array}$

$\begin{array}{llllll}9 & 2 & 0 & 0 & 0 & 0\end{array}$

$\begin{array}{lllllll}4 & 3 & 0 & 0 & 0 & 0 \\ 7 & 3 & 0 & 0 & 0 & 0\end{array}$

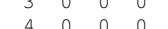

$\begin{array}{llllll}6 & 5 & 0 & 0 & 0 & 0\end{array}$

$\begin{array}{lllllll}11 & 5 & 0 & 0 & 0 & 0\end{array}$

$\begin{array}{llll}0 & 0 & 0 & 0\end{array}$

M END

pentachlorophenol.sdf

cosmotherm

$\begin{array}{llllllllllll}13 & 13 & 0 & 0 & 0 & 0 & 0 & 0 & 0 & 0 & 0 & \mathrm{~V} 2000\end{array}$

$\begin{array}{lllllllllllll}0.4687 & 1.2149 & 0.0000 & \mathrm{C} & 0 & 0 & 0 & 0 & 0 & 0 & 0 & 0 & 0\end{array}$

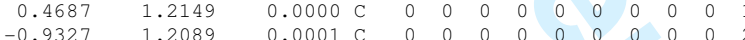

$\begin{array}{lllllllllllll}-0.9327 & 1.2089 & 0.0000 & c & 0 & 0 & 0 & 0 & 0 & 0 & 0 & 0 & 0\end{array}$

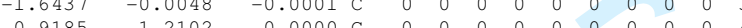

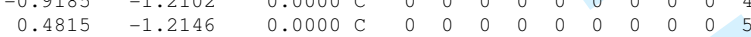

$\begin{array}{lllllllllllll}1.1828 & 0.0030 & -0.0001 & \mathrm{C} & 0 & 0 & 0 & 0 & 0 & 0 & 0 & 0 & 0\end{array}$

$\begin{array}{lllllllllllll}2.9929 & 0.0373 & -0.0006 & 0 & 0 & 0 & 0 & 0 & 0 & 0 & 0 & 0 & 0\end{array}$

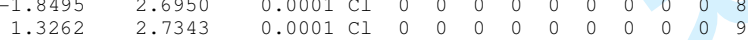

$\begin{array}{llllllllllllll}2.9293 & 0.0096 & -0.0002 & \mathrm{Cl} & 0 & 0 & 0 & 0 & 0 & 0 & 0 & 0 & 0 & 10\end{array}$

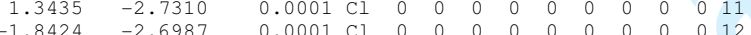

$\begin{array}{llllllllllllll}-1.8424 & -2.6987 & 0.0001 & \mathrm{Cl} & 0 & 0 & 0 & 0 & 0 & 0 & 0 & 0 & 0 & 12 \\ -3.3420 & -0.8815 & 0.0018 & \mathrm{H} & 0 & 0 & 0 & 0 & 0 & 0 & 0 & 0 & 0 & 13\end{array}$

$-3.3420-0.8815$

$\begin{array}{llllllll}1 & 0 & 0 & 0 & 0 & 0 & 0 \\ 9 & 1 & 0 & 0 & 0 & 0\end{array}$

$\begin{array}{lllll}2 & 0 & 0 & 0 & 0 \\ 2 & 0 & 0 & 0 & 0\end{array}$

$\begin{array}{cccccccc}2 & 0 & 0 & 0 & 0\end{array}$

$\begin{array}{llllll}3 & 0 & 0 & 0 & 0 & 0 \\ 4 & 0 & 0 & 0 & 0\end{array}$

$\begin{array}{llllll}5 & 4 & 0 & 0 & 0 & 0\end{array}$

$\begin{array}{llllll}5 & 0 & 0 & 0 & 0\end{array}$

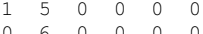

$\begin{array}{lllll}13 & 0 & 0 & 0\end{array}$

END

phenol-anion.sdf

COSMOtherm .sdf

$\begin{array}{lllllllllllll}12 & 12 & 0 & 0 & 0 & 0 & 0 & 0 & 0 & 0 & 0 & \mathrm{v} 2000\end{array}$

$\begin{array}{llllllllll}0 & 0 & 0 & 0 & 0 & 0 & 0 & 0 & 0 & 1\end{array}$

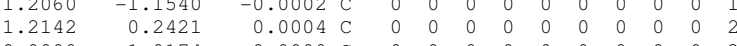

$\begin{array}{lllllllllllll}0.0000 & 1.0174 & 0.0000 & \mathrm{C} & 0 & 0 & 0 & 0 & 0 & 0 & 0 & 0 & 0\end{array}$

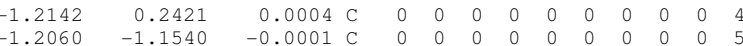

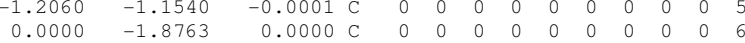

$\begin{array}{llllllllllllll}0.0000 & 2.3153 & -0.0003 & 0 & 0 & 0 & 0 & 0 & 0 & 0 & 0 & 0 & 0 & 7\end{array}$

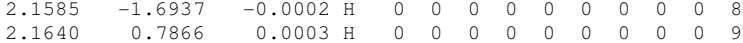

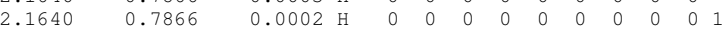

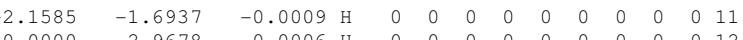

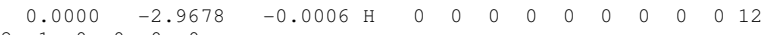

$\begin{array}{lllllllll}2 & 1 & 0 & 0 & 0 & 0\end{array}$

$\begin{array}{cccccccc}1 & 0 & 0 & 0 & 0\end{array}$

$\begin{array}{lllllll}8 & 1 & 0 & 0 & 0 & 0\end{array}$

$\begin{array}{lllll}2 & 0 & 0 & 0 & 0\end{array}$

$\begin{array}{lllll}3 & 0 & 0 & 0\end{array}$

$\begin{array}{llllll}3 & 0 & 0 & 0 & 0 & -1 \\ 5 & 0 & 0 & 0 & 0\end{array}$

$\begin{array}{llllll}4 & 0 & 0 & 0 & 0\end{array}$

$\begin{array}{rllll}5 & 0 & 0 & 0 & 0 \\ 5 & 0 & 0 & 0 & 0\end{array}$

$\begin{array}{llllll}6 & 12 & 0 & 0 & 0 & 0\end{array}$

END

\$\$\$ $\$$ phenol.sd

COSMOtherm

$3 \mathrm{D}$

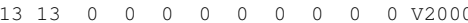

$\begin{array}{cccccccccccccc}-1.8845 & -0.0020 & 0.0001 & \mathrm{C} & 0 & 0 & 0 & 0 & 0 & 0 & 0 & 0 & 0 & 1\end{array}$ 


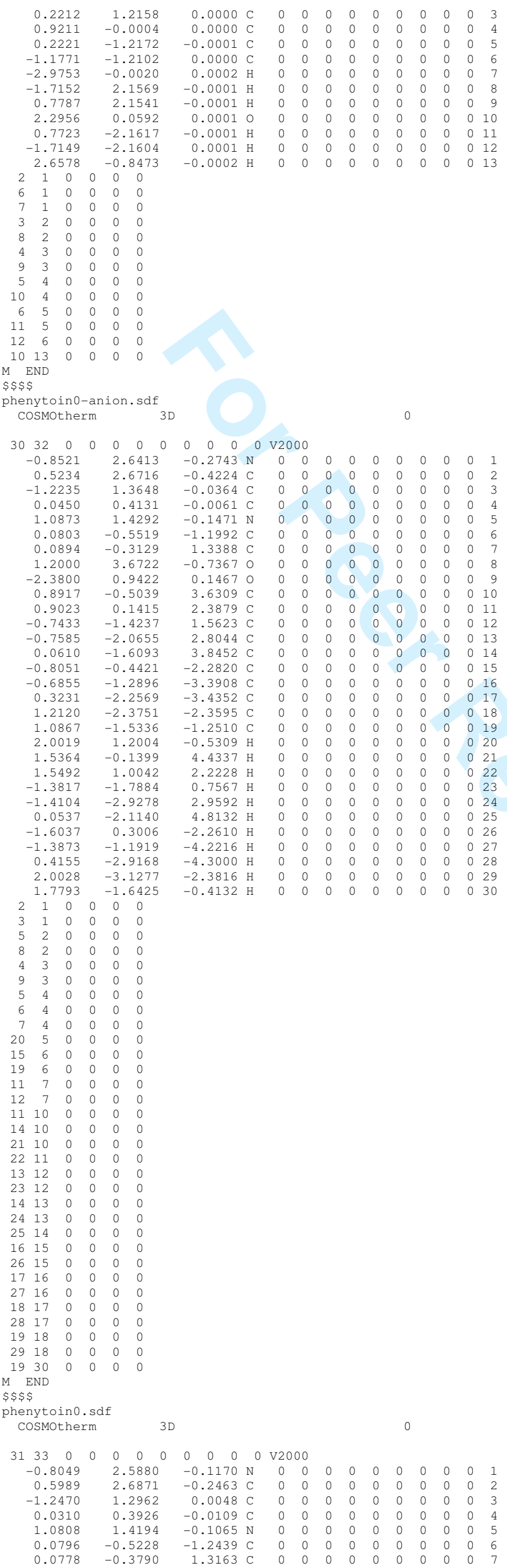




$\begin{array}{rrrrrrrrrrrrrr}1.2230 & 3.7279 & -0.4364 & 0 & 0 & 0 & 0 & 0 & 0 & 0 & 0 & 0 & 0 & 8 \\ -2.4059 & 0.9238 & 0.1190 & 0 & 0 & 0 & 0 & 0 & 0 & 0 & 0 & 0 & 0 & 9 \\ 0.8824 & -0.6491 & 3.5938 & \mathrm{C} & 0 & 0 & 0 & 0 & 0 & 0 & 0 & 0 & 0 & 10 \\ 0.8907 & 0.0440 & 2.3768 & \mathrm{C} & 0 & 0 & 0 & 0 & 0 & 0 & 0 & 0 & 0 & 11 \\ -0.7545 & -1.4971 & 1.4936 & \mathrm{C} & 0 & 0 & 0 & 0 & 0 & 0 & 0 & 0 & 0 & 12 \\ -0.7669 & -2.1828 & 2.7112 & \mathrm{C} & 0 & 0 & 0 & 0 & 0 & 0 & 0 & 0 & 0 & 13 \\ 0.0538 & -1.7623 & 3.7656 & \mathrm{C} & 0 & 0 & 0 & 0 & 0 & 0 & 0 & 0 & 0 & 14 \\ -0.7391 & -0.3098 & -2.3625 & \mathrm{C} & 0 & 0 & 0 & 0 & 0 & 0 & 0 & 0 & 0 & 15 \\ -0.6065 & -1.1142 & -3.5004 & \mathrm{C} & 0 & 0 & 0 & 0 & 0 & 0 & 0 & 0 & 0 & 16 \\ 0.3511 & -2.1322 & -3.5364 & \mathrm{C} & 0 & 0 & 0 & 0 & 0 & 0 & 0 & 0 & 0 & 17 \\ 1.1766 & -2.3450 & -2.4259 & \mathrm{C} & 0 & 0 & 0 & 0 & 0 & 0 & 0 & 0 & 0 & 18 \\ 1.0379 & -1.5495 & -1.2856 & \mathrm{C} & 0 & 0 & 0 & 0 & 0 & 0 & 0 & 0 & 0 & 19 \\ -1.4140 & 3.4047 & -0.1228 & \mathrm{H} & 0 & 0 & 0 & 0 & 0 & 0 & 0 & 0 & 0 & 20 \\ 2.0410 & 1.1991 & -0.3588 & \mathrm{H} & 0 & 0 & 0 & 0 & 0 & 0 & 0 & 0 & 0 & 21 \\ 1.5262 & -0.3137 & 4.4089 & \mathrm{H} & 0 & 0 & 0 & 0 & 0 & 0 & 0 & 0 & 0 & 22 \\ 1.5369 & 0.9133 & 2.2520 & \mathrm{H} & 0 & 0 & 0 & 0 & 0 & 0 & 0 & 0 & 0 & 23 \\ -1.3915 & -1.8359 & 0.6757 & \mathrm{H} & 0 & 0 & 0 & 0 & 0 & 0 & 0 & 0 & 0 & 24 \\ -1.4168 & -3.0508 & 2.8352 & \mathrm{H} & 0 & 0 & 0 & 0 & 0 & 0 & 0 & 0 & 0 & 25 \\ 0.0477 & -2.3018 & 4.7143 & \mathrm{H} & 0 & 0 & 0 & 0 & 0 & 0 & 0 & 0 & 0 & 26 \\ -1.4939 & 0.4780 & -2.3574 & \mathrm{H} & 0 & 0 & 0 & 0 & 0 & 0 & 0 & 0 & 0 & 27 \\ -1.2562 & -0.9415 & -4.3603 & \mathrm{H} & 0 & 0 & 0 & 0 & 0 & 0 & 0 & 0 & 0 & 28 \\ 0.4537 & -2.7583 & -4.4244 & \mathrm{H} & 0 & 0 & 0 & 0 & 0 & 0 & 0 & 0 & 0 & 29 \\ 1.9282 & -3.1363 & -2.4444 & \mathrm{H} & 0 & 0 & 0 & 0 & 0 & 0 & 0 & 0 & 0 & 30 \\ 1.6806 & -1.7300 & -0.4216 & \mathrm{H} & 0 & 0 & 0 & 0 & 0 & 0 & 0 & 0 & 0 & 31\end{array}$




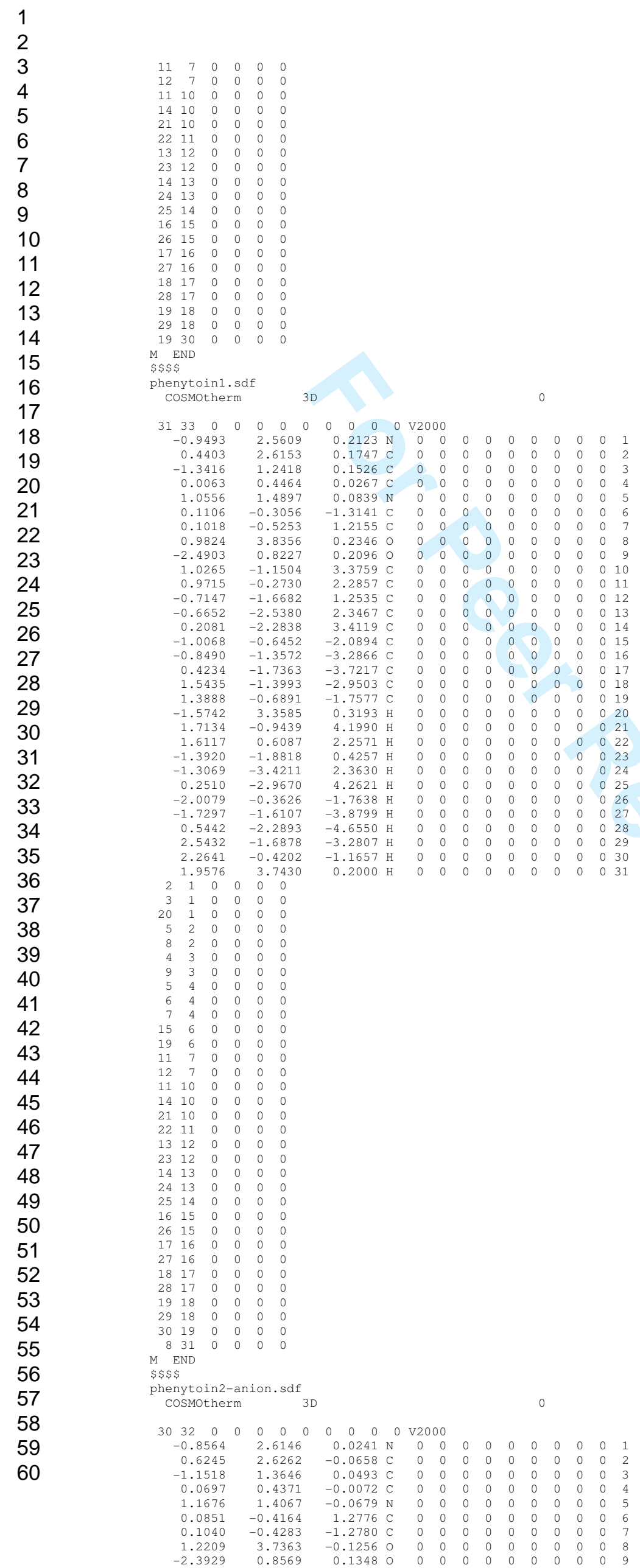




$\begin{array}{rrrrrrrrrrrrrr}1.2144 & -2.1791 & -2.5622 & \mathrm{C} & 0 & 0 & 0 & 0 & 0 & 0 & 0 & 0 & 0 & 10 \\ 1.0841 & -1.4308 & -1.3898 & \mathrm{C} & 0 & 0 & 0 & 0 & 0 & 0 & 0 & 0 & 0 & 11 \\ -0.7474 & -0.2013 & -2.3697 & \mathrm{C} & 0 & 0 & 0 & 0 & 0 & 0 & 0 & 0 & 0 & 12 \\ -0.6207 & -0.9524 & -3.5459 & \mathrm{C} & 0 & 0 & 0 & 0 & 0 & 0 & 0 & 0 & 0 & 13 \\ 0.3594 & -1.9440 & -3.6474 & \mathrm{C} & 0 & 0 & 0 & 0 & 0 & 0 & 0 & 0 & 0 & 14 \\ 0.8828 & -0.0452 & 2.3684 & \mathrm{C} & 0 & 0 & 0 & 0 & 0 & 0 & 0 & 0 & 0 & 15 \\ 0.8605 & -0.7889 & 3.5554 & \mathrm{C} & 0 & 0 & 0 & 0 & 0 & 0 & 0 & 0 & 0 & 16 \\ 0.0400 & -1.9171 & 3.6655 & \mathrm{C} & 0 & 0 & 0 & 0 & 0 & 0 & 0 & 0 & 0 & 17 \\ -0.7575 & -2.2970 & 2.5780 & \mathrm{C} & 0 & 0 & 0 & 0 & 0 & 0 & 0 & 0 & 0 & 18 \\ -0.7333 & -1.5533 & 1.3942 & \mathrm{C} & 0 & 0 & 0 & 0 & 0 & 0 & 0 & 0 & 0 & 19 \\ 1.9848 & -2.9502 & -2.6296 & \mathrm{H} & 0 & 0 & 0 & 0 & 0 & 0 & 0 & 0 & 0 & 20 \\ 1.7535 & -1.6235 & -0.5491 & \mathrm{H} & 0 & 0 & 0 & 0 & 0 & 0 & 0 & 0 & 0 & 21 \\ -1.5227 & 0.5646 & -2.3133 & \mathrm{H} & 0 & 0 & 0 & 0 & 0 & 0 & 0 & 0 & 0 & 22 \\ -1.2968 & -0.7618 & -4.3819 & \mathrm{H} & 0 & 0 & 0 & 0 & 0 & 0 & 0 & 0 & 0 & 23 \\ 0.4561 & -2.5320 & -4.5621 & \mathrm{H} & 0 & 0 & 0 & 0 & 0 & 0 & 0 & 0 & 0 & 24 \\ 1.5247 & 0.8311 & 2.2695 & \mathrm{H} & 0 & 0 & 0 & 0 & 0 & 0 & 0 & 0 & 0 & 25 \\ 1.4880 & -0.4849 & 4.3961 & \mathrm{H} & 0 & 0 & 0 & 0 & 0 & 0 & 0 & 0 & 0 & 26 \\ 0.0222 & -2.4980 & 4.5896 & \mathrm{H} & 0 & 0 & 0 & 0 & 0 & 0 & 0 & 0 & 0 & 27 \\ -1.4001 & -3.1769 & 2.6513 & \mathrm{H} & 0 & 0 & 0 & 0 & 0 & 0 & 0 & 0 & 0 & 28 \\ -1.3583 & -1.8600 & 0.5537 & \mathrm{H} & 0 & 0 & 0 & 0 & 0 & 0 & 0 & 0 & 0 & 29 \\ -3.0292 & 1.6024 & 0.1732 & \mathrm{H} & 0 & 0 & 0 & 0 & 0 & 0 & 0 & 0 & 0 & 30\end{array}$

phenytoin2.sdf
Cosmotherm

$\begin{array}{rrrrrrrrrrrrrrr}31 & 33 & 0 & 0 & 0 & 0 & 0 & 0 & 0 & 0 & \text { V2000 } & & & & \\ -0.8514 & 2.6179 & -0.1383 & \mathrm{~N} & 0 & 0 & 0 & 0 & 0 & 0 & 0 & 0 & 0 & 1 \\ 0.5741 & 2.6842 & -0.2704 & \mathrm{C} & 0 & 0 & 0 & 0 & 0 & 0 & 0 & 0 & 0 & 2 \\ -1.1420 & 1.3630 & -0.0181 & \mathrm{C} & 0 & 0 & 0 & 0 & 0 & 0 & 0 & 0 & 0 & 3 \\ 0.0554 & 0.4007 & -0.0152 & \mathrm{C} & 0 & 0 & 0 & 0 & 0 & 0 & 0 & 0 & 0 & 4 \\ 1.0997 & 1.4247 & -0.1174 & \mathrm{~N} & 0 & 0 & 0 & 0 & 0 & 0 & 0 & 0 & 0 & 5 \\ 0.0866 & -0.3624 & 1.3232 & \mathrm{C} & 0 & 0 & 0 & 0 & 0 & 0 & 0 & 0 & 0 & 6 \\ 0.0933 & -0.5249 & -1.2432 & \mathrm{C} & 0 & 0 & 0 & 0 & 0 & 0 & 0 & 0 & 0 & 7 \\ 1.2042 & 3.7209 & -0.4768 & 0 & 0 & 0 & 0 & 0 & 0 & 0 & 0 & 0 & 0 & 8 \\ -2.3602 & 0.8637 & 0.1207 & 0 & 0 & 0 & 0 & 0 & 0 & 0 & 0 & 0 & 0 & 9 \\ 1.1169 & -2.4073 & -2.3940 & \mathrm{C} & 0 & 0 & 0 & 0 & 0 & 0 & 0 & 0 & 0 & 10 \\ 0.9932 & -1.6030 & -1.2577 & \mathrm{C} & 0 & 0 & 0 & 0 & 0 & 0 & 0 & 0 & 0 & 11 \\ -0.6797 & -0.2666 & -2.3849 & \mathrm{C} & 0 & 0 & 0 & 0 & 0 & 0 & 0 & 0 & 0 & 12 \\ -0.5615 & -1.0781 & -3.5188 & \mathrm{C} & 0 & 0 & 0 & 0 & 0 & 0 & 0 & 0 & 0 & 13 \\ 0.3363 & -2.1500 & -3.5269 & \mathrm{C} & 0 & 0 & 0 & 0 & 0 & 0 & 0 & 0 & 0 & 14 \\ 0.8912 & 0.0748 & 2.3840 & \mathrm{C} & 0 & 0 & 0 & 0 & 0 & 0 & 0 & 0 & 0 & 15 \\ 0.8788 & -0.6061 & 3.6078 & \mathrm{C} & 0 & 0 & 0 & 0 & 0 & 0 & 0 & 0 & 0 & 16 \\ 0.0539 & -1.7210 & 3.7861 & \mathrm{C} & 0 & 0 & 0 & 0 & 0 & 0 & 0 & 0 & 0 & 17 \\ -0.7583 & -2.1557 & 2.7311 & \mathrm{C} & 0 & 0 & 0 & 0 & 0 & 0 & 0 & 0 & 0 & 18 \\ -0.7427 & -1.4813 & 1.5074 & \mathrm{C} & 0 & 0 & 0 & 0 & 0 & 0 & 0 & 0 & 0 & 19 \\ 1.8222 & -3.2404 & -2.3916 & \mathrm{H} & 0 & 0 & 0 & 0 & 0 & 0 & 0 & 0 & 0 & 20 \\ 1.6008 & -1.8180 & -0.3769 & \mathrm{H} & 0 & 0 & 0 & 0 & 0 & 0 & 0 & 0 & 0 & 21 \\ -1.3846 & 0.5665 & -2.4025 & \mathrm{H} & 0 & 0 & 0 & 0 & 0 & 0 & 0 & 0 & 0 & 22 \\ -1.1760 & -0.8698 & -4.3964 & \mathrm{H} & 0 & 0 & 0 & 0 & 0 & 0 & 0 & 0 & 0 & 23 \\ 0.4271 & -2.7829 & -4.4114 & \mathrm{H} & 0 & 0 & 0 & 0 & 0 & 0 & 0 & 0 & 0 & 24 \\ 1.5360 & 0.9440 & 2.2522 & \mathrm{H} & 0 & 0 & 0 & 0 & 0 & 0 & 0 & 0 & 0 & 25 \\ 1.5170 & -0.2603 & 4.4229 & \mathrm{H} & 0 & 0 & 0 & 0 & 0 & 0 & 0 & 0 & 0 & 26 \\ 0.0444 & -2.2510 & 4.7401 & \mathrm{H} & 0 & 0 & 0 & 0 & 0 & 0 & 0 & 0 & 0 & 27 \\ -1.4057 & -3.0249 & 2.8595 & \mathrm{H} & 0 & 0 & 0 & 0 & 0 & 0 & 0 & 0 & 0 & 28 \\ -1.3746 & -1.8329 & 0.6908 & \mathrm{H} & 0 & 0 & 0 & 0 & 0 & 0 & 0 & 0 & 0 & 29 \\ 2.0609 & 1.2066 & -0.3677 & \mathrm{H} & 0 & 0 & 0 & 0 & 0 & 0 & 0 & 0 & 0 & 30 \\ -3.0145 & 1.5964 & 0.1225 & \mathrm{H} & 0 & 0 & 0 & 0 & 0 & 0 & 0 & 0 & 0 & 31\end{array}$




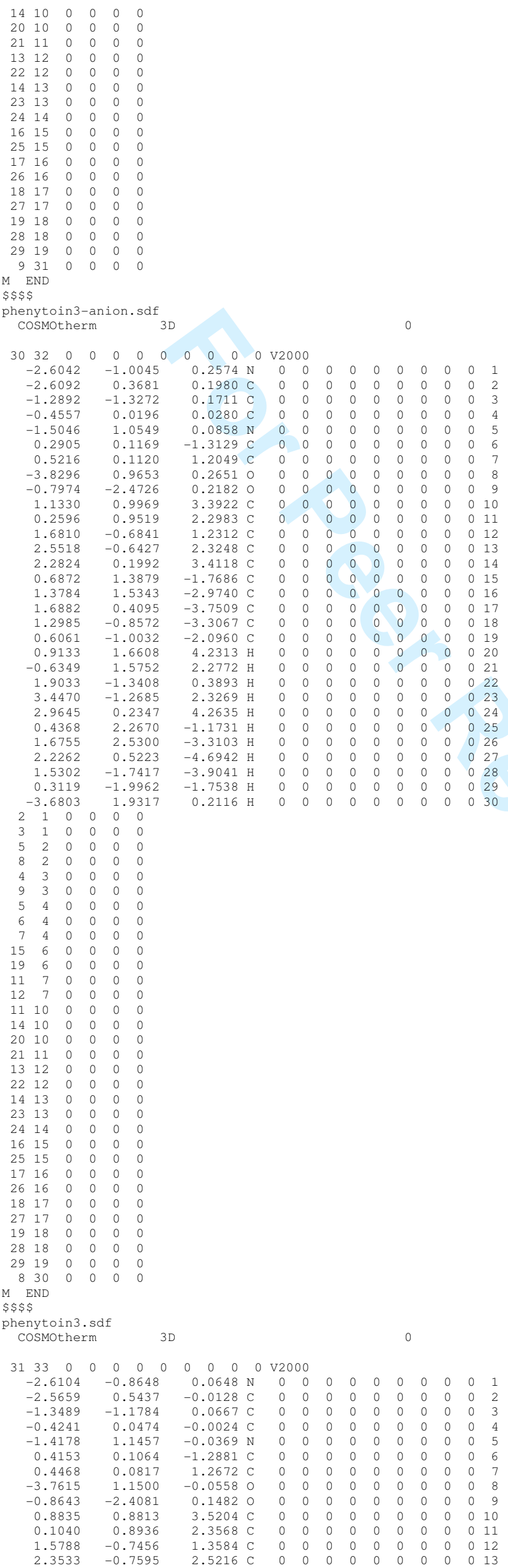




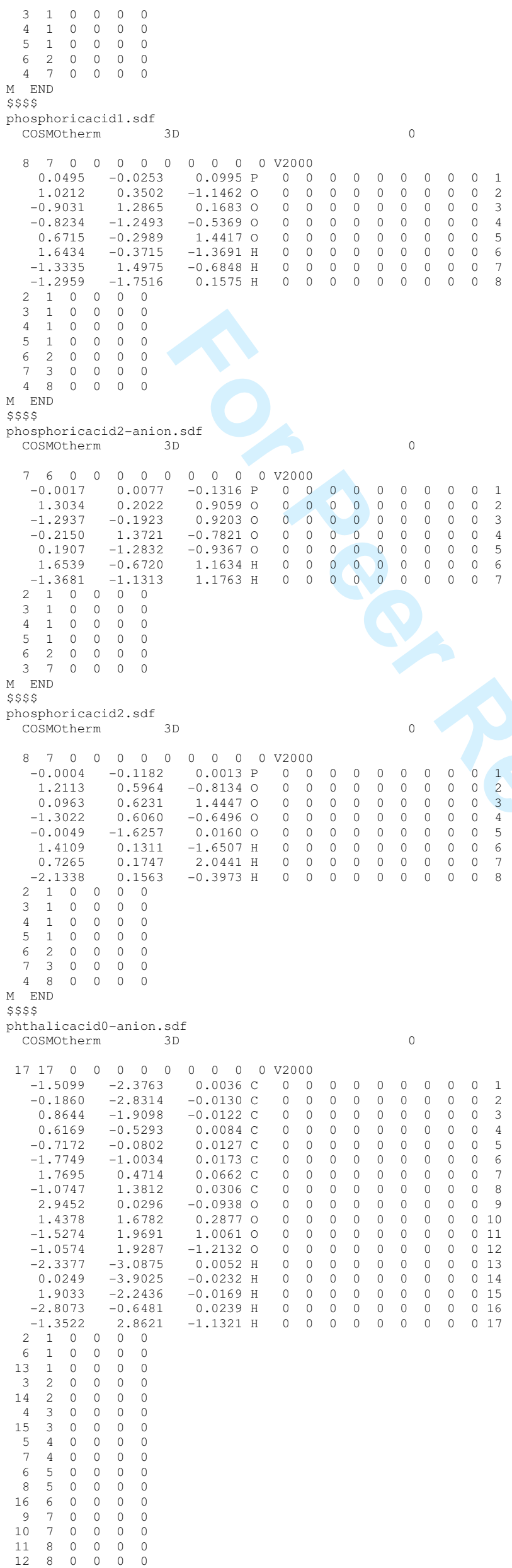




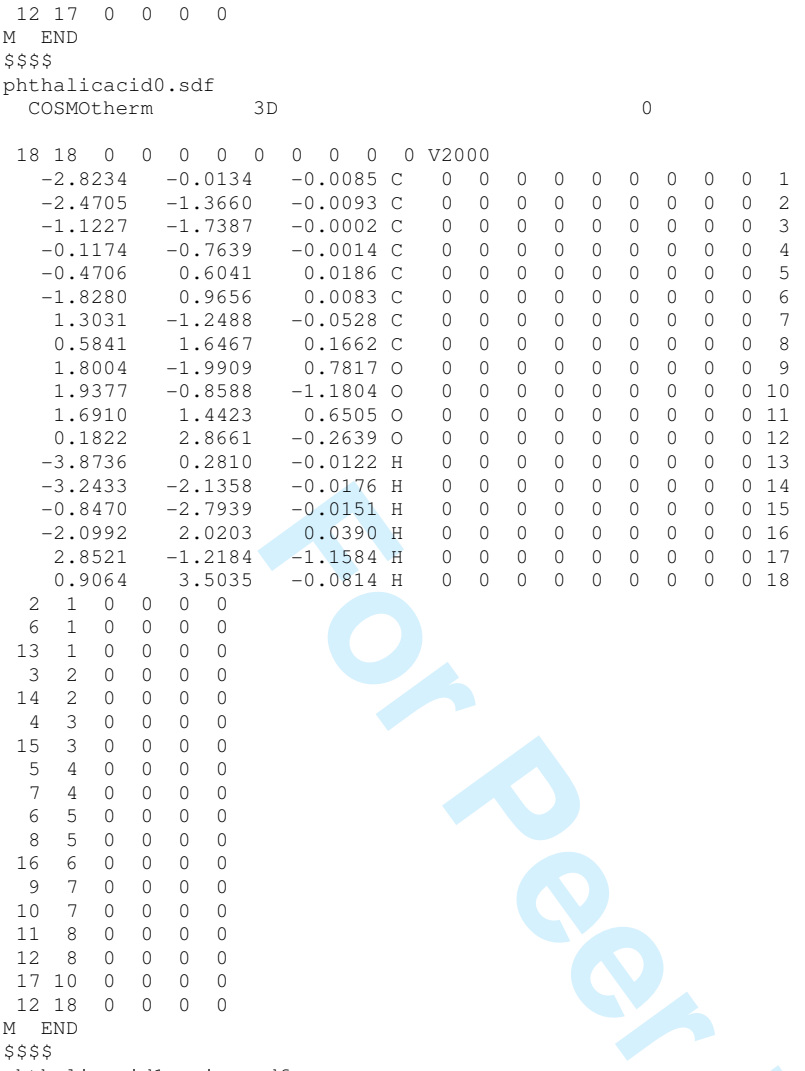

$\$ \$ \$$

Cosmotherm

$\begin{array}{llllllllllll}17 & 17 & 0 & 0 & 0 & 0 & 0 & 0 & 0 & 0 & 0 & \mathrm{~V} 2000\end{array}$

$\begin{array}{llllllllllllll}0.1883 & -2.8314 & -0.0124 & \mathrm{C} & 0 & 0 & 0 & 0 & 0 & 0 & 0 & 0 & 0 & 1\end{array}$

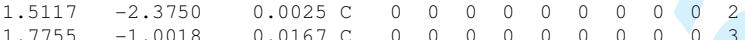

$\begin{array}{llllllllllllll}1.7755 & -1.0018 & 0.0167 & \mathrm{C} & 0 & 0 & 0 & 0 & 0 & 0 & 0 & 0 & 0 & 3 \\ 0.7169 & -0.0797 & 0.0124 & \mathrm{C} & 0 & 0 & 0 & 0 & 0 & 0 & 0 & 0 & 0 & 4\end{array}$

$-0.6167-0.0797$

$-0.6167-0.5300$

$\begin{array}{rr}.0729 & -1.9109 \\ -1.07698 & 1.3821\end{array}$

$1.7698 \quad 0.4703$

$1.5206 \quad 1.9703$

$1.0618 \quad 1.9296$

0.0270

$0.0124 \mathrm{C}$

$\begin{array}{rr}0.0081 & C \\ -0.0097 & C\end{array}$

$\begin{array}{lllll}0 & 0 & 0 & 0 & 6\end{array}$

$0.0317 \mathrm{C}$

.4387

$\begin{array}{rlllllllllllll}-1.0941 & 0 & 0 & 0 & 0 & 0 & 0 & 0 & 0 & 0 & 0 & 11\end{array}$

$-3.0854-0.0033 \mathrm{H}=0$

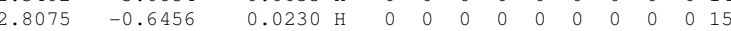

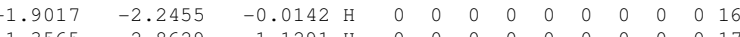




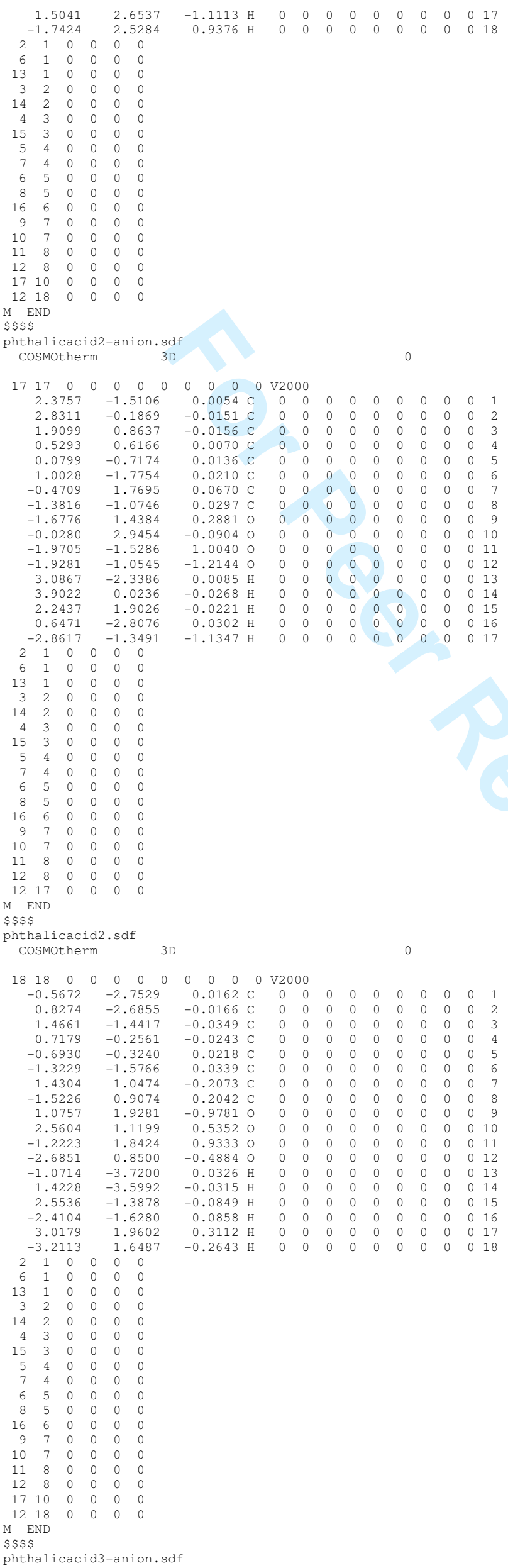




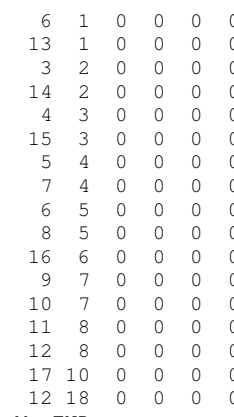




$\begin{array}{rrrrrrrrrrrrrr}1.2447 & 1.1146 & 0.0000 & \mathrm{C} & 0 & 0 & 0 & 0 & 0 & 0 & 0 & 0 & 0 & 7 \\ 1.2447 & -1.1146 & 0.0000 & \mathrm{C} & 0 & 0 & 0 & 0 & 0 & 0 & 0 & 0 & 0 & 8 \\ 2.0502 & 0.0000 & 0.0000 & \mathrm{~N} & 0 & 0 & 0 & 0 & 0 & 0 & 0 & 0 & 0 & 9 \\ 1.6432 & -2.2963 & -0.0001 & 0 & 0 & 0 & 0 & 0 & 0 & 0 & 0 & 0 & 0 & 10 \\ 1.6431 & 2.2963 & 0.0001 & 0 & 0 & 0 & 0 & 0 & 0 & 0 & 0 & 0 & 0 & 11 \\ -3.5555 & 1.2371 & -0.0002 & \mathrm{H} & 0 & 0 & 0 & 0 & 0 & 0 & 0 & 0 & 0 & 12 \\ -3.5555 & -1.2371 & 0.0002 & \mathrm{H} & 0 & 0 & 0 & 0 & 0 & 0 & 0 & 0 & 0 & 13 \\ -1.3971 & -2.5172 & 0.0004 & \mathrm{H} & 0 & 0 & 0 & 0 & 0 & 0 & 0 & 0 & 0 & 14 \\ -1.3971 & 2.5171 & -0.0003 & \mathrm{H} & 0 & 0 & 0 & 0 & 0 & 0 & 0 & 0 & 0 & 15\end{array}$

$-1.3971-2.5171$

$\begin{array}{lllll}2 & 1 & 0 & 0 & 0 \\ 6 & 1 & 0 & 0 & 0\end{array}$

$12 \quad 1100000$

$\begin{array}{llllll}3 & 2 & 0 & 0 & 0 & 0\end{array}$

$\begin{array}{lllllll}3 & 2 & 0 & 0 & 0 & 0\end{array}$

$\begin{array}{llllll}4 & 3 & 0 & 0 & 0 & 0\end{array}$

$\begin{array}{llllllll}5 & 4 & 0 & 0 & 0 & 0\end{array}$

$\begin{array}{llllllll}4 & 0 & 0 & 0 & 0\end{array}$

$\begin{array}{llllll}5 & 0 & 0 & 0 & 0\end{array}$

$\begin{array}{llllllll}7 & 0 & 0 & 0 & 0\end{array}$

$\begin{array}{lllll}7 & 0 & 0 & 0 & 0 \\ 8 & 0 & 0 & 0 & 0\end{array}$

$\begin{array}{rrrrr}8 & 0 & 0 & 0 & 0 \\ 15 & 0 & 0 & 0 & 0\end{array}$

M END

\$\$\$

phthalimide1.sdf
COSMOtherm

$\begin{array}{llllllllllll}16 & 17 & 0 & 0 & 0 & 0 & 0 & 0 & 0 & 0 & 0 & \mathrm{~V} 2000\end{array}$

$\begin{array}{llllllllllllll}-2.5883 & 0.7685 & 0.0004 & \mathrm{C} & 0 & 0 & 0 & 0 & 0 & 0 & 0 & 0 & 0 & 1\end{array}$

$\begin{array}{lllllllllllll}-2.6384 & -0.6312 & -0.0005 & \mathrm{C} & 0 & 0 & 0 & 0 & 0 & 0 & 0 & 0 & 0\end{array}$

$\begin{array}{llllllllllllll}-1.4596 & -1.4030 & -0.0008 & \mathrm{C} & 0 & 0 & 0 & 0 & 0 & 0 & 0 & 0 & 0 & 3\end{array}$

$\begin{array}{rrrrrrrrrrrrrr}-0.2518 & -0.7218 & -0.0002 & \mathrm{C} & 0 & 0 & 0 & 0 & 0 & 0 & 0 & 0 & 0 & 4 \\ -0.2048 & 0.6804 & 0.0005 & \mathrm{C} & 0 & 0 & 0 & 0 & 0 & 0 & 0 & 0 & 0 & 5\end{array}$

$\begin{array}{llllllllllllll}-1.3585 & 1.4540 & 0.0009 & \mathrm{C} & 0 & 0 & 0 & 0 & 0 & 0 & 0 & 0 & 0 & 6\end{array}$

$\begin{array}{llllllllllllll}1.2404 & 0.9976 & 0.0000 & \mathrm{C} & 0 & 0 & 0 & 0 & 0 & 0 & 0 & 0 & 0 & 7\end{array}$

$\begin{array}{llllllllllllll}1.1832 & -1.1859 & 0.0001 & \mathrm{C} & 0 & 0 & 0 & 0 & 0 & 0 & 0 & 0 & 0 & 8\end{array}$

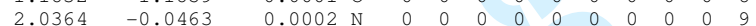

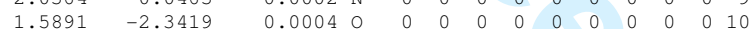

$\begin{array}{llllllllllllll}1.6518 & 2.2538 & -0.0007 & 0 & 0 & 0 & 0 & 0 & 0 & 0 & 0 & 0 & 0 & 11\end{array}$

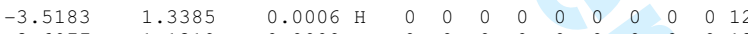

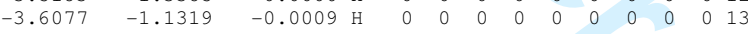

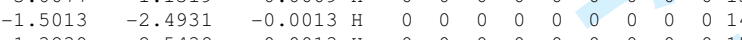

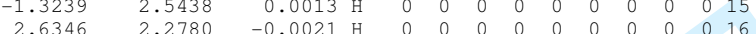

$\begin{array}{llllll}2 & 1 & 0 & 0 & 0 & 0\end{array}$

$\begin{array}{lllllll}1 & 0 & 0 & 0 & 0\end{array}$

$\begin{array}{llllll}1 & 0 & 0 & 0 & 0\end{array}$

$\begin{array}{lllll}2 & 0 & 0 & 0 & 0\end{array}$

$\begin{array}{lllll}2 & 0 & 0 & 0 & 0 \\ 3 & 0 & 0 & 0 & 0\end{array}$

$\begin{array}{lllll}3 & 0 & 0 & 0\end{array}$

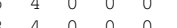

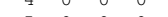

$\begin{array}{lllll}5 & 0 & 0 & 0\end{array}$

$\begin{array}{lllllll}5 & 6 & 0 & 0 & 0 & 0\end{array}$

$\begin{array}{llllll}7 & 0 & 0 & 0 & 0\end{array}$

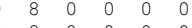

$\begin{array}{lllll}16 & 0 & 0 & 0 & 0\end{array}$

END

salicylicacid-anion $0 . s d f$

cosmotherm 3D

$\begin{array}{llllllllllll}15 & 16 & 0 & 0 & 0 & 0 & 0 & 0 & 0 & 0 & 0 & \mathrm{v} 2000\end{array}$

$\begin{array}{lllllllllllll}1.9959 & -1.4216 & -0.0003 & \mathrm{C} & 0 & 0 & 0 & 0 & 0 & 0 & 0 & 0 & 0\end{array}$

$\begin{array}{llllllllllllll}2.6365 & -0.1708 & 0.0001 & \mathrm{C} & 0 & 0 & 0 & 0 & 0 & 0 & 0 & 0 & 0 & 2\end{array}$

$\begin{array}{lllllllllllll}1.8937 & 1.0093 & 0.0001 & \mathrm{C} & 0 & 0 & 0 & 0 & 0 & 0 & 0 & 0 & 0\end{array}$

$\begin{array}{lllllllllllll}0.4858 & 0.9587 & 0.0002 & \mathrm{C} & 0 & 0 & 0 & 0 & 0 & 0 & 0 & 0 & 0\end{array}$

$\begin{array}{llllllllllllll}-0.1751 & -0.3024 & -0.0002 & \mathrm{C} & 0 & 0 & 0 & 0 & 0 & 0 & 0 & 0 & 0 & 5\end{array}$

$\begin{array}{lllllllllllll}0.6014 & -1.4728 & -0.0002 & \mathrm{C} & 0 & 0 & 0 & 0 & 0 & 0 & 0 & 0 & 0\end{array}$

$\begin{array}{lllllllllllll}-0.2268 & 2.1088 & 0.0003 & 0 & 0 & 0 & 0 & 0 & 0 & 0 & 0 & 0 & 0\end{array}$

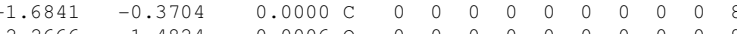

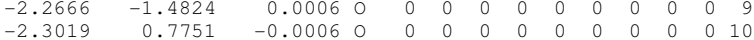

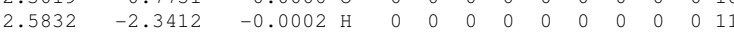

$\begin{array}{llllllllllll}-0.1162 & 0.0001 \mathrm{H} & 0 & 0 & 0 & 0 & 0 & 0 & 0 & 0 & 0 & 1\end{array}$

$\begin{array}{llllllllllllll}0.0777 & -2.4311 & -0.0004 & \mathrm{H} & 0 & 0 & 0 & 0 & 0 & 0 & 0 & 0 & 0 & 14\end{array}$

$-1.2267-1.7479$

$6 \begin{array}{llllll}6 & 1 & 0 & 0 & 0 & 0\end{array}$

$\begin{array}{llllllll}1 & 0 & 0 & 0 & 0\end{array}$

$\begin{array}{lllllll}0 & 0 & 0 & 0\end{array}$

$\begin{array}{llllll}2 & 2 & 0 & 0 & 0 & 0 \\ 4 & 3 & 0 & 0 & 0 & 0\end{array}$

$\begin{array}{llll}0 & 0 & 0 & 0 \\ 0 & 0 & 0 & 0\end{array}$

$\begin{array}{llllll}5 & 4 & 0 & 0 & 0 & 0\end{array}$

$\begin{array}{lllllll}4 & 0 & 0 & 0 & 0\end{array}$

$\begin{array}{ccccc}5 & 0 & 0 & 0 & 0 \\ 5 & 0 & 0 & 0 & 0\end{array}$

$\begin{array}{llllll}4 & 6 & 0 & 0 & 0 & 0 \\ 9 & 8 & 0 & 0 & 0 & 0\end{array}$

$\begin{array}{rrrrrr}0 & 8 & 0 & 0 & 0 & 0 \\ 7 & 15 & 0 & 0 & 0 & 0\end{array}$

1015

\$\$\$

salicylicacid-anion 1. sdf

cosmotherm 


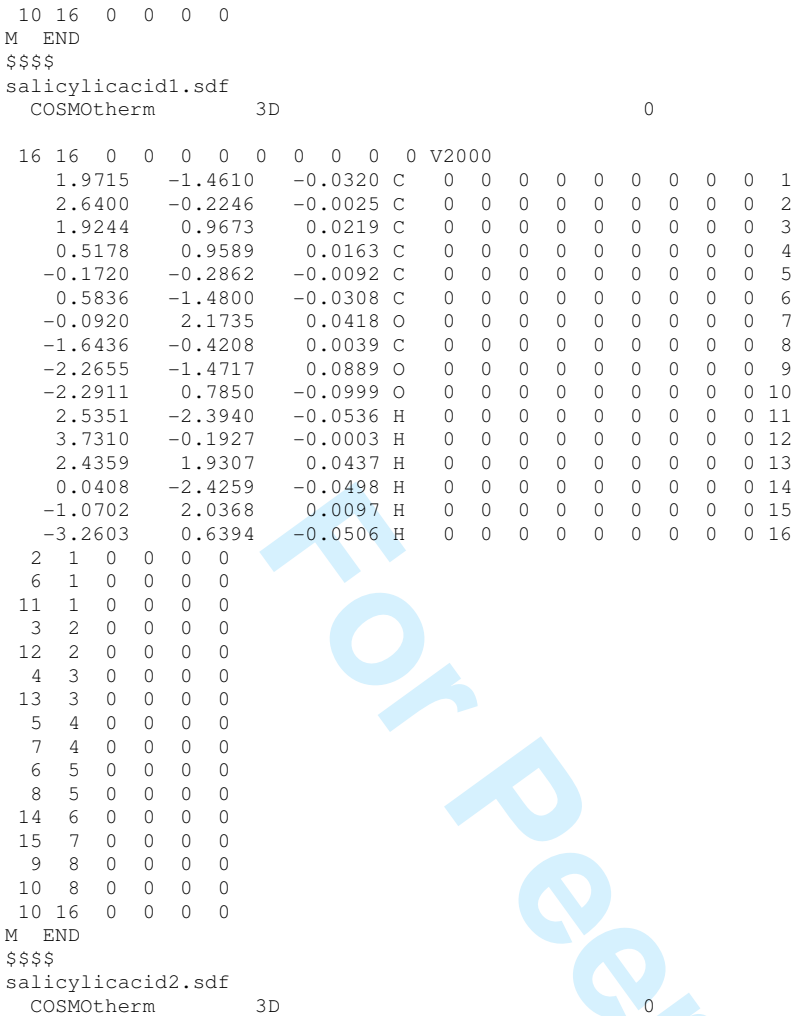

$\underset{\text { cosmotherm }}{\text { salicylicacidf }}$ 3D

$\begin{array}{llllllllllll}16 & 16 & 0 & 0 & 0 & 0 & 0 & 0 & 0 & 0 & 0 & \mathrm{~V} 2000\end{array}$

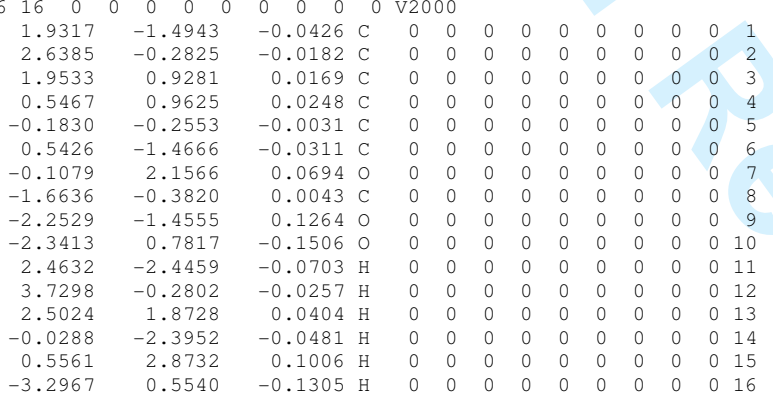

$6 \begin{array}{llllll}2 & 1 & 0 & 0 & 0\end{array}$

$\begin{array}{lllllll}1 & 1 & 0 & 0 & 0 & 0\end{array}$

$\begin{array}{lllllll}3 & 2 & 0 & 0 & 0 & 0 \\ 1 & 2 & 0 & 0 & 0 & 0\end{array}$

$\begin{array}{lllllll}3 & 3 & 0 & 0 & 0 & 0\end{array}$

$\begin{array}{lllllll}5 & 4 & 0 & 0 & 0 & 0 & 0 \\ 7 & 4 & 0 & 0 & 0 & 0\end{array}$

$\begin{array}{cccccc}5 & 4 & 0 & 0 & 0 & 0 \\ 8 & 0 & 0 & 0 & 0\end{array}$

$\begin{array}{lllllll}8 & 5 & 0 & 0 & 0 & 0\end{array}$

$\begin{array}{lllllll}4 & 6 & 0 & 0 & 0 & 0\end{array}$

$\begin{array}{llllll}5 & 7 & 0 & 0 & 0 & 0\end{array}$

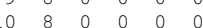

$\begin{array}{lllllll}10 & 16 & 0 & 0 & 0 & 0\end{array}$

END

succinimide0-anion.sdf

$\begin{array}{llllllllllll}11 & 11 & 0 & 0 & 0 & 0 & 0 & 0 & 0 & 0 & 0 & \mathrm{v} 2000\end{array}$

$\begin{array}{llllllllllll}0.0003 & \mathrm{C} & 0 & 0 & 0 & 0 & 0 & 0 & 0 & 0 & 0 & 1\end{array}$

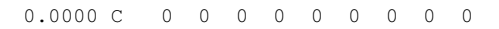

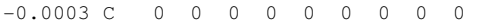

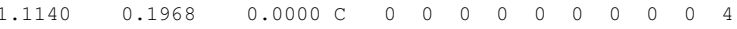

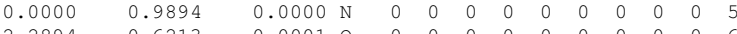

$\begin{array}{llllllllllllll}2.2894 & 0.6213 & 0.0001 & 0 & 0 & 0 & 0 & 0 & 0 & 0 & 0 & 0 & 0 & 6\end{array}$

$\begin{array}{llllllllllllll}-2.2894 & 0.6213 & -0.0001 & 0 & 0 & 0 & 0 & 0 & 0 & 0 & 0 & 0 & 0 & 7\end{array}$

$\begin{array}{llllllllllllll}1.2087 & -1.7807 & -0.8848 & \mathrm{H} & 0 & 0 & 0 & 0 & 0 & 0 & 0 & 0 & 0 & 8\end{array}$

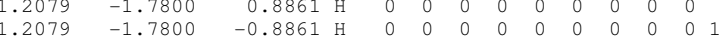

$\begin{array}{llllllllllllll}1.2087 & -1.7806 & 0.8848 & \mathrm{H} & 0 & 0 & 0 & 0 & 0 & 0 & 0 & 0 & 0 & 11\end{array}$

$\begin{array}{llllll}2 & 1 & 0 & 0 & 0 & 0\end{array}$

$\begin{array}{lllll}1 & 0 & 0 & 0 & 0\end{array}$

$\begin{array}{llllll}8 & 1 & 0 & 0 & 0 & 0\end{array}$

$\begin{array}{llllll}5 & 0 & 0 & 0 & 0\end{array}$

$\begin{array}{llllll}10 & 3 & 0 & 0 & 0 & 0\end{array}$

$\begin{array}{lllll}4 & 0 & 0 & 0 & 0\end{array}$

$\begin{array}{llllllllll}6 & 11 & 0 & 0 & 0 & 0\end{array}$ 


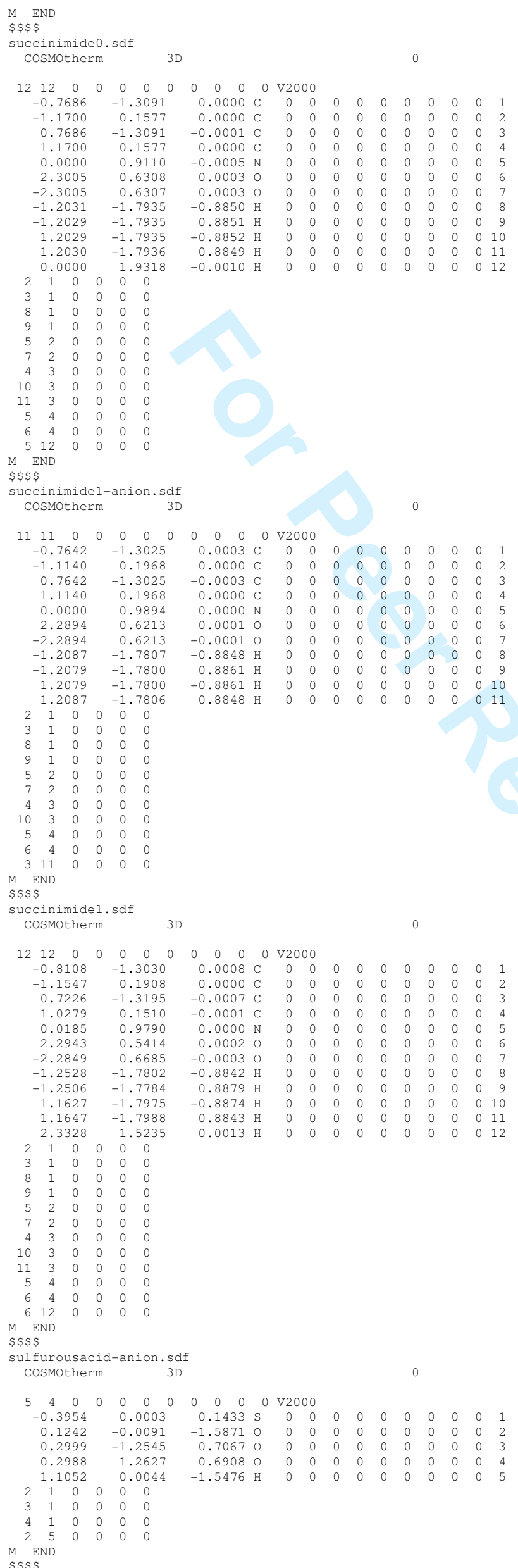




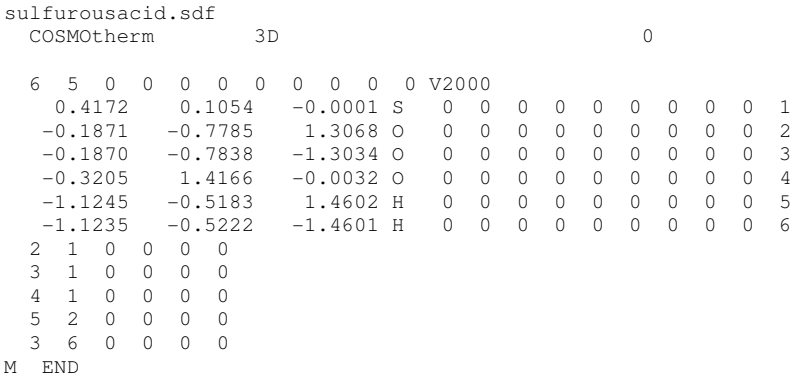




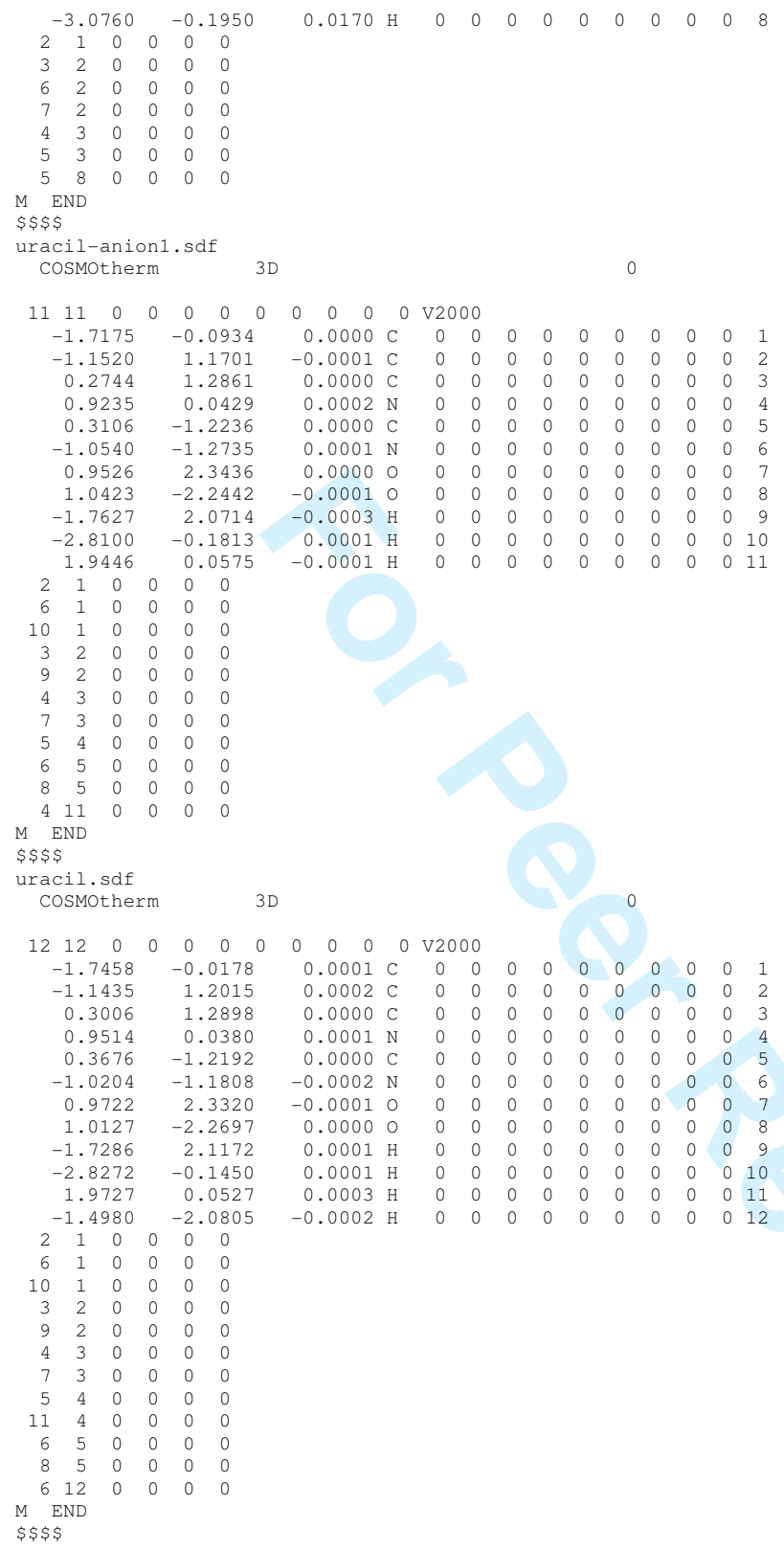

S4 b) Mono-solvated Acids \& Anions

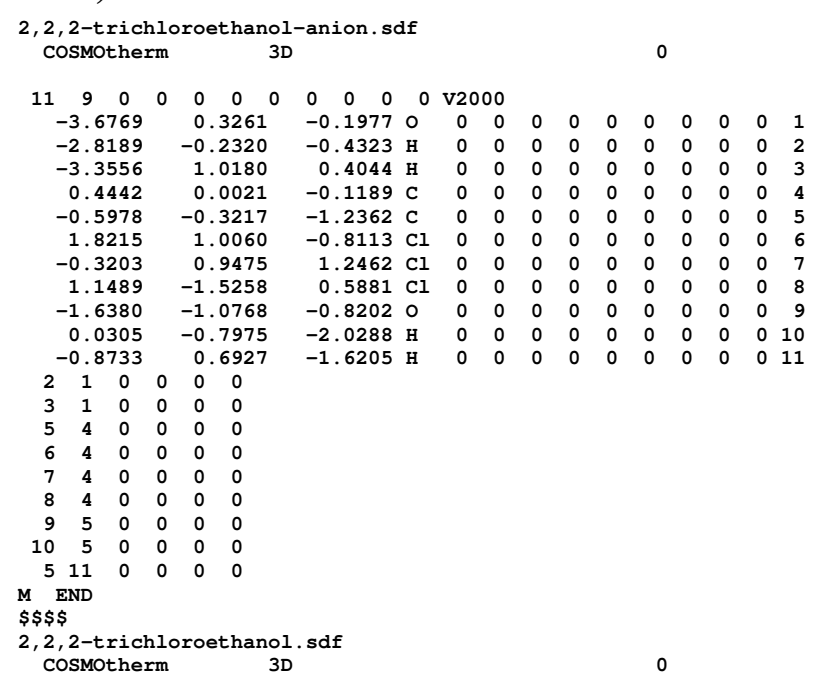




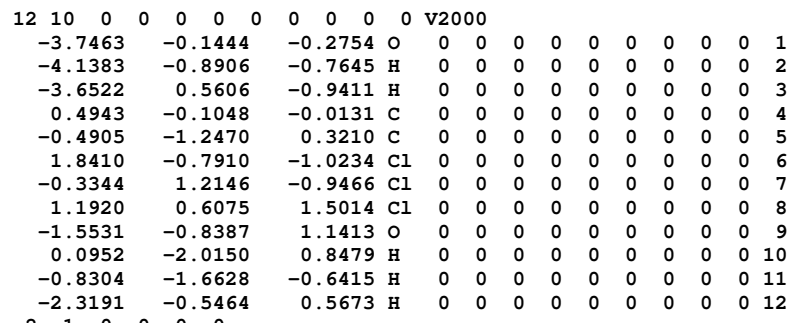

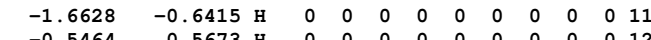

$\begin{array}{lllllll}2 & 1 & 0 & 0 & 0 & 0\end{array}$

$3 \quad 1 \quad 000000$

$5 \quad 4 \quad 0 \quad 0 \quad 000$

$6 \begin{array}{lllllll}6 & 4 & 0 & 0 & 0 & 0\end{array}$

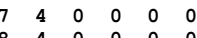

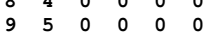

$\begin{array}{rrrrrr}9 & 5 & 0 & 0 & 0 & 0 \\ 10 & 5 & 0 & 0 & 0 & 0\end{array}$

$\begin{array}{rrrrrr}11 & 5 & 0 & 0 & 0 & 0 \\ 9 & 12 & 0 & 0 & 0 & 0\end{array}$

END

\$\$\$

2,2-dimethylpropanoicacid-anion.sdf
CosMOtherm $3 \mathrm{D}$

$\begin{array}{llllllllllll}19 & 17 & 0 & 0 & 0 & 0 & 0 & 0 & 0 & 0 & 0 & \mathrm{v} 2000\end{array}$

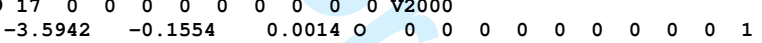

$\begin{array}{lllllllllll}-2.6125 & -0.4207 & -0.0002 & \text { H } & 0 & 0 & 0 & 0 & 0 & 0 & 0\end{array}$

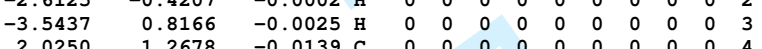

$\begin{array}{llllllllllllll}2.0250 & 1.2678 & -0.0139 & \mathrm{C} & 0 & 0 & 0 & 0 & 0 & 0 & 0 & 0 & 0 & 4\end{array}$

$\begin{array}{llllllllllllll}1.2526 & -0.0598 & 0.0001 & \text { C } & 0 & 0 & 0 & 0 & 0 & 0 & 0 & 0 & 0 & 5 \\ 1.6186 & -0.8592 & 1.2686 & \text { C } & 0 & 0 & 0 & 0 & 0 & 0 & 0 & 0 & 0 & 6\end{array}$

$\begin{array}{lllllllllllllll}1.6210 & -0.8871 & -1.2495 & \mathrm{C} & 0 & 0 & 0 & 0 & 0 & 0 & 0 & 0 & 0 & 7\end{array}$

$\begin{array}{llllllllllllll}-0.2917 & 0.1845 & -0.0023 & \mathrm{C} & 0 & 0 & 0 & 0 & 0 & 0 & 0 & 0 & 0 & 8\end{array}$

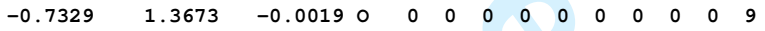

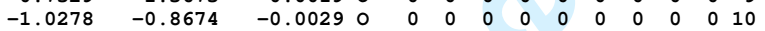

$\begin{array}{llllllllllllll}3.1096 & 1.0743 & -0.0116 & \mathrm{H} & 0 & 0 & 0 & 0 & 0 & 0 & 0 & 0 & 0 & 11\end{array}$

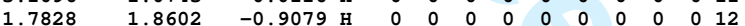

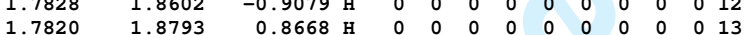

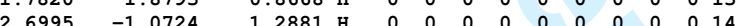

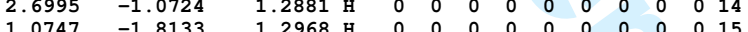

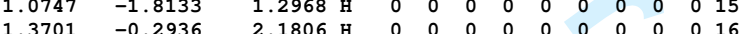

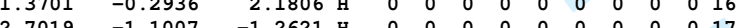

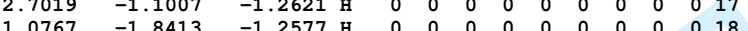

$1.0767-1.8413-1.2577$ H 0

$\begin{array}{cccccccccccccc}1.3744 & -0.3418 & -2.1743 & \mathrm{H} & 0 & 0 & 0 & 0 & 0 & 0 & 0 & 0 & 0 & 19\end{array}$

$\begin{array}{llllll}2 & 1 & 0 & 0 & 0 & 0 \\ 3 & 1 & 0 & 0 & 0 & 0\end{array}$

$\begin{array}{llllll}3 & 1 & 0 & 0 & 0 & 0 \\ 5 & 4 & 0 & 0 & 0 & 0\end{array}$

$11 \quad 4 \quad 000000$

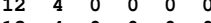

$\begin{array}{rrrrrrr}13 & 4 & 0 & 0 & 0 & 0 \\ 6 & 5 & 0 & 0 & 0 & 0\end{array}$

7 5 $50 \begin{array}{llll}0 & 0 & 0\end{array}$

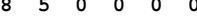

1466000000

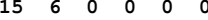

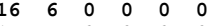

$\begin{array}{llllll}17 & 7 & 0 & 0 & 0 & 0 \\ 17 & 7 & 0 & 0 & 0 & 0\end{array}$

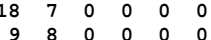

$\begin{array}{llllll}8 & 0 & 0 & 0 & 0\end{array}$

$\begin{array}{lllllll}7 & 19 & 0 & 0 & 0 & 0\end{array}$

M END

\$\$\$

2,2-dimethylpropanoicacid.sdf

cosmotherm 3D

$\begin{array}{llllllllllll}20 & 18 & 0 & 0 & 0 & 0 & 0 & 0 & 0 & 0 & 0 & \mathrm{~V} 2000\end{array}$

$\begin{array}{llllllllllllll}-3.6236 & -0.0263 & 0.1980 & 0 & 0 & 0 & 0 & 0 & 0 & 0 & 0 & 0 & 0 & 1\end{array}$

$\begin{array}{llllllllllllll}-3.8516 & -0.5483 & -0.5932 & \mathrm{H} & 0 & 0 & 0 & 0 & 0 & 0 & 0 & 0 & 0 & 2\end{array}$

$\begin{array}{rrrrrrrrrrrrrr}-3.8582 & -0.5483 & -0.5932 & \mathrm{H} & 0 & 0 & 0 & 0 & 0 & 0 & 0 & 0 & 0 & 1 \\ -3.986 & 0.0180 & \mathrm{H} & 0 & 0 & 0 & 0 & 0 & 0 & 0 & 0 & 0 & 3\end{array}$

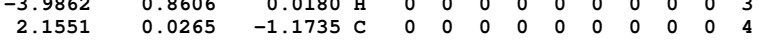

$\begin{array}{llllllllllllll}1.2852 & -0.0005 & 0.0924 & \text { C } & 0 & 0 & 0 & 0 & 0 & 0 & 0 & 0 & 0 & 5\end{array}$

$\begin{array}{rrrrrrrrrrrrrrr}1.2852 & -0.0005 & 0.0924 & C & 0 & 0 & 0 & 0 & 0 & 0 & 0 & 0 & 0 & 5 \\ 1.5802 & 1.2441 & 0.9616 & C & 0 & 0 & 0 & 0 & 0 & 0 & 0 & 0 & 0 & 6\end{array}$

$\begin{array}{rrrrrrrrrrrrrr}1.5802 & 1.2441 & 0.9616 & \text { C } & 0 & 0 & 0 & 0 & 0 & 0 & 0 & 0 & 0 & 6 \\ 1.5755 & -1.2846 & 0.9045 & \text { C } & 0 & 0 & 0 & 0 & 0 & 0 & 0 & 0 & 0 & 7\end{array}$

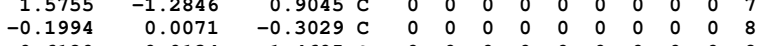

$\begin{array}{lllllllllllllr}-0.6120 & 0.0134 & -1.4605 & 0 & 0 & 0 & 0 & 0 & 0 & 0 & 0 & 0 & 0 & 9\end{array}$

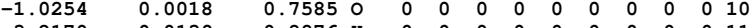

$\begin{array}{lllllllllllll}3.2170 & 0.0139 & -0.8876 \mathrm{H} & 0 & 0 & 0 & 0 & 0 & 0 & 0 & 0 & 0 & 11\end{array}$

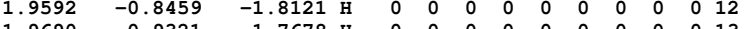

$\begin{array}{lllllllllllll}1.9690 & 0.9321 & -1.7678 \mathrm{H} & 0 & 0 & 0 & 0 & 0 & 0 & 0 & 0 & 0 & 13\end{array}$

$\begin{array}{lllllllllllll}2.6458 & 1.2522 & 1.2353 \mathrm{H} & 0 & 0 & 0 & 0 & 0 & 0 & 0 & 0 & 0 & 14\end{array}$

$\begin{array}{lllllllllllll}0.9859 & 1.2371 & 1.8847 \mathrm{H} & 0 & 0 & 0 & 0 & 0 & 0 & 0 & 0 & 0 & 15\end{array}$

$\begin{array}{llllllllllllllll}1.3631 & 2.1732 & 0.4134 \mathrm{H} & 0 & 0 & 0 & 0 & 0 & 0 & 0 & 0 & 0 & 16\end{array}$

$\begin{array}{lllllllllllll}2.6409 & -1.3089 & 1.1783 \mathrm{H} & 0 & 0 & 0 & 0 & 0 & 0 & 0 & 0 & 0 & 17\end{array}$

$\begin{array}{lllllllllllllll}0.9802 & -1.3171 & 1.8264 & \mathrm{H} & 0 & 0 & 0 & 0 & 0 & 0 & 0 & 0 & 0 & 18\end{array}$

$\begin{array}{llllllllllllll}1.3556 & -2.1871 & 0.3145 \mathrm{H} & 0 & 0 & 0 & 0 & 0 & 0 & 0 & 0 & 0 & 19\end{array}$

$\begin{array}{lllllllllllll}-1.9947 & 0.0035 & 0.4452 \mathrm{H} & 0 & 0 & 0 & 0 & 0 & 0 & 0 & 0 & 0 & 20\end{array}$

211000000

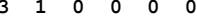

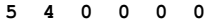

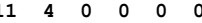

$12 \quad 4 \quad 00001000$

$\begin{array}{llllll}13 & 4 & 0 & 0 & 0 & 0\end{array}$

$6 \begin{array}{llllll}6 & 5 & 0 & 0 & 0 & 0\end{array}$

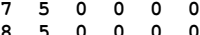

$1460 \begin{array}{llllll}8 & 6 & 0 & 0 & 0 & 0\end{array}$

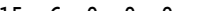

$16 \quad 6 \quad 000000$

$\begin{array}{llllll}17 & 7 & 0 & 0 & 0 & 0\end{array}$

$\begin{array}{llllll}18 & 7 & 0 & 0 & 0 & 0\end{array}$ 


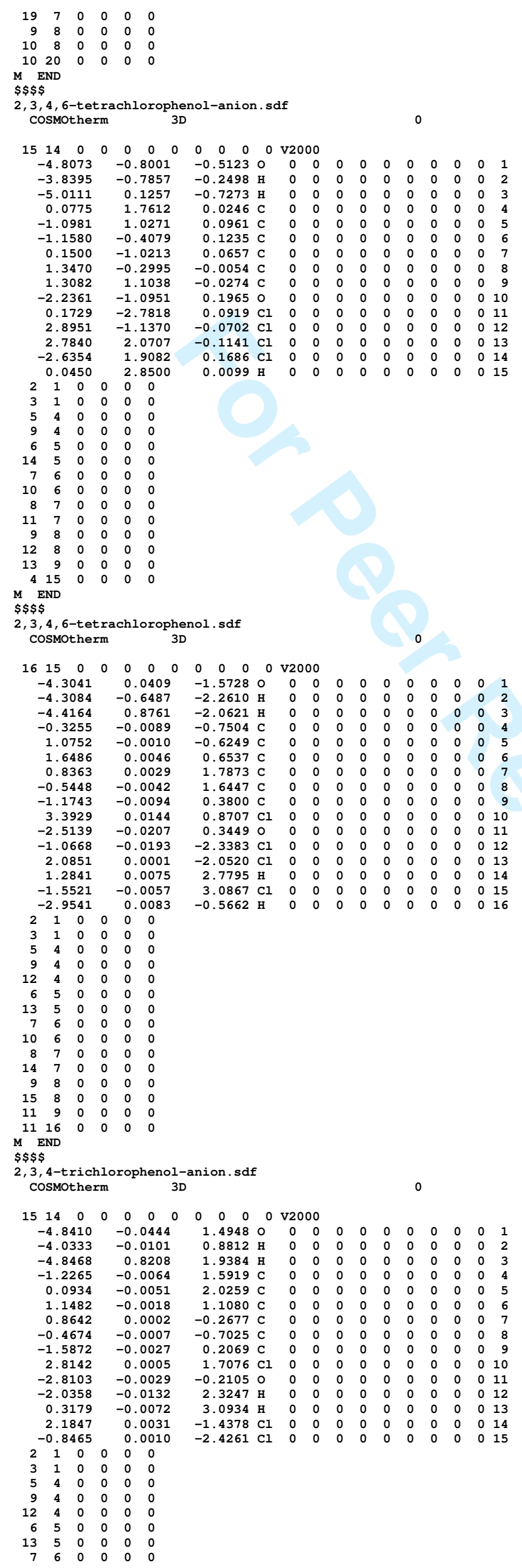




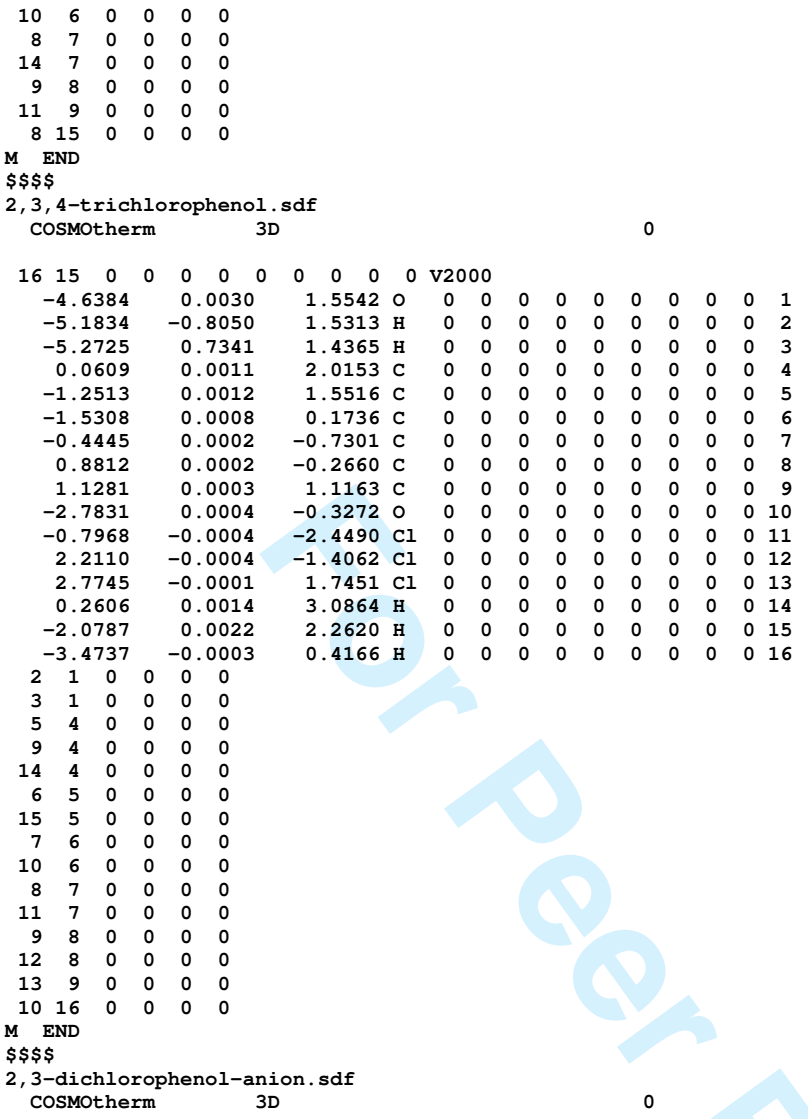

, 3-dichlorophenol-anion.sdf

$1514 \quad 0 \quad 0 \quad 0000000000$ v2000

$\begin{array}{ccccccccccccccc}-4.6680 & -0.0414 & -0.1072 & 0 & 0 & 0 & 0 & 0 & 0 & 0 & 0 & 0 & 0 & 1\end{array}$

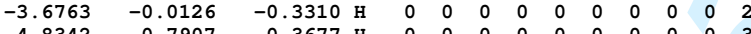

$\begin{array}{rrrrrrrrrrrrrrr}-4.8342 & 0.7907 & 0.3677 & \text { H } & 0 & 0 & 0 & 0 & 0 & 0 & 0 & 0 & 0 & 3 \\ -1.4122 & -0.0080 & 1.4709 & \text { C } & 0 & 0 & 0 & 0 & 0 & 0 & 0 & 0 & 0 & 4\end{array}$

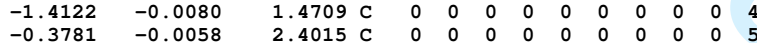

$\begin{array}{rrrrrrrrrrrrrr}-0.3781 & -0.0058 & 2.4015 & \mathrm{C} & 0 & 0 & 0 & 0 & 0 & 0 & 0 & 0 & 0 & 5 \\ 0.9666 & -0.0016 & 2.0031 & \mathrm{C} & 0 & 0 & 0 & 0 & 0 & 0 & 0 & 0 & 0 & 6\end{array}$

$\begin{array}{rrrrrrrrrrrrrrr}0.9666 & -0.0016 & 2.0031 & \mathrm{C} & 0 & 0 & 0 & 0 & 0 & 0 & 0 & 0 & 0 & 5 \\ 1.2403 & 0.0003 & 0.6316 & \mathrm{C} & 0 & 0 & 0 & 0 & 0 & 0 & 0 & 0 & 0 & 7 \\ 0.2182 & -0.003 & -0.3267 & \mathrm{C} & 0 & 0 & 0 & 0 & 0 & 0 & 0 & 0 & 0 & 8\end{array}$

$\begin{array}{rrrrrrrrrrrrrr}1.2403 & 0.0003 & 0.6316 & C & 0 & 0 & 0 & 0 & 0 & 0 & 0 & 0 & 0 & 7 \\ 0.2182 & -0.0018 & -0.3207 & C & 0 & 0 & 0 & 0 & 0 & 0 & 0 & 0 & 0 & 8\end{array}$

$\begin{array}{rrrrrrrrrrrrrr}-1.1753 & -0.0029 & 0.0579 & \mathrm{C} & 0 & 0 & 0 & 0 & 0 & 0 & 0 & 0 & 0 & 9\end{array}$

$\begin{array}{llllllllllllll}1.7802 & 0.0010 & 2.7268 \text { н } & 0 & 0 & 0 & 0 & 0 & 0 & 0 & 0 & 0 & 10\end{array}$

$\begin{array}{llllllllllllll}-2.1232 & -0.0011 & -0.8236 & 0 & 0 & 0 & 0 & 0 & 0 & 0 & 0 & 0 & 0 & 11\end{array}$

$\begin{array}{llllllllllllll}-2.4530 & -0.0158 & 1.8022 & \text { H } & 0 & 0 & 0 & 0 & 0 & 0 & 0 & 0 & 0 & 12\end{array}$

$\begin{array}{llllllllllllll}-0.6131 & -0.0087 & 3.4684 & \text { H } & 0 & 0 & 0 & 0 & 0 & 0 & 0 & 0 & 0 & 13 \\ 2.9467 & 0.043 & 0.273 & \mathrm{Cl} & 0 & 0 & 0 & 0 & 0 & 0 & 0 & 0 & 0 & 14\end{array}$

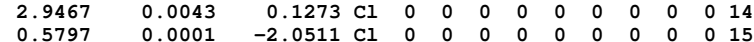
$\begin{array}{lllllll}2 & 1 & 0 & 0 & 0 & 0 \\ 2 & 1 & 0 & 0 & 0 & 0\end{array}$

$\begin{array}{llllll}3 & 1 & 0 & 0 & 0 & 0\end{array}$

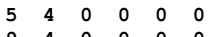

$\begin{array}{rrrrrr}9 & 4 & 0 & 0 & 0 & 0 \\ 12 & 4 & 0 & 0 & 0 & 0\end{array}$

$\begin{array}{llllll}6 & 5 & 0 & 0 & 0 & 0\end{array}$

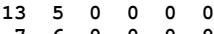

$\begin{array}{llllll}7 & 6 & 6 & 0 & 0 & 0\end{array}$

$\begin{array}{cccccccc}8 & 7 & 0 & 0 & 0 & 0\end{array}$

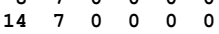

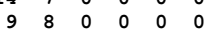

$11 \quad 9600000$

815

$M$ END

2,3-dichlorophenol.sdf

Cosmotherm

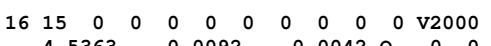

$\begin{array}{llllllllllllll}-4.5363 & -0.0092 & 0.0042 & \circ & 0 & 0 & 0 & 0 & 0 & 0 & 0 & 0 & 0 & 1\end{array}$

$\begin{array}{llllllllllllll}-5.0499 & -0.7579 & -0.3510 \mathrm{H} & 0 & 0 & 0 & 0 & 0 & 0 & 0 & 0 & 0 & 2\end{array}$

$\begin{array}{llllllllllllll}-5.0335 & 0.7842 & -0.2674 & \mathrm{H} & 0 & 0 & 0 & 0 & 0 & 0 & 0 & 0 & 0 & 3\end{array}$

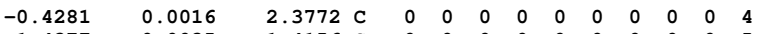

$\begin{array}{llllllllllllll}-1.4377 & 0.0035 & 1.4156 & \mathrm{C} & 0 & 0 & 0 & 0 & 0 & 0 & 0 & 0 & 0 & 5\end{array}$

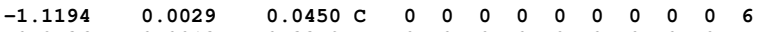

$\begin{array}{llllllllllllll}0.2436 & 0.0013 & -0.3342 & \mathrm{C} & 0 & 0 & 0 & 0 & 0 & 0 & 0 & 0 & 0 & 7\end{array}$

$\begin{array}{llllllllllllll}1.2428 & -0.0004 & 0.6482 & \mathrm{C} & 0 & 0 & 0 & 0 & 0 & 0 & 0 & 0 & 0 & 8\end{array}$

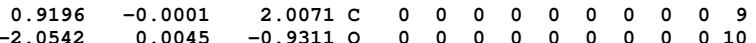

$\begin{array}{llllllllllllll}-2.0542 & 0.0045 & -0.9311 & 0 & 0 & 0 & 0 & 0 & 0 & 0 & 0 & 0 & 0 & 10\end{array}$

$\begin{array}{llllllllllllll}0.6373 & 0.0010 & -2.0468 & \mathrm{Cl} & 0 & 0 & 0 & 0 & 0 & 0 & 0 & 0 & 0 & 11\end{array}$

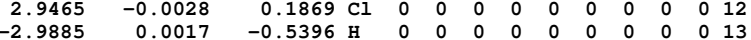

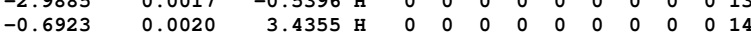

$\begin{array}{lllllllllllllll}-2.4877 & 0.0043 & 1.7114 & \mathrm{H} & 0 & 0 & 0 & 0 & 0 & 0 & 0 & 0 & 0 & 15\end{array}$

$\begin{array}{lllllllllllll}-2.4119 & -0.0014 & 2.7543 \mathrm{H} & 0 & 0 & 0 & 0 & 0 & 0 & 0 & 0 & 0 & 16\end{array}$

$\begin{array}{lllllll}2 & 1 & 0 & 0 & 0 & 0\end{array}$

$\begin{array}{llllll}2 & 1 & 0 & 0 & 0 & 0 \\ 3 & 1 & 0 & 0 & 0 & 0\end{array}$

$\begin{array}{llllll}3 & 1 & 0 & 0 & 0 & 0 \\ 5 & 4 & 0 & 0 & 0 & 0\end{array}$

$9 \begin{array}{llllll}9 & 4 & 0 & 0 & 0 & 0\end{array}$

$14 \quad 4 \quad 0 \quad 0000$ 


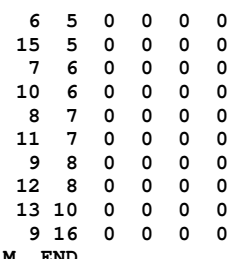

M END

$\$ \$ \$ \$$
$2,4,5-$ trichlorophenol-anion. sdf
CoSMOtherm

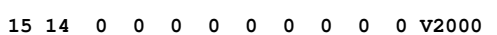

$\begin{array}{cclllllllllllllll}15 & 14 & 0 & 0 & 0 & 0 & 0 & 0 & 0 & 0 & 0 & \mathrm{~V} 2000 \\ -3.3026 & 3.2156 & 0.00655 & 0 & 0 & 0 & 0 & 0 & 0 & 0 & 0 & 0 & 0 & 1\end{array}$

$\begin{array}{rrrrrrrrrrrrrr}-3.3026 & 3.2156 & 0.0065 & \circ & 0 & 0 & 0 & 0 & 0 & 0 & 0 & 0 & 0 & 1 \\ -2.3210 & 2.9607 & -0.0019 & \mathrm{H} & 0 & 0 & 0 & 0 & 0 & 0 & 0 & 0 & 0 & 2\end{array}$

$\begin{array}{llllllllllllll}-3.6270 & 2.9590 & -0.8734 & \mathrm{H} & 0 & 0 & 0 & 0 & 0 & 0 & 0 & 0 & 0 & 3 \\ -1.1848 & 0.3035 & 0.0213 & \mathrm{C} & 0 & 0 & 0 & 0 & 0 & 0 & 0 & 0 & 0 & 4\end{array}$

$\begin{array}{rrrrrrrrrrrrrr}-1.1848 & 0.3035 & 0.0213 & \mathrm{C} & 0 & 0 & 0 & 0 & 0 & 0 & 0 & 0 & 0 & 4 \\ -0.7324 & -1.0105 & 0.0109 & \mathrm{C} & 0 & 0 & 0 & 0 & 0 & 0 & 0 & 0 & 0 & 5\end{array}$

$\begin{array}{rrrrrrrrrrrrrr}-0.7324 & -1.0105 & 0.0109 & \mathrm{C} & 0 & 0 & 0 & 0 & 0 & 0 & 0 & 0 & 0 & 5 \\ 0.6382 & -1.3052 & -0.0039 & \mathrm{C} & 0 & 0 & 0 & 0 & 0 & 0 & 0 & 0 & 0 & 6\end{array}$

$\begin{array}{llllllllllllll}0.6382 & -1.3052 & -0.0039 & \mathrm{C} & 0 & 0 & 0 & 0 & 0 & 0 & 0 & 0 & 0 & 6 \\ 1.5515 & -0.2443 & -0.0072 & \mathrm{C} & 0 & 0 & 0 & 0 & 0 & 0 & 0 & 0 & 0 & 7\end{array}$

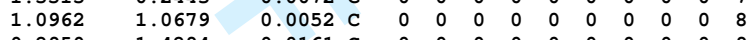

$\begin{array}{llllllllllllll}-0.2950 & 1.4294 & 0.0161 & \mathrm{C} & 0 & 0 & 0 & 0 & 0 & 0 & 0 & 0 & 0 & 9\end{array}$

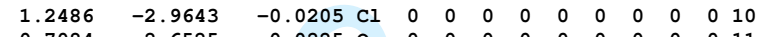

$\begin{array}{llllllllllllll}-0.7084 & 2.6525 & 0.0225 & 0 & 0 & 0 & 0 & 0 & 0 & 0 & 0 & 0 & 0 & 11\end{array}$

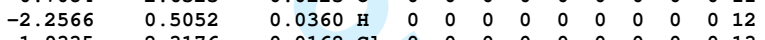

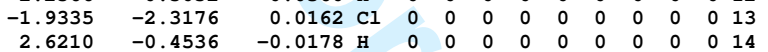

$\begin{array}{rrrrrrrrrrrrrrr}2.2900 & 2.3823 & 0.0012 & \text { Cl } & 0 & 0 & 0 & 0 & 0 & 0 & 0 & 0 & 0 & 15\end{array}$

21000000

$\begin{array}{llllll}3 & 1 & 0 & 0 & 0 & 0\end{array}$

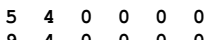

9 \begin{tabular}{llllll}
9 & 4 & 0 & 0 & 0 & 0 \\
\hline & 4 & 0 & 0 & 0 & 0
\end{tabular}

$\begin{array}{rrrrrr}12 & 4 & 0 & 0 & 0 & 0 \\ 6 & 5 & 0 & 0 & 0 & 0\end{array}$

13551000000

$\begin{array}{lllllll}10 & 6 & 0 & 0 & 0 & 0\end{array}$

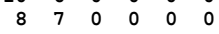

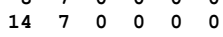

$\begin{array}{llllll}9 & 8 & 0 & 0 & 0 & 0 \\ 1 & 9 & 0 & 0 & 0 & 0\end{array}$

$\begin{array}{rrrrrr}1 & 9 & 0 & 0 & 0 & 0 \\ 8 & 15 & 0 & 0 & 0 & 0\end{array}$

815
$M \quad$ END

$M$ END
\$\$\$

2, 4,5-trichlorophenol.sdf

4, -trichlorophenol.s
CosMotherm

$\begin{array}{llllllllllll}16 & 15 & 0 & 0 & 0 & 0 & 0 & 0 & 0 & 0 & 0 & \mathrm{v} 2000\end{array}$

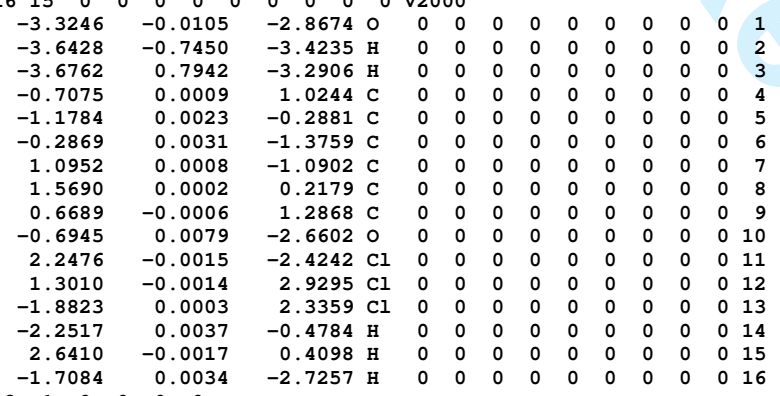

$\begin{array}{llllll}2 & 1 & 0 & 0 & 0 & 0 \\ 3 & 1 & 0 & 0 & 0 & 0\end{array}$

$\begin{array}{llllll}3 & 1 & 0 & 0 & 0 & 0 \\ 5 & 4 & 0 & 0 & 0 & 0\end{array}$

$\begin{array}{llllll}9 & 4 & 0 & 0 & 0 & 0\end{array}$

$\begin{array}{rrrrrr}9 & 4 & 0 & 0 & 0 & 0 \\ 13 & 4 & 0 & 0 & 0 & 0\end{array}$

$\begin{array}{rrrrrrr}13 & 4 & 0 & 0 & 0 & 0 \\ 6 & 5 & 0 & 0 & 0 & 0\end{array}$

1455000000

$\begin{array}{rrrrrr}14 & 5 & 0 & 0 & 0 & 0 \\ 7 & 6 & 0 & 0 & 0 & 0\end{array}$

$10 \quad 6 \quad 000000$

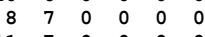

$\begin{array}{rrrrrrr}11 & 7 & 0 & 0 & 0 & 0 \\ 9 & 8 & 0 & 0 & 0 & 0\end{array}$

$\begin{array}{rrrrrr}9 & 8 & 0 & 0 & 0 & 0 \\ 15 & 8 & 0 & 0 & 0 & 0\end{array}$

$\begin{array}{rrrrrr}12 & 9 & 0 & 0 & 0 & 0 \\ 10 & 16 & 0 & 0 & 0 & 0\end{array}$

$M$ END

$\$ \$ \$ \$$ 2, $4,6-$ trichlorophenol-anion.sdf

CosMotherm 3D

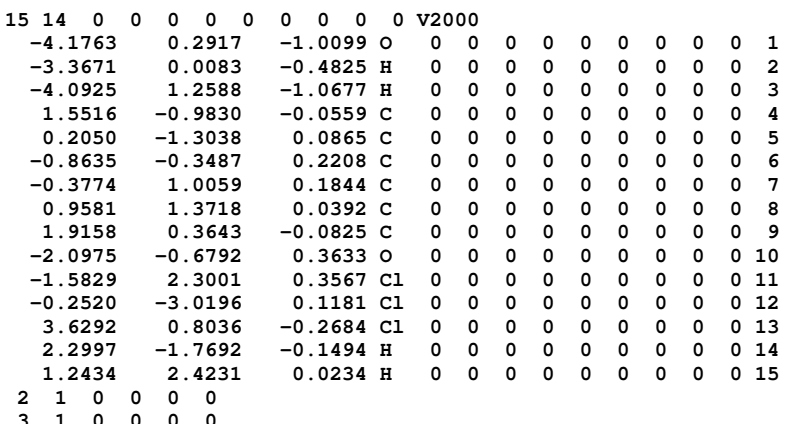

$\begin{array}{llllll}2 & 1 & 0 & 0 & 0 & 0 \\ 3 & 1 & 0 & 0 & 0 & 0\end{array}$ 


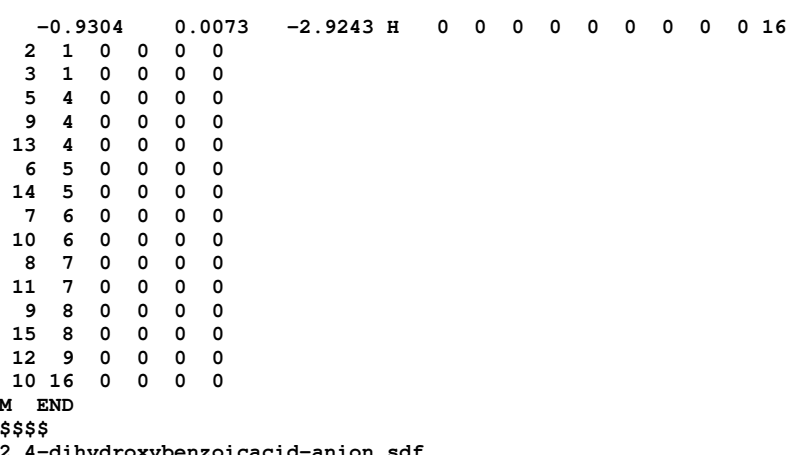




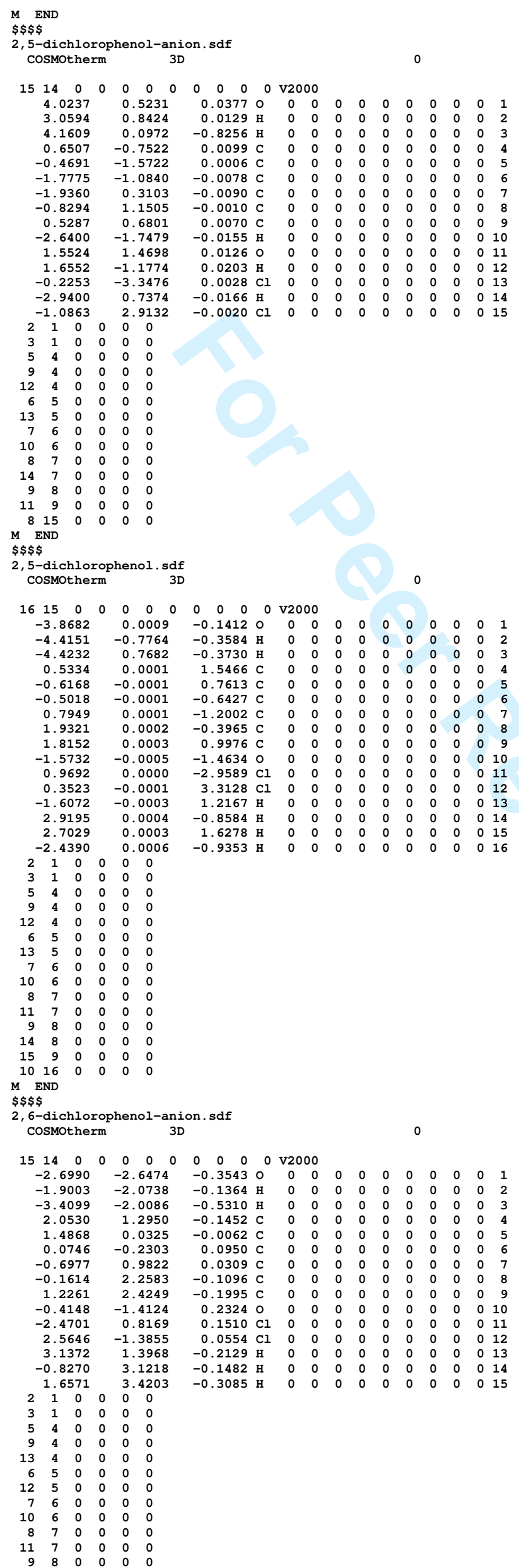




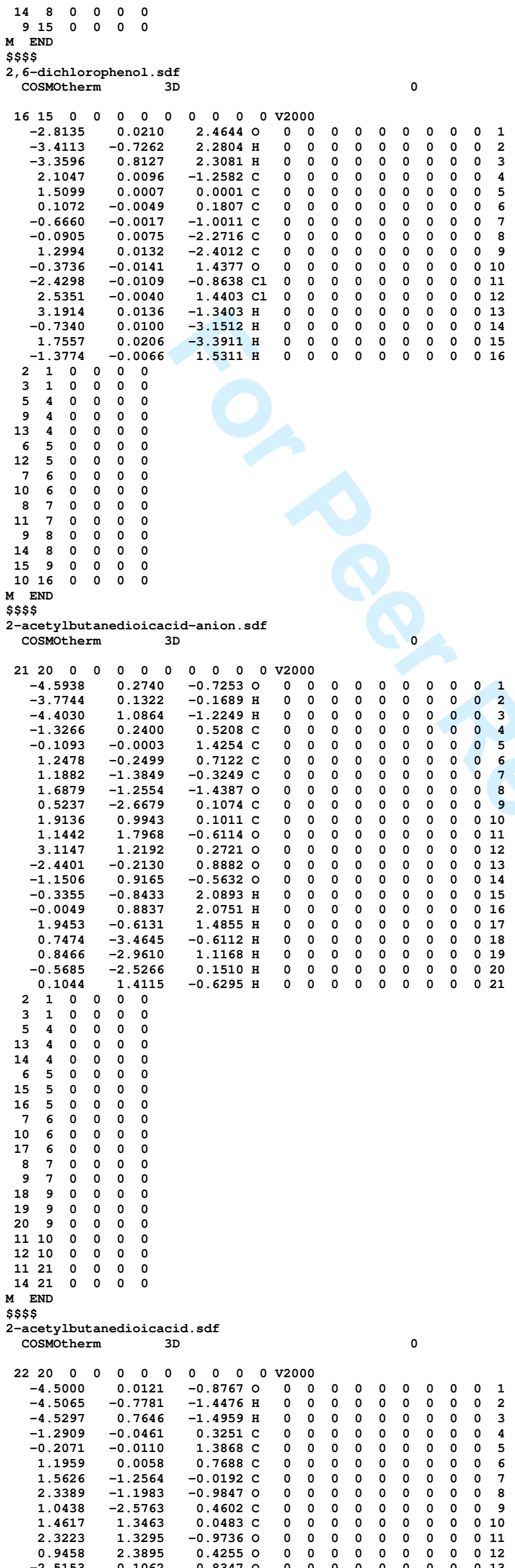




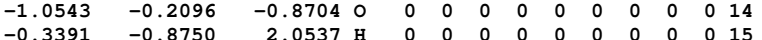

$\begin{array}{llllllllllllll}-0.3487 & 0.8794 & 2.0537 & \mathrm{H} & 0 & 0 & 0 & 0 & 0 & 0 & 0 & 0 & 0 & 15 \\ 1.9177 & -0.0020 & 1.6125 & \mathrm{H} & 0 & 0 & 0 & 0 & 0 & 0 & 0 & 0 & 0 & 16\end{array}$

$\begin{array}{llllllllllllll}1.9177 & -0.0020 & 1.6103 \mathrm{H} & 0 & 0 & 0 & 0 & 0 & 0 & 0 & 0 & 0 & 17\end{array}$

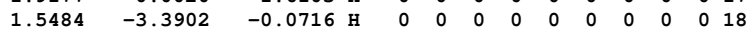

$\begin{array}{rrrrrrrrrrrrrrr}1.1943 & -2.6807 & 1.5452 \mathrm{H} & 0 & 0 & 0 & 0 & 0 & 0 & 0 & 0 & 0 & 19 \\ -0.0497 & -2.6457 & 0.2790 & \text { H } & 0 & 0 & 0 & 0 & 0 & 0 & 0 & 0 & 0 & 20\end{array}$

$\begin{array}{lllllllllllllll}2.5411 & 0.3469 & -1.1614 \mathrm{H} & 0 & 0 & 0 & 0 & 0 & 0 & 0 & 0 & 0 & 20 \\ 2 & 0.0585 & 0.045 & 0 & 0 & 0 & 0 & 0 & 0 & 0 & 0 & 0 & 22\end{array}$

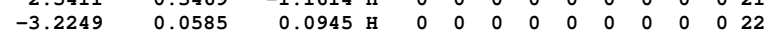

21000000

$\begin{array}{lllllll}3 & 1 & 0 & 0 & 0 & 0 \\ 3 & 4 & 0 & 0 & 0 & 0\end{array}$

$\begin{array}{llllll}3 & 1 & 0 & 0 & 0 & 0 \\ 5 & 4 & 0 & 0 & 0 & 0\end{array}$

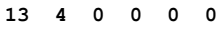

14

6 5 510000100

$\begin{array}{llllll}15 & 5 & 0 & 0 & 0 & 0 \\ 16 & 5 & 0 & 0 & 0 & 0\end{array}$

$\begin{array}{rrrrrrr}16 & 5 & 0 & 0 & 0 & 0 \\ 7 & 6 & 0 & 0 & 0 & 0\end{array}$

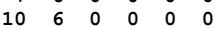

$17 \quad 6 \quad \begin{array}{llllll}17 & 6 & 0 & 0 & 0\end{array}$

$8 \begin{array}{llllll}8 & 7 & 0 & 0 & 0 & 0 \\ 9 & 7 & 0 & 0 & 0 & 0\end{array}$

$\begin{array}{rrrrrr}9 & 7 & 0 & 0 & 0 & 0 \\ 18 & 9 & 0 & 0 & 0 & 0\end{array}$

$\begin{array}{llllll}19 & 9 & 0 & 0 & 0 & 0 \\ 20 & 9 & 0 & 0 & 0 & 0\end{array}$

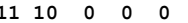

1210 12 10000

$\begin{array}{llllll}21 & 11 & 0 & 0 & 0 & 0 \\ 13 & 22 & 0 & 0 & 0 & 0\end{array}$

$M \quad$ END

\$\$\$

2-chlorophenol-anion.sdf

CosMotherm 3D

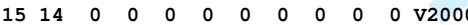

$\begin{array}{llllllllllllll}-3.9304 & -0.5244 & 0.0299 & 0 & 0 & 0 & 0 & 0 & 0 & 0 & 0 & 0 & 0 & 1\end{array}$

$\begin{array}{lllllllllllllll}-2.9164 & -0.6410 & -0.0026 \text { н } & 0 & 0 & 0 & 0 & 0 & 0 & 0 & 0 & 0 & 2\end{array}$

$\begin{array}{llllllllllllll}-4.0944 & -0.0481 & 0.8615 \text { н } & 0 & 0 & 0 & 0 & 0 & 0 & 0 & 0 & 0 & 3\end{array}$

$\begin{array}{lllllllllllllll}-0.8842 & 1.4030 & -0.0546 \mathrm{C} & 0 & 0 & 0 & 0 & 0 & 0 & 0 & 0 & 0 & 4 \\ 1.448 & 2.4511 & -0.0156 \mathrm{C} & 0 & 0 & 0 & 0 & 0 & 0 & 0 & 0 & 0 & 5\end{array}$

$\begin{array}{lllllllllllll}0.0349 & 2.4511 & -0.0156 \mathrm{C} & 0 & 0 & 0 & 0 & 0 & 0 & 0 & 0 & 0 & 5\end{array}$

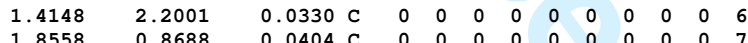

$\begin{array}{llllllllllllll}1.8558 & 0.8688 & 0.0404 & C & 0 & 0 & 0 & 0 & 0 & 0 & 0 & 0 & 0 & 6 \\ 0.9298 & -0.1699 & 0.0017 & C & 0 & 0 & 0 & 0 & 0 & 0 & 0 & 0 & 0 & 7\end{array}$

$\begin{array}{rrrrrrrrrrrrrr}1.9298 & -0.1699 & 0.0017 & \mathrm{C} & 0 & 0 & 0 & 0 & 0 & 0 & 0 & 0 & 0 & 8 \\ -0.4918 & 0.0228 & -0.0481 & \mathrm{C} & 0 & 0 & 0 & 0 & 0 & 0 & 0 & 0 & 0 & 9\end{array}$

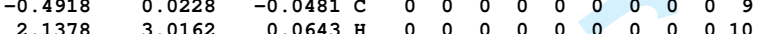

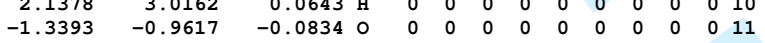

$\begin{array}{rrrrrrrrrrrrrrr}-1.3393 & -0.9617 & -0.0834 & \text { Н } & 0 & 0 & 0 & 0 & 0 & 0 & 0 & 0 & 0 & 11 \\ -1.9563 & 1.6125 & -0.0964 & \text { H } & 0 & 0 & 0 & 0 & 0 & 0 & 0 & 0 & 0 & 12\end{array}$

$\begin{array}{llllllllllllll}-1.953 & 1.6125 & -0.0964 & \mathrm{H} & 0 & 0 & 0 & 0 & 0 & 0 & 0 & 0 & 0 & 11 \\ -0.3300 & 3.4815 & -0.0230 \mathrm{H} & 0 & 0 & 0 & 0 & 0 & 0 & 0 & 0 & 0 & 13\end{array}$

$\begin{array}{llllllllllllll}2.9224 & 0.6387 & 0.0778 \text { H } & 0 & 0 & 0 & 0 & 0 & 0 & 0 & 0 & 0 & 14\end{array}$

$\begin{array}{lllllllllllll}1.5299 & -1.8541 & 0.0136 \mathrm{Cl} & 0 & 0 & 0 & 0 & 0 & 0 & 0 & 0 & 0 & 15\end{array}$

$\begin{array}{llllll}2 & 1 & 0 & 0 & 0 & 0 \\ 3 & 1 & 0 & 0 & 0 & 0\end{array}$

$\begin{array}{llllll}3 & 1 & 0 & 0 & 0 & 0 \\ 5 & 4 & 0 & 0 & 0 & 0\end{array}$

$9 \begin{array}{lllllll}9 & 4 & 0 & 0 & 0 & 0\end{array}$

12

$\begin{array}{lllllll}6 & 5 & 0 & 0 & 0 & 0 \\ 6 & 5 & 0 & 0 & -1\end{array}$

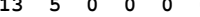

$\begin{array}{lllllll}7 & 6 & 0 & 0 & 0 & 0\end{array}$

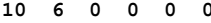

$8 \begin{array}{lllllll}8 & 7 & 0 & 0 & 0 & 0\end{array}$

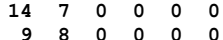

$9 \begin{array}{rrrrrrrr}9 & 8 & 0 & 0 & 0 & 0 \\ 11 & 9 & 0 & 0 & 0 & 0\end{array}$

$\begin{array}{rrrrrrr}8 & 15 & 0 & 0 & 0 & 0 \\ 8 & 15 & 0\end{array}$

$M$ END

$\$ \$ \$ \$$

2-chlorophenol.sdf

cosmotherm 3D

$\begin{array}{llllllllllll}16 & 15 & 0 & 0 & 0 & 0 & 0 & 0 & 0 & 0 & 0 & \mathrm{v} 2000\end{array}$

$\begin{array}{llllllllllllll}-3.8343 & -0.0003 & -0.5149 & 0 & 0 & 0 & 0 & 0 & 0 & 0 & 0 & 0 & 0 & 1 \\ -4.2977 & -0.7268 & -0.9707 & \mathrm{H} & 0 & 0 & 0 & 0 & 0 & 0 & 0 & 0 & 0 & 2\end{array}$

$\begin{array}{rrrrrrrrrrrrrr}-4.2977 & -0.7268 & -0.9707 & \mathrm{H} & 0 & 0 & 0 & 0 & 0 & 0 & 0 & 0 & 0 & 1 \\ -4.2730 & 0.8100 & -0.8332 & \mathrm{H} & 0 & 0 & 0 & 0 & 0 & 0 & 0 & 0 & 0 & 3\end{array}$

$\begin{array}{lrrrrrrrrrrrrr}-4.2730 & 0.8100 & -0.8332 & \mathrm{H} & 0 & 0 & 0 & 0 & 0 & 0 & 0 & 0 & 0 & 3 \\ -0.0820 & -0.0016 & 2.4354 & \mathrm{C} & 0 & 0 & 0 & 0 & 0 & 0 & 0 & 0 & 0 & 4\end{array}$

$\begin{array}{llllllllllllll}-0.0820 & -0.0016 & 2.4354 & \mathrm{C} & 0 & 0 & 0 & 0 & 0 & 0 & 0 & 0 & 0 & 4 \\ -0.9441 & -0.0037 & 1.3365 & \mathrm{C} & 0 & 0 & 0 & 0 & 0 & 0 & 0 & 0 & 0 & 5\end{array}$

$\begin{array}{llllllllllllll}-0.9441 & -0.0037 & 1.3365 & \mathrm{C} & 0 & 0 & 0 & 0 & 0 & 0 & 0 & 0 & 0 & 5 \\ -0.4387 & -0.0026 & 0.0216 & \mathrm{C} & 0 & 0 & 0 & 0 & 0 & 0 & 0 & 0 & 0 & 6\end{array}$

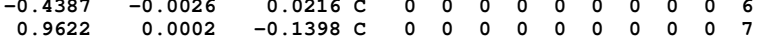

$\begin{array}{lrrrrrrrrrrrrr}0.9622 & 0.0002 & -0.1398 & \mathrm{C} & 0 & 0 & 0 & 0 & 0 & 0 & 0 & 0 & 0 & 7 \\ 1.8272 & 0.0024 & 0.9535 & \mathrm{C} & 0 & 0 & 0 & 0 & 0 & 0 & 0 & 0 & 0 & 8\end{array}$

$\begin{array}{llllllllllllll}1.8272 & 0.0024 & 0.9535 & \mathrm{C} & 0 & 0 & 0 & 0 & 0 & 0 & 0 & 0 & 0 & 8 \\ 1.3054 & 0.0016 & 2.2518 & \mathrm{C} & 0 & 0 & 0 & 0 & 0 & 0 & 0 & 0 & 0 & 9\end{array}$

$\begin{array}{rrrrrrrrrrrrrr}1.3054 & 0.0016 & 2.2518 & \mathrm{C} & 0 & 0 & 0 & 0 & 0 & 0 & 0 & 0 & 0 & 9 \\ -1.2294 & -0.0044 & -1.0787 & 0 & 0 & 0 & 0 & 0 & 0 & 0 & 0 & 0 & 0 & 10\end{array}$

$\begin{array}{lllllllllllllll}1.6335 & 0.0011 & -1.7806 & \mathrm{Cl} & 0 & 0 & 0 & 0 & 0 & 0 & 0 & 0 & 0 & 11\end{array}$

$\begin{array}{lllllllllllllll}-2.2070 & -0.0006 & -0.8227 & \mathrm{H} & 0 & 0 & 0 & 0 & 0 & 0 & 0 & 0 & 0 & 12\end{array}$

$\begin{array}{llllllllllllll}-0.5020 & -0.0024 & 3.4427 & \mathrm{H} & 0 & 0 & 0 & 0 & 0 & 0 & 0 & 0 & 0 & 13\end{array}$

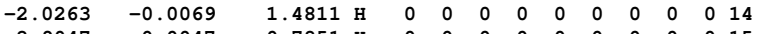

$\begin{array}{llllllllllllll}2.9047 & 0.0047 & 0.7851 & \mathrm{H} & 0 & 0 & 0 & 0 & 0 & 0 & 0 & 0 & 0 & 15 \\ 1.9819 & 0.0034 & 3.1070 & \mathrm{H} & 0 & 0 & 0 & 0 & 0 & 0 & 0 & 0 & 0 & 16\end{array}$

1.98190 .0034

$\begin{array}{llllll}2 & 1 & 0 & 0 & 0 & 0\end{array}$

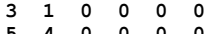

$\begin{array}{llllll}5 & 4 & 0 & 0 & 0 & 0 \\ 9 & 4 & 0 & 0 & 0 & 0\end{array}$

$\begin{array}{llllll}9 & 4 & 0 & 0 & 0 & 0\end{array}$

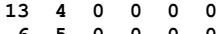

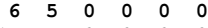

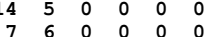

$\begin{array}{lllllll}10 & 6 & 0 & 0 & 0 & 0\end{array}$

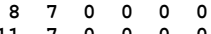

$\begin{array}{llllll}11 & 7 & 0 & 0 & 0 & 0 \\ 9 & 8 & 0 & 0 & 0 & 0\end{array}$

$\begin{array}{rrrrrrr}9 & 8 & 0 & 0 & 0 & 0 \\ 5 & 8 & 0 & 0 & 0 & 0\end{array}$

$1210 \quad 0 \quad 0000$

$9 \begin{array}{llllll}16 & 0 & 0 & 0 & 0\end{array}$

$M$ END

-chloropropanoicacid-anion.sdf 


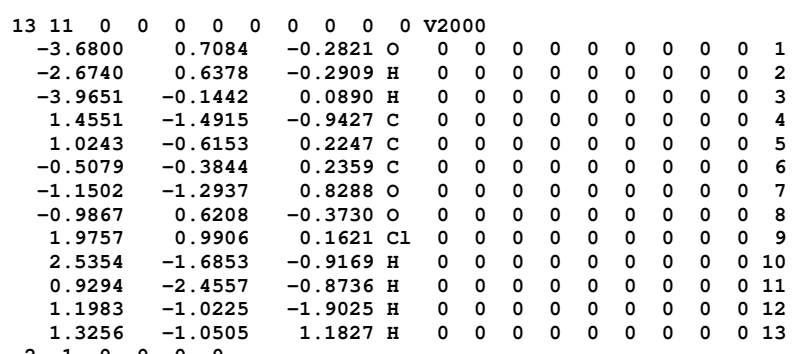

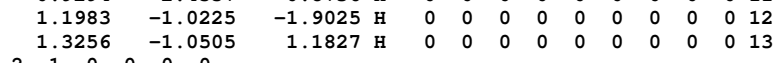
$\begin{array}{llllll}2 & 1 & 0 & 0 & 0 & 0 \\ 3 & 1 & 0 & 0 & 0 & 0 \\ 5 & 4 & 0 & 0 & 0 & 0\end{array}$ 

$\begin{array}{llllllllllllll}2.8660 & -0.3178 & 1.6729 & \mathrm{H} & 0 & 0 & 0 & 0 & 0 & 0 & 0 & 0 & 0 & 10 \\ 1.1943 & -0.3293 & 2.2923 & \mathrm{H} & 0 & 0 & 0 & 0 & 0 & 0 & 0 & 0 & 0 & 11 \\ 1.7288 & -1.5855 & 1.1361 & \mathrm{H} & 0 & 0 & 0 & 0 & 0 & 0 & 0 & 0 & 0 & 12\end{array}$

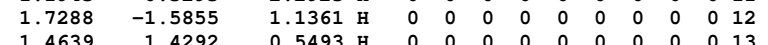

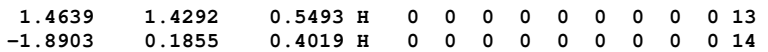
$\begin{array}{lllllllllllllll}1.7064 & -0.2204 & -1.5815 & \mathrm{H} & 0 & 0 & 0 & 0 & 0 & 0 & 0 & 0 & 0 & 15\end{array}$ $\begin{array}{lllllll}2 & 1 & 0 & 0 & 0 & 0\end{array}$

$\begin{array}{llllll}2 & 1 & 0 & 0 & 0 & 0\end{array}$

$\begin{array}{rlllll}5 & 4 & 0 & 0 & 0 & 0 \\ 10 & 4 & 0 & 0 & 0 & 0\end{array}$

104

$\begin{array}{llllll}11 & 4 & 0 & 0 & 0 & 0 \\ 12 & 4 & 0 & 0 & 0 & 0\end{array}$

$\begin{array}{rrllll}12 & 4 & 0 & 0 & 0 & 0 \\ 9 & 5 & 0 & 0 & 0 & 0\end{array}$

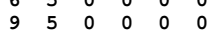

$\begin{array}{llllll}13 & 5 & 0 & 0 & 0 & 0\end{array}$

$7 \begin{array}{lllllll}7 & 5 & 0 & 0 & 0 & 0\end{array}$

$8 \begin{array}{llllll}7 & 6 & 0 & 0 & 0 & 0 \\ 8 & 6 & 0 & 0 & 0 & 0\end{array}$

$\begin{array}{rrrrrr}14 & 8 & 0 & 0 & 0 & 0 \\ 9 & 15 & 0 & 0 & 0 & 0\end{array}$

M END
$\$ \$ \$ \$$
$3,3-d i m e t h$

3,3-dimethylsuccinimide-anion. cosmotherm

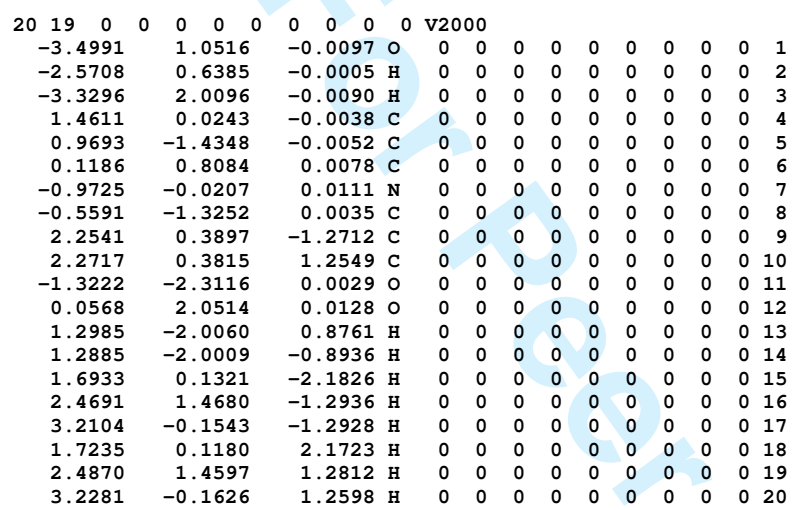

$\begin{array}{llllll}2 & 1 & 0 & 0 & 0 & 0\end{array}$

$\begin{array}{llllll}3 & 1 & 0 & 0 & 0 & 0\end{array}$

$\begin{array}{llllll}5 & 4 & 0 & 0 & 0 & 0\end{array}$

$\begin{array}{llllll}6 & 4 & 0 & 0 & 0 & 0 \\ 9 & 4 & 0 & 0 & 0 & 0\end{array}$

$10 \quad 4 \quad 000000$

855000000

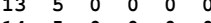

$\begin{array}{rrrrrr}14 & 5 & 0 & 0 & 0 & 0 \\ 7 & 6 & 0 & 0 & 0 & 0\end{array}$

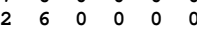

$8 \begin{array}{llllll}8 & 0 & 0 & 0 & 0\end{array}$

$\begin{array}{llllll}11 & 8 & 0 & 0 & 0 & 0\end{array}$

$\begin{array}{llllll}15 & 9 & 0 & 0 & 0 & 0 \\ 16 & 9 & 0 & 0 & 0 & 0\end{array}$

$\begin{array}{llllll}16 & 9 & 0 & 0 & 0 & 0 \\ 17 & 9 & 0 & 0 & 0 & 0\end{array}$

$\begin{array}{rrrrrr}17 & 9 & 0 & 0 & 0 & 0 \\ 18 & 10 & 0 & 0 & 0 & 0\end{array}$

$\begin{array}{llllll}18 & 10 & 0 & 0 & 0 & 0 \\ 19 & 10 & 0 & 0 & 0 & 0\end{array}$

$10 \begin{array}{lllll}10 & 20 & 0 & 0 & 0\end{array}$

M END

$\$ \$ \$ \$$

3,3-dimethylsuccinimide.sdf

cosmotherm 3D

$2120 \quad 0 \quad 00000000000000$

$\begin{array}{llllllllllllll}-3.5316 & -0.0012 & 1.0718 & \circ & 0 & 0 & 0 & 0 & 0 & 0 & 0 & 0 & 0 & 1 \\ -4.0472 & -0.7741 & 0.7785 & \text { H } & 0 & 0 & 0 & 0 & 0 & 0 & 0 & 0 & 0 & 2\end{array}$

$\begin{array}{rrrrrrrrrrrrrr}-4.0472 & -0.7741 & 0.7785 & \mathrm{H} & 0 & 0 & 0 & 0 & 0 & 0 & 0 & 0 & 0 & 1 \\ -4.0571 & 0.7640 & 0.7759 & \mathrm{H} & 0 & 0 & 0 & 0 & 0 & 0 & 0 & 0 & 0 & 3\end{array}$

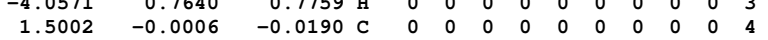

$\begin{array}{rrrrrrrrrrrrrr}1.5002 & -0.0006 & -0.0190 & \text { C } & 0 & 0 & 0 & 0 & 0 & 0 & 0 & 0 & 0 & 4 \\ 0.9240 & 0.0000 & -1.4561 & \text { C } & 0 & 0 & 0 & 0 & 0 & 0 & 0 & 0 & 0 & 5\end{array}$

$\begin{array}{rrrrrrrrrrrrrr}0.2383 & 0.0012 & 0.8659 & \text { C } & 0 & 0 & 0 & 0 & 0 & 0 & 0 & 0 & 0 & 6\end{array}$

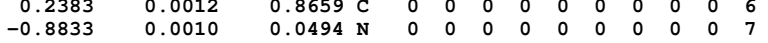

$\begin{array}{llrrrrrrrrrrrr}-0.8833 & 0.0010 & 0.0494 & \mathrm{~N} & 0 & 0 & 0 & 0 & 0 & 0 & 0 & 0 & 0 & 7 \\ -0.5874 & 0.0007 & -1.3062 & \mathrm{C} & 0 & 0 & 0 & 0 & 0 & 0 & 0 & 0 & 0 & 8\end{array}$

$\begin{array}{rrrrrrrrrrrrrr}2.3257 & 1.2644 & 0.2852 & \mathrm{C} & 0 & 0 & 0 & 0 & 0 & 0 & 0 & 0 & 0 & 9\end{array}$

$\begin{array}{llllllllllllll}2.3218 & -1.2681 & 0.2854 & \mathrm{C} & 0 & 0 & 0 & 0 & 0 & 0 & 0 & 0 & 0 & 10\end{array}$

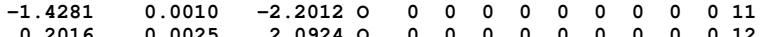

$\begin{array}{rrrrrrrrrrrrrr}0.2016 & 0.0025 & 2.0924 & 0 & 0 & 0 & 0 & 0 & 0 & 0 & 0 & 0 & 0 & 12 \\ -1.8617 & 0.0022 & 0.4195 & \mathrm{H} & 0 & 0 & 0 & 0 & 0 & 0 & 0 & 0 & 0 & 13\end{array}$

$\begin{array}{rrrrrrrrrrrrrr}-1.8617 & 0.0022 & 0.4195 & \mathrm{H} & 0 & 0 & 0 & 0 & 0 & 0 & 0 & 0 & 0 & 13 \\ 1.2216 & -0.8840 & -2.0382 & \mathrm{H} & 0 & 0 & 0 & 0 & 0 & 0 & 0 & 0 & 0 & 14\end{array}$

$\begin{array}{rrrrrrrrrrrrrr}1.2216 & -0.8840 & -2.0382 & \mathrm{H} & 0 & 0 & 0 & 0 & 0 & 0 & 0 & 0 & 0 & 14 \\ 1.2222 & 0.8842 & -2.0376 & \mathrm{H} & 0 & 0 & 0 & 0 & 0 & 0 & 0 & 0 & 0 & 15\end{array}$

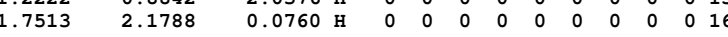

$\begin{array}{llllllllllllll}2.6326 & 1.2802 & 1.3403 & \mathrm{H} & 0 & 0 & 0 & 0 & 0 & 0 & 0 & 0 & 0 & 17\end{array}$

$\begin{array}{lllllllllllll}3.2299 & 1.2747 & -0.3398 & 0 & 0 & 0 & 0 & 0 & 0 & 0 & 0 & 0 & 18\end{array}$

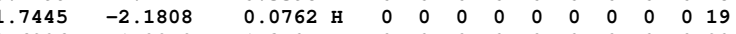

$\begin{array}{lllllllllllll}2.6286 & -1.2848 & 1.3404 \mathrm{H} & 0 & 0 & 0 & 0 & 0 & 0 & 0 & 0 & 0 & 20\end{array}$

$\begin{array}{lllllllllllll}3.2260 & -1.2813 & -0.3396 \mathrm{H} & 0 & 0 & 0 & 0 & 0 & 0 & 0 & 0 & 0 & 21\end{array}$

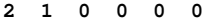

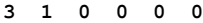

$5 \quad 4 \quad 0 \quad 00000$

$6 \begin{array}{llllll}6 & 4 & 0 & 0 & 0 & 0\end{array}$

$9 \begin{array}{llllll}9 & 4 & 0 & 0 & 0 & 0 \\ 0 & 4 & 0 & 0 & 0 & 0\end{array}$

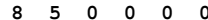

14 5 510000100

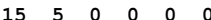

$\begin{array}{llllll}7 & 6 & 0 & 0 & 0 & 0\end{array}$

$\begin{array}{rrrrrr}12 & 6 & 0 & 0 & 0 & 0 \\ 8 & 7 & 0 & 0 & 0 & 0\end{array}$

$\begin{array}{llllll}13 & 7 & 0 & 0 & 0 & 0\end{array}$

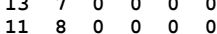




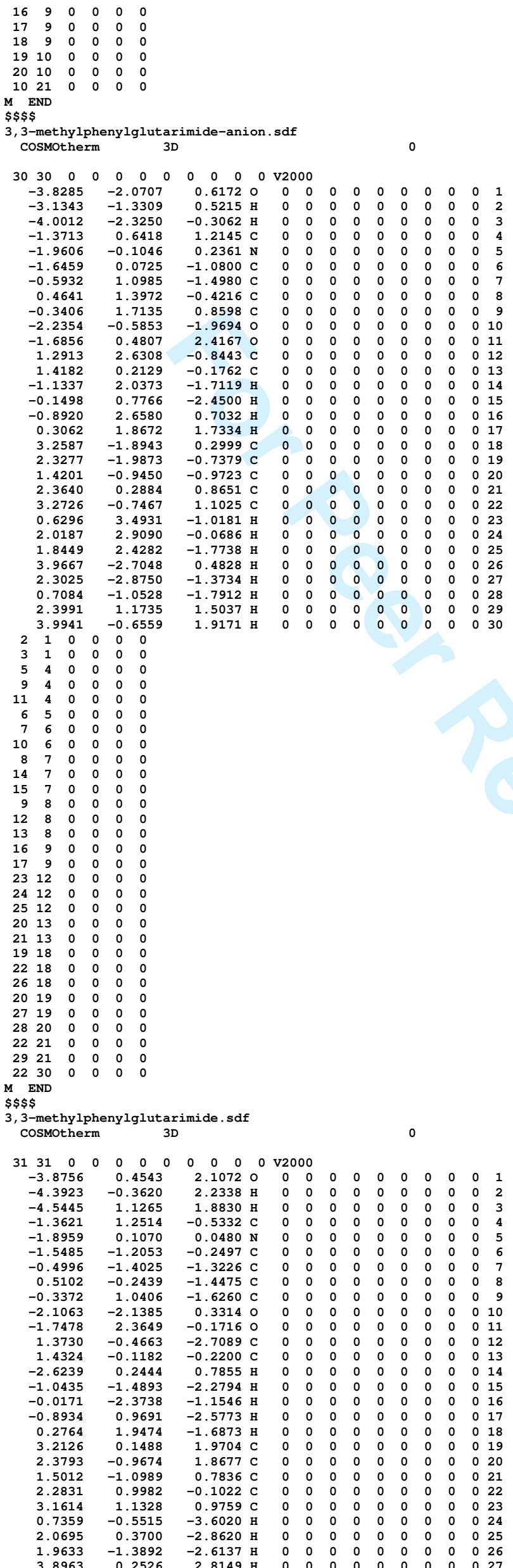




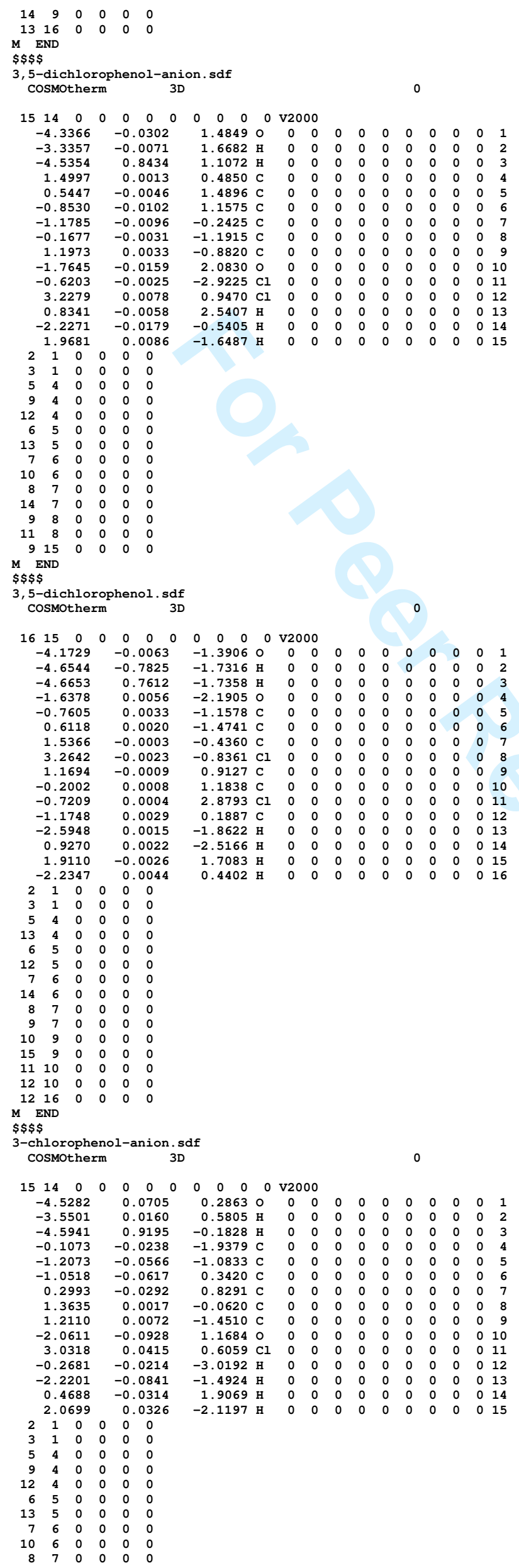




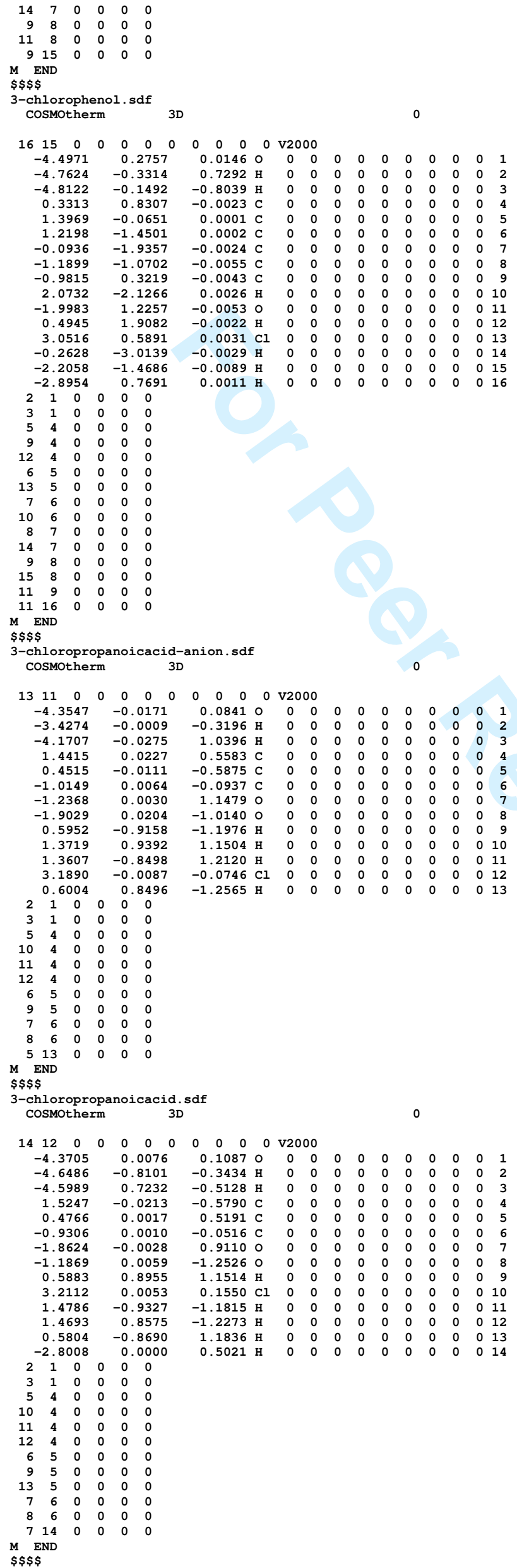




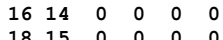

$\begin{array}{llllll}18 & 15 & 0 & 0 & 0 & 0 \\ 19 & 17 & 0 & 0 & 0 & 0 \\ 13 & 25 & 0 & 0 & 0 & 0\end{array}$

M END

3-hydroxybenzoicacid-anion.sdf

cosmotherm

$\begin{array}{llllllllllll}18 & 17 & 0 & 0 & 0 & 0 & 0 & 0 & 0 & 0 & 0 & \mathrm{v} 2000\end{array}$

$\begin{array}{llllllllllllll}-4.6337 & -0.8075 & 0.0085 & 0 & 0 & 0 & 0 & 0 & 0 & 0 & 0 & 0 & 0 & 1 \\ -3.6218 & -0.8227 & 0.0028 & \mathrm{H} & 0 & 0 & 0 & 0 & 0 & 0 & 0 & 0 & 0 & 2\end{array}$

$\begin{array}{rrrrrrrrrrrrrr}-3.6218 & -0.8227 & 0.0028 & \mathrm{H} & 0 & 0 & 0 & 0 & 0 & 0 & 0 & 0 & 0 & 2 \\ -4.8357 & 0.1438 & 0.0361 \mathrm{H} & 0 & 0 & 0 & 0 & 0 & 0 & 0 & 0 & 0 & 3\end{array}$

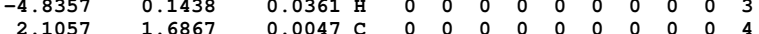

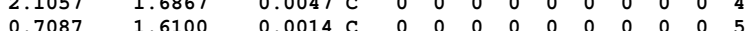

$\begin{array}{rrrllllllllllll}0.0742 & 0.3574 & -0.0021 & \mathrm{C} & 0 & 0 & 0 & 0 & 0 & 0 & 0 & 0 & 0 & 6\end{array}$

$\begin{array}{lrrrrrrrrrrrrr}0.0742 & 0.3574 & -0.0021 & C & 0 & 0 & 0 & 0 & 0 & 0 & 0 & 0 & 0 & 6 \\ 0.8530 & -0.8098 & -0.0027 & C & 0 & 0 & 0 & 0 & 0 & 0 & 0 & 0 & 0 & 7\end{array}$

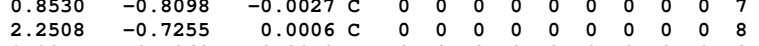

$\begin{array}{rrrrrrrrrrrrrr}2.2508 & -0.7255 & 0.0006 & \mathrm{C} & 0 & 0 & 0 & 0 & 0 & 0 & 0 & 0 & 0 & 8 \\ 2.8847 & 0.5260 & 0.0042 & \mathrm{C} & 0 & 0 & 0 & 0 & 0 & 0 & 0 & 0 & 0 & 9\end{array}$

$\begin{array}{rrrrrrrrrrrrrrrr}-1.4506 & 0.2643 & -0.0052 & \mathrm{C} & 0 & 0 & 0 & 0 & 0 & 0 & 0 & 0 & 0 & 10\end{array}$

$\begin{array}{llllllllllllll}3.0560 & -1.8432 & 0.0004 & 0 & 0 & 0 & 0 & 0 & 0 & 0 & 0 & 0 & 0 & 11\end{array}$

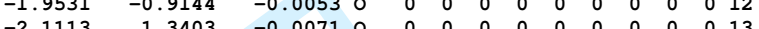

$\begin{array}{lllllllllllllll}-2.1113 & 1.3403 & -0.0071 & 0 & 0 & 0 & 0 & 0 & 0 & 0 & 0 & 0 & 0 & 13\end{array}$

$\begin{array}{llllllllllllll}2.5998 & 2.6606 & 0.0080 & \mathrm{H} & 0 & 0 & 0 & 0 & 0 & 0 & 0 & 0 & 0 & 14\end{array}$

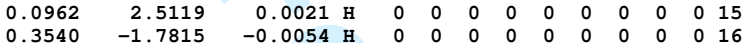

$\begin{array}{rrrrrrrrrrrrrr}0.3540 & -1.7815 & -0.0054 & \text { H } & 0 & 0 & 0 & 0 & 0 & 0 & 0 & 0 & 0 & 16 \\ 3.9750 & 0.5774 & 0.0068 & \text { H } & 0 & 0 & 0 & 0 & 0 & 0 & 0 & 0 & 0 & 17\end{array}$

$\begin{array}{llllllllllllll}2.4950 & -2.6420 & -0.0032 & \mathrm{H} & 0 & 0 & 0 & 0 & 0 & 0 & 0 & 0 & 0 & 18\end{array}$

210000100

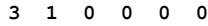

$5 \begin{array}{llllll}5 & 4 & 0 & 0 & 0 & 0 \\ 9 & 4 & 0 & 0 & 0 & 0\end{array}$

$\begin{array}{rrrrrr}9 & 4 & 0 & 0 & 0 & 0\end{array}$

$14 \quad 4 \quad 0010000$

$\begin{array}{rrrrrr}6 & 5 & 0 & 0 & 0 & 0 \\ 15 & 5 & 0 & 0 & 0 & 0\end{array}$

$\begin{array}{llllll}7 & 6 & 0 & 0 & 0 & 0\end{array}$

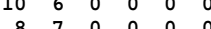

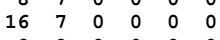

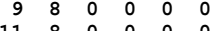

$\begin{array}{llllll}11 & 8 & 0 & 0 & 0 & 0 \\ 17 & 9 & 0 & 0 & 0 & 0\end{array}$

$1210 \quad 00000$

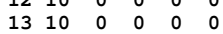

$\begin{array}{llllll}11 & 18 & 0 & 0 & 0 & 0\end{array}$

$M$ END

3-hydroxybenzoicacid. sdf

CosMotherm

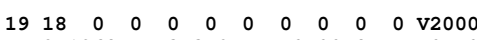

$\begin{array}{llllllllllllll}2.1269 & -3.3585 & 0.0059 & 0 & 0 & 0 & 0 & 0 & 0 & 0 & 0 & 0 & 0 & 1 \\ 2.6851 & -3.0513 & 0.7431 & \mathrm{H} & 0 & 0 & 0 & 0 & 0 & 0 & 0 & 0 & 0 & 2\end{array}$

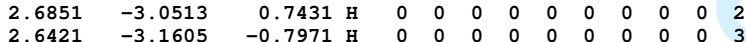

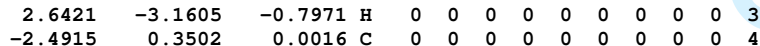

$\begin{array}{llllllllllllll}-2.4915 & 0.3502 & 0.0016 & \text { C } & 0 & 0 & 0 & 0 & 0 & 0 & 0 & 0 & 0 & 4 \\ -1.5191 & 1.3533 & 0.0011 & C & 0 & 0 & 0 & 0 & 0 & 0 & 0 & 0 & 0 & 5\end{array}$

$\begin{array}{llrrrrrrrrrrrr}-1.51611 & 0.9926 & -0.0012 & C & 0 & 0 & 0 & 0 & 0 & 0 & 0 & 0 & 0 & 6\end{array}$

$\begin{array}{llllllllllllll}0.2165 & -0.3615 & -0.0030 & \mathrm{C} & 0 & 0 & 0 & 0 & 0 & 0 & 0 & 0 & 0 & 7\end{array}$

$\begin{array}{llllllllllllll}-0.7672 & -1.3631 & -0.0013 & \mathrm{C} & 0 & 0 & 0 & 0 & 0 & 0 & 0 & 0 & 0 & 8 \\ -2.1267 & -0.9979 & 0.0005 & \mathrm{C} & 0 & 0 & 0 & 0 & 0 & 0 & 0 & 0 & 0 & 9\end{array}$

$\begin{array}{lllllllllllllr}-2.1267 & -0.9979 & 0.0005 & \mathrm{C} & 0 & 0 & 0 & 0 & 0 & 0 & 0 & 0 & 0 & 9\end{array}$

$\begin{array}{llllllllllllll}0.8580 & 2.0773 & -0.0006 & \mathrm{C} & 0 & 0 & 0 & 0 & 0 & 0 & 0 & 0 & 0 & 10\end{array}$

$\begin{array}{llllllllllllll}-0.4665 & -2.6909 & -0.0008 & \circ & 0 & 0 & 0 & 0 & 0 & 0 & 0 & 0 & 0 & 11\end{array}$

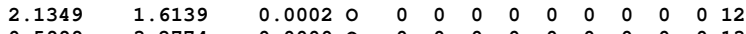

$\begin{array}{llllllllllllll}0.5999 & 3.2774 & 0.0000 & 0 & 0 & 0 & 0 & 0 & 0 & 0 & 0 & 0 & 0 & 13\end{array}$

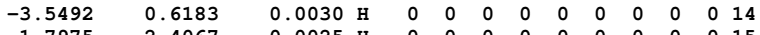

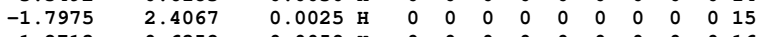

$\begin{array}{llllllllllllll}1.2713 & -0.6352 & -0.0058 & \mathrm{H} & 0 & 0 & 0 & 0 & 0 & 0 & 0 & 0 & 0 & 16\end{array}$

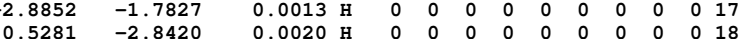

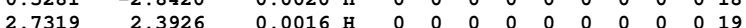

$\begin{array}{lllllll}2 & 1 & 0 & 0 & 0 & 0\end{array}$

$\begin{array}{llllll}2 & 1 & 0 & 0 & 0 & 0 \\ 3 & 1 & 0 & 0 & 0 & 0\end{array}$

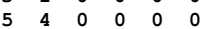

$9 \quad 4 \quad 000000$

$14 \quad 4 \quad 000000$

$6 \begin{array}{cccccc}6 & 5 & 0 & 0 & 0 & 0\end{array}$

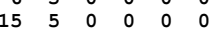

$7 \quad 6 \quad 000000$

$\begin{array}{llllll}0 & 6 & 0 & 0 & 0 & 0\end{array}$

$8 \begin{array}{llllll}8 & 7 & 0 & 0 & 0 & 0 \\ 10 & 7 & 0 & 0 & 0 & 0\end{array}$

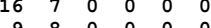

$\begin{array}{rrrrrrrr}9 & 8 & 0 & 0 & 0 & 0 \\ 11 & 8 & 0 & 0 & 0 & 0\end{array}$

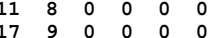

$\begin{array}{rrrrrr}17 & 9 & 0 & 0 & 0 & 0 \\ 12 & 10 & 0 & 0 & 0 & 0\end{array}$

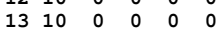

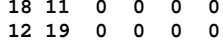

1219

$M$ END

4-(1,1-dimethylethyl) -benzoicacid-anion.sdf
CosmOtherm 3 D

$\begin{array}{llllllllllll}29 & 28 & 0 & 0 & 0 & 0 & 0 & 0 & 0 & 0 & 0 & \mathrm{v} 2000\end{array}$

$\begin{array}{llllllllllllll}-5.8865 & -0.4862 & -0.0059 & \circ & 0 & 0 & 0 & 0 & 0 & 0 & 0 & 0 & 0 & 1\end{array}$

$\begin{array}{llllllllllllll}-4.8840 & -0.6315 & -0.0039 & \text { н } & 0 & 0 & 0 & 0 & 0 & 0 & 0 & 0 & 0 & 2\end{array}$

$\begin{array}{llllllllllllll}-5.9617 & 0.4839 & 0.0082 & \text { H } & 0 & 0 & 0 & 0 & 0 & 0 & 0 & 0 & 0 & 3\end{array}$

$\begin{array}{llllllllllllll}1.0772 & 1.2472 & -0.0020 & \mathrm{C} & 0 & 0 & 0 & 0 & 0 & 0 & 0 & 0 & 0 & 4\end{array}$

$\begin{array}{llllllllllllll}-0.3211 & 1.3020 & -0.0009 & \mathrm{C} & 0 & 0 & 0 & 0 & 0 & 0 & 0 & 0 & 0 & 5\end{array}$

$\begin{array}{llrllllllllllll}-1.0915 & 0.1311 & 0.0014 & C & 0 & 0 & 0 & 0 & 0 & 0 & 0 & 0 & 0 & 6\end{array}$

$\begin{array}{llllllllllllll}-0.4165 & -1.1014 & 0.0029 & \mathrm{C} & 0 & 0 & 0 & 0 & 0 & 0 & 0 & 0 & 0 & 7\end{array}$

$\begin{array}{rrrrrrrrrrrrrr}0.9780 & -1.1540 & 0.0022 & \mathrm{C} & 0 & 0 & 0 & 0 & 0 & 0 & 0 & 0 & 0 & 8 \\ 1.7615 & 0.0189 & -0.0003 & \mathrm{C} & 0 & 0 & 0 & 0 & 0 & 0 & 0 & 0 & 0 & 9\end{array}$

$\begin{array}{rrrrrrrrrrrrrr}1.7615 & 0.0189 & -0.0003 & C & 0 & 0 & 0 & 0 & 0 & 0 & 0 & 0 & 0 & 9 \\ -2.6127 & 0.2028 & 0.0022 & C & 0 & 0 & 0 & 0 & 0 & 0 & 0 & 0 & 0 & 10\end{array}$

$\begin{array}{rrrrrrrrrrrrrr}2.6127 & 0.2028 & 0.0022 & \text { C } & 0 & 0 & 0 & 0 & 0 & 0 & 0 & 0 & 0 & 10 \\ 3.2967 & -0.0808 & -0.0008 & \text { C } & 0 & 0 & 0 & 0 & 0 & 0 & 0 & 0 & 0 & 11\end{array}$ 


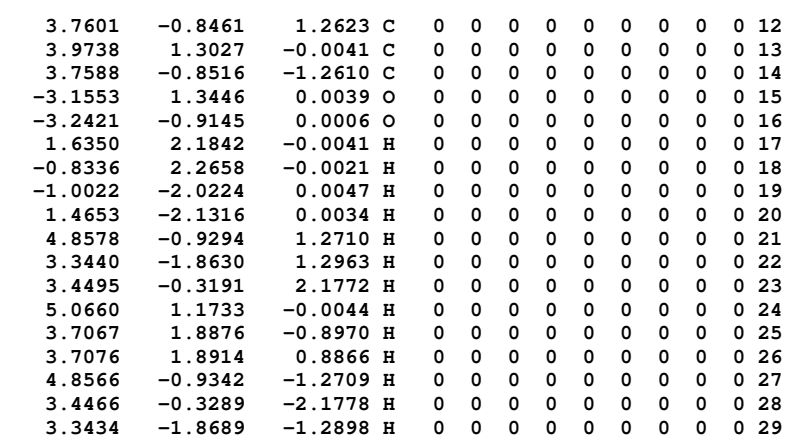


$\begin{array}{lllllll}25 & 13 & 0 & 0 & 0 & 0 \\ 26 & 13 & 0 & 0 & 0 & 0\end{array}$

2613 1 000000

2714 1 000 c 000

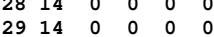

$1630 \quad 00000$

$M$ END

4-(1-methylethyl)-benzoicacid-anion. sdf
CosMotherm

0

$\begin{array}{llllllllllll}26 & 25 & 0 & 0 & 0 & 0 & 0 & 0 & 0 & 0 & 0 & \mathrm{v} 2000\end{array}$

$\begin{array}{llllllllllll}-5.6228 & 0.0118 & 0.2988 & 0 & 0 & 0 & 0 & 0 & 0 & 0 & 0 & 0\end{array}$

$\begin{array}{lrrrrrrrrrrrrr}-5.6228 & 0.0118 & 0.2988 & 0 & 0 & 0 & 0 & 0 & 0 & 0 & 0 & 0 & 0 & 1 \\ -4.6272 & -0.0014 & 0.4867 & \mathrm{H} & 0 & 0 & 0 & 0 & 0 & 0 & 0 & 0 & 0 & 2\end{array}$

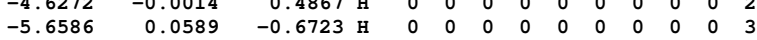

$\begin{array}{rrrrrrrrrrrrr}-5.6586 & 0.0589 & -0.6723 & \mathrm{H} & 0 & 0 & 0 & 0 & 0 & 0 & 0 & 0 & 0 \\ 1.1906 & 0.0204 & 1.3603 \mathrm{C} & 0 & 0 & 0 & 0 & 0 & 0 & 0 & 0 & 0 & 4\end{array}$

$\begin{array}{llllllllllllllll}-0.2009 & 0.0147 & 1.2200 & \mathrm{C} & 0 & 0 & 0 & 0 & 0 & 0 & 0 & 0 & 0 & 5\end{array}$

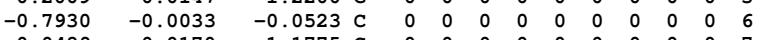

$\begin{array}{llllllllllllll}0.0489 & -0.0170 & -1.1775 & \mathrm{C} & 0 & 0 & 0 & 0 & 0 & 0 & 0 & 0 & 0 & 7 \\ 1.4377 & -0.0132 & -1.0351 & \mathrm{C} & 0 & 0 & 0 & 0 & 0 & 0 & 0 & 0 & 0 & 8\end{array}$

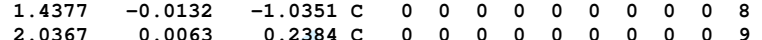

$\begin{array}{rrrrrrrrrrrrrr}2.0367 & 0.0063 & 0.2384 & \mathrm{C} & 0 & 0 & 0 & 0 & 0 & 0 & 0 & 0 & 0 & 9 \\ -2.3071 & -0.0076 & -0.2212 & \mathrm{C} & 0 & 0 & 0 & 0 & 0 & 0 & 0 & 0 & 0 & 10\end{array}$

$\begin{array}{rrrrrrrrrrrrrr}-2.3071 & -0.0076 & -0.2212 & \mathrm{C} & 0 & 0 & 0 & 0 & 0 & 0 & 0 & 0 & 0 & 10 \\ 3.5494 & 0.0105 & 0.4047 & \mathrm{C} & 0 & 0 & 0 & 0 & 0 & 0 & 0 & 0 & 0 & 11\end{array}$

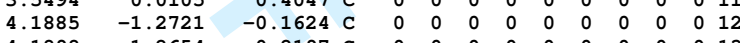

$\begin{array}{llllllllllllll}4.1898 & 1.2654 & -0.2197 & \mathrm{C} & 0 & 0 & 0 & 0 & 0 & 0 & 0 & 0 & 0 & 13\end{array}$

$\begin{array}{llllllllllllll}-3.0058 & -0.0174 & 0.8545 & 0 & 0 & 0 & 0 & 0 & 0 & 0 & 0 & 0 & 0 & 14\end{array}$

$\begin{array}{llllllllllllll}-2.7756 & -0.0010 & -1.3947 & 0 & 0 & 0 & 0 & 0 & 0 & 0 & 0 & 0 & 0 & 15\end{array}$

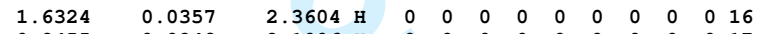

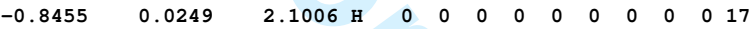

$\begin{array}{llllllllllllll}-0.4066 & -0.0305 & -2.1694 & \text { H } & 0 & 0 & 0 & 0 & 0 & 0 & 0 & 0 & 0 & 18\end{array}$

$\begin{array}{llllllllllllll}2.0641 & -0.0252 & -1.9306 & \text { H } & 0 & 0 & 0 & 0 & 0 & 0 & 0 & 0 & 0 & 19\end{array}$

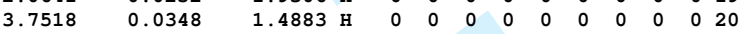

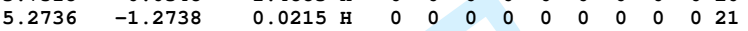

$\begin{array}{llllllllllllll}3.7608 & -2.1719 & 0.3038 & \mathrm{H} & 0 & 0 & 0 & 0 & 0 & 0 & 0 & 0 & 0 & 22\end{array}$

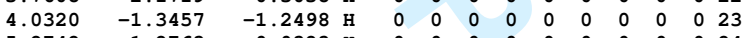

$\begin{array}{lllllllllllll}5.2742 & 1.2763 & -0.0323 \mathrm{H} & 0 & 0 & 0 & 0 & 0 & 0 & 0 & 0 & 0 & 24\end{array}$

$\begin{array}{llllllllllllll}3.7602 & 2.1860 & 0.2021 & \mathrm{H} & 0 & 0 & 0 & 0 & 0 & 0 & 0 & 0 & 0 & 25 \\ 3.0375 & 1.2878 & -1.3099 & \mathrm{H} & 0 & 0 & 0 & 0 & 0 & 0 & 0 & 0 & 0 & 26\end{array}$ $4.0375 \quad 1.2878$

$\begin{array}{lllllll}2 & 1 & 0 & 0 & 0 & 0\end{array}$

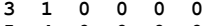

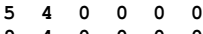

$\begin{array}{llllllllll}16 & 4 & 0 & 0 & 0 & 0\end{array}$

$\begin{array}{rlllll}16 & 4 & 0 & 0 & 0 & 0 \\ 6 & 5 & 0 & 0 & 0 & 0\end{array}$

$\begin{array}{cccccc}6 & 5 & 0 & 0 & 0 & 0 \\ 17 & 5 & 0 & 0 & 0 & 0\end{array}$

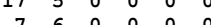

$\begin{array}{rrrrrr}7 & 6 & 0 & 0 & 0 & 0 \\ 10 & 6 & 0 & 0 & 0 & 0\end{array}$

$\begin{array}{rrrrrr}10 & 6 & 0 & 0 & 0 & 0 \\ 8 & 7 & 0 & 0 & 0 & 0\end{array}$

$\begin{array}{cccccc}18 & 7 & 0 & 0 & 0 & 0\end{array}$

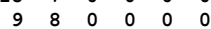

198000000

$\begin{array}{rrrrrr}11 & 9 & 0 & 0 & 0 & 0\end{array}$

15100000000

12 11 11000000

$1311 \quad 00000$

$2011 \quad 000000$

$2112 \quad 000000$

2212 12 1200000

$2312 \quad 0 \quad 00100$

$24 \quad 13 \quad 00001000$

2513 1 000000

$\begin{array}{lllll}1326 & 0 & 0 & 0 & 0 \\ M \text { END } & & & & \end{array}$

$\$ \$ \$$

4-(1-methylethyl) -benzoicacid.sdf

cosMotherm

$\begin{array}{llllllllllll}27 & 26 & 0 & 0 & 0 & 0 & 0 & 0 & 0 & 0 & 0 & \mathrm{v} 2000\end{array}$

$\begin{array}{llllllllllllll}-5.4969 & -0.0215 & 0.8029 & 0 & 0 & 0 & 0 & 0 & 0 & 0 & 0 & 0 & 0 & 1\end{array}$

$\begin{array}{llllllllllllll}-5.8656 & -0.3928 & -0.0199 & \text { H } & 0 & 0 & 0 & 0 & 0 & 0 & 0 & 0 & 0 & 2\end{array}$

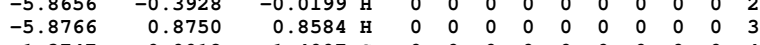

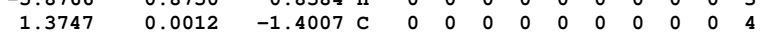

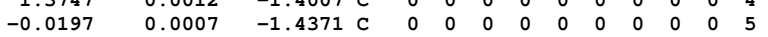

$\begin{array}{llllllllllllll}-0.0197 & 0.0007 & -1.4371 & \mathrm{C} & 0 & 0 & 0 & 0 & 0 & 0 & 0 & 0 & 0 & 5 \\ -0.7609 & -0.0021 & -0.2437 & \mathrm{C} & 0 & 0 & 0 & 0 & 0 & 0 & 0 & 0 & 0 & 6\end{array}$

$\begin{array}{llllllllllllllll}-0.76099 & -0.0021 & -0.2437 & C & 0 & 0 & 0 & 0 & 0 & 0 & 0 & 0 & 0 & 6 \\ -0.0739 & -0.0045 & 0.9845 & C & 0 & 0 & 0 & 0 & 0 & 0 & 0 & 0 & 0 & 7\end{array}$

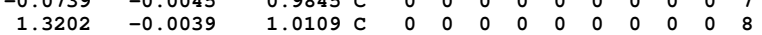

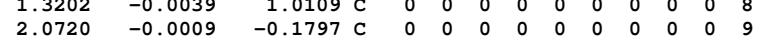

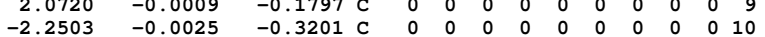

$\begin{array}{rrrrrrrrrrrrrr}-2.2503 & -0.0025 & -0.3201 & \mathrm{C} & 0 & 0 & 0 & 0 & 0 & 0 & 0 & 0 & 0 & 10 \\ 3.5921 & 0.0009 & -0.1563 & \mathrm{C} & 0 & 0 & 0 & 0 & 0 & 0 & 0 & 0 & 0 & 11\end{array}$

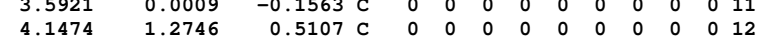

$\begin{array}{rrrrrrrrrrrrrr}4.1474 & 1.2746 & 0.5107 & \mathrm{C} & 0 & 0 & 0 & 0 & 0 & 0 & 0 & 0 & 0 & 12 \\ 4.1524 & -1.2654 & 0.5201 & \mathrm{C} & 0 & 0 & 0 & 0 & 0 & 0 & 0 & 0 & 0 & 13\end{array}$

$\begin{array}{llllllllllllll}-2.8764 & 0.0026 & -1.3824 & 0 & 0 & 0 & 0 & 0 & 0 & 0 & 0 & 0 & 0 & 14\end{array}$

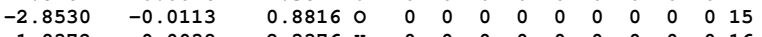

$\begin{array}{llllllllllllll}1.9372 & 0.0038 & -2.3376 & \mathrm{H} & 0 & 0 & 0 & 0 & 0 & 0 & 0 & 0 & 0 & 16\end{array}$

$\begin{array}{llllllllllllll}-0.5482 & 0.0031 & -2.3916 & \mathrm{H} & 0 & 0 & 0 & 0 & 0 & 0 & 0 & 0 & 0 & 17\end{array}$

$\begin{array}{llllllllllllll}-0.6361 & -0.0064 & 1.9186 & \mathrm{H} & 0 & 0 & 0 & 0 & 0 & 0 & 0 & 0 & 0 & 18\end{array}$

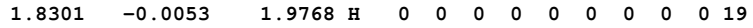

$\begin{array}{llllllllllllll}3.9282 & -0.0023 & -1.2063 & \mathrm{H} & 0 & 0 & 0 & 0 & 0 & 0 & 0 & 0 & 0 & 20\end{array}$

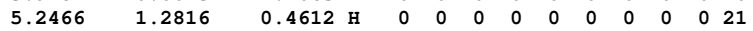

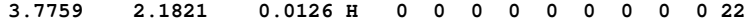

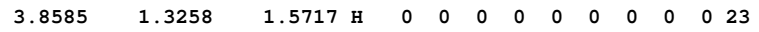

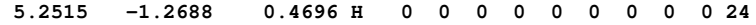

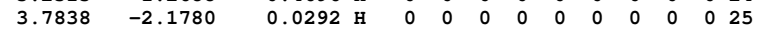

$\begin{array}{llllllllllllll}3.8647 & -1.3097 & 1.5817 & \mathrm{H} & 0 & 0 & 0 & 0 & 0 & 0 & 0 & 0 & 0 & 26\end{array}$

$\begin{array}{lllllllllllll}-3.8665 & -0.0043 & 0.7601 \mathrm{H} & 0 & 0 & 0 & 0 & 0 & 0 & 0 & 0 & 0 & 27\end{array}$

$2 \begin{array}{llllll}2 & 1 & 0 & 0 & 0 & 0\end{array}$

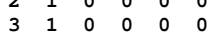

$5 \begin{array}{llllll}5 & 4 & 0 & 0 & 0 & 0 \\ 9 & 4 & 0 & 0 & 0 & 0\end{array}$

$\begin{array}{lllllll}16 & 4 & 0 & 0 & 0 & 0\end{array}$

$\begin{array}{llllll}6 & 5 & 0 & 0 & 0 & 0\end{array}$

$\begin{array}{llllll}17 & 5 & 0 & 0 & 0 & 0\end{array}$

$\begin{array}{llllll}7 & 6 & 0 & 0 & 0 & 0\end{array}$

$\begin{array}{llllll}10 & 6 & 0 & 0 & 0 & 0\end{array}$ 


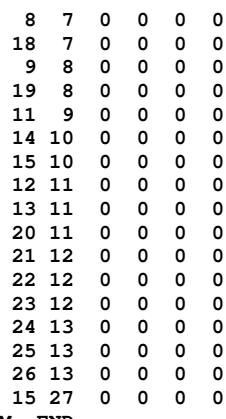




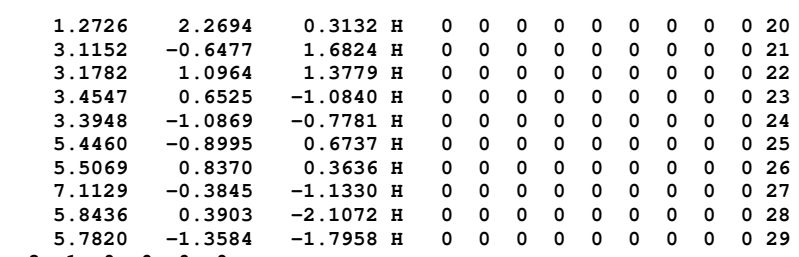


4-carbamoylbenzoicacid-anion.sdf

$\begin{array}{llllllllllll}21 & 20 & 0 & 0 & 0 & 0 & 0 & 0 & 0 & 0 & 0 & \mathrm{v} 2000\end{array}$

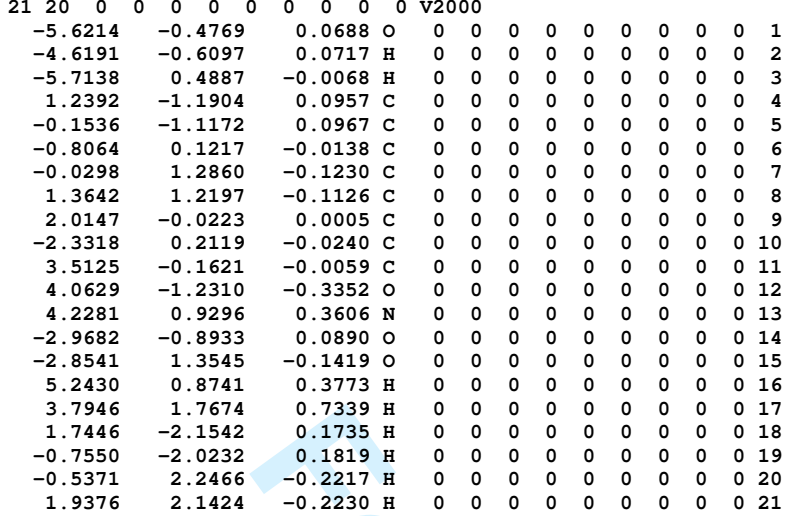

$\begin{array}{cccccc}2 & 1 & 0 & 0 & 0 & 0\end{array}$

$\begin{array}{llllll}3 & 1 & 0 & 0 & 0 & 0 \\ 5 & 4 & 0 & 0 & 0 & 0\end{array}$

$\begin{array}{llllll}5 & 4 & 0 & 0 & 0 & 0 \\ 9 & 4 & 0 & 0 & 0 & 0\end{array}$

$\begin{array}{rrrrrr}9 & 4 & 0 & 0 & 0 & 0 \\ 18 & 4 & 0 & 0 & 0 & 0\end{array}$

$\begin{array}{rrrrrr}6 & 5 & 0 & 0 & 0 & 0 \\ 19 & 5 & 0 & 0 & 0 & 0\end{array}$

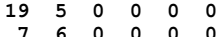

$\begin{array}{rlllll}7 & 6 & 0 & 0 & 0 & 0 \\ 10 & 6 & 0 & 0 & 0 & 0 \\ 8 & 7 & 0 & 0 & 0 & 0\end{array}$

$\begin{array}{rrrrrr}10 & 6 & 0 & 0 & 0 & 0 \\ 8 & 7 & 0 & 0 & 0 & 0\end{array}$

$\begin{array}{rrrrrr}20 & 7 & 0 & 0 & 0 & 0 \\ 9 & 8 & 0 & 0 & 0 & 0\end{array}$

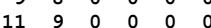

$\begin{array}{lrllll}14 & 10 & 0 & 0 & 0 & 0\end{array}$

$\begin{array}{llllll}15 & 10 & 0 & 0 & 0 & 0\end{array}$

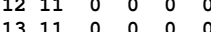

$\begin{array}{lllllll}16 & 13 & 0 & 0 & 0 & 0\end{array}$

$\begin{array}{llllll}17 & 13 & 0 & 0 & 0 & 0\end{array}$

$8 \begin{array}{llllll}81 & 21 & 0 & 0 & 0\end{array}$

$M$ END

$M \quad E$

4-carbamoylbenzoicacid. sdf

cosmotherm $3 \mathrm{D}$

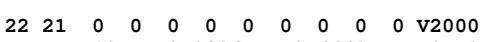

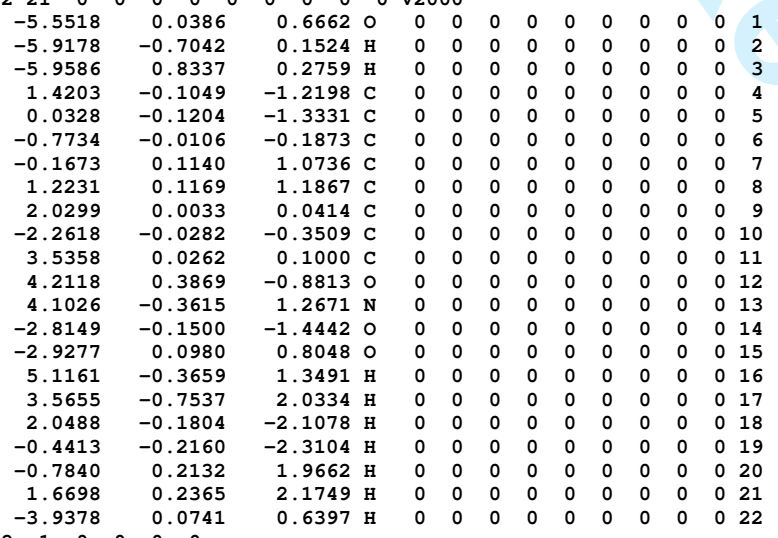

$\begin{array}{llllll}2 & 1 & 0 & 0 & 0 & 0 \\ 3 & 1 & 0 & 0 & 0 & 0\end{array}$

$\begin{array}{llllll}3 & 1 & 0 & 0 & 0 & 0 \\ 5 & 4 & 0 & 0 & 0 & 0\end{array}$

$\begin{array}{llllll}5 & 4 & 0 & 0 & 0 & 0 \\ 9 & 4 & 0 & 0 & 0 & 0\end{array}$

$\begin{array}{rrrrrr}9 & 4 & 0 & 0 & 0 & 0 \\ 18 & 4 & 0 & 0 & 0 & 0\end{array}$

6 5 51000000

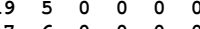

$\begin{array}{lllll}6 & 0 & 0 & 0 & 0\end{array}$

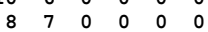

$\begin{array}{llllll}20 & 7 & 0 & 0 & 0 & 0\end{array}$

$\begin{array}{rrrrrr}9 & 8 & 0 & 0 & 0 & 0 \\ 21 & 8 & 0 & 0 & 0 & 0\end{array}$

$\begin{array}{llllll}21 & 8 & 0 & 0 & 0 & 0 \\ 11 & 9 & 0 & 0 & 0 & 0\end{array}$

$\begin{array}{lrrrrr}11 & 9 & 0 & 0 & 0 & 0\end{array}$

$\begin{array}{llllll}14 & 10 & 0 & 0 & 0 & 0 \\ 15 & 10 & 0 & 0 & 0 & 0\end{array}$

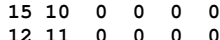

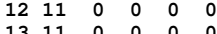

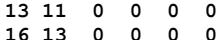

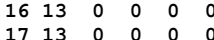

$1522 \quad 00000$

END

$\$ \$ \$$

-chlorobenzoicacid-anion.sdf

cosMotherm

$\begin{array}{llllllllllll}17 & 16 & 0 & 0 & 0 & 0 & 0 & 0 & 0 & 0 & 0 & \text { v } 2000\end{array}$

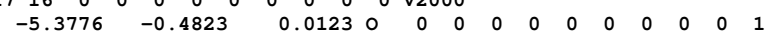

$\begin{array}{llllllllllllll}-5.3776 & -0.4823 & 0.0123 & \text { O } & 0 & 0 & 0 & 0 & 0 & 0 & 0 & 0 & 0 & 1 \\ -4.3740 & -0.6091 & 0.0004 & \text { H } & 0 & 0 & 0 & 0 & 0 & 0 & 0 & 0 & 0 & 2\end{array}$

$\begin{array}{lllllllllllllll}-5.4767 & 0.4854 & 0.0000 \mathrm{H} & 0 & 0 & 0 & 0 & 0 & 0 & 0 & 0 & 0 & 3\end{array}$

$\begin{array}{llllllllllllll}1.4781 & -1.2227 & -0.0002 & \mathrm{C} & 0 & 0 & 0 & 0 & 0 & 0 & 0 & 0 & 0 & 4 \\ 0.0834 & -1.1294 & -0.0021 & \mathrm{C} & 0 & 0 & 0 & 0 & 0 & 0 & 0 & 0 & 0 & 5\end{array}$ 


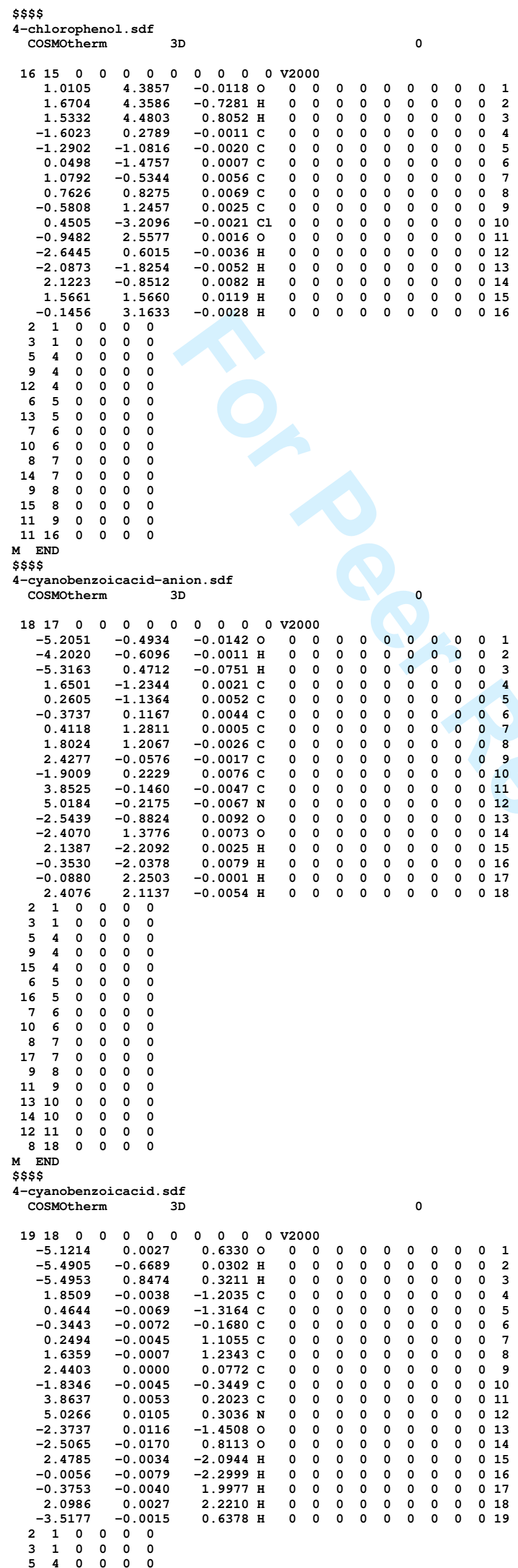




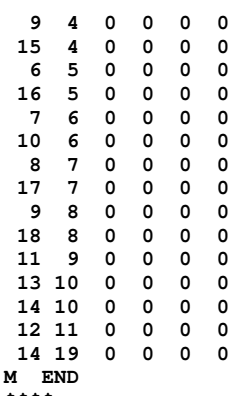

\section{$M \quad$ END}

$\$ \$ \$$

-dimethylaminobenzoicacid-anion sdf

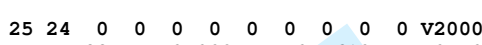

\begin{tabular}{|c|c|c|c|c|c|c|c|c|c|c|c|c|c|}
\hline-5.5995 & 0.0024 & -0.4610 & 0 & 0 & 0 & 0 & 0 & 0 & 0 & 0 & 0 & 0 & 1 \\
\hline-4.5940 & -0.0107 & -0.6059 & H & 0 & 0 & 0 & 0 & 0 & 0 & 0 & 0 & 0 & 2 \\
\hline-5.6763 & -0.0419 & 0.5080 & H & 0 & 0 & 0 & 0 & 0 & 0 & 0 & 0 & 0 & 3 \\
\hline 1.2472 & 0.0260 & -1.2032 & c & 0 & 0 & 0 & 0 & 0 & 0 & 0 & 0 & 0 & 4 \\
\hline-0.1426 & 0.0096 & -1.1199 & c & 0 & 0 & 0 & 0 & 0 & 0 & 0 & 0 & & 5 \\
\hline-0.8138 & 0.0086 & 0.1162 & c & 0 & 0 & 0 & 0 & 0 & 0 & 0 & 0 & 0 & 6 \\
\hline-0.0206 & 0.0191 & 1.2777 & c & 0 & 0 & 0 & 0 & 0 & 0 & 0 & 0 & 0 & 7 \\
\hline 1.3700 & 0.0368 & 1.2210 & C & 0 & 0 & 0 & 0 & 0 & 0 & 0 & 0 & 0 & 8 \\
\hline 2.0508 & 0.0558 & -0.0288 & c & 0 & 0 & 0 & 0 & 0 & 0 & 0 & 0 & 0 & 9 \\
\hline-2.3223 & -0.0150 & 0.2046 & C & 0 & 0 & 0 & 0 & 0 & 0 & 0 & 0 & & 10 \\
\hline 3.4287 & 0.1125 & -0.0980 & $\mathbf{N}$ & 0 & 0 & 0 & 0 & 0 & 0 & 0 & 0 & 0 & 11 \\
\hline 4.2235 & -0.0702 & 1.1140 & C & 0 & 0 & 0 & 0 & 0 & 0 & 0 & 0 & 0 & 12 \\
\hline 4.0990 & -0.0816 & -1.3810 & C & 0 & 0 & 0 & 0 & 0 & 0 & 0 & 0 & 0 & 13 \\
\hline-2.9719 & -0.0084 & -0.9060 & 0 & 0 & 0 & 0 & 0 & 0 & 0 & 0 & 0 & 0 & 14 \\
\hline-2.8602 & -0.0397 & 1.3515 & 0 & 0 & 0 & 0 & 0 & 0 & 0 & 0 & 0 & 0 & 15 \\
\hline 1.7151 & 0.0153 & -2.1867 & $\mathrm{H}$ & 0 & 0 & 0 & 0 & 0 & 0 & 0 & 0 & 0 & 16 \\
\hline-0.7347 & -0.0099 & -2.0370 & H & 0 & 0 & 0 & 0 & 0 & 0 & 0 & 0 & 0 & 17 \\
\hline-0.5200 & 0.0063 & 2.2486 & H & 0 & 0 & 0 & 0 & 0 & 0 & 0 & 0 & 0 & 18 \\
\hline 1.9341 & 0.0354 & 2.1529 & $\mathrm{H}$ & 0 & 0 & 0 & 0 & 0 & 0 & 0 & 0 & 0 & 19 \\
\hline 5.2805 & 0.0766 & 0.8668 & & 0 & 0 & 0 & 0 & 0 & 0 & 0 & 0 & 0 & 20 \\
\hline 3.9516 & 0.6713 & 1.8792 & $\mathrm{H}$ & 0 & 0 & 0 & 0 & 0 & 0 & 0 & 0 & 0 & 21 \\
\hline 4.1037 & -1.0788 & 1.5509 & $\mathrm{H}$ & 0 & 0 & 0 & 0 & 0 & 0 & 0 & 0 & 0 & 22 \\
\hline 5.1749 & 0.0699 & -1.2421 & $\mathrm{H}$ & 0 & 0 & 0 & 0 & 0 & 0 & 0 & 0 & 0 & 23 \\
\hline 3.9392 & -1.0950 & -1.7932 & $\Omega$ & 0 & 0 & 0 & 0 & 0 & 0 & 0 & 0 & 0 & 24 \\
\hline 3.7503 & 0.6514 & -2.1231 & H & 0 & 0 & 0 & 0 & 0 & 0 & 0 & 0 & 0 & 25 \\
\hline
\end{tabular}
$\begin{array}{lllllllllllllll}3.7503 & 0.6514 & -2.1231 \mathrm{H} & 0 & 0 & 0 & 0 & 0 & 0 & 0 & 0 & 0 & 25\end{array}$ $\begin{array}{llllll}2 & 1 & 0 & 0 & 0 & 0 \\ 3 & 1 & 0 & 0 & 0 & 0\end{array}$

$\begin{array}{llllll}3 & 1 & 0 & 0 & 0 & 0 \\ 5 & 4 & 0 & 0 & 0 & 0\end{array}$

$\begin{array}{llllll}5 & 4 & 0 & 0 & 0 & 0 \\ 9 & 4 & 0 & 0 & 0 & 0\end{array}$

$\begin{array}{rrrrrr}9 & 4 & 0 & 0 & 0 & 0 \\ 16 & 4 & 0 & 0 & 0 & 0\end{array}$

$\begin{array}{rrrrrr}6 & 5 & 0 & 0 & 0 & 0\end{array}$

$\begin{array}{llllll}17 & 5 & 0 & 0 & 0 & 0\end{array}$

$\begin{array}{rrrrrr}7 & 6 & 0 & 0 & 0 & 0 \\ 10 & 6 & 0 & 0 & 0 & 0\end{array}$

$\begin{array}{rrrrrr}10 & 6 & 0 & 0 & 0 & 0 \\ 8 & 7 & 0 & 0 & 0 & 0\end{array}$

$\begin{array}{llllll}18 & 7 & 0 & 0 & 0 & 0\end{array}$

$\begin{array}{rrrrrr}9 & 8 & 0 & 0 & 0 & 0\end{array}$

$\begin{array}{llllll}19 & 8 & 0 & 0 & 0 & 0 \\ 11 & 9 & 0 & 0 & 0 & 0\end{array}$

$\begin{array}{rrrrrr}11 & 9 & 0 & 0 & 0 & 0\end{array}$

$\begin{array}{llllll}14 & 10 & 0 & 0 & 0 & 0 \\ 15 & 10 & 0 & 0 & 0 & 0\end{array}$

$\begin{array}{llllll}15 & 10 & 0 & 0 & 0 & 0\end{array}$

$\begin{array}{llllll}12 & 11 & 0 & 0 & 0 & 0\end{array}$

$\begin{array}{llllll}13 & 11 & 0 & 0 & 0 & 0 \\ 20 & 12 & 0 & 0 & 0 & 0\end{array}$

$\begin{array}{llllll}21 & 12 & 0 & 0 & 0 & 0\end{array}$

$\begin{array}{llllll}22 & 12 & 0 & 0 & 0 & 0\end{array}$

$\begin{array}{llllll}23 & 13 & 0 & 0 & 0 & 0\end{array}$

$\begin{array}{llllll}13 & 25 & 0 & 0 & 0 & 0\end{array}$

M END

\$\$\$

4-dimethylaminobenzoicacid.sdf
CoSMOtherm

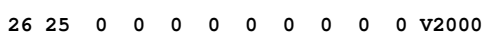

\begin{tabular}{|c|c|c|c|c|c|c|c|c|c|c|c|c|c|}
\hline-5.5824 & -0.0258 & 0.6270 & 0 & 0 & 0 & 0 & 0 & 0 & 0 & 0 & 0 & 0 & 1 \\
\hline .9239 & -0.5663 & -0.1089 & H & 0 & 0 & 0 & 0 & 0 & 0 & 0 & 0 & 0 & 2 \\
\hline-5.9621 & 0.8590 & 0.4760 & H & 0 & 0 & 0 & 0 & 0 & 0 & 0 & 0 & 0 & 3 \\
\hline 1.4302 & -0.0023 & -1.2330 & c & 0 & 0 & 0 & 0 & 0 & 0 & 0 & 0 & 0 & 4 \\
\hline 0.0477 & -0.0008 & -1.3306 & C & 0 & 0 & 0 & 0 & 0 & 0 & 0 & 0 & 0 & 5 \\
\hline-0.7753 & 0.0020 & -0.1847 & C & & 0 & 0 & 0 & 0 & 0 & 0 & 0 & 0 & 6 \\
\hline-0.1442 & 0.0037 & 1.0776 & C & 0 & 0 & 0 & 0 & 0 & 0 & 0 & 0 & 0 & 7 \\
\hline 1.2379 & 0.0026 & 1.1962 & C & & 0 & 0 & 0 & 0 & 0 & 0 & 0 & 0 & 8 \\
\hline 2.0741 & 0.0002 & 0.0405 & C & 0 & 0 & 0 & 0 & 0 & 0 & 0 & 0 & 0 & 9 \\
\hline-2.2401 & 0.0038 & -0.3440 & C & & & 0 & 0 & 0 & 0 & 0 & 0 & 0 & 10 \\
\hline 3.4376 & -0.0005 & 0.1481 & $\mathbf{N}$ & 0 & 0 & 0 & 0 & 0 & 0 & 0 & 0 & 0 & 11 \\
\hline 4.0754 & 0.0013 & 1.4634 & C & & & 0 & 0 & 0 & 0 & 0 & 0 & 0 & 12 \\
\hline 4.2754 & -0.0045 & -1.0499 & C & 0 & 0 & 0 & 0 & 0 & 0 & 0 & 0 & 0 & 13 \\
\hline-2.8193 & 0.0144 & -1.4384 & 0 & & & 0 & 0 & 0 & 0 & 0 & 0 & 0 & 14 \\
\hline-2.9204 & -0.0102 & 0.8258 & 0 & 0 & 0 & 0 & 0 & 0 & 0 & 0 & 0 & 0 & 15 \\
\hline 2.0232 & -0.0053 & -2.1463 & H & 0 & 0 & 0 & 0 & 0 & 0 & 0 & 0 & 0 & 16 \\
\hline-0.4214 & -0.0022 & -2.3161 & H & 0 & 0 & 0 & 0 & 0 & 0 & 0 & 0 & 0 & 17 \\
\hline-0.7523 & 0.0056 & 1.9829 & H & 0 & 0 & 0 & 0 & 0 & 0 & 0 & 0 & 0 & 18 \\
\hline 1.6804 & 0.0042 & 2.1909 & H & 0 & 0 & 0 & 0 & 0 & 0 & 0 & 0 & 0 & 19 \\
\hline 5.1612 & 0.003 & 1.3286 & H & 0 & 0 & 0 & 0 & 0 & 0 & 0 & 0 & & 20 \\
\hline 3.7990 & 0.8950 & 2.0464 & $\mathrm{H}$ & 0 & 0 & 0 & 0 & 0 & 0 & 0 & 0 & 0 & 21 \\
\hline 3.8023 & -0.8927 & 2.0476 & H & 0 & 0 & 0 & 0 & 0 & 0 & 0 & 0 & 0 & 22 \\
\hline 5.3262 & -0.0029 & -0.7446 & H & 0 & 0 & 0 & 0 & 0 & 0 & 0 & 0 & 0 & 23 \\
\hline 4.0966 & -0.9003 & -1.6664 & H & 0 & 0 & 0 & 0 & 0 & 0 & 0 & 0 & 0 & 24 \\
\hline 4.0960 & 0.8869 & -1.6726 & H & 0 & 0 & 0 & 0 & 0 & 0 & 0 & 0 & 0 & 25 \\
\hline-3.9179 & -0.0058 & 0.6382 & H & 0 & 0 & 0 & 0 & 0 & 0 & 0 & 0 & & 26 \\
\hline 1 & $0 \quad 0$ & & & & & & & & & & & & \\
\hline 0 & 0 & & & & & & & & & & & & \\
\hline 4 & & & & & & & & & & & & & \\
\hline
\end{tabular}




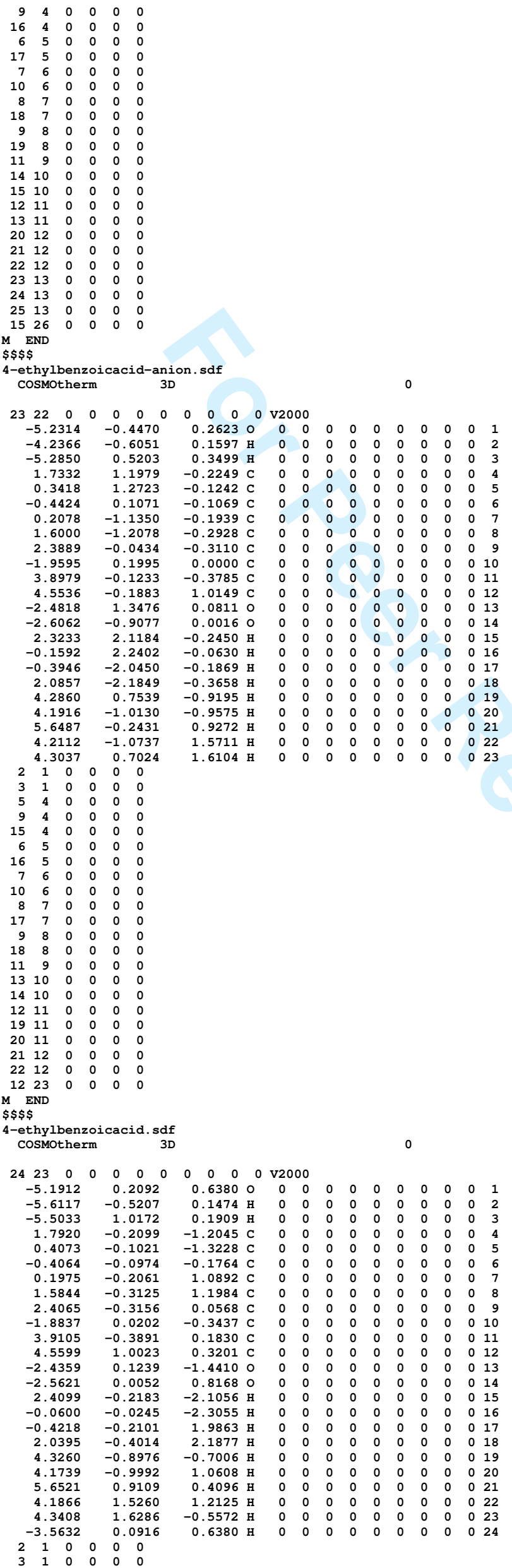




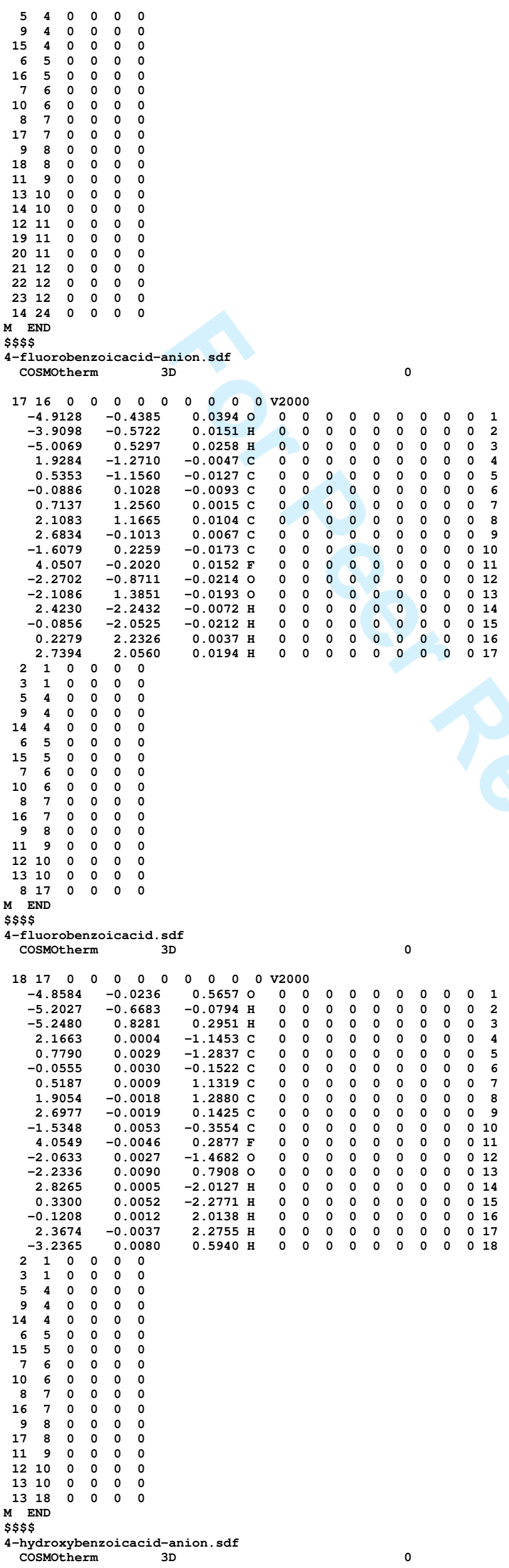


$\begin{array}{llllllllllll}18 & 17 & 0 & 0 & 0 & 0 & 0 & 0 & 0 & 0 & 0 & \mathrm{v} 2000\end{array}$

\begin{tabular}{|c|c|c|c|c|c|c|c|c|c|c|c|c|}
\hline-4.8622 & 0.0133 & -0.4164 & & 0 & 0 & 0 & 0 & 0 & D & & & \\
\hline-3.8599 & 0.0061 & -0.5684 & H & 0 & 0 & 0 & 0 & b & b & D & D & 0 \\
\hline-4.9343 & -0.0378 & 0.5526 & $\mathrm{H}$ & 0 & 0 & 0 & 0 & b & D & D & & \\
\hline 2.1479 & 0.0041 & 1.1586 & c & 0 & 0 & 0 & 0 & b & b & b & 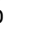 & b \\
\hline 0.7567 & 0.0021 & 1.2468 & & 0 & 0 & 0 & 0 & b & b & b & & \\
\hline-0.0505 & -0.0012 & 0.0950 & & 0 & 0 & 0 & 0 & b & b & b & & \\
\hline 0.5833 & -0.0033 & -1.1588 & & 0 & 0 & 0 & 0 & b & b & b & & \\
\hline 1.9754 & -0.0023 & -1.2669 & & 0 & 0 & 0 & 0 & b & b & b & & \\
\hline 2.7619 & 0.0018 & -0.1039 & & & 0 & 0 & 0 & b & b & & & \\
\hline-1.5652 & -0.0035 & 0.2147 & & 0 & 0 & 0 & 0 & o & b & D & & \\
\hline-2.2305 & -0.0059 & -0.8830 & & 0 & 0 & 0 & 0 & o & b & & & \\
\hline-2.0731 & -0.0074 & 1.3726 & & 0 & 0 & 0 & 0 & D & b & D & & \\
\hline 4.1344 & 0.0034 & -0.1421 & & 0 & 0 & 0 & 0 & b & b & D & & \\
\hline 4.4322 & 0.0021 & -1.0718 & & & 0 & 0 & 0 & b & & & & \\
\hline 2.7691 & 0.0072 & 2.0561 & & 0 & 0 & 0 & 0 & D & D & D & & \\
\hline 0.2711 & 0.0037 & 2.2240 & & 0 & 0 & 0 & 0 & b & D & & & \\
\hline-0.0321 & -0.0063 & -2.0598 & & 0 & U & 0 & 0 & b & b & D & & \\
\hline 2.4563 & -0.0044 & -2.2487 & & & & & & & & & & \\
\hline
\end{tabular}

$\begin{array}{lllllllllllllll}0.0321-0.0063 & -2.0598 & \mathrm{H} & 0 & 0 & 0 & 0 & 0 & 0 & 0 & 0 & 0 & 17\end{array}$

$2 \begin{array}{ccccc}2 & 0 & 0 & 0 & 0\end{array}$

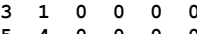

$\begin{array}{llllll}5 & 4 & 0 & 0 & 0 & 0 \\ 9 & 4 & 0 & 0 & 0 & 0\end{array}$

$\begin{array}{rrrrrr}9 & 4 & 0 & 0 & 0 & 0 \\ 15 & 4 & 0 & 0 & 0 & 0\end{array}$

$\begin{array}{rrrrrrr}6 & 5 & 0 & 0 & 0 & 0\end{array}$

$16 \quad 5 \quad 000000$

$\begin{array}{llllll}7 & 6 & 0 & 0 & 0 & 0\end{array}$

$\begin{array}{rrrrrr}10 & 6 & 0 & 0 & 0 & 0 \\ 8 & 7 & 0 & 0 & 0 & 0\end{array}$

$\begin{array}{rrrrrr}8 & 7 & 0 & 0 & 0 & 0 \\ 17 & 7 & 0 & 0 & 0 & 0\end{array}$

$\begin{array}{rrrrrr}17 & 7 & 0 & 0 & 0 & 0 \\ 9 & 8 & 0 & 0 & 0 & 0\end{array}$

$13 \quad 9 \quad 000000$

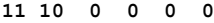

$1210 \quad 00000$

$14 \quad 13 \quad 000000$

818

$\$ \$ \$$

4-hydroxybenzoicacid.sdf

cosmotherm 3 D

$\begin{array}{llllllllllll}19 & 18 & 0 & 0 & 0 & 0 & 0 & 0 & 0 & 0 & 0 & \mathrm{v} 2000\end{array}$

$\begin{array}{lllllllllllllll}-1.1619 & 0.0031 & -4.4682 & 0 & 0 & 0 & 0 & 0 & 0 & 0 & 0 & 0 & 0 & 1\end{array}$

$\begin{array}{llllllllllllllll}-1.1619 & 0.0031 & -4.4682 & 0 & 0 & 0 & 0 & 0 & 0 & 0 & 0 & 0 & 0 & 1 \\ -1.1716 & -0.7809 & -5.0475 & \mathrm{H} & 0 & 0 & 0 & 0 & 0 & 0 & 0 & 0 & 0 & 2\end{array}$

$\begin{array}{rrrrrrrrrrrrrr}-1.1716 & -0.7809 & -5.0475 & \mathrm{H} & 0 & 0 & 0 & 0 & 0 & 0 & 0 & 0 & 0 & 2 \\ -1.1441 & 0.7620 & -5.0798 & \mathrm{H} & 0 & 0 & 0 & 0 & 0 & 0 & 0 & 0 & 0 & 3\end{array}$

$\begin{array}{rlllllllllllll}-1.1441 & 0.7620 & -5.0798 & \mathrm{H} & 0 & 0 & 0 & 0 & 0 & 0 & 0 & 0 & 0 & 3 \\ 1.6542 & 0.0004 & -0.5782 & \mathrm{C} & 0 & 0 & 0 & 0 & 0 & 0 & 0 & 0 & 0 & 4\end{array}$

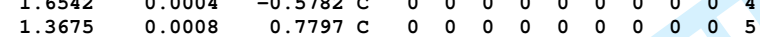

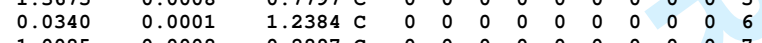

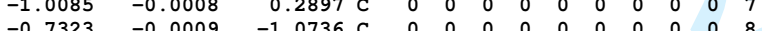

$\begin{array}{rrrrrrrrrrrrrr}-0.7323 & -0.0009 & -1.0736 & \mathrm{C} & 0 & 0 & 0 & 0 & 0 & 0 & 0 & 0 & 0 & 8 \\ 0.6055 & -0.0005 & -1.5222 & \mathrm{C} & 0 & 0 & 0 & 0 & 0 & 0 & 0 & 0 & 0 & 9\end{array}$

$\begin{array}{rrrrrrrrrrrrrr}0.6055 & -0.0005 & -1.5222 & \text { C } & 0 & 0 & 0 & 0 & 0 & 0 & 0 & 0 & 0 & 9 \\ -0.2197 & 0.0002 & 2.6917 & C & 0 & 0 & 0 & 0 & 0 & 0 & 0 & 0 & 0 & 10\end{array}$

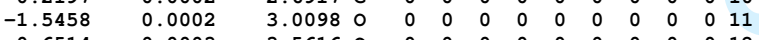

$\begin{array}{rrrrrrrrrrrrrr}0.6514 & 0.0003 & 3.5616 & 0 & 0 & 0 & 0 & 0 & 0 & 0 & 0 & 0 & 0 & 12 \\ 0.9391 & -0.0019 & -2.8332 & 0 & 0 & 0 & 0 & 0 & 0 & 0 & 0 & 0 & 0 & 13\end{array}$

$\begin{array}{rrrrrrrrrrrrrr}0.9391 & -0.0019 & -2.8332 & 0 & 0 & 0 & 0 & 0 & 0 & 0 & 0 & 0 & 0 & 13 \\ -1.6102 & 0.0009 & 3.9886 & H & 0 & 0 & 0 & 0 & 0 & 0 & 0 & 0 & 0 & 14\end{array}$

$\begin{array}{llllllllllllll}0.1194 & 0.0007 & -3.4263 & \mathrm{H} & 0 & 0 & 0 & 0 & 0 & 0 & 0 & 0 & 0 & 15\end{array}$

$\begin{array}{llllllllllllll}2.6861 & 0.0007 & -0.9322 & \mathrm{H} & 0 & 0 & 0 & 0 & 0 & 0 & 0 & 0 & 0 & 16\end{array}$

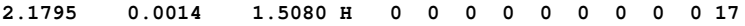

$\begin{array}{rrrrrrrrrrrrrr}-2.0460 & -0.0014 & 0.6234 & \mathrm{H} & 0 & 0 & 0 & 0 & 0 & 0 & 0 & 0 & 0 & 18 \\ -1.5461 & -0.0019 & -1.8005 & \mathrm{H} & 0 & 0 & 0 & 0 & 0 & 0 & 0 & 0 & 0 & 19\end{array}$

$\begin{array}{llllll}2 & 1 & 0 & 0 & 0 & 0 \\ 3 & 1 & 0 & 0 & 0 & 0\end{array}$

$5 \quad 4 \quad 000000$

$\begin{array}{llllll}9 & 4 & 0 & 0 & 0 & 0\end{array}$

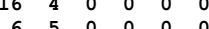

$\begin{array}{rrrrrr}6 & 5 & 0 & 0 & 0 & 0 \\ 17 & 5 & 0 & 0 & 0 & 0\end{array}$

$7 \quad 6 \quad 0 \quad 0 \quad 000$

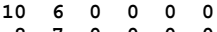

$\begin{array}{lllllll}18 & 7 & 0 & 0 & 0 & 0\end{array}$

$\begin{array}{lllllll}9 & 8 & 0 & 0 & 0 & 0\end{array}$

$\begin{array}{lllllll}13 & 9 & 0 & 0 & 0 & 0\end{array}$

$\begin{array}{lllllll}13 & 9 & 0 & 0 & 0 & 0\end{array}$

121000000

14

15 13 1300000

$\begin{array}{lllllll}8 & 19 & 0 & 0 & 0 & 0\end{array}$

END

\$\$\$

-hydroxymethylbenzoicacid-anion.sdf
COSMOtherm

$\begin{array}{llllllllllll}21 & 20 & 0 & 0 & 0 & 0 & 0 & 0 & 0 & 0 & 0 & \mathrm{v} 2000\end{array}$

$\begin{array}{rrrrrlllllllll}-5.2857 & -0.4589 & -0.2023 & 0 & 0 & 0 & 0 & 0 & 0 & 0 & 0 & 0 & 0 & 1 \\ -4.2846 & -0.5943 & -0.1372 & \mathrm{H} & 0 & 0 & 0 & 0 & 0 & 0 & 0 & 0 & 0 & 2 \\ -5.3712 & 0.5075 & -0.2755 & \mathrm{H} & 0 & 0 & 0 & 0 & 0 & 0 & 0 & 0 & 0 & 3 \\ 1.5515 & -1.2231 & 0.2693 & \mathrm{C} & 0 & 0 & 0 & 0 & 0 & 0 & 0 & 0 & 0 & 4 \\ 0.1606 & -1.1397 & 0.1614 & \mathrm{C} & 0 & 0 & 0 & 0 & 0 & 0 & 0 & 0 & 0 & 5 \\ -0.4779 & 0.1092 & 0.0914 & \mathrm{C} & 0 & 0 & 0 & 0 & 0 & 0 & 0 & 0 & 0 & 6 \\ 0.3096 & 1.2717 & 0.1343 & \mathrm{C} & 0 & 0 & 0 & 0 & 0 & 0 & 0 & 0 & 0 & 7 \\ 1.6993 & 1.1878 & 0.2446 & \mathrm{C} & 0 & 0 & 0 & 0 & 0 & 0 & 0 & 0 & 0 & 8 \\ 2.3388 & -0.0610 & 0.3130 & \mathrm{C} & 0 & 0 & 0 & 0 & 0 & 0 & 0 & 0 & 0 & 9 \\ -1.9965 & 0.2130 & -0.0211 & \mathrm{C} & 0 & 0 & 0 & 0 & 0 & 0 & 0 & 0 & 0 & 10 \\ 3.8401 & -0.1501 & 0.3964 & \mathrm{C} & 0 & 0 & 0 & 0 & 0 & 0 & 0 & 0 & 0 & 11 \\ 4.3773 & -0.1370 & -0.9529 & 0 & 0 & 0 & 0 & 0 & 0 & 0 & 0 & 0 & 0 & 12 \\ -2.6470 & -0.8910 & -0.0312 & 0 & 0 & 0 & 0 & 0 & 0 & 0 & 0 & 0 & 0 & 13 \\ -2.5082 & 1.3650 & -0.0963 & 0 & 0 & 0 & 0 & 0 & 0 & 0 & 0 & 0 & 0 & 14 \\ 5.3481 & -0.1785 & -0.8709 & \mathrm{H} & 0 & 0 & 0 & 0 & 0 & 0 & 0 & 0 & 0 & 15 \\ 2.0348 & -2.2019 & 0.3261 & \mathrm{H} & 0 & 0 & 0 & 0 & 0 & 0 & 0 & 0 & 0 & 16 \\ -0.4489 & -2.0442 & 0.1340 & \mathrm{H} & 0 & 0 & 0 & 0 & 0 & 0 & 0 & 0 & 0 & 17 \\ -0.1877 & 2.2417 & 0.0856 & \mathrm{H} & 0 & 0 & 0 & 0 & 0 & 0 & 0 & 0 & 0 & 18 \\ 2.2978 & 2.1015 & 0.2835 & \mathrm{H} & 0 & 0 & 0 & 0 & 0 & 0 & 0 & 0 & 0 & 19\end{array}$




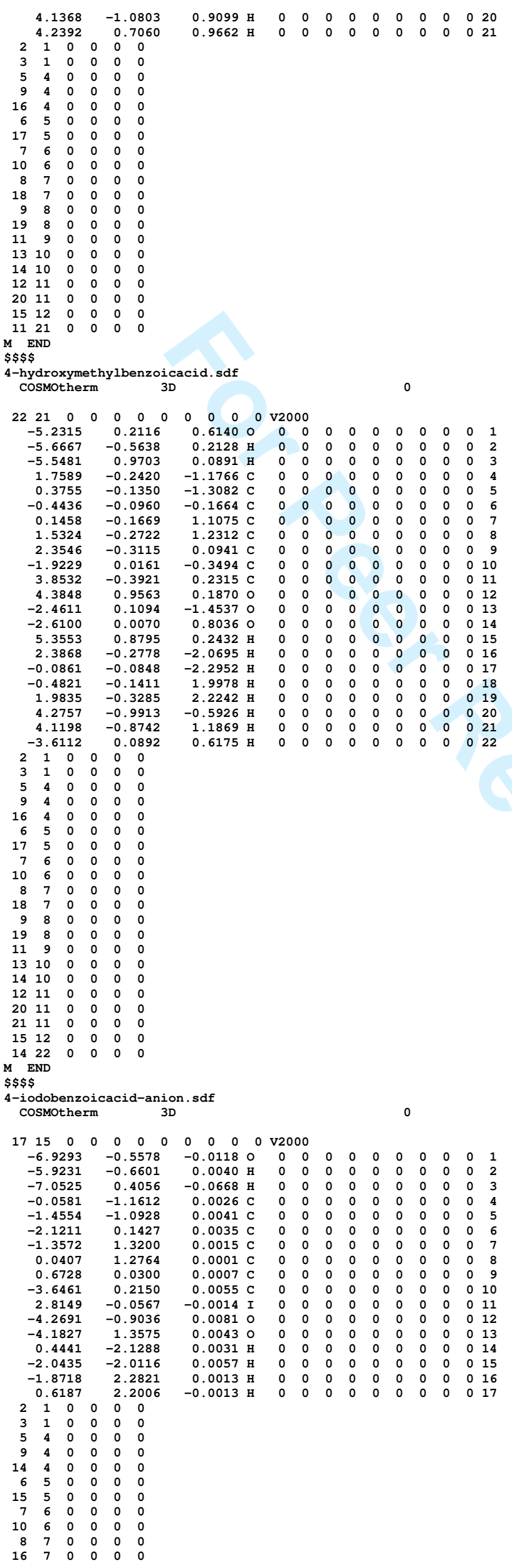




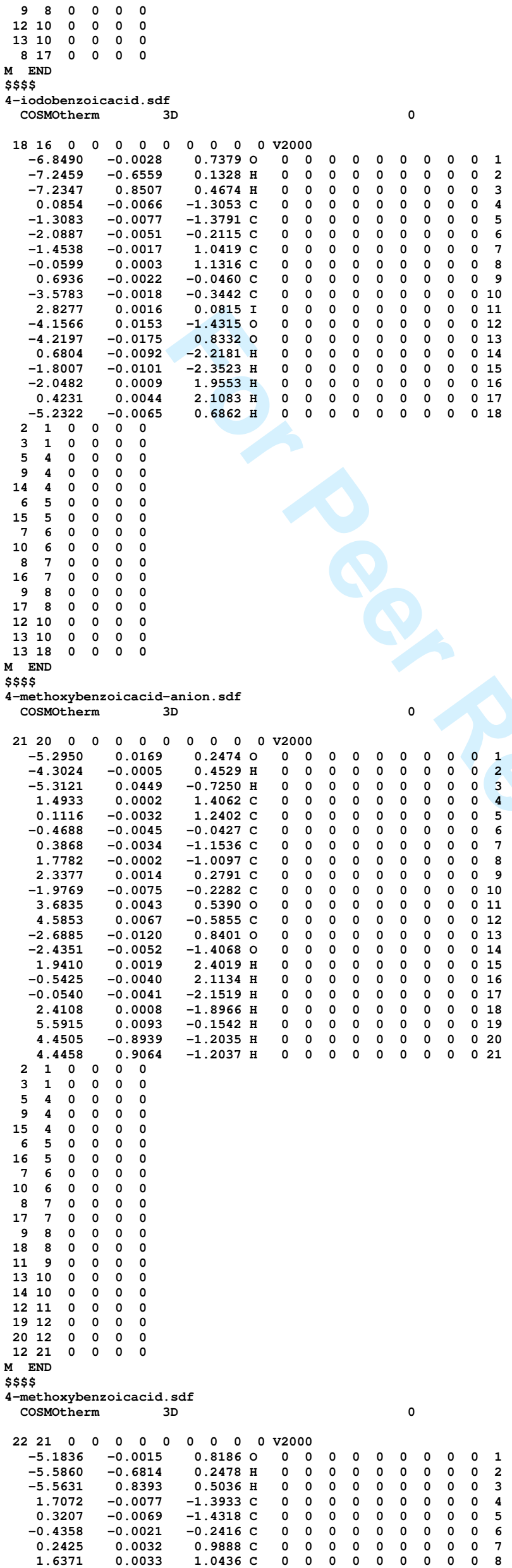




$\begin{array}{rrrrrrrrrrrrrr}2.3775 & -0.0024 & -0.1525 & \mathrm{C} & 0 & 0 & 0 & 0 & 0 & 0 & 0 & 0 & 0 & 9 \\ -1.9181 & -0.0003 & -0.3306 & \mathrm{C} & 0 & 0 & 0 & 0 & 0 & 0 & 0 & 0 & 0 & 10 \\ 3.7391 & -0.0042 & -0.2143 & \mathrm{O} & 0 & 0 & 0 & 0 & 0 & 0 & 0 & 0 & 0 & 11 \\ 4.4760 & 0.0124 & 1.0279 & \mathrm{C} & 0 & 0 & 0 & 0 & 0 & 0 & 0 & 0 & 0 & 12 \\ -2.5370 & 0.0218 & -1.3985 & \mathrm{O} & 0 & 0 & 0 & 0 & 0 & 0 & 0 & 0 & 0 & 13 \\ -2.5346 & -0.0273 & 0.8662 & \mathrm{O} & 0 & 0 & 0 & 0 & 0 & 0 & 0 & 0 & 0 & 14 \\ 2.2951 & -0.0125 & -2.3123 & \mathrm{H} & 0 & 0 & 0 & 0 & 0 & 0 & 0 & 0 & 0 & 15 \\ -0.1985 & -0.0102 & -2.3911 & \mathrm{H} & 0 & 0 & 0 & 0 & 0 & 0 & 0 & 0 & 0 & 16 \\ -0.3252 & 0.0074 & 1.9193 & \mathrm{H} & 0 & 0 & 0 & 0 & 0 & 0 & 0 & 0 & 0 & 17 \\ 2.1347 & 0.0080 & 2.0122 & \mathrm{H} & 0 & 0 & 0 & 0 & 0 & 0 & 0 & 0 & 0 & 18 \\ 5.5317 & 0.0148 & 0.7392 & \mathrm{H} & 0 & 0 & 0 & 0 & 0 & 0 & 0 & 0 & 0 & 19 \\ 4.2476 & 0.9185 & 1.6081 & \mathrm{H} & 0 & 0 & 0 & 0 & 0 & 0 & 0 & 0 & 0 & 20 \\ 4.2569 & -0.8834 & 1.6272 & \mathrm{H} & 0 & 0 & 0 & 0 & 0 & 0 & 0 & 0 & 0 & 21 \\ -3.5448 & -0.0171 & 0.7358 & \mathrm{H} & 0 & 0 & 0 & 0 & 0 & 0 & 0 & 0 & 0 & 22\end{array}$
$\begin{array}{llllllllllllll}-3.5448 & -0.0171 & 0.7358 & \mathrm{H} & 0 & 0 & 0 & 0 & 0 & 0 & 0 & 0 & 0 & 22\end{array}$ $\begin{array}{llllll}2 & 1 & 0 & 0 & 0 & 0 \\ 3 & 1 & 0 & 0 & 0 & 0\end{array}$ $\begin{array}{llllll}3 & 1 & 0 & 0 & 0 & 0 \\ 5 & 4 & 0 & 0 & 0 & 0\end{array}$ $\begin{array}{llllll}5 & 4 & 0 & 0 & 0 & 0 \\ 9 & 4 & 0 & 0 & 0 & 0\end{array}$ $\begin{array}{rrllll}9 & 4 & 0 & 0 & 0 & 0 \\ 15 & 4 & 0 & 0 & 0 & 0\end{array}$ $\begin{array}{rrrrrr}6 & 5 & 0 & 0 & 0 & 0\end{array}$

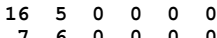
$\begin{array}{rrrrrr}7 & 6 & 0 & 0 & 0 & 0 \\ 10 & 6 & 0 & 0 & 0 & 0\end{array}$ $\begin{array}{llllll}8 & 7 & 0 & 0 & 0 & 0\end{array}$ $\begin{array}{rrrrrr}17 & 7 & 0 & 0 & 0 & 0 \\ 9 & 8 & 0 & 0 & 0 & 0\end{array}$ $\begin{array}{rrrrrr}9 & 8 & 0 & 0 & 0 & 0 \\ 18 & 8 & 0 & 0 & 0 & 0\end{array}$

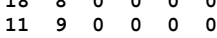
$\begin{array}{llllll}13 & 10 & 0 & 0 & 0 & 0\end{array}$ $\begin{array}{llllll}14 & 10 & 0 & 0 & 0 & 0\end{array}$ $\begin{array}{llllll}12 & 11 & 0 & 0 & 0 & 0\end{array}$ $\begin{array}{llllll}19 & 12 & 0 & 0 & 0 & 0 \\ 20 & 12 & 0 & 0 & 0 & 0\end{array}$ $\begin{array}{llllll}21 & 12 & 0 & 0 & 0 & 0 \\ 14 & 22 & 0 & 0 & 0 & 0\end{array}$ $M$ END

4-methylaminobenzoicacid-anion.sdf

cosmotherm

$\begin{array}{cccccccccccccccccc}22 & 21 & 0 & 0 & 0 & 0 & 0 & 0 & 0 & 0 & 0 & \mathrm{v} 2000 \\ -5.2730 & -0.2012 & 0.0272 & 0 & 0 & 0 & 0 & 0 & 0 & 0 & 0 & 0 & 0 & 1\end{array}$ $\begin{array}{llllllllllllll}-5.2730 & -0.2012 & 0.0272 & \circ & 0 & 0 & 0 & 0 & 0 & 0 & 0 & 0 & 0 & 1 \\ -4.2862 & -0.4420 & 0.0178 & \text { H } & 0 & 0 & 0 & 0 & 0 & 0 & 0 & 0 & 0 & 2\end{array}$ $\begin{array}{rrrrrrrrrrrrrr}-4.2862 & -0.4420 & 0.0178 & \mathrm{H} & 0 & 0 & 0 & 0 & 0 & 0 & 0 & 0 & 0 & 2 \\ -5.2481 & 0.7716 & 0.0300 & \mathrm{H} & 0 & 0 & 0 & 0 & 0 & 0 & 0 & 0 & 0 & 3\end{array}$ $\begin{array}{rrrrrrrrrrrrrr}-5.2481 & 0.7716 & 0.0300 & \mathrm{H} & 0 & 0 & 0 & 0 & 0 & 0 & 0 & 0 & 0 & 3 \\ 1.4991 & -1.4168 & -0.0013 & \mathrm{C} & 0 & 0 & 0 & 0 & 0 & 0 & 0 & 0 & 0 & 4\end{array}$ $\begin{array}{rrrrrrrrrrrrrr}1.4991 & -1.4168 & -0.0013 & \mathrm{C} & 0 & 0 & 0 & 0 & 0 & 0 & 0 & 0 & 0 & 4 \\ 0.1197 & -1.2515 & 0.0078 & \mathrm{C} & 0 & 0 & 0 & 0 & 0 & 0 & 0 & 0 & 0 & 5\end{array}$ $\begin{array}{rrrrrrrrrrrrrr}0.1197 & -1.2515 & 0.0078 & \mathrm{C} & 0 & 0 & 0 & 0 & 0 & 0 & 0 & 0 & 0 & 5 \\ -0.4699 & 0.0295 & -0.0098 & \mathrm{C} & 0 & 0 & 0 & 0 & 0 & 0 & 0 & 0 & 0 & 6\end{array}$ $\begin{array}{llllllllllllll}0.3912 & 1.1395 & -0.0336 & \mathrm{C} & 0 & 0 & 0 & 0 & 0 & 0 & 0 & 0 & 0 & 7 \\ 1.7787 & 0.9967 & -0.0420 & \mathrm{C} & 0 & 0 & 0 & 0 & 0 & 0 & 0 & 0 & 0 & 8\end{array}$ $\begin{array}{rrrrrrrrrrrrrr}1.7787 & 0.9967 & -0.0420 & \mathrm{C} & 0 & 0 & 0 & 0 & 0 & 0 & 0 & 0 & 0 & 8 \\ 2.3659 & -0.2926 & -0.0317 & \mathrm{C} & 0 & 0 & 0 & 0 & 0 & 0 & 0 & 0 & 0 & 9\end{array}$ $\begin{array}{rrrrrrrrrrrrrr}2.3659 & -0.2926 & -0.0317 & \mathrm{C} & 0 & 0 & 0 & 0 & 0 & 0 & 0 & 0 & 0 & 9 \\ -1.9707 & 0.2124 & -0.0006 & \mathrm{C} & 0 & 0 & 0 & 0 & 0 & 0 & 0 & 0 & 0 & 10\end{array}$ $\begin{array}{rrrrrrrrrrrrrr}-1.9707 & 0.2124 & -0.0006 & \mathrm{C} & 0 & 0 & 0 & 0 & 0 & 0 & 0 & 0 & 0 & 10 \\ 3.7299 & -0.4766 & -0.0816 & \mathrm{~N} & 0 & 0 & 0 & 0 & 0 & 0 & 0 & 0 & 0 & 11\end{array}$ $\begin{array}{llllllllllllll}4.6838 & 0.6053 & 0.1117 & \mathrm{C} & 0 & 0 & 0 & 0 & 0 & 0 & 0 & 0 & 0 & 12\end{array}$ $\begin{array}{rrrrrrrrrrrrrr}-2.6878 & -0.8558 & 0.0069 & 0 & 0 & 0 & 0 & 0 & 0 & 0 & 0 & 0 & 0 & 13 \\ -2.4363 & 1.3908 & -0.0012 & 0 & 0 & 0 & 0 & 0 & 0 & 0 & 0 & 0 & 0 & 14\end{array}$ $\begin{array}{lllllllllllll}4.0606 & -1.4096 & 0.1466 \mathrm{H} & 0 & 0 & 0 & 0 & 0 & 0 & 0 & 0 & 0 & 15\end{array}$

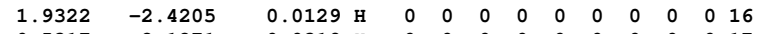

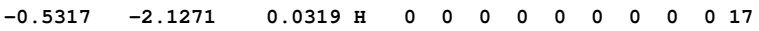

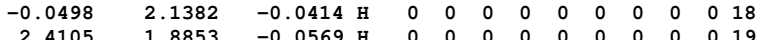

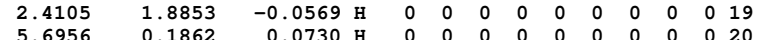

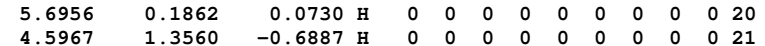
$\begin{array}{llrrrrrrrrrrrrr}4.5538 & 1.1202 & 1.0810 \mathrm{H} & 0 & 0 & 0 & 0 & 0 & 0 & 0 & 0 & 0 & 22\end{array}$

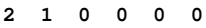
$3 \begin{array}{llllll}3 & 1 & 0 & 0 & 0 & 0\end{array}$ $5 \begin{array}{llllll}5 & 4 & 0 & 0 & 0 & 0\end{array}$ $\begin{array}{rrrrrr}9 & 4 & 0 & 0 & 0 & 0\end{array}$ $\begin{array}{rrrrrr}16 & 4 & 0 & 0 & 0 & 0 \\ 6 & 5 & 0 & 0 & 0 & 0\end{array}$

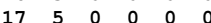
$\begin{array}{llllll}7 & 6 & 0 & 0 & 0 & 0\end{array}$ 1060600100

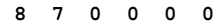
$18 \quad 7 \quad 000000$ $\begin{array}{llllll}9 & 8 & 0 & 0 & 0 & 0\end{array}$ $\begin{array}{llllll}19 & 8 & 0 & 0 & 0 & 0\end{array}$ $\begin{array}{rrrrrr}11 & 9 & 0 & 0 & 0 & 0 \\ 13 & 10 & 0 & 0 & 0 & 0\end{array}$

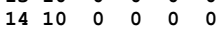
$1211 \quad 000000$ $1511 \quad 000000$ $\begin{array}{lllllll}20 & 12 & 0 & 0 & 0 & 0\end{array}$ $\begin{array}{llllll}21 & 12 & 0 & 0 & 0 & 0 \\ 12 & 22 & 0 & 0 & 0 & 0\end{array}$ END

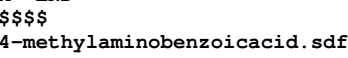
cosmotherm 3D 0

$\begin{array}{lllllllllll}2322 & 0 & 0 & 0 & 0 & 0 & 0 & 0 & 0 & 0 & \mathrm{v} 2000\end{array}$

\begin{tabular}{|c|c|c|c|c|c|c|c|c|c|c|c|c|c|}
\hline 192 & -0.0057 & 0.8235 & 0 & 0 & 0 & 0 & 0 & 0 & 0 & 0 & 0 & 0 & 1 \\
\hline 5.5839 & -0.5868 & 0.1459 & $\mathrm{H}$ & 0 & 0 & 0 & 0 & 0 & 0 & 0 & 0 & 0 & 2 \\
\hline-5.5661 & 0.8747 & 0.6353 & $\mathrm{H}$ & 0 & 0 & 0 & 0 & 0 & 0 & 0 & 0 & 0 & 3 \\
\hline 1.7063 & -0.0047 & -1.4106 & $\mathrm{C}$ & 0 & 0 & 0 & 0 & 0 & 0 & 0 & 0 & 0 & 4 \\
\hline 0.3236 & -0.0049 & -1.4378 & $\mathrm{C}$ & 0 & 0 & 0 & 0 & 0 & 0 & 0 & 0 & 0 & 5 \\
\hline-0.4352 & -0.0038 & -0.2444 & $\mathrm{c}$ & 0 & 0 & 0 & 0 & 0 & 0 & 0 & 0 & 0 & 6 \\
\hline 0.2602 & -0.0010 & 0.9820 & & 0 & & 0 & 0 & 0 & 0 & 0 & 0 & 0 & 7 \\
\hline 1.6491 & 0.0011 & 1.0280 & $\mathrm{c}$ & 0 & 0 & 0 & 0 & 0 & 0 & 0 & 0 & 0 & 8 \\
\hline 2.4098 & -0.0010 & -0.1723 & & 0 & 0 & 0 & 0 & 0 & 0 & 0 & 0 & 0 & 9 \\
\hline-1.9067 & -0.0022 & -0.3247 & $\mathrm{C}$ & 0 & 0 & 0 & 0 & 0 & 0 & 0 & 0 & & 10 \\
\hline 3.7724 & -0.0009 & -0.1627 & & 0 & & 0 & 0 & 0 & 0 & 0 & 0 & & 11 \\
\hline 4.5916 & 0.0110 & 1.0382 & $2 \mathrm{c}$ & 0 & 0 & 0 & 0 & 0 & 0 & 0 & 0 & & 12 \\
\hline-2.5430 & 0.0185 & -1.3864 & & 0 & & 0 & 0 & 0 & 0 & 0 & 0 & 0 & 13 \\
\hline-2.5240 & -0.0278 & 0.8795 & & 0 & & 0 & 0 & 0 & 0 & 0 & 0 & & 14 \\
\hline 4.2493 & 0.0047 & -1.0585 & & & & & & & & & & & \\
\hline
\end{tabular}


$\begin{array}{llllllllllllll}2.2741 & -0.0067 & -2.3441 & \mathrm{H} & 0 & 0 & 0 & 0 & 0 & 0 & 0 & 0 & 0 & 16\end{array}$

$\begin{array}{lllllllllllllll}-0.2001 & -0.0066 & -2.3954 & \mathrm{H} & 0 & 0 & 0 & 0 & 0 & 0 & 0 & 0 & 0 & 17 \\ -0.3012 & 0.0010 & 1.9170 & \mathrm{H} & 0 & 0 & 0 & 0 & 0 & 0 & 0 & 0 & 0 & 18\end{array}$ $\begin{array}{llllllllllllll}2.1541 & 0.0010 & 1.9170 & \mathrm{H} & 0 & 0 & 0 & 0 & 0 & 0 & 0 & 0 & 0 & 18 \\ 5.6445 & 0.0056 & 1.9939 & \mathrm{H} & 0 & 0 & 0 & 0 & 0 & 0 & 0 & 0 & 0 & 19\end{array}$ $\begin{array}{lllllllllllll}5.6445 & -0.0012 & 0.7368 \mathrm{H} & 0 & 0 & 0 & 0 & 0 & 0 & 0 & 0 & 0 & 20\end{array}$ $\begin{array}{rrrrrrrrrrrrrr}4.4147 & 0.9145 & 1.6446 & \mathrm{H} & 0 & 0 & 0 & 0 & 0 & 0 & 0 & 0 & 0 & 21 \\ 4.4016 & -0.8724 & 1.6691 & \mathrm{H} & 0 & 0 & 0 & 0 & 0 & 0 & 0 & 0 & 0 & 22\end{array}$

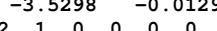

$\begin{array}{llllll}2 & 1 & 0 & 0 & 0 & 0 \\ 3 & 1 & 0 & 0 & 0 & 0\end{array}$

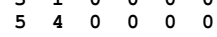

$9 \begin{array}{llllll}5 & 4 & 0 & 0 & 0 & 0\end{array}$

$16 \quad 4 \quad 0 \quad 00000$

6 6 51000000

$\begin{array}{llllll}17 & 5 & 0 & 0 & 0 & 0\end{array}$

$\begin{array}{rrrrrrr}7 & 6 & 0 & 0 & 0 & 0 \\ 10 & 6 & 0 & 0 & 0 & 0\end{array}$

$\begin{array}{rrrrrr}7 & 6 & 0 & 0 & 0 & 0 \\ 8 & 7 & 0 & 0 & 0 & 0\end{array}$

$18 \quad 7 \quad 000000$

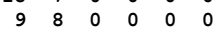

$\begin{array}{llllll}19 & 8 & 0 & 0 & 0 & 0\end{array}$

$\begin{array}{rrrrrr}11 & 9 & 0 & 0 & 0 & 0 \\ 13 & 10 & 0 & 0 & 0 & 0\end{array}$

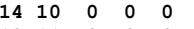

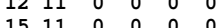

$\begin{array}{llllll}15 & 11 & 0 & 0 & 0 & 0 \\ 20 & 12 & 0 & 0 & 0 & 0\end{array}$

$2112 \quad 0 \quad 0000$

$\begin{array}{llllll}22 & 12 & 0 & 0 & 0 & 0 \\ 14 & 23 & 0 & 0 & 0 & 0\end{array}$

$M$ END

$\$ \$ \$$

-methylbenzoicacid-anion.sdf

CosMOtherm

$\begin{array}{llllllllllll}20 & 19 & 0 & 0 & 0 & 0 & 0 & 0 & 0 & 0 & 0 & \mathrm{v} 2000\end{array}$

$\begin{array}{llllllllllllll}-4.8384 & -0.0034 & -0.4293 & \circ & 0 & 0 & 0 & 0 & 0 & 0 & 0 & 0 & 0 & 1\end{array}$

$\begin{array}{llllllllllllll}-3.8348 & 0.0002 & -0.5695 & \mathrm{H} & 0 & 0 & 0 & 0 & 0 & 0 & 0 & 0 & 0\end{array}$

$\begin{array}{llllllllllllll}-4.9240 & -0.0094 & 0.5398 & \mathrm{H} & 0 & 0 & 0 & 0 & 0 & 0 & 0 & 0 & 0 & 3\end{array}$

$\begin{array}{lllllllllllll}2.0063 & -0.0082 & -1.2579 & C & 0 & 0 & 0 & 0 & 0 & 0 & 0 & 0 & 0\end{array}$

$\begin{array}{llllllllllllll}0.6124 & -0.0019 & -1.1570 & C & 0 & 0 & 0 & 0 & 0 & 0 & 0 & 0 & 0 & 5\end{array}$

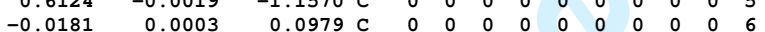

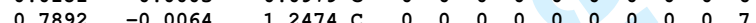

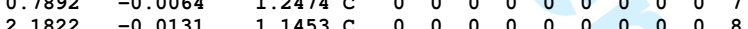

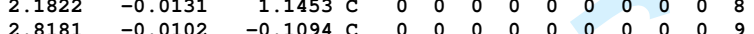

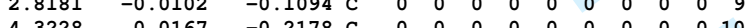

$\begin{array}{rrrrrrrrrrrrrr}4.3228 & 0.0167 & -0.2178 & \mathrm{C} & 0 & 0 & 0 & 0 & 0 & 0 & 0 & 0 & 0 & 10 \\ -1.5366 & 0.0050 & 0.2201 & \mathrm{C} & 0 & 0 & 0 & 0 & 0 & 0 & 0 & 0 & 0 & 11\end{array}$

$\begin{array}{llllllllllllll}-1.5366 & 0.0050 & 0.2201 & 0 & 0 & 0 & 0 & 0 & 0 & 0 & 0 & 0 & 0 & 11 \\ -2.2018 & 0.0035 & -0.8763 & 0 & 0 & 0 & 0 & 0 & 0 & 0 & 0 & 0 & 0 & 12\end{array}$

$\begin{array}{rrrrrrrrrrrrrr}-2.2018 & 0.0035 & -0.8763 & 0 & 0 & 0 & 0 & 0 & 0 & 0 & 0 & 0 & 0 & 12 \\ -2.0409 & 0.0105 & 1.3789 & 0 & 0 & 0 & 0 & 0 & 0 & 0 & 0 & 0 & 0 & 13\end{array}$

$\begin{array}{rrrrrrrrrrrrrr}-2.0409 & 0.0105 & 1.3789 & 0 & 0 & 0 & 0 & 0 & 0 & 0 & 0 & 0 & 0 & 13 \\ 2.4759 & -0.0140 & -2.2452 & \mathrm{H} & 0 & 0 & 0 & 0 & 0 & 0 & 0 & 0 & 0 & 14\end{array}$

$\begin{array}{rrrrrrrrrrrrrr}2.4759 & -0.0140 & -2.2452 & \mathrm{H} & 0 & 0 & 0 & 0 & 0 & 0 & 0 & 0 & 0 & 14 \\ -0.0063 & -0.0023 & -2.0561 & \mathrm{H} & 0 & 0 & 0 & 0 & 0 & 0 & 0 & 0 & 0 & 15\end{array}$

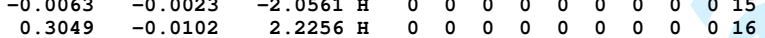

$\begin{array}{lllllllllllll}0.3049 & -0.0102 & 2.2256 \mathrm{H} & 0 & 0 & 0 & 0 & 0 & 0 & 0 & 0 & 0 & 16 \\ 2.7898 & -0.0229 & 2.0541 \mathrm{H} & 0 & 0 & 0 & 0 & 0 & 0 & 0 & 0 & 0 & 17\end{array}$

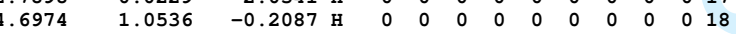

$\begin{array}{lllllllllllll}4.6670 & -0.4457 & -1.1533 \mathrm{H} & 0 & 0 & 0 & 0 & 0 & 0 & 0 & 0 & 0 & 19\end{array}$

$\begin{array}{lllllllllllll}4.7956 & -0.5065 & 0.6251 \mathrm{H} & 0 & 0 & 0 & 0 & 0 & 0 & 0 & 0 & 0 & 20\end{array}$

$\begin{array}{lllllll}2 & 1 & 0 & 0 & 0 & 0\end{array}$

$\begin{array}{llllll}3 & 1 & 0 & 0 & 0 & 0\end{array}$

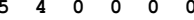

$9 \begin{array}{llllll}9 & 4 & 0 & 0 & 0\end{array}$

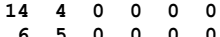

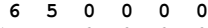

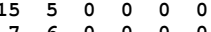

$\begin{array}{lllllll}1 & 6 & 0 & 0 & 0 & 0\end{array}$

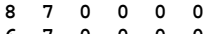

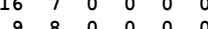

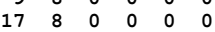

$\begin{array}{llllll}10 & 9 & 0 & 0 & 0 & 0\end{array}$

$18 \quad 10 \quad 0 \quad 0000$

$\begin{array}{llllll}19 & 10 & 0 & 0 & 0 & 0\end{array}$

121110000000

$\begin{array}{llllll}13 & 11 & 0 & 0 & 0 & 0 \\ 10 & 20 & 0 & 0 & 0 & 0\end{array}$

$M$ END

\$\$\$

4-methylbenzoicacid.sdf

cosmotherm

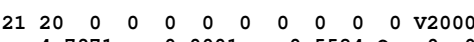

$\begin{array}{lllllllllllll}-0.0001 & 0.5584 & \circ & 0 & 0 & 0 & 0 & 0 & 0 & 0 & 0 & 0\end{array}$

$\begin{array}{llllllllllllll}-5.1508 & -0.5968 & -0.1265 & \mathrm{H} & 0 & 0 & 0 & 0 & 0 & 0 & 0 & 0 & 0 & 2\end{array}$

$\begin{array}{lllllllllllll}-5.1632 & 0.8752 & 0.3345 \mathrm{H} & 0 & 0 & 0 & 0 & 0 & 0 & 0 & 0 & 0 & 3\end{array}$

$\begin{array}{llllllllllllllll}2.2418 & -0.0039 & -1.1225 & \mathrm{C} & 0 & 0 & 0 & 0 & 0 & 0 & 0 & 0 & 0 & 4\end{array}$

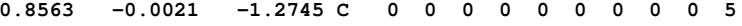

$\begin{array}{lllllllllllllll}0.0160 & -0.0045 & -0.1475 & \mathrm{C} & 0 & 0 & 0 & 0 & 0 & 0 & 0 & 0 & 0 & 6\end{array}$

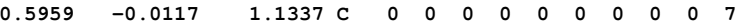

$\begin{array}{llllllllllllll}1.9835 & -0.0135 & 1.2771 & \mathrm{C} & 0 & 0 & 0 & 0 & 0 & 0 & 0 & 0 & 0 & 8\end{array}$

$\begin{array}{llllllllllllll}2.8320 & -0.0059 & 0.1550 & \mathrm{C} & 0 & 0 & 0 & 0 & 0 & 0 & 0 & 0 & 0 & 9\end{array}$

$\begin{array}{llllllllllllll}4.3299 & 0.0253 & 0.3160 & \mathrm{C} & 0 & 0 & 0 & 0 & 0 & 0 & 0 & 0 & 0 & 10\end{array}$

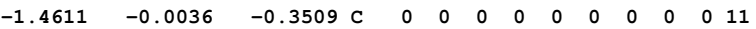

$\begin{array}{llllllllllllll}-1.9938 & 0.0018 & -1.4629 & 0 & 0 & 0 & 0 & 0 & 0 & 0 & 0 & 0 & 0 & 12\end{array}$

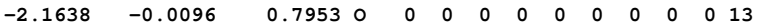

$\begin{array}{llllllllllllll}2.8801 & -0.0064 & -2.0089 & \mathrm{H} & 0 & 0 & 0 & 0 & 0 & 0 & 0 & 0 & 0 & 14\end{array}$

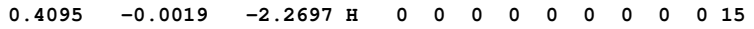

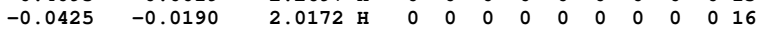

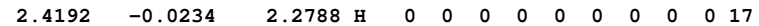

$\begin{array}{lllllllllllll}4.6910 & 1.0655 & 0.3694 \mathrm{H} & 0 & 0 & 0 & 0 & 0 & 0 & 0 & 0 & 0 & 18\end{array}$

$\begin{array}{llllllllllllll}4.8352 & -0.4524 & -0.5344 \mathrm{H} & 0 & 0 & 0 & 0 & 0 & 0 & 0 & 0 & 0 & 19 \\ 4.6452 & -0.4767 & 1.2411 & \mathrm{H} & 0 & 0 & 0 & 0 & 0 & 0 & 0 & 0 & 0 & 20\end{array}$

$\begin{array}{rrrrrrrrrrrrrrrr}4.6452 & -0.4767 & 1.2411 & \text { H } & 0 & 0 & 0 & 0 & 0 & 0 & 0 & 0 & 0 & 20\end{array}$

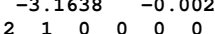

$3 \begin{array}{llllll}2 & 1 & 0 & 0 & 0 & 0\end{array}$

$\begin{array}{llllll}5 & 4 & 0 & 0 & 0 & 0\end{array}$ 


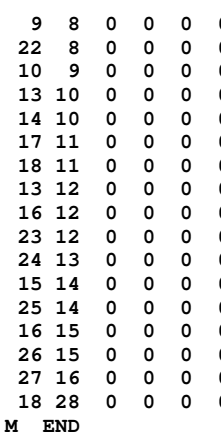




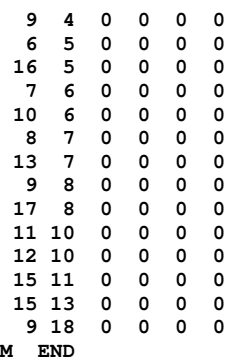

$$
\begin{aligned}
& M \quad \text { END } \\
& \$ \$ \$
\end{aligned}
$$

-iodo-2-hydroxybenzoicacid sdf

cosmotherm

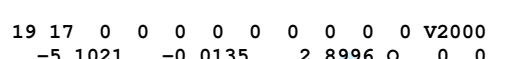

$\begin{array}{llllllllllllll}-5.1021 & -0.0135 & 2.8996 & 0 & 0 & 0 & 0 & 0 & 0 & 0 & 0 & 0 & 0 & 1 \\ -5.6507 & -0.8081 & 2.7647 & & 0 & 0 & 0 & 0 & 0 & 0 & 0 & 0 & 0 & 2\end{array}$

$\begin{array}{llllllllllllll}-5.6507 & -0.8081 & 2.7647 & \text { н } & 0 & 0 & 0 & 0 & 0 & 0 & 0 & 0 & 0 & 2\end{array}$

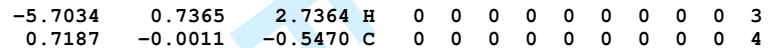

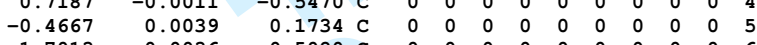

$\begin{array}{rrrrrrrrrrrrrr}-1.7013 & 0.0036 & -0.5080 & \text { C } & 0 & 0 & 0 & 0 & 0 & 0 & 0 & 0 & 0 & 6 \\ -1.7171 & -0.0018 & -1.9302 & \text { C } & 0 & 0 & 0 & 0 & 0 & 0 & 0 & 0 & 0 & 7\end{array}$

$\begin{array}{llllllllllllll}-1.7171 & -0.0018 & -1.9302 & \mathrm{C} & 0 & 0 & 0 & 0 & 0 & 0 & 0 & 0 & 0 & 7 \\ -0.4997 & -0.0073 & -2.6339 & \mathrm{C} & 0 & 0 & 0 & 0 & 0 & 0 & 0 & 0 & 0 & 8\end{array}$

$\begin{array}{lllllllllllllll}0.7118 & -0.0070 & -1.9508 & \mathrm{C} & 0 & 0 & 0 & 0 & 0 & 0 & 0 & 0 & 0 & 9\end{array}$

$\begin{array}{rrrrrrrrrrrrrrr}-2.9839 & 0.0084 & 0.2238 & \mathrm{C} & 0 & 0 & 0 & 0 & 0 & 0 & 0 & 0 & 0 & 10\end{array}$

$\begin{array}{llrlllllllllll}-2.8886 & 0.0108 & 1.5470 & 0 & 0 & 0 & 0 & 0 & 0 & 0 & 0 & 0 & 0 & 11 \\ -4.0818 & 0.0099 & -0.3746 & 0 & 0 & 0 & 0 & 0 & 0 & 0 & 0 & 0 & 0 & 12\end{array}$

$\begin{array}{lrrrrrrrrrrrrr}-4.0818 & 0.0099 & -0.3746 & 0 & 0 & 0 & 0 & 0 & 0 & 0 & 0 & 0 & 0 & 12 \\ -2.8701 & -0.0021 & -2.6345 & 0 & 0 & 0 & 0 & 0 & 0 & 0 & 0 & 0 & 0 & 13\end{array}$

$\begin{array}{llllllllllllll}2.5899 & 0.0001 & 0.4931 & \text { I } & 0 & 0 & 0 & 0 & 0 & 0 & 0 & 0 & 0 & 14\end{array}$

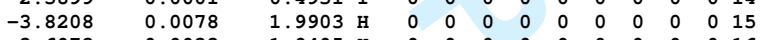

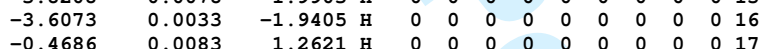

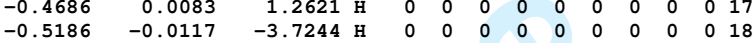

$\begin{array}{ccccccccccccccc}-0.5186 & -0.0117 & -3.7244 & \text { H } & 0 & 0 & 0 & 0 & 0 & 0 & 0 & 0 & 0 & 18 \\ 1.6448 & -0.0113 & -2.5141 & \text { H } & 0 & 0 & 0 & 0 & 0 & 0 & 0 & 0 & 0 & 19\end{array}$

$\begin{array}{llllll}2 & 1 & 0 & 0 & 0 & 0\end{array}$

$\begin{array}{llllll}3 & 1 & 0 & 0 & 0 & 0\end{array}$

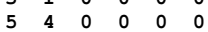

$\begin{array}{llllll}9 & 4 & 0 & 0 & 0 & 0\end{array}$

$\begin{array}{llllll}6 & 5 & 0 & 0 & 0 & 0\end{array}$

$\begin{array}{llllll}17 & 5 & 0 & 0 & 0 & 0\end{array}$

$\begin{array}{lllllll}7 & 6 & 0 & 0 & 0 & 0\end{array}$

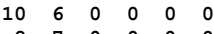

$\begin{array}{rrrrrr}8 & 7 & 0 & 0 & 0 & 0 \\ 13 & 7 & 0 & 0 & 0 & 0\end{array}$

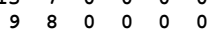

$\begin{array}{llllll}18 & 8 & 0 & 0 & 0 & 0\end{array}$

$\begin{array}{llllll}11 & 10 & 0 & 0 & 0 & 0\end{array}$

$\begin{array}{llllll}12 & 10 & 0 & 0 & 0 & 0\end{array}$

$\begin{array}{llllll}15 & 11 & 0 & 0 & 0 & 0\end{array}$

$\begin{array}{rrrrrr}16 & 13 & 0 & 0 & 0 & 0 \\ 9 & 19 & 0 & 0 & 0 & 0\end{array}$

M END

\$\$\$

5-nitrouracil-anion.sdf
CosMOtherm

$\begin{array}{llllllllllll}16 & 15 & 0 & 0 & 0 & 0 & 0 & 0 & 0 & 0 & 0 & \mathrm{v} 2000\end{array}$

$\begin{array}{rrrrrrrrrrrrrr}-3.7511 & -0.1078 & -2.0922 & \mathrm{O} & 0 & 0 & 0 & 0 & 0 & 0 & 0 & 0 & 0 & 1 \\ -2.9974 & -0.0414 & -1.4342 & \mathrm{H} & 0 & 0 & 0 & 0 & 0 & 0 & 0 & 0 & 0 & 2 \\ -3.9499 & 0.8161 & -2.3221 & \mathrm{H} & 0 & 0 & 0 & 0 & 0 & 0 & 0 & 0 & 0 & 3 \\ -0.3357 & 0.0270 & -0.9117 & \mathrm{C} & 0 & 0 & 0 & 0 & 0 & 0 & 0 & 0 & 0 & 4 \\ 0.8822 & 0.0047 & -0.2132 & \mathrm{C} & 0 & 0 & 0 & 0 & 0 & 0 & 0 & 0 & 0 & 5 \\ 0.8708 & -0.0123 & 1.2434 & \mathrm{C} & 0 & 0 & 0 & 0 & 0 & 0 & 0 & 0 & 0 & 6 \\ -0.4431 & -0.0018 & 1.7386 & \mathrm{~N} & 0 & 0 & 0 & 0 & 0 & 0 & 0 & 0 & 0 & 7 \\ -1.6402 & 0.0194 & 1.0183 & \mathrm{C} & 0 & 0 & 0 & 0 & 0 & 0 & 0 & 0 & 0 & 8 \\ -1.5436 & 0.0315 & -0.3659 & \mathrm{~N} & 0 & 0 & 0 & 0 & 0 & 0 & 0 & 0 & 0 & 9 \\ 1.8200 & -0.0332 & 2.0364 & \mathrm{O} & 0 & 0 & 0 & 0 & 0 & 0 & 0 & 0 & 0 & 10 \\ -2.7273 & 0.0260 & 1.6124 & \mathrm{O} & 0 & 0 & 0 & 0 & 0 & 0 & 0 & 0 & 0 & 11 \\ 2.0948 & 0.0037 & -0.9617 & \mathrm{~N} & 0 & 0 & 0 & 0 & 0 & 0 & 0 & 0 & 0 & 12 \\ -0.2947 & 0.0368 & -2.0035 & \mathrm{H} & 0 & 0 & 0 & 0 & 0 & 0 & 0 & 0 & 0 & 13 \\ -0.5268 & -0.0119 & 2.7569 & \mathrm{H} & 0 & 0 & 0 & 0 & 0 & 0 & 0 & 0 & 0 & 14 \\ 2.0286 & 0.0234 & -2.2196 & 0 & 0 & 0 & 0 & 0 & 0 & 0 & 0 & 0 & 0 & 15 \\ 3.1919 & -0.0171 & -0.3610 & 0 & 0 & 0 & 0 & 0 & 0 & 0 & 0 & 0 & 0 & 16\end{array}$

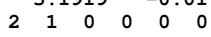

$\begin{array}{llllll}3 & 1 & 0 & 0 & 0 & 0 \\ 5 & 4 & 0 & 0 & 0 & 0\end{array}$

$\begin{array}{llllll}5 & 4 & 0 & 0 & 0 & 0 \\ 9 & 4 & 0 & 0 & 0 & 0\end{array}$

$13 \quad 4 \quad 000000$

$\begin{array}{llllll}6 & 5 & 0 & 0 & 0 & 0\end{array}$

$\begin{array}{llllll}12 & 5 & 0 & 0 & 0 & 0\end{array}$

$\begin{array}{rrrrrr}7 & 6 & 0 & 0 & 0 & 0 \\ 10 & 6 & 0 & 0 & 0 & 0\end{array}$

$\begin{array}{llllll}10 & 6 & 0 & 0 & 0 & 0 \\ 0 & 7 & 0 & 0 & 0 & 0\end{array}$

$\begin{array}{rrrrrr}8 & 7 & 0 & 0 & 0 & 0 \\ 14 & 7 & 0 & 0 & 0 & 0\end{array}$

$\begin{array}{llllll}14 & 7 & 0 & 0 & 0 & 0 \\ 9 & 8 & 0 & 0 & 0 & 0\end{array}$

$\begin{array}{rrrrrr}9 & 8 & 0 & 0 & 0 & 0 \\ 11 & 8 & 0 & 0 & 0 & 0\end{array}$

$\begin{array}{llllll}15 & 12 & 0 & 0 & 0 & 0 \\ 12 & 16 & 0 & 0 & 0 & 0\end{array}$

$M$ END

$\$ \$ \$$

5-nitrouracil.sdf
CosMOtherm

$\begin{array}{llllllllllll}17 & 16 & 0 & 0 & 0 & 0 & 0 & 0 & 0 & 0 & 0 & \mathrm{v} 2000\end{array}$

$\begin{array}{lllllllllllllll}-2.1028 & -3.5784 & 0.0025 & 0 & 0 & 0 & 0 & 0 & 0 & 0 & 0 & 0 & 0 & 1\end{array}$

$\begin{array}{llllllllllllll}-2.1028 & -3.5784 & 0.0025 & \mathrm{O} & 0 & 0 & 0 & 0 & 0 & 0 & 0 & 0 & 0 & 1 \\ -1.9729 & -4.2456 & 0.7011 & \mathrm{H} & 0 & 0 & 0 & 0 & 0 & 0 & 0 & 0 & 0 & 2\end{array}$

$\begin{array}{llrlllllllllll}-1.9729 & -4.2456 & 0.7011 & \mathrm{H} & 0 & 0 & 0 & 0 & 0 & 0 & 0 & 0 & 0 & 2 \\ -2.1392 & -4.0909 & -0.8260 & \mathrm{H} & 0 & 0 & 0 & 0 & 0 & 0 & 0 & 0 & 0 & 3\end{array}$

$\begin{array}{lllllllllllllll}-0.9333 & -0.2794 & 0.0012 & \mathrm{C} & 0 & 0 & 0 & 0 & 0 & 0 & 0 & 0 & 0 & \mathbf{4} \\ -0.2068 & 0.8915 & 0.0017 & \mathrm{C} & 0 & 0 & 0 & 0 & 0 & 0 & 0 & 0 & 0 & 5\end{array}$

$\begin{array}{rrrrrrrrrrrrrr}-0.2068 & 0.8915 & 0.0017 & \mathrm{C} & 0 & 0 & 0 & 0 & 0 & 0 & 0 & 0 & 0 & 5 \\ 1.2588 & 0.8513 & 0.0034 & \mathrm{C} & 0 & 0 & 0 & 0 & 0 & 0 & 0 & 0 & 0 & 6\end{array}$ 
$\begin{array}{llllll}2 & 1 & 0 & 0 & 0 & 0\end{array}$

$\begin{array}{llllll}3 & 1 & 0 & 0 & 0 & 0\end{array}$

$\begin{array}{llllll}5 & 4 & 0 & 0 & 0 & 0\end{array}$

$\begin{array}{rrrrrr}9 & 4 & 0 & 0 & 0 & 0 \\ 13 & 4 & 0 & 0 & 0 & 0\end{array}$

$\begin{array}{rrrrrr}13 & 4 & 0 & 0 & 0 & 0 \\ 6 & 5 & 0 & 0 & 0 & 0\end{array}$

125050000

$\begin{array}{llllll}7 & 6 & 0 & 0 & 0 & 0 \\ 10 & 6 & 0 & 0 & 0 & 0\end{array}$

$\begin{array}{rrrrrr}10 & 6 & 0 & 0 & 0 & 0 \\ 8 & 7 & 0 & 0 & 0 & 0\end{array}$

$\begin{array}{rrrrrr}8 & 7 & 0 & 0 & 0 & 0 \\ 14 & 7 & 0 & 0 & 0 & 0\end{array}$

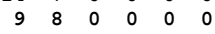

$\begin{array}{llllll}11 & 8 & 0 & 0 & 0 & 0 \\ 15 & 9 & 0 & 0 & 0 & 0\end{array}$

$\begin{array}{llllll}16 & 12 & 0 & 0 & 0 & 0 \\ 12 & 17 & 0 & 0 & 0 & 0\end{array}$

$M$ END

$\$ \$ \$$

cosmotherm $3 \mathrm{D}$

$\begin{array}{llllllllllll}10 & 8 & 0 & 0 & 0 & 0 & 0 & 0 & 0 & 0 & 0 & \mathrm{v} 2000\end{array}$

$\begin{array}{llllllllllllll}-2.6444 & -0.0635 & -0.0058 & \circ & 0 & 0 & 0 & 0 & 0 & 0 & 0 & 0 & 0 & 1\end{array}$

$\begin{array}{llllllllllllll}-1.6798 & -0.3817 & 0.0017 & \mathrm{H} & 0 & 0 & 0 & 0 & 0 & 0 & 0 & 0 & 0 & 2\end{array}$

$\begin{array}{llllllllllllll}-2.5492 & 0.9048 & -0.0039 & \text { H } & 0 & 0 & 0 & 0 & 0 & 0 & 0 & 0 & 0 & 3\end{array}$

$\begin{array}{llllllllllllll}2.1955 & -0.3112 & -0.0073 & \mathrm{C} & 0 & 0 & 0 & 0 & 0 & 0 & 0 & 0 & 0 & 4\end{array}$

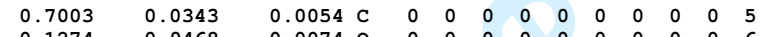

$\begin{array}{llllllllllllll}-0.1274 & -0.9468 & 0.0074 & 0 & 0 & 0 & 0 & 0 & 0 & 0 & 0 & 0 & 0 & 6\end{array}$

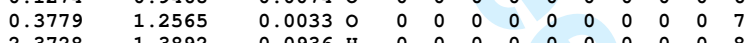

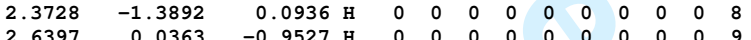

$\begin{array}{llrlllllllllll}2.6397 & 0.0363 & -0.9527 & \mathrm{H} & 0 & 0 & 0 & 0 & 0 & 0 & 0 & 0 & 0 & 8 \\ 2.7089 & 0.2225 & 0.8058 & \mathrm{H} & 0 & 0 & 0 & 0 & 0 & 0 & 0 & 0 & 0 & 9\end{array}$

2.7089
2

$\begin{array}{llllll}2 & 1 & 0 & 0 & 0 & 0 \\ 3 & 1 & 0 & 0 & 0 & 0\end{array}$

$\begin{array}{lllllll}3 & 1 & 0 & 0 & 0 & 0\end{array}$

$8 \begin{array}{lllllll}5 & 4 & 0 & 0 & 0 & 0 \\ 8 & 4 & 0 & 0 & 0 & 0\end{array}$

$\begin{array}{lllll}4 & 0 & 0 & 0 & 0\end{array}$

$\begin{array}{lllll}5 & 0 & 0 & 0 & 0\end{array}$

$\begin{array}{rllll}5 & 0 & 0 & 0 & 0 \\ 10 & 0 & 0 & 0 & 0\end{array}$

$4 \quad 10$
END

$\$ \$ \$ \$$

aceticacid.sdf

CosMOtherm 3D

$\begin{array}{llllllllllll} & 9 & 0 & 0 & 0 & 0 & 0 & 0 & 0 & 0 & 0 & \mathrm{v} 2000\end{array}$

$\begin{array}{llllllllllllll}-2.6632 & -0.0231 & 0.0932 & 0 & 0 & 0 & 0 & 0 & 0 & 0 & 0 & 0 & 0 & 1\end{array}$

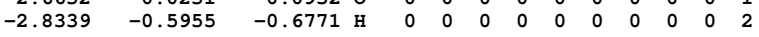

$\begin{array}{llllllllllllll}-2.9948 & 0.8534 & -0.1753 & \mathrm{H} & 0 & 0 & 0 & 0 & 0 & 0 & 0 & 0 & 0 & 3\end{array}$

$\begin{array}{lllllllllllllll}2.2026 & -0.0055 & 0.4247 & \mathrm{C} & 0 & 0 & 0 & 0 & 0 & 0 & 0 & 0 & 0 & 4\end{array}$

$\begin{array}{llllllllllllll}0.7914 & 0.0037 & -0.1040 & \mathrm{C} & 0 & 0 & 0 & 0 & 0 & 0 & 0 & 0 & 0 & 5\end{array}$

$\begin{array}{llllllllllllll}0.4952 & 0.0091 & -1.2971 & 0 & 0 & 0 & 0 & 0 & 0 & 0 & 0 & 0 & 0 & 6\end{array}$

$\begin{array}{llllllllllllll}2.9163 & 0.0324 & -0.4042 & \mathrm{H} & 0 & 0 & 0 & 0 & 0 & 0 & 0 & 0 & 0 & 8\end{array}$

$\begin{array}{lllllllllllll}2.3613 & 0.8523 & 1.0931 \mathrm{H} & 0 & 0 & 0 & 0 & 0 & 0 & 0 & 0 & 0 & 9\end{array}$

$\begin{array}{rrrrrrrrrrrrrr}2.3721 & -0.9165 & 1.0166 & \mathrm{H} & 0 & 0 & 0 & 0 & 0 & 0 & 0 & 0 & 0 & 10 \\ -1.0668 & 0.0031 & 0.4835 & \mathrm{H} & 0 & 0 & 0 & 0 & 0 & 0 & 0 & 0 & 0 & 11\end{array}$

$-1.0668,0.0031$

$\begin{array}{llllll}2 & 1 & 0 & 0 & 0 & 0\end{array}$

$\begin{array}{llllll}3 & 1 & 0 & 0 & 0 & 0\end{array}$

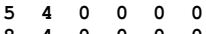

$\begin{array}{llllll}5 & 4 & 0 & 0 & 0 & 0 \\ 9 & 4 & 0 & 0 & 0 & 0\end{array}$

$\begin{array}{llllll}9 & 4 & 0 & 0 & 0 & 0\end{array}$

$\begin{array}{llllll}6 & 4 & 0 & 0 & 0 & 0 \\ 6 & 5 & 0 & 0 & 0 & 0\end{array}$

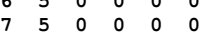

$\begin{array}{lllll}11 & 0 & 0 & 0 & 0\end{array}$

M END

$\$ \$ \$$

acrylicacid-anion.sdf

cosmotherm 3D

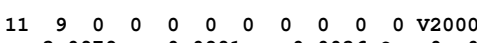

$\begin{array}{llllllllllllll}-3.0979 & 0.0891 & -0.0036 & 0 & 0 & 0 & 0 & 0 & 0 & 0 & 0 & 0 & 0 & 1\end{array}$

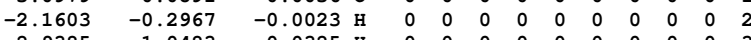

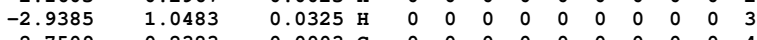

$\begin{array}{llllllllllllll}2.7509 & 0.2383 & 0.0003 & \mathrm{C} & 0 & 0 & 0 & 0 & 0 & 0 & 0 & 0 & 0 & 4\end{array}$

$\begin{array}{llllllllllllll}1.6817 & -0.5680 & -0.0029 & \mathrm{C} & 0 & 0 & 0 & 0 & 0 & 0 & 0 & 0 & 0 & 5\end{array}$

$\begin{array}{llllllllllllll}0.2534 & -0.0659 & 0.0002 & \mathrm{C} & 0 & 0 & 0 & 0 & 0 & 0 & 0 & 0 & 0 & 6\end{array}$

$\begin{array}{llllllllllllll}-0.6462 & -0.9855 & 0.0038 & 0 & 0 & 0 & 0 & 0 & 0 & 0 & 0 & 0 & 0 & 7\end{array}$

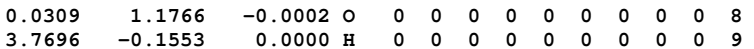

$\begin{array}{rrrrrrrrrrrrr}2.6250 & 1.3241 & 0.0036 \mathrm{H} & 0 & 0 & 0 & 0 & 0 & 0 & 0 & 0 & 0 & 10\end{array}$

$\begin{array}{llllllllllllll}1.8040 & -1.6556 & -0.0048 \mathrm{H} & 0 & 0 & 0 & 0 & 0 & 0 & 0 & 0 & 0 & 11\end{array}$

$\begin{array}{llllll}2 & 1 & 0 & 0 & 0 & 0\end{array}$

$\begin{array}{llllll}3 & 1 & 0 & 0 & 0 & 0\end{array}$

$\begin{array}{llllll}5 & 4 & 0 & 0 & 0 & 0\end{array}$

$\begin{array}{llllll}9 & 4 & 0 & 0 & 0 & 0\end{array}$

$\begin{array}{rlllll}10 & 4 & 0 & 0 & 0 & 0\end{array}$

$7 \quad 6 \quad 000000$

86600000

$5 \begin{array}{rrrrrr}8 & 11 & 0 & 0 & 0 & 0\end{array}$

$M$ END 


$\begin{array}{rrrrrrrrrrrrrr}-3.4957 & 0.5498 & 0.1903 & \mathrm{C} & 0 & 0 & 0 & 0 & 0 & 0 & 0 & 0 & 0 & 9 \\ 0.2475 & 0.2296 & -0.1203 & \mathrm{C} & 0 & 0 & 0 & 0 & 0 & 0 & 0 & 0 & 0 & 10 \\ 0.4198 & -1.1739 & -0.0612 & \mathrm{C} & 0 & 0 & 0 & 0 & 0 & 0 & 0 & 0 & 0 & 11 \\ -0.7518 & -2.0202 & 0.0659 & \mathrm{C} & 0 & 0 & 0 & 0 & 0 & 0 & 0 & 0 & 0 & 12 \\ -2.0222 & -1.4329 & 0.1473 & \mathrm{C} & 0 & 0 & 0 & 0 & 0 & 0 & 0 & 0 & 0 & 13 \\ 1.6997 & -1.8098 & -0.1587 & \mathrm{C} & 0 & 0 & 0 & 0 & 0 & 0 & 0 & 0 & 0 & 14 \\ 1.8169 & -3.1805 & -0.1199 & \mathrm{C} & 0 & 0 & 0 & 0 & 0 & 0 & 0 & 0 & 0 & 15 \\ 0.6649 & -4.0084 & 0.0156 & \mathrm{C} & 0 & 0 & 0 & 0 & 0 & 0 & 0 & 0 & 0 & 16 \\ -0.5839 & -3.4409 & 0.1059 & \mathrm{C} & 0 & 0 & 0 & 0 & 0 & 0 & 0 & 0 & 0 & 17 \\ 1.4459 & 1.1228 & -0.3038 & \mathrm{C} & 0 & 0 & 0 & 0 & 0 & 0 & 0 & 0 & 0 & 18 \\ 1.6481 & 1.7697 & -1.3289 & \mathrm{O} & 0 & 0 & 0 & 0 & 0 & 0 & 0 & 0 & 0 & 19 \\ 2.2564 & 1.1426 & 0.7607 & \mathrm{O} & 0 & 0 & 0 & 0 & 0 & 0 & 0 & 0 & 0 & 20 \\ -4.6518 & 2.3548 & 0.2201 & \mathrm{H} & 0 & 0 & 0 & 0 & 0 & 0 & 0 & 0 & 0 & 21 \\ -2.6579 & 3.8450 & -0.0208 & \mathrm{H} & 0 & 0 & 0 & 0 & 0 & 0 & 0 & 0 & 0 & 22 \\ -0.3971 & 2.9015 & -0.1962 & \mathrm{H} & 0 & 0 & 0 & 0 & 0 & 0 & 0 & 0 & 0 & 23 \\ -4.3596 & -0.1095 & 0.3003 & \mathrm{H} & 0 & 0 & 0 & 0 & 0 & 0 & 0 & 0 & 0 & 24 \\ -2.9001 & -2.0758 & 0.2510 & \mathrm{H} & 0 & 0 & 0 & 0 & 0 & 0 & 0 & 0 & 0 & 25 \\ 2.5964 & -1.1976 & -0.2607 & \mathrm{H} & 0 & 0 & 0 & 0 & 0 & 0 & 0 & 0 & 0 & 26 \\ 2.8032 & -3.6418 & -0.1961 & \mathrm{H} & 0 & 0 & 0 & 0 & 0 & 0 & 0 & 0 & 0 & 27 \\ 0.7809 & -5.0931 & 0.0456 & \mathrm{H} & 0 & 0 & 0 & 0 & 0 & 0 & 0 & 0 & 0 & 28 \\ -1.4742 & -4.0657 & 0.2046 & \mathrm{H} & 0 & 0 & 0 & 0 & 0 & 0 & 0 & 0 & 0 & 29 \\ 3.0615 & 1.7588 & 0.5926 & \mathrm{H} & 0 & 0 & 0 & 0 & 0 & 0 & 0 & 0 & 0 & 30\end{array}$

$\begin{array}{llllll}2 & 1 & 0 & 0 & 0 & 0\end{array}$

$\begin{array}{llllll}3 & 1 & 0 & 0 & 0 & 0 \\ 5 & 4 & 0 & 0 & 0 & 0\end{array}$

$\begin{array}{llllll}5 & 4 & 0 & 0 & 0 & 0 \\ 9 & 4 & 0 & 0 & 0 & 0\end{array}$

$\begin{array}{rrrrrr}9 & 4 & 0 & 0 & 0 & 0 \\ 21 & 4 & 0 & 0 & 0 & 0\end{array}$

$\begin{array}{rrrrrr}21 & 4 & 0 & 0 & 0 & 0 \\ 6 & 5 & 0 & 0 & 0 & 0\end{array}$

$\begin{array}{rlllll}22 & 5 & 0 & 0 & 0 & 0 \\ 7 & 6 & 0 & 0 & 0 & 0\end{array}$

$\begin{array}{rrrrrr}7 & 6 & 0 & 0 & 0 & 0 \\ 23 & 6 & 0 & 0 & 0 & 0\end{array}$

$\begin{array}{rrrrrr}23 & 6 & 0 & 0 & 0 & 0 \\ 8 & 7 & 0 & 0 & 0 & 0\end{array}$

$\begin{array}{rrrrrr}10 & 7 & 0 & 0 & 0 & 0\end{array}$

$\begin{array}{rrrrrr}9 & 8 & 0 & 0 & 0 & 0\end{array}$

$\begin{array}{llllll}13 & 8 & 0 & 0 & 0 & 0\end{array}$

$\begin{array}{lrllll}24 & 9 & 0 & 0 & 0 & 0 \\ 11 & 10 & 0 & 0 & 0 & 0\end{array}$

$\begin{array}{llllll}18 & 10 & 0 & 0 & 0 & 0\end{array}$

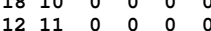

$\begin{array}{llllll}14 & 11 & 0 & 0 & 0 & 0\end{array}$

$\begin{array}{llllll}13 & 12 & 0 & 0 & 0 & 0\end{array}$

$\begin{array}{lllll}25 & 13 & 0 & 0\end{array}$

$\begin{array}{llllll}25 & 13 & 0 & 0 & 0 & 0 \\ 15 & 14 & 0 & 0 & 0 & 0\end{array}$

$\begin{array}{llllll}15 & 14 & 0 & 0 & 0 & 0 \\ 26 & 14 & 0 & 0 & 0 & 0\end{array}$

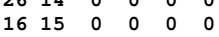

$\begin{array}{llllll}16 & 15 & 0 & 0 & 0 & 0 \\ 27 & 15 & 0 & 0 & 0 & 0\end{array}$

$17 \begin{array}{llllll}16 & 0 & 0 & 0 & 0\end{array}$

$\begin{array}{llllll}17 & 16 & 0 & 0 & 0 & 0 \\ 28 & 16 & 0 & 0 & 0 & 0\end{array}$

$\begin{array}{llllll}28 & 16 & 0 & 0 & 0 & 0 \\ 29 & 17 & 0 & 0 & 0 & 0\end{array}$

$19 \begin{array}{lllll}18 & 0 & 0 & 0 & 0\end{array}$

$\begin{array}{llllll}20 & 18 & 0 & 0 & 0 & 0\end{array}$

2030

$M$ END

benzoicacid-anion.sdf

CosMOtherm

$\begin{array}{llllllllllll}17 & 16 & 0 & 0 & 0 & 0 & 0 & 0 & 0 & 0 & 0 & \mathrm{v} 2000\end{array}$

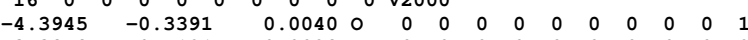

$\begin{array}{llllllllllllll}-3.3979 & -0.5181 & 0.0029 & \text { H } & 0 & 0 & 0 & 0 & 0 & 0 & 0 & 0 & 0 & 2\end{array}$

$\begin{array}{llllllllllllll}-4.4394 & 0.6325 & 0.0276 & \mathrm{H} & 0 & 0 & 0 & 0 & 0 & 0 & 0 & 0 & 0 & 3\end{array}$

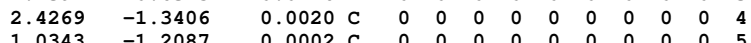

$\begin{array}{llllllllllllll}1.0343 & -1.2087 & 0.0002 & \mathrm{C} & 0 & 0 & 0 & 0 & 0 & 0 & 0 & 0 & 0 & 5 \\ 0.4357 & 0.0624 & -0.0013 & \mathrm{C} & 0 & 0 & 0 & 0 & 0 & 0 & 0 & 0 & 0 & 6\end{array}$

$\begin{array}{llllllllllllll}0.4357 & 0.0624 & -0.0013 & \mathrm{C} & 0 & 0 & 0 & 0 & 0 & 0 & 0 & 0 & 0 & 6 \\ 1.2605 & 1.1998 & -0.0010 & \mathrm{C} & 0 & 0 & 0 & 0 & 0 & 0 & 0 & 0 & 0 & 7\end{array}$

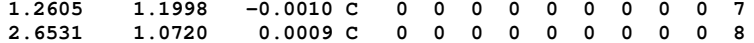

$\begin{array}{rrrrrrrrrrrrrr}3.2403 & -0.1999 & 0.0025 & \text { C } & 0 & 0 & 0 & 0 & 0 & 0 & 0 & 0 & 0 & 9\end{array}$

$\begin{array}{llllllllllllll}-1.0830 & 0.2161 & -0.0028 & \mathrm{C} & 0 & 0 & 0 & 0 & 0 & 0 & 0 & 0 & 0 & 10\end{array}$

$\begin{array}{llllllllllllll}-1.7684 & -0.8670 & -0.0060 & \circ & 0 & 0 & 0 & 0 & 0 & 0 & 0 & 0 & 0 & 11\end{array}$

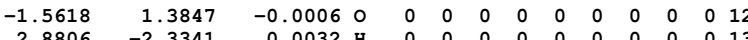

$\begin{array}{lllll}1.2 .3847 & 0.0032 \mathrm{H} & 0 & 0\end{array}$

$\begin{array}{lllllllllllll}0.0909 & -0.0005 & \mathrm{H} & 0 & 0 & 0 & 0 & 0 & 0 & 0 & 0 & 0 & 14\end{array}$

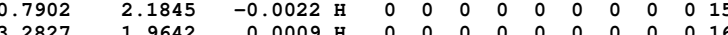

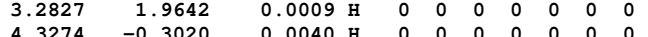

$\begin{array}{ll}4.3274 & -0.3020\end{array}$

$\begin{array}{llllll}2 & 1 & 0 & 0 & 0 & 0 \\ 3 & 1 & 0 & 0 & 0 & 0\end{array}$

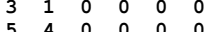

$\begin{array}{llllll}5 & 4 & 0 & 0 & 0 & 0 \\ 9 & 4 & 0 & 0 & 0 & 0\end{array}$

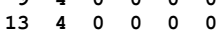

$\begin{array}{llllll}6 & 5 & 0 & 0 & 0 & 0\end{array}$

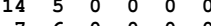

$\begin{array}{rrrrrr}7 & 6 & 0 & 0 & 0 & 0 \\ 10 & 6 & 0 & 0 & 0 & 0\end{array}$

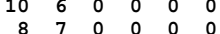

$\begin{array}{rrrrrr}8 & 7 & 0 & 0 & 0 & 0 \\ 15 & 7 & 0 & 0 & 0 & 0\end{array}$

$\begin{array}{rrrrrr}15 & 7 & 0 & 0 & 0 & 0 \\ 9 & 8 & 0 & 0 & 0 & 0\end{array}$

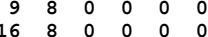

$\begin{array}{rrrrrr}16 & 8 & 0 & 0 & 0 & 0 \\ 11 & 10 & 0 & 0 & 0 & 0\end{array}$

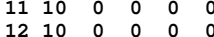

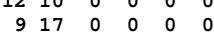

\section{$M$ END}

$\$ \$ \$$

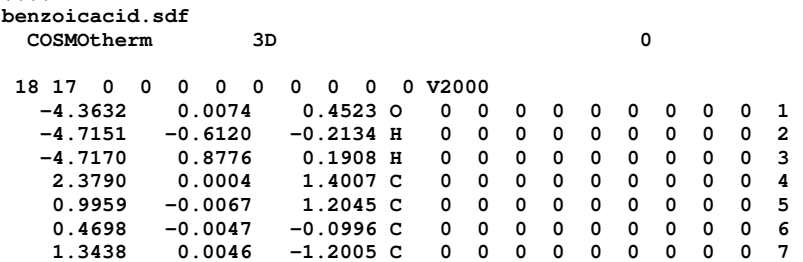


$\begin{array}{rlllllllllllll}-4.6437 & 0.7622 & -0.8494 & \mathrm{H} & 0 & 0 & 0 & 0 & 0 & 0 & 0 & 0 & 0 & 3 \\ 1.8363 & 0.0052 & -0.0963 & \mathrm{Br} & 0 & 0 & 0 & 0 & 0 & 0 & 0 & 0 & 0 & 4\end{array}$

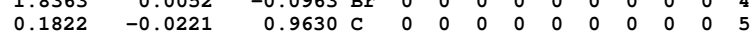

$\begin{array}{llllllllllllll}-1.0784 & -0.0094 & 0.1094 & \mathrm{C} & 0 & 0 & 0 & 0 & 0 & 0 & 0 & 0 & 0 & 6 \\ -2.1470 & 0.0093 & 0.9126 & 0 & 0 & 0 & 0 & 0 & 0 & 0 & 0 & 0 & 0 & 7\end{array}$

\begin{tabular}{llllllllllllll}
-1.1183 & -0.0191 & -1.1115 & 0 & 0 & 0 & 0 & 0 & 0 & 0 & 0 & 0 & 0 & 7 \\
\hline
\end{tabular}

$\begin{array}{rrrrrrrrrrrrrrr}0.2079 & 0.8556 & 1.6167 & \mathrm{H} & 0 & 0 & 0 & 0 & 0 & 0 & 0 & 0 & 0 & 9\end{array}$

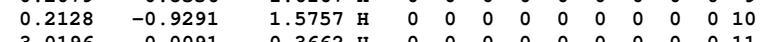

20.0091

$\begin{array}{llllll}2 & 1 & 0 & 0 & 0 & 0 \\ 3 & 1 & 0 & 0 & 0 & 0\end{array}$

$\begin{array}{llllll}3 & 1 & 0 & 0 & 0 & 0 \\ 6 & 5 & 0 & 0 & 0 & 0\end{array}$

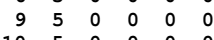

$\begin{array}{rrrrrr}10 & 5 & 0 & 0 & 0 & 0 \\ 7 & 6 & 0 & 0 & 0 & 0\end{array}$

$\begin{array}{llllll}7 & 6 & 0 & 0 & 0 & 0 \\ 8 & 6 & 0 & 0 & 0 & 0\end{array}$

$\begin{array}{rrrrrr}7 & 6 & 0 & 0 & 0 & 0 \\ 7 & 11 & 0 & 0 & 0 & 0\end{array}$

END

$\$ \$ \$ \$$

arbonicacid-anion sdf

cosmotherm

$\begin{array}{llllllllllll}8 & 0 & 0 & 0 & 0 & 0 & 0 & 0 & 0 & 0 & \mathrm{v} 2000\end{array}$

$\begin{array}{llllllllllllll}-2.7019 & -0.0378 & -0.0070 & 0 & 0 & 0 & 0 & 0 & 0 & 0 & 0 & 0 & 0 & 1\end{array}$

$\begin{array}{llllllllllllll}-1.7426 & -0.3446 & -0.0030 & \text { H } & 0 & 0 & 0 & 0 & 0 & 0 & 0 & 0 & 0 & 2\end{array}$

$\begin{array}{llllllllllllll}-2.6325 & 0.9275 & 0.0888 & \text { H } & 0 & 0 & 0 & 0 & 0 & 0 & 0 & 0 & 0 & 3\end{array}$

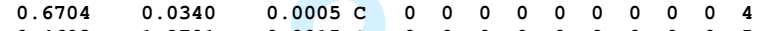

$\begin{array}{llllllllllllll}0.4603 & 1.2701 & -0.0015 & 0 & 0 & 0 & 0 & 0 & 0 & 0 & 0 & 0 & 0 & 5\end{array}$

$\begin{array}{llllllllllllll}2.0126 & -0.3983 & -0.0014 & 0 & 0 & 0 & 0 & 0 & 0 & 0 & 0 & 0 & 0 & 6\end{array}$

$\begin{array}{rrrrrrrrrrrrrr}-0.1600 & -0.9222 & 0.0044 & 0 & 0 & 0 & 0 & 0 & 0 & 0 & 0 & 0 & 0 & 7 \\ 2.5600 & 0.4114 & -0.0037 & \text { H } & 0 & 0 & 0 & 0 & 0 & 0 & 0 & 0 & 0 & 8\end{array}$

$\begin{array}{llllll}2 & 1 & 0 & 0 & 0 & 0\end{array}$

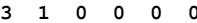

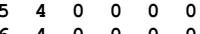

$\begin{array}{lllll}4 & 0 & 0 & 0 & 0\end{array}$

M END

$\$ \$ \$$

carbonicacid.sdf

cosmotherm

$\begin{array}{lllllllllllll}9 & 7 & 0 & 0 & 0 & 0 & 0 & 0 & 0 & 0 & 0 & \mathrm{v} 2000\end{array}$

$\begin{array}{lllllllllllll}-2.6404 & 0.0053 & 0.0548 & 0 & 0 & 0 & 0 & 0 & 0 & 0 & 0 & 0 & 0\end{array}$

$\begin{array}{llllllllllllll}-2.9165 & -0.7935 & -0.4317 & \mathrm{H} & 0 & 0 & 0 & 0 & 0 & 0 & 0 & 0 & 0 & 2\end{array}$

$\begin{array}{lllllllllllllllll}-2.8856 & 0.7493 & -0.5258 \mathrm{H} & 0 & 0 & 0 & 0 & 0 & 0 & 0 & 0 & 0 & 3\end{array}$

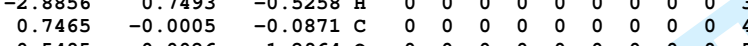

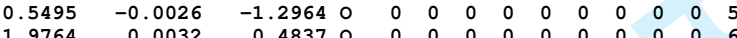

$\begin{array}{rrrrrrrrrrrrrr}1.9764 & 0.0032 & 0.4837 & 0 & 0 & 0 & 0 & 0 & 0 & 0 & 0 & 0 & 0 & 6 \\ -0.1760 & -0.0023 & 0.8699 & 0 & 0 & 0 & 0 & 0 & 0 & 0 & 0 & 0 & 0 & 7\end{array}$

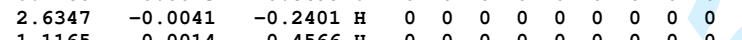
$\begin{array}{lll}-1.1165 & -0.0014\end{array}$

$\begin{array}{llllll}2 & 1 & 0 & 0 & 0 & 0\end{array}$

$\begin{array}{llllll}3 & 1 & 0 & 0 & 0 & 0 \\ 5 & 4 & 0 & 0 & 0 & 0\end{array}$

$\begin{array}{llllll}5 & 4 & 0 & 0 & 0 & 0 \\ 6 & 4 & 0 & 0 & 0 & 0\end{array}$

$7 \begin{array}{lllllll}7 & 4 & 0 & 0 & 0 & 0\end{array}$

$\begin{array}{llllll}8 & 6 & 0 & 0 & 0 & 0 \\ 7 & 9 & 0 & 0 & 0 & 0\end{array}$

chloroaceticacid-anion.sdf

cosmotherm 3D

$\begin{array}{llllllllllll}10 & 8 & 0 & 0 & 0 & 0 & 0 & 0 & 0 & 0 & 0 & \mathrm{~V} 2000\end{array}$

$\begin{array}{llllllllllllll}-3.4582 & -0.8948 & 0.0042 & 0 & 0 & 0 & 0 & 0 & 0 & 0 & 0 & 0 & 0 & 1\end{array}$

$\begin{array}{llllllllllllll}-2.4577 & -0.7761 & -0.0009 & \text { H } & 0 & 0 & 0 & 0 & 0 & 0 & 0 & 0 & 0 & 2\end{array}$

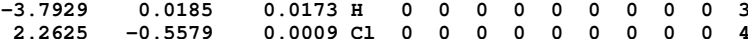

$\begin{array}{llllllllllll}1.1148 & 0.8849 & 0.0045 \mathrm{C} & 0 & 0 & 0 & 0 & 0 & 0 & 0 & 0 & 0\end{array}$

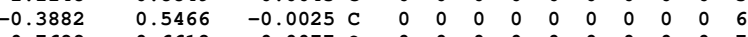

$\begin{array}{llllllllllllll}-0.7688 & -0.6618 & -0.0077 & 0 & 0 & 0 & 0 & 0 & 0 & 0 & 0 & 0 & 0 & 7\end{array}$

$\begin{array}{llllllllllllll}-1.1100 & 1.5812 & -0.0021 & 0 & 0 & 0 & 0 & 0 & 0 & 0 & 0 & 0 & 0 & 8\end{array}$

$\begin{array}{lllllllllllllll}1.3678 & 1.4704 & -0.8854 & \mathrm{H} & 0 & 0 & 0 & 0 & 0 & 0 & 0 & 0 & 0 & 9\end{array}$

$\begin{array}{lllllllllllll}1.3614 & 1.4607 & 0.9026 \mathrm{H} & 0 & 0 & 0 & 0 & 0 & 0 & 0 & 0 & 0 & 10\end{array}$

$\begin{array}{lllllll}2 & 1 & 0 & 0 & 0 & 0\end{array}$

$\begin{array}{llllll}3 & 1 & 0 & 0 & 0 & 0\end{array}$

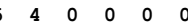

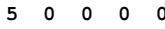

$\begin{array}{llllll}2 & 0 & 0 & 0 & 0\end{array}$

$\begin{array}{llllll}6 & 0 & 0 & 0 & 0\end{array}$

$\begin{array}{lllll}10 & 0 & 0 & 0 & 0\end{array}$

END

\$\$\$

chloroaceticacid_270.sdf
CoSMOtherm 3D

$\begin{array}{llllllllllll}11 & 9 & 0 & 0 & 0 & 0 & 0 & 0 & 0 & 0 & 0 & \mathrm{v} 2000\end{array}$

$\begin{array}{llllllllllllll}-3.7212 & -0.0019 & -0.1683 & 0 & 0 & 0 & 0 & 0 & 0 & 0 & 0 & 0 & 0 & 1\end{array}$

$\begin{array}{llllllllllllll}-3.9278 & -0.7977 & -0.6924 & \text { н } & 0 & 0 & 0 & 0 & 0 & 0 & 0 & 0 & 0 & 2\end{array}$

$\begin{array}{llllllllllllll}-3.9107 & 0.7459 & -0.7646 \mathrm{H} & 0 & 0 & 0 & 0 & 0 & 0 & 0 & 0 & 0 & 3\end{array}$

$\begin{array}{llllllllllllll}2.4612 & -0.0025 & -0.1807 & \mathrm{Cl} & 0 & 0 & 0 & 0 & 0 & 0 & 0 & 0 & 0 & 4\end{array}$

$\begin{array}{llllllllllllll}0.9862 & 0.0007 & 0.8665 & \mathrm{C} & 0 & 0 & 0 & 0 & 0 & 0 & 0 & 0 & 0 & 5\end{array}$

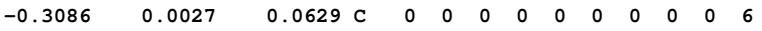

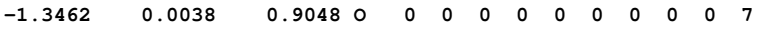

$\begin{array}{lllllllllllllll}-0.3898 & 0.0039 & -1.1559 & 0 & 0 & 0 & 0 & 0 & 0 & 0 & 0 & 0 & 0 & 8\end{array}$

$\begin{array}{llllllllllllll}1.0317 & 0.8929 & 1.5007 & \mathrm{H} & 0 & 0 & 0 & 0 & 0 & 0 & 0 & 0 & 0 & 9\end{array}$

$\begin{array}{rrrrrrrrrrrrrr}1.0285 & -0.8909 & 1.5017 & \mathrm{H} & 0 & 0 & 0 & 0 & 0 & 0 & 0 & 0 & 0 & 10 \\ -2.2388 & 0.0037 & 0.3914 & \mathrm{H} & 0 & 0 & 0 & 0 & 0 & 0 & 0 & 0 & 0 & 11\end{array}$

$-2.2388 \quad 0.0037$

$\begin{array}{lllllll}2 & 1 & 0 & 0 & 0 & 0\end{array}$

$\begin{array}{llllll}3 & 1 & 0 & 0 & 0 & 0\end{array}$

$6 \begin{array}{llllll}6 & 5 & 0 & 0 & 0 & 0\end{array}$

$9 \begin{array}{llllll}5 & 0 & 0 & 0 & 0\end{array}$ 


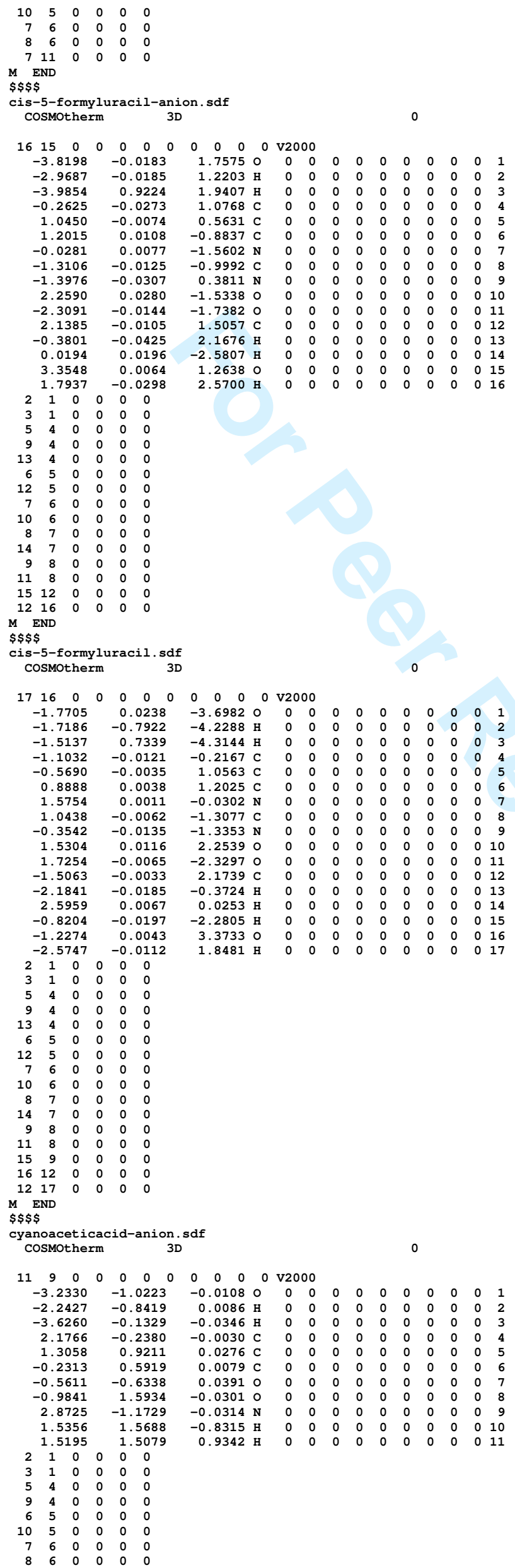




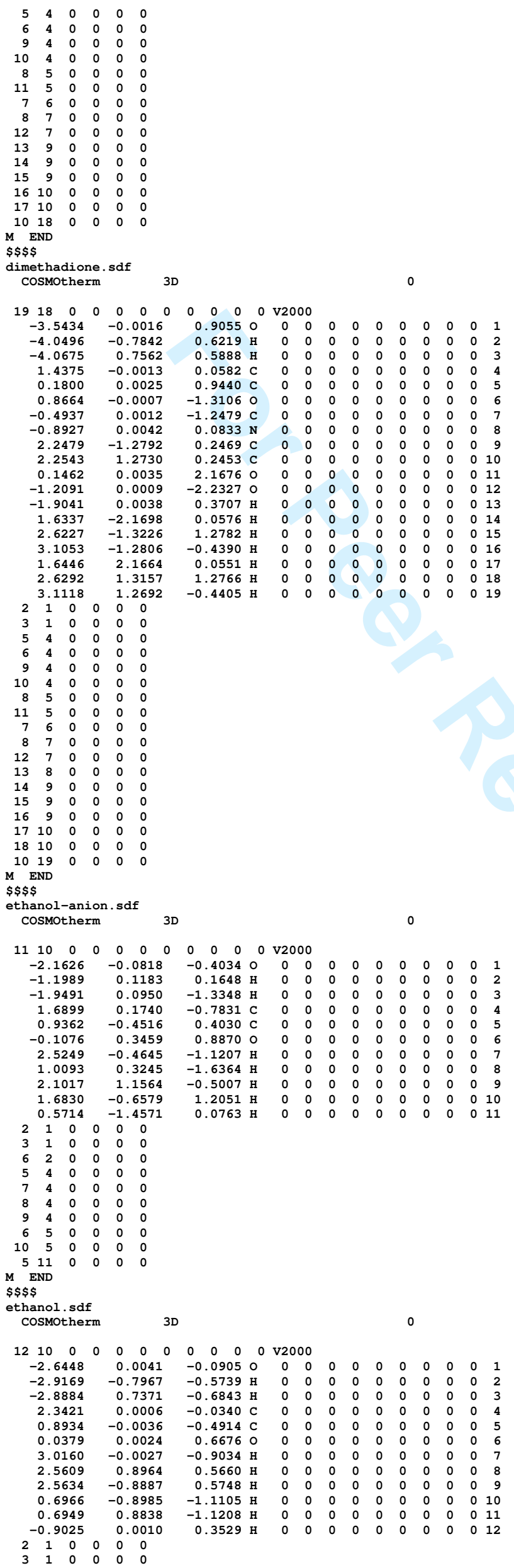




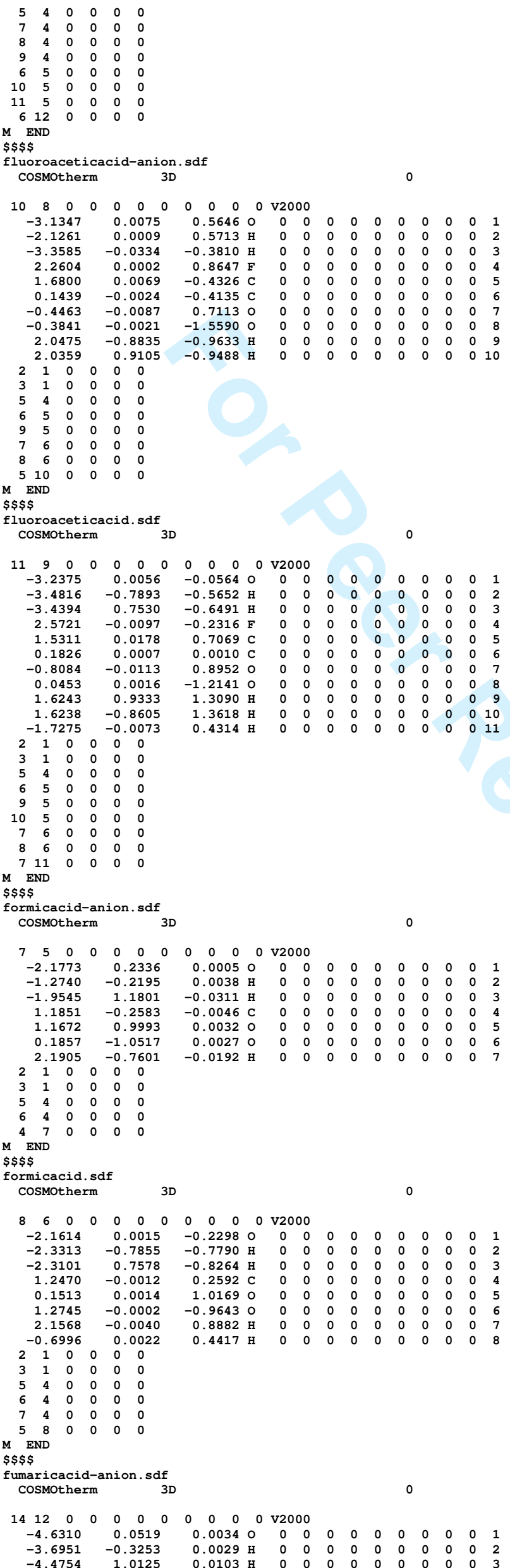


$\begin{array}{rrrrrrrrrrrrrr}-2.2723 & -0.0113 & 0.3352 & 0 & 0 & 0 & 0 & 0 & 0 & 0 & 0 & 0 & 0 & 1 \\ -2.8788 & -0.6891 & -0.0155 & \mathrm{H} & 0 & 0 & 0 & 0 & 0 & 0 & 0 & 0 & 0 & 2\end{array}$

$\begin{array}{llllllllllllll}-2.7569 & 0.8289 & 0.2402 & \mathrm{H} & 0 & 0 & 0 & 0 & 0 & 0 & 0 & 0 & 0 & 3\end{array}$

$\begin{array}{llllllllllllll}0.0126 & 0.0079 & -0.9742 & 0 & 0 & 0 & 0 & 0 & 0 & 0 & 0 & 0 & 0 & 4\end{array}$

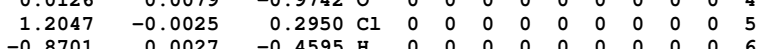

$-0.8701 \quad 0.0027$

$\begin{array}{llllll}2 & 1 & 0 & 0 & 0 & 0 \\ 3 & 1 & 0 & 0 & 0 & 0\end{array}$

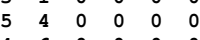

END

$\$ \$ \$ \$$

hypoiodousacid-anion.sd

Cosmotousacid-anion.s

$\begin{array}{llllllllllll}5 & 2 & 0 & 0 & 0 & 0 & 0 & 0 & 0 & 0 & 0 & \mathrm{v} 2000\end{array}$

$\begin{array}{lllllllrllllllll}-3.2394 & -0.0573 & -0.4281 & 0 & 0 & 0 & 0 & 0 & 0 & 0 & 0 & 0 & 0 & 1\end{array}$

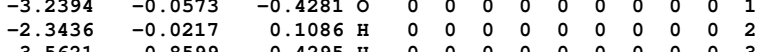

$\begin{array}{lrrrrrrrrrrrrr}-3.5621 & 0.8599 & -0.4295 & \mathrm{H} & 0 & 0 & 0 & 0 & 0 & 0 & 0 & 0 & 0 & 3 \\ -1.1204 & -0.0008 & 1.0103 & 0 & 0 & 0 & 0 & 0 & 0 & 0 & 0 & 0 & 0 & 4\end{array}$

$\begin{array}{rrrrrrrrrrrrrr}-1.1204 & -0.0008 & 1.0103 & 0 & 0 & 0 & 0 & 0 & 0 & 0 & 0 & 0 & 0 & 4 \\ 0.5966 & 0.0007 & -0.0709 & \mathrm{I} & 0 & 0 & 0 & 0 & 0 & 0 & 0 & 0 & 0 & 5\end{array}$

$\begin{array}{ccccc}1 & 0 & 0 & 0 & 0\end{array}$

301100000

M END

hypoiodousacid.sdf

cosmotherm 3D 0

$6 \begin{array}{ccccccccccc}3 & 0 & 0 & 0 & 0 & 0 & 0 & 0 & 0 & 0 & \mathrm{v} 2000\end{array}$

$\begin{array}{llllllllllllll}-3.1660 & 0.0074 & 0.4760 & 0 & 0 & 0 & 0 & 0 & 0 & 0 & 0 & 0 & 0 & 1\end{array}$

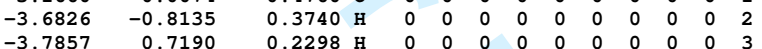

$\begin{array}{rrrrrrrrrrrrrr}-3.7857 & 0.7190 & 0.2298 & \text { H } & 0 & 0 & 0 & 0 & 0 & 0 & 0 & 0 & 0 & 3 \\ -1.0344 & -0.0044 & -1.1480 & 0 & 0 & 0 & 0 & 0 & 0 & 0 & 0 & 0 & 0 & 4\end{array}$

$\begin{array}{rrrrrrrrrrrrrr}0.6034 & 0.0004 & 0.0840 & \text { I } & 0 & 0 & 0 & 0 & 0 & 0 & 0 & 0 & 0 & 5 \\ -1.8226 & 0.0014 & -0.5153 & \text { H } & 0 & 0 & 0 & 0 & 0 & 0 & 0 & 0 & 0 & 6\end{array}$

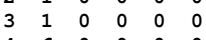

$\begin{array}{llllll}4 & 6 & 0 & 0 & 0 & 0\end{array}$

M END

hypophosphorousacid-anion sdf

COSMOtherm $3 \mathrm{D}$

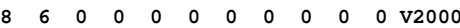

$\begin{array}{llllllllllllll}-2.8099 & -0.1697 & 0.3622 & 0 & 0 & 0 & 0 & 0 & 0 & 0 & 0 & 0 & 0 & 1\end{array}$

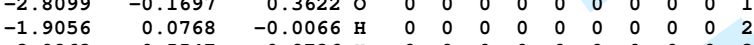

$\begin{array}{lllllllllllll}-3.0263 & 0.5547 & 0.9736 \mathrm{H} & 0 & 0 & 0 & 0 & 0 & 0 & 0 & 0 & 0 & 3\end{array}$

$\begin{array}{lllllllllllllllllllll}-0.4335 & 0.4850 & -0.6914 & 0 & 0 & 0 & 0 & 0 & 0 & 0 & 0 & 0 & 0 & 4\end{array}$

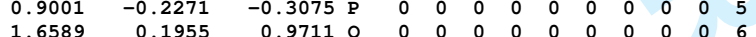

$\begin{array}{llllllllllllllll}1.6589 & 0.6487 & -1.6457 & -0.2622 & \mathrm{H} & 0 & 0 & 0 & 0 & 0 & 0 & 0 & 0 & 0 & 6\end{array}$

$\begin{array}{llllllllllllll}1.7764 & -0.1164 & -1.4447 & \mathrm{H} & 0 & 0 & 0 & 0 & 0 & 0 & 0 & 0 & 0 & 8\end{array}$

$2 \begin{array}{llllll}2 & 1 & 0 & 0 & 0 & 0\end{array}$

$\begin{array}{llllll}3 & 1 & 0 & 0 & 0 & 0 \\ 5 & 4 & 0 & 0 & 0 & 0\end{array}$

$\begin{array}{lllllll}5 & 4 & 0 & 0 & 0 & 0 \\ 6 & 5 & 0 & 0 & 0 & 0\end{array}$

$\begin{array}{lllll}5 & 0 & 0 & 0 & 0\end{array}$

$\begin{array}{llllll}5 & 8 & 0 & 0 & 0 & 0\end{array}$

$M \quad$ END

$\$ \$ \$$

hypophosphorousacid.sde

$\begin{array}{llllllllllll}9 & 7 & 0 & 0 & 0 & 0 & 0 & 0 & 0 & 0 & 0 & \mathrm{v} 2000\end{array}$

$\begin{array}{llllllllllllll}-0.4413 & 2.5662 & -0.1387 & 0 & 0 & 0 & 0 & 0 & 0 & 0 & 0 & 0 & 0 & 1 \\ 0.1073 & 3.2172 & -0.6141 & \mathrm{H} & 0 & 0 & 0 & 0 & 0 & 0 & 0 & 0 & 0 & 2\end{array}$

$\begin{array}{llllllllllllll}0.1073 & 3.2172 & -0.6141 & \mathrm{H} & 0 & 0 & 0 & 0 & 0 & 0 & 0 & 0 & 0 & 2\end{array}$

$\begin{array}{lllllllllllll}0.2747 & -0.6548 & -1.6453 & \mathrm{H} & 0 & 0 & 0 & 0 & 0 & 0 & 0 & 0 & 0 \\ 0.2783 & -0.9412 & -0.2484 & 0 & 0 & 0 & 0 & 0 & 0 & 0 & 0 & 0 & 0\end{array}$

$\begin{array}{llllllllllllll}0.2783 & -0.9412 & -0.2484 & \mathrm{P} & 0 & 0 & 0 & 0 & 0 & 0 & 0 & 0 & 0 & 5\end{array}$

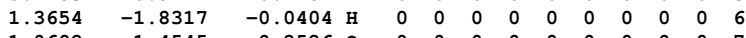

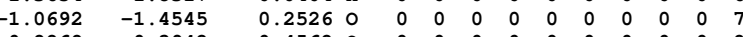

$\begin{array}{rrrrrrrrrrrrrr}0.8868 & 0.3942 & 0.4562 & 0 & 0 & 0 & 0 & 0 & 0 & 0 & 0 & 0 & 0 & 8\end{array}$

$\begin{array}{lllllllllllll}0.3675 & 1.2478 & 0.2356 \mathrm{H} & 0 & 0 & 0 & 0 & 0 & 0 & 0 & 0 & 0 & 9\end{array}$

$2 \quad 1 \quad 0 \quad 0 \quad 000$

$2 \begin{array}{llllll}2 & 1 & 0 & 0 & 0 & 0 \\ 3 & 1 & 0 & 0 & 0 & 0\end{array}$

$\begin{array}{llllllll}3 & 4 & 0 & 0 & 0 & 0\end{array}$

$\begin{array}{lllllll}5 & 0 & 0 & 0 & 0\end{array}$

$5 \quad 0 \quad 0 \quad 000$

$\begin{array}{llllll}5 & 0 & 0 & 0 & 0\end{array}$

MND

odoaceticacid-anion.sdf

CosMOtherm 3D

$\begin{array}{llllllllllll}10 & 7 & 0 & 0 & 0 & 0 & 0 & 0 & 0 & 0 & 0 & \mathrm{v} 2000\end{array}$

$\begin{array}{lllllllllllll}-4.5085 & -1.2652 & 0.0007 & 0 & 0 & 0 & 0 & 0 & 0 & 0 & 0 & 0 & 0\end{array}$

$\begin{array}{lllllllllllll}-3.5440 & -0.9744 & -0.0006 & \mathrm{H} & 0 & 0 & 0 & 0 & 0 & 0 & 0 & 0 & 0\end{array}$

$\begin{array}{llllllllllllll}-4.9996 & -0.4258 & 0.0231 & \mathrm{H} & 0 & 0 & 0 & 0 & 0 & 0 & 0 & 0 & 0 & 3\end{array}$

$\begin{array}{llllllllllllll}1.3945 & -0.1694 & 0.0000 & \text { I } & 0 & 0 & 0 & 0 & 0 & 0 & 0 & 0 & 0 & 4\end{array}$

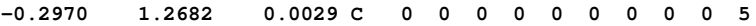

$\begin{array}{llllllllllllll}-1.7130 & 0.6738 & -0.0011 & \mathrm{C} & 0 & 0 & 0 & 0 & 0 & 0 & 0 & 0 & 0 & 6\end{array}$

$\begin{array}{llllllllllllll}-1.8960 & -0.5784 & -0.0025 & 0 & 0 & 0 & 0 & 0 & 0 & 0 & 0 & 0 & 0 & 7 \\ -2.5925 & 1.5809 & -0.0019 & 0 & 0 & 0 & 0 & 0 & 0 & 0 & 0 & 0 & 0 & 8\end{array}$

$\begin{array}{llllllllllllll}-2.5925 & 1.5809 & -0.0019 & \text { O } & 0 & 0 & 0 & 0 & 0 & 0 & 0 & 0 & 0 & 8 \\ -0.1307 & 1.8801 & -0.8888 & \text { H } & 0 & 0 & 0 & 0 & 0 & 0 & 0 & 0 & 0 & 9\end{array}$

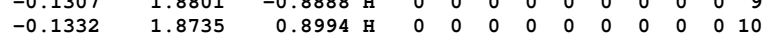

$2 \quad 1 \quad 000000$

$3 \begin{array}{llllll}3 & 1 & 0 & 0 & 0 & 0\end{array}$

$6 \begin{array}{llllll}6 & 5 & 0 & 0 & 0 & 0 \\ 9 & 5 & 0 & 0 & 0 & 0\end{array}$

$\begin{array}{lllllll}7 & 5 & 0 & 0 & 0 & 0\end{array}$

86600000

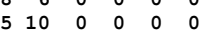

END

$\$ \$ \$ \$$ 


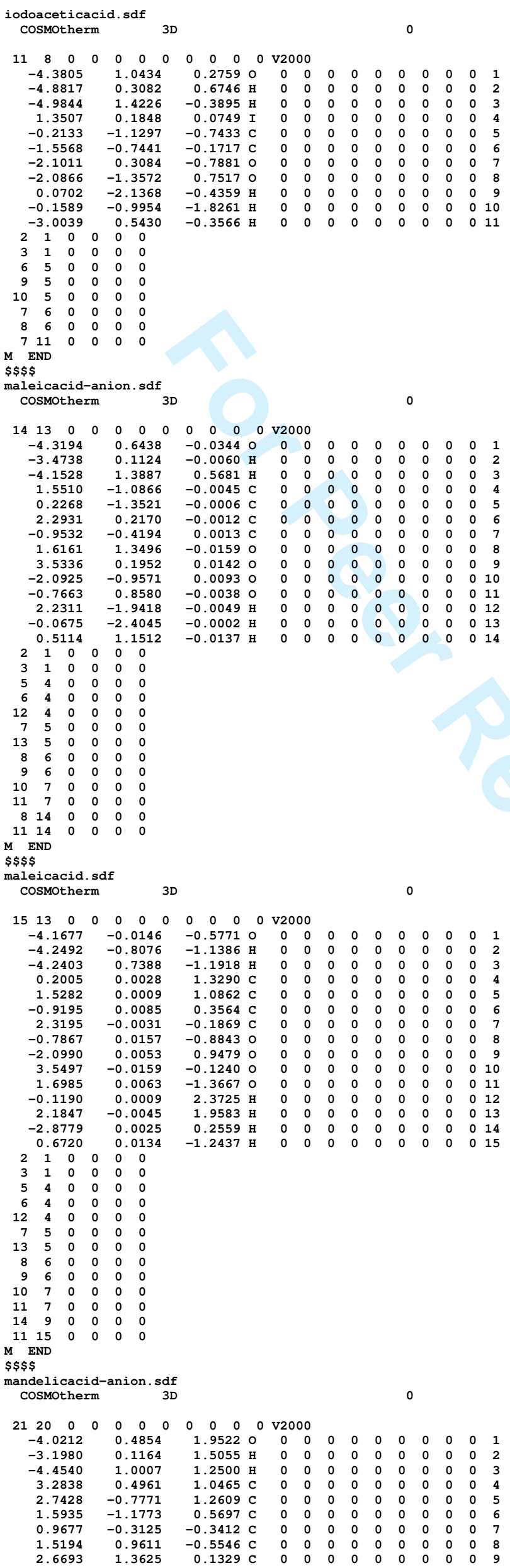




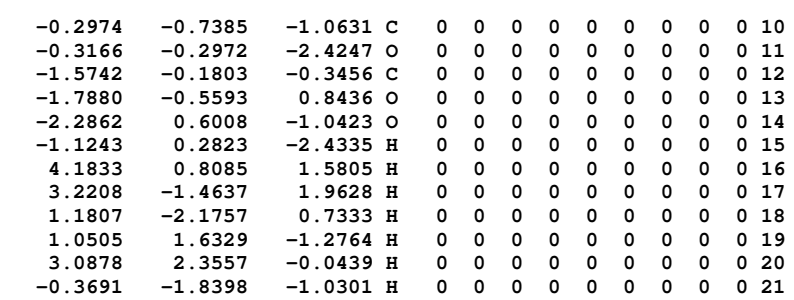




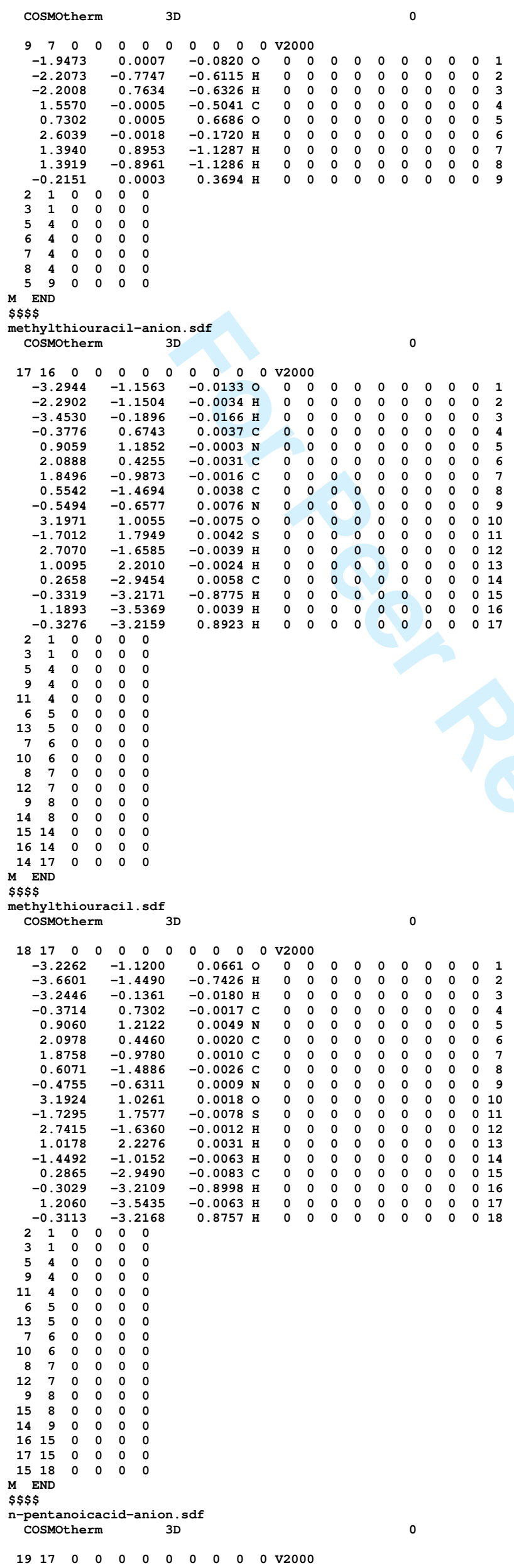




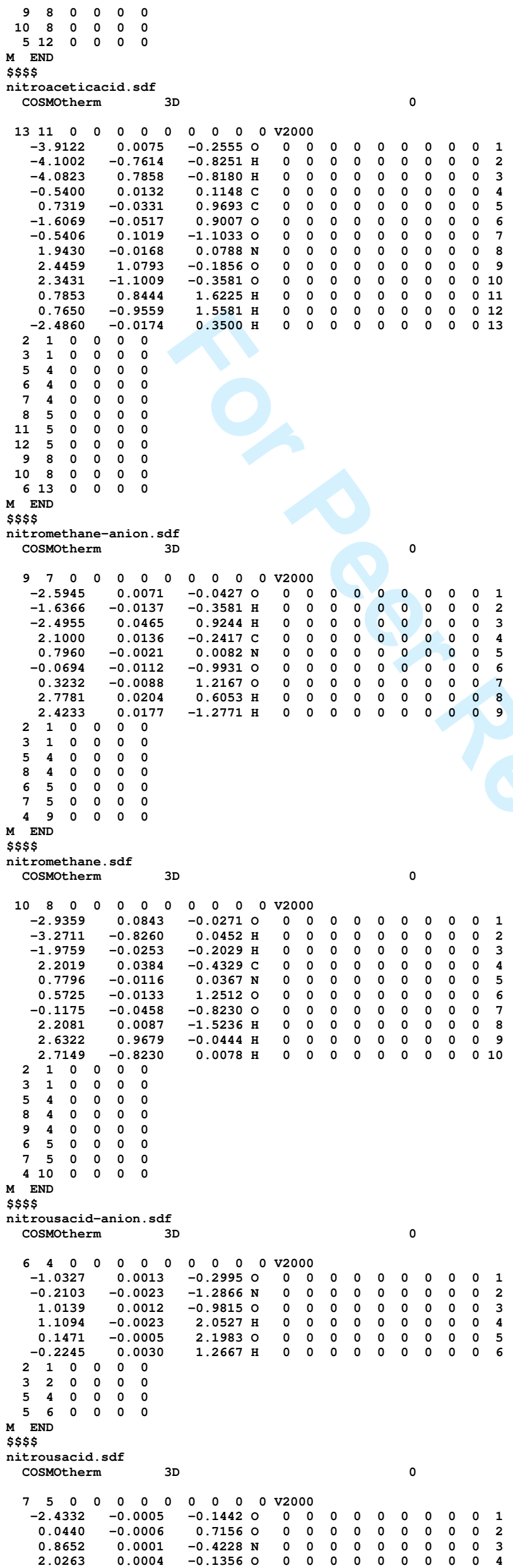


$\begin{array}{rrrrrrrrrrrrrr}-2.6739 & -0.7678 & -0.6952 & \text { H } & 0 & 0 & 0 & 0 & 0 & 0 & 0 & 0 & 0 & 5 \\ -2.6802 & 0.7776 & -0.6771 & \text { H } & 0 & 0 & 0 & 0 & 0 & 0 & 0 & 0 & 0 & 6 \\ -0.9092 & -0.0004 & 0.3304 & \text { H } & 0 & 0 & 0 & 0 & 0 & 0 & 0 & 0 & 0 & 7\end{array}$ $5 \quad 1 \quad 0010000$

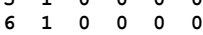

$\begin{array}{llllll}3 & 2 & 0 & 0 & 0 & 0 \\ 4 & 3 & 0 & 0 & 0 & 0\end{array}$

$\begin{array}{lllll}7 & 0 & 0 & 0 & 0\end{array}$

$M \quad$ END

$\$ \$ \$ \$$

COSMOtherm

$\begin{array}{llllllllllll}5 & 4 & 0 & 0 & 0 & 0 & 0 & 0 & 0 & 0 & 0 & \mathrm{v} 2000\end{array}$

$\begin{array}{llllllllllllll}-1.2254 & -0.0372 & -0.0452 & 0 & 0 & 0 & 0 & 0 & 0 & 0 & 0 & 0 & 0 & 1\end{array}$

$\begin{array}{llllllllllllll}-1.2254 & -0.0372 & -0.0452 & 0 & 0 & 0 & 0 & 0 & 0 & 0 & 0 & 0 & 0 & 1 \\ -0.0275 & -0.0006 & -0.0162 & \mathrm{H} & 0 & 0 & 0 & 0 & 0 & 0 & 0 & 0 & 0 & 2\end{array}$

$\begin{array}{llllllllllllll}-1.5188 & 0.4761 & 0.7263 & \mathrm{H} & 0 & 0 & 0 & 0 & 0 & 0 & 0 & 0 & 0 & 3\end{array}$

$\begin{array}{rrrrrrrrrrrrrr}1.5209 & -0.4761 & 0.7262 & \text { H } & 0 & 0 & 0 & 0 & 0 & 0 & 0 & 0 & 0 & 4 \\ 1.2270 & 0.0372 & -0.0453 & 0 & 0 & 0 & 0 & 0 & 0 & 0 & 0 & 0 & 0 & 5\end{array}$

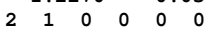

$3 \begin{array}{llllll}3 & 1 & 0 & 0 & 0 & 0 \\ 2 & 5 & 0 & 0 & 0 & 0\end{array}$

$\begin{array}{llllll}5 & 0 & 0 & 0 & 0 \\ 5 & 0 & 0 & 0\end{array}$

END

$\$ \$ \$$

CosMOtherm

$\begin{array}{lllllllllllll}10 & 8 & 0 & 0 & 0 & 0 & 0 & 0 & 0 & 0 & 0 & \mathrm{v} 2000\end{array}$

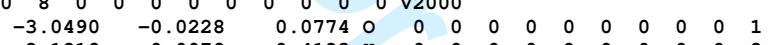

$\begin{array}{llllllllllllll}-2.1819 & -0.0079 & -0.4123 & \text { н } & 0 & 0 & 0 & 0 & 0 & 0 & 0 & 0 & 0 & 2\end{array}$

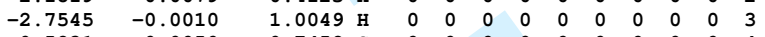

$\begin{array}{llllllllllllll}0.5831 & 0.0050 & 0.7458 & \mathrm{C} & 0 & 0 & 0 & 0 & 0 & 0 & 0 & 0 & 0 & 4\end{array}$

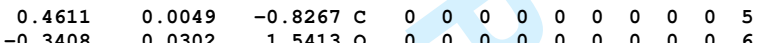

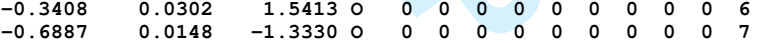

$\begin{array}{llllllllllllll}1.5851 & -0.0026 & -1.4089 & 0 & 0 & 0 & 0 & 0 & 0 & 0 & 0 & 0 & 0 & 8\end{array}$

$\begin{array}{rrrrrrrrrrrrrr}1.8719 & -0.0248 & 1.1307 & 0 & 0 & 0 & 0 & 0 & 0 & 0 & 0 & 0 & 0 & 9\end{array}$

$\begin{array}{lllll}1 & 0 & 0 & 0 & 0\end{array}$

$\begin{array}{lllllll}3 & 1 & 0 & 0 & 0 & 0\end{array}$

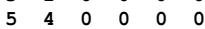

$6 \begin{array}{llllll}6 & 4 & 0 & 0 & 0 & 0\end{array}$

$\begin{array}{llllll}9 & 4 & 0 & 0 & 0 & 0\end{array}$

$\begin{array}{llllll}7 & 5 & 0 & 0 & 0 & 0 \\ 8 & 5 & 0 & 0 & 0 & 0\end{array}$

$\begin{array}{rrrrrr}5 & 0 & 0 & 0 & 0 \\ 10 & 0 & 0 & 0 & 0\end{array}$

END

$\$ \$ \$$

oxalicacid.sdf

3D

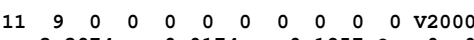

$\begin{array}{llllllllllllll}-3.3974 & -0.0174 & 0.1857 & 0 & 0 & 0 & 0 & 0 & 0 & 0 & 0 & 0 & 0 & 1\end{array}$

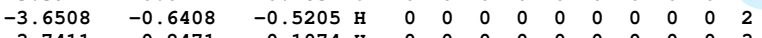

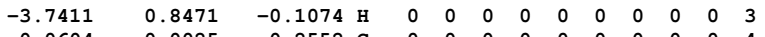

$\begin{array}{llllllllllllll}-0.0604 & 0.0025 & -0.2552 & \mathrm{C} & 0 & 0 & 0 & 0 & 0 & 0 & 0 & 0 & 0 & 4\end{array}$

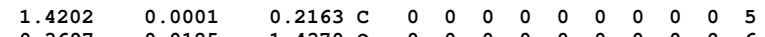

$\begin{array}{llllllllllllll}-0.3697 & 0.0125 & -1.4370 & 0 & 0 & 0 & 0 & 0 & 0 & 0 & 0 & 0 & 0 & 6\end{array}$

$\begin{array}{llllllllllllll}2.2482 & -0.0067 & -0.8385 & 0 & 0 & 0 & 0 & 0 & 0 & 0 & 0 & 0 & 0 & 7\end{array}$

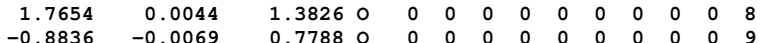

$\begin{array}{rrrrrrrrrrrrrr}-0.8836 & -0.0069 & 0.7788 & 0 & 0 & 0 & 0 & 0 & 0 & 0 & 0 & 0 & 0 & 9\end{array}$

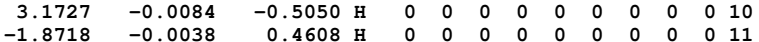

$\begin{array}{llllll}2 & 1 & 0 & 0 & 0 & 0\end{array}$

$\begin{array}{llllll}3 & 1 & 0 & 0 & 0 & 0\end{array}$

$\begin{array}{llllll}5 & 4 & 0 & 0 & 0 & 0\end{array}$

$6 \quad 4 \quad 00000$

$\begin{array}{lllllll}9 & 4 & 0 & 0 & 0 & 0\end{array}$

$\begin{array}{llllll}5 & 0 & 0 & 0 & 0\end{array}$

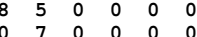

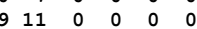

M END

\$\$\$

pamoicacid-anion sdf

cosmotherm

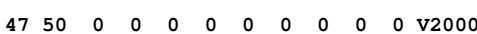

$\begin{array}{llllllllllllll}-7.7945 & 1.1666 & 0.0813 & 0 & 0 & 0 & 0 & 0 & 0 & 0 & 0 & 0 & 0 & 1\end{array}$

$\begin{array}{lllllllllllllll}-7.1328 & 1.1968 & -0.8326 \mathrm{H} & 0 & 0 & 0 & 0 & 0 & 0 & 0 & 0 & 0 & 2\end{array}$

$\begin{array}{llllllllllllll}-7.8014 & 2.0947 & 0.3802 \mathrm{H} & 0 & 0 & 0 & 0 & 0 & 0 & 0 & 0 & 0 & 3\end{array}$

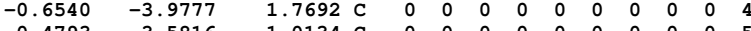

$\begin{array}{lllllllllllllll}0.4793 & -3.5816 & 1.0134 & \mathrm{C} & 0 & 0 & 0 & 0 & 0 & 0 & 0 & 0 & 0 & 5\end{array}$

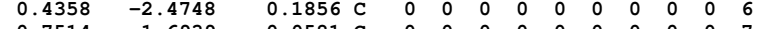

$\begin{array}{llllllllllllll}-0.7514 & -1.6939 & 0.0581 & \mathrm{C} & 0 & 0 & 0 & 0 & 0 & 0 & 0 & 0 & 0 & 7\end{array}$

$\begin{array}{llllllllllllll}-1.9106 & -2.1207 & 0.8072 & \mathrm{C} & 0 & 0 & 0 & 0 & 0 & 0 & 0 & 0 & 0 & 8\end{array}$

$\begin{array}{llllllllllllll}-1.8269 & -3.2609 & 1.6590 & \mathrm{C} & 0 & 0 & 0 & 0 & 0 & 0 & 0 & 0 & 0 & 9\end{array}$

$\begin{array}{llllllllllllll}-0.8249 & -0.5397 & -0.7838 & C & 0 & 0 & 0 & 0 & 0 & 0 & 0 & 0 & 0 & 10\end{array}$

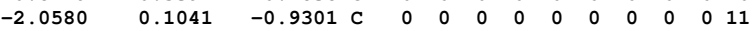

$\begin{array}{llllllllllllll}-3.2193 & -0.3252 & -0.1926 & \mathrm{C} & 0 & 0 & 0 & 0 & 0 & 0 & 0 & 0 & 0 & 12 \\ -3.1225 & -1.4144 & 0.6625 & \mathrm{C} & 0 & 0 & 0 & 0 & 0 & 0 & 0 & 0 & 0 & 13\end{array}$

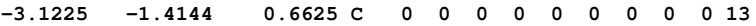

$\begin{array}{llllllllllllll}0.3782 & -0.0399 & -1.5763 & \mathrm{C} & 0 & 0 & 0 & 0 & 0 & 0 & 0 & 0 & 0 & 14\end{array}$

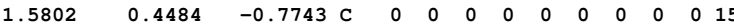

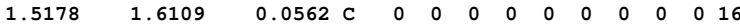

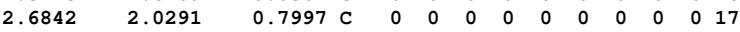

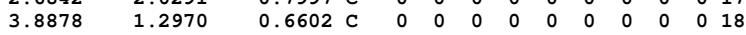

$\begin{array}{llllllllllllll}3.9726 & 0.1974 & -0.1721 & \mathrm{C} & 0 & 0 & 0 & 0 & 0 & 0 & 0 & 0 & 0 & 19\end{array}$

$\begin{array}{llllllllllllll}2.8073 & -0.2153 & -0.9140 & \mathrm{C} & 0 & 0 & 0 & 0 & 0 & 0 & 0 & 0 & 0 & 20 \\ 0.3093 & 3.5241 & 0.9982 & \mathrm{C} & 0 & 0 & 0 & 0 & 0 & 0 & 0 & 0 & 0 & 21\end{array}$

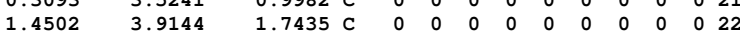

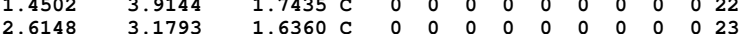

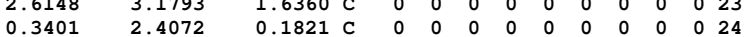

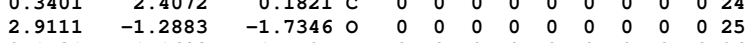

$\begin{array}{rrrrrrrrrrrrrr}2.9111 & -1.2883 & -1.7346 & 0 & 0 & 0 & 0 & 0 & 0 & 0 & 0 & 0 & 0 & 25 \\ -2.1531 & 1.1693 & -1.7727 & 0 & 0 & 0 & 0 & 0 & 0 & 0 & 0 & 0 & 0 & 26\end{array}$ 


$\begin{array}{rrrrllllllllll}5.2812 & -0.5564 & -0.3103 & \mathrm{C} & 0 & 0 & 0 & 0 & 0 & 0 & 0 & 0 & 0 & 27 \\ -4.4965 & 0.4012 & -0.3609 & \mathrm{C} & 0 & 0 & 0 & 0 & 0 & 0 & 0 & 0 & 0 & 28 \\ 3.9320 & -1.5818 & -1.6070 & \mathrm{H} & 0 & 0 & 0 & 0 & 0 & 0 & 0 & 0 & 0 & 29 \\ -3.1142 & 1.4820 & -1.7087 & \mathrm{H} & 0 & 0 & 0 & 0 & 0 & 0 & 0 & 0 & 0 & 30 \\ 5.2702 & -1.5693 & -1.1253 & 0 & 0 & 0 & 0 & 0 & 0 & 0 & 0 & 0 & 0 & 31 \\ 6.2866 & -0.1912 & 0.3427 & \mathrm{O} & 0 & 0 & 0 & 0 & 0 & 0 & 0 & 0 & 0 & 32 \\ -4.6111 & 1.3813 & -1.1302 & \mathrm{O} & 0 & 0 & 0 & 0 & 0 & 0 & 0 & 0 & 0 & 33 \\ -5.5145 & -0.0549 & 0.3593 & \mathrm{O} & 0 & 0 & 0 & 0 & 0 & 0 & 0 & 0 & 0 & 34 \\ -0.5974 & -4.8507 & 2.4214 & \mathrm{H} & 0 & 0 & 0 & 0 & 0 & 0 & 0 & 0 & 0 & 35 \\ 1.4005 & -4.1638 & 1.0837 & \mathrm{H} & 0 & 0 & 0 & 0 & 0 & 0 & 0 & 0 & 0 & 36 \\ 1.3167 & -2.2103 & -0.3984 & \mathrm{H} & 0 & 0 & 0 & 0 & 0 & 0 & 0 & 0 & 0 & 37 \\ -2.7170 & -3.5593 & 2.2178 & \mathrm{H} & 0 & 0 & 0 & 0 & 0 & 0 & 0 & 0 & 0 & 38 \\ -3.9996 & -1.7357 & 1.2253 & \mathrm{H} & 0 & 0 & 0 & 0 & 0 & 0 & 0 & 0 & 0 & 39 \\ 0.0342 & 0.7683 & -2.2368 & \mathrm{H} & 0 & 0 & 0 & 0 & 0 & 0 & 0 & 0 & 0 & 40 \\ 0.7270 & -0.8483 & -2.2352 & \mathrm{H} & 0 & 0 & 0 & 0 & 0 & 0 & 0 & 0 & 0 & 41 \\ 4.7756 & 1.6019 & 1.2187 & \mathrm{H} & 0 & 0 & 0 & 0 & 0 & 0 & 0 & 0 & 0 & 42 \\ -0.6062 & 4.1159 & 1.0662 & \mathrm{H} & 0 & 0 & 0 & 0 & 0 & 0 & 0 & 0 & 0 & 43 \\ 1.4083 & 4.7953 & 2.3864 & \mathrm{H} & 0 & 0 & 0 & 0 & 0 & 0 & 0 & 0 & 0 & 44 \\ 3.5095 & 3.4732 & 2.1906 & \mathrm{H} & 0 & 0 & 0 & 0 & 0 & 0 & 0 & 0 & 0 & 45 \\ -0.5463 & 2.1449 & -0.3940 & \mathrm{H} & 0 & 0 & 0 & 0 & 0 & 0 & 0 & 0 & 0 & 46 \\ -6.3669 & 0.4967 & 0.1828 & \mathrm{H} & 0 & 0 & 0 & 0 & 0 & 0 & 0 & 0 & 0 & 47\end{array}$


$\begin{array}{llllllllllllll}2.7023 & 1.0108 & -1.7037 & \mathrm{H} & 0 & 0 & 0 & 0 & 0 & 0 & 0 & 0 & 0 & 29\end{array}$

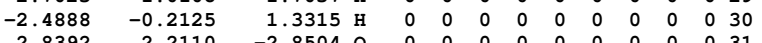
$\begin{array}{llllllllllllll}2.8392 & 2.2110 & -2.8504 & 0 & 0 & 0 & 0 & 0 & 0 & 0 & 0 & 0 & 0 & 31\end{array}$

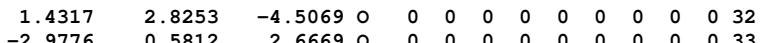
$\begin{array}{llllllllllllll}-2.9776 & 0.5812 & 2.6669 & 0 & 0 & 0 & 0 & 0 & 0 & 0 & 0 & 0 & 0 & 33 \\ -1.8111 & 1.2698 & 4.4741 & 0 & 0 & 0 & 0 & 0 & 0 & 0 & 0 & 0 & 0 & 34\end{array}$ $\begin{array}{llllllllllllll}5.0384 & -0.7561 & 3.8914 & \mathrm{H} & 0 & 0 & 0 & 0 & 0 & 0 & 0 & 0 & 0 & 35\end{array}$

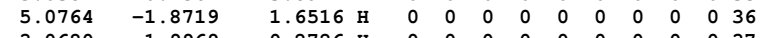

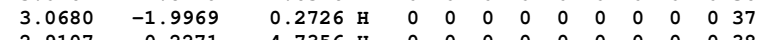

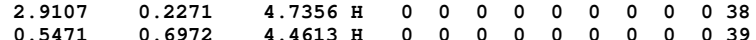
$\begin{array}{rrrrrrrrrrrrrr}0.5471 & 0.6972 & 4.4613 & \mathrm{H} & 0 & 0 & 0 & 0 & 0 & 0 & 0 & 0 & 0 & 39 \\ -0.2666 & -2.5383 & -0.3549 & \mathrm{H} & 0 & 0 & 0 & 0 & 0 & 0 & 0 & 0 & 0 & 40\end{array}$ $\begin{array}{llllllllllllllll}1.4290 & -2.2151 & -0.6508 & \mathrm{H} & 0 & 0 & 0 & 0 & 0 & 0 & 0 & 0 & 0 & 41\end{array}$

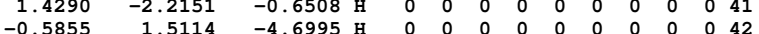
$\begin{array}{lllllllllllllllll}-0.5855 & 1.5114 & -4.6995 & \mathrm{H} & 0 & 0 & 0 & 0 & 0 & 0 & 0 & 0 & 0 & 42\end{array}$

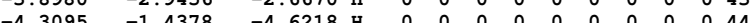
$\begin{array}{llllllllllllll}-4.3095 & -1.4378 & -4.6218 & \mathrm{H} & 0 & 0 & 0 & 0 & 0 & 0 & 0 & 0 & 0 & 43 \\ -2.6609 & 0.3513 & -5.1586 & \mathrm{H} & 0 & 0 & 0 & 0 & 0 & 0 & 0 & 0 & 0 & 45\end{array}$

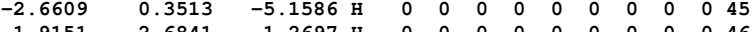

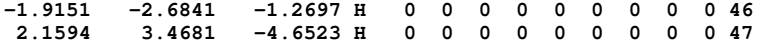

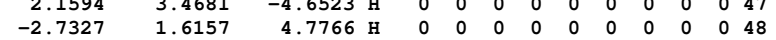
$2 \begin{array}{ccccc}1 & 0 & 0 & 0 & 0\end{array}$ $\begin{array}{llllll}3 & 1 & 0 & 0 & 0 & 0\end{array}$

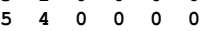
$\begin{array}{rrrrrr}9 & 4 & 0 & 0 & 0 & 0 \\ 35 & 4 & 0 & 0 & 0 & 0\end{array}$

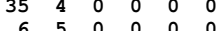
$\begin{array}{rrrrrr}6 & 5 & 0 & 0 & 0 & 0 \\ 36 & 5 & 0 & 0 & 0 & 0\end{array}$

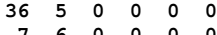
$\begin{array}{rrrrrrr}7 & 6 & 0 & 0 & 0 & 0 \\ 37 & 6 & 0 & 0 & 0 & 0\end{array}$ $\begin{array}{rrrrrr}37 & 6 & 0 & 0 & 0 & 0 \\ 8 & 7 & 0 & 0 & 0 & 0\end{array}$ $107 \begin{array}{llllll}10 & 7 & 0 & 0 & 0 & 0\end{array}$ $\begin{array}{rrrrrr}9 & 8 & 0 & 0 & 0 & 0 \\ 13 & 8 & 0 & 0 & 0 & 0\end{array}$

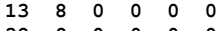
$\begin{array}{lrrrrr}38 & 9 & 0 & 0 & 0 & 0\end{array}$ $1110 \begin{array}{lllll}11 & 10 & 0 & 0 & 0\end{array}$ 141010010000

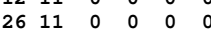
$\begin{array}{llllll}13 & 12 & 0 & 0 & 0 & 0\end{array}$ $\begin{array}{llllll}28 & 12 & 0 & 0 & 0 & 0\end{array}$ $\begin{array}{llllll}39 & 13 & 0 & 0 & 0 & 0\end{array}$ $1514 \quad 000000$ $\begin{array}{llllll}40 & 14 & 0 & 0 & 0 & 0\end{array}$ $\begin{array}{llllll}41 & 14 & 0 & 0 & 0 & 0\end{array}$ $\begin{array}{llllll}16 & 15 & 0 & 0 & 0 & 0\end{array}$ $\begin{array}{llllll}20 & 15 & 0 & 0 & 0 & 0\end{array}$ $\begin{array}{llllll}17 & 16 & 0 & 0 & 0 & 0\end{array}$ $\begin{array}{llllll}18 & 17 & 0 & 0 & 0 & 0\end{array}$ $\begin{array}{lllllll}18 & 17 & 0 & 0 & 0 & 0 \\ 23 & 17 & 0 & 0 & 0 & 0\end{array}$ $\begin{array}{llllll}19 & 18 & 0 & 0 & 0 & 0\end{array}$ $42 \quad 18 \quad 000000$ $\begin{array}{llllll}20 & 19 & 0 & 0 & 0 & 0\end{array}$ $\begin{array}{llllll}27 & 19 & 0 & 0 & 0 & 0 \\ 25 & 20 & 0 & 0 & 0 & 0\end{array}$ $\begin{array}{llllll}25 & 20 & 0 & 0 & 0 & 0 \\ 22 & 21 & 0 & 0 & 0 & 0\end{array}$ $\begin{array}{llllll}22 & 21 & 0 & 0 & 0 & 0 \\ 24 & 21 & 0 & 0 & 0 & 0\end{array}$ $\begin{array}{lllllll}24 & 21 & 0 & 0 & 0 & 0 \\ 43 & 21 & 0 & 0 & 0 & 0\end{array}$ $23 \quad 22 \quad 0 \quad 000$ $4422 \quad 0 \quad 000$ $\begin{array}{llllll}45 & 23 & 0 & 0 & 0 & 0\end{array}$ $\begin{array}{llllll}46 & 24 & 0 & 0 & 0 & 0\end{array}$ $\begin{array}{llllll}29 & 25 & 0 & 0 & 0 & 0\end{array}$ $\begin{array}{llllll}30 & 26 & 0 & 0 & 0 & 0\end{array}$ $\begin{array}{llllll}31 & 27 & 0 & 0 & 0 & 0\end{array}$ $\begin{array}{llllll}32 & 27 & 0 & 0 & 0 & 0\end{array}$ $\begin{array}{lllllll}33 & 28 & 0 & 0 & 0 & 0\end{array}$ $\begin{array}{lllllll}47 & 32 & 0 & 0 & 0 & 0\end{array}$ $34 \quad 48 \quad 0 \quad 0000$ $M$ MND

pentachlorophenol-anion.sdf

CosMOtherm

$\begin{array}{llllllllllll}15 & 14 & 0 & 0 & 0 & 0 & 0 & 0 & 0 & 0 & 0 & \mathrm{v} 2000\end{array}$

\begin{tabular}{|c|c|c|c|c|c|c|c|c|c|c|c|c|c|}
\hline-4.8565 & -1.1301 & 0.5175 & & 0 & 0 & 0 & 0 & 0 & 0 & 0 & 0 & 0 & 1 \\
\hline-3.8898 & -1.1144 & 0.2587 & H & 0 & 0 & 0 & 0 & 0 & 0 & 0 & 0 & 0 & 2 \\
\hline 5.0477 & -0.2182 & 0.7945 & H & 0 & 0 & 0 & 0 & 0 & 0 & 0 & 0 & 0 & 3 \\
\hline 0.1156 & 1.3459 & -0.0242 & & 0 & 0 & 0 & 0 & 0 & 0 & 0 & 0 & 0 & 4 \\
\hline 1.0940 & 0.6512 & -0.0971 & c & & & 0 & 0 & 0 & 0 & 0 & & 0 & 5 \\
\hline-1.1774 & -0.7869 & -0.1214 & & 0 & 0 & 0 & 0 & 0 & 0 & 0 & 0 & 0 & \\
\hline 0.1053 & -1.4435 & -0.0678 & c & & & 0 & 0 & 0 & 0 & 0 & 0 & 0 & \\
\hline 1.3 & -0.7538 & 0.0035 & & 0 & 0 & 0 & 0 & 0 & 0 & 0 & 0 & 0 & 8 \\
\hline 1.3384 & 0.6530 & 0.0281 & C & & & & 0 & 0 & 0 & 0 & 0 & & 9 \\
\hline-2.2752 & -1.4377 & -0.1891 & & & & 0 & 0 & 0 & 0 & 0 & 0 & & 10 \\
\hline-2.6206 & 1.5227 & -0.1760 & $\mathrm{Cl}$ & 0 & & 0 & 0 & 0 & 0 & 0 & 0 & 0 & 11 \\
\hline 0.1205 & 3.1046 & 0.0011 & Fl & 0 & & 0 & 0 & 0 & 0 & 0 & 0 & 0 & 12 \\
\hline 2.8603 & 1.5245 & 0.1204 & C] & 0 & & 0 & 0 & 0 & 0 & 0 & 0 & 0 & 13 \\
\hline 2.8342 & -1.6408 & 0.0665 & ב & 0 & & 0 & 0 & 0 & 0 & 0 & 0 & 0. & 14 \\
\hline 0.0734 & -3.2011 & -0.0957 & $\mathrm{Cl}$ & 0 & & 0 & 0 & 0 & 0 & 0 & 0 & & 15 \\
\hline
\end{tabular}
$\begin{array}{llrrrrrrrrrrrrr}0.0734 & -3.2011 & -0.0957 & \mathrm{Cl} & 0 & 0 & 0 & 0 & 0 & 0 & 0 & 0 & 0 & 15\end{array}$ $\begin{array}{llllll}2 & 1 & 0 & 0 & 0 & 0\end{array}$ $\begin{array}{llllll}3 & 1 & 0 & 0 & 0 & 0 \\ 5 & 4 & 0 & 0 & 0 & 0\end{array}$

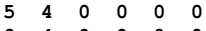
$\begin{array}{llllll}9 & 4 & 0 & 0 & 0 & 0\end{array}$ $\begin{array}{llllll}12 & 4 & 0 & 0 & 0 & 0\end{array}$ $\begin{array}{llllll}6 & 5 & 0 & 0 & 0 & 0 \\ 1 & 5 & 0 & 0 & 0 & 0\end{array}$ $\begin{array}{llllll}7 & 6 & 0 & 0 & 0 & 0\end{array}$ $\begin{array}{llllll}10 & 6 & 0 & 0 & 0 & 0\end{array}$ $\begin{array}{llllll}8 & 7 & 0 & 0 & 0 & 0\end{array}$ $\begin{array}{llllll}9 & 8 & 0 & 0 & 0 & 0\end{array}$ $\begin{array}{llllll}14 & 8 & 0 & 0 & 0 & 0\end{array}$ $\begin{array}{llllll}13 & 9 & 0 & 0 & 0 & 0\end{array}$ $\begin{array}{rrrrrr}7 & 15 & 0 & 0 & 0 & 0\end{array}$ 


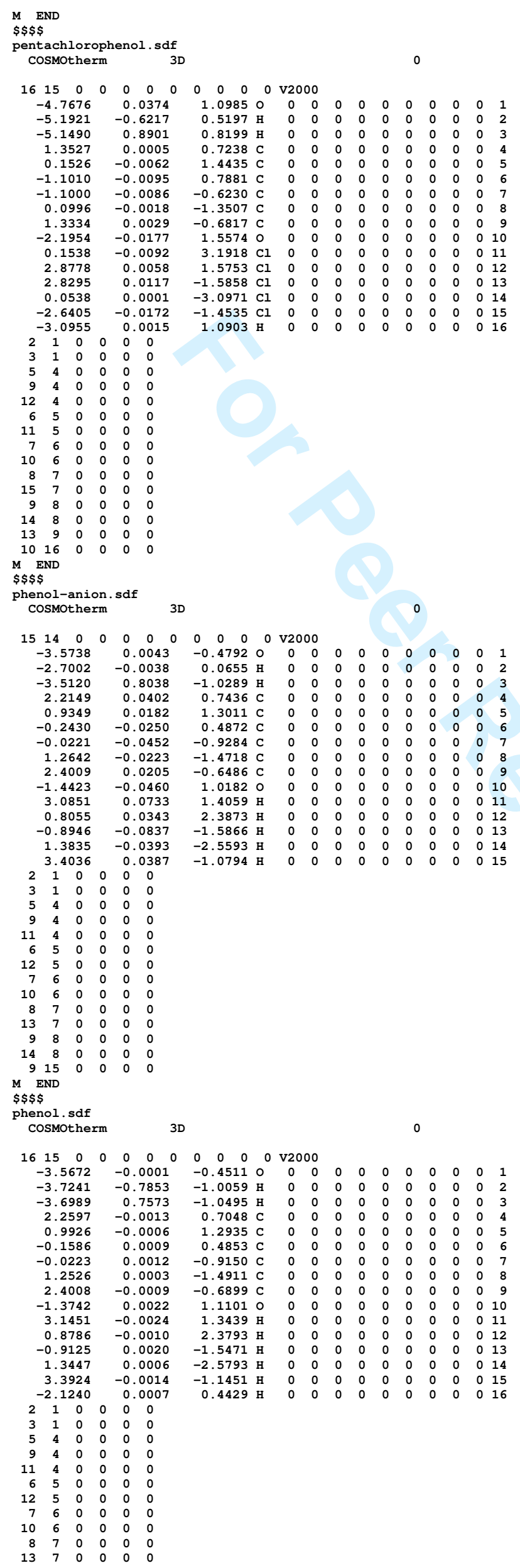


$\begin{array}{rrrrrr}9 & 8 & 0 & 0 & 0 & 0 \\ 14 & 8 & 0 & 0 & 0 & 0\end{array}$

$\begin{array}{rrrrrr}15 & 9 & 0 & 0 & 0 & 0 \\ 10 & 16 & 0 & 0 & 0 & 0\end{array}$

M END

$\$ \$ \$$

phenytoin-anion.sdf

cosmotherm $3 \mathrm{D}$

0

$\begin{array}{llllllllllll}33 & 34 & 0 & 0 & 0 & 0 & 0 & 0 & 0 & 0 & 0 & \mathrm{v} 2000\end{array}$

$\begin{array}{lllllllllllll}-4.8650 & -1.2640 & 0.8897 & 0 & 0 & 0 & 0 & 0 & 0 & 0 & 0 & 0 & 0\end{array}$

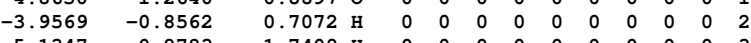

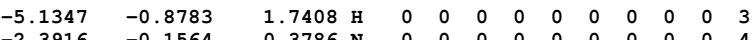

$\begin{array}{llllllllllllll}-2.3916 & -0.1564 & 0.3786 & \mathrm{~N} & 0 & 0 & 0 & 0 & 0 & 0 & 0 & 0 & 0 & \mathbf{4} \\ -2.1674 & 1.1978 & 0.1775 & \mathrm{C} & 0 & 0 & 0 & 0 & 0 & 0 & 0 & 0 & 0 & 5\end{array}$

$\begin{array}{lrllllllllllll}-2.1674 & 1.1978 & 0.1775 & \mathrm{C} & 0 & 0 & 0 & 0 & 0 & 0 & 0 & 0 & 0 & 5 \\ -1.2079 & -0.8091 & 0.3120 & \mathrm{C} & 0 & 0 & 0 & 0 & 0 & 0 & 0 & 0 & 0 & 6\end{array}$

$\begin{array}{rrrrrrrrrrrrrr}-1.2079 & -0.8091 & 0.3120 & \mathrm{C} & 0 & 0 & 0 & 0 & 0 & 0 & 0 & 0 & 0 & 6 \\ -0.0363 & 0.2205 & 0.0373 & \mathrm{C} & 0 & 0 & 0 & 0 & 0 & 0 & 0 & 0 & 0 & 7\end{array}$

$\begin{array}{llllllllllllll}-0.8051 & 1.4635 & 0.1115 & \mathrm{~N} & 0 & 0 & 0 & 0 & 0 & 0 & 0 & 0 & 0 & 8\end{array}$

$\begin{array}{rrrrrrrrrrrrrr}-0.8051 & 1.4635 & 0.1115 & \mathrm{~N} & 0 & 0 & 0 & 0 & 0 & 0 & 0 & 0 & 0 & 8 \\ 0.5664 & 0.0549 & -1.3657 & \mathrm{C} & 0 & 0 & 0 & 0 & 0 & 0 & 0 & 0 & 0 & 9\end{array}$

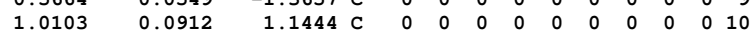

$\begin{array}{llllllllllllll}-3.0453 & 2.0760 & 0.0879 & 0 & 0 & 0 & 0 & 0 & 0 & 0 & 0 & 0 & 0 & 11\end{array}$

$\begin{array}{llllllllllllll}-1.0159 & -2.0286 & 0.4425 & 0 & 0 & 0 & 0 & 0 & 0 & 0 & 0 & 0 & 0 & 12\end{array}$

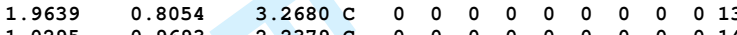

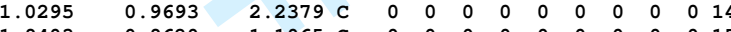

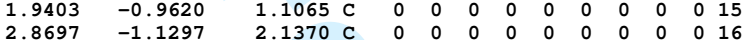

$\begin{array}{llllllllllllll}2.8697 & -1.1297 & 2.1370 & \mathrm{C} & 0 & 0 & 0 & 0 & 0 & 0 & 0 & 0 & 0 & 16 \\ 2.8868 & -0.2449 & 3.2228 & \mathrm{C} & 0 & 0 & 0 & 0 & 0 & 0 & 0 & 0 & 0 & 17\end{array}$

$\begin{array}{rrrrrrrrrrrrrr}2.8868 & -0.2449 & 3.2228 & \mathrm{C} & 0 & 0 & 0 & 0 & 0 & 0 & 0 & 0 & 0 & 17 \\ -0.0151 & -0.7689 & -2.3416 & \mathrm{C} & 0 & 0 & 0 & 0 & 0 & 0 & 0 & 0 & 0 & 18\end{array}$

$\begin{array}{llllllllllllll}-0.0151 & -0.7689 & -2.3416 & \mathrm{C} & 0 & 0 & 0 & 0 & 0 & 0 & 0 & 0 & 0 & 18 \\ 0.5187 & -0.8276 & -3.6355 & \mathrm{C} & 0 & 0 & 0 & 0 & 0 & 0 & 0 & 0 & 0 & 19\end{array}$

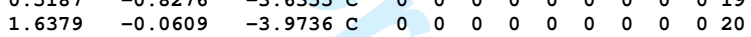

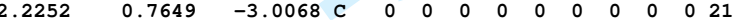

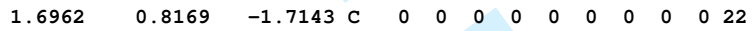

$\begin{array}{llllllllllllll}-0.4867 & 2.3107 & -0.3543 & \mathrm{H} & 0 & 0 & 0 & 0 & 0 & 0 & 0 & 0 & 0 & 23\end{array}$

$\begin{array}{llllllllllllll}1.9693 & 1.5028 & 4.1083 & \mathrm{H} & 0 & 0 & 0 & 0 & 0 & 0 & 0 & 0 & 0 & 24\end{array}$

$\begin{array}{llllllllllllll}0.3093 & 1.7879 & 2.2766 \mathrm{H} & 0 & 0 & 0 & 0 & 0 & 0 & 0 & 0 & 0 & 25\end{array}$

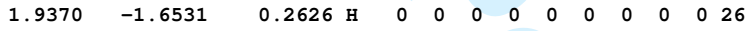

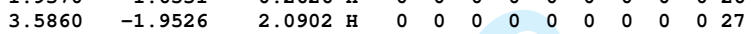

$\begin{array}{llllllllllllll}3.6170 & -0.3719 & 4.0244 & \mathrm{H} & 0 & 0 & 0 & 0 & 0 & 0 & 0 & 0 & 0 & 28\end{array}$

$\begin{array}{lllllllllllllll}-0.8839 & -1.3806 & -2.0951 & \mathrm{H} & 0 & 0 & 0 & 0 & 0 & 0 & 0 & 0 & 0 & 29\end{array}$

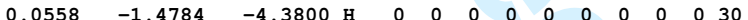

$\begin{array}{llllllllllllll}2.0533 & -0.1081 & -4.9819 & \mathrm{H} & 0 & 0 & 0 & 0 & 0 & 0 & 0 & 0 & 0 & 31\end{array}$

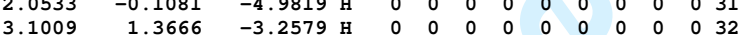

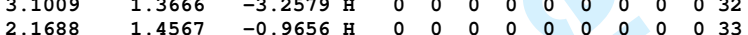

2.168801 .4567

$\begin{array}{lllllll}2 & 1 & 0 & 0 & 0 & 0 \\ 3 & 1 & 0 & 0 & 0 & 0\end{array}$

$\begin{array}{lllllll}3 & 1 & 0 & 0 & 0 & 0 \\ 5 & 4 & 0 & 0 & 0 & 0\end{array}$

$\begin{array}{llllll}5 & 4 & 0 & 0 & 0 & 0 \\ 6 & 4 & 0 & 0 & 0 & 0\end{array}$

8 5 $50 \begin{array}{llll}5 & 0 & 0 & 0\end{array}$

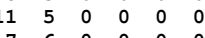

$\begin{array}{llllll}7 & 6 & 0 & 0 & 0 & 0\end{array}$

$\begin{array}{llllll}2 & 6 & 0 & 0 & 0 & 0 \\ 8 & 7 & 0 & 0 & 0 & 0\end{array}$

$\begin{array}{llllll}7 & 7 & 0 & 0 & 0 & 0\end{array}$

$\begin{array}{rrrrrr}10 & 7 & 0 & 0 & 0\end{array}$

$\begin{array}{llllll}23 & 8 & 0 & 0 & 0 & 0\end{array}$

$\begin{array}{llllll}18 & 9 & 0 & 0 & 0 & 0 \\ 22 & 9 & 0 & 0 & 0 & 0\end{array}$

$\begin{array}{lrrrrr}22 & 9 & 0 & 0 & 0 & 0\end{array}$

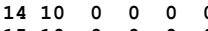

$\begin{array}{llllll}15 & 10 & 0 & 0 & 0 & 0 \\ 14 & 13 & 0 & 0 & 0 & 0\end{array}$

$1413 \quad 00001000$

1713 1 13 1 00100

$2413 \quad 00001000$

25145000000

$\begin{array}{llllll}16 & 15 & 0 & 0 & 0 & 0\end{array}$

2615 15 150000

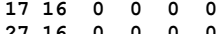

$28 \quad 17 \quad 00000$

$\begin{array}{llllll}19 & 18 & 0 & 0 & 0 & 0\end{array}$

$2918 \quad 0 \quad 0 \quad 000$

2019 19 19000

$30 \begin{array}{lllll}39 & 0 & 0 & 0 & 0\end{array}$

$2120 \quad 00000$

3120000000

$\begin{array}{llllll}22 & 21 & 0 & 0 & 0 & 0\end{array}$

$\begin{array}{llllll}32 & 21 & 0 & 0 & 0 & 0 \\ 22 & 33 & 0 & 0 & 0 & 0\end{array}$

END

\$\$\$

phenytoin.sdf

Cosmotherm 3D

$\begin{array}{llllllllllll}34 & 35 & 0 & 0 & 0 & 0 & 0 & 0 & 0 & 0 & 0 & \mathrm{v} 2000\end{array}$

$\begin{array}{llllllllllllll}-4.9128 & -0.4614 & -1.2180 & 0 & 0 & 0 & 0 & 0 & 0 & 0 & 0 & 0 & 0 & 1 \\ -5.0153 & -1.2380 & -1.7978 & \mathrm{H} & 0 & 0 & 0 & 0 & 0 & 0 & 0 & 0 & 0 & 2\end{array}$

$\begin{array}{rrrrrrrrrrrrrr}-5.0153 & -1.2380 & -1.7978 & \mathrm{H} & 0 & 0 & 0 & 0 & 0 & 0 & 0 & 0 & 0 & 2 \\ -5.1697 & 0.2938 & -1.7777 & \mathrm{H} & 0 & 0 & 0 & 0 & 0 & 0 & 0 & 0 & 0 & 3\end{array}$

$\begin{array}{rrrrrrrrrrrrrr}-5.1697 & 0.2938 & -1.7777 & \text { H } & 0 & 0 & 0 & 0 & 0 & 0 & 0 & 0 & 0 & 3 \\ -2.3532 & -0.1929 & -0.1021 & \mathrm{~N} & 0 & 0 & 0 & 0 & 0 & 0 & 0 & 0 & 0 & 4\end{array}$

$\begin{array}{llllllllllllll}-2.3532 & -0.1929 & -0.1021 & \mathbf{N} & 0 & 0 & 0 & 0 & 0 & 0 & 0 & 0 & 0 & 4 \\ -2.1391 & -0.0434 & 1.2838 & \mathrm{C} & 0 & 0 & 0 & 0 & 0 & 0 & 0 & 0 & 0 & 5\end{array}$

$\begin{array}{llrlllllllllll}-2.1391 & -0.0434 & 1.2838 & \mathrm{C} & 0 & 0 & 0 & 0 & 0 & 0 & 0 & 0 & 0 & 5 \\ -1.1890 & -0.1637 & -0.8212 & \mathrm{C} & 0 & 0 & 0 & 0 & 0 & 0 & 0 & 0 & 0 & 6\end{array}$

$\begin{array}{lllllllllllllll}-0.0217 & -0.0078 & 0.2136 & \mathrm{C} & 0 & 0 & 0 & 0 & 0 & 0 & 0 & 0 & 0 & 7\end{array}$

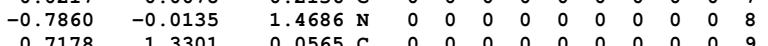

$\begin{array}{lllllllllllllr}0.7178 & 1.3301 & 0.0565 & \mathrm{C} & 0 & 0 & 0 & 0 & 0 & 0 & 0 & 0 & 0 & 9\end{array}$

$\begin{array}{llllllllllllll}0.9014 & -1.2253 & 0.0734 & \mathrm{C} & 0 & 0 & 0 & 0 & 0 & 0 & 0 & 0 & 0 & 10\end{array}$

$\begin{array}{llllllllllllll}-3.0216 & 0.0342 & 2.1374 & \circ & 0 & 0 & 0 & 0 & 0 & 0 & 0 & 0 & 0 & 11\end{array}$

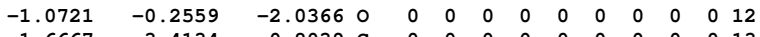

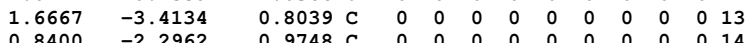

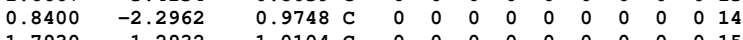

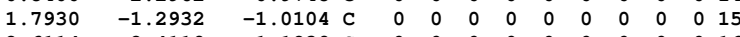

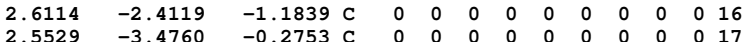

$\begin{array}{lllllllllllllll}2.5529 & -3.4760 & -0.2753 & C & 0 & 0 & 0 & 0 & 0 & 0 & 0 & 0 & 0 & 16 \\ 0.1607 & 2.4059 & -0.6516 & C & 0 & 0 & 0 & 0 & 0 & 0 & 0 & 0 & 0 & 17\end{array}$

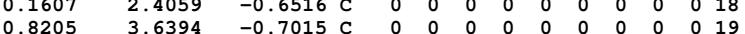

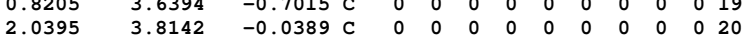

$\begin{array}{rrrrrrrrrrrrrr}2.0395 & 3.8142 & -0.0389 & C & 0 & 0 & 0 & 0 & 0 & 0 & 0 & 0 & 0 & 20 \\ 2.5982 & 2.7469 & 0.6741 & C & 0 & 0 & 0 & 0 & 0 & 0 & 0 & 0 & 0 & 21\end{array}$

$\begin{array}{llllllllllllll}2.5982 & 2.7469 & 0.6741 & \mathrm{C} & 0 & 0 & 0 & 0 & 0 & 0 & 0 & 0 & 0 & 21 \\ 1.9437 & 1.5126 & 0.7177 & \mathrm{C} & 0 & 0 & 0 & 0 & 0 & 0 & 0 & 0 & 0 & 22\end{array}$ 
$\begin{array}{llllll}2 & 1 & 0 & 0 & 0 & 0 \\ 3 & 1 & 0 & 0 & 0 & 0\end{array}$

$\begin{array}{llllll}3 & 1 & 0 & 0 & 0 & 0 \\ 5 & 4 & 0 & 0 & 0 & 0\end{array}$

$\begin{array}{llllll}5 & 4 & 0 & 0 & 0 & 0 \\ 9 & 4 & 0 & 0 & 0 & 0\end{array}$

$\begin{array}{rrrrrr}16 & 4 & 0 & 0 & 0 & 0\end{array}$

$\begin{array}{rrrrrr}6 & 5 & 0 & 0 & 0 & 0 \\ 17 & 5 & 0 & 0 & 0 & 0\end{array}$

$7 \begin{array}{llllll}7 & 6 & 0 & 0 & 0 & 0\end{array}$

$\begin{array}{llllll}18 & 6 & 0 & 0 & 0 & 0\end{array}$

$8 \begin{array}{llllll}8 & 7 & 0 & 0 & 0 & 0\end{array}$

$\begin{array}{cccccc}10 & 7 & 0 & 0 & 0 & 0 \\ 9 & 8 & 0 & 0 & 0 & 0\end{array}$

$\begin{array}{rrrrrr}9 & 8 & 0 & 0 & 0 & 0 \\ 11 & 8 & 0 & 0 & 0 & 0\end{array}$

$\begin{array}{llllll}11 & 8 & 0 & 0 & 0 & 0 \\ 19 & 9 & 0 & 0 & 0 & 0\end{array}$

$1210 \quad 00000$

$1310 \quad 000000$

$\begin{array}{llllll}14 & 11 & 0 & 0 & 0 & 0\end{array}$

$\begin{array}{llllll}15 & 11 & 0 & 0 & 0 & 0\end{array}$

$\begin{array}{llllll}15 & 20 & 0 & 0 & 0 & 0\end{array}$

END

$\$ \$ \$$

phthalicacid.sdf

cosmotherm

$\begin{array}{llllllllllll}21 & 21 & 0 & 0 & 0 & 0 & 0 & 0 & 0 & 0 & 0 & \mathrm{v} 2000\end{array}$

$\begin{array}{lllllllllllllll}-4.6943 & 0.1929 & 0.0120 & 0 & 0 & 0 & 0 & 0 & 0 & 0 & 0 & 0 & 0 & 1 \\ -4.9121 & -0.6006 & -0.5114 & \mathrm{H} & 0 & 0 & 0 & 0 & 0 & 0 & 0 & 0 & 0 & 2\end{array}$

$\begin{array}{rrrrrrrrrrrrrr}-4.9121 & -0.6006 & -0.5114 & \text { H } & 0 & 0 & 0 & 0 & 0 & 0 & 0 & 0 & 0 & 2 \\ -4.9676 & 0.9455 & -0.5448 & \text { H } & 0 & 0 & 0 & 0 & 0 & 0 & 0 & 0 & 0 & 3\end{array}$

$\begin{array}{rrrrrrrrrrrrrrrrr}-4.9676 & 0.9455 & -0.5448 & \mathrm{H} & 0 & 0 & 0 & 0 & 0 & 0 & 0 & 0 & 0 & 3 \\ 1.3605 & -0.1049 & 2.6736 \mathrm{C} & 0 & 0 & 0 & 0 & 0 & 0 & 0 & 0 & 0 & 4\end{array}$

$\begin{array}{llllllllllllll}1.3605 & -0.1049 & 2.6736 & \mathrm{C} & 0 & 0 & 0 & 0 & 0 & 0 & 0 & 0 & 0 & 4 \\ 2.5437 & -0.0755 & 1.9337 & \mathrm{C} & 0 & 0 & 0 & 0 & 0 & 0 & 0 & 0 & 0 & 5\end{array}$

$\begin{array}{llllllllllllll}2.5437 & -0.0755 & 1.9337 & \mathrm{C} & 0 & 0 & 0 & 0 & 0 & 0 & 0 & 0 & 0 & 5 \\ 2.4840 & -0.0081 & 0.5426 & \mathrm{C} & 0 & 0 & 0 & 0 & 0 & 0 & 0 & 0 & 0 & 6\end{array}$

$\begin{array}{rrrrrrrrrrrrrr}2.4840 & -0.0081 & 0.5426 & \mathrm{C} & 0 & 0 & 0 & 0 & 0 & 0 & 0 & 0 & 0 & 6 \\ 1.2642 & -0.0035 & -0.1569 & \mathrm{C} & 0 & 0 & 0 & 0 & 0 & 0 & 0 & 0 & 0 & 7\end{array}$

$\begin{array}{rrrrrrrrrrrrrr}1.2642 & -0.0035 & -0.1569 & \mathrm{C} & 0 & 0 & 0 & 0 & 0 & 0 & 0 & 0 & 0 & 7 \\ 0.0559 & -0.0377 & 0.6025 & \mathrm{C} & 0 & 0 & 0 & 0 & 0 & 0 & 0 & 0 & 0 & 8\end{array}$

$\begin{array}{lllllllllllll}0.1367 & -0.0736 & 2.0086 & \mathrm{C} & 0 & 0 & 0 & 0 & 0 & 0 & 0 & 0 & 0\end{array}$

$\begin{array}{rrrrrrrrrrrrrr}1.4386 & 0.0955 & -1.6656 & \mathrm{C} & 0 & 0 & 0 & 0 & 0 & 0 & 0 & 0 & 0 & 10 \\ -1.3269 & -0.0744 & 0.0281 & \mathrm{C} & 0 & 0 & 0 & 0 & 0 & 0 & 0 & 0 & 0 & 11\end{array}$

$\begin{array}{lllllllllllllll}2.4835 & 0.5374 & -2.1414 & \circ & 0 & 0 & 0 & 0 & 0 & 0 & 0 & 0 & 0 & 12\end{array}$

$\begin{array}{llllllllllllll}0.4804 & -0.3502 & -2.4758 & 0 & 0 & 0 & 0 & 0 & 0 & 0 & 0 & 0 & 0 & 13\end{array}$

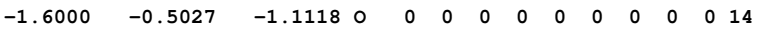

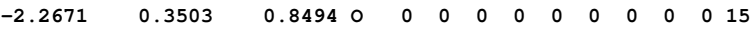

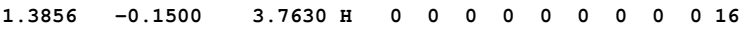

$\begin{array}{lllllllllllll}3.5124 & -0.0973 & 2.4348 \mathrm{H} & 0 & 0 & 0 & 0 & 0 & 0 & 0 & 0 & 0 & 17\end{array}$

$\begin{array}{llllllllllllll}3.4034 & 0.0418 & -0.0395 & \mathrm{H} & 0 & 0 & 0 & 0 & 0 & 0 & 0 & 0 & 0 & 18\end{array}$

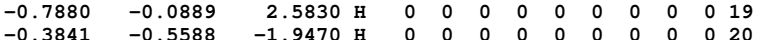

$\begin{array}{lrrrrrrrrrrrrr}-0.3841 & -0.5588 & -1.9470 & \mathrm{H} & 0 & 0 & 0 & 0 & 0 & 0 & 0 & 0 & 0 & 20 \\ -3.2122 & 0.2563 & 0.4252 & \mathrm{H} & 0 & 0 & 0 & 0 & 0 & 0 & 0 & 0 & 0 & 21\end{array}$

$\begin{array}{llllll}2 & 1 & 0 & 0 & 0 & 0 \\ 3 & 1 & 0 & 0 & 0 & 0\end{array}$

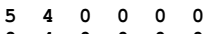

$9 \begin{array}{lllllll}9 & 0 & 0 & 0 & 0\end{array}$

$16 \quad 4 \quad 000000$

$\begin{array}{rlllll}6 & 5 & 0 & 0 & 0 & 0 \\ 17 & 5 & 0 & 0 & 0 & 0\end{array}$

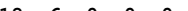

$\begin{array}{lllllll}7 & 6 & 0 & 0 & 0 & 0 \\ 8 & 7 & 0 & 0 & 0 & 0\end{array}$

$\begin{array}{lllllll}8 & 7 & 0 & 0 & 0 & 0\end{array}$

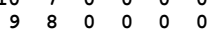

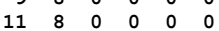

$19 \quad 9 \quad 0 \quad 00100$

$1210 \quad 000000$

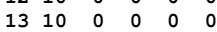

$1411 \quad 00000$

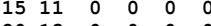

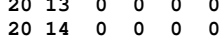

$1521 \quad 0 \quad 0000$

$M$ END

$\$ \$ \$$

phthalimide-anion.sdf

cosMOtherm

$\begin{array}{llllllllllll}18 & 18 & 0 & 0 & 0 & 0 & 0 & 0 & 0 & 0 & 0 & \mathrm{v} 2000\end{array}$

$\begin{array}{llllllllllllll}-4.2768 & 0.0218 & 0.0036 & 0 & 0 & 0 & 0 & 0 & 0 & 0 & 0 & 0 & 0 & 1\end{array}$

$\begin{array}{llllllllllllll}-3.2636 & 0.0020 & -0.0042 & \text { H } & 0 & 0 & 0 & 0 & 0 & 0 & 0 & 0 & 0 & 2\end{array}$

$\begin{array}{llllllllllllll}-4.4963 & 0.9634 & 0.1078 & \text { н } & 0 & 0 & 0 & 0 & 0 & 0 & 0 & 0 & 0 & 3\end{array}$

$\begin{array}{lllllllllllllll}3.1273 & 0.0312 & -0.6872 & \mathrm{C} & 0 & 0 & 0 & 0 & 0 & 0 & 0 & 0 & 0 & 4\end{array}$

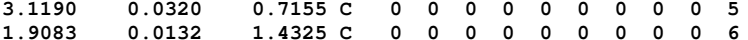

$\begin{array}{llllllllllllll}0.7276 & -0.0038 & 0.6990 & \mathrm{C} & 0 & 0 & 0 & 0 & 0 & 0 & 0 & 0 & 0 & 7\end{array}$

$\begin{array}{lllllllllllllll}0.7357 & -0.0067 & -0.6989 & \mathrm{C} & 0 & 0 & 0 & 0 & 0 & 0 & 0 & 0 & 0 & 8\end{array}$

$\begin{array}{llllllllllllll}1.9251 & 0.0108 & -1.4184 & \mathrm{C} & 0 & 0 & 0 & 0 & 0 & 0 & 0 & 0 & 0 & 9\end{array}$

$\begin{array}{llllllllllllll}1.9251 & 0.0108 & -1.4184 & C & 0 & 0 & 0 & 0 & 0 & 0 & 0 & 0 & 0 & \\ -0.7137 & -0.0273 & -1.1295 & \mathrm{C} & 0 & 0 & 0 & 0 & 0 & 0 & 0 & 0 & 0 & 10\end{array}$

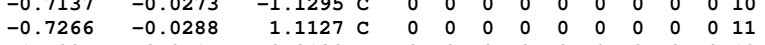

$\begin{array}{llllllllllllll}-1.5225 & -0.0415 & -0.0130 & \mathbf{N} & 0 & 0 & 0 & 0 & 0 & 0 & 0 & 0 & 0 & 11 \\ -1.1335 & -0.0373 & 2.2879 & 0 & 0 & 0 & 0 & 0 & 0 & 0 & 0 & 0 & 0 & 12\end{array}$ 


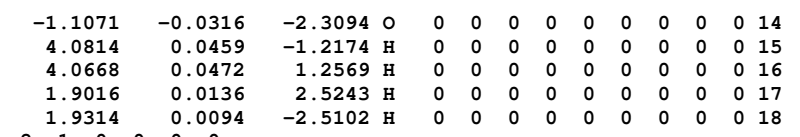




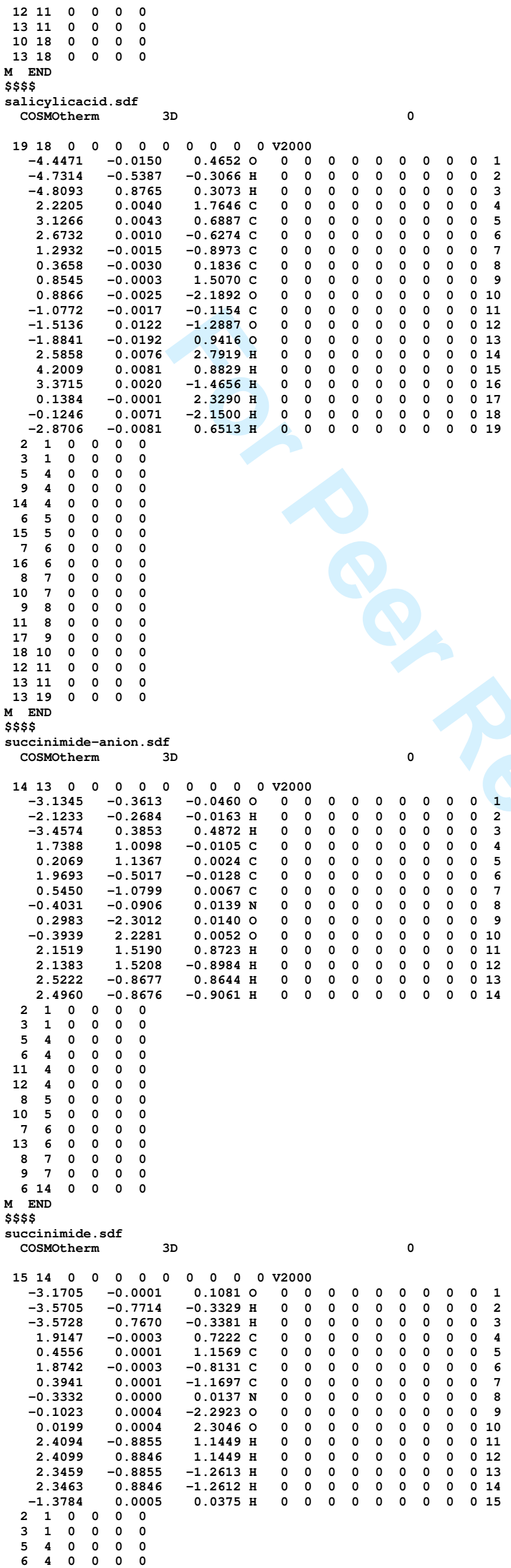




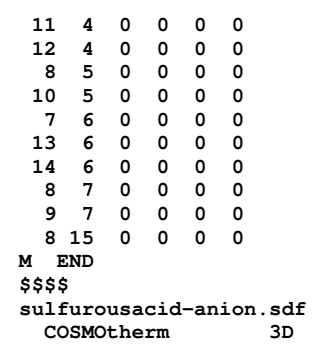


$\begin{array}{rrrrrrrrrrrrrr}0.6815 & -2.1592 & -0.3109 & \mathrm{H} & 0 & 0 & 0 & 0 & 0 & 0 & 0 & 0 & 0 & 9 \\ 2.1727 & 0.8631 & 1.3633 & \mathrm{H} & 0 & 0 & 0 & 0 & 0 & 0 & 0 & 0 & 0 & 10\end{array}$

$\begin{array}{lrrrrrrrrrrrrr}2.1727 & 0.8631 & 1.3633 & \mathrm{H} & 0 & 0 & 0 & 0 & 0 & 0 & 0 & 0 & 0 & 10 \\ 1.3726 & -1.2730 & -1.6973 & \mathrm{H} & 0 & 0 & 0 & 0 & 0 & 0 & 0 & 0 & 0 & 11\end{array}$

$\begin{array}{llllllllllllll}-0.3820 & 1.3111 & -1.3652 & \mathrm{H} & 0 & 0 & 0 & 0 & 0 & 0 & 0 & 0 & 0 & 12\end{array}$

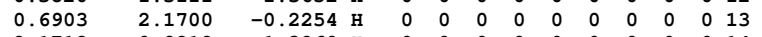

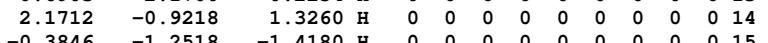

$\begin{array}{cccccccccccccc}-0.3846 & -1.2518 & -1.4180 & \mathrm{H} & 0 & 0 & 0 & 0 & 0 & 0 & 0 & 0 & 0 & 15 \\ 2.9214 & -0.0015 & -0.0049 & \mathrm{H} & 0 & 0 & 0 & 0 & 0 & 0 & 0 & 0 & 0 & 16\end{array}$

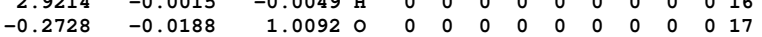

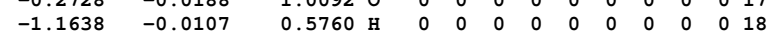

2.1000000

$\begin{array}{llllll}2 & 1 & 0 & 0 & 0 & 0 \\ 3 & 1 & 0 & 0 & 0 & 0\end{array}$

$\begin{array}{llllll}5 & 4 & 0 & 0 & 0 & 0\end{array}$

$\begin{array}{llllll}5 & 4 & 0 & 0 & 0 & 0 \\ 6 & 4 & 0 & 0 & 0 & 0\end{array}$

$7 \begin{array}{llllll}7 & 4 & 0 & 0 & 0 & 0\end{array}$

$17 \quad 4 \quad 0000000$

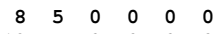

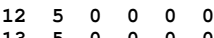

$\begin{array}{cccccccc}13 & 5 & 0 & 0 & 0 & 0\end{array}$

$\begin{array}{rrrrrrrrr}9 & 6 & 0 & 0 & 0 & 0\end{array}$

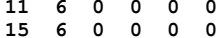

$10 \begin{array}{llllll}15 & 0 & 0 & 0 & 0\end{array}$

$\begin{array}{llllll}14 & 7 & 0 & 0 & 0 & 0 \\ 16 & 7 & 0 & 0 & 0 & 0\end{array}$

$17 \quad 18 \quad 0 \quad 0000$

M END

$\$ \$ \$$

cosmotherm

$\begin{array}{llllllllllll}17 & 16 & 0 & 0 & 0 & 0 & 0 & 0 & 0 & 0 & 0 & \mathrm{v} 2000\end{array}$

$\begin{array}{llllllllllllll}-3.7378 & -0.0331 & -1.2111 & 0 & 0 & 0 & 0 & 0 & 0 & 0 & 0 & 0 & 0 & 1\end{array}$

$\begin{array}{llllllllllllll}-2.7966 & -0.0211 & -0.8297 & \mathrm{H} & 0 & 0 & 0 & 0 & 0 & 0 & 0 & 0 & 0 & 2\end{array}$

$\begin{array}{llllllllllllll}-3.9778 & 0.9064 & -1.2859 & \text { н } & 0 & 0 & 0 & 0 & 0 & 0 & 0 & 0 & 0 & 3\end{array}$

$\begin{array}{llllllllllllll}-0.1453 & -0.0164 & -1.2008 & \mathrm{C} & 0 & 0 & 0 & 0 & 0 & 0 & 0 & 0 & 0 & 4\end{array}$

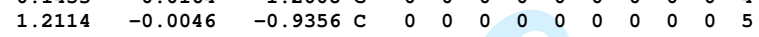

$\begin{array}{llllllllllllll}1.6108 & 0.0075 & 0.4488 & \mathrm{C} & 0 & 0 & 0 & 0 & 0 & 0 & 0 & 0 & 0 & 6\end{array}$

$\begin{array}{llllllllllllll}0.5393 & 0.0056 & 1.3502 & \mathrm{~N} & 0 & 0 & 0 & 0 & 0 & 0 & 0 & 0 & 0 & 7\end{array}$

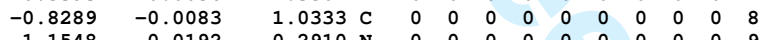

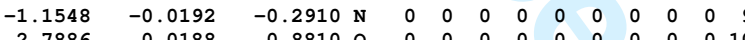

0.88100

$\begin{array}{rrrrrrrrrrrrrr}-1.6703 & -0.0102 & 1.9637 & 0 & 0 & 0 & 0 & 0 & 0 & 0 & 0 & 0 & 0 & 11 \\ 2.2619 & -0.0025 & -2.0100 & \mathrm{C} & 0 & 0 & 0 & 0 & 0 & 0 & 0 & 0 & 0 & 12\end{array}$

$\begin{array}{llllllllllllllll}2.2619 & -0.0025 & -2.0100 & \mathrm{C} & 0 & 0 & 0 & 0 & 0 & 0 & 0 & 0 & 0 & 12 \\ -0.4755 & -0.0249 & -2.2461 & \mathrm{H} & 0 & 0 & 0 & 0 & 0 & 0 & 0 & 0 & 0 & 13\end{array}$

$\begin{array}{lllllllllllllllll}0.4755 & 0.0249 & -2.2461 & H & 0 & 0 & 0 & 0 & 0 & 0 & 0 & 0 & 0 & 12 \\ 0.7771 & 0.0134 & 2.3432 & \text { H } & 0 & 0 & 0 & 0 & 0 & 0 & 0 & 0 & 0 & 13\end{array}$

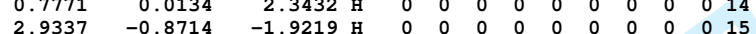

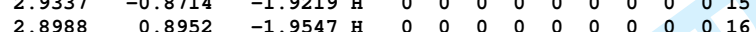

$\begin{array}{llllllllllllll}1.7983 & -0.0295 & -3.0059 & \mathrm{H} & 0 & 0 & 0 & 0 & 0 & 0 & 0 & 0 & 0 & 17\end{array}$

2 1 1 0 00.029

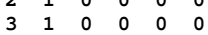

$5 \quad 4 \quad 00000$

$\begin{array}{lllllll}9 & 4 & 0 & 0 & 0 & 0\end{array}$

$\begin{array}{rrrrrr}13 & 4 & 0 & 0 & 0 & 0 \\ 6 & 5 & 0 & 0 & 0 & 0\end{array}$

125000000

$\begin{array}{llllll}10 & 6 & 0 & 0 & 0 & 0\end{array}$

$8 \begin{array}{llllll}8 & 7 & 0 & 0 & 0 & 0\end{array}$

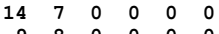

998000000

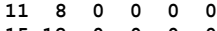

$\begin{array}{llllll}15 & 12 & 0 & 0 & 0 & 0 \\ 16 & 12 & 0 & 0 & 0 & 0\end{array}$

$12 \quad 17 \quad 00000$

$M$ END

$\$ \$ \$$

thymine.sdf

3D

$\begin{array}{llllllllllll}18 & 17 & 0 & 0 & 0 & 0 & 0 & 0 & 0 & 0 & 0 & \mathrm{v} 2000\end{array}$

$\begin{array}{llllllllllllll}-3.7154 & -0.0052 & 1.2303 & \circ & 0 & 0 & 0 & 0 & 0 & 0 & 0 & 0 & 0 & 1 \\ -4.2437 & -0.7725 & 0.9453 & \mathrm{H} & 0 & 0 & 0 & 0 & 0 & 0 & 0 & 0 & 0 & 2\end{array}$

$\begin{array}{rrrrrrrrrrrrrr}-4.2437 & -0.7725 & 0.9453 & \mathrm{H} & 0 & 0 & 0 & 0 & 0 & 0 & 0 & 0 & 0 & 2 \\ -4.2467 & 0.7668 & 0.9637 & \mathrm{H} & 0 & 0 & 0 & 0 & 0 & 0 & 0 & 0 & 0 & 3\end{array}$

$\begin{array}{llllllllllllll}-4.2467 & 0.7668 & 0.9637 & \mathrm{H} & 0 & 0 & 0 & 0 & 0 & 0 & 0 & 0 & 0 & 3 \\ -0.1090 & 0.0032 & 1.2297 & \mathrm{C} & 0 & 0 & 0 & 0 & 0 & 0 & 0 & 0 & 0 & 4\end{array}$

$\begin{array}{llllllllllllll}1.2269 & 0.0013 & 0.9517 & \mathrm{C} & 0 & 0 & 0 & 0 & 0 & 0 & 0 & 0 & 0 & 5\end{array}$

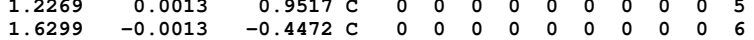

$\begin{array}{llllllllllllll}1.6299 & -0.0013 & -0.4472 & \mathrm{C} & 0 & 0 & 0 & 0 & 0 & 0 & 0 & 0 & 0 & 6 \\ 0.5667 & -0.0016 & -1.3677 & \mathrm{~N} & 0 & 0 & 0 & 0 & 0 & 0 & 0 & 0 & 0 & 7\end{array}$

$\begin{array}{rrrrrrrrrrrrrr}0.5667 & -0.0016 & -1.3677 & \mathrm{~N} & 0 & 0 & 0 & 0 & 0 & 0 & 0 & 0 & 0 & 7 \\ -0.7943 & 0.0018 & -1.0914 & \mathrm{C} & 0 & 0 & 0 & 0 & 0 & 0 & 0 & 0 & 0 & 8\end{array}$

$\begin{array}{rrrrrrrrrrrrrr}-0.7943 & 0.0018 & -1.0914 & \mathrm{C} & 0 & 0 & 0 & 0 & 0 & 0 & 0 & 0 & 0 & 8 \\ -1.0804 & 0.0035 & 0.2592 & \mathbf{N} & 0 & 0 & 0 & 0 & 0 & 0 & 0 & 0 & 0 & 9\end{array}$

$\begin{array}{lllllllllllllr}2.8011 & -0.0039 & -0.8593 & 0 & 0 & 0 & 0 & 0 & 0 & 0 & 0 & 0 & 0 & 10\end{array}$

$\begin{array}{llllllllllllll}-1.6594 & 0.0029 & -1.9749 & 0 & 0 & 0 & 0 & 0 & 0 & 0 & 0 & 0 & 0 & 11\end{array}$

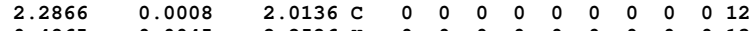

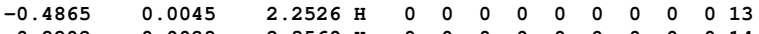

$\begin{array}{llllllllllllll}0.8202 & -0.0028 & -2.3569 & \mathrm{H} & 0 & 0 & 0 & 0 & 0 & 0 & 0 & 0 & 0 & 14\end{array}$

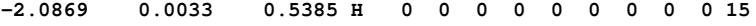

$\begin{array}{lllllllllllllll}2.9358 & 0.8853 & 1.9255 \mathrm{H} & 0 & 0 & 0 & 0 & 0 & 0 & 0 & 0 & 0 & 16\end{array}$

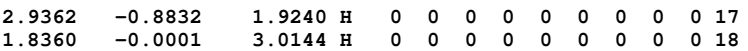

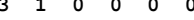

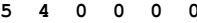

$9 \begin{array}{llllll}5 & 4 & 0 & 0 & 0 & 0\end{array}$

$\begin{array}{lllllll}13 & 4 & 0 & 0 & 0 & 0\end{array}$

$\begin{array}{llllll}6 & 5 & 0 & 0 & 0 & 0\end{array}$

$\begin{array}{llllll}2 & 5 & 0 & 0 & 0 & 0 \\ 7 & 6 & 0 & 0 & 0 & 0\end{array}$

1060600100

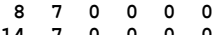

$\begin{array}{cccccccc}9 & 8 & 0 & 0 & 0 & 0\end{array}$

118000000

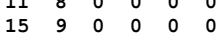

$\begin{array}{lllllll}16 & 12 & 0 & 0 & 0 & 0\end{array}$

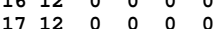




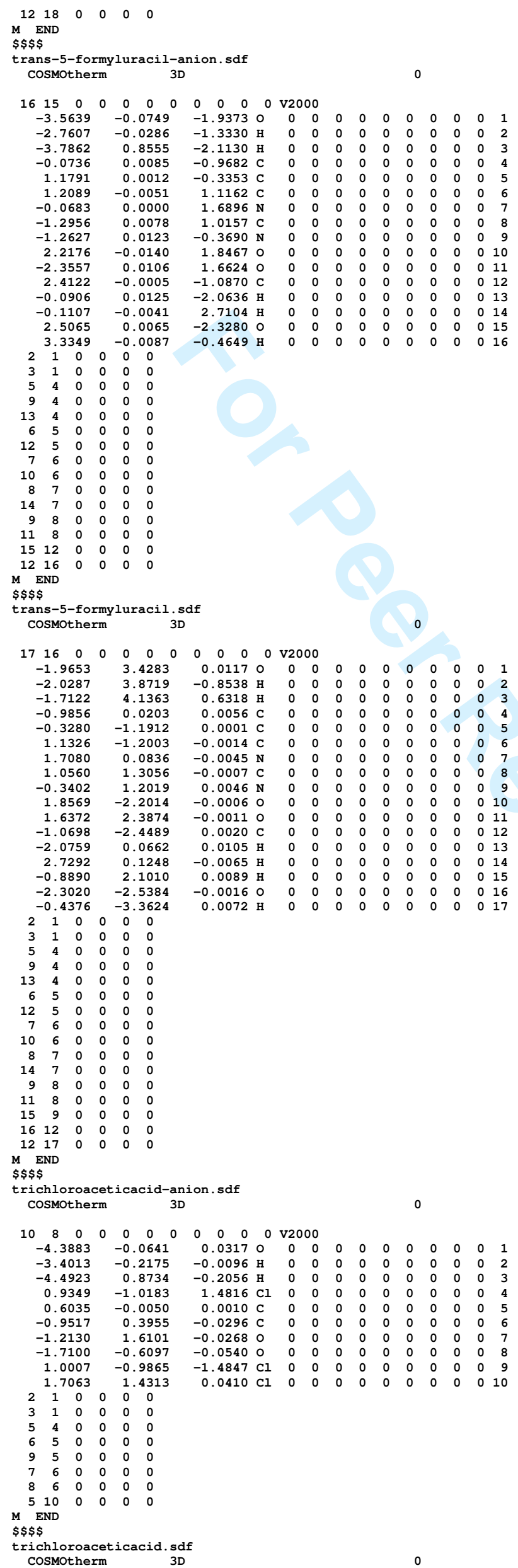



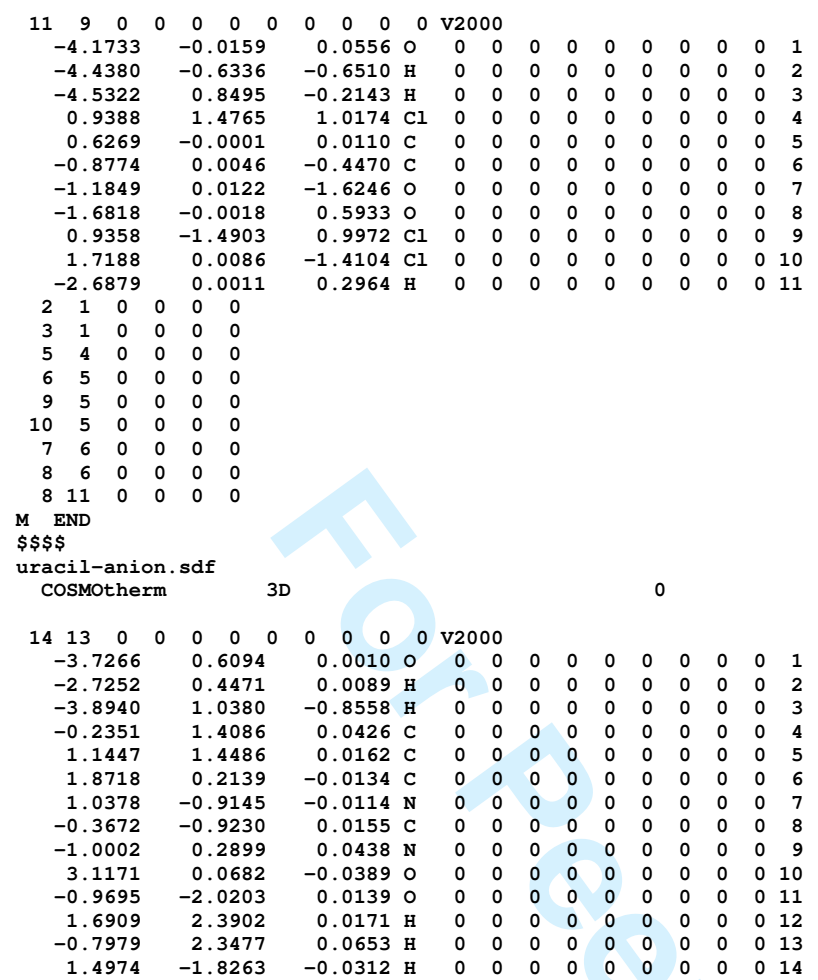

$1.4974-1.8263$

$\begin{array}{llllll}2 & 1 & 0 & 0 & 0 & 0 \\ 3 & 1 & 0 & 0 & 0 & 0\end{array}$

$\begin{array}{llllll}3 & 1 & 0 & 0 & 0 & 0 \\ 5 & 4 & 0 & 0 & 0 & 0\end{array}$

$\begin{array}{llllll}5 & 4 & 0 & 0 & 0 & 0 \\ 9 & 4 & 0 & 0 & 0 & 0\end{array}$

$\begin{array}{rrrrrr}13 & 4 & 0 & 0 & 0 & 0\end{array}$

$\begin{array}{rrrrrr}6 & 5 & 0 & 0 & 0 & 0 \\ 12 & 5 & 0 & 0 & 0 & 0\end{array}$

$\begin{array}{rlllll}12 & 5 & 0 & 0 & 0 & 0 \\ 7 & 6 & 0 & 0 & 0 & 0\end{array}$

$\begin{array}{llllll}10 & 6 & 0 & 0 & 0 & 0\end{array}$

$\begin{array}{llllll}8 & 7 & 0 & 0 & 0 & 0\end{array}$

$\begin{array}{llllll}9 & 8 & 0 & 0 & 0 & 0 \\ 1 & 8 & 0 & 0 & 0 & 0\end{array}$

$\begin{array}{rrrrrr}11 & 8 & 0 & 0 & 0 & 0 \\ 7 & 14 & 0 & 0 & 0 & 0\end{array}$

M END

\$\$\$

uracil.sdf

cosmotherm 3D

$\begin{array}{llllllllllll}15 & 14 & 0 & 0 & 0 & 0 & 0 & 0 & 0 & 0 & 0 & \mathrm{v} 2000\end{array}$

$\begin{array}{rrrrrrrrrrrrrr}-3.6919 & 0.0025 & 0.6561 & 0 & 0 & 0 & 0 & 0 & 0 & 0 & 0 & 0 & 0 & 1 \\ -4.1805 & -0.6598 & 0.1345 & \mathrm{H} & 0 & 0 & 0 & 0 & 0 & 0 & 0 & 0 & 0 & 2 \\ -4.1114 & 0.8510 & 0.4241 & \mathrm{H} & 0 & 0 & 0 & 0 & 0 & 0 & 0 & 0 & 0 & 3 \\ -0.1782 & -0.0119 & 1.4571 & \mathrm{C} & 0 & 0 & 0 & 0 & 0 & 0 & 0 & 0 & 0 & 4 \\ 1.1837 & -0.0032 & 1.4533 & \mathrm{C} & 0 & 0 & 0 & 0 & 0 & 0 & 0 & 0 & 0 & 5 \\ 1.8946 & 0.0037 & 0.1947 & \mathrm{C} & 0 & 0 & 0 & 0 & 0 & 0 & 0 & 0 & 0 & 6 \\ 1.0522 & 0.0039 & -0.9355 & \mathrm{~N} & 0 & 0 & 0 & 0 & 0 & 0 & 0 & 0 & 0 & 7 \\ -0.3362 & -0.0038 & -0.9562 & \mathrm{C} & 0 & 0 & 0 & 0 & 0 & 0 & 0 & 0 & 0 & 8 \\ -0.9140 & -0.0137 & 0.3032 & \mathrm{~N} & 0 & 0 & 0 & 0 & 0 & 0 & 0 & 0 & 0 & 9 \\ 3.1270 & 0.0097 & 0.0451 & 0 & 0 & 0 & 0 & 0 & 0 & 0 & 0 & 0 & 0 & 10 \\ -0.9926 & -0.0027 & -2.0018 & 0 & 0 & 0 & 0 & 0 & 0 & 0 & 0 & 0 & 0 & 11 \\ 1.7506 & -0.0030 & 2.3807 & \mathrm{H} & 0 & 0 & 0 & 0 & 0 & 0 & 0 & 0 & 0 & 12 \\ -0.7604 & -0.0182 & 2.3782 & \mathrm{H} & 0 & 0 & 0 & 0 & 0 & 0 & 0 & 0 & 0 & 13 \\ 1.5100 & 0.0092 & -1.8485 & \mathrm{H} & 0 & 0 & 0 & 0 & 0 & 0 & 0 & 0 & 0 & 14 \\ -1.9588 & -0.0144 & 0.3523 & \mathrm{H} & 0 & 0 & 0 & 0 & 0 & 0 & 0 & 0 & 0 & 15\end{array}$

$\begin{array}{llllll}2 & 1 & 0 & 0 & 0 & 0\end{array}$

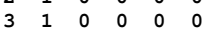

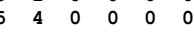

$\begin{array}{llllll}9 & 4 & 0 & 0 & 0 & 0 \\ 3 & 4 & 0 & 0 & 0 & 0\end{array}$

$6 \begin{array}{llllll}6 & 5 & 0 & 0 & 0 & 0\end{array}$

25100000

$\begin{array}{llllll}7 & 6 & 0 & 0 & 0 & 0\end{array}$

$\begin{array}{rrrrrr}10 & 6 & 0 & 0 & 0 & 0 \\ 8 & 7 & 0 & 0 & 0 & 0\end{array}$

$\begin{array}{rrrrrr}8 & 7 & 0 & 0 & 0 & 0 \\ 14 & 7 & 0 & 0 & 0 & 0\end{array}$

$\begin{array}{rrrrrr}14 & 7 & 0 & 0 & 0 & 0 \\ 9 & 8 & 0 & 0 & 0 & 0\end{array}$

$\begin{array}{rrrrrr}9 & 8 & 0 & 0 & 0 & 0 \\ 11 & 8 & 0 & 0 & 0 & 0\end{array}$

$9 \begin{array}{lllll}15 & 0 & 0 & 0 & 0\end{array}$

$M$ END
\$\$\$

S4 c) Di-solvated Acids \& Anions

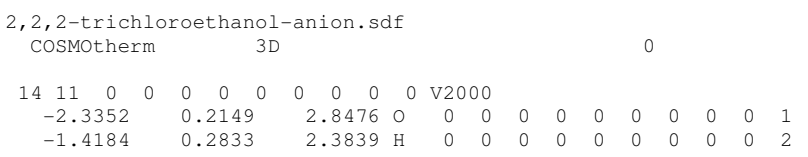




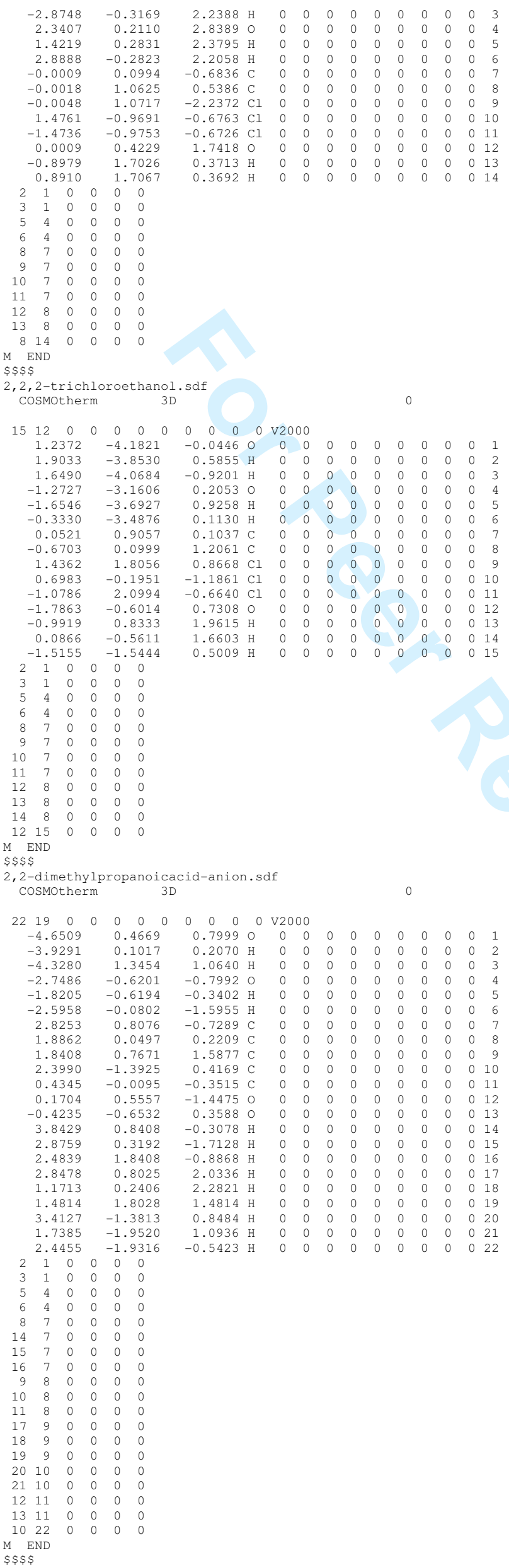




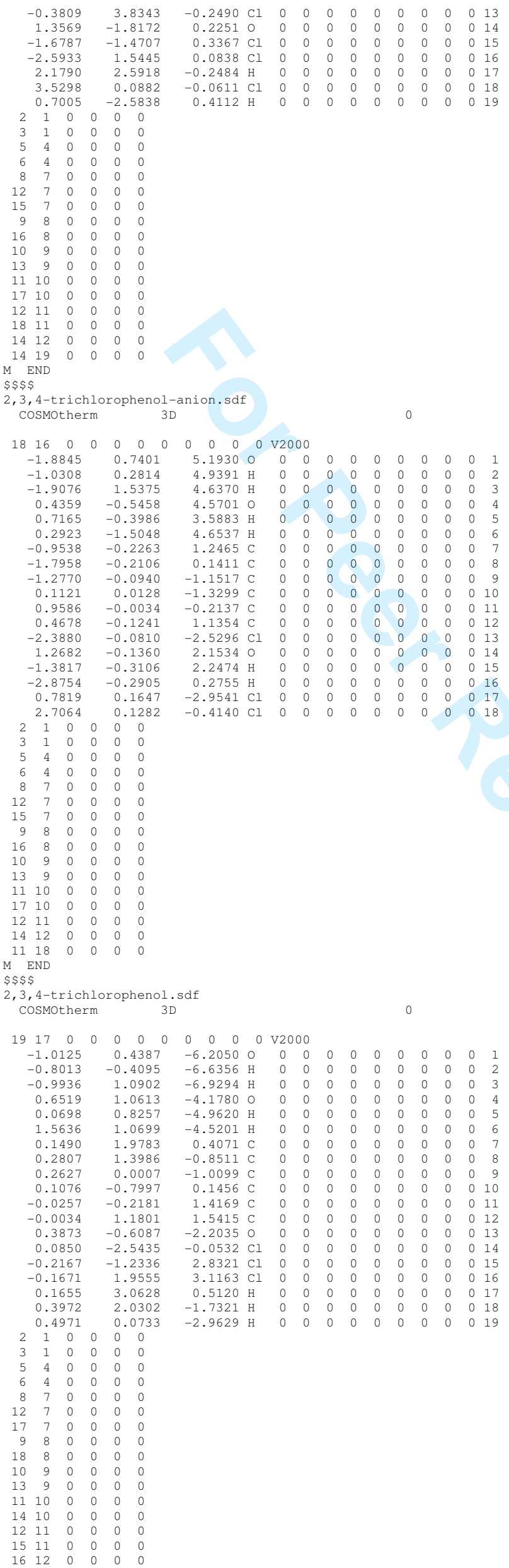




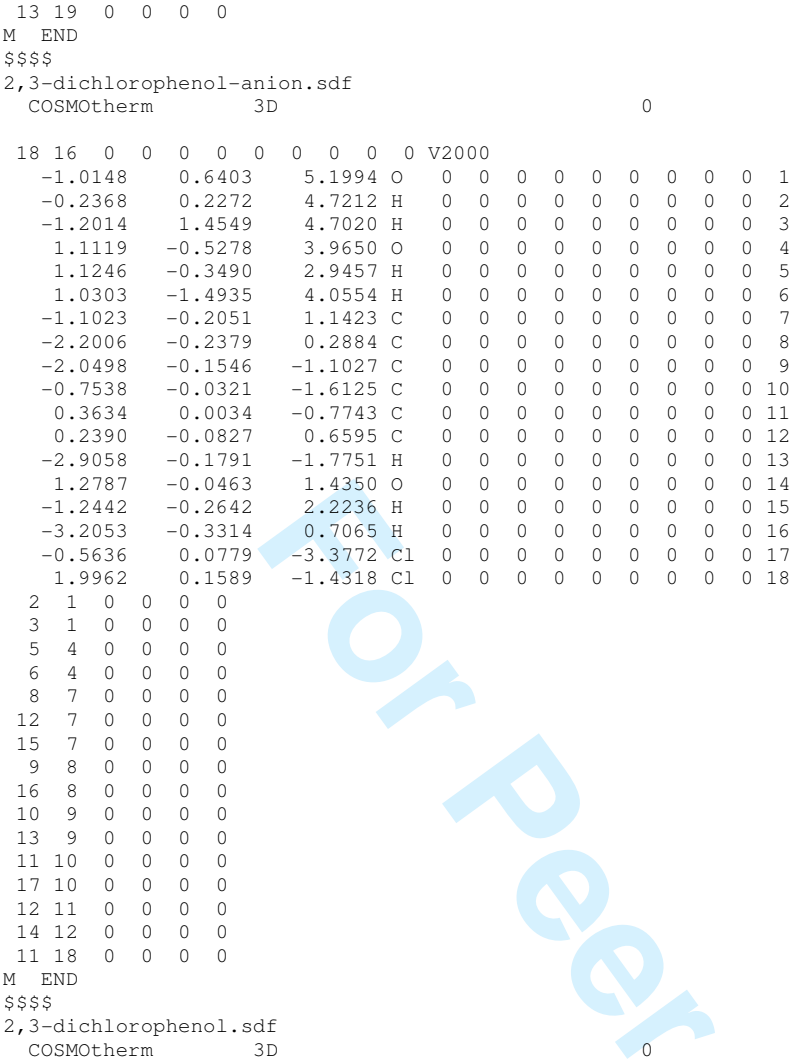

,3-dichlorophenol.sdf

$\begin{array}{llllllllllll}19 & 17 & 0 & 0 & 0 & 0 & 0 & 0 & 0 & 0 & 0 & \text { v2 } 2000\end{array}$

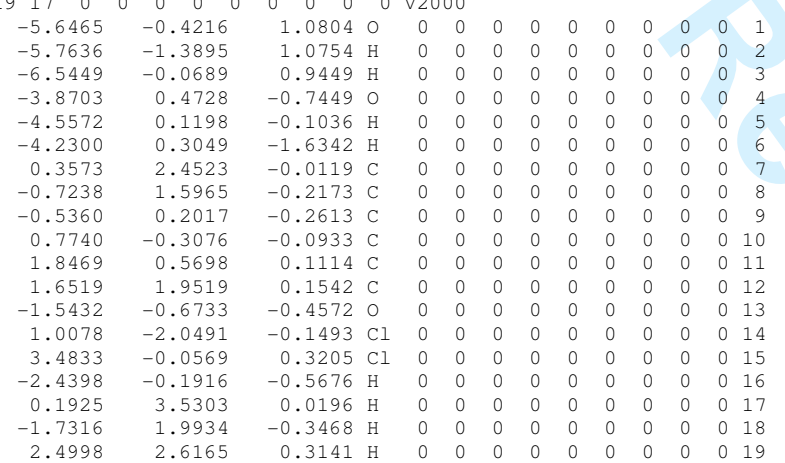

$2.4998 \quad 2.6165$

$\begin{array}{llllll}2 & 1 & 0 & 0 & 0 & 0 \\ 3 & 1 & 0 & 0 & 0 & 0\end{array}$

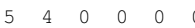

$6 \begin{array}{llllll}6 & 4 & 0 & 0 & 0 & 0\end{array}$

$\begin{array}{lllll}0 & 0 & 0 & 0 & -1 \\ 0 & 0 & 0 & 0\end{array}$

$\begin{array}{cccccccc}1 & 7 & 7 & 0 & 0 & 0 & 0 \\ 17 & 7 & 0 & 0 & 0 & 0\end{array}$

$\begin{array}{lllllll}9 & 8 & 0 & 0 & 0 & 0\end{array}$

$\begin{array}{lllllll}8 & 8 & 0 & 0 & 0 & 0\end{array}$

$\begin{array}{lllll}9 & 0 & 0 & 0\end{array}$

$\begin{array}{lllllll}3 & 9 & 0 & 0 & 0 & 0\end{array}$

$\begin{array}{lllllllll}1 & 10 & 0 & 0 & 0 & 0 & 0\end{array}$

$\begin{array}{lllllll}11 & 10 & 0 & 0 & 0 & 0 \\ 12 & 11 & 0 & 0 & 0 & 0\end{array}$

$\begin{array}{llllll}5 & 11 & 0 & 0 & 0 & 0\end{array}$

$\begin{array}{llllll}6 & 13 & 0 & 0 & 0 & 0\end{array}$

1219

$M$ END

2,4,5-trichlorophenol-anion.sdf

Cosmotherm

$\begin{array}{llllllllllll}18 & 16 & 0 & 0 & 0 & 0 & 0 & 0 & 0 & 0 & 0 & \text { V } 2000\end{array}$

$\begin{array}{rrrrllllllllll}2.4166 & -4.5640 & 1.0951 & 0 & 0 & 0 & 0 & 0 & 0 & 0 & 0 & 0 & 0 & 1 \\ 1.6990 & -4.3802 & 0.4229 & \mathrm{H} & 0 & 0 & 0 & 0 & 0 & 0 & 0 & 0 & 0 & 2 \\ 2.1216 & -5.3726 & 1.5479 & \mathrm{H} & 0 & 0 & 0 & 0 & 0 & 0 & 0 & 0 & 0 & 3 \\ 0.4649 & -4.1138 & -0.7541 & 0 & 0 & 0 & 0 & 0 & 0 & 0 & 0 & 0 & 0 & 4 \\ -0.1446 & -3.3026 & -0.5815 & \mathrm{H} & 0 & 0 & 0 & 0 & 0 & 0 & 0 & 0 & 0 & 5 \\ 0.8346 & -3.9791 & -1.6438 \mathrm{H} & 0 & 0 & 0 & 0 & 0 & 0 & 0 & 0 & 0 & 6 \\ 0.5846 & -0.5454 & -0.2584 \mathrm{C} & 0 & 0 & 0 & 0 & 0 & 0 & 0 & 0 & 0 & 7 \\ 0.9947 & 0.7722 & -0.1430 \mathrm{C} & 0 & 0 & 0 & 0 & 0 & 0 & 0 & 0 & 0 & 8 \\ 0.0662 & 1.8081 & 0.0613 \mathrm{C} & 0 & 0 & 0 & 0 & 0 & 0 & 0 & 0 & 0 & 9 \\ -1.2918 & 1.4794 & 0.1477 & \mathrm{C} & 0 & 0 & 0 & 0 & 0 & 0 & 0 & 0 & 0 & 10 \\ -1.6980 & 0.1560 & 0.0323 & \mathrm{C} & 0 & 0 & 0 & 0 & 0 & 0 & 0 & 0 & 0 & 11 \\ -0.7916 & -0.9374 & -0.1784 & \mathrm{C} & 0 & 0 & 0 & 0 & 0 & 0 & 0 & 0 & 0 & 12 \\ 0.5509 & 3.5012 & 0.2107 & \mathrm{Cl} & 0 & 0 & 0 & 0 & 0 & 0 & 0 & 0 & 0 & 13 \\ -1.1838 & -2.1662 & -0.2843 & 0 & 0 & 0 & 0 & 0 & 0 & 0 & 0 & 0 & 0 & 14 \\ 1.3291 & -1.3263 & -0.4160 \mathrm{H} & 0 & 0 & 0 & 0 & 0 & 0 & 0 & 0 & 0 & 15 \\ 2.7278 & 1.1378 & -0.2592 \mathrm{Cl} & 0 & 0 & 0 & 0 & 0 & 0 & 0 & 0 & 0 & 16\end{array}$




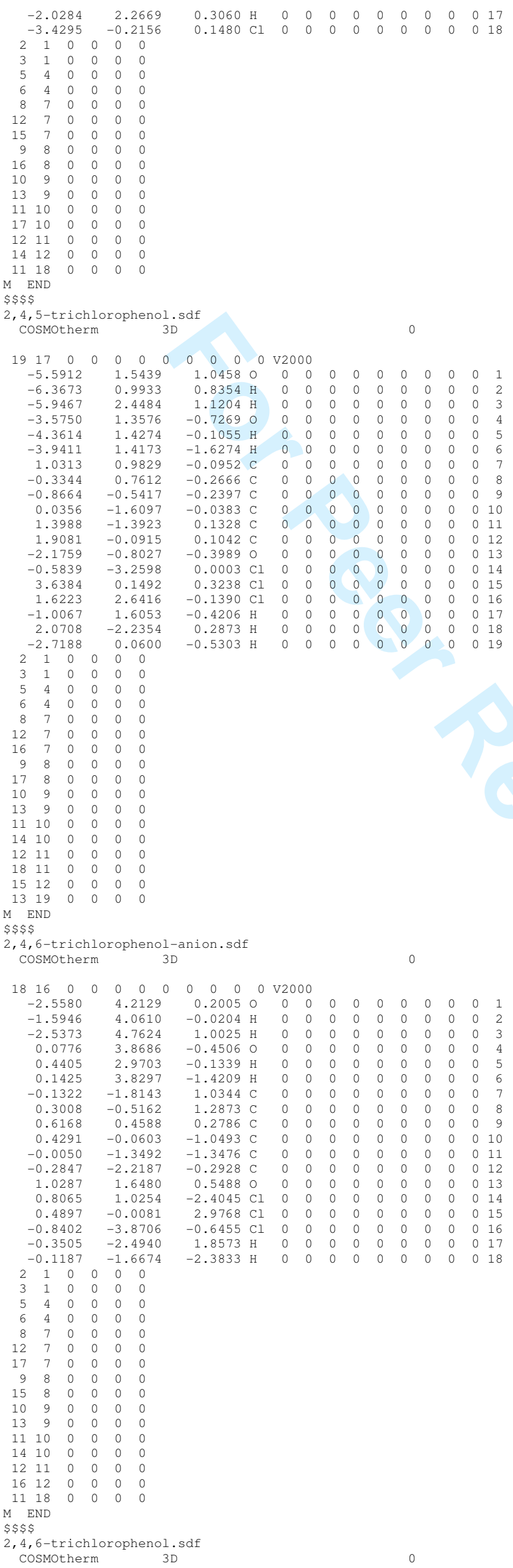




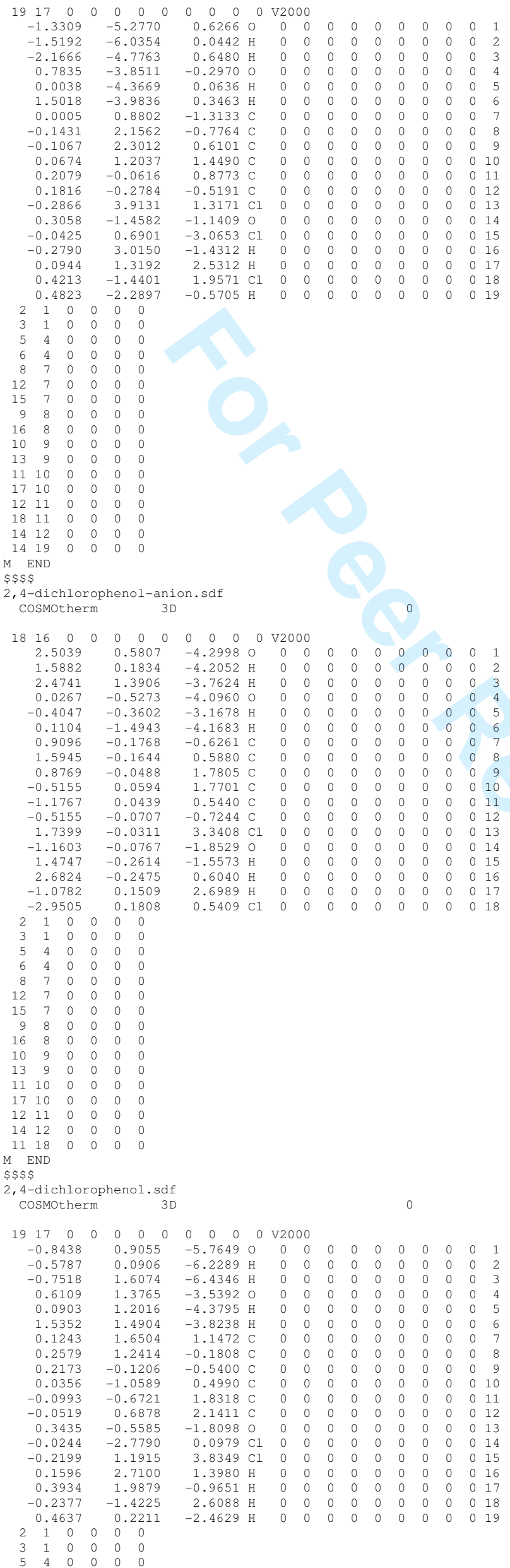


$\begin{array}{rrrrrr}6 & 4 & 0 & 0 & 0 & 0 \\ 8 & 7 & 0 & 0 & 0 & 0 \\ 12 & 7 & 0 & 0 & 0 & 0 \\ 16 & 7 & 0 & 0 & 0 & 0 \\ 9 & 8 & 0 & 0 & 0 & 0 \\ 17 & 8 & 0 & 0 & 0 & 0 \\ 10 & 9 & 0 & 0 & 0 & 0 \\ 13 & 9 & 0 & 0 & 0 & 0 \\ 11 & 10 & 0 & 0 & 0 & 0 \\ 14 & 10 & 0 & 0 & 0 & 0 \\ 12 & 11 & 0 & 0 & 0 & 0 \\ 18 & 11 & 0 & 0 & 0 & 0 \\ 15 & 12 & 0 & 0 & 0 & 0 \\ 13 & 19 & 0 & 0 & 0 & 0\end{array}$ END

2, 4-dihydroxybenzoicacid-anion.sdf

cosmotherm

$\begin{array}{llllllllllll}22 & 21 & 0 & 0 & 0 & 0 & 0 & 0 & 0 & 0 & 0 & \mathrm{~V} 2000\end{array}$

$\begin{array}{llllllllllllll}0.9480 & 5.2316 & -0.0160 & 0 & 0 & 0 & 0 & 0 & 0 & 0 & 0 & 0 & 0 & 1\end{array}$

$\begin{array}{llllllllllllll}1.5959 & 5.1705 & -0.7412 & \mathrm{H} & 0 & 0 & 0 & 0 & 0 & 0 & 0 & 0 & 0 & 2 \\ 1.4874 & 5.2881 & 0.7938 & \mathrm{H} & 0 & 0 & 0 & 0 & 0 & 0 & 0 & 0 & 0 & 3\end{array}$

$\begin{array}{rrrrrrrrrrrrrr}1.4874 & 5.2881 & 0.7938 & \mathrm{H} & 0 & 0 & 0 & 0 & 0 & 0 & 0 & 0 & 0 & 3 \\ -0.1190 & -5.5108 & -0.0178 & 0 & 0 & 0 & 0 & 0 & 0 & 0 & 0 & 0 & 0 & 4\end{array}$

$\begin{array}{llllllllllllll}-0.3823 & -4.5397 & -0.0105 & \mathrm{H} & 0 & 0 & 0 & 0 & 0 & 0 & 0 & 0 & 0 & 5\end{array}$

$\begin{array}{rrrrrrrrrrrrrr}0.8527 & -5.4815 & 0.0126 & \mathrm{H} & 0 & 0 & 0 & 0 & 0 & 0 & 0 & 0 & 0 & 6 \\ -1.8457 & 1.2280 & 0.0032 & \mathrm{C} & 0 & 0 & 0 & 0 & 0 & 0 & 0 & 0 & 0 & 7\end{array}$

$\begin{array}{rrrrrrrrrrrrrr}-1.8457 & 1.2280 & 0.0032 & \mathrm{C} & 0 & 0 & 0 & 0 & 0 & 0 & 0 & 0 & 0 & 7 \\ -1.5704 & -0.1342 & 0.0053 & \mathrm{C} & 0 & 0 & 0 & 0 & 0 & 0 & 0 & 0 & 0 & 8\end{array}$

$\begin{array}{llllllllllllll}-0.2539 & -0.6305 & 0.0046 & \mathrm{C} & 0 & 0 & 0 & 0 & 0 & 0 & 0 & 0 & 0 & 9\end{array}$

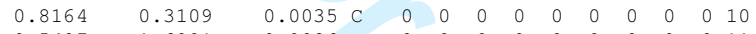

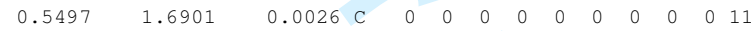

$\begin{array}{llllllllllllll}-0.7747 & 2.1491 & 0.0014 & \mathrm{C} & 0 & 0 & 0 & 0 & 0 & 0 & 0 & 0 & 0 & 12\end{array}$

$\begin{array}{llllllllllllll}0.0333 & -2.0978 & 0.0048 & \mathrm{C} & 0 & 0 & 0 & 0 & 0 & 0 & 0 & 0 & 0 & 13\end{array}$

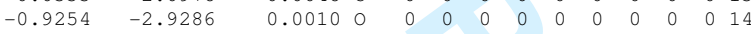

$\begin{array}{llllllllllllll}1.2805 & -2.4495 & 0.0082 & 0 & 0 & 0 & 0 & 0 & 0 & 0 & 0 & 0 & 0 & 15\end{array}$

$\begin{array}{rrrrrrrrrrrrrr}1.1013 & -0.1130 & 0.0043 & 0 & 0 & 0 & 0 & 0 & 0 & 0 & 0 & 0 & 0 & 16 \\ -1.0878 & 3.4749 & -0.0028 & 0 & 0 & 0 & 0 & 0 & 0 & 0 & 0 & 0 & 0 & 17\end{array}$

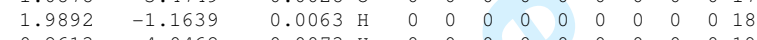

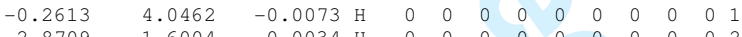

$\begin{array}{llllllllllllll}-2.8709 & 1.6004 & 0.0034 & \mathrm{H} & 0 & 0 & 0 & 0 & 0 & 0 & 0 & 0 & 0 & 20 \\ -2.3891 & -0.8562 & 0.0068 & \mathrm{H} & 0 & 0 & 0 & 0 & 0 & 0 & 0 & 0 & 0 & 21\end{array}$

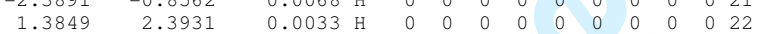

$\begin{array}{llllll}2 & 1 & 0 & 0 & 0 & 0\end{array}$

$\begin{array}{lllllll}3 & 1 & 0 & 0 & 0 & 0\end{array}$

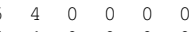

$8 \begin{array}{llllll}6 & 7 & 0 & 0 & 0 & 0\end{array}$

$\begin{array}{lllllll}12 & 7 & 0 & 0 & 0 & 0\end{array}$

$\begin{array}{rrrrrr}20 & 7 & 0 & 0 & 0 & 0 \\ 9 & 8 & 0 & 0 & 0 & 0\end{array}$

$\begin{array}{lllllll}21 & 8 & 0 & 0 & 0 & 0\end{array}$

$\begin{array}{llllll}10 & 9 & 0 & 0 & 0\end{array}$

$\begin{array}{rrrrrr}13 & 9 & 0 & 0 & 0 \\ 11 & 10 & 0 & 0 & 0\end{array}$

$\begin{array}{llllll}11 & 10 & 0 & 0 & 0 & 0\end{array}$

121100000

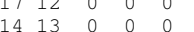

$\begin{array}{lllllll}15 & 13 & 0 & 0 & 0 & 0\end{array}$

$\begin{array}{lllllll}18 & 15 & 0 & 0 & 0 & 0 \\ 18 & 16 & 0 & 0 & 0 & 0\end{array}$

$\begin{array}{lllllll}19 & 17 & 0 & 0 & 0 & 0\end{array}$

$\begin{array}{lllllll}11 & 22 & 0 & 0 & 0 & 0\end{array}$

$\$ \$ \$ \$$

2,4-dihydroxybenzoicacid.sdf

cosMotherm

$\begin{array}{llllllllllll}23 & 21 & 0 & 0 & 0 & 0 & 0 & 0 & 0 & 0 & 0 & \mathrm{v} 2000\end{array}$

$\begin{array}{lllll}-0.9255 & 0.0060 & -5.1222 & 0 & 0\end{array}$

$\begin{array}{lll}-0.9255 & 0.0060 & -5.1222 \\ -0.9328 & -0.7807 & -5.6979 \\ -0.9017 & 0.7623 & -5.7370\end{array}$

$\begin{array}{llllllllll}0 & 0 & 0 & 0 & 0 & 0 & 0 & 0 & 0 & 1 \\ 0 & 0 & 0 & 0 & 0 & 0 & 0 & 0 & 0 & 2\end{array}$

$\begin{array}{llllllllllll}5.7370 & \mathrm{H} & 0 & 0 & 0 & 0 & 0 & 0 & 0 & 0 & 0 & 3\end{array}$

$\begin{array}{lllllllllllll}-0.0101 & 5.4344 & 0 & 0 & 0 & 0 & 0 & 0 & 0 & 0 & 0 & 0 & 4\end{array}$

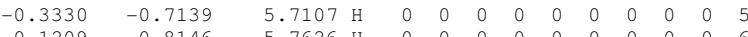

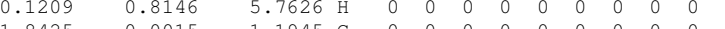

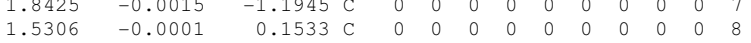

$\begin{array}{llllllllllllll}0.1911 & 0.0011 & 0.6057 & C & 0 & 0 & 0 & 0 & 0 & 0 & 0 & 0 & 0 & 9\end{array}$

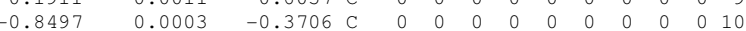

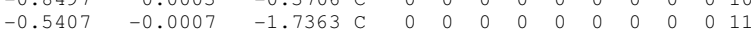

$\begin{array}{lllllllllllllll}0.7987 & -0.0022 & -2.1519 & \mathrm{C} & 0 & 0 & 0 & 0 & 0 & 0 & 0 & 0 & 0 & 12\end{array}$

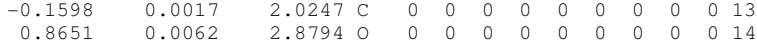

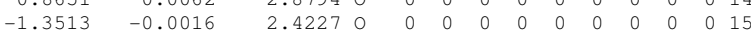

$\begin{array}{llllllllllllll}-2.1555 & 0.0005 & -0.0081 & 0 & 0 & 0 & 0 & 0 & 0 & 0 & 0 & 0 & 0 & 16\end{array}$

$\begin{array}{llllllllllllll}1.1545 & -0.0050 & -3.4584 & 0 & 0 & 0 & 0 & 0 & 0 & 0 & 0 & 0 & 0 & 0\end{array}$

$\begin{array}{llllllllllllll}0.5290 & 0.0039 & 3.8476 & \mathrm{H} & 0 & 0 & 0 & 0 & 0 & 0 & 0 & 0 & 0 & 18\end{array}$

$\begin{array}{llllllllllllll}-2.1458 & -0.0001 & 1.0063 & \mathrm{H} & 0 & 0 & 0 & 0 & 0 & 0 & 0 & 0 & 0 & 19\end{array}$

$\begin{array}{llllllllllllll}0.3450 & -0.0006 & -4.0640 & \mathrm{H} & 0 & 0 & 0 & 0 & 0 & 0 & 0 & 0 & 0 & 20\end{array}$

$\begin{array}{llllllllllll}0 & 0 \\ .8348 & \mathrm{H} & 0 & 0 & 0 & 0 & 0 & 0 & 0 & 0 & 0 & 21\end{array}$

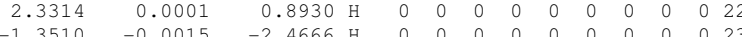

$.3510-0.0015$

$\begin{array}{ccccccc}1 & 0 & 0 & 0 & 0 & 0 \\ 3 & 1 & 0 & 0 & 0 & 0 & 0\end{array}$

$\begin{array}{llllll}5 & 4 & 0 & 0 & 0 & 0\end{array}$

$\begin{array}{lllll}4 & 0 & 0 & 0 & 0 \\ 7 & 0 & 0 & 0 & 0\end{array}$

$\begin{array}{rrrrrr}8 & 7 & 0 & 0 & 0 & 0 \\ 2 & 7 & 0 & 0 & 0 & 0\end{array}$

$\begin{array}{rrrrrrrr}21 & 7 & 0 & 0 & 0 & 0 \\ 9 & 8 & 0 & 0 & 0 & 0\end{array}$

$\begin{array}{llllll}22 & 8 & 0 & 0 & 0\end{array}$

$\begin{array}{llllll}10 & 9 & 0 & 0 & 0 & 0\end{array}$

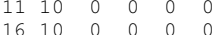


$\begin{array}{llllll}12 & 11 & 0 & 0 & 0 & 0\end{array}$

$\begin{array}{llllll}17 & 12 & 0 & 0 & 0 & 0\end{array}$

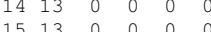

$\begin{array}{llllll}18 & 14 & 0 & 0 & 0 & 0\end{array}$

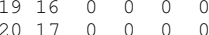

$\begin{array}{llllll}11 & 23 & 0 & 0 & 0 & 0\end{array}$

M END

2,5-dichlorophenol-anion.sd
Cosmotherm

$\begin{array}{llllllllllll}18 & 16 & 0 & 0 & 0 & 0 & 0 & 0 & 0 & 0 & 0 & \mathrm{v} 2000\end{array}$

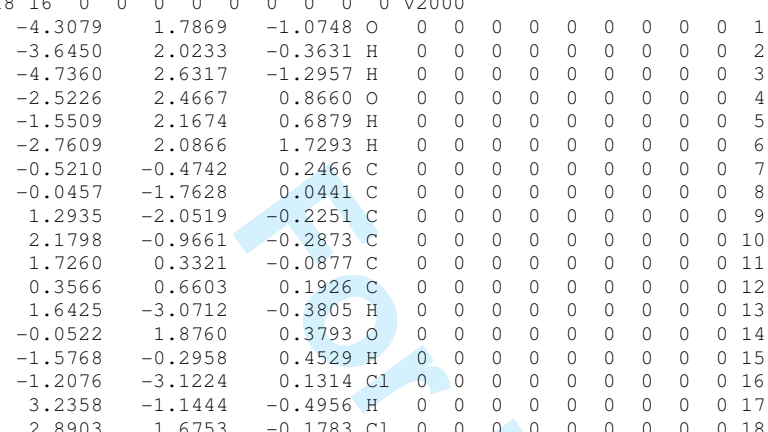

$\begin{array}{lllll}1 & 0 & 0 & 0 & 0\end{array}$

$\begin{array}{llllll}3 & 1 & 0 & 0 & 0 & 0\end{array}$

$\begin{array}{lllll}4 & 0 & 0 & 0 & 0\end{array}$

$\begin{array}{lllll}4 & 0 & 0 & 0 & 0\end{array}$

$\begin{array}{lllllll}2 & 7 & 0 & 0 & 0 & 0\end{array}$

$\begin{array}{lllll}7 & 0 & 0 & 0 & 0\end{array}$

$\begin{array}{llllll}9 & 8 & 0 & 0 & 0 & 0 \\ 16 & 8 & 0 & 0 & 0 & 0\end{array}$

$\begin{array}{lllllll}0 & 9 & 0 & 0 & 0 & 0\end{array}$

$\begin{array}{lllll}9 & 0 & 0 & 0\end{array}$

$\begin{array}{llllll}1 & 10 & 0 & 0 & 0 & 0\end{array}$

$17 \begin{array}{llllll}10 & 0 & 0 & 0 & 0\end{array}$

$1412 \quad 00000$

$\begin{array}{llllll}18 & 0 & 0 & 0 & 0\end{array}$

END

2,5-dichlorophenol.sdf

$9 \begin{array}{lllllllllll}17 & 0 & 0 & 0 & 0 & 0 & 0 & 0 & 0 & 0 & \mathrm{~V} 2000\end{array}$

$\begin{array}{llllllllllllll}-5.0823 & 1.1694 & 0.9141 & 0 & 0 & 0 & 0 & 0 & 0 & 0 & 0 & 0 & 0 & 1 \\ -5.8208 & 0.5750 & 0.6890 & \mathrm{H} & 0 & 0 & 0 & 0 & 0 & 0 & 0 & 0 & 0 & 2\end{array}$

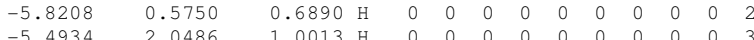

$\begin{array}{rrrrrrrrrrrrrr}-5.4934 & 2.0486 & 1.0013 & \mathrm{H} & 0 & 0 & 0 & 0 & 0 & 0 & 0 & 0 & 0 & 3 \\ -3.0461 & 1.1240 & -0.8480 & 0 & 0 & 0 & 0 & 0 & 0 & 0 & 0 & 0 & 0 & 4\end{array}$

$\begin{array}{llllllllllll}-3.8375 & 1.1402 & -0.2307 \mathrm{H} & 0 & 0 & 0 & 0 & 0 & 0 & 0 & 0 & 0\end{array}$

$1.1165-1.7503 \mathrm{H} \quad 0 \quad 0$

$\begin{array}{lllllllllllll}1.5659 & 1.1285 & 0.0426 & \mathrm{C} & 0 & 0 & 0 & 0 & 0 & 0 & 0 & 0 & 0\end{array}$

$\begin{array}{llllllllllllll}0.2321 & 0.8179 & -0.2079 & \mathrm{C} & 0 & 0 & 0 & 0 & 0 & 0 & 0 & 0 & 0 & 8\end{array}$

$\begin{array}{llllllllllllll}-0.1837 & -0.5294 & -0.2080 & \mathrm{C} & 0 & 0 & 0 & 0 & 0 & 0 & 0 & 0 & 0 & 9\end{array}$

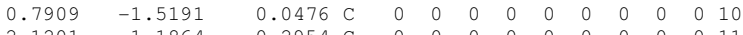

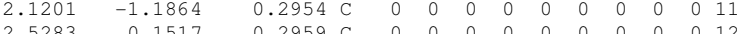

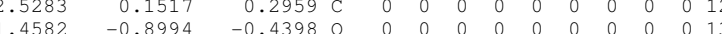

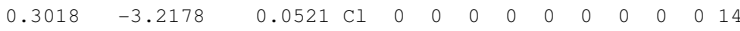

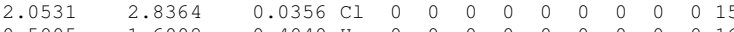

$\begin{array}{llllllllllllll}-0.5005 & 1.6008 & -0.4040 & \mathrm{H} & 0 & 0 & 0 & 0 & 0 & 0 & 0 & 0 & 0 & 16\end{array}$

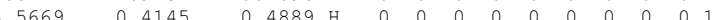

$\begin{array}{llllllllllllll}-2.0611 & -0.0864 & -0.6021 \mathrm{H} & 0 & 0 & 0 & 0 & 0 & 0 & 0 & 0 & 0 & 18\end{array}$

$-2.0611-0.0864$

$\begin{array}{llllllll}3 & 0 & 0 & 0 & 0\end{array}$

$\begin{array}{lllll}4 & 0 & 0 & 0 & 0\end{array}$

$\begin{array}{llllll}4 & 0 & 0 & 0 & 0\end{array}$

$\begin{array}{lllllllll}7 & 7 & 0 & 0 & 0 & 0\end{array}$

$9 \begin{array}{lllllll}9 & 8 & 0 & 0 & 0 & 0\end{array}$

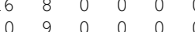

$\begin{array}{llllll}3 & 9 & 0 & 0 & 0\end{array}$

$\begin{array}{llllll}11 & 10 & 0 & 0 & 0 & 0\end{array}$

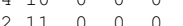

11 r

$\begin{array}{llllll}13 & 19 & 0 & 0 & 0 & 0\end{array}$

(S\$\$

2,6-dichlorophenol-anion.sdf

$\begin{array}{llllllllllll}8 & 16 & 0 & 0 & 0 & 0 & 0 & 0 & 0 & 0 & 0 & \mathrm{v} 2000\end{array}$

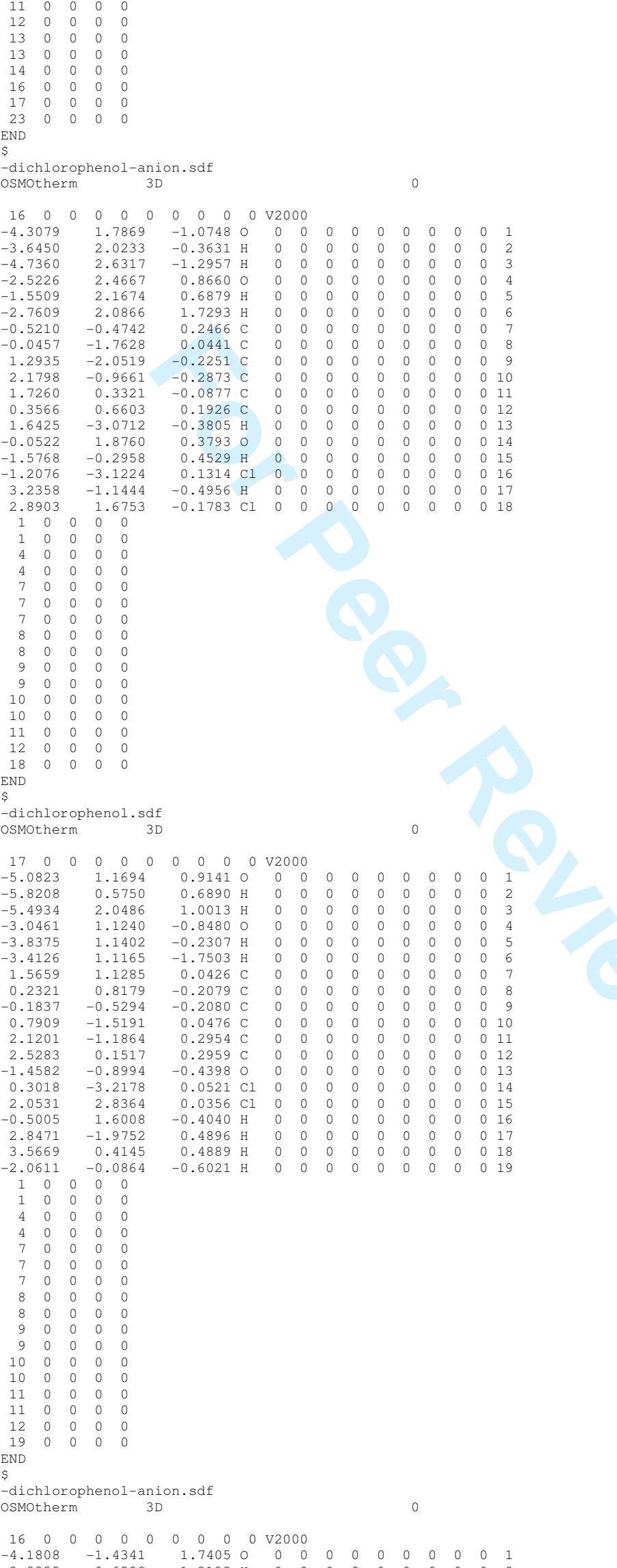

$\begin{array}{llllllllllllll}-4.1808 & -1.4341 & 1.7405 & 0 & 0 & 0 & 0 & 0 & 0 & 0 & 0 & 0 & 0 & 1 \\ -3.8355 & -0.6528 & 1.2193 & \mathrm{H} & 0 & 0 & 0 & 0 & 0 & 0 & 0 & 0 & 0 & 2\end{array}$

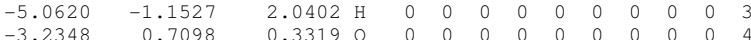

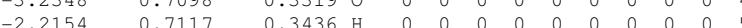

$\begin{array}{llllllllllllll}-3.4712 & 0.6069 & -0.6059 \mathrm{H} & 0 & 0 & 0 & 0 & 0 & 0 & 0 & 0 & 0 & 6\end{array}$

$\begin{array}{lllllllllllllll}2.7724 & -0.5524 & -0.6555 & \mathrm{C} & 0 & 0 & 0 & 0 & 0 & 0 & 0 & 0 & 0 & 7\end{array}$

$\begin{array}{lllllllllllll}1.6121 & -0.0206 & 0.6950 & \mathrm{C} & 0 & 0 & 0 & 0 & 0 & 0 & 0 & 0 & 0\end{array}$

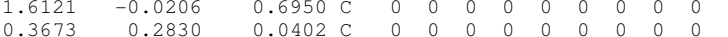




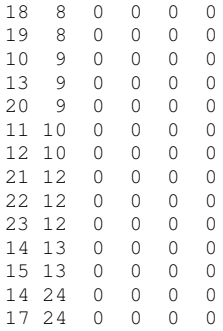

$\begin{array}{llllll}14 & 24 & 0 & 0 & 0 & 0 \\ 17 & 24 & 0 & 0 & 0 & 0\end{array}$ M END

$\$ \$ \$ \$$

-acetylbutanedioicacid.sdf

PD

$\begin{array}{llllllllllll}25 & 22 & 0 & 0 & 0 & 0 & 0 & 0 & 0 & 0 & 0 & \mathrm{~V} 2000\end{array}$

$\begin{array}{llllllllllllll}-0.5714 & 3.0738 & -2.0986 & 0 & 0 & 0 & 0 & 0 & 0 & 0 & 0 & 0 & 0 & 1\end{array}$

$\begin{array}{llllllllllllll}-0.4355 & 2.2051 & -1.6384 & \mathrm{H} & 0 & 0 & 0 & 0 & 0 & 0 & 0 & 0 & 0 & 2\end{array}$

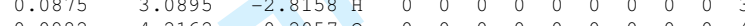

$\begin{array}{llllllllllllll}0.0982 & 4.2162 & 0.2057 & 0 & 0 & 0 & 0 & 0 & 0 & 0 & 0 & 0 & 0 & 4\end{array}$

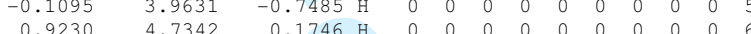

$\begin{array}{llllllllllllll}0.9230 & 4.7342 & 0.1746 \mathrm{H} & 0 & 0 & 0 & 0 & 0 & 0 & 0 & 0 & 0 & \\ 0.1382 & 0.9189 & 0.7781 & \mathrm{C} & 0 & 0 & 0 & 0 & 0 & 0 & 0 & 0 & 0\end{array}$

$\begin{array}{lllllllllllll}0.2772 & -0.3543 & 1.5908 & \mathrm{C} & 0 & 0 & 0 & 0 & 0 & 0 & 0 & 0 & 0\end{array}$

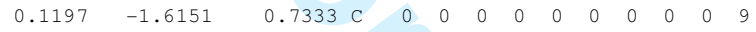

$\begin{array}{llllllllllllll}-1.2710 & -1.8039 & 0.1162 & \mathrm{C} & 0 & 0 & 0 & 0 & 0 & 0 & 0 & 0 & 0 & 10\end{array}$

$\begin{array}{llllllllllllll}-1.4089 & -2.3967 & -0.9639 & 0 & 0 & 0 & 0 & 0 & 0 & 0 & 0 & 0 & 0 & 11\end{array}$

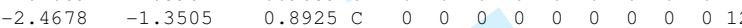

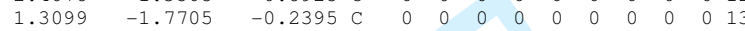

$\begin{array}{llllllllllllll}1.0862 & -2.4419 & -1.3729 & 0 & 0 & 0 & 0 & 0 & 0 & 0 & 0 & 0 & 0 & 14\end{array}$

$\begin{array}{llllllllllllll}2.4222 & -1.3423 & 0.0347 & 0 & 0 & 0 & 0 & 0 & 0 & 0 & 0 & 0 & 0 & 15\end{array}$

$\begin{array}{rrrrrrrrrrrrrr}0.4233 & 2.0097 & 1.4676 & 0 & 0 & 0 & 0 & 0 & 0 & 0 & 0 & 0 & 0 & 16 \\ -0.2221 & 0.8985 & -0.4078 & 0 & 0 & 0 & 0 & 0 & 0 & 0 & 0 & 0 & 0 & 17\end{array}$

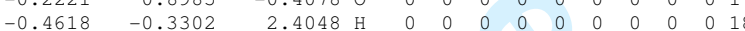

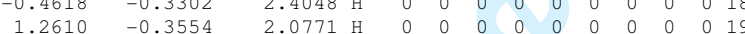

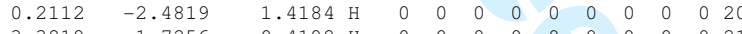

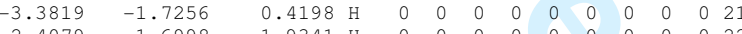

$\begin{array}{lllllllllllll}-2.4071 .6998 & 1.9341 \mathrm{H} & 0 & 0 & 0 & 0 & 0 & 0 & 0 & 0 & 0 & 2\end{array}$

$\begin{array}{lllllllllllll}0 & 0 & 0 & 0 & 0 & 0 & 0 & 0 & 234\end{array}$

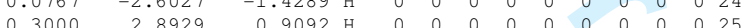

$\begin{array}{lllllll}2 & 1 & 0 & 0 & 0 & 0\end{array}$

$\begin{array}{llllll}3 & 1 & 0 & 0 & 0 & 0\end{array}$

$\begin{array}{llllll}4 & 0 & 0 & 0 & 0\end{array}$

$\begin{array}{lllll}4 & 0 & 0 & 0\end{array}$

$\begin{array}{lllllll}16 & 7 & 0 & 0 & 0 & 0\end{array}$

$\begin{array}{lllllll}17 & 7 & 0 & 0 & 0 & 0\end{array}$

$\begin{array}{llll}0 & 0 & 0\end{array}$

$\begin{array}{llllll}8 & 8 & 0 & 0 & 0 & 0\end{array}$

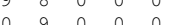

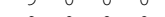

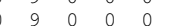

$\begin{array}{llllll}1 & 10 & 0 & 0 & 0\end{array}$

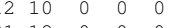

$\begin{array}{llllll}1 & 12 & 0 & 0 & 0 & 0\end{array}$

$\begin{array}{llllll}22 & 12 & 0 & 0 & 0 & 0\end{array}$

$\begin{array}{llllll}23 & 12 & 0 & 0 & 0 & 0\end{array}$

$\begin{array}{lllllll}15 & 13 & 0 & 0 & 0 & 0\end{array}$

$\begin{array}{llllll}24 & 14 & 0 & 0 & 0 & 0\end{array}$

$M \quad$ END

2-chlorophenol-anion.sdf

cosmotherm 3D

$\begin{array}{llllllllllll}18 & 16 & 0 & 0 & 0 & 0 & 0 & 0 & 0 & 0 & 0 & \mathrm{~V} 2000\end{array}$

$\begin{array}{rrrrrrrrrrrrrr}3.0533 & 1.4364 & 0.5681 & 0 & 0 & 0 & 0 & 0 & 0 & 0 & 0 & 0 & 0 & 1 \\ 2.3141 & 0.8205 & 0.2741 & \mathrm{H} & 0 & 0 & 0 & 0 & 0 & 0 & 0 & 0 & 0 & 2 \\ 2.6296 & 2.3107 & 0.6046 & \mathrm{H} & 0 & 0 & 0 & 0 & 0 & 0 & 0 & 0 & 0 & 3 \\ 2.8373 & -2.5587 & -0.2786 & \mathrm{O} & 0 & 0 & 0 & 0 & 0 & 0 & 0 & 0 & 0 & 4 \\ 2.2128 & -1.7632 & -0.2337 & \mathrm{H} & 0 & 0 & 0 & 0 & 0 & 0 & 0 & 0 & 0 & 5 \\ 2.7416 & -2.9910 & 0.5872 & \mathrm{H} & 0 & 0 & 0 & 0 & 0 & 0 & 0 & 0 & 0 & 6 \\ -0.6690 & -1.7928 & 0.0528 & \mathrm{C} & 0 & 0 & 0 & 0 & 0 & 0 & 0 & 0 & 0 & 7 \\ -2.0497 & -1.9299 & 0.1884 & \mathrm{C} & 0 & 0 & 0 & 0 & 0 & 0 & 0 & 0 & 0 & 8 \\ -2.8860 & -0.8037 & 0.2081 & \mathrm{C} & 0 & 0 & 0 & 0 & 0 & 0 & 0 & 0 & 0 & 9 \\ -2.3078 & 0.4658 & 0.0821 & \mathrm{C} & 0 & 0 & 0 & 0 & 0 & 0 & 0 & 0 & 0 & 10 \\ -0.9265 & 0.5924 & -0.0540 & \mathrm{C} & 0 & 0 & 0 & 0 & 0 & 0 & 0 & 0 & 0 & 11 \\ -0.0269 & -0.5205 & -0.0757 & \mathrm{C} & 0 & 0 & 0 & 0 & 0 & 0 & 0 & 0 & 0 & 12 \\ -3.9670 & -0.9036 & 0.3144 & \mathrm{H} & 0 & 0 & 0 & 0 & 0 & 0 & 0 & 0 & 0 & 13 \\ 1.2692 & -0.4027 & -0.2092 & \mathrm{O} & 0 & 0 & 0 & 0 & 0 & 0 & 0 & 0 & 0 & 14 \\ -0.0268 & -2.6768 & 0.0351 & \mathrm{H} & 0 & 0 & 0 & 0 & 0 & 0 & 0 & 0 & 0 & 15 \\ -2.4799 & -2.9303 & 0.2827 & \mathrm{H} & 0 & 0 & 0 & 0 & 0 & 0 & 0 & 0 & 0 & 16 \\ -2.9313 & 1.3616 & 0.0878 & \mathrm{H} & 0 & 0 & 0 & 0 & 0 & 0 & 0 & 0 & 0 & 17 \\ -0.2415 & 2.2321 & -0.2278 & \mathrm{Cl} & 0 & 0 & 0 & 0 & 0 & 0 & 0 & 0 & 0 & 18\end{array}$

$-0.2415-2.2321$

$\begin{array}{lllllll}2 & 1 & 0 & 0 & 0 & 0 \\ 3 & 1 & 0 & 0 & 0 & 0\end{array}$

$\begin{array}{lllllll}5 & 4 & 0 & 0 & 0 & 0\end{array}$

$\begin{array}{llllllll}6 & 4 & 0 & 0 & 0 & 0\end{array}$

$\begin{array}{lllllll}6 & 7 & 0 & 0 & 0 & 0 & 0 \\ 12 & 7 & 0 & 0 & 0 & 0\end{array}$

$\begin{array}{lllllll}15 & 7 & 0 & 0 & 0 & 0\end{array}$

$\begin{array}{rrrrrrrr}9 & 8 & 0 & 0 & 0 & 0 \\ 16 & 8 & 0 & 0 & 0 & 0\end{array}$

$\begin{array}{llllll}10 & 9 & 0 & 0 & 0\end{array}$

$\begin{array}{llllll}13 & 9 & 0 & 0 & 0 & 0\end{array}$

$\begin{array}{llllll}1 & 10 & 0 & 0 & 0 & 0\end{array}$

URL: http://mc.manuscriptcentral.com/tandf/tmph 


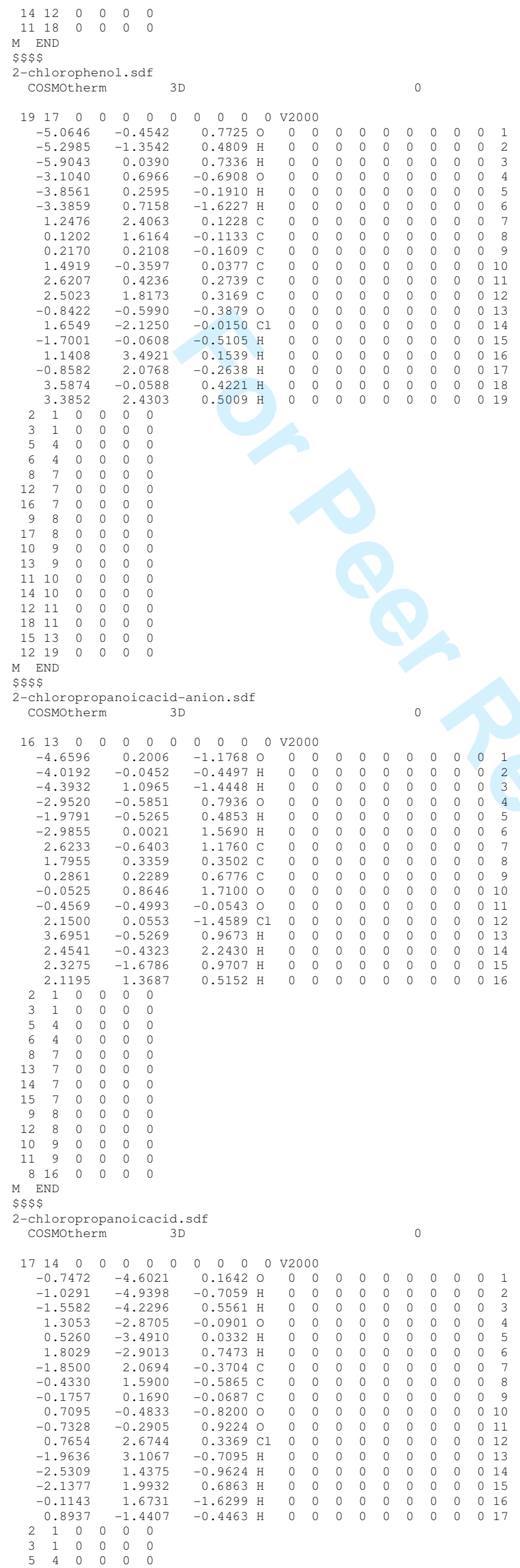




$\begin{array}{rrrrrrrrrrrrrr}2.7588 & 0.1279 & -1.2831 & \mathrm{C} & 0 & 0 & 0 & 0 & 0 & 0 & 0 & 0 & 0 & 13 \\ -1.3808 & 1.7887 & 0.0120 & 0 & 0 & 0 & 0 & 0 & 0 & 0 & 0 & 0 & 0 & 14 \\ 1.0897 & -2.0662 & 0.0101 & 0 & 0 & 0 & 0 & 0 & 0 & 0 & 0 & 0 & 0 & 15 \\ 1.2147 & 2.1844 & -0.8567 & \mathrm{H} & 0 & 0 & 0 & 0 & 0 & 0 & 0 & 0 & 0 & 16 \\ 1.2155 & 2.1540 & 0.9121 & \mathrm{H} & 0 & 0 & 0 & 0 & 0 & 0 & 0 & 0 & 0 & 17 \\ 2.2187 & 0.2112 & 2.1689 & \mathrm{H} & 0 & 0 & 0 & 0 & 0 & 0 & 0 & 0 & 0 & 18 \\ 3.2997 & -0.8679 & 1.2498 & \mathrm{H} & 0 & 0 & 0 & 0 & 0 & 0 & 0 & 0 & 0 & 19 \\ 3.5828 & 0.8930 & 1.2472 & \mathrm{H} & 0 & 0 & 0 & 0 & 0 & 0 & 0 & 0 & 0 & 20 \\ 2.1389 & 0.2365 & -2.1859 & \mathrm{H} & 0 & 0 & 0 & 0 & 0 & 0 & 0 & 0 & 0 & 21 \\ 3.2518 & -0.8543 & -1.3198 & \mathrm{H} & 0 & 0 & 0 & 0 & 0 & 0 & 0 & 0 & 0 & 22 \\ 3.5367 & 0.9063 & -1.3070 & \mathrm{H} & 0 & 0 & 0 & 0 & 0 & 0 & 0 & 0 & 0 & 23\end{array}$


$\begin{array}{rr}1.7690 & 0.1033 \\ -0.1520 & -0.1445\end{array}$

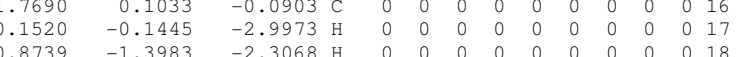

$\begin{array}{llllllllllllll}0.8739 & -1.3983 & -2.3068 & \mathrm{H} & 0 & 0 & 0 & 0 & 0 & 0 & 0 & 0 & 0 & 18 \\ -0.4517 & 2.0795 & -1.8955 & \mathrm{H} & 0 & 0 & 0 & 0 & 0 & 0 & 0 & 0 & 0 & 19\end{array}$

$\begin{array}{llllllllllllll}0.3649 & 2.4635 & -0.3778 & \mathrm{H} & 0 & 0 & 0 & 0 & 0 & 0 & 0 & 0 & 0 & 20\end{array}$

$\begin{array}{llllllllllllll}3.2377 & -0.7070 & 2.1989 & \mathrm{C} & 0 & 0 & 0 & 0 & 0 & 0 & 0 & 0 & 0 & 21\end{array}$

$\begin{array}{llllllllllllll}2.6018 & -1.6548 & 1.3928 & \mathrm{C} & 0 & 0 & 0 & 0 & 0 & 0 & 0 & 0 & 0 & 22\end{array}$

$\begin{array}{llllllllllllll}1.8781 & -1.2527 & 0.2616 & \mathrm{C} & 0 & 0 & 0 & 0 & 0 & 0 & 0 & 0 & 0 & 23\end{array}$

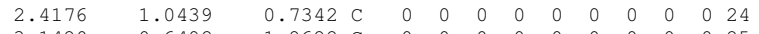

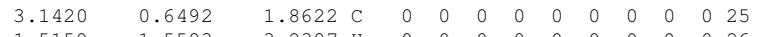

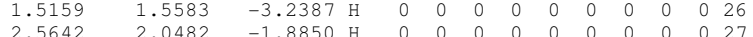

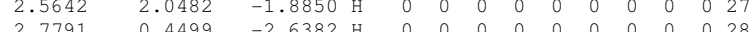

$\begin{array}{rrrrrrrrrrrrrr}2.7791 & 0.4499 & -2.6382 & \mathrm{H} & 0 & 0 & 0 & 0 & 0 & 0 & 0 & 0 & 0 & 28 \\ 3.8024 & -1.0191 & 3.0793 & \mathrm{H} & 0 & 0 & 0 & 0 & 0 & 0 & 0 & 0 & 0 & 28\end{array}$

$\begin{array}{llllllllllllll}3.8024 & -1.0191 & 3.0793 & \mathrm{H} & 0 & 0 & 0 & 0 & 0 & 0 & 0 & 0 & 0 & 28 \\ 2.6657 & -2.7167 & 1.6403 & \mathrm{H} & 0 & 0 & 0 & 0 & 0 & 0 & 0 & 0 & 0 & 30\end{array}$

$\begin{array}{llllllllllllllll}2.6657 & -1.0191 & 3.0793 & \mathrm{H} & 0 & 0 & 0 & 0 & 0 & 0 & 0 & 0 & 0 & 29 \\ 1.3962 & -2.0207 & -0.6403 & \mathrm{H} & 0 & 0 & 0 & 0 & 0 & 0 & 0 & 0 & 0 & 30\end{array}$

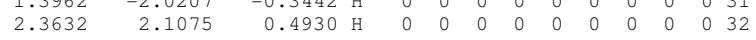

3.634101 .4036

$\begin{array}{llllll}2 & 1 & 0 & 0 & 0 & 0 \\ 3 & 1 & 0 & 0 & 0 & 0\end{array}$

$\begin{array}{llllll}3 & 1 & 0 & 0 & 0 & 0 \\ 5 & 4 & 0 & 0 & 0 & 0\end{array}$

$\begin{array}{llllll}6 & 4 & 0 & 0 & 0 & 0\end{array}$

$\begin{array}{rrrrrr}8 & 7 & 0 & 0 & 0 & 0 \\ 12 & 7 & 0 & 0 & 0 & 0\end{array}$

$\begin{array}{llllll}14 & 7 & 0 & 0 & 0\end{array}$

$\begin{array}{llllll}9 & 8 & 0 & 0 & 0 & 0\end{array}$

$\begin{array}{llllll}10 & 9 & 0 & 0 & 0 & 0 \\ 13 & 9 & 0 & 0 & 0 & 0\end{array}$

$\begin{array}{llllll}11 & 10 & 0 & 0 & 0\end{array}$

$\begin{array}{llllll}17 & 10 & 0 & 0 & 0 & 0\end{array}$

$\begin{array}{llllll}18 & 10 & 0 & 0 & 0 & 0 \\ 12 & 11 & 0 & 0 & 0 & 0\end{array}$

$\begin{array}{llllll}15 & 11 & 0 & 0 & 0 & 0\end{array}$

$\begin{array}{llllll}16 & 11 & 0 & 0 & 0 & 0\end{array}$

$\begin{array}{llllll}19 & 12 & 0 & 0 & 0 & 0\end{array}$

$\begin{array}{lllllllll}26 & 15 & 0 & 0 & 0 & 0\end{array}$

$\begin{array}{llllll}27 & 15 & 0 & 0 & 0\end{array}$

$\begin{array}{lllll}28 & 15 & 0 & 0 & 0\end{array}$

$\begin{array}{llllll}23 & 16 & 0 & 0 & 0 & 0\end{array}$

$\begin{array}{llllll}25 & 21 & 0 & 0 & 0 & 0\end{array}$

$\begin{array}{llllll}29 & 21 & 0 & 0 & 0\end{array}$

$\begin{array}{llllll}3 & 22 & 0 & 0 & 0 & 0\end{array}$

$\begin{array}{llllll}30 & 22 & 0 & 0 & 0 & 0\end{array}$

$\begin{array}{llllll}31 & 23 & 0 & 0 & 0 & 0 \\ 25 & 24 & 0 & 0 & 0 & 0 \\ 32 & 24 & 0 & 0 & 0 & 0\end{array}$

$\begin{array}{llllll}32 & 24 & 0 & 0 & 0 & 0\end{array}$

253

0.49307

,3-methylphenylglutarimide.sdf

cosmotherm

$\begin{array}{llllllllllll}34 & 33 & 0 & 0 & 0 & 0 & 0 & 0 & 0 & 0 & 0 & \mathrm{~V} 2000\end{array}$

$\begin{array}{llllll}-5.8549 & -0.9267 & 0.7630 & 0 & 0\end{array}$

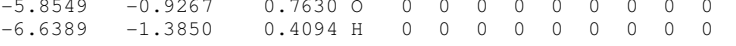

$\begin{array}{llllllllllllll}-6.1151 & 0.0120 & 0.7853 & \mathrm{H} & 0 & 0 & 0 & 0 & 0 & 0 & 0 & 0 & 0\end{array}$

$\begin{array}{lllllllllllll}-3.5970 & -1.3928 & -0.7205 & 0 & 0 & 0 & 0 & 0 & 0 & 0 & 0 & 0 & 0\end{array}$

$\begin{array}{llllllllllllll}-3.8418 & -1.2735 & -1.6552 & \mathrm{H} & 0 & 0 & 0 & 0 & 0 & 0 & 0 & 0 & 0 & 5\end{array}$

$\begin{array}{llllllllllllll}-4.4331 & -1.2038 & -0.2087 & \mathrm{H} & 0 & 0 & 0 & 0 & 0 & 0 & 0 & 0 & 0 & 6\end{array}$

$\begin{array}{llllllllllllll}-0.9813 & 0.4810 & 1.1277 & \mathrm{C} & 0 & 0 & 0 & 0 & 0 & 0 & 0 & 0 & 0 & 7\end{array}$

$\begin{array}{llllllllllllll}-1.4009 & 0.3131 & -0.1865 & \mathrm{~N} & 0 & 0 & 0 & 0 & 0 & 0 & 0 & 0 & 0 & 8\end{array}$

$\begin{array}{llllllllllllll}-0.8196 & 0.8867 & -1.3098 & \mathrm{C} & 0 & 0 & 0 & 0 & 0 & 0 & 0 & 0 & 0 & 9\end{array}$

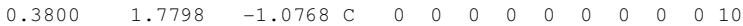

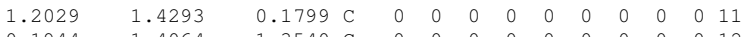

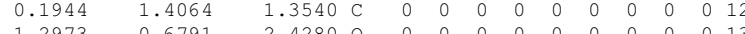

$\begin{array}{llllllllllll}0 & 0 & 0 & 0 & 0 & 0 & 0 & 0 & 0 & 0 & 13\end{array}$

$\begin{array}{llllllllllllllll}2.2476 & 2.5408 & 0.4205 \mathrm{C} & 0 & 0 & 0 & 0 & 0 & 0 & 0 & 0 & 0 & 14\end{array}$

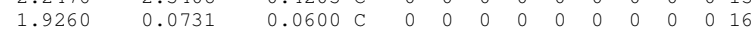

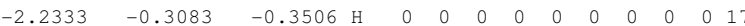

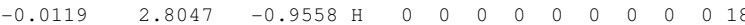

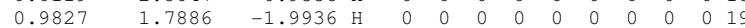

$\begin{array}{lllllllllllllllllllllllll}-0.2181 & 2.4221 & 1.4871 & \mathrm{H} & 0 & 0 & 0 & 0 & 0 & 0 & 0 & 0 & 0 & 20\end{array}$

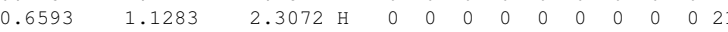

$\begin{array}{lllllllllllllllllll}3.3354 & -2.3826 & -0.0965 & \mathrm{C} & 0 & 0 & 0 & 0 & 0 & 0 & 0 & 0 & 0 & 22\end{array}$

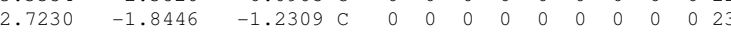

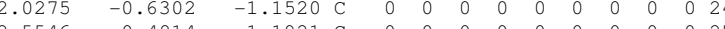

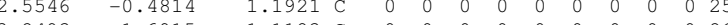

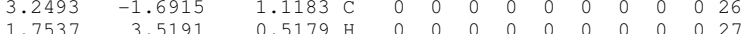

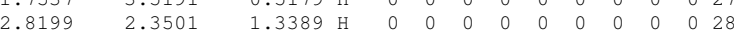

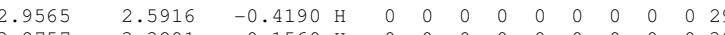

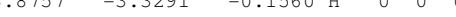

$\begin{array}{llllllllllllll}2.7820 & -2.3682 & -2.1870 & \mathrm{H} & 0 & 0 & 0 & 0 & 0 & 0 & 0 & 0 & 0 & 31\end{array}$

$\begin{array}{llllllllllllll}1.5680 & -0.2404 & -2.0605 & \mathrm{H} & 0 & 0 & 0 & 0 & 0 & 0 & 0 & 0 & 0 & 32\end{array}$

$3.7250-2.0958$

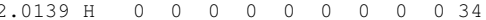

$0 \begin{array}{llll}0 & 0 & 0\end{array}$

$\begin{array}{lllllll}3 & 1 & 0 & 0 & 0 & 0 \\ 2 & 4 & 0 & 0 & 0 & 0\end{array}$

$\begin{array}{lllllll}6 & 4 & 0 & 0 & 0 & 0\end{array}$

\begin{tabular}{llllll}
8 & 7 & 0 & 0 & 0 & 0 \\
\hline & 7 & 0 & 0 & 0 & 0
\end{tabular}

$14 \quad 7 \begin{array}{llllll}14 & 7 & 0 & 0 & 0\end{array}$

$\begin{array}{llllll}9 & 8 & 0 & 0 & 0 & 0 \\ 17 & 8 & 0 & 0 & 0 & 0\end{array}$

$\begin{array}{cccccccc}17 & 8 & 0 & 0 & 0 & 0 \\ 10 & 9 & 0 & 0 & 0 & 0\end{array}$

$\begin{array}{llllllll}3 & 9 & 0 & 0 & 0 & 0\end{array}$

$\begin{array}{lllllll}1 & 10 & 0 & 0 & 0 & 0\end{array}$

$1910 \quad 000000$

$\begin{array}{llllll}12 & 11 & 0 & 0 & 0 & 0\end{array}$ 


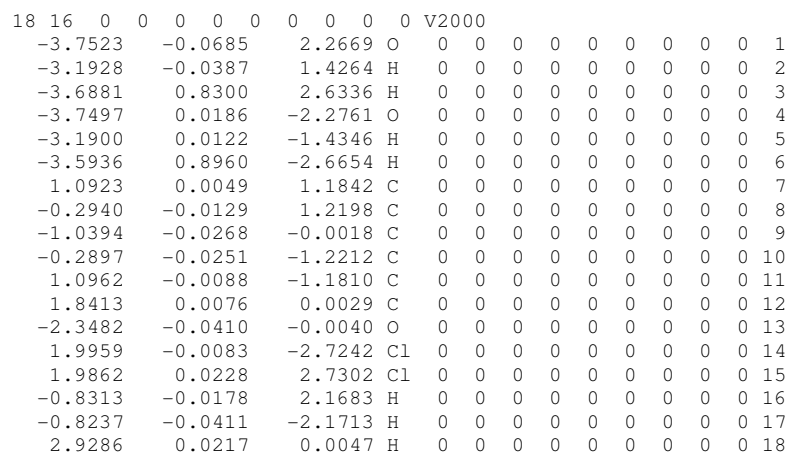


$\begin{array}{rrrrrr}8 & 7 & 0 & 0 & 0 & 0 \\ 12 & 7 & 0 & 0 & 0 & 0 \\ 15 & 7 & 0 & 0 & 0 & 0 \\ 9 & 8 & 0 & 0 & 0 & 0 \\ 16 & 8 & 0 & 0 & 0 & 0 \\ 10 & 9 & 0 & 0 & 0 & 0 \\ 13 & 9 & 0 & 0 & 0 & 0 \\ 11 & 10 & 0 & 0 & 0 & 0 \\ 17 & 10 & 0 & 0 & 0 & 0 \\ 12 & 11 & 0 & 0 & 0 & 0 \\ 14 & 11 & 0 & 0 & 0 & 0 \\ 12 & 18 & 0 & 0 & 0 & 0\end{array}$ \begin{tabular}{rr}
12 & 18 \\
\hline & END
\end{tabular}

M\$\$\$

\$\$\$
3-chlorophenol.sdf

cosmotherm

$\begin{array}{llllllllllll}19 & 17 & 0 & 0 & 0 & 0 & 0 & 0 & 0 & 0 & 0 & \mathrm{~V} 2000\end{array}$

$\begin{array}{llllllllllllll}-0.7238 & 1.1120 & -5.0254 & 0 & 0 & 0 & 0 & 0 & 0 & 0 & 0 & 0 & 0 & 1\end{array}$

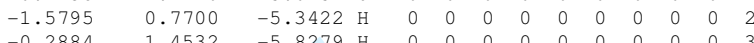

$\begin{array}{rrrrrrrrrrrrrr}-0.2884 & 1.4532 & -5.8279 & \mathrm{H} & 0 & 0 & 0 & 0 & 0 & 0 & 0 & 0 & 0 & 3 \\ 0.8399 & -0.6880 & -3.7461 & 0 & 0 & 0 & 0 & 0 & 0 & 0 & 0 & 0 & 0 & 4\end{array}$

$\begin{array}{llllllllllllll}0.8399 & -0.6880 & -3.7461 & 0 & 0 & 0 & 0 & 0 & 0 & 0 & 0 & 0 & 0 & 4 \\ 0.2306 & -0.0590 & -4.2338 & \mathrm{H} & 0 & 0 & 0 & 0 & 0 & 0 & 0 & 0 & 0 & 5\end{array}$

$\begin{array}{llllllllllllll}0.2306 & -0.0590 & -4.2338 & \mathrm{H} & 0 & 0 & 0 & 0 & 0 & 0 & 0 & 0 & 0 & 5 \\ 0.5041 & -1.5824 & -3.9323 & \mathrm{H} & 0 & 0 & 0 & 0 & 0 & 0 & 0 & 0 & 0 & 6\end{array}$

$\begin{array}{llllllllllllll}0.8682 & 0.0681 & 1.0114 & \mathrm{C} & 0 & 0 & 0 & 0 & 0 & 0 & 0 & 0 & 0\end{array}$

$\begin{array}{rrrrrrrrrrrrrr}-0.1197 & 0.0384 & 1.9914 & \mathrm{C} & 0 & 0 & 0 & 0 & 0 & 0 & 0 & 0 & 0 & 8 \\ -1.4557 & -0.2529 & 1.7094 & \mathrm{C} & 0 & 0 & 0 & 0 & 0 & 0 & 0 & 0 & 0 & 9\end{array}$

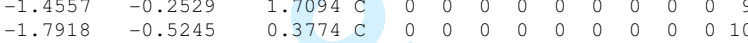

$\begin{array}{lllllllllllllll}-0.8303 & -0.5051 & -0.6354 & \mathrm{C} & 0 & 0 & 0 & 0 & 0 & 0 & 0 & 0 & 0 & 1\end{array}$

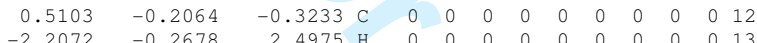

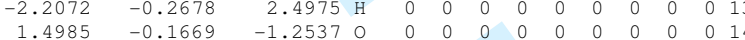

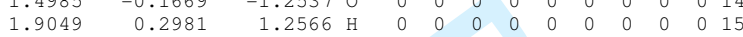

$\begin{array}{lllllllllllll}0.3465 & 0.3877 & 3.6735 \mathrm{Cl} & 0 & 0 & 0 & 0 & 0 & 0 & 0 & 0 & 0 & 16\end{array}$

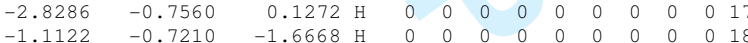

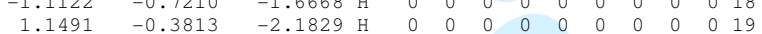

$\begin{array}{llllll}2 & 1 & 0 & 0 & 0 & 0\end{array}$

$\begin{array}{lllllll}5 & 4 & 0 & 0 & 0 & 0\end{array}$

$\begin{array}{llllll}6 & 4 & 0 & 0 & 0\end{array}$

$\begin{array}{lllll}7 & 0 & 0 & 0 & 0\end{array}$

$\begin{array}{lllllll}12 & 7 & 0 & 0 & 0 & 0 \\ 15 & 7 & 0 & 0 & 0 & 0\end{array}$

$\begin{array}{lllll}8 & 0 & 0 & 0 & 0\end{array}$

$\begin{array}{cccccc}16 & 8 & 0 & 0 & 0 & 0 \\ 10 & 9 & 0 & 0 & 0 & 0\end{array}$

$\begin{array}{lllllll}13 & 9 & 0 & 0 & 0 & 0\end{array}$

$\begin{array}{llllll}11 & 10 & 0 & 0 & 0 & 0\end{array}$

$\begin{array}{llllll}17 & 10 & 0 & 0 & 0 & 0\end{array}$

$1811 \quad 000000$

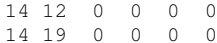

$14 \quad 19$

MS\$

3-chloropropanoicacid-anion.sdf

cosmotherm

$\begin{array}{llllllllllll}16 & 13 & 0 & 0 & 0 & 0 & 0 & 0 & 0 & 0 & 0 & \mathrm{~V} 2000\end{array}$

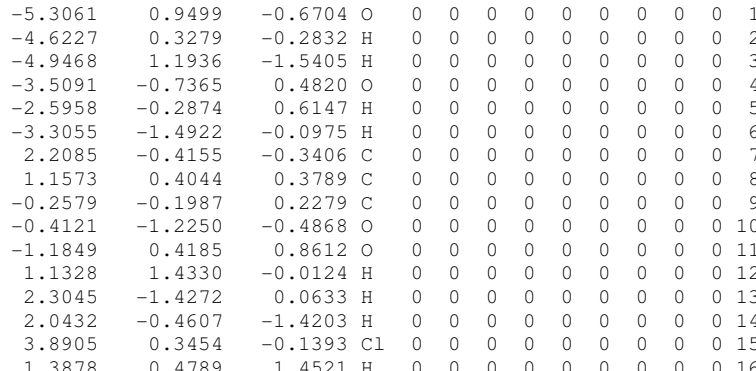

$\begin{array}{llllll}2 & 1 & 0 & 0 & 0 & 0 \\ 3 & 1 & 0 & 0 & 0 & 0\end{array}$

$\begin{array}{llllll}1 & 0 & 0 & 0 & 0 \\ 5 & 0 & 0 & 0 & 0\end{array}$

$\begin{array}{llllll}4 & 0 & 0 & 0 & 0\end{array}$

$\begin{array}{llllll}8 & 7 & 0 & 0 & 0 & 0\end{array}$

$\begin{array}{lllllll}13 & 7 & 0 & 0 & 0 & 0 & 0 \\ 14 & 7 & 0 & 0 & 0 & 0\end{array}$

$\begin{array}{llllll}5 & 7 & 0 & 0 & 0\end{array}$

$\begin{array}{lllllll}9 & 8 & 0 & 0 & 0 & 0 \\ 12 & 8 & 0 & 0 & 0 & 0\end{array}$

$\begin{array}{lllllll}10 & 9 & 0 & 0 & 0 & 0\end{array}$

$\begin{array}{rrrrrr}1 & 9 & 0 & 0 & 0 & 0 \\ 8 & 16 & 0 & 0 & 0 & 0\end{array}$

END

3-chloropropanoicacid.sdf

$\begin{array}{llllllllllll}17 & 14 & 0 & 0 & 0 & 0 & 0 & 0 & 0 & 0 & 0 & \mathrm{~V} 2000\end{array}$

$\begin{array}{rrrrrrrrrrrrrr}-1.2779 & -0.5624 & -4.7637 & 0 & 0 & 0 & 0 & 0 & 0 & 0 & 0 & 0 & 0 & 1 \\ -1.0874 & -1.0765 & -5.5696 & \mathrm{H} & 0 & 0 & 0 & 0 & 0 & 0 & 0 & 0 & 0 & 2 \\ -1.8706 & 0.1499 & -5.0650 & \mathrm{H} & 0 & 0 & 0 & 0 & 0 & 0 & 0 & 0 & 0 & 3 \\ 0.9917 & 0.2836 & -3.5747 & 0 & 0 & 0 & 0 & 0 & 0 & 0 & 0 & 0 & 0 & 4 \\ 1.0911 & 1.2367 & -3.7469 & \mathrm{H} & 0 & 0 & 0 & 0 & 0 & 0 & 0 & 0 & 0 & 5 \\ 0.1356 & 0.0145 & -4.0251 & \mathrm{H} & 0 & 0 & 0 & 0 & 0 & 0 & 0 & 0 & 0 & 6 \\ -0.5467 & 0.2883 & 2.1256 & \mathrm{C} & 0 & 0 & 0 & 0 & 0 & 0 & 0 & 0 & 0 & 7 \\ 0.5348 & -0.2391 & 1.2001 & \mathrm{C} & 0 & 0 & 0 & 0 & 0 & 0 & 0 & 0 & 0 & 8 \\ 0.2877 & 0.1738 & -0.2421 & \mathrm{C} & 0 & 0 & 0 & 0 & 0 & 0 & 0 & 0 & 0 & 9 \\ 1.1991 & -0.3363 & -1.0741 & 0 & 0 & 0 & 0 & 0 & 0 & 0 & 0 & 0 & 0 & 10\end{array}$

URL: http://mc.manuscriptcentral.com/tandf/tmph 


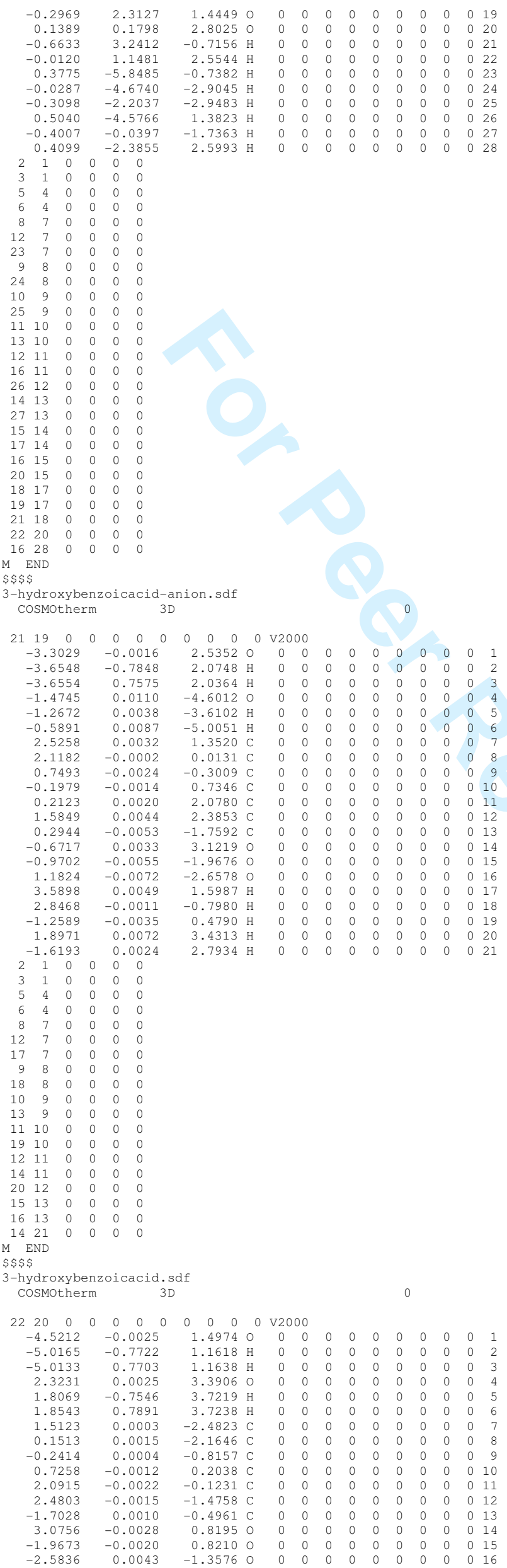




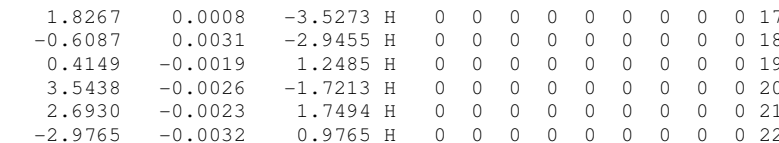




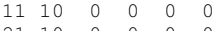

$\begin{array}{llllll}21 & 10 & 0 & 0 & 0 & 0\end{array}$

$\begin{array}{llllll}12 & 11 & 0 & 0 & 0 & 0 \\ 22 & 11 & 0 & 0 & 0 & 0\end{array}$

$14 \quad 12$ 0 120000

$17 \begin{array}{lllll}13 & 0 & 0 & 0 & 0\end{array}$

18 13 13 1 001400

$16 \quad 14 \quad 0 \quad 0000$

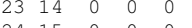

25 15 15000000

$\begin{array}{llllll}26 & 15 & 0 & 0 & 0 & 0\end{array}$

$27-16 \begin{array}{llllll}27 & 0 & 0 & 0\end{array}$

$\begin{array}{llllll}28 & 16 & 0 & 0 & 0 & 0 \\ 16 & 29 & 0 & 0 & 0 & 0\end{array}$

END

$4-(1-$ methylethyl)-benzoicacid.sdf

cosmotherm

$\begin{array}{llllllllllll}30 & 28 & 0 & 0 & 0 & 0 & 0 & 0 & 0 & 0 & 0 & \mathrm{v} 2000\end{array}$

$\begin{array}{llllllllllllll}-1.2292 & 5.1902 & 0.1499 & 0 & 0 & 0 & 0 & 0 & 0 & 0 & 0 & 0 & 0\end{array}$

$\begin{array}{lllllllllllll}-1.2901 & 4.2040 & 0.0273 & \mathrm{H} & 0 & 0 & 0 & 0 & 0 & 0 & 0 & 0 & 0\end{array}$

$\begin{array}{lllllllllllll}-1.6843 & 5.5750 & -0.6209 & \mathrm{H} & 0 & 0 & 0 & 0 & 0 & 0 & 0 & 0 & 0\end{array}$

$\begin{array}{llllllllllllll}1.4017 & 4.8443 & 0.0018 & 0 & 0 & 0 & 0 & 0 & 0 & 0 & 0 & 0 & 0 & 4 \\ 0.4489 & 5.1717 & 0.0031 & \mathrm{H} & 0 & 0 & 0 & 0 & 0 & 0 & 0 & 0 & 0 & 5\end{array}$

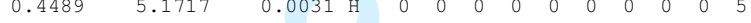

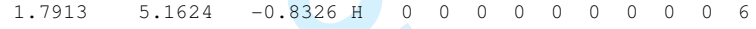

$\begin{array}{llllllllllllll}-1.3884 & -1.7532 & -0.0081 & \mathrm{C} & 0 & 0 & 0 & 0 & 0 & 0 & 0 & 0 & 0 & 7\end{array}$

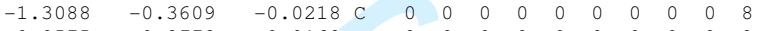

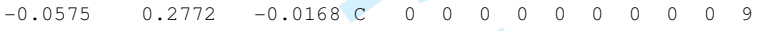

$\begin{array}{lllllllllllllll}1.1094 & -0.5095 & -0.0018 & \mathrm{C} & 0 & 0 & 0 & 0 & 0 & 0 & 0 & 0 & 0 & 10 \\ 1.0194 & -1.9003 & 0.0081 & \mathrm{C} & 0 & 0 & 0 & 0 & 0 & 0 & 0 & 0 & 0 & 11\end{array}$

$\begin{array}{llllllllllllll}1.0194 & -1.9003 & -0.0081 & \mathrm{C} & 0 & 0 & 0 & 0 & 0 & 0 & 0 & 0 & 0 & 11 \\ -0.2297 & -2.5496 & 0.0068 & \mathrm{C} & 0 & 0 & 0 & 0 & 0 & 0 & 0 & 0 & 0 & 12\end{array}$

$\begin{array}{lllllllllllllll}-0.0009 & 1.7650 & -0.0261 & \mathrm{C} & 0 & 0 & 0 & 0 & 0 & 0 & 0 & 0 & 0 & 13\end{array}$

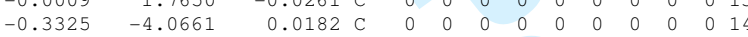

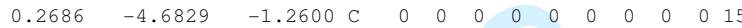

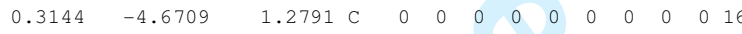

$\begin{array}{llllllllllllll}-1.0285 & 2.4628 & -0.0768 & 0 & 0 & 0 & 0 & 0 & 0 & 0 & 0 & 0 & 0 & 17\end{array}$

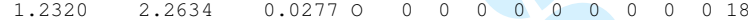

$\begin{array}{llllllllllllll}-2.3685 & -2.2359 & -0.0087 & \mathrm{H} & 0 & 0 & 0 & 0 & 0 & 0 & 0 & 0 & 0 & 19\end{array}$

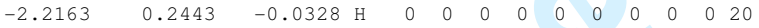

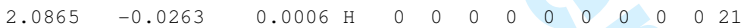

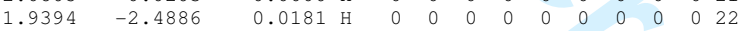

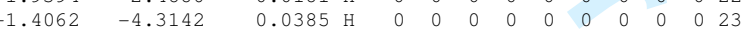

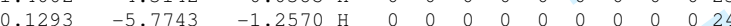

$\begin{array}{llllllllllllll}-0.2108 & -4.2787 & -2.1634 & \mathrm{H} & 0 & 0 & 0 & 0 & 0 & 0 & 0 & 0 & 0 & 25\end{array}$

$\begin{array}{llllllllllllll}1.3488 & -4.4814 & -1.3279 & \mathrm{H} & 0 & 0 & 0 & 0 & 0 & 0 & 0 & 0 & 0 & 26\end{array}$

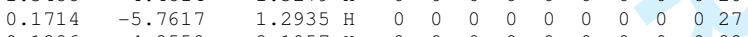

$\begin{array}{llllllllllllll}-0.1286 & -4.2550 & 2.1957 & 0 & 0 & 0 & 0 & 0 & 0 & 0 & 0 & 0 & 0 & 28\end{array}$

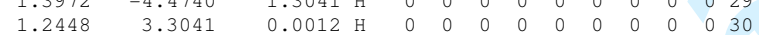

1.24480 .304

$\begin{array}{lllll}1 & 0 & 0 & 0 & 0\end{array}$

$\begin{array}{lllllll}5 & 4 & 0 & 0 & 0 & 0 & 0 \\ 6 & 4 & 0 & 0 & 0 & 0\end{array}$

$\begin{array}{llllll}7 & 0 & 0 & 0 & 0\end{array}$

$\begin{array}{llll}0 & 0 & 0\end{array}$

$\begin{array}{llllll}19 & 7 & 0 & 0 & 0 & 0\end{array}$

$\begin{array}{llllllll}2 & 8 & 0 & 0 & 0 & 0\end{array}$

$\begin{array}{lllll}0 & 0 & 0 & 0 \\ 0 & 0 & 0 & 0 & 0\end{array}$

$\begin{array}{llllll}3 & 9 & 0 & 0 & 0 & 0\end{array}$

$\begin{array}{llllll}1 & 10 & 0 & 0 & 0 & 0\end{array}$

$\begin{array}{llllll}21 & 10 & 0 & 0 & 0 & 0\end{array}$

$\begin{array}{llllll}2 & 11 & 0 & 0 & 0 & 0\end{array}$

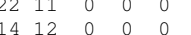

$\begin{array}{llllll}7 & 13 & 0 & 0 & 0 & 0\end{array}$

$\begin{array}{llllll}8 & 13 & 0 & 0 & 0 & 0\end{array}$

$\begin{array}{lllllll}5 & 14 & 0 & 0 & 0\end{array}$

$\begin{array}{lll}0 & 0 & 0 \\ 0 & 0 & 0\end{array}$

$\begin{array}{llllll}14 & 14 & 0 & 0 & 0 & 0\end{array}$

$\begin{array}{llllllllll}25 & 15 & 0 & 0 & 0 & 0\end{array}$

$\begin{array}{lllllll}26 & 15 & 0 & 0 & 0 & 0\end{array}$

$\begin{array}{llllll}27 & 16 & 0 & 0 & 0 & 0\end{array}$

$\begin{array}{llllll}28 & 16 & 0 & 0 & 0 & 0\end{array}$

$\begin{array}{llllll}9 & 16 & 0 & 0 & 0 & 0\end{array}$

8 (1)

\$\$

-aminobenzoicacid-anion.sdf

osMotherm 3D

$\begin{array}{llllllllllll}22 & 20 & 0 & 0 & 0 & 0 & 0 & 0 & 0 & 0 & 0 & \mathrm{~V} 2000\end{array}$

$\begin{array}{llllllll}0 & 0 & 0 & 0 & 0 & 0 & 0 & 1\end{array}$

$\begin{array}{lllllllllllll}-5.1102 & 0.3308 & 0.5853 & \mathrm{H} & 0 & 0 & 0 & 0 & 0 & 0 & 0 & 0 & 0\end{array}$

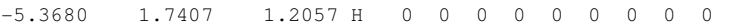

$\begin{array}{llllllllllllll}-4.0970 & -0.6410 & -0.3936 & 0 & 0 & 0 & 0 & 0 & 0 & 0 & 0 & 0 & 0\end{array}$

$\begin{array}{llllllllllllll}-3.1141 & -0.5884 & -0.0837 & \mathrm{H} & 0 & 0 & 0 & 0 & 0 & 0 & 0 & 0 & 0 & 5\end{array}$

$\begin{array}{lllllllllllll}-4.0583 & -0.3127 & -1.3096 & \mathrm{H} & 0 & 0 & 0 & 0 & 0 & 0 & 0 & 0 & 0\end{array}$

$\begin{array}{llllllllllllll}2.4836 & -0.2409 & 1.3501 & \mathrm{C} & 0 & 0 & 0 & 0 & 0 & 0 & 0 & 0 & 0 & 7\end{array}$

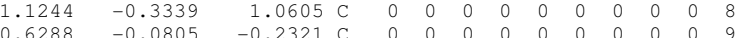

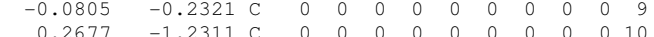

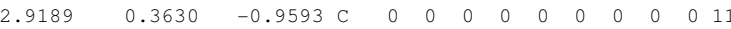

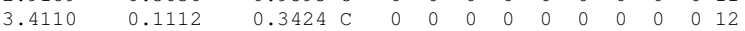

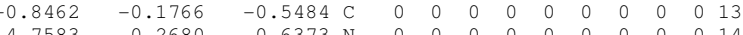

$\begin{array}{llllllllllllll}-1.6240 & -0.5298 & 0.4165 & 0 & 0 & 0 & 0 & 0 & 0 & 0 & 0 & 0 & 0 & 15\end{array}$

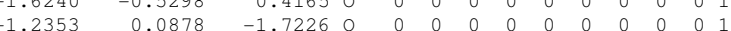

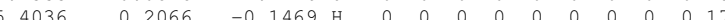

$\begin{array}{llllllllllllll}5.1001 & -0.2161 & 1.4643 & \mathrm{H} & 0 & 0 & 0 & 0 & 0 & 0 & 0 & 0 & 0 & 18\end{array}$

URL: http://mc.manuscriptcentral.com/tandf/tmph 


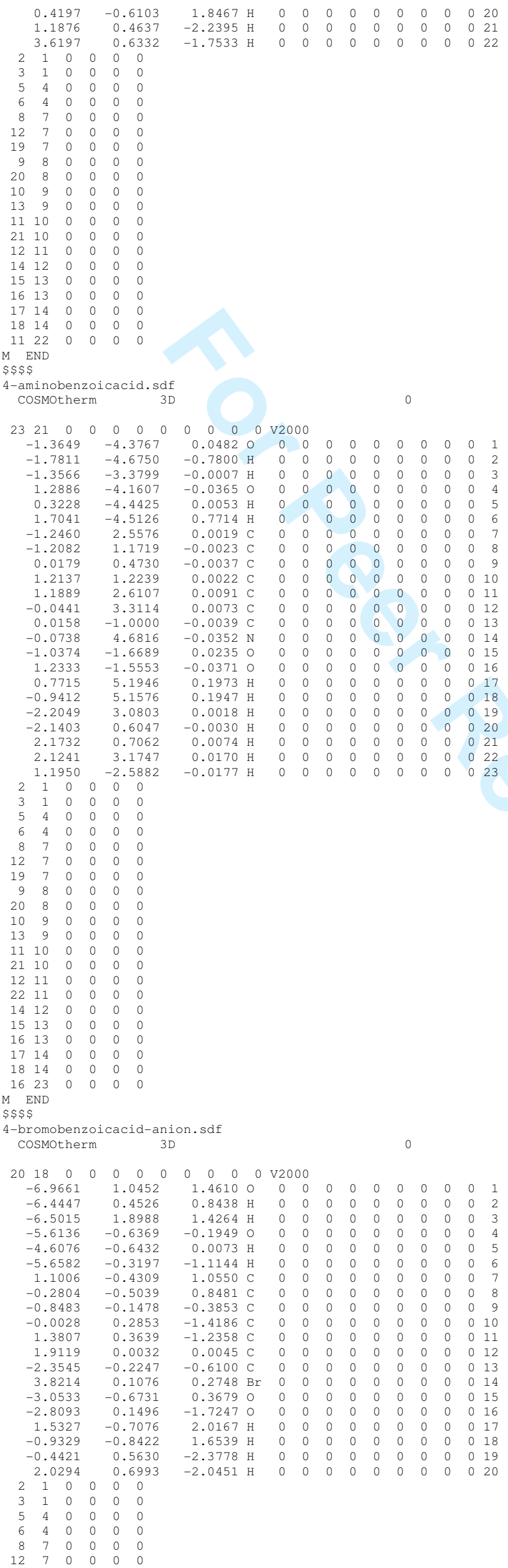


$\begin{array}{lllllll}17 & 7 & 0 & 0 & 0 & 0\end{array}$

$\begin{array}{rrrrrr}9 & 8 & 0 & 0 & 0 & 0 \\ 18 & 8 & 0 & 0 & 0 & 0\end{array}$

$\begin{array}{llllll}10 & 9 & 0 & 0 & 0 & 0\end{array}$

$\begin{array}{rrrrrr}13 & 9 & 0 & 0 & 0 & 0 \\ 11 & 10 & 0 & 0 & 0 & 0\end{array}$

$1910 \quad 00000$

$1211 \quad 00000$

$1513 \quad 000000$

11 END

$\$ \$ \$ \$$

-bromobenzoicacid.sdf

Cosmotherm 3D

$\begin{array}{lllllllllllll}21 & 19 & 0 & 0 & 0 & 0 & 0 & 0 & 0 & 0 & 0 & \mathrm{~V} 2000\end{array}$

$\begin{array}{lllllllllllll}-0.1834 & -1.5466 & -6.8430 & 0 & 0 & 0 & 0 & 0 & 0 & 0 & 0 & 0 & 0\end{array}$

$\begin{array}{lllllllllllll}-0.0994 & -1.4206 & -7.8056 & \mathrm{H} & 0 & 0 & 0 & 0 & 0 & 0 & 0 & 0 & 0\end{array}$

$\begin{array}{llllllllllllll}-0.9512 & -2.1385 & -6.7448 & \mathrm{H} & 0 & 0 & 0 & 0 & 0 & 0 & 0 & 0 & 0 & 3\end{array}$

$\begin{array}{llllllllllllll}-0.5763 & 0.7906 & -5.5368 & 0 & 0 & 0 & 0 & 0 & 0 & 0 & 0 & 0 & 0 & 4\end{array}$

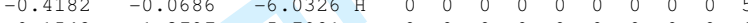

$\begin{array}{llllllllllllll}0.1542 & 1.3797 & -5.7981 & \mathrm{H} & 0 & 0 & 0 & 0 & 0 & 0 & 0 & 0 & 0 & 6\end{array}$

$\begin{array}{rrrrrrrrrrrrrr}1.2775 & -0.0491 & 1.3465 & \mathrm{C} & 0 & 0 & 0 & 0 & 0 & 0 & 0 & 0 & 0 & 7 \\ 1.3797 & 0.0857 & -0.0382 & \mathrm{C} & 0 & 0 & 0 & 0 & 0 & 0 & 0 & 0 & 0 & 8\end{array}$

$\begin{array}{llllllllllllll}1.3797 & 0.0857 & -0.0382 & \mathrm{C} & 0 & 0 & 0 & 0 & 0 & 0 & 0 & 0 & 0 & 8 \\ 0.2326 & 0.2598 & -0.8296 & \mathrm{C} & 0 & 0 & 0 & 0 & 0 & 0 & 0 & 0 & 0 & 9\end{array}$

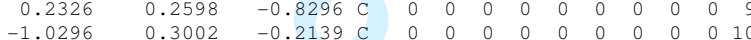

1.1712 C 0 C 0 a

$\begin{array}{llllllllllllll}0.0096 & -0.0055 & 1.9321 & \mathrm{C} & 0 & 0 & 0 & 0 & 0 & 0 & 0 & 0 & 0 & 12\end{array}$

$\begin{array}{llllllllllllll}0.3959 & 0.3972 & -2.3112 & \mathrm{C} & 0 & 0 & 0 & 0 & 0 & 0 & 0 & 0 & 0 & 13\end{array}$

$\begin{array}{llllllllllllll}-0.1449 & -0.1913 & 3.8422 & \mathrm{Br} & 0 & 0 & 0 & 0 & 0 & 0 & 0 & 0 & 0 & 14\end{array}$

$\begin{array}{llllllllllllll}1.4973 & 0.3848 & -2.8647 & 0 & 0 & 0 & 0 & 0 & 0 & 0 & 0 & 0 & 0 & 15\end{array}$

$\begin{array}{llllllllllllll}-0.7617 & 0.5287 & -2.9685 & 0 & 0 & 0 & 0 & 0 & 0 & 0 & 0 & 0 & 0 & 16\end{array}$

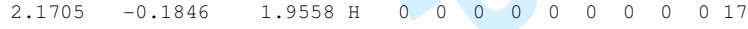

$\begin{array}{llllllllllllll}2.3594 & 0.0562 & -0.5163 & \mathrm{H} & 0 & 0 & 0 & 0 & 0 & 0 & 0 & 0 & 0 & 18\end{array}$

$\begin{array}{llllllllllllll}-1.9266 & 0.4344 & -0.8178 & \text { H } & 0 & 0 & 0 & 0 & 0 & 0 & 0 & 0 & 0 & 19\end{array}$

$\begin{array}{llrlllllllllll}-2.1294 & 0.1998 & 1.6455 & \mathrm{H} & 0 & 0 & 0 & 0 & 0 & 0 & 0 & 0 & 0 & 20 \\ -0.5952 & 0.6202 & -3.9881 & \mathrm{H} & 0 & 0 & 0 & 0 & 0 & 0 & 0 & 0 & 0 & 21\end{array}$

$\begin{array}{lllllll}2 & 1 & 0 & 0 & 0 & 0\end{array}$

$\begin{array}{lllllll}2 & 1 & 0 & 0 & 0 & 0 \\ 3 & 1 & 0 & 0 & 0 & 0\end{array}$

5 . $4 \begin{array}{llllll} & 4 & 0 & 0 & 0 & 0\end{array}$

$\begin{array}{lllll}4 & 0 & 0 & 0\end{array}$

$\begin{array}{lllll}7 & 0 & 0 & 0\end{array}$

$\begin{array}{lllllll}12 & 7 & 0 & 0 & 0 & 0 \\ 17 & 7 & 0 & 0 & 0 & 0\end{array}$

$\begin{array}{llllllll}9 & 8 & 0 & 0 & 0 & 0\end{array}$

$\begin{array}{llllllllll}18 & 8 & 0 & 0 & 0 & 0\end{array}$

$\begin{array}{llllll}10 & 9 & 0 & 0 & 0\end{array}$

$\begin{array}{llllll}1 & 10 & 0 & 0 & 0 & 0\end{array}$

$\begin{array}{lllllll}1 & 10 & 10 & 0 & 0 & 0 & 0\end{array}$

211100000

$\begin{array}{lllllll}4 & 12 & 0 & 0 & 0 & 0 & 0\end{array}$

$\begin{array}{llllll}13 & 0 & 0 & 0 & 0 & 0\end{array}$

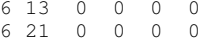

END

-butylbenzoicacid-anion.sdf

Cosmotherm

$\begin{array}{llllllllllll}32 & 30 & 0 & 0 & 0 & 0 & 0 & 0 & 0 & 0 & 0 & \mathrm{~V} 2000\end{array}$

$\begin{array}{llllllllllllll}-7.0625 & 0.5921 & 1.3293 & 0 & 0 & 0 & 0 & 0 & 0 & 0 & 0 & 0 & 0 & 1\end{array}$

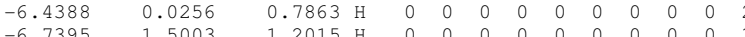

$\begin{array}{rrrrrrrrrrrrrr}-5.4093 & -1.0348 & -0.0851 & 0 & 0 & 0 & 0 & 0 & 0 & 0 & 0 & 0 & 0 & 4\end{array}$

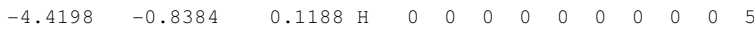

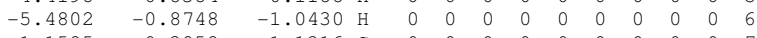

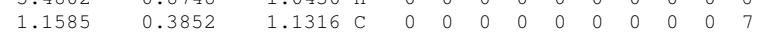

$\begin{array}{llllllllllllll}-0.1927 & 0.0822 & 0.9459 & \mathrm{C} & 0 & 0 & 0 & 0 & 0 & 0 & 0 & 0 & 0 & 8\end{array}$

$\begin{array}{llllllllllllll}-0.7605 & 0.0913 & -0.3389 & \mathrm{C} & 0 & 0 & 0 & 0 & 0 & 0 & 0 & 0 & 0 & 9\end{array}$

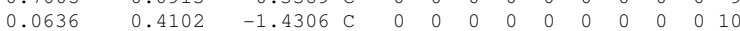

$\begin{array}{lllllllllllll}1.4155 & 0.7076 & -1.2434 \mathrm{C} & 0 & 0 & 0 & 0 & 0 & 0 & 0 & 0 & 0 & 11\end{array}$

$\begin{array}{llllllllllllllll}1.9884 & 0.7021 & 0.0411 \mathrm{C} & 0 & 0 & 0 & 0 & 0 & 0 & 0 & 0 & 0 & 12\end{array}$

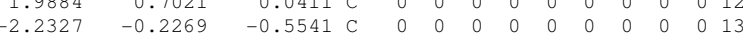

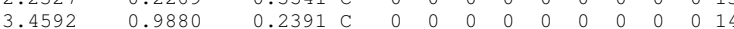

$\begin{array}{lllllllllllllll}4.3320 & -0.2847 & 0.1874 & C & 0 & 0 & 0 & 0 & 0 & 0 & 0 & 0 & 0 & 15\end{array}$

$\begin{array}{llllllllllllllll}5.8253 & 0.0029 & 0.3866 \mathrm{C} & 0 & 0 & 0 & 0 & 0 & 0 & 0 & 0 & 0 & 16\end{array}$

$\begin{array}{llllllllllllll}6.6909 & -1.2619 & 0.3471 & \mathrm{C} & 0 & 0 & 0 & 0 & 0 & 0 & 0 & 0 & 0 & 17\end{array}$

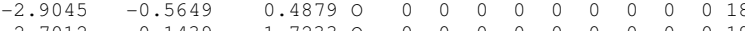

$\begin{array}{llllllllllllllll}-2.7012 & -0.1439 & -1.7233 & 0 & 0 & 0 & 0 & 0 & 0 & 0 & 0 & 0 & 0 & 18 \\ 1.5788 & 0.3822 & 2.1410 & 0 & 0 & 0 & 0 & 0 & 0 & 0 & 0 & 0 & 0 & 19\end{array}$

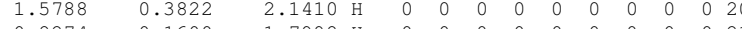

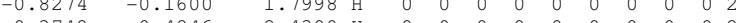

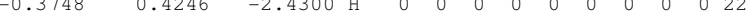

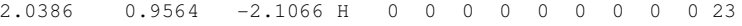

$3.6108-1.4839-1.2119 \mathrm{H}=0$

$1.6896-0.5370 \mathrm{H}$

$\begin{array}{llllllllllllll}3.9863 & -0.9909 & 0.9619 & \mathrm{H} & 0 & 0 & 0 & 0 & 0 & 0 & 0 & 0 & 0 & 27\end{array}$

$\begin{array}{lllll}5.9682 & 0.5182 & 1.3519 \mathrm{H} & 0 & \\ 6.1647 & 0.7077 & -0.3917 & & 0\end{array}$

$\begin{array}{llllllllllllll}6.1647 & 0.7077 & -0.3917 & \mathrm{H} & 0 & 0 & 0 & 0 & 0 & 0 & 0 & 0 & 0 & 29\end{array}$

$\begin{array}{llllllllllllll}7.7554 & -1.0243 & 0.4921 & \mathrm{H} & 0 & 0 & 0 & 0 & 0 & 0 & 0 & 0 & 0 & 30\end{array}$

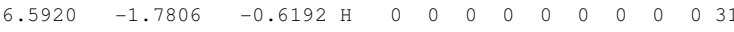
$6.3949-1.9694$

1.1373

$\begin{array}{lllllll}2 & 1 & 0 & 0 & 0 & 0 \\ 3 & 1 & 0 & 0 & 0 & 0\end{array}$

$\begin{array}{llllll}5 & 4 & 0 & 0 & 0\end{array}$

$\begin{array}{lllll}4 & 0 & 0 & 0 \\ 7 & 0 & 0 & 0\end{array}$

$\begin{array}{llllllllll}12 & 7 & 0 & 0 & 0\end{array}$

$\begin{array}{lllllll}20 & 7 & 0 & 0 & 0 & 0\end{array}$

$\begin{array}{lllll}9 & 8 & 0 & 0 & 0 \\ 1 & 8 & 0 & 0 & 0\end{array}$ 


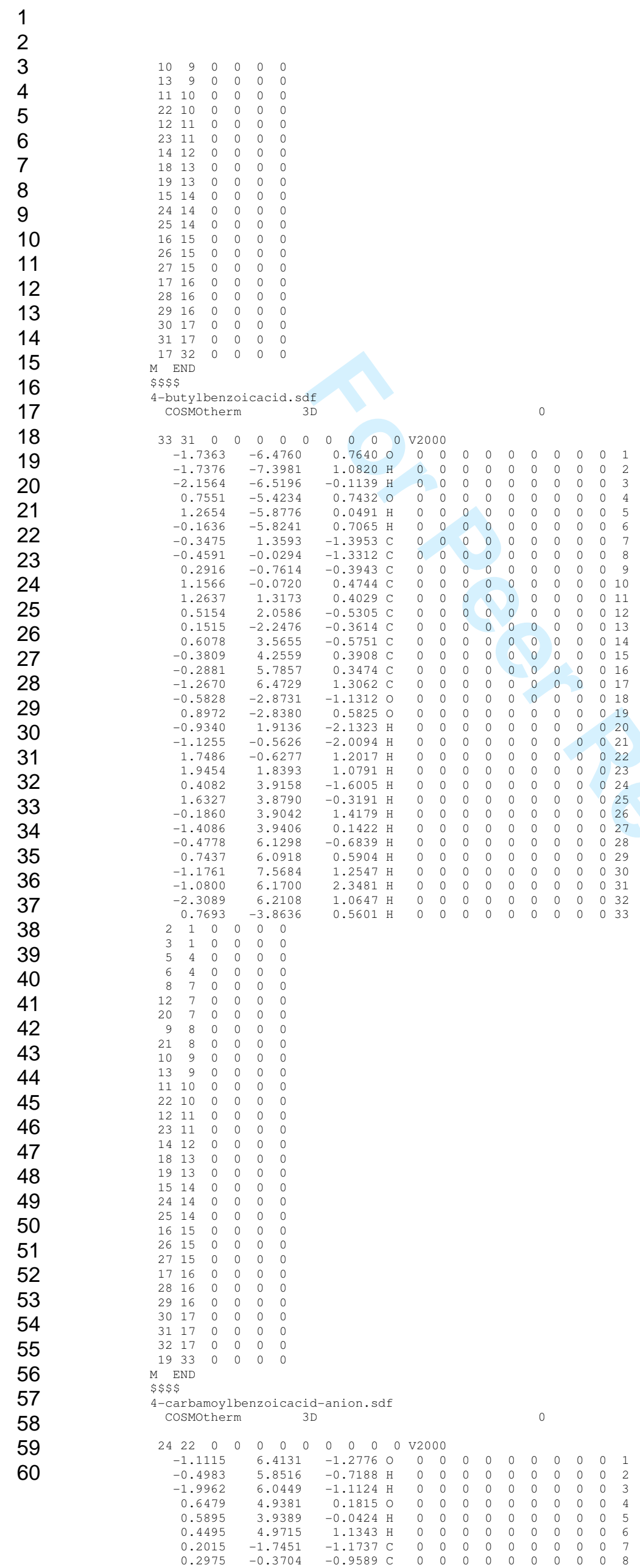




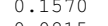

$\begin{array}{rrrrrrrrrrrrr}0.7112 & 1.4034 & \mathrm{C} & 0 & 0 & 0 & 0 & 0 & 0 & 0 & 0 & 0 & 10\end{array}$

$\begin{array}{llrlllllllllll}-0.0486 & -2.6188 & -0.1928 & \mathrm{C} & 0 & 0 & 0 & 0 & 0 & 0 & 0 & 0 & 0 & 11 \\ 0.2648 & 1.6666 & 0.5820 & \mathrm{C} & 0 & 0 & 0 & 0 & 0 & 0 & 0 & 0 & 0 & 12\end{array}$

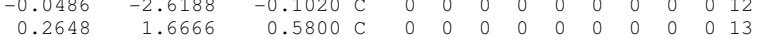

$\begin{array}{llllllllllllll}-0.1292 & -4.0930 & -0.3918 & \mathrm{C} & 0 & 0 & 0 & 0 & 0 & 0 & 0 & 0 & 0 & 14\end{array}$

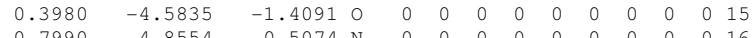

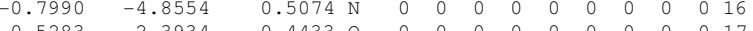

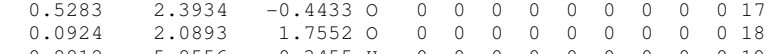

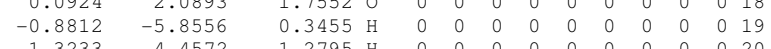

$\begin{array}{rrrrrrrrrrrrrr}-1.3233 & -4.4572 & 1.2795 & \mathrm{H} & 0 & 0 & 0 & 0 & 0 & 0 & 0 & 0 & 0 & 20 \\ 0.3165 & -2.1592 & -2.1764 & \mathrm{H} & 0 & 0 & 0 & 0 & 0 & 0 & 0 & 0 & 0 & 21\end{array}$

$\begin{array}{rrrrrrrrrrrrrr}0.3165 & -2.1592 & -2.1764 & \mathrm{H} & 0 & 0 & 0 & 0 & 0 & 0 & 0 & 0 & 0 & 21 \\ 0.4846 & 0.3077 & -1.7923 & \mathrm{H} & 0 & 0 & 0 & 0 & 0 & 0 & 0 & 0 & 0 & 22\end{array}$

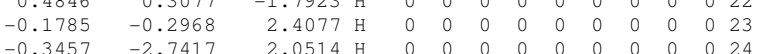

$\begin{array}{lllllll}3 & -1 & 0 & 0 & 0 & 0\end{array}$

$\begin{array}{llllll}5 & 4 & 0 & 0 & 0 & 0\end{array}$

$\begin{array}{lllll}4 & 0 & 0 & 0 & 0\end{array}$

$\begin{array}{rrrrrr}8 & 7 & 0 & 0 & 0 & 0 \\ 12 & 7 & 0 & 0 & 0 & 0 \\ 21 & 7 & 0 & 0 & 0 & 0\end{array}$

$\begin{array}{cccccc}12 & 7 & 0 & 0 & 0 & 0\end{array}$

$\begin{array}{rrrrrr}9 & 8 & 0 & 0 & 0 & 0\end{array}$

$\begin{array}{lllllll}22 & 8 & 0 & 0 & 0 & 0\end{array}$

$\begin{array}{lllllllll}10 & 9 & 0 & 0 & 0 & 0 & 0 \\ 13 & 9 & 0 & 0 & 0 & 0\end{array}$

$\begin{array}{lllllllll}11 & 10 & 0 & 0 & 0 & 0\end{array}$

$\begin{array}{lllllll}11 & 10 & 10 & 0 & 0 & 0 & 0\end{array}$

$\begin{array}{llllll}12 & 11 & 0 & 0 & 0 & 0\end{array}$

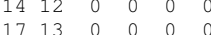

$\begin{array}{llllll}18 & 13 & 0 & 0 & 0 & 0\end{array}$

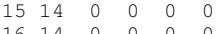

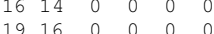

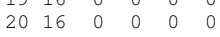

1124

$\$ \$ \$$

4-carbamoylbenzoicacid.sdf

Cosmotherm $3 \mathrm{D}$

$\begin{array}{lllllllllllll}25 & 23 & 0 & 0 & 0 & 0 & 0 & 0 & 0 & 0 & 0 & \mathrm{v} 2000\end{array}$

$\begin{array}{llllllllllllll}-0.8195 & -1.3390 & -6.2495 & 0 & 0 & 0 & 0 & 0 & 0 & 0 & 0 & 0 & 0\end{array}$

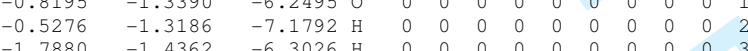

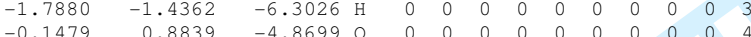

$\begin{array}{lllllllllllll}-0.1479 & 0.8839 & -4.8699 & 0 & 0 & 0 & 0 & 0 & 0 & 0 & 0 & 0 & 0 \\ -0.3744 & 0.0612 & -5.3999 & \mathrm{H} & 0 & 0 & 0 & 0 & 0 & 0 & 0 & 0 & 0\end{array}$

$\begin{array}{cccccccccccccc}-0.3744 & 0.0612 & -5.3999 & \mathrm{H} & 0 & 0 & 0 & 0 & 0 & 0 & 0 & 0 & 0 & 5 \\ 0.7726 & 1.1010 & -5.1028 & \mathrm{H} & 0 & 0 & 0 & 0 & 0 & 0 & 0 & 0 & 0 & 6\end{array}$

$\begin{array}{lrrrrrrrrrrrrr}1.1567 & -0.3674 & 2.0658 & \mathrm{C} & 0 & 0 & 0 & 0 & 0 & 0 & 0 & 0 & 0 & 7\end{array}$

$\begin{array}{llllllllllllll}1.3548 & -0.2678 & 0.6912 & \mathrm{C} & 0 & 0 & 0 & 0 & 0 & 0 & 0 & 0 & 0 & 8 \\ 0.3088 & 0.1525 & -0.1474 & \mathrm{C} & 0 & 0 & 0 & 0 & 0 & 0 & 0 & 0 & 0 & 9\end{array}$

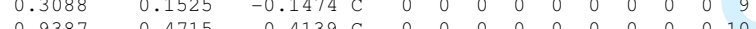

$\begin{array}{llllllllllllll}-0.9387 & 0.4715 & 0.4139 & \mathrm{C} & 0 & 0 & 0 & 0 & 0 & 0 & 0 & 0 & 0 & 10 \\ -1.1380 & 0.3644 & 1.7906 & \mathrm{C} & 0 & 0 & 0 & 0 & 0 & 0 & 0 & 0 & 0 & 11\end{array}$

$\begin{array}{rrrrrrrrrrrrrr}-1.1380 & 0.3644 & 1.7906 & \mathrm{C} & 0 & 0 & 0 & 0 & 0 & 0 & 0 & 0 & 0 & 11 \\ -0.0933 & -0.0603 & 2.6294 & \mathrm{C} & 0 & 0 & 0 & 0 & 0 & 0 & 0 & 0 & 0 & 12\end{array}$

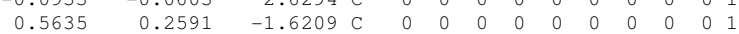

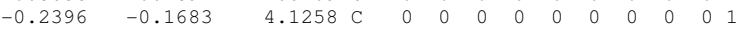

$\begin{array}{llllllllllllll}0.7544 & -0.1163 & 4.8739 & 0 & 0 & 0 & 0 & 0 & 0 & 0 & 0 & 0 & 0 & 15\end{array}$

$\begin{array}{llllllllllllll}1.6660 & 0.0363 & -2.1247 & 0 & 0 & 0 & 0 & 0 & 0 & 0 & 0 & 0 & 0 & 17\end{array}$

$\begin{array}{llllllllllllll}-0.5134 & 0.6193 & -2.3254 & 0 & 0 & 0 & 0 & 0 & 0 & 0 & 0 & 0 & 0 & 18\end{array}$

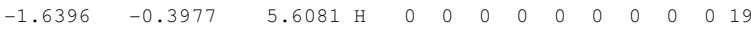

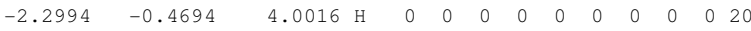

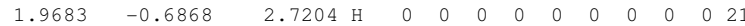

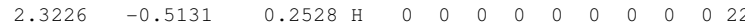

$\begin{array}{lllllllllllll}0.8103 & -0.2278 \mathrm{H} & 0 & 0 & 0 & 0 & 0 & 0 & 0 & 0 & 0 & 23\end{array}$

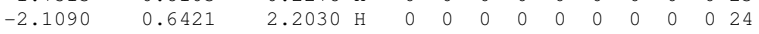

$-0.2854 \quad 0.6889$

$\begin{array}{lllllllllll}-3.3357 \mathrm{H} & 0 & 0 & 0 & 0 & 0 & 0 & 0 & 0 & 0 & 25\end{array}$

$\begin{array}{llllll}2 & 1 & 0 & 0 & 0 & 0 \\ 3 & 1 & 0 & 0 & 0 & 0\end{array}$

$\begin{array}{llllll}5 & 4 & 0 & 0 & 0 & 0\end{array}$

$\begin{array}{lllll}4 & 0 & 0 & 0 & 0\end{array}$

$\begin{array}{llllll}8 & 7 & 0 & 0 & 0\end{array}$

$\begin{array}{llllll}12 & 7 & 0 & 0 & 0 \\ 21 & 7 & 0 & 0 & 0 & 0\end{array}$

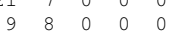

$\begin{array}{llllll}22 & 8 & 0 & 0 & 0 & 0\end{array}$

$\begin{array}{llllll}10 & 9 & 0 & 0 & 0 & 0\end{array}$

$\begin{array}{llllll}3 & 9 & 0 & 0 & 0 & 0\end{array}$

$\begin{array}{llllll}23 & 10 & 0 & 0 & 0 & 0\end{array}$

$\begin{array}{llllll}2 & 11 & 0 & 0 & 0\end{array}$

$\begin{array}{llllll}4 & 11 & 0 & 0 & 0\end{array}$

$\begin{array}{llllll}14 & 12 & 0 & 0 & 0 & 0\end{array}$

$\begin{array}{llllll}17 & 13 & 0 & 0 & 0\end{array}$

$\begin{array}{llllll}5 & 14 & 0 & 0 & 0\end{array}$

$\begin{array}{llllll}16 & 14 & 0 & 0 & 0 & 0\end{array}$

$19-16 \quad 0 \quad 00000$

$\begin{array}{llllll}8 & 25 & 0 & 0 & 0 & 0\end{array}$

END

-chlorobenzoicacid-anion.sdf

cosmotherm

$\begin{array}{llllllllllll}20 & 18 & 0 & 0 & 0 & 0 & 0 & 0 & 0 & 0 & 0 & \mathrm{~V} 2000\end{array}$

$\begin{array}{llllllllllll}1.3241 & 0 & 0 & 0 & 0 & 0 & 0 & 0 & 0 & 0 & 0 & 1\end{array}$

$\begin{array}{llllllllllllll}-5.5888 & 0.4039 & 0.7207 & \mathrm{H} & 0 & 0 & 0 & 0 & 0 & 0 & 0 & 0 & 0 & 2\end{array}$

$\begin{array}{lllllllllllll}-5.7084 & 1.8406 & 1.3187 & \mathrm{H} & 0 & 0 & 0 & 0 & 0 & 0 & 0 & 0 & 0\end{array}$

$\begin{array}{llllllllllllll}-4.6808 & -0.6624 & -0.2759 & 0 & 0 & 0 & 0 & 0 & 0 & 0 & 0 & 0 & 0 & 4 \\ -3.6841 & -0.6296 & -0.0335 & \mathrm{H} & 0 & 0 & 0 & 0 & 0 & 0 & 0 & 0 & 0 & 5\end{array}$

$\begin{array}{rrrrrrrrrrrrrr}-3.6841 & -0.6296 & -0.0335 & \mathrm{H} & 0 & 0 & 0 & 0 & 0 & 0 & 0 & 0 & 0 & 5 \\ -4.6994 & -0.3617 & -1.2019 & \mathrm{H} & 0 & 0 & 0 & 0 & 0 & 0 & 0 & 0 & 0 & 6 \\ 1.9828 & -0.3447 & 1.1982 & \mathrm{C} & 0 & 0 & 0 & 0 & 0 & 0 & 0 & 0 & 0 & 7\end{array}$ 


$\begin{array}{rrrrrrrrrrrrrr}0.6103 & -0.4306 & 0.9483 & \mathrm{C} & 0 & 0 & 0 & 0 & 0 & 0 & 0 & 0 & 0 & 8 \\ 0.0825 & -0.1159 & -0.3141 & \mathrm{C} & 0 & 0 & 0 & 0 & 0 & 0 & 0 & 0 & 0 & 9 \\ 0.9607 & 0.2894 & -1.3315 & \mathrm{C} & 0 & 0 & 0 & 0 & 0 & 0 & 0 & 0 & 0 & 10 \\ 2.3363 & 0.3811 & -1.1047 & \mathrm{C} & 0 & 0 & 0 & 0 & 0 & 0 & 0 & 0 & 0 & 11 \\ 2.8273 & 0.0613 & 0.1629 & \mathrm{C} & 0 & 0 & 0 & 0 & 0 & 0 & 0 & 0 & 0 & 12 \\ -1.4146 & -0.2066 & -0.5860 & \mathrm{C} & 0 & 0 & 0 & 0 & 0 & 0 & 0 & 0 & 0 & 13 \\ 4.5731 & 0.1744 & 0.4665 & \mathrm{Cl} & 0 & 0 & 0 & 0 & 0 & 0 & 0 & 0 & 0 & 14 \\ -2.1460 & -0.6090 & 0.3885 & 0 & 0 & 0 & 0 & 0 & 0 & 0 & 0 & 0 & 0 & 15 \\ -1.8324 & 0.1133 & -1.7320 & 0 & 0 & 0 & 0 & 0 & 0 & 0 & 0 & 0 & 0 & 16 \\ 2.3879 & -0.5886 & 2.1805 & \mathrm{H} & 0 & 0 & 0 & 0 & 0 & 0 & 0 & 0 & 0 & 17 \\ -0.0679 & -0.7460 & 1.7419 & \mathrm{H} & 0 & 0 & 0 & 0 & 0 & 0 & 0 & 0 & 0 & 18 \\ 0.5525 & 0.5346 & -2.3129 & \mathrm{H} & 0 & 0 & 0 & 0 & 0 & 0 & 0 & 0 & 0 & 19 \\ 3.0141 & 0.6950 & -1.8988 & \mathrm{H} & 0 & 0 & 0 & 0 & 0 & 0 & 0 & 0 & 0 & 20\end{array}$




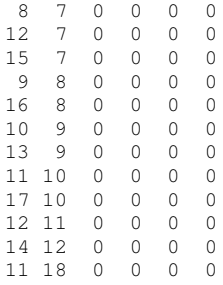

1118

$M$ E

4-chlorophenol.sdf

cosmotherm $3 \mathrm{D}$

$\begin{array}{llllllllllll}19 & 17 & 0 & 0 & 0 & 0 & 0 & 0 & 0 & 0 & 0 & \text { v2 } 2000\end{array}$

$\begin{array}{llllllllllllll}-1.7217 & 4.7271 & 0.8187 & 0 & 0 & 0 & 0 & 0 & 0 & 0 & 0 & 0 & 0\end{array}$

$\begin{array}{llllllllllllll}-2.5087 & 4.1585 & 0.8985 & \mathrm{H} & 0 & 0 & 0 & 0 & 0 & 0 & 0 & 0 & 0 & 2\end{array}$

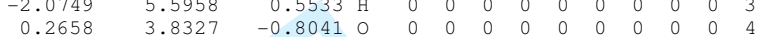

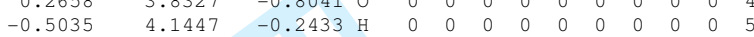

$\begin{array}{llllllllllllll}-0.0691 & 3.7923 & -1.7173 \text { H } & 0 & 0 & 0 & 0 & 0 & 0 & 0 & 0 & 0 & 6\end{array}$

$\begin{array}{llllllllllllll}1.7447 & -0.5877 & 0.4549 & \mathrm{C} & 0 & 0 & 0 & 0 & 0 & 0 & 0 & 0 & 0 & 7 \\ 1.1630 & -1.8570 & 0.4527 & \mathrm{C} & 0 & 0 & 0 & 0 & 0 & 0 & 0 & 0 & 0 & 8\end{array}$

$\begin{array}{lllllllllllll}-0.1501 & -2.0072 & -0.0020 & \mathrm{C} & 0 & 0 & 0 & 0 & 0 & 0 & 0 & 0 & 0\end{array}$

$\begin{array}{llllllllllllll}-0.8861 & -0.9111 & -0.4525 & \text { C } & 0 & 0 & 0 & 0 & 0 & 0 & 0 & 0 & 0 & 10\end{array}$

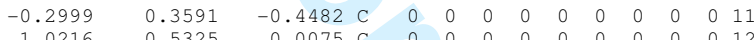

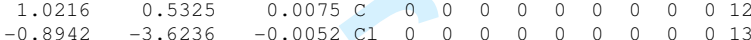

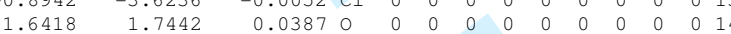

$\begin{array}{lllllllllllllll}2.7696 & -0.4560 & 0.8068 \mathrm{H} & 0 & 0 & 0 & 0 & 0 & 0 & 0 & 0 & 0 & 15\end{array}$

$\begin{array}{lllllllllllll}1.7320 & -2.7200 & 0.8018 \text { H } & 0 & 0 & 0 & 0 & 0 & 0 & 0 & 0 & 0 & 16\end{array}$

$\begin{array}{llllllllllllll}-1.9092 & -1.0373 & -0.8072 & \mathrm{H} & 0 & 0 & 0 & 0 & 0 & 0 & 0 & 0 & 0 & 16 \\ -0.8749 & 1.2174 & -0.8000 & \mathrm{H} & 0 & 0 & 0 & 0 & 0 & 0 & 0 & 0 & 0 & 18\end{array}$

$\begin{array}{lllllllllllll}1.0402 & 2.4831 & -0.3057 \mathrm{H} & 0 & 0 & 0 & 0 & 0 & 0 & 0 & 0 & 0 & 19\end{array}$

$\begin{array}{llllll}2 & 1 & 0 & 0 & 0 & 0 \\ 3 & 1 & 0 & 0 & 0 & 0\end{array}$

$\begin{array}{lllllll}3 & 1 & 0 & 0 & 0 & 0\end{array}$

$\begin{array}{llllll}4 & 0 & 0 & 0 & 0\end{array}$

$\begin{array}{lllll}0 & 0 & 0\end{array}$

$\begin{array}{llllllll}5 & 7 & 0 & 0 & 0 & 0\end{array}$

$\begin{array}{llllll}8 & 0 & 0 & 0 & 0\end{array}$

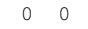

$\begin{array}{lllllll}3 & 9 & 0 & 0 & 0 & 0\end{array}$

$\begin{array}{llllll}1 & 10 & 0 & 0 & 0 & 0\end{array}$

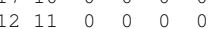

$\begin{array}{llllll}11 & 0 & 0 & 0 & 0\end{array}$

$\begin{array}{llllll}14 & 12 & 0 & 0 & 0 & 0 \\ 14 & 19 & 0 & 0 & 0 & 0\end{array}$

END

$\$ \$ \$$

4-cyanobenzoicacid-anion.sdf
Cosmotherm 3 D

$\begin{array}{llllllllllll}21 & 19 & 0 & 0 & 0 & 0 & 0 & 0 & 0 & 0 & 0 & \mathrm{~V} 2000\end{array}$

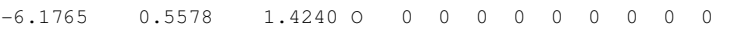

$\begin{array}{llllllllllllll}-5.5352 & 0.1903 & 0.7488 & \text { H } & 0 & 0 & 0 & 0 & 0 & 0 & 0 & 0 & 0 & 2\end{array}$

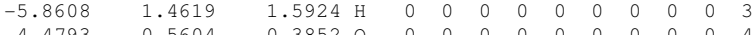

$\begin{array}{llllllllllllll}-4.4793 & -0.5604 & -0.3852 & 0 & 0 & 0 & 0 & 0 & 0 & 0 & 0 & 0 & 0 & 3 \\ -3.4937 & -0.4754 & -0.1249 & \mathrm{H} & 0 & 0 & 0 & 0 & 0 & 0 & 0 & 0 & 0 & 5\end{array}$

$\begin{array}{llllllllllllll}-4.5277 & -0.1442 & -1.2639 \mathrm{H} & 0 & 0 & 0 & 0 & 0 & 0 & 0 & 0 & 0 & 6\end{array}$

$\begin{array}{llllllllllllll}2.1389 & -0.1456 & 1.2824 & \mathrm{C} & 0 & 0 & 0 & 0 & 0 & 0 & 0 & 0 & 0 & 7\end{array}$

$\begin{array}{llllllllllllll}0.7754 & -0.1813 & 0.9993 & \mathrm{C} & 0 & 0 & 0 & 0 & 0 & 0 & 0 & 0 & 0 & 8\end{array}$

$\begin{array}{lllllllllllll}0.3089 & -0.0282 & -0.3166 & \mathrm{C} & 0 & 0 & 0 & 0 & 0 & 0 & 0 & 0 & 0\end{array}$

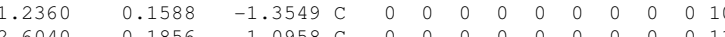

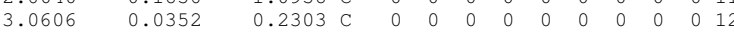

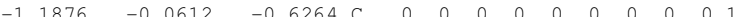

$4.4606=0.0645$
5.6061

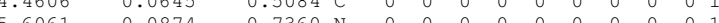

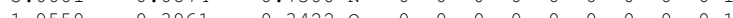

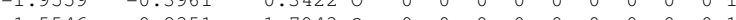

$\begin{array}{llllllllllllll}-1.5546 & -0.2351 & -1.7943 & 0 & 0 & 0 & 0 & 0 & 0 & 0 & 0 & 0 & 0 & 16 \\ 0.0967 & -0.2590 & -2.3060 & 0 & 0 & 0 & 0 & 0 & 0 & 0 & 0 & 0 & 0 & 17\end{array}$

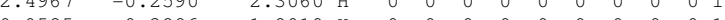

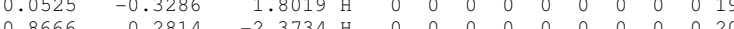

$\begin{array}{lllllllllllllllll}3.3204 & 0.28233 & -1.9061 & \mathrm{H} & 0 & 0 & 0 & 0 & 0 & 0 & 0 & 0 & 0 & 21\end{array}$

$\begin{array}{lllllll}2 & 1 & 0 & 0 & 0 & 0 & 0\end{array}$

$\begin{array}{llllll}1 & 1 & 0 & 0 & 0 & 0\end{array}$

$\begin{array}{llllll}4 & 0 & 0 & 0 & 0\end{array}$

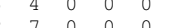

$\begin{array}{llllllll}8 & 7 & 0 & 0 & 0 & 0\end{array}$

$\begin{array}{lllllll}7 & 0 & 0 & 0 & 0 & 0 \\ 9 & 8 & 0 & 0 & 0 & 0 & 0\end{array}$

$\begin{array}{lllll}0 & 0 & 0 & 0 & 0\end{array}$

$\begin{array}{lllllll}19 & 8 & 0 & 0 & 0 & 0\end{array}$

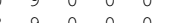

$\begin{array}{lllllll}2 & 0 & 0 & 0 & 0 & 0 \\ 0 & 10 & 0 & 0 & 0 & 0 & 0\end{array}$

$\begin{array}{lllllll}20 & 10 & 0 & 0 & 0 & 0\end{array}$

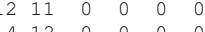

$\begin{array}{llllll}6 & 13 & 0 & 0 & 0 & 0\end{array}$

$\begin{array}{llllll}17 & 13 & 0 & 0 & 0 & 0 \\ 15 & 14 & 0 & 0 & 0 & 0\end{array}$

$\begin{array}{llllll}11 & 21 & 0 & 0 & 0 & 0\end{array}$

END

-cyanobenzoicacid.sdf 


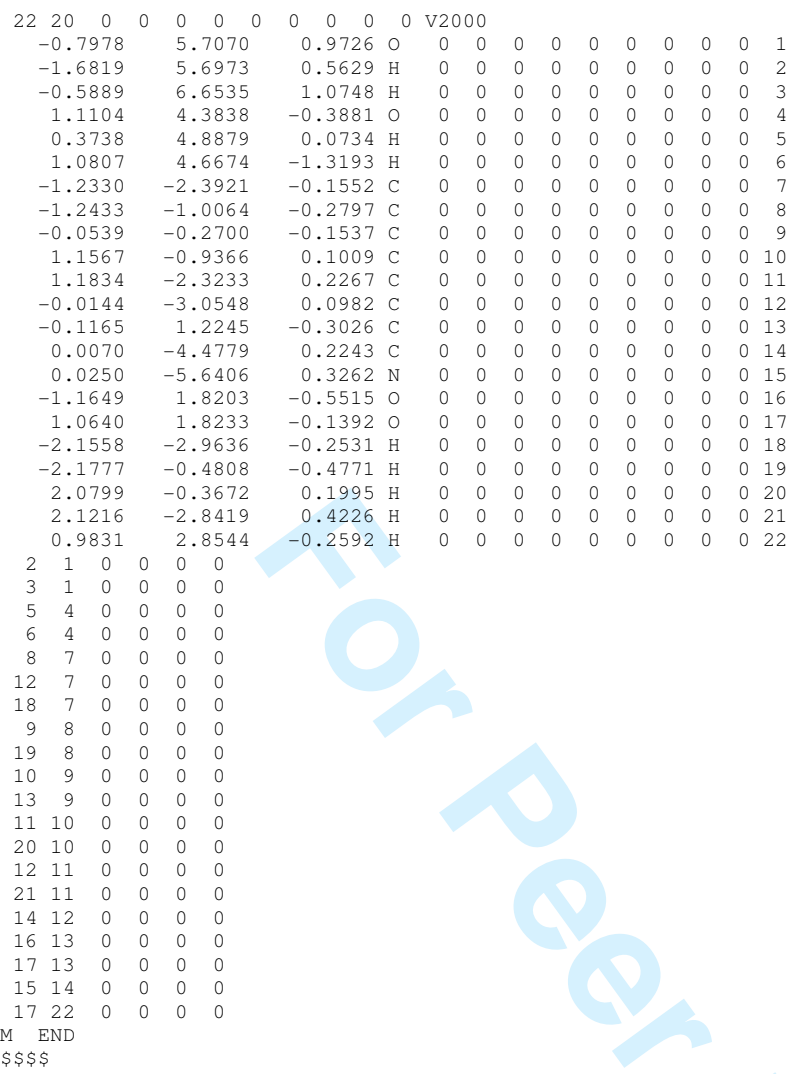


-dimethylaminobenzoicacid.sdf

$\begin{array}{llllllllllll}29 & 27 & 0 & 0 & 0 & 0 & 0 & 0 & 0 & 0 & 0 & \mathrm{v} 2000\end{array}$

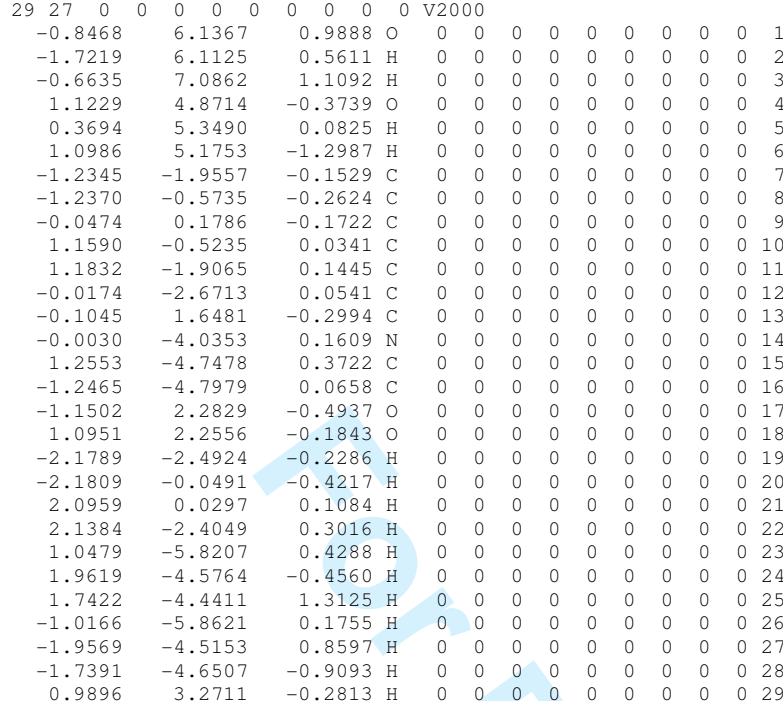

$\begin{array}{rrrrrrrrrrrrrr}-1.0166 & -5.8621 & 0.1755 & \mathrm{H} & 0 & 0 & 0 & 0 & 0 & 0 & 0 & 0 & 0 & 26 \\ -1.9569 & -4.5153 & 0.8597 & \mathrm{H} & 0 & 0 & 0 & 0 & 0 & 0 & 0 & 0 & 0 & 27 \\ -1.7391 & -4.6507 & -0.9093 & \mathrm{H} & 0 & 0 & 0 & 0 & 0 & 0 & 0 & 0 & 0 & 28 \\ 0.9896 & 3.2711 & -0.2813 & \mathrm{H} & 0 & 0 & 0 & 0 & 0 & 0 & 0 & 0 & 0 & 29 \\ 2 & 1 & 0 & 0 & 0 & 0 & & & & & & & & \end{array}$

$\begin{array}{llllll}3 & 1 & 0 & 0 & 0 & 0\end{array}$

$\begin{array}{llllll}5 & 4 & 0 & 0 & 0 & 0\end{array}$

$\begin{array}{lllllll}5 & 4 & 0 & 0 & 0 & 0 & \\ 8 & 7 & 0 & 0 & 0 & 0\end{array}$

$\begin{array}{rrrrrr}8 & 7 & 0 & 0 & 0 \\ 12 & 7 & 0 & 0 & 0 & 0 \\ 19 & 7 & 0 & 0 & 0 & 0\end{array}$

$\begin{array}{llllll}19 & 7 & 0 & 0 & 0 & 0\end{array}$

$\begin{array}{lllll}9 & 8 & 0 & 0 & 0\end{array}$

$\begin{array}{llllll}20 & 8 & 0 & 0 & 0 & 0\end{array}$

$\begin{array}{llllll}13 & 9 & 0 & 0 & 0 & 0\end{array}$

$\begin{array}{llllll}11 & 10 & 0 & 0 & 0 & 0\end{array}$

$\begin{array}{lll}0 & 0 & 0\end{array}$

$\begin{array}{llllll}12 & 11 & 0 & 0 & 0 & 0\end{array}$

$\begin{array}{llllll}14 & 12 & 0 & 0 & 0 & 0\end{array}$

$\begin{array}{llllll}17 & 13 & 0 & 0 & 0 & 0\end{array}$

$\begin{array}{llllll}15 & 14 & 0 & 0 & 0 & 0\end{array}$

$\begin{array}{llllll}16 & 14 & 0 & 0 & 0 & 0\end{array}$

$\begin{array}{lllll}23 & 15 & 0 & 0 & 0\end{array}$

$\begin{array}{lllllll}24 & 15 & 0 & 0 & 0 & 0 \\ 25 & 15 & 0 & 0 & 0 & 0\end{array}$

$\begin{array}{llllll}25 & 15 & 0 & 0 & 0 & 0\end{array}$

$\begin{array}{llllll}26 & 16 & 0 & 0 & 0 & 0 \\ 27 & 16 & 0 & 0 & 0 & 0\end{array}$

$\begin{array}{llllll}28 & 16 & 0 & 0 & 0 & 0 \\ 18 & 29 & 0 & 0 & 0 & 0\end{array}$

18 END

4-ethylbenzoicacid-anion.sdf

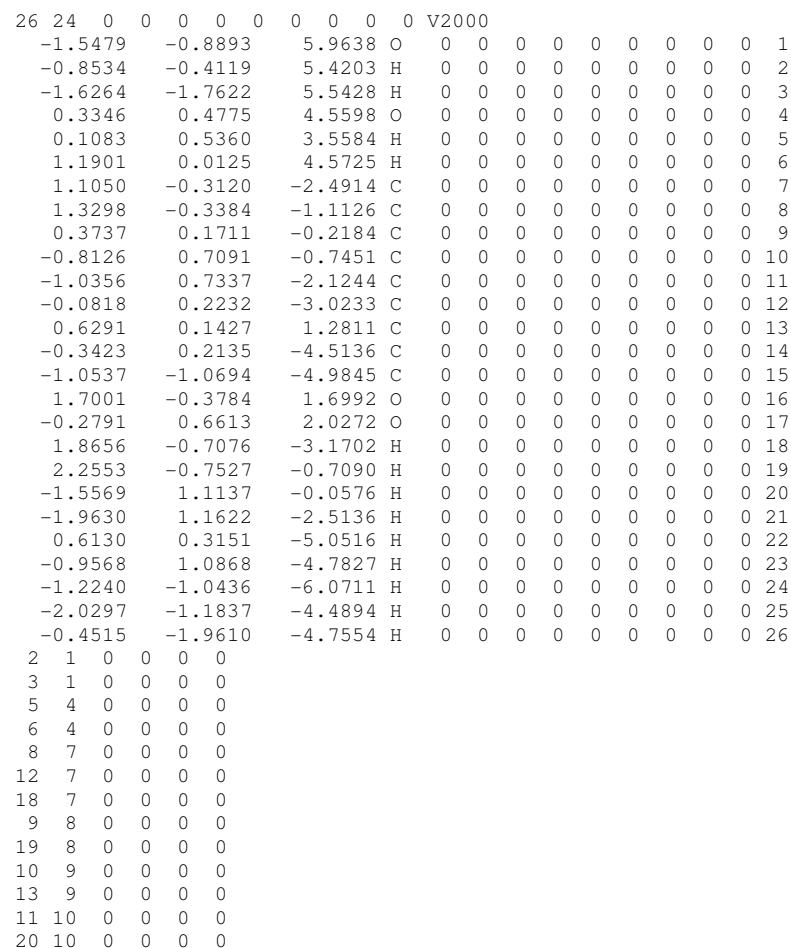




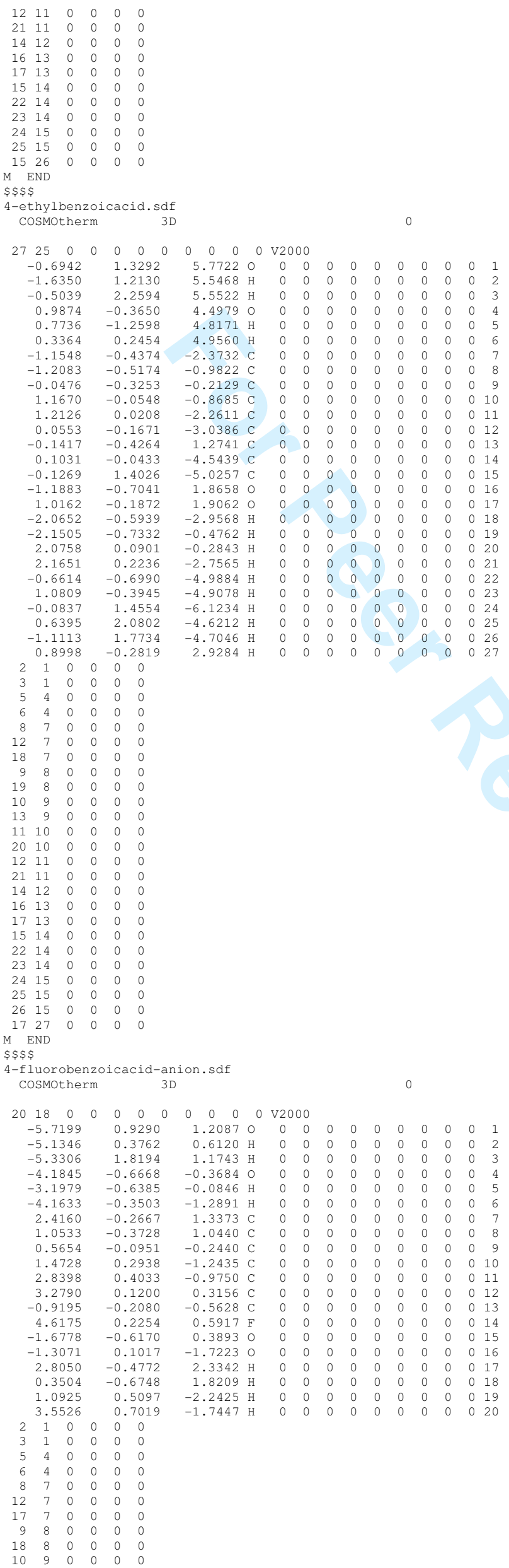




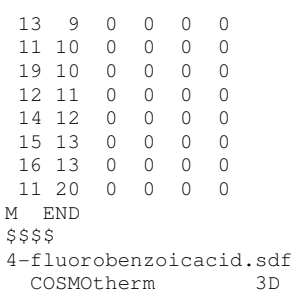

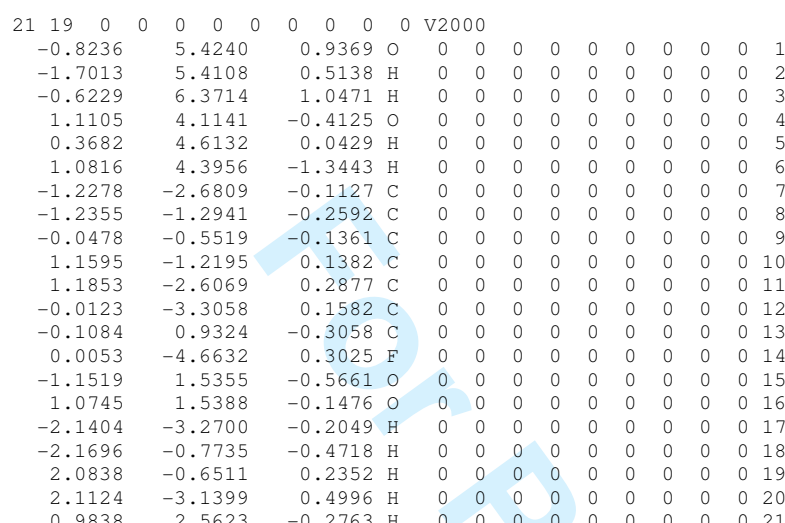
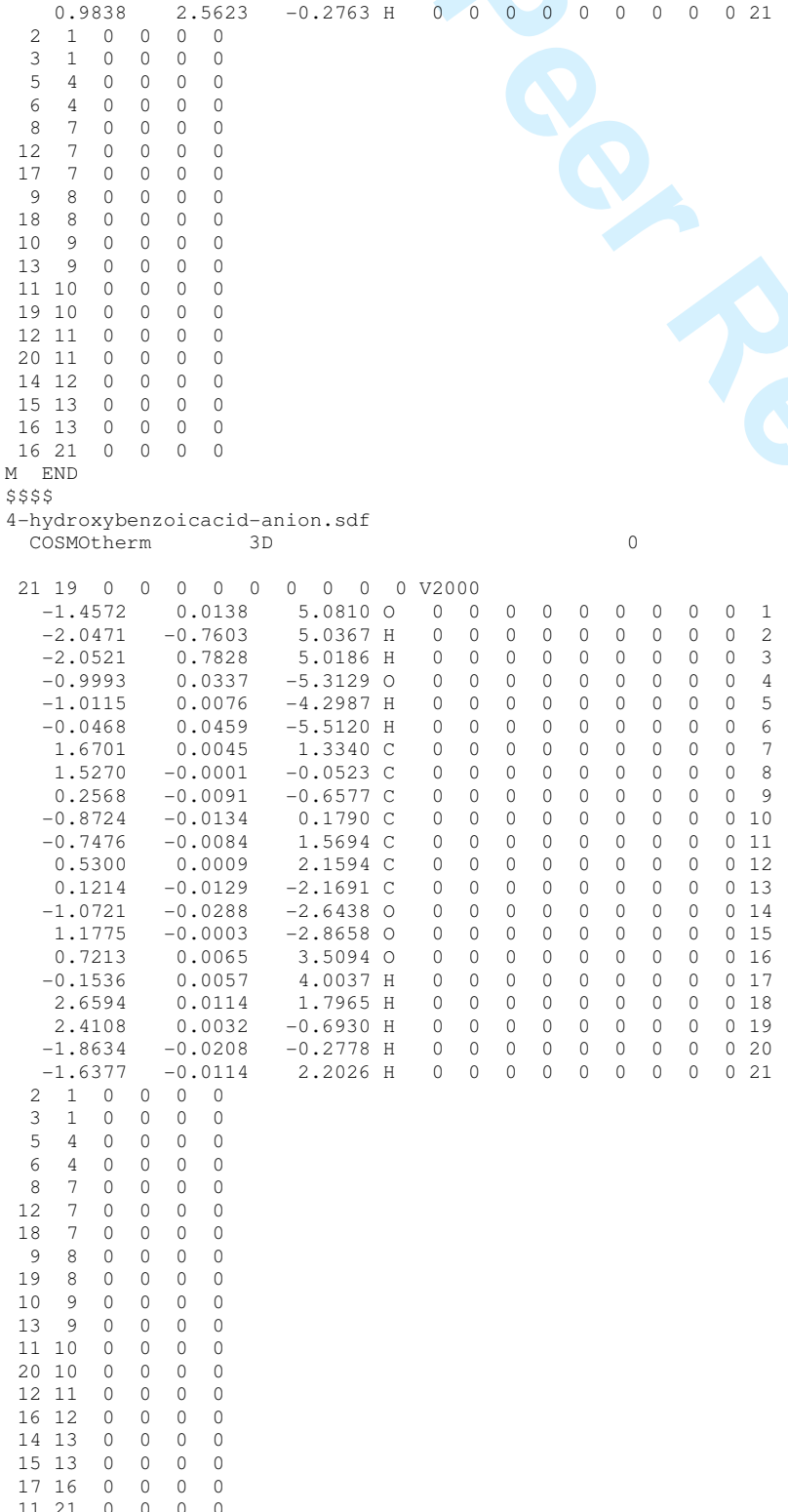

4-hydroxybenzoicacid.sdf

cosmotherm 


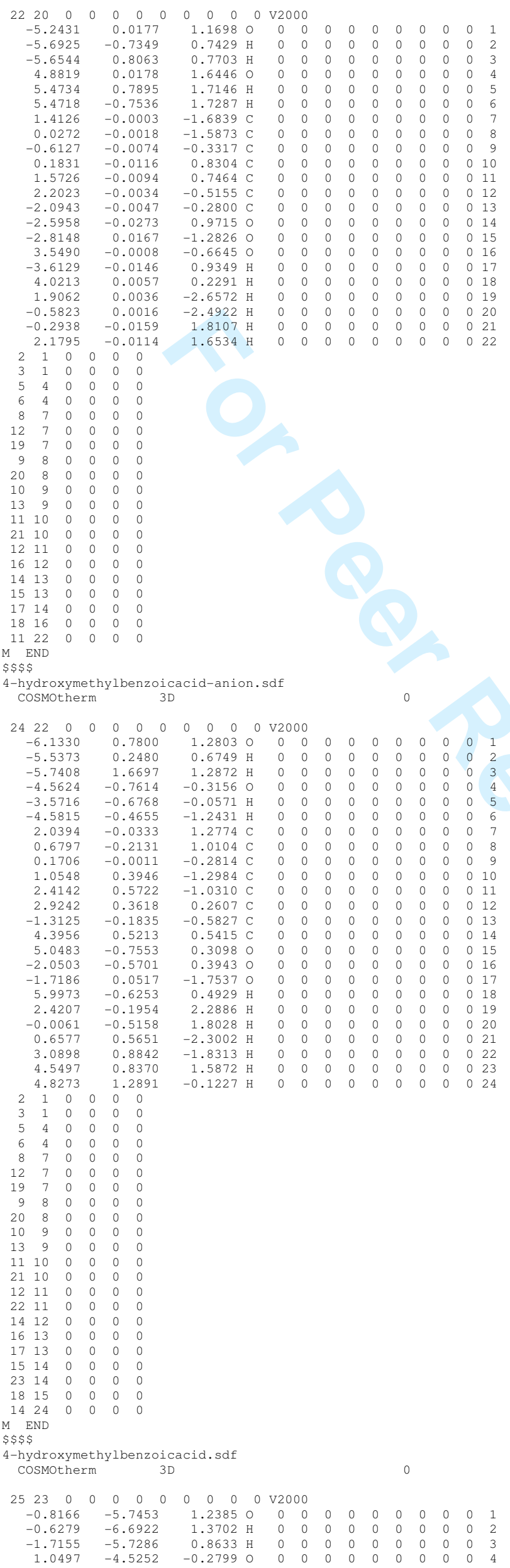


$\begin{array}{llllllllllllll}0.9554 & -4.8517 & -1.1925 & \mathrm{H} & 0 & 0 & 0 & 0 & 0 & 0 & 0 & 0 & 0 & 5\end{array}$

$\begin{array}{rrrrrrrrrrrrrr}0.3331 & -4.9894 & 0.2481 & \mathrm{H} & 0 & 0 & 0 & 0 & 0 & 0 & 0 & 0 & 0 & 6 \\ -1.1493 & 2.3179 & -0.4656 & \mathrm{C} & 0 & 0 & 0 & 0 & 0 & 0 & 0 & 0 & 0 & 7\end{array}$

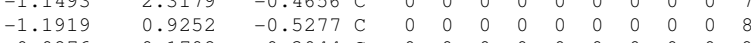

$\begin{array}{llllllllllllll}-0.0276 & 0.1702 & -0.3044 & \mathrm{C} & 0 & 0 & 0 & 0 & 0 & 0 & 0 & 0 & 0 & 9 \\ 1.1788 & 0.8335 & -0.0193 & \mathrm{C} & 0 & 0 & 0 & 0 & 0 & 0 & 0 & 0 & 0 & 10\end{array}$

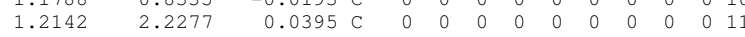

$\begin{array}{llllllllllllll}0.0535 & 2.9861 & -0.1830 & \mathrm{C} & 0 & 0 & 0 & 0 & 0 & 0 & 0 & 0 & 0 & 12\end{array}$

$\begin{array}{llllllllllllll}-0.1090 & -1.3224 & -0.3924 & \mathrm{C} & 0 & 0 & 0 & 0 & 0 & 0 & 0 & 0 & 0 & 13\end{array}$

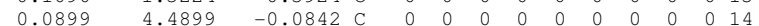

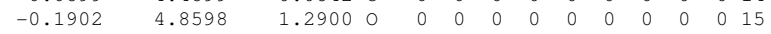

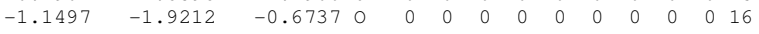

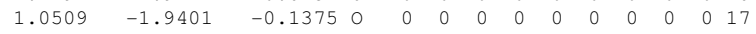

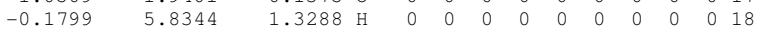

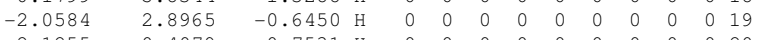

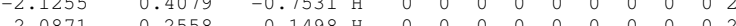

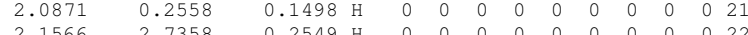

$\begin{array}{lllllllllllllllllll}-0.6658 & 4.73306 & -0.7557 & \mathrm{H} & 0 & 0 & 0 & 0 & 0 & 0 & 0 & 0 & 0 & 22\end{array}$

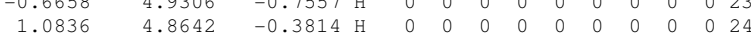

$\begin{array}{lll}0.9449 & -2.9687 & -0.2193 \mathrm{H}\end{array}$

15

16

17

18

19

20

21

$\begin{array}{llll}0 & 0 & 0\end{array}$

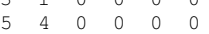

$\begin{array}{llllll}6 & 4 & 0 & 0 & 0 & 0 \\ 8 & 7 & 0 & 0 & 0 & 0\end{array}$

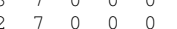

$\begin{array}{lllllll}2 & 7 & 0 & 0 & 0 & 0 & 0 \\ 9 & 7 & 0 & 0 & 0 & 0\end{array}$

$\begin{array}{lllllll}9 & 8 & 0 & 0 & 0 & 0\end{array}$

$\begin{array}{llllll}9 & 8 & 0 & 0 & 0 & 0 \\ 10 & 9 & 0 & 0 & 0 & 0\end{array}$

$\begin{array}{llllll}3 & 9 & 0 & 0 & 0 & 0\end{array}$

$\begin{array}{lllllll}11 & 10 & 0 & 0 & 0 & 0\end{array}$

$\begin{array}{llllll}12 & 11 & 0 & 0 & 0 & 0\end{array}$

$\begin{array}{llllll}22 & 11 & 0 & 0 & 0 & 0\end{array}$

$\begin{array}{llllll}16 & 13 & 0 & 0 & 0 & 0\end{array}$

$\begin{array}{llllll}17 & 13 & 0 & 0 & 0 & 0\end{array}$

$\begin{array}{lllllll}15 & 14 & 0 & 0 & 0 & 0 \\ 23 & 14 & 0 & 0 & 0 & 0\end{array}$

$\begin{array}{llllll}24 & 14 & 0 & 0 & 0 & 0\end{array}$

$\begin{array}{lllllll}8 & 15 & 0 & 0 & 0 & 0\end{array}$

$\begin{array}{lllll}25 & 0 & 0 & 0 & 0\end{array}$

END

4-iodobenzoicacid-anion.sdf

cosmotherm

$\begin{array}{llllllllllll}20 & 17 & 0 & 0 & 0 & 0 & 0 & 0 & 0 & 0 & 0 & \mathrm{~V} 2000\end{array}$

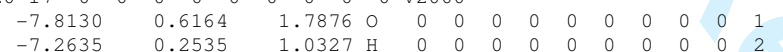

$\begin{array}{llllllllllllll}-7.2635 & 0.2535 & 1.0327 & \mathrm{H} & 0 & 0 & 0 & 0 & 0 & 0 & 0 & 0 & 0 & 2\end{array}$

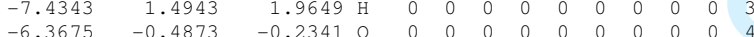

$\begin{array}{llllllllllllll}-6.3675 & -0.4873 & -0.2341 & 0 & 0 & 0 & 0 & 0 & 0 & 0 & 0 & 0 & 0 & 4 \\ -5.3561 & -0.4419 & -0.0685 & \mathrm{H} & 0 & 0 & 0 & 0 & 0 & 0 & 0 & 0 & 0 & 5\end{array}$

$\begin{array}{llllllllllllll}-5.3561 & -0.4419 & -0.0685 & \mathrm{H} & 0 & 0 & 0 & 0 & 0 & 0 & 0 & 0 & 0 & 5\end{array}$

$\begin{array}{llllllllllllll}-6.4914 & -0.0316 & -1.0852 & \mathrm{H} & 0 & 0 & 0 & 0 & 0 & 0 & 0 & 0 & 0 & 6 \\ 0.3522 & -0.2771 & 0.9984 & \mathrm{C} & 0 & 0 & 0 & 0 & 0 & 0 & 0 & 0 & 0 & 7\end{array}$

$\begin{array}{rrrrrrrrrrrrrr}0.3522 & -0.2771 & 0.9984 & \mathrm{C} & 0 & 0 & 0 & 0 & 0 & 0 & 0 & 0 & 0 & 7 \\ -1.0295 & -0.3071 & 0.7820 & \mathrm{C} & 0 & 0 & 0 & 0 & 0 & 0 & 0 & 0 & 0 & 8\end{array}$

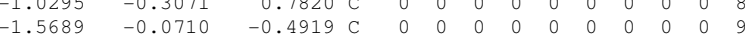

$\begin{array}{llllllllll}0 & 0 & 0 & 0 & 0 & 0 & 0 & 0 & 10 \\ 0 & 0 & 0 & 11\end{array}$

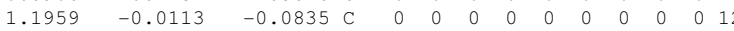

$\begin{array}{llllllllllllll}-3.0757 & -0.0968 & -0.7251 & \mathrm{C} & 0 & 0 & 0 & 0 & 0 & 0 & 0 & 0 & 0 & 13\end{array}$

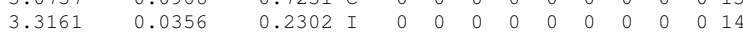

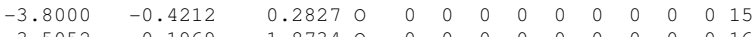

$\begin{array}{lllllllllllll}0.1969 & -1.8734 & 0 & 0 & 0 & 0 & 0 & 0 & 0 & 0 & 0 & 0 & 16\end{array}$

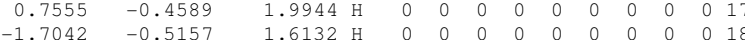

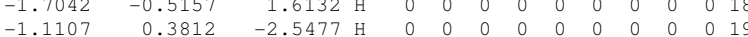

$\begin{array}{ll}1.3563 & 0.4307\end{array}$

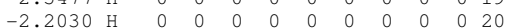

$\begin{array}{lllllll}2 & 1 & 0 & 0 & 0 & 0\end{array}$

$\begin{array}{llllll}3 & 1 & 0 & 0 & 0 & 0\end{array}$

$\begin{array}{lllll}4 & 0 & 0 & 0\end{array}$

$\begin{array}{lllll}4 & 0 & 0 & 0\end{array}$

$\begin{array}{lllllllll}2 & 7 & 0 & 0 & 0 & 0\end{array}$

$\begin{array}{lllll}7 & 0 & 0 & 0 & 0\end{array}$

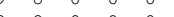

$\begin{array}{llllll}10 & 9 & 0 & 0 & 0 & 0\end{array}$

$\begin{array}{lllllll}3 & 9 & 0 & 0 & 0 & 0\end{array}$

$10 \begin{array}{lllll}10 & 0 & 0 & 0\end{array}$

$\begin{array}{llllll}10 & 0 & 0 & 0 & 0 & 0\end{array}$

$\begin{array}{llllllll}15 & 13 & 0 & 0 & 0 & 0\end{array}$

$\begin{array}{llllll}6 & 13 & 0 & 0 & 0\end{array}$

$\begin{array}{llllll}1 & 20 & 0 & 0 & 0 & 0\end{array}$

M END

Cosmotherm

$\begin{array}{llllllllllll}21 & 18 & 0 & 0 & 0 & 0 & 0 & 0 & 0 & 0 & 0 & \mathrm{~V} 2000\end{array}$

$\begin{array}{rrrrrrrrrrrrrr}-0.0919 & -1.5447 & -7.5842 & 0 & 0 & 0 & 0 & 0 & 0 & 0 & 0 & 0 & 0 & 1 \\ -0.0551 & -1.4210 & -8.5507 & \mathrm{H} & 0 & 0 & 0 & 0 & 0 & 0 & 0 & 0 & 0 & 2 \\ -0.7827 & -2.2201 & -7.4545 & \mathrm{H} & 0 & 0 & 0 & 0 & 0 & 0 & 0 & 0 & 0 & 3 \\ -0.6872 & 0.7389 & -6.2671 & 0 & 0 & 0 & 0 & 0 & 0 & 0 & 0 & 0 & 0 & 4 \\ -0.4465 & -0.0963 & -6.7708 & \mathrm{H} & 0 & 0 & 0 & 0 & 0 & 0 & 0 & 0 & 0 & 5 \\ -0.0496 & 1.4147 & -6.5593 & \mathrm{H} & 0 & 0 & 0 & 0 & 0 & 0 & 0 & 0 & 0 & 6 \\ 1.3120 & 0.0278 & 0.6022 & \mathrm{C} & 0 & 0 & 0 & 0 & 0 & 0 & 0 & 0 & 0 & 7 \\ 1.3954 & 0.1436 & -0.7863 & \mathrm{C} & 0 & 0 & 0 & 0 & 0 & 0 & 0 & 0 & 0 & 8 \\ 0.2355 & 0.2696 & -1.5678 & \mathrm{C} & 0 & 0 & 0 & 0 & 0 & 0 & 0 & 0 & 0 & 9 \\ -1.0204 & 0.2789 & -0.9385 & \mathrm{C} & 0 & 0 & 0 & 0 & 0 & 0 & 0 & 0 & 0 & 10 \\ -1.1203 & 0.1662 & 0.4505 & \mathrm{C} & 0 & 0 & 0 & 0 & 0 & 0 & 0 & 0 & 0 & 11 \\ 0.0501 & 0.0421 & 1.2046 & & 0 & 0 & 0 & 0 & 0 & 0 & 0 & 0 & 0 & 12\end{array}$




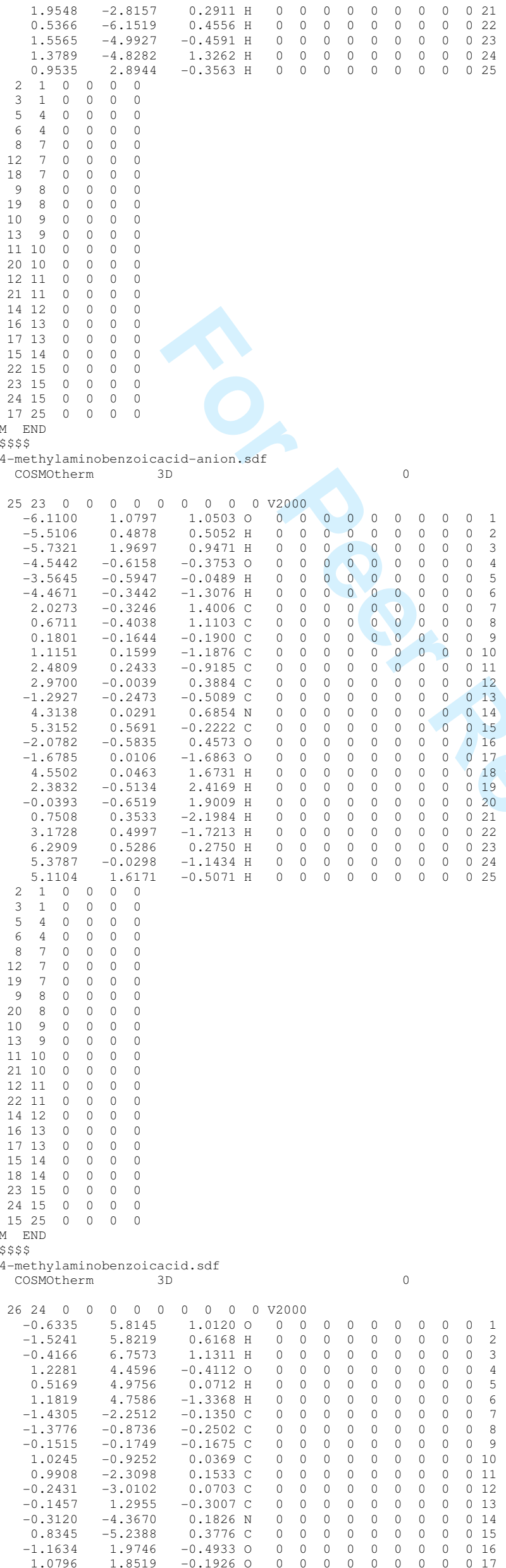




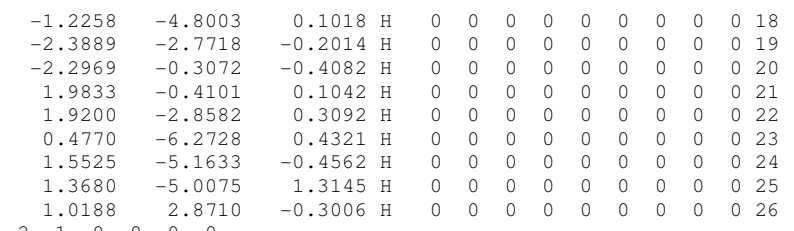


$-2.1644$

$-3.3264$

$\begin{array}{rrrrrrrrrrrrr}2.0785 & -0.7370 & 0.2680 \mathrm{H} & 0 & 0 & 0 & 0 & 0 & 0 & 0 & 0 & 0 & 19 \\ 2.1030 & -3.1980 & 0.5409 \mathrm{H} & 0 & 0 & 0 & 0 & 0 & 0 & 0 & 0 & 0 & 20\end{array}$

$\begin{array}{llllllllllll}-0.2056 & \mathrm{H} & 0 & 0 & 0 & 0 & 0 & 0 & 0 & 0 & 0 & 17\end{array}$

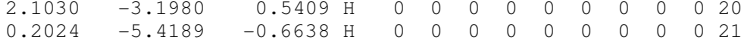

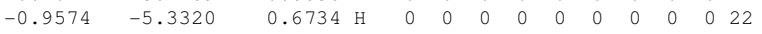

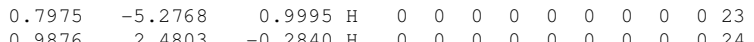

$2.9876 \quad 2.4803$

$\begin{array}{lllll}4 & 0 & 0 & 0 & 0\end{array}$

$\begin{array}{llllll}7 & 0 & 0 & 0 & 0\end{array}$

$\begin{array}{llllll}2 & 7 & 0 & 0 & 0 & 0\end{array}$

$\begin{array}{lllllll}7 & 0 & 0 & 0 & 0 \\ 9 & 8 & 0 & 0 & 0 & 0\end{array}$

$\begin{array}{lllllll}8 & 8 & 0 & 0 & 0 & 0\end{array}$

$\begin{array}{lllllll}18 & 9 & 0 & 0 & 0 & 0\end{array}$

$\begin{array}{llllll}4 & 9 & 0 & 0 & 0 & 0\end{array}$

$\begin{array}{llllll}11 & 10 & 0 & 0 & 0 & 0 \\ 19 & 10 & 0 & 0 & 0 & 0\end{array}$

$\begin{array}{llllll}12 & 11 & 0 & 0 & 0 & 0\end{array}$

$\begin{array}{llllll}20 & 11 & 0 & 0 & 0 & 0 \\ 13 & 12 & 0 & 0 & 0 & 0\end{array}$

$\begin{array}{llllll}13 & 12 & 0 & 0 & 0 & 0\end{array}$

$\begin{array}{llllll}22 & 13 & 0 & 0 & 0 \\ 23 & 13 & 0 & 0 & 0\end{array}$

$\begin{array}{lllllll}23 & 13 & 0 & 0 & 0 & 0 \\ 15 & 14 & 0 & 0 & 0 & 0\end{array}$

$\begin{array}{llllll}16 & 14 & 0 & 0 & 0 & 0 \\ 16 & 24 & 0 & 0 & 0 & 0\end{array}$

END

4-nitrobenzoicacid-anion.sdf

$2 \begin{array}{lllllllllll}20 & 0 & 0 & 0 & 0 & 0 & 0 & 0 & 0 & 0 & \mathrm{v} 2000\end{array}$

$\begin{array}{llllllllllllll}-6.4162 & 0.9784 & 1.3881 & 0 & 0 & 0 & 0 & 0 & 0 & 0 & 0 & 0 & 0 & 1\end{array}$ $\begin{array}{llllllllllllll}-5.8628 & 0.4204 & 0.7677 & \mathrm{H} & 0 & 0 & 0 & 0 & 0 & 0 & 0 & 0 & 0 & 2\end{array}$ $\begin{array}{lllllllllllll}-6.0119 & 1.8618 & 1.3460 & \mathrm{H} & 0 & 0 & 0 & 0 & 0 & 0 & 0 & 0 & 0\end{array}$ $\begin{array}{llllllllllllll}-4.9617 & -0.6345 & -0.2556 & 0 & 0 & 0 & 0 & 0 & 0 & 0 & 0 & 0 & 0 & 4\end{array}$

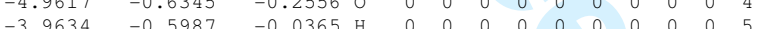
$\begin{array}{lllllllllllllll}-5.0068 & -0.3416 & -1.1831 \mathrm{H} & 0 & 0 & 0 & 0 & 0 & 0 & 0 & 0 & 0 & 6\end{array}$ $\begin{array}{lllllllllllllllllllllll}2.0816 & 0.2654 & -1.1791 & C & 0 & 0 & 0 & 0 & 0 & 0 & 0 & 0 & 0 & 7\end{array}$ $\begin{array}{lllllllllllllllll}0.7091 & 0.1817 & -1.4007 & C & 0 & 0 & 0 & 0 & 0 & 0 & 0 & 0 & 0 & 0 & 0 & 7\end{array}$

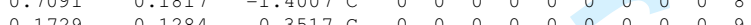
$\begin{array}{lllllllllllllllll}0.1729 & -0.1284 & -0.3517 & \mathrm{C} & 0 & 0 & 0 & 0 & 0 & 0 & 0 & 0 & 0 & 0 & 9\end{array}$

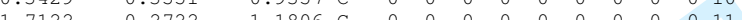
$\begin{array}{llllllllllllllll}1.7122 & -0.2722 & 1.1806 & \mathrm{C} & 0 & 0 & 0 & 0 & 0 & 0 & 0 & 0 & 0 & 11 \\ 2.5634 & 0.0377 & 0.1146 & \mathrm{C} & 0 & 0 & 0 & 0 & 0 & 0 & 0 & 0 & 0 & 12\end{array}$ $\begin{array}{lllllllllllllllllll}1.712534 & 0.0377 & 0.1146 & \mathrm{C} & 0 & 0 & 0 & 0 & 0 & 0 & 0 & 0 & 0 & 12\end{array}$

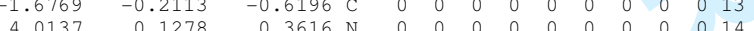

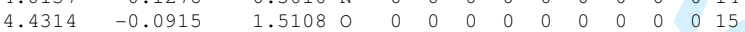

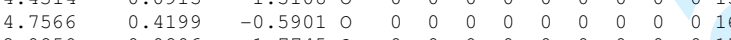
$\begin{array}{llllllllllllllllll}4.7566 & 0.41996 & -0.5901 & 0 & 0 & 0 & 0 & 0 & 0 & 0 & 0 & 0 & 0 & 16\end{array}$ $\begin{array}{rrrrrrrrrrrrrr}-2.4047 & -0.5761 & 0.3685 & 0 & 0 & 0 & 0 & 0 & 0 & 0 & 0 & 0 & 0 & 18 \\ 2.7718 & 0.5028 & -1.9869 & H & 0 & 0 & 0 & 0 & 0 & 0 & 0 & 0 & 0 & 19\end{array}$

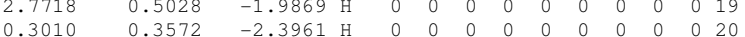

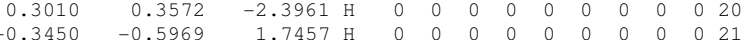

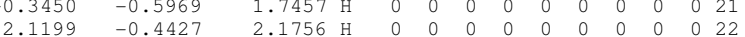

$\begin{array}{llllll}2 & 1 & 0 & 0 & 0 & 0\end{array}$

$\begin{array}{llllll}3 & 1 & 0 & 0 & 0 & 0\end{array}$

$\begin{array}{llllll}5 & 4 & 0 & 0 & 0 & 0\end{array}$

$\begin{array}{lllll}4 & 0 & 0 & 0 & 0\end{array}$

$\begin{array}{lllllll}2 & 7 & 0 & 0 & 0 & 0\end{array}$

$\begin{array}{llllll}9 & 7 & 0 & 0 & 0 & 0 \\ 9 & 8 & 0 & 0 & 0 & 0\end{array}$

$\begin{array}{lllllll}2 & 8 & 0 & 0 & 0 & 0 \\ 2 & 8 & 0 & 0 & 0 & 0\end{array}$

$\begin{array}{lllllll}10 & 9 & 0 & 0 & 0 & 0\end{array}$

$\begin{array}{llllll}13 & 9 & 0 & 0 & 0 & 0\end{array}$

$\begin{array}{llllll}1 & 10 & 0 & 0 & 0\end{array}$

$\begin{array}{llllll}1 & 10 & 0 & 0 & 0\end{array}$

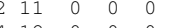

$18 \begin{array}{llllll}13 & 0 & 0 & 0 & 0\end{array}$

$\begin{array}{lllllll}15 & 14 & 0 & 0 & 0 & 0\end{array}$

$1614 \quad 000000$

1122

$\$ \$ \$ \$$

-nitrobenzoicacid.sdf

osmotherm

$\begin{array}{llllllllllll}23 & 21 & 0 & 0 & 0 & 0 & 0 & 0 & 0 & 0 & 0 & \mathrm{v} 2000\end{array}$

\begin{tabular}{|c|c|c|c|c|c|c|c|c|c|c|c|c|}
\hline-0.6482 & 6.1465 & 1.1548 & 0 & 0 & 0 & 0 & 0 & 0 & 0 & 0 & 0 & 0 \\
\hline-1.5720 & 6.2031 & 0.8501 & $\mathrm{H}$ & 0 & 0 & 0 & 0 & 0 & 0 & 0 & 0 & 0 \\
\hline 0.3849 & 7.0730 & 1.3036 & $\mathrm{H}$ & 0 & 0 & 0 & 0 & 0 & 0 & 0 & 0 & 0 \\
\hline 1.0305 & 4.8472 & -0.4974 & 0 & 0 & 0 & 0 & 0 & 0 & 0 & 0 & 0 & 0 \\
\hline 0.3751 & 5.3499 & 0.0759 & $\mathrm{H}$ & 0 & & 0 & 0 & 0 & 0 & 0 & 0 & 0 \\
\hline 0.8489 & 5.1280 & -1.4121 & $\mathrm{H}$ & 0 & ( & 0 & 0 & 0 & 0 & 0 & 0 & 0 \\
\hline$-1.238 \mathrm{~s}$ & -1.9461 & -0.1433 & C & 0 & & 0 & 0 & 0 & 0 & 0 & 0 & 0 \\
\hline .2669 & -0.5597 & -0.2682 & $\mathrm{C}$ & 0 & 0 & 0 & 0 & 0 & 0 & 0 & 0 & 0 \\
\hline 0.0793 & 0.1866 & -0.1908 & C & 0 & & 0 & 0 & 0 & 0 & 0 & 0 & 0 \\
\hline 1.1474 & -0.4680 & 0.0148 & C & 0 & 0 & 0 & 0 & 0 & 0 & 0 & 0 & 0 \\
\hline 1918 & -1.8550 & 0.1404 & & 0 & 0 & 0 & 0 & 0 & 0 & 0 & 0 & \\
\hline .0051 & -2.5713 & 0.0587 & C & & 0 & 0 & 0 & 0 & 0 & 0 & 0 & 0 \\
\hline .1610 & 1.6814 & -0.3389 & C & & 0 & 0 & 0 & 0 & 0 & 0 & 0 & \\
\hline 0.0345 & -4.0447 & 0.1892 & $\mathrm{~N}$ & 0 & & 0 & 0 & 0 & 0 & 0 & 0 & 0 \\
\hline 1.1347 & -4.5862 & 0.3738 & 0 & 0 & 0 & 0 & 0 & 0 & 0 & 0 & 0 & \\
\hline 1.0341 & -4.6690 & 0.1074 & 0 & & & 0 & 0 & 0 & 0 & 0 & 0 & 0 \\
\hline 1.2249 & 2.2652 & -0.5449 & 0 & & & 0 & 0 & 0 & 0 & 0 & 0 & 0 \\
\hline .0192 & 2.2898 & -0.2268 & & & & 0 & 0 & 0 & 0 & 0 & 0 & \\
\hline . & -2.5356 & -0.2017 & & 0 & 0 & 0 & 0 & 0 & 0 & 0 & 0 & \\
\hline & -0.0434 & -0.4280 & & 0 & & 0 & 0 & 0 & 0 & 0 & 0 & 0 \\
\hline & & & & & & & & & & & & \\
\hline
\end{tabular}




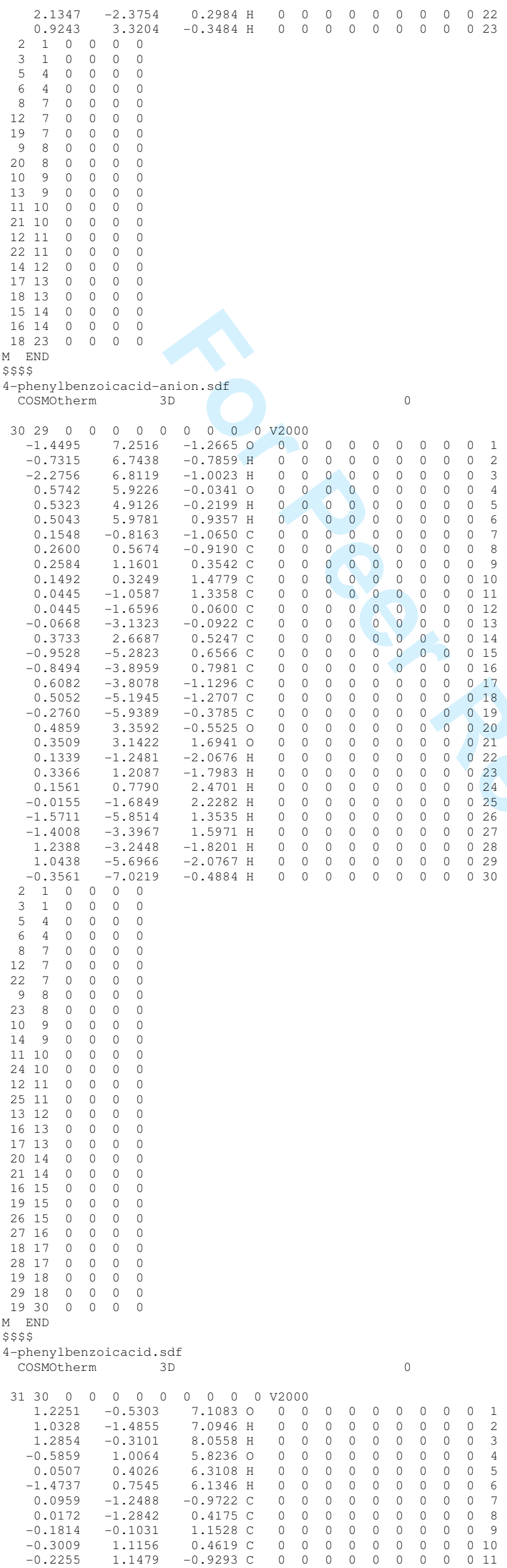




$\begin{array}{rrrrllllllllll}-0.0251 & -0.0318 & -1.6759 & \mathrm{C} & 0 & 0 & 0 & 0 & 0 & 0 & 0 & 0 & 0 & 12 \\ 0.0546 & 0.0056 & -3.1570 & \mathrm{C} & 0 & 0 & 0 & 0 & 0 & 0 & 0 & 0 & 0 & 13 \\ -0.2636 & -0.1834 & 2.6428 & \mathrm{C} & 0 & 0 & 0 & 0 & 0 & 0 & 0 & 0 & 0 & 14 \\ 0.7121 & 1.1379 & -5.2178 & \mathrm{C} & 0 & 0 & 0 & 0 & 0 & 0 & 0 & 0 & 0 & 15 \\ 0.6371 & 1.1032 & -3.8229 & \mathrm{C} & 0 & 0 & 0 & 0 & 0 & 0 & 0 & 0 & 0 & 16 \\ -0.4512 & -1.0551 & -3.9359 & \mathrm{C} & 0 & 0 & 0 & 0 & 0 & 0 & 0 & 0 & 0 & 17 \\ -0.3785 & -1.0182 & -5.3308 & \mathrm{C} & 0 & 0 & 0 & 0 & 0 & 0 & 0 & 0 & 0 & 18 \\ 0.2041 & 0.0779 & -5.9785 & \mathrm{C} & 0 & 0 & 0 & 0 & 0 & 0 & 0 & 0 & 0 & 19 \\ -0.2029 & -1.2483 & 3.2625 & 0 & 0 & 0 & 0 & 0 & 0 & 0 & 0 & 0 & 0 & 20 \\ -0.4071 & 1.0078 & 3.2385 & 0 & 0 & 0 & 0 & 0 & 0 & 0 & 0 & 0 & 0 & 21 \\ 0.2754 & -2.1756 & -1.5194 & \mathrm{H} & 0 & 0 & 0 & 0 & 0 & 0 & 0 & 0 & 0 & 22 \\ 0.1189 & -2.2306 & 0.9505 & \mathrm{H} & 0 & 0 & 0 & 0 & 0 & 0 & 0 & 0 & 0 & 23 \\ -0.4645 & 2.0389 & 1.0176 & \mathrm{H} & 0 & 0 & 0 & 0 & 0 & 0 & 0 & 0 & 0 & 24 \\ -0.3465 & 2.1013 & -1.4462 & \mathrm{H} & 0 & 0 & 0 & 0 & 0 & 0 & 0 & 0 & 0 & 25 \\ 1.1760 & 1.9935 & -5.7123 & \mathrm{H} & 0 & 0 & 0 & 0 & 0 & 0 & 0 & 0 & 0 & 26 \\ 1.0568 & 1.9274 & -3.2434 & \mathrm{H} & 0 & 0 & 0 & 0 & 0 & 0 & 0 & 0 & 0 & 27 \\ -0.9278 & -1.9067 & -3.4469 & \mathrm{H} & 0 & 0 & 0 & 0 & 0 & 0 & 0 & 0 & 0 & 28 \\ -0.7854 & -1.8460 & -5.9146 & \mathrm{H} & 0 & 0 & 0 & 0 & 0 & 0 & 0 & 0 & 0 & 29 \\ 0.2618 & 0.1057 & -7.0680 & \mathrm{H} & 0 & 0 & 0 & 0 & 0 & 0 & 0 & 0 & 0 & 30 \\ -0.4706 & 0.8995 & 4.2657 & \mathrm{H} & 0 & 0 & 0 & 0 & 0 & 0 & 0 & 0 & 0 & 31\end{array}$




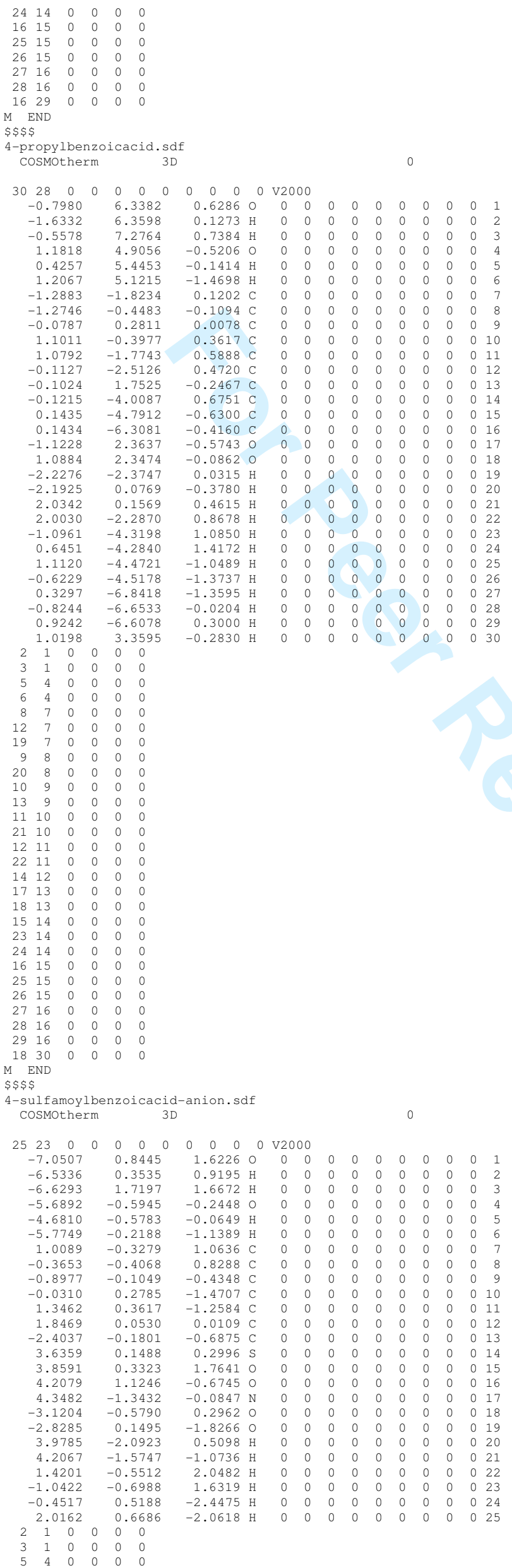


$\begin{array}{llllll}6 & 4 & 0 & 0 & 0 & 0\end{array}$

$\begin{array}{rrrrrr}8 & 7 & 0 & 0 & 0 & 0 \\ 12 & 7 & 0 & 0 & 0 & 0\end{array}$

$\begin{array}{rrrrrr}22 & 7 & 0 & 0 & 0 & 0 \\ 9 & 8 & 0 & 0 & 0 & 0\end{array}$

$\begin{array}{rrrrrr}9 & 8 & 0 & 0 & 0 & 0 \\ 23 & 8 & 0 & 0 & 0 & 0\end{array}$

$\begin{array}{llllll}10 & 9 & 0 & 0 & 0 & 0\end{array}$

$\begin{array}{lrrrrr}13 & 9 & 0 & 0 & 0 & 0\end{array}$

$\begin{array}{llllll}24 & 10 & 0 & 0 & 0 & 0\end{array}$

$1211 \quad 00000$

$\begin{array}{llll}0 & 0 & 0\end{array}$

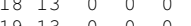

$\begin{array}{llllll}15 & 14 & 0 & 0 & 0 & 0\end{array}$

$\begin{array}{llllll}16 & 14 & 0 & 0 & 0 & 0\end{array}$

$\begin{array}{llllll}17 & 14 & 0 & 0 & 0 & 0 \\ 20 & 17 & 0 & 0 & 0 & 0\end{array}$

$\begin{array}{llllll}21 & 17 & 0 & 0 & 0 & 0\end{array}$

$\begin{array}{llllll}11 & 25 & 0 & 0 & 0 & 0\end{array}$

END

-sulfamoylbenzoicacid.sdf

$\begin{array}{llllllllllll}26 & 24 & 0 & 0 & 0 & 0 & 0 & 0 & 0 & 0 & 0 & v 2000\end{array}$

$\begin{array}{lllllllllllll}-0.8280 & 6.7706 & 1.0794 & 0 & 0 & 0 & 0 & 0 & 0 & 0 & 0 & 0 & 0\end{array}$

$\begin{array}{lllllllllllll}-1.7122 & 6.7583 & 0.6701 & \mathrm{H} & 0 & 0 & 0 & 0 & 0 & 0 & 0 & 0 & 0\end{array}$

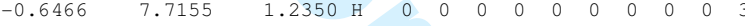

$\begin{array}{llllllllllllll}1.1013 & 5.5688 & -0.3622 & 0 & 0 & 0 & 0 & 0 & 0 & 0 & 0 & 0 & 0 & 4\end{array}$

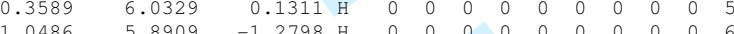

$\begin{array}{rrrrrrrrrrrrrr}1.0486 & 5.8909 & -1.2798 & \mathrm{H} & 0 & 0 & 0 & 0 & 0 & 0 & 0 & 0 & 0 & 6 \\ -1.2339 & -1.2051 & -0.2264 & \mathrm{C} & 0 & 0 & 0 & 0 & 0 & 0 & 0 & 0 & 0 & 7\end{array}$

$\begin{array}{llllllllllllll}-1.2339 & 0.1827 & -0.3606 & \mathrm{C} & 0 & 0 & 0 & 0 & 0 & 0 & 0 & 0 & 0 & 8\end{array}$

$\begin{array}{rrrrrrrrrrrrrr}-0.0428 & 0.9124 & -0.2158 & \mathrm{C} & 0 & 0 & 0 & 0 & 0 & 0 & 0 & 0 & 0 & 9 \\ 1.1587 & 0.2410 & 0.06555 & \mathrm{C} & 0 & 0 & 0 & 0 & 0 & 0 & 0 & 0 & 0 & 10\end{array}$

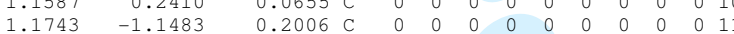

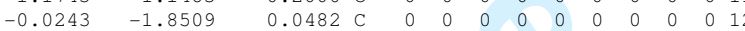

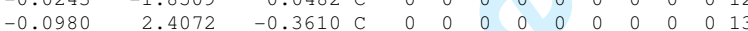

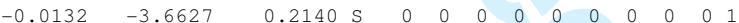
$\begin{array}{lllllllllllllll}-1.3791 & -4.0903 & 0.6344 & 0 & 0 & 0 & 0 & 0 & 0 & 0 & 0 & 0 & 0 & 15\end{array}$

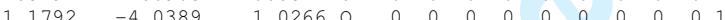
$\begin{array}{lllllllllllllll}1.1792 & -4.0389 & 1.02166 & 0 & 0 & 0 & 0 & 0 & 0 & 0 & 0 & 0 & 0 & 16 & 0\end{array}$ $\begin{array}{lllllllllllllll}-1.1428 & 3.0082 & -0.6142 & 0 & 0 & 0 & 0 & 0 & 0 & 0 & 0 & 0 & 0 & 18\end{array}$

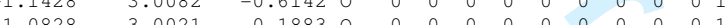
$\begin{array}{lllllllllllllllll}1.0828 & 3.0021 & -0.1883 & 0 & 0 & 0 & 0 & 0 & 0 & 0 & 0 & 0 & 0 & 0 & 19\end{array}$

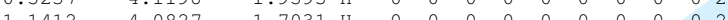
$\begin{array}{llllllllllllllllll}1.1412 & -4.0827 & -1.7031 & \mathrm{H} & 0 & 0 & 0 & 0 & 0 & 0 & 0 & 0 & 0 & 0 & 0 & 21\end{array}$

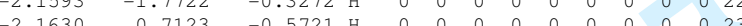
$\begin{array}{lllllllllllllllll}-2.1630 & 0.7123 & -0.5721 & 1 & 0 & 0 & 0 & 0 & 0 & 0 & 0 & 0 & 0 & 23\end{array}$

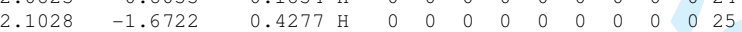

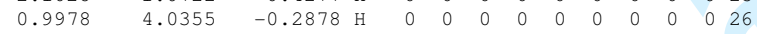

$\begin{array}{lllll}1 & 0 & 0 & 0 & 0\end{array}$

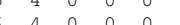

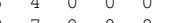

$\begin{array}{llllll}7 & 0 & 0 & 0 & 0\end{array}$

$\begin{array}{llllll}22 & 7 & 0 & 0 & 0 & 0\end{array}$

$\begin{array}{lllllll}2 & 8 & 0 & 0 & 0 & 0 & 0\end{array}$

$\begin{array}{lllll}0 & 9 & 0 & 0 & 0\end{array}$

$\begin{array}{llllll}3 & 9 & 0 & 0 & 0 & 0\end{array}$

$\begin{array}{lllllll}1 & 10 & 10 & 0 & 0 & 0 & 0\end{array}$

$\begin{array}{llllll}12 & 11 & 0 & 0 & 0 & 0\end{array}$

$\begin{array}{llllll}25 & 11 & 0 & 0 & 0 & 0\end{array}$

$\begin{array}{lllllll}18 & 13 & 0 & 0 & 0\end{array}$

$\begin{array}{llllll}19 & 13 & 0 & 0 & 0 & 0\end{array}$

$\begin{array}{llllll}15 & 14 & 0 & 0 & 0 & 0\end{array}$

$\begin{array}{llll}0 & 0 & 0 & 0 \\ 0 & 0 & 0 & 0\end{array}$

$\begin{array}{llllll}21 & 17 & 0 & 0 & 0 & 0\end{array}$

$\begin{array}{llllll}19 & 26 & 0 & 0 & 0 & 0\end{array}$

END

trifluoromethylbenzoicacid-anion.sdf coswotherm

$\begin{array}{llllllllllll}23 & 21 & 0 & 0 & 0 & 0 & 0 & 0 & 0 & 0 & 0 & \mathrm{v} 2000\end{array}$

$\begin{array}{lllllllllllll}-6.8334 & 0.9642 & 1.4738 & 0 & 0 & 0 & 0 & 0 & 0 & 0 & 0 & 0 & 0\end{array}$

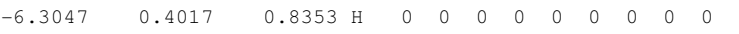

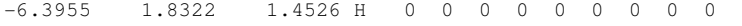

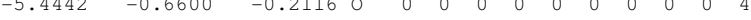

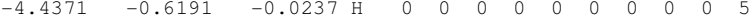

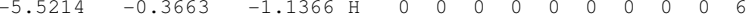

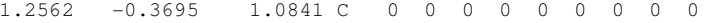

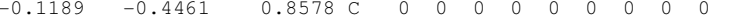

$\begin{array}{lllllllllllll}-0.6602 & -0.1156 & -0.3940 & \mathrm{C} & 0 & 0 & 0 & 0 & 0 & 0 & 0 & 0 & 0\end{array}$

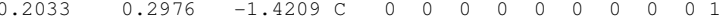

$\begin{array}{llllllllllllll}1.5796 & 0.3778 & -1.2080 & \mathrm{C} & 0 & 0 & 0 & 0 & 0 & 0 & 0 & 0 & 0 & 11\end{array}$

$\begin{array}{llllllllllllll}2.1056 & 0.0486 & 0.0502 & \mathrm{C} & 0 & 0 & 0 & 0 & 0 & 0 & 0 & 0 & 0 & 12\end{array}$

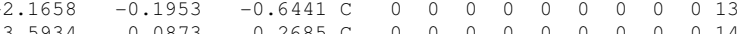

$\begin{array}{rrrrrrrrrrrrrr}3.5934 & 0.0873 & 0.2685 & \mathrm{C} & 0 & 0 & 0 & 0 & 0 & 0 & 0 & 0 & 0 & 1 \\ 4.2047 & -1.0804 & -0.1273 & \mathrm{~F} & 0 & 0 & 0 & 0 & 0 & 0 & 0 & 0 & 0 & 15 \\ 3.9308 & 0.2669 & 1.5790 & \mathrm{~F} & 0 & 0 & 0 & 0 & 0 & 0 & 0 & 0 & 0 & 16\end{array}$

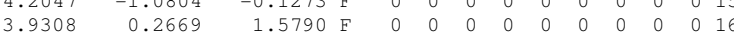

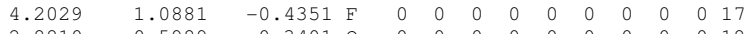

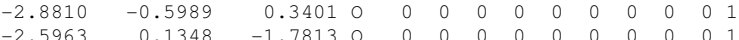

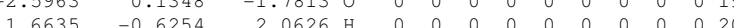

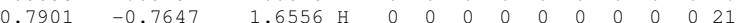

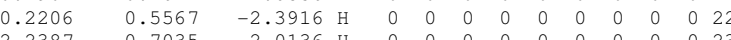


$\begin{array}{rrrrrr}2 & 1 & 0 & 0 & 0 & 0 \\ 3 & 1 & 0 & 0 & 0 & 0 \\ 5 & 4 & 0 & 0 & 0 & 0 \\ 6 & 4 & 0 & 0 & 0 & 0 \\ 8 & 7 & 0 & 0 & 0 & 0 \\ 12 & 7 & 0 & 0 & 0 & 0 \\ 20 & 7 & 0 & 0 & 0 & 0 \\ 9 & 8 & 0 & 0 & 0 & 0 \\ 21 & 8 & 0 & 0 & 0 & 0 \\ 10 & 9 & 0 & 0 & 0 & 0 \\ 13 & 9 & 0 & 0 & 0 & 0 \\ 11 & 10 & 0 & 0 & 0 & 0 \\ 22 & 10 & 0 & 0 & 0 & 0 \\ 12 & 11 & 0 & 0 & 0 & 0 \\ 14 & 12 & 0 & 0 & 0 & 0 \\ 18 & 13 & 0 & 0 & 0 & 0 \\ 19 & 13 & 0 & 0 & 0 & 0 \\ 15 & 14 & 0 & 0 & 0 & 0 \\ 16 & 14 & 0 & 0 & 0 & 0 \\ 17 & 14 & 0 & 0 & 0 & 0 \\ 11 & 23 & 0 & 0 & 0 & 0 \\ 1 & 14 & & & & \end{array}$ $M$ END \$\$\$
4-trifluoromethylbenzoicacid.sdf
CosMotherm 3 D

$\begin{array}{llllllllllll}24 & 22 & 0 & 0 & 0 & 0 & 0 & 0 & 0 & 0 & 0 & \mathrm{~V} 2000\end{array}$

$\begin{array}{llllllllllllll}-1.3701 & -5.6476 & 0.0630 & 0 & 0 & 0 & 0 & 0 & 0 & 0 & 0 & 0 & 0 & 1\end{array}$ $\begin{array}{llllllllllllll}-1.7761 & -5.9650 & -0.7636 & \text { H } & 0 & 0 & 0 & 0 & 0 & 0 & 0 & 0 & 0 & 2\end{array}$ $\begin{array}{llllllllllllll}-1.4012 & -4.6556 & 0.0098 & \mathrm{H} & 0 & 0 & 0 & 0 & 0 & 0 & 0 & 0 & 0 & 3\end{array}$ $\begin{array}{llllllllllllll}1.2723 & -5.3791 & -0.0001 & 0 & 0 & 0 & 0 & 0 & 0 & 0 & 0 & 0 & 0 & 4\end{array}$

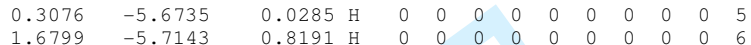
$\begin{array}{llllllllllllll}-1.2141 & 1.3132 & -0.0410 & \mathrm{C} & 0 & 0 & 0 & 0 & 0 & 0 & 0 & 0 & 0 & 7\end{array}$ $\begin{array}{llllllllllllll}-1.1907 & -0.0799 & -0.0357 & \mathrm{C} & 0 & 0 & 0 & 0 & 0 & 0 & 0 & 0 & 0 & 8 \\ 0.0326 & -0.7687 & -0.0299 & \mathrm{C} & 0 & 0 & 0 & 0 & 0 & 0 & 0 & 0 & 0 & 9\end{array}$ $\begin{array}{lllllllllllllll}1.2375 & -0.0470 & -0.0342 & \mathrm{C} & 0 & 0 & 0 & 0 & 0 & 0 & 0 & 0 & 0 & 10\end{array}$ $\begin{array}{llllllllllllll}1.2201 & 1.3474 & -0.0384 & \mathrm{C} & 0 & 0 & 0 & 0 & 0 & 0 & 0 & 0 & 0 & 11\end{array}$

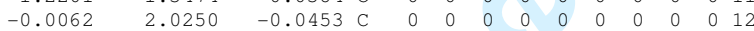
$\begin{array}{llllllllllllll}0.0178 & -2.2668 & -0.0141 & \mathrm{C} & 0 & 0 & 0 & 0 & 0 & 0 & 0 & 0 & 0 & 13\end{array}$ $\begin{array}{rrrrrrrrrrrrrr}-0.0289 & 3.5314 & 0.0075 & \mathrm{C} & 0 & 0 & 0 & 0 & 0 & 0 & 0 & 0 & 0 & 14\end{array}$ $\begin{array}{lllllllllllllll}-0.0739 & 3.9925 & 1.3003 & \mathrm{~F} & 0 & 0 & 0 & 0 & 0 & 0 & 0 & 0 & 0 & 1.5\end{array}$ $\begin{array}{lllllllllllll}-1.1149 & 4.0613 & -0.6256 & \mathrm{~F} & 0 & 0 & 0 & 0 & 0 & 0 & 0 & 0 & 0 \\ 1.0753 & 4.0927 & -0.5621 & \end{array}$ $\begin{array}{llllllllllllll}-1.11493 & 4.09137 & -0.5625 & \mathrm{~F} & 0 & 0 & 0 & 0 & 0 & 0 & 0 & 0 & 0 & 0\end{array}$

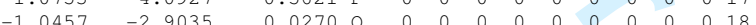
$\begin{array}{llllllllllllllllll}-1.0457 & -2.9035 & 0.0275 & 0 & 0 & 0 & 0 & 0 & 0 & 0 & 0 & 0 & 0 & 18\end{array}$ $\begin{array}{lllllllllllllll}1.2233 & -2.81649 & -0.04574 \mathrm{H} & 0 & 0 & 0 & 0 & 0 & 0 & 0 & 0 & 0 & 190\end{array}$ $\begin{array}{llllllllllllllllll}-2.1680 & 1.8409 & -0.0484 & 1 & 0 & 0 & 0 & 0 & 0 & 0 & 0 & 0 & 0 & 20\end{array}$ $\begin{array}{llllllllllllllllllllll}2.1233 & -0.6439 & -0.0352 & \mathrm{H} & 0 & 0 & 0 & 0 & 0 & 0 & 0 & 0 & 0 & 21\end{array}$

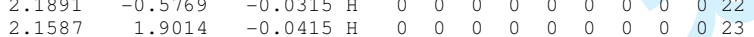
$\begin{array}{llllllllllllll}1.1954 & -3.8654 & -0.0076 & \mathrm{H} & 0 & 0 & 0 & 0 & 0 & 0 & 0 & 0 & 0 & 24\end{array}$ $\begin{array}{lllllll}2 & 1 & 0 & 0 & 0 & 0\end{array}$

$\begin{array}{llllllll}3 & 1 & 0 & 0 & 0 & 0 \\ 5 & 4 & 0 & 0 & 0 & 0\end{array}$

$\begin{array}{lllll}4 & 0 & 0 & 0\end{array}$

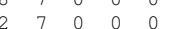

$\begin{array}{lllllll}20 & 7 & 0 & 0 & 0 & 0\end{array}$

$\begin{array}{llllll}9 & 8 & 0 & 0 & 0 & 0\end{array}$

$\begin{array}{lllllll}1 & 9 & 0 & 0 & 0 & 0\end{array}$

$\begin{array}{llllll}13 & 9 & 0 & 0 & 0 & 0\end{array}$

$\begin{array}{lllllll}11 & 10 & 0 & 0 & 0 & 0\end{array}$

$1211 \quad 00000$

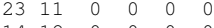

$\begin{array}{lllllll}14 & 12 & 0 & 0 & 0 & 0 \\ 18 & 13 & 0 & 0 & 0\end{array}$

$1913 \quad 0 \quad 0000$

$\begin{array}{llllll}5 & 14 & 0 & 0 & 0 \\ 6 & 14 & 0 & 0 & 0 & 0\end{array}$

$17 \begin{array}{lllll}14 & 0 & 0 & 0 & 0\end{array}$

$\begin{array}{llllll}9 & 24 & 0 & 0 & 0 & 0\end{array}$

$M$ END

$\$ \$ \$ \$$

fluorouracil-anion.sdf

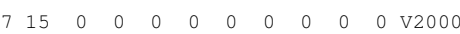

$\begin{array}{llllllllllllll}-1.4634 & -0.6132 & -4.5588 & 0 & 0 & 0 & 0 & 0 & 0 & 0 & 0 & 0 & 0 & 1\end{array}$

$\begin{array}{llllllllllllll}-0.6911 & -0.1839 & -4.0891 & \mathrm{H} & 0 & 0 & 0 & 0 & 0 & 0 & 0 & 0 & 0 & 2\end{array}$

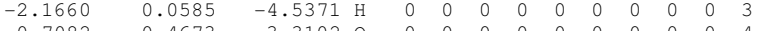

$\begin{array}{llllllllllllll}0.7082 & 0.4673 & -3.3102 & 0 & 0 & 0 & 0 & 0 & 0 & 0 & 0 & 0 & 0 & 4 \\ 0.6448 & 0.3714 & -2.2909 & \mathrm{H} & 0 & 0 & 0 & 0 & 0 & 0 & 0 & 0 & 0 & 5\end{array}$

$\begin{array}{llllllllllllll}0.7809 & 1.4239 & -3.4727 & \mathrm{H} & 0 & 0 & 0 & 0 & 0 & 0 & 0 & 0 & 0\end{array}$

$\begin{array}{llllllllllllll}1.4978 & -0.0378 & 0.1959 & \mathrm{C} & 0 & 0 & 0 & 0 & 0 & 0 & 0 & 0 & 0 & 7\end{array}$

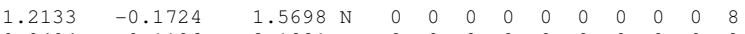

$\begin{array}{rrrrrrrrrrrrrr}-0.0404 & -0.1106 & 2.1881 & \mathrm{C} & 0 & 0 & 0 & 0 & 0 & 0 & 0 & 0 & 0 & 9 \\ -1.0823 & 0.1157 & 1.2279 & \mathrm{C} & 0 & 0 & 0 & 0 & 0 & 0 & 0 & 0 & 0 & 10\end{array}$

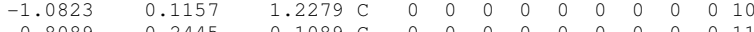

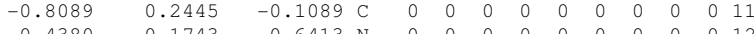

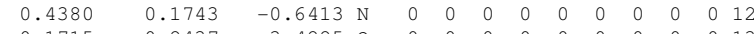

$\begin{array}{llllllllllllll}-0.1715 & -0.2437 & 3.4225 & 0 & 0 & 0 & 0 & 0 & 0 & 0 & 0 & 0 & 0 & 13\end{array}$

$\begin{array}{llllllllllllll}2.6846 & -0.1172 & -0.1897 & 0 & 0 & 0 & 0 & 0 & 0 & 0 & 0 & 0 & 0 & 14\end{array}$

$\begin{array}{rrrrrrrrrrrrrr}2.3680 & 0.1985 & 1.6958 & \mathrm{~F} & 0 & 0 & 0 & 0 & 0 & 0 & 0 & 0 & 0 & 15 \\ 2.0131 & -0.3309 & 2.1851 & \mathrm{H} & 0 & 0 & 0 & 0 & 0 & 0 & 0 & 0 & 0 & 16\end{array}$

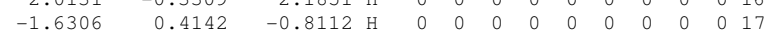

$\begin{array}{llllll}2 & 1 & 0 & 0 & 0 & 0 \\ 3 & 1 & 0 & 0 & 0 & 0\end{array}$

$\begin{array}{llllll}5 & 4 & 0 & 0 & 0 \\ 6 & 4 & 0 & 0 & 0 & 0\end{array}$

$8 \begin{array}{llllll}8 & 7 & 0 & 0 & 0 & 0\end{array}$

$\begin{array}{llllll}12 & 7 & 0 & 0 & 0 & 0\end{array}$

$\begin{array}{lllllll}9 & 8 & 0 & 0 & 0 & 0\end{array}$ 
$\begin{array}{lllllll}10 & 9 & 0 & 0 & 0 & 0\end{array}$

$\begin{array}{cccccc}13 & 9 & 0 & 0 & 0 & 0\end{array}$

$\begin{array}{llllll}11 & 10 & 0 & 0 & 0 & 0 \\ 15 & 10 & 0 & 0 & 0 & 0\end{array}$

$\begin{array}{llllll}12 & 11 & 0 & 0 & 0 & 0\end{array}$

1117

$\$ \$ \$$

5-fluorouracil.sdf

cosmotherm 3 D

$\begin{array}{lllllllllllll}18 & 16 & 0 & 0 & 0 & 0 & 0 & 0 & 0 & 0 & 0 & \mathrm{~V} 2000\end{array}$

$\begin{array}{llllllllllllll}-4.8889 & 0.9926 & -0.1165 & 0 & 0 & 0 & 0 & 0 & 0 & 0 & 0 & 0 & 0 & 1\end{array}$

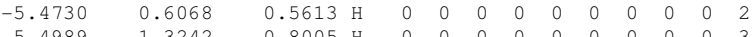

$\begin{array}{llllllllllllllllll}-5.4989 & 1.3242 & -0.8005 & H & 0 & 0 & 0 & 0 & 0 & 0 & 0 & 0 & 0 & 0 & 3\end{array}$

$\begin{array}{lllllllllllllllll}-3.0587 & -0.7593 & -1.1039 & 0 & 0 & 0 & 0 & 0 & 0 & 0 & 0 & 0 & 0 & 3\end{array}$

$\begin{array}{llllllllllllll}-3.7602 & -0.1531 & -0.7265 & \mathrm{H} & 0 & 0 & 0 & 0 & 0 & 0 & 0 & 0 & 0 & 5 \\ -3.3850 & -1.6627 & -0.9459 & \mathrm{H} & 0 & 0 & 0 & 0 & 0 & 0 & 0 & 0 & 0 & 6\end{array}$

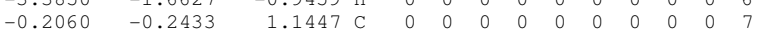

$\begin{array}{llllllllllllll}1.1430 & 0.0036 & 1.4009 & \mathrm{~N} & 0 & 0 & 0 & 0 & 0 & 0 & 0 & 0 & 0 & 8\end{array}$

$\begin{array}{lllllllllllll}2.1839 & 0.1665 & 0.4755 & \mathrm{C} & 0 & 0 & 0 & 0 & 0 & 0 & 0 & 0 & 0\end{array}$

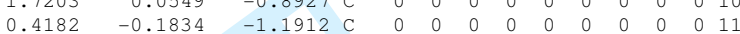

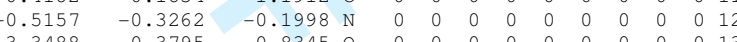

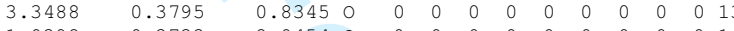

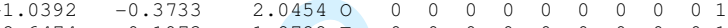

$\begin{array}{llllllllllllll}2.6474 & 0.1973 & -1.8728 & \mathrm{~F} & 0 & 0 & 0 & 0 & 0 & 0 & 0 & 0 & 0 & 15\end{array}$

$\begin{array}{llllllllllllll}1.4014 & 0.0704 & 2.3870 & \mathrm{H} & 0 & 0 & 0 & 0 & 0 & 0 & 0 & 0 & 0 & 16\end{array}$

$\begin{array}{llllllllllllll}-1.5228 & -0.5073 & -0.4657 & \mathrm{H} & 0 & 0 & 0 & 0 & 0 & 0 & 0 & 0 & 0 & 17 \\ 0.0614 & -0.2708 & -2.2165 & \mathrm{H} & 0 & 0 & 0 & 0 & 0 & 0 & 0 & 0 & 0 & 18\end{array}$

$\begin{array}{llllll}2 & 1 & 0 & 0 & 0 & 0\end{array}$

$\begin{array}{llllll}3 & 1 & 0 & 0 & 0 & 0\end{array}$

$\begin{array}{lllllll}5 & 4 & 0 & 0 & 0 & 0\end{array}$

$\begin{array}{lllll}4 & 0 & 0 & 0 & 0\end{array}$

$\begin{array}{llllll}2 & 7 & 0 & 0 & 0 & 0\end{array}$

$\begin{array}{llllll}4 & 7 & 0 & 0 & 0 & 0\end{array}$

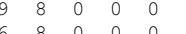

$\begin{array}{lllllll}0 & 9 & 0 & 0 & 0 & 0\end{array}$

$\begin{array}{llllllllll}5 & 10 & 0 & 0 & 0 & 0\end{array}$

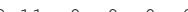

$\begin{array}{llllll}7 & 12 & 0 & 0 & 0 & 0\end{array}$

$\begin{array}{llllll}18 & 0 & 0 & 0 & 0\end{array}$

END

iodo-2-hydroxybenzoicacid-anion.sd

Po

$\begin{array}{lllllllllllll}21 & 19 & 0 & 0 & 0 & 0 & 0 & 0 & 0 & 0 & 0 & \mathrm{v} 2000\end{array}$

$\begin{array}{llllllllllllll}-3.3919 & 0.8547 & 6.0064 & 0 & 0 & 0 & 0 & 0 & 0 & 0 & 0 & 0 & 0 & 1\end{array}$

$\begin{array}{llllllllllllll}-2.5722 & 0.3248 & 5.7924 & \mathrm{H} & 0 & 0 & 0 & 0 & 0 & 0 & 0 & 0 & 0 & 2\end{array}$

$\begin{array}{rrrrrrrrrrrrrr}-3.0940 & 1.7801 & 5.9880 & \mathrm{H} & 0 & 0 & 0 & 0 & 0 & 0 & 0 & 0 & 0 & 3 \\ -1.2228 & -0.6954 & 5.4098 & 0 & 0 & 0 & 0 & 0 & 0 & 0 & 0 & 0 & 0 & 4\end{array}$

$\begin{array}{llllllllllllll}-1.2228 & -0.6954 & 5.4098 & 0 & 0 & 0 & 0 & 0 & 0 & 0 & 0 & 0 & 0 & 4 \\ -0.9401 & -0.5891 & 4.4411 & \mathrm{H} & 0 & 0 & 0 & 0 & 0 & 0 & 0 & 0 & 0 & 5\end{array}$

$\begin{array}{llllllllllllll}-0.9401 & -0.5891 & 4.4411 & \mathrm{H} & 0 & 0 & 0 & 0 & 0 & 0 & 0 & 0 & 0 & 5 \\ -0.4268 & -0.4705 & 5.9222 & \mathrm{H} & 0 & 0 & 0 & 0 & 0 & 0 & 0 & 0 & 0 & 6\end{array}$

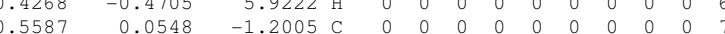

$\begin{array}{llllllllllllllll}-0.0548 & -1.2005 & c & 0 & 0 & 0 & 0 & 0 & 0 & 0 & 0 & 0 & 7 \\ -0.0583 & 0.1150 & c & 0 & 0 & 0 & 0 & 0 & 0 & 0 & 0 & 0\end{array}$

$\begin{array}{llllllllllllll}1.0765 & -0.0583 & 1.1608 & \mathrm{C} & 0 & 0 & 0 & 0 & 0 & 0 & 0 & 0 & 0 & 9\end{array}$

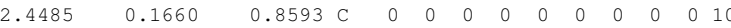

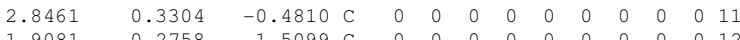

$\begin{array}{rrrrrrrrrrrrrr}1.9081 & 0.2758 & -1.5099 & \mathrm{C} & 0 & 0 & 0 & 0 & 0 & 0 & 0 & 0 & 0 & 12 \\ 0.6363 & -0.2357 & 2.5921 & \mathrm{C} & 0 & 0 & 0 & 0 & 0 & 0 & 0 & 0 & 0 & 13 \\ 1.5570 & -0.1684 & 3.4909 & 0 & 0 & 0 & 0 & 0 & 0 & 0 & 0 & 0 & 0 & 14\end{array}$

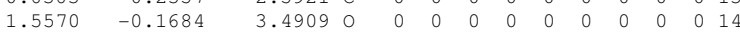

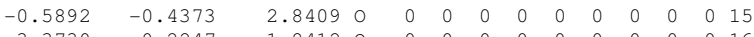

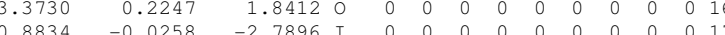

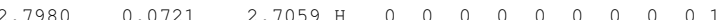

$\begin{array}{rrrrrrrrrrrrr}-0.9050 & -0.2846 & 0.3689 \mathrm{H} & 0 & 0 & 0 & 0 & 0 & 0 & 0 & 0 & 0 & 19\end{array}$

$\begin{array}{llllllllllllll}3.9001 & 0.5014 & -0.7066 & \mathrm{H} & 0 & 0 & 0 & 0 & 0 & 0 & 0 & 0 & 0 & 20\end{array}$

$\begin{array}{llllllllllllllllllllll}2.2337 & 0.4063 & -2.5422 & \mathrm{H} & 0 & 0 & 0 & 0 & 0 & 0 & 0 & 0 & 0 & 21\end{array}$

$\begin{array}{llllll}2 & 1 & 0 & 0 & 0 & 0\end{array}$

$\begin{array}{llllll}5 & 4 & 0 & 0 & 0 & 0\end{array}$

$\begin{array}{llllll}6 & 4 & 0 & 0 & 0 & 0\end{array}$

$\begin{array}{lllll}7 & 0 & 0 & 0\end{array}$

$\begin{array}{lllllll}8 & 0 & 0 & 0 & 0\end{array}$

$\begin{array}{lllllll}9 & 8 & 0 & 0 & 0 & 0\end{array}$

$\begin{array}{llllll}9 & 0 & 0 & 0 & 0\end{array}$

$\begin{array}{lllllll}1 & 10 & 0 & 0 & 0 & 0\end{array}$

$\begin{array}{llllll}20 & 11 & 0 & 0 & 0\end{array}$

$\begin{array}{llllllll}2 & 11 & 0 & 0 & 0 & 0 \\ 13 & 0 & 0 & 0 & 0 & 0\end{array}$

$\begin{array}{llllllll}0 & 0 & 0 & 0 & 0\end{array}$

$\begin{array}{llllll}18 & 14 & 0 & 0 & 0 & 0\end{array}$

$\begin{array}{llllll}12 & 21 & 0 & 0 & 0 & 0\end{array}$

EN

5-iodo-2-hydroxybenzoicacid.sdf

cosmotherm

$\begin{array}{llllllllllll}22 & 19 & 0 & 0 & 0 & 0 & 0 & 0 & 0 & 0 & 0 & \mathrm{~V} 2000\end{array}$

$\begin{array}{llllllllllllll}1.2575 & -1.7159 & -6.6209 & 0 & 0 & 0 & 0 & 0 & 0 & 0 & 0 & 0 & 0 & 1 \\ 1.1299 & -1.0496 & -7.3210 & \mathrm{H} & 0 & 0 & 0 & 0 & 0 & 0 & 0 & 0 & 0 & 2\end{array}$

$\begin{array}{lllllllllllll}1.4577 & -2.5394 & -7.1029 & \mathrm{H} & 0 & 0 & 0 & 0 & 0 & 0 & 0 & 0 & 0\end{array}$

$\begin{array}{llllllllllllll}-0.8158 & -2.0121 & -4.9498 & 0 & 0 & 0 & 0 & 0 & 0 & 0 & 0 & 0 & 0 & 4\end{array}$

$\begin{array}{lllllllllllll}-0.0658 & -1.8808 & -5.6075 & \mathrm{H} & 0 & 0 & 0 & 0 & 0 & 0 & 0 & 0 & 0 \\ -1.6199 & -1.6910 & -5.3963 & \mathrm{H} & 0 & 0 & 0 & 0 & 0 & 0 & 0 & 0 & 0\end{array}$

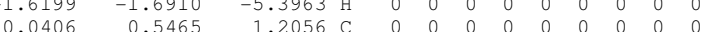




$\begin{array}{rrrrrrrrrrrrrr}-0.1337 & 0.0017 & -0.0586 & \mathrm{C} & 0 & 0 & 0 & 0 & 0 & 0 & 0 & 0 & 0 & 8 \\ -0.1438 & 0.8431 & -1.1896 & \mathrm{C} & 0 & 0 & 0 & 0 & 0 & 0 & 0 & 0 & 0 & 9 \\ 0.0230 & 2.2456 & -1.0212 & \mathrm{C} & 0 & 0 & 0 & 0 & 0 & 0 & 0 & 0 & 0 & 10 \\ 0.2001 & 2.7696 & 0.2720 & \mathrm{C} & 0 & 0 & 0 & 0 & 0 & 0 & 0 & 0 & 0 & 11 \\ 0.2089 & 1.9296 & 1.3800 & \mathrm{C} & 0 & 0 & 0 & 0 & 0 & 0 & 0 & 0 & 0 & 12 \\ -0.3268 & 0.2987 & -2.5526 & \mathrm{C} & 0 & 0 & 0 & 0 & 0 & 0 & 0 & 0 & 0 & 13 \\ -0.4632 & -1.0132 & -2.6349 & 0 & 0 & 0 & 0 & 0 & 0 & 0 & 0 & 0 & 0 & 14 \\ -0.3473 & 1.0488 & -3.5557 & 0 & 0 & 0 & 0 & 0 & 0 & 0 & 0 & 0 & 0 & 15 \\ 0.0173 & 3.0968 & -2.0706 & 0 & 0 & 0 & 0 & 0 & 0 & 0 & 0 & 0 & 0 & 16 \\ 0.0521 & -0.7354 & 2.9195 & \mathrm{I} & 0 & 0 & 0 & 0 & 0 & 0 & 0 & 0 & 0 & 17 \\ -0.6002 & -1.3315 & -3.62744 & \mathrm{H} & 0 & 0 & 0 & 0 & 0 & 0 & 0 & 0 & 0 & 18 \\ -0.1260 & 2.5101 & -2.8849 & \mathrm{H} & 0 & 0 & 0 & 0 & 0 & 0 & 0 & 0 & 0 & 19 \\ -0.2662 & -1.0696 & -0.2026 & \mathrm{H} & 0 & 0 & 0 & 0 & 0 & 0 & 0 & 0 & 0 & 20 \\ 0.3294 & 3.8459 & 0.3942 & \mathrm{H} & 0 & 0 & 0 & 0 & 0 & 0 & 0 & 0 & 0 & 21 \\ 0.3468 & 2.3538 & 2.37444 \mathrm{H} & 0 & 0 & 0 & 0 & 0 & 0 & 0 & 0 & 0 & 22\end{array}$




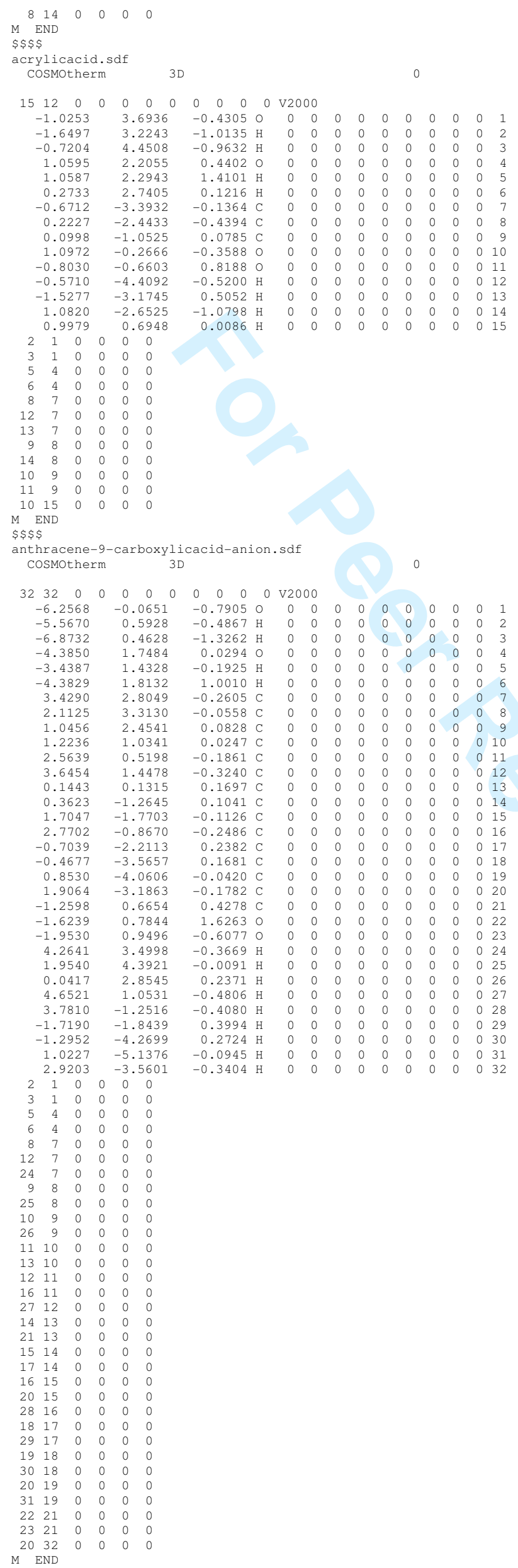


$\$ \$ \$$

anthracene-9-carboxylicacid.sdf

$\begin{array}{llllllllllll}33 & 33 & 0 & 0 & 0 & 0 & 0 & 0 & 0 & 0 & 0 & \mathrm{v} 2000\end{array}$

$\begin{array}{llllllllllllll}-1.2258 & -4.8980 & -0.5371 & 0 & 0 & 0 & 0 & 0 & 0 & 0 & 0 & 0 & 0 & 1\end{array}$

$\begin{array}{llllllllllllll}-1.2277 & -5.2535 & -1.4437 & \mathrm{H} & 0 & 0 & 0 & 0 & 0 & 0 & 0 & 0 & 0 & 2\end{array}$

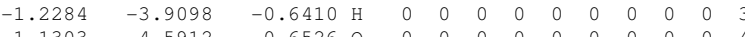

$\begin{array}{lllllllllllll}1.1303 & -4.5912 & 0.6526 & 0 & 0 & 0 & 0 & 0 & 0 & 0 & 0 & 0 & 0\end{array}$

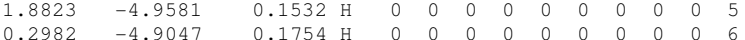

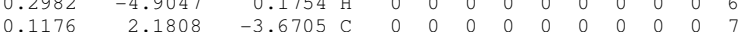

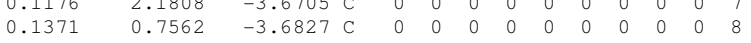

$\begin{array}{llllllllllllll}0.1371 & 0.7562 & -3.6827 & \mathrm{C} & 0 & 0 & 0 & 0 & 0 & 0 & 0 & 0 & 0 & \\ 0.0967 & 0.0394 & -2.5084 & \mathrm{C} & 0 & 0 & 0 & 0 & 0 & 0 & 0 & 0 & 0 & 9\end{array}$

$\begin{array}{lllllllllllll}0.0931 & 0.7013 & -1.2398 \mathrm{C} & 0 & 0 & 0 & 0 & 0 & 0 & 0 & 0 & 0 & 10\end{array}$

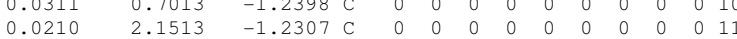

$\begin{array}{lllllllllllllll}0.0616 & 2.8570 & -2.4750 & \mathrm{C} & 0 & 0 & 0 & 0 & 0 & 0 & 0 & 0 & 0 & 12\end{array}$

$\begin{array}{lllllllllllllll}0.0140 & 0.0087 & -0.0040 & \mathrm{C} & 0 & 0 & 0 & 0 & 0 & 0 & 0 & 0 & 0 & 13\end{array}$

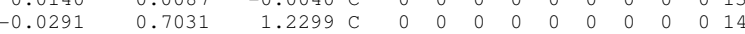

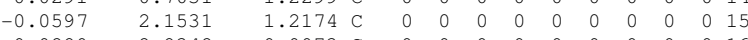

$\begin{array}{llllllllllllll}-0.0280 & 2.8342 & -0.0073 & C & 0 & 0 & 0 & 0 & 0 & 0 & 0 & 0 & 0 & 16\end{array}$

$\begin{array}{llllllllllllll}-0.0853 & 0.0434 & 2.5003 & \mathrm{C} & 0 & 0 & 0 & 0 & 0 & 0 & 0 & 0 & 0 & 17 \\ -0.1489 & 0.7617 & 3.6727 & \mathrm{C} & 0 & 0 & 0 & 0 & 0 & 0 & 0 & 0 & 0 & 18\end{array}$

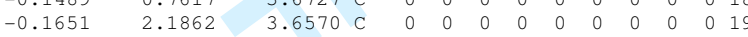

$\begin{array}{llllllllllllll}-0.1220 & 2.8606 & 2.4599 & \mathrm{C} & 0 & 0 & 0 & 0 & 0 & 0 & 0 & 0 & 0 & 20\end{array}$

$\begin{array}{llllllllllllll}0.0128 & -1.4951 & -0.0130 & \mathrm{C} & 0 & 0 & 0 & 0 & 0 & 0 & 0 & 0 & 0 & 21\end{array}$

$\begin{array}{llllllllllllll}-0.9295 & -2.1408 & -0.4939 & 0 & 0 & 0 & 0 & 0 & 0 & 0 & 0 & 0 & 0 & 22\end{array}$

$\begin{array}{llllllllllllll}1.0948 & -2.0335 & 0.5293 & 0 & 0 & 0 & 0 & 0 & 0 & 0 & 0 & 0 & 0 & 23\end{array}$

$\begin{array}{llllllllllllll}0.1502 & 2.7295 & -4.6131 & \mathrm{H} & 0 & 0 & 0 & 0 & 0 & 0 & 0 & 0 & 0 & 24 \\ 0.1870 & 0.2283 & -4.6368 & \mathrm{H} & 0 & 0 & 0 & 0 & 0 & 0 & 0 & 0 & 0 & 25\end{array}$

$\begin{array}{llllllllllllll}0.1870 & 0.2283 & -4.6368 & \mathrm{H} & 0 & 0 & 0 & 0 & 0 & 0 & 0 & 0 & 0 & 25 \\ 0.1091 & -1.0502 & -2.5439 & \mathrm{H} & 0 & 0 & 0 & 0 & 0 & 0 & 0 & 0 & 0 & 26\end{array}$

$\begin{array}{llllllllllllll}0.1091 & -1.0502 & -2.5439 & \mathrm{H} & 0 & 0 & 0 & 0 & 0 & 0 & 0 & 0 & 0 & 26\end{array}$

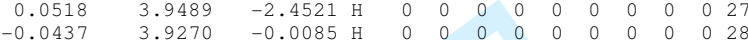

$\begin{array}{llllllllllllll}-0.0727 & -1.0459 & 2.5401 & \mathrm{H} & 0 & 0 & 0 & 0 & 0 & 0 & 0 & 0 & 0 & 29\end{array}$

$\begin{array}{llllllllllllll}-0.1914 & 0.2349 & 4.6277 & \mathrm{H} & 0 & 0 & 0 & 0 & 0 & 0 & 0 & 0 & 0 & 30\end{array}$

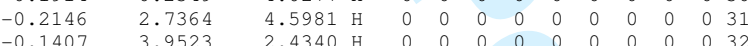

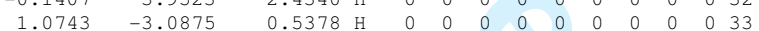

$\begin{array}{lllllll}2 & 1 & 0 & 0 & 0 & 0\end{array}$

$3 \begin{array}{llllll}3 & 1 & 0 & 0 & 0 & 0\end{array}$

$\begin{array}{llllll}5 & 0 & 0 & 0\end{array}$

$\begin{array}{llllll}8 & 7 & 0 & 0 & 0\end{array}$

$\begin{array}{lllllll}12 & 7 & 0 & 0 & 0 & 0\end{array}$

$\begin{array}{llllll}24 & 7 & 0 & 0 & 0 & 0\end{array}$

$\begin{array}{lllllllll}25 & 8 & 0 & 0 & 0 & 0\end{array}$

$\begin{array}{lllllllll}10 & 9 & 0 & 0 & 0 & 0\end{array}$

$\begin{array}{llllll}6 & 9 & 0 & 0 & 0 & 0\end{array}$

$\begin{array}{lllllll}3 & 10 & 0 & 0 & 0 & 0\end{array}$

$\begin{array}{llllll}2 & 11 & 0 & 0 & 0\end{array}$

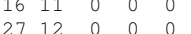

$\begin{array}{llllllll}14 & 13 & 0 & 0 & 0 & 0\end{array}$

131300000

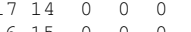

$1615 \quad 0 \quad 000$

$\begin{array}{llllll}8 & 16 & 0 & 0 & 0\end{array}$

$\begin{array}{llllll}18 & 17 & 0 & 0 & 0\end{array}$

$\begin{array}{llllll}29 & 17 & 0 & 0 & 0 & 0 \\ 9 & 18 & 0 & 0 & 0 & 0\end{array}$

$\begin{array}{llllll}30 & 18 & 0 & 0 & 0 & 0\end{array}$

$\begin{array}{llllll}20 & 19 & 0 & 0 & 0 & 0\end{array}$

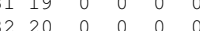

$2 \begin{array}{llllll}21 & 0 & 0 & 0 & 0\end{array}$

$\begin{array}{llllll}3 & 33 & 0 & 0 & 0 & 0\end{array}$

END

$\$ \$ \$ \$$

nzoicacid-anion.sdf

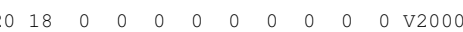

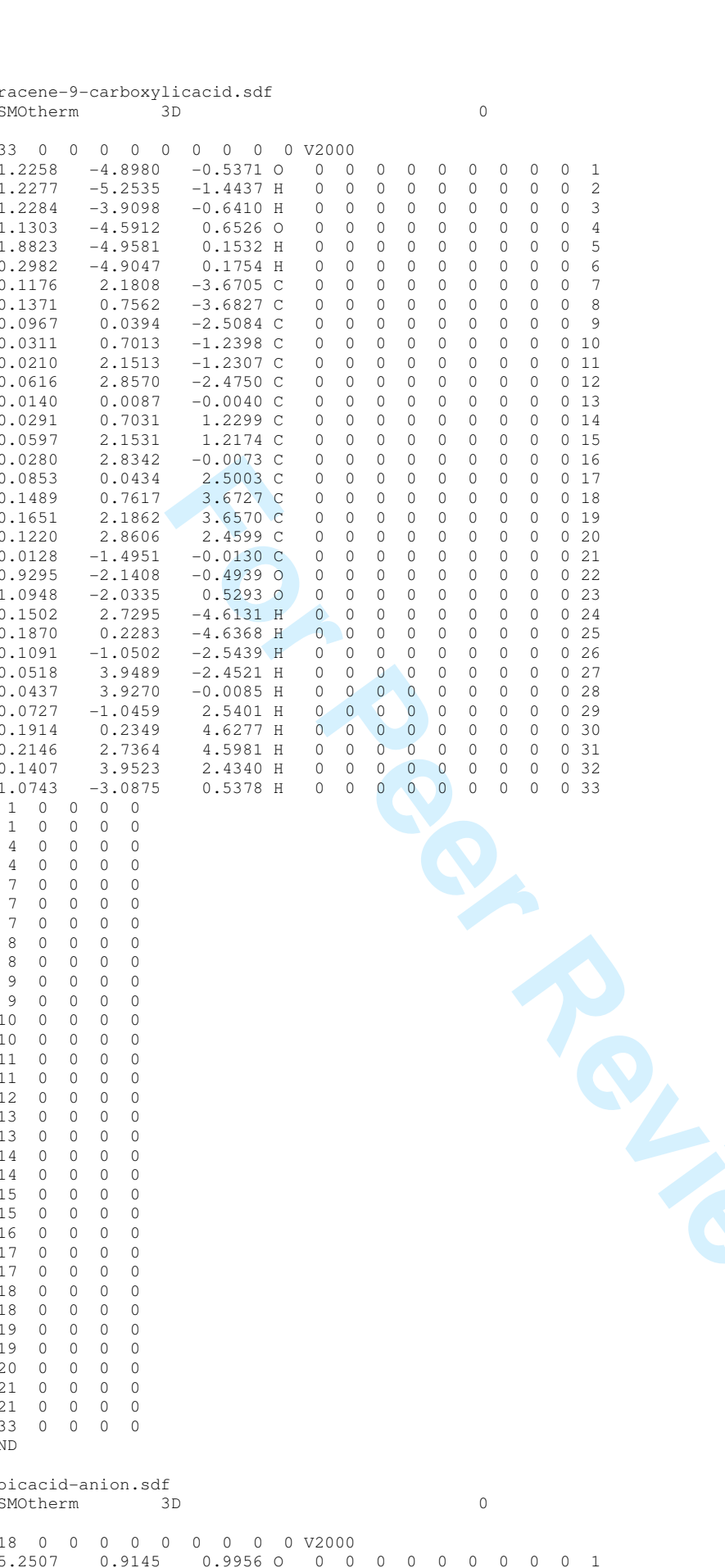

$\begin{array}{llllllllllll}0 & 0 & 0 & 0 & 0 & 0 & 0 & 0 & 0 & 0 & 1\end{array}$

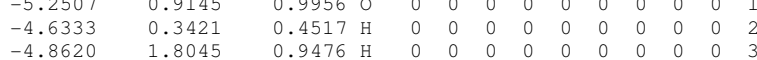

$\begin{array}{rrrrrrrrrrrrrr}-4.8620 & 1.8045 & 0.9476 & \mathrm{H} & 0 & 0 & 0 & 0 & 0 & 0 & 0 & 0 & 0 & 3 \\ -3.6291 & -0.7256 & -0.4436 & 0 & 0 & 0 & 0 & 0 & 0 & 0 & 0 & 0 & 0 & 4\end{array}$

$\begin{array}{llllllllllllll}-3.6291 & -0.7256 & -0.4436 & 0 & 0 & 0 & 0 & 0 & 0 & 0 & 0 & 0 & 0 & 4 \\ -2.6552 & -0.6693 & -0.1196 & \mathrm{H} & 0 & 0 & 0 & 0 & 0 & 0 & 0 & 0 & 0 & 5\end{array}$

$\begin{array}{llllllllllllll}-2.6552 & -0.6693 & -0.1196 & \mathrm{H} & 0 & 0 & 0 & 0 & 0 & 0 & 0 & 0 & 0 & 5 \\ -3.5737 & -0.4436 & -1.3740 & \mathrm{H} & 0 & 0 & 0 & 0 & 0 & 0 & 0 & 0 & 0 & 6\end{array}$

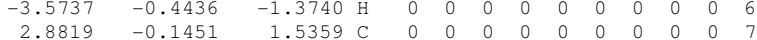

$\begin{array}{lllllllllllll}2.8819 & -0.1451 & 1.5359 & \mathrm{C} & 0 & 0 & 0 & 0 & 0 & 0 & 0 & 0 & 0 \\ 1.5330 & -0.2878 & 1.1937 & \mathrm{C} & 0 & 0 & 0 & 0 & 0 & 0 & 0 & 0 & 0\end{array}$

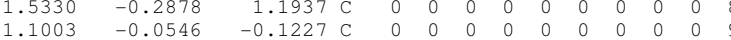

$\begin{array}{lllllllllllllll}2.0459 & 0.3224 & -1.0910 & \mathrm{C} & 0 & 0 & 0 & 0 & 0 & 0 & 0 & 0 & 0 & 10\end{array}$

$\begin{array}{llllllllllllll}3.3959 & 0.4614 & -0.7534 & \mathrm{C} & 0 & 0 & 0 & 0 & 0 & 0 & 0 & 0 & 0 & 11\end{array}$

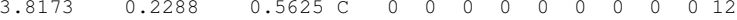

$\begin{array}{lllllllllllll}-0.3677 & -0.2048 & -0.5054 \mathrm{C} & 0 & 0 & 0 & 0 & 0 & 0 & 0 & 0 & 0 & 13\end{array}$

$\begin{array}{llllllllllllll}-1.1615 & -0.6100 & 0.4191 & 0 & 0 & 0 & 0 & 0 & 0 & 0 & 0 & 0 & 0 & 14\end{array}$

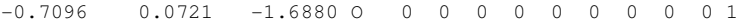

$\begin{array}{llllllllllllll}3.2056 & -0.3262 & 2.5629 & \mathrm{H} & 0 & 0 & 0 & 0 & 0 & 0 & 0 & 0 & 0 & 16\end{array}$

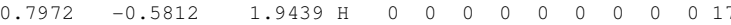

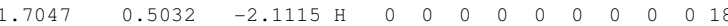

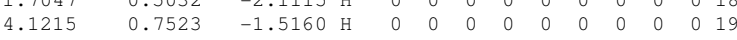
$4.8706-0.3388$

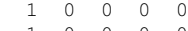

$\begin{array}{lllllll}3 & 1 & 0 & 0 & 0 & 0 & 0 \\ 6 & 4 & 0 & 0 & 0 & 0 & 0\end{array}$

$\begin{array}{lllll}4 & 0 & 0 & 0 & 0\end{array}$

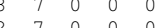

$\begin{array}{lllllll}16 & 7 & 0 & 0 & 0 & 0\end{array}$

$\begin{array}{llllll}8 & 0 & 0 & 0 & 0\end{array}$ 
$\begin{array}{rrrrrr}17 & 8 & 0 & 0 & 0 & 0 \\ 10 & 9 & 0 & 0 & 0 & 0 \\ 13 & 9 & 0 & 0 & 0 & 0 \\ 11 & 10 & 0 & 0 & 0 & 0 \\ 18 & 10 & 0 & 0 & 0 & 0 \\ 12 & 11 & 0 & 0 & 0 & 0 \\ 19 & 11 & 0 & 0 & 0 & 0 \\ 14 & 13 & 0 & 0 & 0 & 0 \\ 15 & 13 & 0 & 0 & 0 & 0 \\ 12 & 20 & 0 & 0 & 0 & 0\end{array}$

$$
\begin{aligned}
& M \text { END } \\
& \$ \$ \$ \$ \\
& \text { benzoicacid.sdf }
\end{aligned}
$$

cosmotherm
Coicacid.sdf

\begin{tabular}{|c|c|c|c|c|c|c|c|c|c|c|c|c|}
\hline-0.803 & 4.9122 & 0.9008 & 0 & 0 & 0 & 0 & 0 & 0 & 0 & 0 & 0 & 0 \\
\hline-1.7008 & 4.8667 & 0.5237 & $\mathrm{H}$ & 0 & 0 & 0 & 0 & 0 & 0 & 0 & 0 & 0 \\
\hline-0.6168 & 5.8665 & 0.9657 & $\mathrm{H}$ & 0 & 0 & 0 & 0 & 0 & 0 & 0 & 0 & 0 \\
\hline 1.0878 & 3.5814 & -0.4899 & 0 & 0 & 0 & 0 & 0 & 0 & 0 & 0 & 0 & 0 \\
\hline 0.3600 & 4.0871 & -0.0188 & $\mathrm{H}$ & 0 & 0 & 0 & 0 & 0 & 0 & 0 & 0 & 0 \\
\hline 1.0141 & 3.8319 & -1.4281 & $\mathrm{H}$ & 0 & 0 & 0 & 0 & 0 & 0 & 0 & 0 & 0 \\
\hline 1.1799 & -3.1259 & 0.4030 & C & 0 & 0 & 0 & 0 & 0 & 0 & 0 & 0 & 0 \\
\hline 1.1617 & -1.7409 & 0.2184 & C & 0 & 0 & 0 & 0 & 0 & 0 & 0 & 0 & 0 \\
\hline-0.0462 & -1.0849 & -0.0797 & C & 0 & 0 & 0 & 0 & 0 & 0 & 0 & 0 & 0 \\
\hline-1.2317 & -1.8327 & -0.1884 & $\mathrm{C}$ & 0 & 0 & 0 & 0 & 0 & 0 & 0 & 0 & 0 \\
\hline-1.2106 & -3.2162 & -0.0019 & C & 0 & 0 & 0 & 0 & 0 & 0 & 0 & 0 & 0 \\
\hline-0.004 & -3.8652 & 0.2 & $\mathrm{C}$ & 0 & 0 & 0 & 0 & 0 & 0 & 0 & 0 & 0 \\
\hline-0.1101 & 0.3960 & -0.2905 & C & 0 & 0 & 0 & 0 & 0 & 0 & 0 & 0 & 0 \\
\hline & 1.0128 & -0.1 & 0 & 0 & 0 & 0 & 0 & 0 & 0 & 0 & 0 & 0 \\
\hline-1.1528 & 0.9888 & -0.5772 & 0 & 0 & 0 & 0 & 0 & 0 & 0 & 0 & 0 & 0 \\
\hline & -3.6302 & 0.6327 & $\mathrm{H}$ & 0 & 0 & 0 & 0 & 0 & 0 & 0 & 0 & 0 \\
\hline 2.0826 & -1.1641 & 0.3034 & $\mathrm{H}$ & 0 & 0 & 0 & 0 & 0 & 0 & 0 & 0 & 0 \\
\hline-2.1645 & -1.3167 & -0.4197 & & 0 & 0 & 0 & 0 & 0 & 0 & 0 & 0 & 0 \\
\hline-2.1345 & -3.7907 & -0.0869 & $\mathrm{H}$ & 0 & 0 & 0 & 0 & 0 & 0 & 0 & 0 & 0 \\
\hline & -4.9469 & 0.4384 & & 0 & 0 & 0 & 0 & 0 & 0 & 0 & 0 & 0 \\
\hline 0.9731 & 2.0302 & -0.3011 & $\mathrm{H}$ & 0 & 0 & 0 & 0 & 0 & 0 & 0 & 0 & 0 \\
\hline
\end{tabular}

$\begin{array}{llllllllllll}21 & 19 & 0 & 0 & 0 & 0 & 0 & 0 & 0 & 0 & 0 & \mathrm{~V} 2000\end{array}$

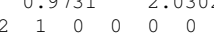

$\begin{array}{llllll}2 & 1 & 0 & 0 & 0 & 0 \\ 3 & 1 & 0 & 0 & 0 & 0\end{array}$

$\begin{array}{llllll}5 & 4 & 0 & 0 & 0 & 0\end{array}$

$6 \begin{array}{lllllll}5 & 4 & 0 & 0 & 0 & 0 & 0 \\ 8 & 7 & 0 & 0 & 0 & 0\end{array}$

$\begin{array}{llllll}8 & 7 & 0 & 0 & 0 & 0 \\ 1 & 7 & 0 & 0 & 0 & 0\end{array}$

$\begin{array}{llllll}16 & 7 & 0 & 0 & 0 & 0\end{array}$

$\begin{array}{llllll}9 & 8 & 0 & 0 & 0 & 0\end{array}$

$\begin{array}{llllll}7 & 8 & 0 & 0 & 0 & 0 \\ 10 & 9 & 0 & 0 & 0 & 0\end{array}$

$\begin{array}{lllllll}3 & 9 & 0 & 0 & 0 & 0\end{array}$

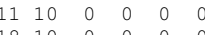

$\begin{array}{llllll}18 & 10 & 0 & 0 & 0 & 0 \\ 12 & 11 & 0 & 0 & 0 & 0\end{array}$

19111000000

$\begin{array}{llllll}20 & 12 & 0 & 0 & 0 & 0 \\ 14 & 13 & 0 & 0 & 0 & 0\end{array}$

$\begin{array}{llllll}15 & 13 & 0 & 0 & 0 & 0 \\ 14 & 21 & 0 & 0 & 0 & 0\end{array}$

$M$ END

$\$ \$ \$$

boricacid-anion.sdf
CosMotherm

$\begin{array}{llllllllllll}12 & 9 & 0 & 0 & 0 & 0 & 0 & 0 & 0 & 0 & 0 & \mathrm{v} 2000\end{array}$

$\begin{array}{llllllllllllll}-2.5583 & -0.0768 & -1.1409 & 0 & 0 & 0 & 0 & 0 & 0 & 0 & 0 & 0 & 0 & 1 \\ -1.5314 & -0.0077 & -1.1310 & \mathrm{H} & 0 & 0 & 0 & 0 & 0 & 0 & 0 & 0 & 0 & 2\end{array}$

$\begin{array}{llllllllllllll}-2.8703 & 0.8015 & -1.4206 & \mathrm{H} & 0 & 0 & 0 & 0 & 0 & 0 & 0 & 0 & 0 & 3\end{array}$

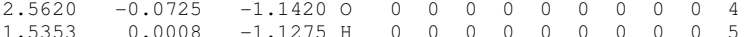

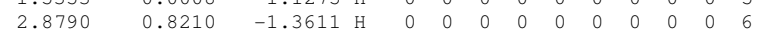

$\begin{array}{llllllllllllll}-0.0010 & 0.0160 & 0.6218 & \text { B } & 0 & 0 & 0 & 0 & 0 & 0 & 0 & 0 & 0 & 7\end{array}$

$\begin{array}{llllllllllllll}-1.1796 & 0.0036 & 1.4016 & 0 & 0 & 0 & 0 & 0 & 0 & 0 & 0 & 0 & 0 & 8\end{array}$

$\begin{array}{rrrrrrrrrrrrrr}0.1738 & -0.0070 & 1.4069 & 0 & 0 & 0 & 0 & 0 & 0 & 0 & 0 & 0 & 0 & 10\end{array}$

$\begin{array}{rrrrrrrrrrrrrr}1.1738 & -0.0070 & 1.4069 & 0 & 0 & 0 & 0 & 0 & 0 & 0 & 0 & 0 & 0 & 10 \\ -1.9436 & 0.0044 & 0.7800 & \mathrm{H} & 0 & 0 & 0 & 0 & 0 & 0 & 0 & 0 & 0 & 11\end{array}$

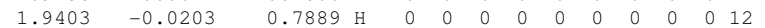

$\begin{array}{lllllll}2 & 1 & 0 & 0 & 0 & 0\end{array}$

$\begin{array}{lllllll}3 & 1 & 0 & 0 & 0 & 0\end{array}$

$\begin{array}{lllll}4 & 0 & 0 & 0 & 0\end{array}$

$\begin{array}{llllll}4 & 0 & 0 & 0 & 0 \\ 8 & 0 & 0 & 0 & 0\end{array}$

$\begin{array}{llllll}7 & 0 & 0 & 0 & 0\end{array}$

$\begin{array}{llllll}0 & 7 & 0 & 0 & 0 & 0\end{array}$

$\begin{array}{rrrrrr}11 & 8 & 0 & 0 & 0 & 0 \\ 10 & 12 & 0 & 0 & 0 & 0\end{array}$

M END

$\$ \$ \$$

boricacid.sdf

cosmotherm 3D

$\begin{array}{llllllllllll}13 & 10 & 0 & 0 & 0 & 0 & 0 & 0 & 0 & 0 & 0 & \mathrm{~V} 2000\end{array}$

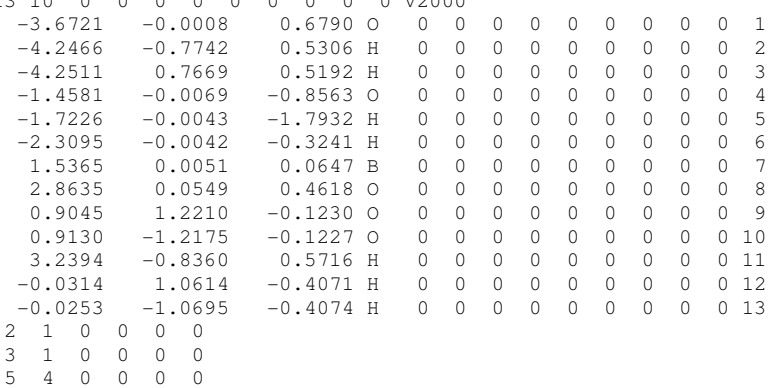

URL: http://mc.manuscriptcentral.com/tandf/tmph 


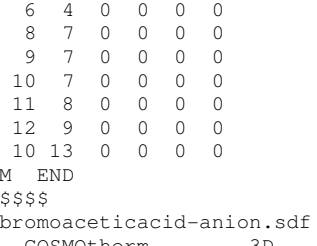

cosmotherm $3 \mathrm{D}$

$\begin{array}{lllllllllllll}13 & 9 & 0 & 0 & 0 & 0 & 0 & 0 & 0 & 0 & 0 & \mathrm{v} 2000\end{array}$

\begin{tabular}{|c|c|c|c|c|c|c|c|c|c|c|c|}
\hline 1.6525 & -0.4721 & 4.9433 & 0 & 0 & 0 & 0 & 0 & 0 & 0 & 0 & 0 \\
\hline 0.8962 & -0.0792 & 4.4192 & $\mathrm{H}$ & 0 & 0 & 0 & 0 & 0 & 0 & 0 & 0 \\
\hline 1.8136 & -1.3363 & 4.5276 & $\mathrm{H}$ & 0 & 0 & 0 & 0 & 0 & 0 & 0 & 0 \\
\hline-0.3742 & 0.7087 & 3.5508 & 0 & 0 & 0 & 0 & 0 & 0 & 0 & 0 & 0 \\
\hline-0.3003 & 0.5506 & 2.5461 & $\mathrm{H}$ & 0 & 0 & 0 & 0 & 0 & 0 & 0 & 0 \\
\hline-1.2454 & 0.3458 & 3.7889 & $\mathrm{H}$ & 0 & 0 & 0 & 0 & 0 & 0 & 0 & 0 \\
\hline 0.5840 & 0.0316 & -2.1064 & $\mathrm{Br}$ & 0 & 0 & 0 & 0 & 0 & 0 & 0 & 0 \\
\hline-1.1373 & -0.3308 & -1.1480 & $\mathrm{C}$ & 0 & 0 & 0 & 0 & 0 & 0 & 0 & 0 \\
\hline-1.1396 & -0.0759 & 0.3675 & C & 0 & 0 & 0 & 0 & 0 & 0 & 0 & 0 \\
\hline-2.2598 & -0.3324 & 0.8868 & & 0 & 0 & 0 & 0 & 0 & 0 & 0 & 0 \\
\hline-0.0952 & 0.3435 & 0.9516 & 0 & 0 & 0 & 0 & 0 & 0 & 0 & 0 & 0 \\
\hline-1.3616 & -1.3815 & -1.3563 & $\mathrm{H}$ & 0 & 0 & 0 & 0 & 0 & 0 & 0 & 0 \\
\hline-1.8763 & 0.3078 & -1.6417 & $\mathrm{H}$ & 0 & 0 & 0 & 0 & 0 & 0 & 0 & 0 \\
\hline
\end{tabular}

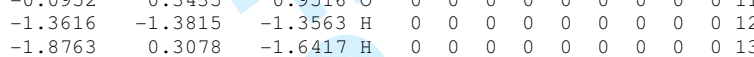

$2 \begin{array}{llllll}2 & 1 & 0 & 0 & 0 & 0\end{array}$

$\begin{array}{llllll}3 & 1 & 0 & 0 & 0 & 0\end{array}$

$\begin{array}{llllll}5 & 4 & 0 & 0 & 0 & 0 \\ 6 & 4 & 0 & 0 & 0 & 0\end{array}$

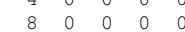

$\begin{array}{llllll}2 & 8 & 0 & 0 & 0 & 0 \\ 0 & 9 & 0 & 0 & 0 & 0\end{array}$

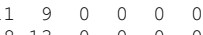

813

$\$ \$ \$$

bromoaceticacid.sdf

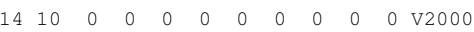

$\begin{array}{lllllllllllllll}-1.4283 & -4.8120 & 0.5141 & 0 & 0 & 0 & 0 & 0 & 0 & 0 & 0 & 0 & 0 & 1\end{array}$

$\begin{array}{llllllllllllll}-1.4283 & -4.8120 & 0.5141 & 0 & 0 & 0 & 0 & 0 & 0 & 0 & 0 & 0 & 0 & 1 \\ -1.6518 & -5.6884 & 0.1504 & \mathrm{H} & 0 & 0 & 0 & 0 & 0 & 0 & 0 & 0 & 0 & \end{array}$

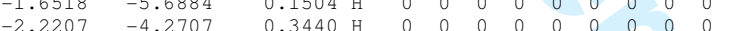

$\begin{array}{llllllllllllllllll}-2.2207 & -4.2787 & 0.3440 & 0 & 0 & 0 & 0 & 0 & 0 & 0 & 0 & 0 & 0 & 2 \\ 0.8735 & -3.8164 & -0.4469 & 0 & 0 & 0 & 0 & 0 & 0 & 0 & 0 & 0 & 0 & 3\end{array}$

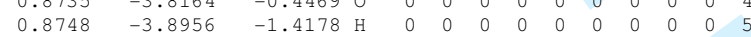

$\begin{array}{lllllllllllll}-0.0128 & -4.1837 & -0.1443 & \mathrm{H} & 0 & 0 & 0 & 0 & 0 & 0 & 0 & 0 & 0\end{array}$

$\begin{array}{lllllllllllll}0.2885 & 2.3278 & 0.0035 & \mathrm{Br} & 0 & 0 & 0 & 0 & 0 & 0 & 0 & 0 & 0\end{array}$

$\begin{array}{rrrrrrrrrrrrrrr}0.92940 & -0.4897 & -0.0688 & \mathrm{C} & 0 & 0 & 0 & 0 & 0 & 0 & 0 & 0 & 0 & 9\end{array}$

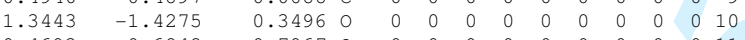

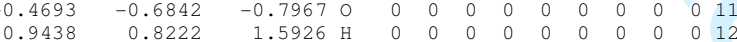

$\begin{array}{llllllllllllll}0.9438 & 0.8222 & 1.5926 & \mathrm{H} & 0 & 0 & 0 & 0 & 0 & 0 & 0 & 0 & 0 & 12 \\ 1.9140 & 1.1279 & 0.1256 & \mathrm{H} & 0 & 0 & 0 & 0 & 0 & 0 & 0 & 0 & 0 & 13\end{array}$

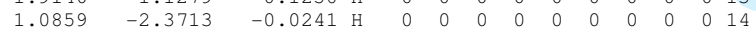

$\begin{array}{lllllll}2 & 1 & 0 & 0 & 0 & 0\end{array}$

$\begin{array}{llllll}3 & 1 & 0 & 0 & 0 & 0\end{array}$

$\begin{array}{lllllll}4 & 4 & 0 & 0 & 0 & 0\end{array}$

$\begin{array}{llllll}4 & 0 & 0 & 0 & 0\end{array}$

$\begin{array}{llllll}2 & 8 & 0 & 0 & 0 & 0 \\ 2 & 8 & 0 & 0 & 0 & 0\end{array}$

$\begin{array}{llllll}3 & 8 & 0 & 0 & 0 & 0\end{array}$

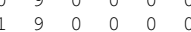

$\begin{array}{llllll}10 & 14 & 0 & 0 & 0 & 0\end{array}$

END

carbonicacid-anion.sdf

Cosmotherm

$\begin{array}{llllllllllll}11 & 8 & 0 & 0 & 0 & 0 & 0 & 0 & 0 & 0 & 0 & \mathrm{~V} 2000\end{array}$

$\begin{array}{llllllllllllll}-3.8027 & 0.4365 & -0.5538 & 0 & 0 & 0 & 0 & 0 & 0 & 0 & 0 & 0 & 0 & 1\end{array}$

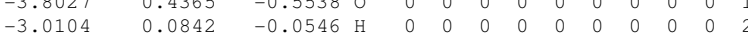

$\begin{array}{lllllllllllll}-3.6415 & 1.3931 & -0.6205 & \mathrm{H} & 0 & 0 & 0 & 0 & 0 & 0 & 0 & 0 & 0\end{array}$

$\begin{array}{llllllllllll}3 & 0.7910 & 0 & 0 & 0 & 0 & 0 & 0 & 0 & 0 & 0 & 0\end{array}$

$\begin{array}{llllllllllllll}-0.8159 & -0.5602 & 0.2607 & \mathrm{H} & 0 & 0 & 0 & 0 & 0 & 0 & 0 & 0 & 0 & 5 \\ -1.5041 & -0.1612 & 1.6224 & \mathrm{H} & 0 & 0 & 0 & 0 & 0 & 0 & 0 & 0 & 0 & 6\end{array}$

$\begin{array}{rrrrrrrrrrrrrr}-1.5041 & -0.1612 & 1.6224 & \mathrm{H} & 0 & 0 & 0 & 0 & 0 & 0 & 0 & 0 & 0 & 6 \\ 1.4924 & 0.0360 & -0.0193 & \mathrm{C} & 0 & 0 & 0 & 0 & 0 & 0 & 0 & 0 & 0 & 7\end{array}$

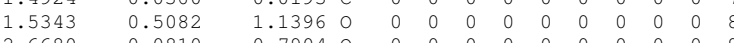

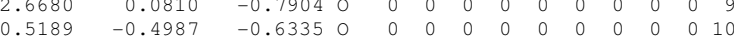

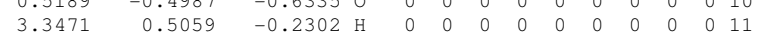

$\begin{array}{llllll}2 & 1 & 0 & 0 & 0 & 0 \\ 3 & 1 & 0 & 0 & 0 & 0\end{array}$

$\begin{array}{lllllll}3 & 1 & 0 & 0 & 0 & 0\end{array}$

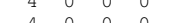

$\begin{array}{llllll}4 & 0 & 0 & 0 & 0 & 0 \\ 7 & 0 & 0 & 0\end{array}$

$\begin{array}{lllll}7 & 0 & 0 & 0\end{array}$

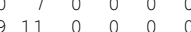

END

carbonicacid.sdf

cosmotherm

$\begin{array}{llllllllllll}12 & 9 & 0 & 0 & 0 & 0 & 0 & 0 & 0 & 0 & 0 & \mathrm{~V} 2000\end{array}$

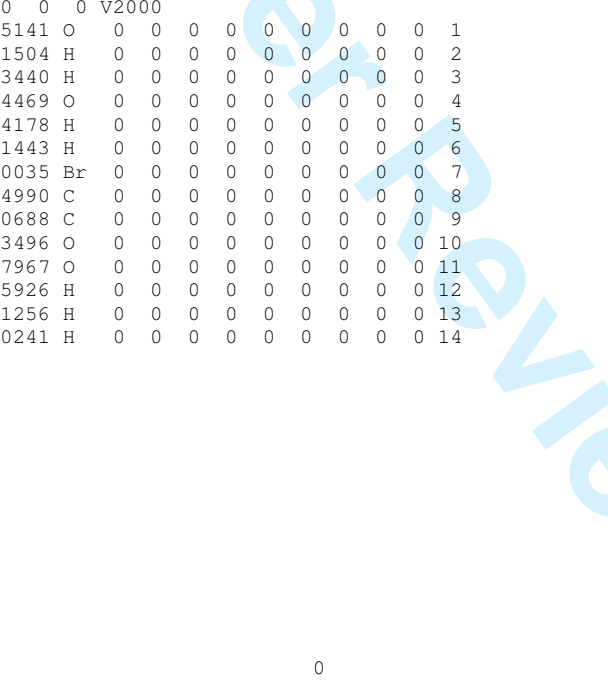

$\begin{array}{llllllllllllll}-3.3851 & 0.0055 & -0.3588 & 0 & 0 & 0 & 0 & 0 & 0 & 0 & 0 & 0 & 0 & 1\end{array}$

-0.9644 H $0 \begin{array}{llllllllll}0 & 0 & 0 & 0 & 0 & 0 & 0 & 0 & 2\end{array}$

$\begin{array}{llllllllllllll}3.3848 & -0.0033 & -0.3596 & 0 & 0 & 0 & 0 & 0 & 0 & 0 & 0 & 0 & 0 & \end{array}$

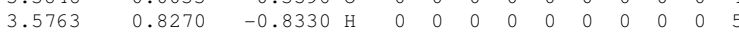

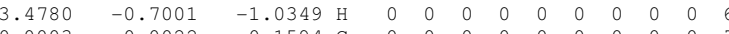

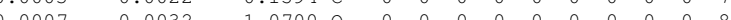

$\begin{array}{rrrrrrrrrrrrrr}0.0007 & -0.0032 & -1.0700 & 0 & 0 & 0 & 0 & 0 & 0 & 0 & 0 & 0 & 0 & 8 \\ -1.0949 & 0.0025 & 0.9300 & 0 & 0 & 0 & 0 & 0 & 0 & 0 & 0 & 0 & 0 & 9\end{array}$ 


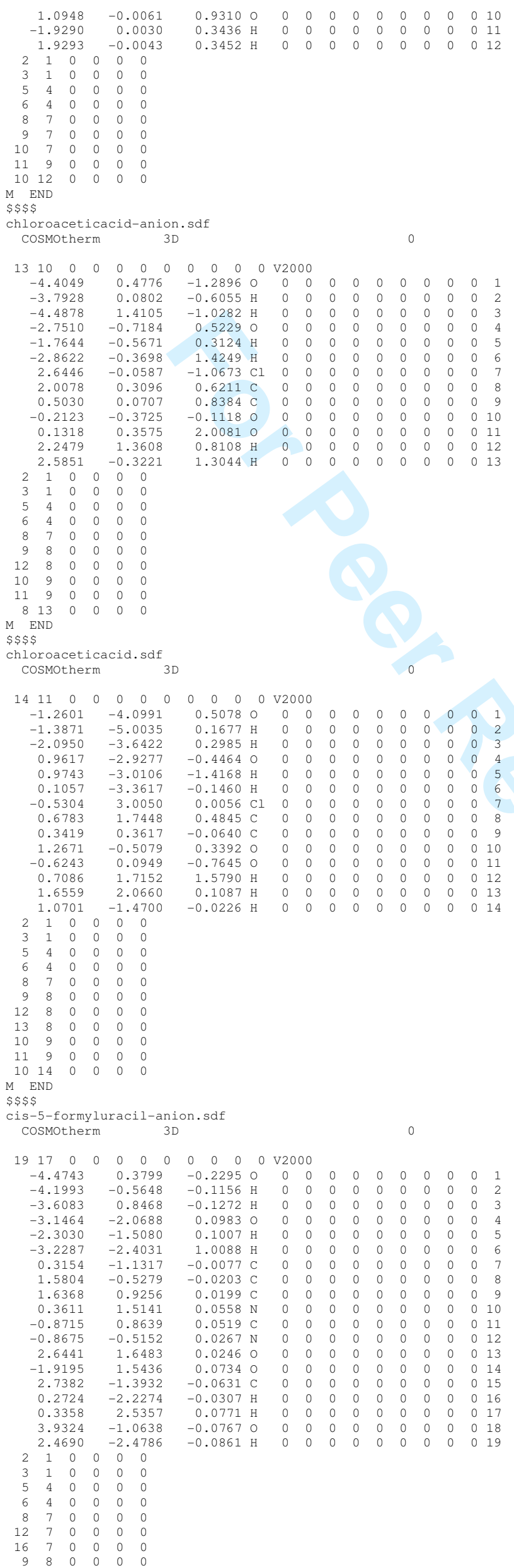


$\begin{array}{llllll}15 & 8 & 0 & 0 & 0 & 0\end{array}$

$\begin{array}{llllll}10 & 9 & 0 & 0 & 0 & 0 \\ 13 & 9 & 0 & 0 & 0 & 0\end{array}$

$\begin{array}{llllll}11 & 10 & 0 & 0 & 0 & 0 \\ 17 & 10 & 0 & 0 & 0 & 0\end{array}$

$\begin{array}{llllll}17 & 10 & 0 & 0 & 0 & 0 \\ 12 & 11 & 0 & 0 & 0 & 0\end{array}$

$\begin{array}{llllll}14 & 11 & 0 & 0 & 0 & 0 \\ 18 & 15 & 0 & 0 & 0 & 0\end{array}$

$\begin{array}{llllll}15 & 19 & 0 & 0 & 0 & 0\end{array}$

END

is-5-formyluracil.sdf

$\begin{array}{llllllllllll}20 & 18 & 0 & 0 & 0 & 0 & 0 & 0 & 0 & 0 & 0 & \mathrm{~V} 2000\end{array}$

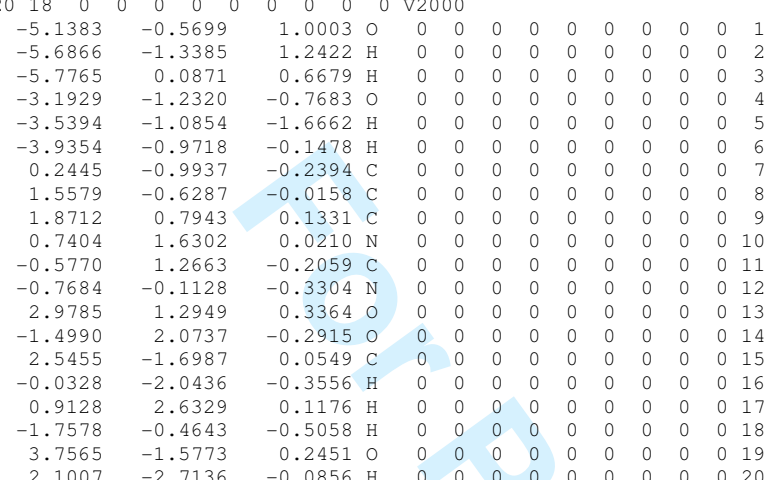

$\begin{array}{llllll}2 & 1 & 0 & 0 & 0 & 0\end{array}$

$\begin{array}{lllllll}3 & 1 & 0 & 0 & 0 & 0\end{array}$

$\begin{array}{lllllll}5 & 4 & 0 & 0 & 0 & 0\end{array}$

$\begin{array}{lllll}4 & 0 & 0 & 0 \\ 7 & 0 & 0 & 0 & 0\end{array}$

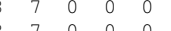

$\begin{array}{llllll}6 & 7 & 0 & 0 & 0\end{array}$

$\begin{array}{llllll}9 & 8 & 0 & 0 & 0\end{array}$

$\begin{array}{lllll}5 & 8 & 0 & 0 & 0\end{array}$

$\begin{array}{llllll}0 & 9 & 0 & 0 & 0\end{array}$

$\begin{array}{llllll}11 & 10 & 0 & 0 & 0 & 0\end{array}$

$\begin{array}{lllllll}12 & 11 & 0 & 0 & 0 & 0\end{array}$

$\begin{array}{lllllll}14 & 11 & 0 & 0 & 0 & 0\end{array}$

$\begin{array}{llllll}8 & 12 & 0 & 0 & 0 & 0\end{array}$

$\begin{array}{llllll}19 & 15 & 0 & 0 & 0 & 0 \\ -5 & 20 & 0 & 0 & 0 & 0\end{array}$

END

cyanoaceticacid-anion.sdf
cosmotherm 3D

$\begin{array}{llllllllllll}14 & 11 & 0 & 0 & 0 & 0 & 0 & 0 & 0 & 0 & 0 & V 2000\end{array}$

$\begin{array}{lllllllllllll}-4.2229 & 0.4596 & -1.2793 & 0 & 0 & 0 & 0 & 0 & 0 & 0 & 0 & 0 & 0\end{array}$

$\begin{array}{llllllllllllll}-3.6001 & 0.0791 & -0.5957 & \mathrm{H} & 0 & 0 & 0 & 0 & 0 & 0 & 0 & 0 & 0 & 2\end{array}$

$\begin{array}{lllllllllllll}-4.3134 & 1.3952 & -1.0303 & \mathrm{H} & 0 & 0 & 0 & 0 & 0 & 0 & 0 & 0 & 0\end{array}$

$\begin{array}{llllllllllllll}-2.5394 & -0.6940 & 0.5347 & 0 & 0 & 0 & 0 & 0 & 0 & 0 & 0 & 0 & 0 & 4\end{array}$

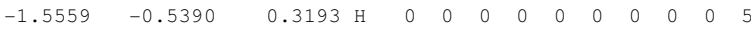

$\begin{array}{llllllllllllll}-2.6489 & -0.3449 & 1.4366 & \mathrm{H} & 0 & 0 & 0 & 0 & 0 & 0 & 0 & 0 & 0 & 6\end{array}$

$\begin{array}{llllllllllllll}2.6399 & 0.0401 & -0.8402 & \mathrm{C} & 0 & 0 & 0 & 0 & 0 & 0 & 0 & 0 & 0 & 7\end{array}$

$\begin{array}{llllllllllllll}2.2580 & 0.2685 & 0.5403 & \mathrm{C} & 0 & 0 & 0 & 0 & 0 & 0 & 0 & 0 & 0 & 8 \\ 0.7313 & 0.0665 & 0.8450 & \mathrm{C} & 0 & 0 & 0 & 0 & 0 & 0 & 0 & 0 & 0 & 9\end{array}$

$\begin{array}{llllllllllllll}-0.0003 & -0.3389 & -0.1131 & 0 & 0 & 0 & 0 & 0 & 0 & 0 & 0 & 0 & 0 & 10\end{array}$

$\begin{array}{llllllllllllll}0.3854 & 0.3229 & 2.0213 & 0 & 0 & 0 & 0 & 0 & 0 & 0 & 0 & 0 & 0 & 11\end{array}$

1.2918

$\begin{array}{llllllllllllllllll}2.5405 & 1.2918 & 0.8290 & \mathrm{H} & 0 & 0 & 0 & 0 & 0 & 0 & 0 & 0 & 0 & 0 & 13\end{array}$

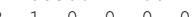

$\begin{array}{lllllll}3 & 1 & 0 & 0 & 0 & 0\end{array}$

$\begin{array}{lllll}4 & 0 & 0 & 0 & 0\end{array}$

$\begin{array}{llllll}4 & 0 & 0 & 0 & 0\end{array}$

$\begin{array}{lllllllll}7 & 0 & 0 & 0 & 0\end{array}$

$\begin{array}{llllll}2 & 0 & 0 & 0 & 0 & 0\end{array}$

$8 \quad 0 \quad 001000$

$\begin{array}{llllll}9 & 0 & 0 & 0 & 0\end{array}$

$\begin{array}{llllll}8 & 14 & 0 & 0 & 0 & 0\end{array}$

END

cyanoaceticacid.sdf

Cosmotherm 3D

$\begin{array}{lllllllllllll}15 & 12 & 0 & 0 & 0 & 0 & 0 & 0 & 0 & 0 & 0 & \mathrm{v} 2000\end{array}$

$\begin{array}{llllllll}0 & 0 & 0 & 0 & 0 & 0 & 1\end{array}$

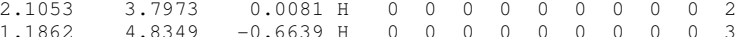

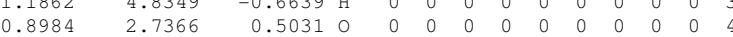

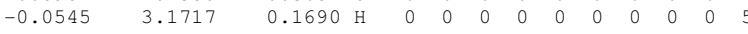

$\begin{array}{llllllllllllll}-0.8766 & 2.8210 & 1.4731 & \mathrm{H} & 0 & 0 & 0 & 0 & 0 & 0 & 0 & 0 & 0 & 6\end{array}$

$\begin{array}{llllllllllllll}0.2690 & -2.9507 & -0.1632 & \mathrm{C} & 0 & 0 & 0 & 0 & 0 & 0 & 0 & 0 & 0 & 7\end{array}$

$\begin{array}{lllllllllllll}-0.3580 & -0.5465 & 0.0575 & \mathrm{C} & 0 & 0 & 0 & 0 & 0 & 0 & 0 & 0 & 0 \\ -0.6253 & -0.3233 & 0.7514 & 0 & 0 & 0 & 0 & 0 & 0 & 0 & 0 & 0 & 0\end{array}$

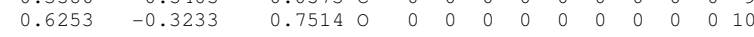

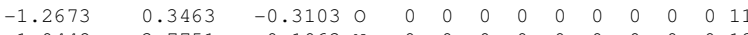

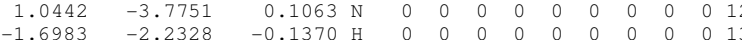

$\begin{array}{lllllllllllllll}-0.7915 & -1.8633 & -1.6054 \mathrm{H} & 0 & 0 & 0 & 0 & 0 & 0 & 0 & 0 & 0 & 14\end{array}$

$\begin{array}{rrrrrrrrrrrrrr}-1.0434 & 1.3003 & 0.0689 \mathrm{H} & 0 & 0 & 0 & 0 & 0 & 0 & 0 & 0 & 0 & 15\end{array}$ 


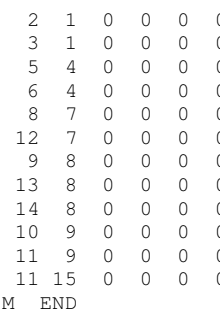




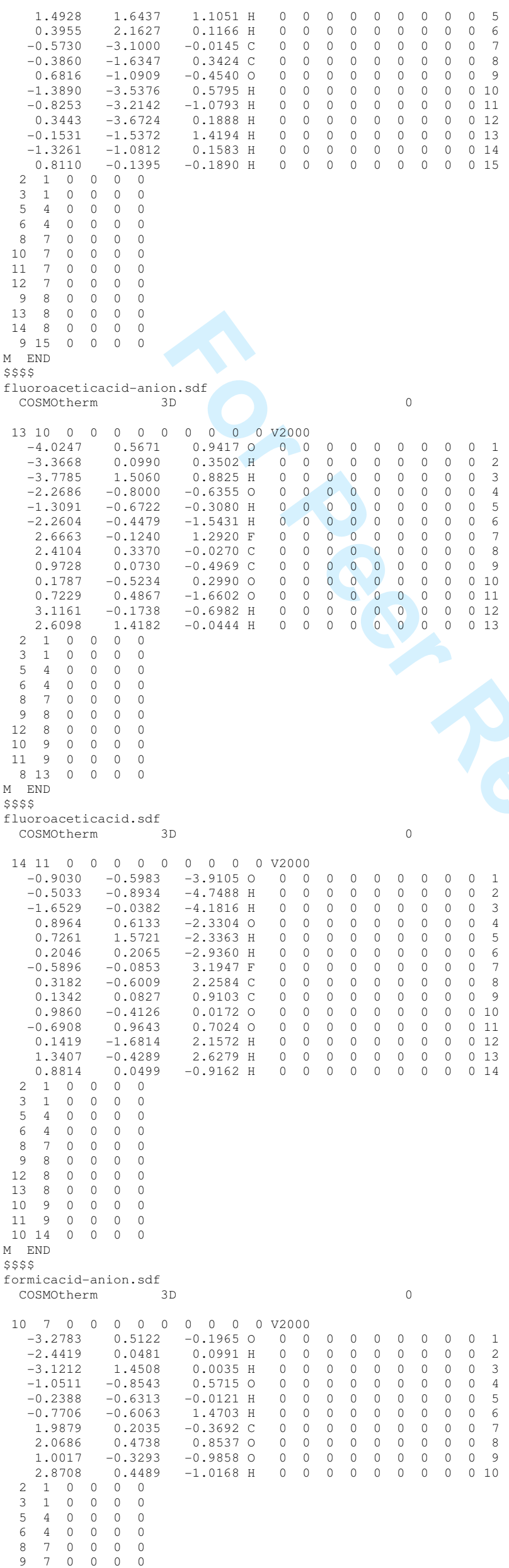


$\begin{array}{rrrrrr}7 & 10 & 0 & 0 & 0 & 0 \\ \text { M END } & & & & \\ \$ \$ \$ \$ \\ \text { formicacid.sdf } \\ \text { COSMOtherm }\end{array}$

$\begin{array}{llllllllllll}11 & 8 & 0 & 0 & 0 & 0 & 0 & 0 & 0 & 0 & 0 & \mathrm{~V} 2000\end{array}$

$\begin{array}{llllllllllllll}-3.0716 & -0.5713 & -0.3489 & 0 & 0 & 0 & 0 & 0 & 0 & 0 & 0 & 0 & 0 & 1\end{array}$ $\begin{array}{llllllllllllll}-2.8136 & -1.4068 & -0.7796 & \mathrm{H} & 0 & 0 & 0 & 0 & 0 & 0 & 0 & 0 & 0 & 2\end{array}$

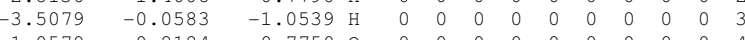

$\begin{array}{llllllllllllll}-1.0579 & 0.8184 & 0.7750 & 0 & 0 & 0 & 0 & 0 & 0 & 0 & 0 & 0 & 0 & 4\end{array}$

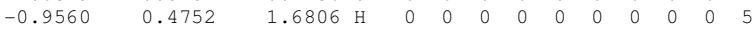

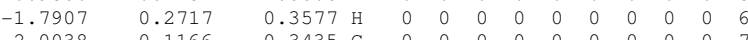

$\begin{array}{rrrrrrrrrrrrrr}2.0038 & -0.1166 & -0.3435 & \mathrm{C} & 0 & 0 & 0 & 0 & 0 & 0 & 0 & 0 & 0 & 7 \\ 1.1317 & 0.8542 & -0.5960 & 0 & 0 & 0 & 0 & 0 & 0 & 0 & 0 & 0 & 0 & 8\end{array}$

$\begin{array}{rrrrrrrrrrrrr}1.8644 & -1.0157 & 0.4771 & 0 & 0 & 0 & 0 & 0 & 0 & 0 & 0 & 0 & 0\end{array}$

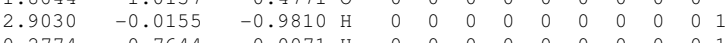

$\begin{array}{lllll}1 & 0 & 0 & 0 & 0\end{array}$

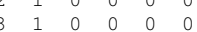

$\begin{array}{lllll}4 & 0 & 0 & 0 & 0\end{array}$

$\begin{array}{llllll}4 & 0 & 0 & 0 & 0\end{array}$

$\begin{array}{llllll}7 & 0 & 0 & 0 & 0\end{array}$

$\begin{array}{lrllll}8 & 11 & 0 & 0 & 0 & 0\end{array}$

END

$\$ \$ \$$

fumaricacid-anion.sdf

Cosmotherm

$\begin{array}{llllllllllll}17 & 14 & 0 & 0 & 0 & 0 & 0 & 0 & 0 & 0 & 0 & \text { V2 } 2000\end{array}$

$\begin{array}{lllllllllllll}-5.3168 & -0.0065 & 0.1831 & 0 & 0 & 0 & 0 & 0 & 0 & 0 & 0 & 0 & 0\end{array}$

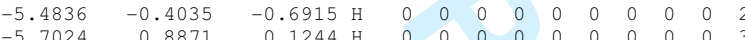

$\begin{array}{llllllllllllll}5.3716 & 0.0431 & 0.0689 & 0 & 0 & 0 & 0 & 0 & 0 & 0 & 0 & 0 & 0 & 4\end{array}$

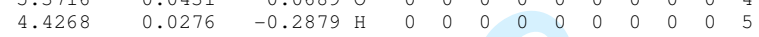

$\begin{array}{llllllllllllll}5.2428 & 0.0251 & 1.0331 & \mathrm{H} & 0 & 0 & 0 & 0 & 0 & 0 & 0 & 0 & 0 & 6\end{array}$

$\begin{array}{llllll}-0.0124 & 0.3631 \mathrm{C} & 0 & 0 & 0 & 0 \\ -0.0167 & -0.4735 \mathrm{C} & 0 & 0 & 0\end{array}$

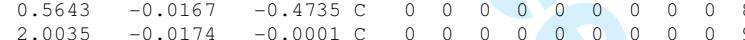

$\begin{array}{llllllllllllllll}-1.8879 & -0.0043 & -0.1228 & \mathrm{C} & 0 & 0 & 0 & 0 & 0 & 0 & 0 & 0 & 0 & 10\end{array}$

$\begin{array}{lllllllllllllllll}-1.8279 & -0.00142 & -0.1228 & 0 & 0 & 0 & 0 & 0 & 0 & 0 & 0 & 0 & 0 & 10\end{array}$

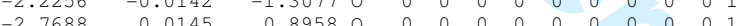

$\begin{array}{llllllllllllllllll}-2.7688 & 0.0145 & 0.8958 & 0 & 0 & 0 & 0 & 0 & 0 & 0 & 0 & 0 & 0 & 0 & 0 & 11\end{array}$

$\begin{array}{lllllllllllllllllllllll}2.8765 & 0.01456 & -0.9402 & 0 & 0 & 0 & 0 & 0 & 0 & 0 & 0 & 0 & 0 & 0 & 13\end{array}$

$\begin{array}{lllllllllllll}-0.3262 & -0.0120 & 1.4435 \mathrm{H} & 0 & 0 & 0 & 0 & 0 & 0 & 0 & 0 & 0 & 15\end{array}$

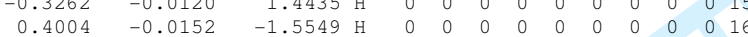

$\begin{array}{llllllllllll}-3.7240 & 0.0231 & 0.5341 \mathrm{H} & 0 & 0 & 0 & 0 & 0 & 0 & 0 & 0 & 0\end{array}$

$\begin{array}{llllll}2 & 1 & 0 & 0 & 0 & 0\end{array}$

$\begin{array}{lllllllll}2 & 4 & 0 & 0 & 0 & 0\end{array}$

$\begin{array}{lllllll}4 & 0 & 0 & 0 & 0\end{array}$

$\begin{array}{lllll}7 & 0 & 0 & 0\end{array}$

$\begin{array}{lllllllll}1 & 7 & 7 & 0 & 0 & 0 & 0 & 2 & 2\end{array}$

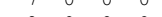

$\begin{array}{lllllll}16 & 8 & 0 & 0 & 0\end{array}$

$\begin{array}{llllll}13 & 9 & 0 & 0 & 0\end{array}$

$\begin{array}{llllll}4 & 9 & 0 & 0 & 0\end{array}$

$\begin{array}{llllll}1 & 10 & 0 & 0 & 0 & 0\end{array}$

$\begin{array}{llllll}2 & 10 & 0 & 0 & 0 & 0 \\ 17 & 17 & 0 & 0 & 0\end{array}$

M END

fumaricacid.sdf

Cosmotherm

$\begin{array}{llllllllllll}18 & 15 & 0 & 0 & 0 & 0 & 0 & 0 & 0 & 0 & 0 & \mathrm{~V} 2000\end{array}$

$-5.3501-0.0220$

$\begin{array}{rr}-5.3501 & 0.0220 \\ -5.6451 & -0.7885\end{array}$

$\begin{array}{rr}-5.5944 & 0.7478\end{array}$

$5.3524 \quad 0.0227$

$\begin{array}{rr}5.6497 & -0.7785 \\ 5.5949 & 0.7595\end{array}$

$\begin{array}{rr}5.5949 & 0.7595 \\ -0.5340 & -0.0073\end{array}$

0.5332

$-0.0104$

.9234

$-0.0061$

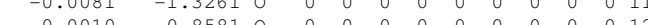

$\begin{array}{llllllllllllll}2.8303 & -0.0003 & -0.8578 & 0 & 0 & 0 & 0 & 0 & 0 & 0 & 0 & 0 & 0 & 13\end{array}$

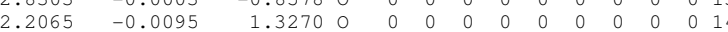

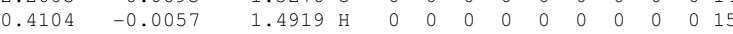

$\begin{array}{llllllllllllll}0.4093 & -0.0126 & -1.4903 & \mathrm{H} & 0 & 0 & 0 & 0 & 0 & 0 & 0 & 0 & 0 & 16\end{array}$

0.78310 .0062

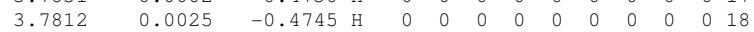

$\begin{array}{lllllll}2 & 1 & 0 & 0 & 0 & 0\end{array}$

$\begin{array}{lllllllll}3 & 1 & 0 & 0 & 0 & 0\end{array}$

$\begin{array}{llllll}5 & 4 & 0 & 0 & 0 & 0\end{array}$

$\begin{array}{cccccc}7 & 7 & 0 & 0 & 0 & 0\end{array}$

$\begin{array}{lllllll}10 & 7 & 0 & 0 & 0 & 0\end{array}$

$\begin{array}{llllll}1 & 7 & 0 & 0 & 0 & 0\end{array}$

$\begin{array}{llllll}9 & 8 & 0 & 0 & 0 & 0 \\ 6 & 8 & 0 & 0 & 0 & 0\end{array}$

$\begin{array}{llllll}3 & 9 & 0 & 0 & 0 & 0\end{array}$

$\begin{array}{lllllll}1 & 9 & 0 & 0 & 0 & 0\end{array}$

$\begin{array}{llllll}11 & 10 & 0 & 0 & 0 & 0\end{array}$

$\begin{array}{llllll}17 & 12 & 0 & 0 & 0 & 0\end{array}$

1318

hypobromousacid-anion.sdf 


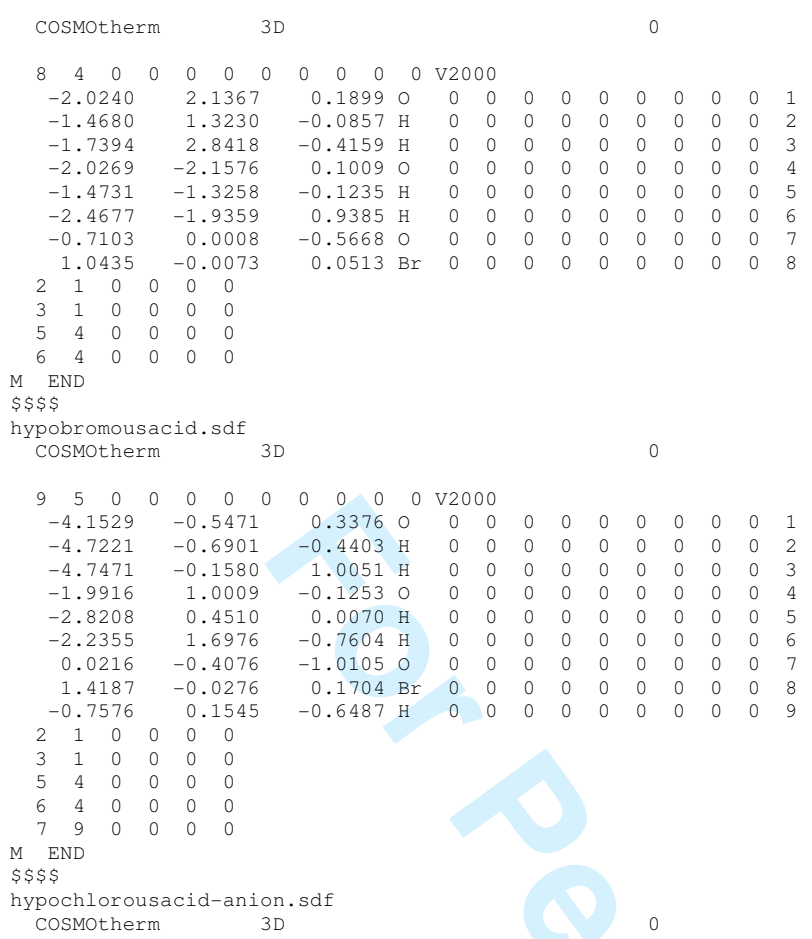




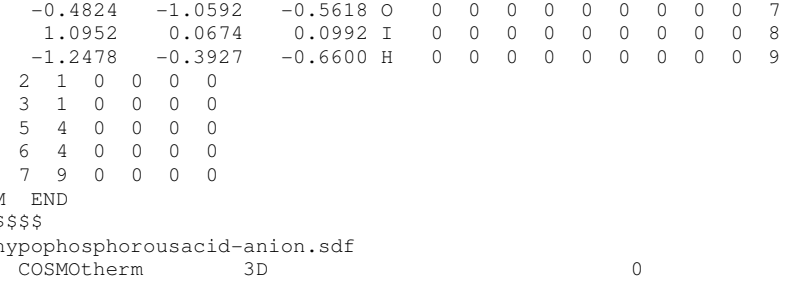




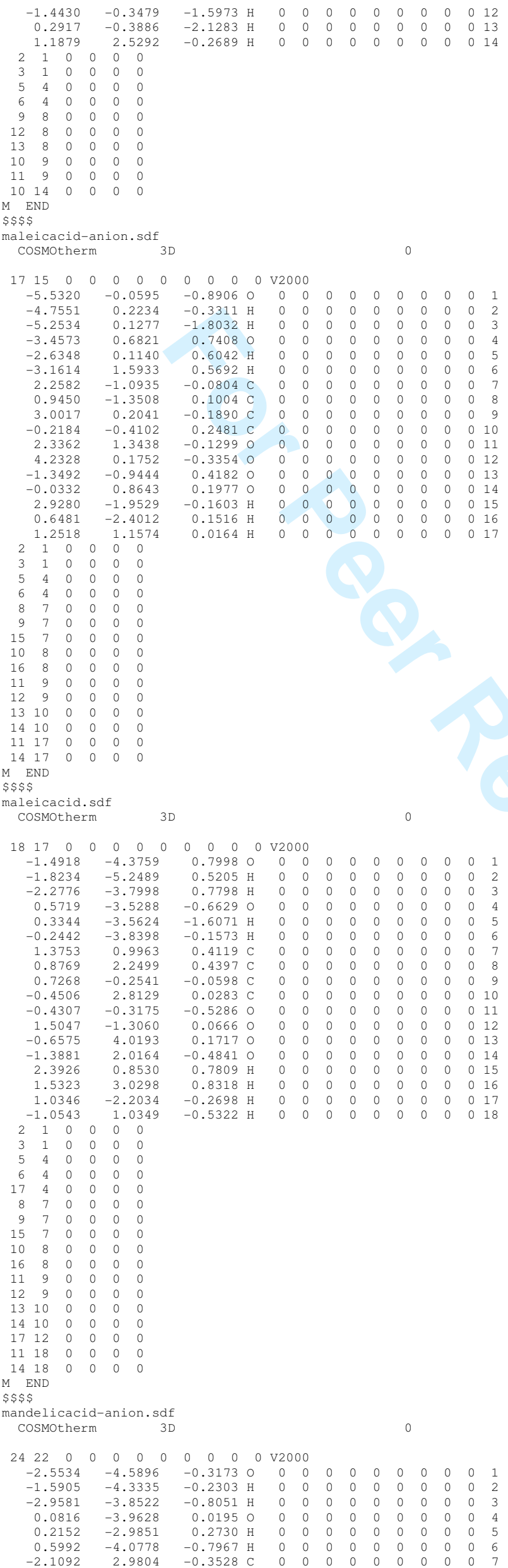


$-1.9096$ 2.4859

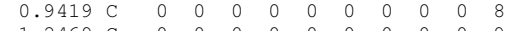

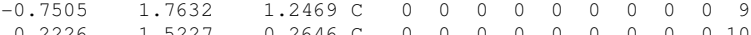

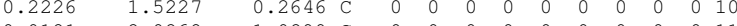

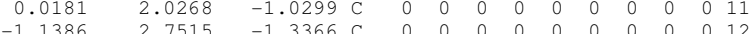

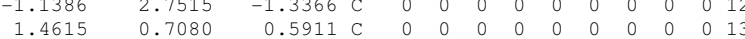
$\begin{array}{llllllllllllll}2.6285 & 1.2112 & -0.0660 & 0 & 0 & 0 & 0 & 0 & 0 & 0 & 0 & 0 & 0 & 14\end{array}$ $\begin{array}{llllllllllllll}1.2718 & -0.7910 & 0.1805 & \mathrm{C} & 0 & 0 & 0 & 0 & 0 & 0 & 0 & 0 & 0 & 15\end{array}$

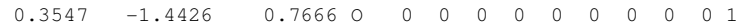
$\begin{array}{llllllllllll}2.0687 & -1.2180 & -0.7028 & 0 & 0 & 0 & 0 & 0 & 0 & 0 & 0 & 0 \\ 2.06 & 0 & 17\end{array}$ $\begin{array}{llllllllllllll}2.8885 & 0.4387 & -0.6340 & \mathrm{H} & 0 & 0 & 0 & 0 & 0 & 0 & 0 & 0 & 0 & 18\end{array}$ $\begin{array}{lllllllllllllllll}-3.0104 & 3.5486 & -0.5915 & \mathrm{H} & 0 & 0 & 0 & 0 & 0 & 0 & 0 & 0 & 0 & 0 & 19\end{array}$

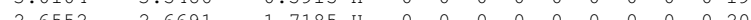

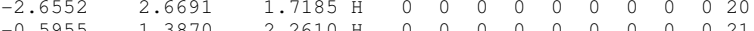

$\begin{array}{llllllllllllll}-1.7936 & 3.8610 & -1.7944 & \mathrm{H} & 0 & 0 & 0 & 0 & 0 & 0 & 0 & 0 & 0 & 22 \\ 1.6172 & 0.7235 & -2.3475 & \mathrm{H} & 0 & 0 & 0 & 0 & 0 & 0 & 0 & 0 & 0 & 23\end{array}$

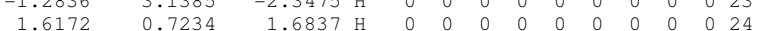




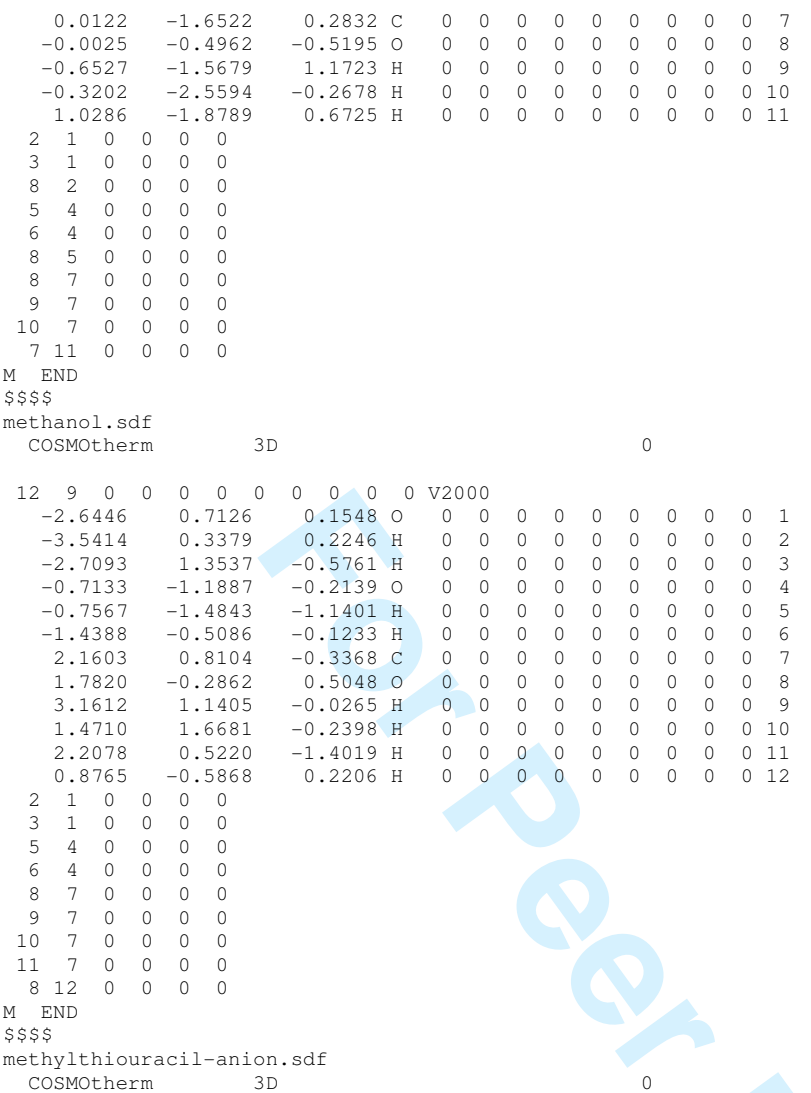

methylthiouracil-anion.sdf

$\begin{array}{llllllllllll}20 & 18 & 0 & 0 & 0 & 0 & 0 & 0 & 0 & 0 & 0 & \mathrm{v} 2000\end{array}$

$\begin{array}{llllllllllllll}-4.5520 & 0.8673 & 1.0900 & 0 & 0 & 0 & 0 & 0 & 0 & 0 & 0 & 0 & 0 & 1 \\ -3.9097 & 0.4665 & 0.4342 & \mathrm{H} & 0 & 0 & 0 & 0 & 0 & 0 & 0 & 0 & 0 & 2\end{array}$

$\begin{array}{llllllllllllll}-3.9097 & 0.4665 & 0.4342 & \mathrm{H} & 0 & 0 & 0 & 0 & 0 & 0 & 0 & 0 & 0 & 2 \\ -4.0861 & 0.8260 & 1.9424 & \mathrm{H} & 0 & 0 & 0 & 0 & 0 & 0 & 0 & 0 & 0 & 3\end{array}$

$\begin{array}{rrrrrrrrrrrrrr}-4.0861 & 0.8260 & 1.9424 & \mathrm{H} & 0 & 0 & 0 & 0 & 0 & 0 & 0 & 0 & 0 & 3 \\ -2.8641 & -0.1917 & -0.7668 & 0 & 0 & 0 & 0 & 0 & 0 & 0 & 0 & 0 & 0 & 4\end{array}$

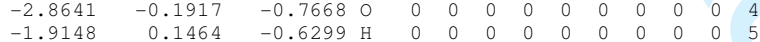

$\begin{array}{rrrrrrrrrrrrrr}-1.9148 & 0.1464 & -0.6299 & \mathrm{H} & 0 & 0 & 0 & 0 & 0 & 0 & 0 & 0 & 0 \\ -2.7134 & -1.1490 & -0.6125 & \mathrm{H} & 0 & 0 & 0 & 0 & 0 & 0 & 0 & 0 & 0 & 6\end{array}$

$\begin{array}{llllllllllllll}0.3883 & -0.8643 & -0.0902 & \mathrm{C} & 0 & 0 & 0 & 0 & 0 & 0 & 0 & 0 & 0 & 7 \\ 1.7378 & -0.8684 & 0.2021 & \mathrm{~N} & 0 & 0 & 0 & 0 & 0 & 0 & 0 & 0 & 0 & 8\end{array}$

$\begin{array}{rrrrrrrrrrrrrr}1.7378 & -0.8684 & 0.2021 & \mathrm{~N} & 0 & 0 & 0 & 0 & 0 & 0 & 0 & 0 & 0 & 8 \\ 2.5625 & 0.2683 & 0.2758 & \mathrm{C} & 0 & 0 & 0 & 0 & 0 & 0 & 0 & 0 & 0 & 9\end{array}$

$\begin{array}{rrrrrrrrrrrrrr}2.5625 & 0.2683 & 0.2758 & \mathrm{C} & 0 & 0 & 0 & 0 & 0 & 0 & 0 & 0 & 0 & 9 \\ 1.8614 & 1.4908 & 0.0146 & \mathrm{C} & 0 & 0 & 0 & 0 & 0 & 0 & 0 & 0 & 0 & 10\end{array}$

$\begin{array}{llllllllllllll}0.5108 & 1.4655 & -0.2763 \mathrm{C} & 0 & 0 & 0 & 0 & 0 & 0 & 0 & 0 & 0 & 11\end{array}$

$\begin{array}{llll}-0.2210 & 0.3085 & -0.3309 \mathrm{~N} & 0 \\ 3.7756 & 0.1361 & 0.5482 & 0\end{array}$

$\begin{array}{llllllllllllll}3.7756 & 0.1361 & 0.5482 & 0 & 0 & 0 & 0 & 0 & 0 & 0 & 0 & 0 & 0 & 13\end{array}$

$\begin{array}{llllllllllllll}-0.4468 & -2.3825 & -0.1304 & \mathrm{~S} & 0 & 0 & 0 & 0 & 0 & 0 & 0 & 0 & 0 & 14\end{array}$

$\begin{array}{llllllllllllll}2.4183 & 2.4258 & 0.0498 & \mathrm{H} & 0 & 0 & 0 & 0 & 0 & 0 & 0 & 0 & 0 & 15\end{array}$

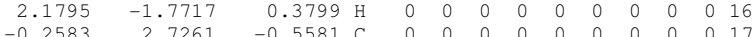

$\begin{array}{rrrrrrrrrrrrrr}-0.2583 & 2.7261 & -0.5581 & \mathrm{C} & 0 & 0 & 0 & 0 & 0 & 0 & 0 & 0 & 0 & 17 \\ -1.0923 & 2.8298 & 0.1525 & \mathrm{H} & 0 & 0 & 0 & 0 & 0 & 0 & 0 & 0 & 0 & 18\end{array}$

$\begin{array}{llllllllllllll}0.3812 & 3.6137 & -0.4847 & \mathrm{H} & 0 & 0 & 0 & 0 & 0 & 0 & 0 & 0 & 0 & 19\end{array}$

$\begin{array}{llllllll}2 & 1 & 0 & 0 & 0 & 0\end{array}$

$\begin{array}{llllll}3 & 1 & 0 & 0 & 0 & 0\end{array}$

$\begin{array}{lllllll}5 & 4 & 0 & 0 & 0 & 0\end{array}$

$\begin{array}{llllll}6 & 4 & 0 & 0 & 0 & 0\end{array}$

$\begin{array}{llllll}12 & 7 & 0 & 0 & 0 \\ 14 & 7 & 0 & 0 & 0 & 0\end{array}$

$\begin{array}{lllllll}9 & 8 & 0 & 0 & 0 & 0\end{array}$

$\begin{array}{llllll}8 & 0 & 0 & 0\end{array}$

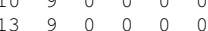

1110000000

$\begin{array}{llllll}15 & 10 & 0 & 0 & 0 & 0\end{array}$

$\begin{array}{llllll}12 & 11 & 0 & 0 & 0 & 0\end{array}$

$\begin{array}{llllll}18 & 17 & 0 & 0 & 0 & 0\end{array}$

$\begin{array}{llllll}19 & 17 & 0 & 0 & 0 & 0 \\ 17 & 20 & 0 & 0 & 0 & 0\end{array}$

$M$ END

\$\$\$

methylthiouracil.sdf
CosMotherm

$\begin{array}{rrrrrrrrrrrrrrr}21 & 19 & 0 & 0 & 0 & 0 & 0 & 0 & 0 & 0 & 0 & V 2000 & & & \\ -4.6676 & 1.0172 & -0.7490 & 0 & 0 & 0 & 0 & 0 & 0 & 0 & 0 & 0 & 0 & 1 \\ -4.3496 & 1.9383 & -0.7447 & \mathrm{H} & 0 & 0 & 0 & 0 & 0 & 0 & 0 & 0 & 0 & 2 \\ -5.4383 & 1.0268 & -0.1526 & \mathrm{H} & 0 & 0 & 0 & 0 & 0 & 0 & 0 & 0 & 0 & 3 \\ -2.7913 & -0.8269 & 0.0031 & 0 & 0 & 0 & 0 & 0 & 0 & 0 & 0 & 0 & 0 & 4 \\ -3.4683 & -0.1320 & -0.2361 & \mathrm{H} & 0 & 0 & 0 & 0 & 0 & 0 & 0 & 0 & 0 & 5 \\ -2.5923 & -0.6839 & 0.9554 & \mathrm{H} & 0 & 0 & 0 & 0 & 0 & 0 & 0 & 0 & 0 & 6 \\ 0.4055 & -0.0810 & 0.9003 & \mathrm{C} & 0 & 0 & 0 & 0 & 0 & 0 & 0 & 0 & 0 & 7 \\ 1.7442 & 0.1910 & 0.9331 & \mathrm{~N} & 0 & 0 & 0 & 0 & 0 & 0 & 0 & 0 & 0 & 8 \\ 2.6153 & 0.2780 & -0.1796 & \mathrm{C} & 0 & 0 & 0 & 0 & 0 & 0 & 0 & 0 & 0 & 9 \\ 1.9682 & 0.0467 & -1.4460 & \mathrm{C} & 0 & 0 & 0 & 0 & 0 & 0 & 0 & 0 & 0 & 10 \\ 0.6285 & -0.2243 & -1.5077 & \mathrm{C} & 0 & 0 & 0 & 0 & 0 & 0 & 0 & 0 & 0 & 11 \\ -0.1153 & -0.2796 & -0.3459 & \mathrm{~N} & 0 & 0 & 0 & 0 & 0 & 0 & 0 & 0 & 0 & 12 \\ 3.8139 & 0.5332 & 0.0055 & 0 & 0 & 0 & 0 & 0 & 0 & 0 & 0 & 0 & 0 & 13\end{array}$




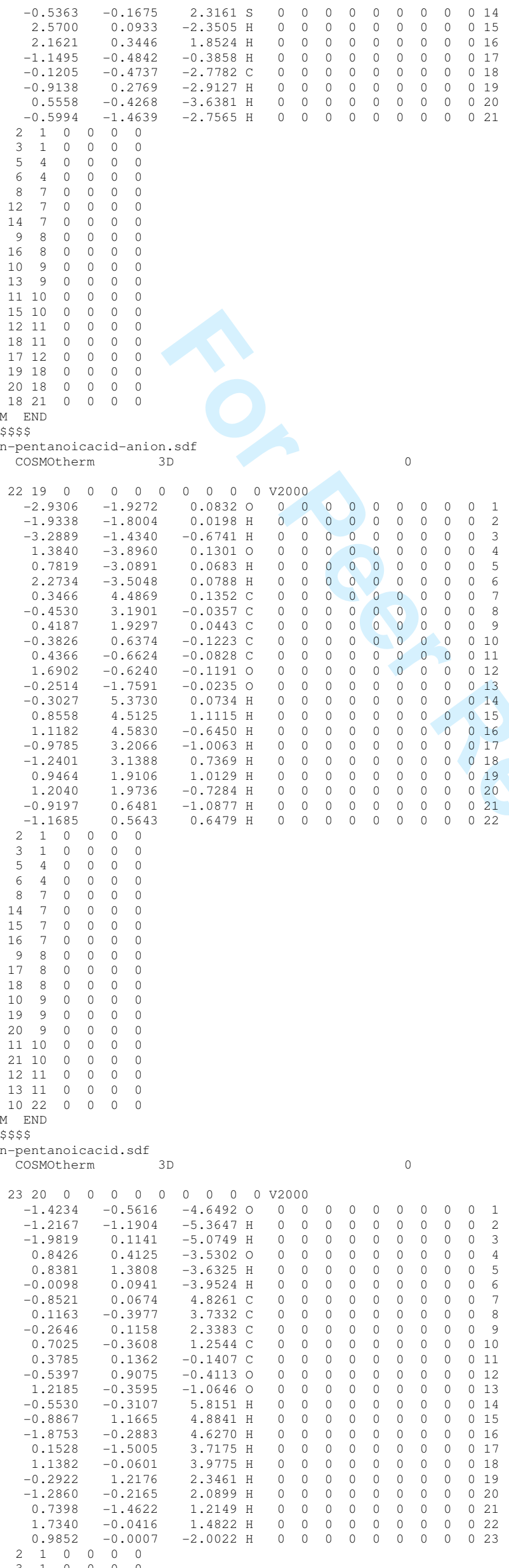




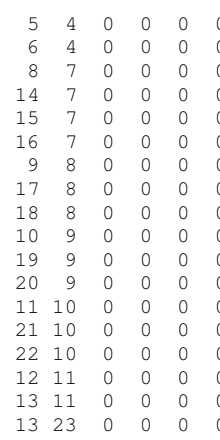

$\mathrm{M}$ END

$\$ \$ \$$

nitroaceticacid-anion.sdf

$\begin{array}{llllllllllll}15 & 12 & 0 & 0 & 0 & 0 & 0 & 0 & 0 & 0 & 0 & \mathrm{v} 2000\end{array}$

$\begin{array}{llllllllllllll}-4.4766 & -0.3355 & 1.4496 & 0 & 0 & 0 & 0 & 0 & 0 & 0 & 0 & 0 & 0 & 1 \\ -3.9128 & -0.0009 & 0.6957 & \mathrm{H} & 0 & 0 & 0 & 0 & 0 & 0 & 0 & 0 & 0 & 2\end{array}$

$\begin{array}{llllllllllllll}-3.9128 & -0.0009 & 0.6957 & \mathrm{H} & 0 & 0 & 0 & 0 & 0 & 0 & 0 & 0 & 0 & 2 \\ -4.5251 & -1.2962 & 1.3078 & \mathrm{H} & 0 & 0 & 0 & 0 & 0 & 0 & 0 & 0 & 0 & 3\end{array}$

$\begin{array}{llllllllllllll}-2.9446 & 0.7001 & -0.5656 & 0 & 0 & 0 & 0 & 0 & 0 & 0 & 0 & 0 & 0 & 4\end{array}$

$\begin{array}{llllllllllllll}-1.9593 & 0.5091 & -0.4213 & \mathrm{H} & 0 & 0 & 0 & 0 & 0 & 0 & 0 & 0 & 0 & 5\end{array}$

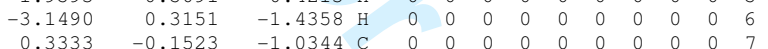

$\begin{array}{llllllllllllll}1.8656 & -0.3766 & -0.7708 & \mathrm{C} & 0 & 0 & 0 & 0 & 0 & 0 & 0 & 0 & 0 & 8\end{array}$

$\begin{array}{lllllllllllllr}-0.3638 & 0.2897 & -0.0745 & 0 & 0 & 0 & 0 & 0 & 0 & 0 & 0 & 0 & 0 & 9\end{array}$

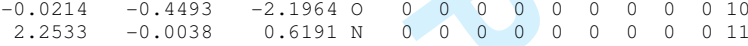

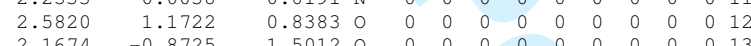

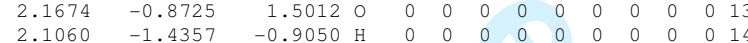

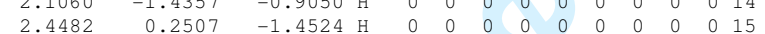

$\begin{array}{llllll}2 & 1 & 0 & 0 & 0 & 0\end{array}$

$\begin{array}{llllll}3 & 1 & 0 & 0 & 0 & 0\end{array}$

5 (1)

$8 \begin{array}{lllllll}6 & 4 & 0 & 0 & 0 & 0 \\ 8 & 7 & 0 & 0 & 0 & 0\end{array}$

$\begin{array}{lllllll}9 & 7 & 0 & 0 & 0 & 0\end{array}$

$\begin{array}{lll}0 & 0 & 0\end{array}$

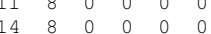

$\begin{array}{llllll}2 & 11 & 0 & 0 & 0 & 0\end{array}$

$8 \begin{array}{llllll}13 & 15 & 0 & 0 & 0 & 0\end{array}$

END

\$\$\$

nitroaceticacid.sdf
CosMotherm

$\begin{array}{lllllllllllll}16 & 14 & 0 & 0 & 0 & 0 & 0 & 0 & 0 & 0 & 0 & \mathrm{~V} 2000\end{array}$

$\begin{array}{llllllllllllll}1.4238 & 4.2127 & 0.1637 & 0 & 0 & 0 & 0 & 0 & 0 & 0 & 0 & 0 & 0 & 1 \\ 1.3770 & 5.1668 & 0.3582 & \mathrm{H} & 0 & 0 & 0 & 0 & 0 & 0 & 0 & 0 & 0 & 2\end{array}$

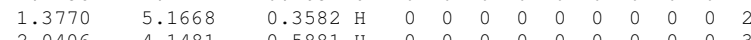

$\begin{array}{llllllllllllll}1.370406 & 4.1481 & -0.5881 & \mathrm{H} & 0 & 0 & 0 & 0 & 0 & 0 & 0 & 0 & 0 & 3 \\ -0.9991 & 3.1659 & -0.2609 & 0 & 0 & 0 & 0 & 0 & 0 & 0 & 0 & 0 & 0 & \end{array}$

$\begin{array}{lllllllllllll}-1.2353 & 3.2805 & -1.1991 & \mathrm{H} & 0 & 0 & 0 & 0 & 0 & 0 & 0 & 0 & 0 \\ -0.0805 & 3.5667 & -0.1534 & \mathrm{H} & 0 & 0 & 0 & 0 & 0 & 0 & 0 & 0 & 0\end{array}$

$\begin{array}{llllllllllllll}-0.0805 & 3.5667 & -0.1534 & \mathrm{H} & 0 & 0 & 0 & 0 & 0 & 0 & 0 & 0 & 0 & 6\end{array}$

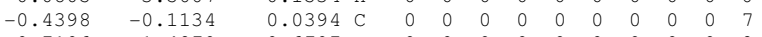

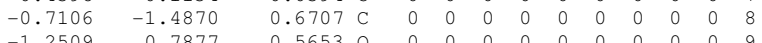

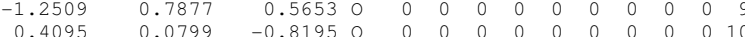

$\begin{array}{llllllllllllll}0.2205 & -2.5089 & 0.0823 & \mathrm{~N} & 0 & 0 & 0 & 0 & 0 & 0 & 0 & 0 & 0 & 11\end{array}$

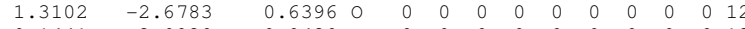

$\begin{array}{llllllllllllll}-0.1441 & -3.0930 & -0.9430 & 0 & 0 & 0 & 0 & 0 & 0 & 0 & 0 & 0 & 0 & 13\end{array}$

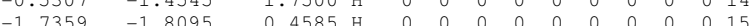

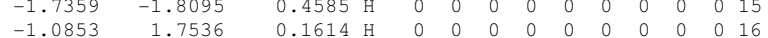

$2-1100000$

$\begin{array}{lllllll}2 & 1 & 0 & 0 & 0 & 0 \\ 3 & 1 & 0 & 0 & 0 & 0\end{array}$

$5.4 \begin{array}{lllll}5 & 0 & 0 & 0 & 0\end{array}$

$\begin{array}{llllll}4 & 0 & 0 & 0 & 0\end{array}$

$\begin{array}{llllll}7 & 0 & 0 & 0 & 0\end{array}$

$\begin{array}{llllll}10 & 7 & 0 & 0 & 0 & 0\end{array}$

$\begin{array}{llllll}4 & 8 & 0 & 0 & 0 & 0\end{array}$

$\begin{array}{llllll}5 & 8 & 0 & 0 & 0\end{array}$

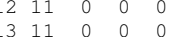

$\begin{array}{lllll}16 & 0 & 0 & 0 & 0\end{array}$

$\begin{array}{llllll}9 & 16 & 0 & 0 & 0 & 0\end{array}$

M END
$\$ \$ \$ \$$

nitromethane-anion.sdf

Cosmotherm $3 \mathrm{D}$

$\begin{array}{llllllllllll}12 & 9 & 0 & 0 & 0 & 0 & 0 & 0 & 0 & 0 & 0 & \mathrm{~V} 2000\end{array}$

$\begin{array}{rrrrrrrrrrrrrr}-3.6863 & 0.4711 & -0.5248 & 0 & 0 & 0 & 0 & 0 & 0 & 0 & 0 & 0 & 0 & 1 \\ -2.8901 & 0.0898 & -0.0514 & \mathrm{H} & 0 & 0 & 0 & 0 & 0 & 0 & 0 & 0 & 0 & 2 \\ -3.5125 & 1.4271 & -0.5613 & \mathrm{H} & 0 & 0 & 0 & 0 & 0 & 0 & 0 & 0 & 0 & 3 \\ -1.5699 & -0.6739 & 0.7442 & 0 & 0 & 0 & 0 & 0 & 0 & 0 & 0 & 0 & 0 & 4 \\ -0.7103 & -0.6086 & 0.1948 & \mathrm{H} & 0 & 0 & 0 & 0 & 0 & 0 & 0 & 0 & 0 & 5 \\ -1.3386 & -0.2084 & 1.5677 & \mathrm{H} & 0 & 0 & 0 & 0 & 0 & 0 & 0 & 0 & 0 & 6 \\ 2.7580 & 0.2315 & -0.6599 & \mathrm{C} & 0 & 0 & 0 & 0 & 0 & 0 & 0 & 0 & 0 & 7 \\ 1.5786 & 0.0543 & -0.0799 & \mathrm{~N} & 0 & 0 & 0 & 0 & 0 & 0 & 0 & 0 & 0 & 8 \\ 0.6056 & -0.5628 & -0.7395 & 0 & 0 & 0 & 0 & 0 & 0 & 0 & 0 & 0 & 0 & 9 \\ 1.3259 & 0.4623 & 1.1241 & 0 & 0 & 0 & 0 & 0 & 0 & 0 & 0 & 0 & 0 & 10 \\ 3.5305 & 0.7383 & -0.0907 & \mathrm{H} & 0 & 0 & 0 & 0 & 0 & 0 & 0 & 0 & 0 & 11 \\ 2.8923 & -0.1364 & -1.6715 & \mathrm{H} & 0 & 0 & 0 & 0 & 0 & 0 & 0 & 0 & 0 & 12\end{array}$

URL: http://mc.manuscriptcentral.com/tandf/tmph 


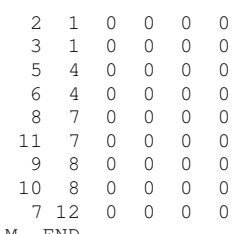




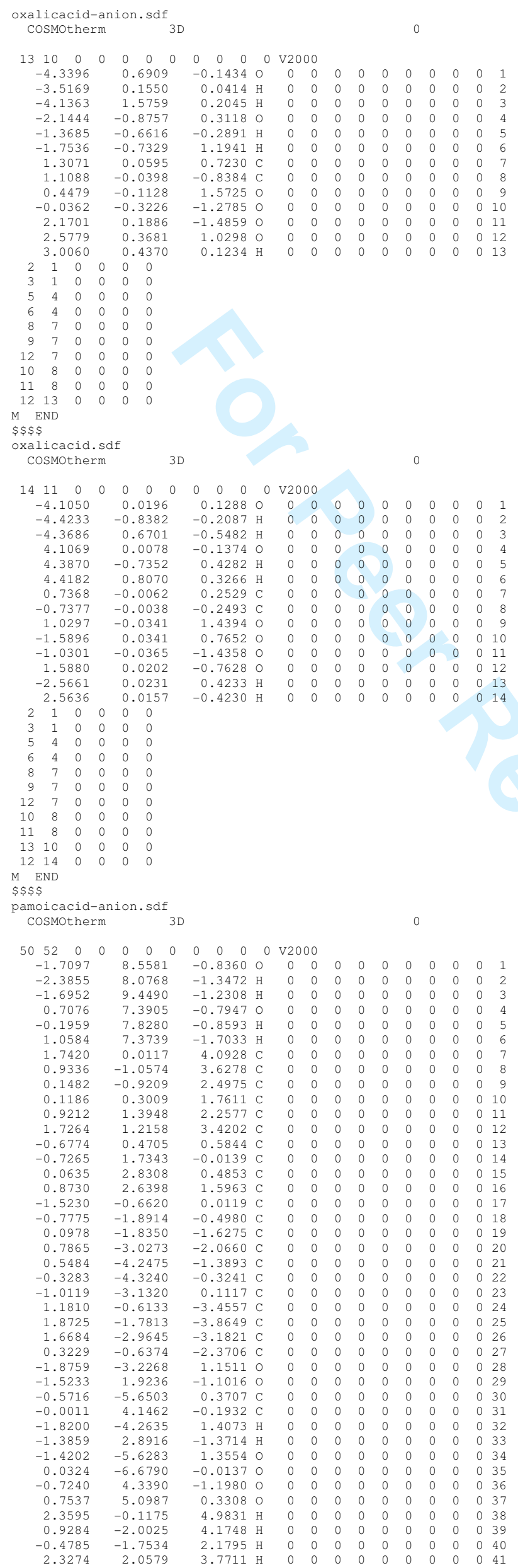




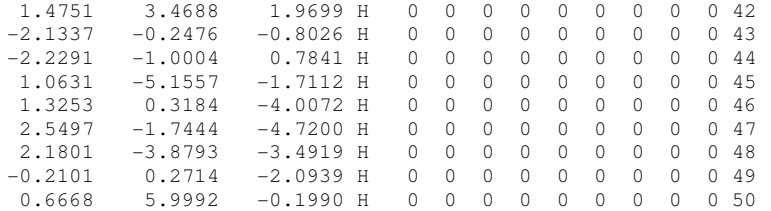




$\begin{array}{rrrrrrrrrrrrrr}-4.7815 & -2.7214 & -1.4584 & \mathrm{H} & 0 & 0 & 0 & 0 & 0 & 0 & 0 & 0 & 0 & 39 \\ -2.5948 & -2.6214 & -0.3823 & \mathrm{H} & 0 & 0 & 0 & 0 & 0 & 0 & 0 & 0 & 0 & 40 \\ -3.4860 & 0.0479 & -4.5012 & \mathrm{H} & 0 & 0 & 0 & 0 & 0 & 0 & 0 & 0 & 0 & 41 \\ -1.2201 & 0.9158 & -4.4755 & \mathrm{H} & 0 & 0 & 0 & 0 & 0 & 0 & 0 & 0 & 0 & 42 \\ 0.8262 & -2.6294 & -0.2874 & \mathrm{H} & 0 & 0 & 0 & 0 & 0 & 0 & 0 & 0 & 0 & 43 \\ -0.8293 & -2.6295 & 0.2831 & \mathrm{H} & 0 & 0 & 0 & 0 & 0 & 0 & 0 & 0 & 0 & 44 \\ 1.2198 & 0.9107 & 4.4747 & \mathrm{H} & 0 & 0 & 0 & 0 & 0 & 0 & 0 & 0 & 0 & 45 \\ 4.7783 & -2.7258 & 1.4536 & \mathrm{H} & 0 & 0 & 0 & 0 & 0 & 0 & 0 & 0 & 0 & 46 \\ 5.2649 & -1.4000 & 3.5199 & \mathrm{H} & 0 & 0 & 0 & 0 & 0 & 0 & 0 & 0 & 0 & 47 \\ 3.4842 & 0.0393 & 4.5008 & \mathrm{H} & 0 & 0 & 0 & 0 & 0 & 0 & 0 & 0 & 0 & 48 \\ 2.5915 & -2.6233 & 0.3777 & \mathrm{H} & 0 & 0 & 0 & 0 & 0 & 0 & 0 & 0 & 0 & 49 \\ -1.7693 & 2.4543 & 5.0883 & \mathrm{H} & 0 & 0 & 0 & 0 & 0 & 0 & 0 & 0 & 0 & 50 \\ 1.7732 & 2.4538 & -5.0885 & \mathrm{H} & 0 & 0 & 0 & 0 & 0 & 0 & 0 & 0 & 0 & 51\end{array}$




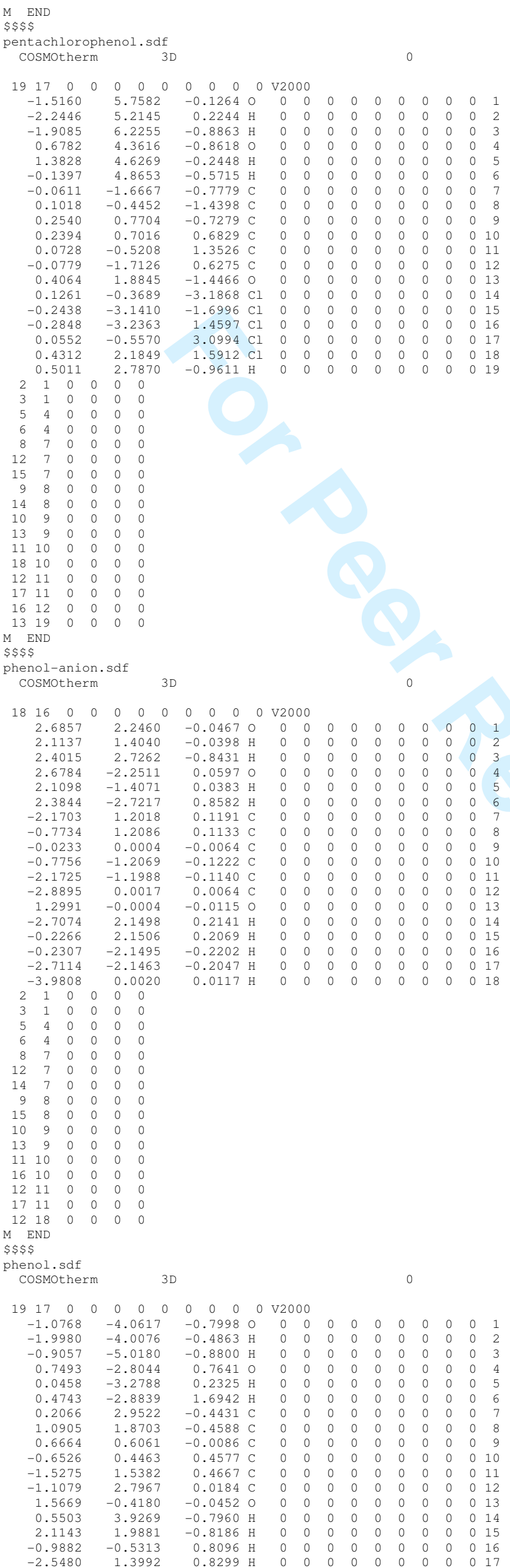




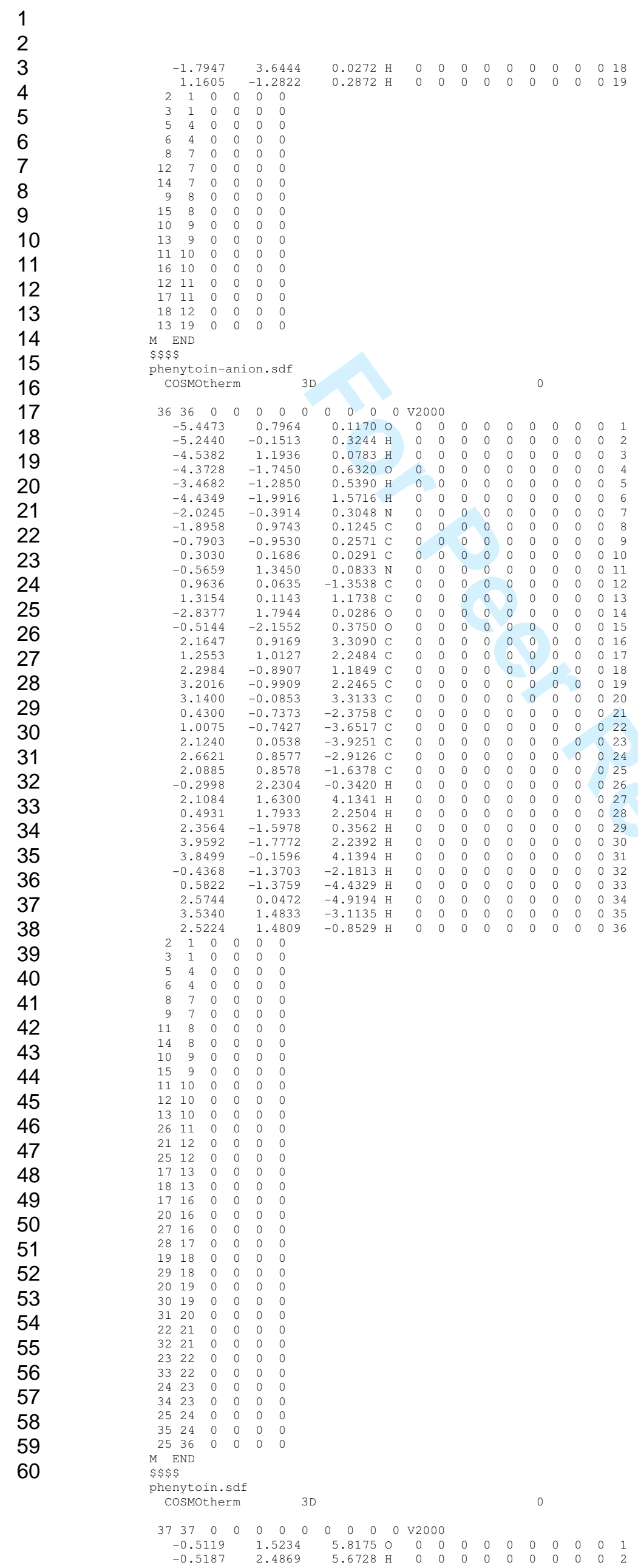




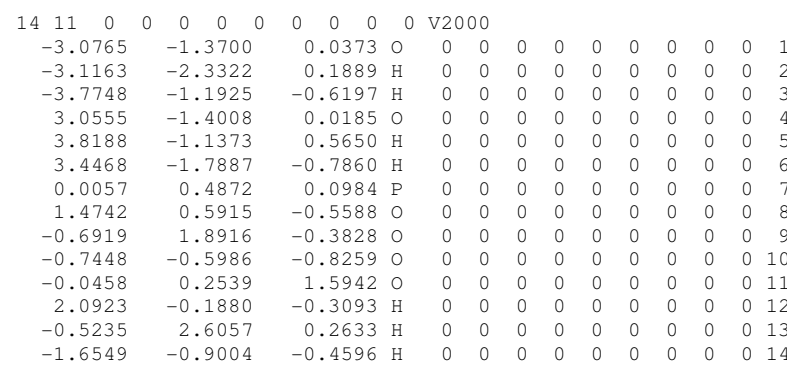




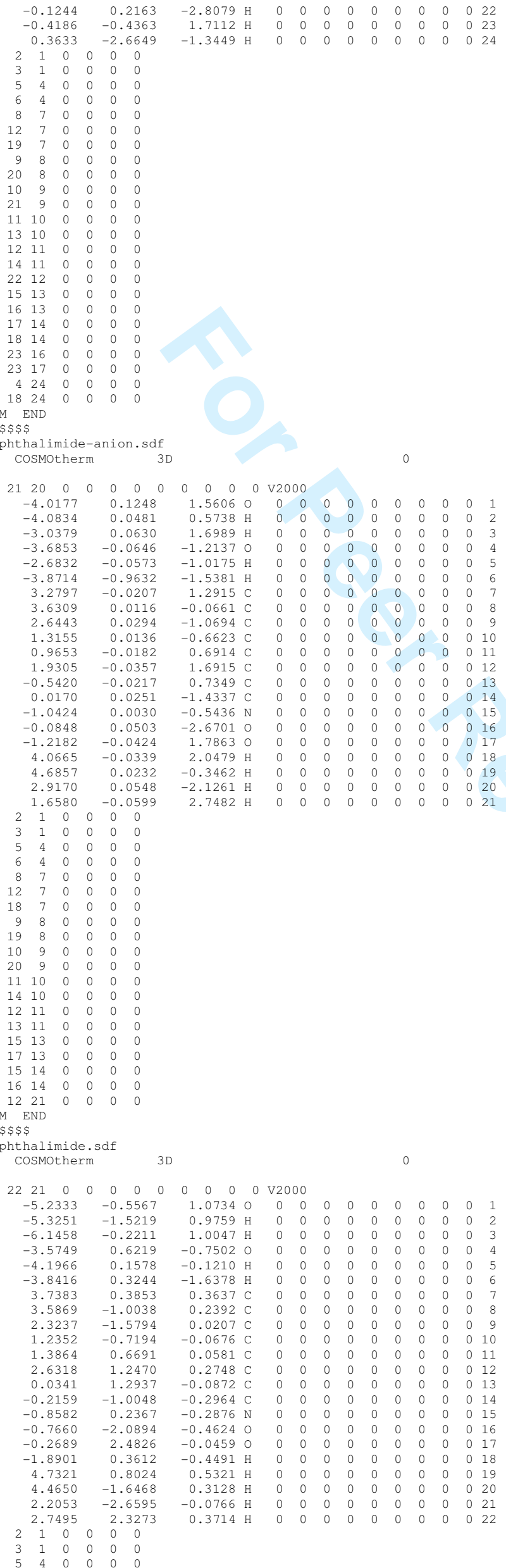




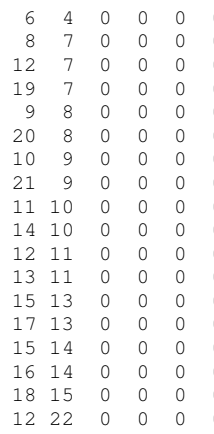

END

salicylicacid-anion.sdf

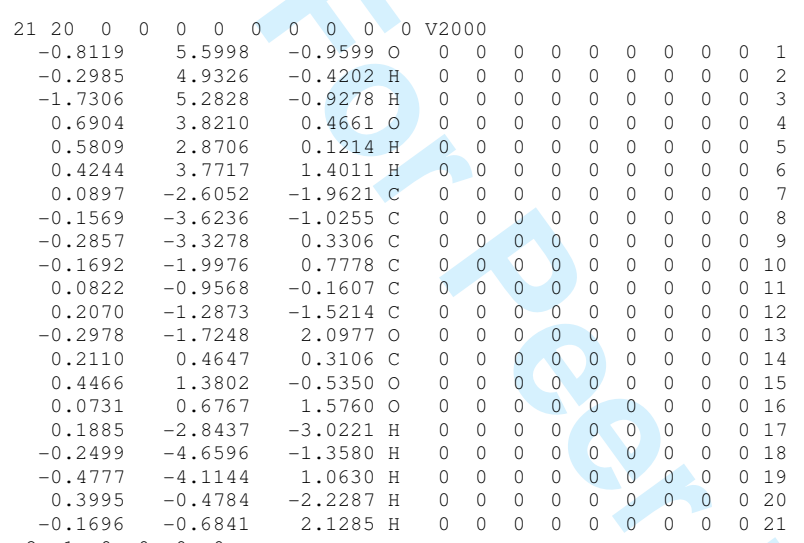

$\begin{array}{lllllllllllllllll}-2.2287 \mathrm{H} & 0 & 0 & 0 & 0 & 0 & 0 & 0 & 0 & 0 & 20\end{array}$

$\begin{array}{llllll}2 & 1 & 0 & 0 & 0 & 0 \\ 3 & 1 & 0 & 0 & 0 & 0\end{array}$

$\begin{array}{lllllllll}3 & 1 & 0 & 0 & 0 & 0\end{array}$

$\begin{array}{llllll}6 & 4 & 0 & 0 & 0 & 0\end{array}$

$\begin{array}{llllll}7 & 0 & 0 & 0 & 0\end{array}$

$\begin{array}{llllll}12 & 7 & 0 & 0 & 0 & 0\end{array}$

$\begin{array}{rrrrrr}17 & 7 & 0 & 0 & 0 & 0 \\ 9 & 8 & 0 & 0 & 0 & 0\end{array}$

$\begin{array}{lllllll}18 & 8 & 0 & 0 & 0 & 0\end{array}$

$\begin{array}{llllll}10 & 9 & 0 & 0 & 0 & 0 \\ 19 & 9 & 0 & 0 & 0 & 0\end{array}$

1900000

$\begin{array}{llllll}11 & 10 & 0 & 0 & 0\end{array}$

$1211 \quad 000000$

$14 \begin{array}{llllll}11 & 0 & 0 & 0 & 0\end{array}$

$\begin{array}{llllll}20 & 12 & 0 & 0 & 0 & 0\end{array}$

$\begin{array}{llllll}15 & 14 & 0 & 0 & 0 & 0 \\ 16 & 14 & 0 & 0 & 0 & 0\end{array}$

$\begin{array}{llllll}13 & 21 & 0 & 0 & 0 & 0\end{array}$

$\begin{array}{llllll}16 & 21 & 0 & 0 & 0 & 0\end{array}$

END

salicylicacid.sdf

cosmotherm

$\begin{array}{llllllllllll}22 & 20 & 0 & 0 & 0 & 0 & 0 & 0 & 0 & 0 & 0 & \mathrm{v} 2000\end{array}$

$\begin{array}{lllll}0.7930 & 5.0128 & -0.7948 & 0 & 0 \\ 1.6519 & 4.9969 & -0.3347 & 0 & 0\end{array}$

$0.7930-5.0128$

$\begin{array}{ll}1.6519 & 4.9969\end{array}$

$\begin{array}{lll}0.5950-5.9607 & -0.3347 \\ -1.1974 & 3.9042 & 0.9050\end{array}$

$\begin{array}{cccccccccccc}9 & \mathrm{H} & 0 & 0 & 0 & 0 & 0 & 0 & 0 & 0 & 0 & 3\end{array}$

$\begin{array}{lllllllllllll}-0.4271 & 4.1991 & 0.0333 & \mathrm{H} & 0 & 0 & 0 & 0 & 0 & 0 & 0 & 0 & 0\end{array}$

$\begin{array}{rrrrrrrrrrrrrr}-1.2049 & 3.9644 & 1.3871 & \mathrm{H} & 0 & 0 & 0 & 0 & 0 & 0 & 0 & 0 & 0 & 6 \\ -1.5297 & -2.9927 & -0.3039 & \mathrm{C} & 0 & 0 & 0 & 0 & 0 & 0 & 0 & 0 & 0 & 7\end{array}$

$\begin{array}{llllllllllllll}-1.5297 & -2.9927 & -0.3039 & \mathrm{C} & 0 & 0 & 0 & 0 & 0 & 0 & 0 & 0 & 0 & 7 \\ -0.3457 & -3.7511 & -0.2744 & \mathrm{C} & 0 & 0 & 0 & 0 & 0 & 0 & 0 & 0 & 0 & 8\end{array}$

$\begin{array}{ccccccccccccccc}-0.3457 & -3.7511 & -0.2744 & \mathrm{C} & 0 & 0 & 0 & 0 & 0 & 0 & 0 & 0 & 0 & 8 \\ 0.8911 & -3.1380 & -0.0948 & \mathrm{C} & 0 & 0 & 0 & 0 & 0 & 0 & 0 & 0 & 0 & 9\end{array}$

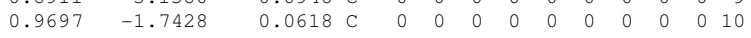

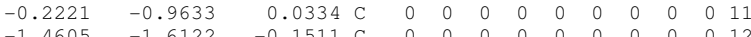

$\begin{array}{rrrrrrrrrrrrrr}-1.4605 & -1.6122 & -0.1511 & C & 0 & 0 & 0 & 0 & 0 & 0 & 0 & 0 & 0 & 12 \\ 2.1881 & -1.1773 & 0.2360 & 0 & 0 & 0 & 0 & 0 & 0 & 0 & 0 & 0 & 0 & 13\end{array}$

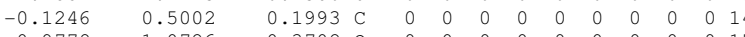

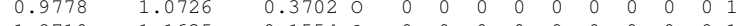

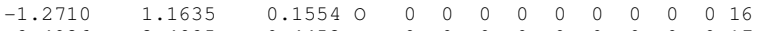

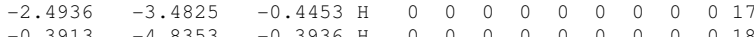

$\begin{array}{llllllllllllll}-0.3913 & -4.8353 & -0.3936 \mathrm{H} & 0 & 0 & 0 & 0 & 0 & 0 & 0 & 0 & 0 & 18\end{array}$

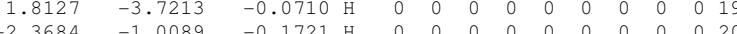

$\begin{array}{lllllllllllll}-1.0089 & -0.1721 & \mathrm{H} & 0 & 0 & 0 & 0 & 0 & 0 & 0 & 0 & 0 & 19 \\ -0.1842 & 0.3287 & 0 & 0 & 0 & 0 & 0 & 0 & 0 & 0 & 0 & 21 \\ 2.1909 & 0.2870 & 0 & 0 & 0 & 0 & 0 & 0 & 0 & 0 & 0 & 22\end{array}$

$\begin{array}{llllllllllllll}-1.1300 & 2.1909 & 0.2870 & \mathrm{H} & 0 & 0 & 0 & 0 & 0 & 0 & 0 & 0 & 0 & 22\end{array}$

$\begin{array}{llllll}2 & 1 & 0 & 0 & 0 & 0\end{array}$

\begin{tabular}{llllll}
3 & 1 & 0 & 0 & 0 & 0 \\
\hline & 4 & 0 & 0 & 0 & 0
\end{tabular}

$\begin{array}{lllll}4 & 0 & 0 & 0\end{array}$

$\begin{array}{llllll}8 & 7 & 0 & 0 & 0 & 0 \\ 1 & 7 & 0 & 0 & 0 & 0\end{array}$

$\begin{array}{lllllll}12 & 7 & 7 & 0 & 0 & 0 & 0\end{array}$

$\begin{array}{lllllll}9 & 8 & 0 & 0 & 0 & 0\end{array}$

$\begin{array}{llllll}18 & 8 & 0 & 0 & 0 & 0\end{array}$

$\begin{array}{lllllll}19 & 9 & 0 & 0 & 0 & 0\end{array}$

$\begin{array}{llllll}11 & 10 & 0 & 0 & 0 & 0\end{array}$ 
$\begin{array}{llllll}13 & 10 & 0 & 0 & 0 & 0\end{array}$

$\begin{array}{llllll}12 & 11 & 0 & 0 & 0 & 0 \\ 14 & 11 & 0 & 0 & 0 & 0\end{array}$

$\begin{array}{llllll}14 & 11 & 0 & 0 & 0 & 0 \\ 20 & 12 & 0 & 0 & 0 & 0\end{array}$

$\begin{array}{llllll}21 & 13 & 0 & 0 & 0 & 0 \\ 15 & 14 & 0 & 0 & 0 & 0\end{array}$

$\begin{array}{lllllllll}15 & 14 & 0 & 0 & 0 & 0\end{array}$

$\begin{array}{llllll}16 & 22 & 0 & 0 & 0 & 0\end{array}$

$M$ END

succinimide-anion.sdf

cosmotherm

$\begin{array}{llllllllllll}17 & 15 & 0 & 0 & 0 & 0 & 0 & 0 & 0 & 0 & 0 & \mathrm{v} 2000\end{array}$

$\begin{array}{rrrrrrrrrrrrrr}-3.9456 & -1.0950 & 0.0939 & \mathrm{O} & 0 & 0 & 0 & 0 & 0 & 0 & 0 & 0 & 0 & 1 \\ -3.3483 & -0.2914 & 0.0405 & \mathrm{H} & 0 & 0 & 0 & 0 & 0 & 0 & 0 & 0 & 0 & 2 \\ -4.6745 & -0.8156 & 0.6736 & \mathrm{H} & 0 & 0 & 0 & 0 & 0 & 0 & 0 & 0 & 0 & 3 \\ -2.3398 & 1.0978 & -0.0551 & \mathrm{O} & 0 & 0 & 0 & 0 & 0 & 0 & 0 & 0 & 0 & 4 \\ -1.3455 & 0.8180 & -0.0171 & \mathrm{H} & 0 & 0 & 0 & 0 & 0 & 0 & 0 & 0 & 0 & 5 \\ -2.4479 & 1.5024 & -0.9338 & \mathrm{H} & 0 & 0 & 0 & 0 & 0 & 0 & 0 & 0 & 0 & 6 \\ 2.3285 & -0.5102 & -0.7983 & \mathrm{C} & 0 & 0 & 0 & 0 & 0 & 0 & 0 & 0 & 0 & 7 \\ 0.9302 & 0.0380 & -1.1215 & \mathrm{C} & 0 & 0 & 0 & 0 & 0 & 0 & 0 & 0 & 0 & 8 \\ 2.3753 & -0.4880 & 0.7300 & \mathrm{C} & 0 & 0 & 0 & 0 & 0 & 0 & 0 & 0 & 0 & 9 \\ 1.0020 & 0.0801 & 1.1227 & \mathrm{C} & 0 & 0 & 0 & 0 & 0 & 0 & 0 & 0 & 0 & 10 \\ 0.2358 & 0.3497 & 0.0187 & \mathrm{~N} & 0 & 0 & 0 & 0 & 0 & 0 & 0 & 0 & 0 & 11 \\ 0.6431 & 0.2659 & 2.3004 & \mathrm{O} & 0 & 0 & 0 & 0 & 0 & 0 & 0 & 0 & 0 & 12 \\ 0.4938 & 0.1804 & -2.2789 & \mathrm{O} & 0 & 0 & 0 & 0 & 0 & 0 & 0 & 0 & 0 & 13 \\ 3.0871 & 0.1287 & -1.2730 & \mathrm{H} & 0 & 0 & 0 & 0 & 0 & 0 & 0 & 0 & 0 & 14 \\ 2.4327 & -1.5161 & -1.2302 & \mathrm{H} & 0 & 0 & 0 & 0 & 0 & 0 & 0 & 0 & 0 & 15 \\ 3.1664 & 0.1559 & 1.1404 & \mathrm{H} & 0 & 0 & 0 & 0 & 0 & 0 & 0 & 0 & 0 & 16 \\ 2.4978 & -1.4829 & 1.1824 & \mathrm{H} & 0 & 0 & 0 & 0 & 0 & 0 & 0 & 0 & 0 & 17\end{array}$

$\begin{array}{llllll}2 & 1 & 0 & 0 & 0 & 0\end{array}$

$\begin{array}{llllll}1 & 1 & 0 & 0 & 0 & 0\end{array}$

$\begin{array}{lllllll}5 & 4 & 0 & 0 & 0 & 0\end{array}$

$\begin{array}{lllll}4 & 0 & 0 & 0 & 0 \\ 7 & 0 & 0 & 0\end{array}$

$\begin{array}{lllllll}9 & 7 & 0 & 0 & 0 & 0\end{array}$

$\begin{array}{lllllll}4 & 7 & 0 & 0 & 0 & 0 \\ 5 & 7 & 0 & 0 & 0 & 0\end{array}$

$\begin{array}{lllllll}11 & 8 & 0 & 0 & 0 & 0\end{array}$

$\begin{array}{llllll}3 & 8 & 0 & 0 & 0\end{array}$

$\begin{array}{llllll}10 & 9 & 0 & 0 & 0 & 0\end{array}$

$\begin{array}{llllll}1 & 10 & 0 & 0 & 0 & 0 \\ 2 & 10 & 0 & 0 & 0 & 0\end{array}$

$\begin{array}{lllllll}9 & 17 & 0 & 0 & 0 & 0\end{array}$

END

succinimide.sdf

$3 \mathrm{D}$

$\begin{array}{llllllllllll}18 & 16 & 0 & 0 & 0 & 0 & 0 & 0 & 0 & 0 & 0 & v 2000\end{array}$

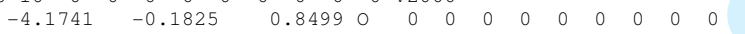

$\begin{array}{llllllllllllll}-4.7265 & -0.9148 & 0.5214 & \mathrm{H} & 0 & 0 & 0 & 0 & 0 & 0 & 0 & 0 & 0 & 2\end{array}$

$\begin{array}{llllllllllllll}-4.8126 & 0.5193 & 1.0728 & \mathrm{H} & 0 & 0 & 0 & 0 & 0 & 0 & 0 & 0 & 0 & 3\end{array}$

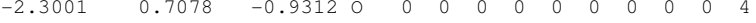

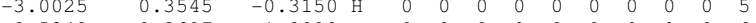

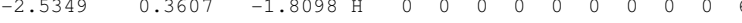

$\begin{array}{lllllllllllllll}2.5824 & 0.4000 & 0.4111 & \mathrm{C} & 0 & 0 & 0 & 0 & 0 & 0 & 0 & 0 & 0 & 7\end{array}$

$\begin{array}{llllllllllllll}1.2626 & 1.0820 & 0.0757 & \mathrm{C} & 0 & 0 & 0 & 0 & 0 & 0 & 0 & 0 & 0 & 8\end{array}$

$\begin{array}{llllllllllllll}1.2626 & 1.0820 & 0.0757 & \mathrm{C} & 0 & 0 & 0 & 0 & 0 & 0 & 0 & 0 & 0 & 8\end{array}$

$\begin{array}{llllllllllllll}-1.1938 & -0.1183 & C & 0 & 0 & 0 & 0 & 0 & 0 & 0 & 0 & 0 & 10\end{array}$

$\begin{array}{llllllllllllll}0.1806 & -2.2131 & -0.3320 & 0 & 0 & 0 & 0 & 0 & 0 & 0 & 0 & 0 & 0 & 11\end{array}$

$\begin{array}{llllllllllllll}1.0341 & 2.2885 & 0.0539 & 0 & 0 & 0 & 0 & 0 & 0 & 0 & 0 & 0 & 0 & 13\end{array}$

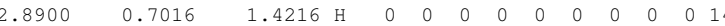

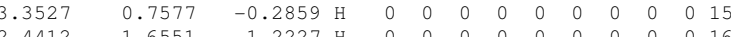

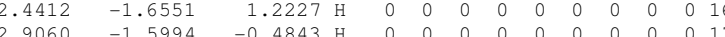

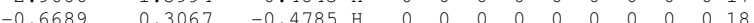

$\begin{array}{llllll}2 & 1 & 0 & 0 & 0 & 0\end{array}$

$\begin{array}{lllllll}3 & 1 & 0 & 0 & 0 & 0\end{array}$

$\begin{array}{lllll}4 & 0 & 0 & 0 & 0\end{array}$

$\begin{array}{lllll}4 & 0 & 0 & 0\end{array}$

$\begin{array}{llll}7 & 0 & 0 & 0\end{array}$

$\begin{array}{lllllll}14 & 7 & 0 & 0 & 0 & 0\end{array}$

$\begin{array}{llllll}5 & 7 & 0 & 0 & 0\end{array}$

$\begin{array}{llllll}1 & 8 & 0 & 0 & 0 & 0\end{array}$

$\begin{array}{lllllllll}13 & 8 & 0 & 0 & 0 & 0\end{array}$

$\begin{array}{lllllll}6 & 9 & 0 & 0 & 0 & 0\end{array}$

$\begin{array}{llllllll}1 & 10 & 0 & 0 & 0 & 0 & 0 \\ 10 & 0 & 0 & 0 & 0 & 0\end{array}$

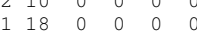

END

sulfurousacid-anion.sd

cosmotherm $3 \mathrm{D}$

$\begin{array}{lllllllllllll}11 & 8 & 0 & 0 & 0 & 0 & 0 & 0 & 0 & 0 & 0 & \mathrm{~V} 2000\end{array}$

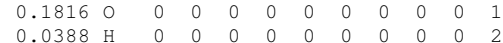

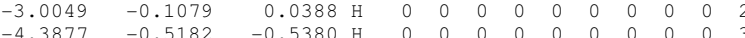

$\begin{array}{llllllllllllll}-4.3877 & -0.5182 & -0.5380 & \mathrm{H} & 0 & 0 & 0 & 0 & 0 & 0 & 0 & 0 & 0 & 3\end{array}$

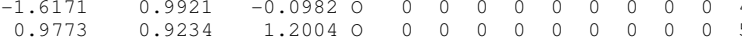

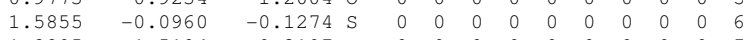

$\begin{array}{lllllllllllll}1.2205 & -1.5194 & 0.3107 & 0 & 0 & 0 & 0 & 0 & 0 & 0 & 0 & 0 & 0\end{array}$

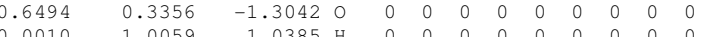

$\begin{array}{llllllllllllllllll}-1.8788 & 1.8924 & -0.3630 & \mathrm{H} & 0 & 0 & 0 & 0 & 0 & 0 & 0 & 0 & 0 & 10\end{array}$

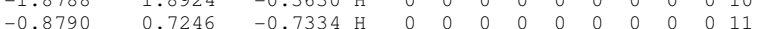

$\begin{array}{llllll}3 & 1 & 0 & 0 & 0 & 0\end{array}$ 


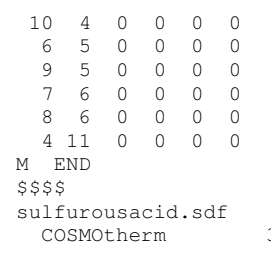




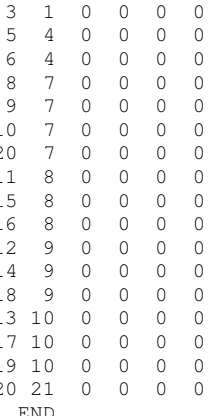

END

thymine-anion. $\begin{array}{ll}\text { Cosmotherm } & 3 D \\ \text { Cond }\end{array}$

$\begin{array}{llllllllllll}20 & 18 & 0 & 0 & 0 & 0 & 0 & 0 & 0 & 0 & 0 & \mathrm{~V} 2000\end{array}$

$\begin{array}{llllllllllllll}-1.4276 & -0.5927 & 4.5599 & 0 & 0 & 0 & 0 & 0 & 0 & 0 & 0 & 0 & 0 & 1 \\ -0.6691 & -0.1679 & 4.0618 & \mathrm{H} & 0 & 0 & 0 & 0 & 0 & 0 & 0 & 0 & 0 & 2\end{array}$

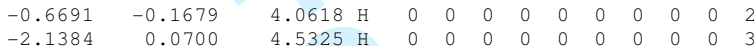

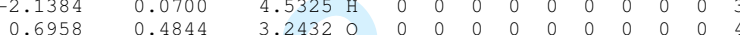

$\begin{array}{llllllllllllll}0.6193 & 0.4844 & 3.2432 & 0 & 0 & 0 & 0 & 0 & 0 & 0 & 0 & 0 & 0 & 4 \\ 0.6193 & 0.3666 & 2.2198 & \mathrm{H} & 0 & 0 & 0 & 0 & 0 & 0 & 0 & 0 & 0 & 5\end{array}$

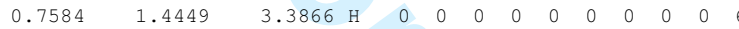

$\begin{array}{llllllllllllll}-0.8426 & 0.2191 & 0.0712 & \mathrm{C} & 0 & 0 & 0 & 0 & 0 & 0 & 0 & 0 & 0 & 7\end{array}$

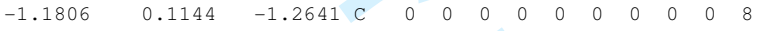

$\begin{array}{llllllllllllll}-0.1046 & -0.0934 & -2.2010 & \mathrm{C} & 0 & 0 & 0 & 0 & 0 & 0 & 0 & 0 & 0 & 9\end{array}$

$\begin{array}{llllllllllllll}1.1627 & -0.1587 & -1.6085 & \mathrm{~N} & 0 & 0 & 0 & 0 & 0 & 0 & 0 & 0 & 0 & 10\end{array}$

$\begin{array}{llllllllllllll}1.4615 & -0.0446 & -0.2419 & \mathrm{C} & 0 & 0 & 0 & 0 & 0 & 0 & 0 & 0 & 0 & 11 \\ 0.4067 & 0.1471 & 0.6021 & \mathrm{~N} & 0 & 0 & 0 & 0 & 0 & 0 & 0 & 0 & 0 & 12\end{array}$

$\begin{array}{llllllllllllll}1.4615 & 0.1471 & 0.6021 & \mathrm{~N} & 0 & 0 & 0 & 0 & 0 & 0 & 0 & 0 & 0 & 12 \\ -0.4183 & 0.147 & -0.2126 & -3.4439 & 0 & 0 & 0 & 0 & 0 & 0 & 0 & 0 & 0 & 0\end{array}$

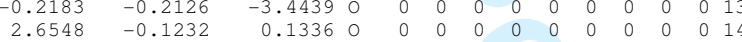

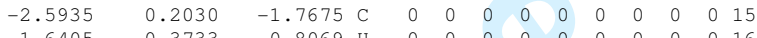

$\begin{array}{llllllllllllllll}1.9544 & -0.3044 & -2.2368 & \mathrm{H} & 0 & 0 & 0 & 0 & 0 & 0 & 0 & 0 & 0 & 0 & 0 & 16\end{array}$

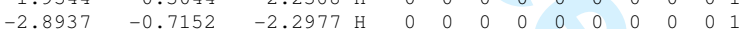

$\begin{array}{llllllllllllllllllllll}-2.7194 & 1.0338 & -2.4801 & \mathrm{H} & 0 & 0 & 0 & 0 & 0 & 0 & 0 & 0 & 0 & 0 & 18\end{array}$

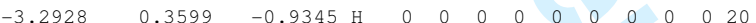

$2 \begin{array}{llllll}2 & 1 & 0 & 0 & 0 & 0\end{array}$

$3.1 \quad 000000$

$\begin{array}{llllll}5 & 0 & 0 & 0\end{array}$

$\begin{array}{lllll}4 & 0 & 0 & 0 & 0\end{array}$

$\begin{array}{llllllll}2 & 7 & 0 & 0 & 0 & 0\end{array}$

$\begin{array}{lllll}7 & 0 & 0 & 0\end{array}$

$\begin{array}{lllllll}5 & 8 & 0 & 0 & 0 & 0\end{array}$

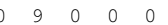

$\begin{array}{llllll}3 & 9 & 0 & 0 & 0 & 0\end{array}$

$\begin{array}{lllllllll}1 & 10 & 0 & 0 & 0 & 0 & 0 & 2 \\ 10 & 0 & 0 & 0 & 0\end{array}$

$\left.\begin{array}{lllllllll}10 & 0 & 0 & 0 & 0 & 0 & 1\end{array}\right)$

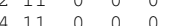

$\begin{array}{llllll}15 & 0 & 0 & 0 & 0\end{array}$

$\begin{array}{llllll}9 & 15 & 0 & 0 & 0 & 0 \\ 2 & 20 & 0 & 0 & 0 & 0\end{array}$

END

$\$ \$ \$$

hymine.sdf

$\begin{array}{llllllllllll}21 & 19 & 0 & 0 & 0 & 0 & 0 & 0 & 0 & 0 & 0 & \mathrm{~V} 2000\end{array}$

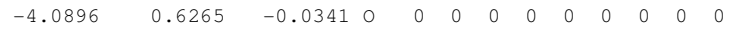

$\begin{array}{llllllllllllll}-4.4871 & 0.8845 & -0.8848 & \mathrm{H} & 0 & 0 & 0 & 0 & 0 & 0 & 0 & 0 & 0 & 2\end{array}$

$\begin{array}{lllllllllllll}-3.1816 & 1.0344 & -0.0343 \mathrm{H} & 0 & 0 & 0 & 0 & 0 & 0 & 0 & 0 & 0 & 3\end{array}$

$\begin{array}{llllllllllllll}-3.1816 & 1.0344 & -0.0343 & \mathrm{H} & 0 & 0 & 0 & 0 & 0 & 0 & 0 & 0 & 0 & 3 \\ -2.9755 & -1.8287 & -0.0091 & 0 & 0 & 0 & 0 & 0 & 0 & 0 & 0 & 0 & 0 & 4\end{array}$

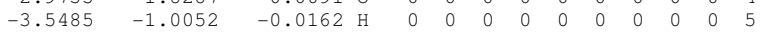

$\begin{array}{llllllllllllll}-3.1871 & -2.2833 & 0.8258 & \mathrm{H} & 0 & 0 & 0 & 0 & 0 & 0 & 0 & 0 & 0 & 6\end{array}$

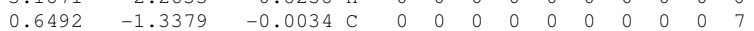

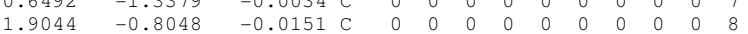

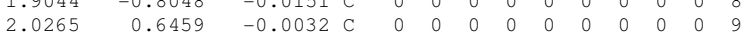

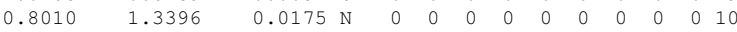

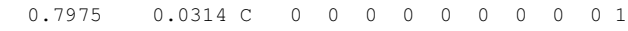

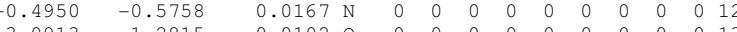

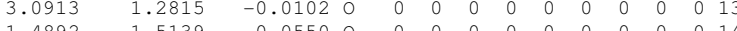

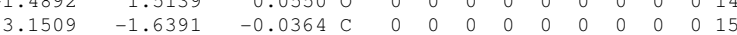

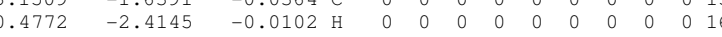

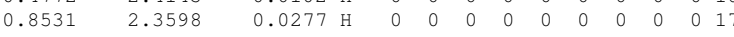

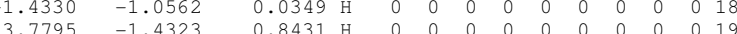

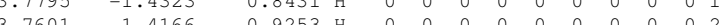

$\begin{array}{llllllllllllll}3.7601 & -1.4166 & -0.9253 & \mathrm{H} & 0 & 0 & 0 & 0 & 0 & 0 & 0 & 0 & 0 & 20 \\ 2.9037 & -2.7084 & -0.0430 & \mathrm{H} & 0 & 0 & 0 & 0 & 0 & 0 & 0 & 0 & 0 & 2\end{array}$

$\begin{array}{llllll}1 & 0 & 0 & 0 & 0\end{array}$

$\begin{array}{lllllllll}3 & 1 & 0 & 0 & 0 & 0 & 0 & 2 & 2 \\ 5 & 4 & 0 & 0 & 0 & 0\end{array}$

$\begin{array}{lllll}4 & 0 & 0 & 0 & 0\end{array}$

$\begin{array}{lllll}4 & 0 & 0 & 0 & 0\end{array}$

$\begin{array}{lllllllll}2 & 7 & 0 & 0 & 0 & 0 & 0 & 2\end{array}$

$\begin{array}{llllllll}2 & 7 & 0 & 0 & 0 & 0\end{array}$

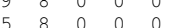

$\begin{array}{llllll}10 & 9 & 0 & 0 & 0 & 0\end{array}$

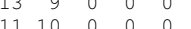

$17 \begin{array}{llllll}10 & 0 & 0 & 0 & 0\end{array}$

12111000000

$\begin{array}{lllllll}4 & 11 & 0 & 0 & 0 & 0\end{array}$

URL: http://mc.manuscriptcentral.com/tandf/tmph 


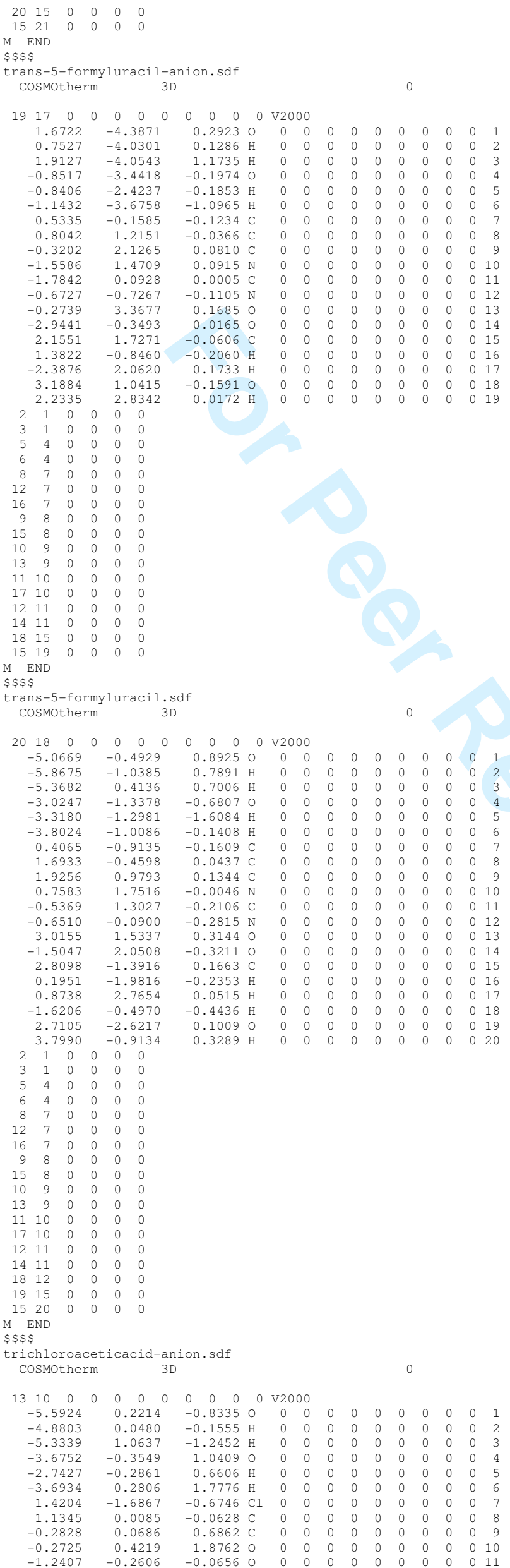




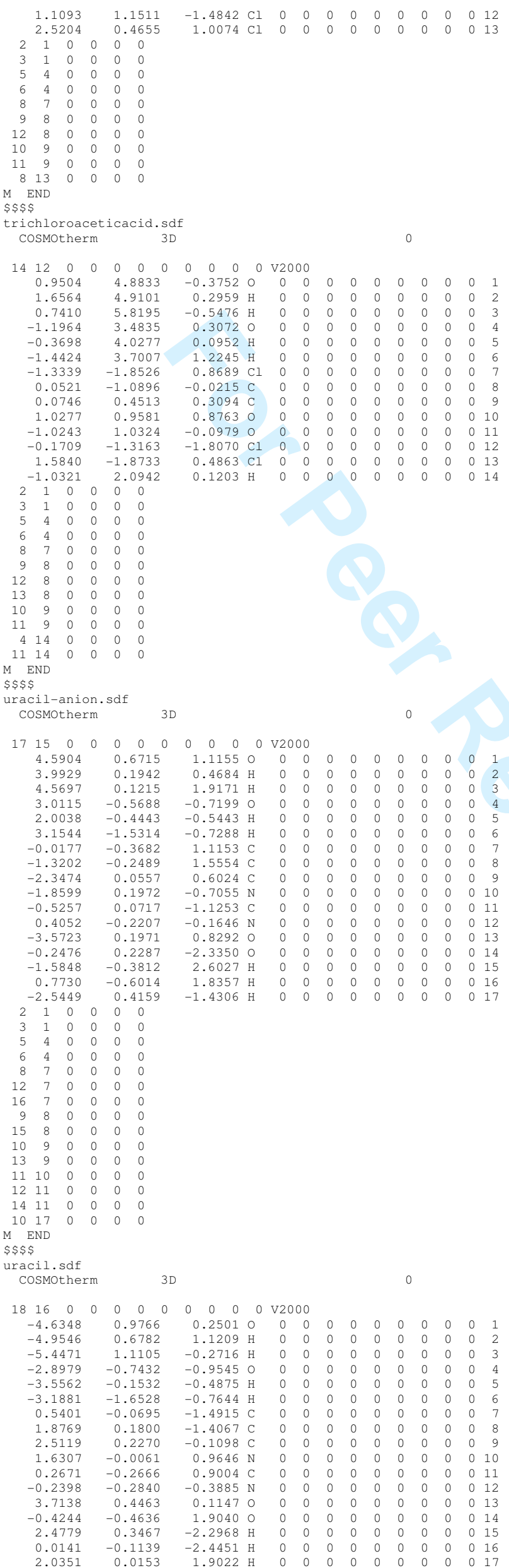



$\begin{array}{lllllllllllll}0.4462 & 1.7120 & \mathrm{C} & 0 & 0 & 0 & 0 & 0 & 0 & 0 & 0 & 0 & 2\end{array}$ $\begin{array}{llllllllllllll}-2.3076 & 0.5441 & 0.5763 & \mathrm{~N} & 0 & 0 & 0 & 0 & 0 & 0 & 0 & 0 & 0 & 3 \\ -2.2551 & 1.7606 & -0.0785 & \mathrm{C} & 0 & 0 & 0 & 0 & 0 & 0 & 0 & 0 & 0 & 4\end{array}$ $\begin{array}{llrrrrrrrrrrrr}-2.9214 & 2.7324 & 0.2729 & 0 & 0 & 0 & 0 & 0 & 0 & 0 & 0 & 0 & 0 & 5\end{array}$ $\begin{array}{llllllllllllll}-1.2814 & 1.8164 & -1.2556 & \mathrm{C} & 0 & 0 & 0 & 0 & 0 & 0 & 0 & 0 & 0 & 6\end{array}$

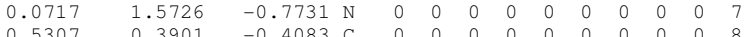
$\begin{array}{llllllllllllll}0.5307 & 0.3901 & -0.4083 & \mathrm{C} & 0 & 0 & 0 & 0 & 0 & 0 & 0 & 0 & 0 & 8 \\ -1.7335 & -0.6521 & 0.0691 & \mathrm{C} & 0 & 0 & 0 & 0 & 0 & 0 & 0 & 0 & 0 & 9\end{array}$ $\begin{array}{lllllllllllll}-0.3761 & -0.7474 & -0.3718 & \mathrm{C} & 0 & 0 & 0 & 0 & 0 & 0 & 0 & 0 & 0\end{array}$ $\begin{array}{lllllllllllllllll}-1.9968 & -3.0434 & -0.3204 & C & 0 & 0 & 0 & 0 & 0 & 0 & 0 & 0 & 0 & 11\end{array}$ $\begin{array}{lllllllllllllllll}-0.6721 & -3.1418 & -0.7679 \mathrm{C} & 0 & 0 & 0 & 0 & 0 & 0 & 0 & 0 & 0 & 11\end{array}$ $\begin{array}{llllllllllllllll}0.1265 & -2.0080 & -0.7792 & \mathrm{C} & 0 & 0 & 0 & 0 & 0 & 0 & 0 & 0 & 0 & 13\end{array}$

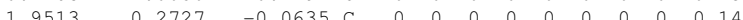

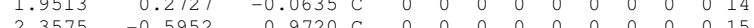

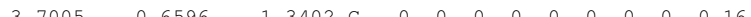

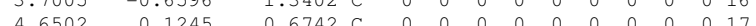
$4.2559=0.19800-0.3629 \mathrm{C}-0$ $\begin{array}{lllllllllllllllll}4.2559 & 0.9800 & -0.3629 & C & 0 & 0 & 0 & 0 & 0 & 0 & 0 & 0 & 0 & 17\end{array}$

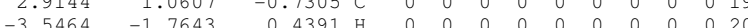
$\begin{array}{lllllllllllllll}-3.5464 & -1.7643 & 0.4391 & \mathrm{H} & 0 & 0 & 0 & 0 & 0 & 0 & 0 & 0 & 0 & 20\end{array}$

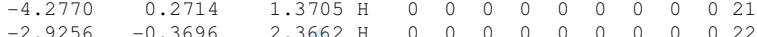

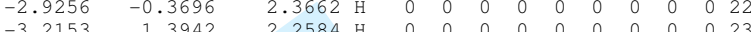
$\begin{array}{lllllllllllllll}-3.2153 & 1.3942 & 2.2584 & \mathrm{H} & 0 & 0 & 0 & 0 & 0 & 0 & 0 & 0 & 0 & 23\end{array}$

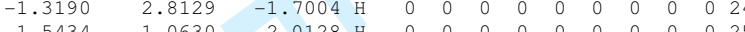

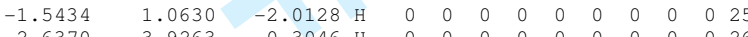
$\begin{array}{llllllllllllll}-2.6370 & -3.9263 & -0.3046 \mathrm{H} & 0 & 0 & 0 & 0 & 0 & 0 & 0 & 0 & 0 & 26\end{array}$ $\begin{array}{llllllllllllll}-0.2705 & -4.0971 & -1.1054 & \mathrm{H} & 0 & 0 & 0 & 0 & 0 & 0 & 0 & 0 & 0 & 27\end{array}$ $\begin{array}{lllllllllllll}1.1544 & -2.0696 & -1.1349 \mathrm{H} & 0 & 0 & 0 & 0 & 0 & 0 & 0 & 0 & 0 & 28\end{array}$

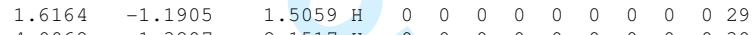

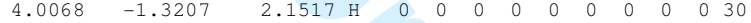

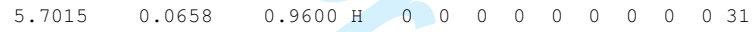

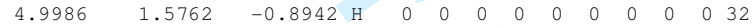
$\begin{array}{llllllllllllll}2.6211 & 1.7013 & -1.5641 & \mathrm{H} & 0 & 0 & 0 & 0 & 0 & 0 & 0 & 0 & 0 & 33\end{array}$ $0.6845 \quad 2.3849$ 


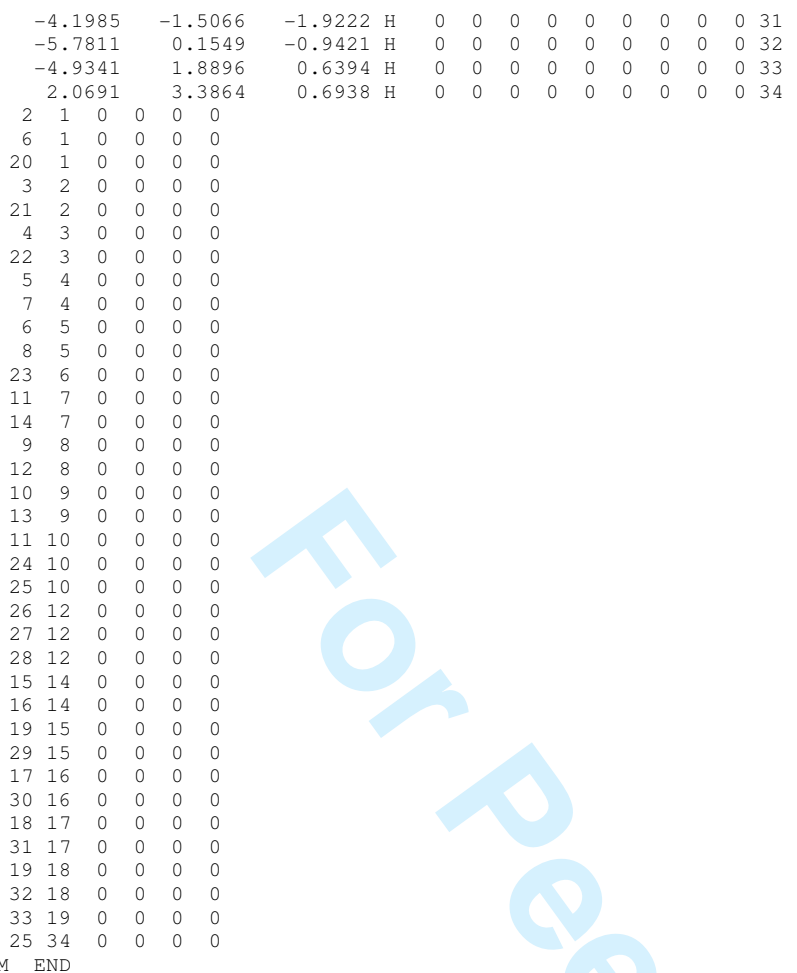

EN

1,3-dihydro-3-hydroxy-1-methyl-5-phenyl-1,4-benzodiazepin-2-one_p1.sdf

cosmotherm $3 \mathrm{D}$

$\begin{array}{lllllllllllll}35 & 37 & 0 & 0 & 0 & 0 & 0 & 0 & 0 & 0 & 0 & \mathrm{~V} 2000\end{array}$

$\begin{array}{llllllllllllll}1.6473 & -3.3298 & 0.6106 & \mathrm{C} & 0 & 0 & 0 & 0 & 0 & 0 & 0 & 0 & 0 & 1\end{array}$

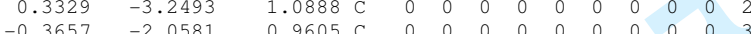

$\begin{array}{rrrrrrrrrrrrrr}-0.3657 & -2.0581 & 0.9605 & \mathrm{C} & 0 & 0 & 0 & 0 & 0 & 0 & 0 & 0 & 0 & 3 \\ 0.2297 & -0.9117 & 0.3792 & \mathrm{C} & 0 & 0 & 0 & 0 & 0 & 0 & 0 & 0 & 0 & 4\end{array}$

$\begin{array}{llllllllllllllll}1.5777 & -0.9991 & -0.0896 & \mathrm{C} & 0 & 0 & 0 & 0 & 0 & 0 & 0 & 0 & 0 & 5\end{array}$

$\begin{array}{llllllllllllll}2.2554 & -2.2230 & 0.0241 & \mathrm{C} & 0 & 0 & 0 & 0 & 0 & 0 & 0 & 0 & 0 & 6\end{array}$

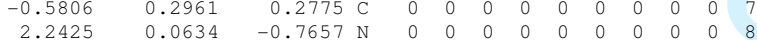

$\begin{array}{llllllllllllll}2.2425 & 0.0634 & -0.7657 & \mathrm{~N} & 0 & 0 & 0 & 0 & 0 & 0 & 0 & 0 & 0 & 8 \\ 2.2815 & 1.3464 & -0.3012 & \mathrm{C} & 0 & 0 & 0 & 0 & 0 & 0 & 0 & 0 & 0 & 9\end{array}$

$\begin{array}{lllllllllllll}1.3875 & 1.6560 & 0.9185 \mathrm{C} & 0 & 0 & 0 & 0 & 0 & 0 & 0 & 0 & 0 & 10\end{array}$

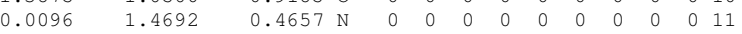

$\begin{array}{llllllllllllll}3.1040 & -0.2584 & -1.9237 & \mathrm{C} & 0 & 0 & 0 & 0 & 0 & 0 & 0 & 0 & 0 & 12 \\ 2.9379 & 2.2575 & -0.8105 & 0 & 0 & 0 & 0 & 0 & 0 & 0 & 0 & 0 & 0 & 13\end{array}$

$\begin{array}{llllllllllllll}2.9379 & 2.2575 & -0.8105 & 0 & 0 & 0 & 0 & 0 & 0 & 0 & 0 & 0 & 0 & 13\end{array}$

$\begin{array}{llllllllllllll}-2.0156 & 0.2550 & -0.0173 & \mathrm{C} & 0 & 0 & 0 & 0 & 0 & 0 & 0 & 0 & 0 & 14\end{array}$

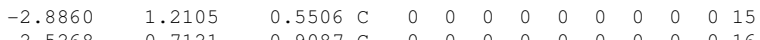

$\begin{array}{llllllllllllll}-2.5268 & -0.7121 & -0.9087 & \mathrm{C} & 0 & 0 & 0 & 0 & 0 & 0 & 0 & 0 & 0 & 16\end{array}$

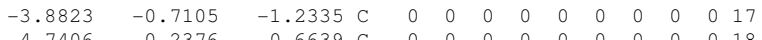

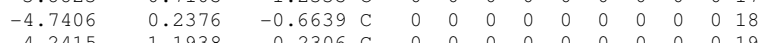

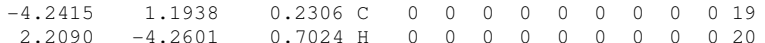

$\begin{array}{llllllllllllllll}-0.1399 & -4.1116 & 1.5585 & \mathrm{H} & 0 & 0 & 0 & 0 & 0 & 0 & 0 & 0 & 0 & 21\end{array}$

$\begin{array}{lllllllllllllll}-1.3844 & -1.9821 & 1.3392 & \mathrm{H} & 0 & 0 & 0 & 0 & 0 & 0 & 0 & 0 & 0 & 22\end{array}$

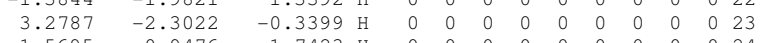

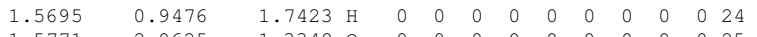

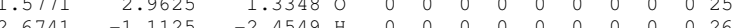

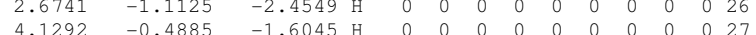

$\begin{array}{llllllllllllll}3.1248 & 0.6158 & -2.5823 & \mathrm{H} & 0 & 0 & 0 & 0 & 0 & 0 & 0 & 0 & 0 & 28\end{array}$

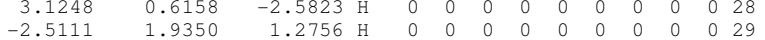

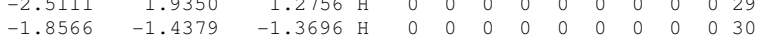

$\begin{array}{llllllllllllll}-1.8566 & -1.4379 & -1.3696 \mathrm{H} & 0 & 0 & 0 & 0 & 0 & 0 & 0 & 0 & 0 & 30 \\ -4.2699 & -1.4493 & -1.9359 \mathrm{H} & 0 & 0 & 0 & 0 & 0 & 0 & 0 & 0 & 0 & 31\end{array}$

$\begin{array}{rrrrrrrrrrrrrr}-4.2699 & -1.4493 & -1.9359 & \mathrm{H} & 0 & 0 & 0 & 0 & 0 & 0 & 0 & 0 & 0 & 31 \\ -5.8026 & 0.2296 & -0.9136 & \mathrm{H} & 0 & 0 & 0 & 0 & 0 & 0 & 0 & 0 & 0 & 32\end{array}$

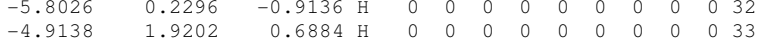

$\begin{array}{rrrrrrrrrrrrrr}-4.9138 & 1.9202 & 0.6884 & \mathrm{H} & 0 & 0 & 0 & 0 & 0 & 0 & 0 & 0 & 0 & 33 \\ 2.1025 & 3.3981 & 0.6193 & \mathrm{H} & 0 & 0 & 0 & 0 & 0 & 0 & 0 & 0 & 0 & 34 \\ -0.5319 & 2.3326 & 0.2879 & \mathrm{H} & 0 & 0 & 0 & 0 & 0 & 0 & 0 & 0 & 0 & 35\end{array}$

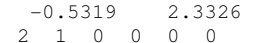

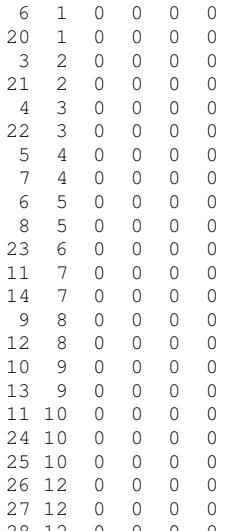

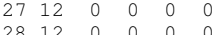


$1514 \quad 000000$

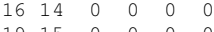

$\begin{array}{lllllll}19 & 15 & 0 & 0 & 0 & 0\end{array}$

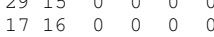

$3016 \quad 0 \quad 0 \quad 000$

$\begin{array}{llllll}18 & 17 & 0 & 0 & 0 & 0 \\ 31 & 17 & 0 & 0 & 0 & 0\end{array}$

$\begin{array}{llllll}19 & 18 & 0 & 0 & 0 & 0\end{array}$

$\begin{array}{llllll}32 & 18 & 0 & 0 & 0 & 0 \\ 33 & 19 & 0 & 0 & 0 & 0 \\ 34 & 25 & 0 & 0 & 0 & 0\end{array}$

$\begin{array}{llllll}34 & 25 & 0 & 0 & 0 & 0\end{array}$

$\begin{array}{lllllll}1 & 35 & 0 & 0 & 0 & 0\end{array}$

END

1,3-dihydro-3-hydroxy-5-phenyl-1,4-benzodiazepin-2-one $s d f$

cosmotherm

$\begin{array}{llllllllllll}31 & 33 & 0 & 0 & 0 & 0 & 0 & 0 & 0 & 0 & 0 & \mathrm{~V} 2000\end{array}$

$\begin{array}{llllllllllllll}0.5145 & -3.2825 & -0.9215 & \mathrm{C} & 0 & 0 & 0 & 0 & 0 & 0 & 0 & 0 & 0 & 1\end{array}$

$\begin{array}{lllllllllllll}-0.1408 & -2.0552 & -0.9137 & c & 0 & 0 & 0 & 0 & 0 & 0 & 0 & 0 & 0\end{array}$

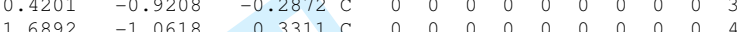

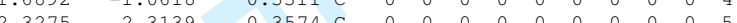

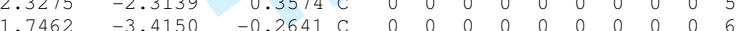

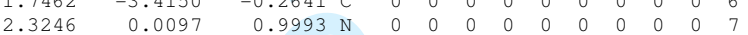

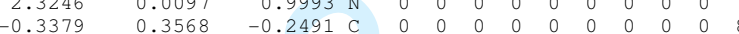

$\begin{array}{lllllllllllll}0.1958 & 1.5303 & -0.3966 & \mathrm{~N} & 0 & 0 & 0 & 0 & 0 & 0 & 0 & 0 & 0\end{array}$

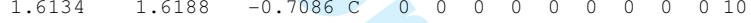

$\begin{array}{llllllllllllll}-1.8101 & 0.3044 & -0.0162 & \mathrm{C} & 0 & 0 & 0 & 0 & 0 & 0 & 0 & 0 & 0 & 11\end{array}$

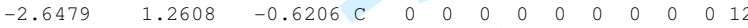

$\begin{array}{llllllllllllll}-2.3806 & -0.6581 & 0.8378 & \mathrm{C} & 0 & 0 & 0 & 0 & 0 & 0 & 0 & 0 & 0 & 13\end{array}$

$\begin{array}{llllllllllllll}-4.0231 & 1.2506 & -0.3811 & \mathrm{C} & 0 & 0 & 0 & 0 & 0 & 0 & 0 & 0 & 0 & 14\end{array}$

$\begin{array}{lllllllllllllll}-4.5814 & 0.2931 & 0.4764 & \mathrm{C} & 0 & 0 & 0 & 0 & 0 & 0 & 0 & 0 & 0 & 15\end{array}$

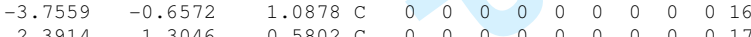

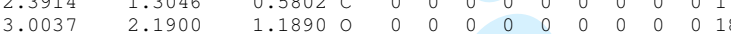

$\begin{array}{lllllllllllllll}1.9545 & 2.9130 & -1.1531 & 0 & 0 & 0 & 0 & 0 & 0 & 0 & 0 & 0 & 0 & 19\end{array}$

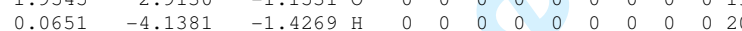

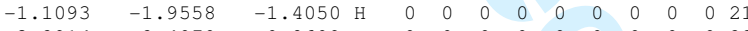

$\begin{array}{lllllllllllll}.2914-2.4070 & 0.8609 & \mathrm{H} & 0 & 0 & 0 & 0 & 0 & 0 & 0 & 0 & 0 & 22\end{array}$

$\begin{array}{llllllllllll}-4.3772 & -0.2455 \mathrm{H} & 0 & 0 & 0 & 0 & 0 & 0 & 0 & 0 & 0 & 23\end{array}$

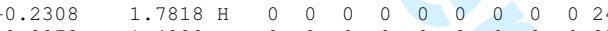

$\begin{array}{llllllllllll}.8973-1.4980 & \mathrm{H} & 0 & 0 & 0 & 0 & 0 & 0 & 0 & 0 & 0 & 25\end{array}$

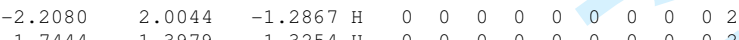

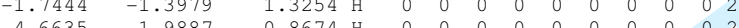

$\begin{array}{llllllllllllll}-4.6635 & 1.9887 & -0.8674 & \mathrm{H} & 0 & 0 & 0 & 0 & 0 & 0 & 0 & 0 & 0 & 27\end{array}$

$\begin{array}{llllllllllllllllllllll}-4.1830 & -1.4009 & 1.7629 & \mathrm{H} & 0 & 0 & 0 & 0 & 0 & 0 & 0 & 0 & 0 & 39\end{array}$

$\begin{array}{lllllllllllllllll}2.3465 & 3.3668 & -0.3722 \mathrm{H} & 0 & 0 & 0 & 0 & 0 & 0 & 0 & 0 & 0 & 31\end{array}$

$\begin{array}{lllllll}2 & 1 & 0 & 0 & 0 & 0\end{array}$

$\begin{array}{llllll}6 & 1 & 0 & 0 & 0 \\ 2 & 1 & 0 & 0\end{array}$

$\begin{array}{llllll}3 & 2 & 0 & 0 & 0 & 0\end{array}$

$\begin{array}{llllllll}3 & 2 & 0 & 0 & 0 & 0\end{array}$

$\begin{array}{lllll}3 & 0 & 0 & 0 & 0\end{array}$

$\begin{array}{llll}3 & 0 & 0 & 0\end{array}$

$\begin{array}{lllll}4 & 0 & 0 & 0\end{array}$

$\begin{array}{llllll}6 & 5 & 0 & 0 & 0 & 0\end{array}$

$\begin{array}{llllll}6 & 5 & 0 & 0 & 0 & 0 \\ 23 & 6 & 0 & 0 & 0\end{array}$

$\begin{array}{lllllll}23 & 6 & 0 & 0 & 0 & 0\end{array}$

$\begin{array}{llllll}4 & 7 & 0 & 0 & 0\end{array}$

$\begin{array}{llllll}9 & 8 & 0 & 0 & 0 & 0\end{array}$

$\begin{array}{lllll}8 & 0 & 0 & 0 & 0\end{array}$

$\begin{array}{rrrrrrr}17 & 10 & 0 & 0 & 0 & 0\end{array}$

$\begin{array}{llllll}19 & 10 & 0 & 0 & 0 & 0\end{array}$

$\begin{array}{llllll}5 & 10 & 0 & 0 & 0 & 0\end{array}$

$\begin{array}{llllll}3 & 11 & 0 & 0 & 0 & 0\end{array}$

$\begin{array}{llllll}26 & 12 & 0 & 0 & 0 & 0 \\ 16 & 13 & 0 & 0 & 0 & 0\end{array}$

$\begin{array}{llllll}27 & 13 & 0 & 0 & 0\end{array}$

$\begin{array}{llllll}15 & 14 & 0 & 0 & 0 & 0\end{array}$

$\begin{array}{lllllll}15 & 14 & 0 & 0 & 0 & 0\end{array}$

$\begin{array}{llllll}6 & 15 & 0 & 0 & 0 & 0\end{array}$

$\begin{array}{llllll}30 & 16 & 0 & 0 & 0 & 0\end{array}$

$\begin{array}{llllll}8 & 17 & 0 & 0 & 0 & 0\end{array}$

1931

\$\$

3-dihydro-3-hydroxy-5-phenyl-1, 4-benzodiazepin-2-one_p1.sdf
Cosmotherm 3 3D

$\begin{array}{llllllllllll}32 & 34 & 0 & 0 & 0 & 0 & 0 & 0 & 0 & 0 & 0 & \mathrm{~V} 2000\end{array}$

$\begin{array}{rrrrrrrrrrrrrr}-3.3101 & -0.4252 & -0.8747 & C & 0 & 0 & 0 & 0 & 0 & 0 & 0 & 0 & 0 & 1 \\ -2.0811 & 0.2156 & -0.8508 & \text { C } & 0 & 0 & 0 & 0 & 0 & 0 & 0 & 0 & 0 & 2\end{array}$

$\begin{array}{lllllllllllll}-0.9402 & -0.3940 & -0.2697 & \mathrm{C} & 0 & 0 & 0 & 0 & 0 & 0 & 0 & 0 & 0\end{array}$

$\begin{array}{llllllllllllll}-1.0790 & -1.7028 & 0.2850 & \mathrm{C} & 0 & 0 & 0 & 0 & 0 & 0 & 0 & 0 & 0 & 4\end{array}$

$\begin{array}{llllllllllllll}-2.3414 & -2.3165 & 0.2928 & \mathrm{C} & 0 & 0 & 0 & 0 & 0 & 0 & 0 & 0 & 0 & 5\end{array}$

$\begin{array}{llllllllllllll}-3.4422 & -1.6897 & -0.2813 & \mathrm{C} & 0 & 0 & 0 & 0 & 0 & 0 & 0 & 0 & 0 & 6\end{array}$

$\begin{array}{llllllllllll}-1.3972 & 0.9266 \mathrm{~N} & 0 & 0 & 0 & 0 & 0 & 0 & 0 & 0 & 0 & \end{array}$

$\begin{array}{lllllllllllll}1.4605 & -0.2324 & -0.3137 & \mathrm{~N} & 0 & 0 & 0 & 0 & 0 & 0 & 0 & 0 & 0\end{array}$

$\begin{array}{llllllllllllll}1.6497 & -1.6491 & -0.6964 & \mathrm{C} & 0 & 0 & 0 & 0 & 0 & 0 & 0 & 0 & 0 & 10\end{array}$

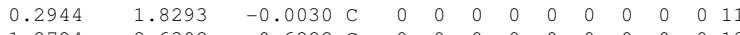

$\begin{array}{llllllllllllll}1.2784 & 2.6302 & -0.6222 & \mathrm{C} & 0 & 0 & 0 & 0 & 0 & 0 & 0 & 0 & 0 & 1\end{array}$

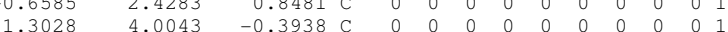

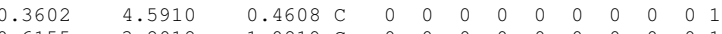

URL: http://mc.manuscriptcentral.com/tandf/tmph 


$\begin{array}{rrrrrrrrrrrrrr}1.2664 & -2.5004 & 0.5318 & \mathrm{C} & 0 & 0 & 0 & 0 & 0 & 0 & 0 & 0 & 0 & 17 \\ 2.1139 & -3.1934 & 1.0928 & \mathrm{O} & 0 & 0 & 0 & 0 & 0 & 0 & 0 & 0 & 0 & 18 \\ 2.9704 & -1.8696 & -1.0470 & \mathrm{O} & 0 & 0 & 0 & 0 & 0 & 0 & 0 & 0 & 0 & 19 \\ -4.1672 & 0.0566 & -1.3447 & \mathrm{H} & 0 & 0 & 0 & 0 & 0 & 0 & 0 & 0 & 0 & 20 \\ -1.9727 & 1.1999 & -1.3051 \mathrm{H} & 0 & 0 & 0 & 0 & 0 & 0 & 0 & 0 & 0 & 21 \\ -2.4441 & -3.3029 & 0.7473 & \mathrm{H} & 0 & 0 & 0 & 0 & 0 & 0 & 0 & 0 & 0 & 22 \\ -4.4086 & -2.1951 & -0.2761 & \mathrm{H} & 0 & 0 & 0 & 0 & 0 & 0 & 0 & 0 & 0 & 23 \\ -0.3124 & -3.0528 & 1.6598 & \mathrm{H} & 0 & 0 & 0 & 0 & 0 & 0 & 0 & 0 & 0 & 24 \\ 0.9736 & -1.8584 & -1.5417 & \mathrm{H} & 0 & 0 & 0 & 0 & 0 & 0 & 0 & 0 & 0 & 25 \\ 1.9934 & 2.1848 & -1.3161 & \mathrm{H} & 0 & 0 & 0 & 0 & 0 & 0 & 0 & 0 & 0 & 26 \\ -1.4047 & 1.8131 & 1.3507 & \mathrm{H} & 0 & 0 & 0 & 0 & 0 & 0 & 0 & 0 & 0 & 27 \\ 2.0515 & 4.6216 & -0.8914 & \mathrm{H} & 0 & 0 & 0 & 0 & 0 & 0 & 0 & 0 & 0 & 28 \\ 0.3846 & 5.6669 & 0.6398 \mathrm{H} & 0 & 0 & 0 & 0 & 0 & 0 & 0 & 0 & 0 & 29 \\ -1.3436 & 4.2579 & 1.7536 \mathrm{H} & 0 & 0 & 0 & 0 & 0 & 0 & 0 & 0 & 0 & 30 \\ 3.3677 & -2.3874 & -0.3057 \mathrm{H} & 0 & 0 & 0 & 0 & 0 & 0 & 0 & 0 & 0 & 31 \\ 2.3257 & 0.2858 & -0.1329 & \mathrm{H} & 0 & 0 & 0 & 0 & 0 & 0 & 0 & 0 & 0 & 32\end{array}$

$\begin{array}{cccccc}2 & 1 & 0 & 0 & 0 & 0\end{array}$

$\begin{array}{rrrrrr}6 & 1 & 0 & 0 & 0 & 0 \\ 20 & 1 & 0 & 0 & 0 & 0\end{array}$

$\begin{array}{llllll}3 & 2 & 0 & 0 & 0 & 0\end{array}$

$\begin{array}{rrrrrr}21 & 2 & 0 & 0 & 0 & 0 \\ 4 & 3 & 0 & 0 & 0 & 0\end{array}$

$\begin{array}{llllll}8 & 3 & 0 & 0 & 0 & 0\end{array}$

$\begin{array}{llllll}5 & 4 & 0 & 0 & 0 & 0 \\ 7 & 4 & 0 & 0 & 0 & 0\end{array}$

$\begin{array}{llllll}7 & 4 & 0 & 0 & 0 \\ 6 & 5 & 0 & 0 & 0 & 0\end{array}$

$\begin{array}{lllllll}22 & 5 & 0 & 0 & 0 & 0 & 0\end{array}$

$\begin{array}{lllllll}23 & 6 & 0 & 0 & 0 & 0 \\ 17 & 7 & 0 & 0 & 0 & 0\end{array}$

$\begin{array}{llllll}17 & 7 & 0 & 0 & 0 \\ 24 & 7 & 0 & 0 & 0 & 0\end{array}$

$\begin{array}{lllllll}9 & 8 & 0 & 0 & 0 & 0\end{array}$

$\begin{array}{cccccc}11 & 8 & 0 & 0 & 0 & 0 \\ 10 & 9 & 0 & 0 & 0 & 0\end{array}$

$\begin{array}{llllll}17 & 10 & 0 & 0 & 0 & 0\end{array}$

$\begin{array}{llllll}19 & 10 & 0 & 0 & 0 & 0 \\ 25 & 10 & 0 & 0 & 0 & 0\end{array}$

$\begin{array}{lllllllll}12 & 11 & 0 & 0 & 0 & 0\end{array}$

$\begin{array}{llllll}13 & 11 & 0 & 0 & 0 & 0 \\ 14 & 12 & 0 & 0 & 0 & 0\end{array}$

$\begin{array}{llllll}12 & 12 & 0 & 0 & 0\end{array}$

$\begin{array}{llllll}16 & 13 & 0 & 0 & 0 & 0\end{array}$

2713 1 010000

128 14 0 r 00 r 0

$\begin{array}{lllllll}28 & 14 & 0 & 0 & 0 & 0 \\ 16 & 15 & 0 & 0 & 0 & 0\end{array}$

$\begin{array}{llllll}16 & 15 & 0 & 0 & 0 & 0 \\ 29 & 15 & 0 & 0 & 0 & 0\end{array}$

$\begin{array}{llllll}30 & 16 & 0 & 0 & 0 & 0\end{array}$

$\begin{array}{llllll}18 & 17 & 0 & 0 & 0 & 0 \\ 31 & 19 & 0 & 0 & 0 & 0\end{array}$

932
$M \quad E N D$

$\$ \$ \$ \$$

,3-dihydro-5-phenyl-1,4-benzodiazepin-2-one.sdf

Cosmotherm 3D

$\begin{array}{llllllllllll}30 & 32 & 0 & 0 & 0 & 0 & 0 & 0 & 0 & 0 & 0 & \mathrm{v} 2000\end{array}$

$\begin{array}{lllllllllllll}2.2544 & -2.9709 & 0.0758 & \mathrm{C} & 0 & 0 & 0 & 0 & 0 & 0 & 0 & 0 & 0 \\ 1.0008 & -3.0625 & 0.6983 & \mathrm{C} & 0 & 0 & 0 & 0 & 0 & 0 & 0 & 0 & 0\end{array}$

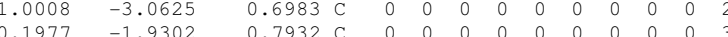

$\begin{array}{cccccccccccc}0.7932 & \mathrm{C} & 0 & 0 & 0 & 0 & 0 & 0 & 0 & 0 & 0 & 3 \\ -0.3069 & \mathrm{C} & 0 & 0 & 0 & 0 & 0 & 0 & 0 & 0 & 0 & 4\end{array}$

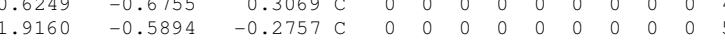

$\begin{array}{llllllllllllll}2.7062 & -1.7464 & -0.4067 & \mathrm{C} & 0 & 0 & 0 & 0 & 0 & 0 & 0 & 0 & 0 & 6\end{array}$

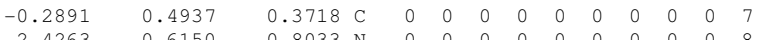

$\begin{array}{llllllllllllll}2.4263 & 0.6150 & -0.8033 & \mathrm{~N} & 0 & 0 & 0 & 0 & 0 & 0 & 0 & 0 & 0 & 8 \\ 2.3493 & 1.8659 & -0.2344 & \mathrm{C} & 0 & 0 & 0 & 0 & 0 & 0 & 0 & 0 & 0 & 9\end{array}$

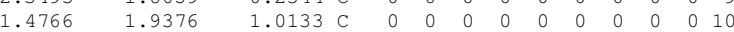

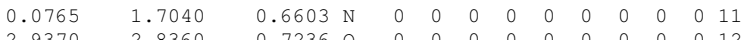

$\begin{array}{llllllllllllll}2.9370 & 2.8360 & -0.7236 & 0 & 0 & 0 & 0 & 0 & 0 & 0 & 0 & 0 & 0 & 12\end{array}$

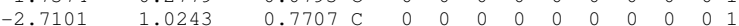

$\begin{array}{llllllllllllll}-2.1540 & -0.6259 & -0.9159 & \mathrm{C} & 0 & 0 & 0 & 0 & 0 & 0 & 0 & 0 & 0 & 15\end{array}$

$\begin{array}{llllllllllllll}-3.5109 & -0.7707 & -1.2198 & \mathrm{C} & 0 & 0 & 0 & 0 & 0 & 0 & 0 & 0 & 0 & 16\end{array}$

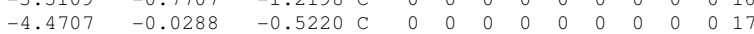

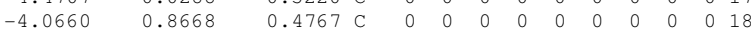

$\begin{array}{llllllllllllll}2.8853 & -3.8554 & -0.0242 \mathrm{H} & 0 & 0 & 0 & 0 & 0 & 0 & 0 & 0 & 0 & 19\end{array}$

$\begin{array}{lllllllllllllllllllllll}0.6509 & -4.0161 & 1.0954 \mathrm{H} & 0 & 0 & 0 & 0 & 0 & 0 & 0 & 0 & 0 & 20\end{array}$

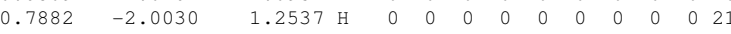

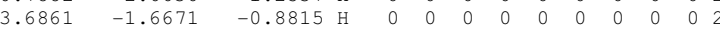

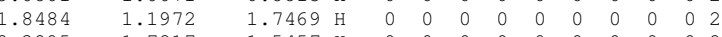

$\begin{array}{rrrrrrrrrrrrrr}-2.3895 & 1.7217 & 1.5457 & \mathrm{H} & 0 & 0 & 0 & 0 & 0 & 0 & 0 & 0 & 0 & 24 \\ -1.4123 & -1.2049 & -1.4682 & \mathrm{H} & 0 & 0 & 0 & 0 & 0 & 0 & 0 & 0 & 0 & 25\end{array}$

$\begin{array}{llllllllllllll}-1.4123 & -1.2049 & -1.4682 & \mathrm{H} & 0 & 0 & 0 & 0 & 0 & 0 & 0 & 0 & 0 & 24\end{array}$

$\begin{array}{llllllllllllll}-5.5307 & -0.1494 & -0.7531 \mathrm{H} & 0 & 0 & 0 & 0 & 0 & 0 & 0 & 0 & 0 & 27\end{array}$

$\begin{array}{lllllllllllll}-4.8112 & 1.4412 & 1.0301 \mathrm{H} & 0 & 0 & 0 & 0 & 0 & 0 & 0 & 0 & 0 & 28\end{array}$

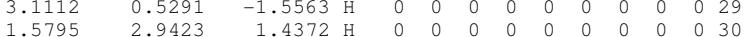

$\begin{array}{lllll}1 & 0 & 0 & 0 & 0\end{array}$

$\begin{array}{lllll}6 & 1 & 0 & 0 & 0\end{array}$

$\begin{array}{lllll}19 & 1 & 0 & 0 & 0\end{array}$

$\begin{array}{llllll}3 & 2 & 0 & 0 & 0\end{array}$

$\begin{array}{lllllll}3 & 2 & 0 & 0 & 0 & 0 & 0\end{array}$

$\begin{array}{lllllll}4 & 3 & 0 & 0 & 0 & 0 \\ 1 & 3 & 0 & 0 & 0 & 0\end{array}$

$\begin{array}{llllll}5 & 4 & 0 & 0 & 0 & 0\end{array}$

$\begin{array}{rrrrrr}4 & 0 & 0 & 0 & 0 \\ 5 & 0 & 0 & 0 & 0 & -1 \\ 5 & 0 & 0 & 0 & 0\end{array}$

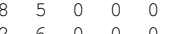

$\begin{array}{llllll}11 & 7 & 0 & 0 & 0 & 0\end{array}$

$\begin{array}{llllll}3 & 7 & 0 & 0 & 0 & 0\end{array}$

$\begin{array}{lllll}8 & 0 & 0 & 0 & 0\end{array}$

$\begin{array}{lllllll}10 & 9 & 0 & 0 & 0 & 0\end{array}$

$\begin{array}{llll}0 & 0 & 0 & 0\end{array}$

$\begin{array}{llllll}11 & 10 & 0 & 0 & 0 & 0\end{array}$ 


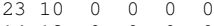

$\begin{array}{llllll}14 & 13 & 0 & 0 & 0 & 0\end{array}$

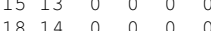

$24 \quad 14 \quad 000000$

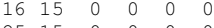

$\begin{array}{llllll}25 & 15 & 0 & 0 & 0 & 0 \\ 17 & 16 & 0 & 0 & 0 & 0\end{array}$

$2616 \quad 0 \quad 0 \quad 0 \quad 0$

$\begin{array}{llllll}18 & 17 & 0 & 0 & 0 & 0 \\ 27 & 17 & 0 & 0 & 0 & 0\end{array}$

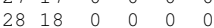

$\begin{array}{llllll}28 & 18 & 0 & 0 & 0 & 0 \\ 10 & 30 & 0 & 0 & 0 & 0\end{array}$

FND

$\$ \$ \$$

3-dihydro-5-phenyl-1,4-benzodiazepin-2-one_p2.sdf

3D

$\begin{array}{llllllllllll}31 & 33 & 0 & 0 & 0 & 0 & 0 & 0 & 0 & 0 & 0 & \mathrm{~V} 2000\end{array}$

$\begin{array}{lllllllllllll}2.1892 & 3.0098 & -0.1068 & \mathrm{C} & 0 & 0 & 0 & 0 & 0 & 0 & 0 & 0 & 0\end{array}$

$\begin{array}{llllllllllllllll}0.9070 & 3.0900 & -0.6731 & c & 0 & 0 & 0 & 0 & 0 & 0 & 0 & 0 & 0 & 0\end{array}$

$\begin{array}{llllllllllllll}0.1202 & 1.9512 & -0.7423 & C & 0 & 0 & 0 & 0 & 0 & 0 & 0 & 0 & 0 & 3\end{array}$

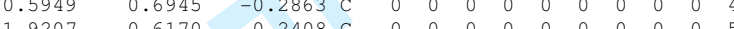

$\begin{array}{llllllllllllll}1.9207 & 0.6170 & 0.2408 & \mathrm{C} & 0 & 0 & 0 & 0 & 0 & 0 & 0 & 0 & 0 & 5\end{array}$

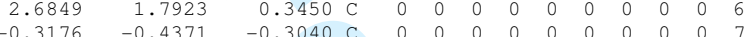

$\begin{array}{lllllllllllll}2.3176 & -0.4371 & -0.3040 & \mathrm{C} & 0 & 0 & 0 & 0 & 0 & 0 & 0 & 0 & 0 \\ 2.4905 & -0.5592 & 0.7583 & \mathrm{~N} & 0 & 0 & 0 & 0 & 0 & 0 & 0 & 0 & 0\end{array}$

$\begin{array}{lllllllllllll}2.4905 & -0.5592 & 0.7583 & \mathrm{~N} & 0 & 0 & 0 & 0 & 0 & 0 & 0 & 0 & 0 \\ 2.4522 & -1.8301 & 0.2276 & \mathrm{C} & 0 & 0 & 0 & 0 & 0 & 0 & 0 & 0 & 0\end{array}$

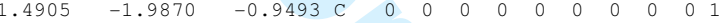

$\begin{array}{llllllllllllll}0.1337 & -1.6573 & -0.5327 & \mathrm{~N} & 0 & 0 & 0 & 0 & 0 & 0 & 0 & 0 & 0 & 11\end{array}$

$\begin{array}{llllllllllllll}3.1395 & -2.7462 & 0.6696 & 0 & 0 & 0 & 0 & 0 & 0 & 0 & 0 & 0 & 0 & 12\end{array}$

$\begin{array}{llllllllllllll}-1.7569 & -0.2784 & -0.0596 & \mathrm{C} & 0 & 0 & 0 & 0 & 0 & 0 & 0 & 0 & 0 & 13\end{array}$

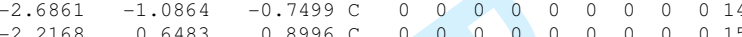

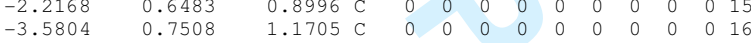

$\begin{array}{llllllllllllll}-4.4969 & -0.0513 & 0.4798 & C & 0 & 0 & 0 & 0 & 0 & 0 & 0 & 0 & 0 & 17\end{array}$

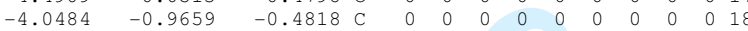

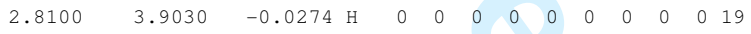

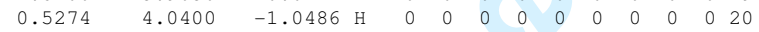

$\begin{array}{llllllllllllll}-0.8785 & 2.0056 & -1.1740 & \mathrm{H} & 0 & 0 & 0 & 0 & 0 & 0 & 0 & 0 & 0 & 21\end{array}$

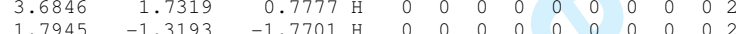

$\begin{array}{lllllllllllllll}1.7945 & -1.3193 & -1.7701 & \mathrm{H} & 0 & 0 & 0 & 0 & 0 & 0 & 0 & 0 & 0 & 0 & 22\end{array}$

$-1.5032-1.2582-1.4538$ H 1.0

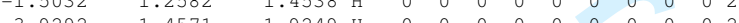

$\begin{array}{llllllllllllllll}-5.9292 & 1.4575 & 1.9249 & -1.00 & 0 & 0 & 0 & 0 & 0 & 0 & 0 & 0 & 0 & 26\end{array}$

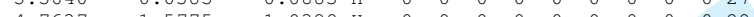

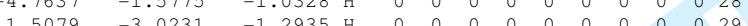

$\begin{array}{lllllllllllll}3.2258 & -0.4236 & 1.4556 \mathrm{H} & 0 & 0 & 0 & 0 & 0 & 0 & 0 & 0 & 0 & 30\end{array}$

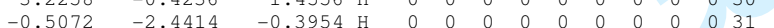

$\begin{array}{llllll}2 & 1 & 0 & 0 & 0 & 0\end{array}$

$\begin{array}{lllllll}6 & 1 & 0 & 0 & 0 & 0\end{array}$

$\begin{array}{llllll}3 & 2 & 0 & 0 & 0 & 0\end{array}$

$\begin{array}{llllll}3 & 2 & 0 & 0 & 0 & 0 \\ 4 & 3 & 0 & 0 & 0 & 0\end{array}$

$\begin{array}{llllll}4 & 3 & 0 & 0 & 0 & 0\end{array}$

$\begin{array}{lllllll}5 & 4 & 0 & 0 & 0 & 0\end{array}$

$\begin{array}{llll}4 & 0 & 0 & 0\end{array}$

$\begin{array}{lllll}5 & 0 & 0 & 0 & 0\end{array}$

$\begin{array}{llll}0 & 0 & 0 & 0 \\ 0 & 0 & 0\end{array}$

$\begin{array}{lllll}1 & 7 & 0 & 0 & 0\end{array}$

$\begin{array}{lllll}7 & 0 & 0 & 0 & 0\end{array}$

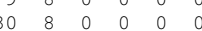

$\begin{array}{llllll}10 & 9 & 0 & 0 & 0 & 0\end{array}$

$\begin{array}{llllll}12 & 9 & 0 & 0 & 0 & 0\end{array}$

$\begin{array}{llllll}11 & 10 & 0 & 0 & 0 & 0\end{array}$

$\begin{array}{llllll}23 & 10 & 0 & 0 & 0 & 0\end{array}$

$\begin{array}{lllll}14 & 13 & 0 & 0 & 0\end{array}$

$\begin{array}{lllll}15 & 13 & 0 & 0 & 0\end{array}$

$\begin{array}{lllll}18 & 14 & 0 & 0 & 0\end{array}$

$\begin{array}{llllll}24 & 14 & 0 & 0 & 0 & 0\end{array}$

$\begin{array}{llllll}16 & 15 & 0 & 0 & 0 & 0\end{array}$

$\begin{array}{llllll}25 & 15 & 0 & 0 & 0 & 0\end{array}$

$\begin{array}{llllll}26 & 16 & 0 & 0 & 0 & 0\end{array}$

$\begin{array}{llllll}18 & 17 & 0 & 0 & 0 & 0\end{array}$

$\begin{array}{llllll}27 & 17 & 0 & 0 & 0 & 0\end{array}$

$\begin{array}{llllll}28 & 18 & 0 & 0 & 0 & 0 \\ 11 & 31 & 0 & 0 & 0 & 0\end{array}$

END

\$\$

-methylindole-cation 0. sdf

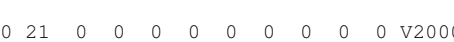

$\begin{array}{rrrrrrrrrrrrrr}-2.1556 & -1.1270 & 0.0657 & \mathrm{C} & 0 & 0 & 0 & 0 & 0 & 0 & 0 & 0 & 0 & 1 \\ -2.5622 & 0.1959 & -0.1619 & \mathrm{C} & 0 & 0 & 0 & 0 & 0 & 0 & 0 & 0 & 0 & 2 \\ -1.6357 & 1.2459 & -0.2123 & \mathrm{C} & 0 & 0 & 0 & 0 & 0 & 0 & 0 & 0 & 0 & 3 \\ -0.2834 & 0.9406 & -0.0289 & \mathrm{C} & 0 & 0 & 0 & 0 & 0 & 0 & 0 & 0 & 0 & 4 \\ 0.0887 & -0.3919 & 0.1966 & \mathrm{C} & 0 & 0 & 0 & 0 & 0 & 0 & 0 & 0 & 0 & 5 \\ -0.8002 & -1.4481 & 0.2536 & \mathrm{C} & 0 & 0 & 0 & 0 & 0 & 0 & 0 & 0 & 0 & 6 \\ 1.5647 & -0.4426 & 0.3564 & \mathrm{~N} & 0 & 0 & 0 & 0 & 0 & 0 & 0 & 0 & 0 & 7 \\ 0.9277 & 1.7585 & -0.0136 & \mathrm{C} & 0 & 0 & 0 & 0 & 0 & 0 & 0 & 0 & 0 & 8 \\ 1.9942 & 0.9767 & 0.2092 & \mathrm{C} & 0 & 0 & 0 & 0 & 0 & 0 & 0 & 0 & 0 & 9 \\ 2.2580 & -1.3673 & -0.6218 & \mathrm{C} & 0 & 0 & 0 & 0 & 0 & 0 & 0 & 0 & 0 & 10 \\ -2.8989 & -1.9239 & 0.1000 & \mathrm{H} & 0 & 0 & 0 & 0 & 0 & 0 & 0 & 0 & 0 & 11 \\ -3.6223 & 0.4108 & -0.3025 & \mathrm{H} & 0 & 0 & 0 & 0 & 0 & 0 & 0 & 0 & 0 & 12 \\ -1.9594 & 2.2721 & -0.3872 & \mathrm{H} & 0 & 0 & 0 & 0 & 0 & 0 & 0 & 0 & 0 & 13 \\ -0.4833 & -2.4749 & 0.4354 & \mathrm{H} & 0 & 0 & 0 & 0 & 0 & 0 & 0 & 0 & 0 & 14 \\ 0.9671 & 2.8353 & -0.1596 & \mathrm{H} & 0 & 0 & 0 & 0 & 0 & 0 & 0 & 0 & 0 & 15 \\ 3.0555 & 1.1836 & 0.2983 & \mathrm{H} & 0 & 0 & 0 & 0 & 0 & 0 & 0 & 0 & 0 & 16\end{array}$

URL: http://mc.manuscriptcentral.com/tandf/tmph 


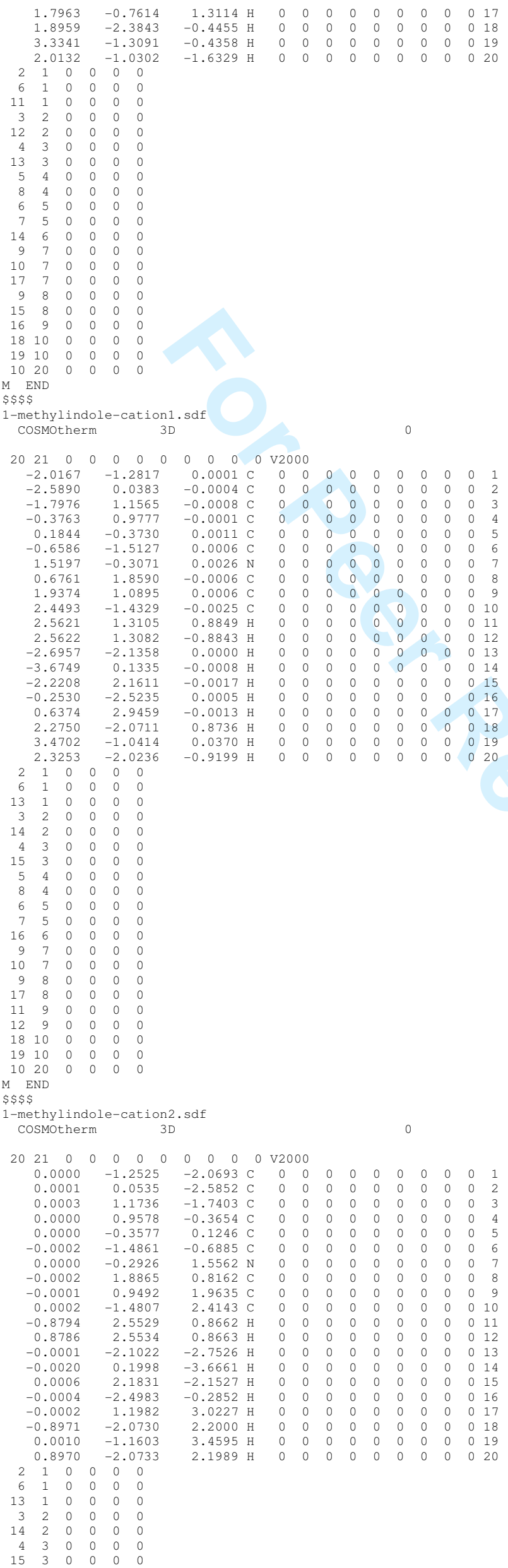




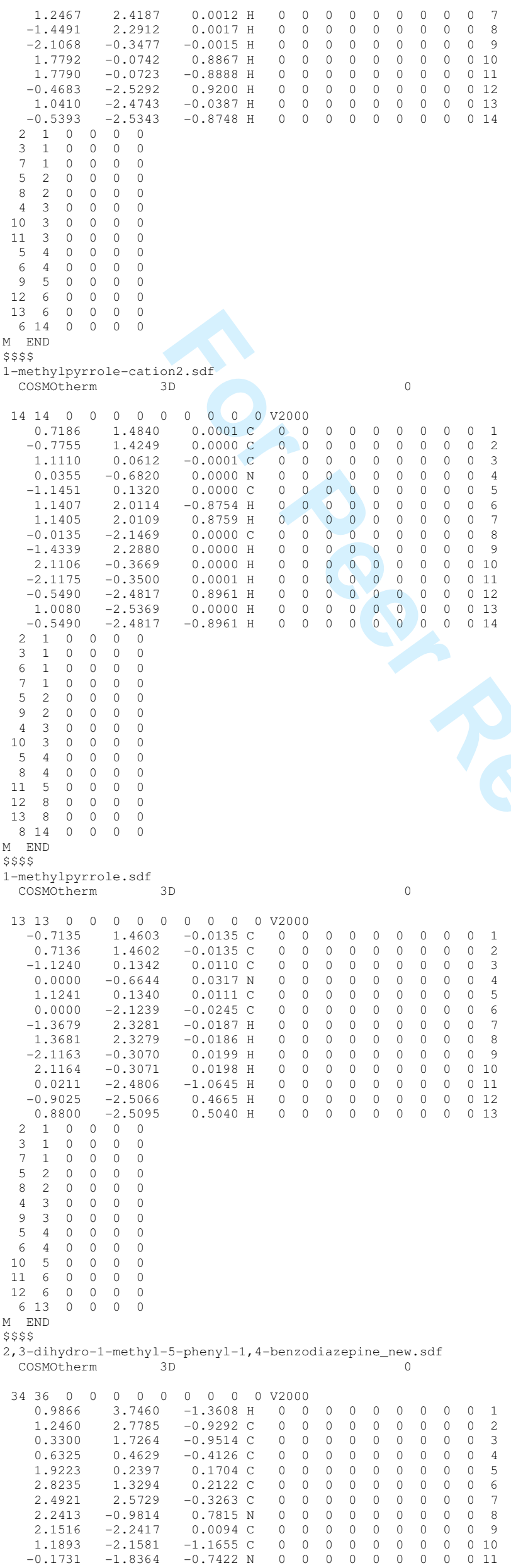




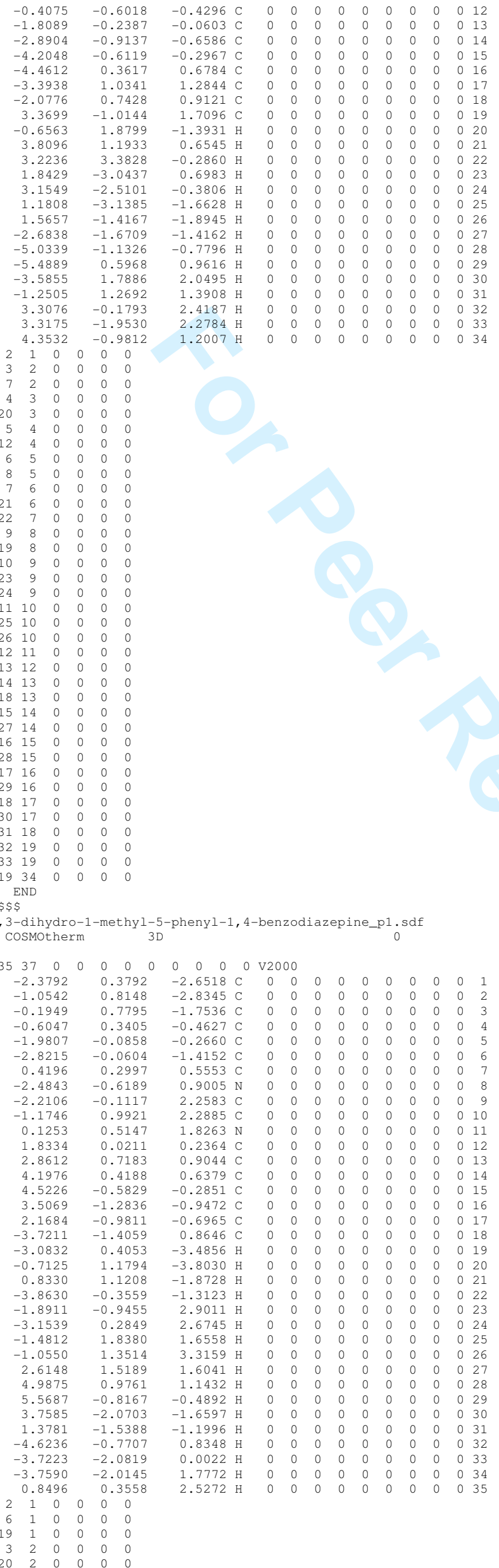




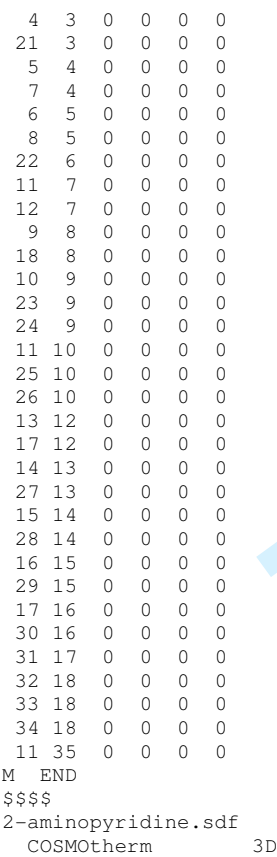

CosMotherm $3 \mathrm{D}$

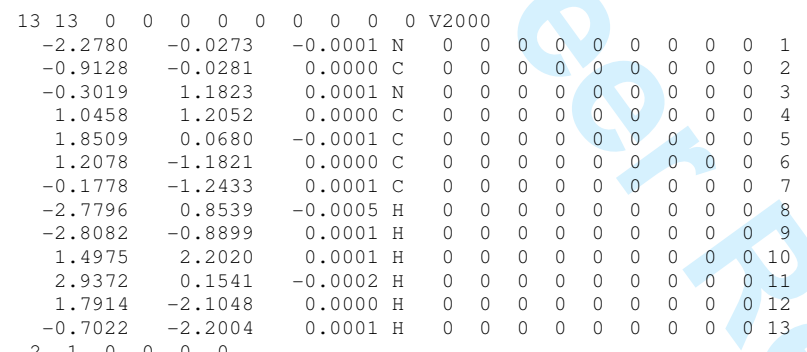

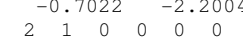

$\begin{array}{llllll}8 & 1 & 0 & 0 & 0 & 0 \\ 9 & 1 & 0 & 0 & 0 & 0 \\ 3 & 2 & 0 & 0 & 0 & 0\end{array}$

$\begin{array}{lllll}9 & 1 & 0 & 0 & 0 \\ 3 & 2 & 0 & 0 & 0\end{array}$

$\begin{array}{lllll}7 & 2 & 0 & 0 & 0 \\ 4 & 3 & 0 & 0 & 0\end{array}$

$\begin{array}{llllll}4 & 3 & 0 & 0 & 0 & 0 \\ 5 & 4 & 0 & 0 & 0 & 0\end{array}$

$\begin{array}{lllll}10 & 4 & 0 & 0 & 0\end{array}$

$\begin{array}{llllll}6 & 5 & 0 & 0 & 0 & 0 \\ 1 & 5 & 0 & 0 & 0 & 0 \\ 7 & 6 & 0 & 0 & 0 & 0\end{array}$

$\begin{array}{llllll}7 & 6 & 0 & 0 & 0 & 0\end{array}$

$\begin{array}{llllll}7 & 13 & 0 & 0 & 0 & 0\end{array}$

M END

2-aminopyridine0.sdf

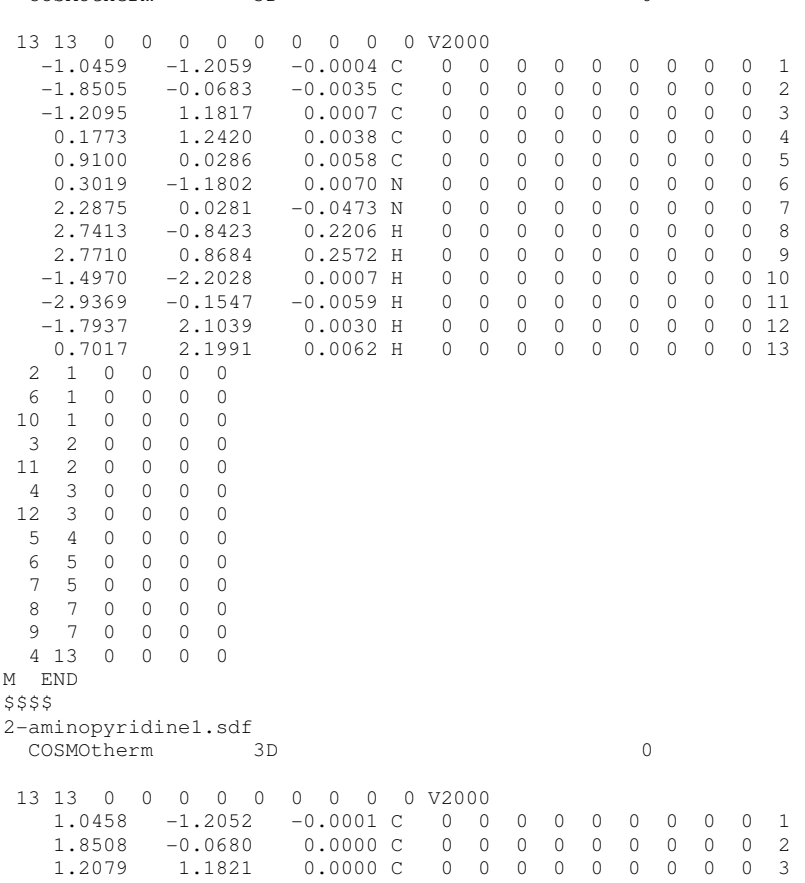




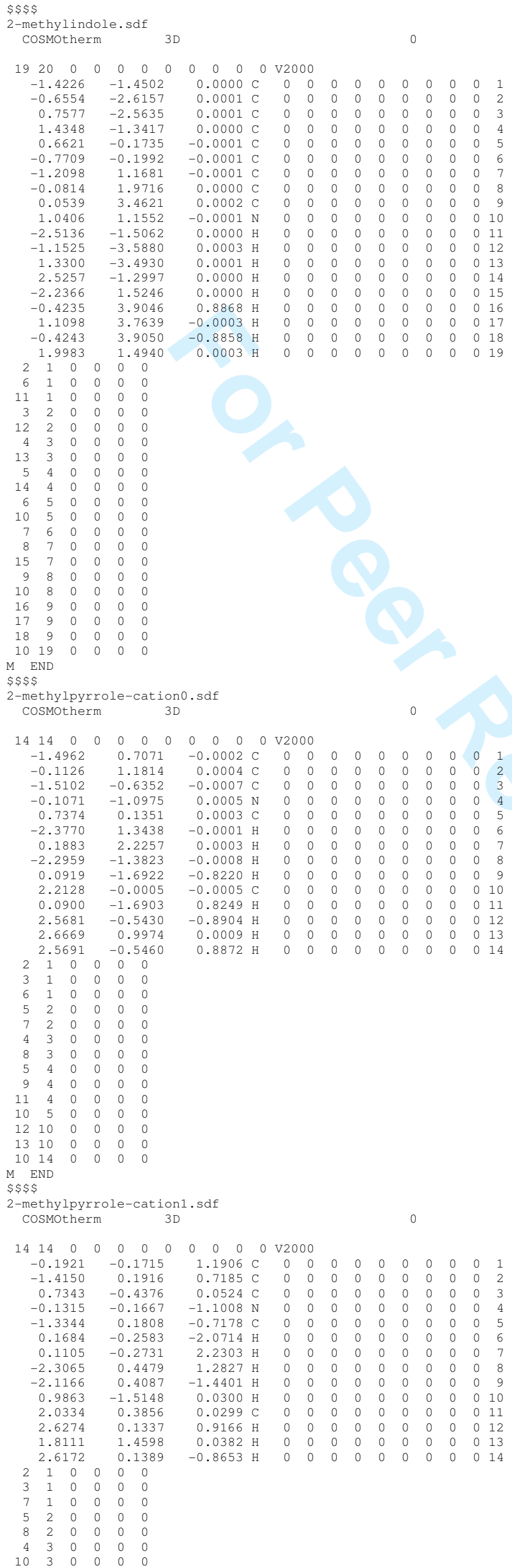




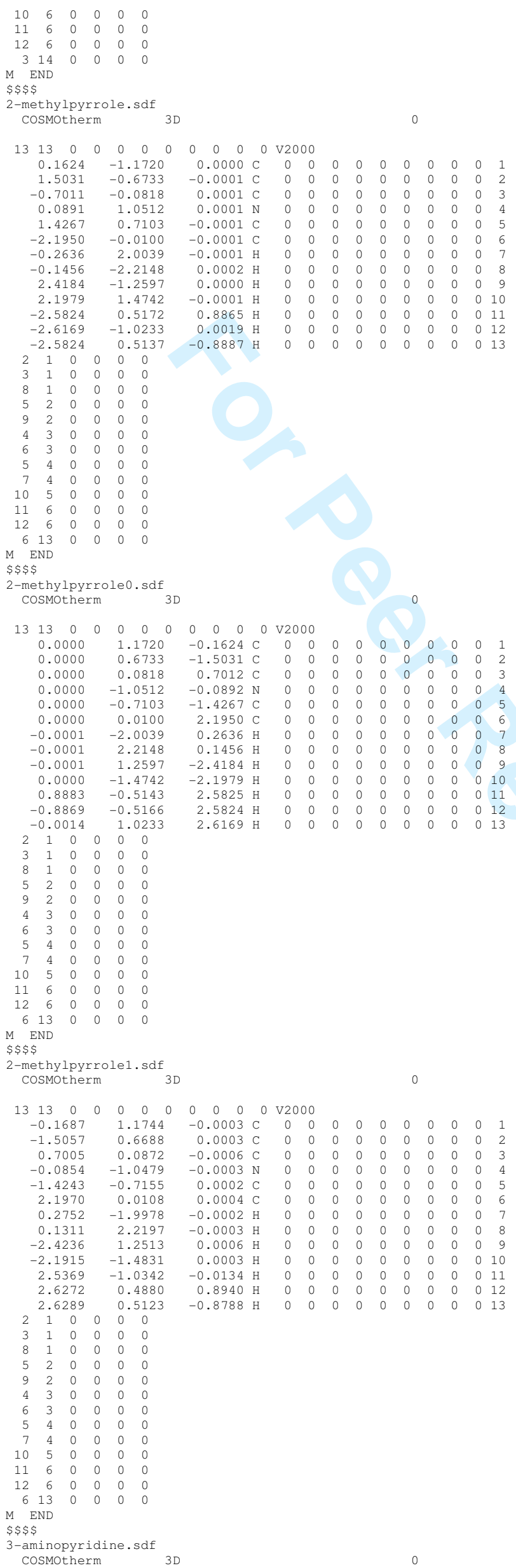




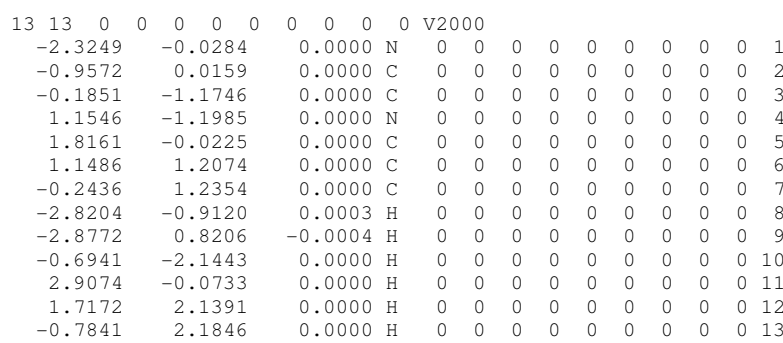




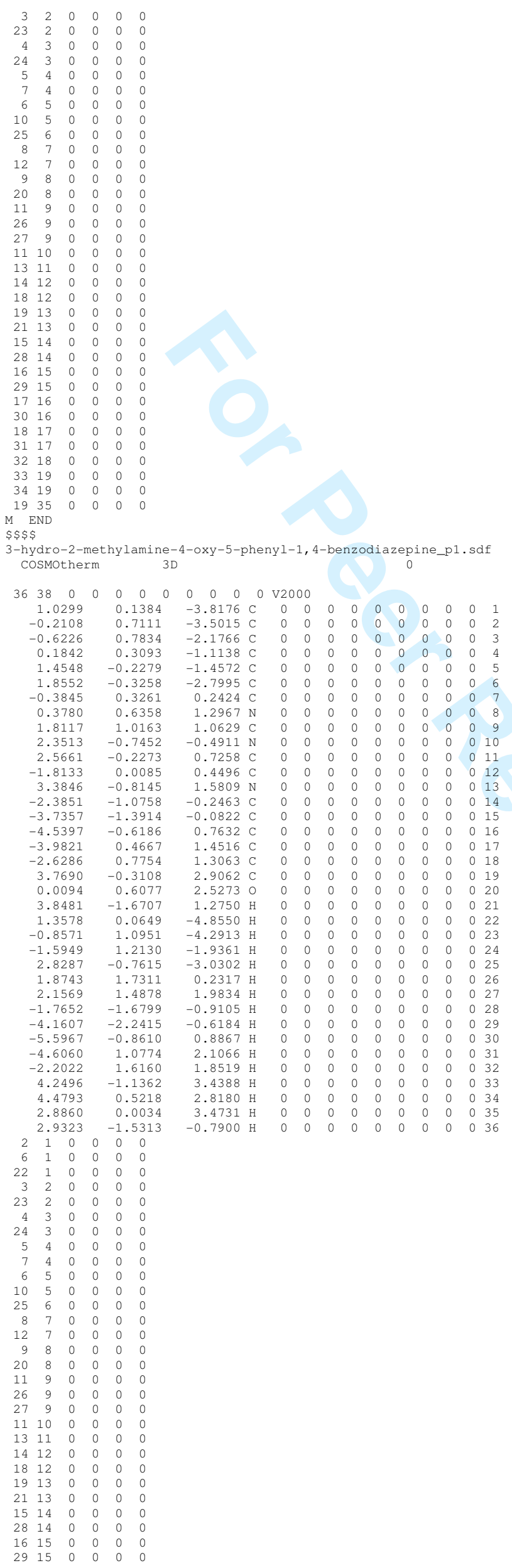


$1716 \quad 000000$

$\begin{array}{llllll}30 & 16 & 0 & 0 & 0 & 0 \\ 18 & 17 & 0 & 0 & 0 & 0\end{array}$

$\begin{array}{lllllll}18 & 17 & 0 & 0 & 0 & 0 \\ 31 & 17 & 0 & 0 & 0 & 0\end{array}$

$\begin{array}{llllll}32 & 18 & 0 & 0 & 0 & 0 \\ 33 & 19 & 0 & 0 & 0 & 0\end{array}$

$34-19-0 \quad 0 \quad 0 \quad 0$

$\begin{array}{llllll}35 & 19 & 0 & 0 & 0 & 0\end{array}$

1036

$\$ \$ \$ \$$

3-hydro-2-methylamine-4-oxy-5-phenyl-1,4-benzodiazepine_t_p1.sdf

cosmotherm $3 \mathrm{D}$

$\begin{array}{lllllllllllll}36 & 38 & 0 & 0 & 0 & 0 & 0 & 0 & 0 & 0 & 0 & \mathrm{v} 2000\end{array}$

$\begin{array}{llllllllllllll}-3.8180 & -0.1392 & 1.0263 & \mathrm{C} & 0 & 0 & 0 & 0 & 0 & 0 & 0 & 0 & 0\end{array}$

$\begin{array}{lllllllllllllll}-0.7102 & -0.2148 & \mathrm{C} & 0 & 0 & 0 & 0 & 0 & 0 & 0 & 0 & 0 & 2\end{array}$

$\begin{array}{llrllllllllllll}-2.1751 & -0.7823 & -0.6251 & \mathrm{C} & 0 & 0 & 0 & 0 & 0 & 0 & 0 & 0 & 0 & 3\end{array}$

$\begin{array}{llllllllllllllll}-1.4581 & 0.2269 & 1.4543 \mathrm{C} & 0 & 0 & 0 & 0 & 0 & 0 & 0 & 0 & 0 & 4\end{array}$

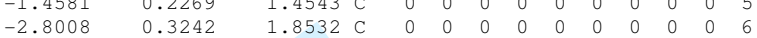

$\begin{array}{llllllllllllll}0.2433 & -0.3245 & -0.3842 & \mathrm{C} & 0 & 0 & 0 & 0 & 0 & 0 & 0 & 0 & 0\end{array}$

$\begin{array}{llrlllllllllll}1.2973 & -0.6336 & 0.3791 & \mathrm{~N} & 0 & 0 & 0 & 0 & 0 & 0 & 0 & 0 & 0 & 8\end{array}$

$\begin{array}{lllllllllllll}1.0624 & -1.0155 & 1.8118 & \mathrm{C} & 0 & 0 & 0 & 0 & 0 & 0 & 0 & 0 & 0\end{array}$

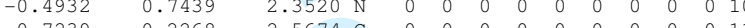

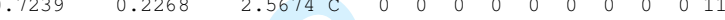

$\begin{array}{llllllllllllll}0.4514 & -0.0074 & -1.8131 & \mathrm{C} & 0 & 0 & 0 & 0 & 0 & 0 & 0 & 0 & 0 & 12\end{array}$

$\begin{array}{lllllllllllll}1.5778 & 0.8136 & 3.3875 \mathrm{~N} & 0 & 0 & 0 & 0 & 0 & 0 & 0 & 0 & 0 & 13\end{array}$

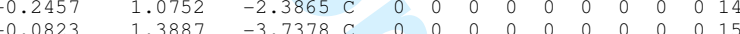

$\begin{array}{lllllllllllll}1.3887 & -3.7378 & \mathrm{C} & 0 & 0 & 0 & 0 & 0 & 0 & 0 & 0 & 0 & 15 \\ 0.6165 & -4.5404 & \mathrm{C} & 0 & 0 & 0 & 0 & 0 & 0 & 0 & 0 & 0 & 16\end{array}$

$\begin{array}{lllllllllllllll}0.7648 & 0.6165 & -4.5404 & \mathrm{C} & 0 & 0 & 0 & 0 & 0 & 0 & 0 & 0 & 0 & 16\end{array}$

$\begin{array}{llllllllllllll}1.4545 & -0.4672 & -3.9811 & \mathrm{C} & 0 & 0 & 0 & 0 & 0 & 0 & 0 & 0 & 0 & 17\end{array}$

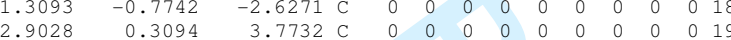

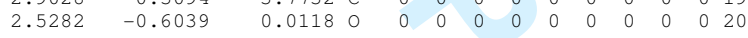

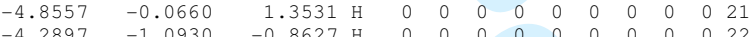

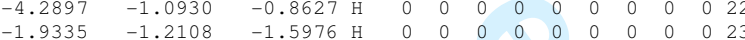

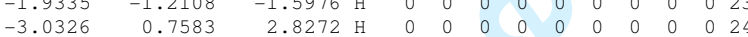

$-1.7308-1.8731 \mathrm{H}-0$

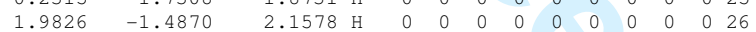

$\begin{array}{llllllllllllllll}-0.9108 & 1.6793 & -1.7676 & \mathrm{H} & 0 & 0 & 0 & 0 & 0 & 0 & 0 & 0 & 0 & 27\end{array}$

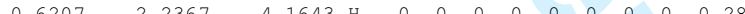

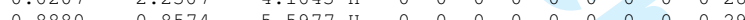

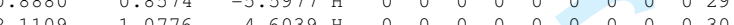

$\begin{array}{llllllllllllllll}1.8550 & -1.6142 & -2.1997 & \mathrm{H} & 0 & 0 & 0 & 0 & 0 & 0 & 0 & 0 & 0 & 30\end{array}$

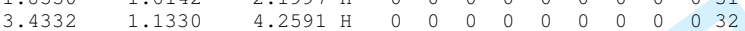

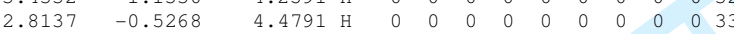

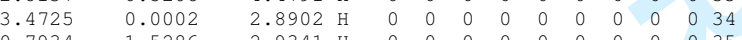

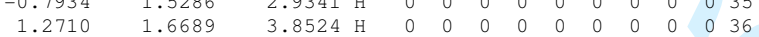

$\begin{array}{lllll}0 & 0 & 0 & 0\end{array}$

$\begin{array}{llllll}6 & 1 & 0 & 0 & 0 & 0\end{array}$

$\begin{array}{lllllll}3 & 1 & 0 & 0 & 0 & 0\end{array}$

$\begin{array}{llllllllll}2 & 2 & 0 & 0 & 0 & 0\end{array}$

$\begin{array}{llllll}3 & 3 & 0 & 0 & 0\end{array}$

$\begin{array}{llllll}4 & 0 & 0 & 0 & 0\end{array}$

$\begin{array}{lllll}5 & 0 & 0 & 0 & 0\end{array}$

$\begin{array}{llllll}0 & 5 & 0 & 0 & 0\end{array}$

$\begin{array}{lllllll}24 & 6 & 0 & 0 & 0 & 0 & 0 \\ 8 & 7 & 0 & 0 & 0 & 0 & 0\end{array}$

$\begin{array}{llllll}12 & 7 & 0 & 0 & 0\end{array}$

$\begin{array}{llllll}9 & 8 & 0 & 0 & 0 & 0\end{array}$

$\begin{array}{lllllll}9 & 8 & 0 & 0 & 0 & 0 & \\ 1 & 9 & 0 & 0 & 0 & 0\end{array}$

$\begin{array}{llllll}25 & 9 & 0 & 0 & 0\end{array}$

$\begin{array}{lllllll}25 & 9 & 0 & 0 & 0 & 0\end{array}$

$\begin{array}{lllll}1 & 10 & 0 & 0 & 0\end{array}$

$\begin{array}{llll}0 & 0 & 0 & 0\end{array}$

$\begin{array}{llllll}4 & 12 & 0 & 0 & 0\end{array}$

$12 \quad 00000$

$\begin{array}{lllll}13 & 0 & 0 & 0\end{array}$

$\begin{array}{llllll}15 & 14 & 0 & 0 & 0\end{array}$

$\begin{array}{llllll}16 & 15 & 0 & 0 & 0 & 0\end{array}$

$\begin{array}{lllllll}28 & 15 & 0 & 0 & 0 & 0\end{array}$

$\begin{array}{lllllll}17 & 16 & 0 & 0 & 0 & 0\end{array}$

$\begin{array}{llllll}29 & 16 & 0 & 0 & 0 & 0\end{array}$

$\begin{array}{lllllll}18 & 17 & 0 & 0 & 0 & 0\end{array}$

$\begin{array}{llllll}1 & 18 & 0 & 0 & 0\end{array}$

$\begin{array}{llllllll}3 & 18 & 0 & 0 & 0 & 0\end{array}$

$\begin{array}{llllll}33 & 19 & 0 & 0 & 0 & 0\end{array}$

$34 \begin{array}{llllll}39 & 0 & 0 & 0 & 0 \\ 3 & 36 & 0 & 0 & 0 & 0\end{array}$

END

-methylindole-cation0.sdf

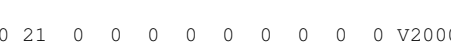

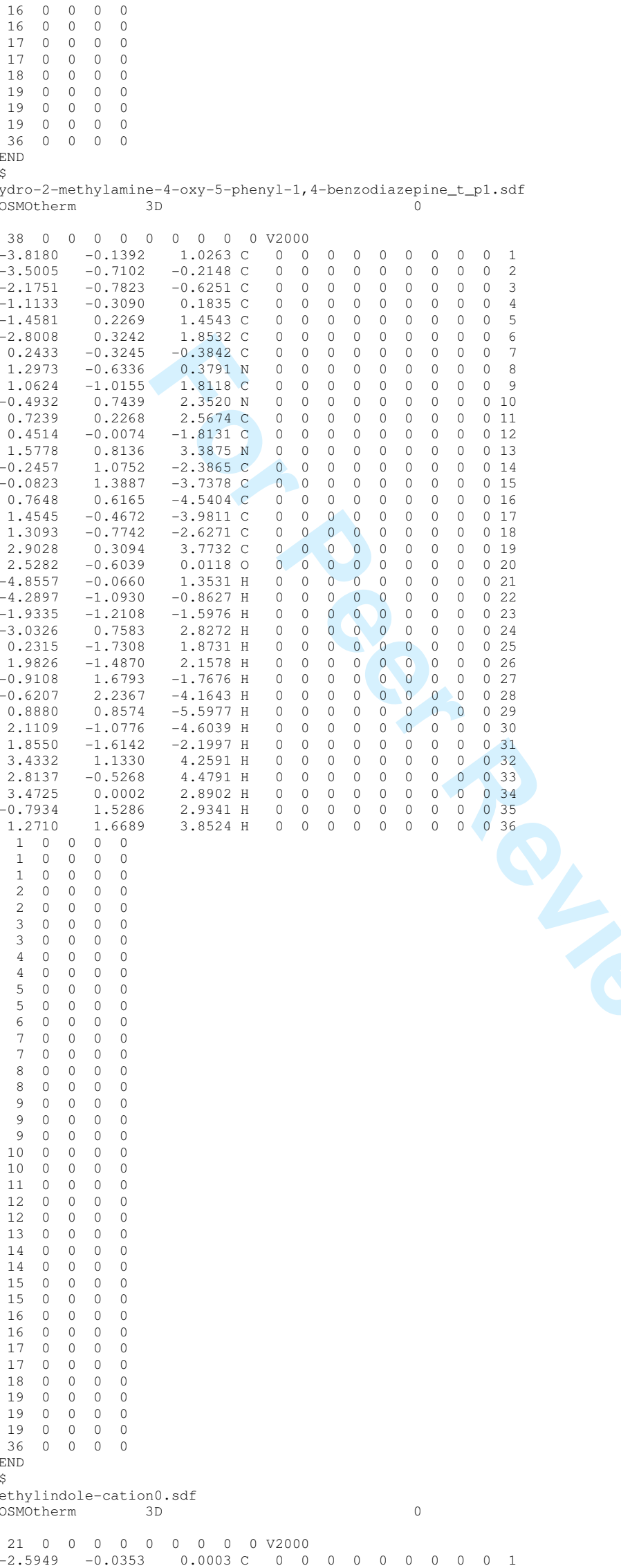

$\begin{array}{rrrrrrrrrrrrrr}-2.5949 & -0.0353 & 0.0003 & \mathrm{C} & 0 & 0 & 0 & 0 & 0 & 0 & 0 & 0 & 0 & 1 \\ -2.0891 & 1.2732 & 0.0001 & \mathrm{C} & 0 & 0 & 0 & 0 & 0 & 0 & 0 & 0 & 0 & 2 \\ -0.7101 & 1.5234 & -0.0002 & \mathrm{C} & 0 & 0 & 0 & 0 & 0 & 0 & 0 & 0 & 0 & 3 \\ 0.1596 & 0.4282 & -0.0003 & \mathrm{C} & 0 & 0 & 0 & 0 & 0 & 0 & 0 & 0 & 0 & 4 \\ -0.3812 & -0.8631 & -0.0004 & \mathrm{C} & 0 & 0 & 0 & 0 & 0 & 0 & 0 & 0 & 0 & 5 \\ -1.7333 & -1.1455 & 0.0001 & \mathrm{C} & 0 & 0 & 0 & 0 & 0 & 0 & 0 & 0 & 0 & 6 \\ 0.7437 & -1.8235 & -0.0003 & \mathrm{~N} & 0 & 0 & 0 & 0 & 0 & 0 & 0 & 0 & 0 & 7 \\ 1.6289 & 0.3265 & 0.0001 & \mathrm{C} & 0 & 0 & 0 & 0 & 0 & 0 & 0 & 0 & 0 & 8 \\ 1.9645 & -0.9779 & 0.0006 & \mathrm{C} & 0 & 0 & 0 & 0 & 0 & 0 & 0 & 0 & 0 & 9 \\ 0.7207 & -2.4448 & -0.8257 & \mathrm{H} & 0 & 0 & 0 & 0 & 0 & 0 & 0 & 0 & 0 & 10\end{array}$




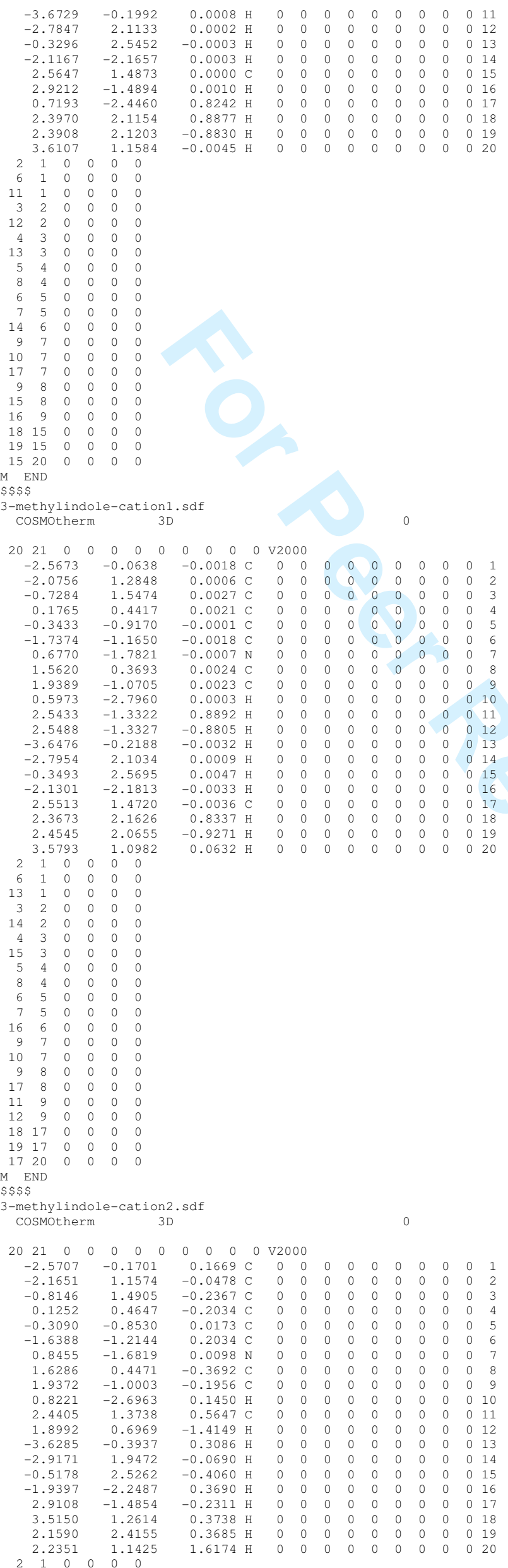


$\begin{array}{llllll}6 & 1 & 0 & 0 & 0 & 0\end{array}$

$\begin{array}{cccccc}13 & 1 & 0 & 0 & 0 & 0 \\ 3 & 2 & 0 & 0 & 0 & 0\end{array}$

$\begin{array}{llllll}14 & 2 & 0 & 0 & 0 & 0\end{array}$

$\begin{array}{rrrrrr}4 & 3 & 0 & 0 & 0 & 0 \\ 15 & 3 & 0 & 0 & 0 & 0\end{array}$

$\begin{array}{llllll}5 & 4 & 0 & 0 & 0 & 0\end{array}$

$\begin{array}{llllll}8 & 4 & 0 & 0 & 0 & 0 \\ 6 & 5 & 0 & 0 & 0 & 0\end{array}$

$\begin{array}{llllll}7 & 5 & 0 & 0 & 0\end{array}$

$6 \begin{array}{lllll}7 & 6 & 0 & 0 & 0\end{array}$

$\begin{array}{llllll}9 & 7 & 0 & 0 & 0\end{array}$

$\begin{array}{llllll}0 & 7 & 0 & 0 & 0 & 0\end{array}$

$\begin{array}{llllll}11 & 8 & 0 & 0 & 0 & 0\end{array}$

$\begin{array}{lllllll}17 & 9 & 0 & 0 & 0 & 0\end{array}$

$\begin{array}{llllll}8 & 11 & 0 & 0 & 0 & 0\end{array}$

$\begin{array}{llllll}11 & 20 & 0 & 0 & 0 & 0\end{array}$

M EN

\$\$

3-methylindole.sdf

$\begin{array}{llllllllllll}9 & 20 & 0 & 0 & 0 & 0 & 0 & 0 & 0 & 0 & 0 & v 2000\end{array}$

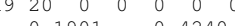

$\begin{array}{rr}-0.1901 & -0.4240 \\ 0.6938 & -1.5229\end{array}$

$2.0688-1.2878$

$\begin{array}{rrrrrrrrrrrrrr}2.5850 & 0.0294 & 0.0000 & \mathrm{C} & 0 & 0 & 0 & 0 & 0 & 0 & 0 & 0 & 0 & 4 \\ 1.7384 & 1.1393 & -0.0001 & \mathrm{C} & 0 & 0 & 0 & 0 & 0 & 0 & 0 & 0 & 0 & 5\end{array}$

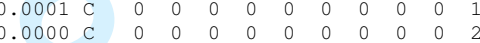

$\begin{array}{llllllllllll}0.0000 & \mathrm{C} & 0 & 0 & 0 & 0 & 0 & 0 & 0 & 0 & 0 & 3\end{array}$

$\begin{array}{llllllllllllll}0.3569 & 0.9005 & 0.0001 & \mathrm{C} & 0 & 0 & 0 & 0 & 0 & 0 & 0 & 0 & 0 & 6\end{array}$

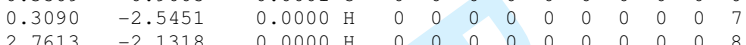

$\begin{array}{rrrrrrrrrrrrrr}2.7613 & -2.1318 & 0.0000 & \mathrm{H} & 0 & 0 & 0 & 0 & 0 & 0 & 0 & 0 & 0 & 8 \\ 3.6661 & 0.1816 & -0.0001 & \mathrm{H} & 0 & 0 & 0 & 0 & 0 & 0 & 0 & 0 & 0 & 9\end{array}$

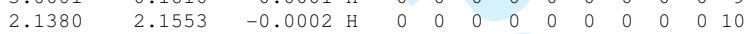

$\begin{array}{llllllllllllll}-1.6277 & -0.2968 & 0.0000 & \mathrm{C} & 0 & 0 & 0 & 0 & 0 & 0 & 0 & 0 & 0 & 11\end{array}$

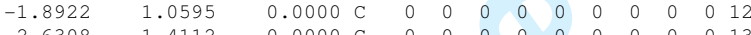

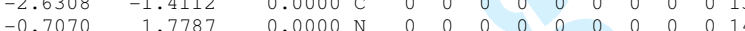

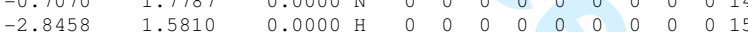

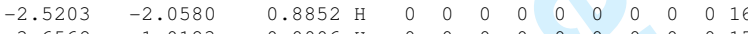

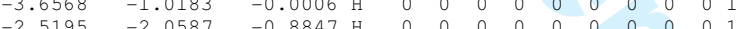

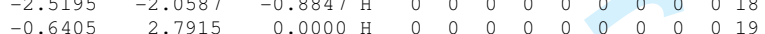

$$
2^{-0.6405} 1000000
$$

$\begin{array}{llllllll}6 & 1 & 0 & 0 & 0 & 0\end{array}$

$\begin{array}{lllllllll}11 & 1 & 0 & 0 & 0 & 0\end{array}$

$\begin{array}{lllllll}3 & 2 & 0 & 0 & 0 & 0\end{array}$

$\begin{array}{lllllllllll}7 & 2 & 0 & 0 & 0 & 0\end{array}$

$\begin{array}{llllllll}3 & 3 & 0 & 0 & 0 & 0\end{array}$

$\begin{array}{lllllll}3 & 4 & 0 & 0 & 0 & 0 & 0\end{array}$

$9 \quad 4 \quad 0 \quad 000$

$\begin{array}{llllllll}10 & 5 & 0 & 0 & 0 & 0\end{array}$

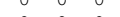

$\begin{array}{llllllll}14 & 6 & 0 & 0 & 0 & 0\end{array}$

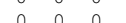

$\begin{array}{llllllll}11 & 0 & 0 & 0 & 0\end{array}$

$\begin{array}{llllll}5 & 12 & 0 & 0 & 0 & 0\end{array}$

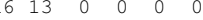

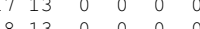

141900000

END

3-methylpyrrole-cationo.sdf

cosmotherm

$\begin{array}{llllllllllll}14 & 14 & 0 & 0 & 0 & 0 & 0 & 0 & 0 & 0 & 0 & \mathrm{~V} 2000\end{array}$

$\begin{array}{llllll}-1.1699 & -0.0939 & 0.0004 & \mathrm{C} & 0 \\ 0.0302 & 0.7632 & 0.0010 & \mathrm{C} & 0 & 0\end{array}$

$\begin{array}{rrr}-1.1699 & -0.0939 & 0.0004 \\ 0.0302 & 0.7632 & 0.0010\end{array}$

$\begin{array}{ccccccccccc}0 & 0 & 0 & 0 & 0 & 0 & 0 & 0 & 0 & 0 & 3\end{array}$

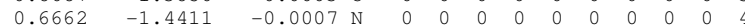

$\begin{array}{llllllllllllll}1.1155 & -0.0365 & 0.0010 & \mathrm{C} & 0 & 0 & 0 & 0 & 0 & 0 & 0 & 0 & 0 & 5\end{array}$

$\begin{array}{lllllllllllll}-2.1942 & 0.2709 & 0.0014 & \mathrm{H} & 0 & 0 & 0 & 0 & 0 & 0 & 0 & 0 & 0\end{array}$

$\begin{array}{llllllllllllll}0.0034 & 2.2543 & -0.0007 & \mathrm{C} & 0 & 0 & 0 & 0 & 0 & 0 & 0 & 0 & 0 & 7\end{array}$

$\begin{array}{llllllllllllll}-1.3576 & -2.3200 & 0.0003 & \mathrm{H} & 0 & 0 & 0 & 0 & 0 & 0 & 0 & 0 & 0 & 8 \\ 1.0227 & -1.9555 & -0.8249 & \mathrm{H} & 0 & 0 & 0 & 0 & 0 & 0 & 0 & 0 & 0 & 9\end{array}$

$\begin{array}{lllllllllllll}2.1826 & 0.1577 & 0.0011 \mathrm{H} & 0 & 0 & 0 & 0 & 0 & 0 & 0 & 0 & 0 & 10\end{array}$

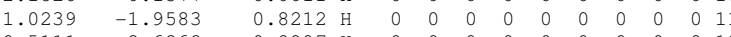

$-0.5111-2.6262$

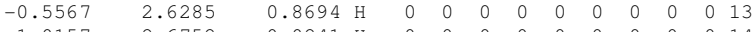

$1.0157-2.6752$

$\begin{array}{llllll}1 & 0 & 0 & 0 & 0\end{array}$

$\begin{array}{llllll}1 & 0 & 0 & 0 & 0\end{array}$

$\begin{array}{lllll}1 & 0 & 0 & 0 & 0 \\ 2 & 0 & 0 & 0 & 0\end{array}$

$\begin{array}{llllllll}2 & 0 & 0 & 0 & 0\end{array}$

$\begin{array}{llllll}3 & 0 & 0 & 0 & 0\end{array}$

$\begin{array}{llllll}3 & 0 & 0 & 0 & 0 & 0 \\ 2 & 4 & 0 & 0 & 0 & 0\end{array}$

$\begin{array}{llllll}4 & 0 & 0 & 0\end{array}$

$\begin{array}{lllll}4 & 0 & 0 & 0 & 0 \\ 5 & 0 & 0 & 0 & 0\end{array}$

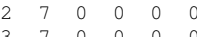

$\begin{array}{llllll}7 & 14 & 0 & 0 & 0 & 0\end{array}$

END

-methylpyrrole-cation 1. sde

$\begin{array}{llllllllllll}14 & 14 & 0 & 0 & 0 & 0 & 0 & 0 & 0 & 0 & 0 & \mathrm{v} 2000\end{array}$ 


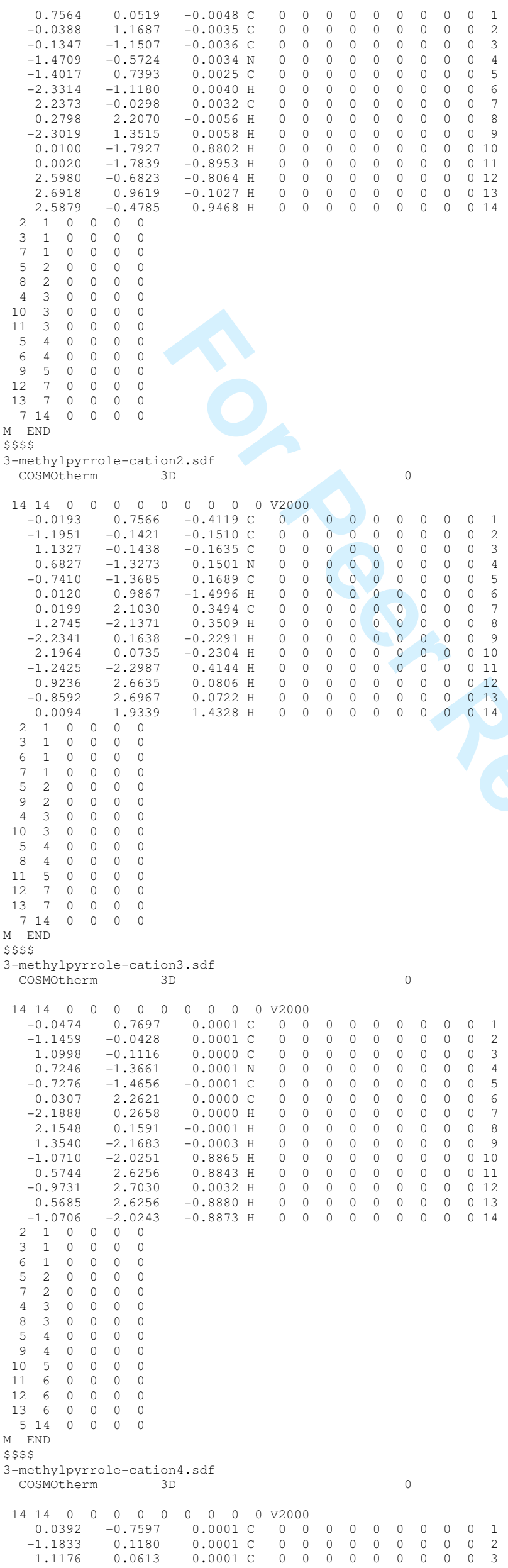


$\begin{array}{llllllllllllll}0.6627 & 1.4052 & 0.0000 & \mathrm{~N} & 0 & 0 & 0 & 0 & 0 & 0 & 0 & 0 & 0 & 4\end{array}$

$\begin{array}{llllllllllllll}-0.6403 & 1.4900 & -0.0002 & \mathrm{C} & 0 & 0 & 0 & 0 & 0 & 0 & 0 & 0 & 0 & 5\end{array}$

$\begin{array}{cccccccccccccc}0.0071 & -2.2528 & -0.0001 & \mathrm{C} & 0 & 0 & 0 & 0 & 0 & 0 & 0 & 0 & 0 & 6 \\ -1.8379 & -0.0480 & -0.8751 & \mathrm{H} & 0 & 0 & 0 & 0 & 0 & 0 & 0 & 0 & 0 & 7\end{array}$

$\begin{array}{lllllllllllllll}2.1844 & -0.1370 & 0.0001 & \mathrm{H} & 0 & 0 & 0 & 0 & 0 & 0 & 0 & 0 & 0 & 8\end{array}$

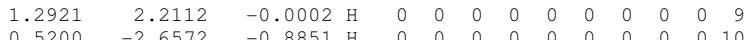

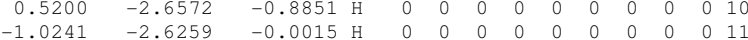

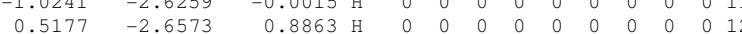

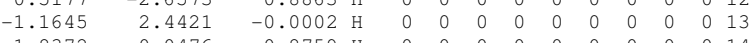

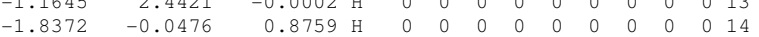




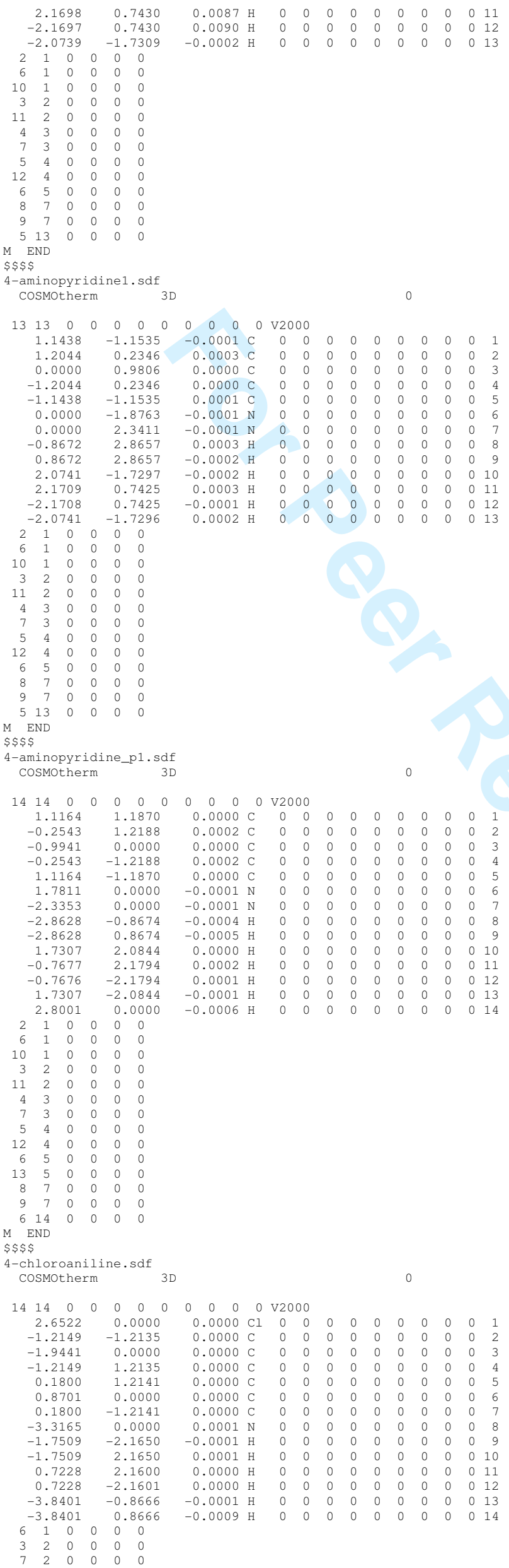




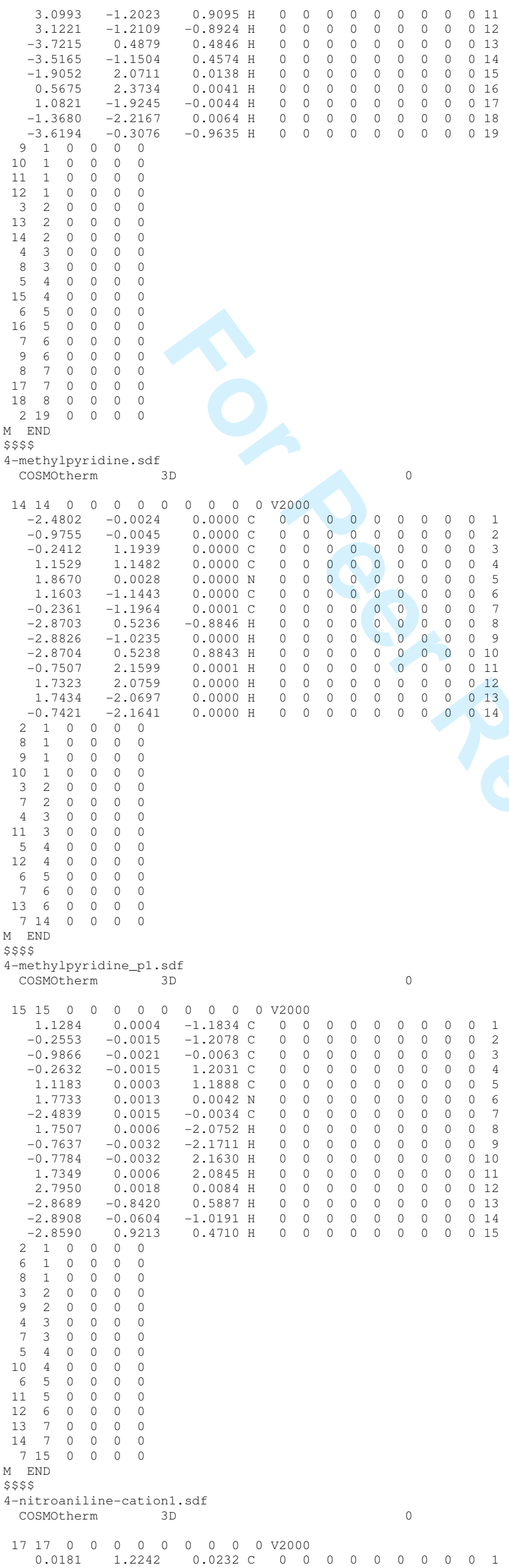




\begin{tabular}{|c|c|c|c|c|c|c|c|c|c|c|}
\hline .0 & & & & & & & & & & \\
\hline 0.0054 & 0.0002 & 2.0861 & c & 0 & 0 & 0 & 0 & 0 & & \\
\hline-0.0145 & -1.2225 & 1.4175 & c & 0 & 0 & 0 & 0 & 0 & & \\
\hline-0.0173 & -1.2242 & 0.0239 & & 0 & 0 & 0 & 0 & & & \\
\hline-0.0018 & 0.0002 & -0.6474 & ( & 0 & 0 & 0 & 0 & 0 & & \\
\hline-0.0012 & 0.0001 & -2.1295 & & 0 & 0 & 0 & 0 & & & \\
\hline-0.0072 & 0.0002 & 3.5603 & & 0 & 0 & 0 & 0 & 0 & & \\
\hline-0.0404 & 1.0927 & -2.7116 & & 0 & 0 & 0 & 0 & 0 & & \\
\hline 0.0381 & -1.0930 & -2.7110 & & 0 & 0 & 0 & 0 & 0 & & \\
\hline 0.5614 & -0.7654 & 3.9503 & & 0 & 0 & 0 & 0 & & & \\
\hline-0.9615 & -0.1100 & 3.9370 & & 0 & 0 & 0 & 0 & & & \\
\hline 0.0342 & 2.1603 & -0.5315 & & 0 & 0 & 0 & 0 & 0 & & \\
\hline 0.0439 & 2.1632 & 1.9671 & & O & 0 & 0 & 0 & 0 & & \\
\hline-0.0259 & -2.1637 & 1.9675 & & 0 & 0 & 0 & 0 & 0 & & \\
\hline-0.029 & -2.1601 & -0.5311 & & & 0 & & 0 & 0 & & \\
\hline & & & & & & & & & & \\
\hline
\end{tabular}




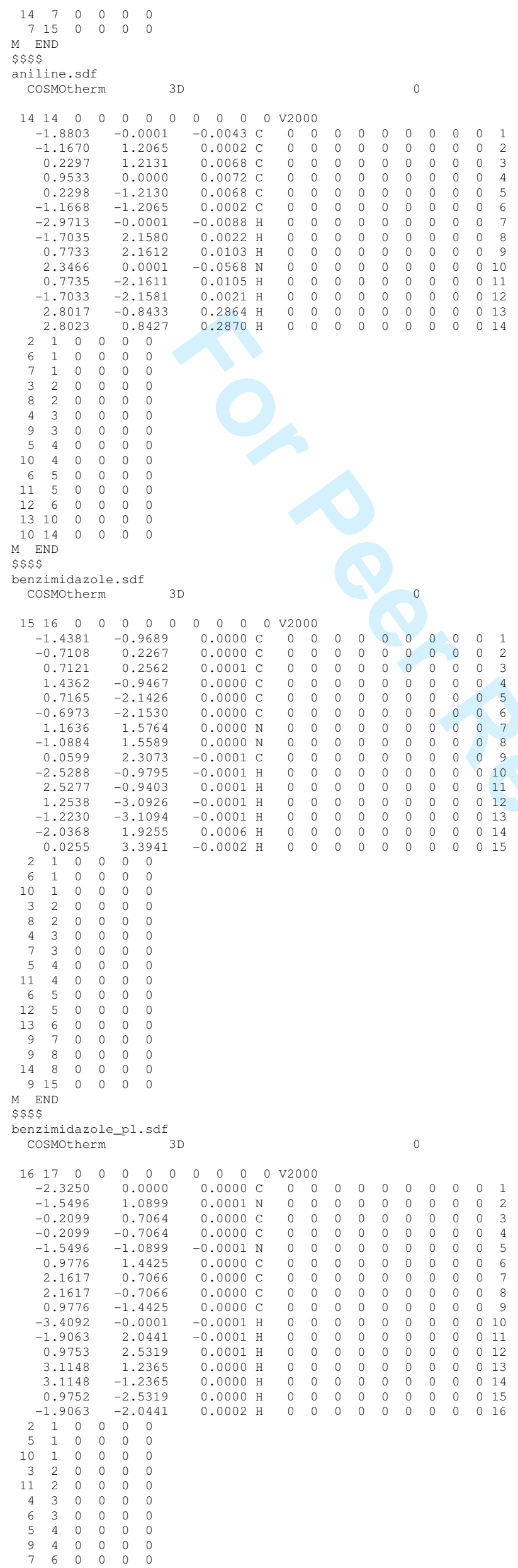


$\begin{array}{llllll}12 & 6 & 0 & 0 & 0 & 0\end{array}$

$\begin{array}{rrrrrr}8 & 7 & 0 & 0 & 0 & 0 \\ 13 & 7 & 0 & 0 & 0 & 0\end{array}$

$\begin{array}{llllll}9 & 8 & 0 & 0 & 0 & 0 \\ 4 & 8 & 0 & 0 & 0 & 0\end{array}$

$\begin{array}{llllll}15 & 9 & 0 & 0 & 0 & 0\end{array}$

516

\$\$\$

clonidine-cation $2 . s d f$

rm

$\begin{array}{llllllllllll}24 & 25 & 0 & 0 & 0 & 0 & 0 & 0 & 0 & 0 & 0 & \mathrm{~V} 2000\end{array}$

\begin{tabular}{|c|c|c|c|c|c|c|c|c|c|c|c|c|}
\hline 2.7823 & 0.9918 & 0.5509 & C & 0 & 0 & 0 & 0 & 0 & 0 & 0 & 0 & 0 \\
\hline 1.5195 & 1.1167 & -0.0268 & C & 0 & 0 & 0 & 0 & 0 & 0 & 0 & 0 & 0 \\
\hline 0.7357 & -0.0120 & -0.3359 & C & 0 & 0 & 0 & 0 & 0 & 0 & 0 & 0 & 0 \\
\hline 1.2745 & -1.2831 & -0.0544 & $\mathrm{C}$ & 0 & 0 & 0 & 0 & 0 & 0 & 0 & 0 & 0 \\
\hline 2.5327 & -1.4268 & 0.5294 & C & 0 & 0 & 0 & 0 & 0 & 0 & 0 & 0 & 0 \\
\hline 3.2786 & -0.2843 & 0.8265 & $\mathrm{C}$ & 0 & 0 & 0 & 0 & 0 & 0 & 0 & 0 & 0 \\
\hline 0.8910 & 2.7212 & -0.3805 & $\mathrm{Cl}$ & 0 & 0 & 0 & 0 & 0 & 0 & 0 & 0 & 0 \\
\hline 0.3468 & -2.7219 & -0.4560 & $\mathrm{Cl}$ & 0 & 0 & 0 & 0 & 0 & 0 & 0 & 0 & 0 \\
\hline-0.5396 & 0.1298 & -0.9530 & $\mathrm{~N}$ & 0 & 0 & 0 & 0 & 0 & 0 & 0 & 0 & 0 \\
\hline-1.6941 & 0.1355 & -0.2665 & $\mathrm{C}$ & 0 & 0 & 0 & 0 & 0 & 0 & 0 & 0 & 0 \\
\hline-3.9581 & 0.0522 & 0.1724 & C & 0 & 0 & 0 & 0 & 0 & 0 & 0 & 0 & 0 \\
\hline-3.1839 & 0.2225 & 1.4955 & $\mathrm{C}$ & 0 & 0 & 0 & 0 & 0 & 0 & 0 & 0 & 0 \\
\hline-2.9010 & 0.3159 & -0.8278 & $\mathrm{~N}$ & 0 & 0 & 0 & 0 & 0 & 0 & 0 & 0 & 0 \\
\hline-1.7943 & -0.0351 & 1.0609 & $\mathrm{~N}$ & 0 & 0 & 0 & 0 & 0 & 0 & 0 & 0 & 0 \\
\hline-3.0556 & 0.1638 & -1.8214 & $\mathrm{H}$ & 0 & 0 & 0 & 0 & 0 & 0 & 0 & 0 & 0 \\
\hline-1.0012 & 0.0900 & 1.6849 & $\mathrm{H}$ & 0 & 0 & 0 & 0 & 0 & 0 & 0 & 0 & 0 \\
\hline 3.3682 & 1.8811 & 0.7796 & $\mathrm{H}$ & 0 & 0 & 0 & 0 & 0 & 0 & 0 & 0 & 0 \\
\hline 2.9261 & -2.4206 & 0.7385 & $\mathrm{H}$ & 0 & 0 & 0 & 0 & 0 & 0 & 0 & 0 & 0 \\
\hline 4.2650 & -0.3899 & 1.2792 & $\mathrm{H}$ & 0 & 0 & 0 & 0 & 0 & 0 & 0 & 0 & 0 \\
\hline-4.3441 & -0.9704 & 0.0519 & $\mathrm{H}$ & 0 & 0 & 0 & 0 & 0 & 0 & 0 & 0 & 0 \\
\hline-4.7789 & 0.7686 & 0.0684 & $\mathrm{H}$ & 0 & 0 & 0 & 0 & 0 & 0 & 0 & 0 & 0 \\
\hline-3.2690 & 1.2440 & 1.8935 & $\mathrm{H}$ & 0 & 0 & 0 & 0 & 0 & 0 & 0 & 0 & 0 \\
\hline-3.4903 & -0.4967 & 2.2615 & & 0 & 0 & & 0 & 0 & 0 & 0 & 0 & 0 \\
\hline-0.5835 & 0.2607 & -1.9627 & 1 & 0 & & & 0 & 0 & 0 & 0 & & 0 \\
\hline
\end{tabular}

$\begin{array}{llllll}2 & 1 & 0 & 0 & 0 & 0 \\ 6 & 1 & 0 & 0 & 0 & 0 \\ 17 & 1 & 0 & 0 & 0 & 0\end{array}$

$\begin{array}{cccccc}17 & 1 & 0 & 0 & 0 & 0\end{array}$

$\begin{array}{llllll}3 & 2 & 0 & 0 & 0\end{array}$

$\begin{array}{lllll}2 & 0 & 0 & 0\end{array}$

$\begin{array}{lllll}3 & 0 & 0 & 0\end{array}$

$\begin{array}{lllll}3 & 0 & 0 & 0 & 0\end{array}$

$\begin{array}{llllll}4 & 0 & 0 & 0 & 0\end{array}$

$\begin{array}{lllll}6 & 5 & 0 & 0 & 0\end{array}$

$\begin{array}{llll}0 & 0 & 0 & 0\end{array}$

$\begin{array}{lllllll}1 & 9 & 0 & 0 & 0 & 0\end{array}$

$\begin{array}{llllll}3 & 10 & 0 & 0 & 0 & 0\end{array}$

$\begin{array}{llllll}4 & 10 & 0 & 0 & 0 & 0\end{array}$

$\begin{array}{llllll}12 & 11 & 0 & 0 & 0 & 0 \\ 3 & 11 & 0 & 0 & 0 & 0\end{array}$

2011 r 00

$\begin{array}{llllll}21 & 11 & 0 & 0 & 0\end{array}$

$\begin{array}{llllll}14 & 12 & 0 & 0 & 0 & 0 \\ 22 & 12 & 0 & 0 & 0 & 0\end{array}$

$\begin{array}{llll}0 & 0 & 0\end{array}$

$\begin{array}{llll}0 & 0 & 0 & 0\end{array}$

$\begin{array}{llllll}6 & 14 & 0 & 0 & 0 & 0\end{array}$

\$\$\$

clonidine.sdf

Cosmotherm 3D

$\begin{array}{llllllllllll}23 & 24 & 0 & 0 & 0 & 0 & 0 & 0 & 0 & 0 & 0 & \mathrm{~V} 2000\end{array}$

$-0.6414-2.8298$

$-0.3588-1.6195$

$-0.0669-1.8560$

$-0.5395 \quad 2.9761$

$-0.9229 \quad 1.7267$

1.7267
0.6961

1.0382

$1.7742 \quad 2.2785$

$0.8182 \quad 3.2524$

2.6036

$\begin{array}{llllllllllllll}1.4001 & 0.5041 & \mathrm{C} & 0 & 0 & 0 & 0 & 0 & 0 & 0 & 0 & 0 & 9 & 9 \\ -0.4855 & 0.8978 & \mathrm{~N} & 0 & 0 & 0 & 0 & 0 & 0 & 0 & 0 & 0 & 1\end{array}$

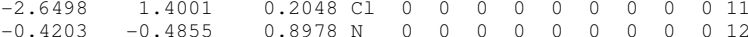

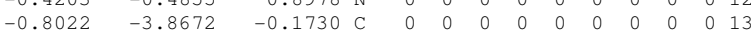

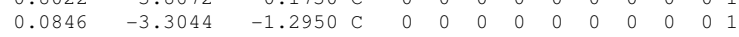

$\begin{array}{llllllllllllll}-1.2746 & -2.8158 & 1.6559 \mathrm{H} & 0 & 0 & 0 & 0 & 0 & 0 & 0 & 0 & 0 & 0 & 14\end{array}$

$\begin{array}{llllllllllllll}0.5515 & -1.2106 & -1.5555 & \mathrm{H} & 0 & 0 & 0 & 0 & 0 & 0 & 0 & 0 & 0 & 16\end{array}$

$\begin{array}{llllllllllllll}-1.2973 & 3.7250 & -0.7290 & \mathrm{H} & 0 & 0 & 0 & 0 & 0 & 0 & 0 & 0 & 0 & 17 \\ 2.8370 & 2.4809 & -0.5177 & \mathrm{H} & 0 & 0 & 0 & 0 & 0 & 0 & 0 & 0 & 0 & 18\end{array}$

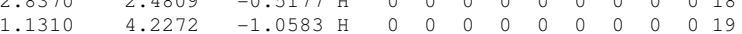

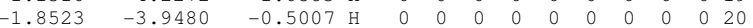

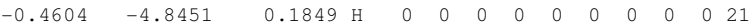

$\begin{array}{llllllllllllll}-0.2616 & -3.5916 & -2.2945 & \mathrm{H} & 0 & 0 & 0 & 0 & 0 & 0 & 0 & 0 & 0 & 22 \\ 1.1338 & -3.6198 & -1.1701 & \mathrm{H} & 0 & 0 & 0 & 0 & 0 & 0 & 0 & 0 & 0 & 23\end{array}$

$1338-3.6198$

$\begin{array}{llllll}2 & 1 & 0 & 0 & 0 & 0\end{array}$

$\begin{array}{llllllll}5 & 1 & 0 & 0 & 0 & 0\end{array}$

$\begin{array}{llllll}3 & 2 & 0 & 0 & 0 & 0\end{array}$

$\begin{array}{llllll}2 & 0 & 0 & 0 & 0\end{array}$

$\begin{array}{llllll}14 & 3 & 0 & 0 & 0 & 0 \\ 16 & 3 & 0 & 0 & 0 & 0\end{array}$

$5 \begin{array}{llllll}5 & 4 & 0 & 0 & 0 & 0 \\ 9 & 4 & 0 & 0 & 0 & 0\end{array}$

$\begin{array}{lllll}17 & 4 & 0 & 0 & 0\end{array}$

$\begin{array}{llllll}5 & 0 & 0 & 0 & 0\end{array}$

$\begin{array}{llllllll}12 & 6 & 0 & 0 & 0 & 0\end{array}$ 
$\begin{array}{rrrrrr}8 & 7 & 0 & 0 & 0 & 0 \\ 10 & 7 & 0 & 0 & 0 & 0 \\ 9 & 8 & 0 & 0 & 0 & 0 \\ 18 & 8 & 0 & 0 & 0 & 0 \\ 19 & 9 & 0 & 0 & 0 & 0 \\ 14 & 13 & 0 & 0 & 0 & 0 \\ 20 & 13 & 0 & 0 & 0 & 0 \\ 21 & 13 & 0 & 0 & 0 & 0 \\ 22 & 14 & 0 & 0 & 0 & 0 \\ 14 & 23 & 0 & 0 & 0 & 0 \\ \text { M END } & & & & \\ \text { \$\$\$ EN } \\ \text { Clonidine0.sdf }\end{array}$

clonidine0.sdf
Cosmotherm

$\begin{array}{lllllllllllll}23 & 24 & 0 & 0 & 0 & 0 & 0 & 0 & 0 & 0 & 0 & \mathrm{~V} 2000\end{array}$

$\begin{array}{lrrrrrrrrrrrrr}-1.7913 & 0.3793 & -2.2634 & \mathrm{C} & 0 & 0 & 0 & 0 & 0 & 0 & 0 & 0 & 0 & 1 \\ -1.3625 & -0.0997 & -1.0264 & \mathrm{C} & 0 & 0 & 0 & 0 & 0 & 0 & 0 & 0 & 0 & 2\end{array}$

$\begin{array}{rrrrrrrrrrrrrr}-1.3625 & -0.0997 & -1.0264 & \mathrm{C} & 0 & 0 & 0 & 0 & 0 & 0 & 0 & 0 & 0 & 2 \\ 0.0037 & -0.2996 & -0.6967 & \mathrm{C} & 0 & 0 & 0 & 0 & 0 & 0 & 0 & 0 & 0 & 3\end{array}$

$\begin{array}{llllllllllllll}0.9122 & 0.0194 & -1.7386 & \mathrm{C} & 0 & 0 & 0 & 0 & 0 & 0 & 0 & 0 & 0 & 4 \\ 0.5146 & 0.4970 & -2.9853 & \mathrm{C} & 0 & 0 & 0 & 0 & 0 & 0 & 0 & 0 & 0 & 5\end{array}$

$\begin{array}{rrrrrrrrrrrrrr}0.5146 & 0.4970 & -2.9853 & \mathrm{C} & 0 & 0 & 0 & 0 & 0 & 0 & 0 & 0 & 0 & 5 \\ -0.8463 & 0.6780 & -3.2479 & \mathrm{C} & 0 & 0 & 0 & 0 & 0 & 0 & 0 & 0 & 0 & 6\end{array}$

$\begin{array}{rrrrrrrrrrrrrr}-0.8463 & 0.6780 & -3.2479 & \mathrm{C} & 0 & 0 & 0 & 0 & 0 & 0 & 0 & 0 & 0 & 6 \\ -2.5943 & -0.5089 & 0.1842 & \mathrm{Cl} & 0 & 0 & 0 & 0 & 0 & 0 & 0 & 0 & 0 & 7\end{array}$

$\begin{array}{llrllllllllllll}2.6429 & -0.1973 & -1.4299 & \mathrm{Cl} & 0 & 0 & 0 & 0 & 0 & 0 & 0 & 0 & 0 & 8\end{array}$

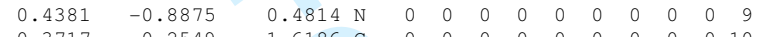

$\begin{array}{rrrrrrrrrrrrrr}0.3717 & -0.2549 & 1.6186 & \mathrm{C} & 0 & 0 & 0 & 0 & 0 & 0 & 0 & 0 & 0 & 10 \\ 0.8241 & 0.1742 & 3.8651 & \mathrm{C} & 0 & 0 & 0 & 0 & 0 & 0 & 0 & 0 & 0 & 11\end{array}$

$\begin{array}{rrrrrrrrrrrrrr}0.8241 & 0.1742 & 3.8651 & \mathrm{C} & 0 & 0 & 0 & 0 & 0 & 0 & 0 & 0 & 0 & 11 \\ -0.0887 & 1.2812 & 3.3149 & \mathrm{C} & 0 & 0 & 0 & 0 & 0 & 0 & 0 & 0 & 0 & 12\end{array}$

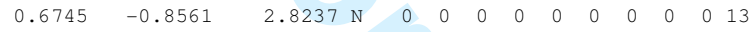

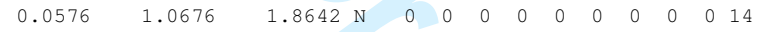

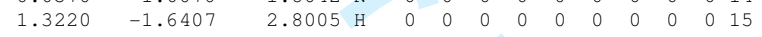

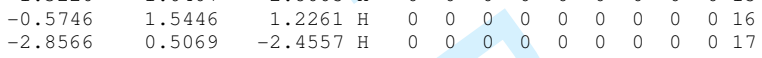

$\begin{array}{llllllllllllll}1.2640 & 0.7260 & -3.7428 & \mathrm{H} & 0 & 0 & 0 & 0 & 0 & 0 & 0 & 0 & 0 & 18\end{array}$

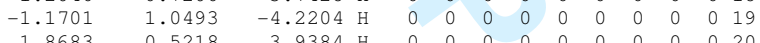

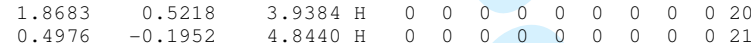

$\begin{array}{rrrrrrrrrrrrrr}1.4976 & -0.1952 & 4.8440 & \mathrm{H} & 0 & 0 & 0 & 0 & 0 & 0 & 0 & 0 & 0 & 20 \\ -1.1330 & 1.1327 & 3.6363 & \mathrm{H} & 0 & 0 & 0 & 0 & 0 & 0 & 0 & 0 & 0 & 22 \\ -1.2386 & 2.2860 & 3.6056 & \mathrm{H} & 0 & 0 & 0 & 0 & 0 & 0 & 0 & 0 & 0 & 23\end{array}$

$\begin{array}{lllllll}2 & 1 & 0 & 0 & 0 & 0 & 0\end{array}$

$\begin{array}{llllll}2 & 1 & 0 & 0 & 0 & 0 \\ 6 & 1 & 0 & 0 & 0 & 0\end{array}$

$\begin{array}{ccccccc}17 & 1 & 0 & 0 & 0 & 0\end{array}$

$\begin{array}{llllll}3 & 2 & 0 & 0 & 0 & 0\end{array}$

$\begin{array}{llllll}2 & 0 & 0 & 0 & 0\end{array}$

$\begin{array}{llllll}4 & 3 & 0 & 0 & 0 & 0\end{array}$

$\begin{array}{lllllll}5 & 4 & 0 & 0 & 0 & 0\end{array}$

$\begin{array}{lllll}4 & 0 & 0 & 0 & 0\end{array}$

$\begin{array}{llllll}8 & 5 & 0 & 0 & 0 & 0\end{array}$

$\begin{array}{llllll}19 & 6 & 0 & 0 & 0 & 0\end{array}$

$\begin{array}{rrrrrr}10 & 9 & 0 & 0 & 0 & 0 \\ 13 & 10 & 0 & 0 & 0 & 0\end{array}$

$1410 \begin{array}{llll}14 & 0 & 0 & 0\end{array}$

$\begin{array}{llllll}12 & 11 & 0 & 0 & 0 \\ 13 & 11 & 0 & 0 & 0 & 0\end{array}$

13 11 110000

$\begin{array}{llllll}20 & 11 & 0 & 0 & 0 & 0 \\ 21 & 11 & 0 & 0 & 0 & 0\end{array}$

$\begin{array}{lllllll}14 & 12 & 0 & 0 & 0 & 0\end{array}$

$\begin{array}{llllll}22 & 12 & 0 & 0 & 0 & 0 \\ 15 & 13 & 0 & 0 & 0 & 0\end{array}$

$\begin{array}{llllll}16 & 14 & 0 & 0 & 0 & 0\end{array}$

$1223 \quad 0 \quad 0 \quad 0 \quad 0$

$M \quad E N D$

clonidine1.sdf

Cosmotherm

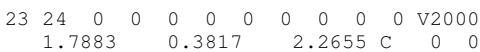

$2.2655 \mathrm{C} \quad 0$

$\begin{array}{ll}1.3620 & -0.1001\end{array}$

$-0.0036-0.3005$

$\begin{array}{lll}-0.9140 & 0.0185 & 1.7367 \\ -0.5190 & 0.4982 & 2.9833 \mathrm{C}\end{array}$

$\begin{array}{llllllllllllll}2.5959 & -0.5090 & -0.1798 & \mathrm{Cl} & 0 & 0 & 0 & 0 & 0 & 0 & 0 & 0 & 0 & 7\end{array}$

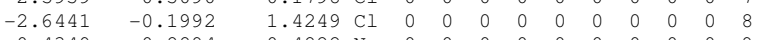

$\begin{array}{llllllllllllll}-0.4349 & -0.8894 & -0.4822 & \mathrm{~N} & 0 & 0 & 0 & 0 & 0 & 0 & 0 & 0 & 0 & 9\end{array}$

$\begin{array}{rrrrrrrrrrrrrr}-0.3698 & -0.2557 & -1.6189 & \mathrm{C} & 0 & 0 & 0 & 0 & 0 & 0 & 0 & 0 & 0 & 10 \\ -0.8202 & 0.1748 & -3.8656 & \mathrm{C} & 0 & 0 & 0 & 0 & 0 & 0 & 0 & 0 & 0 & 11\end{array}$

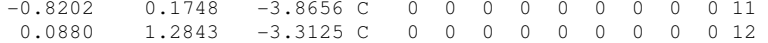

$\begin{array}{llllllllllllllll}-0.6693 & -0.8562 & -2.8251 & \mathrm{~N} & 0 & 0 & 0 & 0 & 0 & 0 & 0 & 0 & 0 & 13\end{array}$

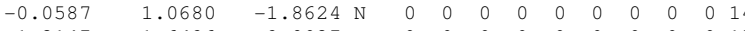

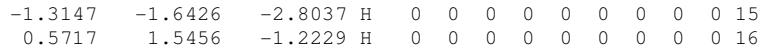

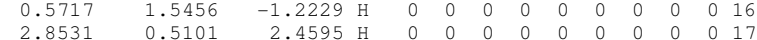

$\begin{array}{rrrrrrrrrrrrrrr}-1.2697 & 0.7276 & 3.7393 \mathrm{H} & 0 & 0 & 0 & 0 & 0 & 0 & 0 & 0 & 0 & 18\end{array}$

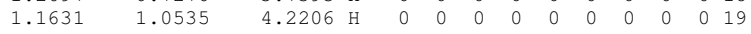

$\begin{array}{llllllllllllll}-1.8653 & 0.5190 & -3.9410 & \mathrm{H} & 0 & 0 & 0 & 0 & 0 & 0 & 0 & 0 & 0 & 20\end{array}$

$\begin{array}{llllllllllllll}-0.4903 & -0.1925 & -4.8441 & \mathrm{H} & 0 & 0 & 0 & 0 & 0 & 0 & 0 & 0 & 0 & 21\end{array}$

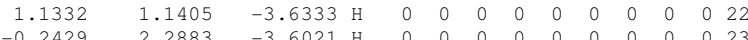

$-0.2429 \quad 2.288$

$\begin{array}{lllllll}2 & 1 & 0 & 0 & 0 & 0 \\ 6 & 1 & 0 & 0 & 0 & 0\end{array}$

$\begin{array}{lllllll}7 & 1 & 0 & 0 & 0 & 0\end{array}$

$\begin{array}{llllll}3 & 2 & 0 & 0 & 0 & 0\end{array}$

$\begin{array}{lllll}2 & 0 & 0 & 0 & 0 \\ 3 & 0 & 0 & 0 & 0\end{array}$

$\begin{array}{lllll}3 & 0 & 0 & 0 & 0\end{array}$

$\begin{array}{lllll}4 & 0 & 0 & 0 & 0\end{array}$

$\begin{array}{llllll}6 & 5 & 0 & 0 & 0 & 0\end{array}$

$\begin{array}{llllll}19 & 6 & 0 & 0 & 0 & 0\end{array}$

$\begin{array}{llllll}10 & 9 & 0 & 0 & 0 & 0\end{array}$ 
$1310 \quad 000000$

$\begin{array}{llllll}14 & 10 & 0 & 0 & 0 & 0\end{array}$

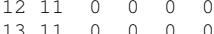

$\begin{array}{llllll}20 & 11 & 0 & 0 & 0 & 0 \\ 21 & 11 & 0 & 0 & 0 & 0\end{array}$

$\begin{array}{llllll}21 & 11 & 0 & 0 & 0 & 0 \\ 14 & 12 & 0 & 0 & 0 & 0\end{array}$

$2212 \quad 00000$

$\begin{array}{llllll}15 & 13 & 0 & 0 & 0 & 0\end{array}$

$\begin{array}{lllllll}16 & 14 & 0 & 0 & 0 & 0 \\ 12 & 23 & 0 & 0 & 0 & 0\end{array}$

$M \quad$ END

$\$ \$ \$$

lonidine_p1.sdf

3D

$\begin{array}{llllllllllll}24 & 25 & 0 & 0 & 0 & 0 & 0 & 0 & 0 & 0 & 0 & \mathrm{~V} 2000\end{array}$

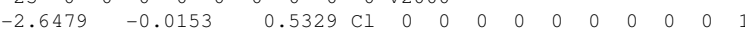

$\begin{array}{lllllllllllll}-0.6424 & -3.2432 & -0.7885 & \mathrm{C} & 0 & 0 & 0 & 0 & 0 & 0 & 0 & 0 & 0\end{array}$

$\begin{array}{lllllllllllllll}-1.6704 & -2.3535 & -0.4699 & \mathrm{C} & 0 & 0 & 0 & 0 & 0 & 0 & 0 & 0 & 0 & 3\end{array}$

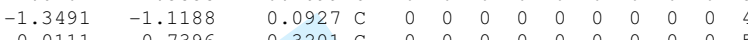

$\begin{array}{llllllllllllll}-0.0111 & -0.7396 & 0.3201 & \mathrm{C} & 0 & 0 & 0 & 0 & 0 & 0 & 0 & 0 & 0 & 5 \\ 0.9978 & -1.6653 & -0.0103 & \mathrm{C} & 0 & 0 & 0 & 0 & 0 & 0 & 0 & 0 & 0 & 6\end{array}$

$\begin{array}{lllllllllllllll}0.6943 & -2.9092 & -0.5615 & \mathrm{C} & 0 & 0 & 0 & 0 & 0 & 0 & 0 & 0 & 0 & 7\end{array}$

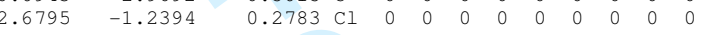

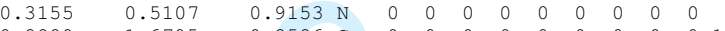

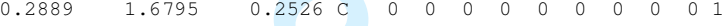

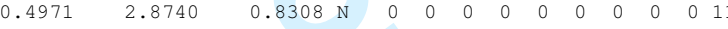

$\begin{array}{lllllllllllll}0.0619 & 1.8106 & -1.0634 \mathrm{~N} & 0 & 0 & 0 & 0 & 0 & 0 & 0 & 0 & 0 & 12\end{array}$

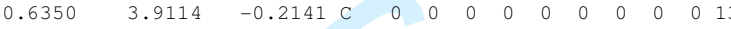

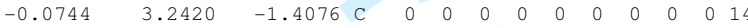

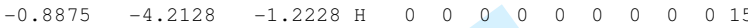

$\begin{array}{llllllllllllll}-2.7128 & -2.6205 & -0.6381 & \mathrm{H} & 0 & 0 & 0 & 0 & 0 & 0 & 0 & 0 & 0 & 16\end{array}$

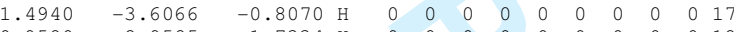

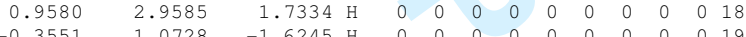

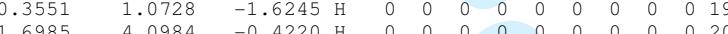

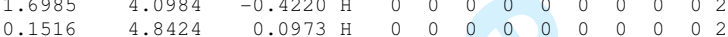

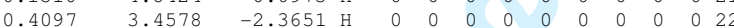

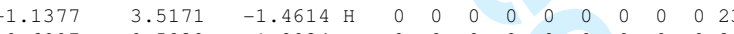

0.5239

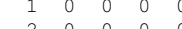

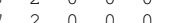

$\begin{array}{llllllll}7 & 2 & 0 & 0 & 0 & 0\end{array}$

$\begin{array}{lllllllll}3 & 0 & 0 & 0 & 0\end{array}$

$\begin{array}{lllll}3 & 0 & 0 & 0 & 0 \\ 5 & 4 & 0 & 0 & 0\end{array}$

$\begin{array}{lllllll}5 & 0 & 0 & 0 & 0\end{array}$

$\begin{array}{lllll}5 & 0 & 0 & 0\end{array}$

$8 \begin{array}{lllllll}7 & 6 & 0 & 0 & 0 & 0\end{array}$

$\begin{array}{llllll}7 & 0 & 0 & 0 & 0 & 0\end{array}$

$\begin{array}{llllll}1 & 0 & 0 & 0 & 0 & 2 \\ 1 & 10 & 0 & 0 & 0 & 0\end{array}$

$\begin{array}{lllllllll}1 & 10 & 0 & 0 & 0 & 0\end{array}$

10 r 00 r 0

$\begin{array}{llllllll}11 & 0 & 0 & 0 & 0 & 1 \\ 11 & 0 & 0 & 0 & 0\end{array}$

$1412 \quad 0 \quad 000$

$\begin{array}{lllllllll}9 & 12 & 0 & 0 & 0\end{array}$

$\begin{array}{lllllll}14 & 13 & 0 & 0 & 0 & 0\end{array}$

$\begin{array}{lllllll}13 & 0 & 0 & 0 & 0\end{array}$

$2 \begin{array}{llllll}13 & 0 & 0 & 0 & 0 \\ 2 & 14 & 0 & 0 & 0 & 0\end{array}$

$\begin{array}{llllll}23 & 14 & 0 & 0 & 0 & 0\end{array}$

$\$ \$ \$$

lonidine_t_p3.sdf

cosmotherm

$\begin{array}{llllllllllll}24 & 25 & 0 & 0 & 0 & 0 & 0 & 0 & 0 & 0 & 0 & \mathrm{~V} 2000\end{array}$

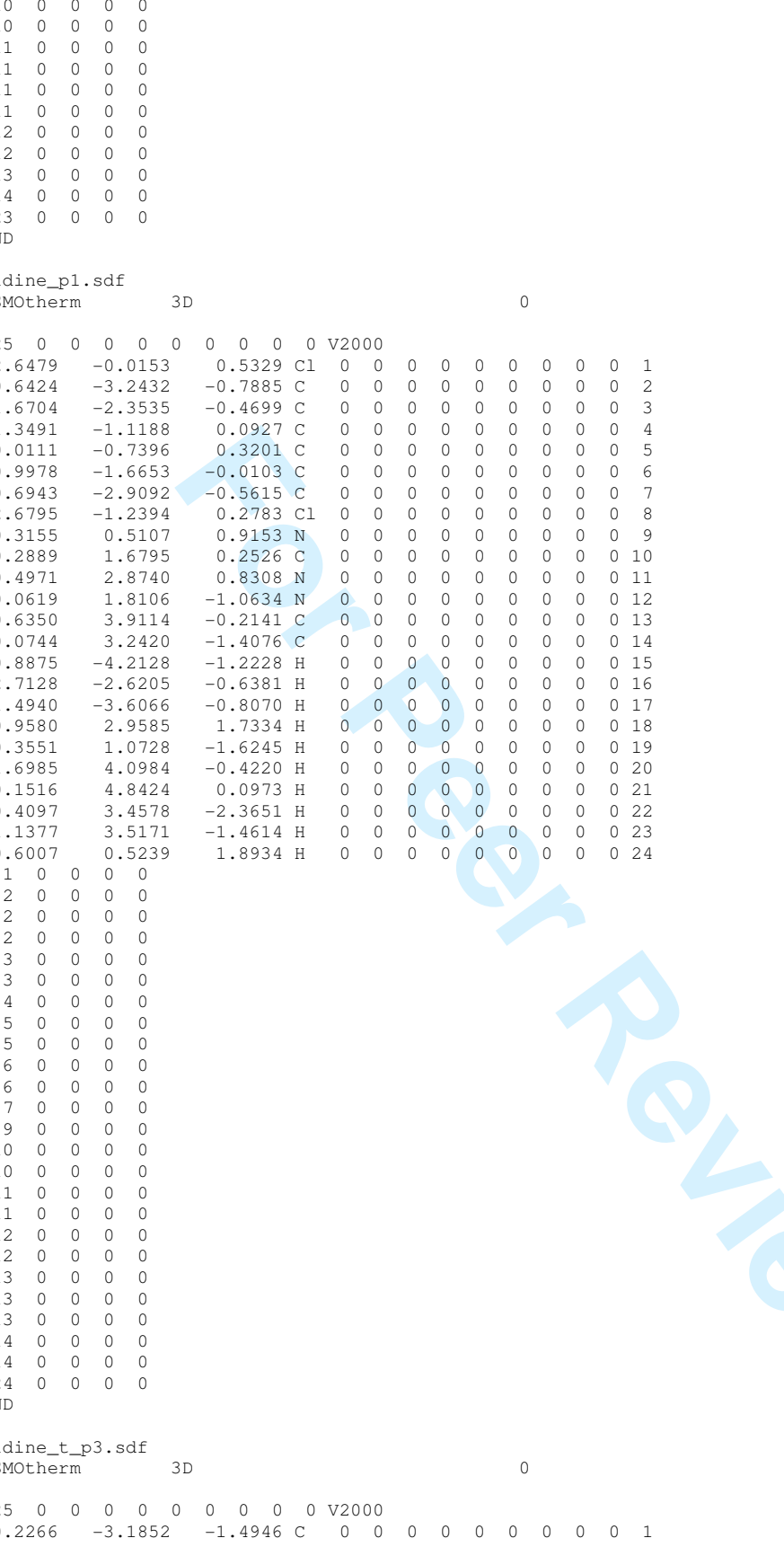

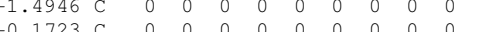

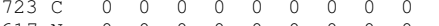

$\begin{array}{llllllllllllll}-0.2840 & 3.2789 & -0.8267 \mathrm{C} & 0 & 0 & 0 & 0 & 0 & 0 & 0 & 0 & 0 & 0\end{array}$

$\begin{array}{rrrrrrrrrrrrrr}.9919 & 2.7821 & -0.5519 & \mathrm{C} & 0 & 0 & 0 & 0 & 0 & 0 & 0 & 0 & 0 & 6 \\ 1.1166 & 1.5200 & 0.0275 & \mathrm{C} & 0 & 0 & 0 & 0 & 0 & 0 & 0 & 0 & 0 & 7\end{array}$

$\begin{array}{llllllllllllll}-0.0121 & 0.7353 & 0.3342 & \mathrm{C} & 0 & 0 & 0 & 0 & 0 & 0 & 0 & 0 & 0 & 8\end{array}$

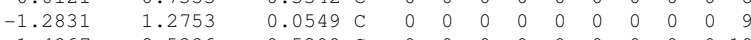

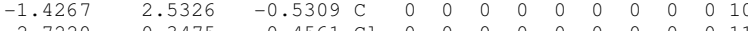

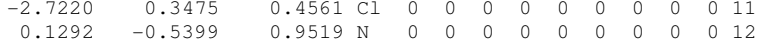

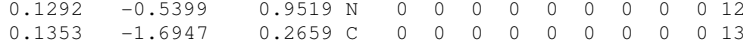

$\begin{array}{llllllllllllll}0.3141 & -2.9015 & 0.8280 & \mathrm{~N} & 0 & 0 & 0 & 0 & 0 & 0 & 0 & 0 & 0 & 14\end{array}$

$\begin{array}{llllllllllllll}-0.4884 & -3.4922 & -2.2642 & \mathrm{H} & 0 & 0 & 0 & 0 & 0 & 0 & 0 & 0 & 0 & 1\end{array}$

$\begin{array}{llllllllllllll}1.2503 & -3.2700 & -1.8869 & \mathrm{H} & 0 & 0 & 0 & 0 & 0 & 0 & 0 & 0 & 0 & 16\end{array}$

$\begin{array}{llllllllllllll}0.7627 & -4.7818 & -0.0657 & \mathrm{H} & 0 & 0 & 0 & 0 & 0 & 0 & 0 & 0 & 0 & 17\end{array}$

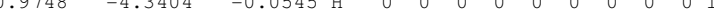

$\begin{array}{llllllllllllll}0.0945 & -1.0027 & -1.6856 & \mathrm{H} & 0 & 0 & 0 & 0 & 0 & 0 & 0 & 0 & 0 & 19\end{array}$

$\begin{array}{lllllllllllllll}0.3894 & 4.2648 & -1.2802 & \mathrm{H} & 0 & 0 & 0 & 0 & 0 & 0 & 0 & 0 & 0 & 20\end{array}$

$\begin{array}{llllllllllllll}1.8812 & 3.3682 & -0.7795 & \mathrm{H} & 0 & 0 & 0 & 0 & 0 & 0 & 0 & 0 & 0 & 21\end{array}$

$\begin{array}{llllllllllllll}-2.4205 & 2.9261 & -0.7398 & \mathrm{H} & 0 & 0 & 0 & 0 & 0 & 0 & 0 & 0 & 0 & 22\end{array}$ $-0.1615-3.0559$

$\begin{array}{llllll}2 & 1 & 0 & 0 & 0 & 0 \\ 3 & 1 & 0 & 0 & 0 & 0\end{array}$

$\begin{array}{lllllll}5 & 1 & 0 & 0 & 0 & 0\end{array}$

$16 \quad 1 \quad 000100$

$\begin{array}{llllll}4 & 2 & 0 & 0 & 0\end{array}$

$\begin{array}{lllll}2 & 0 & 0 & 0 & 0\end{array}$

$\begin{array}{llllllllll}1 & 3 & 3 & 0 & 0 & 0 & 0 & 0\end{array}$ 


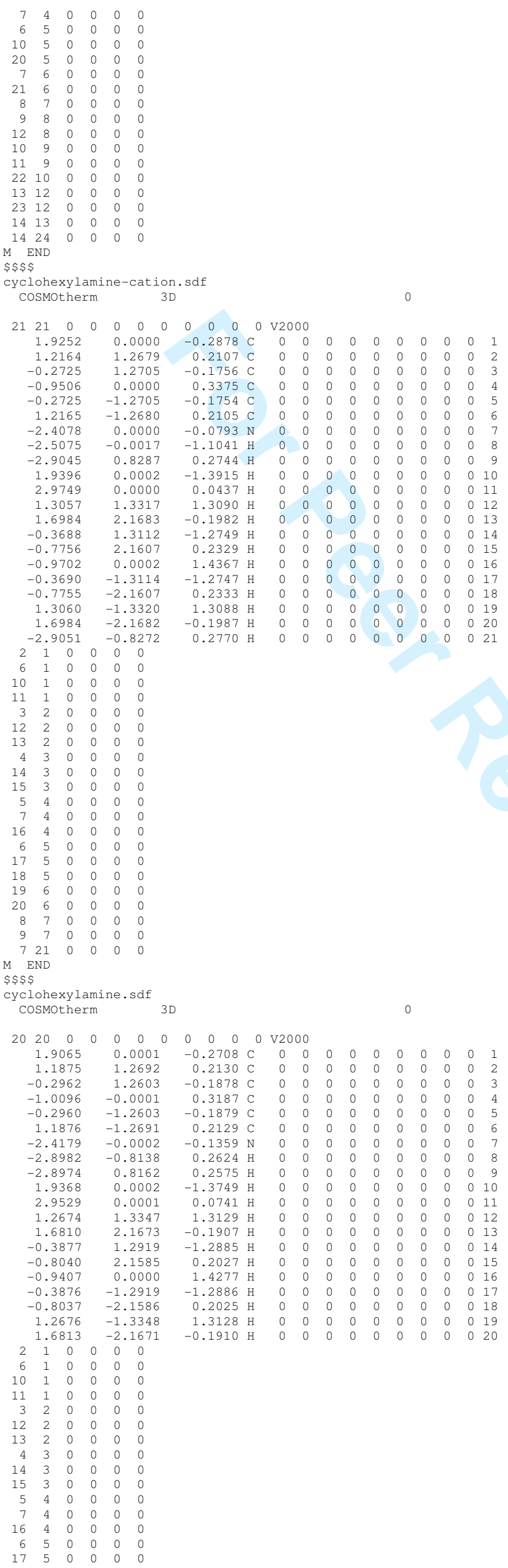


$\begin{array}{rrrrrr}6 & 5 & 0 & 0 & 0 & 0 \\ 8 & 5 & 0 & 0 & 0 & 0 \\ 17 & 6 & 0 & 0 & 0 & 0 \\ 10 & 7 & 0 & 0 & 0 & 0 \\ 18 & 7 & 0 & 0 & 0 & 0 \\ 19 & 7 & 0 & 0 & 0 & 0 \\ 9 & 8 & 0 & 0 & 0 & 0 \\ 20 & 8 & 0 & 0 & 0 & 0 \\ 21 & 8 & 0 & 0 & 0 & 0 \\ 10 & 9 & 0 & 0 & 0 & 0 \\ 22 & 9 & 0 & 0 & 0 & 0 \\ 23 & 9 & 0 & 0 & 0 & 0 \\ 11 & 10 & 0 & 0 & 0 & 0 \\ 12 & 11 & 0 & 0 & 0 & 0 \\ 13 & 11 & 0 & 0 & 0 & 0 \\ 24 & 12 & 0 & 0 & 0 & 0 \\ 25 & 12 & 0 & 0 & 0 & 0 \\ 13 & 26 & 0 & 0 & 0 & 0 \\ 1 & 1 & & & & \end{array}$ $M \quad E N D$

$\$ \$ \$ \$$

debrisoquine_a_p2.sdf
Cosmotherm

$\begin{array}{cccccccccccc}27 & 28 & 0 & 0 & 0 & 0 & 0 & 0 & 0 & 0 & 0 & \mathrm{~V} 2000\end{array}$

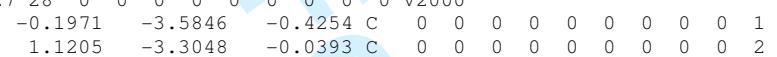

$\begin{array}{rrrrrrrrrrrrrr}1.1205 & -3.3048 & -0.0393 & \mathrm{C} & 0 & 0 & 0 & 0 & 0 & 0 & 0 & 0 & 0 & 2 \\ 1.4740 & -2.0019 & 0.3156 & \mathrm{C} & 0 & 0 & 0 & 0 & 0 & 0 & 0 & 0 & 0 & 3\end{array}$

$\begin{array}{llllllllllllll}0.5245 & -0.9679 & 0.2854 & \mathrm{C} & 0 & 0 & 0 & 0 & 0 & 0 & 0 & 0 & 0 & 4\end{array}$

$\begin{array}{llllllllllllll}-0.7999 & -1.2433 & -0.0981 & \mathrm{C} & 0 & 0 & 0 & 0 & 0 & 0 & 0 & 0 & 0 & 5 \\ -1.1436 & -2.5588 & -0.4542 & \mathrm{C} & 0 & 0 & 0 & 0 & 0 & 0 & 0 & 0 & 0 & 6\end{array}$

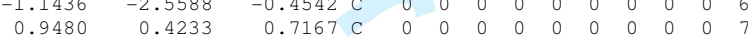

$\begin{array}{rrrrrrrrrrrrrr}-1.8334 & -0.1388 & -0.1458 & \mathrm{C} & 0 & 0 & 0 & 0 & 0 & 0 & 0 & 0 & 0 & 8 \\ -1.4179 & 1.0861 & 0.6726 & \mathrm{C} & 0 & 0 & 0 & 0 & 0 & 0 & 0 & 0 & 0 & 9\end{array}$

$\begin{array}{llllllllllllll}-1.4176 & 1.4515 & 0.3330 & \mathrm{~N} & 0 & 0 & 0 & 0 & 0 & 0 & 0 & 0 & 0 & 10\end{array}$

$\begin{array}{llllllllllllll}0.3177 & 2.6416 & -0.1852 & \mathrm{C} & 0 & 0 & 0 & 0 & 0 & 0 & 0 & 0 & 0 & 11 \\ -0.5515 & 3.6685 & -0.1955 & \mathrm{~N} & 0 & 0 & 0 & 0 & 0 & 0 & 0 & 0 & 0 & 12\end{array}$

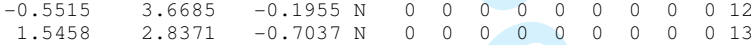

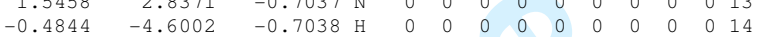

$\begin{array}{llllllllllllll}1.8687 & -4.0990 & -0.0152 & \mathrm{H} & 0 & 0 & 0 & 0 & 0 & 0 & 0 & 0 & 0 & 15\end{array}$

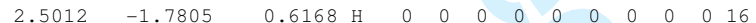

$\begin{array}{rrrrrrrrrrrrrr}1.868 & -1.7805 & 0.6168 & \mathrm{H} & 0 & 0 & 0 & 0 & 0 & 0 & 0 & 0 & 0 & 15 \\ -2.1723 & -2.7767 & -0.7521 & \mathrm{H} & 0 & 0 & 0 & 0 & 0 & 0 & 0 & 0 & 0 & 16\end{array}$

$\begin{array}{llllllllllllllllll}1.9338 & 0.6606 & 0.3049 \mathrm{H} & 0 & 0 & 0 & 0 & 0 & 0 & 0 & 0 & 0 & 18\end{array}$

$\begin{array}{lllllllllllllllllllllll}1.9338 & 0.6606 & 0.3049 & \mathrm{H} & 0 & 0 & 0 & 0 & 0 & 0 & 0 & 0 & 0 & 18\end{array}$

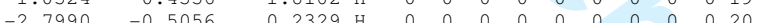

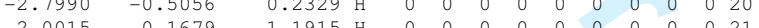

$\begin{array}{llllllllllllllllllll}-2.015 & 0.1679 & -1.1915 & \mathrm{H} & 0 & 0 & 0 & 0 & 0 & 0 & 0 & 0 & 0 & 0 & 21 \\ -1.4601 & 0.8746 & 1.7524 & \mathrm{H} & 0 & 0 & 0 & 0 & 0 & 0 & 0 & 0 & 0 & 22\end{array}$

$\begin{array}{llllllllllllllll}-1.1601-0.8746 & 1.7524 \mathrm{H} & 0 & 0 & 0 & 0 & 0 & 0 & 0 & 0 & 0 & 22\end{array}$

$\begin{array}{lllllllllllllllllllllll}-2.0952 & 1.9189 & 0.4648 & 1 & 0 & 0 & 0 & 0 & 0 & 0 & 0 & 0 & 0 & 23\end{array}$

$\begin{array}{lllllllllllllllllll}-0.3021 & 4.5281 & -0.6742 & \mathrm{H} & 0 & 0 & 0 & 0 & 0 & 0 & 0 & 0 & 0 & 0 & 24 \\ -1.2941 & 3.7348 & 0.4923 & \mathrm{H} & 0 & 0 & 0 & 0 & 0 & 0 & 0 & 0 & 0 & 25\end{array}$

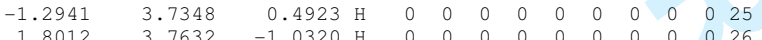

$\begin{array}{llllllllllllll}1.8012 & 3.7632 & -1.0322 & \mathrm{H} & 0 & 0 & 0 & 0 & 0 & 0 & 0 & 0 & 0 & 26 \\ 2.0735 & 2.0655 & -1.0977 & \mathrm{H} & 0 & 0 & 0 & 0 & 0 & 0 & 0 & 0 & 0 & 27\end{array}$

$\begin{array}{llllll}2 & 1 & 0 & 0 & 0 & 0\end{array}$

$\begin{array}{rrrrrr}6 & 1 & 0 & 0 & 0 & 0 \\ 14 & 1 & 0 & 0 & 0 & 0 \\ 3 & 2 & 0 & 0 & 0 & 0\end{array}$

$\begin{array}{llllll}3 & 2 & 0 & 0 & 0\end{array}$

$\begin{array}{llllll}5 & 2 & 0 & 0 & 0 & 0 \\ 4 & 3 & 0 & 0 & 0 & 0\end{array}$

$\begin{array}{llllllll}4 & 3 & 0 & 0 & 0 & 0 & 0 \\ 6 & 3 & 0 & 0 & 0\end{array}$

$\begin{array}{llllll}5 & 4 & 0 & 0 & 0 \\ 7 & 4 & 0 & 0 & 0\end{array}$

$\begin{array}{cccccc}5 & 0 & 0 & 0 & 0\end{array}$

$\begin{array}{lllll}5 & 0 & 0 & 0\end{array}$

$\begin{array}{lllllll}8 & 5 & 0 & 0 & 0 & 0 \\ 17 & 6 & 0 & 0 & 0 & 0 & 0 \\ 10 & 7 & 0 & 0 & 0 & 0\end{array}$

$\begin{array}{lllllll}8 & 7 & 0 & 0 & 0 & 0\end{array}$

$\begin{array}{llllll}9 & 7 & 0 & 0 & 0 & 0\end{array}$

$\begin{array}{rrrrrrr}9 & 8 & 0 & 0 & 0 & 0 \\ 20 & 8 & 0 & 0 & 0 & 0\end{array}$

$\begin{array}{lllllll}21 & 8 & 0 & 0 & 0 & 0\end{array}$

$\begin{array}{llllll}0 & 9 & 0 & 0 & 0 & 0\end{array}$

$\begin{array}{llllll}23 & 9 & 0 & 0 & 0 & 0\end{array}$

$\begin{array}{llllll}1 & 10 & 0 & 0 & 0\end{array}$

$\begin{array}{lllll}2 & 11 & 0 & 0 & 0\end{array}$

$\begin{array}{llllll}13 & 11 & 0 & 0 & 0 & 0 \\ 24 & 12 & 0 & 0 & 0 & 0\end{array}$

$\begin{array}{lllllllll}2 & 12 & 0 & 0 & 0 & 0\end{array}$

$\begin{array}{llllll}26 & 13 & 0 & 0 & 0 & 0\end{array}$

$\begin{array}{llllll}3 & 27 & 0 & 0 & 0 & 0\end{array}$

END

debrisoquine_p2.sdf

$\begin{array}{llllllllllll}27 & 28 & 0 & 0 & 0 & 0 & 0 & 0 & 0 & 0 & 0 & v 2000\end{array}$

\begin{tabular}{|c|c|c|c|c|c|c|c|c|c|c|c|c|}
\hline & $-2.5 / 1 /$ & 0.3303 & c & 0 & 0 & 0 & 0 & 0 & 0 & 0 & 0 & 0 \\
\hline-0.7 & -1.2492 & 0.0383 & C & 0 & 0 & 0 & 0 & 0 & 0 & 0 & 0 & 0 \\
\hline & -0.9637 & -0.1920 & C & 0 & 0 & 0 & 0 & 0 & 0 & 0 & 0 & 0 \\
\hline 1.5156 & -1.9977 & -0.1353 & C & & 0 & 0 & 0 & 0 & 0 & 0 & 0 & 0 \\
\hline $1.12 \mathrm{o}$ & -3.3068 & 0.1563 & $\mathrm{C}$ & & 0 & 0 & 0 & 0 & 0 & 0 & 0 & 0 \\
\hline-0.22 & -3.5 & 0.3922 & C & & 0 & 0 & 0 & 0 & 0 & 0 & 0 & 0 \\
\hline-1.83 & -0 & -0.0 & C & & & 0 & 0 & 0 & 0 & 0 & 0 & 0 \\
\hline 1.3 & & -0.8414 & C & & 0 & 0 & 0 & 0 & 0 & 0 & 0 & 0 \\
\hline 1.059 & 0.4393 & -0.5108 & C & & & 0 & 0 & 0 & 0 & 0 & 0 & 0 \\
\hline 0.0 & & -0 . & $\mathrm{N}$ & & 0 & 0 & 0 & 0 & 0 & 0 & 0 & 0 \\
\hline 0.25 & 2.6597 & 0.1798 & C & & & 0 & 0 & 0 & 0 & 0 & 0 & \\
\hline 0.7 & & & $\mathrm{~N}$ & & 0 & 0 & 0 & 0 & 0 & 0 & & \\
\hline 1.5229 & 3.0846 & 0.3381 & $\mathrm{~N}$ & & & 0 & 0 & 0 & 0 & 0 & 0 & \\
\hline-2.2 & & & $\mathrm{H}$ & & & 0 & & & & & & \\
\hline 2.5692 & -1.7706 & -0.3177 & $\mathrm{H}$ & & & 0 & 0 & 0 & 0 & 0 & 0 & \\
\hline & -4.0 & & & & & 0 & & & & 0 & & \\
\hline 0.5342 & -4.6161 & 0.6192 & $\mathrm{H}$ & & & 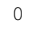 & 0 & ( & 0 & 0 & 0 & 0 \\
\hline & & 9867 & & & & 0 & 0 & 0 & 0 & 0 & 0 & \\
\hline & -0.5298 & 0.4915 & & 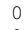 & & & 0 & 0 & 0 & 0 & 0 & \\
\hline 006 & 1.885 & & & & & & & & & & & \\
\hline
\end{tabular}

URL: http://mc.manuscriptcentral.com/tandf/tmph 


\begin{tabular}{|c|c|c|c|c|c|c|c|c|c|}
\hline-1.1900 & 0.7504 & -1.8978 & $\mathrm{H}$ & 0 & 0 & 0 & 0 & & 0 \\
\hline 1.4461 & 0.4776 & -1.5442 & $\mathrm{H}$ & 0 & 0 & 0 & 0 & & 0 \\
\hline 1.8906 & 0.6836 & 0.1636 & $\mathrm{H}$ & 0 & 0 & 0 & 0 & & 0 \\
\hline-0.5419 & 4.4119 & 0.8688 & $\mathrm{H}$ & 0 & 0 & 0 & 0 & & 0 \\
\hline-1.6464 & 3.0903 & 0.8449 & $\mathrm{H}$ & 0 & 0 & 0 & 0 & & 0 \\
\hline 1.7009 & 3.9592 & 0.8220 & $\mathrm{H}$ & 0 & 0 & 0 & 0 & & 0 \\
\hline 2.2677 & 2.7401 & -0.2589 & & 0 & & 0 & 0 & & 0 \\
\hline
\end{tabular}




\begin{tabular}{|c|c|c|c|c|c|c|c|c|c|c|c|}
\hline 2.5079 & 0.0022 & -0.3357 & C & 0 & 0 & 0 & 0 & 0 & 0 & 0 & 0 \\
\hline 2.6500 & -1.4534 & 0.1342 & C & 0 & 0 & 0 & 0 & 0 & 0 & 0 & 0 \\
\hline 1.2229 & 0.6590 & 0.1920 & C & 0 & 0 & 0 & 0 & 0 & 0 & 0 & 0 \\
\hline 0.0000 & -0.0057 & -0.2836 & $\mathrm{~N}$ & 0 & 0 & 0 & 0 & 0 & 0 & 0 & 0 \\
\hline-1.2231 & 0.6594 & 0.1907 & $\mathrm{C}$ & 0 & 0 & 0 & 0 & 0 & 0 & 0 & 0 \\
\hline-2.5080 & 0.0015 & -0.3361 & $C$ & 0 & 0 & 0 & 0 & 0 & 0 & 0 & 0 \\
\hline-3.7305 & 0.8320 & 0.0817 & $\mathrm{C}$ & 0 & 0 & 0 & 0 & 0 & 0 & 0 & 0 \\
\hline-2.6497 & -1.4532 & 0.1367 & C & 0 & 0 & 0 & 0 & 0 & 0 & 0 & 0 \\
\hline 4.6570 & 0.4016 & -0.3251 & $\mathrm{H}$ & 0 & 0 & 0 & 0 & 0 & 0 & 0 & 0 \\
\hline 3.6535 & 1.8704 & -0.2743 & $\mathrm{H}$ & 0 & 0 & 0 & 0 & 0 & 0 & 0 & 0 \\
\hline 3.8296 & 0.8591 & 1.1803 & $\mathrm{H}$ & 0 & 0 & 0 & 0 & 0 & 0 & 0 & 0 \\
\hline 2.4470 & 0.0057 & -1.4386 & $\mathrm{H}$ & 0 & 0 & 0 & 0 & 0 & 0 & 0 & 0 \\
\hline 3.5914 & -1.8915 & -0.2303 & $\mathrm{H}$ & 0 & 0 & 0 & 0 & 0 & 0 & 0 & 0 \\
\hline 1.8323 & -2.0915 & -0.2333 & $\mathrm{H}$ & 0 & 0 & 0 & 0 & 0 & 0 & 0 & 0 \\
\hline 2.6578 & -1.5089 & 1.2356 & $\mathrm{H}$ & 0 & 0 & 0 & 0 & 0 & 0 & 0 & 0 \\
\hline 1.1867 & 1.7048 & -0.1564 & $\mathrm{H}$ & 0 & 0 & 0 & 0 & 0 & 0 & 0 & 0 \\
\hline 1.2712 & 0.6983 & 1.3042 & $\mathrm{H}$ & 0 & 0 & 0 & 0 & 0 & 0 & 0 & 0 \\
\hline-0.0004 & -0.9664 & 0.0694 & $\mathrm{H}$ & 0 & 0 & 0 & 0 & 0 & 0 & 0 & 0 \\
\hline-1.1872 & 1.7046 & -0.1593 & $\mathrm{H}$ & 0 & 0 & 0 & 0 & 0 & 0 & 0 & 0 \\
\hline-1.2716 & 0.7005 & 1.3029 & $\mathrm{H}$ & 0 & 0 & 0 & 0 & 0 & 0 & 0 & 0 \\
\hline-2.4472 & 0.0028 & -1.4390 & $\mathrm{H}$ & 0 & 0 & 0 & 0 & 0 & 0 & 0 & 0 \\
\hline-4.6582 & 0.3953 & -0.3170 & $\mathrm{H}$ & 0 & 0 & 0 & 0 & 0 & 0 & 0 & 0 \\
\hline-3.6582 & 1.8671 & -0.2855 & & 0 & 0 & 0 & 0 & 0 & 0 & 0 & 0 \\
\hline-3.8235 & 0.8690 & 1.1794 & $\mathrm{H}$ & 0 & 0 & 0 & 0 & 0 & 0 & 0 & 0 \\
\hline-3.5900 & -1.8929 & -0.2286 & $\mathrm{H}$ & 0 & 0 & 0 & 0 & 0 & 0 & 0 & 0 \\
\hline-1.8306 & -2.0912 & -0.2279 & & 0 & 0 & 0 & 0 & & 0 & 0 & \\
\hline & -1.5066 & 1.2381 & & & & & & & & & \\
\hline
\end{tabular}




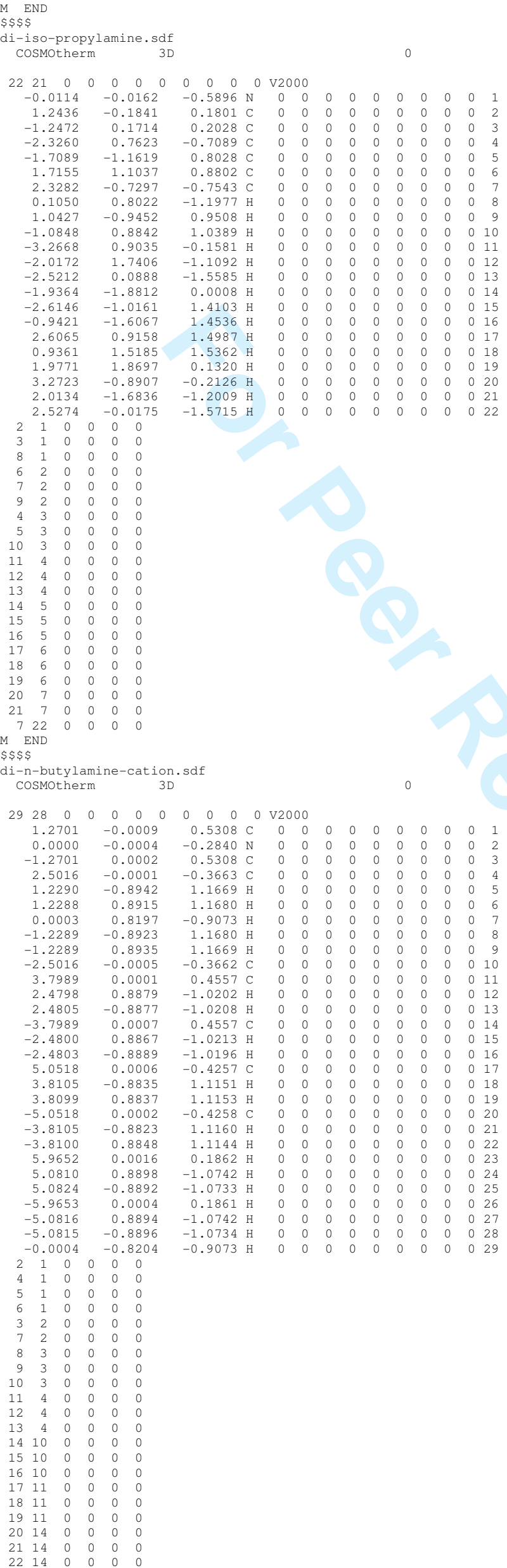


$\begin{array}{llllll}10 & 3 & 0 & 0 & 0 & 0\end{array}$

$\begin{array}{llllll}11 & 4 & 0 & 0 & 0 & 0 \\ 12 & 4 & 0 & 0 & 0 & 0\end{array}$

$\begin{array}{llllll}13 & 4 & 0 & 0 & 0 & 0\end{array}$

$\begin{array}{llllll}14 & 10 & 0 & 0 & 0 & 0 \\ 15 & 10 & 0 & 0 & 0 & 0\end{array}$

$\begin{array}{llllll}16 & 10 & 0 & 0 & 0 & 0\end{array}$

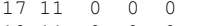

18111000000

$2014 \quad 0 \quad 0000$

$\begin{array}{lllllll}21 & 14 & 0 & 0 & 0 & 0\end{array}$

$22 \quad 14 \quad 0000000$

$M \quad$ END

$\$ \$ \$ \$$

di-n-propylamine.sdf

$\begin{array}{llllllllllll}22 & 21 & 0 & 0 & 0 & 0 & 0 & 0 & 0 & 0 & 0 & \mathrm{~V} 2000\end{array}$

$\begin{array}{lllllllllllll}1.2255 & 0.0141 & -0.3703 & \mathrm{C} & 0 & 0 & 0 & 0 & 0 & 0 & 0 & 0 & 0\end{array}$

$\begin{array}{lllllllllllll}0.0000 & 0.0586 & 0.4414 & \mathrm{~N} & 0 & 0 & 0 & 0 & 0 & 0 & 0 & 0 & 0\end{array}$

$\begin{array}{llllllllllllllll}-1.2255 & 0.0134 & -0.3703 & \mathrm{C} & 0 & 0 & 0 & 0 & 0 & 0 & 0 & 0 & 0 & 3\end{array}$

$\begin{array}{lllllllllllll}2.4820 & 0.0181 & 0.5018 & \mathrm{C} & 0 & 0 & 0 & 0 & 0 & 0 & 0 & 0 & 0\end{array}$

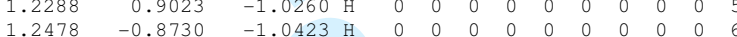

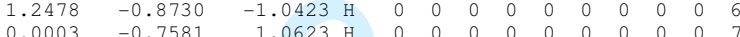

$\begin{array}{rrrrrrrrrrrrr}0.0003 & -0.7581 & 1.0623 & \mathrm{H} & 0 & 0 & 0 & 0 & 0 & 0 & 0 & 0 & 0\end{array}$

$\begin{array}{llllllllllllll}-1.2295 & 0.9018 & -1.0256 & \mathrm{H} & 0 & 0 & 0 & 0 & 0 & 0 & 0 & 0 & 0 & 8\end{array}$

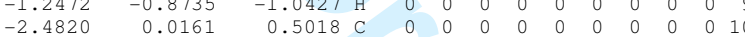

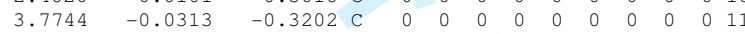

$\begin{array}{llllllllllllll}2.4468 & -0.8483 & 1.1853 \mathrm{H} & 0 & 0 & 0 & 0 & 0 & 0 & 0 & 0 & 0 & 12\end{array}$

$\begin{array}{llllllllllllll}2.4713 & 0.9194 & 1.1363 & \mathrm{H} & 0 & 0 & 0 & 0 & 0 & 0 & 0 & 0 & 0 & 13\end{array}$

$\begin{array}{llllllllllllll}-3.7744 & -0.0299 & -0.3203 & \mathrm{C} & 0 & 0 & 0 & 0 & 0 & 0 & 0 & 0 & 0 & 14 \\ -2.4475 & -0.8518 & 1.1834 & \mathrm{H} & 0 & 0 & 0 & 0 & 0 & 0 & 0 & 0 & 0 & 15\end{array}$

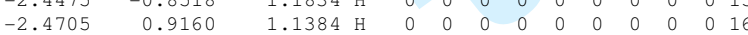

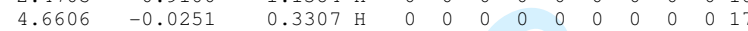

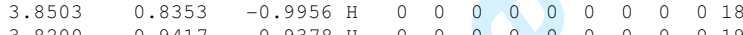

$\begin{array}{lllllllllllllll}3.8200 & -0.9417 & -0.99378 \mathrm{H} & 0 & 0 & 0 & 0 & 0 & 0 & 0 & 0 & 0 & 0 & 18\end{array}$

$\begin{array}{rrrrrrrrrrrrrr}-4.6606 & -0.0258 & 0.3308 & \mathrm{H} & 0 & 0 & 0 & 0 & 0 & 0 & 0 & 0 & 0 & 20 \\ -3.8501 & 0.8392 & -0.9924 & \mathrm{H} & 0 & 0 & 0 & 0 & 0 & 0 & 0 & 0 & 0 & 21\end{array}$

$\begin{array}{llllllllllllll}-3.8204 & -0.9380 & -0.9412 & \mathrm{H} & 0 & 0 & 0 & 0 & 0 & 0 & 0 & 0 & 0 & 22\end{array}$

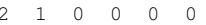

$\begin{array}{llllll}4 & 1 & 0 & 0 & 0 & 0\end{array}$

$\begin{array}{lllll}1 & 0 & 0 & 0 & 0\end{array}$

$\begin{array}{lllll}1 & 0 & 0 & 0 & 0 \\ 2 & 0 & 0 & 0 & 0\end{array}$

$\begin{array}{lllll}7 & 0 & 0 & 0\end{array}$

$\begin{array}{lllll}3 & 0 & 0 & 0\end{array}$

$\begin{array}{lllllll}0 & 3 & 0 & 0 & 0 & 0\end{array}$

$\begin{array}{llllll}1 & 4 & 0 & 0 & 0 & 0\end{array}$

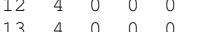

$\begin{array}{lllllll}14 & 4 & 0 & 0 & 0 & 0\end{array}$

$\begin{array}{llllll}15 & 10 & 0 & 0 & 0\end{array}$

$\begin{array}{lllll}0 & 0 & 0 & 0 \\ 0 & 0 & 0\end{array}$

$\begin{array}{llllll}20 & 14 & 0 & 0 & 0 & 0\end{array}$

$\begin{array}{llllll}21 & 14 & 0 & 0 & 0 & 0\end{array}$

1422

\$\$\$

di-sec-butylamine-cation.sdf

\begin{tabular}{|c|c|c|c|c|c|c|c|c|c|c|c|c|c|}
\hline $\begin{array}{lll}29 & 28 & 0\end{array}$ & $\begin{array}{lll}0 & 0 & 0\end{array}$ & $\begin{array}{lll}0 & 0 & 0\end{array}$ & 0 & $\mathrm{~V} 20 \mathrm{C}$ & & & & & & & & & \\
\hline-0.0212 & 0.6220 & -0.5114 & $\mathrm{~N}$ & 0 & 0 & 0 & 0 & 0 & 0 & 0 & 0 & 0 & 1 \\
\hline 1.3787 & 0.4070 & 0.0707 & $\mathrm{C}$ & 0 & 0 & 0 & 0 & 0 & 0 & 0 & 0 & 0 & 2 \\
\hline-1.1996 & -0.0229 & 0.2194 & $\mathrm{C}$ & 0 & 0 & 0 & 0 & 0 & 0 & 0 & 0 & 0 & 3 \\
\hline-1.4818 & 0.7540 & 1.4985 & $\mathrm{C}$ & 0 & 0 & 0 & 0 & 0 & 0 & 0 & 0 & 0 & \\
\hline-2.3812 & -0.0737 & -0.7572 & $\mathrm{C}$ & 0 & 0 & 0 & 0 & 0 & 0 & 0 & 0 & 0 & 5 \\
\hline 2.2783 & 1.5244 & -0.4468 & $\mathrm{C}$ & 0 & 0 & 0 & 0 & 0 & 0 & 0 & 0 & 0 & 6 \\
\hline 1.8577 & -1.0022 & -0.2928 & $\mathrm{~B}$ & 0 & 0 & 0 & 0 & 0 & 0 & 0 & 0 & 0 & 7 \\
\hline-0.2008 & 1.6352 & -0.5735 & $\mathrm{H}$ & 0 & 0 & 0 & 0 & 0 & 0 & 0 & 0 & 0 & 8 \\
\hline 1.2573 & 0.4962 & 1.1585 & $\mathrm{H}$ & 0 & 0 & 0 & 0 & 0 & 0 & 0 & 0 & 0 & 9 \\
\hline-0.8723 & -1.0455 & 0.4531 & $\mathrm{H}$ & 0 & 0 & 0 & 0 & 0 & 0 & 0 & 0 & 0 & 10 \\
\hline-1.8022 & 1.7824 & 1.2709 & $\mathrm{H}$ & 0 & 0 & 0 & 0 & 0 & 0 & 0 & 0 & 0 & 11 \\
\hline-2.2901 & 0.2613 & 2.0527 & $\mathrm{H}$ & 0 & 0 & 0 & 0 & 0 & 0 & 0 & 0 & 0 & 12 \\
\hline-0.6094 & 0.7917 & 2.1641 & $\mathrm{H}$ & 0 & 0 & 0 & 0 & 0 & 0 & 0 & 0 & 0 & 13 \\
\hline-2.0395 & -0.5387 & -1.6964 & $\mathrm{H}$ & 0 & 0 & 0 & 0 & 0 & 0 & 0 & 0 & 0 & 14 \\
\hline-3.5755 & -0.8657 & -0.2141 & - $\mathrm{C}$ & 0 & 0 & 0 & 0 & 0 & 0 & 0 & 0 & 0 & 15 \\
\hline-2.6909 & 0.9559 & -1.0027 & $\mathrm{H}$ & 0 & 0 & 0 & 0 & 0 & 0 & 0 & 0 & 0 & 16 \\
\hline 3.2793 & 1.4255 & -0.0094 & $\mathrm{H}$ & 0 & 0 & 0 & 0 & 0 & 0 & 0 & 0 & 0 & 17 \\
\hline 1.8902 & 2.5135 & -0.1643 & $\mathrm{H}$ & 0 & 0 & 0 & 0 & 0 & 0 & 0 & 0 & 0 & 18 \\
\hline 2.3771 & 1.4784 & -1.5414 & $\mathrm{H}$ & 0 & 0 & 0 & 0 & 0 & 0 & 0 & 0 & 0 & 19 \\
\hline 3.1388 & -1.4043 & 0.4473 & $\mathrm{C}$ & 0 & 0 & 0 & 0 & 0 & 0 & 0 & 0 & 02 & 20 \\
\hline 1.0684 & -1.7306 & -0.0515 & $\mathrm{H}$ & 0 & 0 & 0 & 0 & 0 & 0 & 0 & 0 & 02 & 21 \\
\hline 2.0186 & -1.0514 & -1.3831 & $\mathrm{H}$ & 0 & 0 & 0 & 0 & 0 & 0 & 0 & 0 & 02 & 22 \\
\hline-4.3592 & -0.9365 & -0.9807 & $\mathrm{H}$ & 0 & 0 & 0 & 0 & 0 & 0 & 0 & 0 & 02 & \\
\hline-4.0182 & -0.3865 & 0.6697 & $\mathrm{H}$ & 0 & 0 & 0 & 0 & 0 & 0 & 0 & 0 & 02 & 24 \\
\hline-3.2803 & -1.8891 & 0.0613 & $\mathrm{H}$ & 0 & 0 & 0 & 0 & 0 & 0 & 0 & 0 & 02 & 25 \\
\hline 3.4142 & -2.4355 & 0.1885 & $\mathrm{H}$ & 0 & 0 & 0 & 0 & 0 & 0 & 0 & 0 & 02 & 26 \\
\hline 3.9870 & -0.7577 & 0.1829 & $\mathrm{H}$ & 0 & 0 & 0 & 0 & 0 & 0 & 0 & 0 & 02 & 27 \\
\hline 2.9978 & -1.3562 & 1.5373 & $\mathrm{H}$ & 0 & 0 & 0 & 0 & 0 & 0 & 0 & 0 & 02 & 28 \\
\hline-0.0162 & 0.2881 & -1.4875 & $\mathrm{H}$ & 0 & 0 & 0 & 0 & 0 & 0 & 0 & 0 & 02 & 29 \\
\hline
\end{tabular}




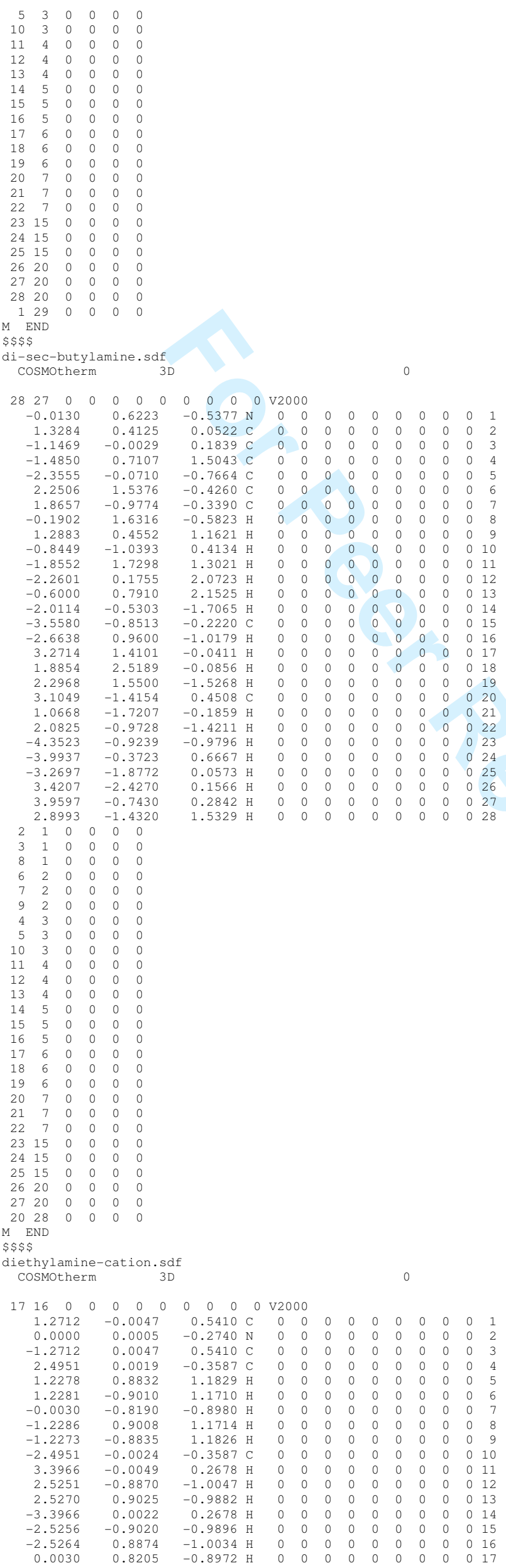




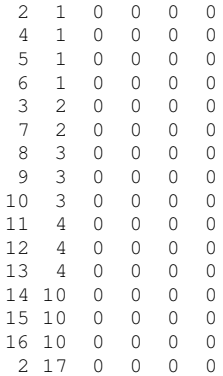


$\$ \$ \$$

dimethyl-iso-butylamine.sdf

$\begin{array}{llllllllllll}22 & 21 & 0 & 0 & 0 & 0 & 0 & 0 & 0 & 0 & 0 & \mathrm{~V} 2000\end{array}$

$\begin{array}{llllllllllllll}1.2288 & -0.1570 & 0.1768 & \mathrm{~N} & 0 & 0 & 0 & 0 & 0 & 0 & 0 & 0 & 0 & 1\end{array}$

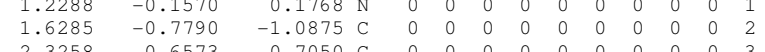

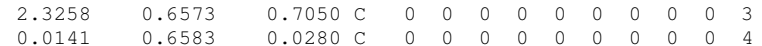

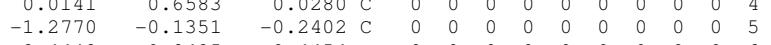

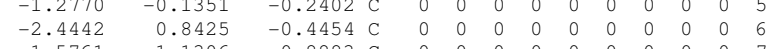

$\begin{array}{rrrrrrrrrrrrrr}-1.5761 & -1.1306 & 0.8883 & \mathrm{C} & 0 & 0 & 0 & 0 & 0 & 0 & 0 & 0 & 0 & 7\end{array}$

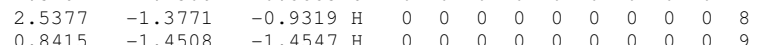

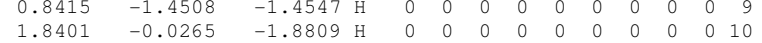

$\begin{array}{rrrrrrrrrrrrrr}1.8401 & -0.0265 & -1.8809 & \mathrm{H} & 0 & 0 & 0 & 0 & 0 & 0 & 0 & 0 & 0 & 10 \\ 2.0400 & 1.0849 & 1.6769 & \mathrm{H} & 0 & 0 & 0 & 0 & 0 & 0 & 0 & 0 & 0 & 11\end{array}$

$\begin{array}{llllllllllllll}2.0400 & 1.0849 & 1.6769 & \mathrm{H} & 0 & 0 & 0 & 0 & 0 & 0 & 0 & 0 & 0 & 11 \\ 2.6042 & 1.4959 & 0.0267 & \mathrm{H} & 0 & 0 & 0 & 0 & 0 & 0 & 0 & 0 & 0 & 12\end{array}$

$\begin{array}{llllllllllllll}2.6042 & 1.4959 & 0.0267 & \mathrm{H} & 0 & 0 & 0 & 0 & 0 & 0 & 0 & 0 & 0 & 12 \\ 3.2174 & 0.0305 & 0.8512 & \mathrm{H} & 0 & 0 & 0 & 0 & 0 & 0 & 0 & 0 & 0 & 13\end{array}$

$\begin{array}{llllllllllllllll}0.1498 & 1.4131 & -0.7829 & \mathrm{H} & 0 & 0 & 0 & 0 & 0 & 0 & 0 & 0 & 0 & 14\end{array}$

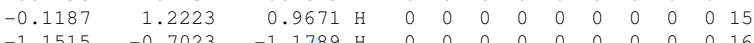

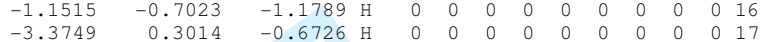

$\begin{array}{llllllllllllll}-2.2458 & 1.5382 & -1.2749 & \mathrm{H} & 0 & 0 & 0 & 0 & 0 & 0 & 0 & 0 & 0 & 18\end{array}$

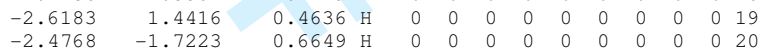

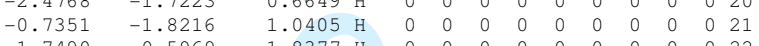

$\begin{array}{llllllllllllll}-1.7490 & -0.5969 & 1.8377 & \mathrm{H} & 0 & 0 & 0 & 0 & 0 & 0 & 0 & 0 & 0 & 22\end{array}$

$\begin{array}{lllllll}2 & 1 & 0 & 0 & 0 & 0\end{array}$

$\begin{array}{llllll}3 & 1 & 0 & 0 & 0 & 0 \\ 4 & 1 & 0 & 0 & 0 & 0\end{array}$

$\begin{array}{lllllll}8 & 2 & 0 & 0 & 0 & 0\end{array}$

$\begin{array}{llllll}9 & 2 & 0 & 0 & 0 & 0 \\ 0 & 2 & 0 & 0 & 0 & 0\end{array}$

$\begin{array}{llllll}11 & 3 & 0 & 0 & 0 & 0\end{array}$

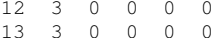

$\begin{array}{llllll}5 & 4 & 0 & 0 & 0 & 0\end{array}$

$\begin{array}{cccccc}14 & 4 & 0 & 0 & 0 & 0 \\ 15 & 4 & 0 & 0 & 0 & 0\end{array}$

$\begin{array}{llllll}6 & 5 & 0 & 0 & 0 & 0\end{array}$

$16 \quad 5 \quad 00000$

$\begin{array}{lllllll}17 & 6 & 0 & 0 & 0 & 0\end{array}$

$\begin{array}{llllll}18 & 6 & 0 & 0 & 0 & 0\end{array}$

$\begin{array}{llllll}19 & 6 & 0 & 0 & 0 & 0 \\ 20 & 7 & 0 & 0 & 0 & 0 \\ 21 & 7 & 0 & 0 & 0 & 0\end{array}$

$\begin{array}{llllll}21 & 7 & 0 & 0 & 0 & 0\end{array}$

M END

dimethyl-iso-propylamine-cation.sdf

cosmotherm

$\begin{array}{llllllllllll}20 & 19 & 0 & 0 & 0 & 0 & 0 & 0 & 0 & 0 & 0 & \text { v } 2000\end{array}$

$\begin{array}{rrrrrrrrrrrrrr}0.6794 & -0.0013 & 0.2861 & \mathrm{~N} & 0 & 0 & 0 & 0 & 0 & 0 & 0 & 0 & 0 & 1 \\ 1.4703 & -1.2293 & -0.0709 & \mathrm{C} & 0 & 0 & 0 & 0 & 0 & 0 & 0 & 0 & 0 & 2\end{array}$

$\begin{array}{llllllllllllll}1.4703 & -1.2293 & -0.0709 & \mathrm{C} & 0 & 0 & 0 & 0 & 0 & 0 & 0 & 0 & 0 & 2 \\ 1.4680 & 1.2306 & -0.0630 & \mathrm{C} & 0 & 0 & 0 & 0 & 0 & 0 & 0 & 0 & 0 & 3\end{array}$

$\begin{array}{rrrrrrrrrrrrrr}1.4680 & 1.2306 & -0.0630 & \mathrm{C} & 0 & 0 & 0 & 0 & 0 & 0 & 0 & 0 & 0 & 3 \\ -0.7319 & -0.0013 & -0.3377 & \mathrm{C} & 0 & 0 & 0 & 0 & 0 & 0 & 0 & 0 & 0 & 4\end{array}$

$\begin{array}{llllllllllllll}-1.4873 & 1.2631 & 0.0652 & \mathrm{C} & 0 & 0 & 0 & 0 & 0 & 0 & 0 & 0 & 0 & 5\end{array}$

$\begin{array}{llllllll}0 & 0 & 0 & 0\end{array}$

$\begin{array}{llllllllllllll}2.4676 & -1.1304 & 0.3678 & \mathrm{H} & 0 & 0 & 0 & 0 & 0 & 0 & 0 & 0 & 0 & 7\end{array}$

$\begin{array}{llllllllllllll}0.9768 & -2.1148 & 0.3348 & \mathrm{H} & 0 & 0 & 0 & 0 & 0 & 0 & 0 & 0 & 0 & 8\end{array}$

$\begin{array}{llllllllllllll}1.5386 & -1.2893 & -1.1622 & \mathrm{H} & 0 & 0 & 0 & 0 & 0 & 0 & 0 & 0 & 0 & 9\end{array}$

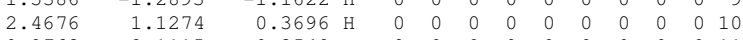

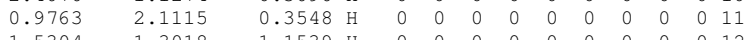

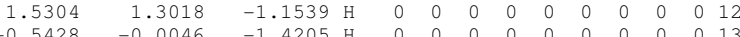

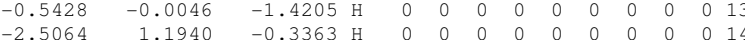

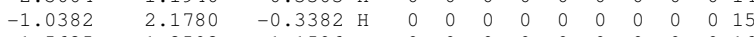

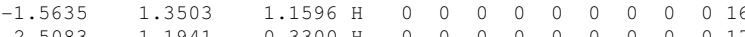

$-1.0417-2.1803$

$\begin{array}{lllllllllllllllll}-1.5670 & -1.3412 & 1.3239 & \mathrm{H} & 0 & 0 & 0 & 0 & 0 & 0 & 0 & 0 & 0 & 18\end{array}$

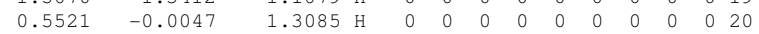

$\begin{array}{llllllll}2 & 1 & 0 & 0 & 0 & 0\end{array}$

$\begin{array}{lllllll}3 & 1 & 0 & 0 & 0 & 0\end{array}$

$\begin{array}{llllll}4 & 1 & 0 & 0 & 0 & 0\end{array}$

$\begin{array}{llllll}2 & 0 & 0 & 0 & 0\end{array}$

$\begin{array}{llllll}2 & 0 & 0 & 0 & 0\end{array}$

$10 \begin{array}{llllll}1 & 3 & 0 & 0 & 0 & 0\end{array}$

$\begin{array}{lllllllll}11 & 3 & 0 & 0 & 0 & 0\end{array}$

5 (4)

$\begin{array}{llllll}6 & 4 & 0 & 0 & 0 & 0\end{array}$

$\begin{array}{lllllll}14 & 5 & 0 & 0 & 0 & 0\end{array}$

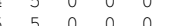

$\begin{array}{lllllllll}14 & 5 & 0 & 0 & 0 & 0\end{array}$

$\begin{array}{lllllll}17 & 6 & 0 & 0 & 0 & 0\end{array}$

1866000100

12000000

END

dimethyl-iso-propylamine.sdf

cosmotherm $3 \mathrm{D}$

$\begin{array}{llllllllllll}19 & 18 & 0 & 0 & 0 & 0 & 0 & 0 & 0 & 0 & 0 & \mathrm{~V} 2000\end{array}$

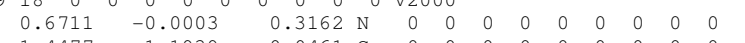

$\begin{array}{lllllllllllllll}1.4477 & -1.1939 & -0.0461 \mathrm{C} & 0 & 0 & 0 & 0 & 0 & 0 & 0 & 0 & 0 & 2\end{array}$

$\begin{array}{rrrrrrrrrrrrrr}1.4473 & 1.1939 & -0.0448 & \mathrm{C} & 0 & 0 & 0 & 0 & 0 & 0 & 0 & 0 & 0 & 3\end{array}$

$\begin{array}{lllllllllllll}-1.4473 & 1.1939 & -0.0448 & c & 0 & 0 & 0 & 0 & 0 & 0 & 0 & 0 & 0\end{array}$

$\begin{array}{rrrrrrrrrrrrrr}-1.4724 & 1.2525 & 0.0696 & \mathrm{C} & 0 & 0 & 0 & 0 & 0 & 0 & 0 & 0 & 0 & 5 \\ -1.4733 & -1.2520 & 0.0709 & \mathrm{C} & 0 & 0 & 0 & 0 & 0 & 0 & 0 & 0 & 0 & 6\end{array}$ 


$\begin{array}{rrrrrrrrrrrrrr}2.4460 & -1.1229 & 0.4072 & \mathrm{H} & 0 & 0 & 0 & 0 & 0 & 0 & 0 & 0 & 0 & 7 \\ 0.9718 & -2.1051 & 0.3349 & \mathrm{H} & 0 & 0 & 0 & 0 & 0 & 0 & 0 & 0 & 0 & 8 \\ 1.5773 & -1.3009 & -1.1468 & \mathrm{H} & 0 & 0 & 0 & 0 & 0 & 0 & 0 & 0 & 0 & 9 \\ 2.4455 & 1.1230 & 0.4089 & \mathrm{H} & 0 & 0 & 0 & 0 & 0 & 0 & 0 & 0 & 0 & 10 \\ 0.9708 & 2.1045 & 0.3366 & \mathrm{H} & 0 & 0 & 0 & 0 & 0 & 0 & 0 & 0 & 0 & 11 \\ 1.5775 & 1.3019 & -1.1453 & \mathrm{H} & 0 & 0 & 0 & 0 & 0 & 0 & 0 & 0 & 0 & 12 \\ -0.5464 & -0.0010 & -1.4253 & \mathrm{H} & 0 & 0 & 0 & 0 & 0 & 0 & 0 & 0 & 0 & 13 \\ -2.4905 & 1.1789 & -0.3377 & \mathrm{H} & 0 & 0 & 0 & 0 & 0 & 0 & 0 & 0 & 0 & 14 \\ -1.0296 & 2.1777 & -0.3207 & \mathrm{H} & 0 & 0 & 0 & 0 & 0 & 0 & 0 & 0 & 0 & 15 \\ -1.5464 & 1.3376 & 1.1653 & \mathrm{H} & 0 & 0 & 0 & 0 & 0 & 0 & 0 & 0 & 0 & 16 \\ -2.4903 & -1.1796 & -0.3395 & \mathrm{H} & 0 & 0 & 0 & 0 & 0 & 0 & 0 & 0 & 0 & 17 \\ -1.0293 & -2.1782 & -0.3156 & \mathrm{H} & 0 & 0 & 0 & 0 & 0 & 0 & 0 & 0 & 0 & 18 \\ -1.5502 & -1.3340 & 1.1667 & \mathrm{H} & 0 & 0 & 0 & 0 & 0 & 0 & 0 & 0 & 0 & 19\end{array}$




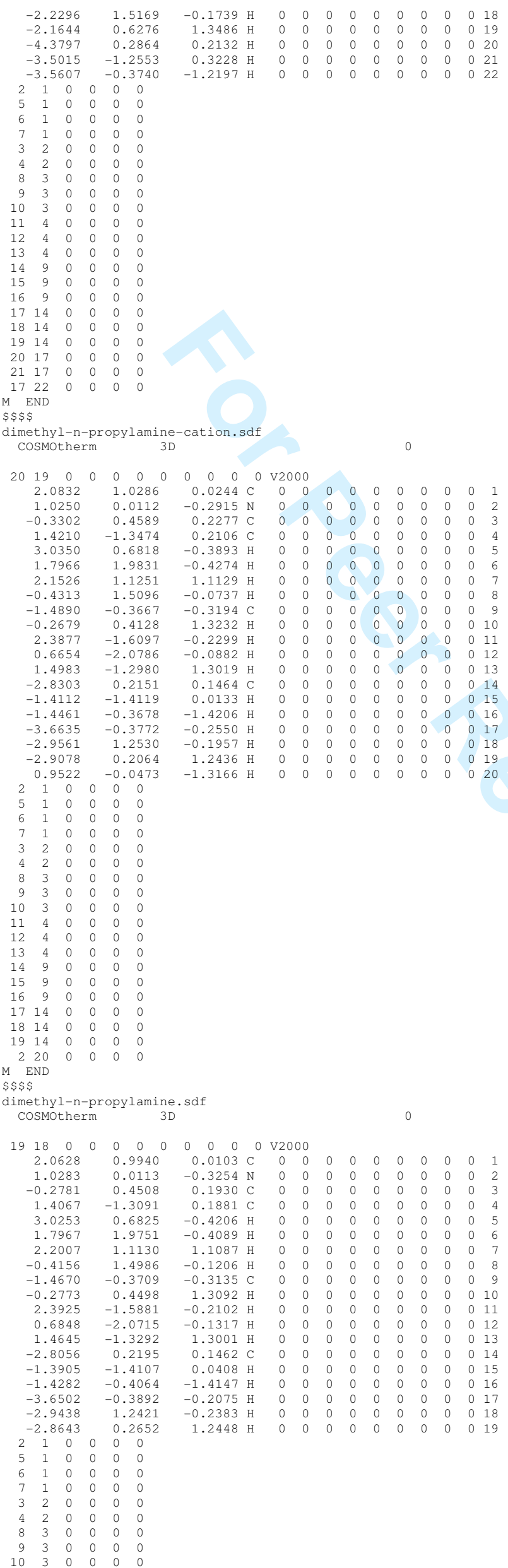


$11 \quad 4 \quad 0000100$

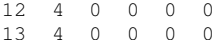

$\begin{array}{llllll}14 & 9 & 0 & 0 & 0 & 0\end{array}$

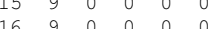

$17 \begin{array}{llllll}14 & 0 & 0 & 0 & 0\end{array}$

$18 \quad 14 \quad 000000$

$14 \quad 19$

$\$ \$ \$ \$$

dimethyl-sec-butylamine-cation.sdf

cosmotherm

$\begin{array}{llllllllllll}23 & 22 & 0 & 0 & 0 & 0 & 0 & 0 & 0 & 0 & 0 & \mathrm{~V} 2000\end{array}$

$\begin{array}{llllllllllllll}1.0889 & 0.2264 & 0.2810 & \mathrm{~N} & 0 & 0 & 0 & 0 & 0 & 0 & 0 & 0 & 0 & 1\end{array}$

$\begin{array}{llllllllllllll}2.2339 & -0.6965 & -0.0296 & \mathrm{C} & 0 & 0 & 0 & 0 & 0 & 0 & 0 & 0 & 0 & 2\end{array}$

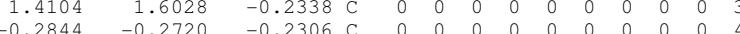

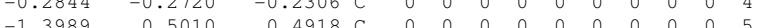

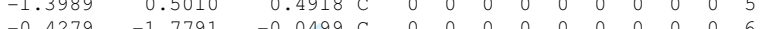

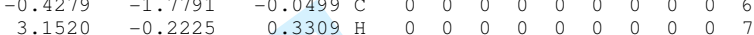

$\begin{array}{lllllllllllll}2.0864 & -1.6501 & 0.4820 \mathrm{H} & 0 & 0 & 0 & 0 & 0 & 0 & 0 & 0 & 0 & 8\end{array}$

$\begin{array}{lllllllllllll}2.2813 & -0.8387 & -1.1139 & \mathrm{H} & 0 & 0 & 0 & 0 & 0 & 0 & 0 & 0 & 0\end{array}$

$\begin{array}{llllllllllllll}2.3306 & 1.9446 & 0.2491 & \mathrm{H} & 0 & 0 & 0 & 0 & 0 & 0 & 0 & 0 & 0 & 10\end{array}$

$\begin{array}{llllllllllllll}0.5925 & 2.2867 & 0.0035 & \mathrm{H} & 0 & 0 & 0 & 0 & 0 & 0 & 0 & 0 & 0 & 11\end{array}$

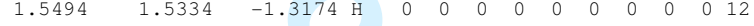

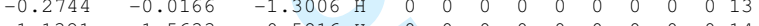

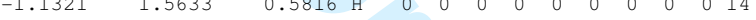

$\begin{array}{llllllllllllll}-2.7479 & 0.4068 & -0.2317 & \mathrm{C} & 0 & 0 & 0 & 0 & 0 & 0 & 0 & 0 & 0 & 15\end{array}$

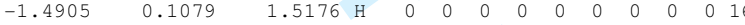

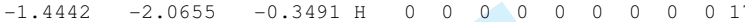

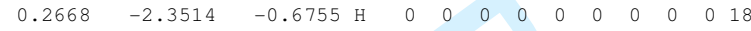

$\begin{array}{llllllllllllll}-0.2970 & -2.0706 & 1.0026 & \mathrm{H} & 0 & 0 & 0 & 0 & 0 & 0 & 0 & 0 & 0 & 19\end{array}$

$\begin{array}{llllllllllllll}-3.5062 & 0.9871 & 0.3112 & \mathrm{H} & 0 & 0 & 0 & 0 & 0 & 0 & 0 & 0 & 0 & 20\end{array}$

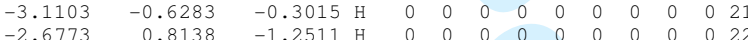

$\begin{array}{lllllllllllll}1.0178 & 0.2865 & 1.3068 \mathrm{H} & 0 & 0 & 0 & 0 & 0 & 0 & 0 & 0 & 0 & 23\end{array}$

$\begin{array}{llllllll}2 & 1 & 0 & 0 & 0 & 0\end{array}$

$\begin{array}{lllllll}3 & 1 & 0 & 0 & 0 & 0\end{array}$

$\begin{array}{llllll}4 & 1 & 0 & 0 & 0\end{array}$

$\begin{array}{lllll}2 & 0 & 0 & 0\end{array}$

$\begin{array}{llll}0 & 0 & 0\end{array}$

$\begin{array}{lllll}3 & 0 & 0 & 0 & 0\end{array}$

$\begin{array}{lllllll}1 & 3 & 0 & 0 & 0 & 0 \\ 2 & 3 & 0 & 0 & 0 & 0\end{array}$

$\begin{array}{llllll}4 & 0 & 0 & 0\end{array}$

$\begin{array}{lllll}4 & 0 & 0 & 0\end{array}$

$\begin{array}{llllll}4 & 5 & 0 & 0 & 0 & 0\end{array}$

$\begin{array}{llllll}5 & 5 & 0 & 0 & 0 & 0\end{array}$

$\begin{array}{lllllll}17 & 6 & 0 & 0 & 0 & 0\end{array}$

$\begin{array}{llllll}18 & 6 & 0 & 0 & 0 & 0\end{array}$

$\begin{array}{llllll}19 & 6 & 0 & 0 & 0\end{array}$

$\begin{array}{llllll}15 & 0 & 0 & 0 & 0\end{array}$

$\begin{array}{llllll}15 & 0 & 0 & 0 & 0\end{array}$

$\begin{array}{lllllll}1 & 23 & 0 & 0 & 0 & 0\end{array}$

END

dimethyl-sec-butylamine.sdf

cosmotherm $3 \mathrm{D}$

$\begin{array}{llllllllllll}22 & 21 & 0 & 0 & 0 & 0 & 0 & 0 & 0 & 0 & 0 & \text { v2000 }\end{array}$

$\begin{array}{rrrrrrrrrrrrrr}1.0908 & 0.2134 & 0.3103 & \mathrm{~N} & 0 & 0 & 0 & 0 & 0 & 0 & 0 & 0 & 0 & 1 \\ 2.2061 & -0.6565 & -0.0869 & \mathrm{C} & 0 & 0 & 0 & 0 & 0 & 0 & 0 & 0 & 0 & 2\end{array}$

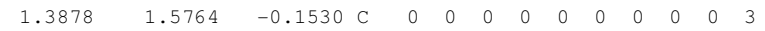

$\begin{array}{llllllllllllll}-0.2252 & -0.2610 & -0.2007 & \mathrm{C} & 0 & 0 & 0 & 0 & 0 & 0 & 0 & 0 & 0 & 4\end{array}$

$\begin{array}{llllllllllllll}-1.3813 & 0.5106 & 0.4722 & \mathrm{C} & 0 & 0 & 0 & 0 & 0 & 0 & 0 & 0 & 0 & 5\end{array}$

$\begin{array}{llllllllllllll}-0.4106 & -1.7678 & 0.0203 & \mathrm{C} & 0 & 0 & 0 & 0 & 0 & 0 & 0 & 0 & 0 & 6\end{array}$

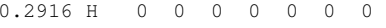

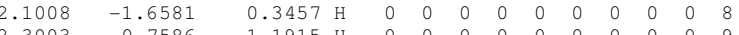

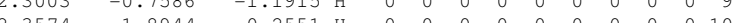

$\begin{array}{lllllllllllllll}2.3574 & 1.8944 & 0.2551 & \mathrm{H} & 0 & 0 & 0 & 0 & 0 & 0 & 0 & 0 & 0 & 10 \\ 0.6314 & 2.2911 & 0.1920 & \mathrm{H} & 0 & 0 & 0 & 0 & 0 & 0 & 0 & 0 & 0 & 11\end{array}$

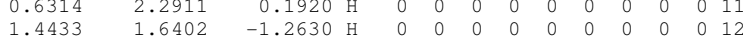

$\begin{array}{lllllllllllllllll}-0.2717 & -0.0651 & -1.2992 \mathrm{H} & 0 & 0 & 0 & 0 & 0 & 0 & 0 & 0 & 0 & 13\end{array}$

$\begin{array}{llllllllllllll}-1.1256 & 1.5767 & -1.5486 \mathrm{H} & 0 & 0 & 0 & 0 & 0 & 0 & 0 & 0 & 0 & 14\end{array}$

$\begin{array}{llllllllllllll}-2.7201 & 0.3925 & -0.2671 & \mathrm{C} & 0 & 0 & 0 & 0 & 0 & 0 & 0 & 0 & 0 & 15\end{array}$

$\begin{array}{llllllllllllll}-1.4854 & 0.1419 & 1.5069 & \mathrm{H} & 0 & 0 & 0 & 0 & 0 & 0 & 0 & 0 & 0 & 16 \\ -1.2557 & -2.3748 & -0.6059 & \mathrm{H} & 0 & 0 & 0 & 0 & 0 & 0 & 0 & 0 & 0 & 17\end{array}$

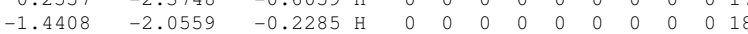

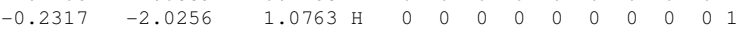

$-3.4988 \quad 0.9779$

$\begin{array}{lll}-3.0758 & -0.6470 & -0.3233 \\ -0.6\end{array}$

$-1.637100 .7735$

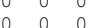

$\begin{array}{lllllll}4 & 1 & 0 & 0 & 0 & 0 & 0\end{array}$

\begin{tabular}{llllll}
7 & 2 & 0 & 0 & 0 & 0 \\
\hline & 2 & 0 & 0 & 0 & 0
\end{tabular}

$\begin{array}{lllll}2 & 0 & 0 & 0\end{array}$

$\begin{array}{lllllll}3 & 3 & 0 & 0 & 0 & 0 \\ 1 & 3 & 0 & 0 & 0 & 0 & 0\end{array}$

$\begin{array}{llllll}4 & 0 & 0 & 0 & 0 & 2\end{array}$

$\begin{array}{llllll}3 & 4 & 0 & 0 & 0 & 0\end{array}$

$\begin{array}{llllll}4 & 5 & 0 & 0 & 0 & 0\end{array}$

$\begin{array}{llllll}14 & 5 & 0 & 0 & 0 & 0\end{array}$

$\begin{array}{lllllll}17 & 6 & 0 & 0 & 0 & 0\end{array}$

$\begin{array}{llllll}18 & 6 & 0 & 0 & 0 & 0\end{array}$ 


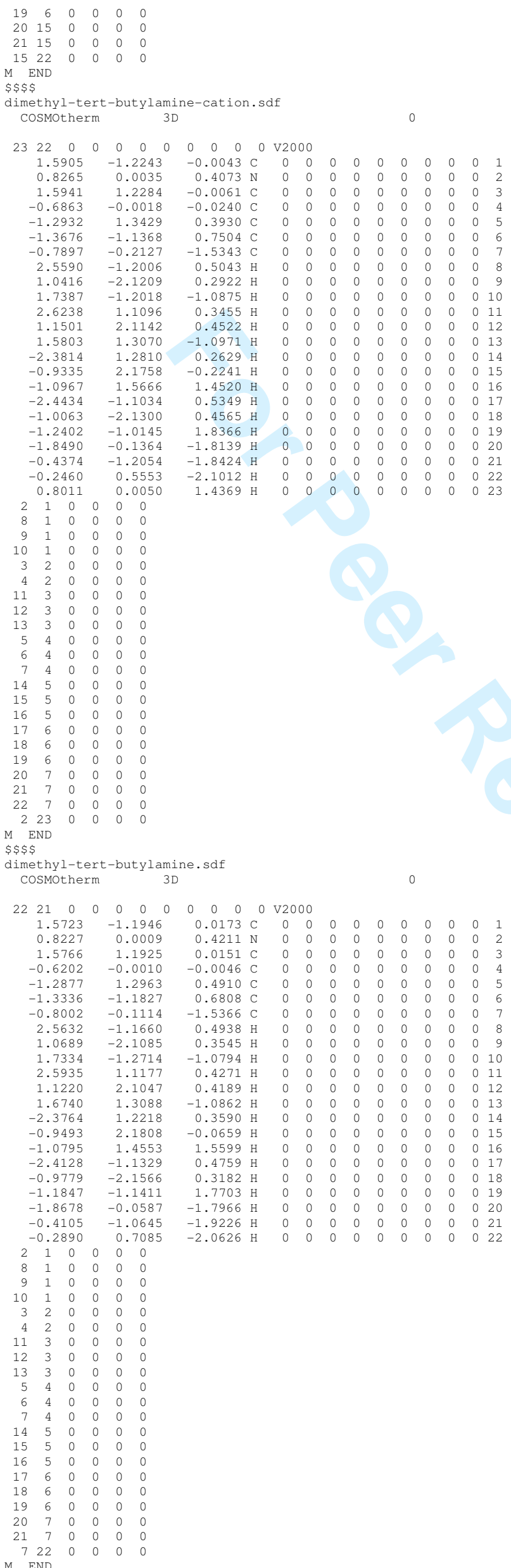


$\$ \$ \$$

dimethylamine-cation.sdf

cosmotherm 3D

$\begin{array}{llllllllllll}11 & 10 & 0 & 0 & 0 & 0 & 0 & 0 & 0 & 0 & 0 & \mathrm{~V} 2000\end{array}$

$\begin{array}{llllllllllllll}1.2556 & 0.0000 & -0.2861 & \mathrm{C} & 0 & 0 & 0 & 0 & 0 & 0 & 0 & 0 & 0 & 1\end{array}$

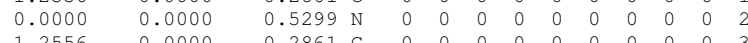

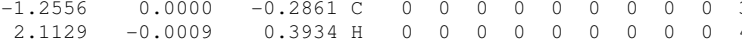

$\begin{array}{lllllllllllll}1.2614 & -0.8988 & -0.9100 \mathrm{H} & 0 & 0 & 0 & 0 & 0 & 0 & 0 & 0 & 0 & 5\end{array}$

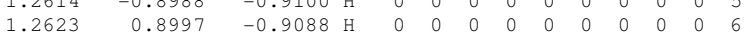

$\begin{array}{rrrrrrrrrrrrrrrrr}1.2623 & 0.8178 & 1.1531 & \mathrm{H} & 0 & 0 & 0 & 0 & 0 & 0 & 0 & 0 & 0 & 7\end{array}$

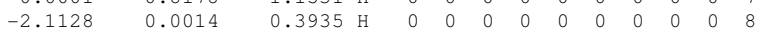

$\begin{array}{lllllllllllll}-1.2626 & -0.8998 & -0.9086 & \mathrm{H} & 0 & 0 & 0 & 0 & 0 & 0 & 0 & 0 & 0\end{array}$

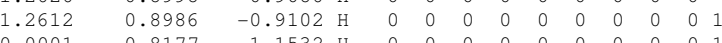

$\begin{array}{llllll}1 & 0 & 0 & 0 & 0\end{array}$

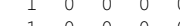

$\begin{array}{llllll}1 & 0 & 0 & 0 & 0\end{array}$

$\begin{array}{lllll}1 & 0 & 0 & 0 & 0\end{array}$

$\begin{array}{lllll}2 & 0 & 0 & 0 & 0\end{array}$

$\begin{array}{lllllll}7 & 2 & 0 & 0 & 0 & 0 \\ 3 & 3 & 0 & 0 & 0 & 0\end{array}$

$\begin{array}{lllll}3 & 0 & 0 & 0 & 0\end{array}$

$2 \begin{array}{rrrrrr}2 & 11 & 0 & 0 & 0 & 0\end{array}$

END

$\$ \$ \$$

dimethylamine.sdf

cosmotherm

$\begin{array}{llllllllllll}10 & 9 & 0 & 0 & 0 & 0 & 0 & 0 & 0 & 0 & 0 & \mathrm{~V} 2000\end{array}$

$\begin{array}{llllllllllllll}1.2168 & 0.0159 & -0.2573 & \mathrm{C} & 0 & 0 & 0 & 0 & 0 & 0 & 0 & 0 & 0 & 1\end{array}$

$\begin{array}{lllllllllllll}0.0000 & -0.0882 & 0.5567 & \mathrm{~N} & 0 & 0 & 0 & 0 & 0 & 0 & 0 & 0 & 0\end{array}$

$\begin{array}{lllllllllllll}1.2168 & 0.0159 & 0.2573 & \mathrm{H} & 0 & 0 & 0 & 0 & 0 & 0 & 0 & 0 & 0 \\ 2.1018 & 0.0109 & 0.3942 & \mathrm{H} & 0 & 0 & 0 & 0 & 0 & 0 & 0 & 0 & 0\end{array}$

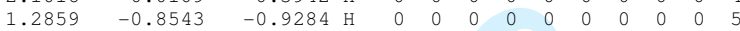

$\begin{array}{llllllllllllll}1.2527 & 0.9295 & -0.8875 & \mathrm{H} & 0 & 0 & 0 & 0 & 0 & 0 & 0 & 0 & 0 & 6\end{array}$

$\begin{array}{lllllllllllll}0.0000 & 0.6739 & 1.2394 & \mathrm{H} & 0 & 0 & 0 & 0 & 0 & 0 & 0 & 0 & 0\end{array}$

$\begin{array}{lrrrrrrrrrrrrr}-2.1018 & 0.0106 & 0.3943 & \mathrm{H} & 0 & 0 & 0 & 0 & 0 & 0 & 0 & 0 & 0 & 8 \\ -1.2858 & -0.8541 & -0.9286 & \mathrm{H} & 0 & 0 & 0 & 0 & 0 & 0 & 0 & 0 & 0 & 9\end{array}$

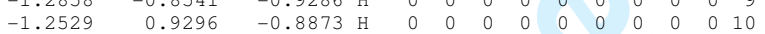

2.1000 .090

$\begin{array}{lllllll}4 & 1 & 0 & 0 & 0 & 0\end{array}$

$\begin{array}{llllll}1 & 0 & 0 & 0 & 0\end{array}$

$\begin{array}{llllll}2 & 0 & 0 & 0 & 0\end{array}$

$\begin{array}{llllll}3 & 0 & 0 & 0 & 0\end{array}$

$\begin{array}{lllll}3 & 0 & 0 & 0 & 0\end{array}$

M END

dimethylethylamine-cation.sdf

CosMotherm

$\begin{array}{llllllllllll}17 & 16 & 0 & 0 & 0 & 0 & 0 & 0 & 0 & 0 & 0 & \mathrm{v} 2000\end{array}$

$\begin{array}{llllllllllllll}1.6899 & 0.8096 & -0.0063 & \mathrm{C} & 0 & 0 & 0 & 0 & 0 & 0 & 0 & 0 & 0 & 1\end{array}$

$\begin{array}{lllllllllllll}0.4590 & -0.0005 & -0.2935 & \mathrm{~N} & 0 & 0 & 0 & 0 & 0 & 0 & 0 & 0 & 0\end{array}$

$\begin{array}{llllllllllllll}-0.7718 & -0.6486 & 0.3187 & \mathrm{C} & 0 & 0 & 0 & 0 & 0 & 0 & 0 & 0 & 0 & 3\end{array}$

$\begin{array}{lllllllllllll}0.6416 & -1.4260 & 0.1394 & \mathrm{C} & 0 & 0 & 0 & 0 & 0 & 0 & 0 & 0 & 0\end{array}$

$\begin{array}{llllllllllllll}2.5434 & 0.3342 & -0.4992 & \mathrm{H} & 0 & 0 & 0 & 0 & 0 & 0 & 0 & 0 & 0 & 5\end{array}$

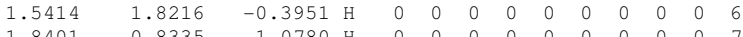

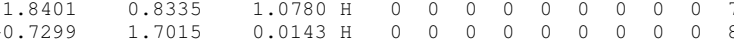

$\begin{array}{llllllllllllll}-2.0701 & 0.0032 & -0.1335 & \mathrm{C} & 0 & 0 & 0 & 0 & 0 & 0 & 0 & 0 & 0 & 9\end{array}$

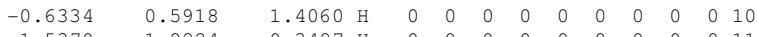

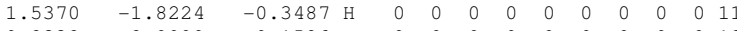

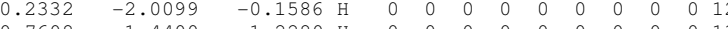

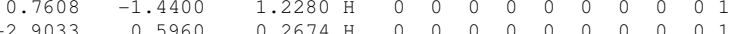

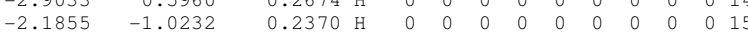

$\begin{array}{llllllllllllll}-2.1571 & 0.0032 & -1.2295 & \mathrm{H} & 0 & 0 & 0 & 0 & 0 & 0 & 0 & 0 & 0 & 16\end{array}$

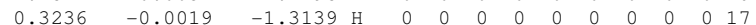

$2 \quad 1 \quad 0 \quad 0000$

$\begin{array}{llllll}5 & 1 & 0 & 0 & 0 & 0\end{array}$

$\begin{array}{llllll}1 & 0 & 0 & 0 & 0\end{array}$

$\begin{array}{llll}2 & 0 & 0 & 0\end{array}$

$\begin{array}{llllll}2 & 0 & 0 & 0 & 0 \\ 3 & 0 & 0 & 0 & 0\end{array}$

$\begin{array}{llllllll}3 & 0 & 0 & 0 & 0\end{array}$

$\begin{array}{llllll}0 & 3 & 0 & 0 & 0\end{array}$

$\begin{array}{lllllll}1 & 4 & 0 & 0 & 0\end{array}$

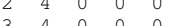

$\begin{array}{llllllll}1 & 9 & 0 & 0 & 0 & 0 & 0 & 1 \\ 15 & 9 & 0 & 0 & 0 & 0 & 0\end{array}$

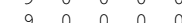

$\begin{array}{rrrrr}9 & 0 & 0 & 0 & 0 \\ 17 & 0 & 0 & 0 & 0\end{array}$

$\$ \$ \$ \$$

dimethylethylamine.sdf

$\begin{array}{llllllllllll}16 & 15 & 0 & 0 & 0 & 0 & 0 & 0 & 0 & 0 & 0 & \mathrm{~V} 2000\end{array}$

$\begin{array}{rrrrrrrrrrrrrr}1.6571 & 0.7843 & -0.0278 & \mathrm{C} & 0 & 0 & 0 & 0 & 0 & 0 & 0 & 0 & 0 & 1 \\ 0.4542 & 0.0010 & -0.3233 & \mathrm{~N} & 0 & 0 & 0 & 0 & 0 & 0 & 0 & 0 & 0 & 2 \\ -0.7258 & 0.6265 & 0.2987 & \mathrm{C} & 0 & 0 & 0 & 0 & 0 & 0 & 0 & 0 & 0 & 3 \\ 0.6378 & -1.3846 & 0.1174 & \mathrm{C} & 0 & 0 & 0 & 0 & 0 & 0 & 0 & 0 & 0 & 4 \\ 2.5247 & 0.3400 & -0.5365 & \mathrm{H} & 0 & 0 & 0 & 0 & 0 & 0 & 0 & 0 & 0 & 5 \\ 1.5316 & 1.8140 & -0.3924 & \mathrm{H} & 0 & 0 & 0 & 0 & 0 & 0 & 0 & 0 & 0 & 6 \\ 1.8830 & 0.8282 & 1.0616 & \mathrm{H} & 0 & 0 & 0 & 0 & 0 & 0 & 0 & 0 & 0 & 7 \\ -0.7135 & 1.6901 & 0.0134 & \mathrm{H} & 0 & 0 & 0 & 0 & 0 & 0 & 0 & 0 & 0 & 8\end{array}$

URL: http://mc.manuscriptcentral.com/tandf/tmph 


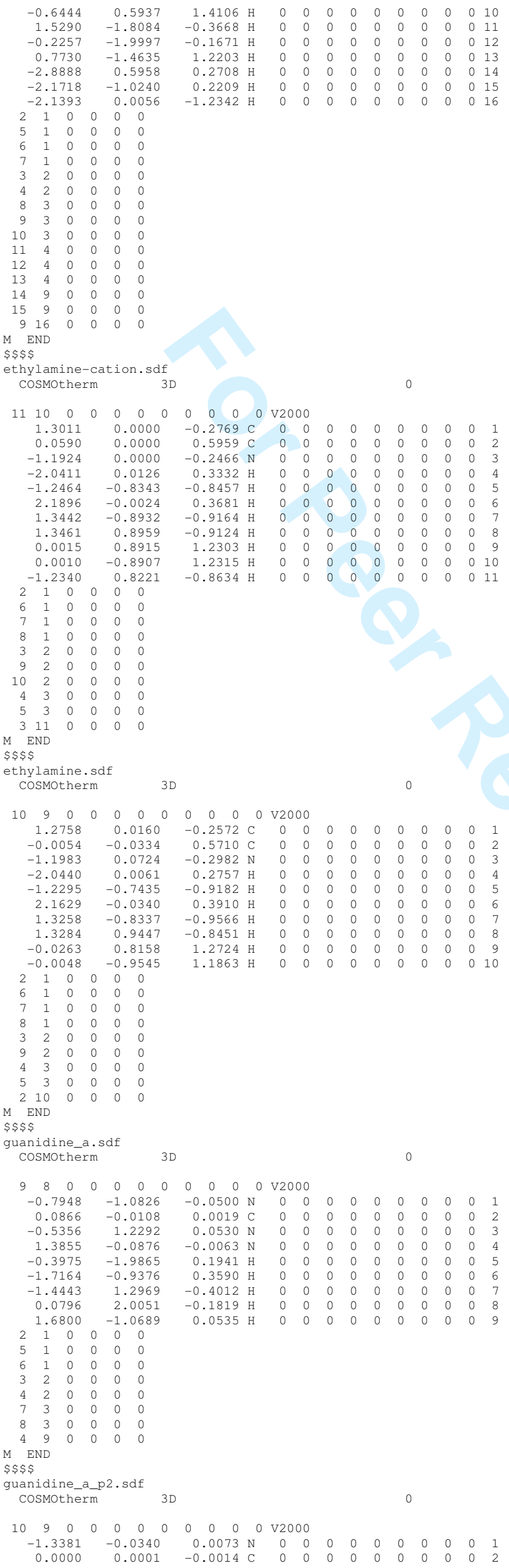




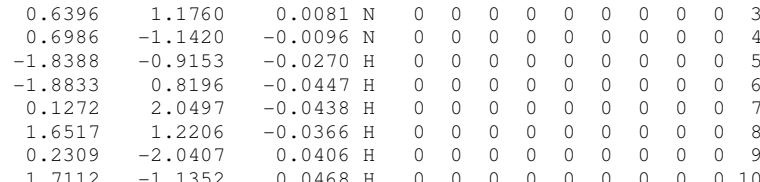




$\begin{array}{rrrrrrrrrrrrrr}1.3381 & -0.3641 & 0.2443 & \mathrm{C} & 0 & 0 & 0 & 0 & 0 & 0 & 0 & 0 & 0 & 10 \\ 3.2651 & 0.8925 & 0.1683 & \mathrm{C} & 0 & 0 & 0 & 0 & 0 & 0 & 0 & 0 & 0 & 11 \\ 3.5266 & -0.5727 & 0.5715 & \mathrm{C} & 0 & 0 & 0 & 0 & 0 & 0 & 0 & 0 & 0 & 12 \\ 1.8073 & 0.9514 & 0.3241 & \mathrm{~N} & 0 & 0 & 0 & 0 & 0 & 0 & 0 & 0 & 0 & 13 \\ 2.2483 & -1.2888 & 0.3959 & \mathrm{~N} & 0 & 0 & 0 & 0 & 0 & 0 & 0 & 0 & 0 & 14 \\ 3.6912 & 1.0843 & -1.1922 & \mathrm{O} & 0 & 0 & 0 & 0 & 0 & 0 & 0 & 0 & 0 & 15 \\ -4.9107 & 1.4827 & -0.0515 & \mathrm{H} & 0 & 0 & 0 & 0 & 0 & 0 & 0 & 0 & 0 & 16 \\ -3.0159 & 3.0269 & 0.3958 & \mathrm{H} & 0 & 0 & 0 & 0 & 0 & 0 & 0 & 0 & 0 & 17 \\ -0.6939 & 2.2202 & 0.4959 & \mathrm{H} & 0 & 0 & 0 & 0 & 0 & 0 & 0 & 0 & 0 & 18 \\ -4.5195 & -0.9480 & -0.4030 & \mathrm{H} & 0 & 0 & 0 & 0 & 0 & 0 & 0 & 0 & 0 & 19 \\ -2.2927 & -3.0138 & -0.5114 & \mathrm{H} & 0 & 0 & 0 & 0 & 0 & 0 & 0 & 0 & 0 & 20 \\ 0.1865 & -2.7290 & -0.2206 & \mathrm{H} & 0 & 0 & 0 & 0 & 0 & 0 & 0 & 0 & 0 & 21 \\ 3.7541 & 1.6344 & 0.8137 & \mathrm{H} & 0 & 0 & 0 & 0 & 0 & 0 & 0 & 0 & 0 & 22 \\ 4.3248 & -1.0093 & -0.0459 & \mathrm{H} & 0 & 0 & 0 & 0 & 0 & 0 & 0 & 0 & 0 & 23 \\ 3.8453 & -0.6305 & 1.6257 & \mathrm{H} & 0 & 0 & 0 & 0 & 0 & 0 & 0 & 0 & 0 & 24 \\ 1.3131 & 1.6880 & -0.1777 & \mathrm{H} & 0 & 0 & 0 & 0 & 0 & 0 & 0 & 0 & 0 & 25 \\ 3.7199 & 2.0430 & -1.3674 & \mathrm{H} & 0 & 0 & 0 & 0 & 0 & 0 & 0 & 0 & 0 & 26\end{array}$




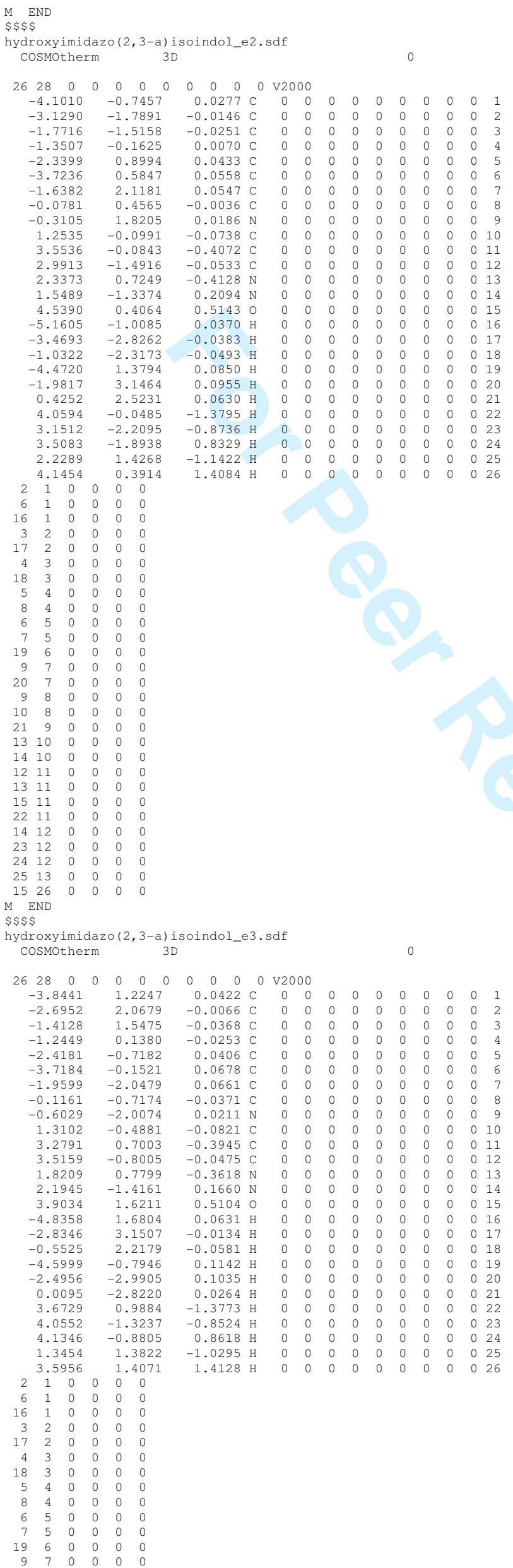


$\begin{array}{rrrrrr}20 & 7 & 0 & 0 & 0 & 0 \\ 9 & 8 & 0 & 0 & 0 & 0 \\ 10 & 8 & 0 & 0 & 0 & 0 \\ 21 & 9 & 0 & 0 & 0 & 0 \\ 13 & 10 & 0 & 0 & 0 & 0 \\ 14 & 10 & 0 & 0 & 0 & 0 \\ 12 & 11 & 0 & 0 & 0 & 0 \\ 13 & 11 & 0 & 0 & 0 & 0 \\ 15 & 11 & 0 & 0 & 0 & 0 \\ 22 & 11 & 0 & 0 & 0 & 0 \\ 14 & 12 & 0 & 0 & 0 & 0 \\ 23 & 12 & 0 & 0 & 0 & 0 \\ 24 & 12 & 0 & 0 & 0 & 0 \\ 25 & 13 & 0 & 0 & 0 & 0 \\ 15 & 26 & 0 & 0 & 0 & 0 \\ 1\end{array}$

$M$ END

imidazo-(2,3-b)-thioxazole_b-cation1.sdf

cosmotherm

$\begin{array}{llllllllllll}18 & 19 & 0 & 0 & 0 & 0 & 0 & 0 & 0 & 0 & 0 & \mathrm{~V} 2000\end{array}$

$\begin{array}{llllllllllllll}3.1474 & 0.2861 & 0.6727 & \mathrm{C} & 0 & 0 & 0 & 0 & 0 & 0 & 0 & 0 & 0 & 1 \\ 1.7392 & 0.2360 & 1.1207 & \mathrm{~N} & 0 & 0 & 0 & 0 & 0 & 0 & 0 & 0 & 0 & 2\end{array}$

$\begin{array}{rrrrrrrrrrrrrr}1.7392 & 0.2360 & 1.1207 & \mathrm{~N} & 0 & 0 & 0 & 0 & 0 & 0 & 0 & 0 & 0 & 2 \\ 3.0564 & -0.3060 & -0.7545 & \mathrm{C} & 0 & 0 & 0 & 0 & 0 & 0 & 0 & 0 & 0 & 3\end{array}$

$\begin{array}{llllllllllll}1.6033 & -0.2502 & -1.0222 \mathrm{~N} & 0 & 0 & 0 & 0 & 0 & 0 & 0 & 0 & 0\end{array}$

$\begin{array}{llllllllllllll}0.9200 & -0.0105 & 0.0981 & C & 0 & 0 & 0 & 0 & 0 & 0 & 0 & 0 & 0 & 5\end{array}$

$\begin{array}{llllllllllllll}-2.6315 & -0.2097 & 1.1747 & \mathrm{~N} & 0 & 0 & 0 & 0 & 0 & 0 & 0 & 0 & 0 & 6 \\ -1.2745 & -0.2280 & 1.3476 & \mathrm{C} & 0 & 0 & 0 & 0 & 0 & 0 & 0 & 0 & 0 & 7\end{array}$

$\begin{array}{llllllllllllll}-2.9389 & 0.0093 & -0.0833 & \mathrm{C} & 0 & 0 & 0 & 0 & 0 & 0 & 0 & 0 & 0 & 8\end{array}$

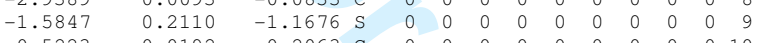

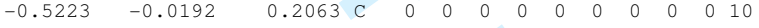

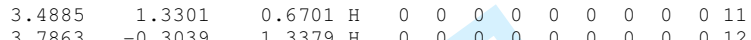

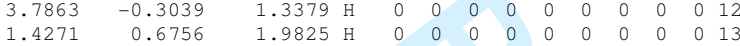

$\begin{array}{llllllllllllll}3.3914 & -1.3512 & -0.7931 & \mathrm{H} & 0 & 0 & 0 & 0 & 0 & 0 & 0 & 0 & 0 & 14\end{array}$

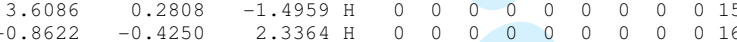

$\begin{array}{llllllllllllll}-3.9547 & 0.0813 & -0.4683 & \mathrm{H} & 0 & 0 & 0 & 0 & 0 & 0 & 0 & 0 & 0 & 17\end{array}$

$1.1874-0.6914$

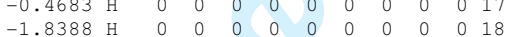

$\begin{array}{llllll}2 & 1 & 0 & 0 & 0 & 0 \\ 3 & 1 & 0 & 0 & 0 & 0\end{array}$

$\begin{array}{lllllll}11 & 1 & 0 & 0 & 0 & 0\end{array}$

$\begin{array}{llllll}11 & 1 & 0 & 0 & 0 & 0\end{array}$

$\begin{array}{lllllll}5 & 2 & 0 & 0 & 0 & 0\end{array}$

$\begin{array}{lllllll}3 & 2 & 0 & 0 & 0 & 0\end{array}$

$\begin{array}{lllllll}4 & 3 & 0 & 0 & 0 & 0 \\ 4 & 3 & 0 & 0 & 0 & 0\end{array}$

$\begin{array}{lllllll}5 & 3 & 0 & 0 & 0 & 0\end{array}$

$\begin{array}{llllll}5 & 4 & 0 & 0 & 0 & 0 \\ 0 & 5 & 0 & 0 & 0 & 0\end{array}$

$\begin{array}{lllll}6 & 0 & 0 & 0 & 0\end{array}$

$\begin{array}{llllll}8 & 6 & 0 & 0 & 0 & 0 \\ 0 & 7 & 0 & 0 & 0 & 0\end{array}$

$\begin{array}{lllll}6 & 7 & 0 & 0 & 0\end{array}$

$\begin{array}{llllll}9 & 8 & 0 & 0 & 0 \\ 7 & 8 & 0 & 0 & 0\end{array}$

$\begin{array}{rrrrrr}0 & 9 & 0 & 0 & 0 & 0 \\ 4 & 18 & 0 & 0 & 0 & 0\end{array}$

END

$\$ \$ \$$

imidazo-(2,3-b)-thioxazole_b.sdf

Cosmotherm 3D

$\begin{array}{llllllllllll}17 & 18 & 0 & 0 & 0 & 0 & 0 & 0 & 0 & 0 & 0 & \mathrm{~V} 2000\end{array}$

$\begin{array}{llllllllllllll}3.0440 & 0.7249 & -0.3622 & \mathrm{C} & 0 & 0 & 0 & 0 & 0 & 0 & 0 & 0 & 0 & 1 \\ 1.6115 & 1.0503 & -0.1741 & \mathrm{~N} & 0 & 0 & 0 & 0 & 0 & 0 & 0 & 0 & 0 & 2\end{array}$

$\begin{array}{llllllllllllllll}1.6115 & 1.0503 & -0.1741 & & 0 & 0 & 0 & 0 & 0 & 0 & 0 & 0 & 0 & 1 \\ 3.1096 & -0.6878 & 0.2711 & \mathrm{C} & 0 & 0 & 0 & 0 & 0 & 0 & 0 & 0 & 0 & \end{array}$

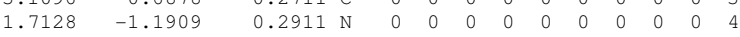

$\begin{array}{llllllllllllll}0.9616 & -0.1654 & 0.0230 & \mathrm{C} & 0 & 0 & 0 & 0 & 0 & 0 & 0 & 0 & 0 & 5\end{array}$

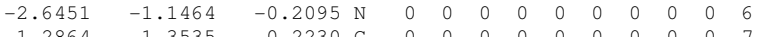

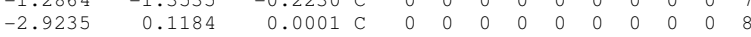

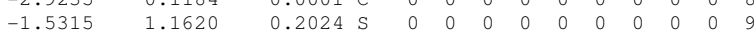

$\begin{array}{llllllllllllll}-0.4988 & -0.2401 & -0.0205 & \mathrm{C} & 0 & 0 & 0 & 0 & 0 & 0 & 0 & 0 & 0 & 10\end{array}$

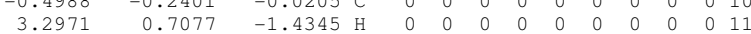

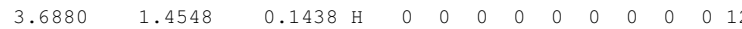

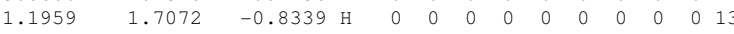

$\begin{array}{lllllllllllll}3.4799 & -0.6441 & 1.3094 \mathrm{H} & 0 & 0 & 0 & 0 & 0 & 0 & 0 & 0 & 0 & 14\end{array}$

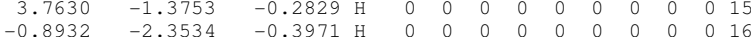

$\begin{array}{llllllllllllll}-3.9291 & 0.5313 & 0.0577 & \mathrm{H} & 0 & 0 & 0 & 0 & 0 & 0 & 0 & 0 & 0 & 17\end{array}$

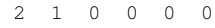

$\begin{array}{rlllll}3 & 1 & 1 & 0 & 0 & 0\end{array}$

$\begin{array}{lllllll}2 & 1 & 0 & 0 & 0 & 0 \\ 5 & 2 & 0 & 0 & 0 & 0 & 0\end{array}$

$\begin{array}{lllllllll}3 & 2 & 0 & 0 & 0 & 0\end{array}$

$\begin{array}{llllll}4 & 3 & 0 & 0 & 0 & 0\end{array}$

$\begin{array}{llllll}4 & 0 & 0 & 0 & 0\end{array}$

$\begin{array}{lllllll}5 & 5 & 0 & 0 & 0 & 0\end{array}$

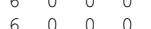

$\begin{array}{lllll}7 & 0 & 0 & 0 & 0\end{array}$

$\begin{array}{rlllll}9 & 0 & 0 & 0 & 0\end{array}$

8 FND

$\$ \$ \$$

imidazole-cation.sdf

$\begin{array}{lllllllllllll}10 & 10 & 0 & 0 & 0 & 0 & 0 & 0 & 0 & 0 & 0 & \text { v2 } 2000\end{array}$ 


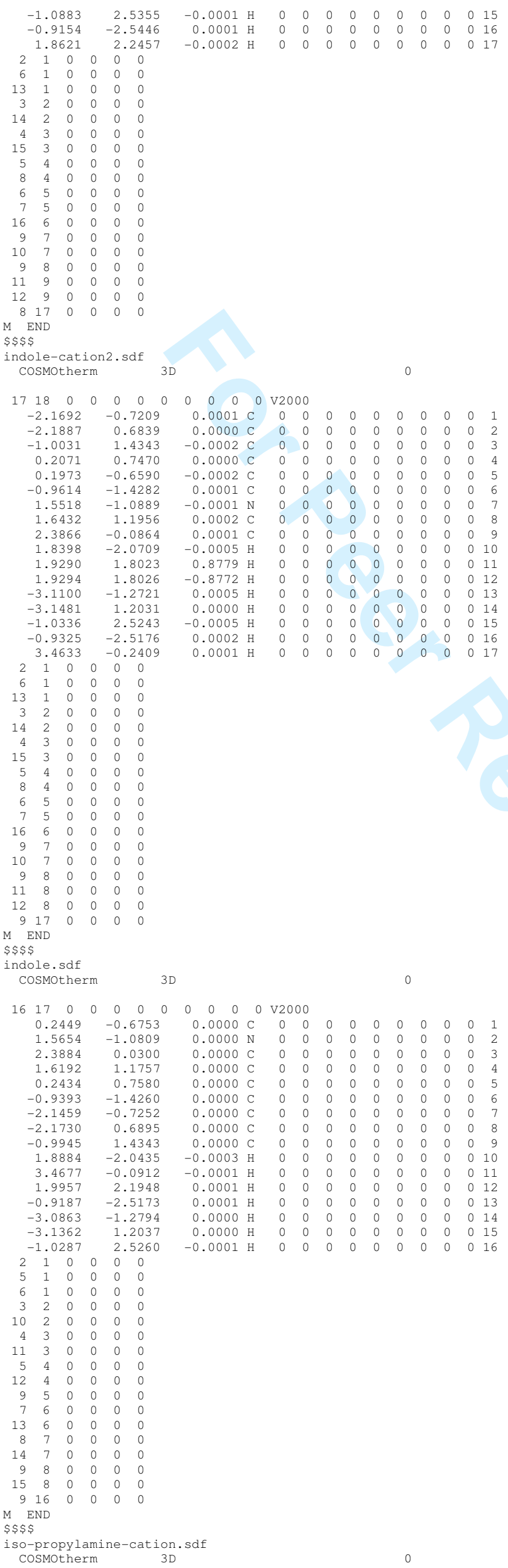




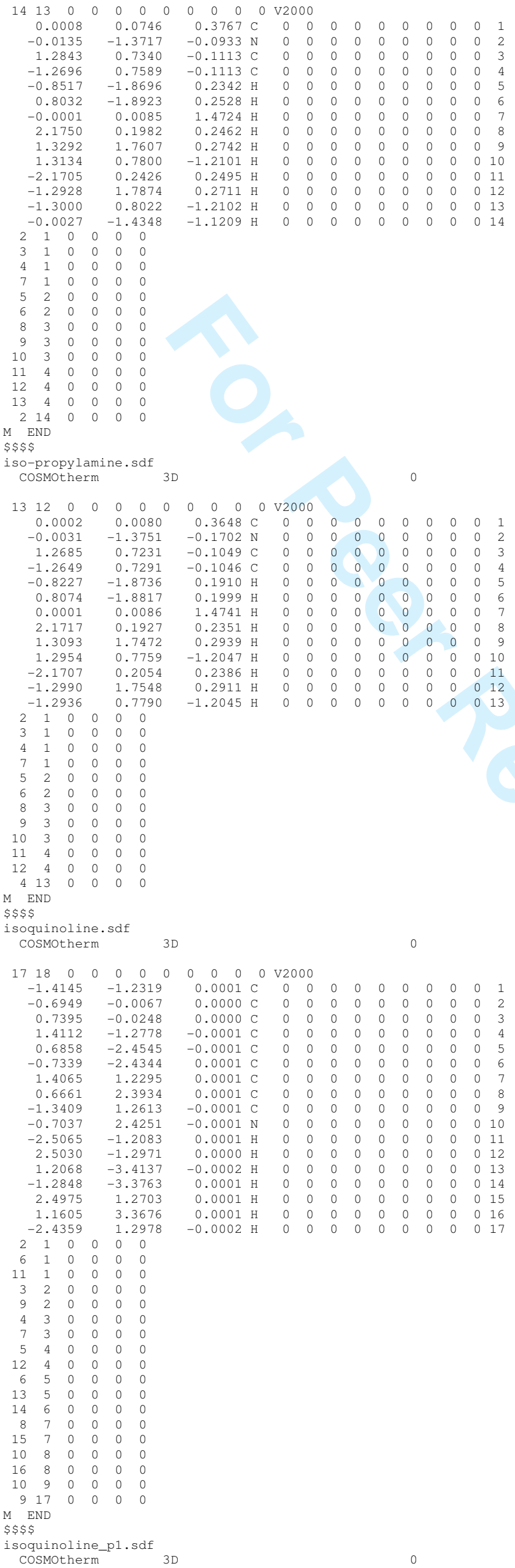




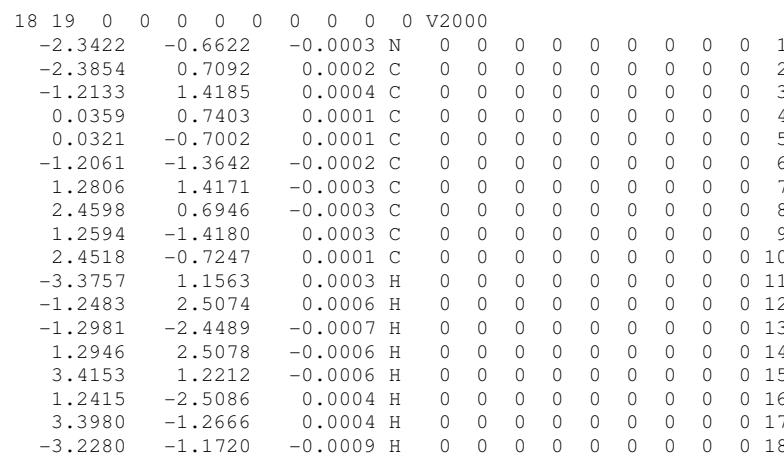




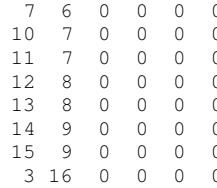




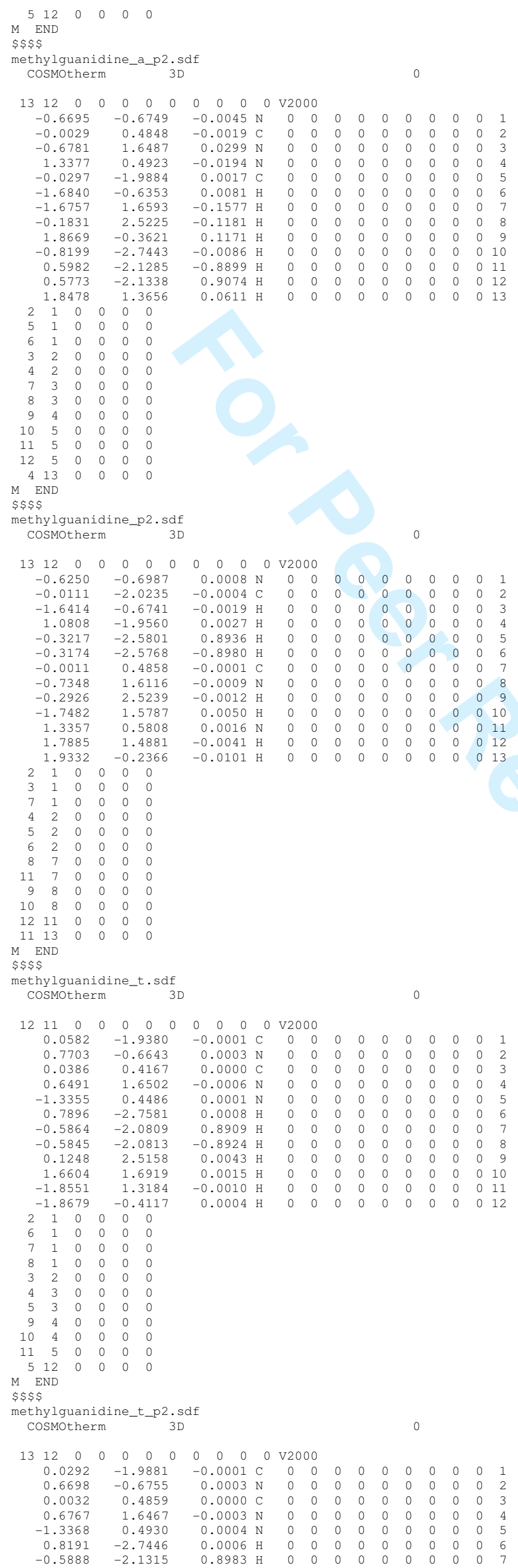


$\begin{array}{llllllllllllll}-0.5875 & -2.1317 & -0.8994 & \mathrm{H} & 0 & 0 & 0 & 0 & 0 & 0 & 0 & 0 & 0 & 8 \\ 0.1881 & 2.5352 & -0.0004 & \mathrm{H} & 0 & 0 & 0 & 0 & 0 & 0 & 0 & 0 & 0 & 9\end{array}$

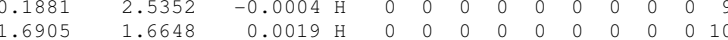

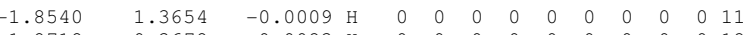

$\begin{array}{lllllllllllll}-1.8718 & -0.3678 & -0.0023 \mathrm{H} & 0 & 0 & 0 & 0 & 0 & 0 & 0 & 0 & 0 & 12\end{array}$

$\begin{array}{llllll}2 & 1 & 0 & 0 & 0 \\ 6 & 1 & 0 & 0 & 0\end{array}$

$\begin{array}{llllll}7 & 1 & 0 & 0 & 0\end{array}$

$\begin{array}{lllll}1 & 0 & 0 & 0 & 0\end{array}$

$\begin{array}{lllll}3 & 0 & 0 & 0\end{array}$

$\begin{array}{llllll}3 & 0 & 0 & 0 & 0 & 0\end{array}$

$10 \quad 4 \quad 0 \quad 0000$

$1 \quad 5 \quad 000000$

$\begin{array}{llllll}5 & 0 & 0 & 0 & 0\end{array}$

END

\$S\$

n-butylamine-cation.sdf

$\begin{array}{llllllllllll}17 & 16 & 0 & 0 & 0 & 0 & 0 & 0 & 0 & 0 & 0 & \mathrm{~V} 2000\end{array}$

$\begin{array}{llllllllllllll}2.6046 & 0.0002 & -0.3317 & \mathrm{C} & 0 & 0 & 0 & 0 & 0 & 0 & 0 & 0 & 0 & 1 \\ 1.3307 & 0.0000 & 0.5192 & \mathrm{C} & 0 & 0 & 0 & 0 & 0 & 0 & 0 & 0 & 0 & 2\end{array}$

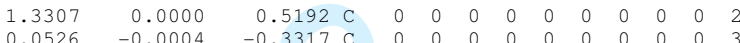

$\begin{array}{llllllllllllll}0.0526 & -0.0004 & -0.3317 & C & 0 & 0 & 0 & 0 & 0 & 0 & 0 & 0 & 0 & 3\end{array}$

$\begin{array}{llllllllllllll}-1.1973 & -0.0001 & 0.5378 & \mathrm{C} & 0 & 0 & 0 & 0 & 0 & 0 & 0 & 0 & 0 & 4\end{array}$

$\begin{array}{llllllllllllll}-2.4473 & 0.0002 & -0.3038 & \mathrm{~N} & 0 & 0 & 0 & 0 & 0 & 0 & 0 & 0 & 0 & 5 \\ -3.2958 & 0.0054 & 0.2767 & \mathrm{H} & 0 & 0 & 0 & 0 & 0 & 0 & 0 & 0 & 0 & \end{array}$

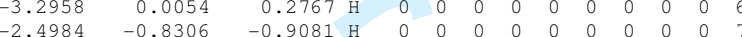

$\begin{array}{rrrrrrrrrrrrrr}3.5030 & 0.0016 & 0.3022 & \mathrm{H} & 0 & 0 & 0 & 0 & 0 & 0 & 0 & 0 & 0 & 8\end{array}$

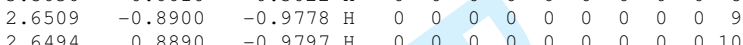

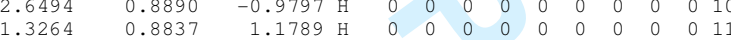

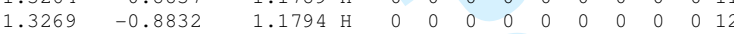

$\begin{array}{llllllllllllll}0.0451 & -0.8882 & -0.9863 & \mathrm{H} & 0 & 0 & 0 & 0 & 0 & 0 & 0 & 0 & 0 & 13\end{array}$

$\begin{array}{lllllllllllll}0.0450 & 0.8871 & -0.9868 \mathrm{H} & 0 & 0 & 0 & 0 & 0 & 0 & 0 & 0 & 0 & 14\end{array}$

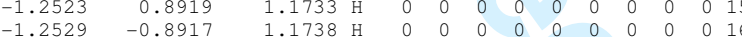

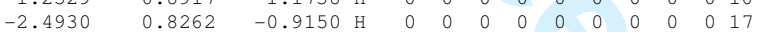

-butylamine.sdf
CosMotherm

$\begin{array}{lllllllllllll}16 & 15 & 0 & 0 & 0 & 0 & 0 & 0 & 0 & 0 & 0 & \mathrm{v} 2000\end{array}$

$\begin{array}{llllllllllllll}2.5761 & 0.0047 & -0.3223 & \mathrm{C} & 0 & 0 & 0 & 0 & 0 & 0 & 0 & 0 & 0 & 1\end{array}$

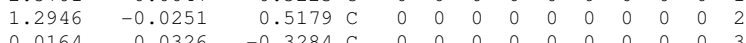

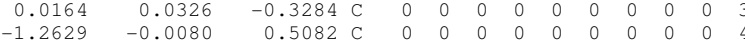

$\begin{array}{lllllllllllllll}-2.4656 & 0.0520 & -0.3511 & \mathrm{~N} & 0 & 0 & 0 & 0 & 0 & 0 & 0 & 0 & 0 & 5\end{array}$

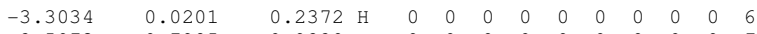

$\begin{array}{llllllllllllll}-2.5073 & -0.7985 & -0.9220 & \mathrm{H} & 0 & 0 & 0 & 0 & 0 & 0 & 0 & 0 & 0 & 7\end{array}$

$\begin{array}{lllllllllllll}3.4738 & -0.0398 & 0.3121 \mathrm{H} & 0 & 0 & 0 & 0 & 0 & 0 & 0 & 0 & 0\end{array}$

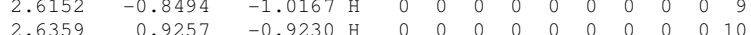

$\begin{array}{lllllllllllll}1.2993 & 0.8218 & 1.2259 \mathrm{H} & 0 & 0 & 0 & 0 & 0 & 0 & 0 & 0 & 0 & 11\end{array}$

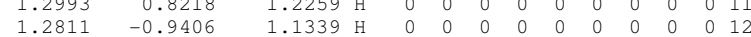

$\begin{array}{lllllllllllll}0.0105 & -0.8175 & -1.0354 \mathrm{H} & 0 & 0 & 0 & 0 & 0 & 0 & 0 & 0 & 0 & 13\end{array}$

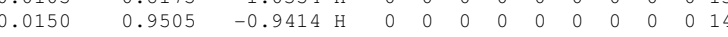

$\begin{array}{llllllllllll}0.8641 & 1.1822 \mathrm{H} & 0 & 0 & 0 & 0 & 0 & 0 & 0 & 0 & 0 & 15\end{array}$

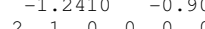

$8 \begin{array}{lllllll}8 & 1 & 0 & 0 & 0 & 0\end{array}$

$9 \quad 1 \quad 000000$

$\begin{array}{lllllll}1 & 1 & 0 & 0 & 0 & 0 \\ 3 & 2 & 0 & 0 & 0 & 0\end{array}$

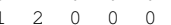

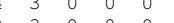

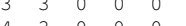

$\begin{array}{llllll}5 & 3 & 0 & 0 & 0 & 0\end{array}$

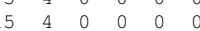

$\begin{array}{rllll}5 & 0 & 0 & 0 & 0 \\ 5 & 0 & 0 & 0 & 0\end{array}$

$\begin{array}{llllll}4 & 16 & 0 & 0 & 0 & 0\end{array}$

$M$ END

n-heptylamine-cation.sdf

cosmotherm

$\begin{array}{llllllllllll}26 & 25 & 0 & 0 & 0 & 0 & 0 & 0 & 0 & 0 & 0 & \mathrm{v} 2000\end{array}$

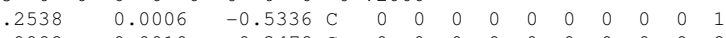

$\begin{array}{llllllllllllll}1.9982 & -0.0010 & 0.3478 & \mathrm{C} & 0 & 0 & 0 & 0 & 0 & 0 & 0 & 0 & 0 & 2\end{array}$

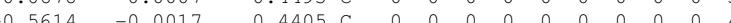

$\begin{array}{rrrrrrrrrrrrrr}-0.5614 & -0.0017 & 0.4405 & C & 0 & 0 & 0 & 0 & 0 & 0 & 0 & 0 & 0 & 4 \\ -1.8697 & -0.0013 & -0.3628 & \text { C } & 0 & 0 & 0 & 0 & 0 & 0 & 0 & 0 & 0 & 5\end{array}$ 


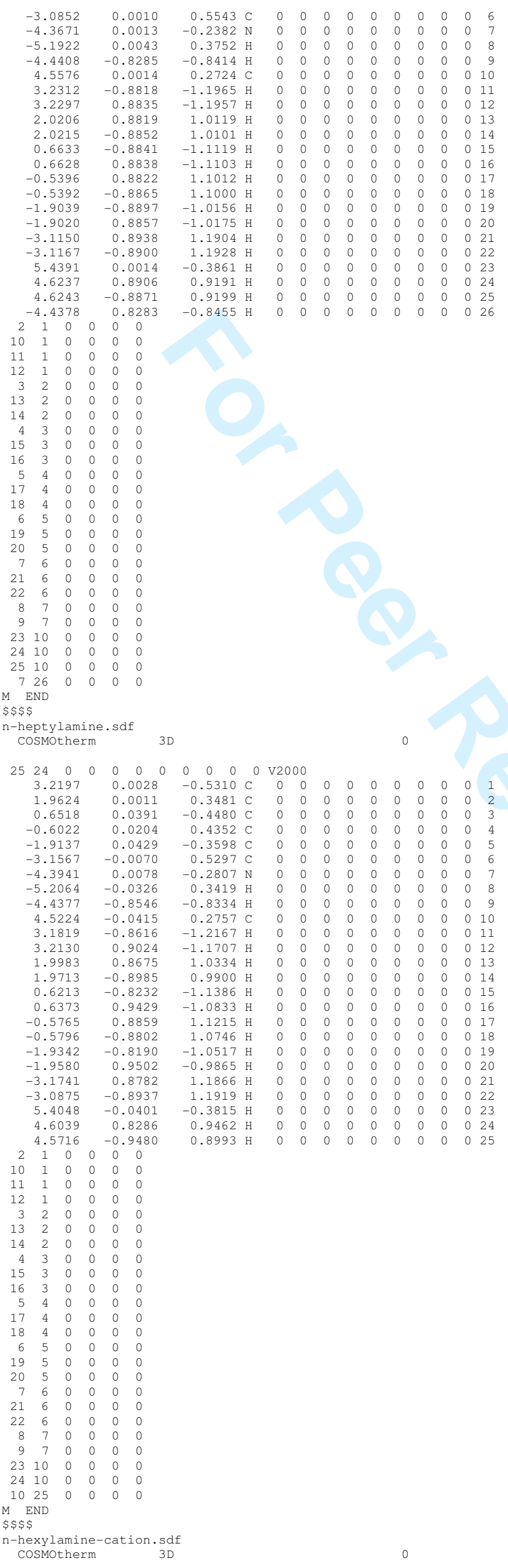


$\begin{array}{llllllllllll}23 & 22 & 0 & 0 & 0 & 0 & 0 & 0 & 0 & 0 & 0 & \mathrm{~V} 2000\end{array}$

$\begin{array}{llllllllllllll}3.9012 & -0.0004 & 0.3550 & \mathrm{C} & 0 & 0 & 0 & 0 & 0 & 0 & 0 & 0 & 0 & 1\end{array}$

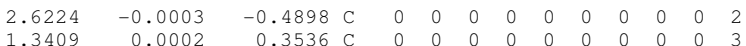

$\begin{array}{llllllllllllll}0.0601 & 0.0008 & -0.4902 & \mathrm{C} & 0 & 0 & 0 & 0 & 0 & 0 & 0 & 0 & 0 & 4\end{array}$

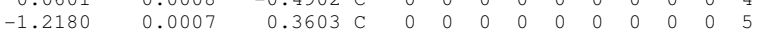

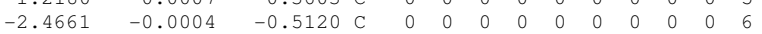

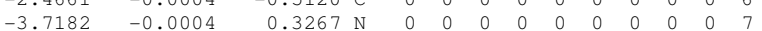

$\begin{array}{llllllllllll}-4.5652 & -0.0027 & -0.2560 \mathrm{H} & 0 & 0 & 0 & 0 & 0 & 0 & 0 & 0 & 0\end{array}$

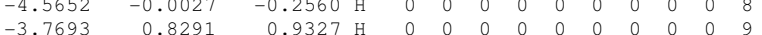

$\begin{array}{llllllllllllll}4.8013 & -0.0026 & -0.2775 & \mathrm{H} & 0 & 0 & 0 & 0 & 0 & 0 & 0 & 0 & 0 & 10\end{array}$

$\begin{array}{lllllllllllllllll}4.8013 & -0.0026 & -0.2775 & \mathrm{H} & 0 & 0 & 0 & 0 & 0 & 0 & 0 & 0 & 0 & 10\end{array}$

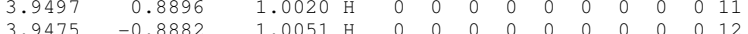

$\begin{array}{lllllllllllllllll}3.9175 & 0.8882 & 1.0051 & \mathrm{H} & 0 & 0 & 0 & 0 & 0 & 0 & 0 & 0 & 0 & 0 & 11 \\ 2.6195 & -0.8833 & -1.1521 & \mathrm{H} & 0 & 0 & 0 & 0 & 0 & 0 & 0 & 0 & 0 & 13\end{array}$

$\begin{array}{llllllllllllllllllll}2.6185 & -0.8833 & -1.1521 & \mathrm{H} & 0 & 0 & 0 & 0 & 0 & 0 & 0 & 0 & 0 & 0 & 13 \\ 2.6190 & 0.8822 & -1.1528 & \mathrm{H} & 0 & 0 & 0 & 0 & 0 & 0 & 0 & 0 & 0 & 14\end{array}$

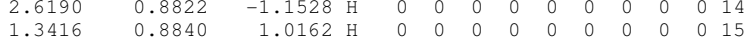

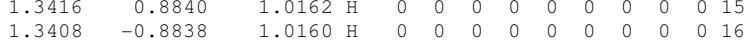

$\begin{array}{lllllllllllll}1.3458 & -0.8838 & 1.0160 \mathrm{H} & 0 & 0 & 0 & 0 & 0 & 0 & 0 & 0 & 0 & 16 \\ 0.0578 & -0.8834 & -1.1509 \mathrm{H} & 0 & 0 & 0 & 0 & 0 & 0 & 0 & 0 & 0 & 17\end{array}$

$\begin{array}{llllllllllllll}0.0582 & 0.8854 & -1.1504 & \mathrm{H} & 0 & 0 & 0 & 0 & 0 & 0 & 0 & 0 & 0 & 18\end{array}$

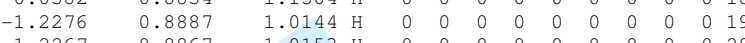

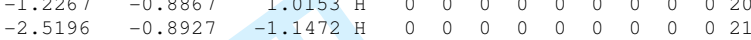

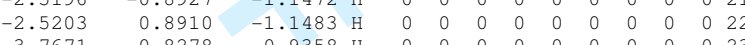

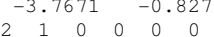

$\begin{array}{lllllll}2 & 1 & 0 & 0 & 0 & 0 & 0 \\ 0 & 1 & 0 & 0 & 0 & 0 & 0\end{array}$

$\begin{array}{lllllll}10 & 1 & 0 & 0 & 0 & 0\end{array}$

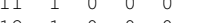

$\begin{array}{llllllll}1 & 1 & 0 & 0 & 0 & 0 & 0 \\ 3 & 2 & 0 & 0 & 0 & 0\end{array}$

$\begin{array}{lllllll}3 & 2 & 0 & 0 & 0 & 0\end{array}$

$\begin{array}{lllllll}4 & 2 & 0 & 0 & 0 & 0 \\ 4 & 3 & 0 & 0 & 0 & 0\end{array}$

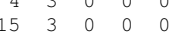

$\begin{array}{llllll}16 & 3 & 0 & 0 & 0 & 0 \\ 5 & 4 & 0 & 0 & 0 & 0\end{array}$

$\begin{array}{lllllll}7 & 4 & 0 & 0 & 0 & 0 \\ 7 & 4 & 0 & 0 & 0 & 0 & 0 \\ 18 & 4 & 0 & 0 & 0 & 0\end{array}$

$\begin{array}{lllllll}8 & 4 & 0 & 0 & 0 & 0\end{array}$

$9 \begin{array}{lllll}9 & 5 & 0 & 0 & 0\end{array}$

$\begin{array}{lllllll}20 & 5 & 0 & 0 & 0 & 0\end{array}$

$\begin{array}{lllll}6 & 0 & 0 & 0 & 0\end{array}$

$\begin{array}{llllllll}6 & 0 & 0 & 0 & 0\end{array}$

$\begin{array}{llllll}7 & 0 & 0 & 0 & 0\end{array}$

$\begin{array}{ccccc}7 & 0 & 0 & 0 & 0\end{array}$

END

-hexylamine, sdf

cosmotherm

$\begin{array}{llllllllllll}22 & 21 & 0 & 0 & 0 & 0 & 0 & 0 & 0 & 0 & 0 & \mathrm{v} 2000\end{array}$

$3.8687-0.0078-0.3493 \mathrm{C}$ V200

$\begin{array}{rr}1.3070 & -0.0264 \\ 1.020258\end{array}$

$0.0207 \quad 0.0014$

$-1.2600 \quad 0.0421$

$-2.5355-0.0058$

$-3.7422 \quad 0.0306$

$-0.0053$

$-3.7759$

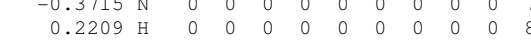

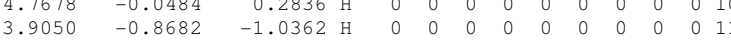

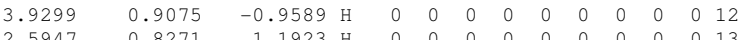

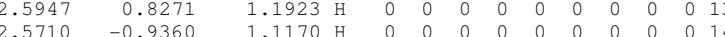

$\begin{array}{llllllllllllll}1.3006 & -0.8275 & -1.0538 \mathrm{H} & 0 & 0 & 0 & 0 & 0 & 0 & 0 & 0 & 0 & 15\end{array}$

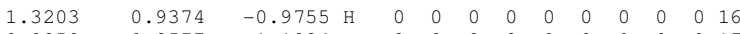

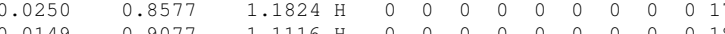

$\begin{array}{llllllllllllll}-1.2615 & -0.8126 & -1.0601 & \mathrm{H} & 0 & 0 & 0 & 0 & 0 & 0 & 0 & 0 & 0 & 19\end{array}$

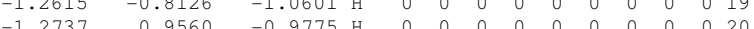

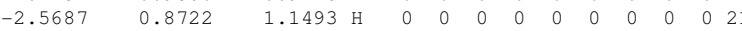

$-2.4995-0.900$

$\begin{array}{llllllllll}2 & 1 & 0 & 0 & 0 & 0\end{array}$

$\begin{array}{lllllll}10 & 1 & 0 & 0 & 0 & 0\end{array}$

$\begin{array}{llllllll}1 & 1 & 0 & 0 & 0 & 0\end{array}$

$\begin{array}{llllll}1 & 0 & 0 & 0 & 0\end{array}$

$\begin{array}{llllllll}13 & 2 & 0 & 0 & 0 & 0\end{array}$

$\begin{array}{llllllll}14 & 2 & 0 & 0 & 0 & 0\end{array}$

$\begin{array}{lllll}3 & 0 & 0 & 0\end{array}$

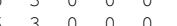

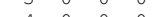

$\begin{array}{llllll}4 & 0 & 0 & 0 & 0\end{array}$

$\begin{array}{lllllll}18 & 4 & 0 & 0 & 0 & 0 & 0 \\ 6 & 5 & 0 & 0 & 0 & 0\end{array}$

$\begin{array}{lllll}5 & 0 & 0 & 0 & 0\end{array}$

$\begin{array}{llllll}5 & 0 & 0 & 0 & 0\end{array}$

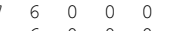

$\begin{array}{lllll}6 & 0 & 0 & 0 & 0\end{array}$

$\begin{array}{lllll}7 & 0 & 0 & 0 & 0\end{array}$

622

$\$ \$ \$$

n-octylamine-cation.sdf

cosmotherm

$\begin{array}{llllllllllll}29 & 28 & 0 & 0 & 0 & 0 & 0 & 0 & 0 & 0 & 0 & \mathrm{~V} 2000\end{array}$

$\begin{array}{lllllllllllll}2.6318 & 0.0002 & 0.3668 & \mathrm{C} & 0 & 0 & 0 & 0 & 0 & 0 & 0 & 0 & 0\end{array}$

$\begin{array}{llrlllllllllll}1.3458 & 0.0014 & -0.4692 & \mathrm{C} & 0 & 0 & 0 & 0 & 0 & 0 & 0 & 0 & 0 & 2 \\ 0.0645 & 0.0016 & 0.3742 & \mathrm{C} & 0 & 0 & 0 & 0 & 0 & 0 & 0 & 0 & 0 & 3\end{array}$ 


$\begin{array}{rrrrllllllllll}-1.2151 & 0.0015 & -0.4715 & \mathrm{C} & 0 & 0 & 0 & 0 & 0 & 0 & 0 & 0 & 0 & 4 \\ -2.4950 & 0.0016 & 0.3762 & \mathrm{C} & 0 & 0 & 0 & 0 & 0 & 0 & 0 & 0 & 0 & 5 \\ -3.7408 & -0.0020 & -0.4994 & \mathrm{C} & 0 & 0 & 0 & 0 & 0 & 0 & 0 & 0 & 0 & 6 \\ -4.9952 & -0.0014 & 0.3357 & \mathrm{~N} & 0 & 0 & 0 & 0 & 0 & 0 & 0 & 0 & 0 & 7 \\ -5.8406 & -0.0041 & -0.2495 & \mathrm{H} & 0 & 0 & 0 & 0 & 0 & 0 & 0 & 0 & 0 & 8 \\ -5.0481 & 0.8286 & 0.9409 & \mathrm{H} & 0 & 0 & 0 & 0 & 0 & 0 & 0 & 0 & 0 & 9 \\ 3.9132 & -0.0007 & -0.4770 \mathrm{C} & 0 & 0 & 0 & 0 & 0 & 0 & 0 & 0 & 0 & 10 \\ 2.6356 & 0.8837 & 1.0306 & \mathrm{H} & 0 & 0 & 0 & 0 & 0 & 0 & 0 & 0 & 0 & 11 \\ 2.6342 & -0.8836 & 1.0301 & \mathrm{H} & 0 & 0 & 0 & 0 & 0 & 0 & 0 & 0 & 0 & 12 \\ 1.3432 & -0.8818 & -1.1331 & \mathrm{H} & 0 & 0 & 0 & 0 & 0 & 0 & 0 & 0 & 0 & 13 \\ 1.3441 & 0.8853 & -1.1323 & \mathrm{H} & 0 & 0 & 0 & 0 & 0 & 0 & 0 & 0 & 0 & 14 \\ 0.0632 & 0.8858 & 1.0361 & \mathrm{H} & 0 & 0 & 0 & 0 & 0 & 0 & 0 & 0 & 0 & 15 \\ 0.0632 & -0.8827 & 1.0361 & \mathrm{H} & 0 & 0 & 0 & 0 & 0 & 0 & 0 & 0 & 0 & 16 \\ -1.2158 & -0.8830 & -1.1319 & \mathrm{H} & 0 & 0 & 0 & 0 & 0 & 0 & 0 & 0 & 0 & 17 \\ -1.2161 & 0.8857 & -1.1322 \mathrm{H} & 0 & 0 & 0 & 0 & 0 & 0 & 0 & 0 & 0 & 18 \\ -2.5071 & 0.8906 & 1.0289 & \mathrm{H} & 0 & 0 & 0 & 0 & 0 & 0 & 0 & 0 & 0 & 19 \\ -2.5047 & -0.8854 & 1.0318 \mathrm{H} & 0 & 0 & 0 & 0 & 0 & 0 & 0 & 0 & 0 & 20 \\ -3.7918 & -0.8954 & -1.1333 \mathrm{H} & 0 & 0 & 0 & 0 & 0 & 0 & 0 & 0 & 0 & 21 \\ -3.7938 & 0.8884 & -1.1374 & \mathrm{H} & 0 & 0 & 0 & 0 & 0 & 0 & 0 & 0 & 0 & 22 \\ 5.1936 & -0.0018 & 0.3656 \mathrm{C} & 0 & 0 & 0 & 0 & 0 & 0 & 0 & 0 & 0 & 23 \\ 3.9083 & -0.8833 & -1.1401 \mathrm{H} & 0 & 0 & 0 & 0 & 0 & 0 & 0 & 0 & 0 & 24 \\ 3.9098 & 0.8819 & -1.1401 & \mathrm{H} & 0 & 0 & 0 & 0 & 0 & 0 & 0 & 0 & 0 & 25 \\ 6.0935 & -0.0037 & -0.2674 & \mathrm{H} & 0 & 0 & 0 & 0 & 0 & 0 & 0 & 0 & 0 & 26 \\ 5.2430 & 0.8874 & 1.0137 & \mathrm{H} & 0 & 0 & 0 & 0 & 0 & 0 & 0 & 0 & 0 & 27 \\ 5.2404 & -0.8901 & 1.0152 \mathrm{H} & 0 & 0 & 0 & 0 & 0 & 0 & 0 & 0 & 0 & 28 \\ -5.0458 & -0.8283 & 0.9453 & \mathrm{H} & 0 & 0 & 0 & 0 & 0 & 0 & 0 & 0 & 0 & 29\end{array}$


$\begin{array}{llllll}21 & 6 & 0 & 0 & 0 & 0\end{array}$

$\begin{array}{llllll}22 & 6 & 0 & 0 & 0 & 0 \\ 8 & 7 & 0 & 0 & 0 & 0\end{array}$

$\begin{array}{llllll}8 & 7 & 0 & 0 & 0 & 0 \\ 9 & 7 & 0 & 0 & 0 & 0\end{array}$

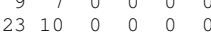

$\begin{array}{llllll}24 & 10 & 0 & 0 & 0 & 0\end{array}$

$\begin{array}{llllll}25 & 10 & 0 & 0 & 0 & 0 \\ 26 & 23 & 0 & 0 & 0 & 0\end{array}$

$\begin{array}{llllll}27 & 23 & 0 & 0 & 0 & 0\end{array}$

$\begin{array}{lll}23 & 28 \\ 2 & & -1\end{array}$

$\$ \$ \$$

n-pentylamine-cation.sdf

3D

\begin{tabular}{|c|c|c|c|c|c|c|c|c|c|c|c|c|}
\hline $\begin{array}{lll}0 & 19 & 0\end{array}$ & $\begin{array}{lll}0 & 0 & 0\end{array}$ & $\begin{array}{lll}0 & 0 & 0\end{array}$ & 0 & $\mathrm{~V} 20$ & & & & & & & & \\
\hline 3.2728 & 0.0009 & 0.2228 & $\mathrm{C}$ & 0 & 0 & 0 & 0 & 0 & 0 & 0 & 0 & 0 \\
\hline 1.9469 & -0.0002 & -0.5457 & $\mathrm{C}$ & 0 & 0 & 0 & 0 & 0 & 0 & 0 & 0 & 0 \\
\hline 0.7220 & -0.0008 & 0.3777 & $\mathrm{C}$ & 0 & 0 & 0 & 0 & 0 & 0 & 0 & 0 & 0 \\
\hline-0.6076 & -0.0009 & -0.3895 & $\mathrm{C}$ & 0 & 0 & 0 & 0 & 0 & 0 & 0 & 0 & 0 \\
\hline-1.7981 & -0.0003 & 0.5596 & C & 0 & 0 & 0 & 0 & 0 & 0 & 0 & 0 & 0 \\
\hline-3.1007 & 0.0009 & -0.1984 & $\mathrm{~N}$ & 0 & 0 & 0 & 0 & 0 & 0 & 0 & 0 & 0 \\
\hline-3.9091 & 0.0036 & 0.4368 & $\mathrm{H}$ & 0 & 0 & 0 & 0 & 0 & 0 & 0 & 0 & 0 \\
\hline-3.1908 & -0.8282 & -0.8003 & $\mathrm{H}$ & 0 & 0 & 0 & 0 & 0 & 0 & 0 & 0 & 0 \\
\hline 4.1331 & 0.0006 & -0.4627 & $\mathrm{H}$ & 0 & 0 & 0 & 0 & 0 & 0 & 0 & 0 & 0 \\
\hline 3.3579 & 0.8906 & 0.8662 & $\mathrm{H}$ & 0 & 0 & 0 & 0 & 0 & 0 & 0 & 0 & 0 \\
\hline 3.3584 & -0.8874 & 0.8678 & $\mathrm{H}$ & 0 & 0 & 0 & 0 & 0 & 0 & 0 & 0 & 0 \\
\hline 1.9028 & -0.8833 & -1.2057 & $\mathrm{H}$ & 0 & 0 & 0 & 0 & 0 & 0 & 0 & 0 & 0 \\
\hline 1.9016 & 0.8826 & -1.2061 & $\mathrm{H}$ & 0 & 0 & 0 & 0 & 0 & 0 & 0 & 0 & 0 \\
\hline 0.7629 & 0.8834 & 1.0373 & $\mathrm{H}$ & 0 & 0 & 0 & 0 & 0 & 0 & 0 & 0 & 0 \\
\hline 0.7634 & -0.8852 & 1.0370 & $\mathrm{H}$ & 0 & 0 & 0 & 0 & 0 & 0 & 0 & 0 & 0 \\
\hline-0.6586 & -0.8887 & -1.0420 & $\mathrm{H}$ & 0 & 0 & 0 & 0 & 0 & 0 & 0 & 0 & 0 \\
\hline-0.6583 & 0.8865 & -1.0425 & $\mathrm{H}$ & 0 & 0 & 0 & 0 & 0 & 0 & 0 & 0 & 0 \\
\hline-1.8111 & 0.8915 & 1.1977 & $\mathrm{H}$ & 0 & 0 & 0 & 0 & 0 & 0 & 0 & 0 & 0 \\
\hline-1.8125 & -0.8923 & 1.1974 & H & 0 & & 0 & 0 & 0 & 0 & 0 & 0 & 0 \\
\hline-3.1874 & 0.8286 & -0.8028 & & 0 & & & & & & & & \\
\hline
\end{tabular}

$1874 \quad 0.828$

$\begin{array}{llllll}2 & 1 & 0 & 0 & 0 & 0 \\ 9 & 1 & 0 & 0 & 0 & 0 \\ 10 & 1 & 0 & 0 & 0 & 0\end{array}$

$\begin{array}{rrrrrrr}9 & 1 & 0 & 0 & 0 & 0 \\ 10 & 1 & 0 & 0 & 0\end{array}$

$\begin{array}{llllll}1 & 1 & 0 & 0 & 0 & 0\end{array}$

$\begin{array}{llllll}3 & 2 & 0 & 0 & 0\end{array}$

$\begin{array}{lllll}2 & 2 & 0 & 0 & 0\end{array}$

$\begin{array}{lllllll}3 & 2 & 0 & 0 & 0\end{array}$

$\begin{array}{llll}3 & 0 & 0 & 0\end{array}$

$\begin{array}{llllll}4 & 3 & 0 & 0 & 0 & 0\end{array}$

$\begin{array}{lllllll}5 & 4 & 0 & 0 & 0 & 0\end{array}$

$\begin{array}{llll}4 & 0 & 0 & 0\end{array}$

$\begin{array}{lllll}5 & 0 & 0 & 0\end{array}$

$\begin{array}{lllll}5 & 0 & 0 & 0 & 0\end{array}$

$\begin{array}{lllll}5 & 0 & 0 & 0 & 0\end{array}$

$\begin{array}{lllll}6 & 0 & 0 & 0 & 0\end{array}$

$\begin{array}{lllll}20 & 0 & 0 & 0 & 0\end{array}$

END

n-pentylamine.sdf

Cosmotherm 3 D

$\begin{array}{llllllllllll}19 & 18 & 0 & 0 & 0 & 0 & 0 & 0 & 0 & 0 & 0 & \mathrm{v} 2000\end{array}$

$\begin{array}{llllllllllllll}3.2401 & -0.0309 & 0.2271 & \mathrm{C} & 0 & 0 & 0 & 0 & 0 & 0 & 0 & 0 & 0 & 1\end{array}$

$\begin{array}{llllllllllllll}1.9152 & 0.0218 & -0.5417 & \mathrm{C} & 0 & 0 & 0 & 0 & 0 & 0 & 0 & 0 & 0 & 2\end{array}$

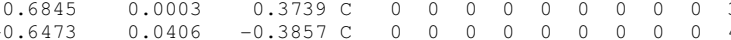

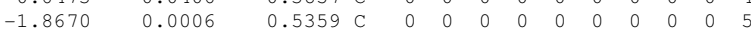

$\begin{array}{llllllllllllll}-3.1250 & 0.0290 & -0.2419 & \mathrm{~N} & 0 & 0 & 0 & 0 & 0 & 0 & 0 & 0 & 0 & 6\end{array}$

$\begin{array}{lllllllllllll}-3.9208 & -0.0036 & 0.4021 & \mathrm{H} & 0 & 0 & 0 & 0 & 0 & 0 & 0 & 0 & 0\end{array}$

$\begin{array}{lllllllllllll}-3.1920 & -0.8322 & -0.7939 & \mathrm{H} & 0 & 0 & 0 & 0 & 0 & 0 & 0 & 0 & 0\end{array}$

$\begin{array}{lllllllllllll}4.1034 & -0.0142 & -0.4548 & \mathrm{H} & 0 & 0 & 0 & 0 & 0 & 0 & 0 & 0 & 0 \\ 3.3375 & 0.8277 & 0.9101 & \mathrm{H} & 0 & 0 & 0 & 0 & 0 & 0 & 0 & 0 & 0\end{array}$

$3.3103-0.9479=0.83$

$\begin{array}{lllllllllllllll}1.8603 & -0.8315 & -1.2399 & \mathrm{H} & 0 & 0 & 0 & 0 & 0 & 0 & 0 & 0 & 0 & 0 & 12\end{array}$

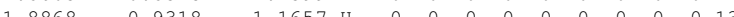

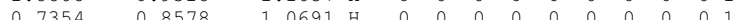

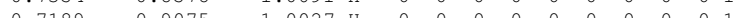

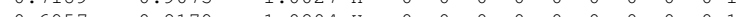

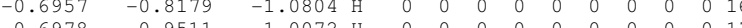

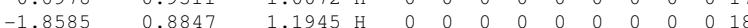

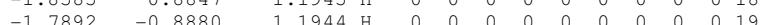

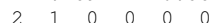

$\begin{array}{lllllll}2 & 1 & 0 & 0 & 0 & 0\end{array}$

$\begin{array}{lllllll}9 & 1 & 0 & 0 & 0 & 0\end{array}$

$\begin{array}{lllll}1 & 1 & 0 & 0 & 0\end{array}$

$\begin{array}{llllll}3 & 2 & 0 & 0 & 0 & 0\end{array}$

$\begin{array}{llllll}3 & 2 & 0 & 0 & 0\end{array}$

$\begin{array}{llll}0 & 0 & 0 & 0\end{array}$

$\begin{array}{llllll}15 & 3 & 0 & 0 & 0 & 0\end{array}$

$\begin{array}{llllll}5 & 4 & 0 & 0 & 0\end{array}$

$\begin{array}{lllllll}1 & 4 & 4 & 0 & 0 & 0 & 0\end{array}$

$\begin{array}{ccccc}5 & 0 & 0 & 0 & 0 \\ 5 & 0 & 0 & 0 & 0\end{array}$

$\begin{array}{lllll}5 & 0 & 0 & 0 & 0 \\ 6 & 0 & 0 & 0 & 0\end{array}$

$\begin{array}{rrrrr}7 & 6 & 0 & 0 & 0\end{array}$

END

-propylamine-cation.sdf

$\begin{array}{llllllllllll}14 & 13 & 0 & 0 & 0 & 0 & 0 & 0 & 0 & 0 & 0 & \mathrm{~V} 2000\end{array}$

URL: http://mc.manuscriptcentral.com/tandf/tmph 


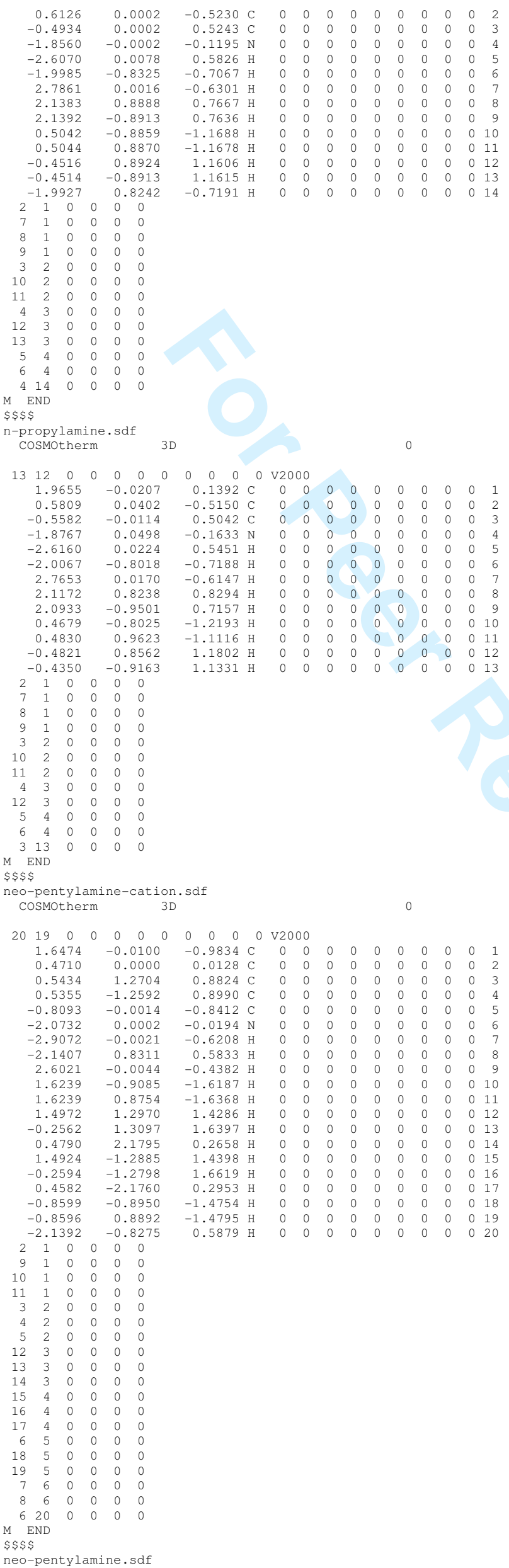




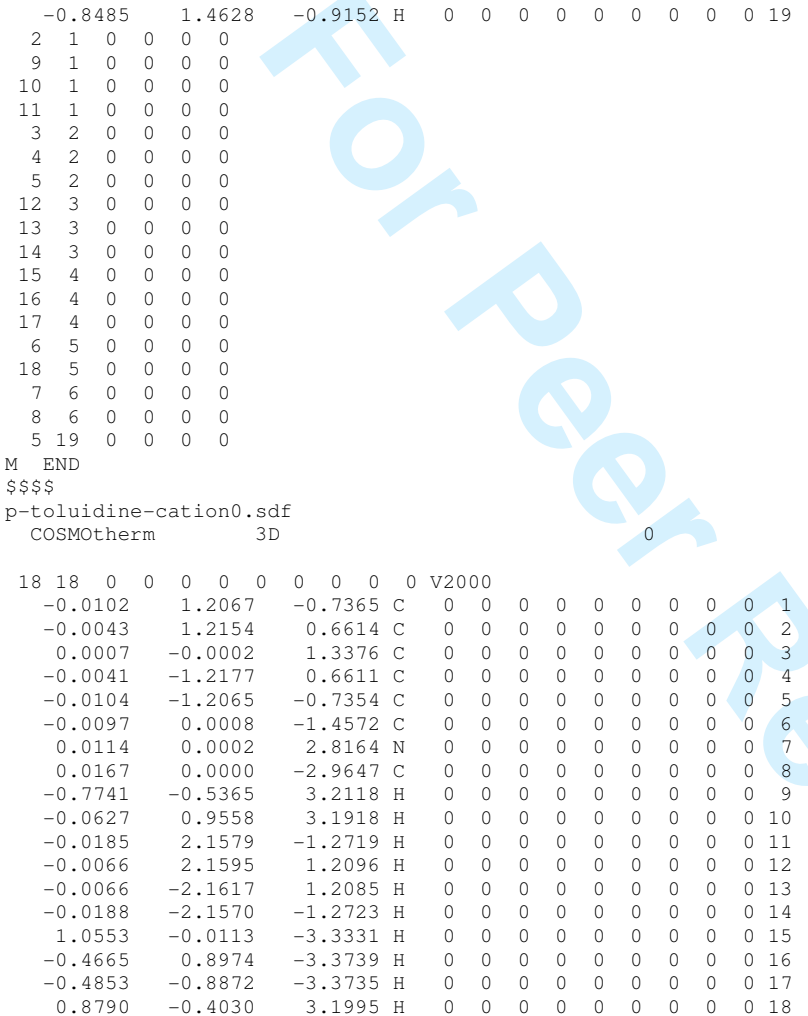




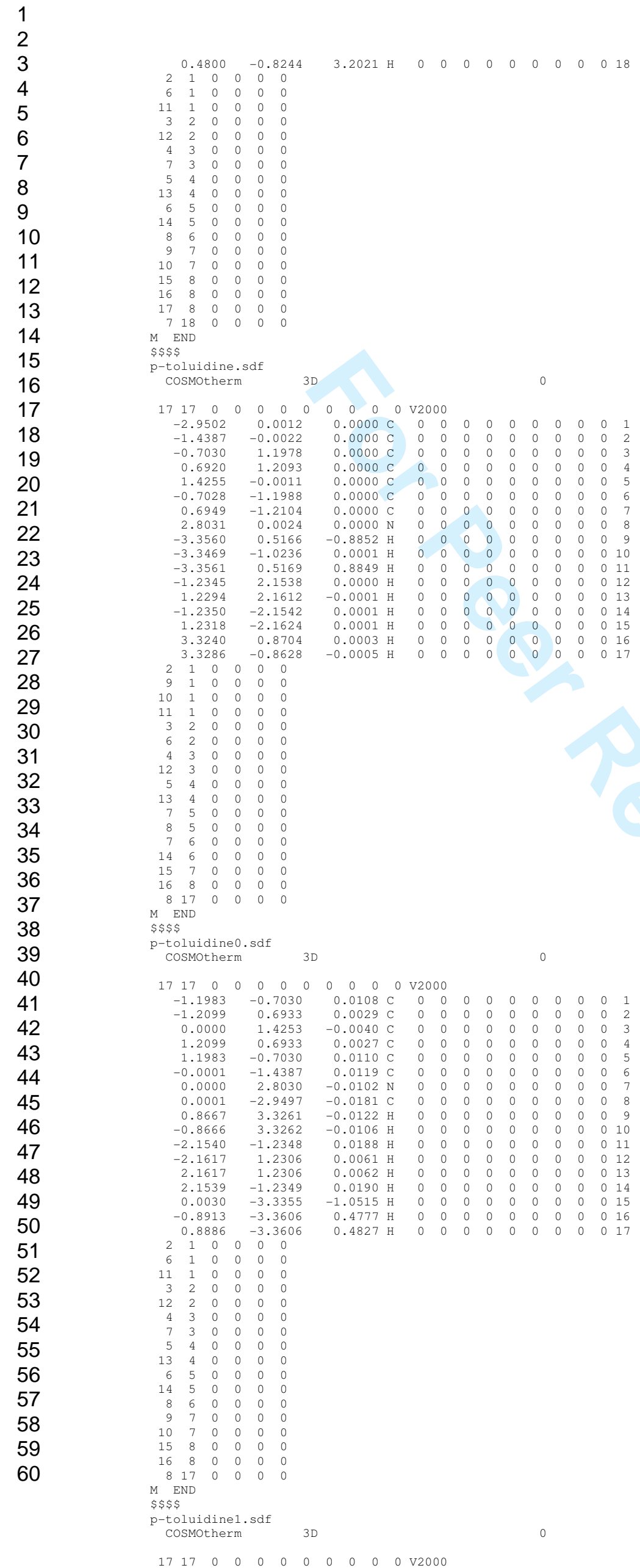

$\begin{array}{rrrrrrrrrrrrrr}-2.9502 & 0.0012 & 0.0000 & \mathrm{C} & 0 & 0 & 0 & 0 & 0 & 0 & 0 & 0 & 0 & 1 \\ -1.4387 & -0.0022 & 0.0000 & \mathrm{C} & 0 & 0 & 0 & 0 & 0 & 0 & 0 & 0 & 0 & 2\end{array}$

$\begin{array}{rrrrrrrrrrrrrr}-1.4387 & -0.0022 & 0.0000 & \mathrm{C} & 0 & 0 & 0 & 0 & 0 & 0 & 0 & 0 & 0 & 2 \\ -0.7030 & 1.1978 & 0.0000 & \mathrm{C} & 0 & 0 & 0 & 0 & 0 & 0 & 0 & 0 & 0 & 3\end{array}$

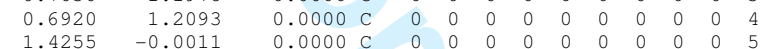

$\begin{array}{rrrrrrrrrrrrrr}1.4255 & -0.0011 & 0.0000 & \mathrm{C} & 0 & 0 & 0 & 0 & 0 & 0 & 0 & 0 & 0 & 5 \\ -0.7028 & -1.1988 & 0.0000 & \mathrm{C} & 0 & 0 & 0 & 0 & 0 & 0 & 0 & 0 & 0 & 6\end{array}$

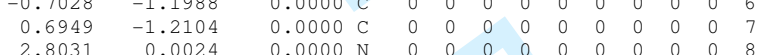

$\begin{array}{rrrrrrrrrrrrrr}2.8031 & 0.0024 & 0.0000 & \mathrm{~N} & 0 & 0 & 0 & 0 & 0 & 0 & 0 & 0 & 0 & 8 \\ -3.3560 & 0.5166 & -0.8852 & \mathrm{H} & 0 & 0 & 0 & 0 & 0 & 0 & 0 & 0 & 0 & 9\end{array}$

$\begin{array}{llllllllllllll}-3.3469 & -1.0236 & 0.0001 & \mathrm{H} & 0 & 0 & 0 & 0 & 0 & 0 & 0 & 0 & 0 & 10\end{array}$

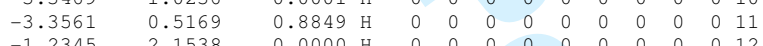

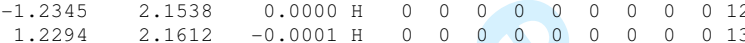

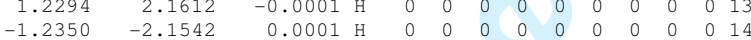

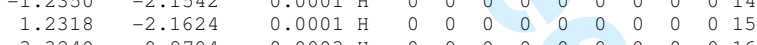

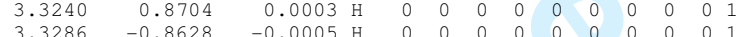

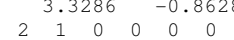

$\begin{array}{llllll}2 & 1 & 0 & 0 & 0 & 0 \\ 9 & 1 & 0 & 0 & 0 & 0\end{array}$

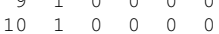

$\begin{array}{lllllll}11 & 1 & 0 & 0 & 0 & 0\end{array}$

$\begin{array}{lllllll}3 & 2 & 0 & 0 & 0 & 0 \\ 6 & 2 & 0 & 0 & 0 & 0\end{array}$

$\begin{array}{llllll}4 & 3 & 0 & 0 & 0\end{array}$

$\begin{array}{llllll}2 & 3 & 0 & 0 & 0 & 0 \\ 5 & 4 & 0 & 0 & 0 & 0\end{array}$

$\begin{array}{lllllll}3 & 4 & 0 & 0 & 0 & 0\end{array}$

$\begin{array}{llllll}7 & 5 & 0 & 0 & 0 \\ 8 & 5 & 0 & 0 & 0\end{array}$

$\begin{array}{lllll}7 & 6 & 0 & 0 & 0 \\ 4 & 6 & 0 & 0 & 0\end{array}$

$\begin{array}{lllll}7 & 0 & 0 & 0 & 0\end{array}$

$\begin{array}{rrrrrr}16 & 8 & 0 & 0 & 0 & 0 \\ 8 & 17 & 0 & 0 & 0 & 0\end{array}$

END

$\$ \$ \$$

p-toluidine0.sdf

Cosmotherm 3D

$\begin{array}{llllllllllll}17 & 17 & 0 & 0 & 0 & 0 & 0 & 0 & 0 & 0 & 0 & V 2000\end{array}$ $\begin{array}{rrrrrrrrrrrrrr}-1.1983 & -0.7030 & 0.0108 & \mathrm{C} & 0 & 0 & 0 & 0 & 0 & 0 & 0 & 0 & 0 & 1 \\ -1.2099 & 0.6933 & 0.0029 & \mathrm{C} & 0 & 0 & 0 & 0 & 0 & 0 & 0 & 0 & 0 & 2\end{array}$ $\begin{array}{llllllllllllll}0.0000 & 1.4253 & -0.0040 & \mathrm{C} & 0 & 0 & 0 & 0 & 0 & 0 & 0 & 0 & 0 & 3\end{array}$

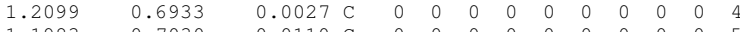

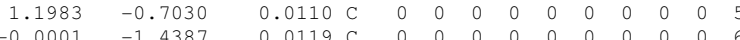

$\begin{array}{llllllllllllll}0.0001 & -1.4387 & 0.0119 & \mathrm{C} & 0 & 0 & 0 & 0 & 0 & 0 & 0 & 0 & 0 & 5 \\ 0.0000 & 2.8030 & -0.0102 & \mathrm{~N} & 0 & 0 & 0 & 0 & 0 & 0 & 0 & 0 & 0 & 6\end{array}$

$\begin{array}{lllllllllllllllll}0.0000 & -1.8030 & -0.0102 & \mathrm{~N} & 0 & 0 & 0 & 0 & 0 & 0 & 0 & 0 & 0 & 6 & 0 & 0 & 0\end{array}$

$\begin{array}{llllllllllllllll}0.0001 & -2.9497 & -0.0181 & 0 & 0 & 0 & 0 & 0 & 0 & 0 & 0 & 0 & 0 & 0 & 8\end{array}$

$\begin{array}{rrrrrrrrrrrrrr}0.8667 & 3.3261 & -0.0122 & \mathrm{H} & 0 & 0 & 0 & 0 & 0 & 0 & 0 & 0 & 0 & 8 \\ -0.8666 & 3.3262 & -0.0106 & \mathrm{H} & 0 & 0 & 0 & 0 & 0 & 0 & 0 & 0 & 0 & 10\end{array}$

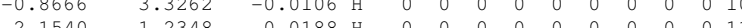

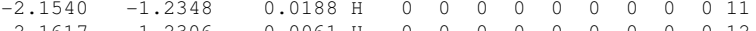

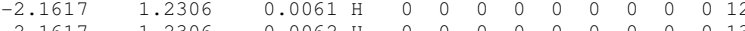

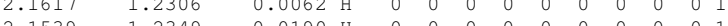

$\begin{array}{lllllllllllllllll}2.1539 & -1.2349 & 0.0190 & \mathrm{H} & 0 & 0 & 0 & 0 & 0 & 0 & 0 & 0 & 0 & 13 \\ 0.0030 & -3.3355 & -1.0515 & \mathrm{H} & 0 & 0 & 0 & 0 & 0 & 0 & 0 & 0 & 14\end{array}$

$\begin{array}{ccccccccccccc}-0.8913 & -3.3606 & -3.4777 \mathrm{H} & 0 & 0 & 0 & 0 & 0 & 0 & 0 & 0 & 0 & 16\end{array}$

$\begin{array}{lllllllllllll}0.8886 & -3.3606 & 0.4827 \mathrm{H} & 0 & 0 & 0 & 0 & 0 & 0 & 0 & 0 & 0 & 17\end{array}$

$\begin{array}{lllll}6 & 1 & 0 & 0 & 0\end{array}$

$\begin{array}{llllll}1 & 1 & 0 & 0 & 0 & 0 \\ 3 & 2 & 0 & 0 & 0 & 0\end{array}$

$\begin{array}{lllllll}2 & 2 & 0 & 0 & 0 & 0 & 0\end{array}$

$\begin{array}{lllllll}4 & 3 & 0 & 0 & 0 & 0 \\ 7 & 3 & 0 & 0 & 0 & 0\end{array}$

$\begin{array}{llllllll}7 & 3 & 0 & 0 & 0 & 0\end{array}$

$\begin{array}{llllll}5 & 4 & 0 & 0 & 0 & 0 \\ 3 & 4 & 0 & 0 & 0 & 0\end{array}$

$\begin{array}{lllllll}6 & 5 & 0 & 0 & 0 & 0\end{array}$

$\begin{array}{lllllll}4 & 5 & 0 & 0 & 0 & 0 \\ 8 & 6 & 0 & 0 & 0 & 0\end{array}$

$\begin{array}{cccccccc}7 & 7 & 0 & 0 & 0 & 0\end{array}$

$\begin{array}{llllll}9 & 7 & 0 & 0 & 0 & 0\end{array}$

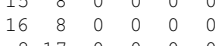

$\begin{array}{cc}8 & 17 \\ 2 & 17\end{array}$

\$\$\$

p-toluidine1.sdf

cosmotherm 3D

$\begin{array}{llllllllllll}17 & 17 & 0 & 0 & 0 & 0 & 0 & 0 & 0 & 0 & 0 & \mathrm{~V} 2000\end{array}$ 


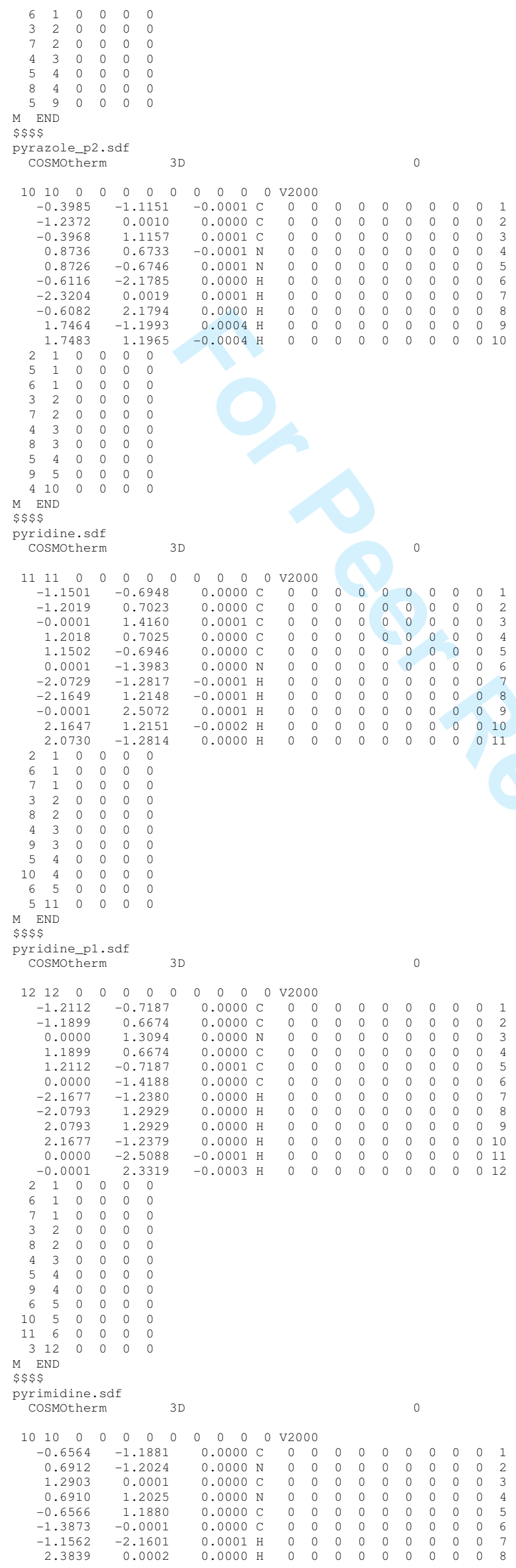




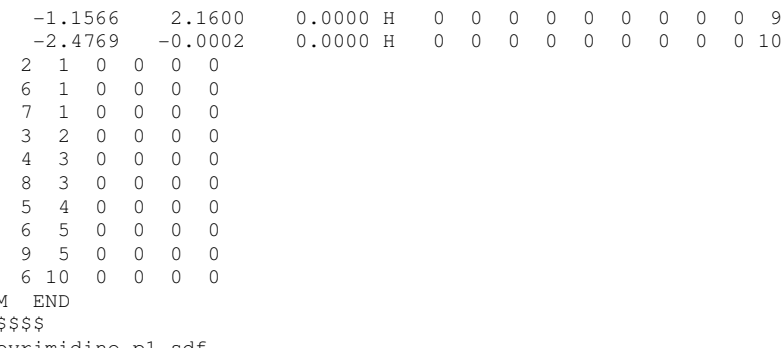




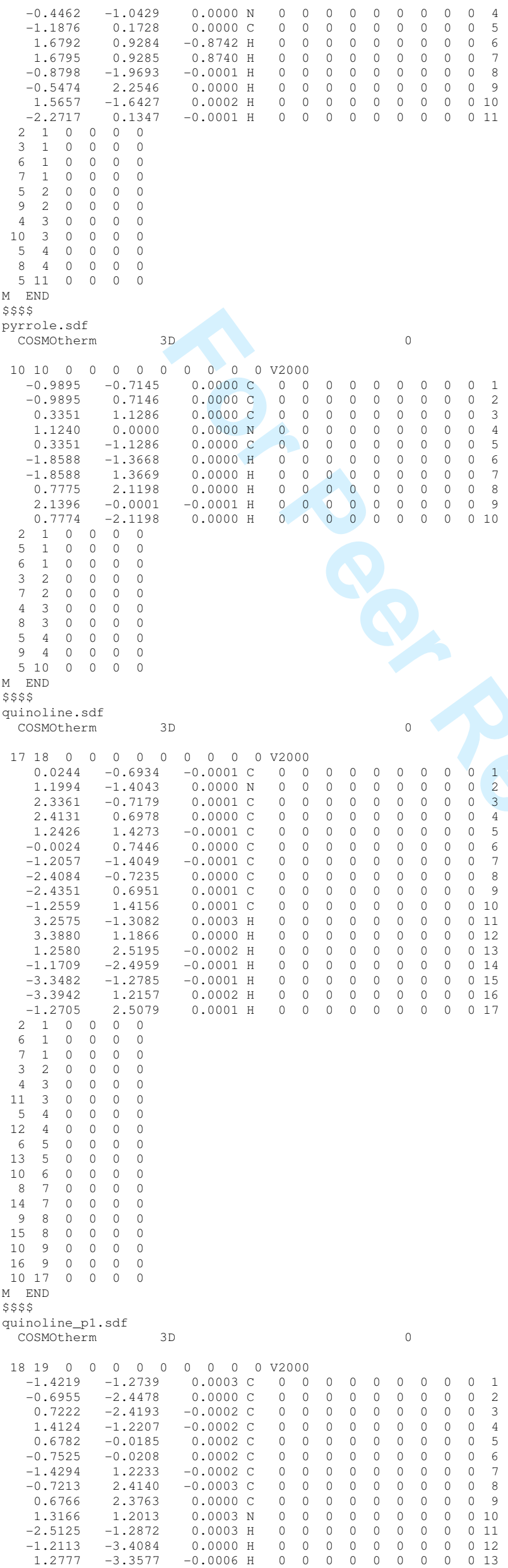




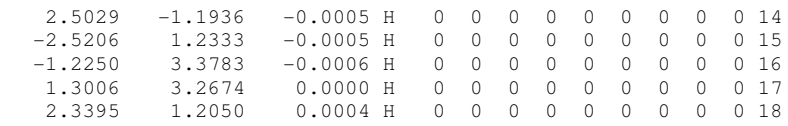




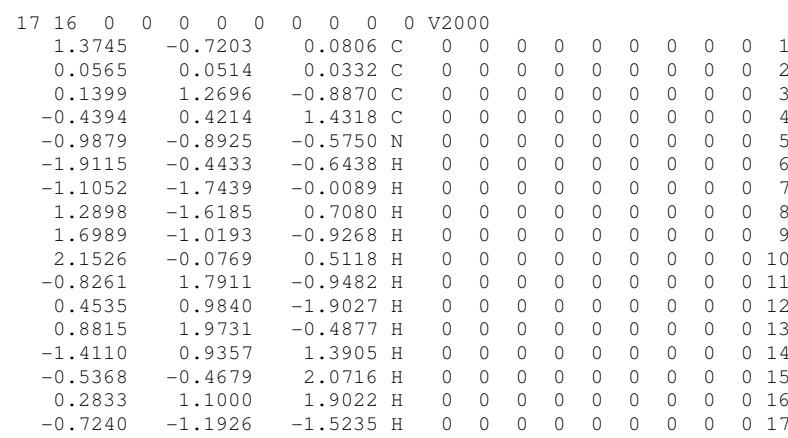




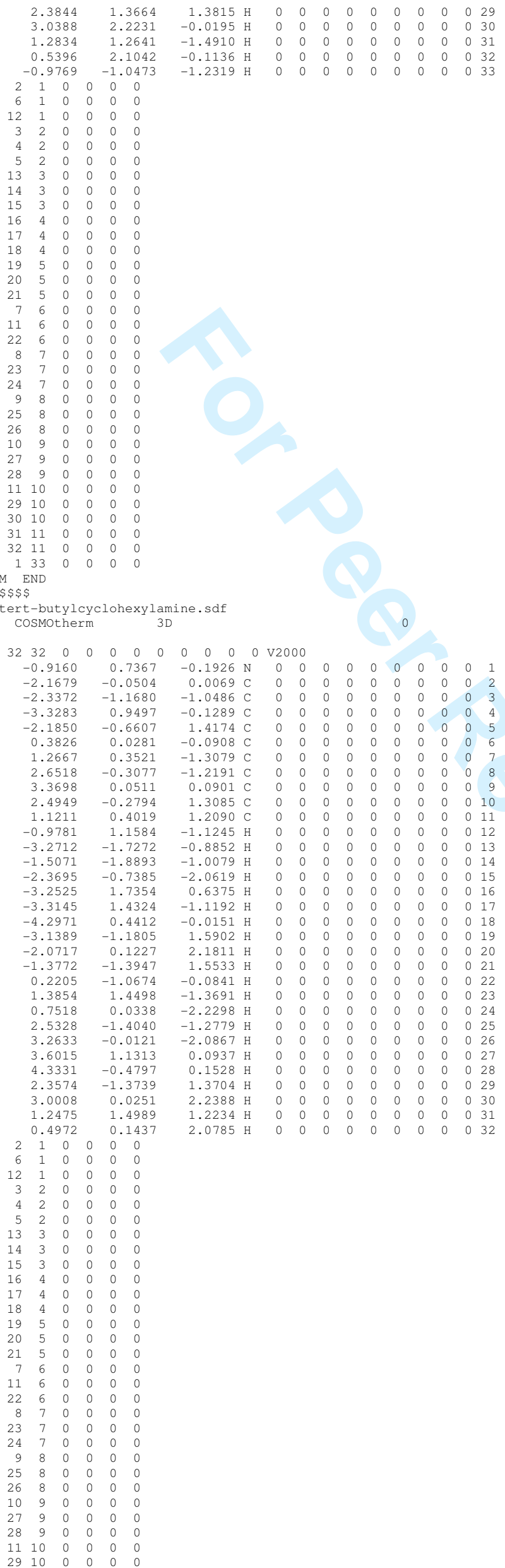




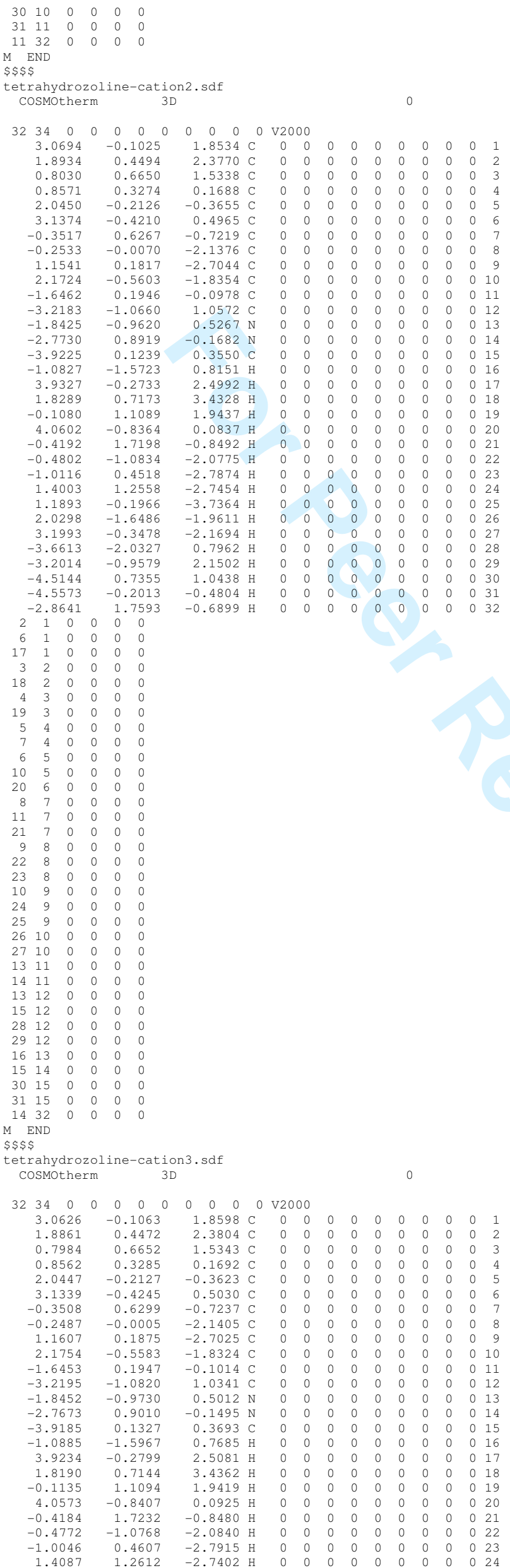




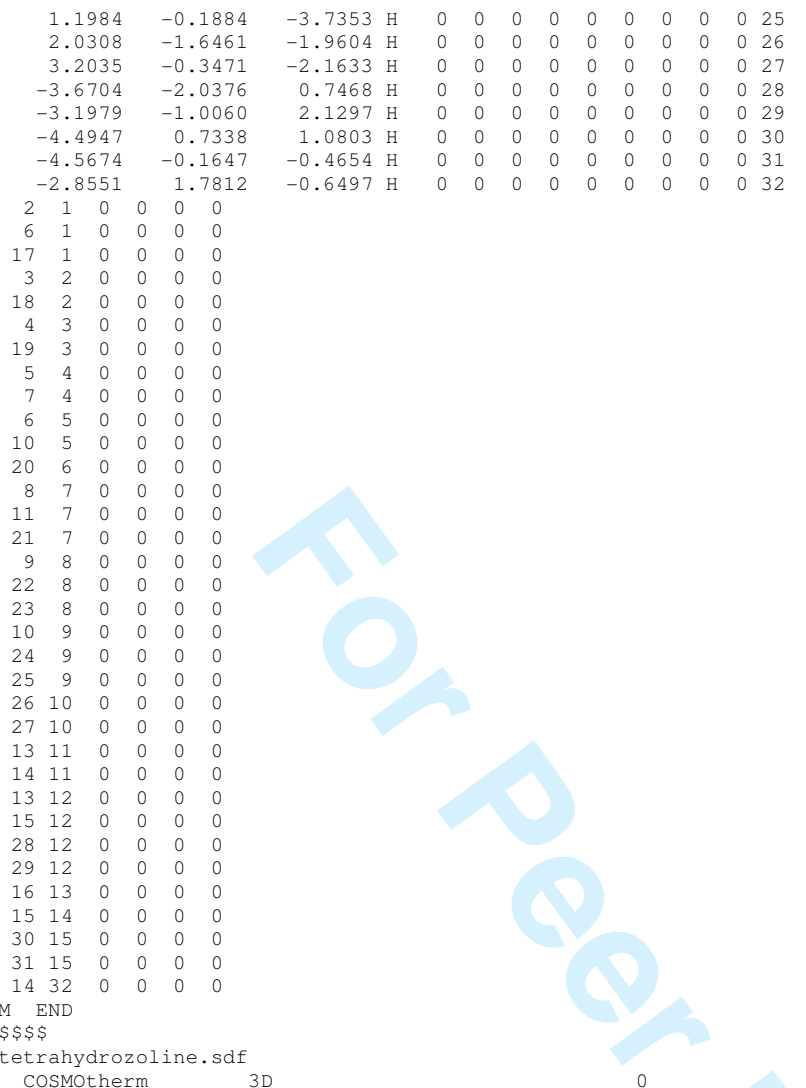

tetrahydrozoline.sdf
cosmotherm

$\begin{array}{llllllllllll}31 & 33 & 0 & 0 & 0 & 0 & 0 & 0 & 0 & 0 & 0 & v 2000\end{array}$

$\begin{array}{lllllllllllll}-0.6581 & 1.5436 & 0.9871 & \mathrm{~N} & 0 & 0 & 0 & 0 & 0 & 0 & 0 & 0 & 0\end{array}$

$\begin{array}{llllllllllllll}-1.1160 & 2.9269 & 1.2888 & C & 0 & 0 & 0 & 0 & 0 & 0 & 0 & 0 & 0 & 2 \\ -0.5134 & 3.8181 & 0.1762 & C & 0 & 0 & 0 & 0 & 0 & 0 & 0 & 0 & 0 & 3\end{array}$

$\begin{array}{lrrrrrrrrrrrrr}-0.5134 & 3.8181 & 0.1762 & \mathrm{C} & 0 & 0 & 0 & 0 & 0 & 0 & 0 & 0 & 0 & 3 \\ -0.2837 & 2.8047 & -0.8737 & \mathrm{~N} & 0 & 0 & 0 & 0 & 0 & 0 & 0 & 0 & 0 & 4\end{array}$

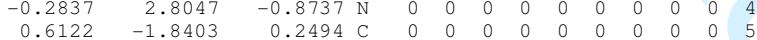

$\begin{array}{llllllllllllll}-0.1730 & -0.9394 & -0.4968 & \mathrm{C} & 0 & 0 & 0 & 0 & 0 & 0 & 0 & 0 & 0\end{array}$

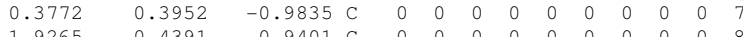

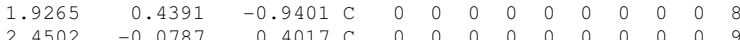

$\begin{array}{llllllllllllll}2.4502 & -0.0787 & 0.4017 & \mathrm{C} & 0 & 0 & 0 & 0 & 0 & 0 & 0 & 0 & 0 & 9 \\ 2.0639 & -1.5496 & 0.5855 & \mathrm{C} & 0 & 0 & 0 & 0 & 0 & 0 & 0 & 0 & 0 & 10\end{array}$

$\begin{array}{llllllllllllll}-0.2048 & 1.5729 & -0.2262 & \mathrm{C} & 0 & 0 & 0 & 0 & 0 & 0 & 0 & 0 & 0 & 10 \\ 0.0388 & -3.059 & 0.6612 & 0 & 0 & 0 & 0 & 0 & 0 & 0 & 0 & 0 & 0 & 11\end{array}$

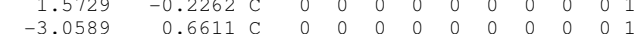

$\begin{array}{lllllllllllllllll}-1.2787 & -3.3909 & 0.3426 & C & 0 & 0 & 0 & 0 & 0 & 0 & 0 & 0 & 0 & 13\end{array}$

$\begin{array}{lllllllllllllll}-2.0548 & -2.4965 & -0.4078 & \mathrm{C} & 0 & 0 & 0 & 0 & 0 & 0 & 0 & 0 & 0 & 14\end{array}$

$\begin{array}{llllllllllllll}-1.4981 & -1.2847 & -0.8182 & \mathrm{C} & 0 & 0 & 0 & 0 & 0 & 0 & 0 & 0 & 0 & 15\end{array}$

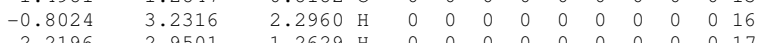

$\begin{array}{lllllllllllll}-2.2196 & 2.9501 & 1.2629 \text { H } & 0 & 0 & 0 & 0 & 0 & 0 & 0 & 0 & 0 & 17\end{array}$

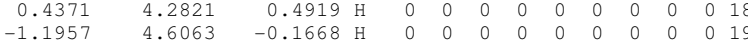

$\begin{array}{cccccccccccccc}-1.1949 & 3.0124 & -1.6050 \mathrm{H} & 0 & 0 & 0 & 0 & 0 & 0 & 0 & 0 & 0 & 20\end{array}$

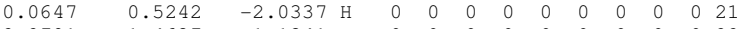

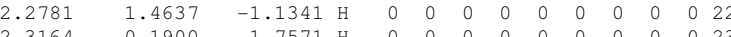

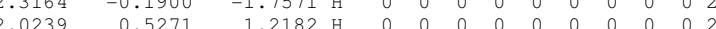

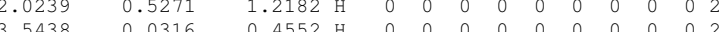

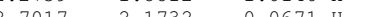

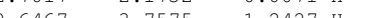

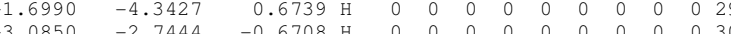

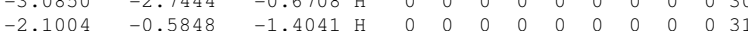

$2 \begin{array}{llllll}2 & 1 & 0 & 0 & 0 & 0\end{array}$

$\begin{array}{lllllll}11 & 1 & 0 & 0 & 0 & 0\end{array}$

$\begin{array}{lllllll}3 & 2 & 0 & 0 & 0 & 0\end{array}$

$\begin{array}{lllllll}16 & 2 & 0 & 0 & 0 & 0 \\ 17 & 2 & 0 & 0 & 0 & 0\end{array}$

$\begin{array}{llllllll}4 & 3 & 0 & 0 & 0 & 0\end{array}$

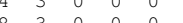

$\begin{array}{lllllllll}1 & 3 & 3 & 0 & 0 & 0 & 0 & 0\end{array}$

$\begin{array}{lllll}3 & 0 & 0 & 0 & 0\end{array}$

$\begin{array}{lllllll}1 & 4 & 0 & 0 & 0 & 0 & 0 \\ 0 & 4 & 0 & 0 & 0 & 0 & 0\end{array}$

$\begin{array}{llllll}6 & 5 & 0 & 0 & 0 & 0\end{array}$

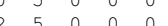

$\begin{array}{llllllll}5 & 0 & 0 & 0 & 0 & 0\end{array}$

$\begin{array}{lllllll}7 & 6 & 0 & 0 & 0 & 0\end{array}$

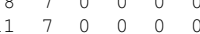

$\begin{array}{lllllll}21 & 7 & 0 & 0 & 0 & 0 \\ 9 & 8 & 0 & 0 & 0 & 0\end{array}$

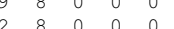

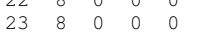

$\begin{array}{lllllll}10 & 9 & 0 & 0 & 0 & 0 & 0\end{array}$

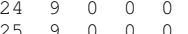

$26 \begin{array}{llllll}26 & 10 & 0 & 0 & 0\end{array}$

$2710 \begin{array}{lllll}10 & 0 & 0 & 0\end{array}$ 


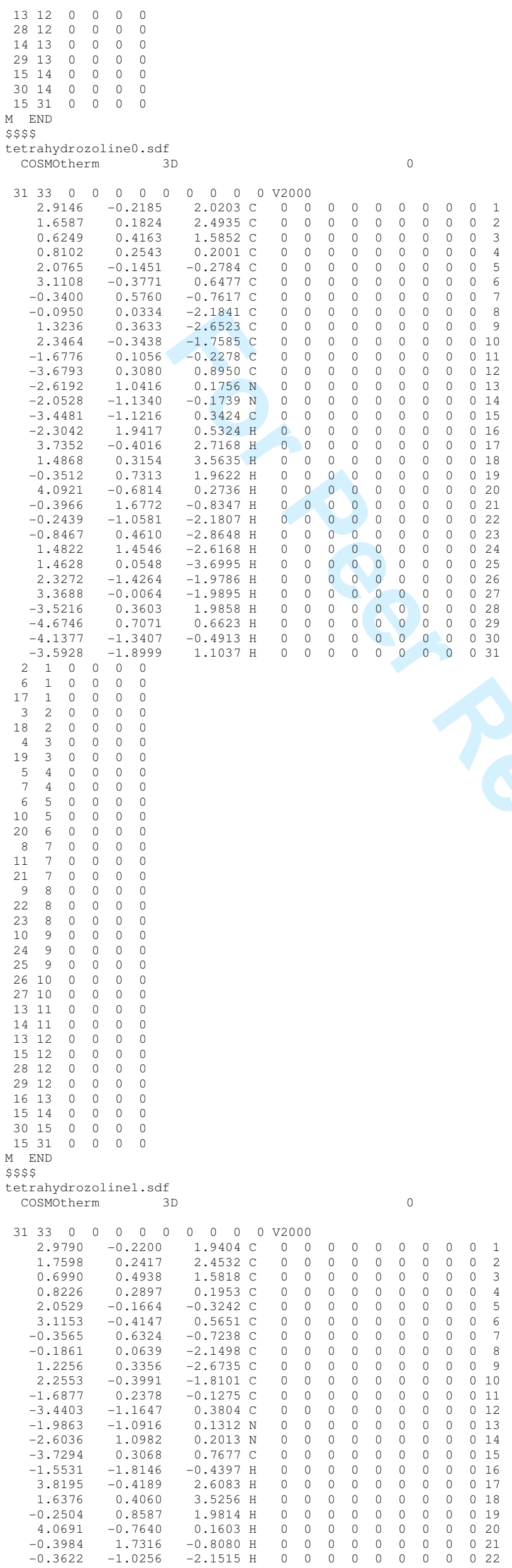




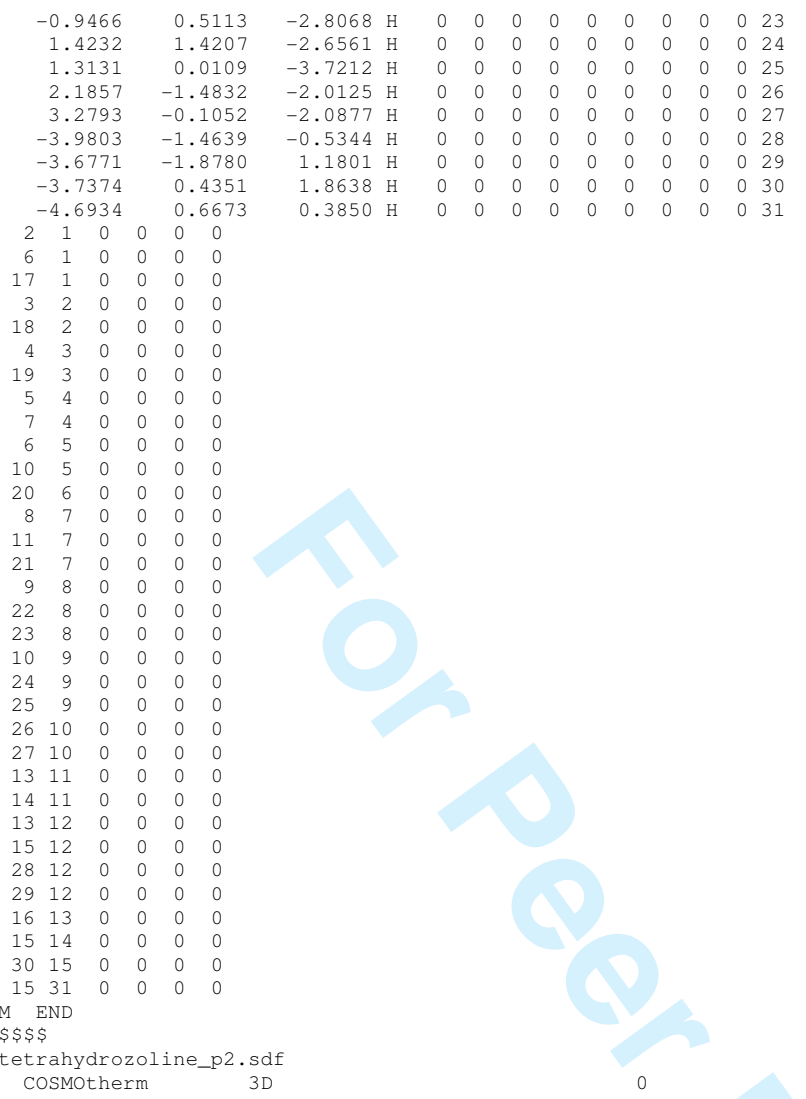

Cosmotherm

\begin{tabular}{|c|c|c|c|c|c|c|c|c|c|c|c|c|}
\hline $34 \quad 0$ & & & & & & & & & & & & \\
\hline 1.5628 & 0.3090 & 0.1184 & $\mathrm{C}$ & 0 & 0 & 0 & 0 & 0 & 0 & & & \\
\hline 2.7937 & 0.8063 & 0.1431 & $\mathrm{~N}$ & 0 & 0 & 0 & 0 & 0 & 0 & 0 & 0 & \\
\hline 3.7738 & -0.1852 & 0.6332 & C & 0 & 0 & 0 & 0 & 0 & 0 & 0 & & \\
\hline 2.8504 & -1.2997 & 1.1914 & $\mathrm{C}$ & 0 & 0 & 0 & 0 & 0 & 0 & & & \\
\hline & -0.9119 & 0.6394 & $\mathrm{~N}$ & 0 & 0 & & 0 & 0 & 0 & & & \\
\hline 0.3758 & 1.0308 & -0.4629 & C & 0 & & & 0 & 0 & 0 & & & \\
\hline 0.9339 & & 0.1450 & C & & & & & c & & & & \\
\hline 1.8247 & -0.2762 & -0.5873 & $\mathrm{C}$ & 0 & & & 0 & 0 & c & & c & \\
\hline & -0.6 & -2.0326 & C & & & & & & & & & \\
\hline 140 & -0.4 & -2.4767 & C & ( & & & c & c & c & & & \\
\hline & & -2.01 & $\mathrm{C}$ & & & & & & & & & \\
\hline-1 & 0 & 1.4652 & C & & & ( & 0 & ( & & & & \\
\hline & 0.4 & 2.0699 & C & & & & & & & & & \\
\hline-3.3 & -0.3 & 1.3495 & C & & & & 0 & ( & & & & \\
\hline-3 . & -0. & 0.0387 & $\mathrm{C}$ & & & & & & & & & \\
\hline & & -0.28 & $\mathrm{H}$ & & & & 0 & & & & & \\
\hline 4. & & 1.4 & $\mathrm{H}$ & & & & & & & & & \\
\hline & -0 . & -0.2 & $\mathrm{H}$ & & & & 0 & & & & & \\
\hline & -1 & 2.28 & $\mathrm{H}$ & & & & & & & & & \\
\hline & -2 . & & $\mathrm{H}$ & & & & & & & & & \\
\hline & & -0.19 & $\mathrm{H}$ & 0 & 0 & 0 & 0 & 0 & 0 & 0 & & 0 \\
\hline & -0.0 & -2.6 & $\mathrm{H}$ & & & & & & & & & \\
\hline-1.8 & -1.7 & -2.1937 & $\mathrm{H}$ & c & 0 & 0 & 0 & 0 & 0 & 0 & & \\
\hline & -0. & -3.5 & $\mathrm{H}$ & & & & 0 & & & & & \\
\hline & -1.2 & -2.0705 & $\mathrm{H}$ & c & 0 & 0 & 0 & 0 & 0 & 0 & 0 & \\
\hline & & -2.3 & $\mathrm{H}$ & & & & 0 & & & & & \\
\hline & 1.12 & -2.39 & $\mathrm{H}$ & c & 0 & 0 & 0 & 0 & 0 & 0 & 0 & \\
\hline & 1.54 & 2.0212 & $\mathrm{H}$ & & & & 0 & 0 & 0 & & & \\
\hline & & & $\mathrm{H}$ & c & 0 & 0 & 0 & 0 & 0 & 0 & 0 & \\
\hline & -0.68 & 1.8100 & $\mathrm{H}$ & 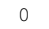 & & 0 & 0 & 0 & 0 & 0 & & \\
\hline-3.7062 & -1.3321 & -0.5227 & $\mathrm{H}$ & 0 & 0 & 0 & 0 & 0 & 0 & & & \\
\hline 0.6682 & -1.4020 & 0.8440 & & & & & & & & & & \\
\hline
\end{tabular}

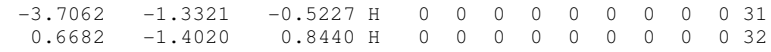
$\begin{array}{llllll}2 & 1 & 0 & 0 & 0 & 0 \\ 5 & 1 & 0 & 0 & 0 & 0\end{array}$ $\begin{array}{llllll}5 & 1 & 0 & 0 & 0 & 0\end{array}$ $\begin{array}{llllll}3 & 2 & 0 & 0 & 0 & 0\end{array}$ $\begin{array}{llllll}6 & 2 & 0 & 0 & 0 & 0\end{array}$ $\begin{array}{llllll}7 & 3 & 0 & 0 & 0 & 0\end{array}$ $\begin{array}{llllll}8 & 3 & 0 & 0 & 0 & 0\end{array}$ $\begin{array}{rlllll}9 & 4 & 0 & 0 & 0 & 0\end{array}$ $\begin{array}{llllllll}4 & 0 & 0 & 0 & 0 & 0\end{array}$ $\begin{array}{lllllll}6 & 0 & 0 & 0 & 0 & 0\end{array}$ $\begin{array}{llllll}1 & 6 & 0 & 0 & 0\end{array}$ $\begin{array}{llllll}8 & 7 & 0 & 0 & 0 & 0\end{array}$ $\begin{array}{lllllll}9 & 8 & 0 & 0 & 0 & 0 \\ 5 & 8 & 0 & 0 & 0 & 0\end{array}$ $\begin{array}{llllll}10 & 8 & 0 & 0 & 0 & 0\end{array}$ $\begin{array}{llllll}22 & 9 & 0 & 0 & 0\end{array}$ $\begin{array}{lllll}23 & 9 & 0 & 0 & 0\end{array}$ $\begin{array}{llllll}11 & 10 & 0 & 0 & 0 & 0\end{array}$ $\begin{array}{lllllll}25 & 10 & 0 & 0 & 0 & 0\end{array}$ $\begin{array}{lllllll}25 & 10 & 0 & 0 & 0 & 0\end{array}$ 


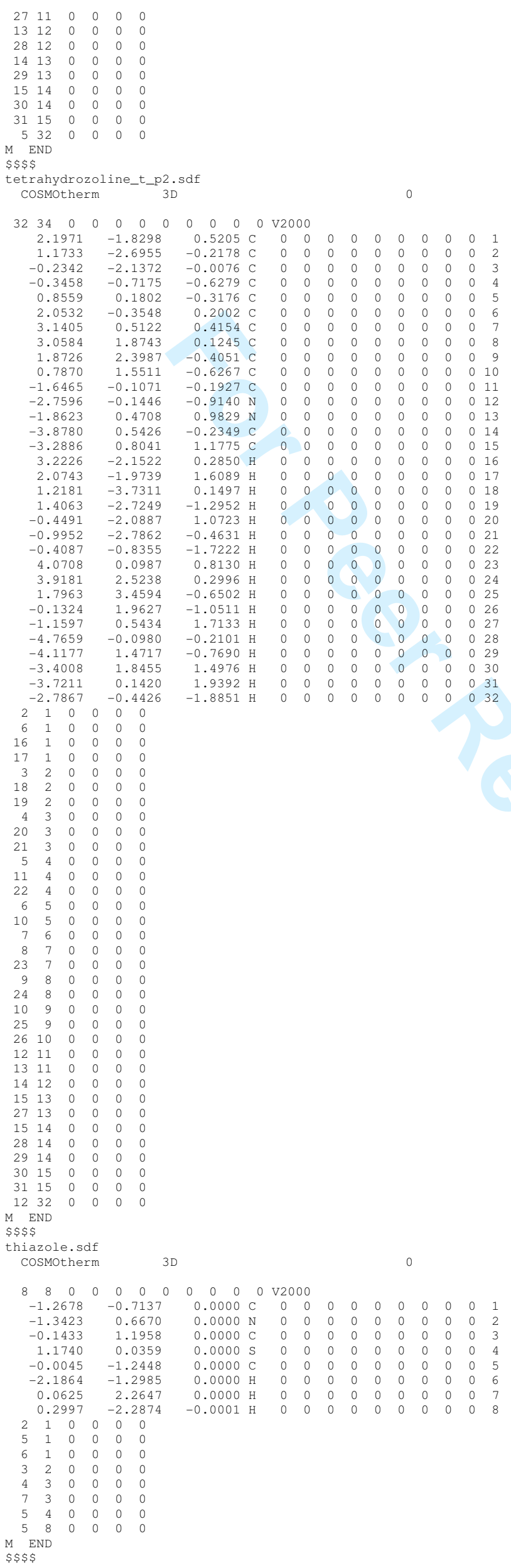




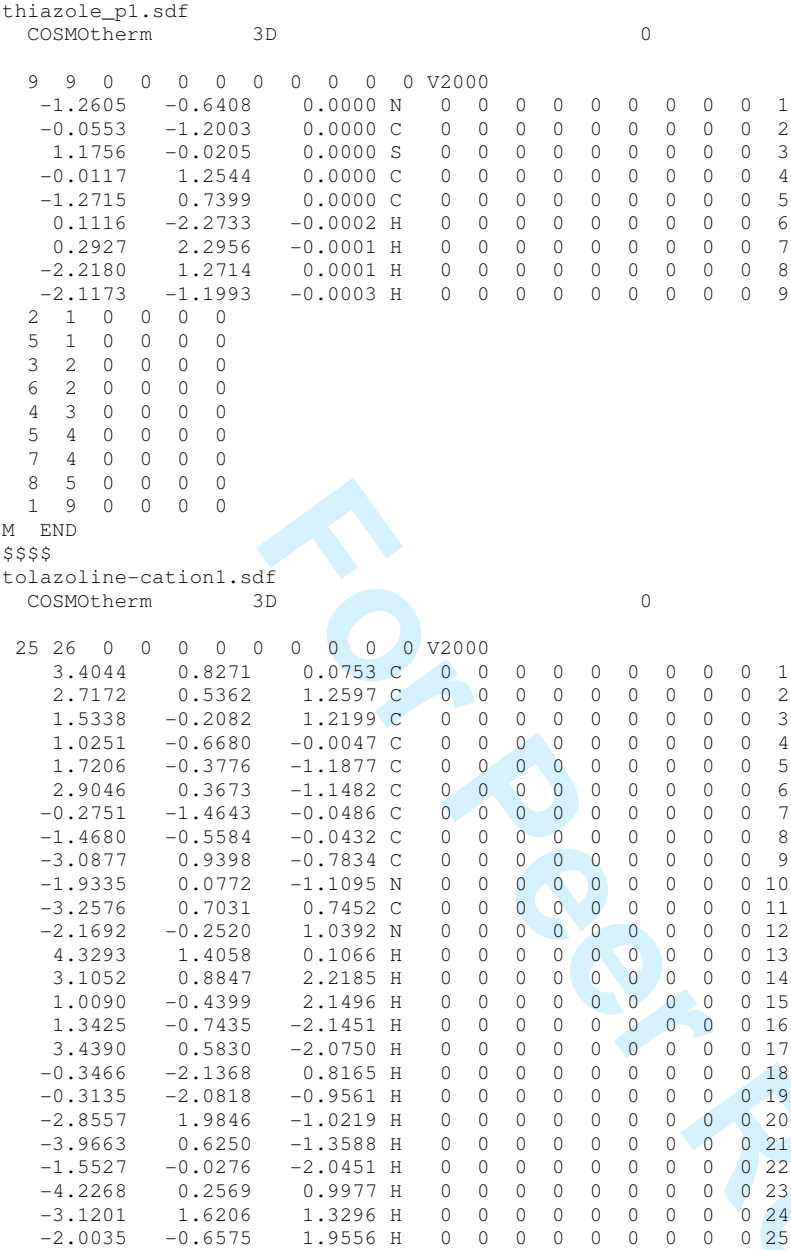




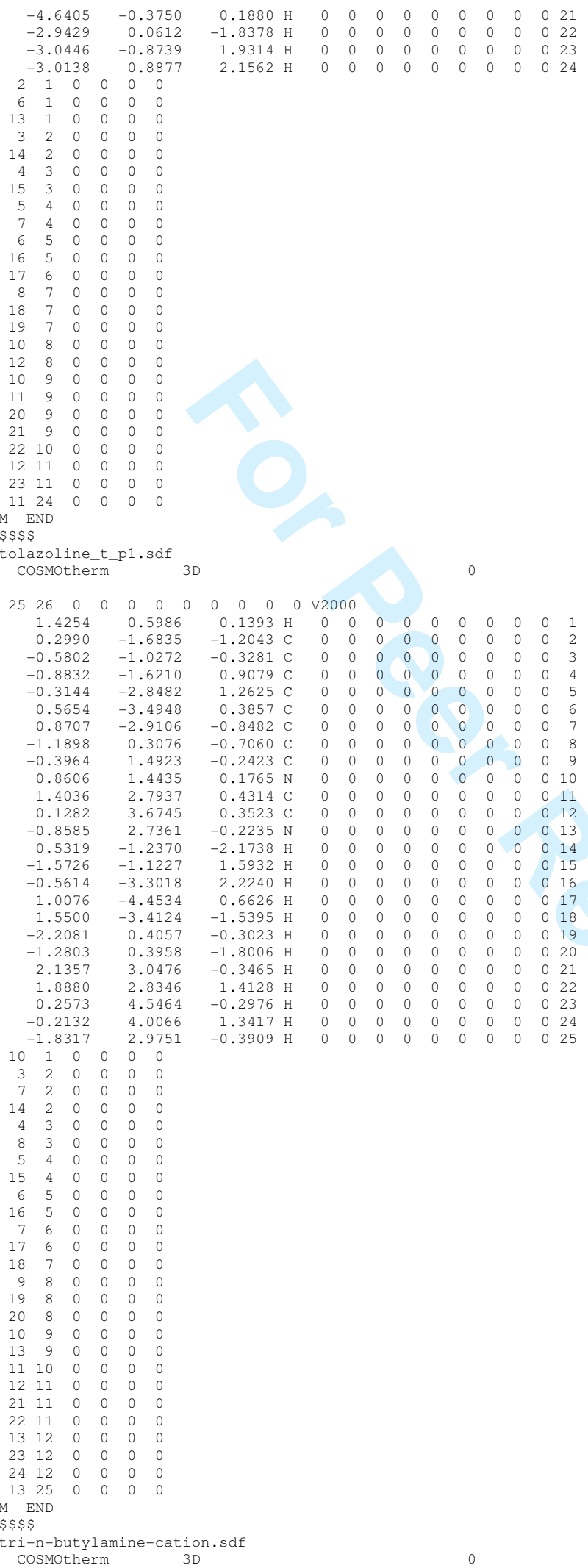

cosmotherm 3D

$\begin{array}{rrrrrrrrrrrrrrr}40 & 0 & 0 & 0 & 0 & 0 & 0 & 0 & 0 & 0 & \text { V2 } 2000 & & & & \\ 2.5400 & 0.8979 & -0.2947 & \mathrm{C} & 0 & 0 & 0 & 0 & 0 & 0 & 0 & 0 & 0 & 1 \\ 1.2514 & 1.0242 & 0.5103 & \mathrm{C} & 0 & 0 & 0 & 0 & 0 & 0 & 0 & 0 & 0 & 2 \\ 0.0035 & 0.6792 & -0.2860 & \mathrm{~N} & 0 & 0 & 0 & 0 & 0 & 0 & 0 & 0 & 0 & 3 \\ -1.2428 & 1.0325 & 0.5090 & \mathrm{C} & 0 & 0 & 0 & 0 & 0 & 0 & 0 & 0 & 0 & 4 \\ -2.5326 & 0.9047 & -0.2938 & \mathrm{C} & 0 & 0 & 0 & 0 & 0 & 0 & 0 & 0 & 0 & 5 \\ -0.0009 & -0.7378 & -0.8428 & \mathrm{C} & 0 & 0 & 0 & 0 & 0 & 0 & 0 & 0 & 0 & 6 \\ -0.0118 & -1.8352 & 0.2123 & \mathrm{C} & 0 & 0 & 0 & 0 & 0 & 0 & 0 & 0 & 0 & 7 \\ 3.7345 & 1.4642 & 0.4896 & \mathrm{C} & 0 & 0 & 0 & 0 & 0 & 0 & 0 & 0 & 0 & 8 \\ 2.7430 & -0.1547 & -0.5450 & \mathrm{H} & 0 & 0 & 0 & 0 & 0 & 0 & 0 & 0 & 0 & 9 \\ 2.4344 & 1.4459 & -1.2463 & \mathrm{H} & 0 & 0 & 0 & 0 & 0 & 0 & 0 & 0 & 0 & 10 \\ 1.1055 & 2.0606 & 0.8410 & \mathrm{H} & 0 & 0 & 0 & 0 & 0 & 0 & 0 & 0 & 0 & 11 \\ 1.2509 & 0.3804 & 1.3979 & \mathrm{H} & 0 & 0 & 0 & 0 & 0 & 0 & 0 & 0 & 0 & 12 \\ -1.0936 & 2.0706 & 0.8332 & \mathrm{H} & 0 & 0 & 0 & 0 & 0 & 0 & 0 & 0 & 0 & 13\end{array}$




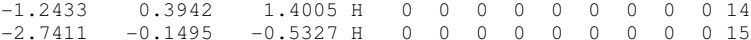
$\begin{array}{rrrrrrrrrrrrrr}-2.7411 & -0.1495 & -0.5327 & \mathrm{H} & 0 & 0 & 0 & 0 & 0 & 0 & 0 & 0 & 0 & 15 \\ -3.7232 & 1.4858 & 0.4855 & \mathrm{C} & 0 & 0 & 0 & 0 & 0 & 0 & 0 & 0 & 0 & 16\end{array}$

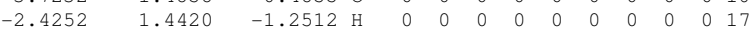
$\begin{array}{llllllllllllll}0.8849 & -0.8112 & -1.4852 & \mathrm{H} & 0 & 0 & 0 & 0 & 0 & 0 & 0 & 0 & 0 & 18 \\ -0.8808 & -0.8021 & -1.4942 & \mathrm{H} & 0 & 0 & 0 & 0 & 0 & 0 & 0 & 0 & 0 & 19\end{array}$

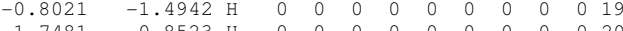
$\begin{array}{cccccccccccccc}-0.9036 & -1.7481 & 0.8523 & \mathrm{H} & 0 & 0 & 0 & 0 & 0 & 0 & 0 & 0 & 0 & 20 \\ 0.8682 & -1.7504 & 0.8687 & \mathrm{H} & 0 & 0 & 0 & 0 & 0 & 0 & 0 & 0 & 0 & 21\end{array}$

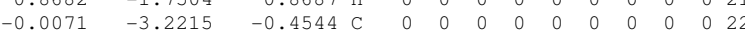

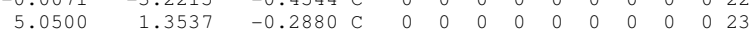
$\begin{array}{llllllllllllll}3.5372 & 2.5200 & 0.7378 & \mathrm{H} & 0 & 0 & 0 & 0 & 0 & 0 & 0 & 0 & 0 & 24 \\ 3.8253 & 0.9288 & 1.4491 & \mathrm{H} & 0 & 0 & 0 & 0 & 0 & 0 & 0 & 0 & 0 & 25\end{array}$ $\begin{array}{lllllllllllllll}3.8253 & 0.9288 & 1.4491 \mathrm{H} & 0 & 0 & 0 & 0 & 0 & 0 & 0 & 0 & 0 & 25\end{array}$

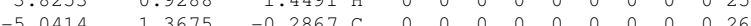

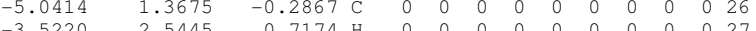

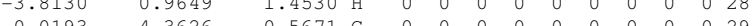

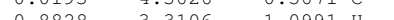
$\begin{array}{lllllllllllllllll}0.8828 & -3.3106 & -1.0991 & H & 0 & 0 & 0 & 0 & 0 & 0 & 0 & 0 & 0 & 30\end{array}$

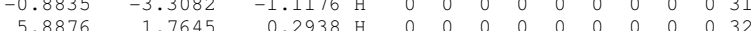

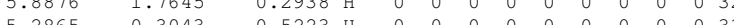
$\begin{array}{llllllllllllll}5.2865 & 0.3043 & -0.5223 & \mathrm{H} & 0 & 0 & 0 & 0 & 0 & 0 & 0 & 0 & 0 & 33\end{array}$

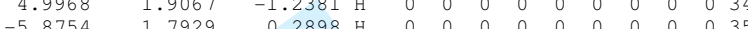

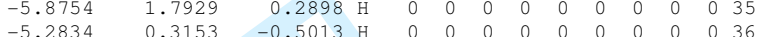
$\begin{array}{llllllllllllll}-5.2834 & 0.3153 & -0.5013 & \mathrm{H} & 0 & 0 & 0 & 0 & 0 & 0 & 0 & 0 & 0 & 36 \\ -4.9881 & 1.9032 & -1.2467 & \mathrm{H} & 0 & 0 & 0 & 0 & 0 & 0 & 0 & 0 & 0 & 37\end{array}$

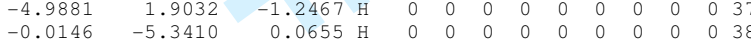
$\begin{array}{llllllllllllll}-0.9157 & -4.3156 & 1.2043 & \mathrm{H} & 0 & 0 & 0 & 0 & 0 & 0 & 0 & 0 & 0 & 39\end{array}$ $0.8631--4.3172-1.2238$ H $\begin{array}{llllllllllllll}0.0060 & 1.2978 & -1.1098 \mathrm{H} & 0 & 0 & 0 & 0 & 0 & 0 & 0 & 0 & 0 & 41\end{array}$ $\begin{array}{llllll}2 & 1 & 0 & 0 & 0 & 0\end{array}$

$8 \begin{array}{llllll}8 & 1 & 0 & 0 & 0 & 0\end{array}$

$\begin{array}{llllll}2 & 1 & 0 & 0 & 0 & 0 \\ 0 & 1 & 0 & 0 & 0 & 0\end{array}$

$\begin{array}{llllll}1 & 0 & 0 & 0 & 0 \\ 3 & 2 & 0 & 0 & 0 & 0\end{array}$

$\begin{array}{llllll}1 & 2 & 0 & 0 & 0 & 0\end{array}$

$\begin{array}{llllll}2 & 2 & 0 & 0 & 0 & 0\end{array}$ $\begin{array}{llllll}3 & 0 & 0 & 0 & 0 \\ 3 & 0 & 0 & 0 & 0\end{array}$ $\begin{array}{lllllll}5 & 4 & 0 & 0 & 0 & 0\end{array}$ $\begin{array}{llllll}4 & 4 & 0 & 0 & 0 & 0\end{array}$ $\begin{array}{lllllll}5 & 5 & 0 & 0 & 0 & 0\end{array}$ $\begin{array}{lllllll}16 & 5 & 0 & 0 & 0 & 0\end{array}$

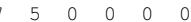
$7 \begin{array}{llllll}6 & 0 & 0 & 0 & 0\end{array}$ 18 6 6100100 $207 \begin{array}{llllll}20 & 7 & 0 & 0 & 0 & 0\end{array}$ $\begin{array}{lllllll}21 & 7 & 0 & 0 & 0 & 0\end{array}$ $2 \begin{array}{llllll}2 & 7 & 0 & 0 & 0 & 0\end{array}$ $\begin{array}{lllllll}3 & 8 & 0 & 0 & 0 & 0\end{array}$ $\begin{array}{lllllll}5 & 8 & 0 & 0 & 0 & 0\end{array}$ $\begin{array}{llllll}25 & 16 & 0 & 0 & 0 & 0\end{array}$ $\begin{array}{llllll}27 & 16 & 0 & 0 & 0 & 0\end{array}$ $\begin{array}{llllll}16 & 16 & 0 & 0 \\ 28 & 16 & 0 & 0 & 0\end{array}$ $\begin{array}{llllllll}28 & 16 & 0 & 0 & 0 & 0\end{array}$

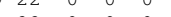
$\begin{array}{llllll}30 & 22 & 0 & 0 & 0 & 0\end{array}$ $\begin{array}{lllllll}32 & 23 & 0 & 0 & 0 & 0\end{array}$ $\begin{array}{llllll}3 & 23 & 0 & 0 & 0 & 0\end{array}$ $\begin{array}{llllll}34 & 23 & 0 & 0 & 0 & 0\end{array}$ $\begin{array}{llllll}35 & 26 & 0 & 0 & 0 & 0\end{array}$ $\begin{array}{llllll}36 & 26 & 0 & 0 & 0 & 0\end{array}$ $\begin{array}{llllll}37 & 26 & 0 & 0 & 0 & 0\end{array}$ $\begin{array}{llllll}38 & 29 & 0 & 0 & 0 & 0\end{array}$ $\begin{array}{llllll}4 & 29 & 0 & 0 & 0 & 0\end{array}$ $\begin{array}{llllll}3 & 41 & 0 & 0 & 0 & 0\end{array}$ END

tri-n-butylamine, sdf i-n-butylamine.sdf
cosmotherm $\begin{array}{llllllllllll}40 & 39 & 0 & 0 & 0 & 0 & 0 & 0 & 0 & 0 & 0 & \text { v2 } 2000\end{array}$

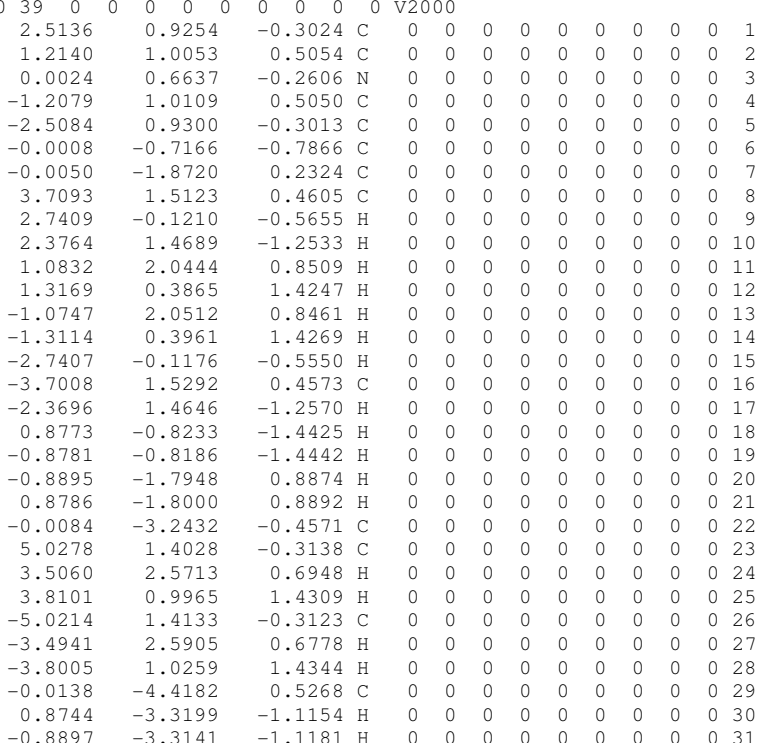


$\begin{array}{lllllll}21 & 7 & 0 & 0 & 0 & 0\end{array}$

$\begin{array}{llllll}22 & 7 & 0 & 0 & 0 & 0 \\ 23 & 8 & 0 & 0 & 0 & 0\end{array}$

$\begin{array}{llllll}24 & 8 & 0 & 0 & 0 & 0\end{array}$

$\begin{array}{lrrrrrr}25 & 8 & 0 & 0 & 0 & 0 \\ 26 & 16 & 0 & 0 & 0 & 0\end{array}$

$27 \begin{array}{lllll}16 & 0 & 0 & 0 & 0\end{array}$

$\begin{array}{lllllll}28 & 16 & 0 & 0 & 0 & 0 & 0 \\ 29 & 22 & 0 & 0 & 0 & 0 & 0\end{array}$

$\begin{array}{llllll}30 & 22 & 0 & 0 & 0 & 0\end{array}$

$\begin{array}{llllll}1 & 22 & 0 & 0 & 0 & 0\end{array}$

332

$\$ \$ \$$

tri-n-propylamine.sdf

Cosmotherm

$\begin{array}{lllllllllllll}31 & 30 & 0 & 0 & 0 & 0 & 0 & 0 & 0 & 0 & 0 & \mathrm{v} 2000\end{array}$

$\begin{array}{lllllllllllll}2.5169 & 0.6977 & -0.4412 & \mathrm{C} & 0 & 0 & 0 & 0 & 0 & 0 & 0 & 0 & 0\end{array}$

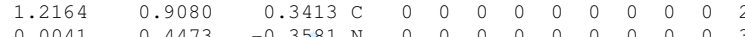

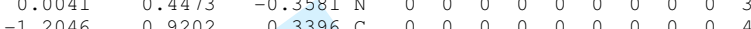

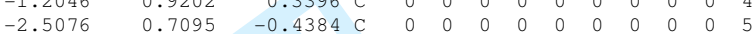

$\begin{array}{lllllllllllllll}-0.0030 & -1.0005 & -0.6487 & C & 0 & 0 & 0 & 0 & 0 & 0 & 0 & 0 & 0 & 6\end{array}$

$\begin{array}{lllllllllllll}-0.0165 & -1.9733 & 0.5472 & \mathrm{C} & 0 & 0 & 0 & 0 & 0 & 0 & 0 & 0 & 0\end{array}$

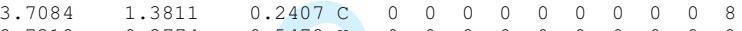

$\begin{array}{llllllllllllll}2.7318 & -0.3774 & -0.5472 & \mathrm{H} & 0 & 0 & 0 & 0 & 0 & 0 & 0 & 0 & 0 & 9\end{array}$

$\begin{array}{llllllllllllll}2.3849 & 1.0966 & -1.4606 & \mathrm{H} & 0 & 0 & 0 & 0 & 0 & 0 & 0 & 0 & 0 & 10\end{array}$

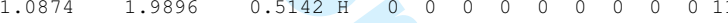

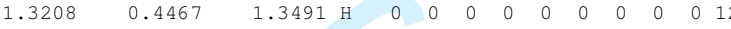

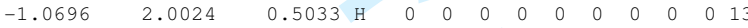

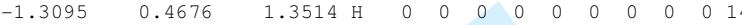

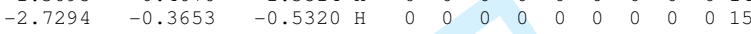

$\begin{array}{llllllllllllll}-3.6935 & 1.4080 & 0.2379 & \mathrm{C} & 0 & 0 & 0 & 0 & 0 & 0 & 0 & 0 & 0 & 16\end{array}$

$\begin{array}{llllllllllllll}-2.3751 & 1.0962 & -1.4625 & \mathrm{H} & 0 & 0 & 0 & 0 & 0 & 0 & 0 & 0 & 0 & 17\end{array}$

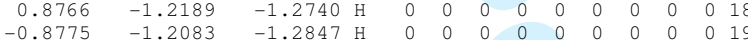

$\begin{array}{llllllllllllll}-0.9031 & -1.7856 & 1.1739 & \mathrm{H} & 0 & 0 & 0 & 0 & 0 & 0 & 0 & 0 & 0 & 20\end{array}$

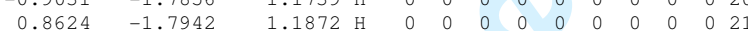

$\begin{array}{llllllllllllllll}-0.0202 & -3.4339 & 0.0808 & C & 0 & 0 & 0 & 0 & 0 & 0 & 0 & 0 & 0 & 22\end{array}$

$\begin{array}{llllllllllllll}4.6392 & 1.2074 & -0.3184 \mathrm{H} & 0 & 0 & 0 & 0 & 0 & 0 & 0 & 0 & 0 & 23\end{array}$

$\begin{array}{llllllllllllllllll}3.5569 & 2.4694 & 0.3116 & \mathrm{H} & 0 & 0 & 0 & 0 & 0 & 0 & 0 & 0 & 0 & 0 & 0 & 23\end{array}$

$\begin{array}{llllllllllllllll}3.8565 & 0.9976 & 1.2624 \mathrm{H} & 0 & 0 & 0 & 0 & 0 & 0 & 0 & 0 & 0 & 24\end{array}$

$\begin{array}{llllllllllllllll}-4.6268 & 1.2323 & -0.3164 \mathrm{H} & 0 & 0 & 0 & 0 & 0 & 0 & 0 & 0 & 0 & 26\end{array}$

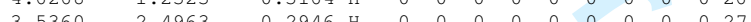

$\begin{array}{lllllllllllllll}-3.8409 & 1.0383 & 1.2648 \mathrm{H} & 0 & 0 & 0 & 0 & 0 & 0 & 0 & 0 & 0 & 28\end{array}$

$\begin{array}{llllllllllllll}-0.0323 & -4.1288 & 0.9335 \mathrm{H} & 0 & 0 & 0 & 0 & 0 & 0 & 0 & 0 & 0 & 29\end{array}$

$\begin{array}{llllllllllllll}0.8726 & -3.6605 & -0.5229 & \mathrm{H} & 0 & 0 & 0 & 0 & 0 & 0 & 0 & 0 & 0 & 30\end{array}$

$\begin{array}{llllllllllll}-0.9038 & -3.6505 & -0.5399 \mathrm{H} & 0 & 0 & 0 & 0 & 0 & 0 & 0 & 0 & 0\end{array}$

$\begin{array}{llllll}2 & 0 & 0 & 0 & 0\end{array}$

$\begin{array}{llllll}1 & 0 & 0 & 0 & 0\end{array}$

$\begin{array}{llllll}9 & 1 & 0 & 0 & 0 & 0\end{array}$

$\begin{array}{llll}2 & 0 & 0 & 0\end{array}$

$\begin{array}{llllll}11 & 2 & 0 & 0 & 0 & 0 \\ 12 & 2 & 0 & 0 & 0 & 0\end{array}$

$\begin{array}{lllll}3 & 0 & 0 & 0 & 0\end{array}$

$\begin{array}{llll}3 & 0 & 0 & 0\end{array}$

$\begin{array}{llllll}4 & 0 & 0 & 0 & 0\end{array}$

$\begin{array}{lllll}4 & 0 & 0 & 0 & 0 \\ 4 & 0 & 0 & 0\end{array}$

$\begin{array}{lllll}5 & 0 & 0 & 0\end{array}$

$\begin{array}{lllll}5 & 0 & 0 & 0 & 0\end{array}$

$\begin{array}{llll}6 & 0 & 0 & 0\end{array}$

$\begin{array}{llllll}18 & 6 & 0 & 0 & 0 & 0\end{array}$

$\begin{array}{llllll}19 & 6 & 0 & 0 & 0 & 0\end{array}$

$\begin{array}{rlllll}21 & 7 & 0 & 0 & 0 & 0\end{array}$

$\begin{array}{llllll}21 & 7 & 0 & 0 & 0 & 0\end{array}$

580000

$\begin{array}{llllll}26 & 16 & 0 & 0 & 0 & 0\end{array}$

$\begin{array}{llllll}27 & 16 & 0 & 0 & 0 & 0\end{array}$

$\begin{array}{llllll}28 & 16 & 0 & 0 & 0 & 0\end{array}$

$\begin{array}{llllll}9 & 22 & 0 & 0 & 0 & 0\end{array}$

$\begin{array}{lllll}22 & 0 & 0 & 0 & 0\end{array}$

END

iethylamine-cation.sd

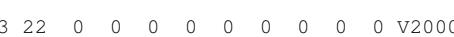

\begin{tabular}{|c|c|c|c|c|c|c|c|c|c|c|c|c|}
\hline 2.5323 & 5836 & -0.2812 & C & 0 & 0 & 0 & 0 & 0 & 0 & 0 & 0 & 0 \\
\hline 1.2514 & 0.7976 & 0.5080 & $\mathrm{C}$ & 0 & 0 & 0 & 0 & 0 & 0 & 0 & 0 & 0 \\
\hline 0.0029 & 0.3797 & -0.2523 & $\mathrm{~N}$ & 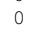 & 0 & 0 & 0 & 0 & 0 & 0 & 0 & 0 \\
\hline-1.2432 & 0.8082 & 0.5058 & $C^{\circ}$ & & 0 & 0 & 0 & 0 & 0 & 0 & 0 & 0 \\
\hline-2.525 & 0.5967 & -0.2821 & $\mathrm{C}$ & & & 0 & 0 & 0 & 0 & 0 & 0 & 0 \\
\hline-0.002 & -1.0861 & -0.6666 & & 0 & 0 & 0 & 0 & 0 & 0 & 0 & 0 & 0 \\
\hline-0.01 & -2.060 & 0.4971 & C & & & 0 & 0 & 0 & 0 & 0 & 0 & 0 \\
\hline 3.359 & 1.0347 & 0.2837 & $\mathrm{H}$ & 0 & 0 & 0 & 0 & 0 & 0 & 0 & 0 & 0 \\
\hline 2.7717 & -0.4776 & -0.4267 & $\mathrm{H}$ & & 0 & 0 & 0 & 0 & 0 & 0 & 0 & 0 \\
\hline 2.48 & & -1.2616 & $\mathrm{H}$ & 0 & 0 & 0 & 0 & 0 & 0 & 0 & 0 & 0 \\
\hline 1.1072 & 1.8604 & 0.7366 & $\mathrm{H}$ & & 0 & 0 & 0 & 0 & 0 & 0 & 0 & 0 \\
\hline & & 1.4 & $\mathrm{H}$ & & & 0 & 0 & 0 & 0 & 0 & 0 & \\
\hline-1.0932 & 1.8715 & 0.7287 & $\mathrm{H}$ & 0 & 0 & 0 & 0 & 0 & 0 & 0 & 0 & 0 \\
\hline-1.2 & & 1.45 & $\mathrm{H}$ & & & 0 & 0 & 0 & 0 & 0 & 0 & \\
\hline-3.3495 & 1.0564 & 0.2798 & $\mathrm{H}$ & 0 & 0 & 0 & 0 & 0 & 0 & 0 & 0 & 0 \\
\hline-2.7707 & -0.4640 & -0.4207 & & & & 0 & 0 & 0 & 0 & 0 & 0 & \\
\hline-2.4781 & 1.0857 & -1.2657 & $\mathrm{H}$ & 0 & 0 & 0 & 0 & 0 & 0 & 0 & 0 & 0 \\
\hline 0.8820 & -1.2227 & -1.2981 & & & & 0 & 0 & 0 & 0 & 0 & 0 & ( \\
\hline-0.8820 & -1.2127 & -1.3082 & & & & 0 & 0 & 0 & 0 & 0 & 0 & 0 \\
\hline 0.0159 & -3.0769 & 0.0803 & & & & 0 & 0 & 0 & 0 & 0 & 0 & \\
\hline
\end{tabular}

URL: http://mc.manuscriptcentral.com/tandf/tmph 


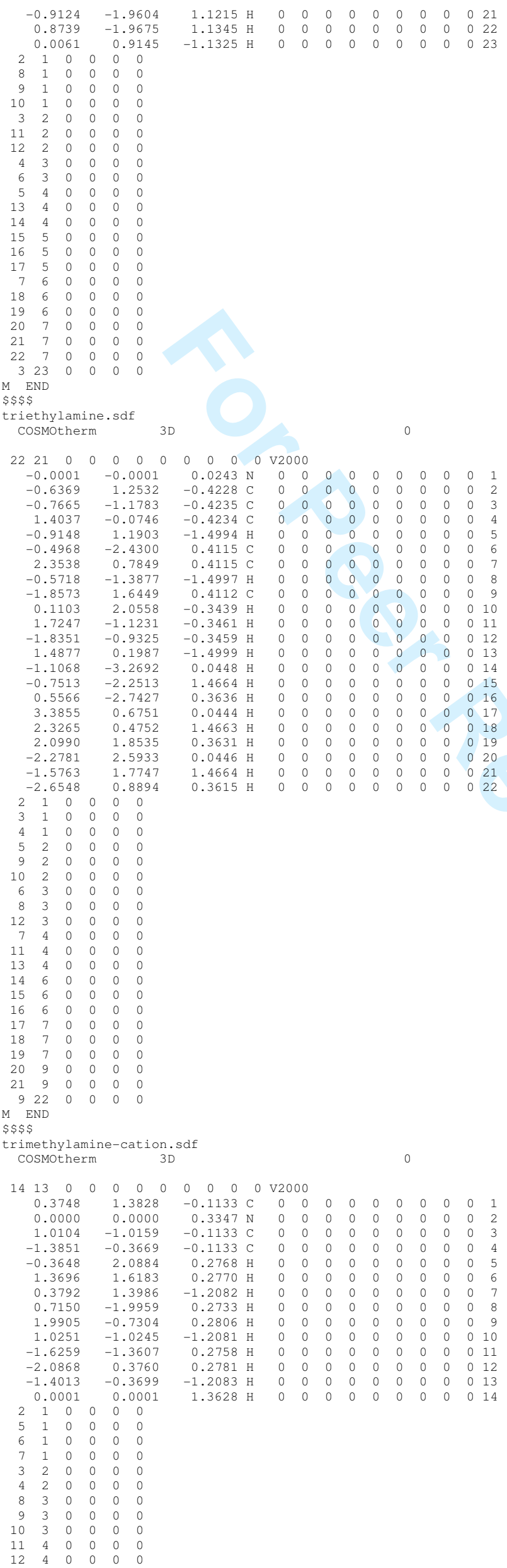




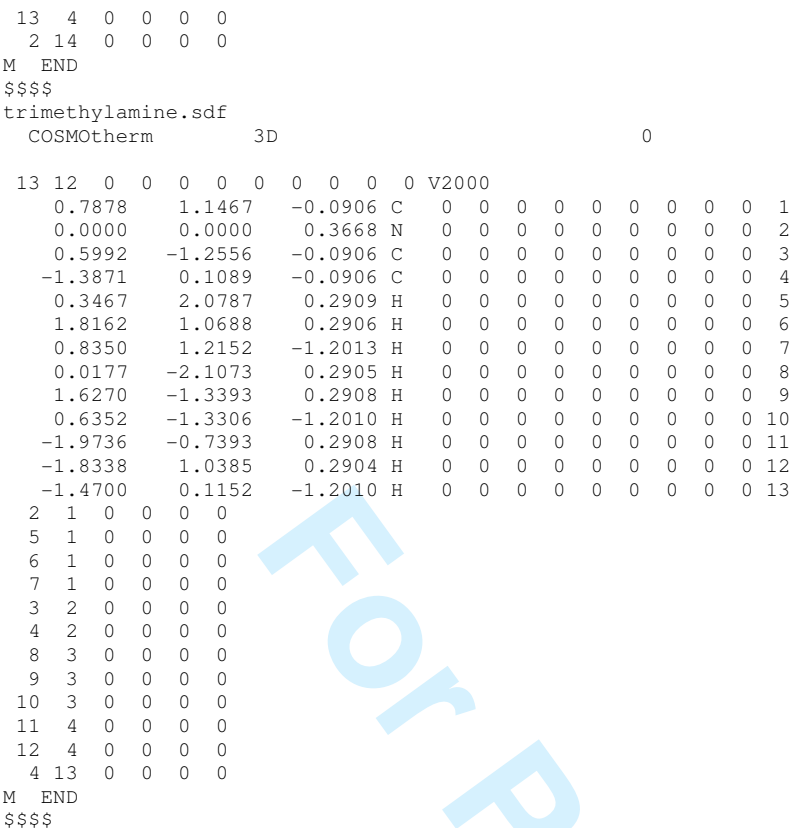

S4 e) Mono-solvated Bases \& Cations

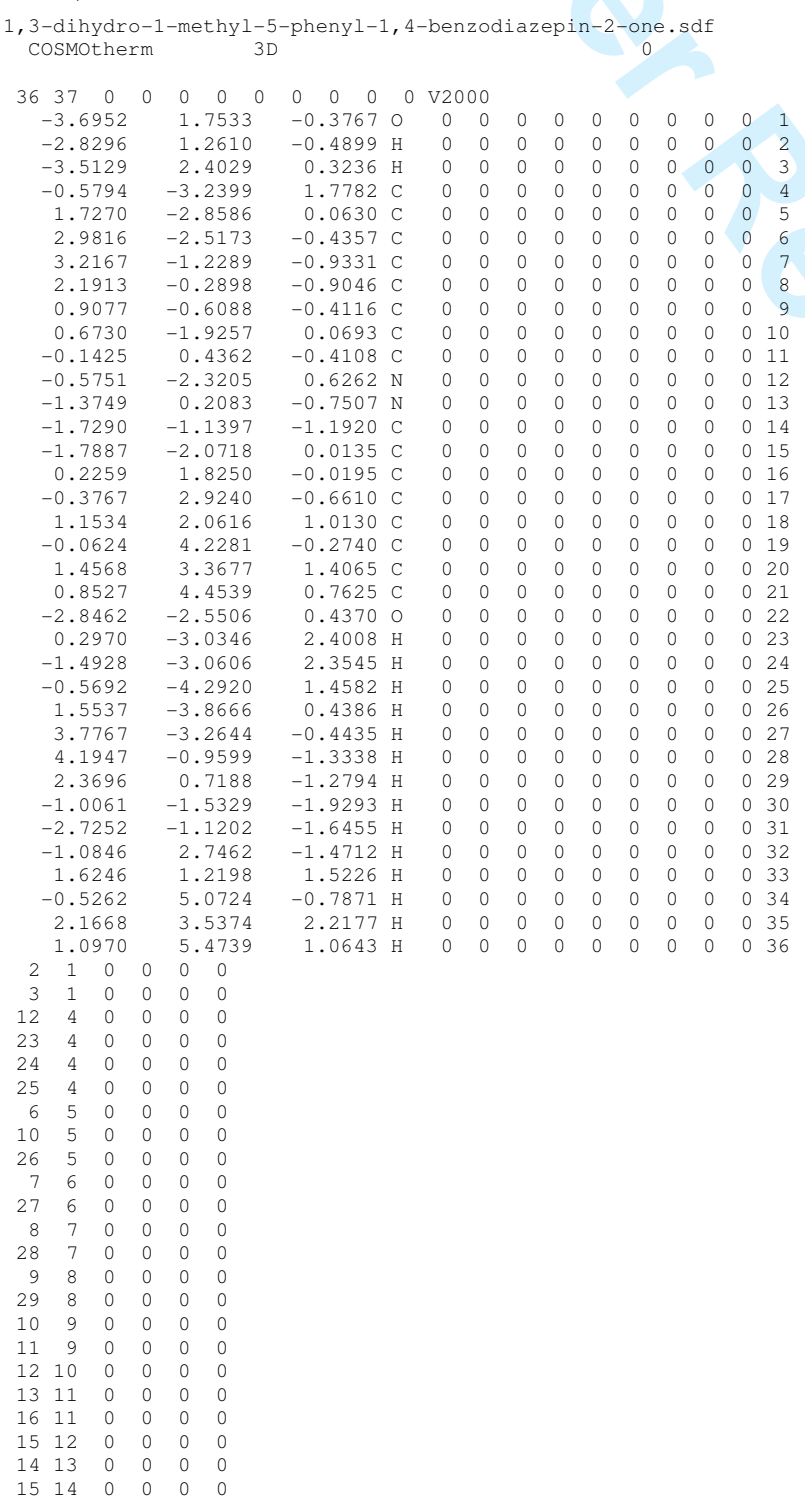




\begin{tabular}{|c|c|c|c|c|c|c|c|c|c|c|}
\hline 3.4105 & -1.0596 & 0.8997 & C & 0 & & 0 & & & & \\
\hline 2.3097 & -0.9834 & 0.0526 & $\mathrm{C}$ & 0 & & 0 & O & 0 & & \\
\hline 1.0962 & -0.3938 & 0.4676 & C & 0 & 0 & 0 & 0 & 0 & & \\
\hline 1.0178 & 0.1366 & 1.7840 & C & 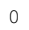 & & 0 & & & & \\
\hline 2.1462 & 0.0828 & 2.6214 & C & & & 0 & & & & \\
\hline-0.0426 & -0.3433 & -0.4804 & C & & & & & & & \\
\hline-0.1506 & 0.7939 & 2.2704 & $\mathrm{~N}$ & & & & & & & \\
\hline 1.4075 & 0.2794 & 2.1192 & $\mathrm{C}$ & & & & & & & \\
\hline-1.5243 & -0.9866 & 1.2510 & C & 0 & & & & & & \\
\hline-1.2705 & -0.58 & -0.1284 & $\mathrm{~N}$ & & & & & & & \\
\hline 0.0037 & 1.9 & 3.1258 & C & & & & & & & \\
\hline-2.4264 & 0.7938 & 2.5996 & 0 & & & & & & & \\
\hline 0.2242 & -0.01 & -1.9076 & C & 0 & & 0 & & & & \\
\hline-0.5238 & -0.6412 & -2.9247 & C & & & & & & & \\
\hline 1.2002 & 0.9 & -2.2615 & C & 0 & & & & & & \\
\hline 1.4106 & 1.2653 & -3.6024 & C & & & & & & & \\
\hline 0.6633 & & -4 & C & & & & & & & \\
\hline-0.3019 & -0.31 & -4.2643 & C & & & & & & & \\
\hline 4.18 & -0.5 & & $\mathrm{H}$ & & & & & & & \\
\hline 4.3304 & -1.5 & 0.5593 & $\mathrm{H}$ & & & & & & & \\
\hline 2.37 & -1.3 & -0.9 & $\mathrm{H}$ & & & & & & & \\
\hline 2.0892 & & 3.6271 & $\mathrm{H}$ & & & & & & & \\
\hline-0.79 & -1.7 & 1.5774 & $\mathrm{H}$ & & & & & & & \\
\hline-2.8171 & -1.5 & 1.3871 & 0 & & & & & & & \\
\hline 0.8871 & & 2.80 & $\mathrm{H}$ & & & & & & & \\
\hline 0.1076 & 1.7 & 4.1841 & $\mathrm{H}$ & & & 0 & & & & \\
\hline-0.8899 & 2.60 & & 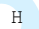 & & & & & & & \\
\hline-1.2687 & -1.3912 & -2.6564 & $\mathrm{H}$ & 0 & & 0 & & & & \\
\hline 1.7831 & 1.4 & & & & & & & & & \\
\hline 2.1602 & 0 & -3.8628 & $\mathrm{H}$ & 0 & 0 & 0 & & & & \\
\hline 0.8352 & 0.89 & -5.6546 & 1 & & & & & & & \\
\hline & -0.8161 & -5.0452 & $\mathrm{H}$ & & & 0 & & 0 & & \\
\hline-3.3694 & -0.7924 & 1.7471 & $\mathrm{H}$ & & & & & & & \\
\hline
\end{tabular}

$\begin{array}{cccccc}2 & 1 & 0 & 0 & 0 & 0 \\ 3 & 1 & 0 & 0 & 0 & 0\end{array}$

$\begin{array}{llllll}5 & 4 & 0 & 0 & 0 & 0\end{array}$

$\begin{array}{lllllll}9 & 4 & 0 & 0 & 0 & 0 & 0\end{array}$

$\begin{array}{llllll}3 & 4 & 0 & 0 & 0 \\ 6 & 5 & 0 & 0 & 0\end{array}$

2455000

$\begin{array}{llllll}7 & 6 & 0 & 0 & 0\end{array}$

$\begin{array}{llllll}5 & 6 & 0 & 0 & 0 & 0\end{array}$

$\begin{array}{llllll}8 & 7 & 0 & 0 & 0 & 0\end{array}$

$\begin{array}{llllll}8 & 7 & 0 & 0 & 0 & 0 \\ 9 & 8 & 0 & 0 & 0 & 0\end{array}$

$\begin{array}{lllllll}1 & 8 & 0 & 0 & 0 & 0\end{array}$

$\begin{array}{llllll}26 & 9 & 0 & 0 & 0\end{array}$

$\begin{array}{llllll}14 & 10 & 0 & 0 & 0\end{array}$

$\begin{array}{llllll}17 & 10 & 0 & 0 & 0 & 0\end{array}$

$\begin{array}{llllll}15 & 11 & 0 & 0 & 0 & 0\end{array}$

1312000

$\begin{array}{llllll}13 & 12 & 12 & 0 & 0\end{array}$

$\begin{array}{lllll}14 & 13 & 0 & 0 & 0\end{array}$

$\begin{array}{llllll}27 & 13 & 0 & 0 & 0 & 0 \\ 28 & 13 & 0 & 0 & 0 & 0\end{array}$

$\begin{array}{llllll}28 & 13 & 0 & 0 & 0\end{array}$

$\begin{array}{llllll}29 & 15 & 0 & 0 & 0 & 0\end{array}$

$\begin{array}{lllll}31 & 15 & 0 & 0 & 0\end{array}$

$\begin{array}{lllll}18 & 17 & 0 & 0 & 0\end{array}$

$\begin{array}{llllll}19 & 17 & 0 & 0 & 0 & 0\end{array}$

$\begin{array}{llllll}22 & 18 & 0 & 0 & 0 & 0\end{array}$

$\begin{array}{llllll}32 & 18 & 0 & 0 & 0 & 0 \\ 20 & 19 & 0 & 0 & 0 & 0\end{array}$

$\begin{array}{llllll}33 & 19 & 0 & 0 & 0 & 0\end{array}$

$\begin{array}{llllll}21 & 20 & 0 & 0 & 0 & 0\end{array}$

$\begin{array}{llllll}34 & 20 & 0 & 0 & 0 & 0 \\ 22 & 21 & 0 & 0 & 0 & 0\end{array}$

$\begin{array}{llllll}35 & 21 & 0 & 0 & 0 & 0\end{array}$

$\begin{array}{llllll}36 & 22 & 0 & 0 & 0 & 0\end{array}$

END

\$\$\$

3-dihydro-3-hydroxy-1-methyl-5-phenyl-1,4-benzodiazepin-2-one-cation.sd

cosmotherm

\begin{tabular}{|c|c|c|c|c|c|c|c|c|c|c|c|c|}
\hline $\begin{array}{ccc}8 & 39 & 0 \\
& -3.5131\end{array}$ & $\begin{array}{l}0 \\
0.2785^{0} \\
0.27\end{array}$ & $\begin{array}{ccc}0 & 0 & 0 \\
-1.3959\end{array}$ & 0 & 0 & 0 & 0 & 0 & 0 & 0 & 0 & 0 & \\
\hline-4.0854 & -0.4027 & -1.7936 & $\mathrm{H}$ & 0 & 0 & 0 & 0 & 0 & 0 & 0 & 0 & 0 \\
\hline-3.4378 & 0.9697 & -2.0788 & $\mathrm{H}$ & 0 & 0 & 0 & 0 & 0 & 0 & 0 & 0 & \\
\hline 3.3716 & -0.5273 & 2.0958 & $\mathrm{C}$ & 0 & 0 & 0 & 0 & 0 & 0 & 0 & 0 & 0 \\
\hline 3.4393 & -1.0276 & 0.7888 & $\mathrm{C}$ & 0 & 0 & 0 & 0 & 0 & 0 & 0 & 0 & 0 \\
\hline 2.3249 & -0.9381 & -0.0331 & $\mathrm{C}$ & 0 & 0 & 0 & 0 & 0 & 0 & 0 & 0 & 0 \\
\hline 1.1096 & -0.3781 & 0.4297 & $\mathrm{C}$ & 0 & 0 & & 0 & 0 & 0 & 0 & 0 & 0 \\
\hline 1.0449 & 0.1115 & 1.7710 & $\mathrm{C}$ & 0 & 0 & 0 & 0 & 0 & 0 & 0 & 0 & 0 \\
\hline 2.1937 & 0.0399 & 2.5753 & $\mathrm{C}$ & 0 & & & 0 & 0 & 0 & 0 & 0 & 0 \\
\hline-0.0092 & -0.3002 & -0.5074 & $\mathrm{C}$ & 0 & 0 & 0 & 0 & 0 & 0 & 0 & 0 & 0 \\
\hline-0.1028 & 0.7595 & 2.3116 & $\mathrm{~N}$ & 0 & & & 0 & 0 & 0 & 0 & 0 & \\
\hline-1.3666 & 0.2522 & 2.2126 & C & 0 & 0 & 0 & 0 & 0 & 0 & 0 & 0 & 0 \\
\hline-1.5325 & -0.9750 & 1.2934 & C & 0 & & & 0 & 0 & 0 & 0 & 0 & 0 \\
\hline-1.2352 & -0.5101 & -0.0783 & $\mathrm{~N}$ & 0 & & 0 & 0 & 0 & 0 & 0 & 0 & 0 \\
\hline 0.0896 & 1.9253 & 3.2003 & C & 0 & & & 0 & 0 & 0 & 0 & 0 & 0 \\
\hline-2.3602 & 0.7338 & 2.7633 & 0 & 0 & & & 0 & 0 & 0 & 0 & 0 & 0 \\
\hline 0.2024 & -0.0024 & -1.9302 & C & 0 & 0 & & 0 & 0 & 0 & 0 & 0 & 0 \\
\hline-0.5868 & -0.6345 & -2.9146 & C & 0 & & & 0 & 0 & 0 & 0 & 0 & 0 \\
\hline 1.1819 & 0.9354 & -2.3193 & C & 0 & 0 & 0 & 0 & 0 & 0 & 0 & 0 & 0 \\
\hline 517 & 1.2 & -3.6677 & C & 0 & & & 0 & 0 & 0 & 0 & 0 & 0 \\
\hline 0.5666 & 0.6145 & -4.6392 & C & 0 & 0 & 0 & 0 & 0 & 0 & 0 & 0 & 0 \\
\hline-0.3977 & -0.3287 & -4.2611 & C & 0 & 0 & 0 & 0 & 0 & 0 & 0 & & \\
\hline 4.2404 & -0.5871 & 2.7522 & $\mathrm{H}$ & 0 & 0 & 0 & 0 & 0 & 0 & 0 & 0 & 0 \\
\hline 4.3573 & -1.4821 & 0.4166 & $\mathrm{H}$ & 0 & 0 & 0 & 0 & 0 & 0 & 0 & 0 & 0 \\
\hline 2.3656 & -1.3280 & -1.0496 & $\mathrm{H}$ & 0 & 0 & 0 & 0 & 0 & 0 & 0 & 0 & 0 \\
\hline 2.1566 & 0.4194 & 3.5952 & $\mathrm{H}$ & 0 & 0 & 0 & 0 & 0 & 0 & 0 & 0 & 0 \\
\hline-0.8079 & -1.7649 & 1.5471 & $\mathrm{H}$ & 0 & 0 & 0 & 0 & 0 & 0 & 0 & 0 & 0 \\
\hline & & 1.3682 & & & & & & & & & & \\
\hline
\end{tabular}




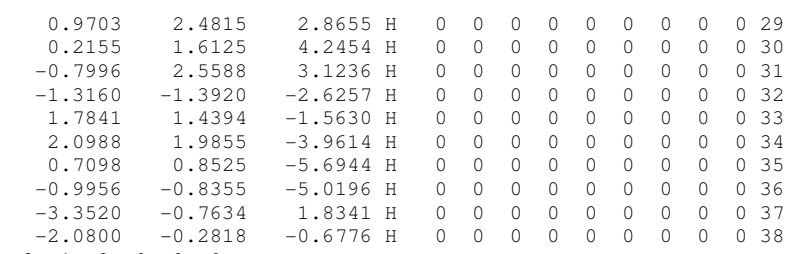




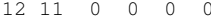

$\begin{array}{llllll}14 & 11 & 0 & 0 & 0 & 0\end{array}$

$\begin{array}{lllllll}13 & 12 & 0 & 0 & 0 & 0 \\ 20 & 13 & 0 & 0 & 0 & 0\end{array}$

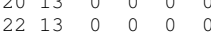

$2813 \quad 000000$

$\begin{array}{llllll}15 & 14 & 0 & 0 & 0 & 0 \\ 16 & 14 & 0 & 0 & 0 & 0\end{array}$

$17 \begin{array}{llllll}15 & 0 & 0 & 0 & 0\end{array}$

$\begin{array}{lllllll}29 & 15 & 0 & 0 & 0 & 0 & 0 \\ 19 & 16 & 0 & 0 & 0 & 0\end{array}$

$\begin{array}{llllll}30 & 16 & 0 & 0 & 0 & 0\end{array}$

$\begin{array}{lllllll}18 & 17 & 0 & 0 & 0 & 0 & 0\end{array}$

$\begin{array}{llllll}31 & 17 & 0 & 0 & 0 & 0 \\ 19 & 18 & 0 & 0 & 0 & 0\end{array}$

$\begin{array}{llllll}32 & 18 & 0 & 0 & 0 & 0\end{array}$

$\begin{array}{llllll}33 & 19 & 0 & 0 & 0 & 0 \\ 21 & 20 & 0 & 0 & 0 & 0\end{array}$

$\begin{array}{lllllll}22 & 34 & 0 & 0 & 0 & 0\end{array}$

$M \quad E N$

1,3-dihydro-3-hydroxy-5-phenyl-1,4-benzodiazepin-2-one-cation.sdf

$\begin{array}{llllllllllll}35 & 36 & 0 & 0 & 0 & 0 & 0 & 0 & 0 & 0 & 0 & \mathrm{~V} 2000\end{array}$

$\begin{array}{lllllllllllll}-3.4973 & -0.4119 & -1.2084 & 0 & 0 & 0 & 0 & 0 & 0 & 0 & 0 & 0 & 0\end{array}$

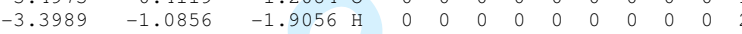

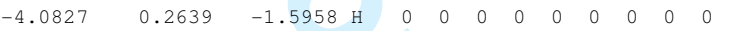

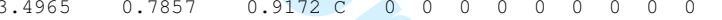

$\begin{array}{lllllllllllll}2.3507 & 0.8126 & 0.1363 & \mathrm{C} & 0 & 0 & 0 & 0 & 0 & 0 & 0 & 0 & 0\end{array}$

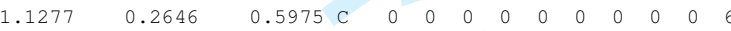

$\begin{array}{llllllllllllll}1.0944 & -0.3119 & 1.9034 & \mathrm{C} & 0 & 0 & 0 & 0 & 0 & 0 & 0 & 0 & 0 & 7\end{array}$

$\begin{array}{llllllllllllll}2.2749 & -0.3739 & 2.6610 & \mathrm{C} & 0 & 0 & 0 & 0 & 0 & 0 & 0 & 0 & 0 & 8\end{array}$

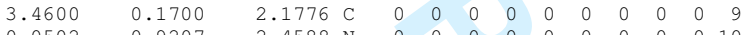

$\begin{array}{llllllllllllll}-0.0502 & -0.9207 & 2.4588 & \mathrm{~N} & 0 & 0 & 0 & 0 & 0 & 0 & 0 & 0 & 0 & 10\end{array}$

$\begin{array}{llllllllllllll}-0.0125 & 0.2391 & -0.3143 & \mathrm{C} & 0 & 0 & 0 & 0 & 0 & 0 & 0 & 0 & 0 & 11 \\ -1.2399 & 0.3833 & 0.1408 & \mathrm{~N} & 0 & 0 & 0 & 0 & 0 & 0 & 0 & 0 & 0 & 12\end{array}$

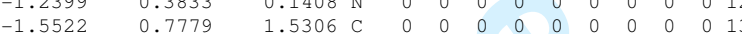

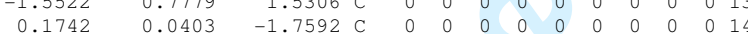

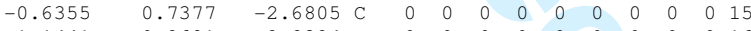

$\begin{array}{llllllllllllll}1.1441 & -0.8681 & -2.2324 & \mathrm{C} & 0 & 0 & 0 & 0 & 0 & 0 & 0 & 0 & 0 & 16\end{array}$

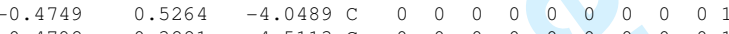

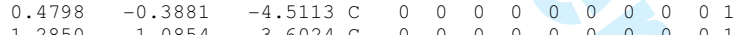

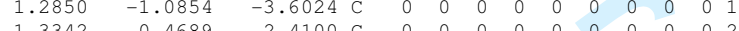

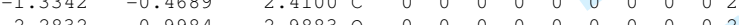

$-2.2832-1.2172-1.625400021$

$\begin{array}{lllllllllllllllll}-2.8640 & 1.2172 & 1.6254 & 0 & 0 & 0 & 0 & 0 & 0 & 0 & 0 & 0 & 0 & 22\end{array}$

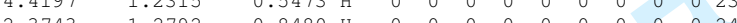

$\begin{array}{lllllllllllllllll}2.3743 & 1.2792 & -0.8480 & \mathrm{H} & 0 & 0 & 0 & 0 & 0 & 0 & 0 & 0 & 0 & 0 & 23 \\ 2.2455 & -0.8458 & -3.6442 & \mathrm{H} & 0 & 0 & 0 & 0 & 0 & 0 & 0 & 0 & 0 & 24\end{array}$

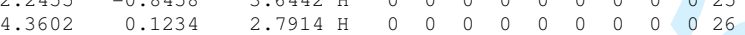

$\begin{array}{llllllllllllll}0.1195 & -1.6699 & 3.1334 \mathrm{H} & 0 & 0 & 0 & 0 & 0 & 0 & 0 & 0 & 0 & 27\end{array}$

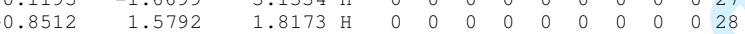

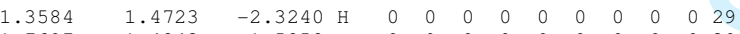

$\begin{array}{llllllllllllll}1.7607 & -1.4243 & -1.5258 & \mathrm{H} & 0 & 0 & 0 & 0 & 0 & 0 & 0 & 0 & 0 & 30\end{array}$

$\begin{array}{llllllllllllll}-1.0892 & 1.0834 & -4.7575 & \mathrm{H} & 0 & 0 & 0 & 0 & 0 & 0 & 0 & 0 & 0 & 31\end{array}$

$\begin{array}{llllllllllllll}0.6003 & -0.5532 & -5.5831 & \mathrm{H} & 0 & 0 & 0 & 0 & 0 & 0 & 0 & 0 & 0 & 32 \\ 2.0245 & -1.8020 & -3.9622 & \mathrm{H} & 0 & 0 & 0 & 0 & 0 & 0 & 0 & 0 & 0 & 33\end{array}$

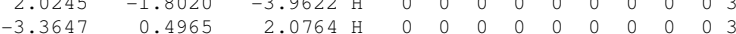

$\begin{array}{rrrrrrrrrrrrrrr}-3.3647 & 0.4965 & 2.0764 & \mathrm{H} & 0 & 0 & 0 & 0 & 0 & 0 & 0 & 0 & 0 & 34 \\ -2.0802 & 0.1606 & -0.4670 & \mathrm{H} & 0 & 0 & 0 & 0 & 0 & 0 & 0 & 0 & 0 & 35\end{array}$

$\begin{array}{llllll}3 & 1 & 0 & 0 & 0 & 0\end{array}$

$\begin{array}{lllllll}5 & 4 & 0 & 0 & 0 & 0\end{array}$

$\begin{array}{llllll}3 & 4 & 0 & 0 & 0\end{array}$

$\begin{array}{lllll}5 & 0 & 0 & 0 & 0\end{array}$

$\begin{array}{llllll}24 & 5 & 0 & 0 & 0 & 0\end{array}$

$\begin{array}{llllll}1 & 6 & 0 & 0 & 0\end{array}$

$\begin{array}{llllll}8 & 7 & 0 & 0 & 0 & 0\end{array}$

$\begin{array}{lllll}0 & 7 & 0 & 0 & 0\end{array}$

$\begin{array}{llllll}9 & 8 & 0 & 0 & 0 & 0\end{array}$

$\begin{array}{llllll}8 & 8 & 0 & 0 & 0\end{array}$

$\begin{array}{llllll}26 & 9 & 0 & 0 & 0 & 0\end{array}$

$\begin{array}{llllll}10 & 0 & 0 & 0\end{array}$

$\begin{array}{llllll}12 & 11 & 0 & 0 & 0\end{array}$

$\begin{array}{llllll}14 & 11 & 0 & 0 & 0 & 0\end{array}$

$\begin{array}{llllll}3 & 12 & 0 & 0 & 0 & 0\end{array}$

$\begin{array}{llllll}22 & 13 & 0 & 0 & 0 & 0\end{array}$

$\begin{array}{llllll}28 & 13 & 0 & 0 & 0 & 0\end{array}$

$\begin{array}{lllll}5 & 13 & 0 & 0 & 0\end{array}$

$\begin{array}{llllll}6 & 14 & 0 & 0 & 0\end{array}$

$\begin{array}{llllll}17 & 15 & 0 & 0 & 0 & 0\end{array}$

$\begin{array}{lllll}15 & 0 & 0 & 0\end{array}$

16 o 0 o

$\begin{array}{lllll}16 & 0 & 0 & 0 & 0\end{array}$

$\begin{array}{lllll}17 & 0 & 0 & 0 & 0\end{array}$

$\begin{array}{llllll}1 & 18 & 0 & 0 & 0\end{array}$

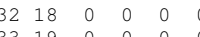

$\begin{array}{llllll}33 & 19 & 0 & 0 & 0 & 0\end{array}$

$\begin{array}{llllll}3 & 20 & 0 & 0 & 0 & 0\end{array}$

$\begin{array}{llllll}12 & 35 & 0 & 0 & 0 & 0\end{array}$

END

1,3-dihydro-5-phenyl-1,4-benzodiazepin-2-one.sdf

cosmotherm

$\begin{array}{llllllllllll}33 & 34 & 0 & 0 & 0 & 0 & 0 & 0 & 0 & 0 & 0 & \mathrm{v} 2000\end{array}$

$\begin{array}{lllllllllllllll}-1.7446 & -3.6345 & -0.1202 & 0 & 0 & 0 & 0 & 0 & 0 & 0 & 0 & 0 & 0 & 1\end{array}$

$\begin{array}{lllllllllllll}-1.1984 & -2.8103 & -0.2830 & \mathrm{H} & 0 & 0 & 0 & 0 & 0 & 0 & 0 & 0 & 0\end{array}$

URL: http://mc.manuscriptcentral.com/tandf/tmph 


\begin{tabular}{|c|c|c|c|c|c|c|c|c|c|c|c|c|}
\hline 2.7947 & 2.8635 & -0.1177 & C & 0 & 0 & 0 & 0 & 0 & 0 & 0 & 0 & 0 \\
\hline 1.5638 & 3.1311 & -0.7351 & C & 0 & 0 & 0 & 0 & 0 & 0 & 0 & 0 & 0 \\
\hline 0.6028 & 2.1287 & -0.8102 & $\mathrm{C}$ & 0 & 0 & 0 & 0 & 0 & 0 & 0 & 0 & 0 \\
\hline 0.8462 & 0.8295 & -0.3117 & $\mathrm{C}$ & 0 & 0 & 0 & 0 & 0 & 0 & 0 & 0 & 0 \\
\hline 2.1150 & 0.5609 & 0.2659 & $\mathrm{C}$ & 0 & 0 & 0 & 0 & 0 & 0 & 0 & 0 & 0 \\
\hline 3.0657 & 1.5921 & 0.3779 & C & & 0 & 0 & 0 & 0 & 0 & 0 & 0 & 0 \\
\hline 0.2349 & -0.1835 & -0.3449 & C & & 0 & 0 & 0 & 0 & 0 & 0 & 0 & 0 \\
\hline 2.4506 & 0.6 & 0.8068 & $\mathrm{~N}$ & & 0 & 0 & 0 & 0 & 0 & 0 & 0 & 0 \\
\hline 2.1913 & 1.9 & 0.2583 & $\mathrm{C}$ & & 0 & 0 & 0 & 0 & 0 & 0 & 0 & 0 \\
\hline 1.2893 & -1.8 & -0.9702 & C & & 0 & 0 & 0 & 0 & 0 & 0 & 0 & 0 \\
\hline-0.049 & -1 & -0. & $\mathrm{~N}$ & & 0 & 0 & 0 & 0 & 0 & 0 & 0 & 0 \\
\hline 2.6533 & -2.9 & 461 & 0 & & 0 & 0 & 0 & 0 & 0 & 0 & 0 & 0 \\
\hline-1.6296 & & -0.0569 & C & & 0 & 0 & 0 & 0 & 0 & 0 & 0 & 0 \\
\hline-2.7081 & -0 . & -0 . & C & 0 & 0 & 0 & 0 & 0 & 0 & 0 & 0 & 0 \\
\hline-1.8939 & 1.218 & 0.9384 & C & 0 & 0 & 0 & 0 & 0 & 0 & 0 & 0 & 0 \\
\hline-3.2090 & 1.5 & 1.23 & C & 0 & 0 & 0 & 0 & 0 & 0 & 0 & 0 & 0 \\
\hline-4.2748 & 1.0150 & 0.5300 & C & 0 & 0 & 0 & 0 & 0 & 0 & 0 & 0 & 0 \\
\hline-4.0204 & & $-0.47 C$ & C & 0 & 0 & 0 & 0 & 0 & 0 & 0 & 0 & 0 \\
\hline 3.5495 & 3.6467 & -0.0323 & $\mathrm{H}$ & 0 & 0 & 0 & 0 & 0 & 0 & 0 & 0 & 0 \\
\hline & & -1.1414 & $\mathrm{H}$ & 0 & 0 & 0 & 0 & 0 & 0 & 0 & 0 & 0 \\
\hline-0.3648 & 2.33 & -1.2669 & $\mathrm{H}$ & 0 & 0 & 0 & 0 & 0 & 0 & 0 & 0 & 0 \\
\hline 4.0267 & & & $\mathrm{H}$ & 0 & 0 & 0 & 0 & 0 & 0 & 0 & 0 & 0 \\
\hline 1.7482 & -1.2 & -1.7297 & $\mathrm{H}$ & & 0 & 0 & 0 & 0 & 0 & 0 & 0 & 0 \\
\hline-2.5072 & -1.0 & -1.5412 & $\mathrm{H}$ & & 0 & 0 & 0 & 0 & 0 & 0 & 0 & 0 \\
\hline-1.0685 & 1.66 & 1.4960 & $\mathrm{H}$ & & 0 & 0 & 0 & 0 & 0 & 0 & 0 & 0 \\
\hline-3.4015 & & & $\mathrm{H}$ & 0 & 0 & 0 & 0 & 0 & 0 & 0 & 0 & 0 \\
\hline & & & & 0 & 0 & 0 & 0 & 0 & 0 & 0 & 0 & 0 \\
\hline-4 . & 0.3 & -1.03 & $\mathrm{H}$ & 0 & 0 & 0 & 0 & 0 & 0 & 0 & 0 & 0 \\
\hline & & & & & 0 & 0 & 0 & 0 & 0 & 0 & 0 & 0 \\
\hline & & & & & & & & & & & & \\
\hline
\end{tabular}




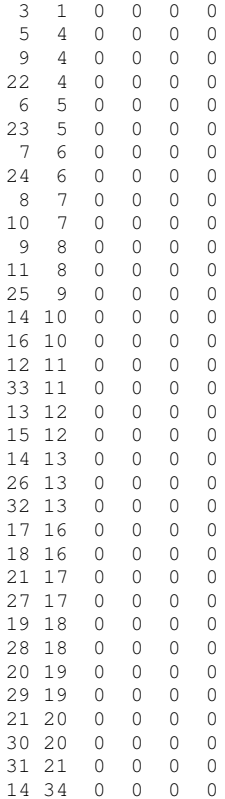
$M \quad E N D$

1 -methylindole-cation 0 sd

cosmotherm

$\begin{array}{llllllllllll}23 & 23 & 0 & 0 & 0 & 0 & 0 & 0 & 0 & 0 & 0 & \mathrm{~V} 2000\end{array}$

$\begin{array}{lllllllllllllll}1.2462 & 0.2039 & -0.6915 & 0 & 0 & 0 & 0 & 0 & 0 & 0 & 0 & 0 & 0 & 1\end{array}$

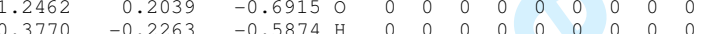

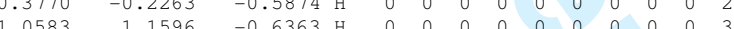

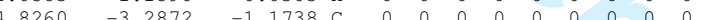

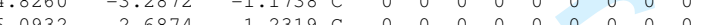

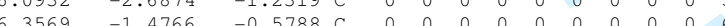

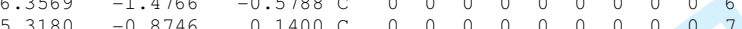
$\begin{array}{llllllllllllll}.3180 & -0.8746 & 0.1400 & \mathrm{C} & 0 & 0 & 0 & 0 & 0 & 0 & 0 & 0 & 0 & 0\end{array}$

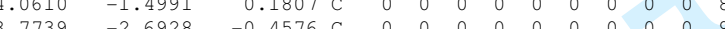

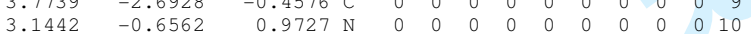
$\begin{array}{llllllllllllll}5.2292 & 0.3579 & 0.9185 & \mathrm{C} & 0 & 0 & 0 & 0 & 0 & 0 & 0 & 0 & 0 & 11\end{array}$

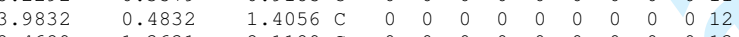

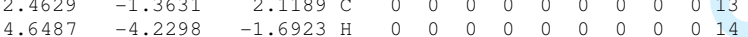

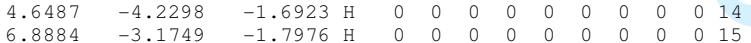
$\begin{array}{llllllllllllll}6.8884 & -3.1749 & -1.7976 & \mathrm{H} & 0 & 0 & 0 & 0 & 0 & 0 & 0 & 0 & 0 & 14 \\ 7.3436 & -1.0151 & -0.6305 & \mathrm{H} & 0 & 0 & 0 & 0 & 0 & 0 & 0 & 0 & 0 & 16\end{array}$

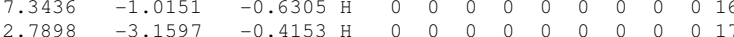

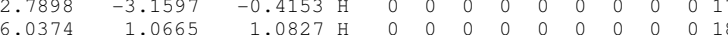
$0.0240 \mathrm{H}$ $\begin{array}{lllllllllllll}2.0240 \mathrm{H} & 0 & 0 & 0 & 0 & 0 & 0 & 0 & 0 & 0 & 19\end{array}$ $\begin{array}{llllllllllllll}2.3555 & -0.2791 & 0.3144 & \mathrm{H} & 0 & 0 & 0 & 0 & 0 & 0 & 0 & 0 & 0 & 20\end{array}$ $\begin{array}{lllllllllllll}-2.1755 & 1.7116 & \mathrm{H} & 0 & 0 & 0 & 0 & 0 & 0 & 0 & 0 & 0 & 21 \\ -0.6372 & 2.6333 & \mathrm{H} & 0 & 0 & 0 & 0 & 0 & 0 & 0 & 0 & 22\end{array}$ $\begin{array}{llllllllllllll}3.2290 & -1.7545 & 2.7333 & \mathrm{H} & 0 & 0 & 0 & 0 & 0 & 0 & 0 & 0 & 0 & 22 \\ 1 & 2.7951 & \mathrm{H} & 0 & 0 & 0 & 0 & 0 & 0 & 0 & 0 & 0 & 23\end{array}$ $\begin{array}{llllll}2 & 1 & 0 & 0 & 0 & 0\end{array}$ $\begin{array}{llllll}3 & 1 & 0 & 0 & 0 & 0\end{array}$

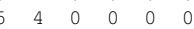
$\begin{array}{lllllll}9 & 4 & 0 & 0 & 0 & 0\end{array}$ $\begin{array}{llllll}4 & 4 & 0 & 0 & 0\end{array}$ $6 \begin{array}{lllll}6 & 5 & 0 & 0 & 0\end{array}$ $\begin{array}{llllll}5 & 5 & 0 & 0 & 0\end{array}$ $\begin{array}{lllll}6 & 0 & 0 & 0\end{array}$ $\begin{array}{lllll}7 & 0 & 0 & 0\end{array}$ $\begin{array}{llll}0 & 0 & 0\end{array}$ $\begin{array}{llllll}10 & 8 & 0 & 0 & 0\end{array}$ $\begin{array}{lllll}9 & 0 & 0 & 0\end{array}$

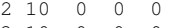
$\begin{array}{llllll}13 & 10 & 0 & 0 & 0 & 0\end{array}$ $\begin{array}{lllllll}2 & 11 & 0 & 0 & 0 & 0\end{array}$ $\begin{array}{llllll}8 & 11 & 0 & 0 & 0 & 0\end{array}$ $\begin{array}{llllll}9 & 12 & 0 & 0 & 0 & 0\end{array}$ $\begin{array}{llllll}1 & 13 & 0 & 0 & 0 & 0\end{array}$ $\begin{array}{llllll}2 & 13 & 0 & 0 & 0 & 0 \\ 3 & 23 & 0 & 0 & 0 & 0\end{array}$

-methylindole-cation1.sdf

$\begin{array}{rrrrrrrrrrrrrrrr}23 & 23 & 0 & 0 & 0 & 0 & 0 & 0 & 0 & 0 & \mathrm{v} 2000 & \\ 0.4865 & -0.1995 & 0.2488 & 0 & 0 & 0 & 0 & 0 & 0 & 0 & 0 & 0 & 0 & 1 \\ 0.1818 & -0.8389 & -0.4203 & \mathrm{H} & 0 & 0 & 0 & 0 & 0 & 0 & 0 & 0 & 0 & 2 \\ -0.1009 & 0.5679 & 0.1255 & \mathrm{H} & 0 & 0 & 0 & 0 & 0 & 0 & 0 & 0 & 0 & 3 \\ 5.5162 & 4.4930 & 0.6148 & \mathrm{C} & 0 & 0 & 0 & 0 & 0 & 0 & 0 & 0 & 0 & 4 \\ 6.1017 & 3.7036 & 1.6644 & \mathrm{C} & 0 & 0 & 0 & 0 & 0 & 0 & 0 & 0 & 0 & 5 \\ 5.8064 & 2.3720 & 1.8021 & \mathrm{C} & 0 & 0 & 0 & 0 & 0 & 0 & 0 & 0 & 0 & 6 \\ 4.8972 & 1.7814 & 0.8672 & \mathrm{C} & 0 & 0 & 0 & 0 & 0 & 0 & 0 & 0 & 0 & 7 \\ 4.3160 & 2.6008 & -0.1929 & \mathrm{C} & 0 & 0 & 0 & 0 & 0 & 0 & 0 & 0 & 0 & 8 \\ 4.6338 & 3.9776 & -0.3094 & \mathrm{C} & 0 & 0 & 0 & 0 & 0 & 0 & 0 & 0 & 0 & 9 \\ 3.5029 & 1.8509 & -0.9459 & \mathrm{~N} & 0 & 0 & 0 & 0 & 0 & 0 & 0 & 0 & 0 & 10 \\ 4.3950 & 0.5088 & 0.7232 & \mathrm{C} & 0 & 0 & 0 & 0 & 0 & 0 & 0 & 0 & 0 & 11 \\ 3.4708 & 0.4917 & -0.4231 & \mathrm{C} & 0 & 0 & 0 & 0 & 0 & 0 & 0 & 0 & 0 & 12 \\ 2.7162 & 2.2989 & -2.0908 & \mathrm{C} & 0 & 0 & 0 & 0 & 0 & 0 & 0 & 0 & 0 & 13\end{array}$




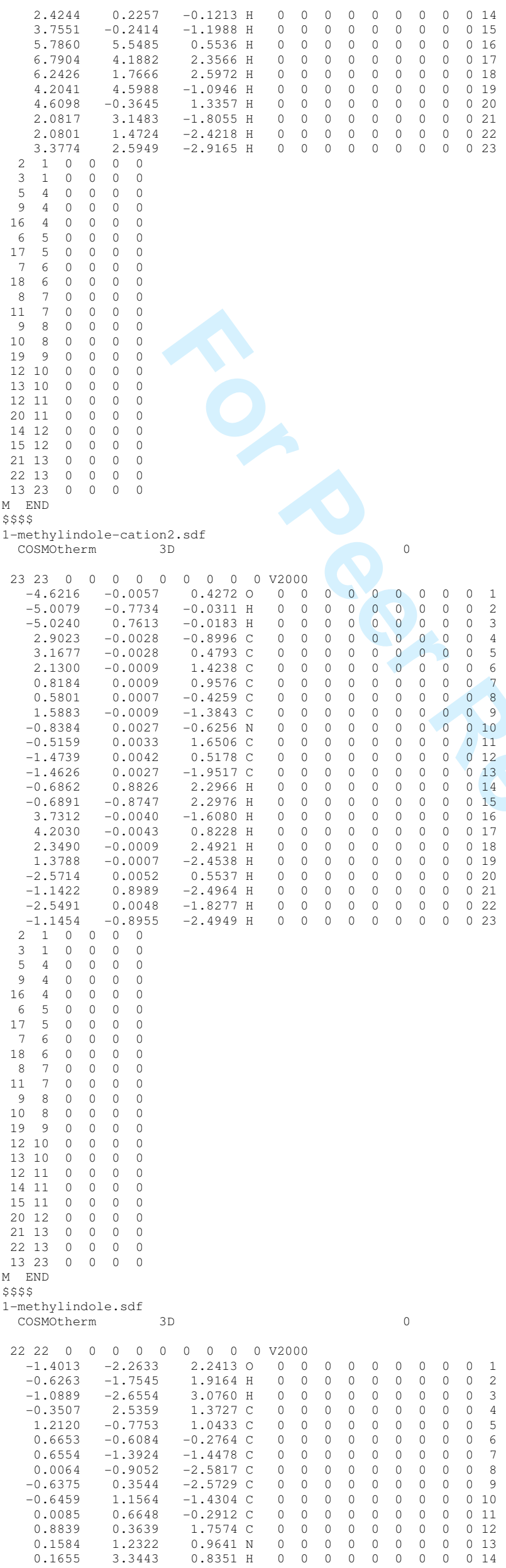




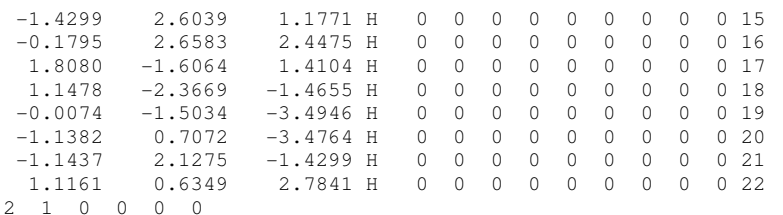




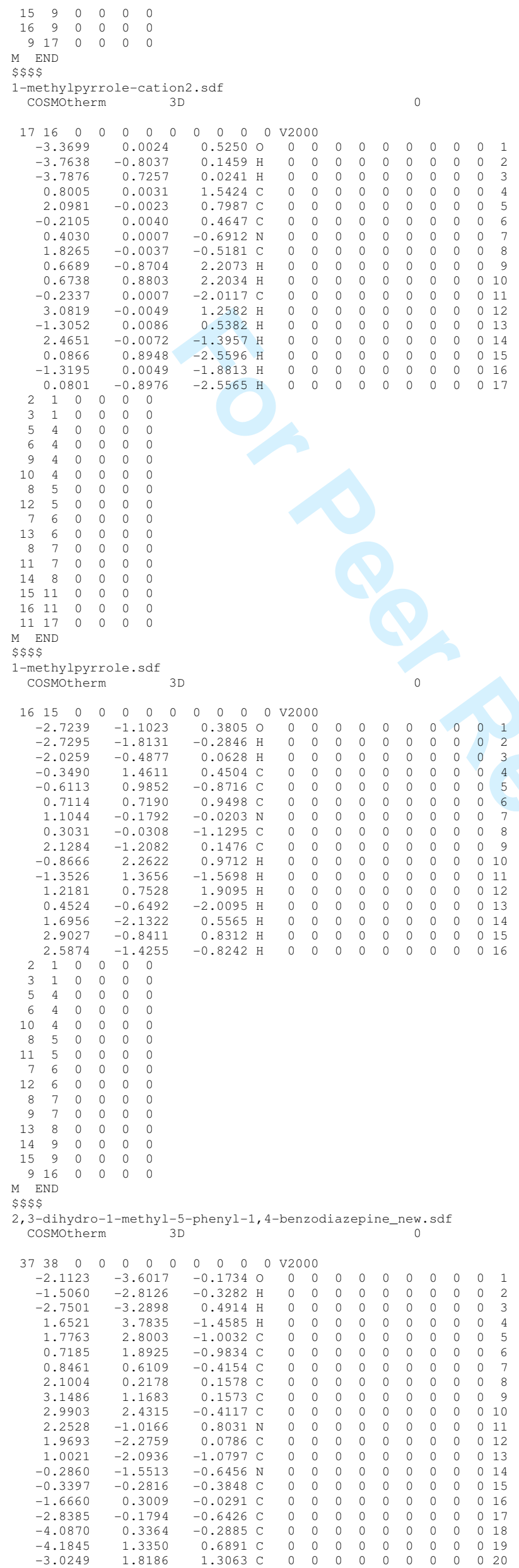




$\begin{array}{rrrrllllllllll}-1.7735 & 1.3128 & 0.9437 & \mathrm{C} & 0 & 0 & 0 & 0 & 0 & 0 & 0 & 0 & 0 & 21 \\ 3.3800 & -1.1816 & 1.7201 & \mathrm{C} & 0 & 0 & 0 & 0 & 0 & 0 & 0 & 0 & 0 & 22 \\ -0.2427 & 2.1754 & -1.4161 & \mathrm{H} & 0 & 0 & 0 & 0 & 0 & 0 & 0 & 0 & 0 & 23 \\ 4.1115 & 0.9035 & 0.5916 & \mathrm{H} & 0 & 0 & 0 & 0 & 0 & 0 & 0 & 0 & 0 & 24 \\ 3.8314 & 3.1278 & -0.4035 & \mathrm{H} & 0 & 0 & 0 & 0 & 0 & 0 & 0 & 0 & 0 & 25 \\ 1.5656 & -3.0030 & 0.8007 & \mathrm{H} & 0 & 0 & 0 & 0 & 0 & 0 & 0 & 0 & 0 & 26 \\ 2.9144 & -2.6969 & -0.3203 & \mathrm{H} & 0 & 0 & 0 & 0 & 0 & 0 & 0 & 0 & 0 & 27 \\ 0.8305 & -3.0782 & -1.5369 & \mathrm{H} & 0 & 0 & 0 & 0 & 0 & 0 & 0 & 0 & 0 & 28 \\ 1.4597 & -1.4435 & -1.8461 & \mathrm{H} & 0 & 0 & 0 & 0 & 0 & 0 & 0 & 0 & 0 & 29 \\ -2.7602 & -0.9543 & -1.4061 & \mathrm{H} & 0 & 0 & 0 & 0 & 0 & 0 & 0 & 0 & 0 & 30 \\ -4.9868 & -0.0355 & -0.7820 & \mathrm{H} & 0 & 0 & 0 & 0 & 0 & 0 & 0 & 0 & 0 & 31 \\ -5.1607 & 1.7373 & 0.9659 & \mathrm{H} & 0 & 0 & 0 & 0 & 0 & 0 & 0 & 0 & 0 & 32 \\ -3.0946 & 2.5932 & 2.0721 & \mathrm{H} & 0 & 0 & 0 & 0 & 0 & 0 & 0 & 0 & 0 & 33 \\ -0.8737 & 1.6924 & 1.4303 & \mathrm{H} & 0 & 0 & 0 & 0 & 0 & 0 & 0 & 0 & 0 & 34 \\ 3.4466 & -0.3255 & 2.4032 & \mathrm{H} & 0 & 0 & 0 & 0 & 0 & 0 & 0 & 0 & 0 & 35 \\ 3.2037 & -2.0860 & 2.3182 & \mathrm{H} & 0 & 0 & 0 & 0 & 0 & 0 & 0 & 0 & 0 & 36 \\ 4.3498 & -1.3029 & 1.1992 & \mathrm{H} & 0 & 0 & 0 & 0 & 0 & 0 & 0 & 0 & 0 & 37\end{array}$




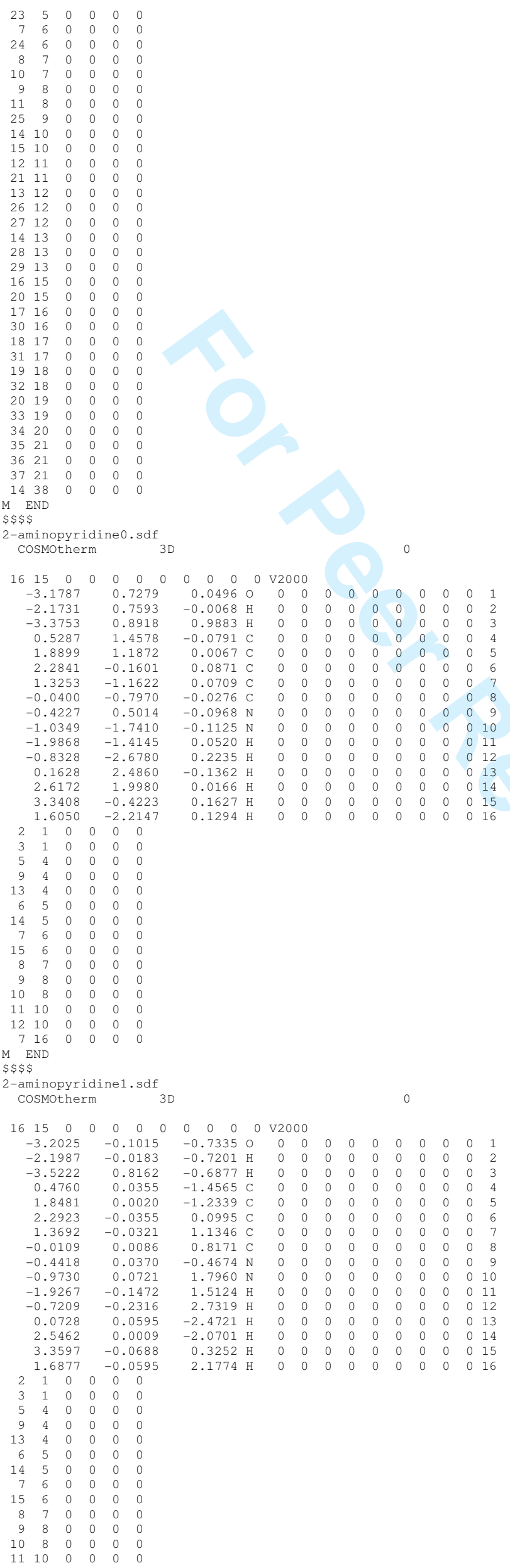




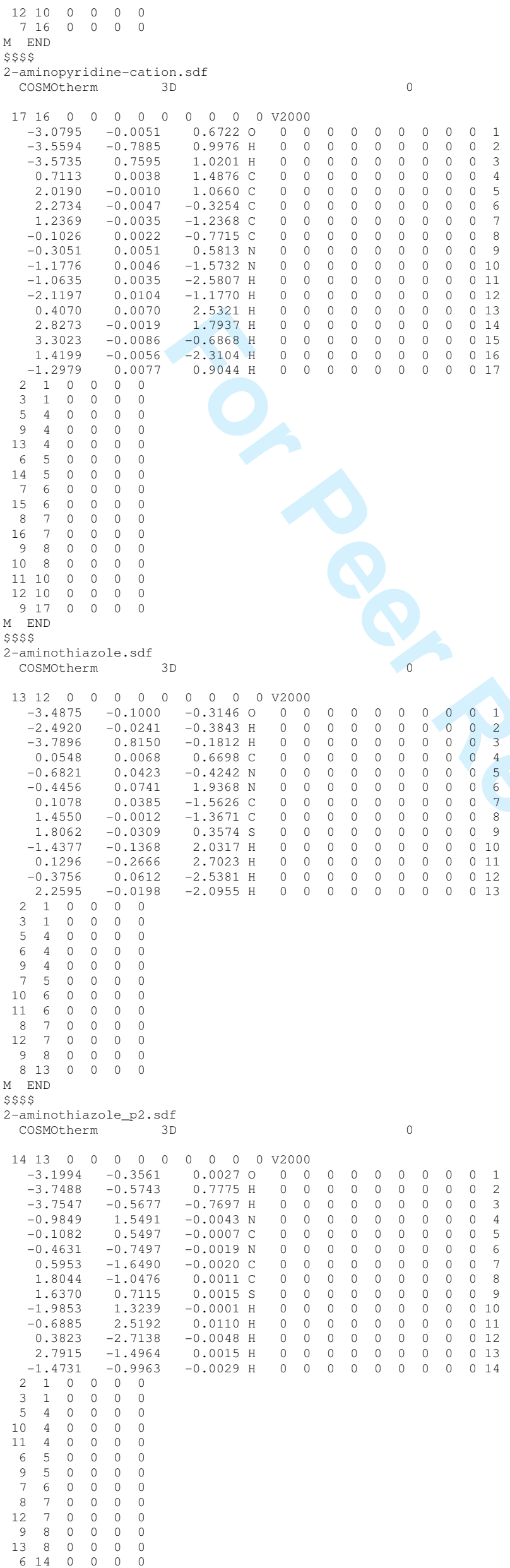




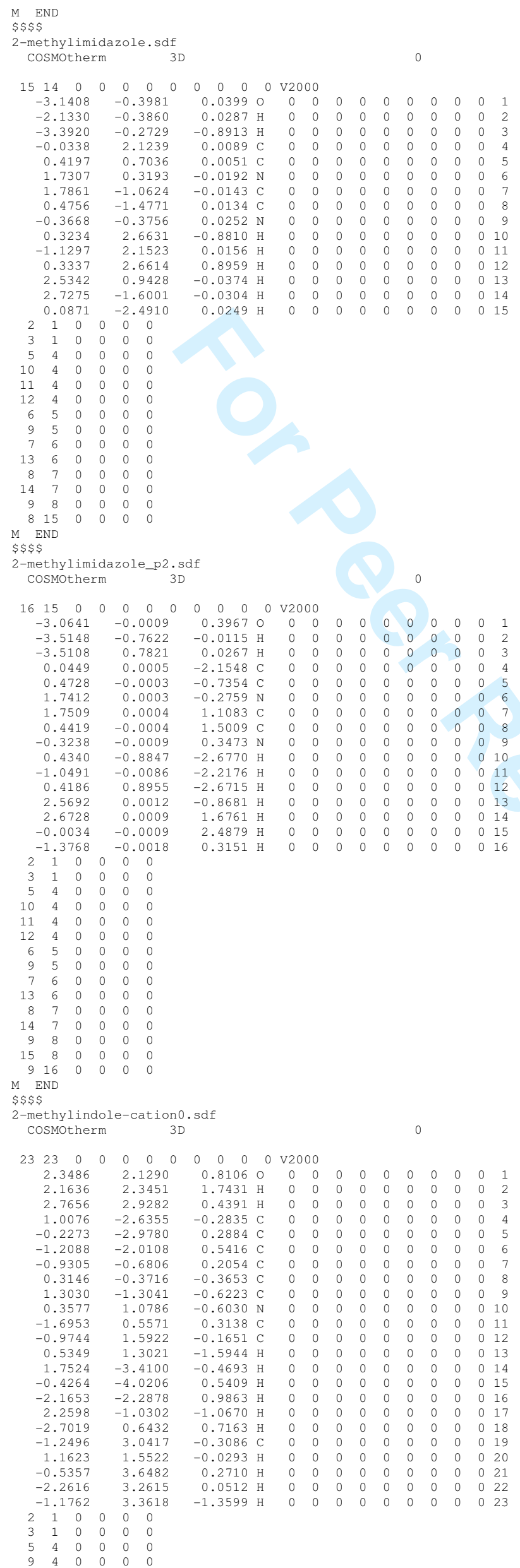




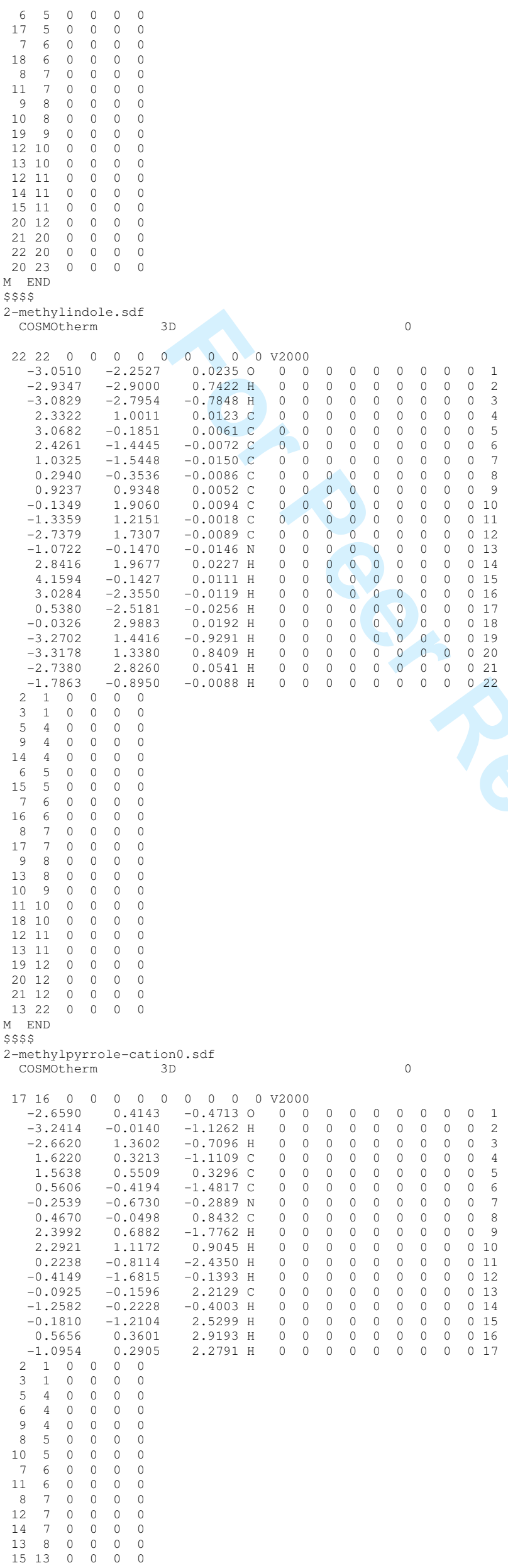




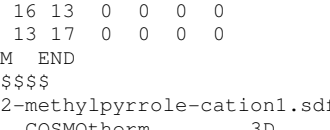

cosmotherm

$\begin{array}{llllllllllll}17 & 16 & 0 & 0 & 0 & 0 & 0 & 0 & 0 & 0 & 0 & \mathrm{~V} 2000\end{array}$

$\begin{array}{llllllllllllll}-3.0859 & 0.0803 & -0.2013 & 0 & 0 & 0 & 0 & 0 & 0 & 0 & 0 & 0 & 0 & 1\end{array}$

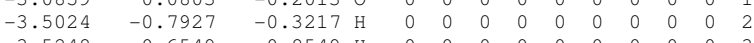

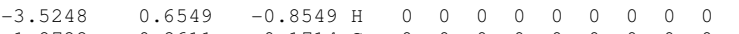

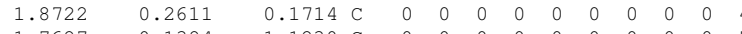

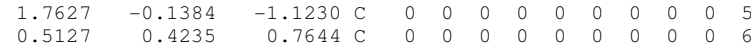

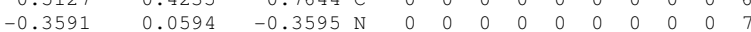

$\begin{array}{llllllllllllll}0.3546 & -0.2502 & -1.4110 & \mathrm{C} & 0 & 0 & 0 & 0 & 0 & 0 & 0 & 0 & 0 & 8\end{array}$

$\begin{array}{llllllllllllll}-1.4170 & 0.0615 & -0.3176 & \mathrm{H} & 0 & 0 & 0 & 0 & 0 & 0 & 0 & 0 & 0 & 9\end{array}$

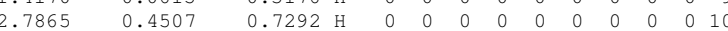

$\begin{array}{lllllllllllll}2.5568 & -0.3435 & -1.8350 \mathrm{H} & 0 & 0 & 0 & 0 & 0 & 0 & 0 & 0 & 0 & 11\end{array}$

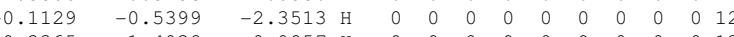

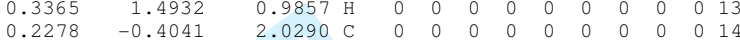

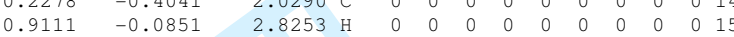

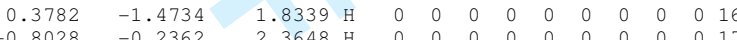

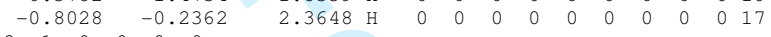

$\begin{array}{llllll}2 & 1 & 0 & 0 & 0 & 0\end{array}$

$\begin{array}{lllllll}3 & 1 & 0 & 0 & 0 & 0 \\ 5 & 4 & 0 & 0 & 0 & 0\end{array}$

$\begin{array}{llllll}6 & 4 & 0 & 0 & 0\end{array}$

1004500000

1 1 5 r

$\begin{array}{llllll}7 & 6 & 0 & 0 & 0 & 0\end{array}$

$\begin{array}{llllll}3 & 6 & 0 & 0 & 0 & 0\end{array}$

$\begin{array}{llllll}4 & 6 & 0 & 0 & 0 & 0 \\ 8 & 7 & 0 & 0 & 0 & 0\end{array}$

$\begin{array}{lllllll}9 & 7 & 0 & 0 & 0 & 0\end{array}$

$\begin{array}{lllll}15 & 14 & 0 & 0 & 0\end{array}$

$\begin{array}{lllllll}6 & 14 & 0 & 0 & 0 & 0\end{array}$

END

-methylpyrrole-cation2.sdf

cosmotherm

$\begin{array}{llllllllllll}17 & 16 & 0 & 0 & 0 & 0 & 0 & 0 & 0 & 0 & 0 & \mathrm{~V} 2000\end{array}$

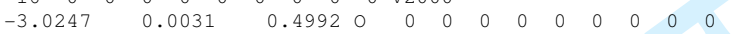

$\begin{array}{lllllllllllll}-3.4819 & -0.8048 & 0.2025 & \mathrm{H} & 0 & 0 & 0 & 0 & 0 & 0 & 0 & 0 & 0\end{array}$

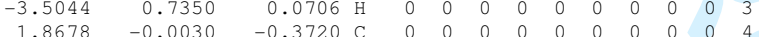

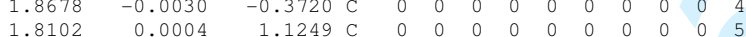

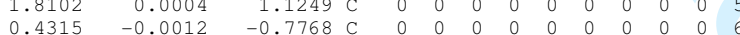

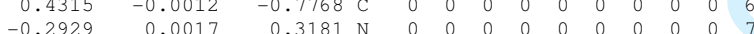

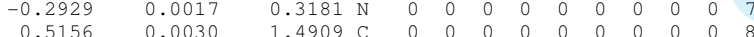

$\begin{array}{lllllllllllll}2.3956 & 0.8713 & -0.7921 & \mathrm{H} & 0 & 0 & 0 & 0 & 0 & 0 & 0 & 0 & 0\end{array}$

$\begin{array}{llllllllllllll}2.3912 & -0.8822 & -0.7875 & \mathrm{H} & 0 & 0 & 0 & 0 & 0 & 0 & 0 & 0 & 0 & 10\end{array}$

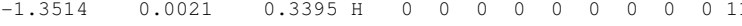

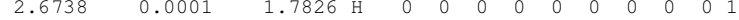

$\begin{array}{llrlllllllllll}0.1222 & 0.0002 & -2.1461 & \mathrm{C} & 0 & 0 & 0 & 0 & 0 & 0 & 0 & 0 & 0 & 13 \\ 0.0387 & 0.0051 & 2.4659 & \mathrm{H} & 0 & 0 & 0 & 0 & 0 & 0 & 0 & 0 & 0 & 1\end{array}$

$\begin{array}{llllllllllll}0 & 0 & 0 & 0 & 0 & 0 & 0 & 0 & 0 & 15\end{array}$

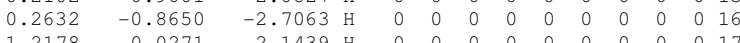

$-1.2178-0.0271$

$\begin{array}{lllllll}2 & 1 & 0 & 0 & 0 & 0 \\ 3 & 1 & 0 & 0 & 0 & 0\end{array}$

$5 \begin{array}{lllllll}5 & 4 & 0 & 0 & 0 & 0\end{array}$

$\begin{array}{llllll}4 & 0 & 0 & 0 & 0\end{array}$

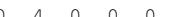

$8 \begin{array}{llllll}8 & 5 & 0 & 0 & 0\end{array}$

$\begin{array}{lllllll}2 & 5 & 0 & 0 & 0 & 0 & 0 \\ 7 & 6 & 0 & 0 & 0 & 0 & 0\end{array}$

$\begin{array}{llllll}7 & 6 & 0 & 0 & 0 & 0\end{array}$

$\begin{array}{lllllll}4 & 8 & 0 & 0 & 0 & 0\end{array}$

$\begin{array}{llllllll}5 & 13 & 0 & 0 & 0 & 0\end{array}$

$16 \begin{array}{lllll}13 & 0 & 0 & 0 & 0\end{array}$

END

-methylpyrrole-cation3.sdf

CosMotherm

$\begin{array}{llllllllllll}17 & 16 & 0 & 0 & 0 & 0 & 0 & 0 & 0 & 0 & 0 & \mathrm{~V} 2000\end{array}$

$\begin{array}{lllllllllll}0 & 0 & v 2000 & 0 & 0 & 0 & 0 & 0 & 0 & 0 & 1\end{array}$

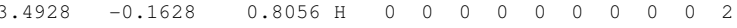

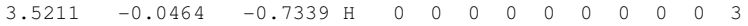

$\begin{array}{llllllllllllll}-0.5326 & -1.5166 & -0.0032 & \mathrm{C} & 0 & 0 & 0 & 0 & 0 & 0 & 0 & 0 & 0 & 4\end{array}$

$\begin{array}{llllllllllllll}0.2949 & -0.3175 & -0.0063 & \mathrm{~N} & 0 & 0 & 0 & 0 & 0 & 0 & 0 & 0 & 0 & 5\end{array}$

$\begin{array}{llllllllllllll}-1.9133 & -0.9656 & 0.0030 & \mathrm{C} & 0 & 0 & 0 & 0 & 0 & 0 & 0 & 0 & 0 & 6\end{array}$

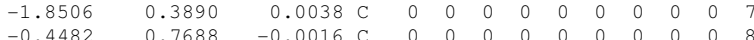

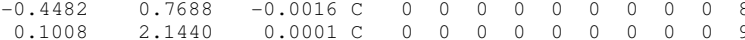

$\begin{array}{llllllllllllll}-0.3132 & -2.1361 & 0.8824 & \mathrm{H} & 0 & 0 & 0 & 0 & 0 & 0 & 0 & 0 & 0 & 10\end{array}$

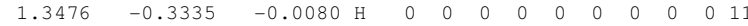

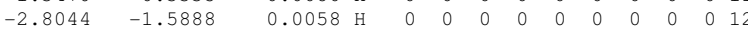

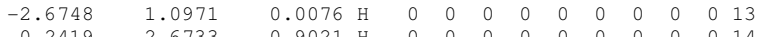

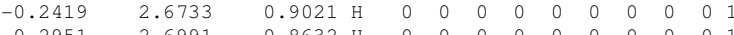

$\begin{array}{llllllllllllll}-0.2951 & 2.6991 & -0.8632 & \mathrm{H} & 0 & 0 & 0 & 0 & 0 & 0 & 0 & 0 & 0 & 15 \\ 1.1959 & 2.1517 & -0.0313 & \mathrm{H} & 0 & 0 & 0 & 0 & 0 & 0 & 0 & 0 & 0 & 16\end{array}$

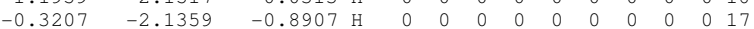

$\begin{array}{lllll}1 & 0 & 0 & 0 & 0\end{array}$ 


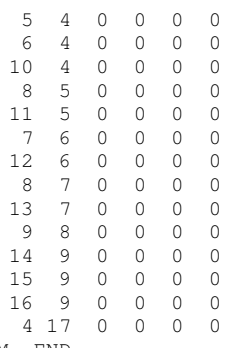




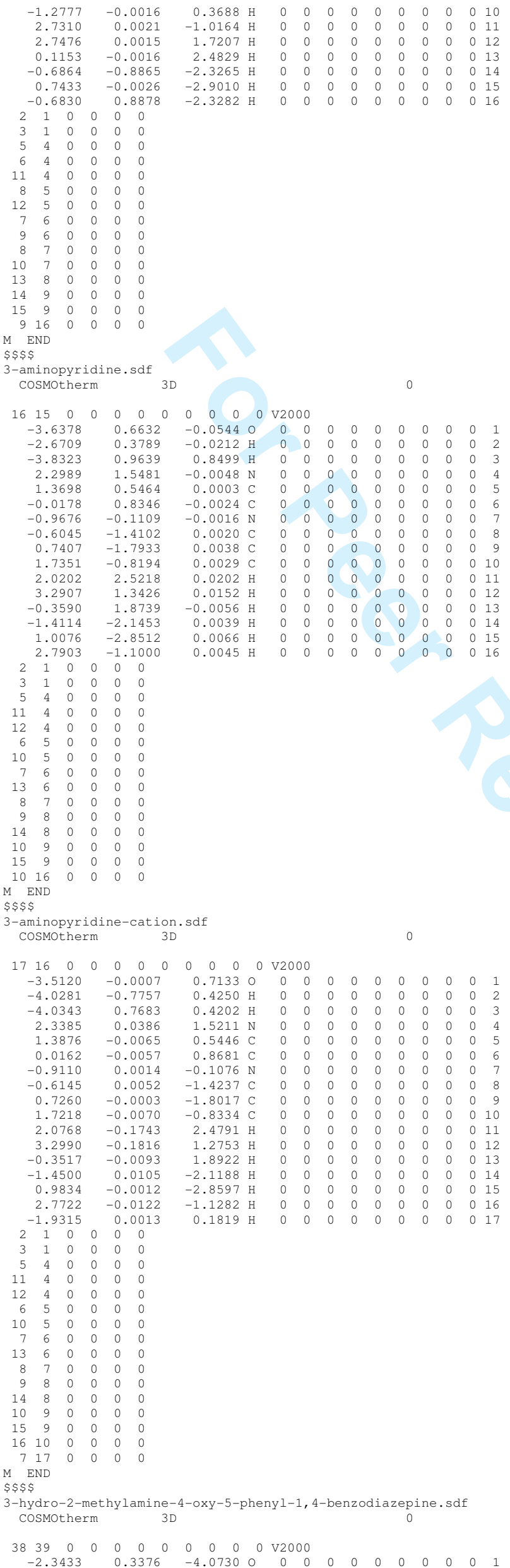




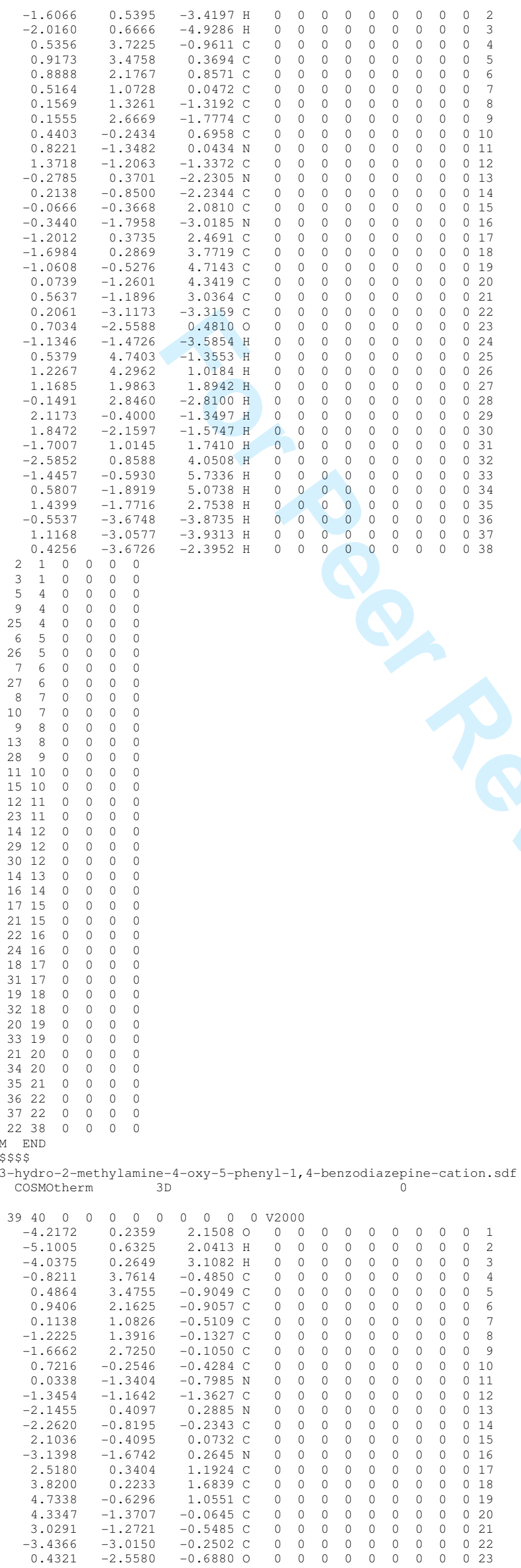




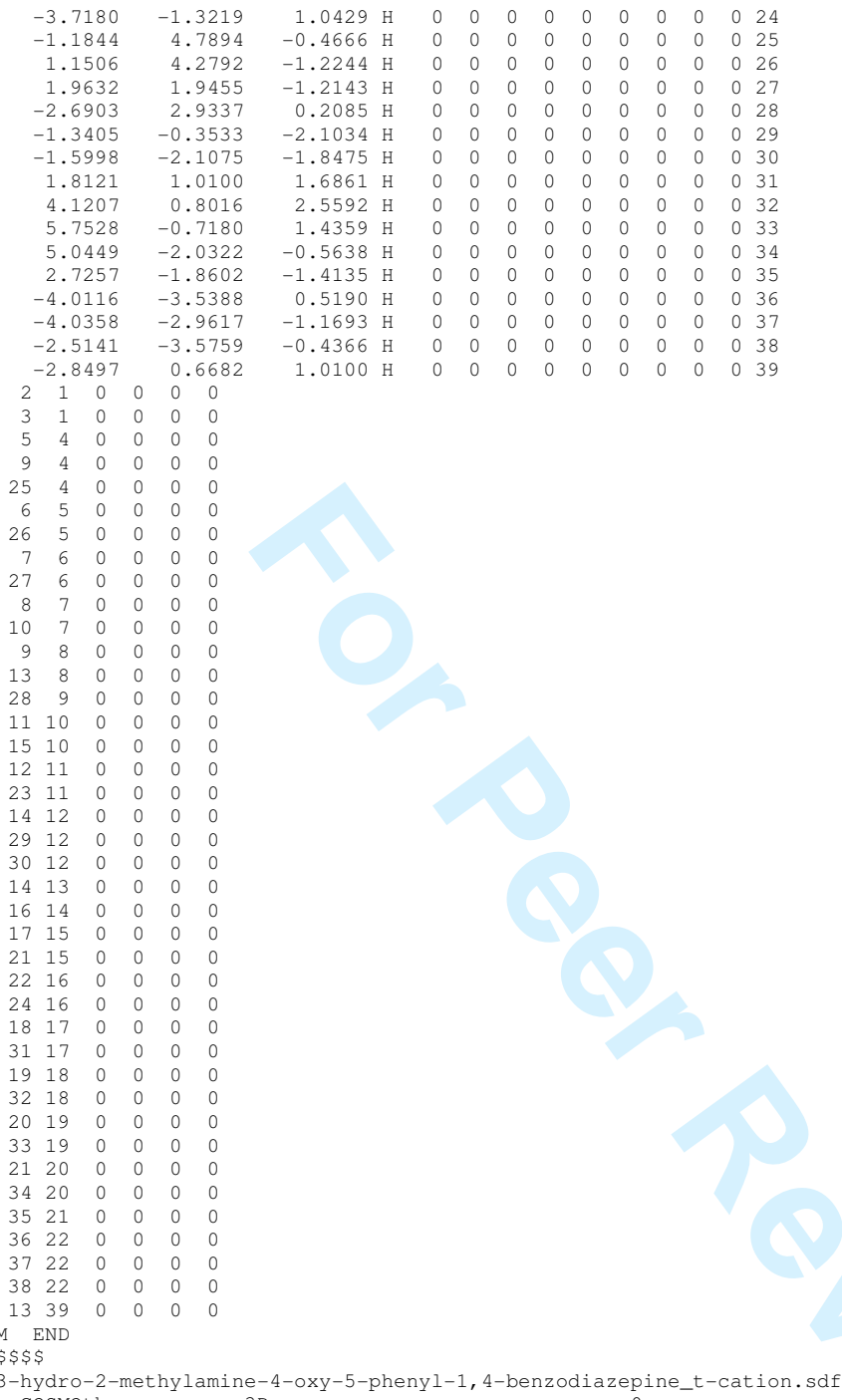

3-hydro-2-methylamine-4-oxy-5-phenyl-1, 4-benzodiazepi
Cosmotherm

$\begin{array}{llllllllllll}39 & 40 & 0 & 0 & 0 & 0 & 0 & 0 & 0 & 0 & 0 & \mathrm{~V} 2000\end{array}$

$\begin{array}{llllllllllllll}-4.2085 & -2.1613 & 0.2194 & 0 & 0 & 0 & 0 & 0 & 0 & 0 & 0 & 0 & 0 & 1\end{array}$

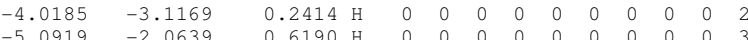

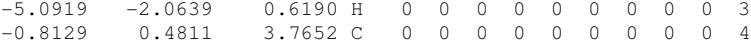

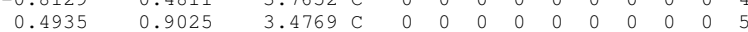

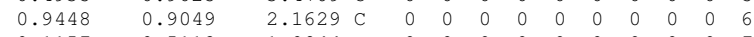

$\begin{array}{lllllllllllll}0.1157 & 0.5112 & 1.0844 & \mathrm{C} & 0 & 0 & 0 & 0 & 0 & 0 & 0 & 0 & 0\end{array}$

$\begin{array}{llllllllllllll}-1.2194 & 0.1317 & 1.3960 & \mathrm{C} & 0 & 0 & 0 & 0 & 0 & 0 & 0 & 0 & 0 & 8\end{array}$

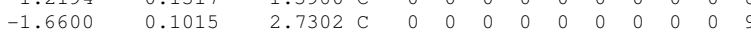

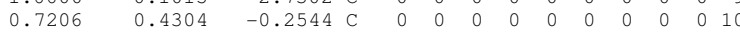

$\begin{array}{lllllllllllll}0.0301 & 0.8005 & -1.3385 \mathrm{~N} & 0 & 0 & 0 & 0 & 0 & 0 & 0 & 0 & 0 & 11\end{array}$

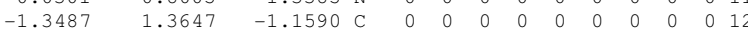

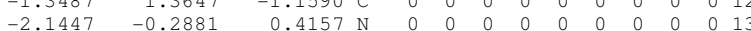

$\begin{array}{llllllllllllll}-2.1447 & -0.2881 & 0.4157 & \mathrm{~N} & 0 & 0 & 0 & 0 & 0 & 0 & 0 & 0 & 0 & 13\end{array}$

$\begin{array}{lllllllllllllll}2.2647 & 0.2364 & -0.8125 & \mathrm{C} & 0 & 0 & 0 & 0 & 0 & 0 & 0 & 0 & 0 & 13 \\ 2.1020 & -0.0720 & -0.4127 & \mathrm{C} & 0 & 0 & 0 & 0 & 0 & 0 & 0 & 0 & 0 & 15\end{array}$

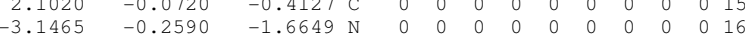

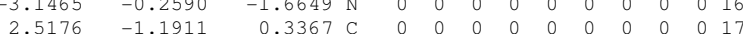

$\begin{array}{llllllllllllll}-3.1465 & -0.2590 & -1.6649 & \mathrm{C} & 0 & 0 & 0 & 0 & 0 & 0 & 0 & 0 & 0 & 16 \\ 3.8191 & -1.1911 & 0.3367 & \mathrm{C} & 0 & 0 & 0 & 0 & 0 & 0 & 0 & 0 & 0 & 17 \\ 4.7311 & -1.6833 & 0.2170 & \mathrm{C} & 0 & 0 & 0 & 0 & 0 & 0 & 0 & 0 & 0 & 18\end{array}$

$\begin{array}{llllllllllllll}4.7311 & -1.0556 & -0.6387 & \mathrm{C} & 0 & 0 & 0 & 0 & 0 & 0 & 0 & 0 & 0 & 19\end{array}$

$\begin{array}{llllllllllllll}4.3307 & 0.0638 & -1.3795 \mathrm{C} & 0 & 0 & 0 & 0 & 0 & 0 & 0 & 0 & 0 & 20\end{array}$

$\begin{array}{llllllllllllll}3.0256 & 0.5486 & -1.2780 & \mathrm{C} & 0 & 0 & 0 & 0 & 0 & 0 & 0 & 0 & 0 & 21\end{array}$

$\begin{array}{lllllllllllllll}-3.4404 & 0.2571 & -3.0060 & \mathrm{C} & 0 & 0 & 0 & 0 & 0 & 0 & 0 & 0 & 0 & 22\end{array}$

$\begin{array}{lllllllllllllll}0.4254 & 0.6900 & -2.5570 & 0 & 0 & 0 & 0 & 0 & 0 & 0 & 0 & 0 & 0 & 23\end{array}$

$\begin{array}{lllllllllllllllll}-1.1739 & 0.4610 & 4.7941 \mathrm{H} & 0 & 0 & 0 & 0 & 0 & 0 & 0 & 0 & 0 & 0 & 0 & 0 & 23 \\ 1.1592 & 1.2217 & 4.2795 & \end{array}$

$\begin{array}{llllllllllllll}1.1592 & 1.2217 & 4.2795 & \mathrm{H} & 0 & 0 & 0 & 0 & 0 & 0 & 0 & 0 & 0 & 25\end{array}$

$\begin{array}{lllllllllllll}1.9667 & 1.2148 & 1.9439 \mathrm{H} & 0 & 0 & 0 & 0 & 0 & 0 & 0 & 0 & 0 & 26\end{array}$

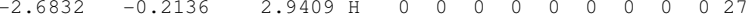

$-1.3416 \quad 2.1055-0.348$

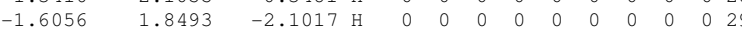

$\begin{array}{llllllllllllll}4.1206 & -2.5584 & 0.7950 & \mathrm{H} & 0 & 0 & 0 & 0 & 0 & 0 & 0 & 0 & 0 & 31\end{array}$

$\begin{array}{llllllllllllll}5.7497 & -1.4368 & -0.7291 & \mathrm{H} & 0 & 0 & 0 & 0 & 0 & 0 & 0 & 0 & 0 & 32\end{array}$

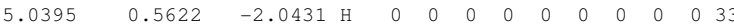

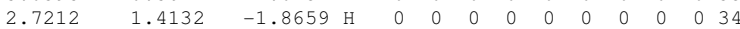

$\begin{array}{llllllllllllll}-4.0555 & -0.4911 & -3.5143 & \mathrm{H} & 0 & 0 & 0 & 0 & 0 & 0 & 0 & 0 & 0 & 35\end{array}$

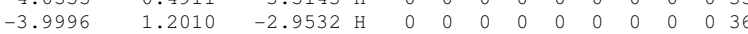

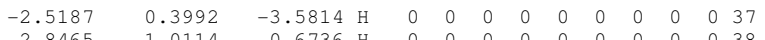

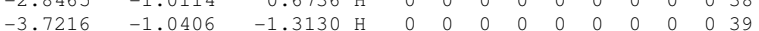

$\begin{array}{lllll}2 & 1 & 0 & 0 & 0\end{array}$

$\begin{array}{lllll}1 & 0 & 0 & 0 & 0\end{array}$

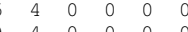




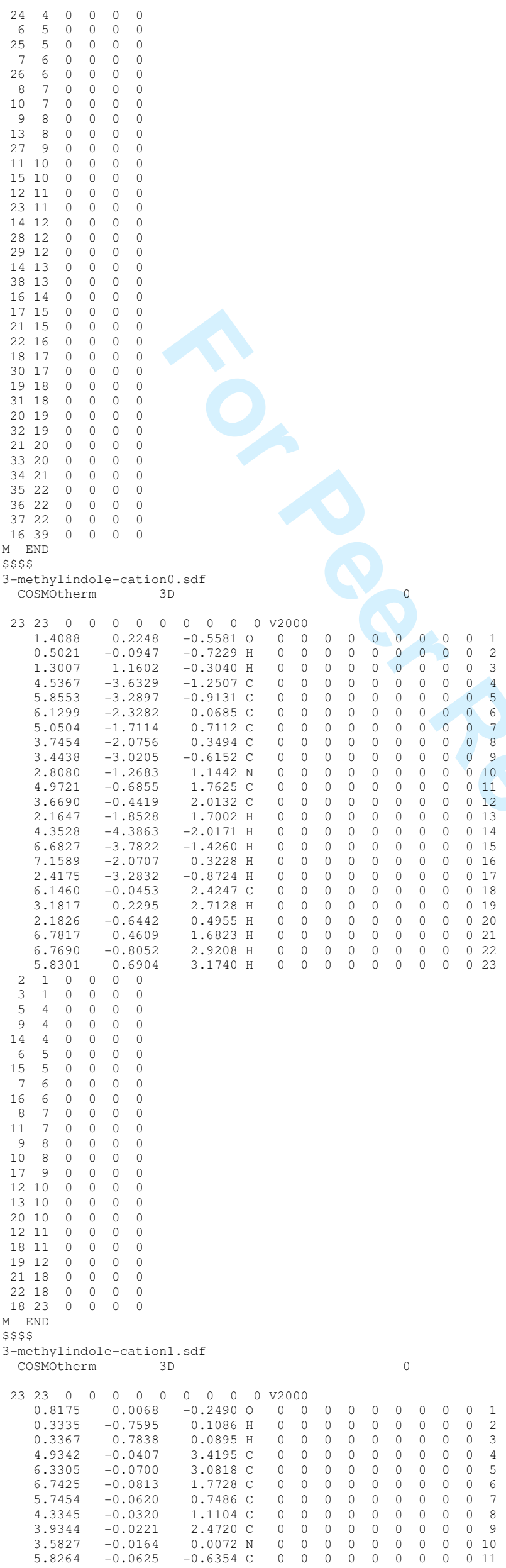




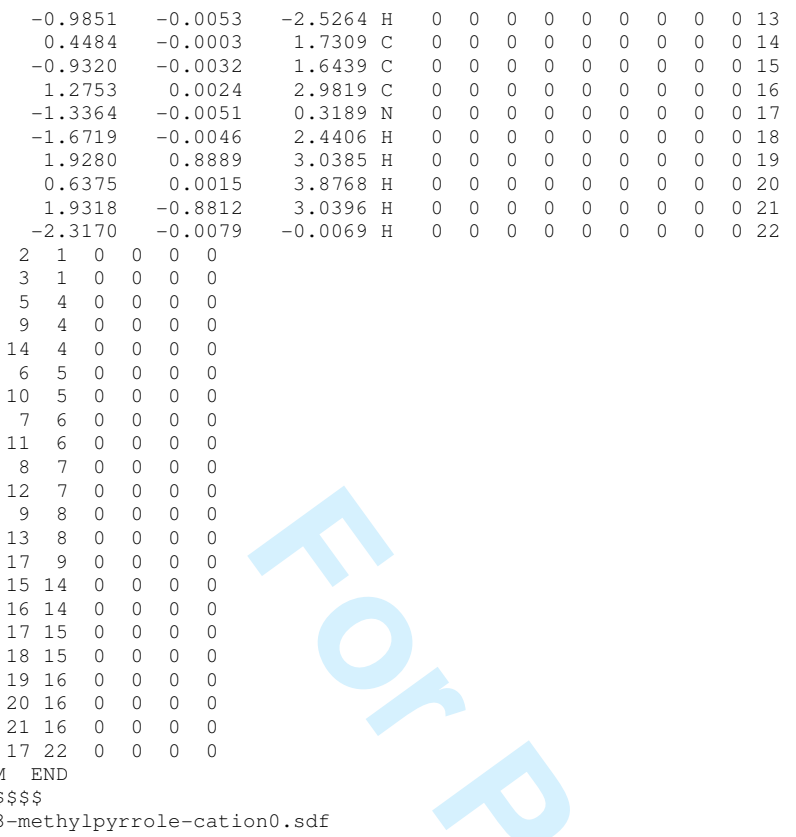

3-methylpyrrole-cationo.sdf

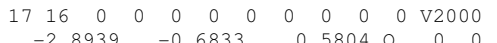

$\begin{array}{lllllllllllll}-2.8939 & -1.5169 & 0.2213 & \mathrm{H} & 0 & 0 & 0 & 0 & 0 & 0 & 0 & 0 & 0\end{array}$

$\begin{array}{llllllllllllll}-3.2521 & -1.5169 & 0.2213 & \mathrm{H} & 0 & 0 & 0 & 0 & 0 & 0 & 0 & 0 & 0 & 2 \\ -3.6568 & -0.0758 & 0.6078 & \mathrm{H} & 0 & 0 & 0 & 0 & 0 & 0 & 0 & 0 & 0 & 3\end{array}$

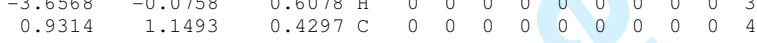

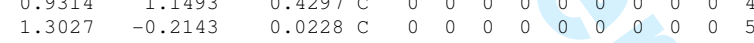

$\begin{array}{rrrrrrrrrrrrrrr}1.3027 & -0.2143 & 0.0228 & \mathrm{C} & 0 & 0 & 0 & 0 & 0 & 0 & 0 & 0 & 0 & 4 \\ -0.2976 & 1.4375 & -0.0342 & \mathrm{C} & 0 & 0 & 0 & 0 & 0 & 0 & 0 & 0 & 0 & 6\end{array}$

$\begin{array}{lllllllllllllll}-0.2976 & 1.4375 & -0.0342 & \mathrm{C} & 0 & 0 & 0 & 0 & 0 & 0 & 0 & 0 & 0 & 0 \\ -0.8066 & 0.2611 & -0.7429 & \mathrm{~N} & 0 & 0 & 0 & 0 & 0 & 0 & 0 & 0 & 0\end{array}$

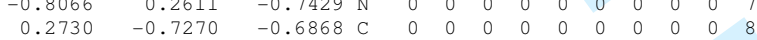

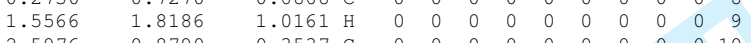

$\begin{array}{rrrrrrrrrrrrrr}2.5976 & -0.8790 & 0.3537 & \mathrm{C} & 0 & 0 & 0 & 0 & 0 & 0 & 0 & 0 & 0 & 10 \\ -0.9285 & 2.3170 & 0.0369 & \mathrm{H} & 0 & 0 & 0 & 0 & 0 & 0 & 0 & 0 & 0 & 11\end{array}$

$\begin{array}{rrrrrrrrrrrrrr}-0.9285 & 2.3170 & 0.0369 & \mathrm{H} & 0 & 0 & 0 & 0 & 0 & 0 & 0 & 0 & 0 & 11 \\ -1.0889 & 0.4822 & -1.7112 & \mathrm{H} & 0 & 0 & 0 & 0 & 0 & 0 & 0 & 0 & 0 & 12\end{array}$

$\begin{array}{llllllllllllllll}0.1235 & -1.6865 & -1.1707 & \mathrm{H} & 0 & 0 & 0 & 0 & 0 & 0 & 0 & 0 & 0 & 13\end{array}$

$\begin{array}{llllllllllllll}-1.7153 & -0.1261 & -0.2442 & \mathrm{H} & 0 & 0 & 0 & 0 & 0 & 0 & 0 & 0 & 0 & 14\end{array}$

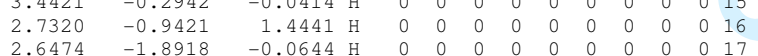

$\begin{array}{lllll}1 & 0 & 0 & 0 & 0\end{array}$

$\begin{array}{llllll}3 & 1 & 0 & 0 & 0 & 0 \\ 5 & 4 & 0 & 0 & 0 & 0\end{array}$

$\begin{array}{lllll}4 & 0 & 0 & 0 & 0 \\ 4 & 0 & 0 & 0 & 0\end{array}$

$\begin{array}{lllll}4 & 0 & 0 & 0 & 0 \\ 5 & 0 & 0 & 0 & 0\end{array}$

$\begin{array}{llllll}0 & 5 & 0 & 0 & 0 & 0\end{array}$

$\begin{array}{lllll}6 & 0 & 0 & 0 & 0 \\ 6 & 0 & 0 & 0 & 0\end{array}$

$\begin{array}{llllll}8 & 7 & 0 & 0 & 0 & 0\end{array}$

$\begin{array}{lllllll}12 & 7 & 0 & 0 & 0 & 0 \\ 14 & 7 & 0 & 0 & 0 & 0\end{array}$

$\begin{array}{lrllll}13 & 8 & 0 & 0 & 0 & 0\end{array}$

$\begin{array}{llllll}5 & 10 & 0 & 0 & 0 & 0 \\ 6 & 10 & 0 & 0 & 0 & 0\end{array}$

$\begin{array}{lllll}16 & 10 & 0 & 0 & 0 \\ 10 & 17 & 0 & 0 & 0\end{array}$

M END

-methylpyrrole-cation1.sdf

3D

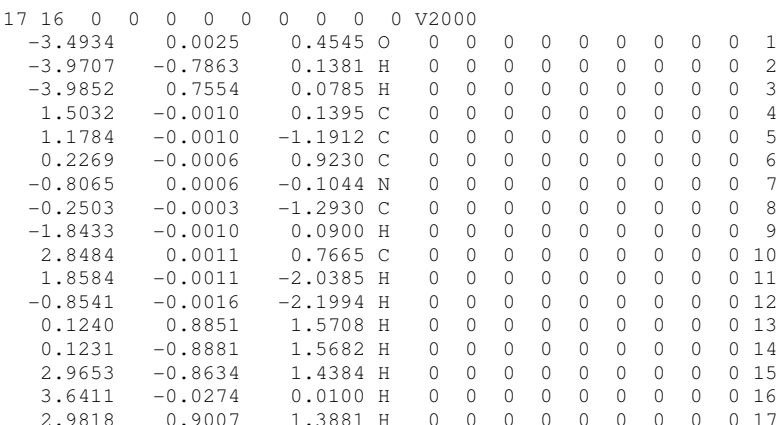

$\begin{array}{llllll}2 & 1 & 0 & 0 & 0 & 0 \\ 3 & 1 & 0 & 0 & 0 & 0\end{array}$

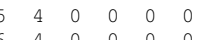

$\begin{array}{llllll}10 & 4 & 0 & 0 & 0 & 0\end{array}$

$\begin{array}{lllll}1 & 5 & 0 & 0 & 0\end{array}$

$7 \begin{array}{llllll}7 & 0 & 0 & 0 & 0\end{array}$

$\begin{array}{llllll}14 & 6 & 0 & 0 & 0 & 0\end{array}$

URL: http://mc.manuscriptcentral.com/tandf/tmph 


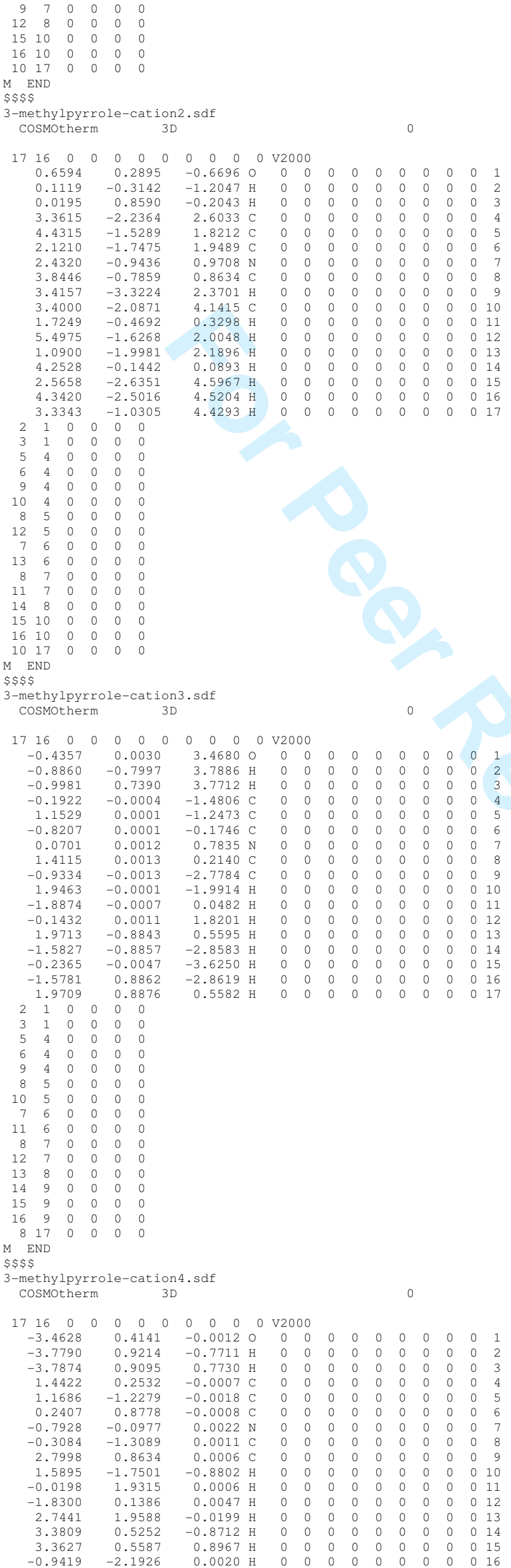




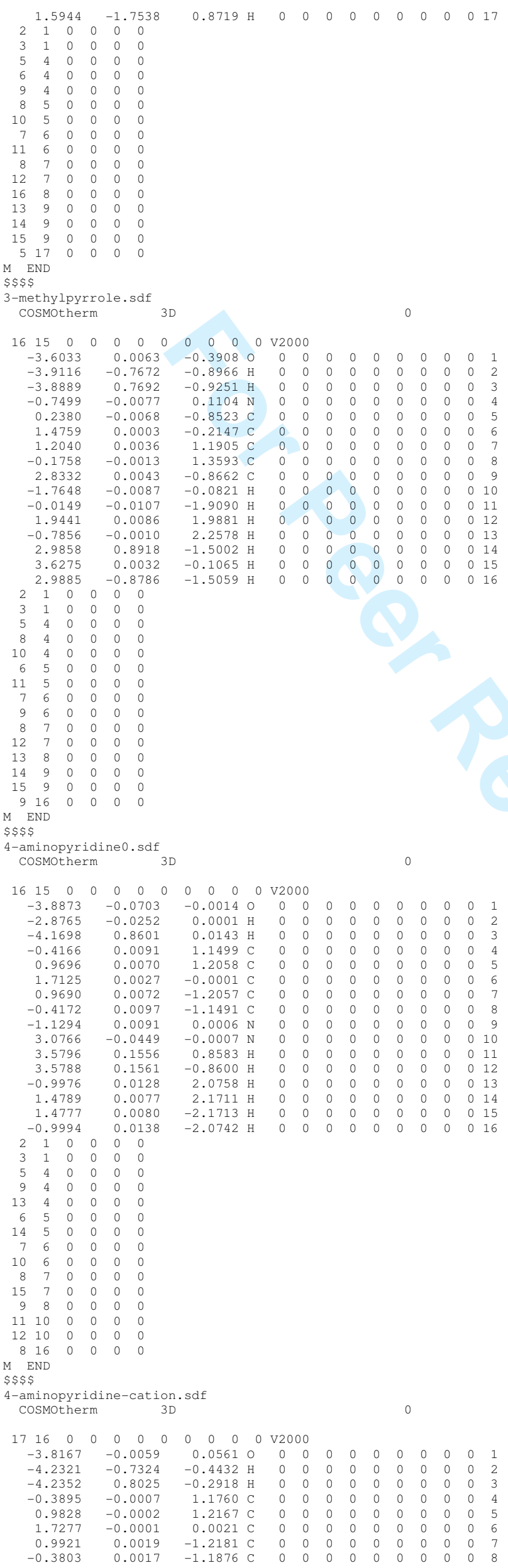




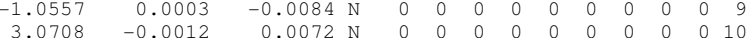

$\begin{array}{llrlllllllllll}3.6012 & -0.0001 & -0.8581 & \mathrm{H} & 0 & 0 & 0 & 0 & 0 & 0 & 0 & 0 & 0 & 11\end{array}$

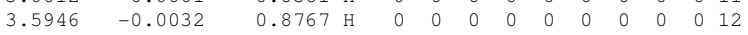

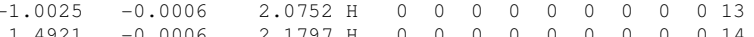

$\begin{array}{llllllllllllll}1.5087 & 0.0032 & -2.1771 & \mathrm{H} & 0 & 0 & 0 & 0 & 0 & 0 & 0 & 0 & 0 & 15\end{array}$

$\begin{array}{lllllllllllllll}-0.9863 & 0.0040 & -2.0917 \mathrm{H} & 0 & 0 & 0 & 0 & 0 & 0 & 0 & 0 & 0 & 16\end{array}$

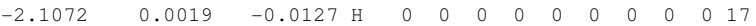

$\begin{array}{lllll}2 & 1 & 0 & 0 & 0\end{array}$

$\begin{array}{llllllll}9 & 4 & 0 & 0 & 0 & 0\end{array}$

$\begin{array}{lllllll}3 & 4 & 0 & 0 & 0 & 0\end{array}$

$6 \begin{array}{llllll}6 & 5 & 0 & 0 & 0 & 0\end{array}$

$\begin{array}{llllll}6 & 0 & 0 & 0 & 0\end{array}$

$\begin{array}{lllll}6 & 0 & 0 & 0 & 0\end{array}$

$\begin{array}{lllllll}8 & 0 & 0 & 0 & 0\end{array}$

$\begin{array}{lllllll}9 & 8 & 0 & 0 & 0 & 0\end{array}$

$\begin{array}{rrrrrr}6 & 8 & 0 & 0 & 0 & 0 \\ 1 & 10 & 0 & 0 & 0 & 0\end{array}$

$\begin{array}{lllllll}2 & 10 & 0 & 0 & 0 & 0\end{array}$

$\begin{array}{lll}0 & 0 & 0\end{array}$

END

-chloroaniline.sd

Cosmotherm 3D

$\begin{array}{llllllllllll}17 & 16 & 0 & 0 & 0 & 0 & 0 & 0 & 0 & 0 & 0 & \mathrm{v} 2000\end{array}$

$\begin{array}{lllllllllllll}-1.4769 & 0.1059 & 4.2036 & 0 & 0 & 0 & 0 & 0 & 0 & 0 & 0 & 0 & 0\end{array}$

$\begin{array}{llllllllllllll}-1.6555 & -0.8058 & 4.4923 & \mathrm{H} & 0 & 0 & 0 & 0 & 0 & 0 & 0 & 0 & 0 & 2\end{array}$

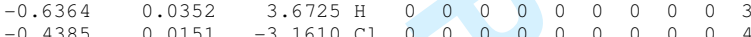

$\begin{array}{rrrrrrrrrrrrrr}0.4385 & 0.0151 & -3.1610 & \mathrm{Cl} & 0 & 0 & 0 & 0 & 0 & 0 & 0 & 0 & 0 & 4 \\ 0.4686 & -1.2285 & 0.5821 & \mathrm{C} & 0 & 0 & 0 & 0 & 0 & 0 & 0 & 0 & 0 & 5\end{array}$

$\begin{array}{lllllllllllll}0.6764 & -0.0236 & 1.2794 & \mathrm{C} & 0 & 0 & 0 & 0 & 0 & 0 & 0 & 0 & 0\end{array}$

$\begin{array}{llllllllllllll}0.5337 & 1.1934 & 0.5867 & \mathrm{C} & 0 & 0 & 0 & 0 & 0 & 0 & 0 & 0 & 0 & 7\end{array}$

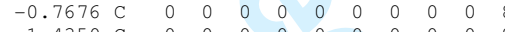

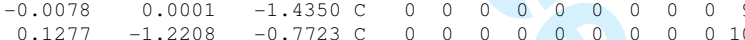

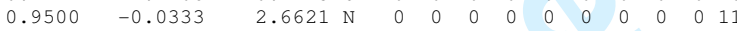

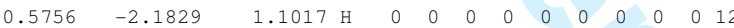

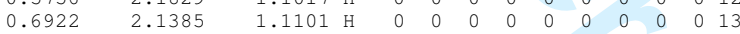

$\begin{array}{llllllllllllll}0.0901 & 2.1584 & -1.2941 & \mathrm{H} & 0 & 0 & 0 & 0 & 0 & 0 & 0 & 0 & 0 & 14\end{array}$

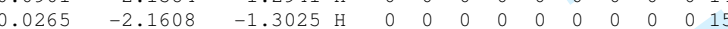

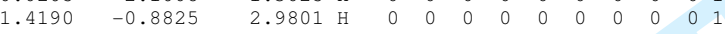

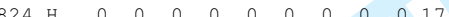

$\begin{array}{llllll}1 & 0 & 0 & 0 & 0\end{array}$

$\begin{array}{lllllllll}3 & 1 & 0 & 0 & 0 & 0\end{array}$

$\begin{array}{cccccc}5 & 0 & 0 & 0 & 0\end{array}$

$\begin{array}{llllll}5 & 0 & 0 & 0 & 0\end{array}$

12500000

$\begin{array}{llllll}6 & 0 & 0 & 0 & 0\end{array}$

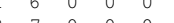

$\begin{array}{llllll}3 & 7 & 0 & 0 & 0 & 0\end{array}$

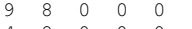

$\begin{array}{lllllll}4 & 8 & 0 & 0 & 0 & 0\end{array}$

$\begin{array}{lllllllll}15 & 10 & 0 & 0 & 0 & 0\end{array}$

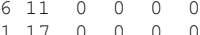

END

4-chloroaniline-cation sde

cosmotherm

$\begin{array}{llllllllllll}18 & 17 & 0 & 0 & 0 & 0 & 0 & 0 & 0 & 0 & 0 & \mathrm{~V} 2000\end{array}$

$\begin{array}{llllll}17 & 0.9729 & 0.9306 & 4.0910 & 0 & 0\end{array}$

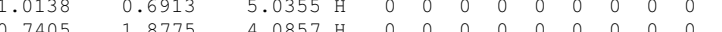

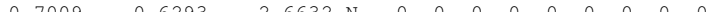

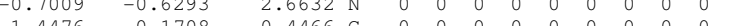

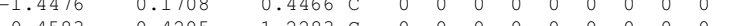

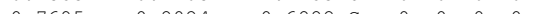

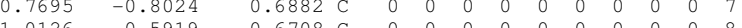

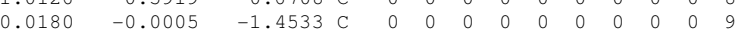

$\begin{array}{lllllllllllllllll}-1.2099 & 0.3847 & -0.9140 & \mathrm{C} & 0 & 0 & 0 & 0 & 0 & 0 & 0 & 0 & 0 & 10\end{array}$

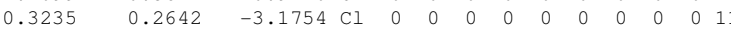

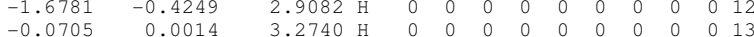

$\begin{array}{lllllllllllll}-2.4042 & 0.4629 & 0.8825 \mathrm{H} & 0 & 0 & 0 & 0 & 0 & 0 & 0 & 0 & 0 & 14\end{array}$

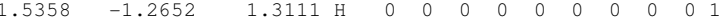

$\begin{array}{llllllllllll}0 & 0 & 0 & 0 & 0 & 0 & 0 & 0 & 16\end{array}$

$\begin{array}{lll}-1.9755 & 0.8426 & -1.5388 \\ -0.5293 & -1.6061 & -2.9373\end{array}$

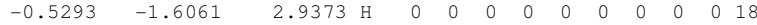

$\begin{array}{llllll}2 & 1 & 0 & 0 & 0 & 0\end{array}$

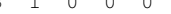

$\begin{array}{llllll}4 & 0 & 0 & 0 & 0\end{array}$

$\begin{array}{lllllll}2 & 4 & 0 & 0 & 0 & 0 & 0 \\ 1 & 4 & 0 & 0 & 0 & 0 & 2\end{array}$

$6 \begin{array}{llllll}5 & 0 & 0 & 0 & 0\end{array}$

\begin{tabular}{llllll}
6 & 5 & 0 & 0 & 0 & 0 \\
\hline & 5 & 0 & 0 & 0 & 0
\end{tabular}

$\begin{array}{lllll}6 & 0 & 0 & 0 & 0\end{array}$

\begin{tabular}{llllll}
8 & 7 & 0 & 0 & 0 & 0 \\
\hline & 7 & 0 & 0 & 0 & 0
\end{tabular}

$\begin{array}{llllllll}9 & 8 & 0 & 0 & 0 & 0\end{array}$

$\begin{array}{llllll}6 & 8 & 0 & 0 & 0 & 0\end{array}$

$10 \begin{array}{llllll}10 & 9 & 0 & 0 & 0 & 0\end{array}$

$\begin{array}{lllll}10 & 0 & 0 & 0 & 0\end{array}$

$\begin{array}{llllllllll}4 & 18 & 0 & 0 & 0 & 0\end{array}$ 


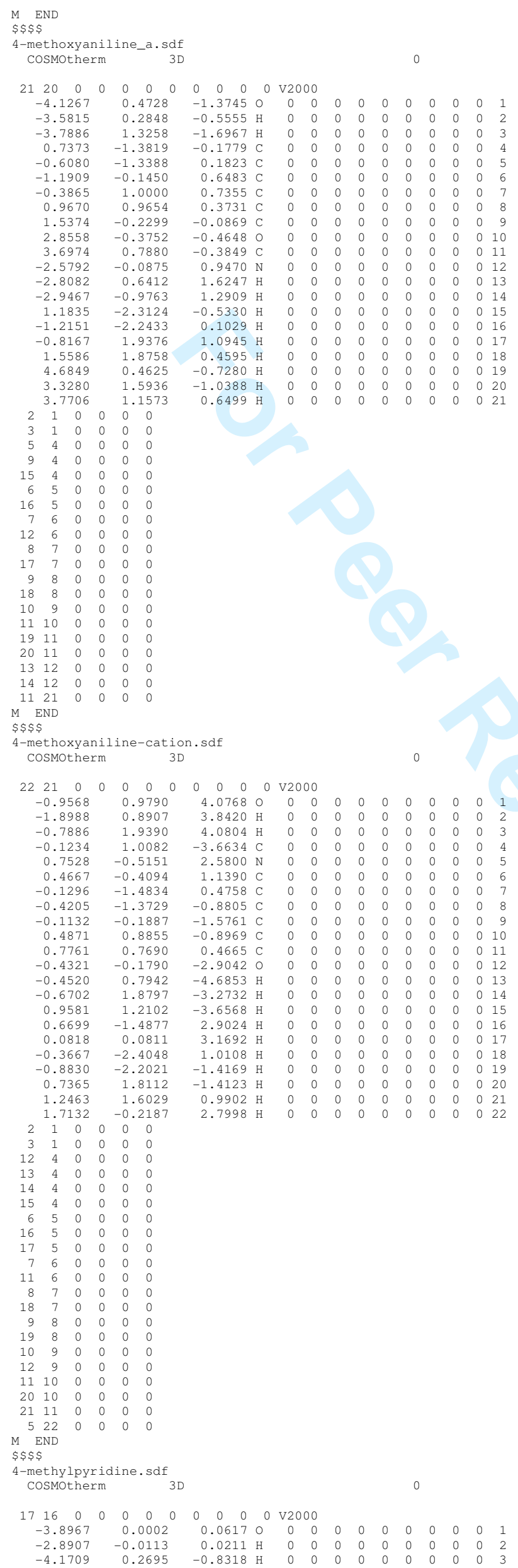




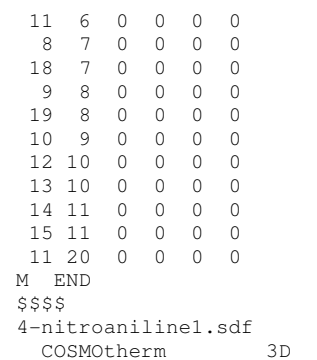

cosmotherm $3 \mathrm{D}$

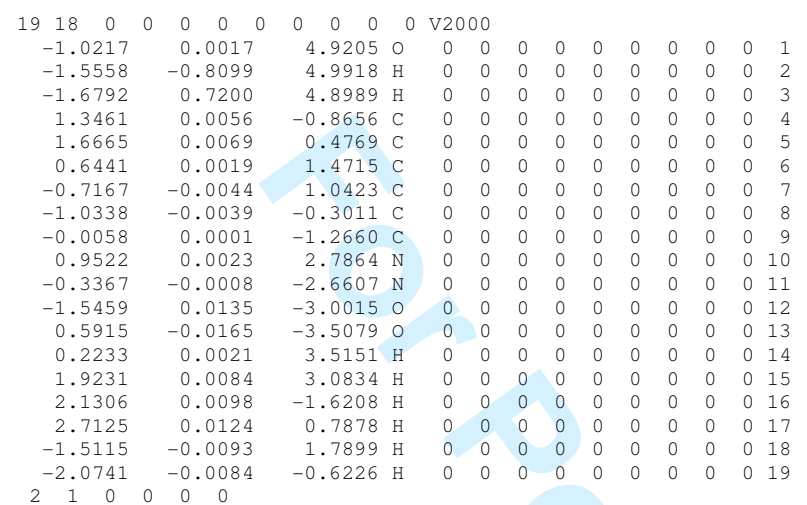


$\begin{array}{rrrrrrrrrrrrrr}0.4379 & -0.3194 & -1.1983 & \mathrm{C} & 0 & 0 & 0 & 0 & 0 & 0 & 0 & 0 & 0 & 5 \\ -0.1963 & -0.5777 & 0.0315 & C & 0 & 0 & 0 & 0 & 0 & 0 & 0 & 0 & 0 & 6\end{array}$

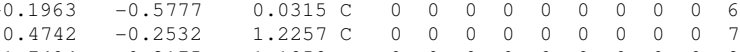

$\begin{array}{rrrrrrrrrrrrrr}1.7494 & 0.3175 & 1.1858 & \mathrm{C} & 0 & 0 & 0 & 0 & 0 & 0 & 0 & 0 & 0 & 8 \\ 2.3798 & 0.5743 & -0.0386 & \mathrm{C} & 0 & 0 & 0 & 0 & 0 & 0 & 0 & 0 & 0 & 9\end{array}$

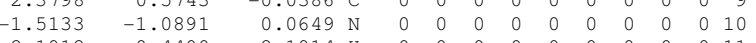

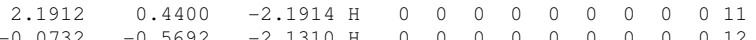

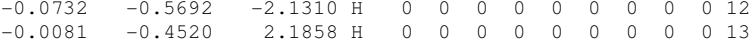

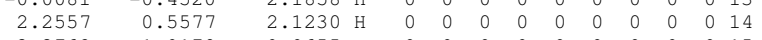

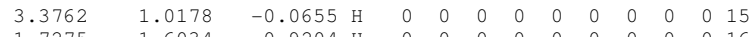

$\begin{array}{rrrrrrrrr}-1.7275 & -1.6034 & 0.9204 \mathrm{H} & 0 & 0 & 0 & 0 & 0 & 0 \\ -1.7446 & -1.6669 & -0.7447 & \mathrm{H} & 0 & 0 & 0 & 0 & 0\end{array}$

$-1.7446 \quad-1.6669$

$\begin{array}{llllll}4 & 0 & 0 & 0 & 0\end{array}$

$\begin{array}{llllll}9 & 4 & 0 & 0 & 0 & 0\end{array}$

$\begin{array}{llllll}5 & 5 & 0 & 0 & 0 & 0\end{array}$

$\begin{array}{lllll}5 & 0 & 0 & 0 & 0\end{array}$

$\begin{array}{llllll}7 & 0 & 0 & 0 & 0\end{array}$

$\begin{array}{lllll}7 & 0 & 0 & 0\end{array}$

$\begin{array}{cccccccc}9 & 8 & 0 & 0 & 0 & 0\end{array}$

$\begin{array}{llllll}4 & 8 & 0 & 0 & 0 & 0\end{array}$

$\begin{array}{rrrrrr}15 & 9 & 0 & 0 & 0 & 0\end{array}$

$\begin{array}{lllll}17 & 0 & 0 & 0 & 0\end{array}$

END

benzimidazole.sdf

cosmotherm $3 \mathrm{D}$

$\begin{array}{llllllllllll}18 & 18 & 0 & 0 & 0 & 0 & 0 & 0 & 0 & 0 & 0 & \mathrm{~V} 2000\end{array}$

$\begin{array}{llllllllllllll}-3.7844 & 1.1119 & -0.0707 & 0 & 0 & 0 & 0 & 0 & 0 & 0 & 0 & 0 & 0 & 1\end{array}$

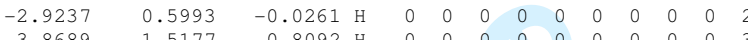

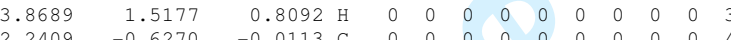

$\begin{array}{lllllllllllllllll}2.2409 & -0.6270 & -0.0113 & \mathrm{C} & 0 & 0 & 0 & 0 & 0 & 0 & 0 & 0 & 0 & 0 & 0 & 4 \\ 0.8601 & -0.8534 & -0.0019 \mathrm{C} & 0 & 0 & 0 & 0 & 0 & 0 & 0 & 0 & 0 & 5\end{array}$

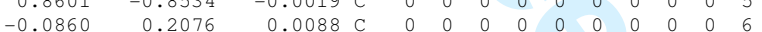

$\begin{array}{llllllllllllll}0.3517 & 1.5410 & 0.0104 & C & 0 & 0 & 0 & 0 & 0 & 0 & 0 & 0 & 0 & 7\end{array}$

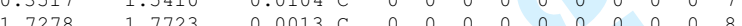

$\begin{array}{llllllllllllllllll}1.7278 & 1.7723 & 0.0013 & c & 0 & 0 & 0 & 0 & 0 & 0 & 0 & 0 & 0 & 0 & 0 & 8\end{array}$

$\begin{array}{lllllllllllllll}1.7 .3781 & -0.3182 & -0.0090 & \mathrm{C} & 0 & 0 & 0 & 0 & 0 & 0 & 0 & 0 & 0 & 0 & 0\end{array}$

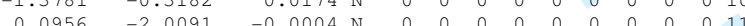

$\begin{array}{llllllllllllllll}-1.0956 & -1.00939 & -0.0004 \mathrm{~N} & 0 & 0 & 0 & 0 & 0 & 0 & 0 & 0 & 0 & 0 & 0 & 0 & 10\end{array}$

$\begin{array}{llllllllllllllllllll}1.2177 & -1.6339 & 0.0110 & \mathrm{C} & 0 & 0 & 0 & 0 & 0 & 0 & 0 & 0 & 0 & 0 & 12\end{array}$

$\begin{array}{lllllllllllllllll}2.9586 & -1.4482 & -0.0198 & \mathrm{H} & 0 & 0 & 0 & 0 & 0 & 0 & 0 & 0 & 0 & 0 & 12 \\ -0.3639 & 2.3646 & 0.0179 & & 0 & 0 & 0 & 0 & 0 & 0 & 0 & 0 & 0 & 0 & 13\end{array}$

$\begin{array}{lllllllllllllllllllll}0.3639 & 2.3646 & 0.0179 & \mathrm{H} & 0 & 0 & 0 & 0 & 0 & 0 & 0 & 0 & 0 & 0 & 13 \\ 2.0988 & 2.7986 & 0.0025 & \mathrm{H} & 0 & 0 & 0 & 0 & 0 & 0 & 0 & 0 & 0 & 14\end{array}$

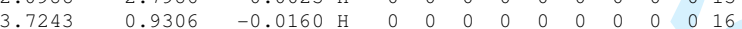

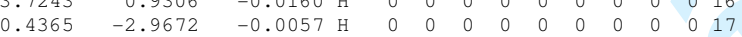

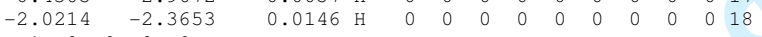


$\begin{array}{rrrrrr}8 & 7 & 0 & 0 & 0 & 0 \\ 12 & 7 & 0 & 0 & 0 & 0 \\ 10 & 9 & 0 & 0 & 0 & 0 \\ 15 & 9 & 0 & 0 & 0 & 0 \\ 11 & 10 & 0 & 0 & 0 & 0 \\ 16 & 10 & 0 & 0 & 0 & 0 \\ 12 & 11 & 0 & 0 & 0 & 0 \\ 17 & 11 & 0 & 0 & 0 & 0 \\ 18 & 12 & 0 & 0 & 0 & 0 \\ 8 & 19 & 0 & 0 & 0 & 0\end{array}$ $M$ END

$\$ \$ \$$
clonidine-cation2.sdf

Cosmotherm

$\begin{array}{llllllllllll}27 & 27 & 0 & 0 & 0 & 0 & 0 & 0 & 0 & 0 & 0 & \text { V2 } 2000\end{array}$

$\begin{array}{rrrrrrrrrrrrrr}1.8027 & -1.9601 & 2.4012 & 0 & 0 & 0 & 0 & 0 & 0 & 0 & 0 & 0 & 0 & 1 \\ 1.8325 & -1.6945 & 3.3384 & \mathrm{H} & 0 & 0 & 0 & 0 & 0 & 0 & 0 & 0 & 0 & 2 \\ 1.7088 & -2.9297 & 2.4184 & \mathrm{H} & 0 & 0 & 0 & 0 & 0 & 0 & 0 & 0 & 0 & 3 \\ -2.6505 & 1.6329 & -0.0583 & \mathrm{C} & 0 & 0 & 0 & 0 & 0 & 0 & 0 & 0 & 0 & 4 \\ -1.3378 & 1.3127 & 0.2870 & \mathrm{C} & 0 & 0 & 0 & 0 & 0 & 0 & 0 & 0 & 0 & 5 \\ -0.8399 & -0.0010 & 0.1692 & \mathrm{C} & 0 & 0 & 0 & 0 & 0 & 0 & 0 & 0 & 0 & 6 \\ -1.7333 & -0.9926 & -0.2840 & \mathrm{C} & 0 & 0 & 0 & 0 & 0 & 0 & 0 & 0 & 0 & 7 \\ -3.0548 & -0.6965 & -0.6141 & \mathrm{C} & 0 & 0 & 0 & 0 & 0 & 0 & 0 & 0 & 0 & 8 \\ -3.5034 & 0.6215 & -0.5045 & \mathrm{C} & 0 & 0 & 0 & 0 & 0 & 0 & 0 & 0 & 0 & 9 \\ -0.2865 & 2.5852 & 0.9040 & \mathrm{Cl} & 0 & 0 & 0 & 0 & 0 & 0 & 0 & 0 & 0 & 10 \\ -1.1642 & -2.6522 & -0.4315 & \mathrm{Cl} & 0 & 0 & 0 & 0 & 0 & 0 & 0 & 0 & 0 & 11 \\ 0.4874 & -0.3341 & 0.5391 & \mathrm{~N} & 0 & 0 & 0 & 0 & 0 & 0 & 0 & 0 & 0 & 12 \\ 1.5747 & 0.0058 & -0.1736 & \mathrm{C} & 0 & 0 & 0 & 0 & 0 & 0 & 0 & 0 & 0 & 13 \\ 3.7745 & 0.0016 & -0.8620 & \mathrm{C} & 0 & 0 & 0 & 0 & 0 & 0 & 0 & 0 & 0 & 14 \\ 2.9643 & 0.9787 & -1.7387 & \mathrm{C} & 0 & 0 & 0 & 0 & 0 & 0 & 0 & 0 & 0 & 15 \\ 2.8265 & -0.2620 & 0.2385 & \mathrm{~N} & 0 & 0 & 0 & 0 & 0 & 0 & 0 & 0 & 0 & 16 \\ 1.5779 & 0.6241 & -1.3664 & \mathrm{~N} & 0 & 0 & 0 & 0 & 0 & 0 & 0 & 0 & 0 & 17 \\ 2.9807 & -0.9616 & 0.9697 & \mathrm{H} & 0 & 0 & 0 & 0 & 0 & 0 & 0 & 0 & 0 & 18 \\ 0.7714 & 1.1243 & -1.7293 & \mathrm{H} & 0 & 0 & 0 & 0 & 0 & 0 & 0 & 0 & 0 & 19 \\ -3.0036 & 2.6585 & 0.0402 & \mathrm{H} & 0 & 0 & 0 & 0 & 0 & 0 & 0 & 0 & 0 & 20 \\ -3.7206 & -1.4866 & -0.9586 & \mathrm{H} & 0 & 0 & 0 & 0 & 0 & 0 & 0 & 0 & 0 & 21 \\ -4.5336 & 0.8635 & -0.7666 & \mathrm{H} & 0 & 0 & 0 & 0 & 0 & 0 & 0 & 0 & 0 & 22 \\ 4.0064 & -0.9311 & -1.3977 & \mathrm{H} & 0 & 0 & 0 & 0 & 0 & 0 & 0 & 0 & 0 & 23 \\ 4.7004 & 0.4452 & -0.4824 & \mathrm{H} & 0 & 0 & 0 & 0 & 0 & 0 & 0 & 0 & 0 & 24 \\ 3.1692 & 2.0283 & -1.4814 & \mathrm{H} & 0 & 0 & 0 & 0 & 0 & 0 & 0 & 0 & 0 & 25 \\ 3.1290 & 0.8249 & -2.8097 & \mathrm{H} & 0 & 0 & 0 & 0 & 0 & 0 & 0 & 0 & 0 & 26 \\ 0.6673 & -0.9771 & 1.3349 & \mathrm{H} & 0 & 0 & 0 & 0 & 0 & 0 & 0 & 0 & 0 & 27 \\ 1.0 & 0 & 0 & & & & & & & & & & \end{array}$

$\begin{array}{cccccc}2 & 1 & 0 & 0 & 0 & 0\end{array}$

$\begin{array}{llllll}3 & 1 & 0 & 0 & 0 & 0\end{array}$

$\begin{array}{llllll}5 & 4 & 0 & 0 & 0 & 0 \\ 9 & 4 & 0 & 0 & 0 & 0\end{array}$

$\begin{array}{llllll}20 & 4 & 0 & 0 & 0 & 0\end{array}$

$\begin{array}{rrrrrr}6 & 5 & 0 & 0 & 0 & 0 \\ 10 & 5 & 0 & 0 & 0 & 0\end{array}$

$\begin{array}{llllll}7 & 6 & 0 & 0 & 0 & 0\end{array}$

$\begin{array}{rrrrrr}12 & 6 & 0 & 0 & 0 & 0 \\ 8 & 7 & 0 & 0 & 0 & 0\end{array}$

$\begin{array}{rrrrrrr}11 & 7 & 0 & 0 & 0 & 0\end{array}$

$\begin{array}{rrrrrr}9 & 8 & 0 & 0 & 0 \\ 21 & 8 & 0 & 0 & 0 & 0\end{array}$

$\begin{array}{lllllll}21 & 8 & 0 & 0 & 0 & 0\end{array}$

$\begin{array}{rrrrrr}13 & 12 & 0 & 0 & 0 & 0\end{array}$

$\begin{array}{llllll}16 & 13 & 0 & 0 & 0 & 0\end{array}$

$\begin{array}{llllll}17 & 13 & 0 & 0 & 0 & 0\end{array}$

$\begin{array}{llllll}15 & 14 & 0 & 0 & 0 & 0\end{array}$

$\begin{array}{llllll}16 & 14 & 0 & 0 & 0 & 0\end{array}$

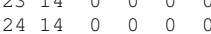

$\begin{array}{llllll}17 & 15 & 0 & 0 & 0 & 0\end{array}$

$\begin{array}{llllll}25 & 15 & 0 & 0 & 0 & 0\end{array}$

$\begin{array}{llllll}26 & 15 & 0 & 0 & 0 & 0 \\ 18 & 16 & 0 & 0 & 0 & 0\end{array}$

$\begin{array}{llllll}19 & 17 & 0 & 0 & 0 & 0\end{array}$

1227

$\$ \$ \$$

\$\$ $\$$
clonidine.sdf

cosmotherm 3D

$\begin{array}{llllllllllll}26 & 26 & 0 & 0 & 0 & 0 & 0 & 0 & 0 & 0 & 0 & \mathrm{~V} 2000\end{array}$

$\begin{array}{rrrrrrrrrrrrrr}-2.2157 & 2.4338 & 1.3631 & 0 & 0 & 0 & 0 & 0 & 0 & 0 & 0 & 0 & 0 & 1 \\ -1.5941 & 1.7934 & 0.9011 & \mathrm{H} & 0 & 0 & 0 & 0 & 0 & 0 & 0 & 0 & 0 & 2 \\ -3.1023 & 2.0588 & 1.2213 & \mathrm{H} & 0 & 0 & 0 & 0 & 0 & 0 & 0 & 0 & 0 & 3 \\ -0.3167 & 0.3478 & 2.8317 & \mathrm{~N} & 0 & 0 & 0 & 0 & 0 & 0 & 0 & 0 & 0 & 4 \\ -0.0339 & -0.1052 & 1.5653 & \mathrm{C} & 0 & 0 & 0 & 0 & 0 & 0 & 0 & 0 & 0 & 5 \\ 0.5724 & -1.3349 & 1.6623 & \mathrm{~N} & 0 & 0 & 0 & 0 & 0 & 0 & 0 & 0 & 0 & 6 \\ -0.5847 & -0.6395 & -3.0386 & \mathrm{C} & 0 & 0 & 0 & 0 & 0 & 0 & 0 & 0 & 0 & 7 \\ -0.9176 & -0.3132 & -1.7255 & \mathrm{C} & 0 & 0 & 0 & 0 & 0 & 0 & 0 & 0 & 0 & 8 \\ 0.0274 & 0.1485 & -0.7760 & \mathrm{C} & 0 & 0 & 0 & 0 & 0 & 0 & 0 & 0 & 0 & 9 \\ 1.3536 & 0.2655 & -1.2634 & \mathrm{C} & 0 & 0 & 0 & 0 & 0 & 0 & 0 & 0 & 0 & 10 \\ 1.7183 & -0.0597 & -2.5693 & \mathrm{C} & 0 & 0 & 0 & 0 & 0 & 0 & 0 & 0 & 0 & 11 \\ 0.7420 & -0.5127 & -3.4597 & \mathrm{C} & 0 & 0 & 0 & 0 & 0 & 0 & 0 & 0 & 0 & 12 \\ 2.6075 & 0.8817 & -0.1719 & \mathrm{C} 1 & 0 & 0 & 0 & 0 & 0 & 0 & 0 & 0 & 0 & 13 \\ -2.6030 & -0.4876 & -1.2128 & \mathrm{Cl} & 0 & 0 & 0 & 0 & 0 & 0 & 0 & 0 & 0 & 14 \\ -0.3683 & 0.5677 & 0.4912 & \mathrm{~N} & 0 & 0 & 0 & 0 & 0 & 0 & 0 & 0 & 0 & 15 \\ -0.1294 & -0.7461 & 3.7990 & \mathrm{C} & 0 & 0 & 0 & 0 & 0 & 0 & 0 & 0 & 0 & 16 \\ 0.9152 & -1.6085 & 3.0705 & \mathrm{C} & 0 & 0 & 0 & 0 & 0 & 0 & 0 & 0 & 0 & 17 \\ -1.0953 & 1.0015 & 2.9273 & \mathrm{H} & 0 & 0 & 0 & 0 & 0 & 0 & 0 & 0 & 0 & 18 \\ 1.1985 & -1.6406 & 0.9219 & \mathrm{H} & 0 & 0 & 0 & 0 & 0 & 0 & 0 & 0 & 0 & 19 \\ -1.3561 & -0.9912 & -3.7233 & \mathrm{H} & 0 & 0 & 0 & 0 & 0 & 0 & 0 & 0 & 0 & 20 \\ 2.7545 & 0.0527 & -2.8871 & \mathrm{H} & 0 & 0 & 0 & 0 & 0 & 0 & 0 & 0 & 0 & 21 \\ 1.0147 & -0.7644 & -4.4847 & \mathrm{H} & 0 & 0 & 0 & 0 & 0 & 0 & 0 & 0 & 0 & 22 \\ -1.0671 & -1.3049 & 3.9559 & \mathrm{H} & 0 & 0 & 0 & 0 & 0 & 0 & 0 & 0 & 0 & 23 \\ 0.2317 & -0.3705 & 4.7629 & \mathrm{H} & 0 & 0 & 0 & 0 & 0 & 0 & 0 & 0 & 0 & 24 \\ 0.8209 & -2.6754 & 3.3014 & \mathrm{H} & 0 & 0 & 0 & 0 & 0 & 0 & 0 & 0 & 0 & 25 \\ 1.9404 & -1.2757 & 3.3004 & \mathrm{H} & 0 & 0 & 0 & 0 & 0 & 0 & 0 & 0 & 0 & 26\end{array}$

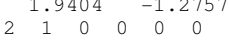

$\begin{array}{llllll}3 & 1 & 0 & 0 & 0 & 0 \\ 5 & 4 & 0 & 0 & 0 & 0\end{array}$ 


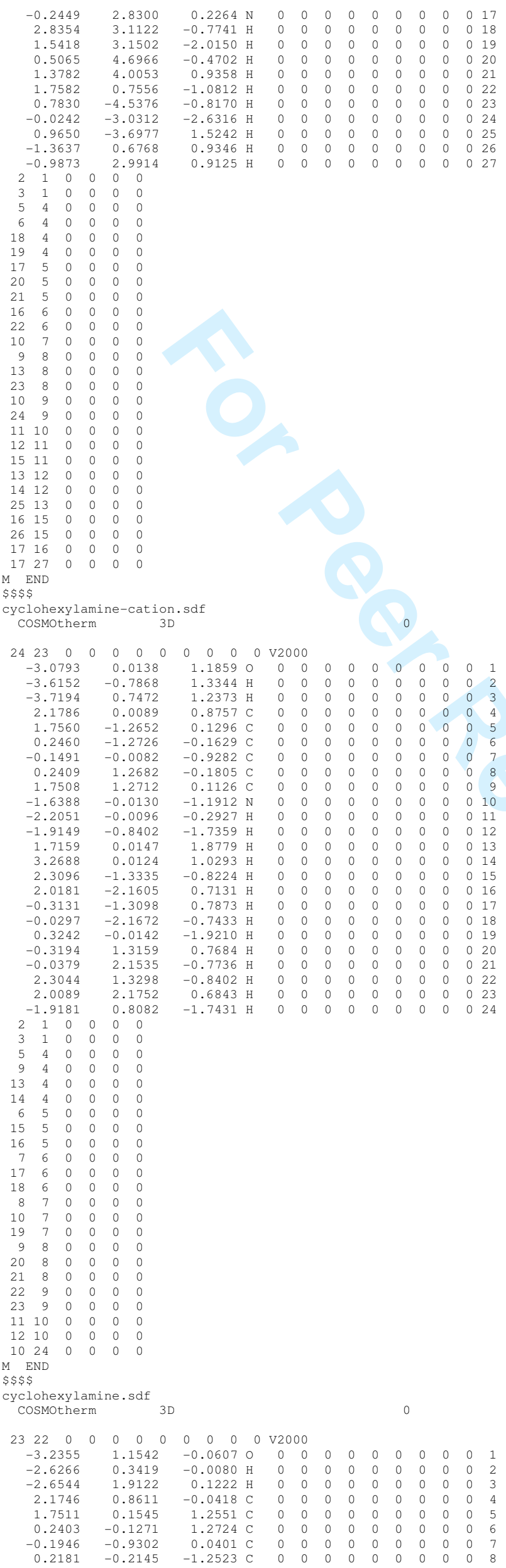


$\begin{array}{rrrrrrrrrrrrrr}1.7285 & 0.0694 & -1.2808 & \mathrm{C} & 0 & 0 & 0 & 0 & 0 & 0 & 0 & 0 & 0 & 9 \\ -1.6660 & -1.1297 & 0.0598 & \mathrm{~N} & 0 & 0 & 0 & 0 & 0 & 0 & 0 & 0 & 0 & 10\end{array}$

$\begin{array}{llllllllllllll}-1.9406 & -1.7243 & -0.7292 & \mathrm{H} & 0 & 0 & 0 & 0 & 0 & 0 & 0 & 0 & 0 & 11\end{array}$

$\begin{array}{llllllllllllll}-1.9280 & -1.6530 & 0.9018 & \mathrm{H} & 0 & 0 & 0 & 0 & 0 & 0 & 0 & 0 & 0 & 12\end{array}$

$\begin{array}{llllllllllllll}1.7197 & 1.8670 & -0.0711 & \mathrm{H} & 0 & 0 & 0 & 0 & 0 & 0 & 0 & 0 & 0 & 13\end{array}$

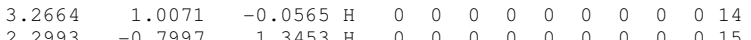

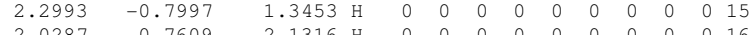

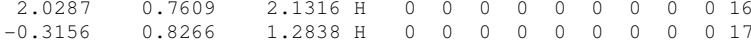

$\begin{array}{lllllllllllll}-0.0386 & -0.6720 & 2.1898 \mathrm{H} & 0 & 0 & 0 & 0 & 0 & 0 & 0 & 0 & 0 & 18\end{array}$

$\begin{array}{llllllllllllllllllll}0.3284 & -1.9065 & 0.0705 & \mathrm{H} & 0 & 0 & 0 & 0 & 0 & 0 & 0 & 0 & 0 & 18\end{array}$

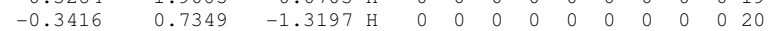

$\begin{array}{llllllllllllll}-0.0751 & -0.8222 & -2.1247 & \mathrm{H} & 0 & 0 & 0 & 0 & 0 & 0 & 0 & 0 & 0 & 21\end{array}$

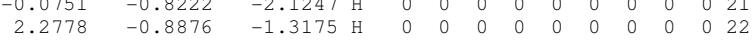

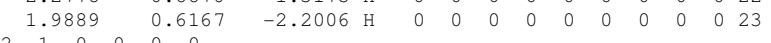

$\begin{array}{lllllll}1 & 0 & 0 & 0 & 0\end{array}$

$\begin{array}{llllll}1 & 0 & 0 & 0 & 0\end{array}$

$\begin{array}{llllll}4 & 0 & 0 & 0 & 0\end{array}$

134500000

$\begin{array}{rrrrrr}14 & 4 & 0 & 0 & 0 & 0 \\ 6 & 5 & 0 & 0 & 0 & 0\end{array}$

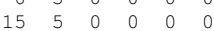

$\begin{array}{rrrrrrrr}16 & 5 & 0 & 0 & 0 & 0 \\ 7 & 6 & 0 & 0 & 0 & 0\end{array}$

$17 \begin{array}{ccccccc}7 & 6 & 0 & 0 & 0 & 0\end{array}$

$\begin{array}{llll}0 & 0 & 0 & 0\end{array}$

$\begin{array}{lllllll}18 & 7 & 0 & 0 & 0 & 0 \\ 10 & 7 & 0 & 0 & 0 & 0\end{array}$

$\begin{array}{llllll}10 & 7 & 0 & 0 & 0 & 0 \\ 19 & 7 & 0 & 0 & 0 & 0\end{array}$

$\begin{array}{lllllll}9 & 8 & 0 & 0 & 0 & 0\end{array}$

$\begin{array}{llllll}20 & 8 & 0 & 0 & 0 & 0 \\ 21 & 8 & 0 & 0 & 0 & 0\end{array}$

$22 \quad 9000000$

$\begin{array}{llllll}11 & 10 & 0 & 0 & 0 & 0 \\ 12 & 10 & 0 & 0 & 0 & 0\end{array}$

$\begin{array}{lllllll}9 & 23 & 0 & 0 & 0 & 0\end{array}$

END

debrisoquine.sdf

Cosmotherm

$\begin{array}{lllllllllllll}29 & 29 & 0 & 0 & 0 & 0 & 0 & 0 & 0 & 0 & 0 & \mathrm{~V} 2000\end{array}$

$\begin{array}{cccccccccccccccc}-3.3937 & 0.4642 & -0.5125 & 0 & 0 & 0 & 0 & 0 & 0 & 0 & 0 & 0 & 0\end{array}$

$\begin{array}{lllllllllllll}-2.5893 & 0.4496 & -1.1302 & \mathrm{H} & 0 & 0 & 0 & 0 & 0 & 0 & 0 & 0 & 0\end{array}$

$\begin{array}{llllllllllllll}-3.0508 & 0.8217 & 0.3247 & \mathrm{H} & 0 & 0 & 0 & 0 & 0 & 0 & 0 & 0 & 0 & 3\end{array}$

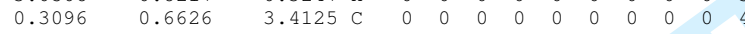

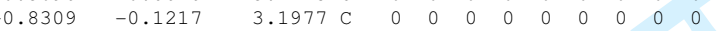

$\begin{array}{llllllllllllll}-1.0193 & -0.7404 & 1.9596 & \mathrm{C} & 0 & 0 & 0 & 0 & 0 & 0 & 0 & 0 & 0 & 6\end{array}$

$\begin{array}{rrrrrrrrrrrrrr}-0.0855 & -0.5809 & 0.9237 & \mathrm{C} & 0 & 0 & 0 & 0 & 0 & 0 & 0 & 0 & 0 & 7\end{array}$

$\begin{array}{rrrrrrrrrrrrrr}1.0649 & 0.2046 & 1.1360 & \mathrm{C} & 0 & 0 & 0 & 0 & 0 & 0 & 0 & 0 & 0 & 8 \\ 1.2447 & 0.8205 & 2.3867 & \mathrm{C} & 0 & 0 & 0 & 0 & 0 & 0 & 0 & 0 & 0 & 9\end{array}$

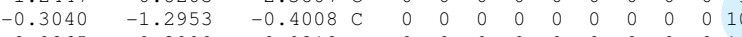

$\begin{array}{llllllllllllll}2.0865 & 0.3990 & 0.0318 & \mathrm{C} & 0 & 0 & 0 & 0 & 0 & 0 & 0 & 0 & 0 & 11 \\ 1.9256 & 0.6376 & -1.0868 & \mathrm{C} & 0 & 0 & 0 & 0 & 0 & 0 & 0 & 0 & 0 & 12\end{array}$

$\begin{array}{llllllllllllll}1.9256 & -0.6376 & -1.0868 & \mathrm{C} & 0 & 0 & 0 & 0 & 0 & 0 & 0 & 0 & 0 & 12\end{array}$

$\begin{array}{rrrrrrrrrrrrrr}0.5110 & -0.7449 & -1.4852 & \mathrm{~N} & 0 & 0 & 0 & 0 & 0 & 0 & 0 & 0 & 0 & 13 \\ -0.0587 & 0.1477 & -2.3756 & \mathrm{C} & 0 & 0 & 0 & 0 & 0 & 0 & 0 & 0 & 0 & 14\end{array}$

$\begin{array}{llllllllllllll}-0.0587 & 0.1477 & -2.3756 & \mathrm{C} & 0 & 0 & 0 & 0 & 0 & 0 & 0 & 0 & 0 & 14 \\ 0.8152 & 0.6962 & -3.3060 & \mathrm{~N} & 0 & 0 & 0 & 0 & 0 & 0 & 0 & 0 & 0 & 14\end{array}$

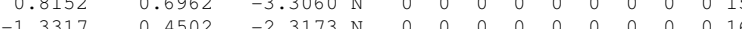

$\begin{array}{llllllllllllll}-1.3317 & 0.4502 & -2.3173 & \mathrm{~N} & 0 & 0 & 0 & 0 & 0 & 0 & 0 & 0 & 0 & 16\end{array}$

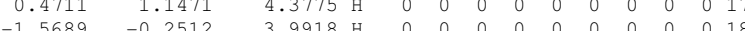

$\begin{array}{lllllllllllll}-1.5689-0.2512 & 3.9918 & \mathrm{H} & 0 & 0 & 0 & 0 & 0 & 0 & 0 & 0 & 0 & 18\end{array}$

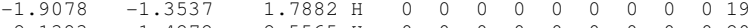

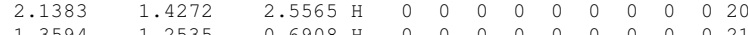

$\begin{array}{llllllllllllll}-1.3594 & -1.2535 & -0.6908 & \mathrm{H} & 0 & 0 & 0 & 0 & 0 & 0 & 0 & 0 & 0 & 21\end{array}$

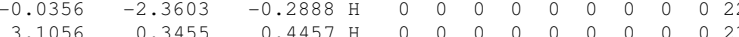

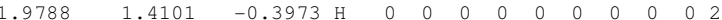

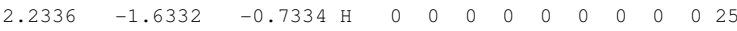

$\begin{array}{llllllllllllll}2.5628 & -0.3929 & -1.9429 & \mathrm{H} & 0 & 0 & 0 & 0 & 0 & 0 & 0 & 0 & 0 & 26\end{array}$

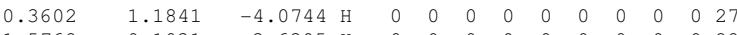

$\begin{array}{llllllll}1.5768 & 0.1031 & -3.6305 \mathrm{H} & 0 & 0 & 0 & -0\end{array}$

$-1.6017 \quad 1.0123$

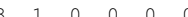

$\begin{array}{llllll}5 & 4 & 0 & 0 & 0 & 0\end{array}$

$\begin{array}{lllllll}9 & 4 & 0 & 0 & 0 & 0\end{array}$

$\begin{array}{llllll}7 & 4 & 0 & 0 & 0 & 0\end{array}$

$\begin{array}{lllll}6 & 5 & 0 & 0 & 0\end{array}$

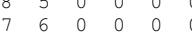

$\begin{array}{llllll}9 & 6 & 0 & 0 & 0 & 0\end{array}$

$\begin{array}{lllll}8 & 7 & 0 & 0 & 0\end{array}$

$\begin{array}{rrllll}1 & 8 & 0 & 0 & 0 & 0\end{array}$

$\begin{array}{cccccc}11 & 8 & 0 & 0 & 0 & 0 \\ 20 & 9 & 0 & 0 & 0 & 0\end{array}$

$\begin{array}{llllll}13 & 10 & 0 & 0 & 0\end{array}$

$\begin{array}{lllllll}10 & 0 & 0 & 0 & 0\end{array}$

$\begin{array}{llllll}21 & 10 & 0 & 0 & 0 & 0\end{array}$

$\begin{array}{llllll}22 & 10 & 0 & 0 & 0 & 0\end{array}$

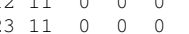

$\begin{array}{llllll}24 & 11 & 0 & 0 & 0 & 0\end{array}$

$\begin{array}{llllll}13 & 12 & 0 & 0 & 0 & 0\end{array}$

$\begin{array}{llllll}25 & 12 & 0 & 0 & 0 & 0\end{array}$

$\begin{array}{llllll}26 & 12 & 0 & 0 & 0 & 0\end{array}$

$\begin{array}{llllll}14 & 13 & 0 & 0 & 0 & 0\end{array}$

$\begin{array}{llllll}16 & 14 & 0 & 0 & 0 & 0\end{array}$

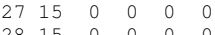

END

ebrisoquine_a.sdf

cosmotherm 


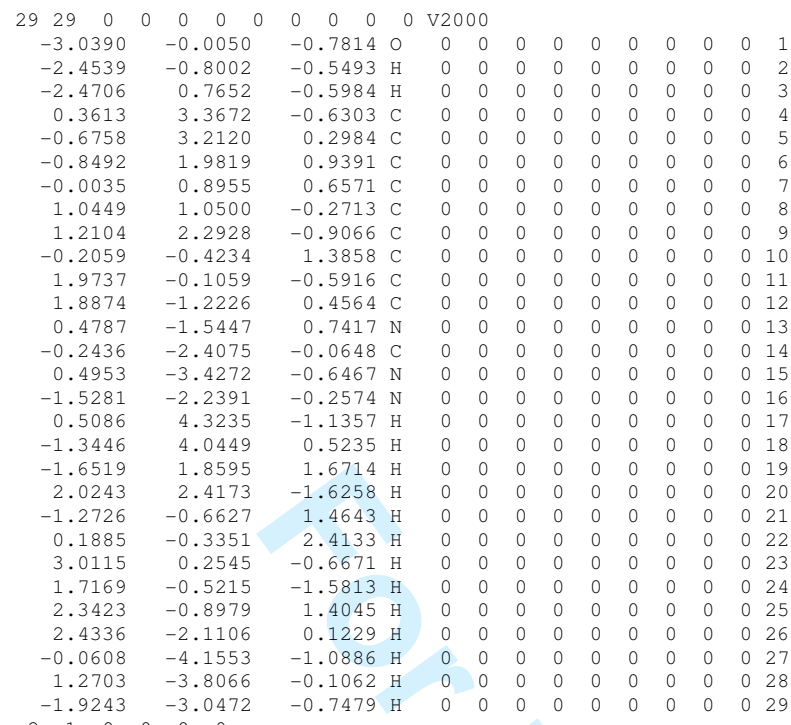


$\begin{array}{lllllll}8 & 7 & 0 & 0 & 0 & 0\end{array}$

$\begin{array}{llllll}8 & 7 & 0 & 0 & 0 & 0 \\ 9 & 8 & 0 & 0 & 0 & 0\end{array}$

$\begin{array}{llllll}11 & 8 & 0 & 0 & 0 & 0 \\ 20 & 9 & 0 & 0 & 0 & 0\end{array}$

$\begin{array}{lrrrrrr}20 & 9 & 0 & 0 & 0 & 0 \\ 13 & 10 & 0 & 0 & 0 & 0\end{array}$

$\begin{array}{llllll}21 & 10 & 0 & 0 & 0 & 0\end{array}$

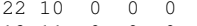

$1211 \quad 000000$

$2411 \quad 0 \quad 0000$

$\begin{array}{lllllll}13 & 12 & 0 & 0 & 0 & 0\end{array}$

$\begin{array}{llllll}25 & 12 & 0 & 0 & 0 & 0\end{array}$

$\begin{array}{lllllll}14 & 13 & 0 & 0 & 0 & 0\end{array}$

$1514 \quad 000000$

$\begin{array}{llllll}16 & 14 & 0 & 0 & 0 & 0\end{array}$

$28 \quad 15 \quad 000000$

$\begin{array}{llllll}29 & 16 & 0 & 0 & 0 & 0\end{array}$

$M$ END

debrisoquine_p2.sd

cosmotherm $3 \mathrm{D}$

$\begin{array}{llllllllllll}30 & 30 & 0 & 0 & 0 & 0 & 0 & 0 & 0 & 0 & 0 & \mathrm{v} 2000\end{array}$

$\begin{array}{llllllllllllll}-5.3329 & 0.7293 & -0.5254 & 0 & 0 & 0 & 0 & 0 & 0 & 0 & 0 & 0 & 0 & 1\end{array}$

$\begin{array}{llllllllllllll}-6.1068 & 0.1406 & -0.5842 & \mathrm{H} & 0 & 0 & 0 & 0 & 0 & 0 & 0 & 0 & 0 & 2\end{array}$

$\begin{array}{llllllllllllll}-5.6819 & 1.6247 & -0.6848 & \mathrm{H} & 0 & 0 & 0 & 0 & 0 & 0 & 0 & 0 & 0 & 3\end{array}$

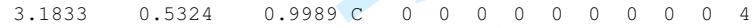

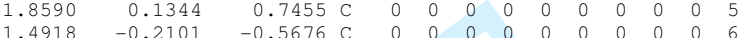

$\begin{array}{llllllllllllll}1.4918 & -0.2101 & -0.5676 & \mathrm{C} & 0 & 0 & 0 & 0 & 0 & 0 & 0 & 0 & 0 & 6 \\ 3.749 & -0.1567 & -1.5956 & \mathrm{C} & 0 & 0 & 0 & 0 & 0 & 0 & 0 & 0 & 0 & 7\end{array}$

$\begin{array}{llllllllllllll}3.7589 & 0.2418 & -1.3320 & \mathrm{C} & 0 & 0 & 0 & 0 & 0 & 0 & 0 & 0 & 0 & 8 \\ 1.1288 & 0.5903 & -0.0260 & \mathrm{C} & 0 & 0 & 0 & 0 & 0 & 0 & 0 & 0 & 0 & 9\end{array}$

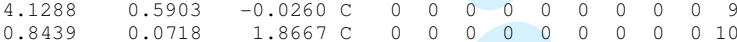

$\begin{array}{llllllllllllll}-0.3085 & -0.8605 & 1.5059 & \mathrm{C} & 0 & 0 & 0 & 0 & 0 & 0 & 0 & 0 & 0 & 11\end{array}$

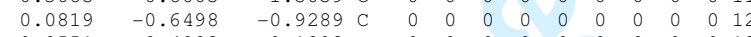

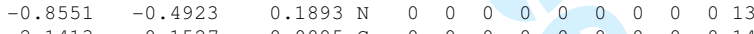

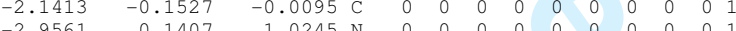

$\begin{array}{llllllllllllllll}-2.6541 & -0.14929 & -1.2540 \mathrm{~N} & 0 & 0 & 0 & 0 & 0 & 0 & 0 & 0 & 0 & 15\end{array}$

$\begin{array}{llllllllllllllll}3.4 .4711 & 0.7949 & 2.0201 \mathrm{H} & 0 & 0 & 0 & 0 & 0 & 0 & 0 & 0 & 0 & 0 & 16\end{array}$

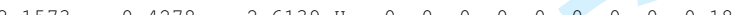

$\begin{array}{lllllllllllllll}4.1589 & 0.4278 & -2.6139 & \mathrm{H} & 0 & 0 & 0 & 0 & 0 & 0 & 0 & 0 & 0 & 0 & 18\end{array}$

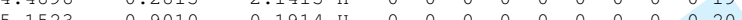

$\begin{array}{llllllllllllllll}5.1523 & 0.9010 & 0.1914 & \mathrm{H} & 0 & 0 & 0 & 0 & 0 & 0 & 0 & 0 & 0 & 0 & 20\end{array}$

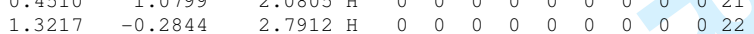

$\begin{array}{lllllllllllll}-1.1006 & -0.8426 & 2.2606 \mathrm{H} & 0 & 0 & 0 & 0 & 0 & 0 & 0 & 0 & 0 & 23\end{array}$

$\begin{array}{lllllllllllll}0.0486 & -1.9008 & 1.4354 \mathrm{H} & 0 & 0 & 0 & 0 & 0 & 0 & 0 & 0 & 0 & 24\end{array}$

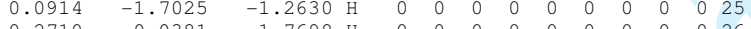

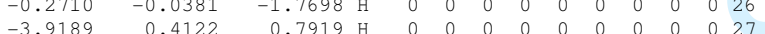

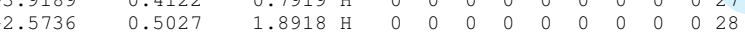

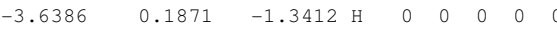

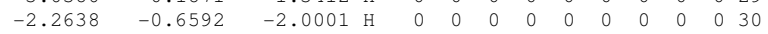

$\begin{array}{llllll}2 & 1 & 0 & 0 & 0 & 0\end{array}$

$\begin{array}{llllll}3 & 1 & 0 & 0 & 0 & 0\end{array}$

$\begin{array}{lllllll}4 & 0 & 0 & 0 & 0\end{array}$

$\begin{array}{lllllll}7 & 4 & 0 & 0 & 0 & 0 & 2\end{array}$

$\begin{array}{lllll}5 & 0 & 0 & 0 & 0\end{array}$

$\begin{array}{lllll}5 & 0 & 0 & 0 \\ 6 & 0 & 0\end{array}$

$\begin{array}{lllllll}2 & 6 & 0 & 0 & 0 & 0\end{array}$

$\begin{array}{lllllll}8 & 7 & 0 & 0 & 0 & 0 & 0 \\ 8 & 7 & 0 & 0 & 0 & -1\end{array}$

$\begin{array}{llllll}9 & 8 & 0 & 0 & 0 & 0\end{array}$

$\begin{array}{lllllll}9 & 8 & 0 & 0 & 0 & 0 & 0\end{array}$

$\begin{array}{llllll}2 & 10 & 0 & 0 & 0 & 0\end{array}$

$\begin{array}{lllllll}13 & 11 & 0 & 0 & 0 & 0 \\ 23 & 11 & 0 & 0 & 0 & 0\end{array}$

$2411 \quad 0 \quad 0000$

$\begin{array}{lllllllll}13 & 12 & 0 & 0 & 0 & 0\end{array}$

$\begin{array}{llllll}26 & 12 & 0 & 0 & 0 & 0\end{array}$

$\begin{array}{llllll}14 & 13 & 0 & 0 & 0 & 0\end{array}$

$\begin{array}{lllllll}15 & 14 & 0 & 0 & 0 & 0\end{array}$

$\begin{array}{llllll}16 & 14 & 0 & 0 & 0\end{array}$

$\begin{array}{lllllll}27 & 15 & 0 & 0 & 0 & 0 & 0 \\ 28 & 15 & 0 & 0 & 0 & 0 & 0\end{array}$

$\begin{array}{llllll}29 & 16 & 0 & 0 & 0 & 0\end{array}$

$\begin{array}{llllll}16 & 30 & 0 & 0 & 0 & 0\end{array}$

$\underset{\$ \$ \$ \$}{M} \operatorname{END}$

di-iso-butylamine-cation.sdf

Cosmotherm 3D $\quad 3$.

$\begin{array}{llllllllllll}32 & 30 & 0 & 0 & 0 & 0 & 0 & 0 & 0 & 0 & 0 & \mathrm{v} 2000\end{array}$

$\begin{array}{llllllllllll}-0.6627 & 0 & 0 & 0 & 0 & 0 & 0 & 0 & 0 & 0 & 0 & 1\end{array}$

$\begin{array}{lllllllllllll}-3.2283 & 0.1954 & -0.0393 & \mathrm{H} & 0 & 0 & 0 & 0 & 0 & 0 & 0 & 0 & 0\end{array}$

$\begin{array}{llllllllllllll}-2.7771 & -0.6687 & -1.2319 & \mathrm{H} & 0 & 0 & 0 & 0 & 0 & 0 & 0 & 0 & 0 & 3\end{array}$

$\begin{array}{lllllllllllllll}1.2532 & 3.6910 & -0.2243 & \mathrm{C} & 0 & 0 & 0 & 0 & 0 & 0 & 0 & 0 & 0 & 4\end{array}$

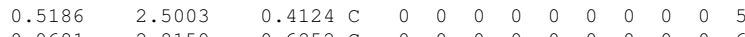

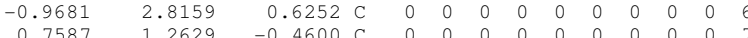

$\begin{array}{lllllllllllllll}0.1842 & -0.0011 & 0.1383 \mathrm{~N} & 0 & 0 & 0 & 0 & 0 & 0 & 0 & 0 & 0\end{array}$

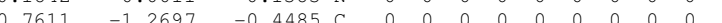

$\begin{array}{llllllllllllllll}0.4889 & -2.5064 & -0.4158 \mathrm{C} & 0 & 0 & 0 & 0 & 0 & 0 & 0 & 0 & 0 & 10\end{array}$

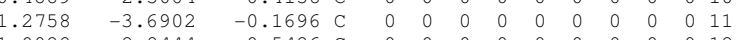

URL: http://mc.manuscriptcentral.com/tandf/tmph 


$\begin{array}{rrrrllllllllll}1.1466 & 4.5841 & 0.4072 & \mathrm{H} & 0 & 0 & 0 & 0 & 0 & 0 & 0 & 0 & 0 & 13 \\ 2.3269 & 3.4856 & -0.3456 \mathrm{H} & 0 & 0 & 0 & 0 & 0 & 0 & 0 & 0 & 0 & 14 \\ 0.8348 & 3.9264 & -1.2156 \mathrm{H} & 0 & 0 & 0 & 0 & 0 & 0 & 0 & 0 & 0 & 15 \\ 0.9808 & 2.3032 & 1.3964 & \mathrm{H} & 0 & 0 & 0 & 0 & 0 & 0 & 0 & 0 & 0 & 16 \\ -1.0763 & 3.7240 & 1.2352 & \mathrm{H} & 0 & 0 & 0 & 0 & 0 & 0 & 0 & 0 & 0 & 17 \\ -1.4990 & 2.0090 & 1.1514 & \mathrm{H} & 0 & 0 & 0 & 0 & 0 & 0 & 0 & 0 & 0 & 18 \\ -1.4758 & 2.9878 & -0.3363 & \mathrm{H} & 0 & 0 & 0 & 0 & 0 & 0 & 0 & 0 & 0 & 19 \\ 1.8354 & 1.0885 & -0.5804 & \mathrm{H} & 0 & 0 & 0 & 0 & 0 & 0 & 0 & 0 & 0 & 20 \\ 0.3071 & 1.3743 & -1.4553 \mathrm{H} & 0 & 0 & 0 & 0 & 0 & 0 & 0 & 0 & 0 & 21 \\ -0.8611 & -0.0027 & -0.0116 & \mathrm{H} & 0 & 0 & 0 & 0 & 0 & 0 & 0 & 0 & 0 & 22 \\ 1.8418 & -1.1041 & -0.5407 & \mathrm{H} & 0 & 0 & 0 & 0 & 0 & 0 & 0 & 0 & 0 & 23 \\ 0.3367 & -1.3750 & -1.4564 & \mathrm{H} & 0 & 0 & 0 & 0 & 0 & 0 & 0 & 0 & 0 & 24 \\ 0.8910 & -2.2978 & 1.4234 & \mathrm{H} & 0 & 0 & 0 & 0 & 0 & 0 & 0 & 0 & 0 & 25 \\ 1.1397 & -4.5829 & 0.4569 & \mathrm{H} & 0 & 0 & 0 & 0 & 0 & 0 & 0 & 0 & 0 & 26 \\ 2.3526 & -3.4729 & -0.2236 \mathrm{H} & 0 & 0 & 0 & 0 & 0 & 0 & 0 & 0 & 0 & 27 \\ 0.9228 & -3.9331 & -1.1841 & \mathrm{H} & 0 & 0 & 0 & 0 & 0 & 0 & 0 & 0 & 0 & 28 \\ -1.1349 & -3.7402 & 1.1659 & \mathrm{H} & 0 & 0 & 0 & 0 & 0 & 0 & 0 & 0 & 0 & 29 \\ -1.5829 & -2.0352 & 1.0101 \mathrm{H} & 0 & 0 & 0 & 0 & 0 & 0 & 0 & 0 & 0 & 30 \\ -1.4419 & -3.0538 & -0.4458 & \mathrm{H} & 0 & 0 & 0 & 0 & 0 & 0 & 0 & 0 & 0 & 31 \\ 0.3658 & 0.0022 & 1.1515 \mathrm{H} & 0 & 0 & 0 & 0 & 0 & 0 & 0 & 0 & 0 & 32\end{array}$


$\begin{array}{llllll}23 & 9 & 0 & 0 & 0 & 0\end{array}$

$\begin{array}{llllll}24 & 9 & 0 & 0 & 0 & 0\end{array}$

$\begin{array}{llllll}11 & 10 & 0 & 0 & 0 & 0\end{array}$

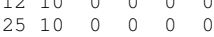

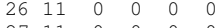

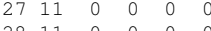

$\begin{array}{lllllll}29 & 12 & 0 & 0 & 0 & 0\end{array}$

$\begin{array}{llllll}30 & 12 & 0 & 0 & 0 & 0\end{array}$

$M \quad$ END

$\$ \$ \$$

di-iso-propylamine-cation. sdf

cosmotherm

$\begin{array}{llllllllllll}26 & 24 & 0 & 0 & 0 & 0 & 0 & 0 & 0 & 0 & 0 & \mathrm{~V} 2000\end{array}$

$\begin{array}{llllllllllllll}-2.5346 & 0.2190 & -1.0061 & 0 & 0 & 0 & 0 & 0 & 0 & 0 & 0 & 0 & 0 & 1\end{array}$

$\begin{array}{lllllllllllll}-2.9874 & 1.0395 & -0.7396 & \mathrm{H} & 0 & 0 & 0 & 0 & 0 & 0 & 0 & 0 & 0\end{array}$

$\begin{array}{llllllllllllll}-3.2134 & -0.4756 & -0.9257 & \mathrm{H} & 0 & 0 & 0 & 0 & 0 & 0 & 0 & 0 & 0 & 3\end{array}$

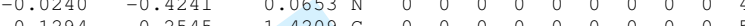

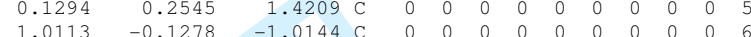

$\begin{array}{llllllllllllll}0.7034 & -1.0221 & -2.2135 & \mathrm{C} & 0 & 0 & 0 & 0 & 0 & 0 & 0 & 0 & 0\end{array}$

$\begin{array}{llllllllllllll}1.0116 & 1.3563 & -1.3623 & \mathrm{C} & 0 & 0 & 0 & 0 & 0 & 0 & 0 & 0 & 0 & 8\end{array}$

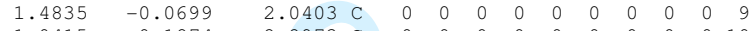

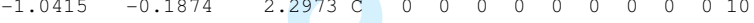

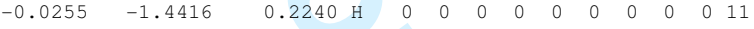

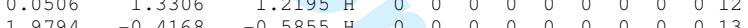

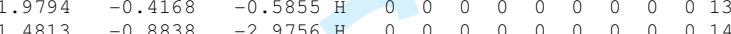

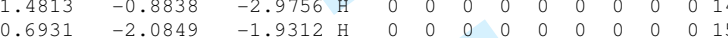

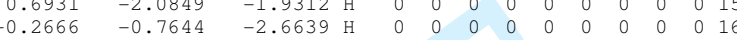

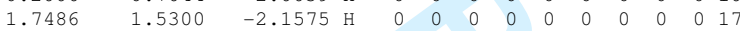

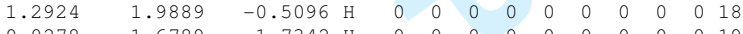

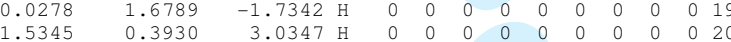

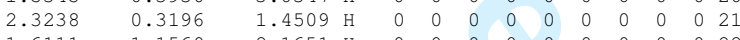

$\begin{array}{llllllllllllll}1.6111 & -1.1560 & 2.1651 & \mathrm{H} & 0 & 0 & 0 & 0 & 0 & 0 & 0 & 0 & 0 & 22\end{array}$

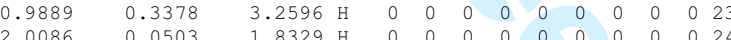

$\begin{array}{lllllllllllllllllllllll}-1.0008 & -1.2691 & 2.49964 \mathrm{H} & 0 & 0 & 0 & 0 & 0 & 0 & 0 & 0 & 0 & 25\end{array}$

$\begin{array}{lllllllllllllllllllll}-1.0008 & -1.2691 & 2.4964 & \mathrm{H} & 0 & 0 & 0 & 0 & 0 & 0 & 0 & 0 & 0 & 0 & 0 & 0 & 0\end{array}$

$-1008-0.18$

$\begin{array}{lllllll}3 & 1 & 0 & 0 & 0 & 0\end{array}$

$5 \quad 4 \quad 000000$

$\begin{array}{llllll}6 & 4 & 0 & 0 & 0 & 0\end{array}$

$\begin{array}{llllll}5 & 0 & 0 & 0 & 0\end{array}$

$\begin{array}{lllllllll}10 & 5 & 0 & 0 & 0 & 0\end{array}$

$\begin{array}{lllllll}2 & 5 & 0 & 0 & 0 & 0\end{array}$

$\begin{array}{lllll}6 & 0 & 0 & 0\end{array}$

$\begin{array}{lllllllll}3 & 6 & 0 & 0 & 0 & 0\end{array}$

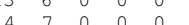

$\begin{array}{llllllll}7 & 7 & 0 & 0 & 0 & 0\end{array}$

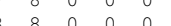

$\begin{array}{lllllll}18 & 8 & 0 & 0 & 0 & 0\end{array}$

$\begin{array}{lllllll}18 & 9 & 0 & 0 & 0 & 0\end{array}$

$\begin{array}{lllllll}21 & 9 & 0 & 0 & 0 & 0\end{array}$

$\begin{array}{lrrrrrr}22 & 9 & 0 & 0 & 0 & 0\end{array}$

2410000000

$2510 \quad 00000$

M END

di-iso-propylamine, sdf

Cosmotherm

$\begin{array}{llllllllllll}25 & 23 & 0 & 0 & 0 & 0 & 0 & 0 & 0 & 0 & 0 & \mathrm{v} 2000\end{array}$

$\begin{array}{rrrrrrrrrrrrrr}-0.3136 & -0.0152 & 0.1632 & 0 & 0 & 0 & 0 & 0 & 0 & 0 & 0 & 0 & 0 & 1 \\ 0.6973 & 0.0054 & 0.0870 & \mathrm{H} & 0 & 0 & 0 & 0 & 0 & 0 & 0 & 0 & 0 & 2\end{array}$

$\begin{array}{ccccccccccccc}-0.5107 & 0.6012 & 0.8899 \mathrm{H} & 0 & 0 & 0 & 0 & 0 & 0 & 0 & 0 & 0 & 3\end{array}$

$\begin{array}{lllllllllllll}2.4800 & 0.0292 & -0.0402 & \mathrm{~N} & 0 & 0 & 0 & 0 & 0 & 0 & 0 & 0 & 0\end{array}$

$\begin{array}{llllllllllllll}1.2355 & -0.7614 & \mathrm{C} & 0 & 0 & 0 & 0 & 0 & 0 & 0 & 0 & 0\end{array}$

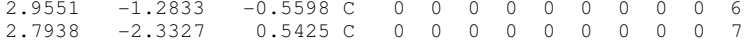

$\begin{array}{llllllllllllll}2.1795 & -1.6735 & -1.8227 & C & 0 & 0 & 0 & 0 & 0 & 0 & 0 & 0 & 0 & 8\end{array}$

$\begin{array}{llllllllllllll}4.4863 & 1.4690 & -0.5862 & \mathrm{C} & 0 & 0 & 0 & 0 & 0 & 0 & 0 & 0 & 0 & 9\end{array}$

$\begin{array}{llllllllllllllll}2.1786 & 2.4577 & -0.2964 & \mathrm{C} & 0 & 0 & 0 & 0 & 0 & 0 & 0 & 0 & 0 & 10\end{array}$

$\begin{array}{llllllllllllll}2.7879 & 0.1114 & 0.9362 & \mathrm{H} & 0 & 0 & 0 & 0 & 0 & 0 & 0 & 0 & 0 & 11\end{array}$

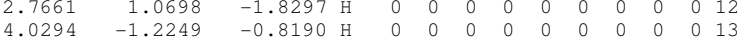

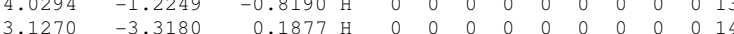

$\begin{array}{llllllllllllll}-3.3180 & 0.1877 \mathrm{H} & 0 & 0 & 0 & 0 & 0 & 0 & 0 & 0 & 0 & 14\end{array}$

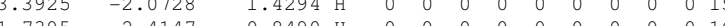

$\begin{array}{lllllllllllllll}1.7395 & -2.4147 & 0.8490 & \mathrm{H} & 0 & 0 & 0 & 0 & 0 & 0 & 0 & 0 & 0 & 16\end{array}$

$\begin{array}{llllllllllllll}1.1134 & -1.8212 & -1.5922 & \mathrm{H} & 0 & 0 & 0 & 0 & 0 & 0 & 0 & 0 & 0 & 17\end{array}$

$\begin{array}{lllllllllllll}-2.6104 & -2.2381 \mathrm{H} & 0 & 0 & 0 & 0 & 0 & 0 & 0 & 0 & 0 & 18\end{array}$

$\begin{array}{llllllllllllll}2.2577 & -0.9054 & -2.6060 & \mathrm{H} & 0 & 0 & 0 & 0 & 0 & 0 & 0 & 0 & 0 & 19\end{array}$

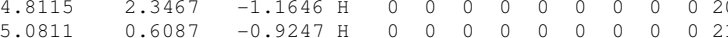

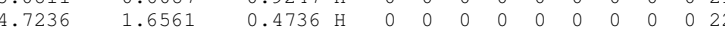

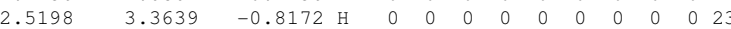

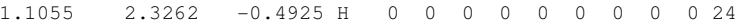
$2.3142 \quad 2.6204$

$-0.4925 \mathrm{H}$

$\begin{array}{llllll}2 & 1 & 0 & 0 & 0 \\ 3 & 1 & 0 & 0 & 0 & 0\end{array}$

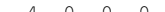

$6 \begin{array}{llllll}5 & 4 & 0 & 0 & 0 & 0\end{array}$

$\begin{array}{lllllll}11 & 4 & 0 & 0 & 0 & 0\end{array}$

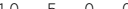




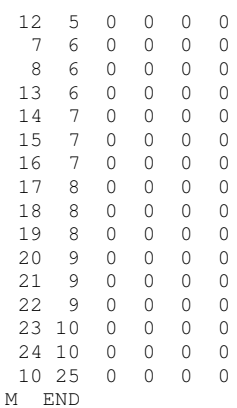

$M \quad$ END

\$\$-n-butylamine-cation.sdf
di-n

cosmotherm 3D

$\begin{array}{llllllllllll}32 & 30 & 0 & 0 & 0 & 0 & 0 & 0 & 0 & 0 & 0 & \mathrm{~V} 2000\end{array}$

$\begin{array}{llllllllllllll}-2.6233 & 0.2222 & 0.0657 & 0 & 0 & 0 & 0 & 0 & 0 & 0 & 0 & 0 & 0 & 1\end{array}$

$\begin{array}{rrrrrrrrrrrrrr}-3.1789 & -0.2974 & -0.5433 & \mathrm{H} & 0 & 0 & 0 & 0 & 0 & 0 & 0 & 0 & 0 & 2 \\ -2.9219 & 1.1426 & -0.0508 & \mathrm{H} & 0 & 0 & 0 & 0 & 0 & 0 & 0 & 0 & 0 & 3\end{array}$

$\begin{array}{lllllllllllllll}0.6902 & 0.3824 & 1.2620 & \mathrm{C} & 0 & 0 & 0 & 0 & 0 & 0 & 0 & 0 & 0 & 4\end{array}$

$\begin{array}{llllllllllllll}0.1231 & -0.2054 & -0.0041 & \mathrm{~N} & 0 & 0 & 0 & 0 & 0 & 0 & 0 & 0 & 0 & 5\end{array}$

$\begin{array}{rrrrrrrrrrrrrr}0.6865 & 0.3881 & -1.2689 & \mathrm{C} & 0 & 0 & 0 & 0 & 0 & 0 & 0 & 0 & 0 & 6 \\ 0.1168 & -0.3005 & 2.4993 & \mathrm{C} & 0 & 0 & 0 & 0 & 0 & 0 & 0 & 0 & 0 & 7\end{array}$

$\begin{array}{rrrrrrrrrrrrrr}0.1168 & -0.3005 & 2.4993 & \mathrm{C} & 0 & 0 & 0 & 0 & 0 & 0 & 0 & 0 & 0 & 7 \\ 0.4520 & 1.4539 & 1.2439 & \mathrm{H} & 0 & 0 & 0 & 0 & 0 & 0 & 0 & 0 & 0 & 8\end{array}$

$\begin{array}{lllllllllllll}1.7806 & 0.2662 & 1.2068 \mathrm{H} & 0 & 0 & 0 & 0 & 0 & 0 & 0 & 0 & 0 & 9\end{array}$

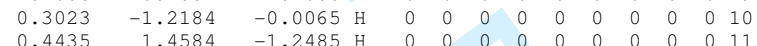

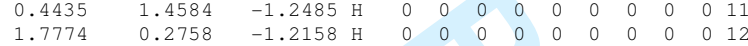

$\begin{array}{llllllllllllll}0.1143 & -0.2967 & -2.5055 & \mathrm{C} & 0 & 0 & 0 & 0 & 0 & 0 & 0 & 0 & 0 & 13\end{array}$

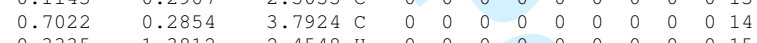

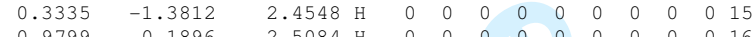

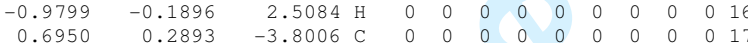

$\begin{array}{llllllllllllll}0.3334 & -1.3769 & -2.4604 & \mathrm{H} & 0 & 0 & 0 & 0 & 0 & 0 & 0 & 0 & 0 & 18\end{array}$

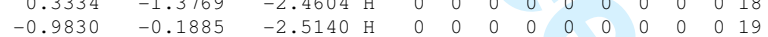

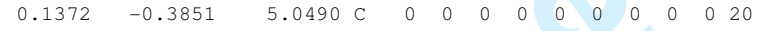

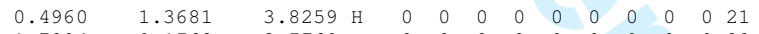

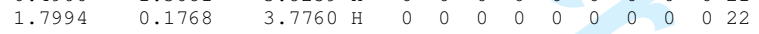

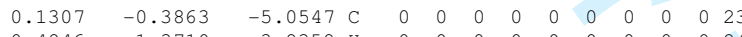

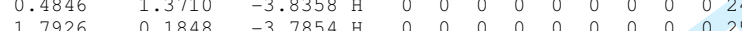

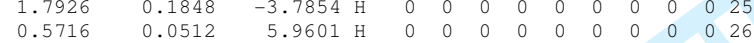

$\begin{array}{lllllllllllll}0.3559 & -1.4640 & 5.0556 \mathrm{H} & 0 & 0 & 0 & 0 & 0 & 0 & 0 & 0 & 0 & 27\end{array}$

$\begin{array}{llllllllllllll}-0.9555 & -0.2637 & 5.1063 & \mathrm{H} & 0 & 0 & 0 & 0 & 0 & 0 & 0 & 0 & 0 & 28\end{array}$

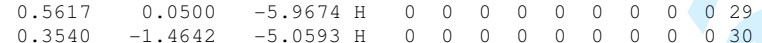

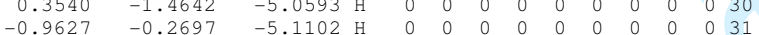

$\begin{array}{llllllllllllll}-0.9293 & -0.0777 & -0.0035 & \mathrm{H} & 0 & 0 & 0 & 0 & 0 & 0 & 0 & 0 & 0 & 32\end{array}$

$\begin{array}{llllll}3 & 1 & 0 & 0 & 0 & 0\end{array}$

$\begin{array}{llllll}4 & 0 & 0 & 0 & 0 & 0 \\ 4 & 0 & 0 & 0\end{array}$

$\begin{array}{llllll}8 & 4 & 0 & 0 & 0 & 0\end{array}$

$\begin{array}{llllll}9 & 4 & 0 & 0 & 0 \\ 6 & 5 & 0 & 0 & 0\end{array}$

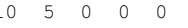

$\begin{array}{llllll}11 & 6 & 0 & 0 & 0 & 0\end{array}$

$\begin{array}{llllllllll}3 & 6 & 0 & 0 & 0 & 0\end{array}$

$\begin{array}{lllllll}14 & 7 & 0 & 0 & 0 & 0\end{array}$

$\begin{array}{llllll}15 & 7 & 0 & 0 & 0 & 0 \\ 16 & 7 & 0 & 0 & 0 & 0\end{array}$

$\begin{array}{llllll}17 & 13 & 0 & 0 & 0 & 0\end{array}$

$18 \quad 13 \quad 000000$

$\begin{array}{llllll}21 & 14 & 0 & 0 & 0 & 0\end{array}$

$2214 \quad 0 \quad 0000$

$\begin{array}{lllll}23 & 17 & 0 & 0 & 0\end{array}$

$\begin{array}{llllll}24 & 17 & 0 & 0 & 0 & 0\end{array}$

$\begin{array}{llllll}25 & 17 & 0 & 0 & 0 & 0 \\ 26 & 20 & 0 & 0 & 0 & 0\end{array}$

$\begin{array}{lllllll}27 & 20 & 0 & 0 & 0 & 0\end{array}$

$\begin{array}{llllll}28 & 20 & 0 & 0 & 0 & 0\end{array}$

$\begin{array}{llllll}29 & 23 & 0 & 0 & 0 & 0\end{array}$

$\begin{array}{llllll}30 & 23 & 0 & 0 & 0 & 0 \\ 31 & 23 & 0 & 0 & 0 & 0\end{array}$

$\begin{array}{llllll}5 & 32 & 0 & 0 & 0 & 0\end{array}$

$\$ \$ \$ \$$

di-n-butylamine.sdf

cosmotherm 3D

$\begin{array}{llllllllllll}31 & 29 & 0 & 0 & 0 & 0 & 0 & 0 & 0 & 0 & 0 & \mathrm{~V} 2000\end{array}$

$\begin{array}{rrrrrrrrrrrrrr}2.6716 & 0.1970 & -0.0524 & 0 & 0 & 0 & 0 & 0 & 0 & 0 & 0 & 0 & 0 & 1 \\ 1.6659 & 0.0434 & -0.0247 & \mathrm{H} & 0 & 0 & 0 & 0 & 0 & 0 & 0 & 0 & 0 & 2 \\ 2.7816 & 0.9910 & -0.6033 & \mathrm{H} & 0 & 0 & 0 & 0 & 0 & 0 & 0 & 0 & 0 & 3 \\ -0.6487 & 0.3893 & -1.2181 & \mathrm{C} & 0 & 0 & 0 & 0 & 0 & 0 & 0 & 0 & 0 & 4 \\ -0.0687 & -0.1920 & 0.0106 & \mathrm{~N} & 0 & 0 & 0 & 0 & 0 & 0 & 0 & 0 & 0 & 5 \\ -0.6385 & 0.3921 & 1.2422 & \mathrm{C} & 0 & 0 & 0 & 0 & 0 & 0 & 0 & 0 & 0 & 6 \\ -0.1299 & -0.3089 & -2.4756 & \mathrm{C} & 0 & 0 & 0 & 0 & 0 & 0 & 0 & 0 & 0 & 7 \\ -0.3756 & 1.4574 & -1.2417 & \mathrm{H} & 0 & 0 & 0 & 0 & 0 & 0 & 0 & 0 & 0 & 8 \\ -1.7565 & 0.3425 & -1.1949 & \mathrm{H} & 0 & 0 & 0 & 0 & 0 & 0 & 0 & 0 & 0 & 9 \\ -0.2664 & -1.1990 & 0.0127 & \mathrm{H} & 0 & 0 & 0 & 0 & 0 & 0 & 0 & 0 & 0 & 10 \\ -0.3779 & 1.4635 & 1.2541 & \mathrm{H} & 0 & 0 & 0 & 0 & 0 & 0 & 0 & 0 & 0 & 11 \\ -1.7460 & 0.3314 & 1.2341 & \mathrm{H} & 0 & 0 & 0 & 0 & 0 & 0 & 0 & 0 & 0 & 12 \\ -0.0942 & -0.2900 & 2.4975 & \mathrm{C} & 0 & 0 & 0 & 0 & 0 & 0 & 0 & 0 & 0 & 13 \\ -0.6899 & 0.2973 & -3.7690 & \mathrm{C} & 0 & 0 & 0 & 0 & 0 & 0 & 0 & 0 & 0 & 14 \\ -0.3946 & -1.3803 & -2.4305 & \mathrm{H} & 0 & 0 & 0 & 0 & 0 & 0 & 0 & 0 & 0 & 15\end{array}$

URL: http://mc.manuscriptcentral.com/tandf/tmph 
$\begin{array}{llllllllllllll}0.9720 & -0.2577 & -2.4890 & \mathrm{H} & 0 & 0 & 0 & 0 & 0 & 0 & 0 & 0 & 0 & 16\end{array}$

$\begin{array}{lllllllllllllll}-0.6839 & 0.2849 & 3.7921 & \mathrm{C} & 0 & 0 & 0 & 0 & 0 & 0 & 0 & 0 & 0 & 17\end{array}$

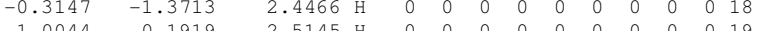

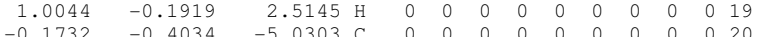

$\begin{array}{lllllllllllllll}-0.4291 & 1.3686 & -3.8096 & \mathrm{H} & 0 & 0 & 0 & 0 & 0 & 0 & 0 & 0 & 0 & 21\end{array}$

$\begin{array}{llllllllllllll}-1.7919 & 0.2488 & -3.7462 & \mathrm{H} & 0 & 0 & 0 & 0 & 0 & 0 & 0 & 0 & 0 & 22\end{array}$

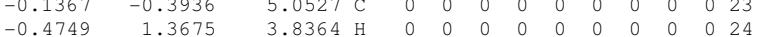

$\begin{array}{lllllllllllllllllllllll}-1.7824 & 0.1838 & 3.7672 & \mathrm{H} & 0 & 0 & 0 & 0 & 0 & 0 & 0 & 0 & 0 & 24\end{array}$

$\begin{array}{lllllllllllllllllllllll}-0.5895 & 0.0516 & -5.9413 \mathrm{H} & 0 & 0 & 0 & 0 & 0 & 0 & 0 & 0 & 0 & 25\end{array}$

$\begin{array}{lllllllllllllll}-0.4480 & -1.4697 & -5.0325 & \mathrm{H} & 0 & 0 & 0 & 0 & 0 & 0 & 0 & 0 & 0 & 26\end{array}$

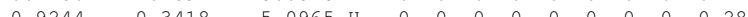

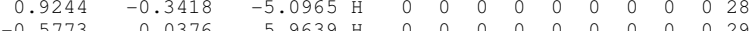

$\begin{array}{lllllllllllllllll}-0.3592 & -1.4721 & 5.0510 & \mathrm{H} & 0 & 0 & 0 & 0 & 0 & 0 & 0 & 0 & 0 & 30\end{array}$

$\begin{array}{llllll}1 & 0 & 0 & 0 & 0\end{array}$

$\begin{array}{lllllllll}3 & 1 & 0 & 0 & 0 & 0\end{array}$

$\begin{array}{llllllll}5 & 4 & 0 & 0 & 0 & 0\end{array}$

$\begin{array}{llllll}8 & 4 & 0 & 0 & 0 & 0\end{array}$

$\begin{array}{lllll}4 & 0 & 0 & 0 & 0\end{array}$

$\begin{array}{lllllll}0 & 5 & 0 & 0 & 0 & 0\end{array}$

$\begin{array}{lllllll}11 & 6 & 0 & 0 & 0 & 0\end{array}$

$\begin{array}{lllllll}12 & 6 & 0 & 0 & 0 & 0\end{array}$

$\begin{array}{lllllll}13 & 6 & 0 & 0 & 0 & 0 \\ 1 & 7 & 0 & 0 & 0 & 0\end{array}$

$\begin{array}{cccccccc}15 & 7 & 0 & 0 & 0 & 0\end{array}$

$\begin{array}{lrrrrr}16 & 7 & 0 & 0 & 0 & 0\end{array}$

$\begin{array}{lllllll}13 & 0 & 0 & 0 & 0\end{array}$

$\begin{array}{llllll}19 & 13 & 0 & 0 & 0 & 0\end{array}$

$\begin{array}{llllll}20 & 14 & 0 & 0 & 0 & 0 \\ 21 & 14 & 0 & 0 & 0 & 0\end{array}$

$\begin{array}{lllllll}21 & 14 & 0 & 0 & 0 & 0 \\ 22 & 14 & 0 & 0 & 0 & 0\end{array}$

$\begin{array}{llllll}23 & 17 & 0 & 0 & 0 & 0\end{array}$

$\begin{array}{llllll}24 & 17 & 0 & 0 & 0 & 0 \\ 25 & 17 & 0 & 0 & 0 & 0\end{array}$

$\begin{array}{llllll}26 & 20 & 0 & 0 & 0 & 0\end{array}$

$\begin{array}{llllll}28 & 23 & 0 & 0 & 0 & 0\end{array}$

$\begin{array}{llllll}30 & 23 & 0 & 0 & 0 & 0\end{array}$

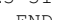

\$\$\$

di-n-propylamine-cation.sdf

cosmotherm

$\begin{array}{llllllllllll}26 & 24 & 0 & 0 & 0 & 0 & 0 & 0 & 0 & 0 & 0 & \text { V2 } 2000\end{array}$

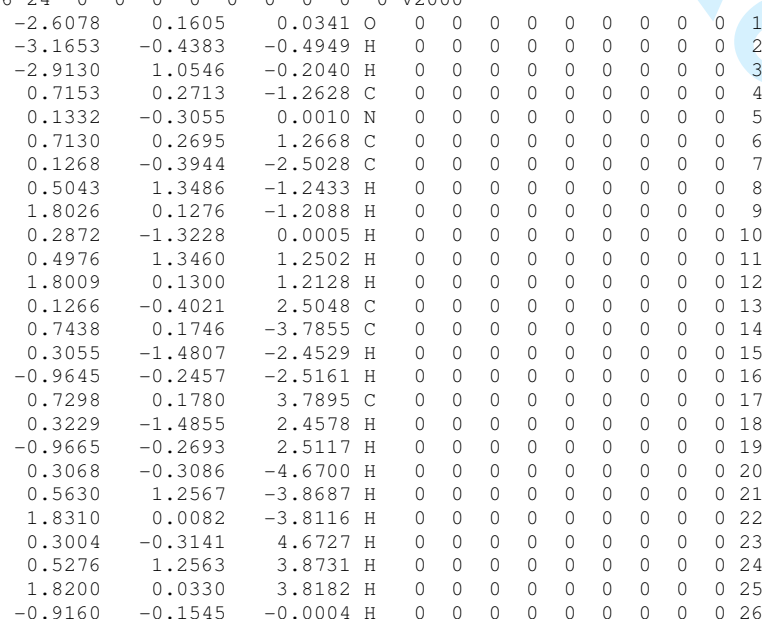

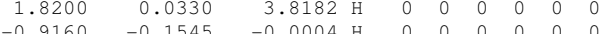

$\begin{array}{lllllll}2 & 1 & 0 & 0 & 0 & 0\end{array}$

$\begin{array}{llllll}3 & 1 & 0 & 0 & 0 & 0\end{array}$

$\begin{array}{llllll}5 & 4 & 0 & 0 & 0 & 0\end{array}$

$\begin{array}{llllll}7 & 4 & 0 & 0 & 0 & 0\end{array}$

$\begin{array}{lllll}4 & 0 & 0 & 0 & 0\end{array}$

$6 \begin{array}{llllll}6 & 5 & 0 & 0 & 0 & 0\end{array}$

$\begin{array}{lllllll}6 & 5 & 0 & 0 & 0 & 0 \\ 10 & 5 & 0 & 0 & 0\end{array}$

$\begin{array}{lllllllll}1 & 6 & 0 & 0 & 0 & 0\end{array}$

$\begin{array}{lllllll}12 & 6 & 0 & 0 & 0 & 0\end{array}$

$\begin{array}{lllllll}13 & 6 & 0 & 0 & 0 & 0\end{array}$

$\begin{array}{lllllll}14 & 7 & 0 & 0 & 0 & 0\end{array}$

$\begin{array}{lllllll}1 & 7 & 7 & 0 & 0 & 0 & 0 \\ 1 & 7 & 0 & 0 & 0\end{array}$

$\begin{array}{llllll}17 & 13 & 0 & 0 & 0 & 0\end{array}$

$\begin{array}{llllll}18 & 13 & 0 & 0 & 0 & 0\end{array}$

$\begin{array}{llllll}19 & 13 & 0 & 0 & 0 & 0 \\ 20 & 14 & 0 & 0 & 0 & 0\end{array}$

$\begin{array}{llllll}21 & 14 & 0 & 0 & 0 & 0\end{array}$

$\begin{array}{llllll}22 & 14 & 0 & 0 & 0 & 0 \\ 23 & 17 & 0 & 0 & 0 & 0\end{array}$

$\begin{array}{llllll}24 & 17 & 0 & 0 & 0 & 0\end{array}$

$\begin{array}{llllll}5 & 17 & 0 & 0 & 0 & 0\end{array}$

$\begin{array}{rl}52 & 26 \\ & \end{array}$

$\$ \$ \$$

di-n-propylamine.sdf

cosmotherm 


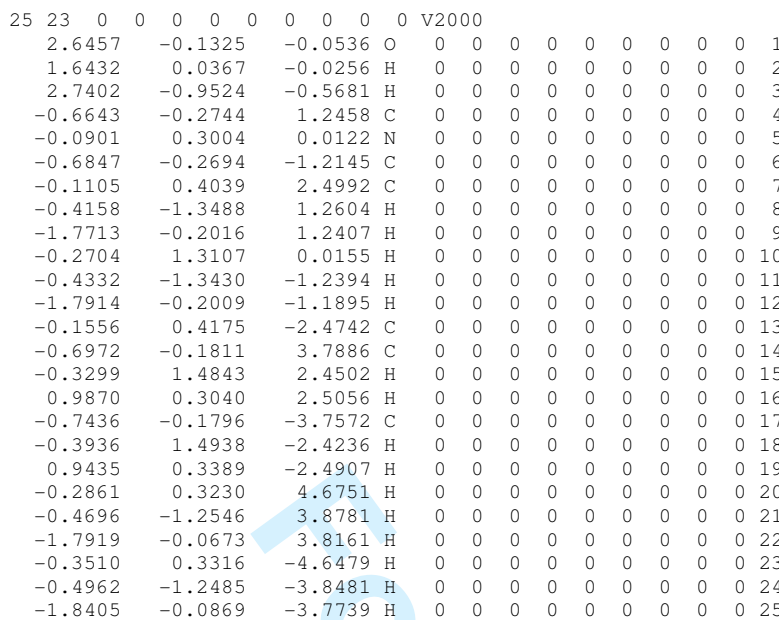




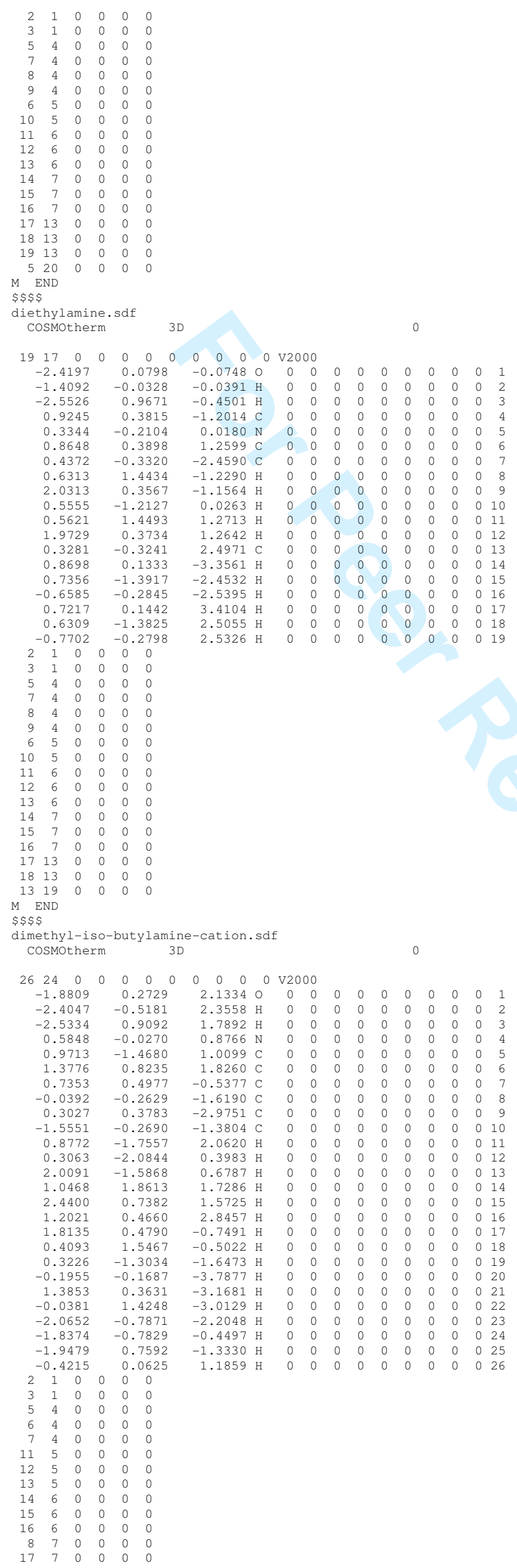


$\begin{array}{lllllll}18 & 7 & 0 & 0 & 0 & 0\end{array}$

$\begin{array}{llllll}9 & 8 & 0 & 0 & 0 & 0 \\ 0 & 8 & 0 & 0 & 0 & 0\end{array}$

$\begin{array}{llllll}19 & 8 & 0 & 0 & 0 & 0\end{array}$

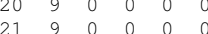

$\begin{array}{llllll}22 & 9 & 0 & 0 & 0 & 0\end{array}$

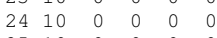

$2510 \quad 000000$

M END

$\$ \$ \$$

dimethyl-iso-butylamine.sdf

cosmotherm

$\begin{array}{llllllllllll}25 & 23 & 0 & 0 & 0 & 0 & 0 & 0 & 0 & 0 & 0 & \mathrm{~V} 2000\end{array}$

$\begin{array}{llllllllllllll}1.9439 & -2.1351 & -0.1430 & 0 & 0 & 0 & 0 & 0 & 0 & 0 & 0 & 0 & 0 & 1\end{array}$

$\begin{array}{lllllllllllll}1.0813 & -1.6050 & -0.1073 & \mathrm{H} & 0 & 0 & 0 & 0 & 0 & 0 & 0 & 0 & 0\end{array}$

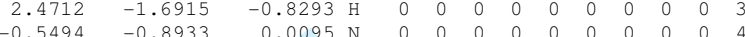

$\begin{array}{lllllllllllll}-0.9866 & -1.0649 & 1.4052 & \mathrm{C} & 0 & 0 & 0 & 0 & 0 & 0 & 0 & 0 & 0\end{array}$

$\begin{array}{lllllllllllll}-1.3050 & -1.8098 & -0.8639 & \mathrm{C} & 0 & 0 & 0 & 0 & 0 & 0 & 0 & 0 & 0\end{array}$

$\begin{array}{llllllllllllll}-0.7472 & 0.4978 & -0.4607 & \mathrm{C} & 0 & 0 & 0 & 0 & 0 & 0 & 0 & 0 & 0 & 7\end{array}$

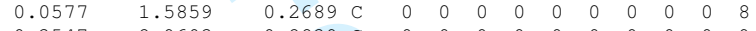

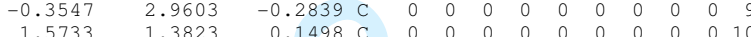

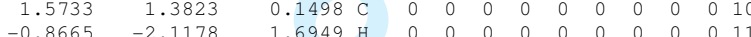

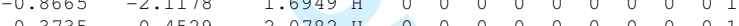

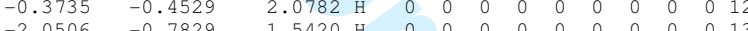

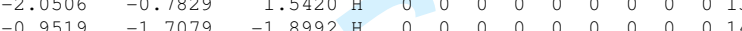

$\begin{array}{llllllllllllll}-0.9519 & -1.7079 & -1.8992 & \mathrm{H} & 0 & 0 & 0 & 0 & 0 & 0 & 0 & 0 & 0 & 14\end{array}$

$\begin{array}{llllllllllllll}-2.3936 & -1.6005 & -0.8407 & \mathrm{H} & 0 & 0 & 0 & 0 & 0 & 0 & 0 & 0 & 0 & 15\end{array}$

$\begin{array}{llllllllllllll}-1.1426 & -2.8465 & -0.5389 & \mathrm{H} & 0 & 0 & 0 & 0 & 0 & 0 & 0 & 0 & 0 & 16\end{array}$

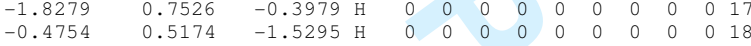

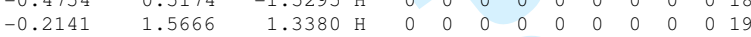

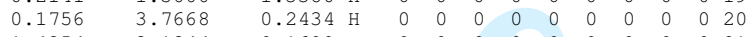

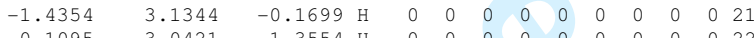

$\begin{array}{llllllllllllll}2.1150 & 3.0421 & -1.3554 & \mathrm{H} & 0 & 0 & 0 & 0 & 0 & 0 & 0 & 0 & 0 & 22\end{array}$

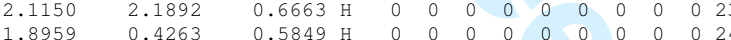

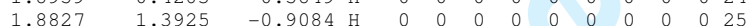

$\begin{array}{llllllll}2 & 1 & 0 & 0 & 0 & 0\end{array}$

$\begin{array}{lllllll}3 & 1 & 0 & 0 & 0 & 0\end{array}$

$\begin{array}{llllll}4 & 0 & 0 & 0 & 0\end{array}$

$\begin{array}{ccccc}4 & 0 & 0 & 0 & 0\end{array}$

$\begin{array}{lllllll}1 & 5 & 0 & 0 & 0 & 0\end{array}$

$\begin{array}{lllllllll}12 & 5 & 0 & 0 & 0 & 0\end{array}$

$\begin{array}{llllll}3 & 5 & 0 & 0 & 0\end{array}$

$\begin{array}{llllllll}15 & 6 & 0 & 0 & 0 & 0\end{array}$

$\begin{array}{lllllll}1 & 6 & 6 & 0 & 0 & 0 & 0\end{array}$

$8 \begin{array}{llllll}8 & 0 & 0 & 0\end{array}$

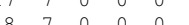

$\begin{array}{lllll}8 & 0 & 0 & 0\end{array}$

$\begin{array}{llllll}9 & 8 & 0 & 0 & 0 & 0\end{array}$

$\begin{array}{lllllll}20 & 9 & 0 & 0 & 0 & 0\end{array}$

$\begin{array}{lllllll}21 & 9 & 0 & 0 & 0 & 0\end{array}$

$\begin{array}{llllll}3 & 10 & 0 & 0 & 0 & 0\end{array}$

$2410 \quad 000100$

1025

$\$ \$ \$$

dimethyl-iso-propylamine-cation.sd

cosmotherm

$\begin{array}{llllllllllll}23 & 21 & 0 & 0 & 0 & 0 & 0 & 0 & 0 & 0 & 0 & \text { V2 } 2000\end{array}$

$\begin{array}{rrrrrrrrrrrrrr}-1.9862 & 1.6111 & 0.0008 & 0 & 0 & 0 & 0 & 0 & 0 & 0 & 0 & 0 & 0 & 1 \\ -2.4674 & 1.7752 & 0.8322 & \mathrm{H} & 0 & 0 & 0 & 0 & 0 & 0 & 0 & 0 & 0 & 2 \\ -2.6824 & 1.5866 & -0.6809 & \mathrm{H} & 0 & 0 & 0 & 0 & 0 & 0 & 0 & 0 & 0 & 3 \\ -0.2710 & -0.5930 & 0.0024 & \mathrm{~N} & 0 & 0 & 0 & 0 & 0 & 0 & 0 & 0 & 0 & 4 \\ -0.6014 & -1.3877 & -1.2267 & \mathrm{C} & 0 & 0 & 0 & 0 & 0 & 0 & 0 & 0 & 0 & 5 \\ -0.5846 & -1.4046 & 1.2253 & \mathrm{C} & 0 & 0 & 0 & 0 & 0 & 0 & 0 & 0 & 0 & 6 \\ 1.1756 & -0.0694 & -0.0037 & \mathrm{C} & 0 & 0 & 0 & 0 & 0 & 0 & 0 & 0 & 0 & 7 \\ 1.4524 & 0.7491 & 1.2573 & \mathrm{C} & 0 & 0 & 0 & 0 & 0 & 0 & 0 & 0 & 0 & 8 \\ 1.4431 & 0.7500 & -1.2662 & \mathrm{C} & 0 & 0 & 0 & 0 & 0 & 0 & 0 & 0 & 0 & 9 \\ -1.6236 & -1.7659 & -1.1269 & \mathrm{H} & 0 & 0 & 0 & 0 & 0 & 0 & 0 & 0 & 0 & 10 \\ -0.5337 & -0.7484 & -2.1099 & \mathrm{H} & 0 & 0 & 0 & 0 & 0 & 0 & 0 & 0 & 0 & 11 \\ 0.1011 & -2.2249 & -1.3067 & \mathrm{H} & 0 & 0 & 0 & 0 & 0 & 0 & 0 & 0 & 0 & 12 \\ -1.6085 & -1.7804 & 1.1340 & \mathrm{H} & 0 & 0 & 0 & 0 & 0 & 0 & 0 & 0 & 0 & 13 \\ -0.5041 & -0.7779 & 2.1164 & \mathrm{H} & 0 & 0 & 0 & 0 & 0 & 0 & 0 & 0 & 0 & 14 \\ 0.1186 & -2.2429 & 1.2834 & \mathrm{H} & 0 & 0 & 0 & 0 & 0 & 0 & 0 & 0 & 0 & 15 \\ 1.7955 & -0.9780 & -0.0060 & \mathrm{H} & 0 & 0 & 0 & 0 & 0 & 0 & 0 & 0 & 0 & 16 \\ 2.4716 & 1.1510 & 1.1878 & \mathrm{H} & 0 & 0 & 0 & 0 & 0 & 0 & 0 & 0 & 0 & 17 \\ 1.3991 & 0.1529 & 2.1759 & \mathrm{H} & 0 & 0 & 0 & 0 & 0 & 0 & 0 & 0 & 0 & 18 \\ 0.7618 & 1.6013 & 1.3407 & \mathrm{H} & 0 & 0 & 0 & 0 & 0 & 0 & 0 & 0 & 0 & 19 \\ 2.4605 & 1.1572 & -1.2007 & \mathrm{H} & 0 & 0 & 0 & 0 & 0 & 0 & 0 & 0 & 0 & 20 \\ 1.3895 & 0.1525 & -2.1838 & \mathrm{H} & 0 & 0 & 0 & 0 & 0 & 0 & 0 & 0 & 0 & 21 \\ 0.7470 & 1.5979 & -1.3467 & \mathrm{H} & 0 & 0 & 0 & 0 & 0 & 0 & 0 & 0 & 0 & 22 \\ -0.9135 & 0.2489 & 0.0117 & \mathrm{H} & 0 & 0 & 0 & 0 & 0 & 0 & 0 & 0 & 0 & 23\end{array}$

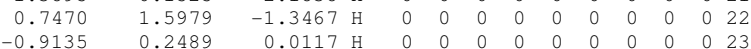

$\begin{array}{llllll}2 & 1 & 0 & 0 & 0 & 0\end{array}$

$\begin{array}{llllll}1 & 0 & 0 & 0 & 0\end{array}$

$\begin{array}{llllll}5 & 4 & 0 & 0 & 0 & 0 \\ 6 & 4 & 0 & 0 & 0 & 0\end{array}$

$\begin{array}{llll}4 & 0 & 0 & 0\end{array}$

$\begin{array}{llllll}12 & 5 & 0 & 0 & 0 & 0\end{array}$

$\begin{array}{lllll}6 & 0 & 0 & 0 & 0\end{array}$

$\begin{array}{llllll}4 & 6 & 0 & 0 & 0 \\ 1 & 6 & 0 & 0 & 0\end{array}$ 


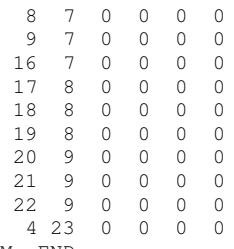




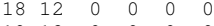

$\begin{array}{llllll}19 & 12 & 0 & 0 & 0 & 0 \\ 20 & 17 & 0 & 0 & 0 & 0\end{array}$

$\begin{array}{lllllll}21 & 17 & 0 & 0 & 0 & 0\end{array}$

$\begin{array}{llllll}22 & 17 & 0 & 0 & 0 & 0 \\ 23 & 20 & 0 & 0 & 0 & 0\end{array}$

$\begin{array}{lllllll}24 & 20 & 0 & 0 & 0 & 0\end{array}$

$\begin{array}{llllll}25 & 20 & 0 & 0 & 0 & 0\end{array}$

526

$M$ END

dimethyl-n-butylamine.sdf

cosmotherm

$\begin{array}{llllllllllll}25 & 23 & 0 & 0 & 0 & 0 & 0 & 0 & 0 & 0 & 0 & \mathrm{~V} 2000\end{array}$

$\begin{array}{lllllllllllll}-2.3702 & -0.1655 & 1.7031 & 0 & 0 & 0 & 0 & 0 & 0 & 0 & 0 & 0 & 0\end{array}$

$\begin{array}{llllllllllllll}-1.3704 & -0.0821 & 1.5374 & \mathrm{H} & 0 & 0 & 0 & 0 & 0 & 0 & 0 & 0 & 0 & 2\end{array}$

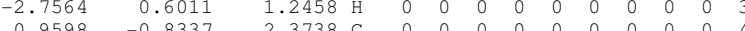

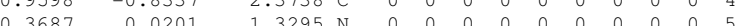

$\begin{array}{llllllllllllll}0.7571 & -0.4744 & -0.0116 & \mathrm{C} & 0 & 0 & 0 & 0 & 0 & 0 & 0 & 0 & 0 & 6\end{array}$

$\begin{array}{lllllllllllll}0.7726 & 1.4211 & 1.5393 & \mathrm{C} & 0 & 0 & 0 & 0 & 0 & 0 & 0 & 0 & 0\end{array}$

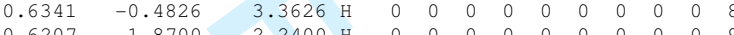

$\begin{array}{llllllllllllll}0.6207 & -1.8700 & 2.2400 & \mathrm{H} & 0 & 0 & 0 & 0 & 0 & 0 & 0 & 0 & 0 & 9\end{array}$

$\begin{array}{lllllllllllll}2.0680 & -0.8193 & 2.3443 \mathrm{H} & 0 & 0 & 0 & 0 & 0 & 0 & 0 & 0 & 0 & 10\end{array}$

$\begin{array}{llllllllllllll}0.5454 & -1.5555 & -0.0264 & \mathrm{H} & 0 & 0 & 0 & 0 & 0 & 0 & 0 & 0 & 0 & 1\end{array}$

$\begin{array}{llllllllllllll}0.0089 & 0.1931 & -1.1678 & \mathrm{C} & 0 & 0 & 0 & 0 & 0 & 0 & 0 & 0 & 0 & 12\end{array}$

$\begin{array}{llllllllllllll}1.8535 & -0.3580 & -0.1543 & \mathrm{H} & 0 & 0 & 0 & 0 & 0 & 0 & 0 & 0 & 0 & 13\end{array}$

$\begin{array}{lllllllllllll}0.4707 & 1.7375 & 2.5468 \mathrm{H} & 0 & 0 & 0 & 0 & 0 & 0 & 0 & 0 & 0 & 14\end{array}$

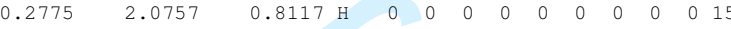

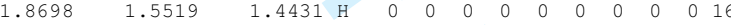

$\begin{array}{llllllllllllll}0.3454 & -0.4476 & -2.5216 & \mathrm{C} & 0 & 0 & 0 & 0 & 0 & 0 & 0 & 0 & 0 & 17\end{array}$

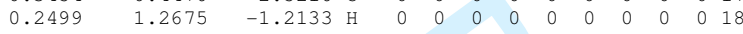

$\begin{array}{llllllllllllll}-1.0762 & 0.1163 & -0.9835 & \mathrm{H} & 0 & 0 & 0 & 0 & 0 & 0 & 0 & 0 & 0 & 19\end{array}$

$\begin{array}{llllllllllllll}-0.3842 & 0.2175 & -3.6941 & \mathrm{C} & 0 & 0 & 0 & 0 & 0 & 0 & 0 & 0 & 0 & 20\end{array}$

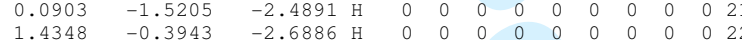

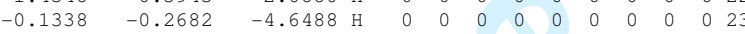

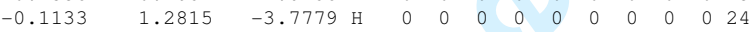

$\begin{array}{llllllllllll}-1.4763 & 0.1606 & -3.5649 \mathrm{H} & 0 & 0 & 0 & 0 & 0 & 0 & 0 & 0 & 0\end{array}$

$\begin{array}{lllllll}2 & 1 & 0 & 0 & 0 & 0\end{array}$

$\begin{array}{lllllll}3 & 1 & 0 & 0 & 0 & 0\end{array}$

$5 \quad 4 \quad 000000$

$\begin{array}{llllll}4 & 0 & 0 & 0 & 0 & 0\end{array}$

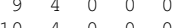

$\begin{array}{lllll}6 & 5 & 0 & 0 & 0\end{array}$

110600000

$\begin{array}{lllllllll}1 & 6 & 0 & 0 & 0 & 0\end{array}$

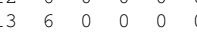

$\begin{array}{lllll}4 & 7 & 0 & 0 & 0\end{array}$

$\begin{array}{llllllll}16 & 7 & 0 & 0 & 0 & 0\end{array}$

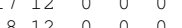

$\begin{array}{lllllllll}18 & 12 & 0 & 0 & 0 & 0 & 0\end{array}$

$\begin{array}{llllllll}19 & 12 & 0 & 0 & 0 & 0 & 0 \\ 17 & 0 & 0 & 0\end{array}$

$\begin{array}{llllll}1 & 17 & 0 & 0 & 0\end{array}$

$2 \begin{array}{llllll}2 & 17 & 0 & 0 & 0 & 0\end{array}$

23201000000

$\begin{array}{lllllll}24 & 20 & 0 & 0 & 0 & 0 \\ 20 & 25 & 0 & 0 & 0 & 0\end{array}$

END

dimethyl-n-propylamine-cation.sdf

Cosmotherm

$\begin{array}{llllllllllll}23 & 21 & 0 & 0 & 0 & 0 & 0 & 0 & 0 & 0 & 0 & \mathrm{~V} 2000\end{array}$

$\begin{array}{llllllllllllllllllll}2.6354 & 1.6268 & 0.7908 & & 0 & 0 & 0 & 0 & 0 & 0 & 0 & 0 & 0 & 0 & 3\end{array}$

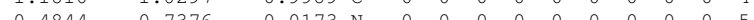

$\begin{array}{lllllllllllll}-0.4844 & -0.73763 & -0.0173 & \mathrm{~N} & 0 & 0 & 0 & 0 & 0 & 0 & 0 & 0 & 0 \\ -0.5481 & -0.7063 & -0.4675 \mathrm{C} & 0 & 0 & 0 & 0 & 0 & 0 & 0 & 0 & 0 & 6\end{array}$

$\begin{array}{lllllllllllll}-1.0234 & 0.9421 & -1.3652 & \mathrm{C} & 0 & 0 & 0 & 0 & 0 & 0 & 0 & 0 & 0\end{array}$

$\begin{array}{llllllllllllll}-1.0234 & . .6421 & 1.3652 & \mathrm{C} & 0 & 0 & 0 & 0 & 0 & 0 & 0 & 0 & 0 & 0 \\ -1.0696 & 2.6681 & -0.6706 & \mathrm{H} & 0 & 0 & 0 & 0 & 0 & 0 & 0 & 0 & 0 & 8\end{array}$

$\begin{array}{llllllllllllll}-1.0696 & 2.6681 & -0.6706 & \mathrm{H} & 0 & 0 & 0 & 0 & 0 & 0 & 0 & 0 & 0 & 8 \\ -0.7255 & 1.4949 & -1.9848 & \mathrm{H} & 0 & 0 & 0 & 0 & 0 & 0 & 0 & 0 & 0 & 9\end{array}$

$\begin{array}{lllllllllllll}-2.2423 & 1.3578 & -1.0324 \mathrm{H} & 0 & 0 & 0 & 0 & 0 & 0 & 0 & 0 & 0 & 10\end{array}$

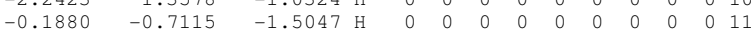

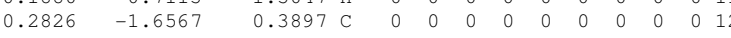

$\begin{array}{llllllllllllll}-1.6108 & -0.9865 & -0.4709 & \mathrm{H} & 0 & 0 & 0 & 0 & 0 & 0 & 0 & 0 & 0 & 13\end{array}$

$\begin{array}{lllllllllllll}-0.9567 & 2.0067 & 1.6097 \mathrm{H} & 0 & 0 & 0 & 0 & 0 & 0 & 0 & 0 & 0 & 14 \\ -0.4310 & 0.3620 & 2.0781 \mathrm{H} & 0 & 0 & 0 & 0 & 0 & 0 & 0 & 0 & 0 & 15\end{array}$

$\begin{array}{llllllllllllll}-0.4310 & 0.3620 & 2.0781 & \mathrm{H} & 0 & 0 & 0 & 0 & 0 & 0 & 0 & 0 & 0 & 15 \\ -2.0695 & 0.6162 & 1.3875 & \mathrm{H} & 0 & 0 & 0 & 0 & 0 & 0 & 0 & 0 & 0 & 16\end{array}$

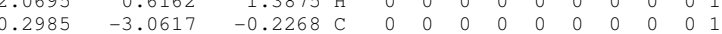

$\begin{array}{llllllllllllll}-0.1270 & -1.7123 & 1.4085 & \mathrm{H} & 0 & 0 & 0 & 0 & 0 & 0 & 0 & 0 & 0 & 18\end{array}$

$\begin{array}{lllllllllllll}1.3128 & -1.2751 & 0.4715 \mathrm{H} & 0 & 0 & 0 & 0 & 0 & 0 & 0 & 0 & 0 & 19\end{array}$

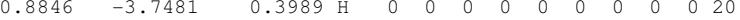

-1.2309 H $\begin{array}{llllllllllllllllll}0 & 0 & 0 & 0 & 0 & 0 & 0 & 0 & 0 & 0 & 21\end{array}$

$\begin{array}{llllllllllllll}.7194 & -3.4699 & -0.3121 & \mathrm{H} & 0 & 0 & 0 & 0 & 0 & 0 & 0 & 0 & 0 & 22\end{array}$

1.025

$\begin{array}{lllllll}1 & 0 & 0 & 0 & 0\end{array}$

$5 \begin{array}{llllll}5 & 4 & 0 & 0 & 0 & 0\end{array}$

$\begin{array}{lllll}4 & 0 & 0 & 0 & 0\end{array}$

$\begin{array}{llllll}0 & 4 & 0 & 0 & 0 & 0 \\ 0 & 4 & 0 & 0 & 0 & 0\end{array}$

$\begin{array}{llllll}5 & 0 & 0 & 0 & 0\end{array}$

$\begin{array}{llllll}6 & 0 & 0 & 0 & 0\end{array}$

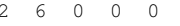

$\begin{array}{llllll}6 & 0 & 0 & 0 & 0\end{array}$

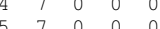




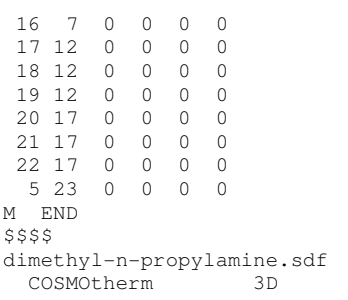

$\begin{array}{rrrrrrrrrrrrrrr}22 & 20 & 0 & 0 & 0 & 0 & 0 & 0 & 0 & 0 & V 2000 & & & & 0 \\ -2.1982 & -0.1277 & 1.5878 & 0 & 0 & 0 & 0 & 0 & 0 & 0 & 0 & 0 & 0 & 1 \\ -1.2380 & -0.0725 & 1.2573 & \mathrm{H} & 0 & 0 & 0 & 0 & 0 & 0 & 0 & 0 & 0 & 2 \\ -2.5764 & 0.7431 & 1.3757 & \mathrm{H} & 0 & 0 & 0 & 0 & 0 & 0 & 0 & 0 & 0 & 3 \\ 1.1621 & -0.9576 & 1.6330 & \mathrm{C} & 0 & 0 & 0 & 0 & 0 & 0 & 0 & 0 & 0 & 4 \\ 0.4427 & -0.0151 & 0.7600 & \mathrm{~N} & 0 & 0 & 0 & 0 & 0 & 0 & 0 & 0 & 0 & 5 \\ 0.5793 & -0.4269 & -0.6557 & \mathrm{C} & 0 & 0 & 0 & 0 & 0 & 0 & 0 & 0 & 0 & 6 \\ 0.9339 & 1.3542 & 0.9863 & \mathrm{C} & 0 & 0 & 0 & 0 & 0 & 0 & 0 & 0 & 0 & 7 \\ 1.0167 & -0.6706 & 2.6835 & \mathrm{H} & 0 & 0 & 0 & 0 & 0 & 0 & 0 & 0 & 0 & 8 \\ 0.7653 & -1.9717 & 1.4889 & \mathrm{H} & 0 & 0 & 0 & 0 & 0 & 0 & 0 & 0 & 0 & 9 \\ 2.2507 & -0.9721 & 1.4224 & \mathrm{H} & 0 & 0 & 0 & 0 & 0 & 0 & 0 & 0 & 0 & 10 \\ 0.3213 & -1.4967 & -0.7033 & \mathrm{H} & 0 & 0 & 0 & 0 & 0 & 0 & 0 & 0 & 0 & 11 \\ -0.3207 & 0.3443 & -1.6245 & \mathrm{C} & 0 & 0 & 0 & 0 & 0 & 0 & 0 & 0 & 0 & 12 \\ 1.6401 & -0.3366 & -0.9765 & \mathrm{H} & 0 & 0 & 0 & 0 & 0 & 0 & 0 & 0 & 0 & 13 \\ 0.8243 & 1.6081 & 2.0494 & \mathrm{H} & 0 & 0 & 0 & 0 & 0 & 0 & 0 & 0 & 0 & 14 \\ 0.3483 & 2.0739 & 0.4010 & \mathrm{H} & 0 & 0 & 0 & 0 & 0 & 0 & 0 & 0 & 0 & 15 \\ 2.0022 & 1.4601 & 0.7081 & \mathrm{H} & 0 & 0 & 0 & 0 & 0 & 0 & 0 & 0 & 0 & 16 \\ -0.2239 & -0.2101 & -3.0505 & \mathrm{C} & 0 & 0 & 0 & 0 & 0 & 0 & 0 & 0 & 0 & 17 \\ -0.0479 & 1.4109 & -1.6326 & \mathrm{H} & 0 & 0 & 0 & 0 & 0 & 0 & 0 & 0 & 0 & 18 \\ -1.3614 & 0.2833 & -1.2664 & \mathrm{H} & 0 & 0 & 0 & 0 & 0 & 0 & 0 & 0 & 0 & 19 \\ -0.8709 & 0.3535 & -3.7378 & \mathrm{H} & 0 & 0 & 0 & 0 & 0 & 0 & 0 & 0 & 0 & 20 \\ -0.5310 & -1.2666 & -3.0896 & \mathrm{H} & 0 & 0 & 0 & 0 & 0 & 0 & 0 & 0 & 0 & 21 \\ 0.8063 & -0.1458 & -3.4341 & \mathrm{H} & 0 & 0 & 0 & 0 & 0 & 0 & 0 & 0 & 0 & 22\end{array}$




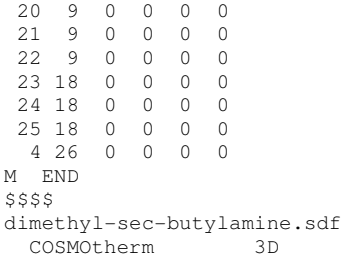




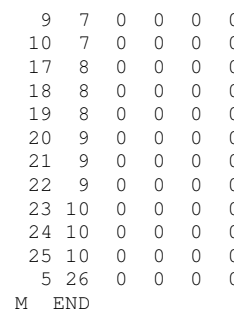




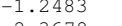

$\begin{array}{rr}-1.2483 & -0.1319 \\ -2.3678 & 0.9792\end{array}$

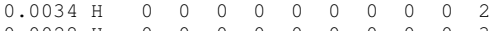

$\begin{array}{llllllllllllll}1.0521 & 0.1655 & -1.2258 & \mathrm{C} & 0 & 0 & 0 & 0 & 0 & 0 & 0 & 0 & 0 & 4\end{array}$

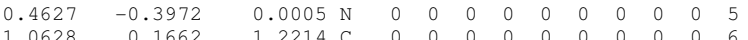

$\begin{array}{llllllllllllll}0.6182 & -0.3312 & -2.1037 & \mathrm{H} & 0 & 0 & 0 & 0 & 0 & 0 & 0 & 0 & 0 & 7\end{array}$

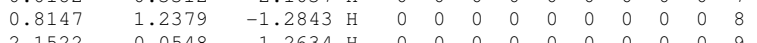

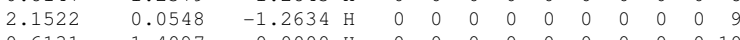

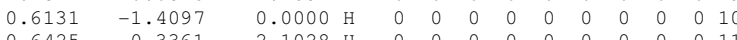

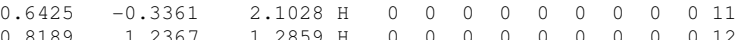

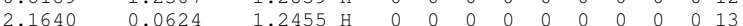

$\begin{array}{lllllll}2 & 1 & 0 & 0 & 0 & 0\end{array}$

$\begin{array}{lllllll}3 & 1 & 0 & 0 & 0 & 0\end{array}$

$5 \quad 4 \quad 0 \quad 0000$

$\begin{array}{lllll}4 & 0 & 0 & 0 \\ 4 & 0 & 0 & 0\end{array}$

$\begin{array}{llllll}4 & 0 & 0 & 0 & 0\end{array}$

$\begin{array}{llllll}5 & 0 & 0 & 0 & 0\end{array}$

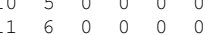

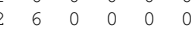

6
13
END

MS\$

dimethylethylamine-cation.sdf

cosmotherm 3D

$\begin{array}{lllllllllllll}20 & 18 & 0 & 0 & 0 & 0 & 0 & 0 & 0 & 0 & 0 & \mathrm{v} 2000\end{array}$

$\begin{array}{llllllllllllll}-2.2949 & -0.0076 & -0.9361 & 0 & 0 & 0 & 0 & 0 & 0 & 0 & 0 & 0 & 0 & 1\end{array}$

$\begin{array}{llllllllllllll}-2.4286 & -0.7089 & -1.5993 & \mathrm{H} & 0 & 0 & 0 & 0 & 0 & 0 & 0 & 0 & 0 & 2\end{array}$

$\begin{array}{llllllllllllll}-2.5544 & 0.8139 & -1.3916 & \mathrm{H} & 0 & 0 & 0 & 0 & 0 & 0 & 0 & 0 & 0 & 3\end{array}$

$\begin{array}{llllllllllllll}-0.2441 & -0.7953 & 1.7321 & \mathrm{C} & 0 & 0 & 0 & 0 & 0 & 0 & 0 & 0 & 0 & 4\end{array}$

$\begin{array}{llllllllllllll}0.0790 & 0.0004 & 0.5050 & \mathrm{~N} & 0 & 0 & 0 & 0 & 0 & 0 & 0 & 0 & 0 & 5\end{array}$

$\begin{array}{rrrrrrrrrrrrrr}1.1884 & -0.6631 & -0.2861 & \mathrm{C} & 0 & 0 & 0 & 0 & 0 & 0 & 0 & 0 & 0 & 6 \\ 0.3866 & 1.4231 & 0.8574 & \mathrm{C} & 0 & 0 & 0 & 0 & 0 & 0 & 0 & 0 & 0 & 7\end{array}$

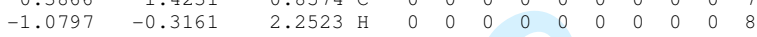

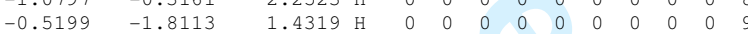

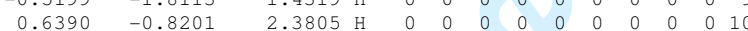

$\begin{array}{lllllllllllll}0.8730 & -1.7054 & -0.4201 \mathrm{H} & 0 & 0 & 0 & 0 & 0 & 0 & 0 & 0 & 0 & 11\end{array}$

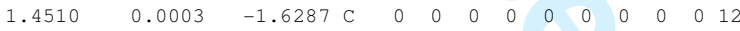

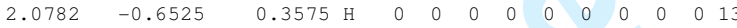

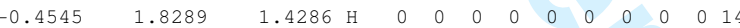

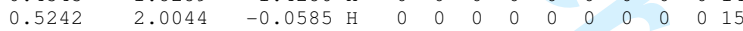

$\begin{array}{lllllllllllll}1.2996 & 1.4463 & 1.4627 \mathrm{H} & 0 & 0 & 0 & 0 & 0 & 0 & 0 & 0 & 0 & 16\end{array}$

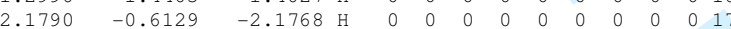

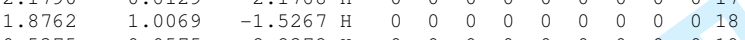

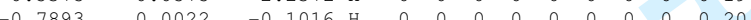

$\begin{array}{llllll}1 & 0 & 0 & 0 & 0\end{array}$

$\begin{array}{llllll}1 & 0 & 0 & 0 & 0\end{array}$

$\begin{array}{llllllll}1 & 0 & 0 & 0 & 0 & 0 & 0\end{array}$

$\begin{array}{lllll}4 & 0 & 0 & 0 & 0\end{array}$

$\begin{array}{lllllll}0 & 4 & 0 & 0 & 0 & 0 & 0 \\ 0 & 4 & 0 & 0 & 0 & 0\end{array}$

$\begin{array}{lllll}5 & 0 & 0 & 0 \\ 5 & 0 & 0 & 0\end{array}$

$\begin{array}{lllllllll}7 & 5 & 0 & 0 & 0 & 0 & 2 & 2 \\ 1 & 6 & 0 & 0 & 0 & 0\end{array}$

$\begin{array}{llllll}1 & 6 & 0 & 0 & 0 & 0 \\ 1 & 0 & 0 & 0\end{array}$

$\begin{array}{llllll}3 & 6 & 0 & 0 & 0 & 0\end{array}$

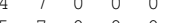

$\begin{array}{llllllll}1 & 7 & 0 & 0 & 0 & 0\end{array}$

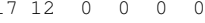

$\begin{array}{llllll}8 & 12 & 0 & 0 & 0 & 0\end{array}$

END

dimethylethylamine, sdf

Cosmotherm

$\begin{array}{llllllllllll}19 & 17 & 0 & 0 & 0 & 0 & 0 & 0 & 0 & 0 & 0 & \mathrm{~V} 2000\end{array}$

$\begin{array}{llllllllllllll}-2.2735 & 1.0303 & -0.0564 & 0 & 0 & 0 & 0 & 0 & 0 & 0 & 0 & 0 & 0 & 1 \\ -1.4097 & 0.4953 & -0.0195 & \mathrm{H} & 0 & 0 & 0 & 0 & 0 & 0 & 0 & 0 & 0 & 2\end{array}$

$\begin{array}{llllllllllllll}-1.2147 & 1.6389 & 0.7000 & \mathrm{H} & 0 & 0 & 0 & 0 & 0 & 0 & 0 & 0 & 0 & 3\end{array}$

$\begin{array}{llllllllllllll}-0.2761 & -1.7040 & -0.7762 & \mathrm{C} & 0 & 0 & 0 & 0 & 0 & 0 & 0 & 0 & 0 & 4\end{array}$

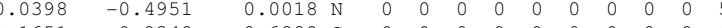

$\begin{array}{rrrrrrrrrrrrrr}1.1651 & 0.2342 & -0.6282 & \mathrm{C} & 0 & 0 & 0 & 0 & 0 & 0 & 0 & 0 & 0 & 6 \\ 0.3308 & -0.8575 & 1.3986 & \mathrm{C} & 0 & 0 & 0 & 0 & 0 & 0 & 0 & 0 & 0 & 7\end{array}$

$\begin{array}{lllllllllllllll}-1.1374 & -2.2165 & -0.3261 & \mathrm{H} & 0 & 0 & 0 & 0 & 0 & 0 & 0 & 0 & 0 & 8\end{array}$

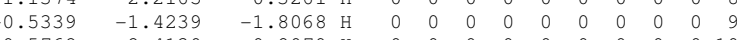

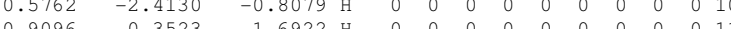

$\begin{array}{llllllllllllll}0.9096 & 0.3523 & -1.6922 & \mathrm{H} & 0 & 0 & 0 & 0 & 0 & 0 & 0 & 0 & 0 & 1.1\end{array}$

$\begin{array}{rrrrrrrrrrrrrr}1.4284 & 1.6088 & -0.0174 & \mathrm{C} & 0 & 0 & 0 & 0 & 0 & 0 & 0 & 0 & 0 & 12 \\ 2.0861 & -0.3849 & -0.5839 & \mathrm{H} & 0 & 0 & 0 & 0 & 0 & 0 & 0 & 0 & 0 & 13\end{array}$

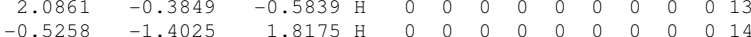

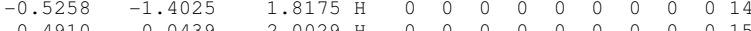

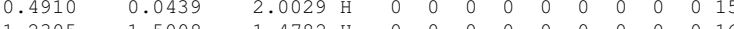

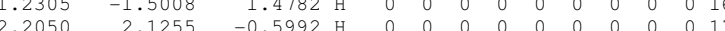

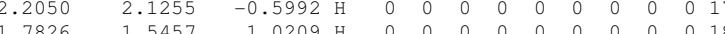

$\begin{array}{lllllllllllll}0.5192 & 2.2271 & -0.0380 & \mathrm{H} & 0 & 0 & 0 & 0 & 0 & 0 & 0 & 0 & 0\end{array}$

$\begin{array}{lllllll}2 & 1 & 0 & 0 & 0 & 0\end{array}$

$\begin{array}{llllll}1 & 0 & 0 & 0 & 0\end{array}$

$\begin{array}{lllllll}4 & 0 & 0 & 0 & 0\end{array}$

$\begin{array}{lllll}4 & 0 & 0 & 0\end{array}$

$\begin{array}{llllll}4 & 0 & 0 & 0 & 0\end{array}$

$\begin{array}{lllll}5 & 0 & 0 & 0 & 0 \\ 5 & 0 & 0 & 0\end{array}$

$\begin{array}{llllll}1 & 6 & 0 & 0 & 0 & 0\end{array}$

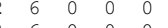

$\begin{array}{lllllll}4 & 7 & 0 & 0 & 0 & 0\end{array}$

$\begin{array}{lllllll}15 & 7 & 0 & 0 & 0 & 0\end{array}$ 


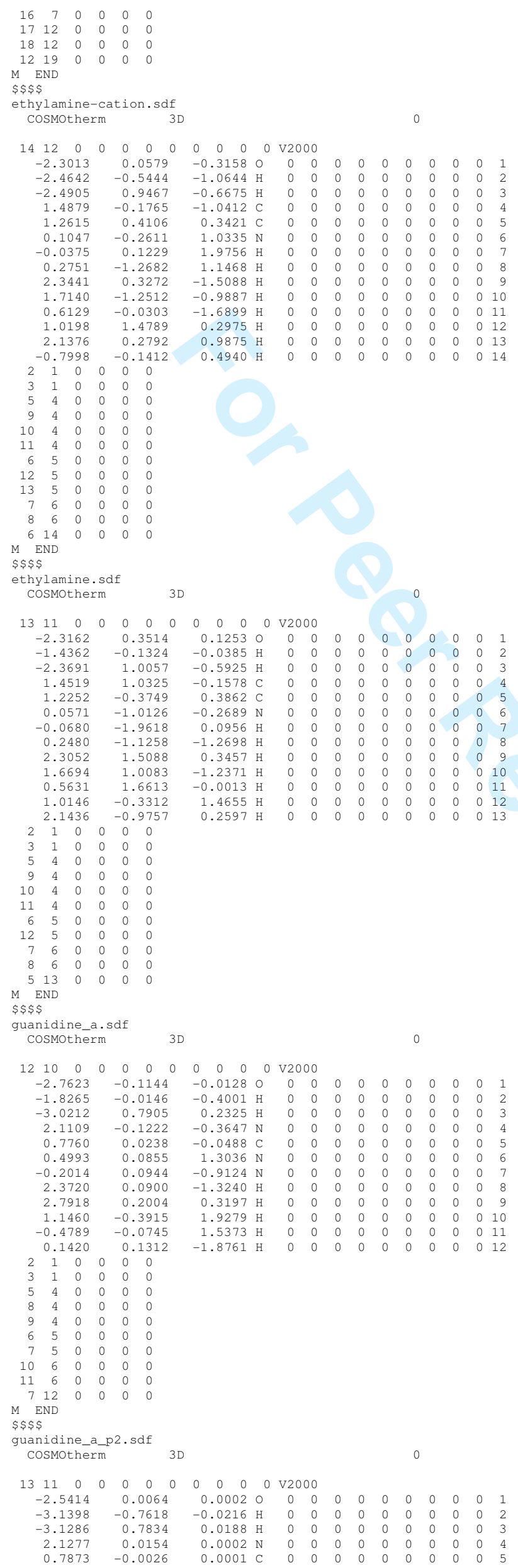


$\begin{array}{llllllllllllll}0.1081 & -0.0044 & 1.1543 & \mathrm{~N} & 0 & 0 & 0 & 0 & 0 & 0 & 0 & 0 & 0 & 6\end{array}$ $\begin{array}{llllllllllllll}0.1086 & -0.0150 & -1.1547 & \mathrm{~N} & 0 & 0 & 0 & 0 & 0 & 0 & 0 & 0 & 0 & 7 \\ 2.6505 & -0.0184 & -0.8679 & \mathrm{H} & 0 & 0 & 0 & 0 & 0 & 0 & 0 & 0 & 0 & 8\end{array}$ $\begin{array}{llllllllllllll}2.6507 & -0.0172 & 0.8682 & \mathrm{H} & 0 & 0 & 0 & 0 & 0 & 0 & 0 & 0 & 0 & 9\end{array}$

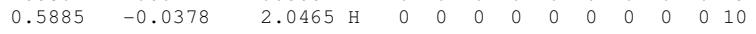

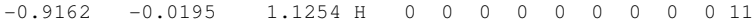

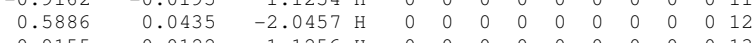

$\begin{array}{llllll}2 & 1 & 0 & 0 & 0 & 0\end{array}$

$\begin{array}{lllllll}3 & 1 & 0 & 0 & 0 & 0\end{array}$

$\begin{array}{llllll}4 & 0 & 0 & 0 & 0\end{array}$

$\begin{array}{llllll}4 & 0 & 0 & 0 & 0\end{array}$

$\begin{array}{lllll}5 & 0 & 0 & 0 & 0\end{array}$

11600000

$\begin{array}{llllllll}13 & 0 & 0 & 0 & 0\end{array}$

M END

hydroxyimidazo $(2,3-a)$ isoindol_e-cation.sdf

$\begin{array}{llllllllllll}30 & 31 & 0 & 0 & 0 & 0 & 0 & 0 & 0 & 0 & 0 & \mathrm{v} 2000\end{array}$

$\begin{array}{lllllllllllll}-3.1366 & -2.0486 & 0.0671 & 0 & 0 & 0 & 0 & 0 & 0 & 0 & 0 & 0 & 0\end{array}$

$\begin{array}{lllllllllllll}-3.6931 & -2.3306 & 0.8158 & \mathrm{H} & 0 & 0 & 0 & 0 & 0 & 0 & 0 & 0 & 0\end{array}$

$\begin{array}{llllllllllllll}-3.6503 & -2.2579 & -0.7342 & \mathrm{H} & 0 & 0 & 0 & 0 & 0 & 0 & 0 & 0 & 0 & 3\end{array}$

$\begin{array}{llllllllllllll}0.6687 & 4.2929 & -0.1892 & \mathrm{C} & 0 & 0 & 0 & 0 & 0 & 0 & 0 & 0 & 0 & 4 \\ 1.7742 & 3.4033 & -0.1318 & \mathrm{C} & 0 & 0 & 0 & 0 & 0 & 0 & 0 & 0 & 0 & 5\end{array}$

$\begin{array}{llllllllllllll}1.7742 & 3.4033 & -0.1318 & \mathrm{C} & 0 & 0 & 0 & 0 & 0 & 0 & 0 & 0 & 0 & 5 \\ 1.6049 & 2.0299 & -0.0265 & \mathrm{C} & 0 & 0 & 0 & 0 & 0 & 0 & 0 & 0 & 0 & 6\end{array}$

$\begin{array}{lllllllllllll}0.2910 & 1.5087 & 0.0270 & \mathrm{C} & 0 & 0 & 0 & 0 & 0 & 0 & 0 & 0 & 0\end{array}$

$\begin{array}{llllllllllllll}-0.8296 & 2.4186 & -0.0396 & \mathrm{C} & 0 & 0 & 0 & 0 & 0 & 0 & 0 & 0 & 0 & 8\end{array}$

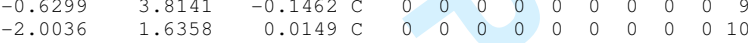

$\begin{array}{llllllllllllll}-0.2599 & 0.1952 & 0.1254 & \mathrm{C} & 0 & 0 & 0 & 0 & 0 & 0 & 0 & 0 & 0 & 11\end{array}$

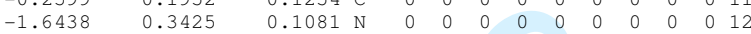

$\begin{array}{lllllllllllllll}0.3571 & -1.0786 & 0.2210 & \mathrm{C} & 0 & 0 & 0 & 0 & 0 & 0 & 0 & 0 & 0 & 13\end{array}$

$\begin{array}{llllllll}0.6580 & -1.07872 & 0.2210 & \mathrm{C} & 0 & 0 & 0 & 0 \\ 1.9872 & -2.6969 & 0.1815 & \mathrm{C} & 0 & 0 & 0 & 0 \\ -0.2869 & -2.2663 & 0.5260 & \mathrm{C} & 0 & 0 & 0 & 0\end{array}$

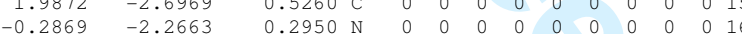

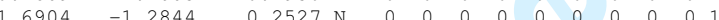

$\begin{array}{llllllllllllll}0.7310-3.9012 & -1.1463 & 0 & 0 & 0 & 0 & 0 & 0 & 0 & 0 & 0 & 0 & 0 & 18\end{array}$

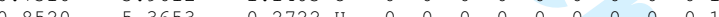

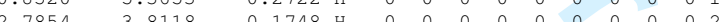

$\begin{array}{llllllllllllll}2.4876 & 1.3903 & -0.0011 \mathrm{H} & 0 & 0 & 0 & 0 & 0 & 0 & 0 & 0 & 0 & 21\end{array}$

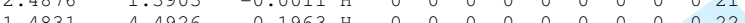

$\begin{array}{lllllllllllllllllll}1.4831 & 4.4926 & -0.1963 & \mathrm{H} & 0 & 0 & 0 & 0 & 0 & 0 & 0 & 0 & 0 & 22\end{array}$

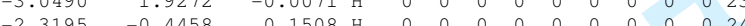

$\begin{array}{llllllllllllll}-2.3195 & -0.4458 & 0.1508 \mathrm{H} & 0 & 0 & 0 & 0 & 0 & 0 & 0 & 0 & 0 & 0 & 24\end{array}$

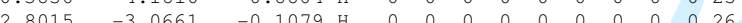

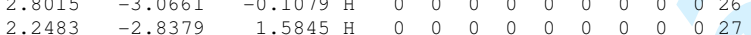

$\begin{array}{rrrrrrrrrrrrrr}2.2483 & -2.8379 & 1.5845 & \mathrm{H} & 0 & 0 & 0 & 0 & 0 & 0 & 0 & 0 & 0 & 27 \\ -1.3016 & -2.3757 & 0.1456 & \mathrm{H} & 0 & 0 & 0 & 0 & 0 & 0 & 0 & 0 & 0 & 28\end{array}$

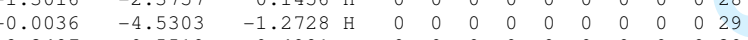

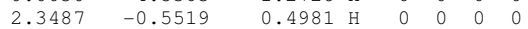

$\begin{array}{lllllll}2 & 1 & 0 & 0 & 0 & 0\end{array}$

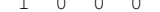

$\begin{array}{lllllll}2 & 4 & 0 & 0 & 0 & 0\end{array}$

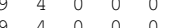

$6 \begin{array}{llllll}5 & 0 & 0 & 0 & 0 & 0\end{array}$

$\begin{array}{llllll}6 & 5 & 0 & 0 & 0 & 0\end{array}$

$\begin{array}{llllll}6 & 0 & 0 & 0 & 0\end{array}$

$\begin{array}{lllllll}8 & 7 & 0 & 0 & 0 & 0\end{array}$

$\begin{array}{llllll}1 & 7 & 0 & 0 & 0 & 0\end{array}$

$\begin{array}{llllllll}2 & 8 & 0 & 0 & 0 & 0 \\ 0 & 8 & 0 & 0 & 0 & 0 & 0 \\ 22 & 9 & 0 & 0 & 0 & 0\end{array}$

$\begin{array}{llllll}22 & 9 & 0 & 0 & 0 & 0\end{array}$

$\begin{array}{llllll}2 & 10 & 0 & 0 & 0 & 0\end{array}$

$\begin{array}{llllll}23 & 10 & 0 & 0 & 0\end{array}$

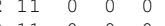

$\begin{array}{llllll}16 & 13 & 0 & 0 & 0\end{array}$

$\begin{array}{llllll}7 & 13 & 0 & 0 & 0\end{array}$

$\begin{array}{llllll}5 & 14 & 0 & 0 & 0 & 0\end{array}$

$\begin{array}{llllll}6 & 14 & 0 & 0 & 0 \\ 8 & 14 & 0 & 0 & 0\end{array}$

25140000

$\begin{array}{lllllll}7 & 15 & 0 & 0 & 0 & 0\end{array}$

$\begin{array}{lllllll}15 & 15 & 0 & 0 & 0 & 0\end{array}$

$\begin{array}{llllll}7 & 15 & 0 & 0 & 0 & 0\end{array}$

$\begin{array}{llllll}28 & 16 & 0 & 0 & 0 & 0\end{array}$

$\begin{array}{llllll}29 & 18 & 0 & 0 & 0 & 0 \\ 7 & 30 & 0 & 0 & 0 & 0\end{array}$

$\$ \$ \$$

hydroxyimidazo $(2,3-a)$ isoindol_eo.sdf

cosmotherm $3 \mathrm{D}$

$\begin{array}{llllllllllll}29 & 30 & 0 & 0 & 0 & 0 & 0 & 0 & 0 & 0 & 0 & \mathrm{~V} 2000\end{array}$

$\begin{array}{rrrrrrrrrrrrrr}-2.7912 & -0.6123 & -4.2850 & 0 & 0 & 0 & 0 & 0 & 0 & 0 & 0 & 0 & 0 & 1 \\ -3.2833 & -1.4150 & -4.0339 & \mathrm{H} & 0 & 0 & 0 & 0 & 0 & 0 & 0 & 0 & 0 & 2 \\ -2.8960 & -0.5582 & -5.2523 & \mathrm{H} & 0 & 0 & 0 & 0 & 0 & 0 & 0 & 0 & 0 & 3 \\ -1.7206 & 0.0858 & 3.9144 & \mathrm{C} & 0 & 0 & 0 & 0 & 0 & 0 & 0 & 0 & 0 & 4 \\ -2.2680 & 0.5198 & 2.6715 & \mathrm{C} & 0 & 0 & 0 & 0 & 0 & 0 & 0 & 0 & 0 & 5 \\ -1.5127 & 0.5378 & 1.5113 & \mathrm{C} & 0 & 0 & 0 & 0 & 0 & 0 & 0 & 0 & 0 & 6 \\ -0.1594 & 0.1115 & 1.5642 & \mathrm{C} & 0 & 0 & 0 & 0 & 0 & 0 & 0 & 0 & 0 & 7 \\ 0.3993 & -0.3164 & 2.8374 & \mathrm{C} & 0 & 0 & 0 & 0 & 0 & 0 & 0 & 0 & 0 & 8 \\ -0.4034 & -0.3249 & 4.0082 & \mathrm{C} & 0 & 0 & 0 & 0 & 0 & 0 & 0 & 0 & 0 & 9 \\ 1.7439 & -0.6578 & 2.6065 & \mathrm{C} & 0 & 0 & 0 & 0 & 0 & 0 & 0 & 0 & 0 & 10 \\ 0.8795 & 0.0037 & 0.6066 & \mathrm{C} & 0 & 0 & 0 & 0 & 0 & 0 & 0 & 0 & 0 & 11 \\ 1.9901 & -0.4534 & 1.2863 & \mathrm{~N} & 0 & 0 & 0 & 0 & 0 & 0 & 0 & 0 & 0 & 12 \\ 0.9704 & 0.2754 & -0.8110 & \mathrm{C} & 0 & 0 & 0 & 0 & 0 & 0 & 0 & 0 & 0 & 13\end{array}$




\begin{tabular}{|c|c|c|c|c|c|c|c|c|c|c|c|c|}
\hline 0.2134 & 0.5017 & -2.9775 & $\mathrm{C}$ & 0 & 0 & 0 & 0 & 0 & 0 & 0 & 0 & 0 \\
\hline 1.7512 & 0.5891 & -2.8682 & & 0 & 0 & 0 & 0 & 0 & 0 & 0 & 0 & 0 \\
\hline-0.1529 & 0.6516 & -1.5554 & $\mathrm{~N}$ & 0 & 0 & 0 & 0 & 0 & 0 & 0 & 0 & 0 \\
\hline 2.0931 & 0.2366 & -1.4771 & $\mathrm{~N}$ & 0 & 0 & 0 & 0 & 0 & 0 & 0 & 0 & 0 \\
\hline-0.1616 & -0.7622 & -3.5186 & 0 & 0 & 0 & 0 & 0 & 0 & 0 & 0 & 0 & 0 \\
\hline-2.3554 & 0.0840 & 4.8023 & $\mathrm{H}$ & 0 & 0 & 0 & 0 & 0 & 0 & 0 & 0 & 0 \\
\hline-3.3084 & 0.8485 & 2.6377 & $\mathrm{H}$ & 0 & 0 & 0 & 0 & 0 & 0 & 0 & 0 & 0 \\
\hline-1.9612 & 0.8930 & 0.5822 & $\mathrm{H}$ & 0 & 0 & 0 & 0 & 0 & 0 & 0 & 0 & 0 \\
\hline 0.0168 & -0.6460 & 4.9635 & $\mathrm{H}$ & 0 & 0 & 0 & 0 & 0 & 0 & 0 & 0 & 0 \\
\hline 2.5151 & -1.0269 & 3.2742 & $\mathrm{H}$ & 0 & 0 & 0 & 0 & 0 & 0 & 0 & 0 & 0 \\
\hline 2.8830 & -0.6143 & 0.8221 & $\mathrm{H}$ & 0 & 0 & 0 & 0 & 0 & 0 & 0 & 0 & 0 \\
\hline-0.2365 & 1.3128 & -3.5685 & $\mathrm{H}$ & 0 & 0 & 0 & 0 & 0 & 0 & 0 & 0 & 0 \\
\hline 2.2296 & -0.0912 & -3.5873 & $\mathrm{H}$ & & 0 & 0 & 0 & 0 & 0 & 0 & 0 & \\
\hline 2.0972 & 1.6127 & -3.0900 & $\mathrm{H}$ & 0 & 0 & 0 & 0 & 0 & 0 & 0 & 0 & 0 \\
\hline-1.0656 & 0.291 & & $\mathrm{H}$ & & & 0 & 0 & 0 & 0 & 0 & 0 & 0 \\
\hline-1.1149 & -0.7123 & -3.8054 & $\mathrm{H}$ & 0 & 0 & 0 & 0 & 0 & 0 & 0 & 0 & ( \\
\hline
\end{tabular}




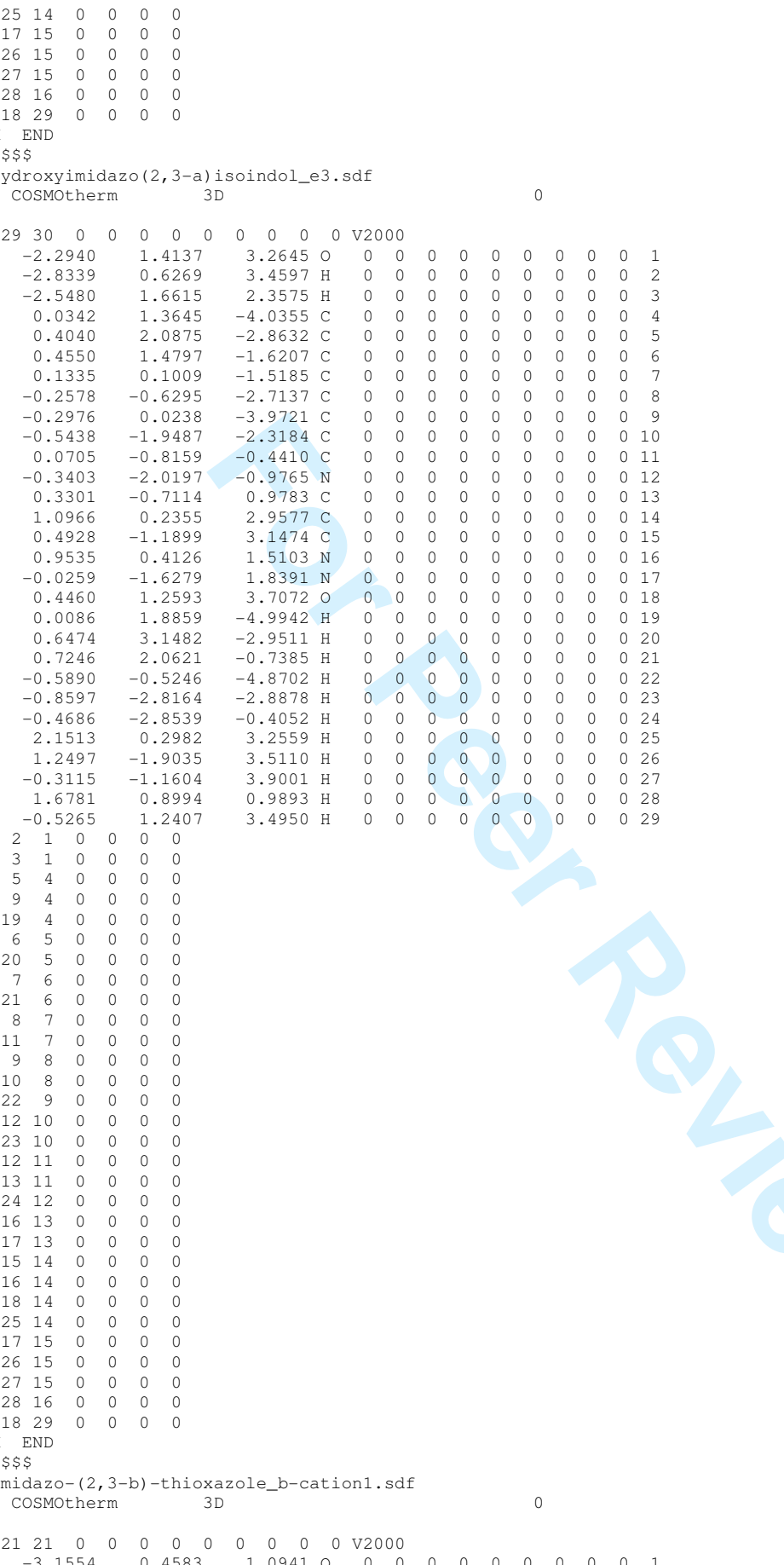

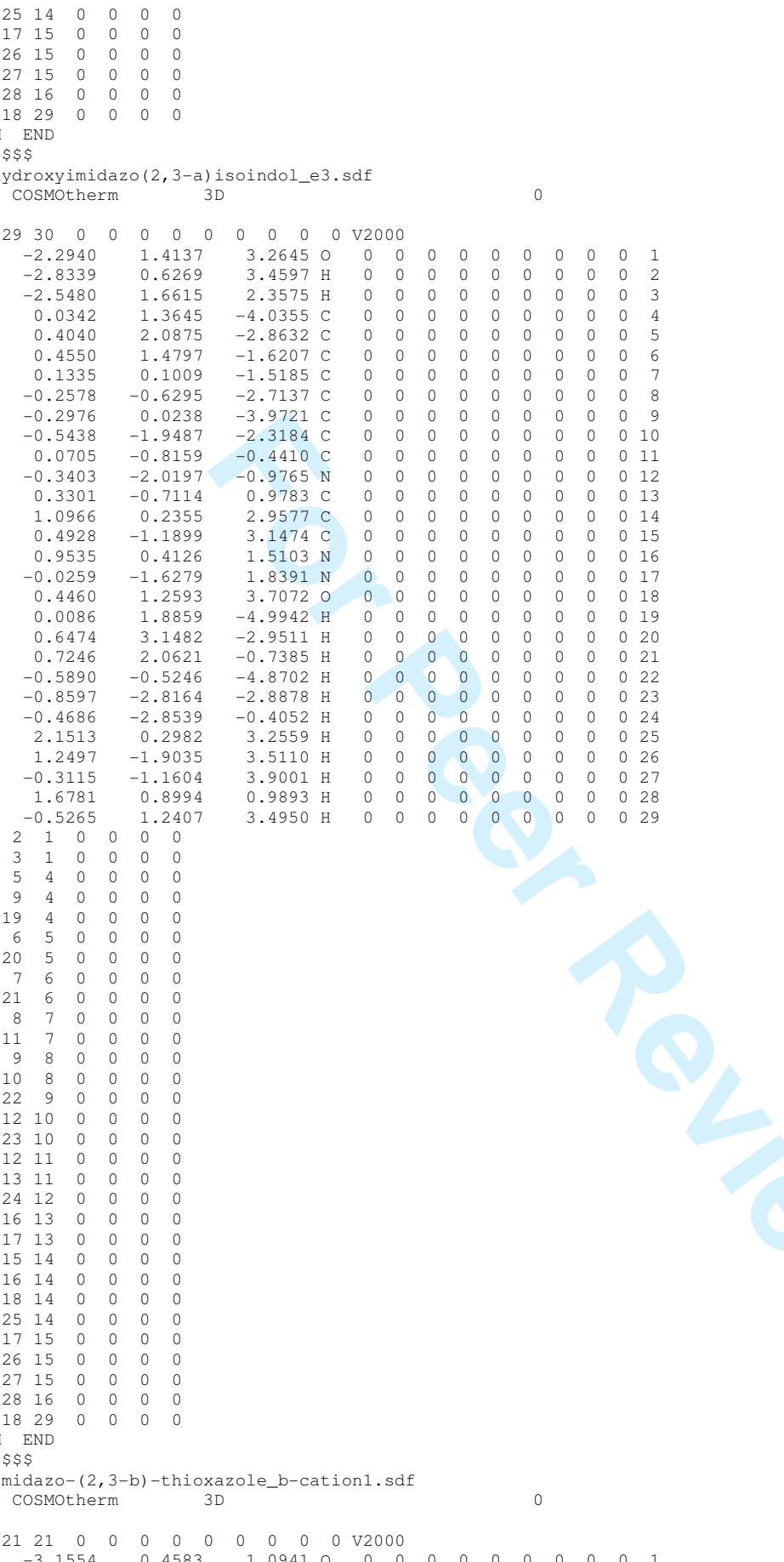

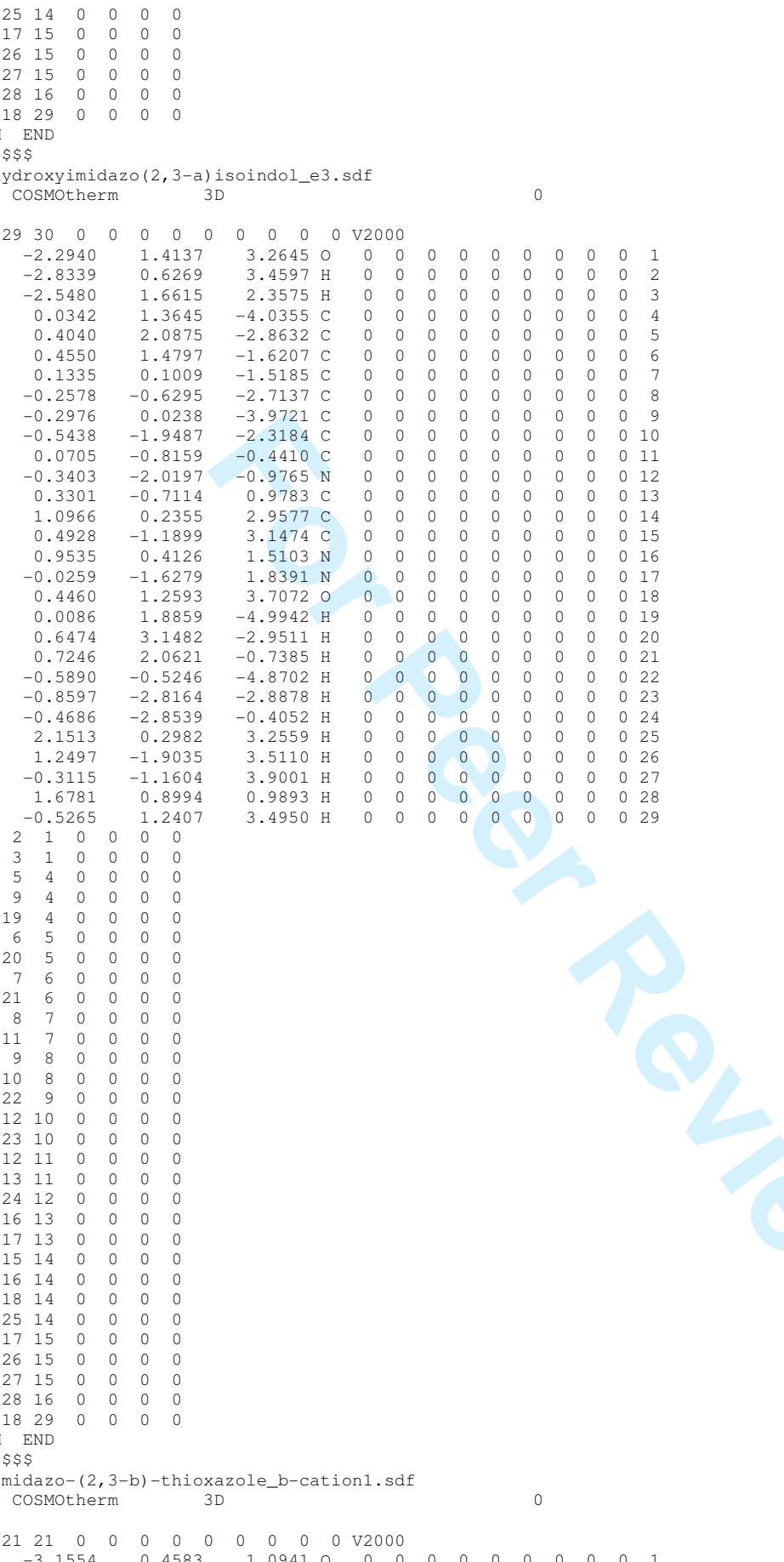

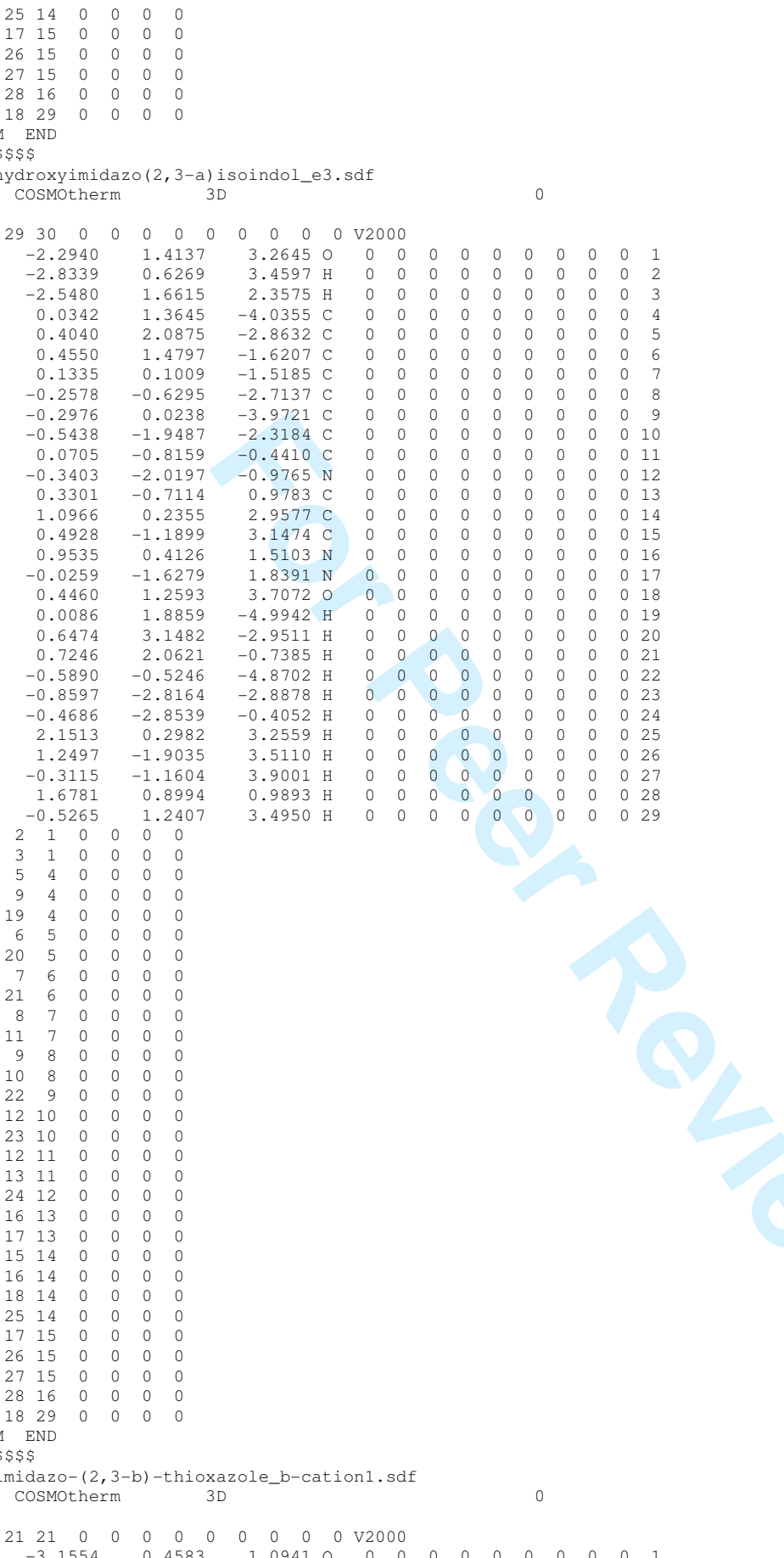

1829

$\$ \$ \$$

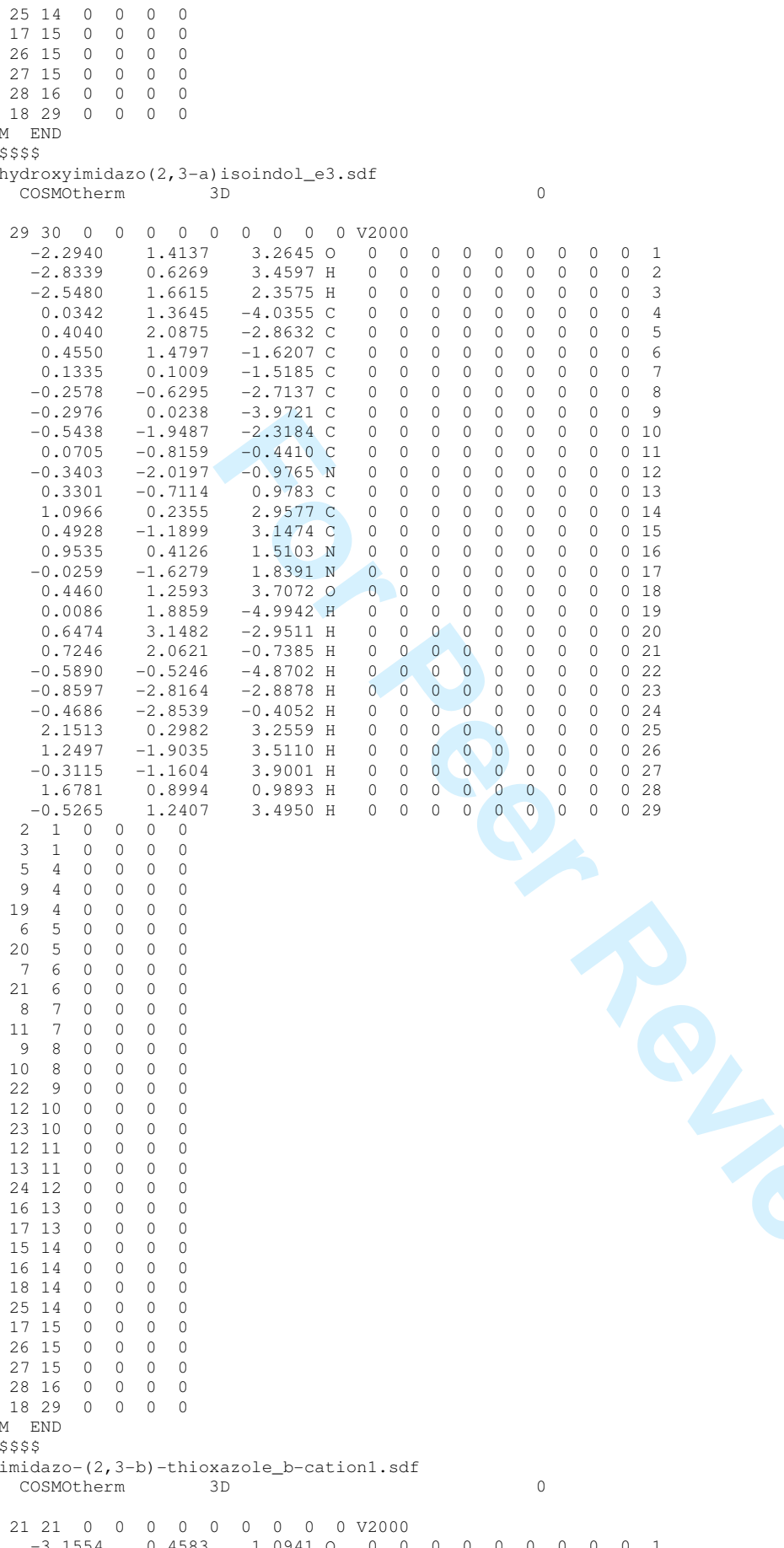

cosmotherm

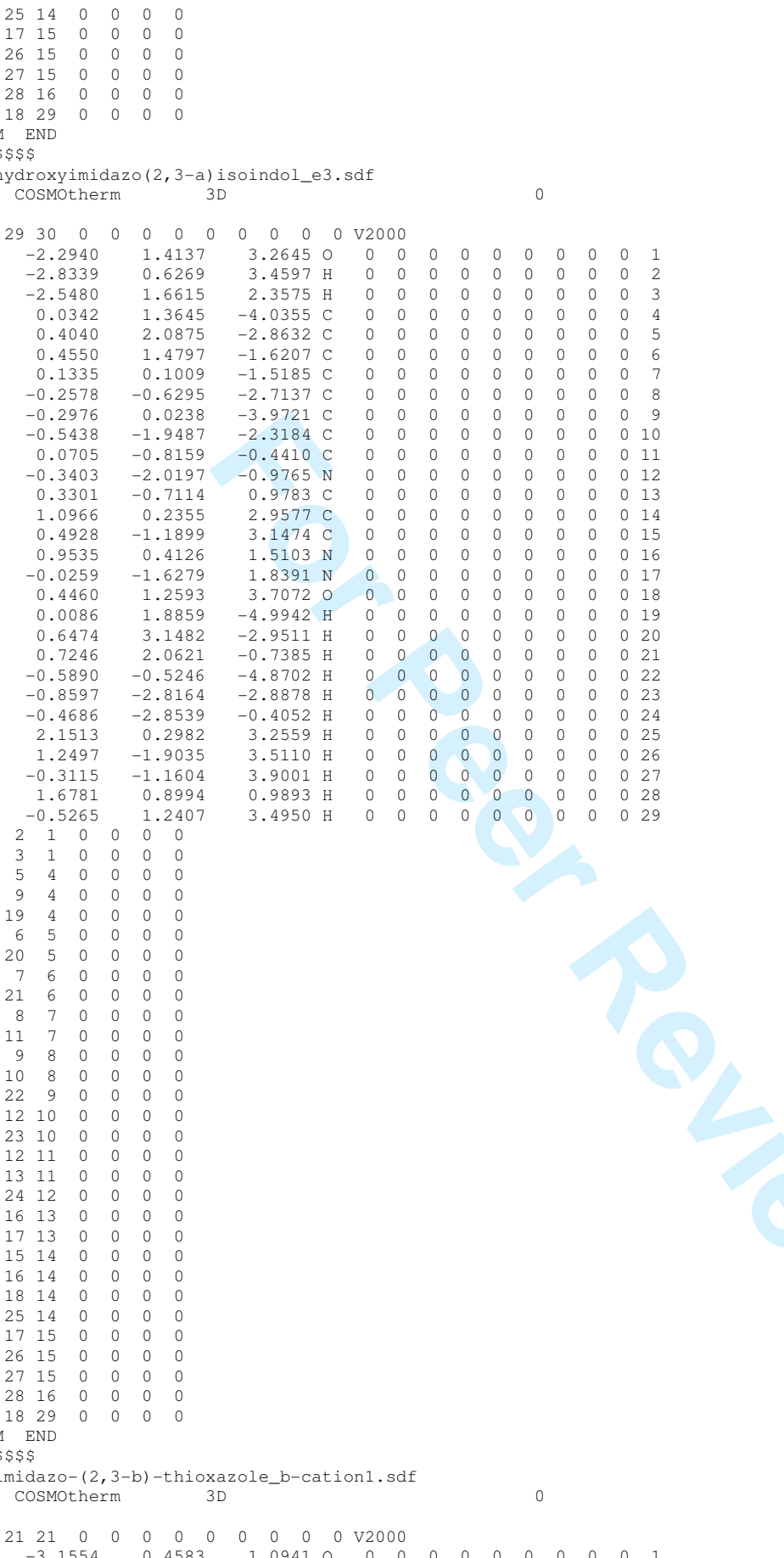

\begin{tabular}{|c|c|c|c|c|c|c|c|c|c|c|c|c|}
\hline & & 3.2645 & 0 & 0 & 0 & 0 & 0 & 0 & 0 & 0 & 0 & 0 \\
\hline-2.8339 & 0.6269 & 3.4597 & $\mathrm{H}$ & 0 & 0 & 0 & 0 & 0 & 0 & 0 & 0 & 0 \\
\hline-2.5480 & 1.6615 & 2.3575 & $\mathrm{H}$ & 0 & 0 & 0 & 0 & 0 & 0 & 0 & 0 & 0 \\
\hline 0.0342 & 1.3645 & -4.0355 & $\mathrm{C}$ & 0 & 0 & 0 & 0 & 0 & 0 & 0 & 0 & 0 \\
\hline 0.4040 & 2.0875 & -2.8632 & C & 0 & 0 & 0 & 0 & 0 & 0 & 0 & 0 & 0 \\
\hline 0.4550 & 1.4797 & -1.6207 & $\mathrm{C}$ & 0 & 0 & 0 & 0 & 0 & 0 & 0 & 0 & 0 \\
\hline 0.1335 & 0.1009 & -1.5185 & C & 0 & 0 & 0 & 0 & 0 & 0 & 0 & 0 & 0 \\
\hline-0.2578 & -0.6295 & -2.7137 & $\mathrm{C}$ & 0 & 0 & 0 & 0 & 0 & 0 & 0 & 0 & 0 \\
\hline-0.2976 & 0.0238 & -3.9721 & C & 0 & 0 & 0 & 0 & 0 & 0 & 0 & 0 & 0 \\
\hline-0.5438 & -1.9487 & -2.3184 & $\mathrm{C}$ & 0 & 0 & 0 & 0 & 0 & 0 & 0 & 0 & 0 \\
\hline 0.0705 & -0.8159 & -0.4410 & C & 0 & 0 & 0 & 0 & 0 & 0 & 0 & 0 & 0 \\
\hline-0.3403 & -2.0197 & -0.9765 & $\mathrm{~N}$ & 0 & 0 & 0 & 0 & 0 & 0 & 0 & 0 & 0 \\
\hline 0.3301 & -0.7114 & 0.9783 & $\mathrm{C}$ & 0 & 0 & 0 & 0 & 0 & 0 & 0 & 0 & 0 \\
\hline 1.0966 & 0.2355 & 2.9577 & $\mathrm{C}$ & 0 & 0 & 0 & 0 & 0 & 0 & 0 & 0 & 0 \\
\hline 0.4928 & -1.1899 & 3.1474 & $\mathrm{C}$ & 0 & 0 & 0 & 0 & 0 & 0 & 0 & 0 & 0 \\
\hline 0.9535 & 0.4126 & 1.5103 & $\mathrm{~N}$ & 0 & 0 & 0 & 0 & 0 & 0 & 0 & 0 & 0 \\
\hline-0.0259 & -1.6279 & 1.8391 & $\mathrm{~N}$ & 0 & 0 & 0 & 0 & 0 & 0 & 0 & 0 & 0 \\
\hline 0.4460 & 1.2593 & 3.7072 & 0 & 0 & 0 & 0 & 0 & 0 & 0 & 0 & 0 & 0 \\
\hline 0.0086 & 1.8859 & -4.9942 & $\mathrm{H}$ & 0 & 0 & 0 & 0 & 0 & 0 & 0 & 0 & 0 \\
\hline 0.6474 & & -2.9511 & $\mathrm{H}$ & 0 & 0 & 0 & 0 & 0 & 0 & 0 & & 0 \\
\hline 0.7246 & 2.0621 & -0.7385 & $\mathrm{H}$ & 0 & 0 & 0 & 0 & 0 & 0 & 0 & 0 & 0 \\
\hline-0.5890 & -0.5246 & -4.8702 & $\mathrm{H}$ & 0 & 0 & 0 & 0 & 0 & 0 & 0 & 0 & 0 \\
\hline-0.8597 & -2.8164 & -2.8878 & $\mathrm{H}$ & 0 & 0 & 0 & 0 & 0 & 0 & 0 & 0 & 0 \\
\hline-0.4686 & -2.8539 & -0.4052 & & 0 & 0 & 0 & 0 & 0 & 0 & 0 & 0 & 0 \\
\hline 2.1513 & 0.2982 & 3.2559 & $\mathrm{H}$ & 0 & 0 & 0 & 0 & 0 & 0 & 0 & 0 & 0 \\
\hline 1.2497 & -1.9035 & 3.5110 & & 0 & 0 & 0 & & 0 & 0 & 0 & 0 & 0 \\
\hline-0.3115 & -1.1604 & 3.9001 & & 0 & 0 & 0 & 0 & 0 & 0 & 0 & 0 & 0 \\
\hline 1.6781 & 0.8994 & 0.9893 & & 0 & 0 & 0 & 0 & 0 & 0 & 0 & 0 & 0 \\
\hline-0.5265 & 1.2407 & 3.4950 & $\mathrm{H}$ & 0 & 0 & 0 & 0 & 0 & 0 & & 0 & 0 \\
\hline
\end{tabular}

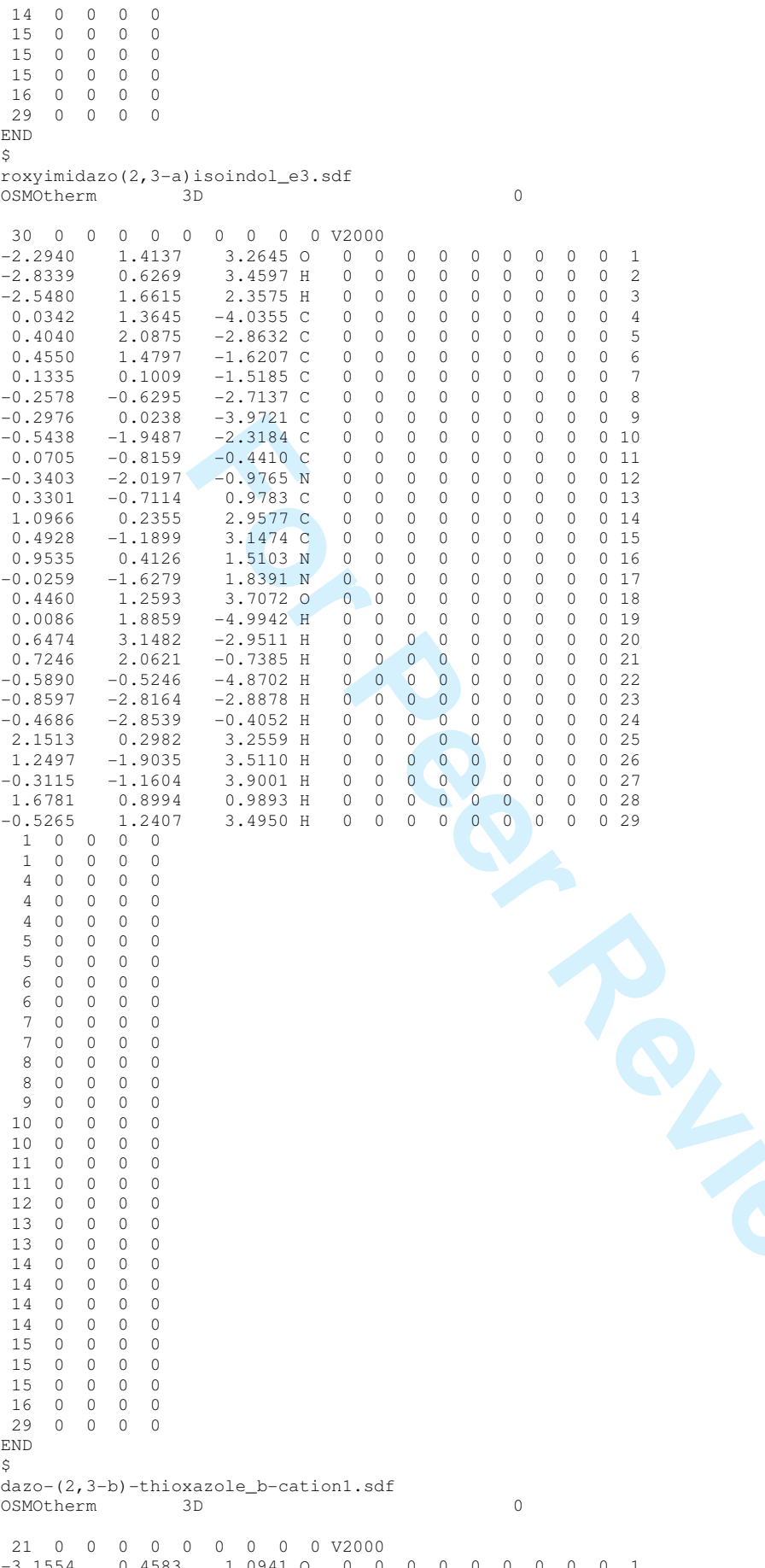

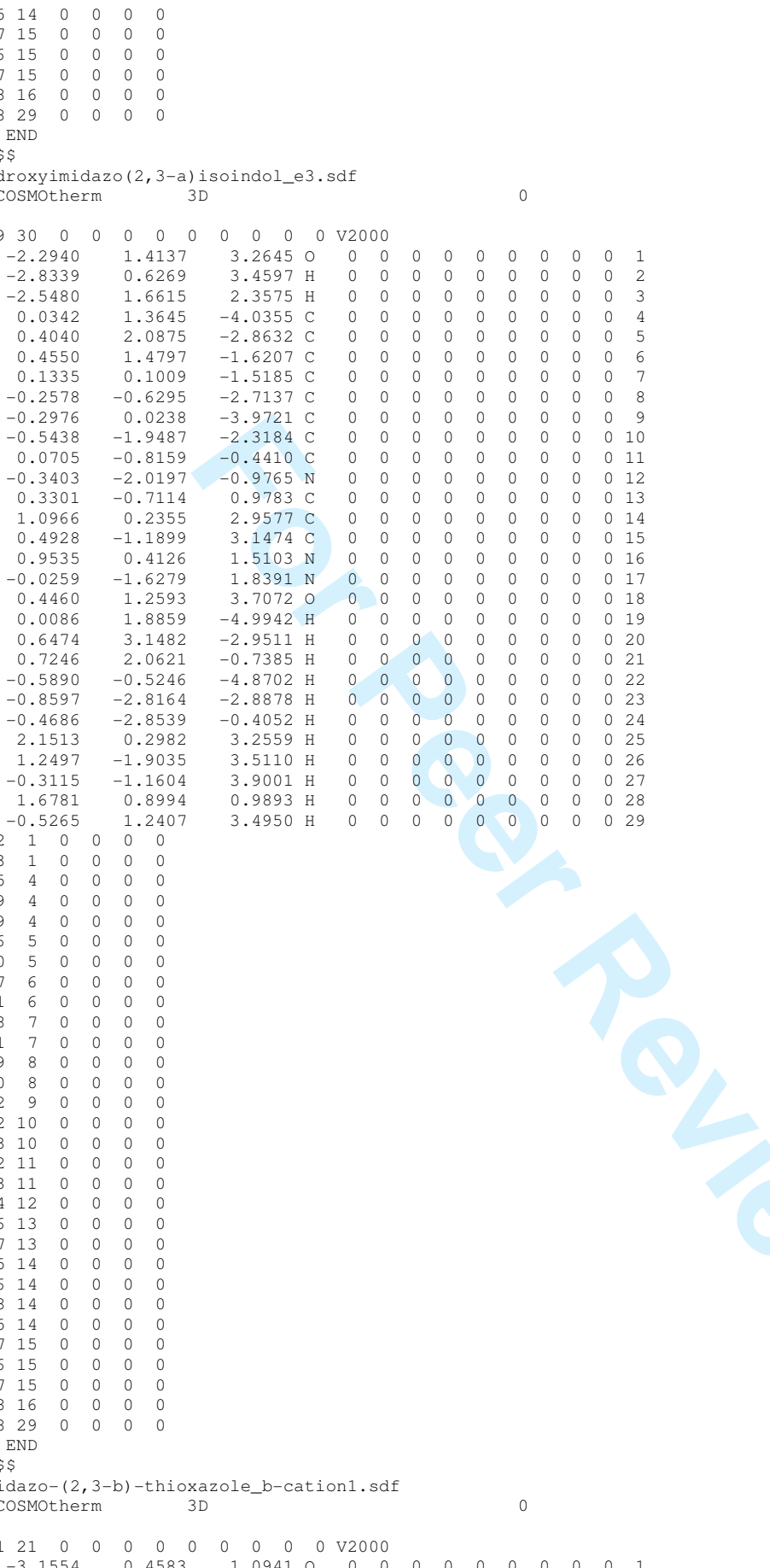

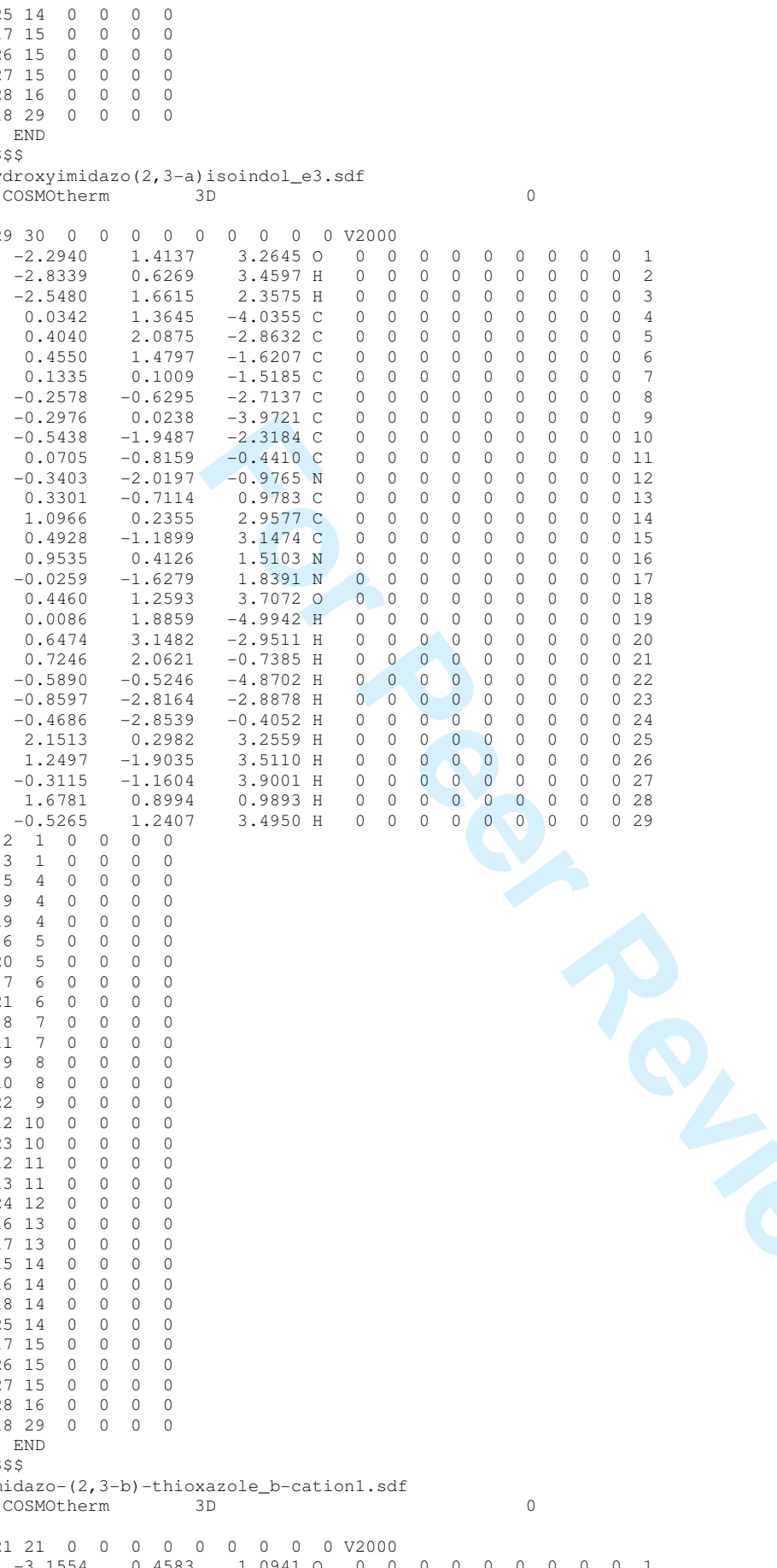

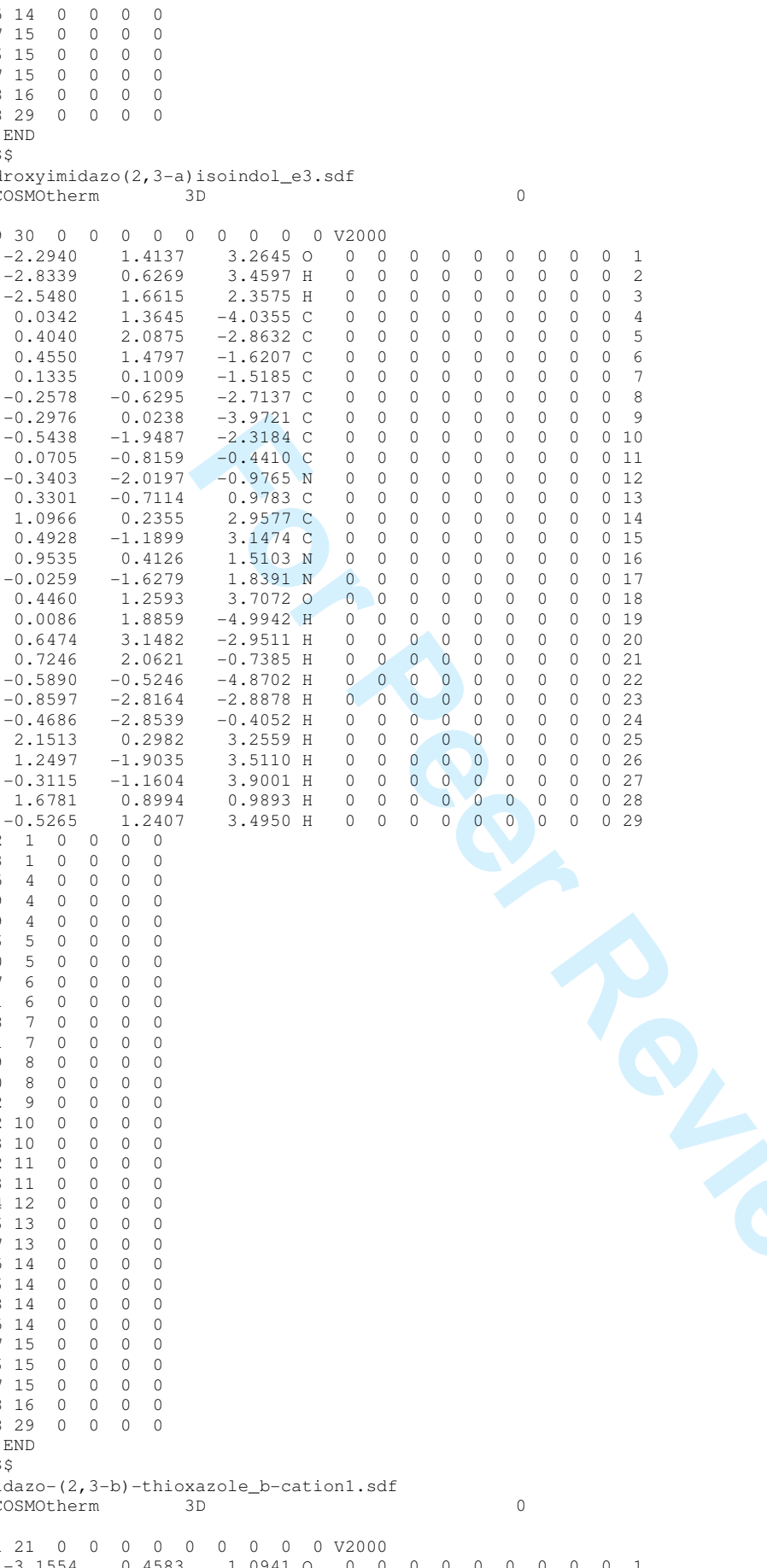

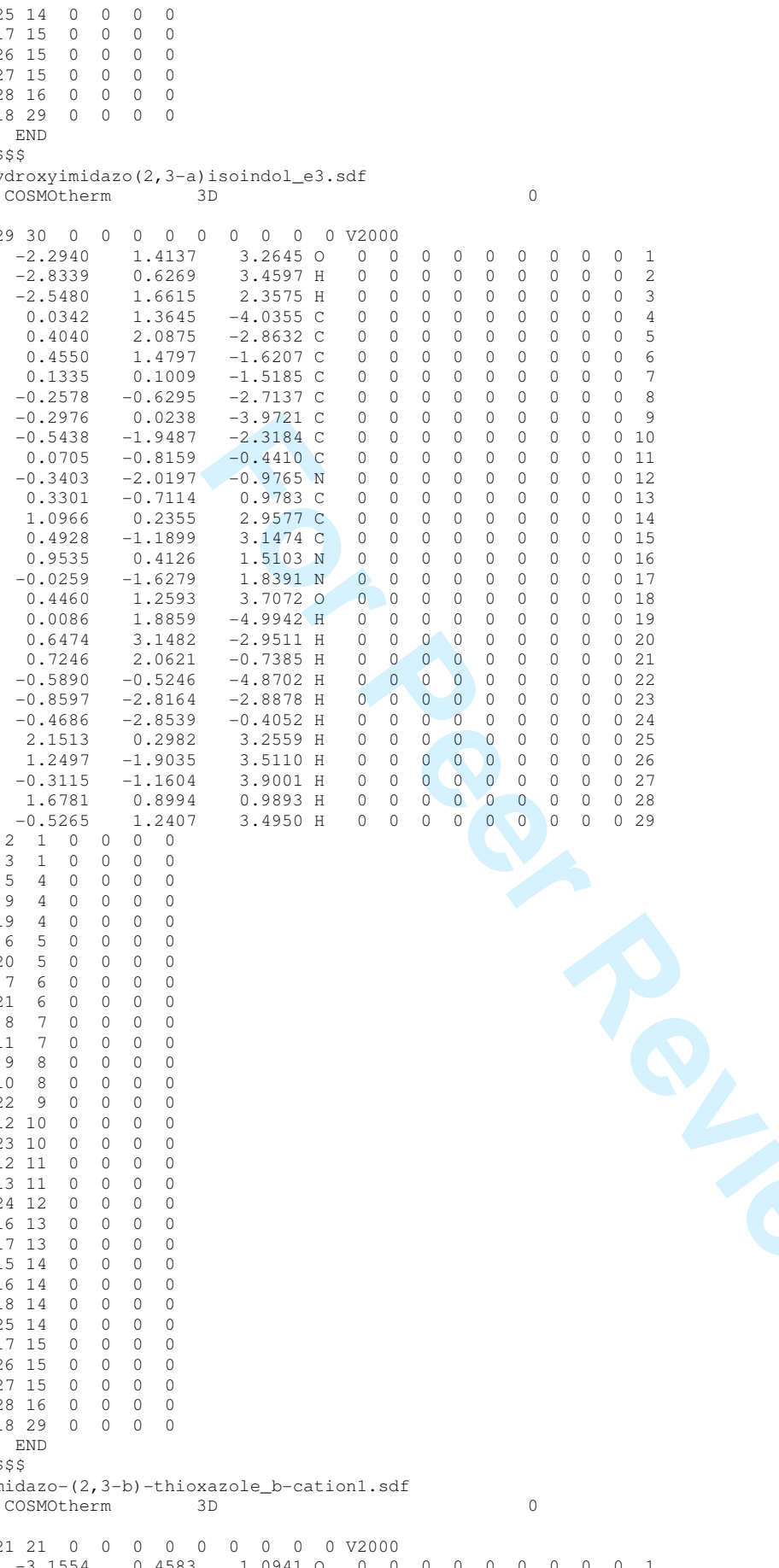

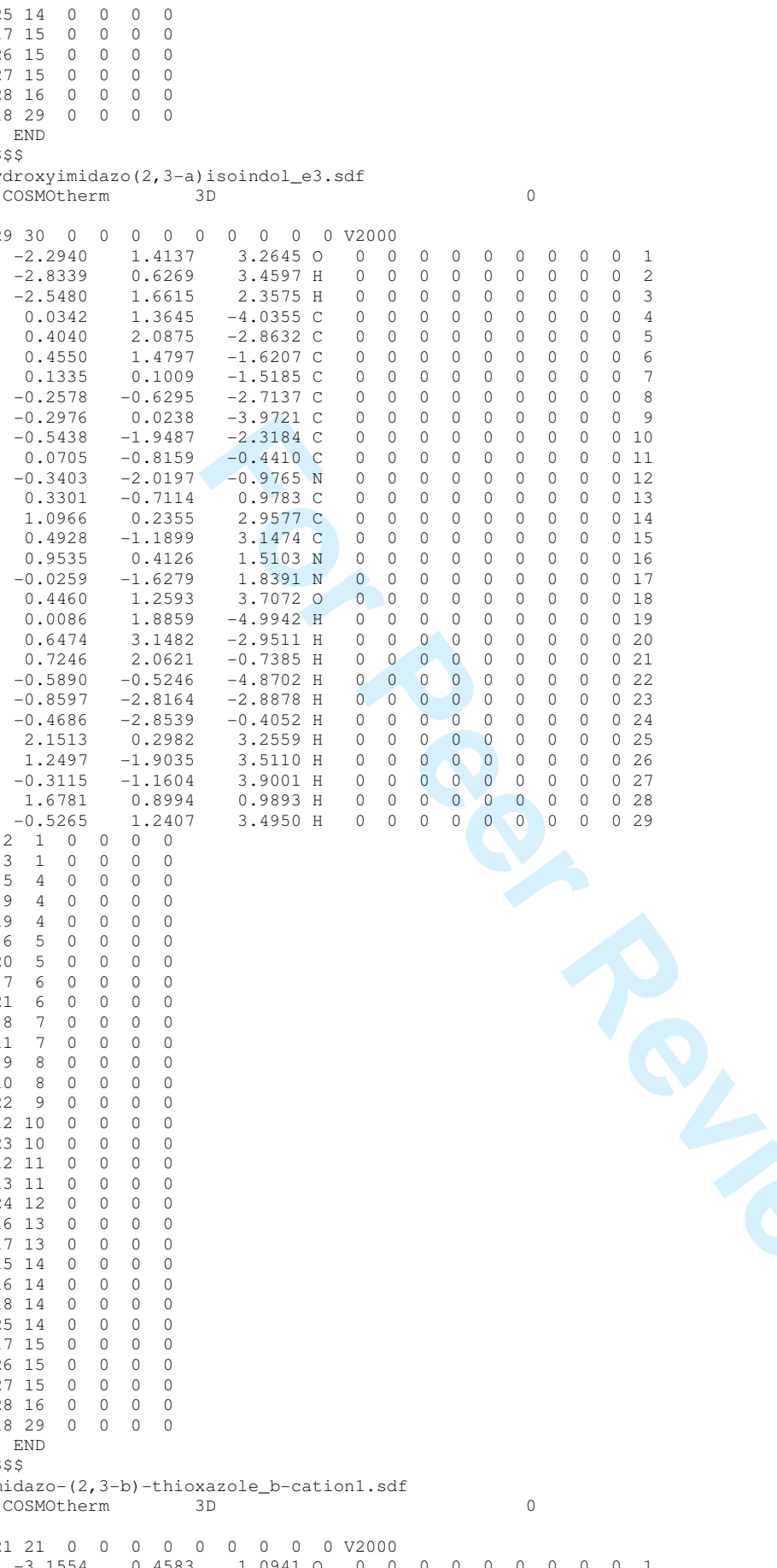

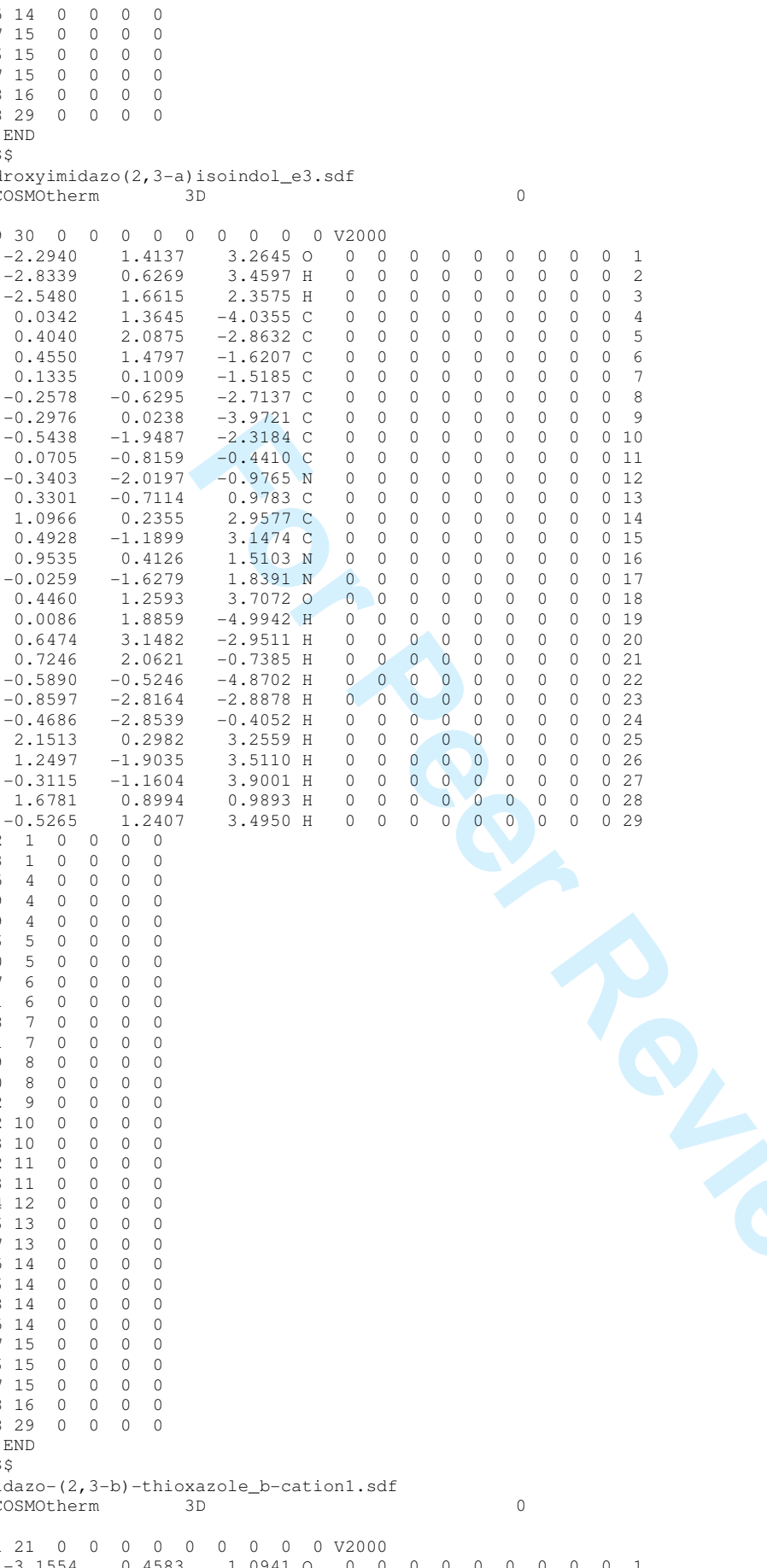

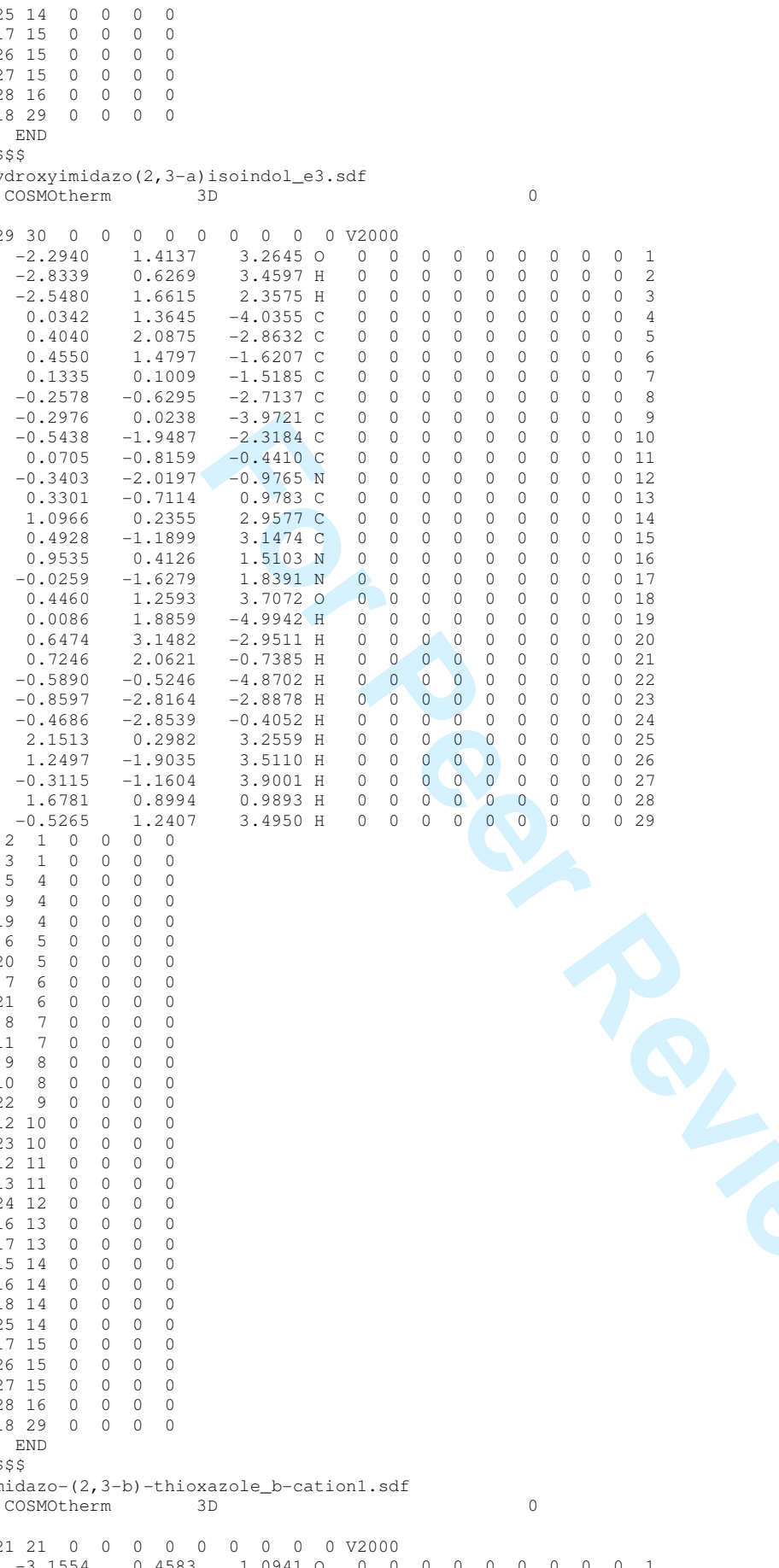

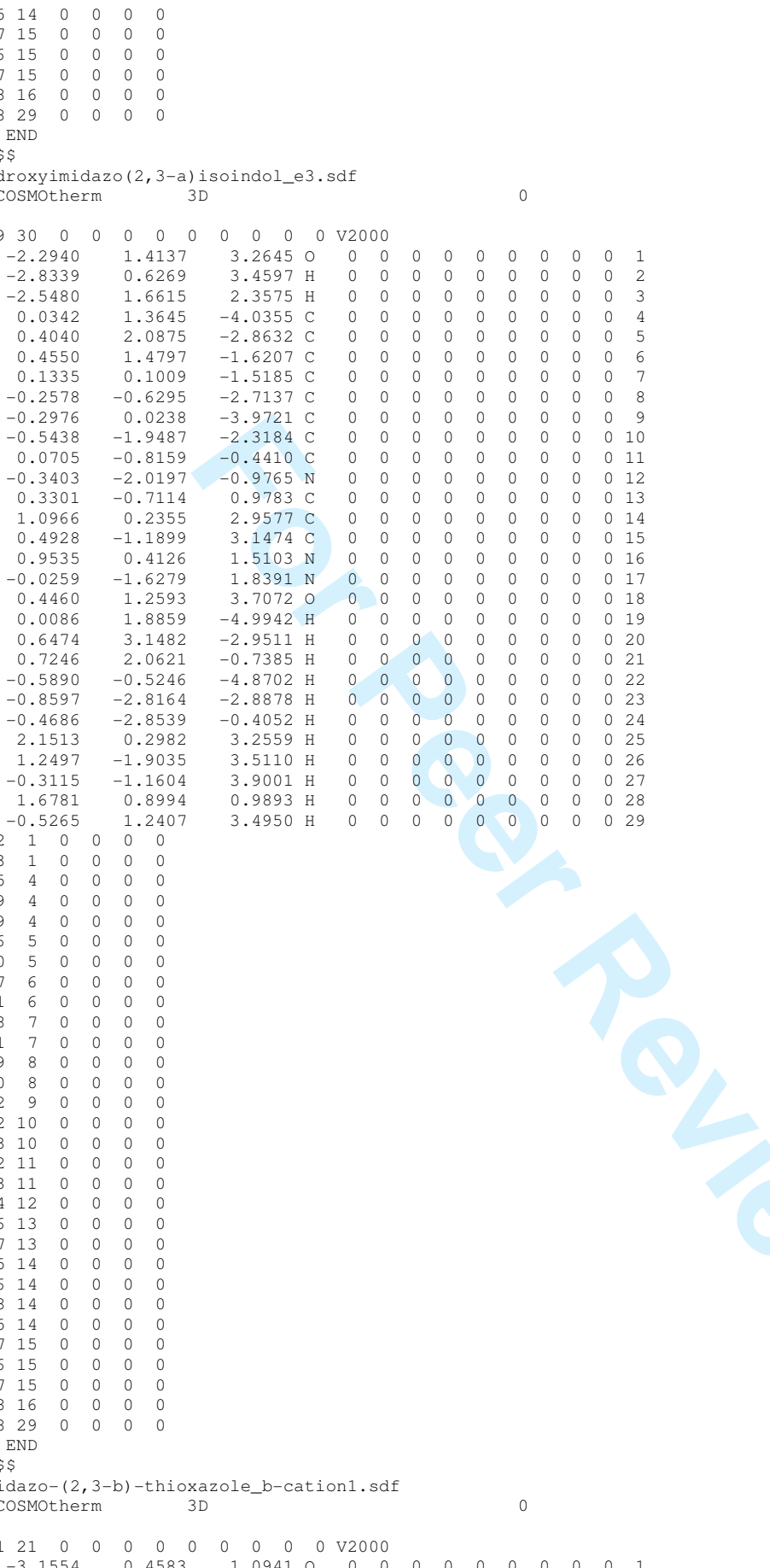

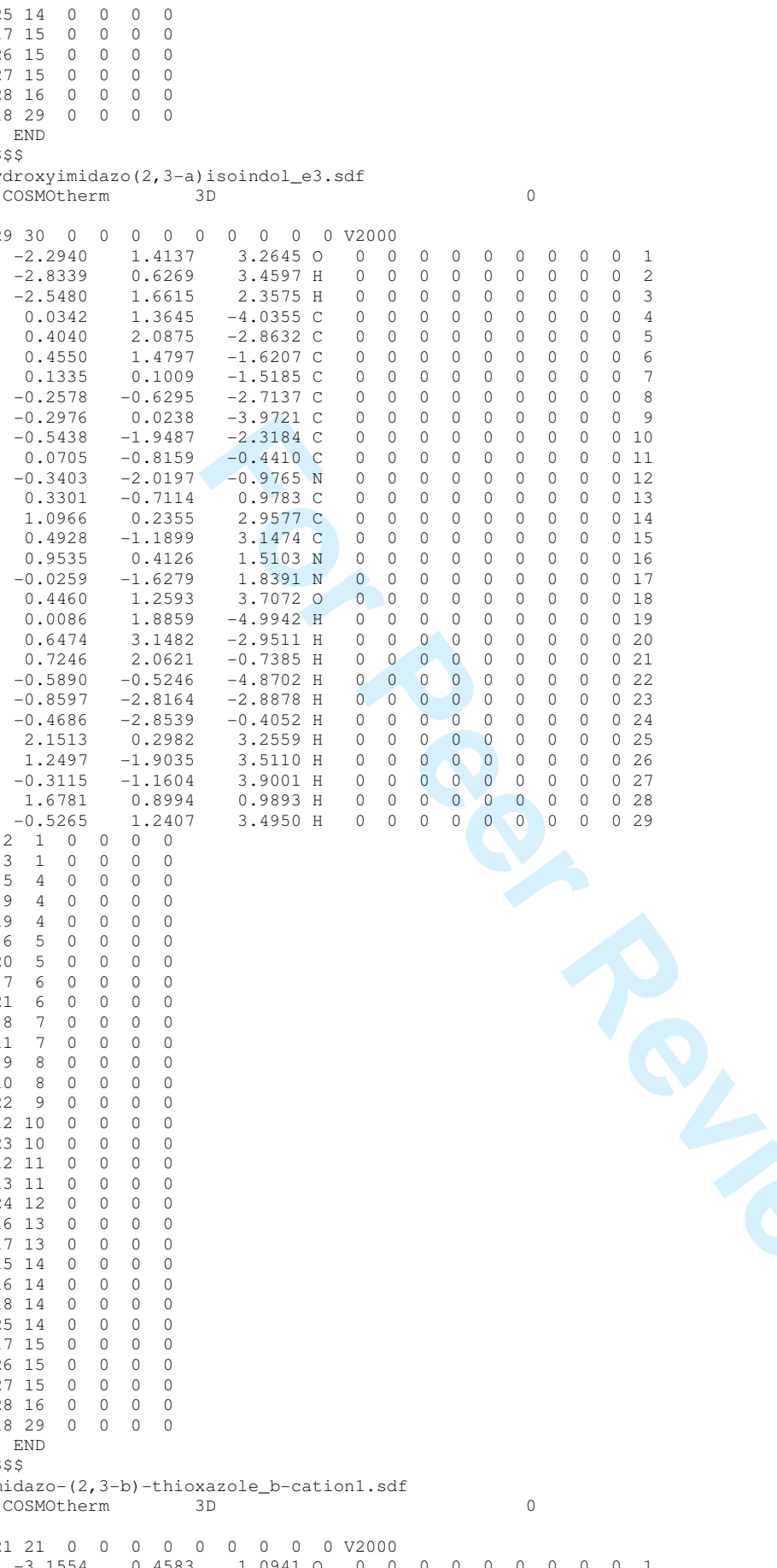

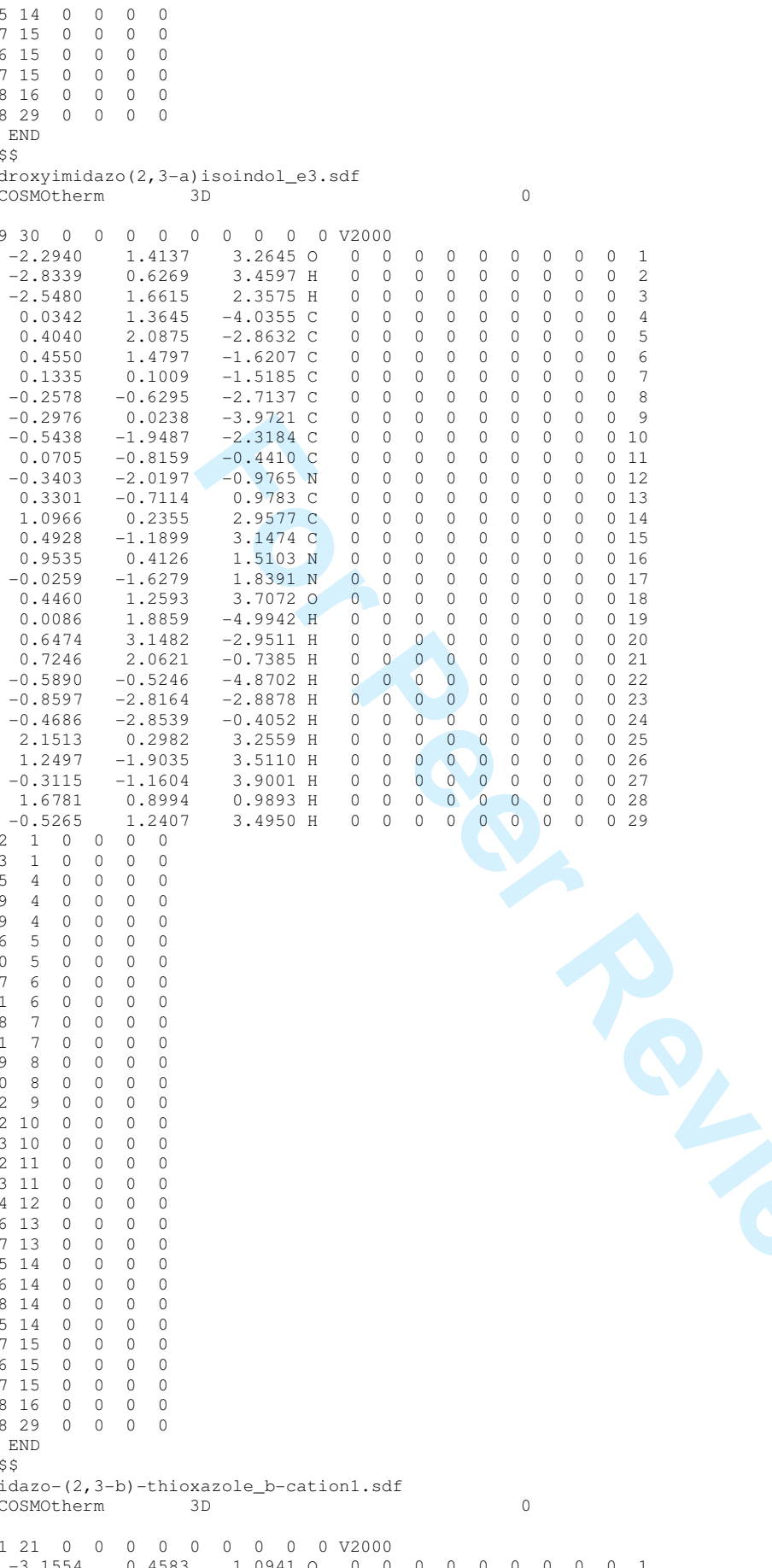

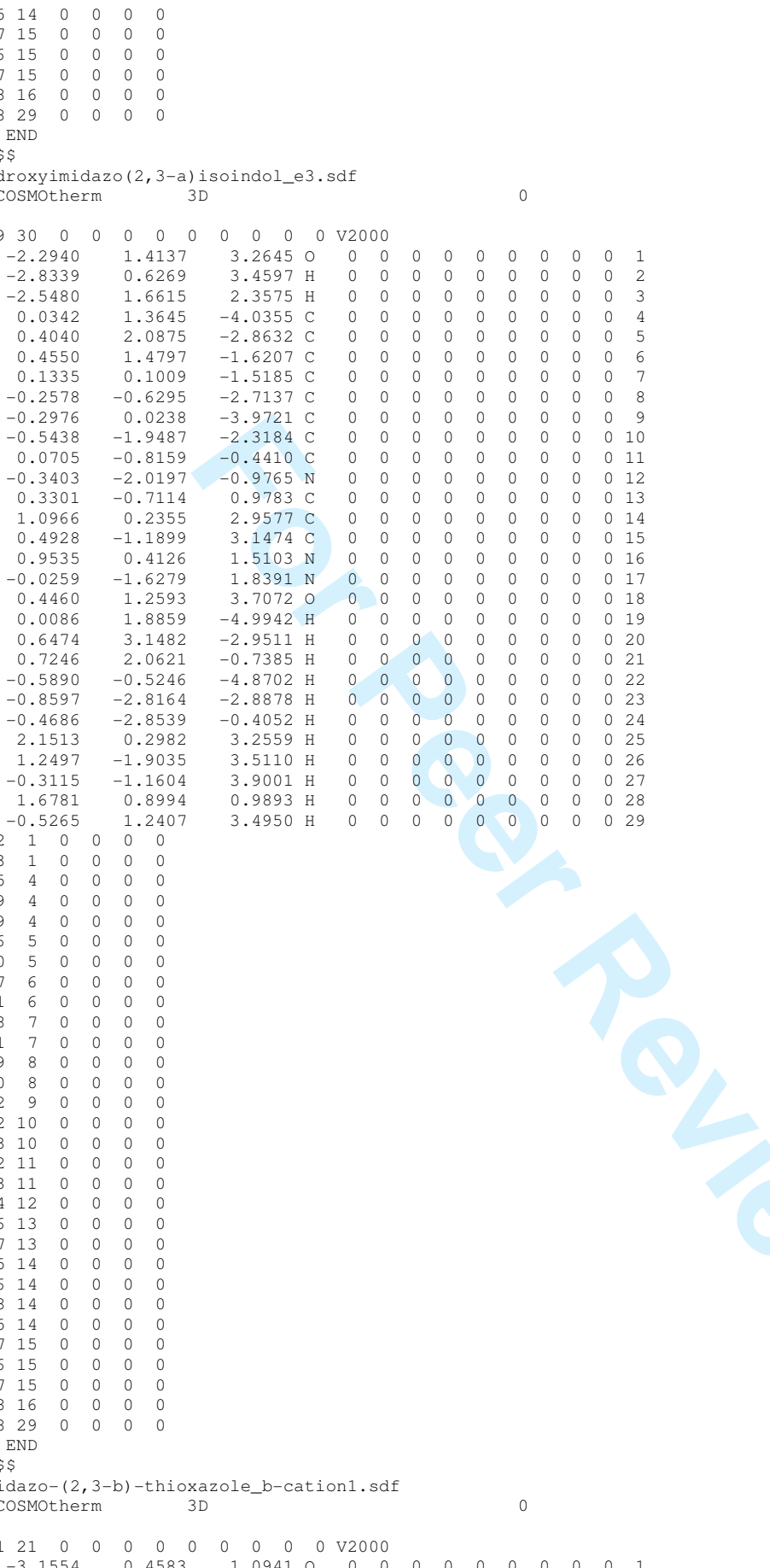

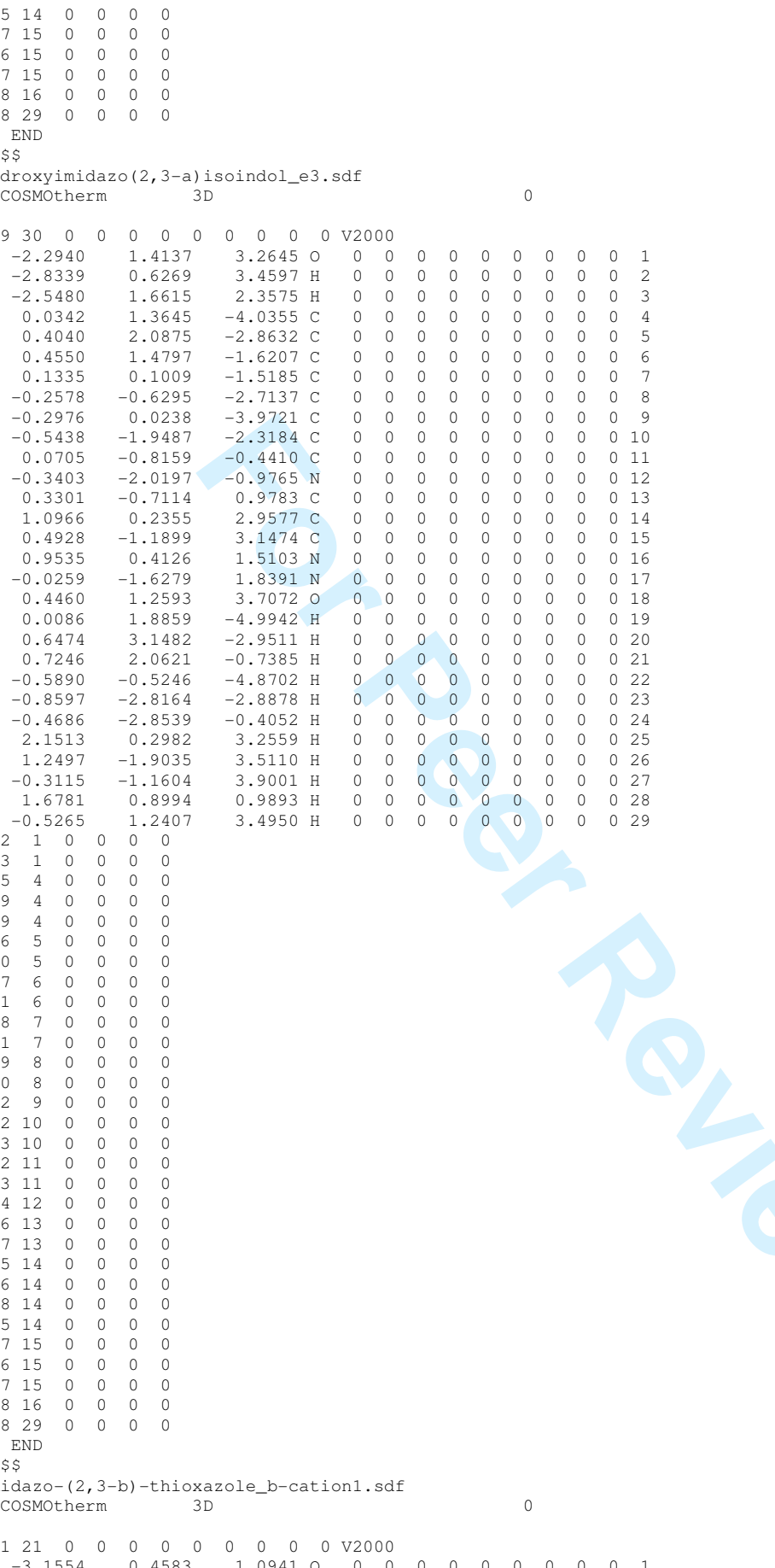

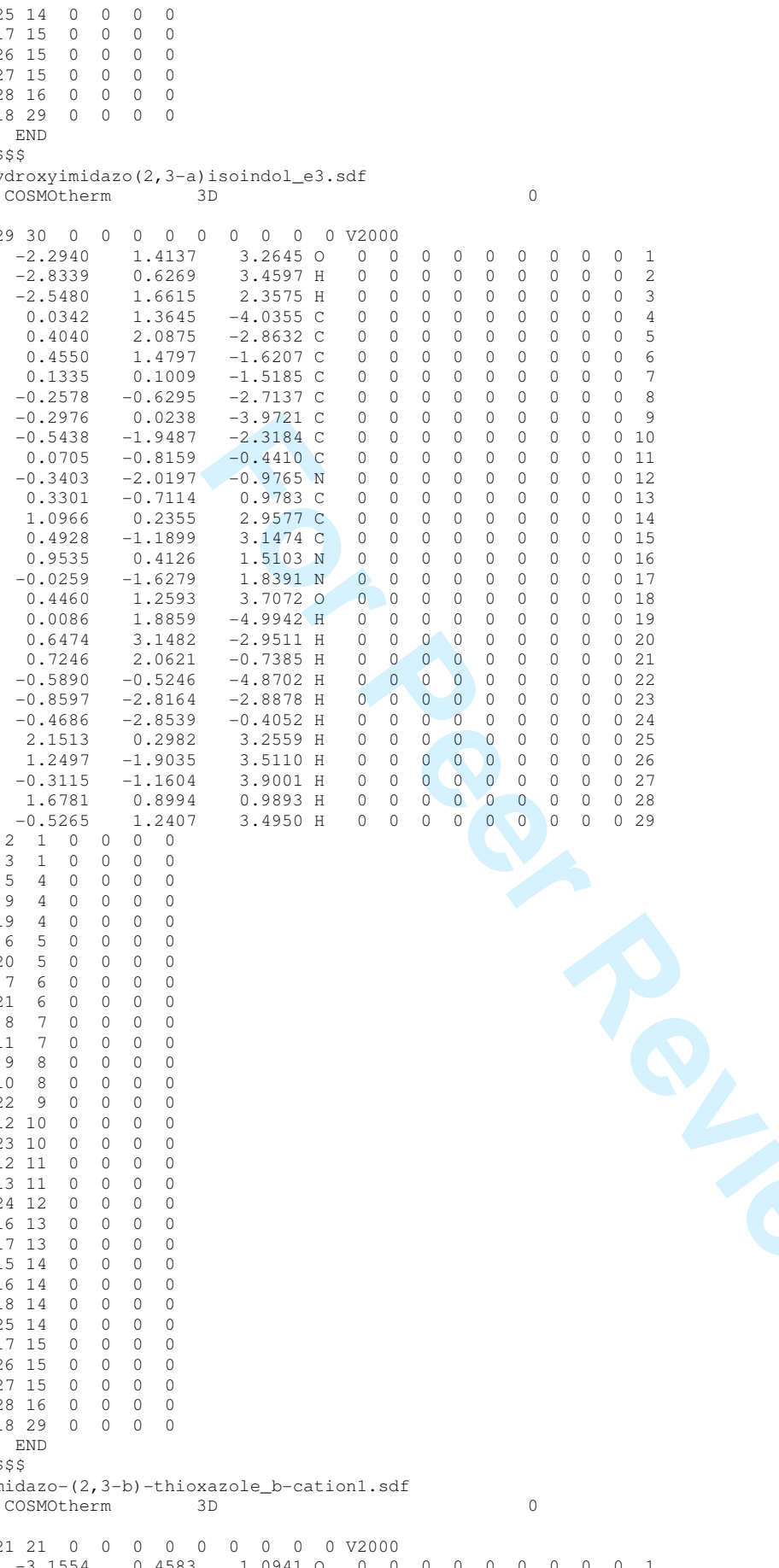

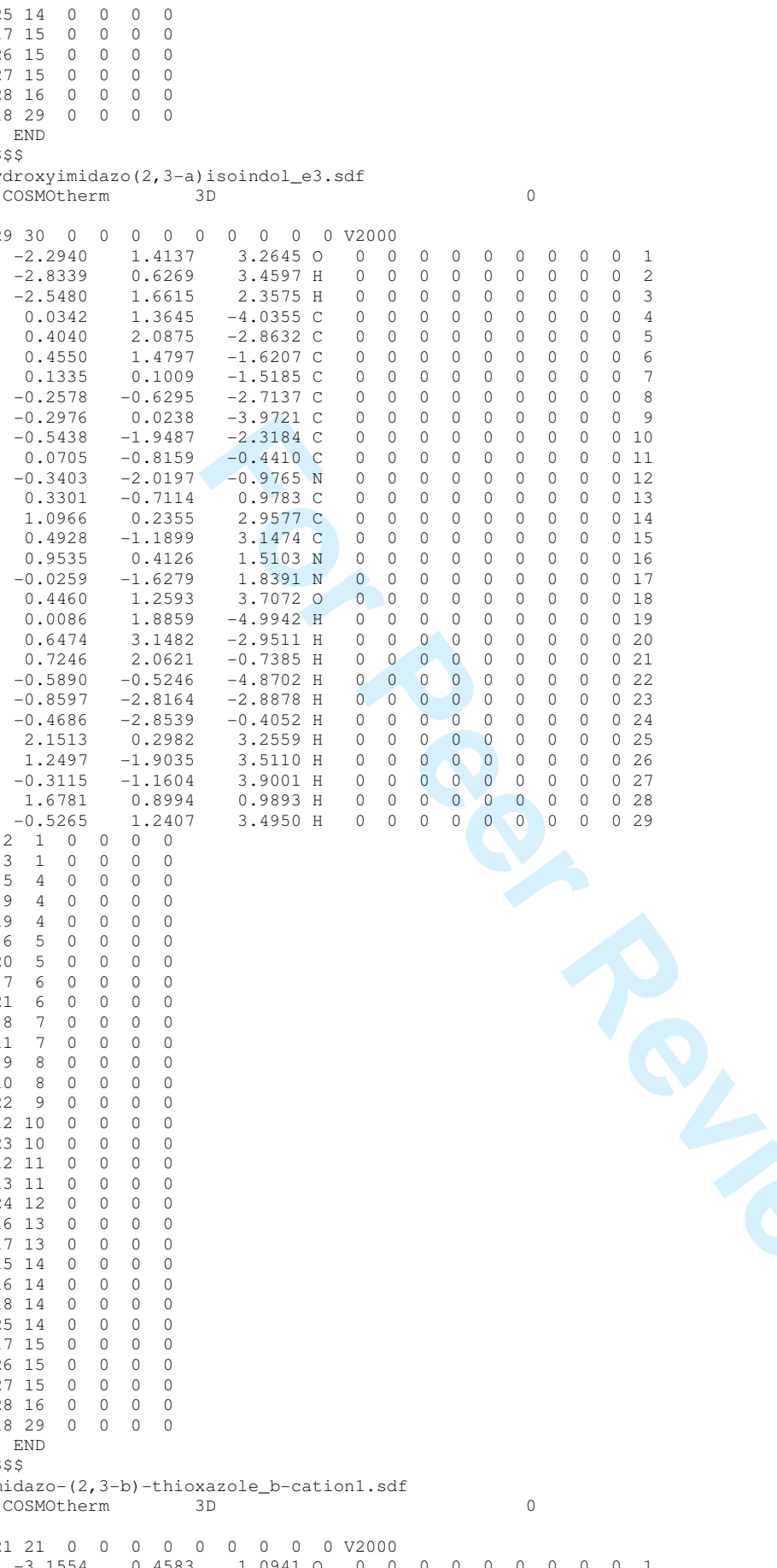

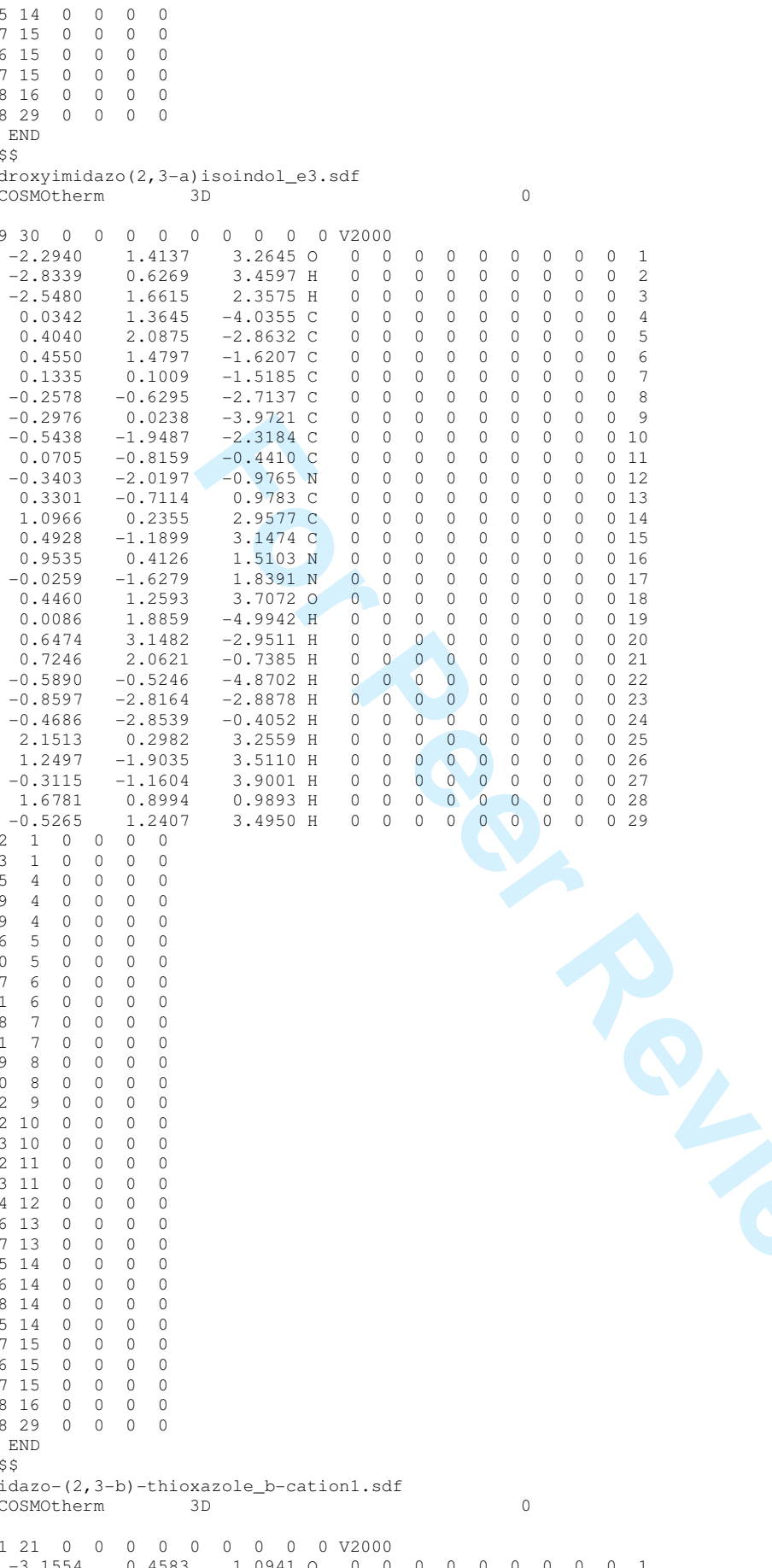

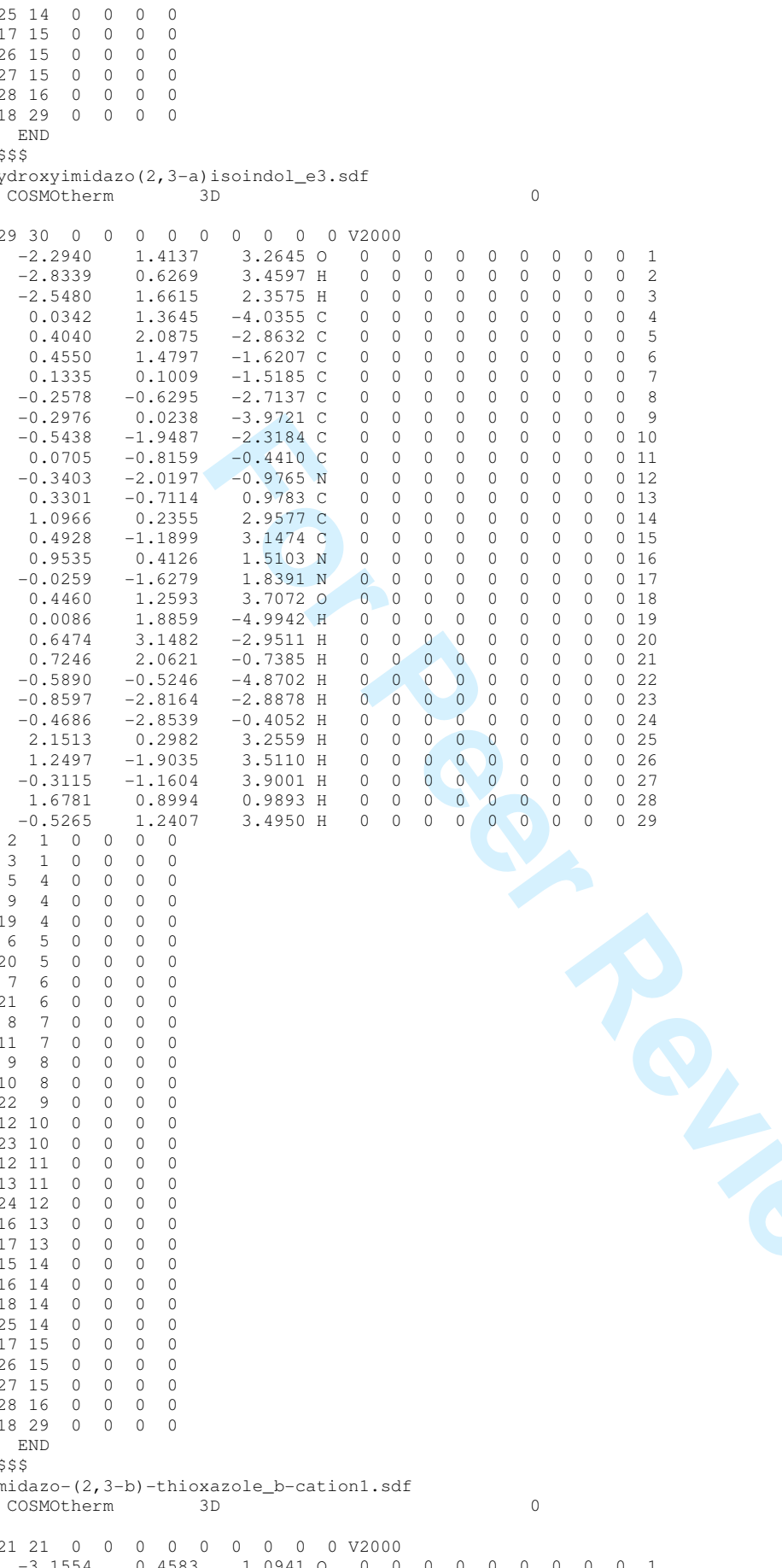

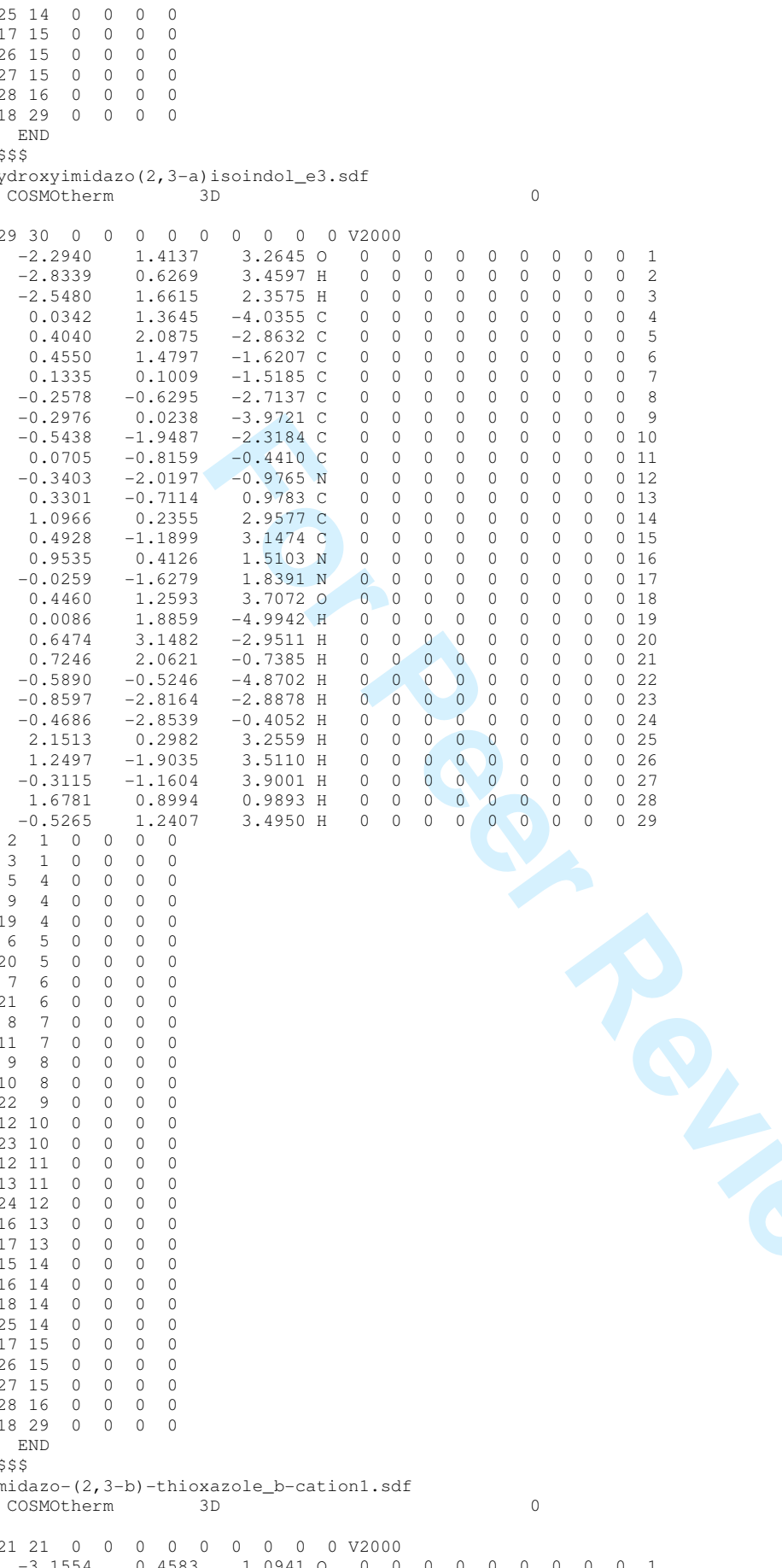

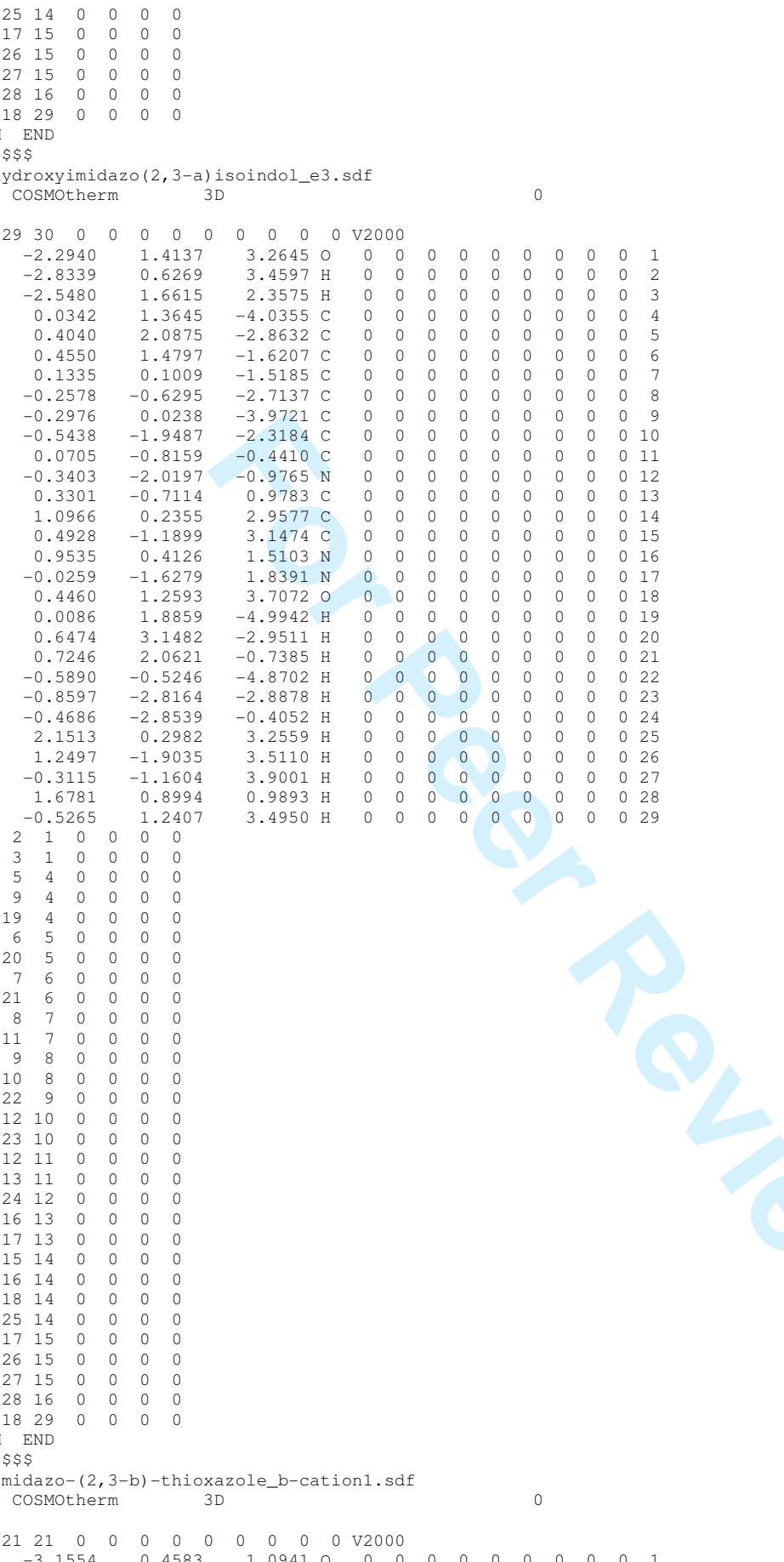

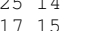

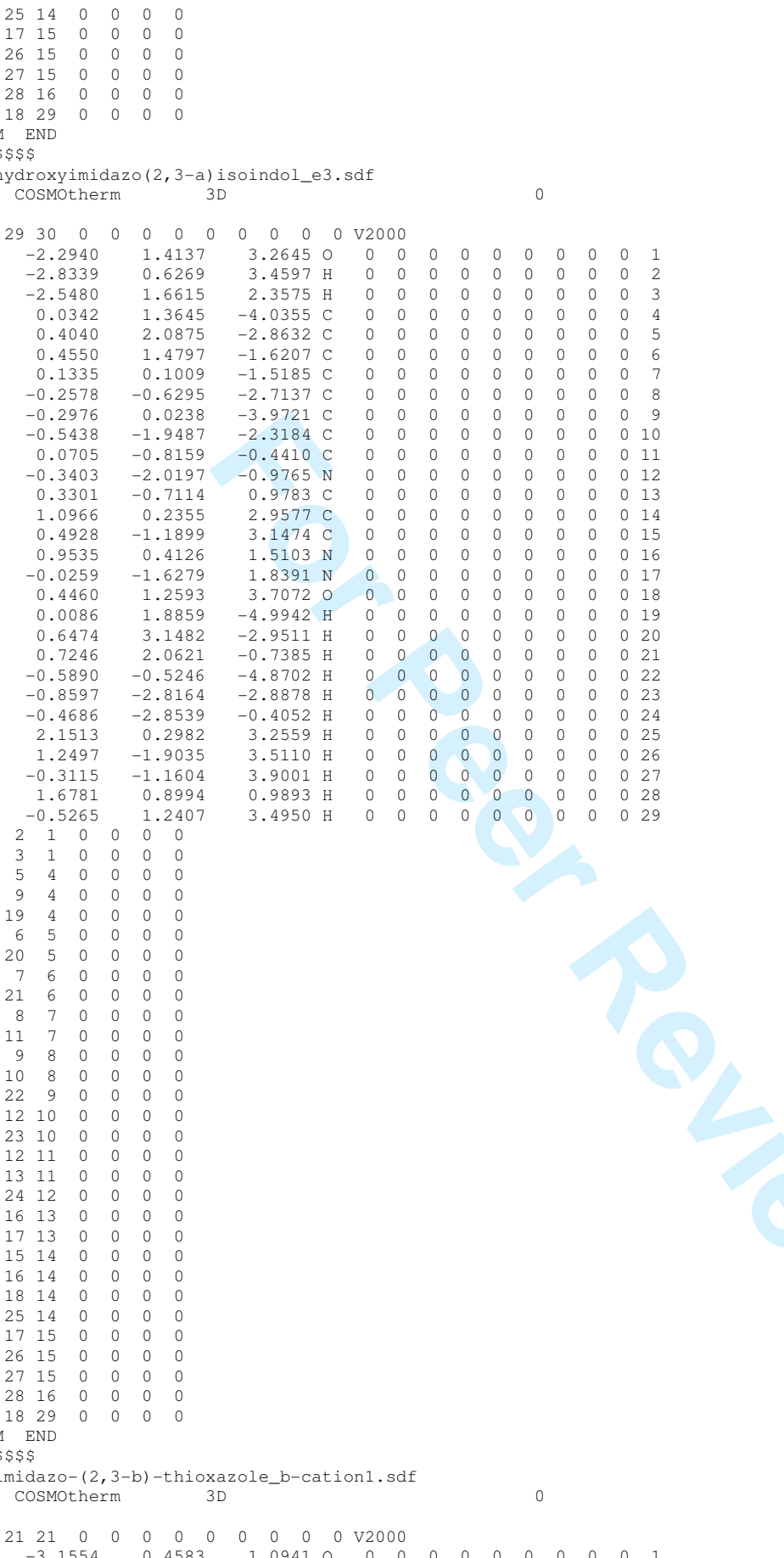

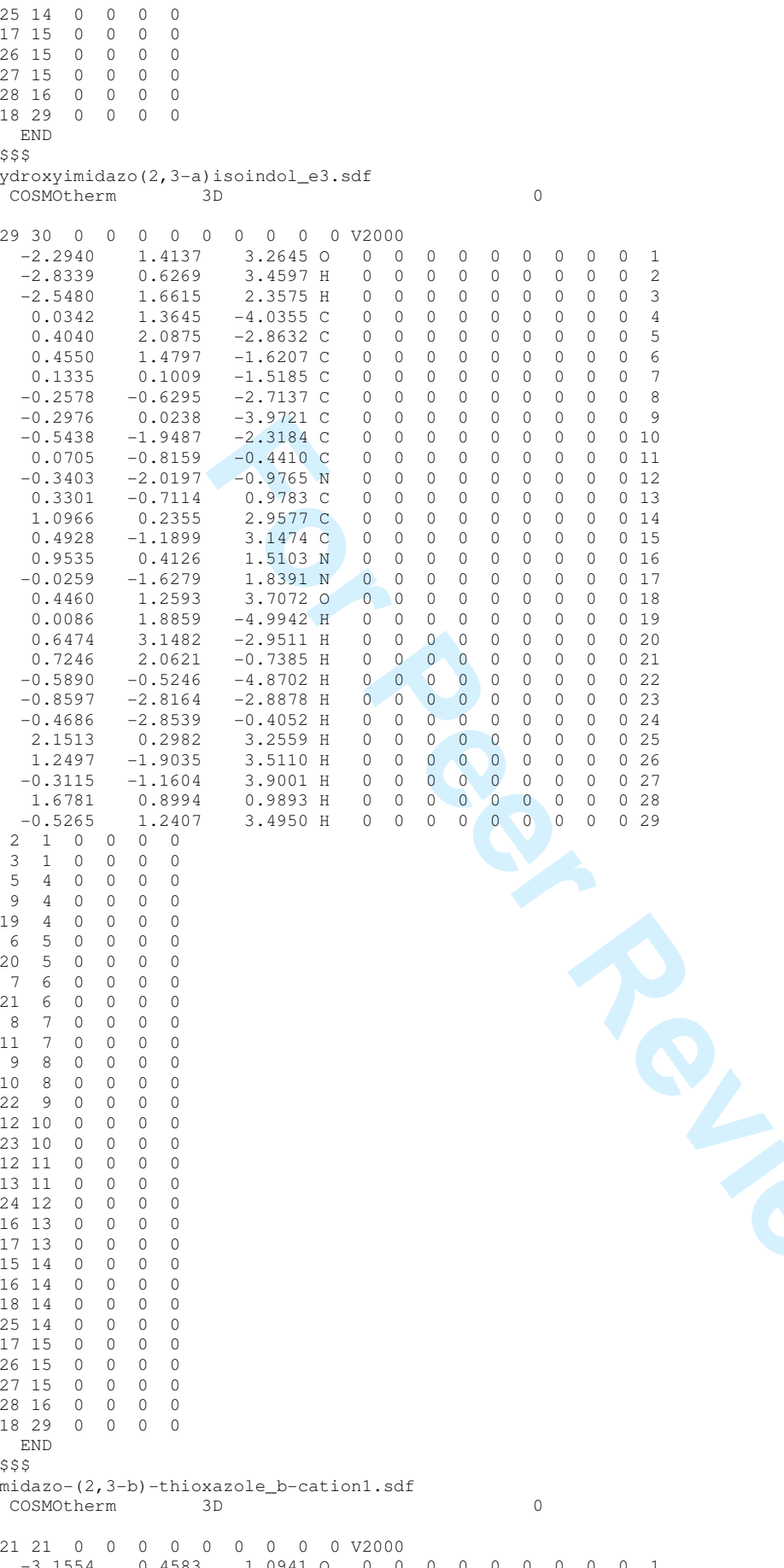

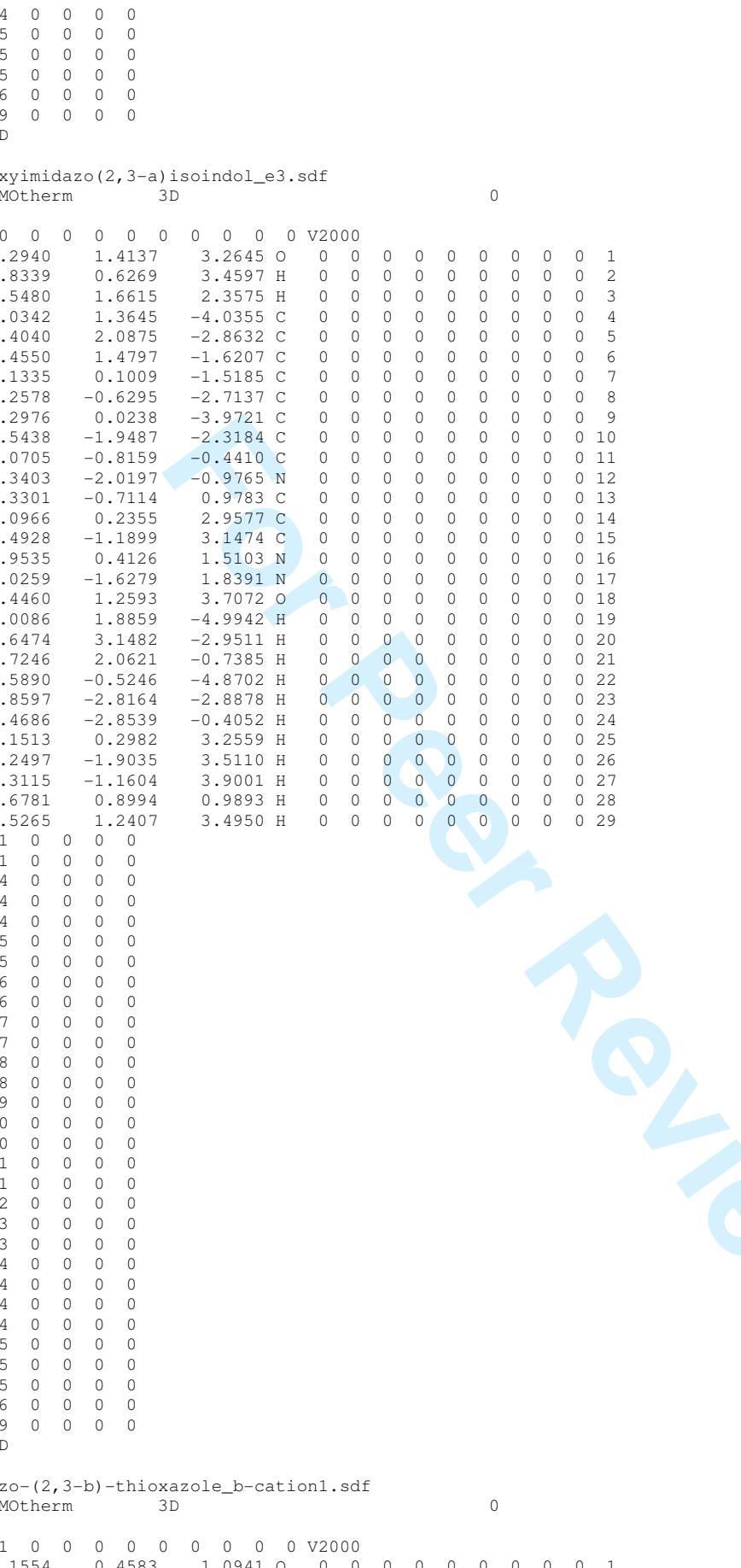

1 END

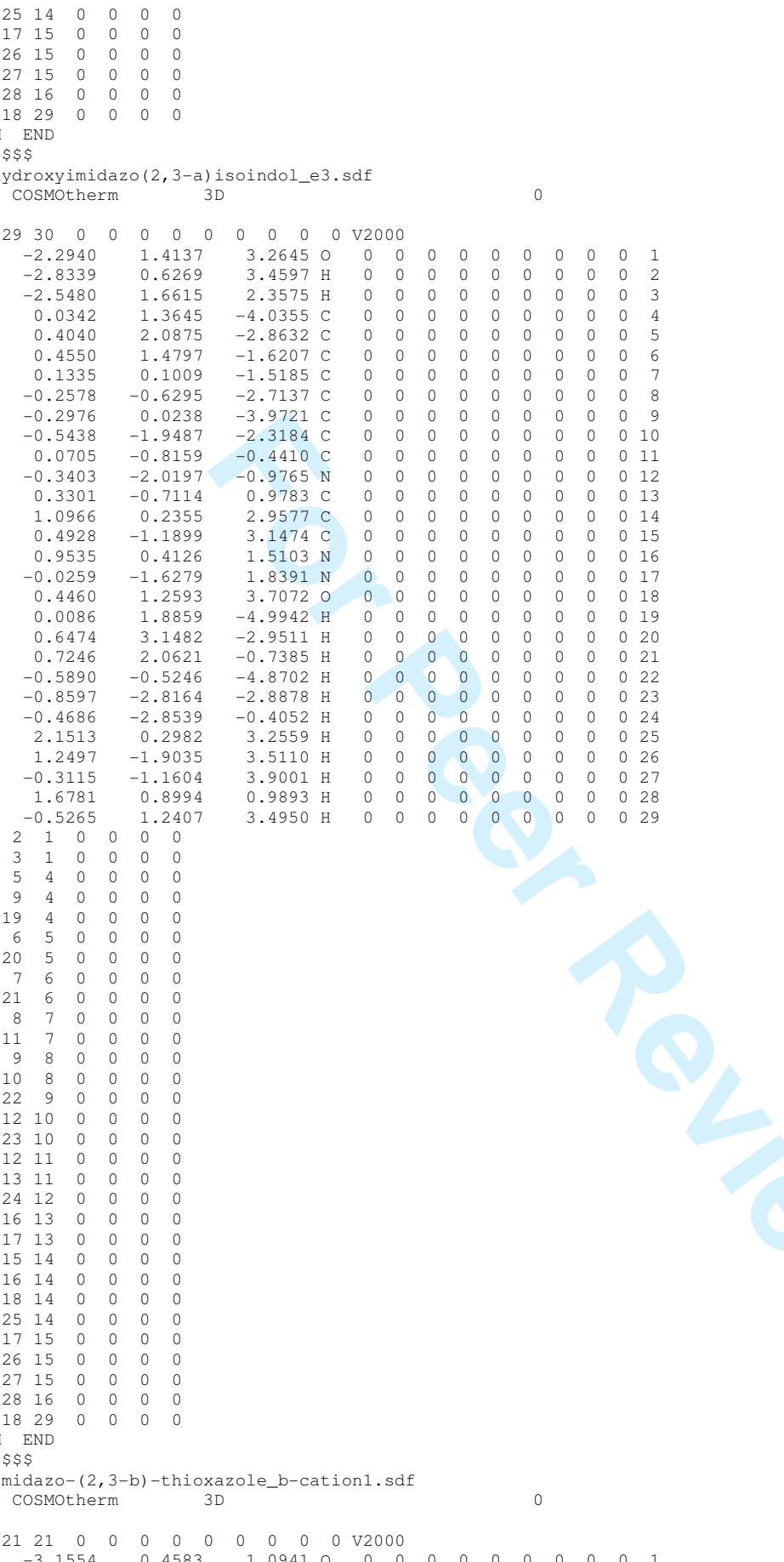

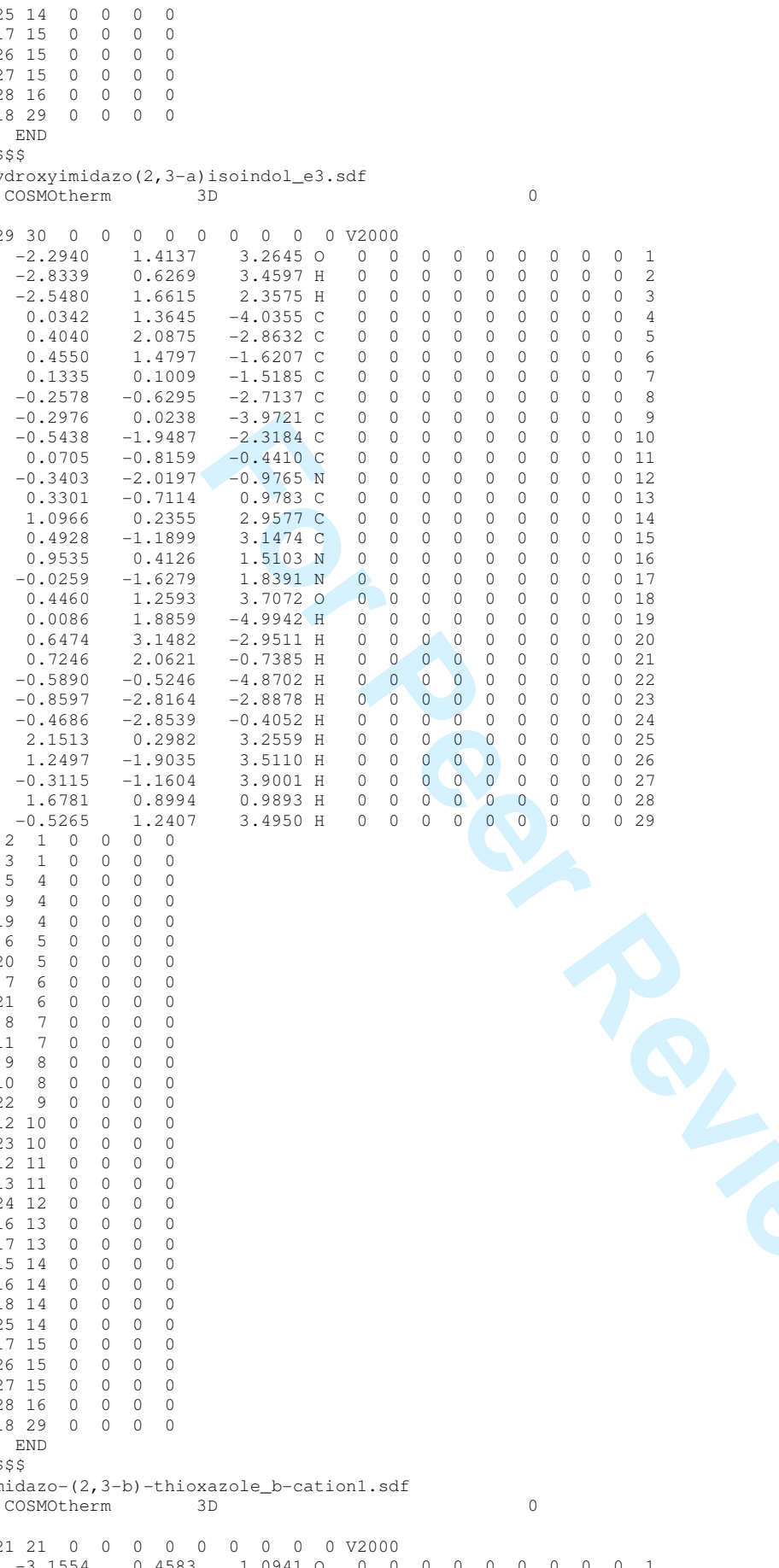

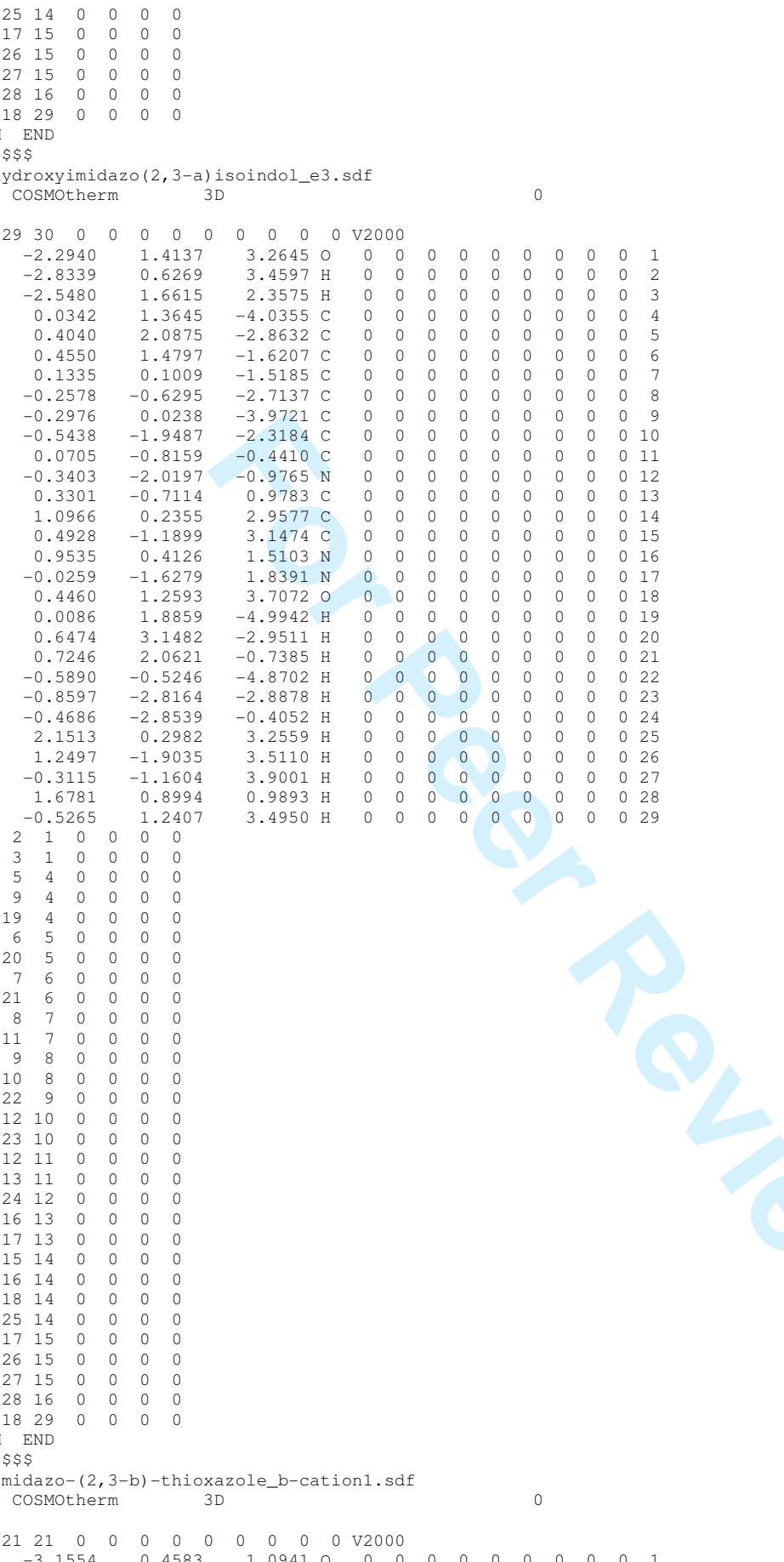

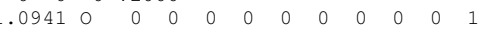

$\begin{array}{llllllllllllll}-3.7273 & -0.2466 & 0.7414 & \mathrm{H} & 0 & 0 & 0 & 0 & 0 & 0 & 0 & 0 & 0 & 2\end{array}$

$\begin{array}{rrrrrrrrrrrrrr}-3.3956 & 1.2528 & 0.5849 & \mathrm{H} & 0 & 0 & 0 & 0 & 0 & 0 & 0 & 0 & 0 & 3 \\ 1.4840 & -0.1992 & 2.9072 & \mathrm{C} & 0 & 0 & 0 & 0 & 0 & 0 & 0 & 0 & 0 & 4\end{array}$

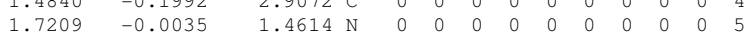

$\begin{array}{rrrrrrrrrrrrrrr}1.70458 & -0.0117 & 3.0132 & \mathrm{C} & 0 & 0 & 0 & 0 & 0 & 0 & 0 & 0 & 0 & 6\end{array}$

$\begin{array}{lllllllllllll}-0.4818 & -0.0779 & 1.6028 \mathrm{~N} & 0 & 0 & 0 & 0 & 0 & 0 & 0 & 0 & 0\end{array}$

$\begin{array}{rrrrrrrrrrrrrr}0.5653 & -0.0241 & 0.7861 & \mathrm{C} & 0 & 0 & 0 & 0 & 0 & 0 & 0 & 0 & 0 & 8 \\ 1.1616 & 0.4131 & -2.8591 & \mathrm{~N} & 0 & 0 & 0 & 0 & 0 & 0 & 0 & 0 & 0 & 9\end{array}$

$\begin{array}{llllllllllllll}1.1616 & 0.4131 & -2.8591 & \mathrm{~N} & 0 & 0 & 0 & 0 & 0 & 0 & 0 & 0 & 0 & \\ 1.4766 & 0.4629 & -1.5283 & \mathrm{C} & 0 & 0 & 0 & 0 & 0 & 0 & 0 & 0 & 0 & 10\end{array}$

$\begin{array}{lllllllllllllll}1.0 .0501 & -0.0636 & -3.0328 \mathrm{C} & 0 & 0 & 0 & 0 & 0 & 0 & 0 & 0 & 0 & 11\end{array}$

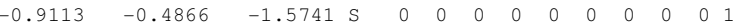

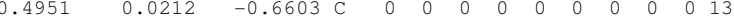

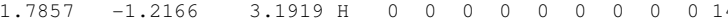

$\begin{array}{llllllllllllll}2.0548 & 0.5266 & 3.4961 & \mathrm{H} & 0 & 0 & 0 & 0 & 0 & 0 & 0 & 0 & 0 & 15\end{array}$

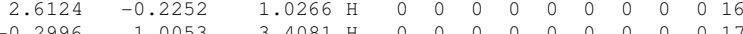

$\begin{array}{llllllllllllll}0.2996 & 1.0053 & 3.4081 & \mathrm{H} & 0 & 0 & 0 & 0 & 0 & 0 & 0 & 0 & 0 & 17\end{array}$

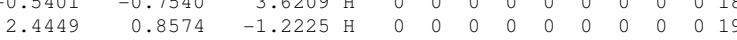
$\begin{array}{ll}-1.4682 & 0.1150\end{array}$

$1.3172 \mathrm{H}$

$\begin{array}{llllll}2 & 1 & 0 & 0 & 0 & 0 \\ 3 & 1 & 0 & 0 & 0 & 0\end{array}$

$\begin{array}{lllll}1 & 0 & 0 & 0 & 0\end{array}$

$\begin{array}{llllll}6 & 4 & 0 & 0 & 0 & 0\end{array}$

$\begin{array}{llllll}4 & 4 & 0 & 0 & 0 & 0\end{array}$

$\begin{array}{llllll}14 & 4 & 0 & 0 & 0 & 0\end{array}$ 
$\begin{array}{rrrrrr}16 & 5 & 0 & 0 & 0 & 0 \\ 7 & 6 & 0 & 0 & 0 & 0 \\ 17 & 6 & 0 & 0 & 0 & 0 \\ 18 & 6 & 0 & 0 & 0 & 0 \\ 8 & 7 & 0 & 0 & 0 & 0 \\ 13 & 8 & 0 & 0 & 0 & 0 \\ 10 & 9 & 0 & 0 & 0 & 0 \\ 11 & 9 & 0 & 0 & 0 & 0 \\ 13 & 10 & 0 & 0 & 0 & 0 \\ 19 & 10 & 0 & 0 & 0 & 0 \\ 12 & 11 & 0 & 0 & 0 & 0 \\ 20 & 11 & 0 & 0 & 0 & 0 \\ 13 & 12 & 0 & 0 & 0 & 0 \\ 7 & 21 & 0 & 0 & 0 & 0 \\ 1\end{array}$ $M \quad E N D$

\$\$\$

imidazo-(2,3-b)-thioxazole_b.sdf

$\begin{array}{llllllllllll}20 & 20 & 0 & 0 & 0 & 0 & 0 & 0 & 0 & 0 & 0 & \mathrm{~V} 2000\end{array}$

$\begin{array}{llllllllllllll}-3.2274 & -1.7469 & 0.2047 & 0 & 0 & 0 & 0 & 0 & 0 & 0 & 0 & 0 & 0 & 1\end{array}$

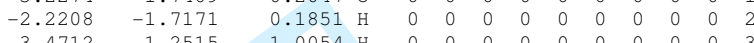

$\begin{array}{rrrrrrrrrrrrrr}-3.4712 & -1.2515 & 1.0054 & \mathrm{H} & 0 & 0 & 0 & 0 & 0 & 0 & 0 & 0 & 0 & 3 \\ 1.7649 & -2.6115 & -0.2912 & \mathrm{C} & 0 & 0 & 0 & 0 & 0 & 0 & 0 & 0 & 0 & 4\end{array}$

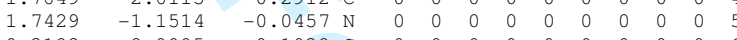

$\begin{array}{llllllllllllll}0.3183 & -2.9995 & 0.1032 & \mathrm{C} & 0 & 0 & 0 & 0 & 0 & 0 & 0 & 0 & 0 & 6\end{array}$

$\begin{array}{llllllllllllll}-0.4566 & -1.7352 & 0.0588 & \mathrm{~N} & 0 & 0 & 0 & 0 & 0 & 0 & 0 & 0 & 0 & 7\end{array}$

$\begin{array}{rrrrrrrrrrrrrr}0.4127 & -0.7693 & -0.0338 & \mathrm{C} & 0 & 0 & 0 & 0 & 0 & 0 & 0 & 0 & 0 & 8 \\ -1.2688 & 2.5596 & -0.3487 & \mathrm{~N} & 0 & 0 & 0 & 0 & 0 & 0 & 0 & 0 & 0 & 9\end{array}$

$\begin{array}{lllllllllllllll}-1.2688 & 2.5596 & -0.3487 & \mathrm{~N} & 0 & 0 & 0 & 0 & 0 & 0 & 0 & 0 & 0 & 8 \\ -1.1872 & 1.1891 & -0.3913 & \mathrm{C} & 0 & 0 & 0 & 0 & 0 & 0 & 0 & 0 & 0 & 10 \\ -0.1191 & 3.0860 & 0.0015 & \mathrm{C} & 0 & 0 & 0 & 0 & 0 & 0 & 0 & 0 & 0 & 11\end{array}$

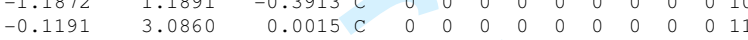

$\begin{array}{llllllllllllll}1.1628 & 1.9352 & 0.3122 & \mathrm{~S} & 0 & 0 & 0 & 0 & 0 & 0 & 0 & 0 & 0 & 12\end{array}$

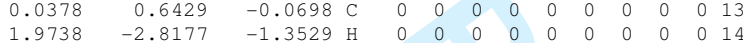

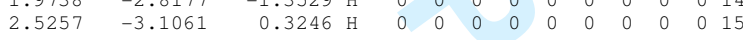

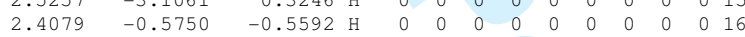

$\begin{array}{llllllllllllll}0.2752 & -3.4022 & 1.1288 & \mathrm{H} & 0 & 0 & 0 & 0 & 0 & 0 & 0 & 0 & 0 & 17\end{array}$

$\begin{array}{lllllllllllll}-0.1221 & -3.7490 & -0.5676 \mathrm{H} & 0 & 0 & 0 & 0 & 0 & 0 & 0 & 0 & 0 & 18\end{array}$

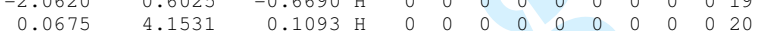

$\begin{array}{llllll}2 & 1 & 0 & 0 & 0 & 0\end{array}$

$\begin{array}{lllllll}3 & 1 & 0 & 0 & 0 & 0\end{array}$

$\begin{array}{llllll}5 & 4 & 0 & 0 & 0 & 0\end{array}$

$\begin{array}{llllll}4 & 4 & 0 & 0 & 0 & 0\end{array}$

$\begin{array}{lllllll}5 & 4 & 0 & 0 & 0 & 0\end{array}$

$\begin{array}{lllllll}6 & 5 & 0 & 0 & 0 & 0\end{array}$

$\begin{array}{llllll}7 & 6 & 0 & 0 & 0 & 0\end{array}$

$\begin{array}{llllll}17 & 6 & 0 & 0 & 0 & 0 \\ 18 & 6 & 0 & 0 & 0 & 0\end{array}$

$\begin{array}{lllll}8 & 7 & 0 & 0 & 0\end{array}$

$\begin{array}{llllll}13 & 8 & 0 & 0 & 0 & 0 \\ 10 & 9 & 0 & 0 & 0 & 0\end{array}$

$\begin{array}{llllll}1 & 9 & 0 & 0 & 0\end{array}$

$\begin{array}{llllll}3 & 10 & 0 & 0 & 0 & 0 \\ 9 & 10 & 0 & 0 & 0 & 0\end{array}$

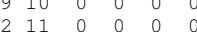

$\begin{array}{llllll}3 & 12 & 0 & 0 & 0 & 0 \\ 1 & 20 & 0 & 0 & 0 & 0\end{array}$

END

$\$ \$ \$$

midazole-cation.sdf

$\begin{array}{llllllllllll}13 & 12 & 0 & 0 & 0 & 0 & 0 & 0 & 0 & 0 & 0 & \mathrm{~V} 2000\end{array}$

$\begin{array}{rrrrrrrrrrrrrr}-3.0497 & 0.0014 & -0.0593 & 0 & 0 & 0 & 0 & 0 & 0 & 0 & 0 & 0 & 0 & 1 \\ -3.4650 & -0.7808 & 0.3480 & \mathrm{H} & 0 & 0 & 0 & 0 & 0 & 0 & 0 & 0 & 0 & 2\end{array}$

$\begin{array}{llllllllllllll}-3.4666 & 0.7619 & 0.3856 & \mathrm{H} & 0 & 0 & 0 & 0 & 0 & 0 & 0 & 0 & 0 & 3 \\ -0.4820 & -0.0005 & 1.1468 & \mathrm{C} & 0 & 0 & 0 & 0 & 0 & 0 & 0 & 0 & 0 & 4\end{array}$

$\begin{array}{llllllllllllll}-0.4820 & -0.0005 & 1.1468 & \mathrm{C} & 0 & 0 & 0 & 0 & 0 & 0 & 0 & 0 & 0 & 4 \\ -0.3294 & -0.0002 & 0.0268 & \mathrm{~N} & 0 & 0 & 0 & 0 & 0 & 0 & 0 & 0 & 0 & 5\end{array}$

$\begin{array}{llllllllllllll}1.7775 & -0.0002 & 0.7025 & \mathrm{C} & 0 & 0 & 0 & 0 & 0 & 0 & 0 & 0 & 0 & 6\end{array}$

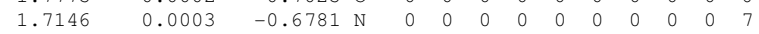

$\begin{array}{llllllllllllll}0.4329 & 0.0004 & -1.0728 & \mathrm{C} & 0 & 0 & 0 & 0 & 0 & 0 & 0 & 0 & 0 & 8\end{array}$

$\begin{array}{lllllllllllll}0.077 & -0.0007 & 2.1511 & \mathrm{H} & 0 & 0 & 0 & 0 & 0 & 0 & 0 & 0 & 0\end{array}$

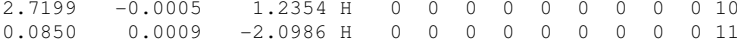

$\begin{array}{llllllllllllll}2.5138 & 0.0006 & -1.3090 & \mathrm{H} & 0 & 0 & 0 & 0 & 0 & 0 & 0 & 0 & 0 & 12\end{array}$

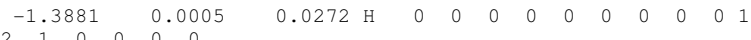

$\begin{array}{llllll}2 & 1 & 0 & 0 & 0 & 0 \\ 3 & 1 & 0 & 0 & 0 & 0\end{array}$

$\begin{array}{llllll}3 & 1 & 0 & 0 & 0 & 0 \\ 5 & 4 & 0 & 0 & 0 & 0\end{array}$

$\begin{array}{lllll}4 & 0 & 0 & 0\end{array}$

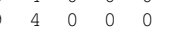

$\begin{array}{lllllll}5 & 5 & 0 & 0 & 0 & 0 & 2 \\ 7 & 6 & 0 & 0 & 0\end{array}$

$\begin{array}{llllll}6 & 0 & 0 & 0 & 0\end{array}$

$\begin{array}{llll}0 & 0 & 0\end{array}$

$\begin{array}{llllll}2 & 7 & 0 & 0 & 0 & 0 \\ 1 & 8 & 0 & 0 & 0 & 0\end{array}$

$\begin{array}{llllll}5 & 13 & 0 & 0 & 0 & 0\end{array}$

$\$$ END

imidazole.sdf

cosmotherm

$\begin{array}{llllllllllll}12 & 11 & 0 & 0 & 0 & 0 & 0 & 0 & 0 & 0 & 0 & \mathrm{~V} 2000\end{array}$

$\begin{array}{llllllllllllll}-3.1604 & -0.0441 & 0.0048 & 0 & 0 & 0 & 0 & 0 & 0 & 0 & 0 & 0 & 0 & 1\end{array}$

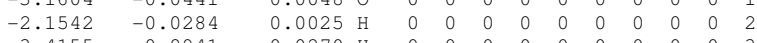

$\begin{array}{lllllllllllll}-3.4155 & 0.8941 & 0.0270 & \mathrm{H} & 0 & 0 & 0 & 0 & 0 & 0 & 0 & 0 & 0\end{array}$

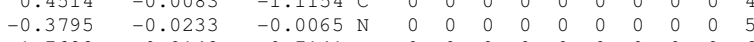

$\begin{array}{llllllllllllll}1.7688 & 0.0142 & -0.7141 & \mathrm{C} & 0 & 0 & 0 & 0 & 0 & 0 & 0 & 0 & 0 & 6\end{array}$

$\begin{array}{rrrrrrrrrrrrrr}1.7310 & 0.0127 & 0.6659 & \mathrm{~N} & 0 & 0 & 0 & 0 & 0 & 0 & 0 & 0 & 0 & 7 \\ 0.4268 & -0.0098 & 1.0524 & \mathrm{C} & 0 & 0 & 0 & 0 & 0 & 0 & 0 & 0 & 0 & 8\end{array}$ 


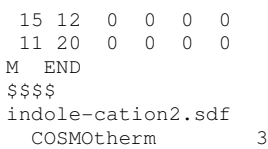

$\begin{array}{llllllllllll}20 & 20 & 0 & 0 & 0 & 0 & 0 & 0 & 0 & 0 & 0 & V 2000\end{array}$

$\begin{array}{cccccccccccccccc}20 & 20 & 0 & 0 & 0 & 0 & 0 & 0 & \mathrm{~V} 2000 \\ -3.5645 & -0.0006 & 1.2070 & 0 & 0 & 0 & 0 & 0 & 0 & 0 & 0 & 0 & 0 & 1 \\ -4.1290 & -0.7873 & 1.0935 & \mathrm{H} & 0 & 0 & 0 & 0 & 0 & 0 & 0 & 0 & 0 & 2\end{array}$

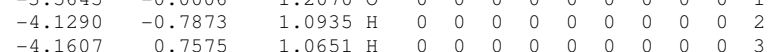

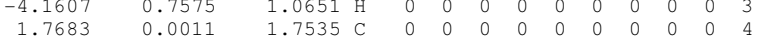

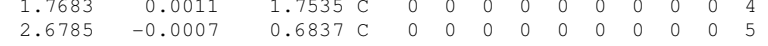

$\begin{array}{llllllllllllll}2.2415 & -0.0028 & -0.6499 & \mathrm{C} & 0 & 0 & 0 & 0 & 0 & 0 & 0 & 0 & 0 & 6\end{array}$

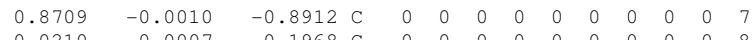

$\begin{array}{rrrrrrrrrrrrrr}-0.0210 & 0.0007 & 0.1968 & \mathrm{C} & 0 & 0 & 0 & 0 & 0 & 0 & 0 & 0 & 0 & 8 \\ 0.3864 & 0.0026 & 1.5278 & \mathrm{C} & 0 & 0 & 0 & 0 & 0 & 0 & 0 & 0 & 0 & 9\end{array}$

$\begin{array}{rrrrrrrrrrrrrr}0.3864 & 0.0026 & 1.5278 & \mathrm{C} & 0 & 0 & 0 & 0 & 0 & 0 & 0 & 0 & 0 & 9 \\ -1.3436 & 0.0011 & -0.3248 & \mathrm{~N} & 0 & 0 & 0 & 0 & 0 & 0 & 0 & 0 & 0 & 10\end{array}$

$\begin{array}{rrrrrrrrrrrrrr}-1.3436 & 0.0011 & -0.3248 & \mathrm{~N} & 0 & 0 & 0 & 0 & 0 & 0 & 0 & 0 & 0 & 10 \\ 0.0503 & 0.0007 & -2.1526 & \mathrm{C} & 0 & 0 & 0 & 0 & 0 & 0 & 0 & 0 & 0 & 11\end{array}$

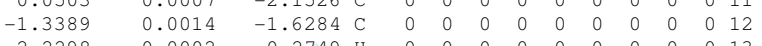

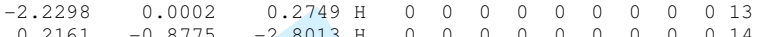

$\begin{array}{rrrrrrrrrrrrrr}0.2161 & -0.8775 & -2.8013 & \mathrm{H} & 0 & 0 & 0 & 0 & 0 & 0 & 0 & 0 & 0 & 14 \\ 0.2184 & 0.8787 & -2.8015 & \mathrm{H} & 0 & 0 & 0 & 0 & 0 & 0 & 0 & 0 & 0 & 15\end{array}$

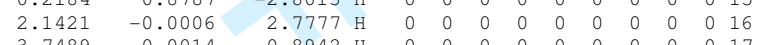

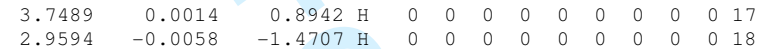

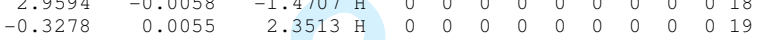

$\begin{array}{lllllllllllllll}-2.2632 & 0.0009 & -2.2027 & \mathrm{H} & 0 & 0 & 0 & 0 & 0 & 0 & 0 & 0 & 0 & 20\end{array}$

$\begin{array}{lllllll}2 & 1 & 0 & 0 & 0 & 0\end{array}$

$\begin{array}{llllll}3 & 1 & 0 & 0 & 0 & 0 \\ 5 & 4 & 0 & 0 & 0 & 0\end{array}$

$\begin{array}{llllll}5 & 4 & 0 & 0 & 0 & 0 \\ 9 & 4 & 0 & 0 & 0 & 0\end{array}$

$\begin{array}{llllll}16 & 4 & 0 & 0 & 0 & 0\end{array}$

$\begin{array}{rrrrrr}6 & 5 & 0 & 0 & 0 & 0 \\ 17 & 5 & 0 & 0 & 0 & 0\end{array}$

$\begin{array}{llllll}7 & 6 & 0 & 0 & 0 & 0\end{array}$

$\begin{array}{ccccccc}18 & 6 & 0 & 0 & 0 & 0 \\ 8 & 7 & 0 & 0 & 0 & 0\end{array}$

$\begin{array}{rrrrrrr}8 & 7 & 0 & 0 & 0 & 0 \\ 11 & 7 & 0 & 0 & 0 & 0\end{array}$

$\begin{array}{rrrrrrr}9 & 8 & 0 & 0 & 0 & 0 \\ 10 & 8 & 0 & 0 & 0 & 0\end{array}$

$\begin{array}{llllll}10 & 8 & 0 & 0 & 0 & 0 \\ 19 & 9 & 0 & 0 & 0 & 0\end{array}$

$\begin{array}{lllllll}12 & 10 & 0 & 0 & 0 & 0\end{array}$

$\begin{array}{llllll}13 & 10 & 0 & 0 & 0 & 0\end{array}$

$1211 \quad 000000$

$\begin{array}{llllll}14 & 11 & 0 & 0 & 0 & 0 \\ 15 & 11 & 0 & 0 & 0 & 0\end{array}$

$\begin{array}{lllllll}2 & 20 & 0 & 0 & 0 & 0\end{array}$

M END

\$\$\$

ndole.sdf

$\begin{array}{llllllllllll}19 & 19 & 0 & 0 & 0 & 0 & 0 & 0 & 0 & 0 & 0 & \mathrm{~V} 2000\end{array}$

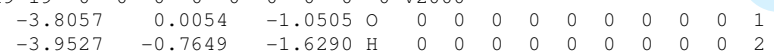

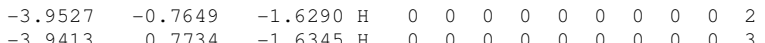

$\begin{array}{rrrrrrrrrrrrrr}-3.9413 & 0.7734 & -1.6345 & \mathrm{H} & 0 & 0 & 0 & 0 & 0 & 0 & 0 & 0 & 0 & 3 \\ -0.0409 & -0.0029 & -0.1758 & \mathrm{C} & 0 & 0 & 0 & 0 & 0 & 0 & 0 & 0 & 0 & 4\end{array}$

$\begin{array}{llllllllllllll}-1.3072 & -0.0047 & 0.3754 & \mathrm{~N} & 0 & 0 & 0 & 0 & 0 & 0 & 0 & 0 & 0 & 5\end{array}$

$\begin{array}{llllll}0 & 0 & 0 & 0\end{array}$

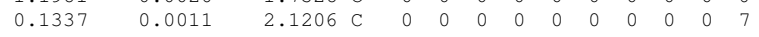

$\begin{array}{llllllllllllll}0.9013 & 0.0011 & 0.9058 & \mathrm{C} & 0 & 0 & 0 & 0 & 0 & 0 & 0 & 0 & 0 & 8\end{array}$

$\begin{array}{llllllllllllll}0.3653 & -0.0043 & -1.5188 & \mathrm{C} & 0 & 0 & 0 & 0 & 0 & 0 & 0 & 0 & 0 & 9\end{array}$

$\begin{array}{llllllllllllll}1.7359 & -0.0013 & -1.7803 & \mathrm{C} & 0 & 0 & 0 & 0 & 0 & 0 & 0 & 0 & 0 & 10\end{array}$

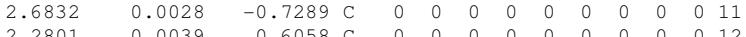

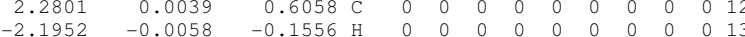

$\begin{array}{llllllllllllll}-2.0913 & -0.0034 & 2.3713 & \mathrm{H} & 0 & 0 & 0 & 0 & 0 & 0 & 0 & 0 & 0 & 14\end{array}$

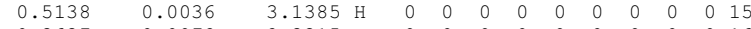

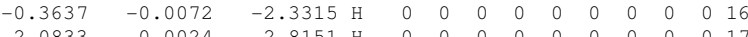

$\begin{array}{llllllllllllllll}3.7478 & 0.0051 & -0.9712 \mathrm{H} & 0 & 0 & 0 & 0 & 0 & 0 & 0 & 0 & 0 & 0 & 18\end{array}$

$\begin{array}{llllllllllllllll}3.0213 & 0.0069 & 1.4083 \mathrm{H} & 0 & 0 & 0 & 0 & 0 & 0 & 0 & 0 & 0 & 18\end{array}$

$\begin{array}{llll}2 & 1 & 0 & 0 \\ 3 & 0 & 0 & 0\end{array}$

$\begin{array}{lllllll}2 & 1 & 0 & 0 & 0 & 0 \\ 3 & 1 & 0 & 0 & 0 & 0\end{array}$

$\begin{array}{llllll}3 & 1 & 0 & 0 & 0 & 0 \\ 5 & 4 & 0 & 0 & 0 & 0\end{array}$

$\begin{array}{llllll}8 & 4 & 0 & 0 & 0\end{array}$

$\begin{array}{lllll}5 & 0 & 0 & 0 & 0\end{array}$

$\begin{array}{llllll}5 & 0 & 0 & 0 & 0\end{array}$

$\begin{array}{lllll}6 & 0 & 0 & 0 & 0\end{array}$

$\begin{array}{cccccc}14 & 6 & 0 & 0 & 0 & 0 \\ 8 & 7 & 0 & 0 & 0 & 0\end{array}$

$\begin{array}{llllll}5 & 7 & 0 & 0 & 0 & 0\end{array}$

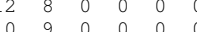

$\begin{array}{lllll}16 & 0 & 0 & 0\end{array}$

$\begin{array}{llllll}7 & 10 & 0 & 0 & 0\end{array}$

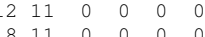

$\begin{array}{llllll}12 & 19 & 0 & 0 & 0 & 0\end{array}$

END

so-propylamine-cation.sdf

cosmotherm 3D

$\begin{array}{llllllllllll}17 & 15 & 0 & 0 & 0 & 0 & 0 & 0 & 0 & 0 & 0 & \mathrm{~V} 2000\end{array}$

$\begin{array}{llllllllllllll}-2.4596 & -0.4111 & -0.0012 & 0 & 0 & 0 & 0 & 0 & 0 & 0 & 0 & 0 & 0 & 1 \\ -3.0174 & -0.2708 & -0.7881 & \mathrm{H} & 0 & 0 & 0 & 0 & 0 & 0 & 0 & 0 & 0 & 2\end{array}$

$\begin{array}{llllllllllllll}-3.0507 & -0.2290 & 0.7522 & \mathrm{H} & 0 & 0 & 0 & 0 & 0 & 0 & 0 & 0 & 0 & 3\end{array}$

$\begin{array}{llllllllllllll}1.0978 & 0.2901 & 0.0007 & \mathrm{C} & 0 & 0 & 0 & 0 & 0 & 0 & 0 & 0 & 0 & 4\end{array}$

$\begin{array}{rrrrrrrrrrrrrr}-0.1520 & 1.1497 & 0.0198 & \mathrm{~N} & 0 & 0 & 0 & 0 & 0 & 0 & 0 & 0 & 0 & 5 \\ 1.1293 & -0.5585 & 1.2666 & \mathrm{C} & 0 & 0 & 0 & 0 & 0 & 0 & 0 & 0 & 0 & 6 \\ 1.1165 & -0.5273 & -1.2858 & \mathrm{C} & 0 & 0 & 0 & 0 & 0 & 0 & 0 & 0 & 0 & 7\end{array}$ 


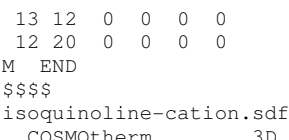

$\begin{array}{llllllllllll}21 & 21 & 0 & 0 & 0 & 0 & 0 & 0 & 0 & 0 & 0 & \mathrm{~V} 2000\end{array}$

$\begin{array}{ccccccccccccccc}21 & 1.4219 & 0.0021 & 0.7252 & 0 & 0 & 0 & 0 & 0 & 0 & 0 & 0 & 0 & 0 & 1 \\ -4.9554 & -0.7444 & 0.3963 & \mathrm{H} & 0 & 0 & 0 & 0 & 0 & 0 & 0 & 0 & 0 & 2\end{array}$

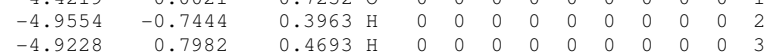

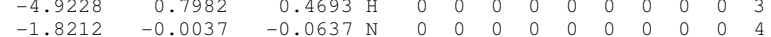

$\begin{array}{lllllllllllllll}-1.8212 & -0.0037 & -0.0637 & \mathrm{~N} & 0 & 0 & 0 & 0 & 0 & 0 & 0 & 0 & 0 & 4 \\ -1.4970 & -0.0018 & -1.3959 & \mathrm{C} & 0 & 0 & 0 & 0 & 0 & 0 & 0 & 0 & 0 & 5\end{array}$

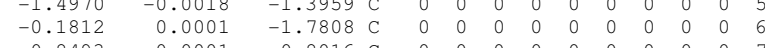

$\begin{array}{rrrrrrrrrrrrrr}0.8493 & 0.0001 & -0.8016 & \mathrm{C} & 0 & 0 & 0 & 0 & 0 & 0 & 0 & 0 & 0 & 7 \\ 0.4695 & -0.0018 & 0.5875 & \mathrm{C} & 0 & 0 & 0 & 0 & 0 & 0 & 0 & 0 & 0 & 8\end{array}$

$\begin{array}{rrrrrrrrrrrrrr}0.4695 & -0.0018 & 0.5875 & \mathrm{C} & 0 & 0 & 0 & 0 & 0 & 0 & 0 & 0 & 0 & 8 \\ -0.9019 & -0.0040 & 0.9024 & \mathrm{C} & 0 & 0 & 0 & 0 & 0 & 0 & 0 & 0 & 0 & 9\end{array}$

$\begin{array}{rrrrrrrrrrrrrr}-0.9019 & -0.0040 & 0.9024 & C & 0 & 0 & 0 & 0 & 0 & 0 & 0 & 0 & 0 & 9 \\ 2.2281 & 0.0025 & -1.1312 & C & 0 & 0 & 0 & 0 & 0 & 0 & 0 & 0 & 0 & 10 \\ 3.1784 & 0.0027 & -0.1268 & & 0 & 0 & 0 & 0 & 0 & 0 & 0 & 0 & 0 & 11\end{array}$

$\begin{array}{lllllllllllllll}3.1784 & 0.0027 & -0.1268 & \mathrm{C} & 0 & 0 & 0 & 0 & 0 & 0 & 0 & 0 & 0 & 11\end{array}$

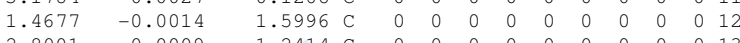

$\begin{array}{llllllllllllll}2.8001 & 0.0009 & 1.2414 & \mathrm{C} & 0 & 0 & 0 & 0 & 0 & 0 & 0 & 0 & 0 & 13\end{array}$

$\begin{array}{rrrrrrrrrrrrrr}-2.3330 & -0.0015 & -2.0913 & \mathrm{H} & 0 & 0 & 0 & 0 & 0 & 0 & 0 & 0 & 0 & 14 \\ 0.0679 & 0.0016 & -2.8416 & \mathrm{H} & 0 & 0 & 0 & 0 & 0 & 0 & 0 & 0 & 0 & 15\end{array}$

$\begin{array}{rrrrrrrrrrrrr}-1.2665 & -0.0058 & 1.9291 \mathrm{H} & 0 & 0 & 0 & 0 & 0 & 0 & 0 & 0 & 0 & 16\end{array}$

$\begin{array}{lllllllllllll}2.5252 & 0.0039 & -2.1808 \mathrm{H} & 0 & 0 & 0 & 0 & 0 & 0 & 0 & 0 & 0 & 17\end{array}$

$\begin{array}{llllllllllllll}4.2382 & 0.0045 & -0.3858 & \mathrm{H} & 0 & 0 & 0 & 0 & 0 & 0 & 0 & 0 & 0 & 18\end{array}$

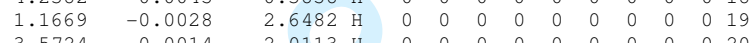

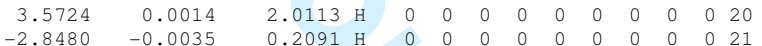

$\begin{array}{llllll}2 & 1 & 0 & 0 & 0 & 0\end{array}$

$\begin{array}{llllll}3 & 1 & 0 & 0 & 0 & 0\end{array}$

$\begin{array}{llllll}5 & 4 & 0 & 0 & 0 & 0 \\ 9 & 4 & 0 & 0 & 0 & 0\end{array}$

$\begin{array}{lllllll}6 & 4 & 0 & 0 & 0 & 0 \\ 6 & 5 & 0 & 0 & 0\end{array}$

1450500000

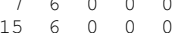

$\begin{array}{rrrrrr}8 & 7 & 0 & 0 & 0 & 0 \\ 10 & 7 & 0 & 0 & 0 & 0\end{array}$

$\begin{array}{lll}0 & 0 \\ 0 & 0 & 0\end{array}$

$\begin{array}{rrrrrr}9 & 8 & 0 & 0 & 0 \\ 12 & 8 & 0 & 0 & 0\end{array}$

$\begin{array}{llllll}16 & 9 & 0 & 0 & 0 & 0\end{array}$

$\begin{array}{llllll}11 & 10 & 0 & 0 & 0 & 0\end{array}$

$\begin{array}{llllll}17 & 10 & 0 & 0 & 0 & 0\end{array}$

$\begin{array}{llllll}13 & 11 & 0 & 0 & 0 & 0 \\ 18 & 11 & 0 & 0 & 0 & 0\end{array}$

$\begin{array}{llllll}13 & 12 & 0 & 0 & 0 & 0\end{array}$

$\begin{array}{llllll}19 & 12 & 0 & 0 & 0 & 0 \\ 20 & 13 & 0 & 0 & 0 & 0\end{array}$

$\begin{array}{lll}0 & 0 & 0\end{array}$

END

melamine.sdf

Cosmotherm

$\begin{array}{llllllllllll}18 & 17 & 0 & 0 & 0 & 0 & 0 & 0 & 0 & 0 & 0 & \text { v2 } 2000\end{array}$

$\begin{array}{rrrrrrrrrrrrr}-3.6154 & 0.0731 & 0.0149 & 0 & 0 & 0 & 0 & 0 & 0 & 0 & 0 & 0 & 0 \\ -2.6488 & 0.0436 & -0.2507 & \mathrm{H} & 0 & 0 & 0 & 0 & 0 & 0 & 0 & 0 & 0\end{array}$

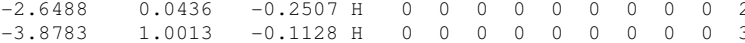

$\begin{array}{lllllllllllll}0.9759 & 0.0077 & 1.3137 & \mathrm{~N} & 0 & 0 & 0 & 0 & 0 & 0 & 0 & 0 & 0\end{array}$

$\begin{array}{llllllllllllll}-0.3367 & -0.0445 & 1.0069 & \mathrm{C} & 0 & 0 & 0 & 0 & 0 & 0 & 0 & 0 & 0 & 5\end{array}$

$\begin{array}{llllllllllllll}-0.8497 & -0.0642 & -0.2497 & \mathrm{~N} & 0 & 0 & 0 & 0 & 0 & 0 & 0 & 0 & 0 & 6\end{array}$

$\begin{array}{llllllllllllll}0.0748 & -0.0303 & -1.2387 & \mathrm{C} & 0 & 0 & 0 & 0 & 0 & 0 & 0 & 0 & 0 & 7\end{array}$

$\begin{array}{llllllllllllll}1.4110 & 0.0207 & -1.0580 & \mathrm{~N} & 0 & 0 & 0 & 0 & 0 & 0 & 0 & 0 & 0 & 8 \\ 1.7973 & 0.0343 & 0.2386 & \mathrm{C} & 0 & 0 & 0 & 0 & 0 & 0 & 0 & 0 & 0 & 9\end{array}$

$\begin{array}{rrrrrrrrrrrrrr}1.7973 & 0.0343 & 0.2386 & \mathrm{C} & 0 & 0 & 0 & 0 & 0 & 0 & 0 & 0 & 0 & 9 \\ 3.1304 & 0.0516 & 0.4831 & \mathrm{~N} & 0 & 0 & 0 & 0 & 0 & 0 & 0 & 0 & 0 & 10\end{array}$

$\begin{array}{llllllllllllll}-0.3796 & -0.0779 & -2.5140 & \mathrm{~N} & 0 & 0 & 0 & 0 & 0 & 0 & 0 & 0 & 0 & 11\end{array}$

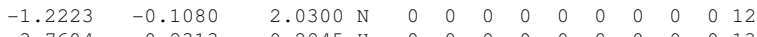

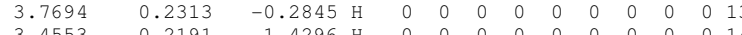

$\begin{array}{rrrrrrrrrrrrrrr}3.4553 & 0.2191 & 1.4296 & \mathrm{H} & 0 & 0 & 0 & 0 & 0 & 0 & 0 & 0 & 0 & 14 \\ -1.3712 & 0.0342 & -2.6959 & \mathrm{H} & 0 & 0 & 0 & 0 & 0 & 0 & 0 & 0 & 0 & 15\end{array}$

$\begin{array}{lllllllllllll}0.2703 & 0.0976 & -3.2732 \mathrm{H} & 0 & 0 & 0 & 0 & 0 & 0 & 0 & 0 & 0 & 16\end{array}$

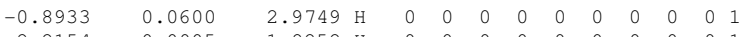

$-2.2154 \quad 0.0005$

$\begin{array}{llllll}2 & 1 & 0 & 0 & 0 & 0 \\ 3 & 1 & 0 & 0 & 0 & 0\end{array}$

5400000

$\begin{array}{lllll}4 & 0 & 0 & 0 & 0\end{array}$

$\begin{array}{lllll}5 & 0 & 0 & 0 & 0\end{array}$

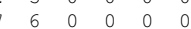

$\begin{array}{llllll}8 & 7 & 0 & 0 & 0\end{array}$

$\begin{array}{rrrrrr}11 & 7 & 0 & 0 & 0 & 0 \\ 9 & 8 & 0 & 0 & 0 & 0\end{array}$

$\begin{array}{llllll}0 & 9 & 0 & 0 & 0\end{array}$

$\begin{array}{llllll}13 & 10 & 0 & 0 & 0 & 0\end{array}$

$\begin{array}{llllll}14 & 10 & 0 & 0 & 0 & 0\end{array}$

$\begin{array}{llllll}5 & 11 & 0 & 0 & 0 & 0\end{array}$

$\begin{array}{llllll}6 & 11 & 0 & 0 & 0 & 0 \\ 17 & 12 & 0 & 0 & 0 & 0\end{array}$

$\begin{array}{llllll}2 & 18 & 0 & 0 & 0 & 0\end{array}$

END

melamine_p2.sdf

cosmotherm 3D

$\begin{array}{llllllllllll}19 & 18 & 0 & 0 & 0 & 0 & 0 & 0 & 0 & 0 & 0 & \mathrm{~V} 2000\end{array}$

$\begin{array}{llllllllllllll}-3.5159 & -0.0198 & 0.0039 & 0 & 0 & 0 & 0 & 0 & 0 & 0 & 0 & 0 & 0 & 1\end{array}$

$\begin{array}{llllllllllllll}-4.0535 & 0.2262 & -0.7708 & \mathrm{H} & 0 & 0 & 0 & 0 & 0 & 0 & 0 & 0 & 0 & 2\end{array}$

$\begin{array}{lllllllllllll}-4.0507 & 0.2321 & 0.7787 & \mathrm{H} & 0 & 0 & 0 & 0 & 0 & 0 & 0 & 0 & 0\end{array}$

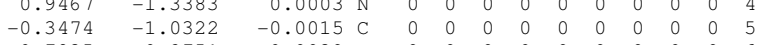

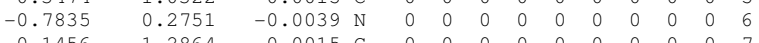

$\begin{array}{rrrrrrrrrrrrrr}0.1456 & 1.2864 & -0.0015 & \mathrm{C} & 0 & 0 & 0 & 0 & 0 & 0 & 0 & 0 & 0 & 7 \\ 1.4518 & 1.0339 & 0.0003 & \mathrm{~N} & 0 & 0 & 0 & 0 & 0 & 0 & 0 & 0 & 0 & 8\end{array}$ 


\begin{tabular}{|c|c|c|c|c|c|c|c|c|c|c|c|c|}
\hline 7994 & -0.2799 & 0.0009 & C & 0 & 0 & & & 0 & & 0 & 0 & 0 \\
\hline 3.1101 & -0.5583 & 0.0022 & & 0 & & 0 & 0 & & & & & $\begin{array}{ll}0 & 10\end{array}$ \\
\hline-1.2845 & -1.9836 & -0.0022 & $\mathrm{~N}$ & 0 & 0 & 0 & 0 & 0 & 0 & 0 & 0 & $\begin{array}{lll}0 & 11\end{array}$ \\
\hline-0.2987 & 2.5466 & 0.0011 & & & & 0 & 0 & 0 & & & & $0 \quad 12$ \\
\hline 3.7921 & 0.1929 & 0.0033 & $\mathrm{H}$ & & & 0 & 0 & 0 & 0 & U & 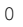 & $\begin{array}{ll}0 & 13\end{array}$ \\
\hline 3.4280 & -1.5218 & 0.0032 & & & & 0 & 0 & 0 & & & & $0 \quad 14$ \\
\hline-1.0019 & -2.9584 & 0.0019 & & & & & & & & & & \\
\hline-2.2758 & -1.7369 & -0.0013 & & & & & & & & 0 & & 016 \\
\hline-1.2877 & 2.7741 & -0.0138 & $\mathrm{H}$ & & & & & & & & 0 & 0 \\
\hline 0.3749 & 3.3060 & -0.0050 & & & & 0 & & & & & & \\
\hline & & & & & & & & & & & & \\
\hline
\end{tabular}




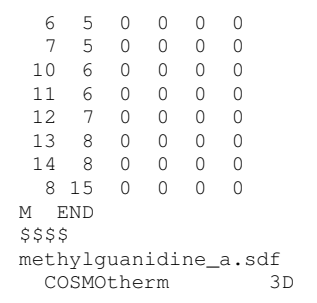


$\begin{array}{llllll}7 & 5 & 0 & 0 & 0 & 0 \\ 9 & 5 & 0 & 0 & 0 & 0\end{array}$

$\begin{array}{llllll}7 & 5 & 0 & 0 & 0 & 0 \\ 9 & 5 & 0 & 0 & 0 & 0\end{array}$

$\begin{array}{llllll}11 & 10 & 0 & 0 & 0 & 0 \\ 14 & 10 & 0 & 0 & 0 & 0\end{array}$

$\begin{array}{lllllll}12 & 11 & 0 & 0 & 0 & 0\end{array}$

131110010000

$1416 \quad 0 \quad 0 \quad 0 \quad 0$

END

methylguanidine_t.sdf

3D

$\begin{array}{llllllllllll}15 & 13 & 0 & 0 & 0 & 0 & 0 & 0 & 0 & 0 & 0 & \mathrm{~V} 2000\end{array}$

$\begin{array}{lllllllllllllll}-2.7457 & -0.1051 & -0.1285 & 0 & 0 & 0 & 0 & 0 & 0 & 0 & 0 & 0 & 0 & 1\end{array}$

$\begin{array}{cccccccccccccc}-1.7898 & -0.0336 & 0.2286 & \mathrm{H} & 0 & 0 & 0 & 0 & 0 & 0 & 0 & 0 & 0 & 2\end{array}$

$\begin{array}{lllllllllllll}0.4076 & 0.0365 & 1.9650 & \mathrm{C} & 0 & 0 & 0 & 0 & 0 & 0 & 0 & 0 & 0\end{array}$

$\begin{array}{lllllllllllllll}-0.1477 & 0.0281 & 0.6149 & \mathrm{~N} & 0 & 0 & 0 & 0 & 0 & 0 & 0 & 0 & 0 & 5\end{array}$

$\begin{array}{llllllllllllll}0.6779 & 0.0070 & -0.3943 & \mathrm{C} & 0 & 0 & 0 & 0 & 0 & 0 & 0 & 0 & 0 & 6\end{array}$

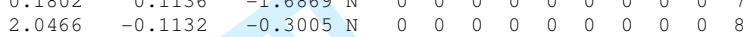

$\begin{array}{cccccccccccccc}-0.4150 & 0.1274 & 2.6859 & \text { H } & 0 & 0 & 0 & 0 & 0 & 0 & 0 & 0 & 0 & 9\end{array}$

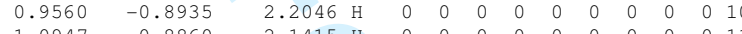

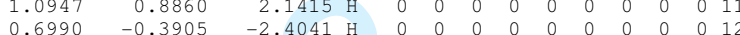

$\begin{array}{cccccccccccccc}-0.6990 & -0.3905 & -2.4041 & \mathrm{H} & 0 & 0 & 0 & 0 & 0 & 0 & 0 & 0 & 0 & 11 \\ -0.8250 & -0.0555 & -1.7417 & \mathrm{H} & 0 & 0 & 0 & 0 & 0 & 0 & 0 & 0 & 0 & 13\end{array}$

$\begin{array}{lllllllllllll}2.6024 & 0.2179 & -1.0854 \text { H } & 0 & 0 & 0 & 0 & 0 & 0 & 0 & 0 & 0 & 14\end{array}$

$2.4676 \quad 0.078$

$\begin{array}{lllllll}1 & 0 & 0 & 0 & 0 & 0 \\ 3 & 1 & 0 & 0 & 0 & 0\end{array}$

$\begin{array}{lllllll}3 & 1 & 0 & 0 & 0 & 0 \\ 5 & 4 & 0 & 0 & 0 & 0\end{array}$

$\begin{array}{cccccccc}5 & 4 & 0 & 0 & 0 & 0 & 0 & 1 \\ 9 & 4 & 0 & 0 & 0 & 0 & 0\end{array}$

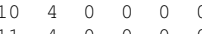

$\begin{array}{lllllll}1 & 4 & 0 & 0 & 0 & 0 \\ 6 & 5 & 0 & 0 & 0 & 0\end{array}$

$76 \begin{array}{lllll}6 & 0 & 0 & 0 \\ 8 & 6 & 0 & 0 & 0\end{array}$

$\begin{array}{lllllll}2 & 7 & 0 & 0 & 0 & 0\end{array}$

$\begin{array}{lllllll}4 & 8 & 0 & 0 & 0 & 0\end{array}$

$\begin{array}{lllllll}8 & 15 & 0 & 0 & 0 & 0\end{array}$

MND

nethylguanidine_t_p2.sdf

3D

$\begin{array}{llllllllllll}16 & 14 & 0 & 0 & 0 & 0 & 0 & 0 & 0 & 0 & 0 & \mathrm{~V} 2000\end{array}$

$\begin{array}{llllllllllllll}-2.9205 & -0.0019 & 0.5632 & 0 & 0 & 0 & 0 & 0 & 0 & 0 & 0 & 0 & 0 & 1\end{array}$

$\begin{array}{lllllllllllll}-0.8743-0.4220 \mathrm{H} & 0 & 0 & 0 & 0 & 0 & 0 & 0 & 0 & 0 & 2\end{array}$

$\begin{array}{llllllllllllll}-3.3523 & 0.5744 & -0.0920 & \mathrm{H} & 0 & 0 & 0 & 0 & 0 & 0 & 0 & 0 & 0 & 3\end{array}$

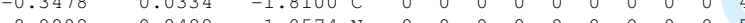

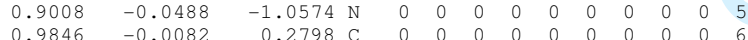

$\begin{array}{cccccccccccccc}0.9846 & -0.0082 & 0.2798 & \mathrm{C} & 0 & 0 & 0 & 0 & 0 & 0 & 0 & 0 & 0 & 6 \\ 2.1986 & 0.0187 & 0.8643 & \mathrm{~N} & 0 & 0 & 0 & 0 & 0 & 0 & 0 & 0 & 0 & 7\end{array}$

$\begin{array}{llllllllllllll}2.1986 & 0.0187 & 0.8643 & \mathrm{~N} & 0 & 0 & 0 & 0 & 0 & 0 & 0 & 0 & 0 & 7\end{array}$

$\begin{array}{rrrrrrrrrrrrrr}-0.1170 & 0.0360 & 1.0361 & \mathrm{~N} & 0 & 0 & 0 & 0 & 0 & 0 & 0 & 0 & 0 & 8 \\ -0.1018 & -0.0483 & -2.8727 & \mathrm{H} & 0 & 0 & 0 & 0 & 0 & 0 & 0 & 0 & 0 & 9\end{array}$

$\begin{array}{llllllllllllll}-1.0260 & -0.7901 & -1.5471 \mathrm{H} & 0 & 0 & 0 & 0 & 0 & 0 & 0 & 0 & 0 & 8 \\ -0.8564 & 0.9937 & -1.6394 & 0 & 0 & 0 & 0 & 0 & 0 & 0 & 0 & 0 & 10\end{array}$

$\begin{array}{llllllllllllll}-0.8564 & 0.9937 & -1.6394 \mathrm{H} & 0 & 0 & 0 & 0 & 0 & 0 & 0 & 0 & 0 & 11\end{array}$

$\begin{array}{llllllllllllll}2.2844 & -0.1030 & 1.8676 & \mathrm{H} & 0 & 0 & 0 & 0 & 0 & 0 & 0 & 0 & 0 & 12\end{array}$

$\begin{array}{lllllllllllllll}3.0389 & -0.1129 & 0.3115 & \mathrm{H} & 0 & 0 & 0 & 0 & 0 & 0 & 0 & 0 & 0 & 13\end{array}$

$\begin{array}{llllllllllllll}-0.0211 & 0.0551 & 2.0463 \mathrm{H} & 0 & 0 & 0 & 0 & 0 & 0 & 0 & 0 & 0 & 14\end{array}$

$\begin{array}{rrrrrrrrrrrrrr}-1.0814 & 0.0147 & 0.6653 \mathrm{H} & 0 & 0 & 0 & 0 & 0 & 0 & 0 & 0 & 0 & 15 \\ 1.7710 & -0.0625 & -1.5801 \mathrm{H} & 0 & 0 & 0 & 0 & 0 & 0 & 0 & 0 & 0 & 16\end{array}$

$\begin{array}{lll}1.7710 & -0.0625\end{array}$ 


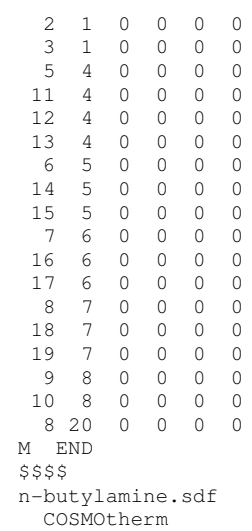

$\begin{array}{lllllllllllll}19 & 17 & 0 & 0 & 0 & 0 & 0 & 0 & 0 & 0 & 0 & \mathrm{~V} 2000\end{array}$

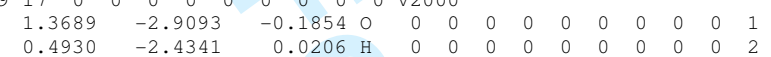

$\begin{array}{llllllllllllll}1.9715 & -2.6228 & 0.5222 & \mathrm{H} & 0 & 0 & 0 & 0 & 0 & 0 & 0 & 0 & 0 & 3\end{array}$

$\begin{array}{llllllllllllll}0.8944 & 2.9876 & 0.1905 & \mathrm{C} & 0 & 0 & 0 & 0 & 0 & 0 & 0 & 0 & 0 & 4\end{array}$

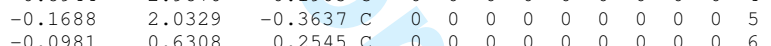

$\begin{array}{rrrrrrrrrrrrrr}-1.1650 & -0.3178 & -0.2923 & \mathrm{C} & 0 & 0 & 0 & 0 & 0 & 0 & 0 & 0 & 0 & 7\end{array}$

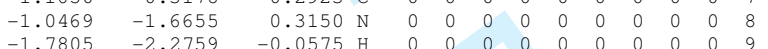

$\begin{array}{rrrrrrrrrrrrrr}-1.7805 & -2.2759 & -0.0575 & \mathrm{H} & 0 & 0 & 0 & 0 & 0 & 0 & 0 & 0 & 0 & 9 \\ -1.2289 & -1.6017 & 1.3217 & \mathrm{H} & 0 & 0 & 0 & 0 & 0 & 0 & 0 & 0 & 0 & 10\end{array}$

$\begin{array}{llrllllllllll}0.8348 & 3.9762 & -0.2884 \mathrm{H} & 0 & 0 & 0 & 0 & 0 & 0 & 0 & 0 & 0 & 11\end{array}$

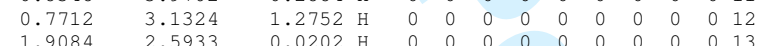

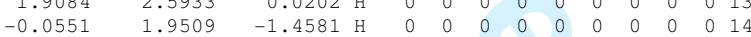

$\begin{array}{llllllllllllll}-1.1721 & 2.4583 & -0.1896 & \mathrm{H} & 0 & 0 & 0 & 0 & 0 & 0 & 0 & 0 & 0 & 15\end{array}$

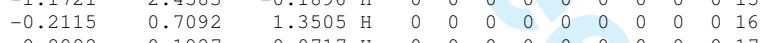

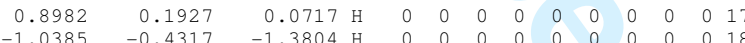

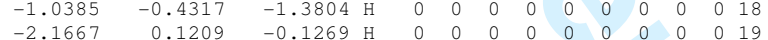

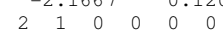

$\begin{array}{llllll}3 & 1 & 0 & 0 & 0 & 0\end{array}$

$\begin{array}{llllll}1 & 4 & 0 & 0 & 0 & 0\end{array}$

$\begin{array}{llllll}2 & 4 & 0 & 0 & 0 & 0\end{array}$

$\begin{array}{llllll}13 & 4 & 0 & 0 & 0 & 0 \\ 6 & 5 & 0 & 0 & 0 & 0\end{array}$

$\begin{array}{llllll}14 & 5 & 0 & 0 & 0 & 0\end{array}$

$\begin{array}{llllll}5 & 5 & 0 & 0 & 0 & 0 \\ 7 & 6 & 0 & 0 & 0 & 0\end{array}$

$\begin{array}{llllll}6 & 6 & 0 & 0 & 0\end{array}$

$\begin{array}{llllll}7 & 6 & 0 & 0 & 0 & 0 \\ 8 & 7 & 0 & 0 & 0 & 0\end{array}$

$\begin{array}{llllllll}8 & 7 & 0 & 0 & 0 & 0 & 0 \\ 9 & 7 & 0 & 0 & 0 & 0\end{array}$

$8 \begin{array}{lllll}8 & 0 & 0 & 0 & 0\end{array}$

$\begin{array}{rrrrrr}10 & 8 & 0 & 0 & 0 & 0 \\ 7 & 19 & 0 & 0 & 0 & 0\end{array}$

END

$\$ \$ \$$

n-heptylamine-cation.sdf

$\begin{array}{llllllllllll}29 & 27 & 0 & 0 & 0 & 0 & 0 & 0 & 0 & 0 & 0 & \text { V2 } 2000\end{array}$

$\begin{array}{lllllllllllll}-1.7848 & -0.1888 & 4.3958 & 0 & 0 & 0 & 0 & 0 & 0 & 0 & 0 & 0 & 0\end{array}$

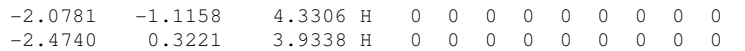

$\begin{array}{rrrrrrrrrrrrrr}-2.4740 & 0.3221 & 3.9338 & \mathrm{H} & 0 & 0 & 0 & 0 & 0 & 0 & 0 & 0 & 0 & 3 \\ -0.5953 & 0.3770 & -3.8375 & \mathrm{C} & 0 & 0 & 0 & 0 & 0 & 0 & 0 & 0 & 0 & 4\end{array}$

$\begin{array}{rrrrrrrrrrrrrr}-0.5953 & 0.3770 & -3.8375 & \mathrm{C} & 0 & 0 & 0 & 0 & 0 & 0 & 0 & 0 & 0 & 4 \\ 0.1851 & -0.2628 & -2.6813 & \mathrm{C} & 0 & 0 & 0 & 0 & 0 & 0 & 0 & 0 & 0 & 5\end{array}$

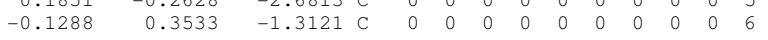

$\begin{array}{rrrrrrrrrrrrrrrr}-0.1288 & 0.3589 & -0.2889 & -0.1639 & \mathrm{C} & 0 & 0 & 0 & 0 & 0 & 0 & 0 & 0 & 0 & 7\end{array}$

$\begin{array}{llllllllllllll}0.3429 & 0.3296 & 1.2044 & \mathrm{C} & 0 & 0 & 0 & 0 & 0 & 0 & 0 & 0 & 0 & 8\end{array}$

$\begin{array}{lllllllllllll}1.1587 & -0.3219 & 2.3151 & \mathrm{C} & 0 & 0 & 0 & 0 & 0 & 0 & 0 & 0 & 0\end{array}$

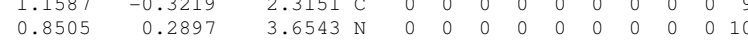

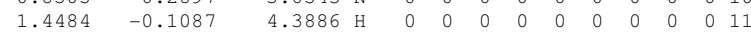

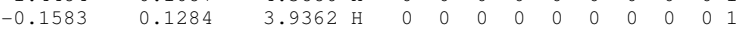

$\begin{array}{llllllllllllll}-0.2749 & -0.2469 & -5.2004 & \mathrm{C} & 0 & 0 & 0 & 0 & 0 & 0 & 0 & 0 & 0 & 13\end{array}$

$\begin{array}{llllllllllllll}-1.6771 & 0.2878 & -3.6365 & \mathrm{H} & 0 & 0 & 0 & 0 & 0 & 0 & 0 & 0 & 0 & 14 \\ -0.3766 & 1.4584 & -3.8663 & \mathrm{H} & 0 & 0 & 0 & 0 & 0 & 0 & 0 & 0 & 0 & 15 \\ 1.2680 & -0.1726 & -2.8814 & \mathrm{H} & 0 & 0 & 0 & 0 & 0 & 0 & 0 & 0 & 0 & 16\end{array}$

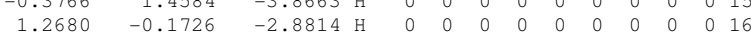

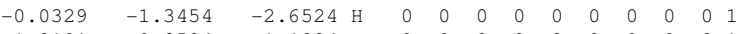

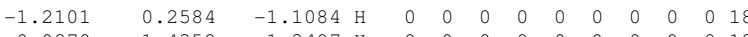

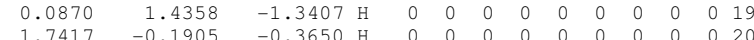

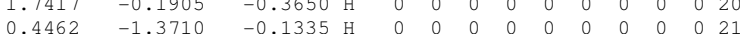

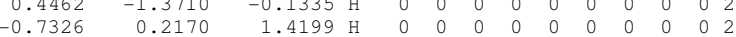

$\begin{array}{llllllllllllll}0.5567 & 1.4114 & 1.1805 \mathrm{H} & 0 & 0 & 0 & 0 & 0 & 0 & 0 & 0 & 0 & 23\end{array}$

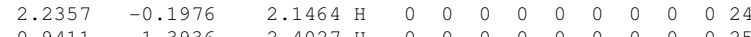

$\begin{array}{llllllllllllll}0.9411 & -1.3936 & 2.4027 & \mathrm{H} & 0 & 0 & 0 & 0 & 0 & 0 & 0 & 0 & 0 & 25\end{array}$

$\begin{array}{llllllllllllll}-0.8486 & 0.2314 & -6.0084 & \mathrm{H} & 0 & 0 & 0 & 0 & 0 & 0 & 0 & 0 & 0 & 26\end{array}$

$\begin{array}{llllllllllllll}0.7945 & -0.1425 & -5.4423 & \mathrm{H} & 0 & 0 & 0 & 0 & 0 & 0 & 0 & 0 & 0 & 27\end{array}$

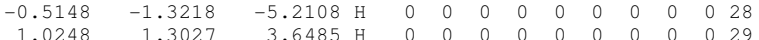

$\begin{array}{llllll}2 & 1 & 0 & 0 & 0 & 0\end{array}$

$\begin{array}{llllll}3 & 1 & 0 & 0 & 0 & 0 \\ 5 & 4 & 0 & 0 & 0 & 0\end{array}$

$\begin{array}{llllllll}3 & 4 & 0 & 0 & 0 & 0\end{array}$

$\begin{array}{llllll}14 & 4 & 0 & 0 & 0 & 0\end{array}$

$\begin{array}{llllll}15 & 4 & 0 & 0 & 0 & 0\end{array}$

$\begin{array}{llllll}6 & 5 & 0 & 0 & 0 & 0\end{array}$

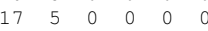

$\begin{array}{llllll}7 & 6 & 0 & 0 & 0 & 0\end{array}$ 


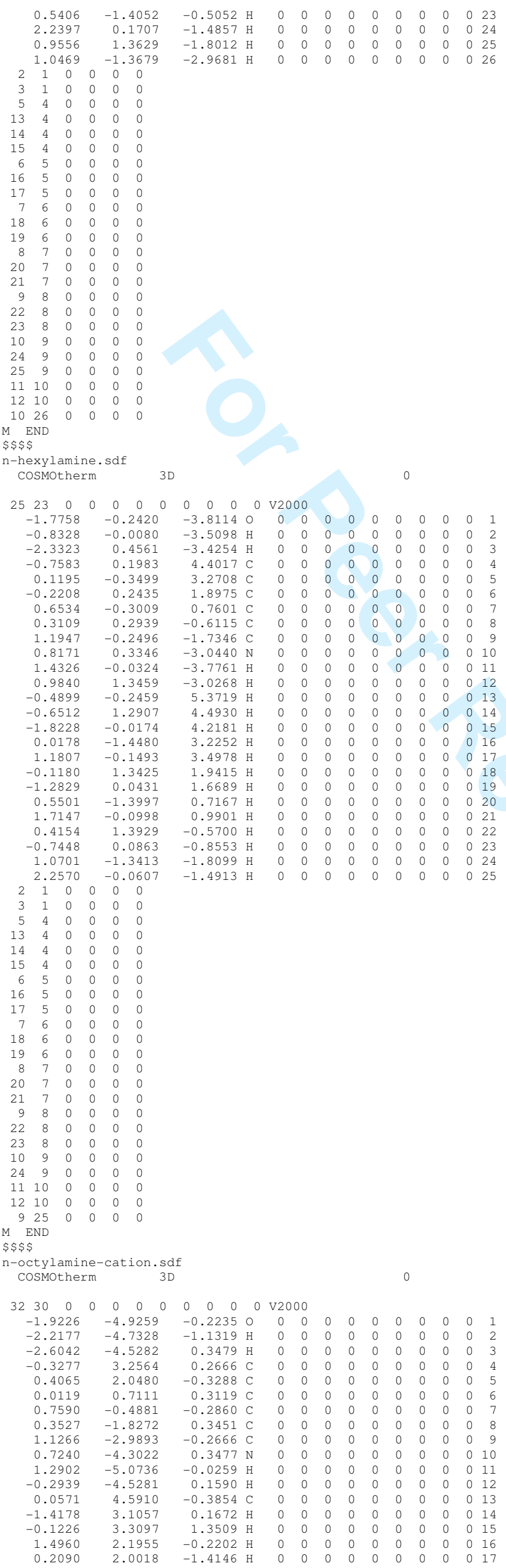




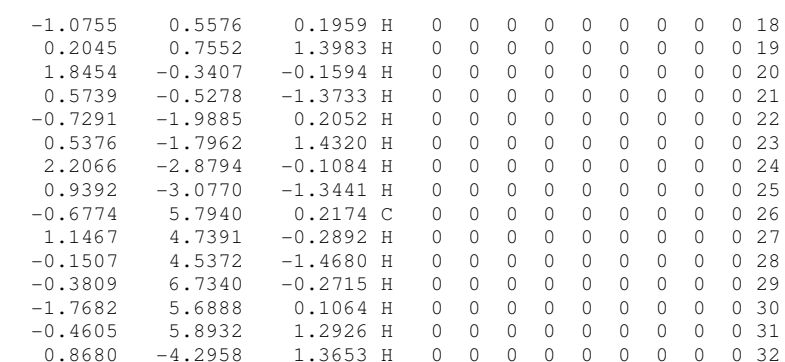




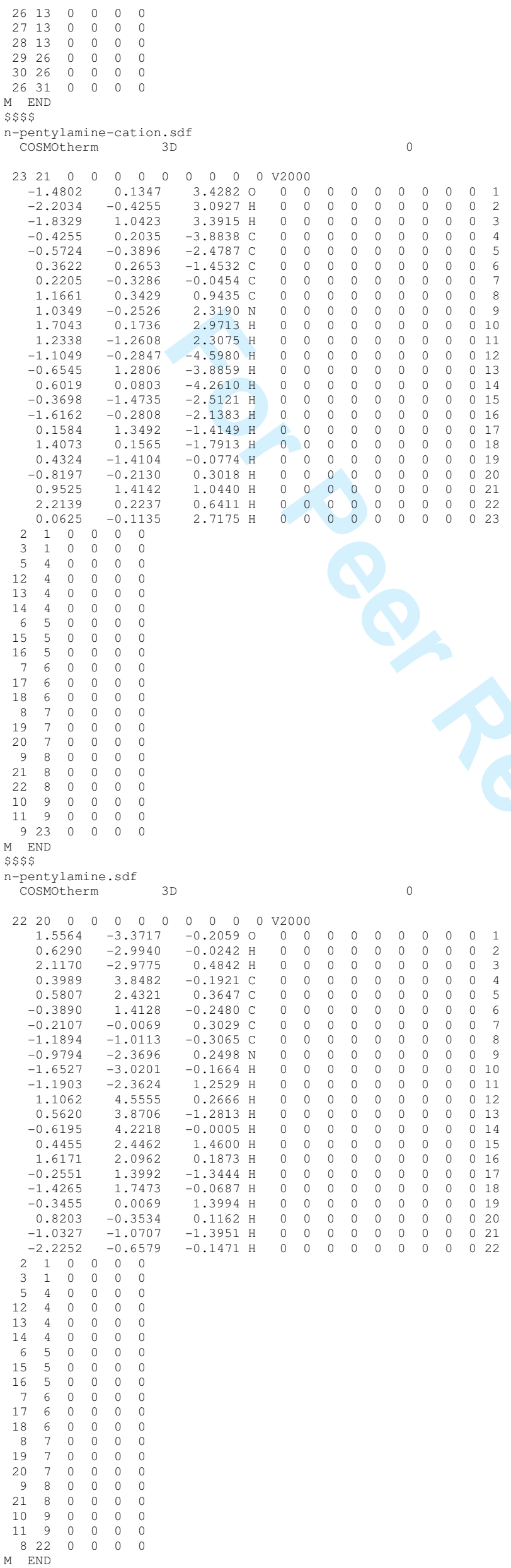




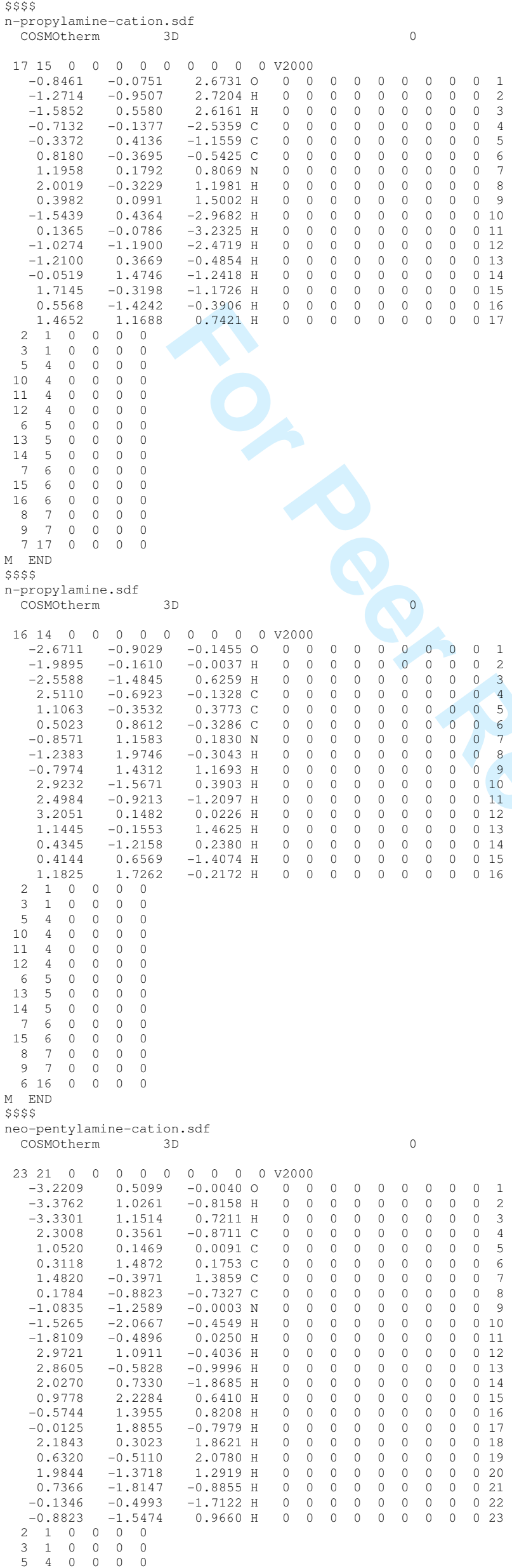


$\begin{array}{rrrrrr}12 & 4 & 0 & 0 & 0 & 0 \\ 13 & 4 & 0 & 0 & 0 & 0 \\ 14 & 4 & 0 & 0 & 0 & 0 \\ 6 & 5 & 0 & 0 & 0 & 0 \\ 7 & 5 & 0 & 0 & 0 & 0 \\ 8 & 5 & 0 & 0 & 0 & 0 \\ 15 & 6 & 0 & 0 & 0 & 0 \\ 16 & 6 & 0 & 0 & 0 & 0 \\ 17 & 6 & 0 & 0 & 0 & 0 \\ 18 & 7 & 0 & 0 & 0 & 0 \\ 19 & 7 & 0 & 0 & 0 & 0 \\ 20 & 7 & 0 & 0 & 0 & 0 \\ 9 & 8 & 0 & 0 & 0 & 0 \\ 21 & 8 & 0 & 0 & 0 & 0 \\ 22 & 8 & 0 & 0 & 0 & 0 \\ 10 & 9 & 0 & 0 & 0 & 0 \\ 11 & 9 & 0 & 0 & 0 & 0 \\ 9 & 23 & 0 & 0 & 0 & 0 \\ & & & & & \end{array}$ $M \quad E N D$

$\$ \$ \$$

neo-pentylamine.sdf

$\begin{array}{llllllllllll}22 & 20 & 0 & 0 & 0 & 0 & 0 & 0 & 0 & 0 & 0 & \mathrm{v} 2000\end{array}$

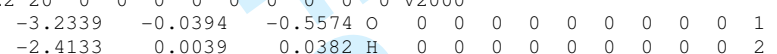

$\begin{array}{rrrrrrrrrrrrrr}-2.4133 & 0.0039 & 0.0382 & \mathrm{H} & 0 & 0 & 0 & 0 & 0 & 0 & 0 & 0 & 0 & 2 \\ -3.0788 & 0.6384 & -1.2373 & \mathrm{H} & 0 & 0 & 0 & 0 & 0 & 0 & 0 & 0 & 0 & 3\end{array}$

$\begin{array}{llllllllllllll}2.3551 & 0.7584 & -0.2400 & \mathrm{C} & 0 & 0 & 0 & 0 & 0 & 0 & 0 & 0 & 0 & 4\end{array}$

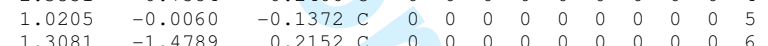

$\begin{array}{rrrrrrrrrrrrrr}1.3081 & -1.4789 & 0.2152 & C & 0 & 0 & 0 & 0 & 0 & 0 & 0 & 0 & 0 & 6 \\ 0.2853 & 0.0741 & -1.4879 & C & 0 & 0 & 0 & 0 & 0 & 0 & 0 & 0 & 0 & 7\end{array}$

$\begin{array}{llllllllllllll}0.1938 & 0.6656 & 0.9833 & \mathrm{C} & 0 & 0 & 0 & 0 & 0 & 0 & 0 & 0 & 0 & 8 \\ -1.1173 & 0.0204 & 1.2387 & \mathrm{~N} & 0 & 0 & 0 & 0 & 0 & 0 & 0 & 0 & 0 & 9\end{array}$

$\begin{array}{rrrrrrrrrrrrrr}-1.1173 & 0.0204 & 1.2387 & \mathrm{~N} & 0 & 0 & 0 & 0 & 0 & 0 & 0 & 0 & 0 & 9 \\ -1.5720 & 0.5081 & 2.0166 & \mathrm{H} & 0 & 0 & 0 & 0 & 0 & 0 & 0 & 0 & 0 & 10\end{array}$

$\begin{array}{llllllllllllll}-0.9682 & -0.9351 & 1.5775 & \mathrm{H} & 0 & 0 & 0 & 0 & 0 & 0 & 0 & 0 & 0 & 11\end{array}$

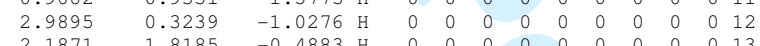

$\begin{array}{llllllllllllll}2.1871 & 1.8185 & -0.4883 & \mathrm{H} & 0 & 0 & 0 & 0 & 0 & 0 & 0 & 0 & 0 & 13\end{array}$

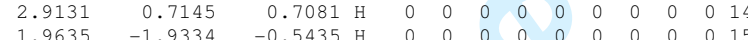

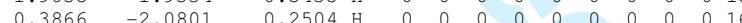

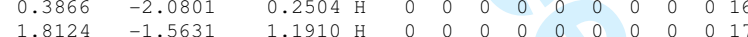

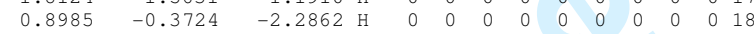

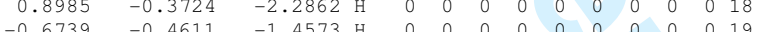

$\begin{array}{lllllllllllllllll}0.0733 & 1.1215 & -1.7630 & \mathrm{H} & 0 & 0 & 0 & 0 & 0 & 0 & 0 & 0 & 0 & 20\end{array}$

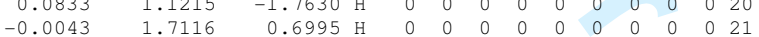

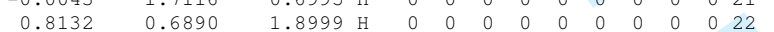

$2 \begin{array}{llllll}2 & 1 & 0 & 0 & 0 & 0\end{array}$

$\begin{array}{lllll}1 & 0 & 0 & 0 & 0\end{array}$

$\begin{array}{lllllll}2 & 4 & 0 & 0 & 0 & 0\end{array}$

$\begin{array}{llllll}3 & 4 & 0 & 0 & 0 & 0\end{array}$

$\begin{array}{llllll}4 & 4 & 0 & 0 & 0 & 0\end{array}$

$\begin{array}{lllll}5 & 0 & 0 & 0 & 0\end{array}$

$\begin{array}{cccccccc}5 & 5 & 0 & 0 & 0 & 0 & 0 & 0 \\ 15 & 6 & 0 & 0 & 0 & 0\end{array}$

$\begin{array}{llllll}6 & 0 & 0 & 0\end{array}$

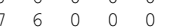

$\begin{array}{lllllll}8 & 7 & 0 & 0 & 0 & 0\end{array}$

$\begin{array}{lllllll}9 & 7 & 0 & 0 & 0 & 0\end{array}$

$9 \begin{array}{llllll}9 & 8 & 0 & 0 & 0 & 0\end{array}$

$\begin{array}{lllll}8 & 0 & 0 & 0 & 0 \\ 9 & 0 & 0 & 0 & 0\end{array}$

$\begin{array}{rrrrrr}1 & 9 & 0 & 0 & 0 & 0 \\ 8 & 22 & 0 & 0 & 0 & 0\end{array}$

END

$\$ \$ \$$

p-toluidine-cationo.sdf

$\begin{array}{llllllllllll}21 & 20 & 0 & 0 & 0 & 0 & 0 & 0 & 0 & 0 & 0 & \mathrm{v} 2000\end{array}$

$\begin{array}{lllllllllllll}-0.8053 & -3.8126 & -0.6898 & 0 & 0 & 0 & 0 & 0 & 0 & 0 & 0 & 0 & 0\end{array}$

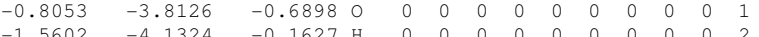

$\begin{array}{llllllllllllll}-1.5602 & -4.1324 & -0.1627 & \mathrm{H} & 0 & 0 & 0 & 0 & 0 & 0 & 0 & 0 & 0 & 2 \\ -1.1938 & -3.5239 & -1.5358 & \mathrm{H} & 0 & 0 & 0 & 0 & 0 & 0 & 0 & 0 & 0 & 3\end{array}$

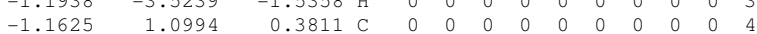

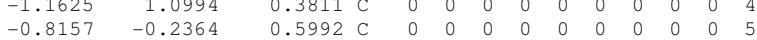

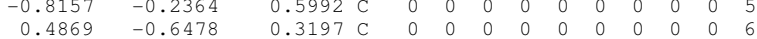

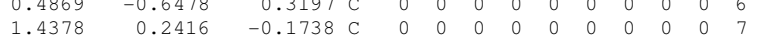

$\begin{array}{llllllllllllll}1.0739 & 1.5745 & -0.3863 & \mathrm{C} & 0 & 0 & 0 & 0 & 0 & 0 & 0 & 0 & 0 & 8\end{array}$

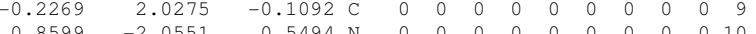

$\begin{array}{rrrrrrrrrrrrrr}0.8599 & -2.0551 & 0.5494 & \mathrm{~N} & 0 & 0 & 0 & 0 & 0 & 0 & 0 & 0 & 0 & 10 \\ -0.6022 & 3.4748 & -0.3079 & \mathrm{C} & 0 & 0 & 0 & 0 & 0 & 0 & 0 & 0 & 0 & 11\end{array}$

$\begin{array}{llllllllllllll}1.8036 & -2.2492 & 0.1906 & \mathrm{H} & 0 & 0 & 0 & 0 & 0 & 0 & 0 & 0 & 0 & 12\end{array}$

$\begin{array}{llllllllllllll}0.1843 & -2.7359 & 0.0692 & \mathrm{H} & 0 & 0 & 0 & 0 & 0 & 0 & 0 & 0 & 0 & 13\end{array}$

$\begin{array}{llllllllllllllll}-2.1827 & 1.4233 & 0.5959 \mathrm{H} & 0 & 0 & 0 & 0 & 0 & 0 & 0 & 0 & 0 & 0 & 14\end{array}$

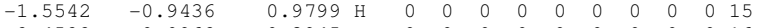

$\begin{array}{llllllllllllll}2.4522 & -0.0962 & -0.3945 \mathrm{H} & 0 & 0 & 0 & 0 & 0 & 0 & 0 & 0 & 0 & 16\end{array}$

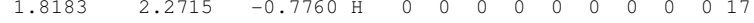

$\begin{array}{llllllllllllll}-0.3923 & 4.0585 & 0.6029 & \mathrm{H} & 0 & 0 & 0 & 0 & 0 & 0 & 0 & 0 & 0 & 18\end{array}$

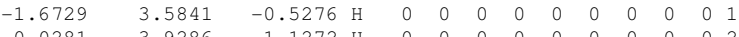

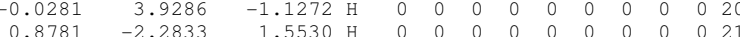

$\begin{array}{lllllll}2 & 1 & 0 & 0 & 0 & 0\end{array}$

$\begin{array}{lllllll}3 & 1 & 0 & 0 & 0 & 0\end{array}$

$\begin{array}{lllll}4 & 0 & 0 & 0 & 0\end{array}$

$\begin{array}{llllll}9 & 4 & 0 & 0 & 0 & 0 \\ 4 & 4 & 0 & 0 & 0 & 0\end{array}$

$\begin{array}{lllllll}6 & 5 & 0 & 0 & 0 & 0\end{array}$

$\begin{array}{lllllllll}7 & 6 & 0 & 0 & 0 & 0 & 0 & 0\end{array}$

$\begin{array}{llllll}0 & 6 & 0 & 0 & 0 & 0\end{array}$

$8 \begin{array}{lllll}7 & 0 & 0 & 0 & 0\end{array}$

$\begin{array}{llllll}16 & 7 & 0 & 0 & 0 & 0 \\ 9 & 8 & 0 & 0 & 0 & 0\end{array}$ 
$\begin{array}{llllll}17 & 8 & 0 & 0 & 0 & 0\end{array}$

1111900010000

$\begin{array}{lllllll}12 & 10 & 0 & 0 & 0 & 0 \\ 13 & 10 & 0 & 0 & 0 & 0\end{array}$

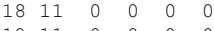

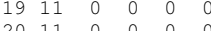

$\begin{array}{llllll}20 & 11 & 0 & 0 & 0 & 0 \\ 10 & 21 & 0 & 0 & 0 & 0\end{array}$

$M$ END

p-toluidine.sde

tosmotherm
coluidine.s.

$\begin{array}{llllllllllll}20 & 19 & 0 & 0 & 0 & 0 & 0 & 0 & 0 & 0 & 0 & \text { v2 } 2000\end{array}$

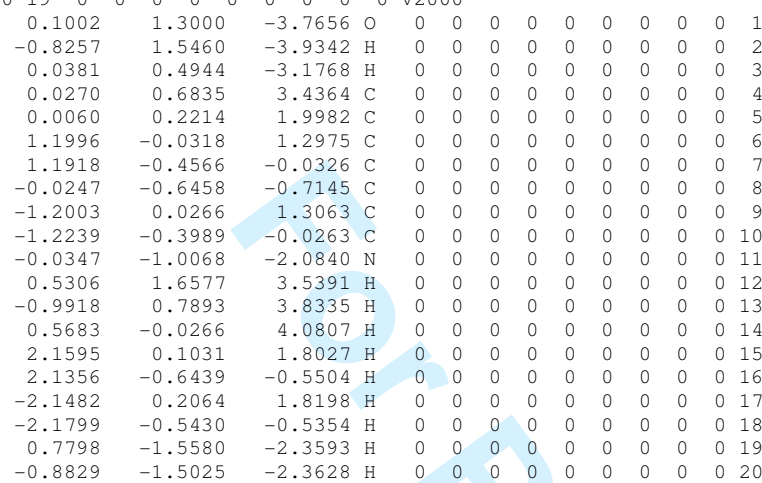

$\begin{array}{llllll}2 & 1 & 0 & 0 & 0 & 0 \\ 3 & 1 & 0 & 0 & 0 & 0\end{array}$

$\begin{array}{lllllll}3 & 1 & 0 & 0 & 0 & 0 \\ 5 & 4 & 0 & 0 & 0 & 0\end{array}$

$\begin{array}{lllllll}2 & 4 & 0 & 0 & 0 & 0\end{array}$

$\begin{array}{lllllll}13 & 4 & 0 & 0 & 0 & 0 & 0 \\ 12 & 4 & 0 & 0 & 0 & 0\end{array}$

$\begin{array}{lllllll}14 & 4 & 0 & 0 & 0 & 0 & 0 \\ 6 & 5 & 0 & 0 & 0 & 0\end{array}$

$\begin{array}{llllll}9 & 5 & 0 & 0 & 0 & 0 \\ 7 & 6 & 0 & 0 & 0 & 0\end{array}$

$\begin{array}{lllllll}15 & 6 & 0 & 0 & 0\end{array}$

$\begin{array}{lllllll}8 & 7 & 0 & 0 & 0 & 0\end{array}$

$\begin{array}{cccccc}18 & 7 & 0 & 0 & 0 & 0\end{array}$

$\begin{array}{lllllll}1 & 8 & 0 & 0 & 0 & 0\end{array}$

$\begin{array}{lllllll}1 & 9 & 0 & 0 & 0 & 0\end{array}$

$\begin{array}{llllll}9 & 0 & 0 & 0 & 0\end{array}$

$\begin{array}{lllllll}9 & 11 & 0 & 0 & 0 & 0\end{array}$

END

$\$ \$ \$ \$$

p-toluidine 0.sdf
Cosmotherm

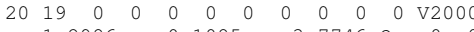

$\begin{array}{lllllllllllll}-1.2986 & 0.1085 & -3.7746 & 0 & 0 & 0 & 0 & 0 & 0 & 0 & 0 & 0 & 0\end{array}$

$\begin{array}{llllllllllllll}-1.5119 & -0.8113 & -4.0086 \mathrm{H} & 0 & 0 & 0 & 0 & 0 & 0 & 0 & 0 & 0 & 2\end{array}$

$\begin{array}{lllllllllllllll}-0.4972 & 0.0341 & -3.1825 & \text { H } & 0 & 0 & 0 & 0 & 0 & 0 & 0 & 0 & 0 & 3\end{array}$

$\begin{array}{llllllllllllll}0.0451 & 1.1999 & 1.3012 & \mathrm{C} & 0 & 0 & 0 & 0 & 0 & 0 & 0 & 0 & 0 & 4\end{array}$

$\begin{array}{llllllllllllll}0.4649 & 1.1894 & -0.0319 & \mathrm{C} & 0 & 0 & 0 & 0 & 0 & 0 & 0 & 0 & 0 & 5\end{array}$

$\begin{array}{llllllllllllll}0.6429 & -0.0277 & -0.7129 & \text { C } & 0 & 0 & 0 & 0 & 0 & 0 & 0 & 0 & 0 & 6\end{array}$

$\begin{array}{llllllllllllll}0.3939 & -1.2261 & -0.0212 & \mathrm{C} & 0 & 0 & 0 & 0 & 0 & 0 & 0 & 0 & 0 & 7\end{array}$

$\begin{array}{rrrrrrrrrrrrrr}-0.0251 & -1.1999 & 1.3120 & \mathrm{C} & 0 & 0 & 0 & 0 & 0 & 0 & 0 & 0 & 0 & 8 \\ -0.2132 & 0.0098 & 2.0029 & \mathrm{C} & 0 & 0 & 0 & 0 & 0 & 0 & 0 & 0 & 0 & 9\end{array}$

$\begin{array}{llllllllllllll}0.9999 & -0.0436 & -2.0835 & \mathrm{~N} & 0 & 0 & 0 & 0 & 0 & 0 & 0 & 0 & 0 & 10\end{array}$

$\begin{array}{cccccccccccccc}-0.6985 & 0.0308 & 3.4332 & \mathrm{C} & 0 & 0 & 0 & 0 & 0 & 0 & 0 & 0 & 0 & 11\end{array}$

$\begin{array}{llllllllllllll}1.4923 & -0.8943 & -2.3605 \mathrm{H} & 0 & 0 & 0 & 0 & 0 & 0 & 0 & 0 & 0 & 12\end{array}$

$\begin{array}{lllllllllllll}1.5532 & 0.7681 & -2.3626 \text { H } & 0 & 0 & 0 & 0 & 0 & 0 & 0 & 0 & 0 & 13\end{array}$

$-0.6794-2.1331-1.8083 \mathrm{H}-0$

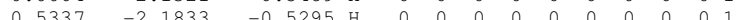

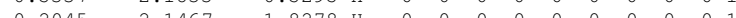

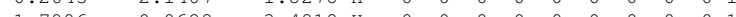

$\begin{array}{llllllllllllll}-1.7996 & 0.0628 & 3.4818 & \mathrm{H} & 0 & 0 & 0 & 0 & 0 & 0 & 0 & 0 & 0 & 18 \\ -0.3240 & 0.9135 & 3.9707 & \mathrm{H} & 0 & 0 & 0 & 0 & 0 & 0 & 0 & 0 & 0 & 19\end{array}$

$\begin{array}{lllllllllllllllllll}-0.3755 & -0.8668 & 3.9795 & \mathrm{H} & 0 & 0 & 0 & 0 & 0 & 0 & 0 & 0 & 0 & 18\end{array}$

$\begin{array}{lllllll}2 & 1 & 0 & 0 & 0 & 0 \\ 3 & 1 & 0 & 0 & 0 & 0\end{array}$

$\begin{array}{llllll}3 & 1 & 0 & 0 & 0 & 0\end{array}$

$\begin{array}{llllll}4 & 0 & 0 & 0 & 0\end{array}$

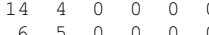

$\begin{array}{llllllll}6 & 5 & 0 & 0 & 0 & 0 & 0 & 0 \\ 15 & 5 & 0 & 0 & 0 & 0\end{array}$

$7 \begin{array}{lllllll}7 & 0 & 0 & 0\end{array}$

$\begin{array}{llllll}15 & 5 & 0 & 0 & 0 & 0 \\ 17 & 0 & 0 & 0 & 0\end{array}$

$\begin{array}{lllllll}7 & 6 & 0 & 0 & 0 & 0 \\ 8 & 7 & 0 & 0 & 0 & 0 & 0\end{array}$

$\begin{array}{lllllll}6 & 7 & 0 & 0 & 0 & 0\end{array}$

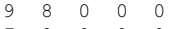

$\begin{array}{llllll}8 & 0 & 0 & 0 & 0 \\ 9 & 0 & 0 & 0 & 0\end{array}$

$\begin{array}{llllll}12 & 10 & 0 & 0 & 0 & 0\end{array}$

$\begin{array}{llllll}13 & 10 & 0 & 0 & 0 & 0 \\ 1 & 11 & 0 & 0 & 0 & 0\end{array}$

$\begin{array}{llllll}19 & 11 & 0 & 0 & 0 & 0\end{array}$

\$\$\$

p-toluidine1.sdf

Cosmotherm

$\begin{array}{ccccccccccccccccc}20 & 19 & 0 & 0 & 0 & 0 & 0 & 0 & 0 & 0 & 0 & \mathrm{v} 2000 \\ -3.7656 & -1.3067 & 0.0814 & 0 & 0 & 0 & 0 & 0 & 0 & 0 & 0 & 0 & 0 & 1\end{array}$ 


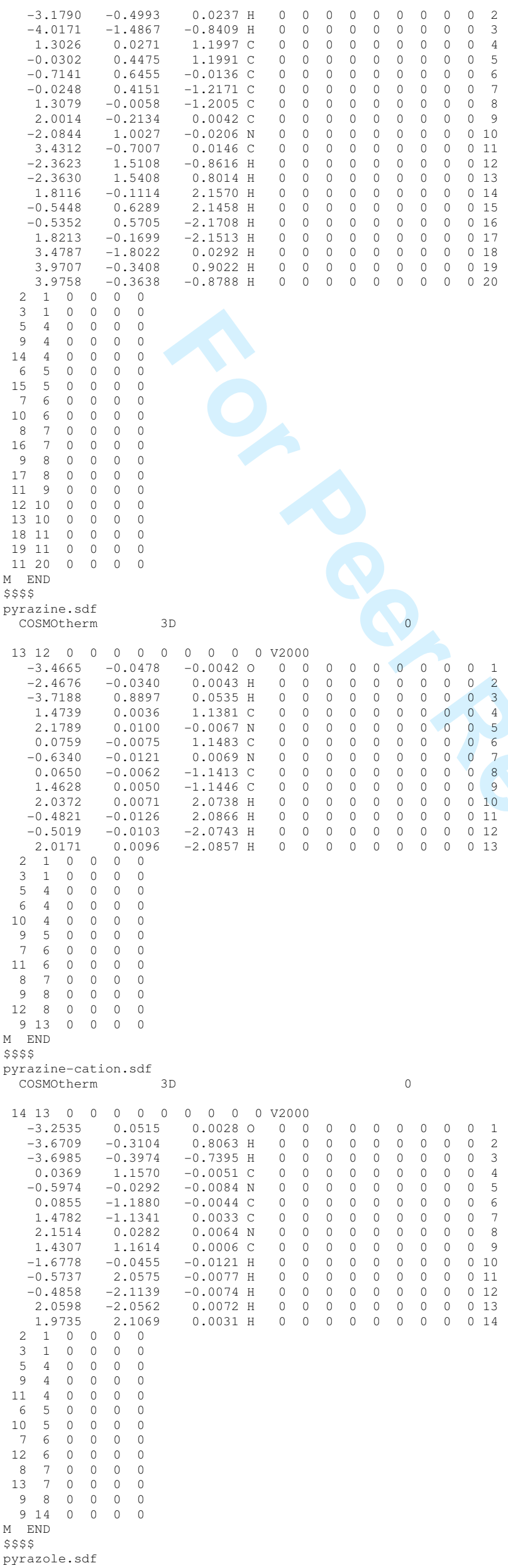


Cosmotherm 3D

$\begin{array}{lllllllllllll}12 & 11 & 0 & 0 & 0 & 0 & 0 & 0 & 0 & 0 & 0 & \mathrm{~V} 2000\end{array}$

$\begin{array}{llllllllllllll}-3.1718 & -0.0525 & 0.0006 & 0 & 0 & 0 & 0 & 0 & 0 & 0 & 0 & 0 & 0 & 1 \\ -2.1739 & -0.0246 & -0.0032 & \mathrm{H} & 0 & 0 & 0 & 0 & 0 & 0 & 0 & 0 & 0 & 2\end{array}$

$\begin{array}{llllllllllllll}-3.4379 & 0.8823 & -0.0331 & \mathrm{H} & 0 & 0 & 0 & 0 & 0 & 0 & 0 & 0 & 0 & 2 \\ 1.8333 & 0.0059 & -0.7022 & \mathrm{C} & 0 & 0 & 0 & 0 & 0 & 0 & 0 & 0 & 0 & 3\end{array}$

$\begin{array}{llllllllllllll}1.8333 & 0.0059 & -0.7022 & \mathrm{C} & 0 & 0 & 0 & 0 & 0 & 0 & 0 & 0 & 0 & 4\end{array}$

$\begin{array}{llllllllllllll}0.4828 & -0.0030 & -1.1120 & \mathrm{C} & 0 & 0 & 0 & 0 & 0 & 0 & 0 & 0 & 0 & 5\end{array}$

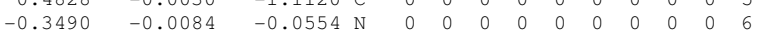

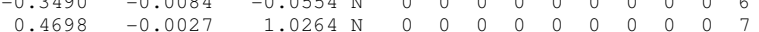

$\begin{array}{llllllllllllll}1.7834 & 0.0067 & 0.6873 & \mathrm{C} & 0 & 0 & 0 & 0 & 0 & 0 & 0 & 0 & 0 & 8\end{array}$

$\begin{array}{llllllllllllll}1.7209 & 0.0111 & -1.3258 & \mathrm{H} & 0 & 0 & 0 & 0 & 0 & 0 & 0 & 0 & 0 & 9\end{array}$

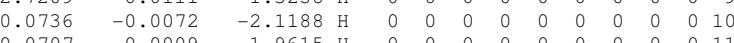

$\begin{array}{lllllllllllllllllllll}0.0736 & -0.0072 & -0.0009 & 1.118615 & \mathrm{H} & 0 & 0 & 0 & 0 & 0 & 0 & 0 & 0 & 0 & 0 & 0 & 11\end{array}$

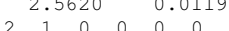

$\begin{array}{lllllll}3 & 1 & 0 & 0 & 0 & 0\end{array}$

$\begin{array}{llllll}1 & 0 & 0 & 0 & 0\end{array}$

$\begin{array}{llllllll}4 & 0 & 0 & 0 & 0\end{array}$

$\begin{array}{lllll}4 & 0 & 0 & 0\end{array}$

$\begin{array}{llllll}5 & 0 & 0 & 0 & 0\end{array}$

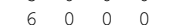

$\begin{array}{lllll}7 & 0 & 0 & 0 & 0\end{array}$

$\begin{array}{rrrrrr}1 & 7 & 0 & 0 & 0 & 0 \\ 8 & 12 & 0 & 0 & 0 & 0\end{array}$

END

$\$ \$ \$$

pyrazole_p2.sdf
Cosmotherm

$\begin{array}{lllllllllllll}13 & 12 & 0 & 0 & 0 & 0 & 0 & 0 & 0 & 0 & 0 & \mathrm{~V} 2000\end{array}$

$\begin{array}{lllllllllllll}-2.9724 & -0.0032 & -0.0464 & 0 & 0 & 0 & 0 & 0 & 0 & 0 & 0 & 0 & 0\end{array}$

$\begin{array}{llllllllllllll}-3.4054 & -0.7273 & 0.4423 & \mathrm{H} & 0 & 0 & 0 & 0 & 0 & 0 & 0 & 0 & 0 & 2\end{array}$

$\begin{array}{llllllllllllll}-3.3915 & 0.8118 & 0.2868 & \mathrm{H} & 0 & 0 & 0 & 0 & 0 & 0 & 0 & 0 & 0 & 3\end{array}$

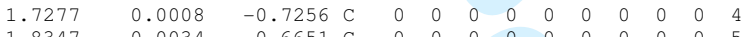

$\begin{array}{llllllllllllll}1.8347 & 0.0034 & 0.6651 & \mathrm{C} & 0 & 0 & 0 & 0 & 0 & 0 & 0 & 0 & 0 & 5\end{array}$

$\begin{array}{rrrrrrrrrrrrrr}0.5224 & 0.0009 & 1.1484 & \mathrm{C} & 0 & 0 & 0 & 0 & 0 & 0 & 0 & 0 & 0 & 6 \\ -0.3183 & -0.0042 & 0.0988 & \mathrm{~N} & 0 & 0 & 0 & 0 & 0 & 0 & 0 & 0 & 0 & 7\end{array}$

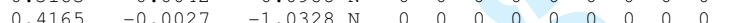

$\begin{array}{lllllllllllll}2.4779 & 0.0022 & -1.5086 & \mathrm{H} & 0 & 0 & 0 & 0 & 0 & 0 & 0 & 0 & 0\end{array}$

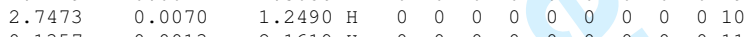

$\begin{array}{llllllllllllllllll}0.7373 & 0.0013 & 2.1619 & \mathrm{H} & 0 & 0 & 0 & 0 & 0 & 0 & 0 & 0 & 0 & 10\end{array}$

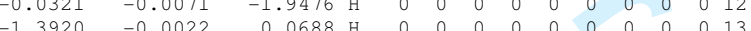

$-1.3920-0.0022$

$\begin{array}{lllllllll}3 & -1 & 0 & 0 & 0 & 0\end{array}$

$\begin{array}{lllll}4 & 0 & 0 & 0 & 0\end{array}$

4.0000

$\begin{array}{lllll}4 & 0 & 0 & 0 & 0\end{array}$

$\begin{array}{lllllll}5 & 0 & 0 & 0 & 0\end{array}$

$\begin{array}{lllllllll}6 & 0 & 0 & 0 & 0\end{array}$

$\begin{array}{llllll}6 & 0 & 0 & 0 & 0\end{array}$

$\begin{array}{lllll}8 & 0 & 0 & 0 & 0\end{array}$

$\begin{array}{lllllll}7 & 13 & 0 & 0 & 0 & 0\end{array}$

$M$ END

\$\$

pyridine.sdf
Cosmotherm

$\begin{array}{llllllllllll}14 & 13 & 0 & 0 & 0 & 0 & 0 & 0 & 0 & 0 & 0 & \mathrm{v} 2000\end{array}$

$\begin{array}{lllllllllllll}-3.4132 & -0.0586 & -0.0007 & 0 & 0 & 0 & 0 & 0 & 0 & 0 & 0 & 0 & 0\end{array}$

$\begin{array}{llllllllllllll}-2.4083 & -0.0245 & -0.0051 & \mathrm{H} & 0 & 0 & 0 & 0 & 0 & 0 & 0 & 0 & 0 & 2\end{array}$

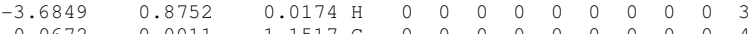

$\begin{array}{llllllllllllll}0.0672 & 0.0011 & 1.1517 & \mathrm{C} & 0 & 0 & 0 & 0 & 0 & 0 & 0 & 0 & 0 & 4 \\ 1.4627 & 0.0006 & 1.2065 & \mathrm{C} & 0 & 0 & 0 & 0 & 0 & 0 & 0 & 0 & 0 & 5\end{array}$

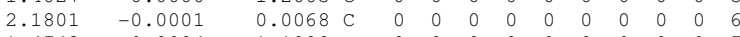

$\begin{array}{llllllllllllll}1.4743 & 0.0004 & -1.1998 & \mathrm{C} & 0 & 0 & 0 & 0 & 0 & 0 & 0 & 0 & 0\end{array}$

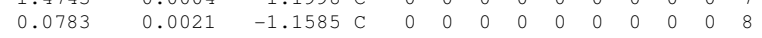

$\begin{array}{lllllllllllll}-0.6216 & 0.0019 & -0.0067 & \mathrm{~N} & 0 & 0 & 0 & 0 & 0 & 0 & 0 & 0 & 0\end{array}$

$\begin{array}{lllllllllll}.0009 & 2.0672 \mathrm{H} & 0 & 0 & 0 & 0 & 0 & 0 & 0 & 0\end{array}$

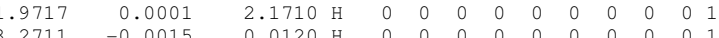

$\begin{array}{llllllllllllllll}1.2711 & -0.0015 & 0.0120 & \mathrm{H} & 0 & 0 & 0 & 0 & 0 & 0 & 0 & 0 & 0 & 11 \\ -0.5926 & 0.0000 & -2.1592 & \mathrm{H} & 0 & 0 & 0 & 0 & 0 & 0 & 0 & 0 & 0 & 13\end{array}$

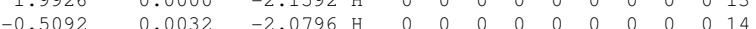

$\begin{array}{llllll}1 & 0 & 0 & 0 & 0\end{array}$

$\begin{array}{lllll}1 & 0 & 0 & 0 & 0\end{array}$

$\begin{array}{llllll}4 & 0 & 0 & 0 & 0\end{array}$

$\begin{array}{lllll}4 & 0 & 0 & 0 & 0\end{array}$

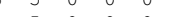

$\begin{array}{llllll}6 & 6 & 0 & 0 & 0\end{array}$

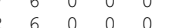

$\begin{array}{lllll}7 & 0 & 0 & 0\end{array}$

$\begin{array}{rrrrr}7 & 0 & 0 & 0 & 0 \\ 8 & 0 & 0 & 0 & 0\end{array}$

$\begin{array}{lllll}14 & 0 & 0 & 0 & 0\end{array}$

$\$ \$ \$ \$$

pyridine-cation.sdf

$\begin{array}{llllllllllll}15 & 14 & 0 & 0 & 0 & 0 & 0 & 0 & 0 & 0 & 0 & \mathrm{~V} 2000\end{array}$

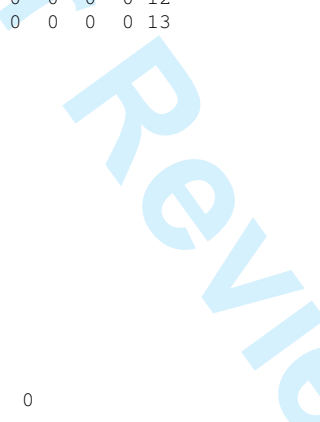

$\begin{array}{rrrrrrrrrrrrrr}-3.2776 & -0.0510 & -0.0018 & 0 & 0 & 0 & 0 & 0 & 0 & 0 & 0 & 0 & 0 & 1 \\ -3.7112 & 0.4354 & -0.7267 & \mathrm{H} & 0 & 0 & 0 & 0 & 0 & 0 & 0 & 0 & 0 & 2 \\ -3.6948 & 0.2880 & 0.8113 & \mathrm{H} & 0 & 0 & 0 & 0 & 0 & 0 & 0 & 0 & 0 & 3\end{array}$

$\begin{array}{llllllllllllll}3.6948 & 0.2880 & 0.8113 & \mathrm{H} & 0 & 0 & 0 & 0 & 0 & 0 & 0 & 0 & 0 & 3 \\ 1.4845 & 1.2018 & 0.0024 & \mathrm{C} & 0 & 0 & 0 & 0 & 0 & 0 & 0 & 0 & 0 & 4\end{array}$

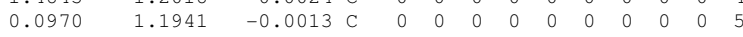

$\begin{array}{lllllllllllll}-0.5679 & 0.0185 & -0.0045 & \mathrm{~N} & 0 & 0 & 0 & 0 & 0 & 0 & 0 & 0 & 0\end{array}$

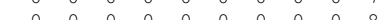

$\begin{array}{llllllllllllll}1.4527 & -1.2186 & 0.0002 & \mathrm{C} & 0 & 0 & 0 & 0 & 0 & 0 & 0 & 0 & 0 & 8 \\ 2.1699 & -0.0176 & 0.0032 & \mathrm{C} & 0 & 0 & 0 & 0 & 0 & 0 & 0 & 0 & 0 & 9\end{array}$ 


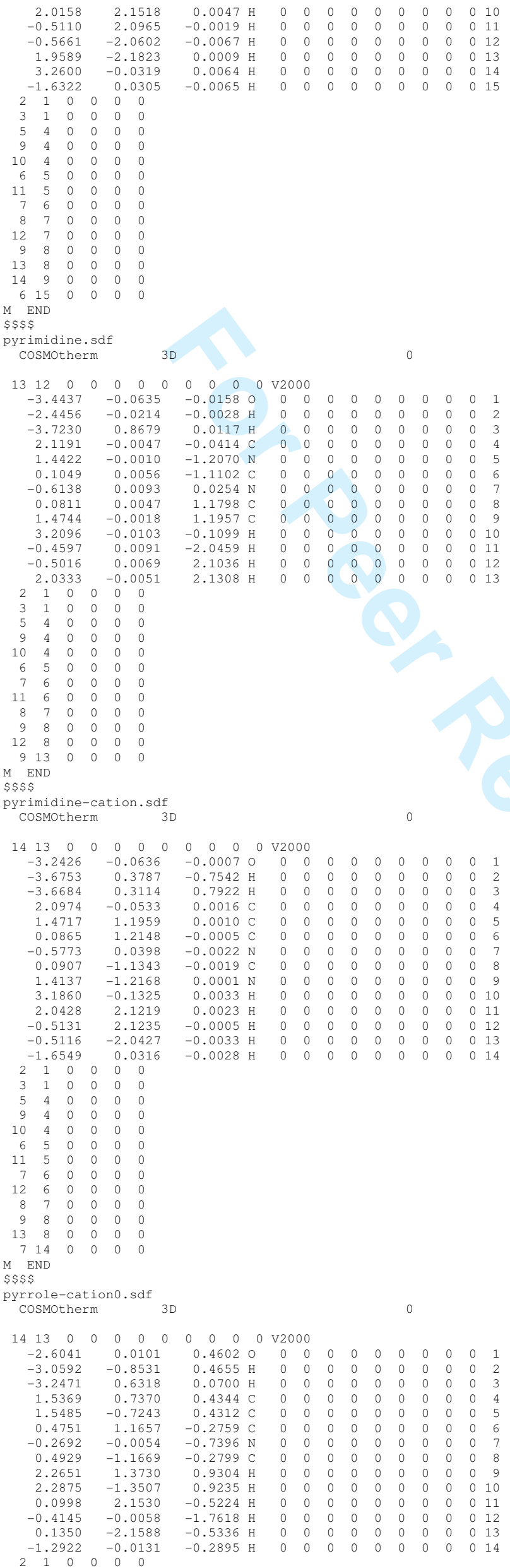




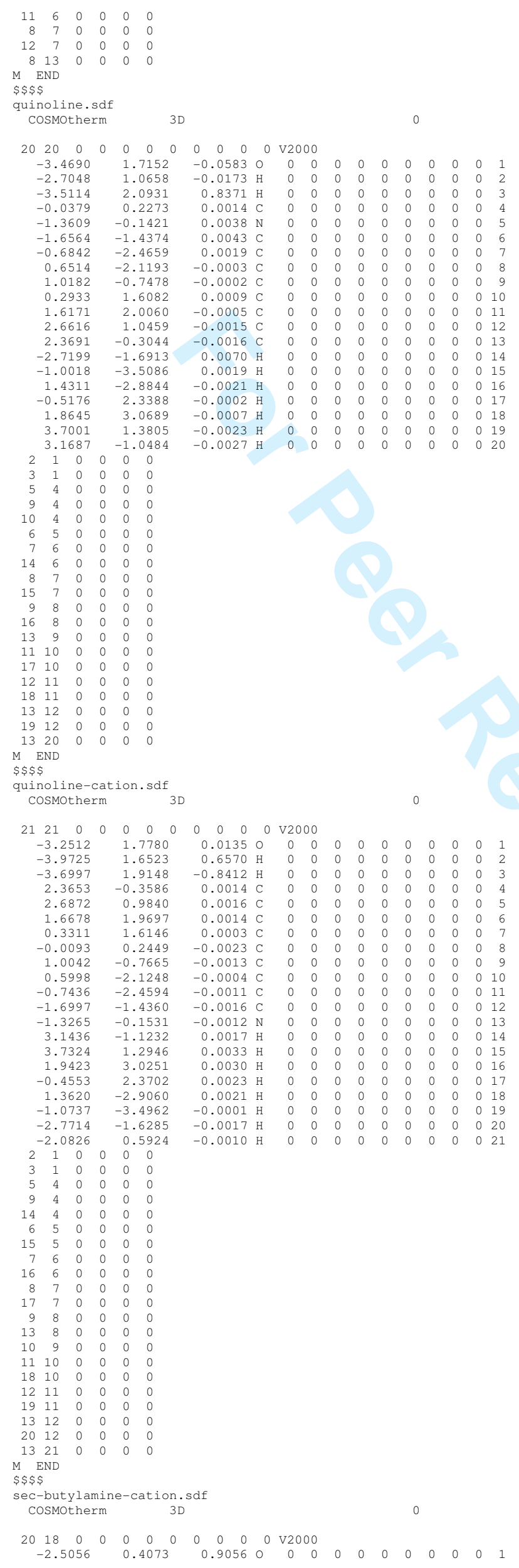



0.3192

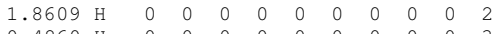
$\begin{array}{rrrrrrrrrrrrrr}-.3654 & 0.2216 & 0.4860 & \mathrm{H} & 0 & 0 & 0 & 0 & 0 & 0 & 0 & 0 & 0 & 3 \\ 0.5942 & -0.2765 & -0.7790 & \mathrm{C} & 0 & 0 & 0 & 0 & 0 & 0 & 0 & 0 & 0 & 4\end{array}$ $\begin{array}{llllllllllllll}-0.0817 & 0.4322 & -1.9477 & \mathrm{C} & 0 & 0 & 0 & 0 & 0 & 0 & 0 & 0 & 0 & 5\end{array}$ $\begin{array}{llllllllllllll}-0.4309 & -1.1524 & -0.0818 & \mathrm{~N} & 0 & 0 & 0 & 0 & 0 & 0 & 0 & 0 & 0 & 6\end{array}$ $\begin{array}{llllllllllllll}1.2249 & 0.6846 & 0.2322 & \mathrm{C} & 0 & 0 & 0 & 0 & 0 & 0 & 0 & 0 & 0 & 7\end{array}$

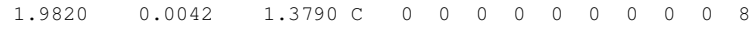

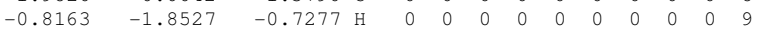

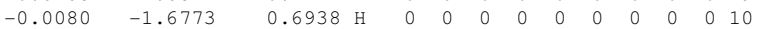
$\begin{array}{lllllllllllllll}1.3586 & -0.9725 & -1.1534 & \mathrm{H} & 0 & 0 & 0 & 0 & 0 & 0 & 0 & 0 & 0 & 11\end{array}$ $\begin{array}{lllllllllllllllll}1.3586 & -0.9725 & -1.1534 & \mathrm{H} & 0 & 0 & 0 & 0 & 0 & 0 & 0 & 0 & 0 & 11\end{array}$ $\begin{array}{llllllllllllllllll}0.6727 & 0.9892 & -2.5189 \mathrm{H} & 0 & 0 & 0 & 0 & 0 & 0 & 0 & 0 & 0 & 13\end{array}$ $\begin{array}{llllllllllllllll}-0.8407 & 1.1462 & -1.5957 \mathrm{H} & 0 & 0 & 0 & 0 & 0 & 0 & 0 & 0 & 0 & 14\end{array}$ $\begin{array}{llllllllllllllllll}-0.8407 & 1.1462 & -1.5957 & \mathrm{H} & 0 & 0 & 0 & 0 & 0 & 0 & 0 & 0 & 0 & 13 \\ 1.9191 & 1.3208 & -0.3374 \mathrm{H} & 0 & 0 & 0 & 0 & 0 & 0 & 0 & 0 & 14\end{array}$

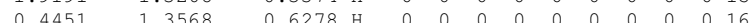
$\begin{array}{llllllllllllllllllll}0.4451 & 1.3568 & 0.6278 & 0 & 0 & 0 & 0 & 0 & 0 & 0 & 0 & 0 & 0 & 16\end{array}$

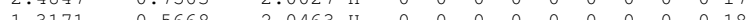
$\begin{array}{llllllllllllll}2.7500 & -0.66844 & 0.9956 & \mathrm{H} & 0 & 0 & 0 & 0 & 0 & 0 & 0 & 0 & 0 & 18\end{array}$ $\begin{array}{llllllllllllllllll}1.3171 & -0.5668 & 2.0463 & \mathrm{H} & 0 & 0 & 0 & 0 & 0 & 0 & 0 & 0 & 0 & 18 \\ -1.2426 & -0.5864 & 0.9956 & \mathrm{H} & 0 & 0 & 0 & 0 & 0 & 0 & 0 & 0 & 0 & 19\end{array}$ $-1.2426-0.58640$ $\begin{array}{llllllll}3 & 1 & 0 & 0 & 0 & 0\end{array}$ $\begin{array}{lllllll}5 & 4 & 0 & 0 & 0 & 0\end{array}$ $\begin{array}{lllllll}6 & 4 & 0 & 0 & 0 & 0\end{array}$ $\begin{array}{llllll}7 & 4 & 0 & 0 & 0 & 0\end{array}$

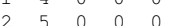
$\begin{array}{lllllllll}12 & 5 & 0 & 0 & 0 & 0 \\ 3 & 5 & 0 & 0 & 0 & 0\end{array}$ $4 \quad 5 \quad 00000$ $9 \begin{array}{llllll}9 & 6 & 0 & 0 & 0 & 0\end{array}$

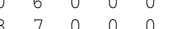
$\begin{array}{lllllll}5 & 7 & 0 & 0 & 0 & 0\end{array}$ $\begin{array}{lllllll}16 & 7 & 0 & 0 & 0 & 0\end{array}$ $\begin{array}{lllllll}17 & 8 & 0 & 0 & 0 & 0\end{array}$ $\begin{array}{llllll}8 & 8 & 0 & 0 & 0 & 0 \\ 9 & 8 & 0 & 0 & 0 & 0\end{array}$ $\begin{array}{lllllll}6 & 20 & 0 & 0 & 0 & 0\end{array}$ END

sec-butylamine.sdf

Cosmotherm

\section{$\begin{array}{llllllllllll}19 & 17 & 0 & 0 & 0 & 0 & 0 & 0 & 0 & 0 & 0 & \mathrm{~V} 2000\end{array}$}

$\begin{array}{llllllllllllll}-1.8215 & -0.5798 & -0.1233 & \mathrm{H} & 0 & 0 & 0 & 0 & 0 & 0 & 0 & 0 & 0 & \end{array}$

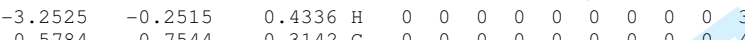

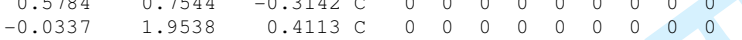

$\begin{array}{lllllllllllll}-0.4899 & 0.0670 & -1.0882 & \mathrm{~N} & 0 & 0 & 0 & 0 & 0 & 0 & 0 & 0 & 0\end{array}$

$\begin{array}{rrrrrrrrrrrrrrr}1.2331 & -0.2287 & 0.6660 & \mathrm{C} & 0 & 0 & 0 & 0 & 0 & 0 & 0 & 0 & 0\end{array}$

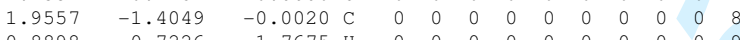

$\begin{array}{llllllllllllll}-0.8898 & 0.7226 & -1.7675 & \mathrm{H} & 0 & 0 & 0 & 0 & 0 & 0 & 0 & 0 & 0 & 9\end{array}$

$\begin{array}{rrrrrrrrrrrrrr}-0.0837 & -0.6881 & -1.6488 & \mathrm{H} & 0 & 0 & 0 & 0 & 0 & 0 & 0 & 0 & 0 & 10 \\ 1.3672 & 1.1259 & -0.9982 & \mathrm{H} & 0 & 0 & 0 & 0 & 0 & 0 & 0 & 0 & 0 & 11\end{array}$

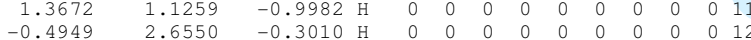

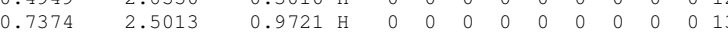

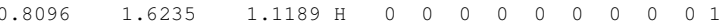

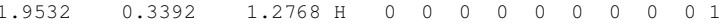

$\begin{array}{lllllllllllllll}1.9532 & 0.3392 & -0.6038 & 1.2768 & \mathrm{H} & 0 & 0 & 0 & 0 & 0 & 0 & 0 & 0 & 0 & 15 \\ 2.4681 & -2.0283 & 0.7474 & \mathrm{H} & 0 & 0 & 0 & 0 & 0 & 0 & 0 & 0 & 0 & 0 & 16\end{array}$

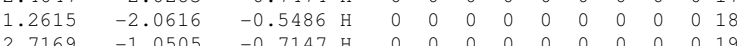

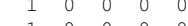

$\begin{array}{lllllll}1 & 0 & 0 & 0 & 0\end{array}$

$\begin{array}{lllll}4 & 0 & 0 & 0 & 0\end{array}$

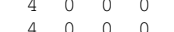

$\begin{array}{lllllll}2 & 5 & 0 & 0 & 0 & 0\end{array}$

$\begin{array}{lllll}5 & 0 & 0 & 0 & 0 \\ 5 & 0 & 0 & 0 & 0\end{array}$

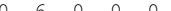

$\begin{array}{lllll}7 & 0 & 0 & 0\end{array}$

$\begin{array}{llllll}15 & 7 & 0 & 0 & 0\end{array}$

$\begin{array}{lllll}7 & 0 & 0 & 0\end{array}$

$\begin{array}{llllll}8 & 0 & 0 & 0 & 0 \\ 8 & 8 & 0 & 0 & 0\end{array}$

$\begin{array}{llllll}19 & 0 & 0 & 0 & 0\end{array}$

END

C-butylamine-cation.sdf

cosmotherm 3D

$\begin{array}{llllllllllll}20 & 18 & 0 & 0 & 0 & 0 & 0 & 0 & 0 & 0 & 0 & \mathrm{v} 2000\end{array}$

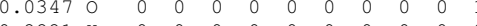

$\begin{array}{lllllllllllll}-3.3787 & -0.7799 & -0.2221 & \mathrm{H} & 0 & 0 & 0 & 0 & 0 & 0 & 0 & 0 & 0\end{array}$

$\begin{array}{lllllllllllll}2.1301 & 0.0061 & -0.6321 & \mathrm{C} & 0 & 0 & 0 & 0 & 0 & 0 & 0 & 0 & 0\end{array}$

$\begin{array}{llllllllllllll}0.7611 & -0.0017 & 0.0538 & \mathrm{C} & 0 & 0 & 0 & 0 & 0 & 0 & 0 & 0 & 0 & 5\end{array}$

$\begin{array}{llllllllllllll}0.5557 & 1.2451 & 0.9189 & \mathrm{C} & 0 & 0 & 0 & 0 & 0 & 0 & 0 & 0 & 0 & 6\end{array}$

$\begin{array}{llllllllllllll}0.5368 & -1.2896 & 0.8514 & \mathrm{C} & 0 & 0 & 0 & 0 & 0 & 0 & 0 & 0 & 0 & 7\end{array}$

$\begin{array}{llllllllllllll}-0.2985 & 0.0354 & -1.0476 & \mathrm{~N} & 0 & 0 & 0 & 0 & 0 & 0 & 0 & 0 & 0 & 8\end{array}$

$\begin{array}{rrrrrrrrrrrrrr}-1.2865 & 0.0235 & -0.6566 & \mathrm{H} & 0 & 0 & 0 & 0 & 0 & 0 & 0 & 0 & 0 & \\ -0.1998 & -0.7698 & -1.6795 & \mathrm{H} & 0 & 0 & 0 & 0 & 0 & 0 & 0 & 0 & 0 & 10\end{array}$

$\begin{array}{llllllllllllll}2.2552 & -0.8716 & -1.2831 & \mathrm{H} & 0 & 0 & 0 & 0 & 0 & 0 & 0 & 0 & 0 & 11\end{array}$

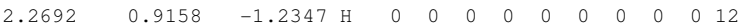

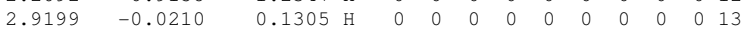

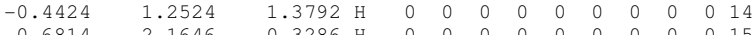

$\begin{array}{lllllllllllllllllllll}1.3025 & 1.2536 & 1.32839 \mathrm{H} & 0 & 0 & 0 & 0 & 0 & 0 & 0 & 0 & 0 & 16\end{array}$

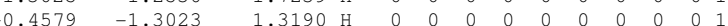

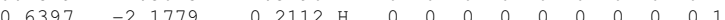

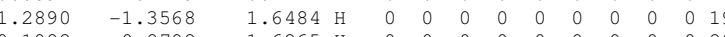




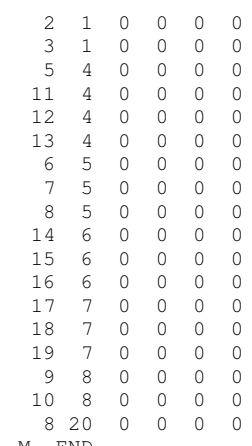$$
\text { M END }
$$$$
\$ \$ \$ \$
$$

ert-butylamine.sdf

$\begin{array}{llllllllllll}19 & 17 & 0 & 0 & 0 & 0 & 0 & 0 & 0 & 0 & 0 & \mathrm{~V} 2000\end{array}$

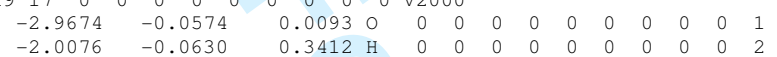

$\begin{array}{rrrrrrrrrrrrrrr}-2.0076 & -0.0630 & 0.3412 & \mathrm{H} & 0 & 0 & 0 & 0 & 0 & 0 & 0 & 0 & 0 & 2 \\ -3.1629 & 0.8819 & -0.1509 & \mathrm{H} & 0 & 0 & 0 & 0 & 0 & 0 & 0 & 0 & 0 & 3\end{array}$

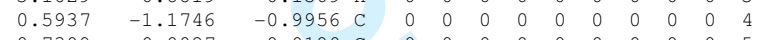

$\begin{array}{llllllllllllll}0.7309 & 0.0027 & -0.0190 & \mathrm{C} & 0 & 0 & 0 & 0 & 0 & 0 & 0 & 0 & 0 & 5 \\ 0.5422 & 1.3364 & -0.7572 & \mathrm{C} & 0 & 0 & 0 & 0 & 0 & 0 & 0 & 0 & 0 & 6\end{array}$

$\begin{array}{rrrrrrrrrrrrrr}0.5422 & 1.3364 & -0.7572 & \mathrm{C} & 0 & 0 & 0 & 0 & 0 & 0 & 0 & 0 & 0 & 6 \\ 2.1107 & -0.0336 & 0.6661 & \mathrm{C} & 0 & 0 & 0 & 0 & 0 & 0 & 0 & 0 & 0 & 7\end{array}$

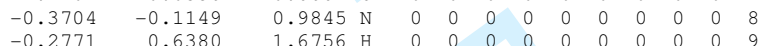

$\begin{array}{rrrrrrrrrrrrrr}-0.2771 & 0.6380 & 1.6756 & \mathrm{H} & 0 & 0 & 0 & 0 & 0 & 0 & 0 & 0 & 0 & 9 \\ -0.2526 & -0.9856 & 1.5146 & \mathrm{H} & 0 & 0 & 0 & 0 & 0 & 0 & 0 & 0 & 0 & 10\end{array}$

$\begin{array}{llllllllllllll}0.6892 & -2.1356 & -0.4664 \mathrm{H} & 0 & 0 & 0 & 0 & 0 & 0 & 0 & 0 & 0 & 11\end{array}$

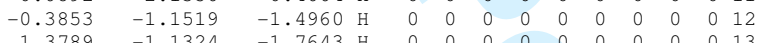

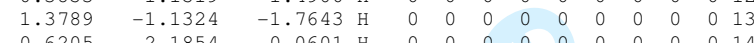

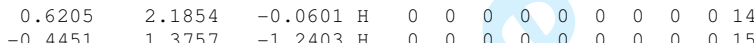

$\begin{array}{lllllllllllll}-0.4451 & 1.3767 & -1.2403 & \mathrm{H} & 0 & 0 & 0 & 0 & 0 & 0 & 0 & 0 & 0 \\ 1.3126 & 1.4627 & -1.5312 \mathrm{H} & 0 & 0 & 0 & 0 & 0 & 0 & 0 & 0 & 0 & 16\end{array}$

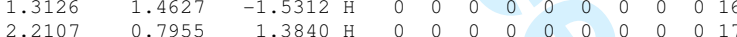

$\begin{array}{llllllllllllll}2.2488 & -0.9794 & 1.2128 & \mathrm{H} & 0 & 0 & 0 & 0 & 0 & 0 & 0 & 0 & 0 & 18\end{array}$

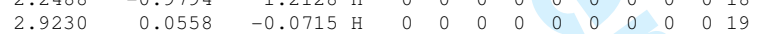

$\begin{array}{llllll}2 & 1 & 0 & 0 & 0 & 0\end{array}$

$\begin{array}{llllll}3 & 1 & 0 & 0 & 0 & 0 \\ 5 & 4 & 0 & 0 & 0 & 0\end{array}$

$\begin{array}{llllll}11 & 4 & 0 & 0 & 0 & 0\end{array}$

$\begin{array}{llllll}12 & 4 & 0 & 0 & 0 & 0 \\ 13 & 4 & 0 & 0 & 0 & 0\end{array}$

$\begin{array}{llllll}5 & 0 & 0 & 0 & 0\end{array}$

$\begin{array}{lllll}5 & 0 & 0 & 0 & 0\end{array}$

$\begin{array}{llllll}8 & 5 & 0 & 0 & 0 & 0 \\ 4 & 6 & 0 & 0 & 0 & 0\end{array}$

$\begin{array}{llllll}5 & 6 & 0 & 0 & 0\end{array}$

$\begin{array}{llllll}16 & 6 & 0 & 0 & 0 & 0 \\ 17 & 7 & 0 & 0 & 0 & 0\end{array}$

$\begin{array}{llllllllll}18 & 7 & 0 & 0 & 0 & 0 & 0 & 2\end{array}$

$\begin{array}{lllllll}9 & 8 & 0 & 0 & 0 & 0\end{array}$

$\begin{array}{rrrrrr}10 & 8 & 0 & 0 & 0 & 0 \\ 7 & 19 & 0 & 0 & 0 & 0\end{array}$

END

$\$ \$ \$$

tert-butylcyclohexylamine-cation.sdf

cosmotherm

$\begin{array}{llllllllllll}36 & 35 & 0 & 0 & 0 & 0 & 0 & 0 & 0 & 0 & 0 & \mathrm{v} 2000\end{array}$

$\begin{array}{lllllllllllll}-2.8269 & 1.1312 & -0.7075 & 0 & 0 & 0 & 0 & 0 & 0 & 0 & 0 & 0 & 0\end{array}$

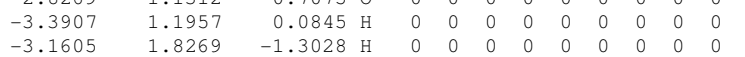

$\begin{array}{lllllllllllll}-3.1605 & 1.8269 & -1.3828 & 0 & 0 & 0 & 0 & 0 & 0 & 0 & 0 & 0 & 2 \\ -0.0254 & 0.8387 & -0.5273 & 0 & 0 & 0 & 0 & 0 & 0 & 0 & 0 & 0\end{array}$

$\begin{array}{llrlllllllllll}0.6057 & 2.0559 & 0.1867 & \mathrm{C} & 0 & 0 & 0 & 0 & 0 & 0 & 0 & 0 & 0 & 5\end{array}$

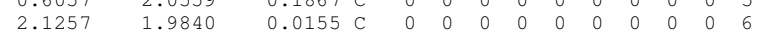

$\begin{array}{lllllllllllllll}0.0411 & 3.2912 & -0.5264 & \mathrm{C} & 0 & 0 & 0 & 0 & 0 & 0 & 0 & 0 & 0 & 7\end{array}$

$\begin{array}{rrrrrrrrrrrrrrr}0.1955 & 2.0375 & 1.6601 & \mathrm{C} & 0 & 0 & 0 & 0 & 0 & 0 & 0 & 0 & 0 & 8\end{array}$

$\begin{array}{rrrrrrrrrrrrrr}0.1955 & 2.0375 & 1.6601 & C & 0 & 0 & 0 & 0 & 0 & 0 & 0 & 0 & 0 & 8 \\ 0.3149 & -0.5627 & -0.0168 & C & 0 & 0 & 0 & 0 & 0 & 0 & 0 & 0 & 0 & 9\end{array}$

$\begin{array}{rrrrrrrrrrrrrr}0.3149 & -0.5627 & -0.0168 & \mathrm{C} & 0 & 0 & 0 & 0 & 0 & 0 & 0 & 0 & 0 & 8 \\ -0.8615 & -1.1730 & 0.7509 & \mathrm{C} & 0 & 0 & 0 & 0 & 0 & 0 & 0 & 0 & 0 & 10\end{array}$

$\begin{array}{lllllllllllllll}-0.8615 & -1.1730 & 0.7509 & \mathrm{C} & 0 & 0 & 0 & 0 & 0 & 0 & 0 & 0 & 0 & 10 \\ -0.5016 & -2.5881 & 1.2345 \mathrm{C} & 0 & 0 & 0 & 0 & 0 & 0 & 0 & 0 & 0 & 11\end{array}$

$\begin{array}{llllllllllllll}-0.5016 & -2.5881 & 1.2345 & \mathrm{C} & 0 & 0 & 0 & 0 & 0 & 0 & 0 & 0 & 0 & 11 \\ -0.0742 & -3.4896 & 0.0679 & \mathrm{C} & 0 & 0 & 0 & 0 & 0 & 0 & 0 & 0 & 0 & 12\end{array}$

$\begin{array}{rrrrrrrrrrrrrr}-0.0742 & -3.4896 & 0.0679 & \mathrm{C} & 0 & 0 & 0 & 0 & 0 & 0 & 0 & 0 & 0 & 12 \\ 1.0822 & -2.8628 & -0.7232 & \mathrm{C} & 0 & 0 & 0 & 0 & 0 & 0 & 0 & 0 & 0 & 13\end{array}$

$\begin{array}{llllllllllllll}1.0822 & -2.8628 & -0.7232 & \mathrm{C} & 0 & 0 & 0 & 0 & 0 & 0 & 0 & 0 & 0 & 13 \\ 0.7355 & -1.4429 & -1.1991 & \mathrm{C} & 0 & 0 & 0 & 0 & 0 & 0 & 0 & 0 & 0 & 14\end{array}$

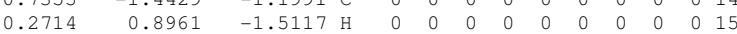

$\begin{array}{rrrrrrrrrrrrrr}0.5741 & 2.8784 & 0.4679 & \mathrm{H} & 0 & 0 & 0 & 0 & 0 & 0 & 0 & 0 & 0 & 16\end{array}$

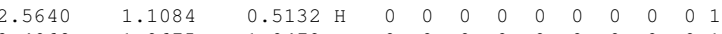

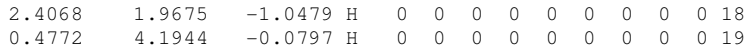

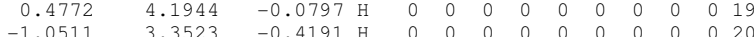

$\begin{array}{rrrrrrrrrrrrrr}-1.0511 & 3.3523 & -0.4191 & \mathrm{H} & 0 & 0 & 0 & 0 & 0 & 0 & 0 & 0 & 0 & 20 \\ 0.2941 & 3.2864 & -1.5969 & \mathrm{H} & 0 & 0 & 0 & 0 & 0 & 0 & 0 & 0 & 0 & 21\end{array}$

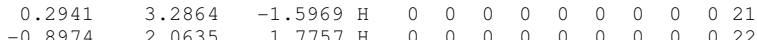

$\begin{array}{llllllllllllll}-0.8974 & 2.0635 & 1.7757 & \mathrm{H} & 0 & 0 & 0 & 0 & 0 & 0 & 0 & 0 & 0 & 22\end{array}$

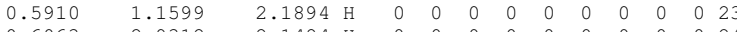

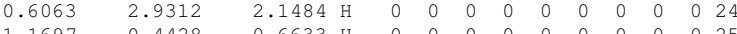

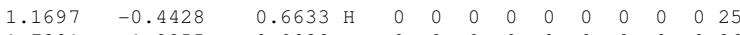

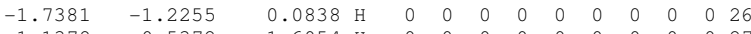

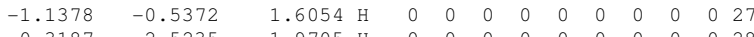

$\begin{array}{llllllllllllll}0.3187 & -2.5235 & 1.9705 & \mathrm{H} & 0 & 0 & 0 & 0 & 0 & 0 & 0 & 0 & 0 & 28\end{array}$

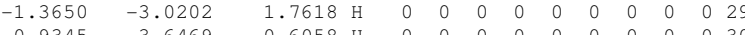

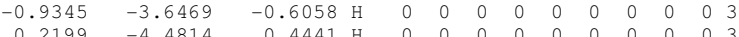

$\begin{array}{rrrrrrrrrrrrrr}0.2199 & -4.4814 & 0.4441 & \mathrm{H} & 0 & 0 & 0 & 0 & 0 & 0 & 0 & 0 & 0 & 31 \\ 1.9829 & -2.8214 & -0.0868 & \mathrm{H} & 0 & 0 & 0 & 0 & 0 & 0 & 0 & 0 & 0 & 32\end{array}$

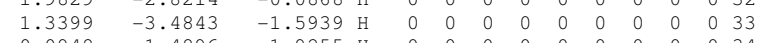

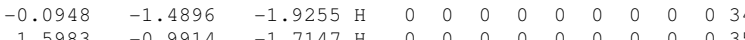

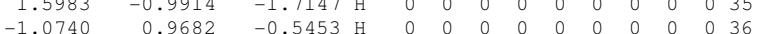

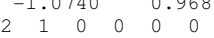

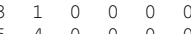




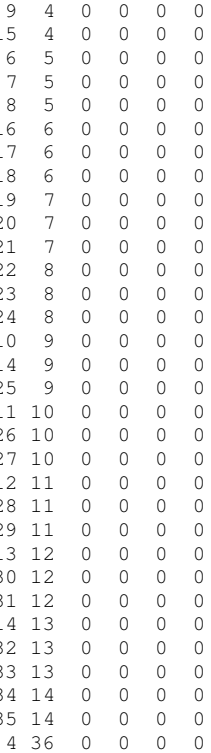

END

$\$ \$ \$$

tert-butylcyclohexylamine.sdf

$\begin{array}{llllllllllll}35 & 34 & 0 & 0 & 0 & 0 & 0 & 0 & 0 & 0 & 0 & \mathrm{~V} 2000\end{array}$

$\begin{array}{lllllllllllll}-2.8318 & -0.7694 & 1.2234 & 0 & 0 & 0 & 0 & 0 & 0 & 0 & 0 & 0 & 0\end{array}$

$\begin{array}{lllllllllllll}-1.8361 & -0.6385 & 1.0765 & \mathrm{H} & 0 & 0 & 0 & 0 & 0 & 0 & 0 & 0 & 0\end{array}$

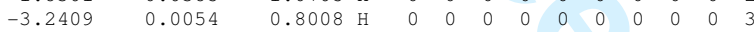

$\begin{array}{lllllllllllll}-0.0673 & -0.5129 & 0.8413 & \mathrm{~N} & 0 & 0 & 0 & 0 & 0 & 0 & 0 & 0 & 0\end{array}$

$\begin{array}{llllllllllllll}0.5716 & 0.1704 & 2.0174 & \mathrm{C} & 0 & 0 & 0 & 0 & 0 & 0 & 0 & 0 & 0 & 5\end{array}$

$\begin{array}{llllllllllllll}2.1117 & 0.0729 & 1.9792 & \mathrm{C} & 0 & 0 & 0 & 0 & 0 & 0 & 0 & 0 & 0 & 6\end{array}$

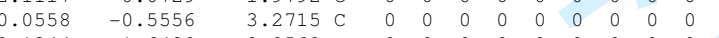

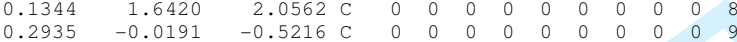

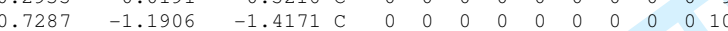

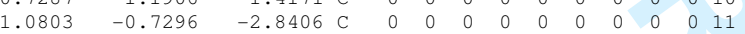

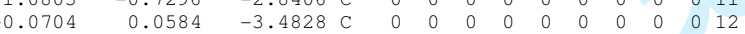

$\begin{array}{llllllllllllll}-0.5053 & 1.2289 & -2.5891 & \mathrm{C} & 0 & 0 & 0 & 0 & 0 & 0 & 0 & 0 & 0 & 13\end{array}$

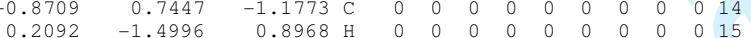

$\begin{array}{lllllllllllll}2.5485 & 0.5547 & 2.8668 \mathrm{H} & 0 & 0 & 0 & 0 & 0 & 0 & 0 & 0 & 0 & 15\end{array}$

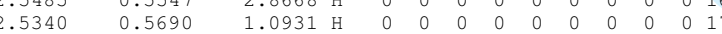

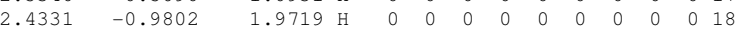

$\begin{array}{llllllllllllll}1.0393 & -0.4876 & 3.3393 & \mathrm{H} & 0 & 0 & 0 & 0 & 0 & 0 & 0 & 0 & 0 & 19\end{array}$

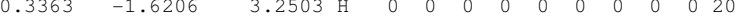

$\begin{array}{llllllllllllll}0.4916 & -0.1105 & 4.1776 \mathrm{H} & 0 & 0 & 0 & 0 & 0 & 0 & 0 & 0 & 0 & 21\end{array}$

$\begin{array}{lllllllllllllll}-0.9622 & 1.7213 & 2.0875 & \mathrm{H} & 0 & 0 & 0 & 0 & 0 & 0 & 0 & 0 & 0 & 23\end{array}$

$0.4988-2.1986-1.1803-\mathrm{H}-0$

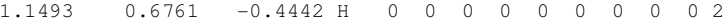

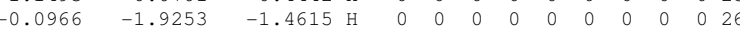

$\begin{array}{llllllllllllll}1.5909 & -1.7031 & -0.9592 & \mathrm{H} & 0 & 0 & 0 & 0 & 0 & 0 & 0 & 0 & 0 & 27\end{array}$

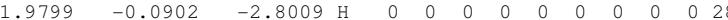

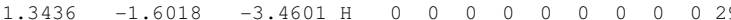

$\begin{array}{llllllllllllll}-0.9307 & -0.6165 & -3.6398 & \mathrm{H} & 0 & 0 & 0 & 0 & 0 & 0 & 0 & 0 & 0 & 30\end{array}$

$\begin{array}{llllllllllllll}0.2294 & 0.4256 & -4.4772 & \mathrm{H} & 0 & 0 & 0 & 0 & 0 & 0 & 0 & 0 & 0 & 31\end{array}$

$\begin{array}{llllllllllllll}0.3179 & 1.9630 & -2.5223 & \mathrm{H} & 0 & 0 & 0 & 0 & 0 & 0 & 0 & 0 & 0 & 32\end{array}$

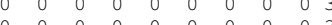

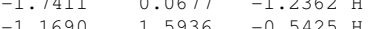

21.16901 .536

$\begin{array}{llllll}2 & 1 & 0 & 0 & 0 & 0 \\ 3 & 1 & 0 & 0 & 0 & 0\end{array}$

$\begin{array}{lllllll}5 & 4 & 0 & 0 & 0 & 0\end{array}$

$\begin{array}{lllllllll}9 & 4 & 0 & 0 & 0 & 0\end{array}$

$\begin{array}{lllllllll}5 & 4 & 0 & 0 & 0 & 0\end{array}$

$\begin{array}{lllll}5 & 0 & 0 & 0\end{array}$

$\begin{array}{llllll}5 & 0 & 0 & 0 & 0\end{array}$

$\begin{array}{llllll}6 & 6 & 0 & 0 & 0\end{array}$

$\begin{array}{llllll}18 & 6 & 0 & 0 & 0 & 0\end{array}$

$\begin{array}{llllll}7 & 7 & 0 & 0 & 0 & 0\end{array}$

$\begin{array}{llll}7 & 0 & 0 & 0\end{array}$

$\begin{array}{lllll}3 & 8 & 0 & 0 & 0\end{array}$

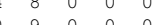

$\begin{array}{lllll}9 & 0 & 0 & 0\end{array}$

$\begin{array}{llllll}14 & 9 & 0 & 0 & 0 & 0\end{array}$

$\begin{array}{llllll}1 & 10 & 0 & 0 & 0 \\ 26 & 10 & 0 & 0 & 0\end{array}$

$\begin{array}{llllll}27 & 10 & 0 & 0 & 0 & 0\end{array}$

$\begin{array}{llllll}2 & 11 & 0 & 0 & 0 & 0 \\ 28 & 11 & 0 & 0 & 0 & 0\end{array}$

$2911 \quad 000000$

$\begin{array}{lllll}1 & 12 & 0 & 0 & 0\end{array}$

$\begin{array}{llllll}31 & 12 & 0 & 0 & 0 & 0\end{array}$

$\begin{array}{llllll}4 & 13 & 0 & 0 & 0 & 0\end{array}$

$\begin{array}{lllll}33 & 13 & 0 & 0 & 0\end{array}$ 


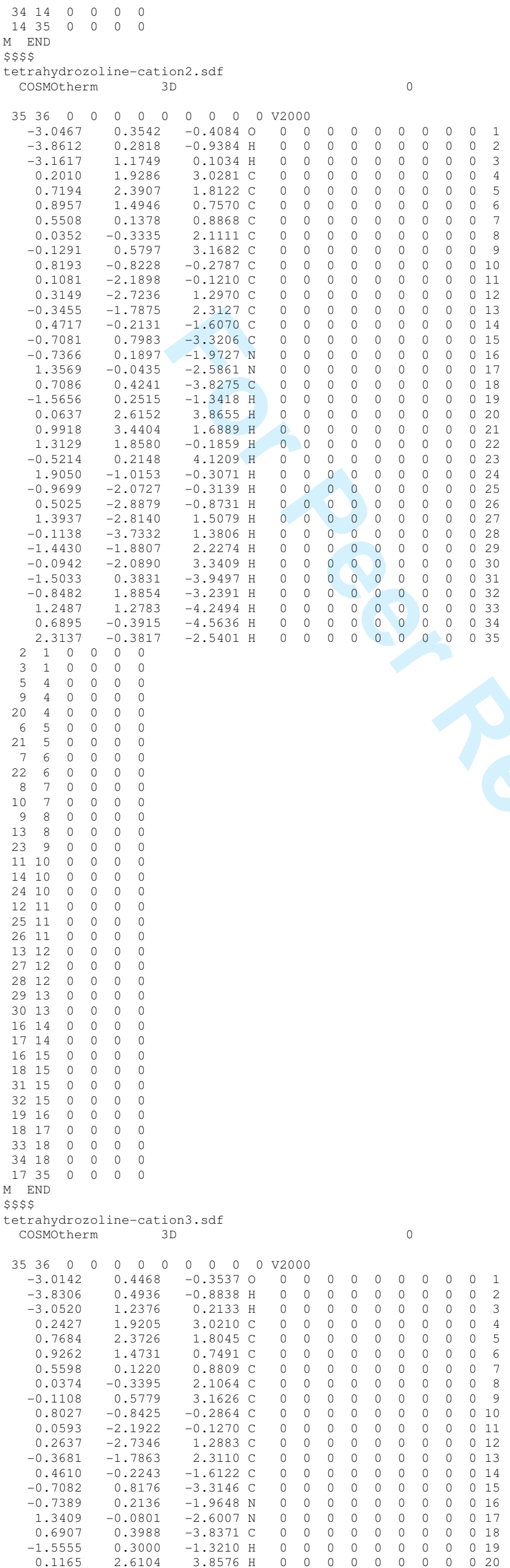


$\begin{array}{llllllllllllll}-2.8392 & -1.5552 & -0.4069 & \mathrm{H} & 0 & 0 & 0 & 0 & 0 & 0 & 0 & 0 & 0 & 2 \\ -3.4982 & -0.2196 & -0.0059 & \mathrm{H} & 0 & 0 & 0 & 0 & 0 & 0 & 0 & 0 & 0 & 3\end{array}$

$\begin{array}{llllllllllllll}-0.5322 & 1.7807 & -0.0059 & \mathrm{H} & 0 & 0 & 0 & 0 & 0 & 0 & 0 & 0 & 0 & 3\end{array}$

$\begin{array}{llllllllllllll}0.0469 & 2.7670 & -1.2521 & \mathrm{C} & 0 & 0 & 0 & 0 & 0 & 0 & 0 & 0 & 0 & 5\end{array}$

$\begin{array}{llllllllllllll}-0.0904 & 2.2015 & 0.1621 & \mathrm{C} & 0 & 0 & 0 & 0 & 0 & 0 & 0 & 0 & 0 & 6 \\ 0.7476 & 0.9039 & 0.3010 & \mathrm{C} & 0 & 0 & 0 & 0 & 0 & 0 & 0 & 0 & 0 & 7\end{array}$

$\begin{array}{llllllllllllll}0.5786 & -0.0570 & -0.8801 & \mathrm{C} & 0 & 0 & 0 & 0 & 0 & 0 & 0 & 0 & 0 & 8\end{array}$

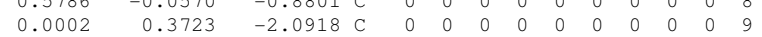

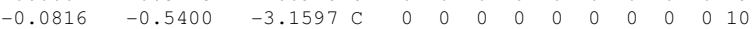

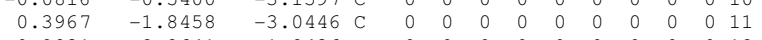

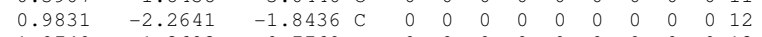

$\begin{array}{lllllllllllll}1.0740 & -1.3693 & -0.7769 \mathrm{C} & 0 & 0 & 0 & 0 & 0 & 0 & 0 & 0 & 0 & 13\end{array}$

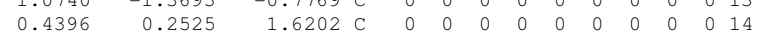

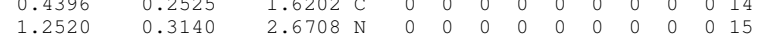

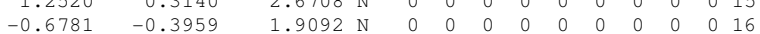

$\begin{array}{cccccccccccccc}-0.6781 & -0.3959 & 1.9092 \mathrm{~N} & 0 & 0 & 0 & 0 & 0 & 0 & 0 & 0 & 0 & 16 \\ 0.6916 & -0.3834 & 3.8448 \mathrm{C} & 0 & 0 & 0 & 0 & 0 & 0 & 0 & 0 & 0 & 17\end{array}$

$\begin{array}{llllllllllllllllllllllll}-0.7032 & -0.8310 & 3.3217 \mathrm{C} & 0 & 0 & 0 & 0 & 0 & 0 & 0 & 0 & 0 & 18\end{array}$

$\begin{array}{llllllllllllll}-0.3299 & 2.1175 & -3.2976 \mathrm{H} & 0 & 0 & 0 & 0 & 0 & 0 & 0 & 0 & 0 & 19\end{array}$

$\begin{array}{lllllllllllllll}-1.6321 & 1.7616 & -2.1671 \mathrm{H} & 0 & 0 & 0 & 0 & 0 & 0 & 0 & 0 & 0 & 20\end{array}$

$\begin{array}{llllllllllllll}-0.4776 & 3.7312 & -1.3190 \mathrm{H} & 0 & 0 & 0 & 0 & 0 & 0 & 0 & 0 & 0 & 21\end{array}$

$\begin{array}{lllllllllllllll}1.1096 & 2.9619 & -1.4738 & \mathrm{H} & 0 & 0 & 0 & 0 & 0 & 0 & 0 & 0 & 0 & 22\end{array}$

$\begin{array}{llllllllllllllll}-1.1510 & 1.9827 & 0.3663 & \mathrm{H} & 0 & 0 & 0 & 0 & 0 & 0 & 0 & 0 & 0 & 23\end{array}$

$\begin{array}{llllllllllllllllll}0.2439 & 2.9261 & 0.9180 & \mathrm{H} & 0 & 0 & 0 & 0 & 0 & 0 & 0 & 0 & 0 & 0 & 24 \\ 1.8096 & 1.1981 & 0.3444 & \end{array}$

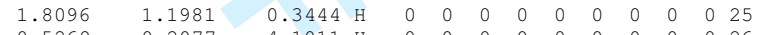

$\begin{array}{lllllllllllllll}-0.5268 & -0.2077 & -4.1011 & \mathrm{H} & 0 & 0 & 0 & 0 & 0 & 0 & 0 & 0 & 0 & 26\end{array}$

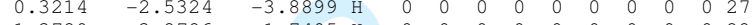

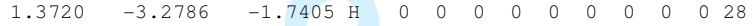

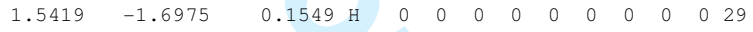

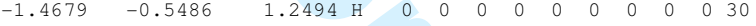

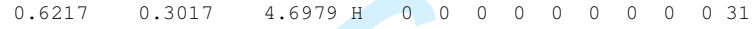

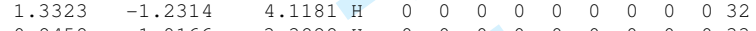

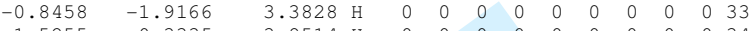

$\begin{array}{llllllllllllll}-1.5255 & -0.3335 & 3.8514 & \mathrm{H} & 0 & 0 & 0 & 0 & 0 & 0 & 0 & 0 & 0 & 34\end{array}$

$2.1796 \quad 0.7269$

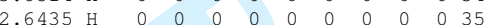

thiazole.sd

cosmotherm

$\begin{array}{llllllllllll}11 & 10 & 0 & 0 & 0 & 0 & 0 & 0 & 0 & 0 & 0 & \mathrm{~V} 2000\end{array}$

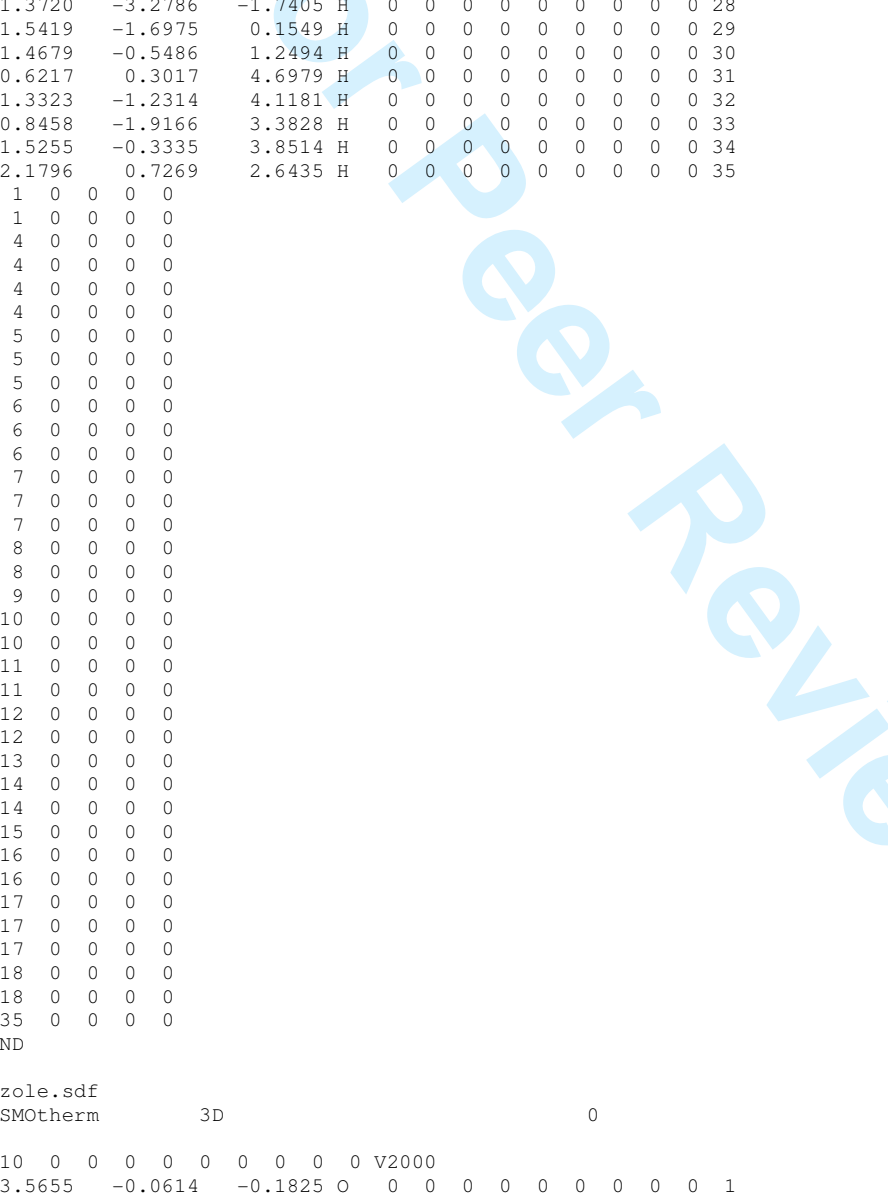

$\begin{array}{lllllllllllll}-0.1825 & 0 & 0 & 0 & 0 & 0 & 0 & 0 & 0 & 0 & 0 & 1\end{array}$

$\begin{array}{rrrrrrrrrrrrrr}-2.5737 & -0.0184 & -0.0789 & \mathrm{H} & 0 & 0 & 0 & 0 & 0 & 0 & 0 & 0 & 0 & 2 \\ -3.8407 & 0.8696 & -0.2439 & \mathrm{H} & 0 & 0 & 0 & 0 & 0 & 0 & 0 & 0 & 0 & 3\end{array}$

$\begin{array}{rrrrrrrrrrrrrr}-3.8407 & 0.8696 & -0.2439 & \mathrm{H} & 0 & 0 & 0 & 0 & 0 & 0 & 0 & 0 & 0 & 3 \\ -0.0354 & 0.0036 & 1.2692 & \mathrm{C} & 0 & 0 & 0 & 0 & 0 & 0 & 0 & 0 & 0 & 4\end{array}$

$\begin{array}{lllllllllllllll}-0.7453 & 0.0064 & 0.0831 \mathrm{~N} & 0 & 0 & 0 & 0 & 0 & 0 & 0 & 0 & 0 & 5\end{array}$

$\begin{array}{llllllllllllll}0.0611 & 0.0037 & -0.9530 & \mathrm{C} & 0 & 0 & 0 & 0 & 0 & 0 & 0 & 0 & 0 & 6 \\ 1.7603 & -0.0016 & -0.5473 & \mathrm{~S} & 0 & 0 & 0 & 0 & 0 & 0 & 0 & 0 & 0 & 7\end{array}$

$\begin{array}{rrrrrrrrrrrrrr}1.7603 & -0.0016 & -0.5473 & \mathrm{~S} & 0 & 0 & 0 & 0 & 0 & 0 & 0 & 0 & 0 & 7 \\ 1.3273 & -0.0008 & 1.1386 & \mathrm{C} & 0 & 0 & 0 & 0 & 0 & 0 & 0 & 0 & 0 & 8\end{array}$

$\begin{array}{llllllllllllll}-0.5736 & 0.0055 & 2.2153 & \mathrm{H} & 0 & 0 & 0 & 0 & 0 & 0 & 0 & 0 & 0 & 9\end{array}$

$-0.2670-0.0053-1.9903-1$

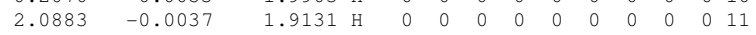

$\begin{array}{lllllll}2 & 1 & 0 & 0 & 0 & 0\end{array}$

$\begin{array}{llllll}3 & 1 & 0 & 0 & 0 & 0\end{array}$

$\begin{array}{llllll}5 & 4 & 0 & 0 & 0 & 0 \\ 8 & 4 & 0 & 0 & 0 & 0\end{array}$

$\begin{array}{lllll}4 & 0 & 0 & 0 & 0\end{array}$

$\begin{array}{ccccc}4 & 0 & 0 & 0 & 0 \\ 5 & 0 & 0 & 0 & 0\end{array}$

$\begin{array}{lllllll}7 & 6 & 0 & 0 & 0 & 0\end{array}$

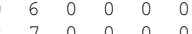

$8 \begin{array}{llllll}11 & 0 & 0 & 0 & 0\end{array}$

END

thiazole-cation.sdf

cosmotherm 3 D

$\begin{array}{llllllllllll}12 & 11 & 0 & 0 & 0 & 0 & 0 & 0 & 0 & 0 & 0 & \mathrm{v} 2000\end{array}$

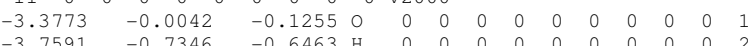




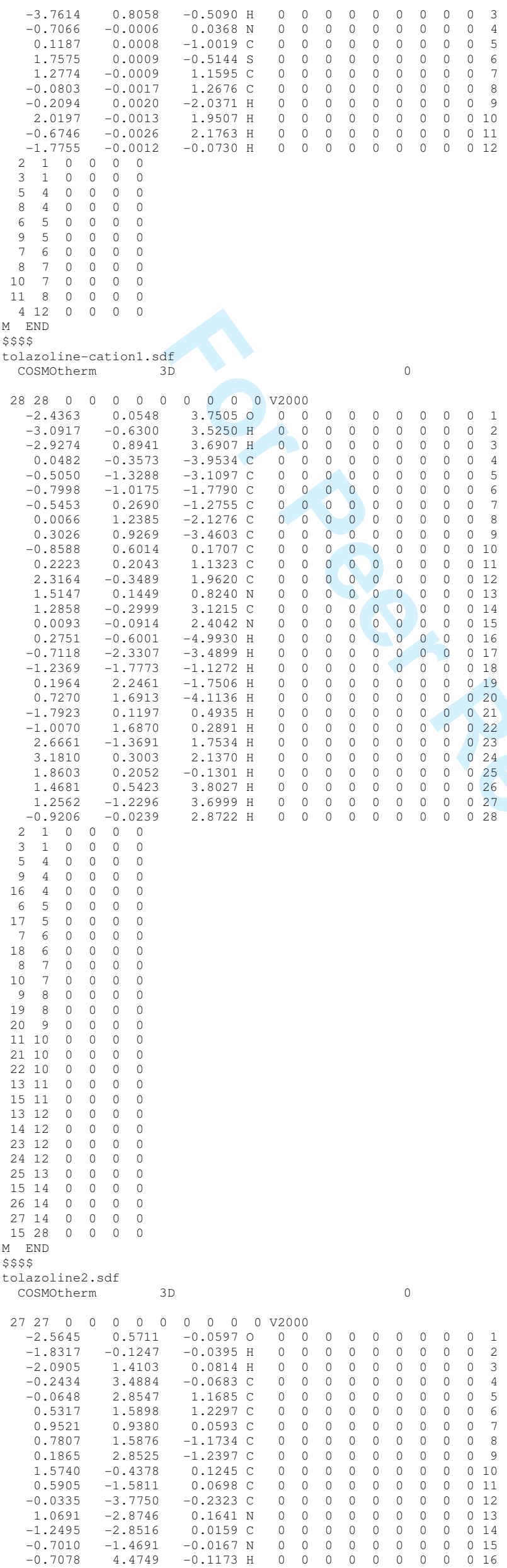




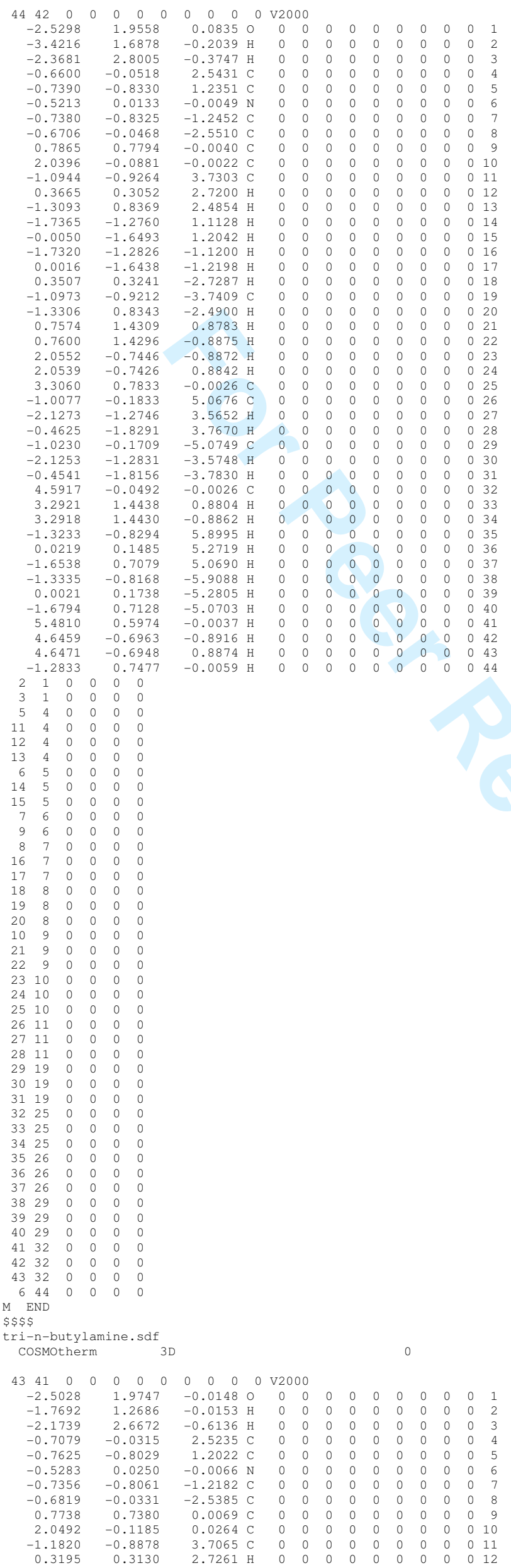


$\begin{array}{llllllllllllll}-1.3366 & 0.8714 & 2.4441 & \mathrm{H} & 0 & 0 & 0 & 0 & 0 & 0 & 0 & 0 & 0 & 13\end{array}$

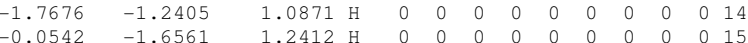

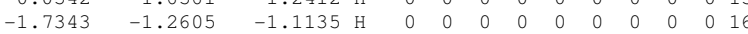

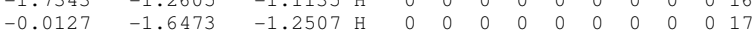

$\begin{array}{llllllllllllll}0.3354 & 0.3500 & -2.7215 & \mathrm{H} & 0 & 0 & 0 & 0 & 0 & 0 & 0 & 0 & 0 & 18\end{array}$

$\begin{array}{llllllllllllll}-1.1028 & -0.9029 & -3.7318 & \mathrm{C} & 0 & 0 & 0 & 0 & 0 & 0 & 0 & 0 & 0 & 19\end{array}$

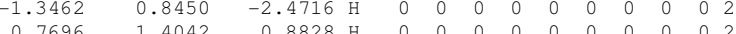

$\begin{array}{lllllllllllllll}0.7930 & 1.3983 & -0.8736 \mathrm{H} & 0 & 0 & 0 & 0 & 0 & 0 & 0 & 0 & 0 & 21\end{array}$

$\begin{array}{lllllllllllllllllll}0.7930 & 1.3983 & -0.8736 & \mathrm{H} & 0 & 0 & 0 & 0 & 0 & 0 & 0 & 0 & 0 & 22 \\ 2.0771 & -0.7789 & -0.8571 & \mathrm{H} & 0 & 0 & 0 & 0 & 0 & 0 & 0 & 0 & 0 & 23\end{array}$

$\begin{array}{llllllllllllllll}2.0500-0.7777 & 0.9112 & \mathrm{H} & 0 & 0 & 0 & 0 & 0 & 0 & 0 & 0 & 0 & 23 \\ 3.3162 & 0.7482 & 0.0452 & 0 & 0 & 0 & 0 & 0 & 0 & 0 & 0 & 0 & 0 & 24\end{array}$

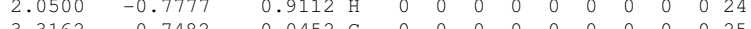

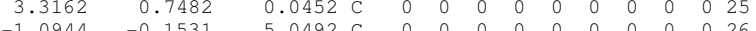

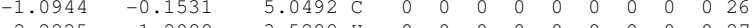

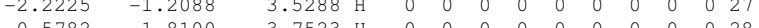

- $-1.8100-3.7523$ H -10

$\begin{array}{lllllllllllllll}-1.0311 & -0.1564 & -5.0689 & \mathrm{C} & 0 & 0 & 0 & 0 & 0 & 0 & 0 & 0 & 0 & 29\end{array}$

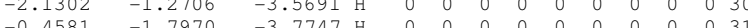

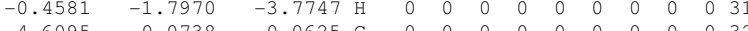

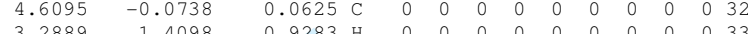

$\begin{array}{llllllllllllllllll}3.2889 & 1.4098 & 0.9283 & \mathrm{H} & 0 & 0 & 0 & 0 & 0 & 0 & 0 & 0 & 0 & 0 & 0 & 32 \\ 3.3143 & 1.4124 & -0.8365 & \mathrm{H} & 0 & 0 & 0 & 0 & 0 & 0 & 0 & 0 & 0 & 34\end{array}$

$\begin{array}{rrrrrrrrrrrrrr}-1.4374 & -0.7907 & 5.8775 & \mathrm{H} & 0 & 0 & 0 & 0 & 0 & 0 & 0 & 0 & 0 & 35\end{array}$

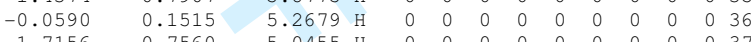

$\begin{array}{llllllllllllll}-1.7156 & 0.7560 & 5.0455 & \mathrm{H} & 0 & 0 & 0 & 0 & 0 & 0 & 0 & 0 & 0 & 37\end{array}$

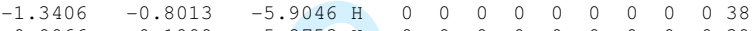

$\begin{array}{llllllllllllll}-0.0066 & 0.1909 & -5.2753 & \mathrm{H} & 0 & 0 & 0 & 0 & 0 & 0 & 0 & 0 & 0 & 39\end{array}$

$\begin{array}{llllllllllllll}-1.6880 & 0.7273 & -5.0651 & \mathrm{H} & 0 & 0 & 0 & 0 & 0 & 0 & 0 & 0 & 0 & 40\end{array}$

$\begin{array}{llllllllllll} & 0 & 0 & 0 & 0 & 0 & 0 & 0 & 0 & 0 & 41\end{array}$

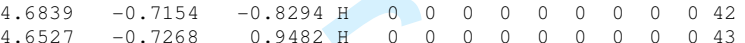

$2 \quad 1 \quad 0 \quad 0000$

$\begin{array}{llllll}3 & 1 & 0 & 0 & 0 & 0 \\ 5 & 4 & 0 & 0 & 0 & 0\end{array}$

$1 \quad 4 \quad 0 \quad 0 \quad 00$

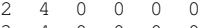

$\begin{array}{lllllll}3 & 4 & 0 & 0 & 0 & 0\end{array}$

$\begin{array}{llllll}6 & 5 & 0 & 0 & 0 & 0 \\ 4 & 5 & 0 & 0 & 0 & 0\end{array}$

$\begin{array}{llllll}5 & 5 & 0 & 0 & 0 & 0\end{array}$

$7 \begin{array}{llllll}7 & 6 & 0 & 0 & 0\end{array}$

$\begin{array}{llllll}6 & 0 & 0 & 0 & 0 & 0 \\ 7 & 0 & 0 & 0 & 0\end{array}$

$\begin{array}{lllllll}16 & 7 & 0 & 0 & 0 & 0\end{array}$

$\begin{array}{lllll}7 & 0 & 0 & 0 & 0\end{array}$

$\begin{array}{lllllll}18 & 8 & 0 & 0 & 0 & 0\end{array}$

$\begin{array}{llllll}10 & 8 & 0 & 0 & 0 & 0\end{array}$

$\begin{array}{lllllll}10 & 9 & 0 & 0 & 0 & 0\end{array}$

$\begin{array}{lllllll}21 & 9 & 0 & 0 & 0 & 0\end{array}$

$\begin{array}{llllll}22 & 9 & 0 & 0 & 0 & 0\end{array}$

$\begin{array}{llllll}23 & 10 & 0 & 0 & 0 & 0\end{array}$

$\begin{array}{llllll}24 & 10 & 0 & 0 & 0 & 0\end{array}$

$\begin{array}{lllllll}25 & 10 & 0 & 0 & 0 & 0\end{array}$

$2711 \quad 0 \quad 000$

28110000

$\begin{array}{llllll}29 & 19 & 0 & 0 & 0 & 0\end{array}$

$\begin{array}{llllll}30 & 19 & 0 & 0 & 0 & 0\end{array}$

$\begin{array}{llllll}31 & 19 & 0 & 0 & 0 & 0\end{array}$

$\begin{array}{llllll}32 & 25 & 0 & 0 & 0 & 0\end{array}$

$\begin{array}{llllll}33 & 25 & 0 & 0 & 0 & 0\end{array}$

$\begin{array}{llllll}34 & 25 & 0 & 0 & 0 & 0 \\ 35 & 26 & 0 & 0 & 0 & 0\end{array}$

$\begin{array}{llllll}36 & 26 & 0 & 0 & 0 & 0\end{array}$

$\begin{array}{llllll}37 & 26 & 0 & 0 & 0 & 0\end{array}$

$\begin{array}{llllll}38 & 29 & 0 & 0 & 0 & 0 \\ 39 & 29 & 0 & 0 & 0 & 0\end{array}$

$\begin{array}{llllll}40 & 29 & 0 & 0 & 0 & 0\end{array}$

$\begin{array}{llllll}41 & 32 & 0 & 0 & 0 & 0\end{array}$

$\begin{array}{llllll}32 & 43 & 0 & 0 & 0 & 0\end{array}$

END

\$\$\$

i-n-propylamine-cation.sdf

cosmotherm -cation

$\begin{array}{llllllllllll}35 & 33 & 0 & 0 & 0 & 0 & 0 & 0 & 0 & 0 & 0 & \mathrm{v} 2000\end{array}$

\begin{tabular}{|c|c|c|c|c|c|c|c|c|c|c|c|c|}
\hline & & 0.0832 & 0 & 0 & 0 & 0 & 0 & 0 & 0 & 0 & 0 & 0 \\
\hline-2.0645 & -2.8283 & -0.3187 & $\mathrm{H}$ & 0 & 0 & 0 & 0 & 0 & 0 & 0 & 0 & 0 \\
\hline-3.1503 & -1.7345 & -0.2629 & $\mathrm{H}$ & 0 & 0 & 0 & 0 & 0 & 0 & 0 & 0 & 0 \\
\hline-0.4455 & 0.0681 & -2.5505 & $\mathrm{C}$ & 0 & 0 & 0 & 0 & 0 & 0 & 0 & 0 & 0 \\
\hline 0.5291 & 0.8510 & -1.2431 & C & 0 & 0 & 0 & 0 & 0 & 0 & 0 & 0 & 0 \\
\hline-0.2922 & 0.0103 & -0.0031 & $\mathrm{~N}$ & 0 & 0 & 0 & 0 & 0 & 0 & 0 & 0 & 0 \\
\hline 0.5193 & 0.8544 & 1.2366 & C & 0 & 0 & 0 & 0 & 0 & 0 & 0 & 0 & 0 \\
\hline 0.427 & 0.0760 & 2.5460 & C & 0 & 0 & 0 & 0 & 0 & 0 & 0 & 0 & 0 \\
\hline 1.0291 & -0.7325 & -0.0071 & C & 0 & 0 & 0 & 0 & 0 & 0 & 0 & 0 & 0 \\
\hline 2.2673 & 0.1567 & -0.0118 & C & 0 & 0 & 0 & 0 & 0 & 0 & 0 & 0 & 0 \\
\hline-0.8908 & 0.9424 & -3.7299 & C & 0 & 0 & 0 & 0 & 0 & 0 & 0 & 0 & 0 \\
\hline 0.5826 & -0.2812 & -2.7262 & $\mathrm{H}$ & 0 & 0 & 0 & 0 & 0 & 0 & 0 & 0 & 0 \\
\hline .0864 & -0.8256 & -2.4882 & $\mathrm{H}$ & 0 & 0 & 0 & 0 & 0 & 0 & 0 & 0 & 0 \\
\hline-1.5331 & 1.2784 & -1.1175 & $\mathrm{H}$ & 0 & 0 & 0 & 0 & 0 & 0 & 0 & 0 & 0 \\
\hline 0 . & 1.6793 & -1.2194 & $\mathrm{H}$ & 0 & 0 & 0 & 0 & 0 & 0 & 0 & 0 & 0 \\
\hline-1.5233 & 1.2833 & 1.1168 & $\mathrm{H}$ & 0 & 0 & 0 & 0 & 0 & 0 & 0 & 0 & 0 \\
\hline & 1.68 & 1.204 & $\mathrm{H}$ & & & 0 & 0 & 0 & 0 & 0 & 0 & 0 \\
\hline 0.6014 & -0.2751 & 2.7149 & $\mathrm{H}$ & 0 & 0 & 0 & 0 & 0 & 0 & 0 & 0 & 0 \\
\hline 1.85 & 0.9574 & 3.7251 & $C_{1}$ & & & 0 & 0 & 0 & 0 & 0 & 0 & 0 \\
\hline-1.0714 & -0.8156 & 2.4916 & $\mathrm{H}$ & 0 & 0 & 0 & 0 & 0 & 0 & 0 & 0 & 0 \\
\hline 1.0110 & -1.3847 & -0.8894 & $\mathrm{H}$ & 0 & 0 & 0 & 0 & 0 & 0 & 0 & 0 & 0 \\
\hline 1.0164 & -1.3834 & 0.8762 & $\mathrm{H}$ & 0 & 0 & 0 & 0 & 0 & 0 & 0 & 0 & 0 \\
\hline 2.2710 & 0.8129 & 0.8721 & $\mathrm{H}$ & 0 & 0 & 0 & 0 & 0 & 0 & 0 & 0 & 0 \\
\hline 2.2663 & 0.8106 & -0.8975 & $\mathrm{H}$ & 0 & 0 & 0 & 0 & 0 & 0 & 0 & 0 & 0 \\
\hline 3.5401 & -0.7000 & -0.0138 & & 0 & 0 & 0 & 0 & 0 & 0 & 0 & 0 & 0 \\
\hline .8191 & 0.3808 & -4.6713 & $\mathrm{H}$ & 0 & 0 & 0 & 0 & 0 & 0 & 0 & 0 & \\
\hline
\end{tabular}

URL: http://mc.manuscriptcentral.com/tandf/tmph 


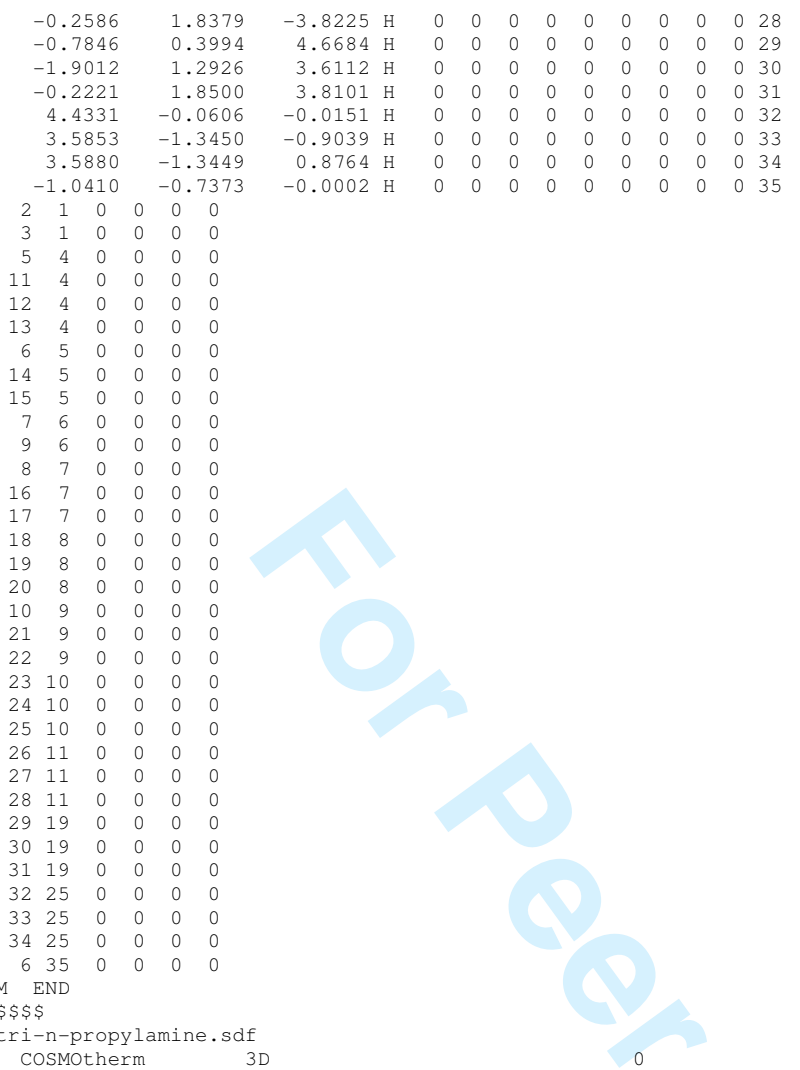

cosmotherm

\begin{tabular}{|c|c|c|c|c|c|c|c|c|c|c|c|c|c|}
\hline $\begin{array}{lll}34 & 32 & 0\end{array}$ & $\begin{array}{lll}0 & 0 & 0\end{array}$ & $\begin{array}{lll}0 & 0 & 0\end{array}$ & 0 & $\mathrm{~V} 20 \mathrm{C}$ & & & & & & & & & \\
\hline 2.2250 & 2.0015 & -0.0034 & 0 & 0 & 0 & 0 & 0 & 0 & 0 & 0 & 0 & 0 & 1 \\
\hline 1.5075 & 1.2794 & -0.0067 & $\mathrm{H}$ & 0 & 0 & 0 & 0 & 0 & 0 & 0 & 0 & 0 & 2 \\
\hline 1.8697 & 2.6991 & -0.5807 & $\mathrm{H}$ & 0 & 0 & 0 & 0 & 0 & 0 & 0 & 0 & 0 & 3 \\
\hline 0.4769 & -0.0468 & -2.5309 & C & 0 & 0 & 0 & 0 & 0 & 0 & 0 & 0 & 0 & \\
\hline 0.5365 & -0.8203 & -1.2109 & C & 0 & 0 & 0 & 0 & 0 & 0 & 0 & 0 & 0 & \\
\hline 0.3007 & 0.0037 & 0.0000 & $\mathrm{~N}$ & 0 & 0 & 0 & 0 & 0 & 0 & 0 & 0 & 0 & \\
\hline 0.5528 & -0.8161 & 1.2105 & $\mathrm{C}$ & 0 & 0 & 0 & 0 & 0 & 0 & 0 & 0 & 0 & \\
\hline 0.4812 & -0.0439 & 2.5310 & $\mathrm{C}$ & 0 & 0 & 0 & 0 & 0 & 0 & 0 & 0 & 0 & \\
\hline-1.0194 & 0.6827 & 0.0077 & $\mathrm{C}$ & 0 & 0 & 0 & 0 & 0 & 0 & 0 & 0 & 0 & \\
\hline-2.2729 & -0.2063 & 0.0159 & $\mathrm{C}$ & 0 & 0 & 0 & 0 & 0 & 0 & 0 & 0 & 0 & 10 \\
\hline 0.9101 & -0.9197 & -3.7149 & $\mathrm{C}$ & 0 & 0 & 0 & 0 & 0 & 0 & 0 & 0 & 0 & \\
\hline-0.5432 & 0.3259 & -2.7130 & $\mathrm{H}$ & 0 & 0 & 0 & 0 & 0 & 0 & 0 & 0 & 0 & 12 \\
\hline 1.1311 & 0.8372 & -2.4589 & $\mathrm{H}$ & 0 & 0 & 0 & 0 & 0 & 0 & 0 & 0 & 0 & 13 \\
\hline 1.5444 & -1.2522 & -1.1002 & $\mathrm{H}$ & 0 & 0 & 0 & 0 & 0 & 0 & 0 & 0 & 0 & 14 \\
\hline-0.1663 & -1.6780 & -1.2516 & $\mathrm{H}$ & 0 & 0 & 0 & 0 & 0 & 0 & 0 & 0 & 0 & 15 \\
\hline 1.5679 & -1.2308 & 1.0975 & $\mathrm{H}$ & 0 & 0 & 0 & 0 & 0 & 0 & 0 & 0 & 0 & 16 \\
\hline-0.1350 & -1.6857 & 1.2523 & $\mathrm{H}$ & 0 & 0 & 0 & 0 & 0 & 0 & 0 & 0 & 0 & 17 \\
\hline-0.5509 & 0.2870 & 2.7260 & $\mathrm{H}$ & 0 & 0 & 0 & 0 & 0 & 0 & 0 & 0 & 0 & 18 \\
\hline 0.9630 & -0.8996 & 3.7086 & $\mathrm{C}$ & 0 & 0 & 0 & 0 & 0 & 0 & 0 & 0 & 0 & 19 \\
\hline 1.0973 & 0.8661 & 2.4504 & $\mathrm{H}$ & 0 & 0 & 0 & 0 & 0 & 0 & 0 & 0 & 0 & 20 \\
\hline-1.0517 & 1.3454 & -0.8707 & $\mathrm{H}$ & 0 & 0 & 0 & 0 & 0 & 0 & 0 & 0 & 0 & 21 \\
\hline-1.0395 & 1.3467 & 0.8853 & $\mathrm{H}$ & 0 & 0 & 0 & 0 & 0 & 0 & 0 & 0 & 0 & 22 \\
\hline-2.2610 & -0.8672 & 0.8978 & $\mathrm{H}$ & 0 & 0 & 0 & 0 & 0 & 0 & 0 & 0 & 0 & 23 \\
\hline-2.2751 & -0.8636 & -0.8688 & $\mathrm{H}$ & 0 & 0 & 0 & 0 & 0 & 0 & 0 & 0 & 0 & 24 \\
\hline-3.5536 & 0.6365 & 0.0274 & $\mathrm{C}$ & 0 & 0 & 0 & 0 & 0 & 0 & 0 & 0 & 0 & 25 \\
\hline 0.8576 & -0.3585 & -4.6589 & $\mathrm{H}$ & 0 & 0 & 0 & 0 & 0 & 0 & 0 & 0 & 0 & 26 \\
\hline 1.9450 & -1.2744 & -3.5914 & $\mathrm{H}$ & 0 & 0 & 0 & 0 & 0 & 0 & 0 & 0 & 0 & 27 \\
\hline 0.2624 & -1.8042 & -3.8153 & $\mathrm{H}$ & 0 & 0 & 0 & 0 & 0 & 0 & 0 & 0 & 0 & 28 \\
\hline 0.8916 & -0.3449 & 4.6552 & $\mathrm{H}$ & 0 & 0 & 0 & 0 & 0 & 0 & 0 & 0 & 0 & 29 \\
\hline 2.0122 & -1.2059 & 3.5763 & $\mathrm{H}$ & 0 & 0 & 0 & 0 & 0 & 0 & 0 & 0 & 0 & \\
\hline 0.3577 & -1.8137 & 3.8106 & $\mathrm{H}$ & 0 & 0 & 0 & 0 & 0 & 0 & 0 & 0 & 0 & 1 \\
\hline-4.4506 & 0.0006 & 0.0287 & $\mathrm{H}$ & 0 & 0 & 0 & 0 & 0 & 0 & 0 & 0 & 0 & \\
\hline-3.6080 & 1.2895 & -0.8578 & $\mathrm{H}$ & 0 & 0 & 0 & 0 & 0 & 0 & 0 & 0 & 0 & 3 \\
\hline-3.5985 & 1.2799 & 0.9200 & $\mathrm{H}$ & 0 & 0 & 0 & 0 & 0 & 0 & 0 & 0 & 0 & 4 \\
\hline
\end{tabular}

$\begin{array}{llllll}2 & 1 & 0 & 0 & 0 & 0\end{array}$

$\begin{array}{llllll}3 & 1 & 0 & 0 & 0 \\ 5 & 4 & 0 & 0 & 0\end{array}$

$\begin{array}{lllll}11 & 4 & 0 & 0 & 0\end{array}$

$\begin{array}{llllll}12 & 4 & 0 & 0 & 0 & 0 \\ 13 & 4 & 0 & 0 & 0 & 0\end{array}$

$\begin{array}{cccccccc}6 & 5 & 0 & 0 & 0 & 0 & 1 \\ 6 & 5 & 0 & 0 & 0 & 0\end{array}$

$\begin{array}{llllll}14 & 5 & 0 & 0 & 0\end{array}$

$\begin{array}{ccccc}15 & 5 & 0 & 0 & 0 \\ 7 & 6 & 0 & 0 & 0\end{array}$

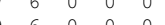

$\begin{array}{llllll}8 & 7 & 0 & 0 & 0\end{array}$

$\begin{array}{llllll}16 & 7 & 0 & 0 & 0 & 0 \\ 17 & 7 & 0 & 0 & 0 & 0\end{array}$

$\begin{array}{lllllll}8 & 8 & 0 & 0 & 0 & 0\end{array}$

$\begin{array}{llllll}9 & 8 & 0 & 0 & 0 & 0 \\ 20 & 8 & 0 & 0 & 0 & 0\end{array}$

$\begin{array}{llllll}10 & 9 & 0 & 0 & 0 & 0\end{array}$

$\begin{array}{lllllll}21 & 9 & 0 & 0 & 0 & 0 & 0 \\ 22 & 9 & 0 & 0 & 0 & 0\end{array}$

$\begin{array}{lllll}23 & 10 & 0 & 0 & 0\end{array}$

$\begin{array}{llllll}24 & 10 & 0 & 0 & 0\end{array}$

$\begin{array}{llllll}25 & 10 & 0 & 0 & 0 & 0 \\ 26 & 11 & 0 & 0 & 0 & 0\end{array}$ 
$\begin{array}{llllll}27 & 11 & 0 & 0 & 0 & 0\end{array}$

$\begin{array}{llllll}28 & 11 & 0 & 0 & 0 & 0 \\ 29 & 19 & 0 & 0 & 0 & 0 \\ 30 & 19 & 0 & 0 & 0 & 0\end{array}$

$\begin{array}{llllll}29 & 19 & 0 & 0 & 0 & 0 \\ 30 & 19 & 0 & 0 & 0 & 0\end{array}$

$\begin{array}{llllll}31 & 19 & 0 & 0 & 0 & 0\end{array}$

$\begin{array}{llllll}32 & 25 & 0 & 0 & 0 & 0 \\ 33 & 25 & 0 & 0 & 0 & 0\end{array}$

$\begin{array}{lllllll}25 & 34 & 0 & 0 & 0 & 0\end{array}$

$\mathrm{M}$ END

triethylamine-cation.sdf

cosmotherm

$\begin{array}{llllllllllll}26 & 24 & 0 & 0 & 0 & 0 & 0 & 0 & 0 & 0 & 0 & \mathrm{v} 2000\end{array}$

\begin{tabular}{|c|c|c|c|c|c|c|c|c|c|c|c|c|}
\hline & -0.7412 & -2.6475 & 0 & 0 & 0 & 0 & 0 & 0 & 0 & 0 & & 0 \\
\hline 0.2544 & -1.6840 & -2.7985 & $\mathrm{H}$ & 0 & 0 & 0 & 0 & 0 & 0 & 0 & & 0 \\
\hline 0.6327 & -0.2681 & -3.2778 & $\mathrm{H}$ & 0 & 0 & 0 & 0 & 0 & 0 & 0 & 0 & \\
\hline 2.5361 & 0.2823 & -0.0242 & C & & & 0 & 0 & 0 & 0 & 0 & 0 & \\
\hline 1.2300 & 1.0338 & 0.1831 & C & & c & 0 & 0 & 0 & 0 & 0 & 0 & \\
\hline-0.0041 & 0.1712 & -0.0018 & $\mathrm{~N}$ & & & 0 & 0 & 0 & 0 & 0 & 0 & \\
\hline & 1.0290 & 0.1025 & & & & 0 & 0 & 0 & 0 & 0 & & \\
\hline 357 & 0.2755 & -0.2036 & C & & & 0 & 0 & 0 & 0 & 0 & 0 & \\
\hline & -1.0579 & 0.8877 & & & & 0 & 0 & 0 & 0 & 0 & & \\
\hline 0.0723 & -0.7540 & 2.3762 & C & & & 0 & 0 & 0 & 0 & 0 & 0 & \\
\hline 3.3567 & 1.0123 & -0.0033 & $\mathrm{H}$ & & & 0 & 0 & 0 & 0 & 0 & 0 & \\
\hline 2.7304 & -0.4555 & 0.7645 & $\mathrm{H}$ & & 0 & 0 & 0 & 0 & 0 & 0 & 0 & \\
\hline 2.5628 & -0.22 & -0.9997 & $\mathrm{H}$ & & & & & 0 & 0 & 0 & & \\
\hline 1.1282 & & -0 . & $\mathrm{H}$ & c & 0 & 0 & 0 & 0 & 0 & 0 & 0 & \\
\hline 1.1684 & 1.4769 & 1.1849 & $\mathrm{H}$ & 0 & 0 & & 0 & 0 & 0 & 0 & & \\
\hline-1.1002 & 1.84 & -0.6137 & $\mathrm{H}$ & 0 & 0 & 0 & 0 & 0 & 0 & 0 & 0 & \\
\hline-1.2608 & 1.4641 & 1.1096 & $\mathrm{H}$ & & & & 0 & 0 & 0 & 0 & 0 & \\
\hline & & -0.2 & & & & 0 & 0 & 0 & & & & \\
\hline-2.7826 & -0.4699 & 0.5629 & & & & 0 & 0 & 0 & 0 & 0 & 0 & \\
\hline-2.4910 & -0.2201 & -1.1838 & & & 0 & 0 & 0 & 0 & & & & \\
\hline 0.8591 & -1.6422 & 0.6249 & & 0 & 0 & 0 & 0 & 0 & 0 & 0 & 0 & 0 \\
\hline & -1.6410 & 0.5752 & & & & 0 & 0 & 0 & 0 & 0 & & \\
\hline .0854 & -1.7098 & 2.9168 & & 0 & c & 0 & 0 & 0 & 0 & 0 & 0 & 0 \\
\hline & -0.19 & 2.6658 & & & & & & 0 & 0 & 0 & 0 & \\
\hline & -0.1948 & 2.7150 & & 0 & & 0 & 0 & 0 & 0 & 0 & 0 & \\
\hline & & & & & & & & & & & & \\
\hline
\end{tabular}

$\begin{array}{ll}0.0303 & -0.1919\end{array}$

$\begin{array}{lllllllll}3 & 1 & 0 & 0 & 0\end{array}$

$\begin{array}{llllllll}5 & 4 & 0 & 0 & 0 & 0\end{array}$

$\begin{array}{lllllll}1 & 4 & 0 & 0 & 0 & 0\end{array}$

$\begin{array}{llllll}2 & 4 & 0 & 0 & 0 & 0\end{array}$

$\begin{array}{llllll}3 & 4 & 0 & 0 & 0 & 0\end{array}$

$\begin{array}{lllllll}4 & 5 & 0 & 0 & 0 & 0\end{array}$

$\begin{array}{lllll}5 & 0 & 0 & 0\end{array}$

$\begin{array}{llllll}9 & 6 & 0 & 0 & 0 & 0\end{array}$

$\begin{array}{lllll}7 & 0 & 0 & 0 & 0\end{array}$

$\begin{array}{llllll}8 & 7 & 0 & 0 & 0\end{array}$

$\begin{array}{lllllll}17 & 7 & 0 & 0 & 0 & 0 \\ 18 & 8 & 0 & 0 & 0 & 0\end{array}$

$\begin{array}{llllllll}9 & 8 & 0 & 0 & 0 & 0 & 0 & 2\end{array}$

$\begin{array}{lllllll}21 & 9 & 0 & 0 & 0 & 0\end{array}$

$\begin{array}{lllllll}10 & 9 & 0 & 0 & 0 & 0\end{array}$

$\begin{array}{llllll}3 & 10 & 0 & 0 & 0 & 0\end{array}$

$2410 \quad 000000$

$\begin{array}{llllll}25 & 10 & 0 & 0 & 0 & 0\end{array}$

END

$\$ \$ \$$

triethylamine.sdf

cosmotherm

$\begin{array}{llllllllllll}25 & 23 & 0 & 0 & 0 & 0 & 0 & 0 & 0 & 0 & 0 & \mathrm{~V} 2000\end{array}$

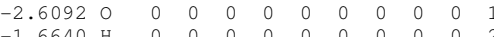

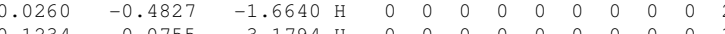

$0.1234-0.0755$

$\begin{array}{lllllllllllllll}2.5205 & 0.2663 & -0.1288 & \mathrm{c} & 0 & 0 & 0 & 0 & 0 & 0 & 0 & 0 & 0 & 3 \\ 1.2068 & 1.0075 & 0.1135 & \mathrm{c} & 0 & 0 & 0 & 0 & 0 & 0 & 0 & 0 & 0 & 4\end{array}$

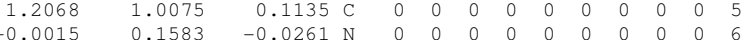

$\begin{array}{rrrrrrrrrrrrrrrrr}-0.0015 & 0.1583 & -0.0261 & N & 0 & 0 & 0 & 0 & 0 & 0 & 0 & 0 & 0 & 5 \\ -1.2148 & 1.0053 & 0.0772 & C & 0 & 0 & 0 & 0 & 0 & 0 & 0 & 0 & 0 & 7\end{array}$

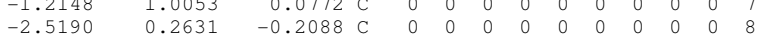

$\begin{array}{lrrrrrrrrrrrrrr}-2.5190 & 0.2631 & -0.2088 & \mathrm{C} & 0 & 0 & 0 & 0 & 0 & 0 & 0 & 0 & 0 & 0 & 8 \\ -0.0142 & -1.0061 & 0.8956 & \mathrm{C} & 0 & 0 & 0 & 0 & 0 & 0 & 0 & 0 & 0 & 9\end{array}$

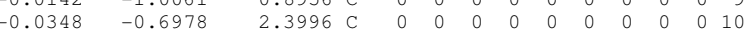

$\begin{array}{llllllllllllll}3.3473 & 0.9908 & -0.1564 \mathrm{H} & 0 & 0 & 0 & 0 & 0 & 0 & 0 & 0 & 0 & 11\end{array}$

$\begin{array}{lllllllllllll}2.7462 & -0.4595 & 0.6646 \mathrm{H} & 0 & 0 & 0 & 0 & 0 & 0 & 0 & 0 & 0 & 1\end{array}$

$\begin{array}{lllllllllllllll}2.4995 & -0.2660 & -1.0912 \mathrm{H} & 0 & 0 & 0 & 0 & 0 & 0 & 0 & 0 & 0 & 13\end{array}$

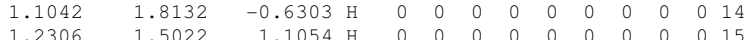

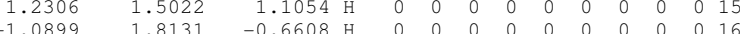

$\begin{array}{rrrrrrrrrrrrrr}1.2709 & 1.4972 & 1.0692 & \mathrm{H} & 0 & 0 & 0 & 0 & 0 & 0 & 0 & 0 & 0 & 17\end{array}$

$\begin{array}{llllllllllllll}-1.2709 & 0.99863 & -0.2580 \mathrm{H} & 0 & 0 & 0 & 0 & 0 & 0 & 0 & 0 & 0 & 18\end{array}$

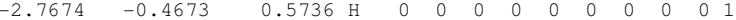

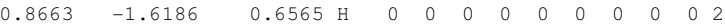

$\begin{array}{llllllllllllll}-0.0435 & -1.6419 & 2.9635 & \mathrm{H} & 0 & 0 & 0 & 0 & 0 & 0 & 0 & 0 & 0 & 23\end{array}$ $0.8513-0.1293$

$\begin{array}{llllll}2 & 1 & 0 & 0 & 0 & 0 \\ 3 & 1 & 0 & 0 & 0 & 0\end{array}$

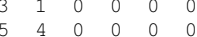

$\begin{array}{lllllll}11 & 4 & 0 & 0 & 0 & 0\end{array}$

$\begin{array}{lllllllll}13 & 4 & 0 & 0 & 0\end{array}$

$6 \begin{array}{llllll}6 & 5 & 0 & 0 & 0 & 0\end{array}$

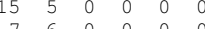




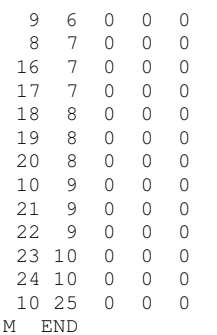

